

\section{DISCLAIMER}

This report was prepared as an account of work sponsored by an agency of the United States Government. Neither the United States Government nor any agency Thereof, nor any of their employees, makes any warranty, express or implied, or assumes any legal liability or responsibility for the accuracy, completeness, or usefulness of any information, apparatus, product, or process disclosed, or represents that its use would not infringe privately owned rights. Reference herein to any specific commercial product, process, or service by trade name, trademark, manufacturer, or otherwise does not necessarily constitute or imply its endorsement, recommendation, or favoring by the United States Government or any agency thereof. The views and opinions of authors expressed herein do not necessarily state or reflect those of the United States Government or any agency thereof. 


\section{DISCLAIMER}

Portions of this document may be illegible in electronic image products. Images are produced from the best available original document. 
PART I

(Physics-Nuclear - TID-4500)

\title{
THE BIBLIOGRAPHY OF
}

\section{INTEGRAL CHARGED PARTICLE NUCLEAR DATA}

\author{
ARCHIVAL EDITION \\ - Literature scanned from January 1, 1976, through March 15, 1980 \\ Index of data in the international exchange format (EXFOR) received between \\ January 1, 1976, and March 15, 1980
}

\section{T.W. BURROWS AND P. DEMPSEY}

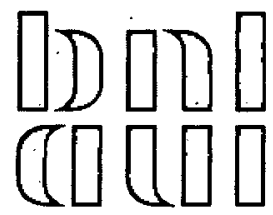

March 1980

\section{NATIONAL NUCLEAR DATA CENTER}

(in support of the International Nuclear Reaction Data Centers Network)

\section{BROOKHAVEN NATIONAL LABORATORY}

ASSOCIATED UNIVERSITIES, INC.

UNDER CONTRACT NO. DE-AC02-76CH00016 WITH THE

UNITED STATES DEPARTMENT OF ENERGY 


\section{DISCLAIMER}

This book was prepared as an account of work sponsored by an agency of the United States Government. Neither the United States Government nor any agency thereof, nor any of their employees, makes any warranty, express or implied, or assumes any legal liability or responsibility for the accuracy, completeness, or usefulness of any information, apparatus, product, or process disclosed, or represents that its use would not infringe privately owned rights. Reference herein to any specific commercial product, process, or service by trade name, trademark, manufacturer, or otherwise, does not necessarily constitute or imply its endorsement, recommendation, or favoring by the United States Government or any agency thereof. The views and opinions of authors expressed herein do not necessarily state or reflect those of the United States Government or any agency thereof.

\section{Printed in the United States of America}

Available from

National Technical Information Service

U.S. Department of Commerce

5285 Port Royal Road

Springfield, VA 22161

Price: Printed Copy $\$ 19.00$; Microfiche $\$ 3.00$ 
THE BIBLIOGRAPHY OF INTEGRAL CHARGEO PARTICLE NUCLEAR DATA, FOURTH EOITION Table of Contents

Toble of Contents

Part 1

Introduction

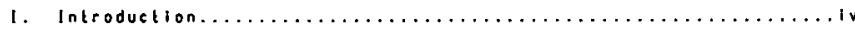

II. Scope........................................ iv

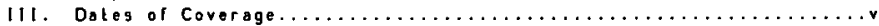

Iv. Secondary Sources of information...............................

V. Organizallon............................................

VI. Campliations and Evaluations..............................

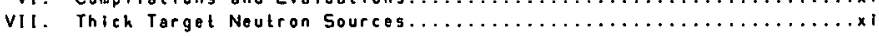

VIII. Methods of Radiolsolope Production........................

Ix. Retrieval Capobilities............................

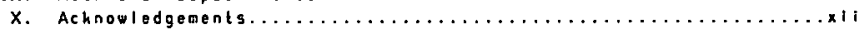

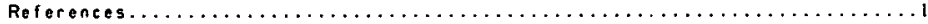

Tobles

1. Other Nuclear Dato Bibliogrophic Sources.................... iv

2. Centers Participating in the Compilation and Exchange

of Charged-Particle Nuclear Data...............................

3. 'Core' Journal Coverage.............................

4. Selected Evaluations. Reviems, and Compilations of

Charged-Partlale Nuclear Data............................

5. Some Recent Reviews of Thick-Target Neutron Yields..............

Figures

1. Description of References Section....................

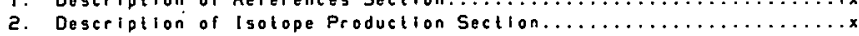

Part 2

Introduction

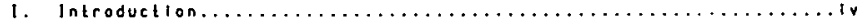

II. Scope.................................

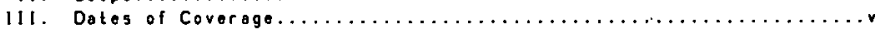

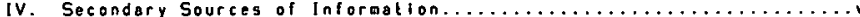

$\checkmark$ Organizotion.................................

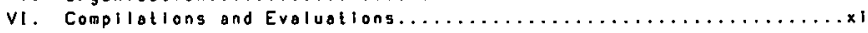

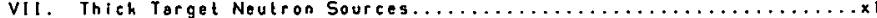

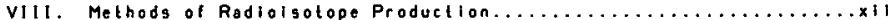

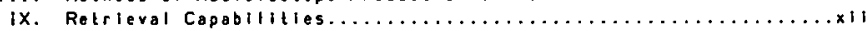

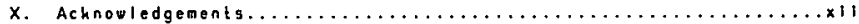

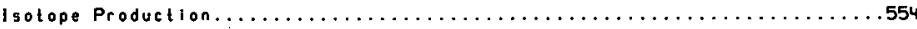

Tables

1. Othor Nuclear Data Btbliographic Sources.................

2. Centers Participating in the Compilation and Exchange

of Charged-Particle Nuclear Data......................

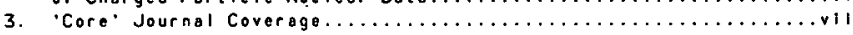

4. Selected Evaluatlons. Revieos, and Compilations of

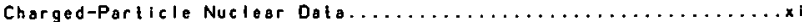

5. Some Recent Reviews of Thich-Target Neutron Yields...........

Figures

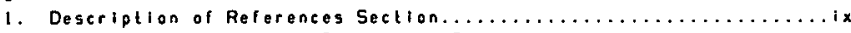

2. Desertiption of lsotope Production Section...................

Appendices

A. Abbreviations for Molecules and Mixtures...................

B. References

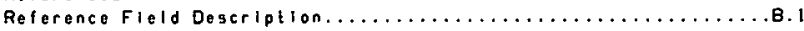

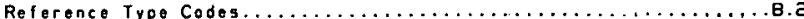

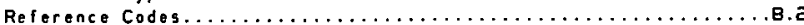

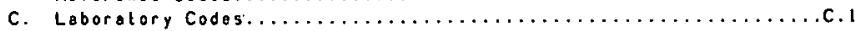

D. Some Abbrevtations Used in Comments .....................

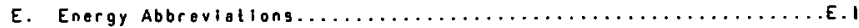

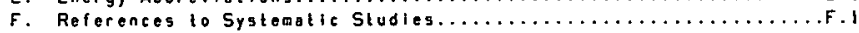


THE BIBLIOGRAPHY OF INTEGRAL CHARGED PARTICLE NUCLEAR DATA. FOURTH EOITION Introduction

\section{INTROOUCTION}

This publication is the fourth annual edition of the National Nuclear Data Center charged-particle blbliography. This edition is cumulative and supersedes the previous editions. The original publication (BNL-NCS-50640) was an outgrouth of a recomendation by the newly-formed international charged-Parlicle Nuclear Data Compilation Network (see Table 2 for members ff the neiwork). The blbliographys primary aims are to satisfy the need expressed by the network for a concise and comprehenstye index of integral charged-particle cross section dala and to provide an index of charged-particle data compiled in the international exchange format. EXFOR. The reader is referred to Table l for o partial list of other nuclear data bibliographies relevant to charged-porticle induced reactions and to A Source List of Nuclear Data Bibllographies. Compllationg, and Evaluationst for a more comprehensive list.

The rapid growth in the size of this publication has forced a change in philosophy. The present publication is an archival volume coverting the first four years of scanning. Future publications will be cumulative supplements to the Fourth Edition.

\section{Toblo 1}

Other Nuclear Dato Bibliographic Sources

1. H.B. Eobanh, el ale Nuclear Structure References. 1969-1974.

Nuc1. Data Sheets 16. Supplement. Academic Press. NY (1975).

'Recent References. 3 lssues/year ith last issue cumulative for year in Nucl. Data Sheets. Academic Press, NY.

2. INIS Alomindex. International Atomic Energy Agency, Vienno.

3. F.K. McGowan and W.T. Milner. Reaction List for Charged-Particle-laduced Reactiong. At. Data Nucl. Data Reprints 2. Academic Press. NY (1974). Nuc 1. Data Tables All. 1. Academic Press. NY. (1972).

At. Data Nucl. Data Tables 12, 499. Acadenlc Pres5. NY 11973).

At. Data Nucl. Data Tables 15, 189. Academic Press, NY 119751

At. Data Nuc1. Data Tables 18.1. Academic Press. NY (1976).

\section{1]. SCOPE}

For the purposes of this bibliography, integral charged particle data may be defined as data which have been integrated over the product angle and energy or dota which may be essily converted lo such a representation.

General criteria for inclusion of data in this bibliography are the following.

1. Excitation functions. thich target. or product yields leading lo the formation of ground or metastable states. This includes such quantilies as fission yieles, isomeric ratios, multiplicilies. and excitation functions for specific particle groups where such dato readily yields information on the excitation functions or thich larget yields for ground or metastable states.

2. The atomic mass and charge of the incident particle must be greater than or equal to 1 .

3. The atomic mass of the target must be greater than or equal to 1.

1. T.W. Burrows and N.E. Holden. A Source List of Nuclear Data Bibliographies. Compilations, and Evaluations. Second Edition." Brookhaven National Laboratory Report BNL-NC5-50702. Secand Edition (1978).

- Thich largel yields are defined as measurements on largets whose thickness is sufficient to degrade the incident particle energy below the reaction threshold. The term product yield is used when in the compilers opinion there is an ambigully in what quantity has been measured. 
THE BIBLIOGRAPHY OF INTEGRAL CHARGED PARTICLE NUCLEAR DATA, FOURTH EDITION Scope

4. The atomic masses of the outgoing and residual nuclei must be greater than or equal to 1 with the exception of processes which do not lead to a definite residual nucleus (eqe lotal crass sections) and of gamma-ray production reactions.

There may be several exceptions to these criteria. Any charged-particle data complied in the EXFOR format are indexed along oith the pertinent references. If a reference includes data salisfying the criteria noled above and additional data satisfyting oll the crileria except energy. these additional data are also indexed.

\section{DATES OF COVERAGE}

For those journals included in the bibliography of the Charged Particle Data Group, Oah Ridge National Laboratory lsee entry 3 of iable l for a cumalative list of their bibliographyl, the present coverage began with the first journal issue after their nominal cui-off date of January, 1976. Coverage of all other journals began with the first lssue bearing a 1976 cover date. The cut-off date for the present edition is March 15. 1980. All other types of references which came to our at lention beteen January 1. 1976. and March 15. 1980, have been scanned.

In addition. all charged-particte data compiled in the EXFOR format have been indexed olong with the pertinent references. Prior work which is related directly to a current paper and which has been cited by the authors has also been included.

Table 3 lists the coverage of 27 'core journals to give a representative sample of the present coverage. Appendix b lists all references contained in this publicalion. Other references have been scanned without finding pertinent entries. A complete list of the coverage may be obtained from the authors.

\section{SECONDARY SOURCES OF INFORMATION}

Since this bibllography is designed to serve both as an archivel and a current-awareness bibliography, abstracts, theses. progress reports, elc. have been included. However, they ore present in this edition only if they have not been superceded by a primary source such as a journal or conference paper. Nevertheless, all references scanned which satisfy the criteria of sections ll and IIl are indexed in the computerized bibliographic file and ma be retrleved on request (see section $1 \times$ for retrieval details). 
THE BIBLIOGRAPHY OF INTEGRAL CHARGED PARTICLE NUCLEAR DATA, FOURTH EOITION

Centers Participating in the Compliation and Exchinge of Charged-Particle Nuclear Da

Toble 2

Centers Porticipating in the Compilation and Exchange

of Charged-Particla Nuclear Dala.

\section{Conter Code}

\section{Canter}

A. CAJad Dr. F.E. Chuhreev

Center for Nuclear Structure and Reaction Data of the USSR State Comitie on the Utilization of Atomic Energy Moscow, USSR

(Hondleg disgemination for the Union of Soviet Socialigt

Republics and Eastern Europel

B. KoChapag Prof. H. Munzel

Charged Particle Nuclear Data Group

Institut fur Radiochemle

Kernforschungzentrum Karlsruhe

Pastfoche 3640

D-7500 Karlspuhe. Federal Republic of Germany

c. NNDC

Dr. S. Pearlstein

National Nucloar Data Center

Brookhoven National Laboratory

Upton. NY 11973, USA

Hondles dissemination for the United States of America and

Conadal

D. NDS Dr. J.J. Schmidt

Nuclear Data Section

IAEA. P.0.B. 590

Karninerring II

1011 Vienns, Austria

(A) countries not covered by centers A, C, G, or I should contact NDS for intormation)

E.

Prof. Hajime Tanoha

Department of Phystcs

Hokkoldo Universily

Sopporo. Hohtoldo. Japan

F. Harwell Dr. G. Dearnaley

Nuctear Physic Division. Hanger 8

Atomic Research Estoblishoent

Harwell, Didcot, Oxll ORA

United KIngdom

G. F $1 Z$

Dr. H. Behrens

Fachinformationszentrun

7514 Eggenstein-Lecpoldshofen 2

Federal Republic of Germony

(Handles dissemination for the Federat Ropublic of Germany)

H. IBJ

Dr. A. Marcinhowski

Nuclear Dalo Group

Inst. Baden Jadrowych

Hoz 69

PL-00-68! Warsaw. Poland

I. NEADB Dr. N. Tubbs

Nuclear Energy Agency Data Bonk

B.P. No. 9

F-9IIgo Gif-gur-YYolto

France

thandles disseginotlon for Nuclear Energy Agency countries

other than USA. Canada. and FRG 
THE BIBL IOGRAPHY OF INTEGRAL CHARGED PARTICLE NUCLEAR DATA. FOURTH.EDITION "Core. Journal Coverage

\section{Toble 3}

'Core' Journal Coverage

\section{Journal}

Anneles de Physique (Parls)

Annals of Physlics (New Yorh)

Australian Journal of Physics

Bulletin of the Acedeny of Sciences of the USSR,

Physical Series

Canadian Journal of Chemistry

Canadian Journal of Physles

Comples Rendus Hepdomadalres des Seances de

1 Academie des Sclences. Series B. Physique

Helvetia Physica Acta

International Journal of Applied Radiation and

Isotopes

lizestia Ahademit Nouk SSSR. Serlya Fizlcheskaya

Journal of lnorganic and Nuclear Chemistry

Journal of the Physical Society of Japan

Journal of Physies $G$

Lotiere oll Nuovo Cimento. Ser: 2

Nuclear Instruments and Methods

Nuctear Physies A

Nuovo Cimento A

Physica Scripta

Physical Revlea C

Physical Revied Letters

Physics Letters $B$

Revue Roumaine de Physique

Soviet Atomic Energy

Soviet Journal of Nuciear Physics.

Uhrains'kit Flzichnil Zhurnal

Yadernaya Fizika"

Zeltschrift Iver Physik A
First Issue Vol (issue)

$9(4)$

$95(2)$

59(1)

$39(5)$

$53(24)$

$53(23)$

$57(23)$

$58(2)$

281 (5)

289(12)

$47(2)$

$52(2)$

$27(1)$

$39(5)$

$30(12)$

431111

$38(2) \quad 42(1)$

$40(1) \quad 47(6)$

(1) 5(12)

$14(14) \quad 26(18)$

$131(1) \quad 167(3)$

ट55(1) $334(2)$

$30(4) \quad 54(2)$

$12(6) \quad 20161$

$13(2) \quad 2113$

$36(7) \quad 44(9)$

$60(2) \quad 89(4)$

$20(10) \quad 24(9)$

$39(3) \quad 46(6)$

$21(6) \quad 29(5)$

$20(10) \quad 24110$

$21(6) \quad 30(9)$

$275(4) \quad 294(1)$

- The original publications were also scanned. Translations are normally issued six months after the original. 
THE BIBL IOGRAPHY OF INTEGRAL CHARGED PARTICLE NUCLEAR DATA, FOURTH EDITION Organization

\section{ORGANIZATION}

This bibliography is divided into two sections. References and lsolope Production. The References section containg alt references salisfying the criteria noted above and is organized as descrlbed in the next paragraph. The Isolope Production gection contains an abroviated referen:e line for a il entries which contoin information on a deitinte residual nuzleus, on particle production, or on mass. charge, or isotoplc distribulions ond is described oelow. Entries in the Reforences section are sequentially numbered. These sequence numbers serve as a link between the two sections.

The References section is ordered by increasing $Z$ and $A$ of the incldent particle: then by increasing $Z$ and $A$ of the target. Within this order. the entrieg are ordered by residual nucleus and outgoing particles or processes; then by quantity (eq. cross section). All entries having the same reaction ore grouped under a common heading. All onteles relating to the same experiment are blocked logether under the same sequence number. Within a bloch the entries are ordered by inverse publication dete with the exception of the matn reference which ig always first and translations and data index lines which ore alway last. The blocks ore ordered by increasing mintmum energy. The reader ls referred to figure l for a detalled desciption of this section.

The Isotope Production section is limited in the information it contatins. Only those references which include data on definite residual nucteus, particle production, or mass. charge, or tsotople distrlbutions are included. The information included ore the isotope produced, quantity, target, incident particle. energy range, laboratory code. first outhor of the first reference in the block. sequence number. and data flag. The sequence number refers lo the bloch in the Reference section where complete blbliographle inforation as be found. The presence of a data flag indicates that the block contains a data index line. The entrleg are ordered by increasing $Z$ and $A$ of the product oilh nass distributions preceding all other entries and charge digtribution preceding the isotope production for that charge. Whthin this grouping the entries are ordered by increasing $Z$ and $A$ of the larget: then by increesing $Z$ and $A$ of lhe fincident particle; and. Finally, by increasing sequence number. The reader is referred to Figure 2 for a detailed description of this section. 
THE BIBLIOGRAPHY OF INTEGRAL CHARGED PARTICLE NUCLEAR DATA, FOURTH EDITION Organtzation

REFERENCES(cont)

No. Lab Work Reference
Type

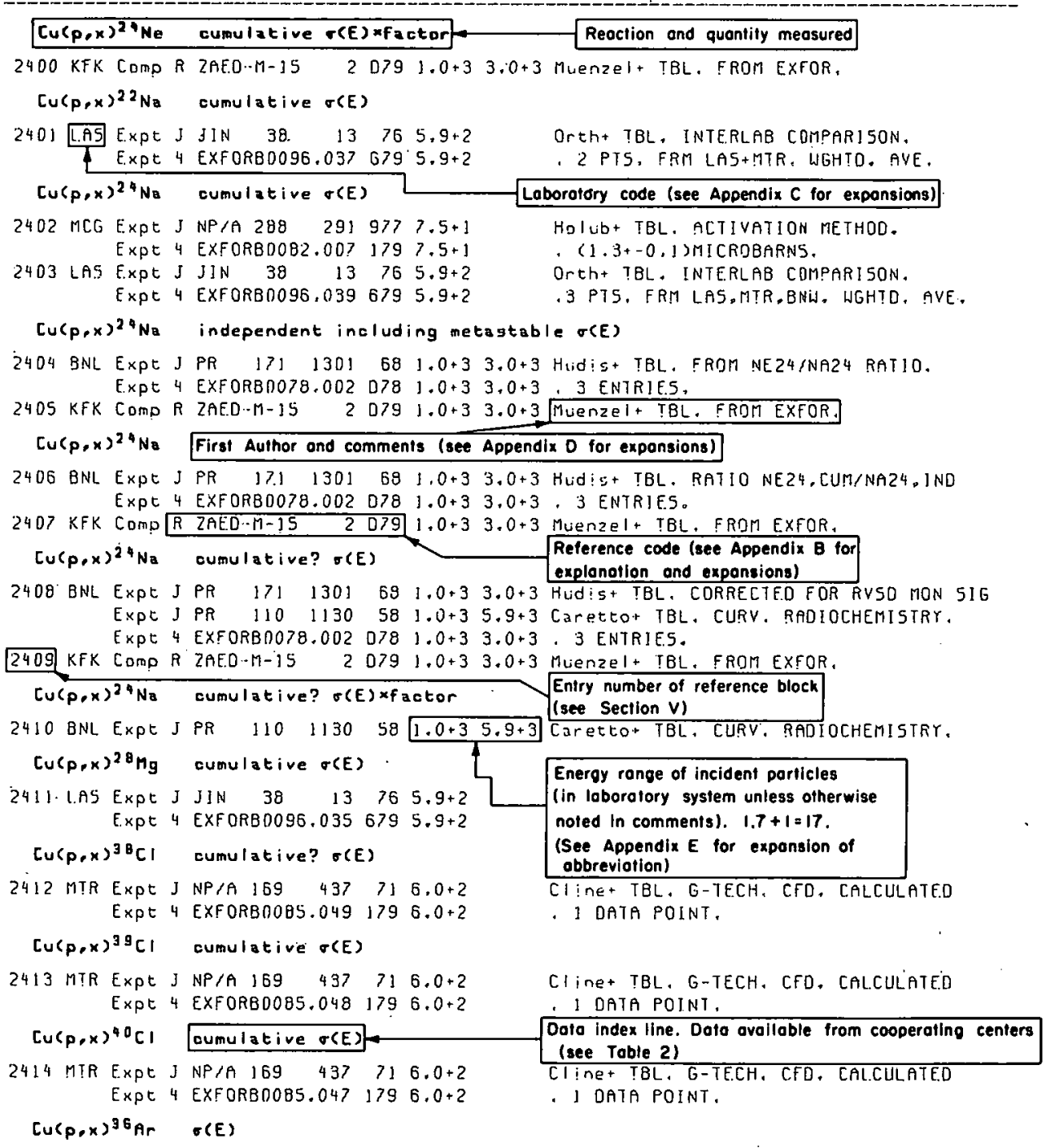

FIGURE I. DESCRIPTION OF REFERENCE SECTION 


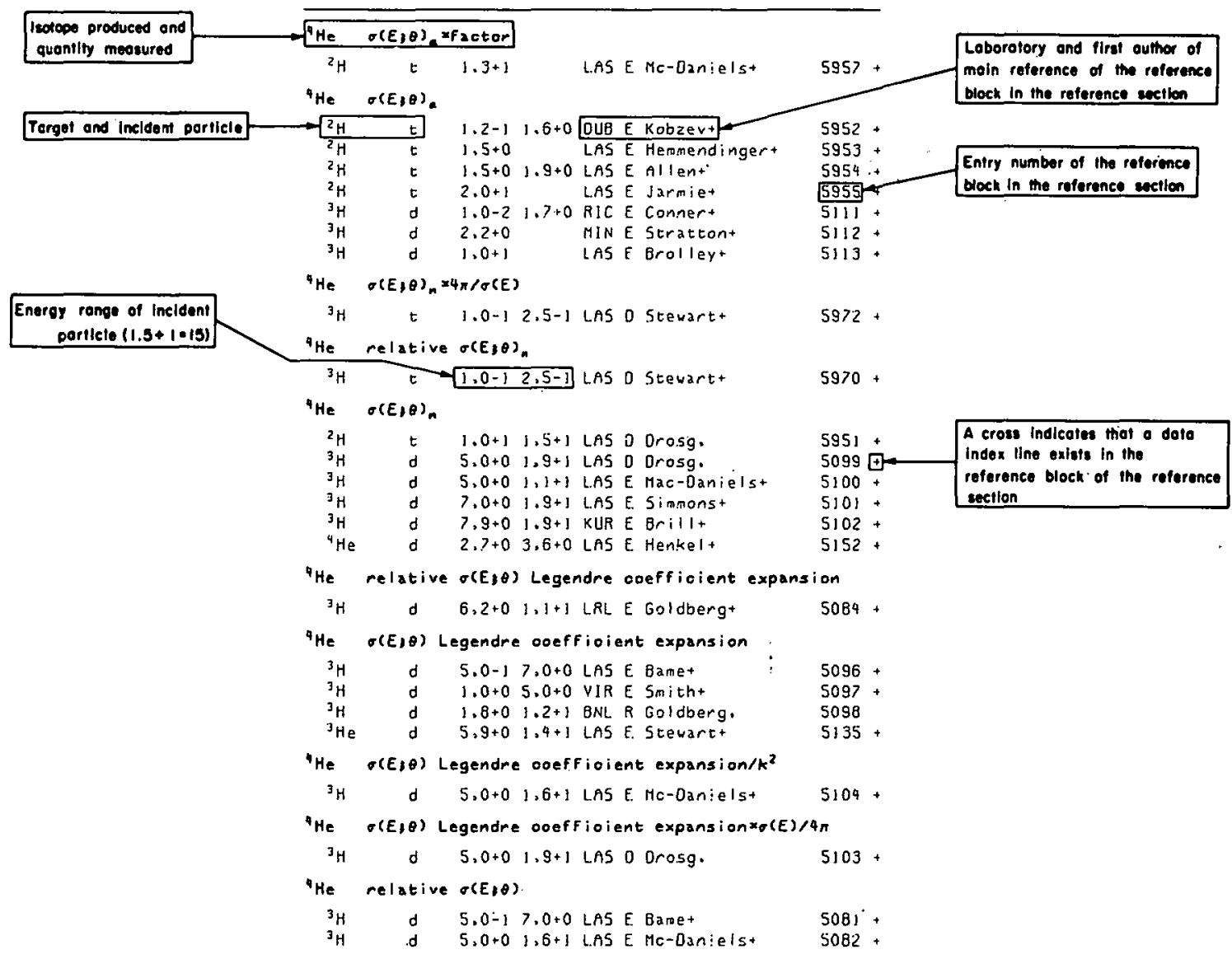


THE BIBLIOGRAPHY OF INTEGRAL CHARGED PARTICLE NUCLEAR DATA. FOURTH EDITION Compilations and Evalualions

\section{COMPILATIONS AND EVALUATIONS}

To enhance the usefulness of this publication several compilations. evaluations and reviews published prior lo the present effort are given in Table 4. - A Source List of Nuclear Dota Btbliographies, Compilations, and Evaluationgli contains more comprehensive 1 ist.

Table 4

Selected Evaluations. Revieos, and Compilations of Charged-Particle Nuclear Data

1. K.A. Keller, et al. Q-Values and Excitation Functions of Nuclear Reactions.' Landholt-Bernstein, Group I, Vol, 5, Parts A-C. Springer-Verlag. Berlin (1973).

2. H.J. Kim, et al. Nuclear Cross Seclions for Charged-Particle-Induced Reactions.' Nucl. Dato Al, 203. Acadenle Pregs. NY (1965). Nucl. Data A2, I. Acadeatic Press, NY (1966). Nucl. Data A3, 123. Acodemlc Press. NY (1967).

3. H. Liskien and A. Paulsen. Neutron Production Cross Sections and Energies for the Reactions $T(p, n)^{3} \mathrm{He}, D(d, n)^{3} \mathrm{He}$, and $T(d, n)^{4} \mathrm{He}$." Nucl. Dala Tables Ll, 569. Acadenlc Press, NY (1973).

Neutron Production Cross Sections and Energies for the Reactions ${ }^{7} \mathrm{~L}(1, p, n)^{7} \mathrm{Be}$ and ${ }^{\mathrm{L} i}(p, n)^{7} \mathrm{Be}^{\circ}$. Al. Data Nuel. Dato Tables 15.57 Acodemic Press. NY (1975).

4. F.K. McGoosan. et. Nuclear Cross Sections for Charged-Particle Induced Reactions Mn. Fe, Co." Oak Ridgo National Laboratory Report ORNL-CPX-1 (1954).

- Nuclear Cross Sections for Charged-Particle Induced Reactions Ni, Cu. Oah Ridge National Loboratory Reporl ORNL-CPX-2 (1964).

5. R.J. de Meijer. et al. Tables for Reaction Gama-Ray Spectroscopy.. At. Data Nuct. Dala Tables 15, 391. Academle Press. NY (1975).

6. W. Neubert. Peah Cross Sectians for (HI,xn) Reactions.' Nucl. Dato Tables All. 531. Acodemic Press, NY (1973).

7. S. Pearlstein. 'Starter Evaluated Charged Particle Data Library." Brookhaven National Laboratory Regort BNL-19148 (1974).

8. E.K. Warburton. et al. Gama-Rays from Fusion-Evaporation Reactions in 32 \& 446 Nuclei. At. Data Nucl. Data Tobles 14.147

\section{THICK TARGET NEUTRON YIELOS}

As has been noted above, this bibllography covers integral data only: that is, dota which has been inlegrated over product angle ond energy. Much of the data on thick target neutron ylelds are presented elther as o-degrea oxcitalian functions or secondary energy distributions and, therefore, fall outside the present scope of this publication. Eseveral recent symposia and articles revieding thick target neutron yield dota and methods are sumarized in Table 5 for the conventence of the reader. Tables 1 and 4 and. A Source List of Nuclear Data Bibliographles, Compliations, and Evaluations.l contain additional references pertineat to neutron sources.

Table 5

Some Recent Reviews of Thich Target Neutron Sources

1. H.H. Barschall. 'Intenge Sources of Fast Neutrong, Ann. Rev. Nucl. Part. Sel. 2B. 207. Annual Revleus. Ine., Palo Alto. CA. II978I.

2. M.R. Bhat and S. Pearlstein, editorg. Sympostum on Neutron Cross Sections from 10 to $40 \mathrm{MeV}$. Brookhoven National Laboratory Report BNL-NCS-50681 (1977). 
THE BIBLIOGRAPHY OF INTEGRAL CHARGEO PARTICLE NUCLEAR DATA, FOURTH EDITION Thich Target Neutron Yields

3. M.A. Lone. Lbed., D. 79 .

4. M.A. Lone, editor. International Speclalists Symposium." Report NBSIR 77-1279 (1977).

5. Neutron Dosimetry for Biology and Medicine. ICRU Report 26. ICRU Publications, Washington, DC. (1977).

6. H. Ullmaier, editor. 'High Energy and High Intengity Neatron Sources. Nucl. Instr. Methods 145, 1. North-Holland Publighing Co.. Amsterdam. Hollond. (1977).

\title{
VIII. METHODS OF RADIOISOTOPE PROQUCTION
}

While this publication ghould be very useful in providing the sources of cross section dats for radioisotope production. It does not index, per se, the techniques of radioisolope or labelled compound production. The Radiopharmaceutical Research Group. Chemistry Dept. Broohhaven Notlonal Laboratory does index such techniques for accelerators. 2 Other bibllographies dealing with such methods may be lound in - A Source tist of Nuclear Data Bibliographles. Compilations, and Evaluationg."

\section{RETRIEVAL CAPABILITIES}

Retrievals may be oblained based on larget nucleus, incident particle, outgoing particles or processes, residual nucleus, quantity. energy range, or any combination of these. Send requests to:

\author{
National Nuclear Data Center \\ Building 1970 \\ Broohhaven National Laboratory \\ Upton. NY 11973 \\ U. A
}

For more detalted information. you should contact the authors.

All Information Analysis Centers supported by the Department of Energy are required to conform with o DOE plan for charging services. There is no charge to DOE and its contractors. However. there cauld be nominal charges for others. Further information will be supplied on request.

\section{ACKNOWLEOGEMENTS}

The authors wish to thank $C . L$. Dunford for his early planning of the format and scope of the computerized charged-particle bibliography. We are also graleful to $G$. Waite for the many hours spent scanning the literalure and to R. Kingey for ald in producting the graphics program necessary for this publication. We wish to achnowiedge the timely transmittals cf data compiled by the Karlsruhe Charged Particle Data Group. The Center for Nuclear Structure and Reaction Data of lhe USSR, and the Nuclear Dota Section. International Atonic Energy Agency and lo than Karlsruhe for malntaining the charged-particle EXFOR library. We thanh the staff of the National Nuclear Data Center for lheir aid, encouragement, and many useful suggestions and the people who have brought our attention to errors or omissions. Finally. we gratefully acknowledge Jean Burt who coauthored this publication in its flist three years of existence.

2. D.R. Christoan and K.l. Karlstrom. Accelerator Produced Nuclides for Use in Biology and Medicine. A Bibliography, 1939 - 1973." Brookhaven National Laboratory Report BNL-50448 (1975).

- Accelerator Produced Nuclides for Use in Blology and Medicine.

A Bibliography, January 1974 - June 1976." Broohhaven National Laboratory Report BNL-50448-Vol. II (1978). 


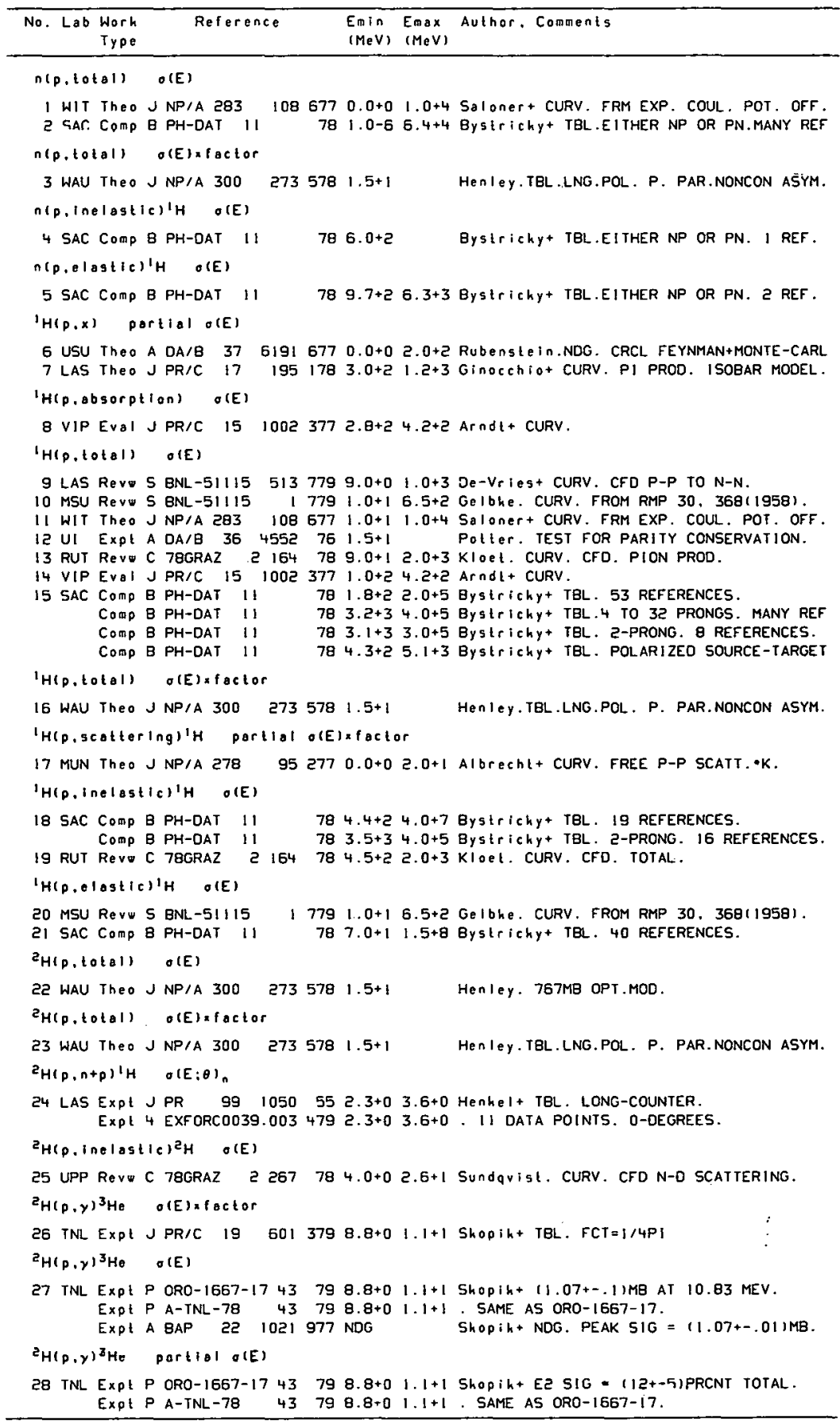


REFERENCES ( c on t )

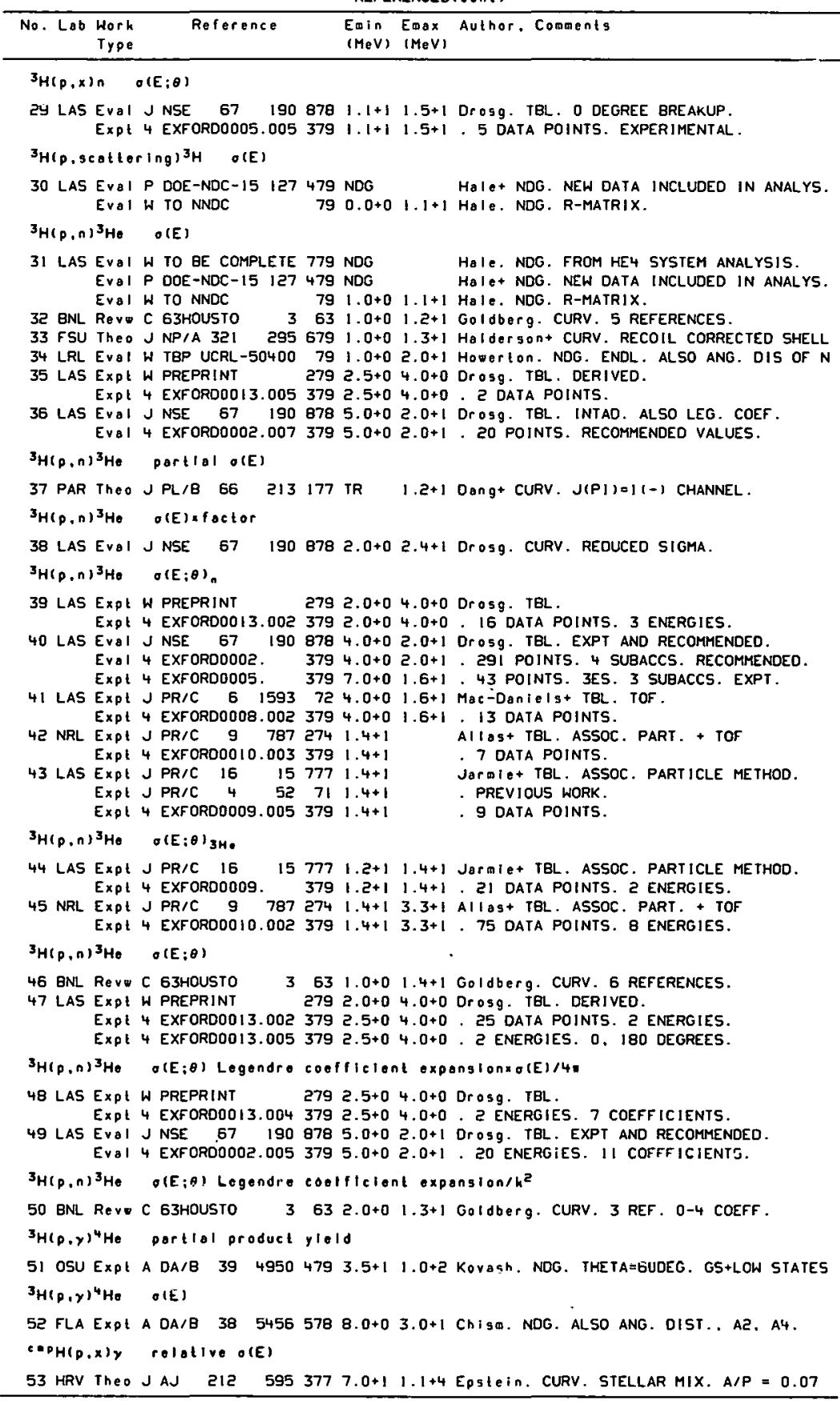


REFERENCES ( cont)

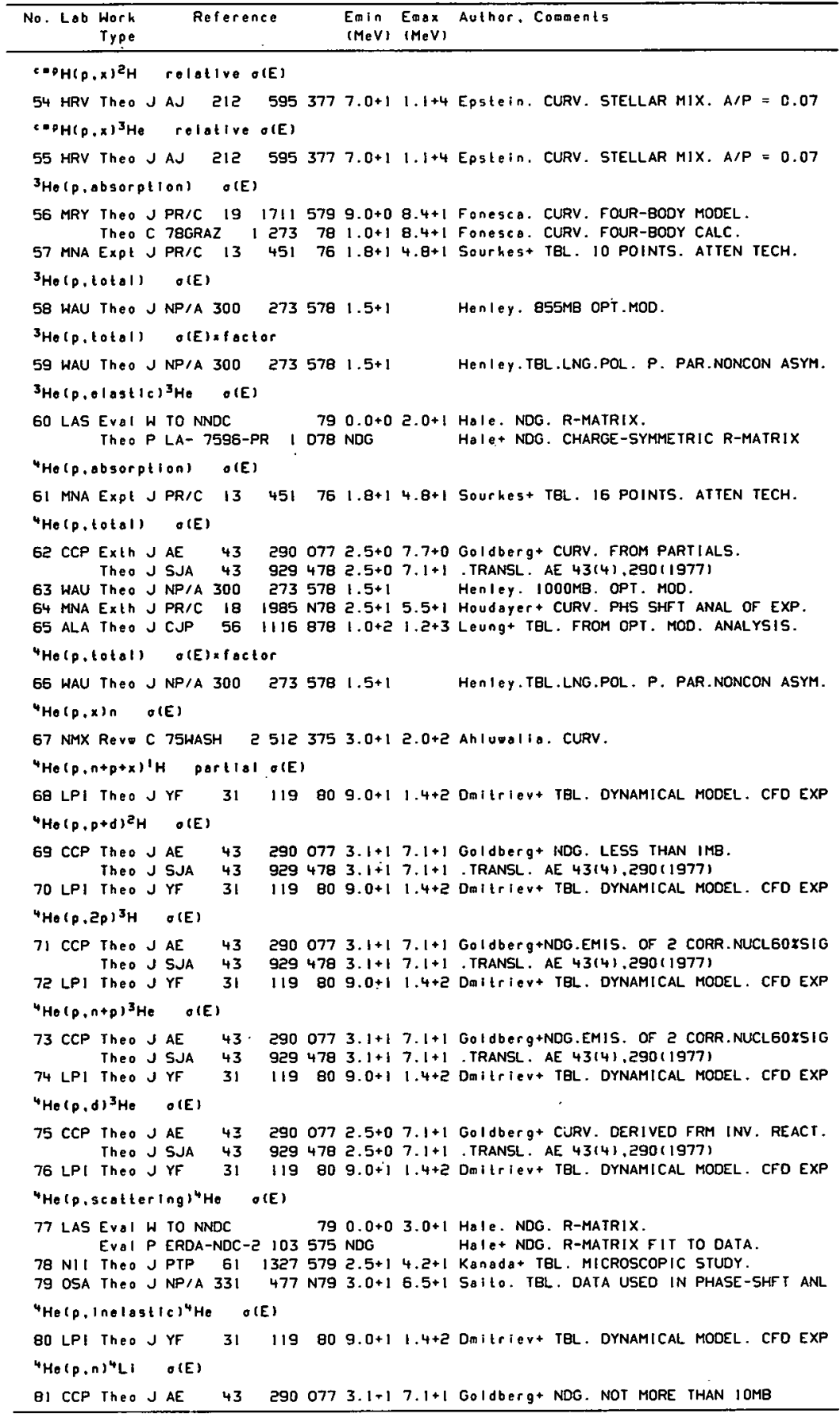




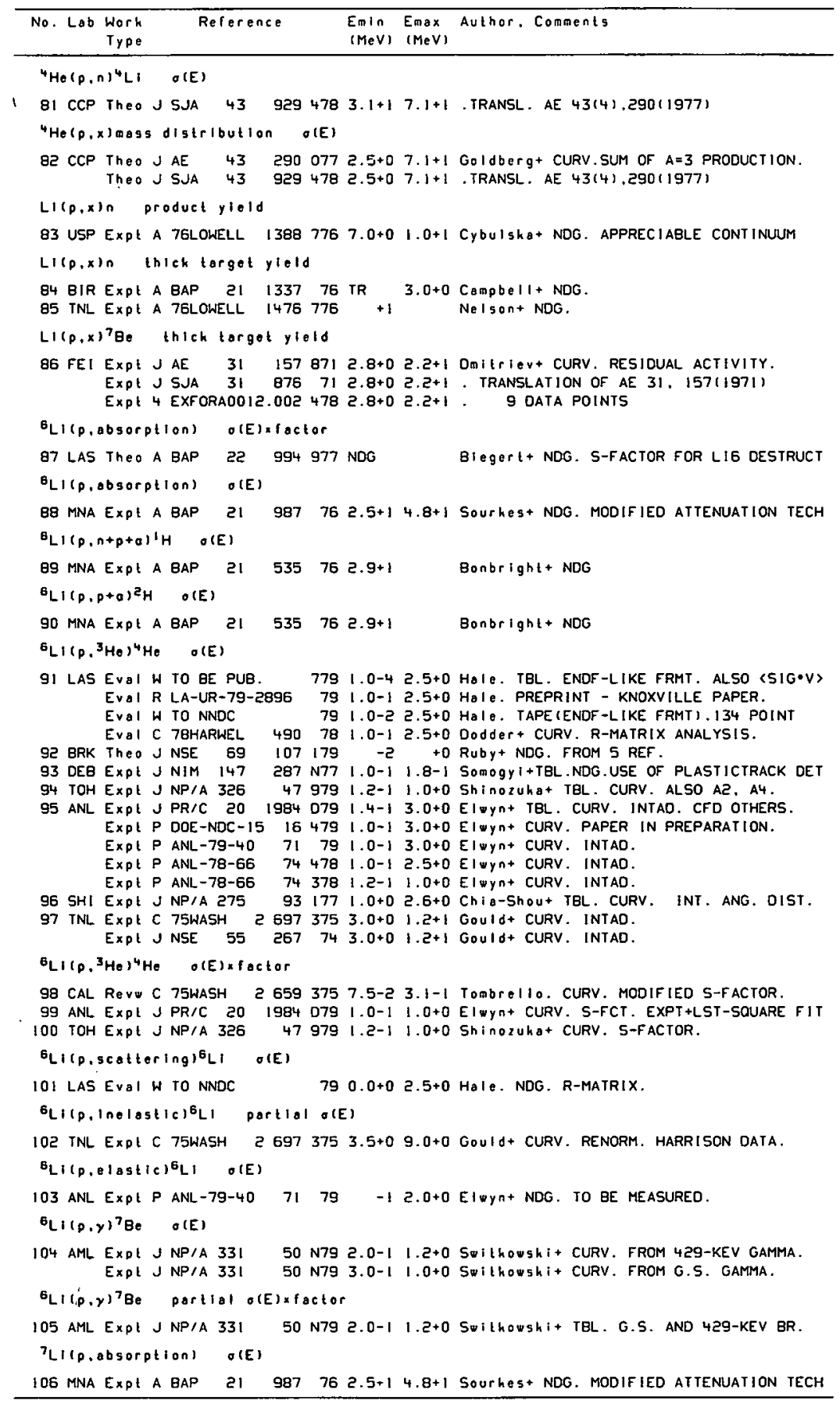


REFERENCES ( cont)

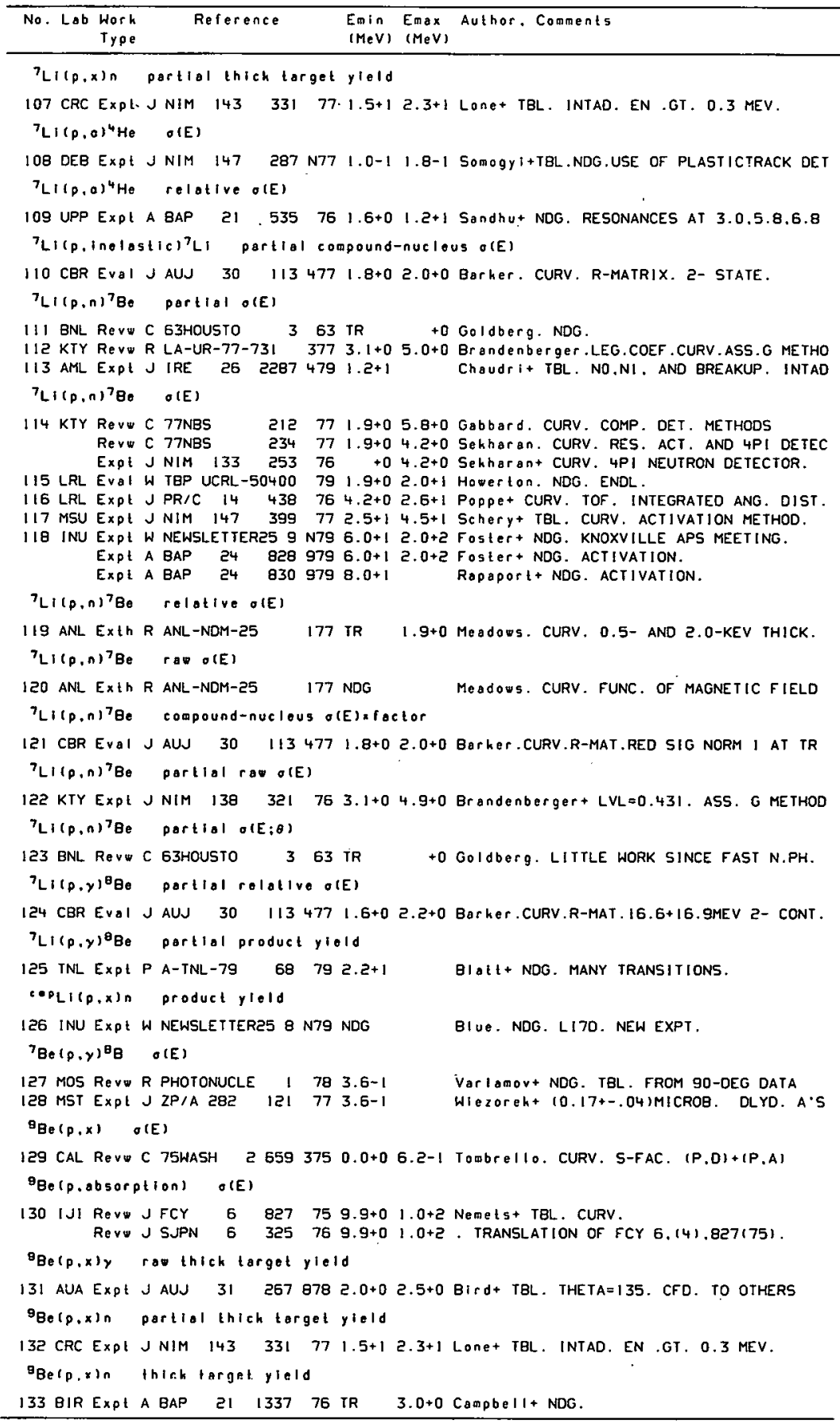




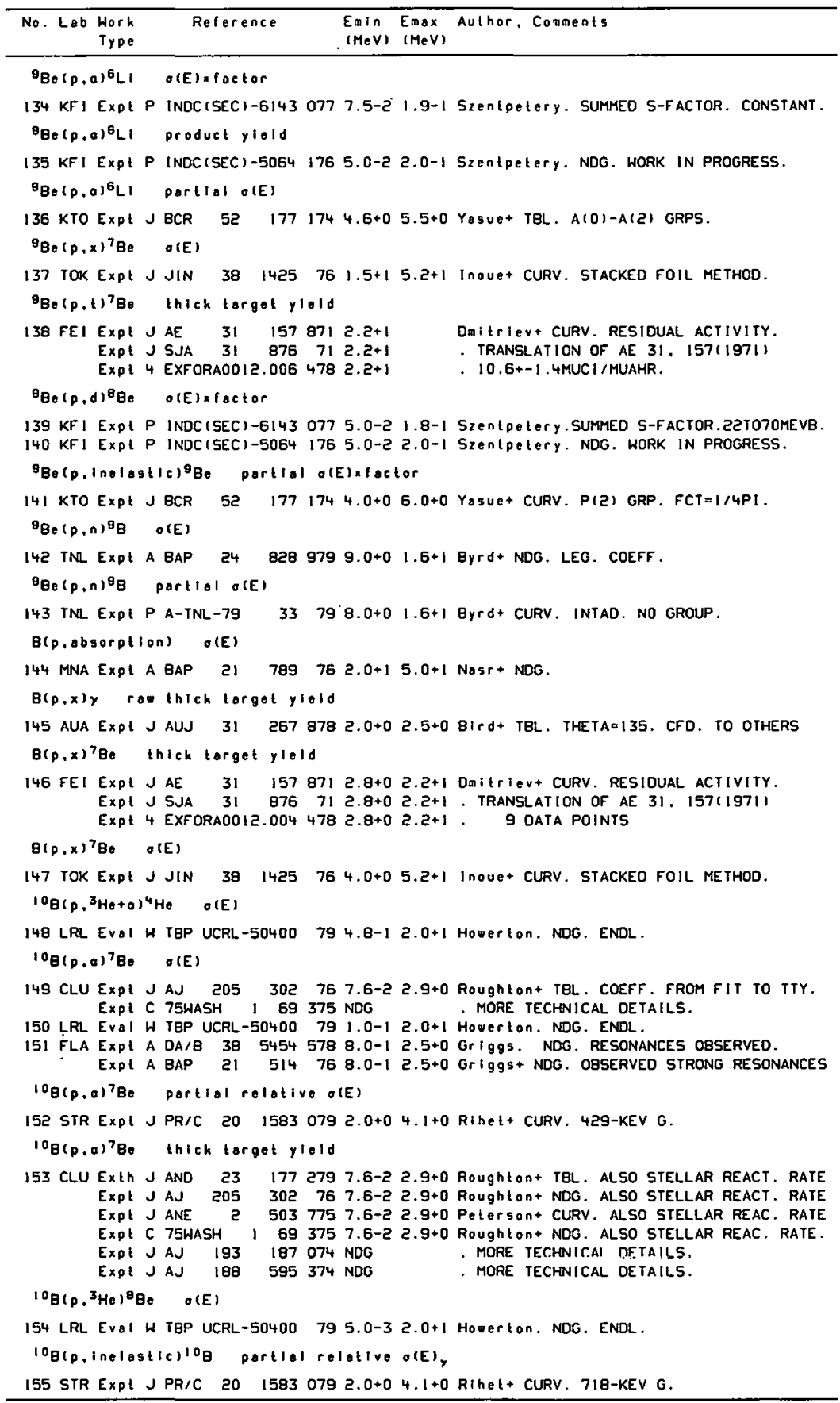


REFERENCES (cont)

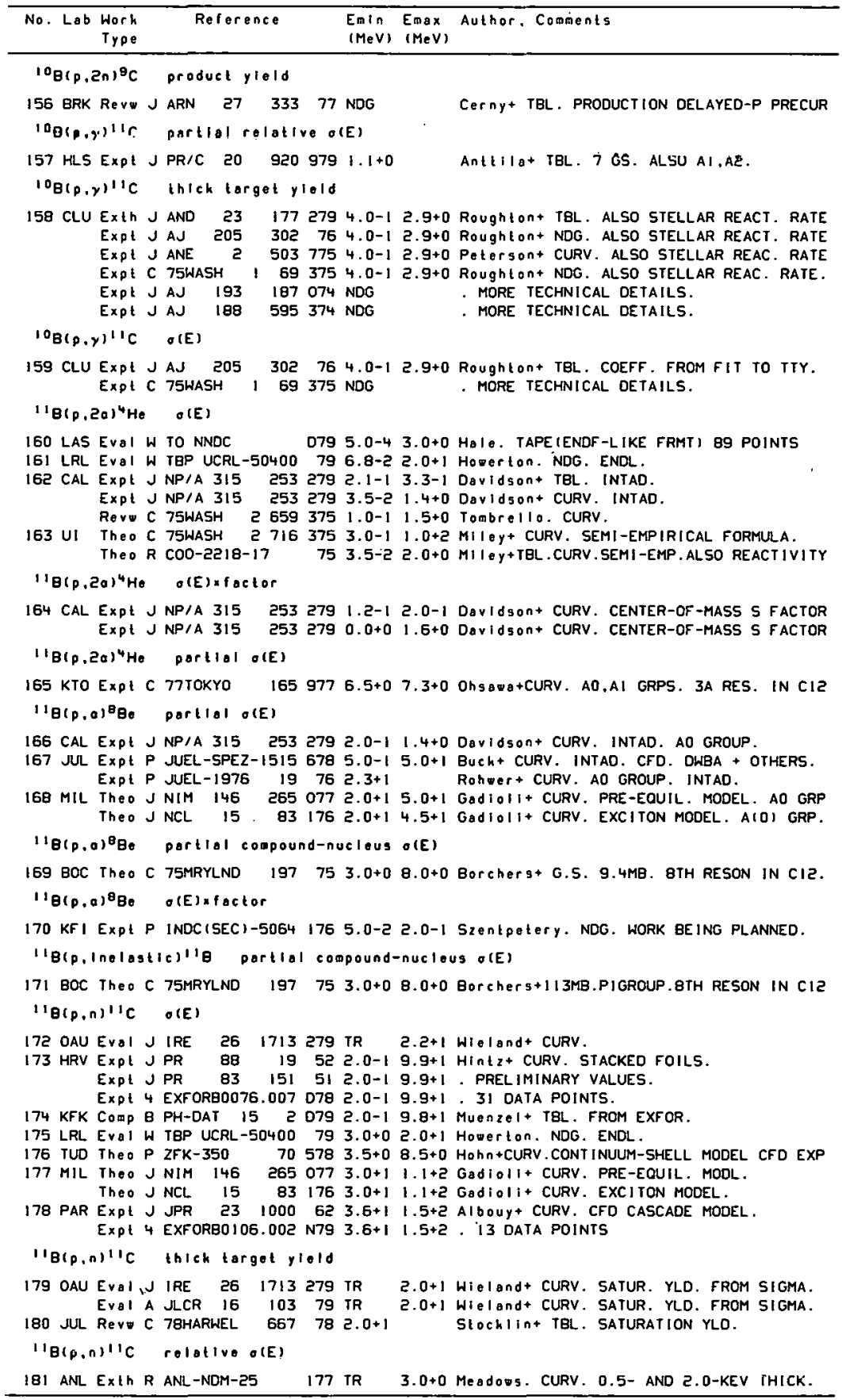




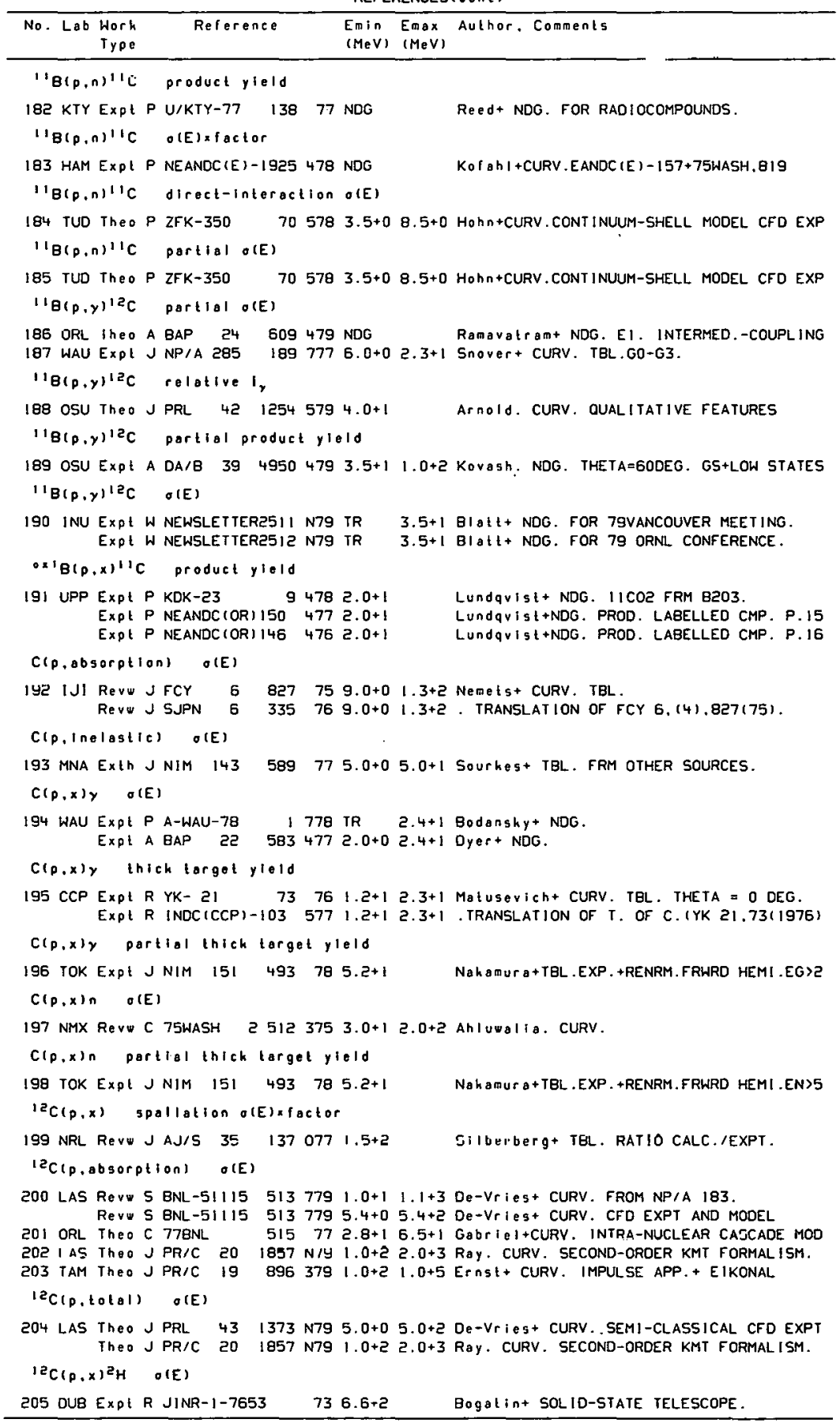




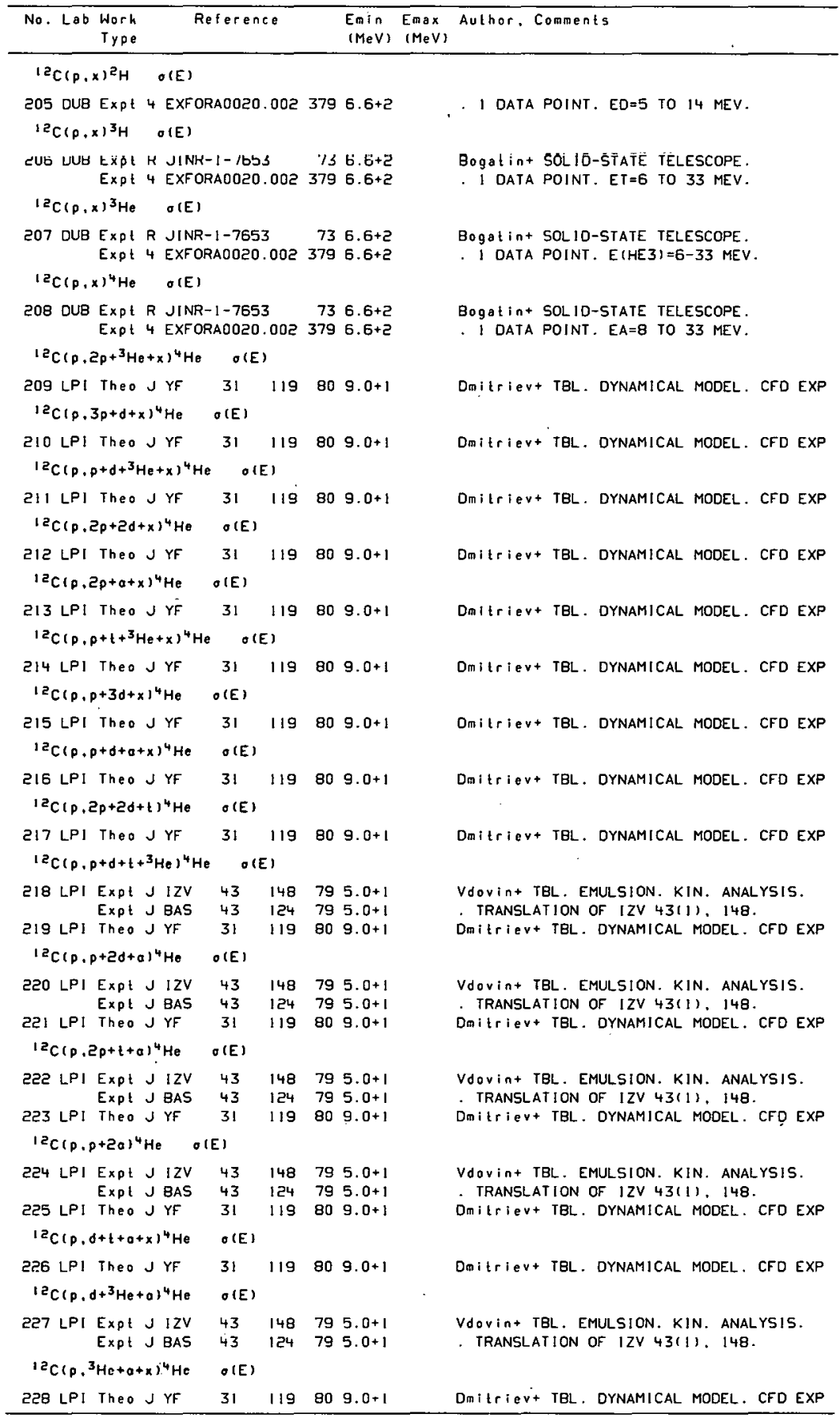


REFERENCES ( c on t)

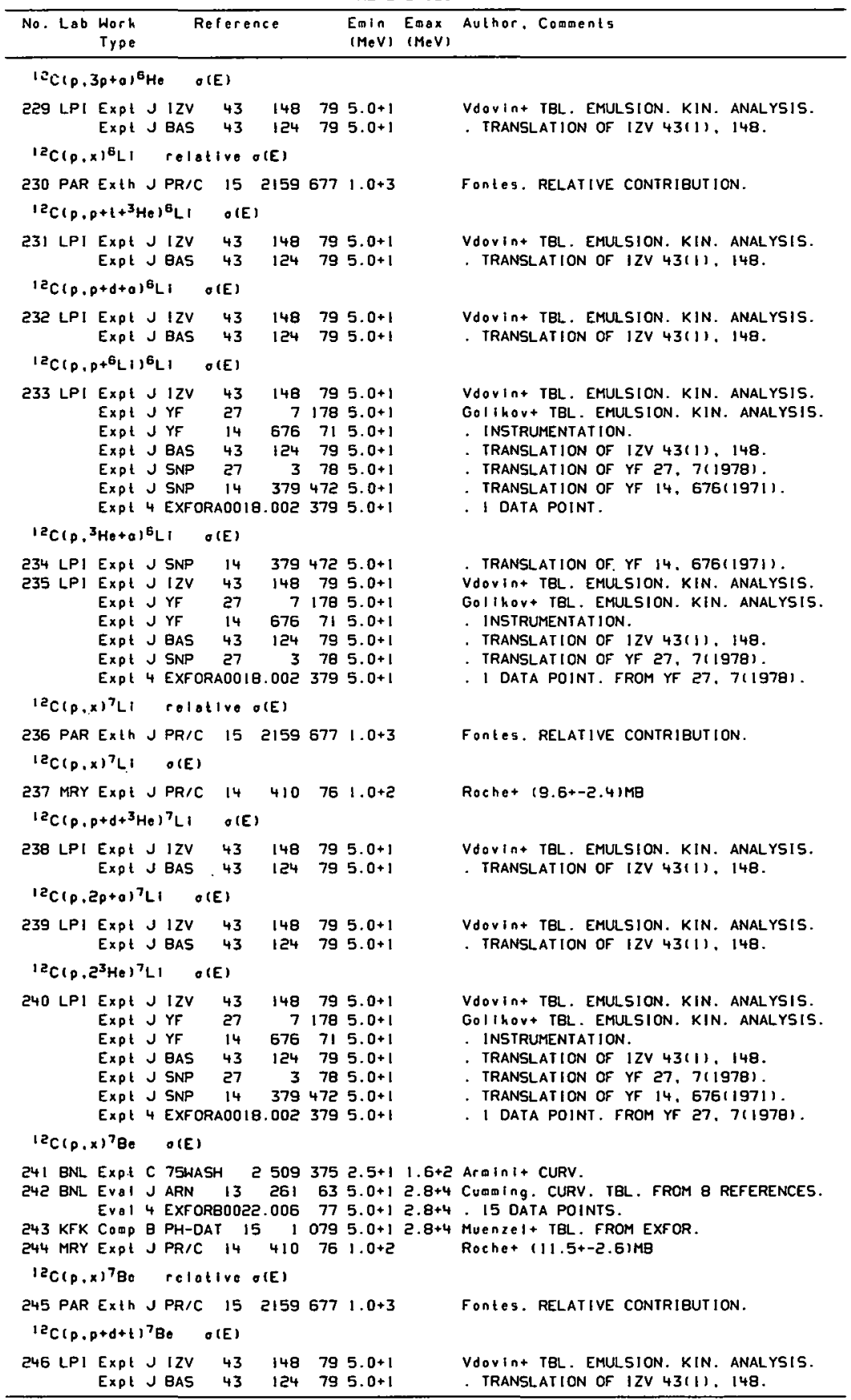


REFERENCES ( c o o l)

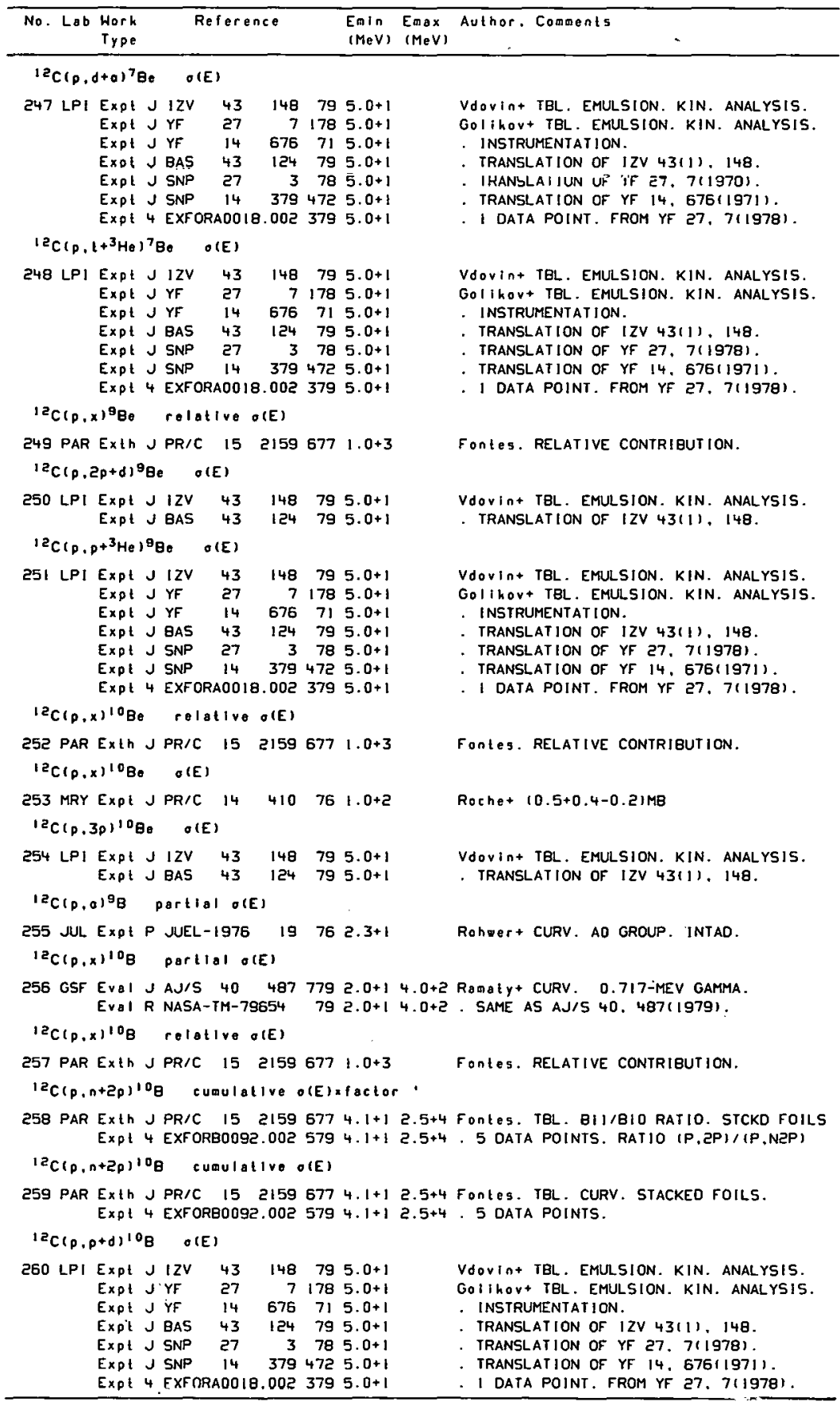


REFERENCES (cont)

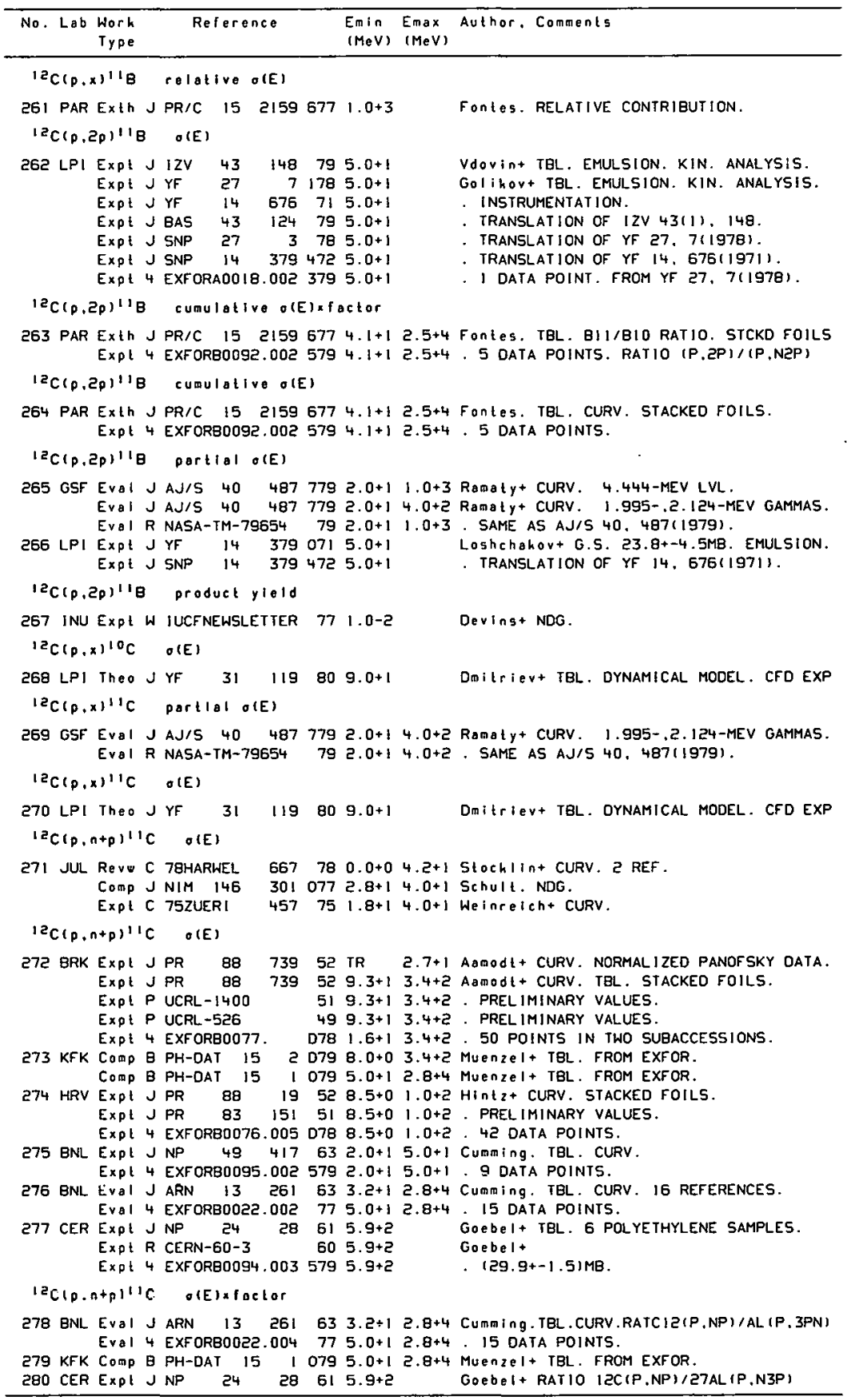




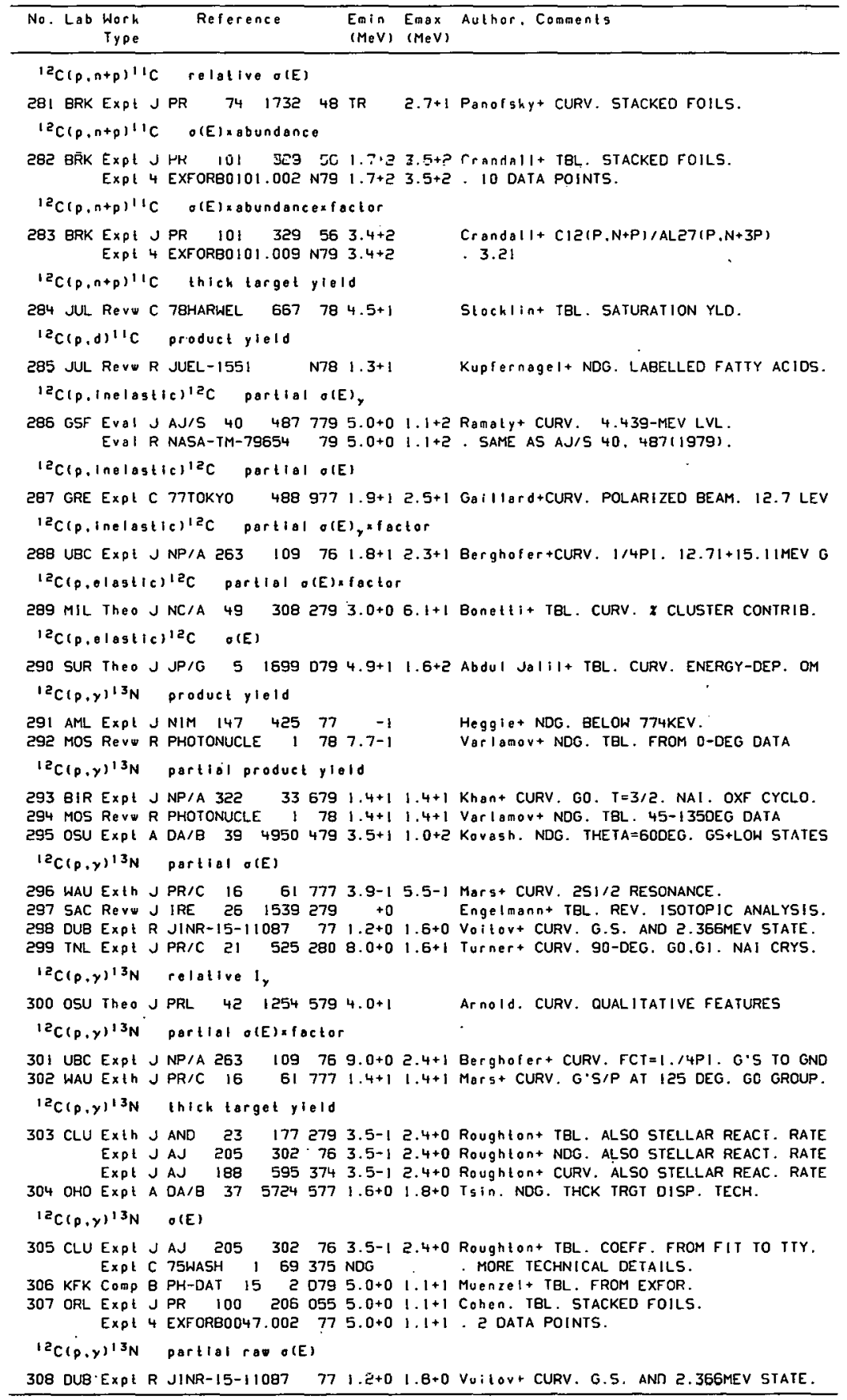




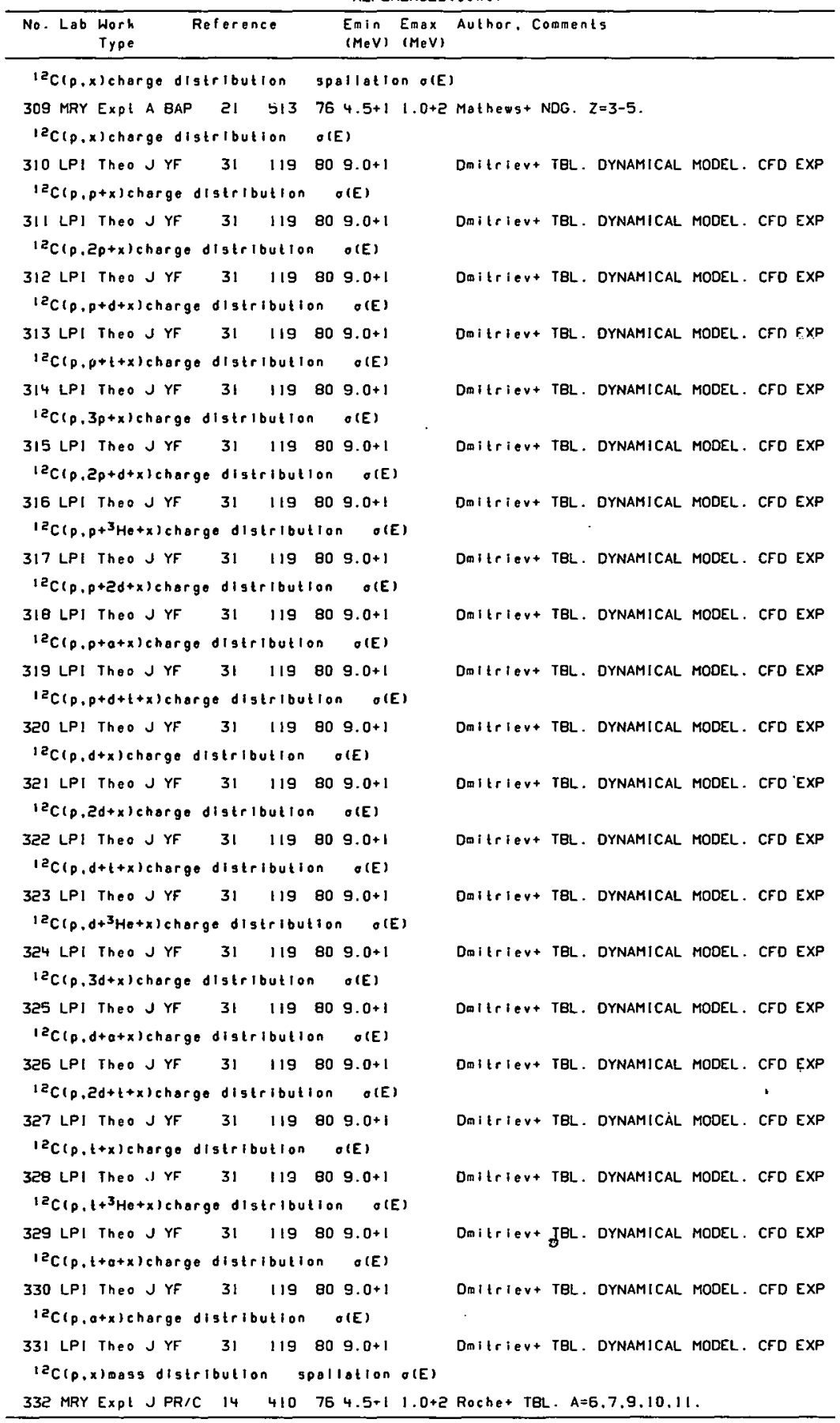


REFERENCES (cont)

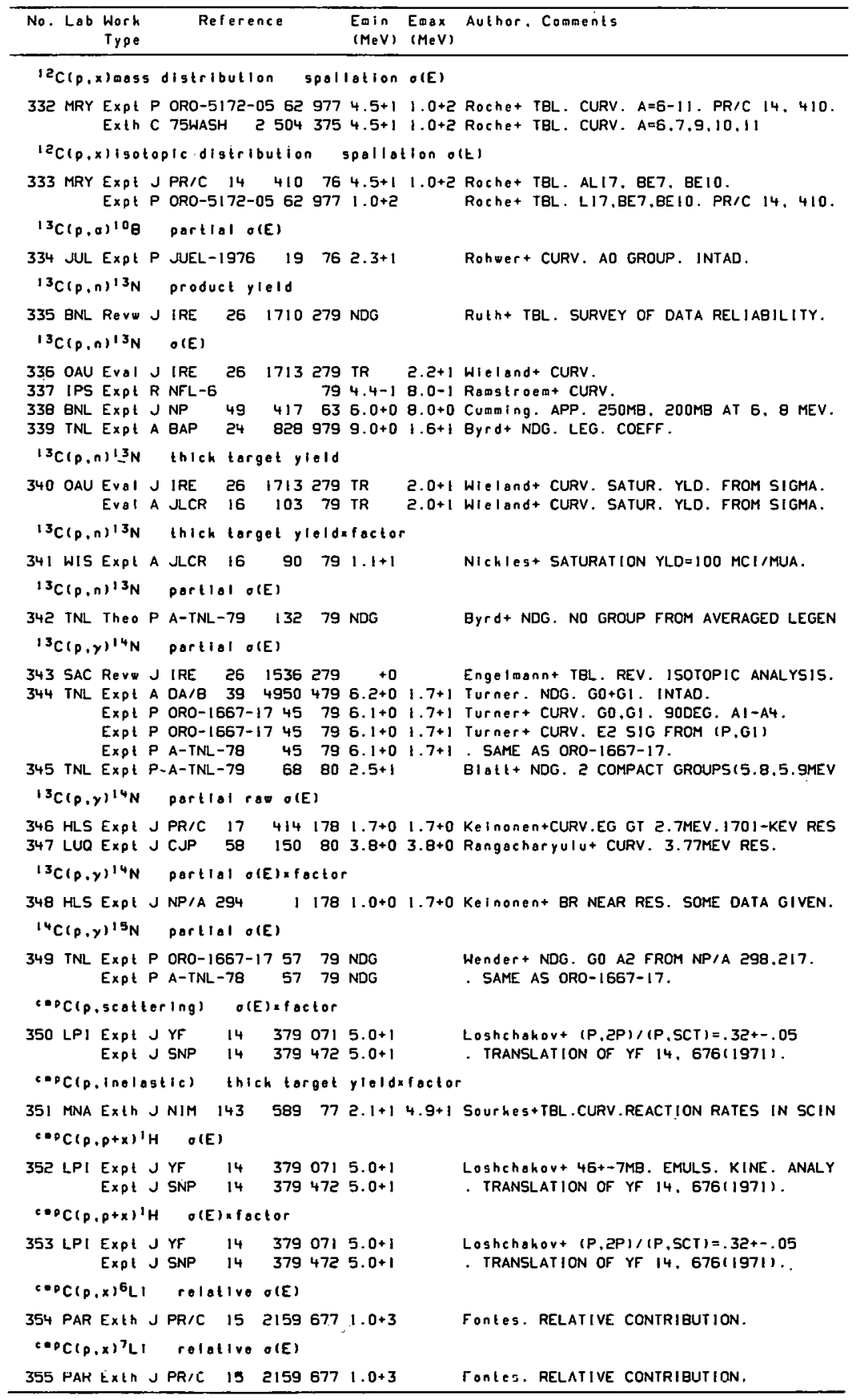


REFERENCES ( c on t )

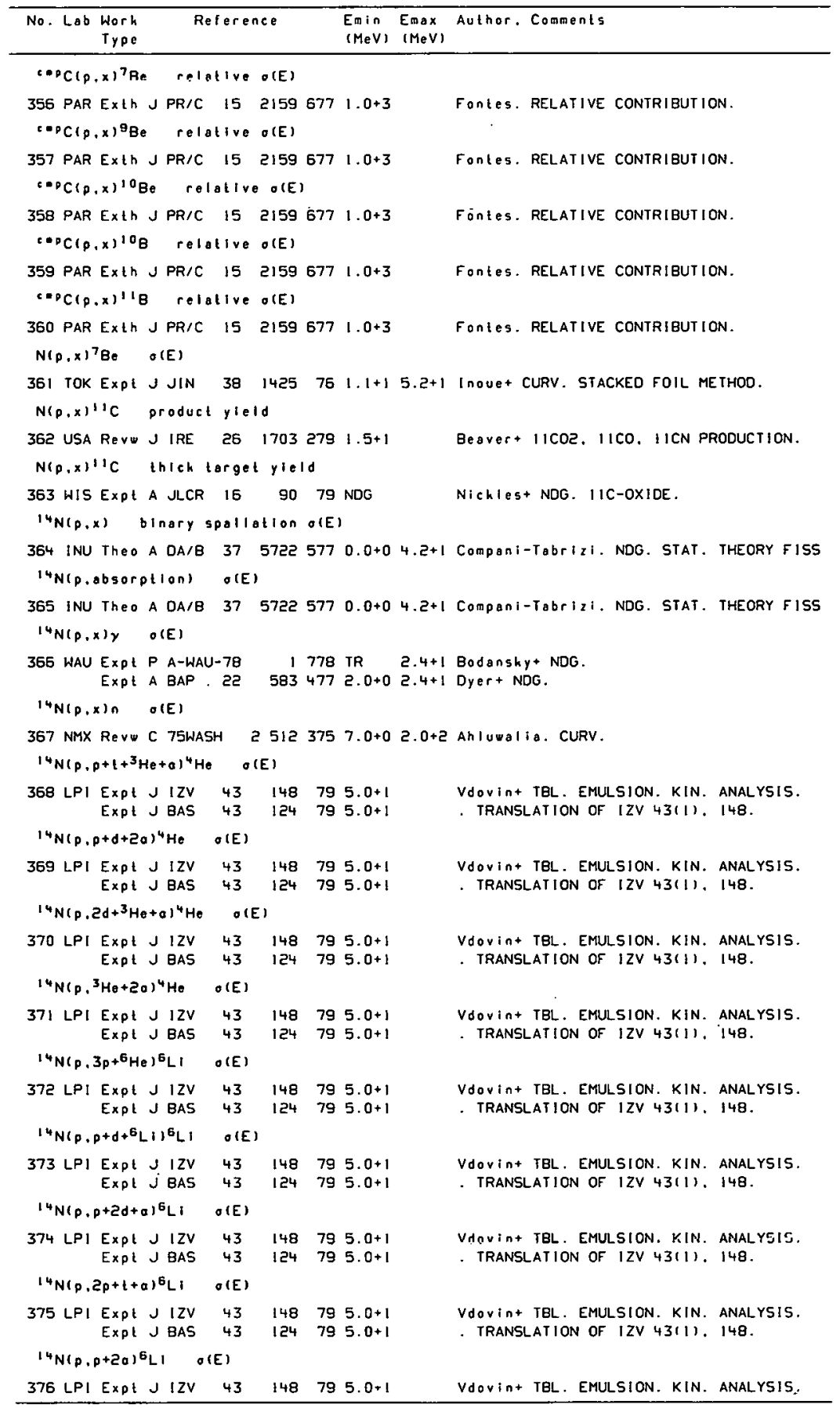


REFERENCES (cont)

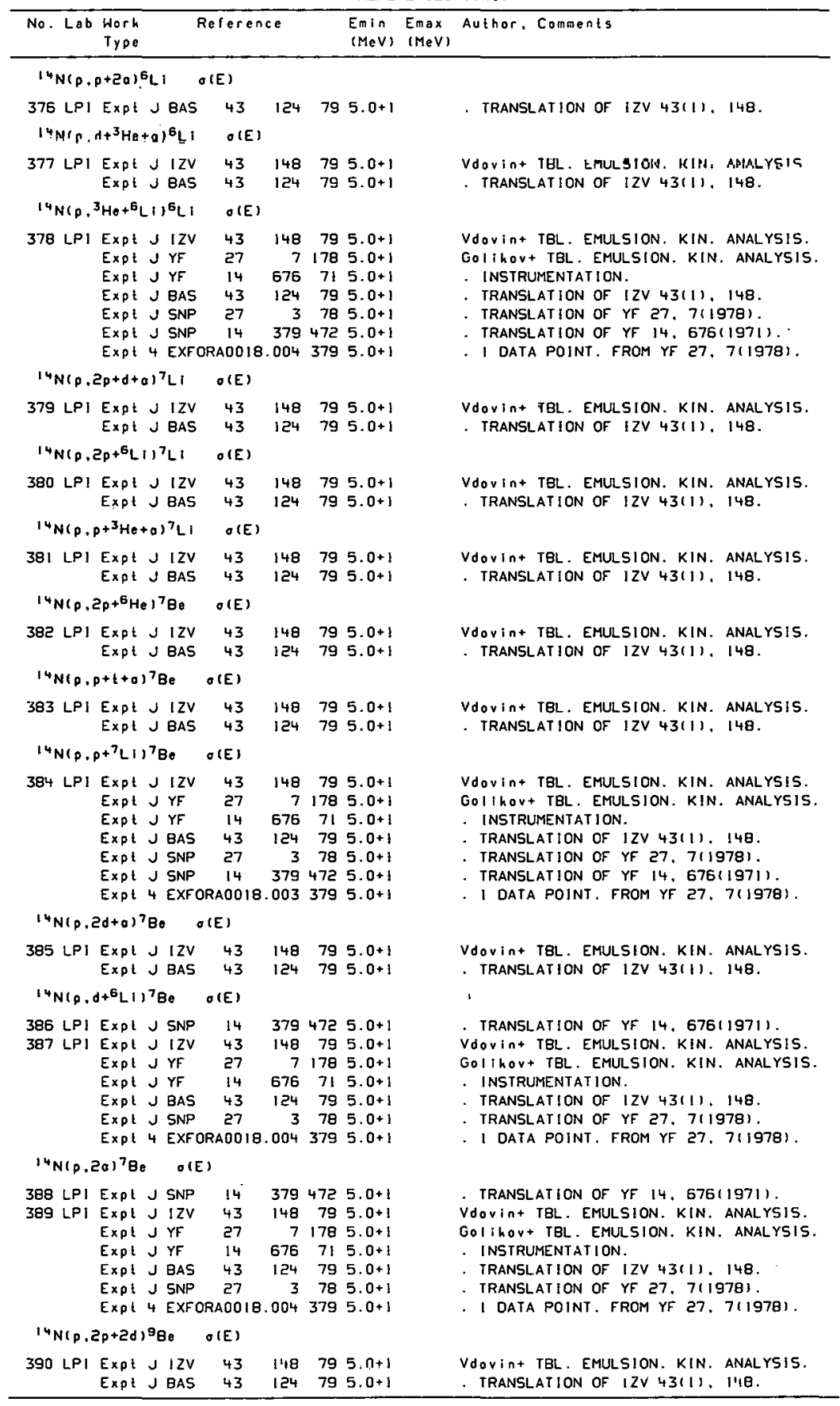


REFERENCES ( c ont )

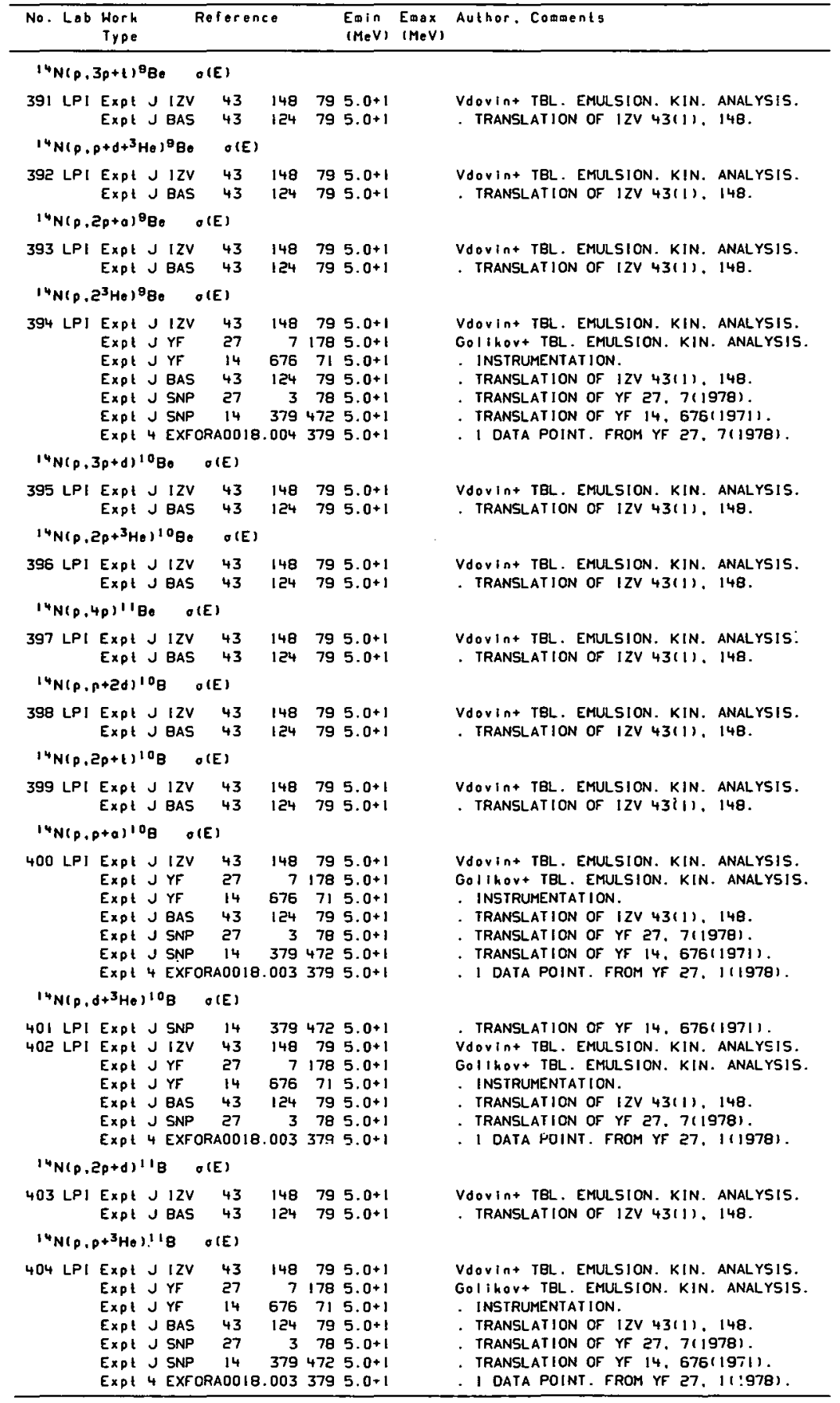


REFERENCES ( cont)

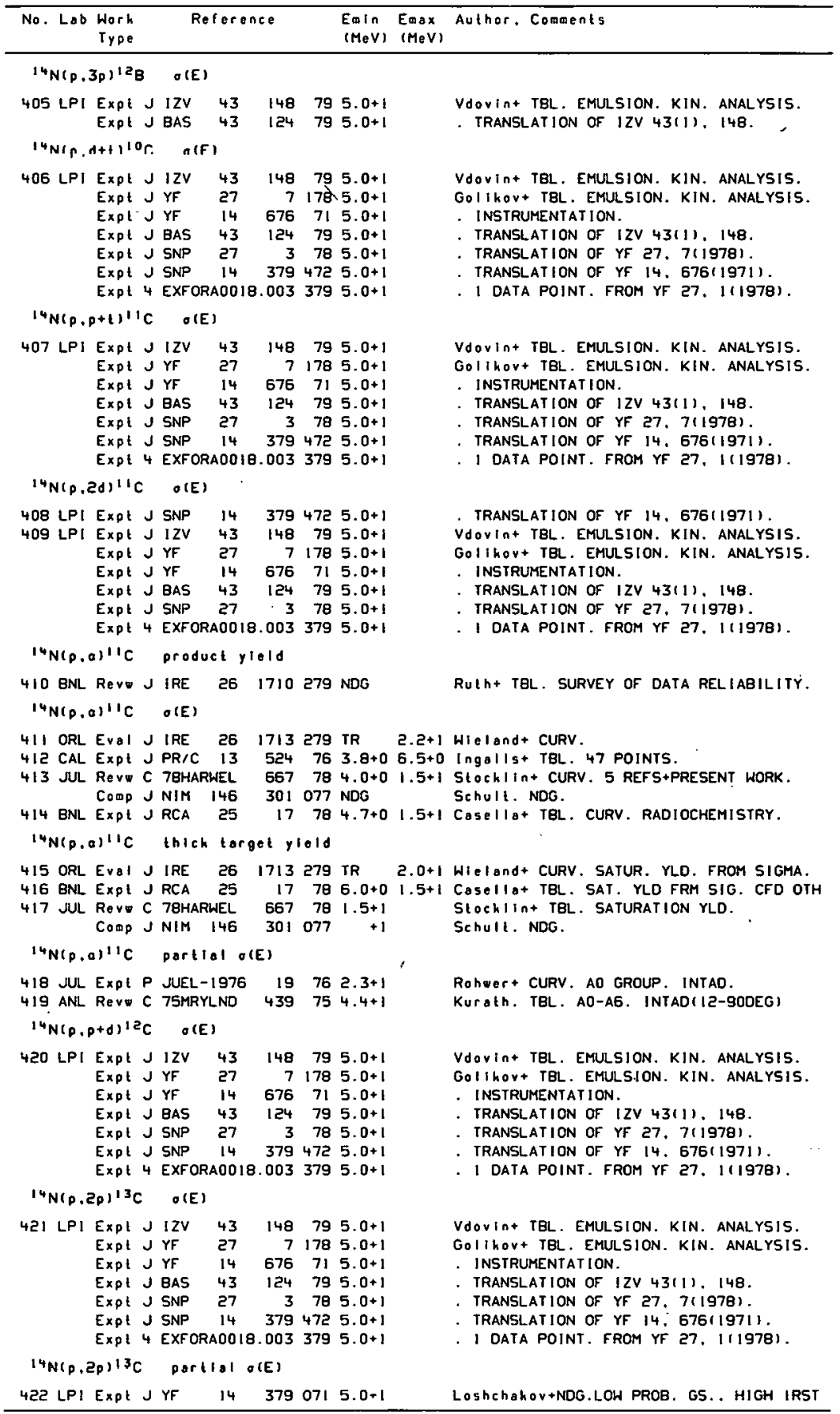




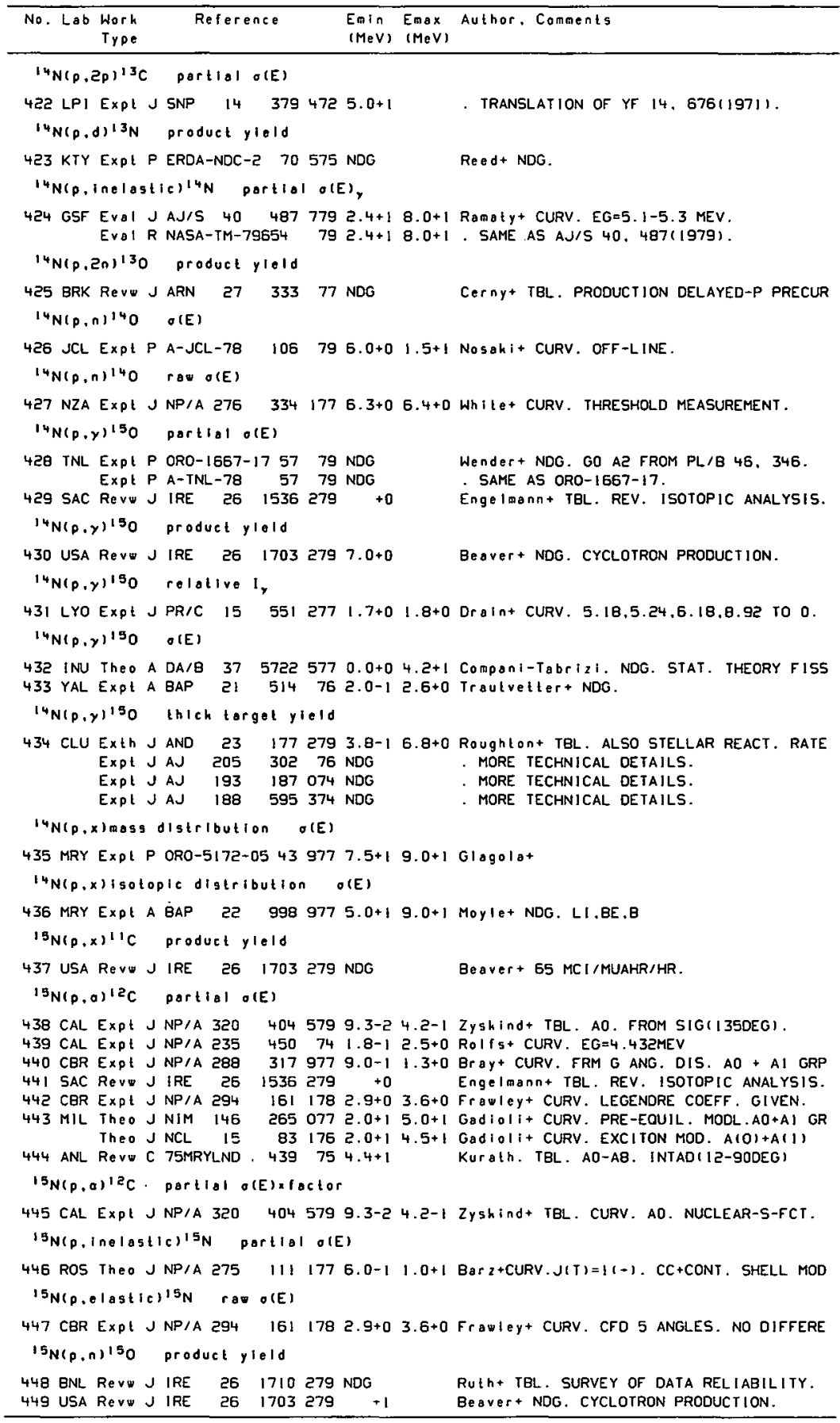


REFERENCES(con ()

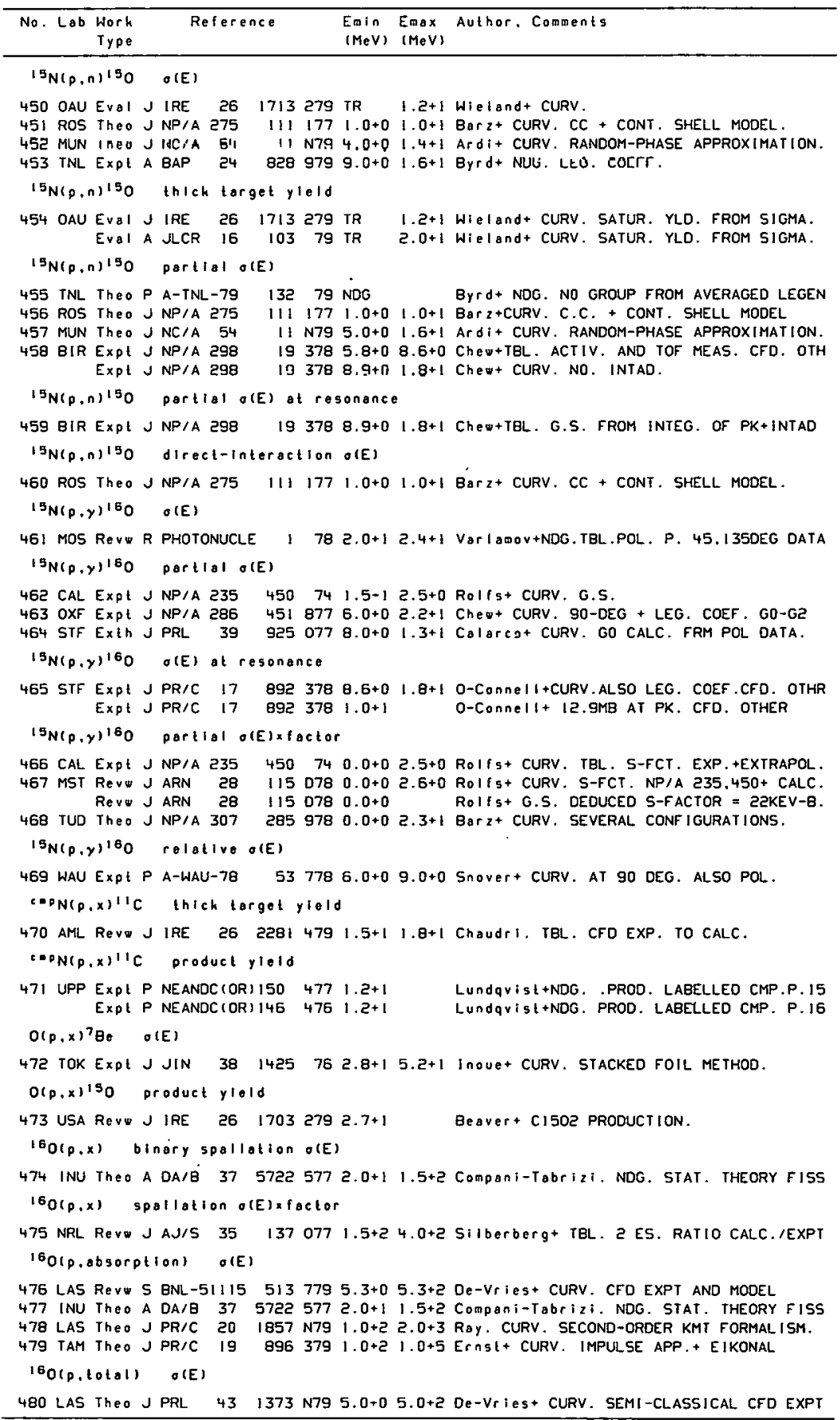


REFERENCES (cont)

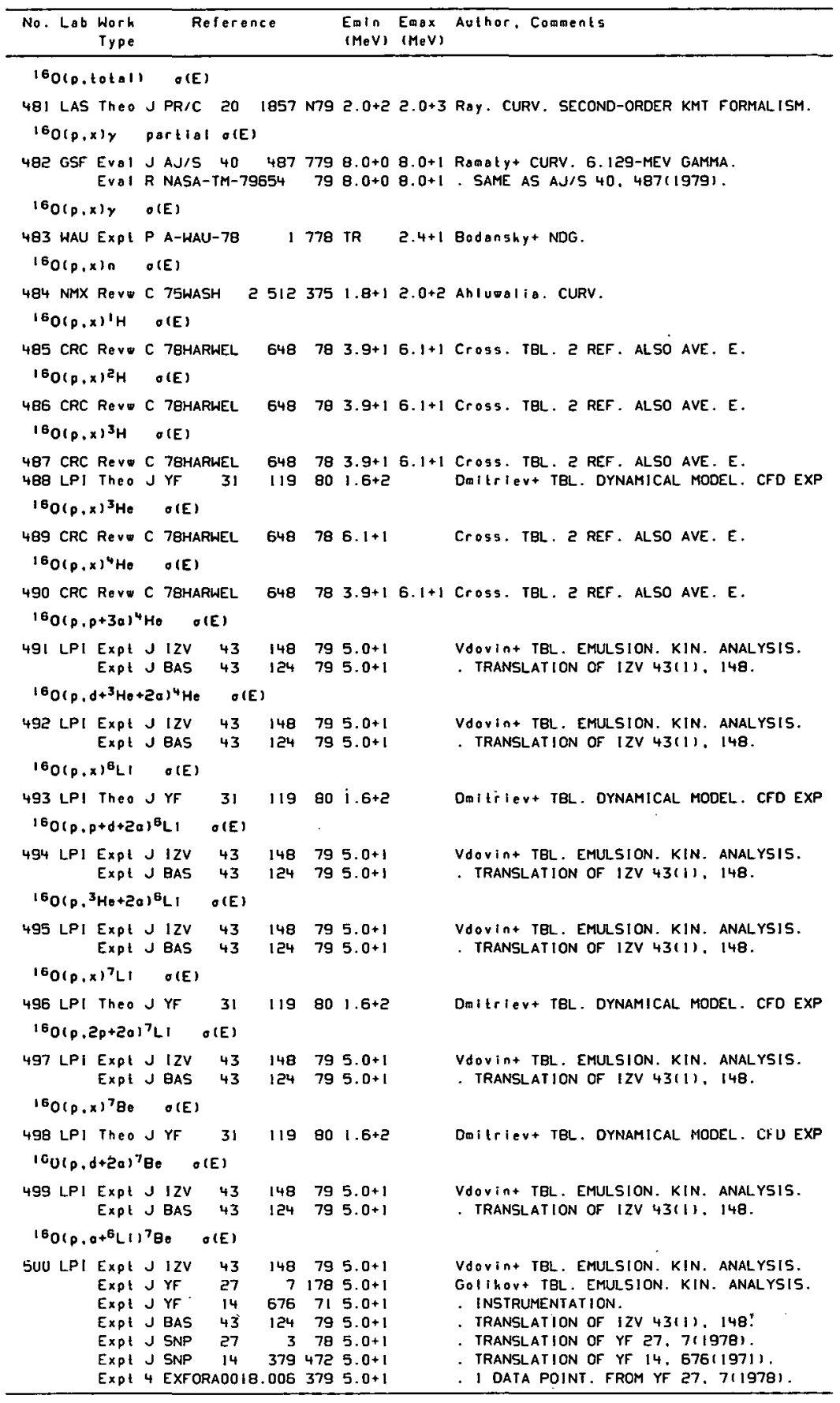


REFERENCES ( $c$ on $t)$

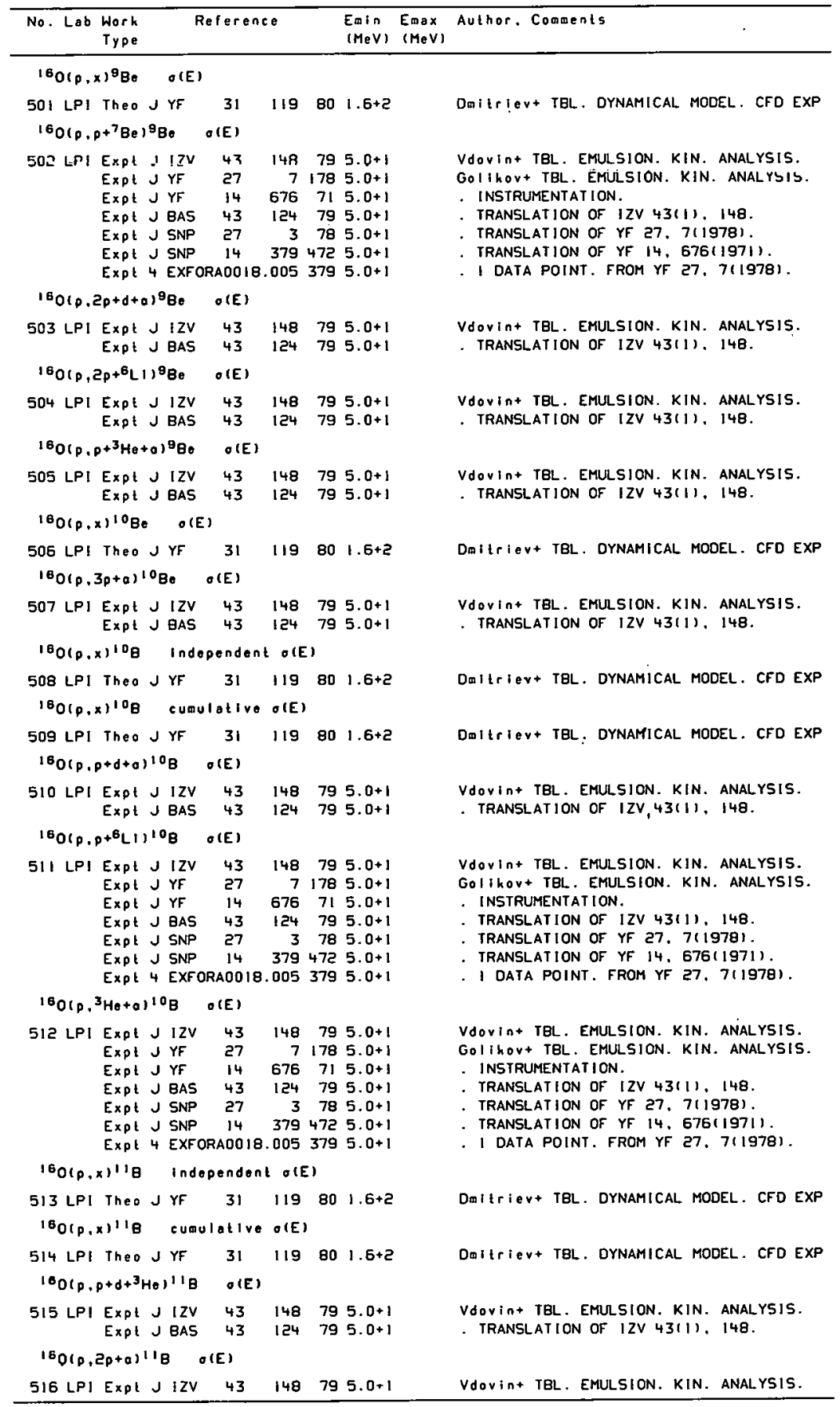




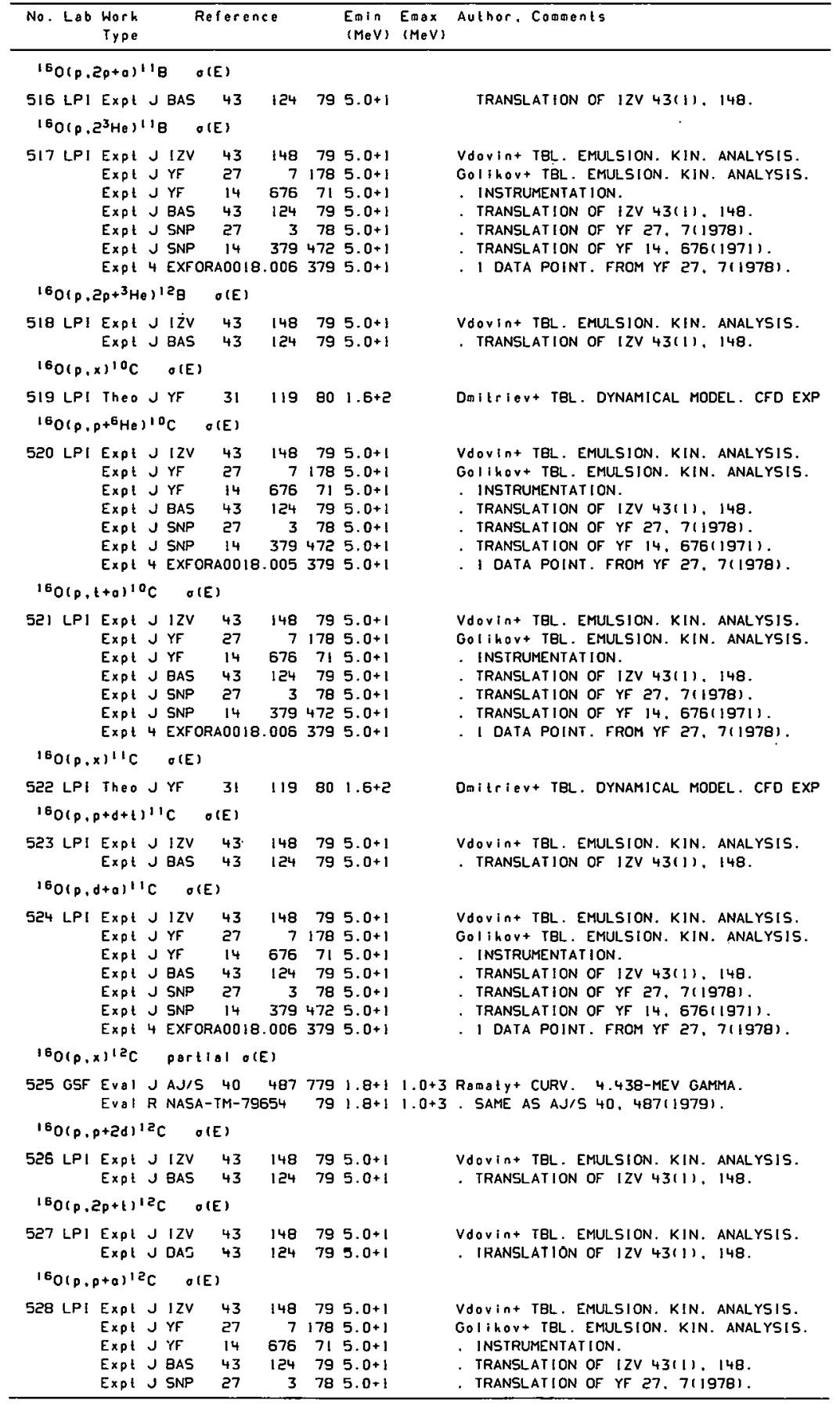


REFERENCES ( cont)

\begin{tabular}{|c|c|c|c|c|c|c|c|c|}
\hline No. $L a b$ & $\begin{array}{l}\text { Work } \\
\text { Type }\end{array}$ & \multicolumn{3}{|c|}{ Reference } & & \multirow[t]{2}{*}{$\begin{array}{l}\text { Enin } \\
(\mathrm{MeV})\end{array}$} & \multirow[t]{2}{*}{$\begin{array}{l}\text { Emax } \\
(M e V)\end{array}$} & \multirow[t]{2}{*}{ Author. Comments } \\
\hline \multicolumn{2}{|c|}{${ }^{16} 0(p, p+a)^{12} c$} & OIE & E) & & & & & \\
\hline $160(p$ & $\begin{array}{l}\text { Expt J } \\
\text { Expt } 4 \\
d+{ }^{3} \mathrm{Hel}^{3}\end{array}$ & $\begin{array}{l}\text { SNP } \\
+ \text { EXF OR } \\
{ }^{2} \mathrm{C}\end{array}$ & $\begin{array}{c}14 \\
\text { RADO1E } \\
O(E)\end{array}$ & $\begin{array}{r}379 \\
9.005\end{array}$ & $\begin{array}{l}472 \\
379\end{array}$ & $\begin{array}{l}5 \cdot 0+1 \\
5 \cdot 0+1\end{array}$ & & $\begin{array}{l}\text { TRANSLATION OF YF } 14,675(1971) \\
1 \text { DATA POINT. FROM YF } 27,711978)\end{array}$ \\
\hline $160(p)$, & $\begin{array}{l}\text { Expt J } \\
\text { Expt J } \\
\text { Expt J } \\
\text { Expt J } \\
\text { Expt J } \\
\text { Expt J } \\
\text { Expt } 4 \\
x+3 C\end{array}$ & $\begin{array}{l}1 Z V \\
Y F \\
Y F \\
\text { J BAS } \\
\text { JNP } \\
\text { JNP } \\
\text { EXFOR } \\
\text { PECtI }\end{array}$ & $\begin{array}{r}43 \\
27 \\
14 \\
43 \\
27 \\
14 \\
\text { RADO18 } \\
18100\end{array}$ & $\begin{array}{r}148 \\
7 \\
676 \\
124 \\
3 \\
379 \\
3.006 \\
\text { (E) }\end{array}$ & $\begin{array}{r}79 \\
178 \\
71 \\
79 \\
78 \\
472 \\
379\end{array}$ & $\begin{array}{l}5 \cdot 0+1 \\
5 \cdot 0+1 \\
5 \cdot 0+1 \\
5 \cdot 0+1 \\
5 \cdot 0+1 \\
5 \cdot 0+1 \\
5 \cdot 0+1\end{array}$ & & $\begin{array}{l}\text { Vdovint TBL. EMULSION. KIN. ANALYSIS. } \\
\text { Golihov+ TBL. EMULSION. KIN. ANALYSIS. } \\
\text {. INSTRUMENTATION. } \\
\text {. TRANSLATION OF IZV } 43(1), 148 . \\
\text {. TRANSLATION OF YF } 27,7(19781 . \\
\text {. TRANSLATION OF YF } 14,676(1971) \\
\text {. I DATA POINT. FROM YF } 27,7(1978) \text {. }\end{array}$ \\
\hline 530 GSF & $\begin{array}{l}\text { Eval J } \\
\text { Eval R }\end{array}$ & $\begin{array}{l}\text { J } A J / S \\
\text { NASA- }\end{array}$ & $\begin{array}{c}40 \\
-T M-70\end{array}$ & $\begin{array}{l}487 \\
9654\end{array}$ & $\begin{array}{r}779 \\
79\end{array}$ & $\begin{array}{l}2.1+1 \\
2.1+1\end{array}$ & $\begin{array}{l}4.0+2 \\
4.0+2\end{array}$ & $\begin{array}{l}\text { Ramaty+ CURV. } 3.684-.3 .853-M E V \text { GAMMAS. } \\
\text { SAME AS AJ/S } 40.487(1979) .\end{array}$ \\
\hline${ }^{16} 06, c^{2}$ & $2 p+d) 13 c$ & $\mathrm{c}$ & (E) & & & & & \\
\hline 531 LPI & $\begin{array}{ll}E x p t J \\
E x p t & J\end{array}$ & $\begin{array}{l}\text { IZV } \\
\text { I BAS }\end{array}$ & $\begin{array}{l}43 \\
43\end{array}$ & $\begin{array}{l}148 \\
124\end{array}$ & $\begin{array}{l}79 \\
79\end{array}$ & $\begin{array}{l}5 \cdot 0+1 \\
5 \cdot 0+1\end{array}$ & & $\begin{array}{l}\text { VdOVIN* TBL. EMULSION. KIN. ANALYSIS. } \\
\text { TRANSLATION OF } I Z V 4311), 148 .\end{array}$ \\
\hline 16010. & $p+3 \mathrm{He}, 13$ & ${ }^{3} \mathrm{C}$ & $\sigma(E)$ & & & & & \\
\hline $\begin{array}{l}532 \text { LPI } \\
1601 p, 3\end{array}$ & $\begin{array}{l}\text { Expl J } \\
\text { Expt J } \\
\text { Expt J } \\
\text { Expt J } \\
\text { Expt J } \\
\text { Expt J } \\
\text { Expl } 4 \\
3 p 1{ }^{14} \mathrm{C}\end{array}$ & $\begin{array}{l}1 Z V \\
Y F \\
Y Y F \\
B A S \\
S N P \\
\text { JNP } \\
\text { EXFOR } \\
\text { O(E) }\end{array}$ & $\begin{array}{r}43 \\
27 \\
14 \\
43 \\
27 \\
14 \\
\text { RAO018 } \\
1\end{array}$ & $\begin{array}{r}148 \\
7 \\
676 \\
124 \\
3 \\
379 \\
.005\end{array}$ & $\begin{array}{r}79 \\
178 \\
71 \\
79 \\
78 \\
472 \\
379\end{array}$ & $\begin{array}{l}5 \cdot 0+1 \\
5 \cdot 0+1 \\
5 \cdot 0+1 \\
5 \cdot 0+1 \\
5 \cdot 0+1 \\
5 \cdot 0+1 \\
5 \cdot 0+1\end{array}$ & & $\begin{array}{l}\text { Vdovin+ TBL. EMULSION, KIN. ANALYSIS. } \\
\text { GOI ikOVt TBL. EMULSION. KIN. ANALYSIS. } \\
\text {. INSTRUMENTATION. } \\
\text {. TRANSLATION OF IZV } 43(1), 148 . \\
\text {. TRANSLATION OF YF } 27,7(1978) \\
\text {. TRANSLATION OF YF } 14.676(1971) . \\
\text {. I DATA POINT. FROM YF } 27.7(1978) .\end{array}$ \\
\hline & $\begin{array}{ll}\text { Expt } J \\
\text { Expt }\end{array}$ & $\begin{array}{l}\text { IZV } \\
\text { BAS }\end{array}$ & $\begin{array}{l}43 \\
43\end{array}$ & $\begin{array}{l}148 \\
124\end{array}$ & $\begin{array}{l}79 \\
79\end{array}$ & $\begin{array}{l}5 \cdot 0+1 \\
5 \cdot 0+1\end{array}$ & & $\begin{array}{l}\text { Vdorint TEL. EMULSION. KIN. ANALYSIS. } \\
\text { TRANSLATION OF } 1 Z V 43(1) .148 .\end{array}$ \\
\hline $\begin{array}{l}534 \text { LPI } \\
16060.0\end{array}$ & $\begin{array}{l}\text { Theo } J \\
d+t) 12^{2}\end{array}$ & $\int Y F$ & 31 & 119 & 80 & $1.6+2$ & & Dmitrievt IBL. DYNAMICAL MODEL. CFD EXP \\
\hline $1601 p, x$ & $\begin{array}{l}\text { Expt J } \\
\text { Expt J } \\
\text { Expt J } \\
\text { Expt J } \\
\text { Expt J } \\
\text { Expt J } \\
\text { Expl } 4 \\
x{ }^{13} N\end{array}$ & $\begin{array}{l}I Z V \\
Y F \\
Y F \\
B A S \\
S N N P \\
J N P \\
\text { JXFOR } \\
\text { a(E) }\end{array}$ & $\begin{array}{r}43 \\
27 \\
14 \\
43 \\
27 \\
14 \\
\text { RAO018 }\end{array}$ & $\begin{array}{r}148 \\
7 \\
676 \\
124 \\
3 \\
379 \\
006\end{array}$ & $\begin{array}{r}79 \\
178 \\
71 \\
79 \\
78 \\
472 \\
379\end{array}$ & $\begin{array}{l}5.0+1 \\
5.0+1 \\
5.0+1 \\
5.0+1 \\
5.0+1 \\
5.0+1 \\
5.0+1\end{array}$ & & $\begin{array}{l}\text { VdOVIN TBL. EMULSION. KIN. ANALYSIS. } \\
\text { GOIIhOV+ TBL. EMULSION. KIN. ANALYSIS. } \\
\text {. INSTRUMENTATION. } \\
\text {. TRANSLATION OF IZV } 43(1), 148 . \\
\text {. TRANSLATION OF YF } 27,7(1978) . \\
\text {. IRANSLATION OF YF } 14,676(1971) . \\
\text { I DATA POINT. FROM YF } 27.7(1978) \text {. }\end{array}$ \\
\hline $\begin{array}{l}536 \text { LPI } \\
1601 P, P\end{array}$ & $\begin{array}{l}\text { Theo } J \\
p+t, 13^{N}\end{array}$ & $\begin{array}{l}\text { Y } \\
\text { OIE }\end{array}$ & 31 & 119 & Bo & $1.6+2$ & & Omitrievt TBL. DYNAMICAL MODEL. CFD EXP \\
\hline $16010 . \bar{c}$ & $\begin{array}{l}\text { Expt J } \\
\text { Expt J } \\
\text { Expt J } \\
\text { Expt J } \\
\text { Expt J } \\
\text { Expt J } \\
\text { Expt } 4 \\
\text { Ed }{ }^{13} \mathrm{~N}\end{array}$ & $\begin{array}{l}\text { IZV } \\
Y F \\
Y F \\
\text { BAS } \\
\text { SNP } \\
\text { SNP } \\
\text { EXFOR } \\
\\
\sigma(E)\end{array}$ & $\begin{array}{r}43 \\
27 \\
14 \\
43 \\
27 \\
14 \\
R A 0018\end{array}$ & $\begin{array}{r}148 \\
7 \\
676 \\
124 \\
3 \\
379 \\
005\end{array}$ & $\begin{array}{r}79 \\
178 \\
71 \\
79 \\
78 \\
472 \\
379\end{array}$ & $\begin{array}{l}5 \cdot 0+1 \\
5 \cdot 0+1 \\
5 \cdot 0+1 \\
5 \cdot 0+1 \\
5 \cdot 0+1 \\
5 \cdot 0+1 \\
5 \cdot 0+1\end{array}$ & & $\begin{array}{l}\text { VdoVint TBL. EMULSION. KIN. ANALYSIS. } \\
\text { GOI IKOV+ TBL. EMULSION. KIN. ANALYSIS. } \\
\text {. INSTRUMENTATION. } \\
\text {. TRANSLATION OF IZV } 43(1), 148 . \\
\text {. TRANSLATION OF YF } 27,7(1978) . \\
\text {. TRANSLATION OF YF } 14,676(1971) . \\
\text {. I DATA POINT. FROM YF } 27,7(1978) .\end{array}$ \\
\hline $\begin{array}{l}538 \mathrm{LPI} \\
539 \mathrm{LPI}\end{array}$ & 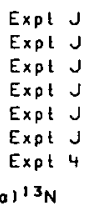 & $\begin{array}{l}S N P \\
I Z V \\
Y F \\
Y F \\
B A S \\
S N P \\
\text { EXFOR } \\
\text { O(E) }\end{array}$ & $\begin{array}{r}14 \\
43 \\
27 \\
14 \\
43 \\
27 \\
\text { RAD0 18 }\end{array}$ & $\begin{array}{r}379 \\
148 \\
7 \\
676 \\
124 \\
3 \\
005\end{array}$ & $\begin{array}{r}472 \\
79 \\
178 \\
71 \\
79 \\
78 \\
379\end{array}$ & $\begin{array}{l}5.0+1 \\
5.0+1 \\
5.0+1 \\
5.0+1 \\
5.0+1 \\
5.0+1 \\
5.0+1\end{array}$ & & $\begin{array}{l}\text {. TRANSLATION OF YF } 14,676(1971) \text {. } \\
\text { VdOVIIN+ TBL. EMULSION. KIN. ANALYSIS. } \\
\text { GOIikOV+ TBL. EMULSION. KIN. ANALYSIS. } \\
\text {. INSTRUMENTATION. } \\
\text {. TRANSLATION OF IZV } 43(1) .148 . \\
\text {. IRANSLATION OF YF } 27,7(1978) \\
\text {. I DATA POINT. FROM YF } 27.7(1978) \text {. }\end{array}$ \\
\hline $\begin{array}{c}540 \text { ORL } \\
541 \mathrm{KLN} \\
160(p,\end{array}$ & $\begin{array}{l}\text { Eval J } \\
\text { Expl J } \\
a)^{13} \mathrm{~N}\end{array}$ & $\begin{array}{l}\text { IRE } \\
\text { JP/A } \\
\text { thlch }\end{array}$ & $\begin{array}{c}26 \\
286 \\
\text { targ }\end{array}$ & $\begin{array}{r}1713 \\
523 \\
\text { get y }\end{array}$ & $\begin{array}{r}279 \\
877 \\
\text { ie Id }\end{array}$ & $\begin{array}{l}\text { TR } \\
6.7+0\end{array}$ & $\begin{array}{l}1.8+1 \\
9 \cdot 2+0\end{array}$ & $\begin{array}{l}\text { Wieland+ CURV. } \\
\text { Gruhle+ CURV. ACTIV. ALSO REACTION RATE }\end{array}$ \\
\hline $542 O R L$ & Eval J & IRE & 26 & 1713 & 279 & TR & $1.8+1$ & Wielandt CURV. SATUR, YLD. FROM SIGMA. \\
\hline
\end{tabular}




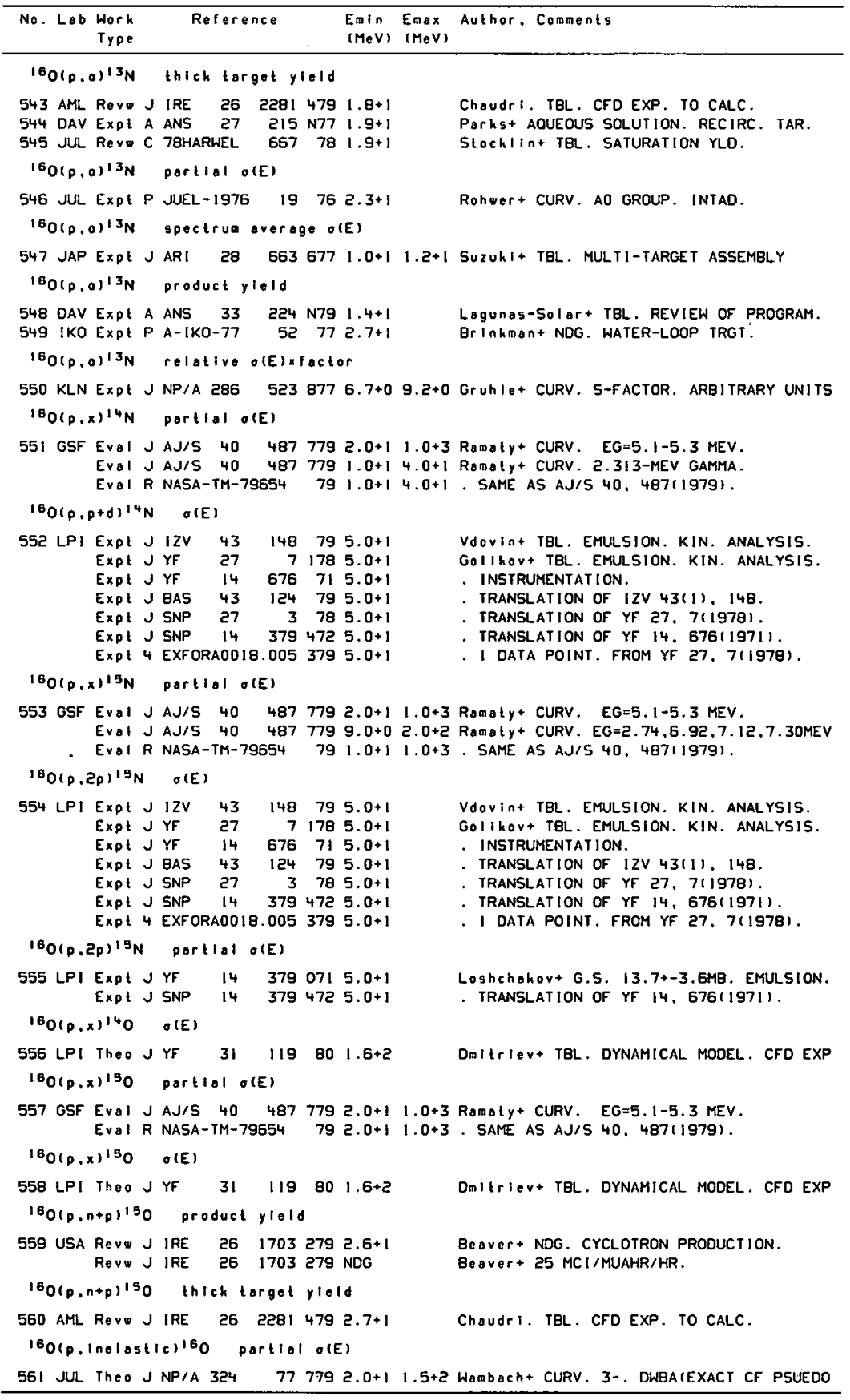


REFERENCES ( c on t)

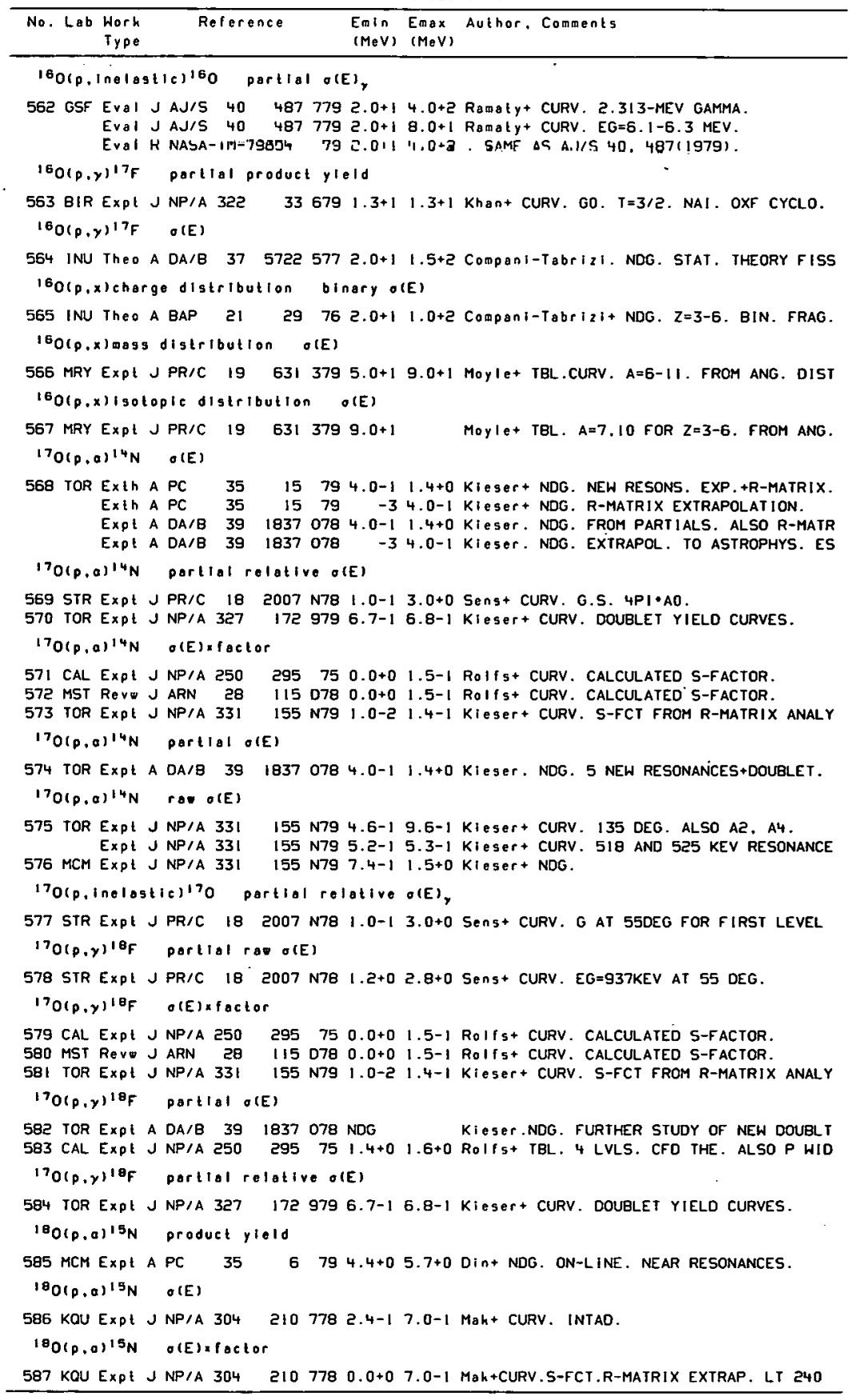


REFERENCES (cont)

\begin{tabular}{cccc}
\hline No. Lab Work & Reference & $\begin{array}{l}\text { Emin Emax Author. Comments } \\
\text { Type }\end{array}$ & (MeV) (MeV) \\
\hline
\end{tabular}

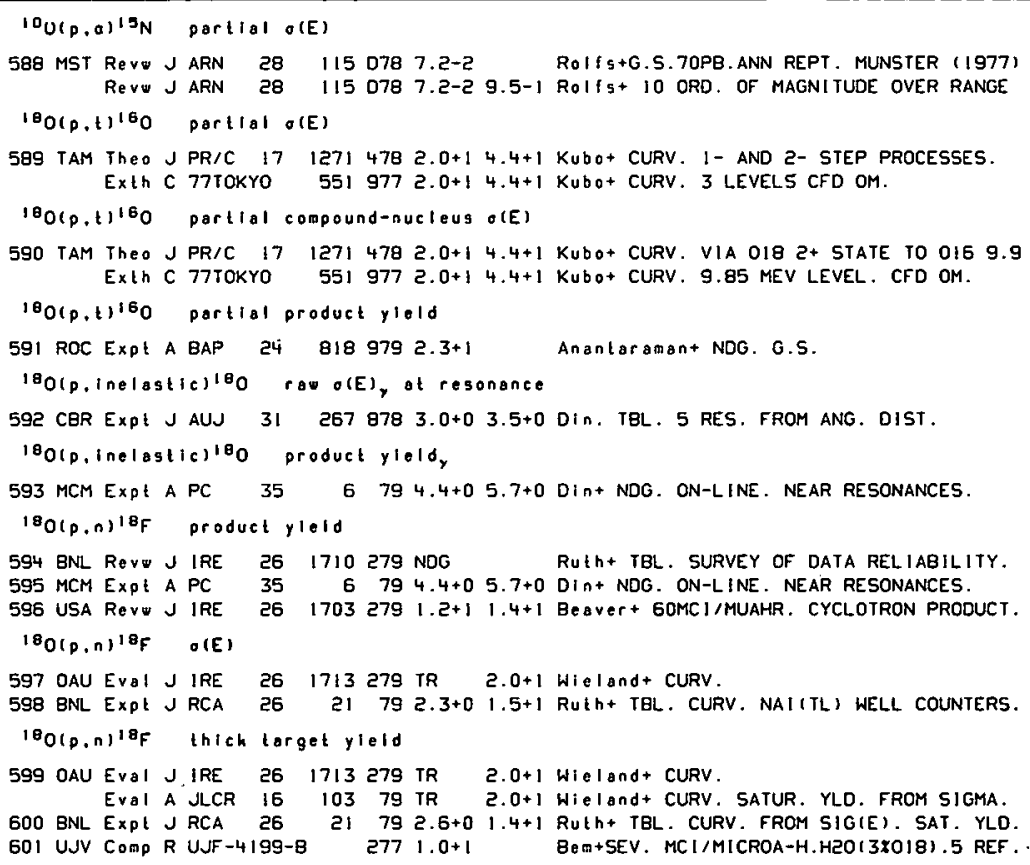




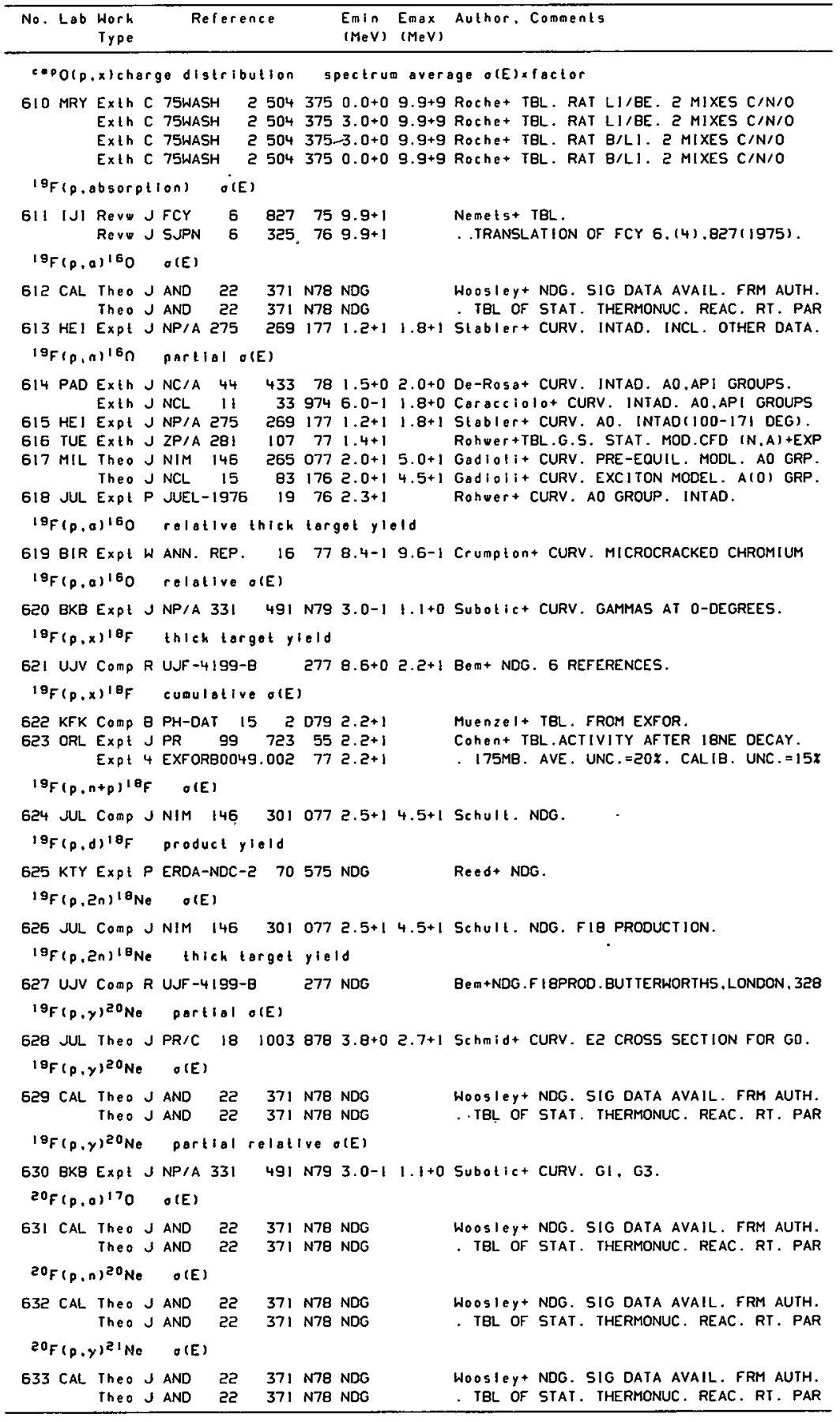


REFERENCES (c ont)

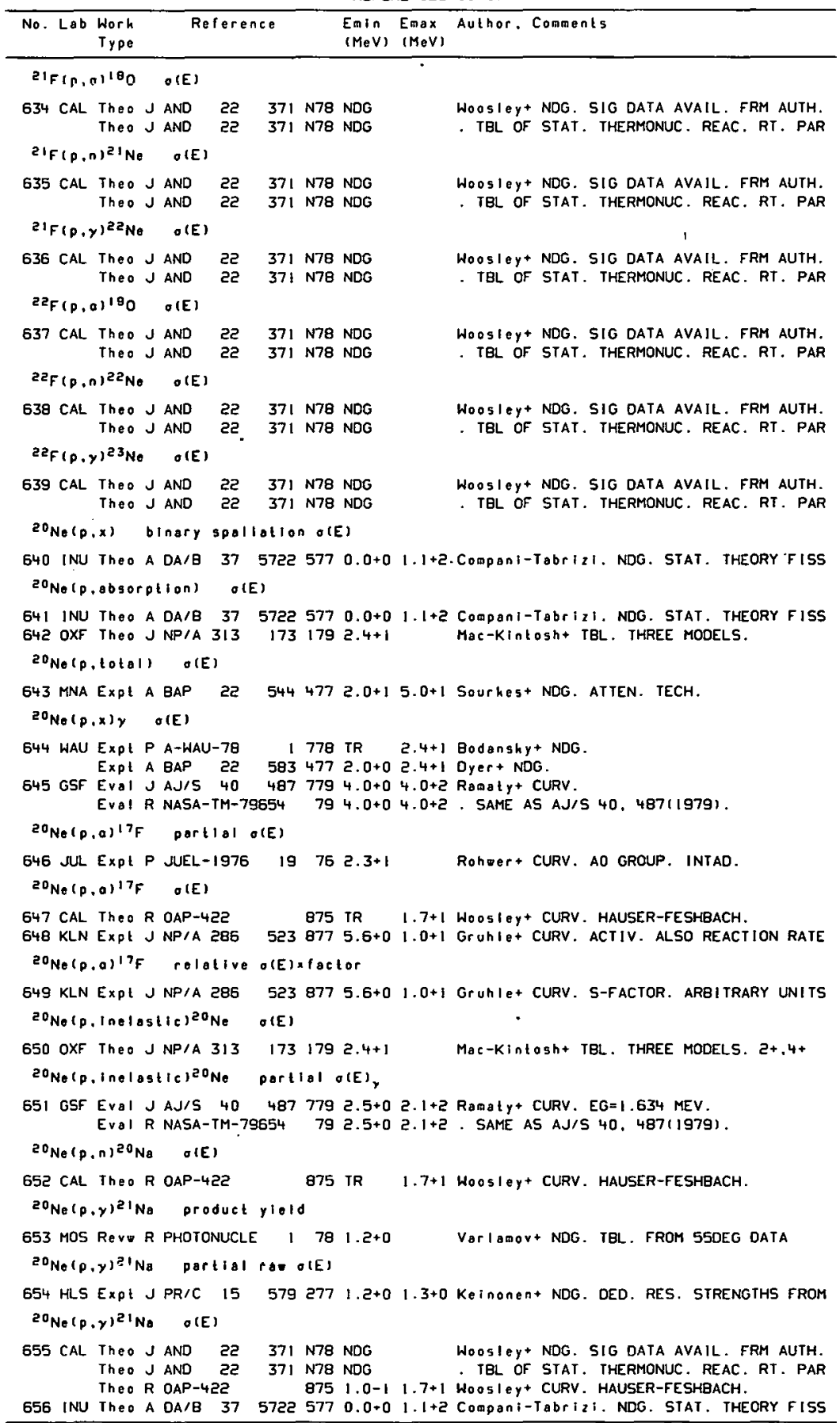




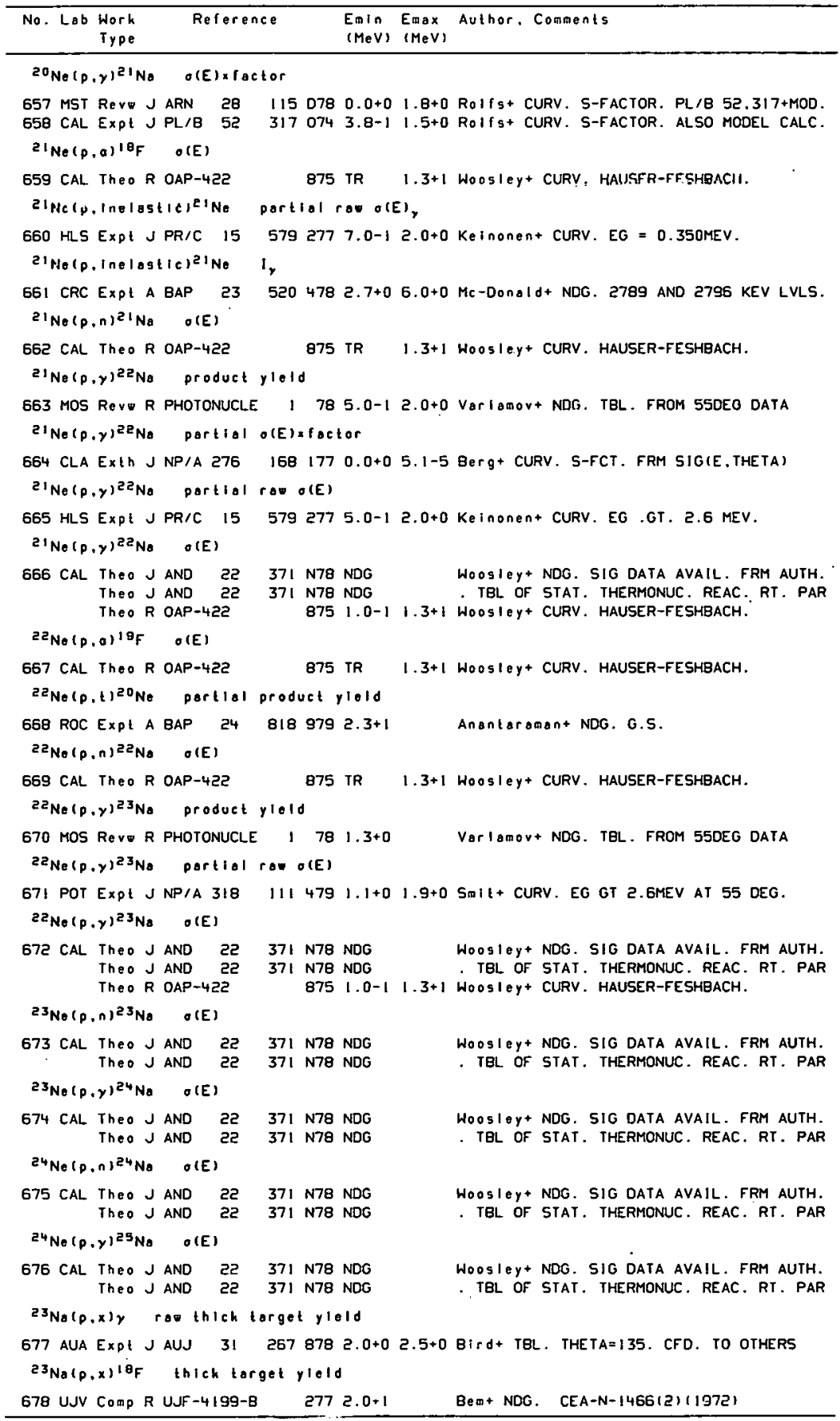


REFERENCES ( c on t)

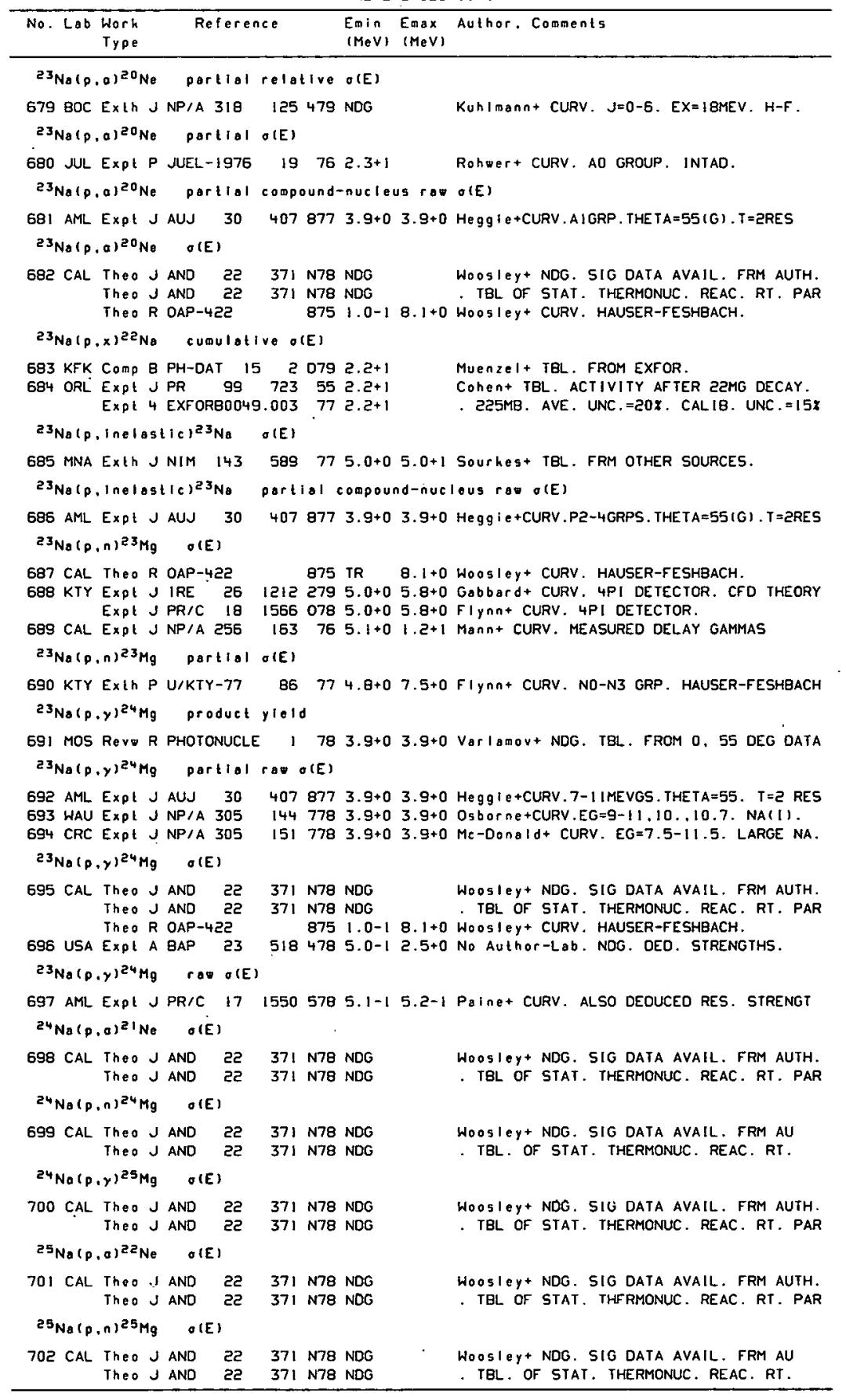


REFERENCES (cont)

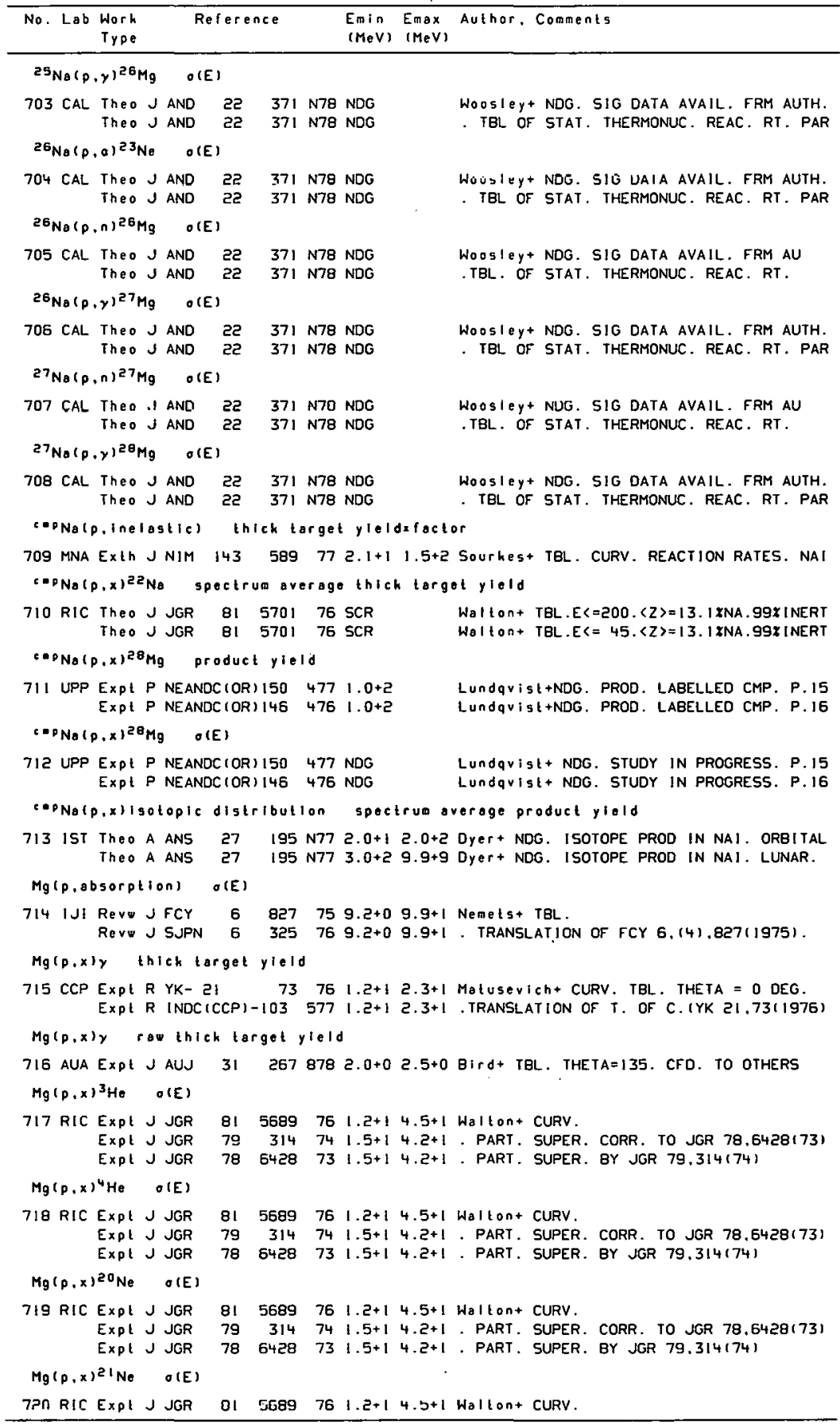




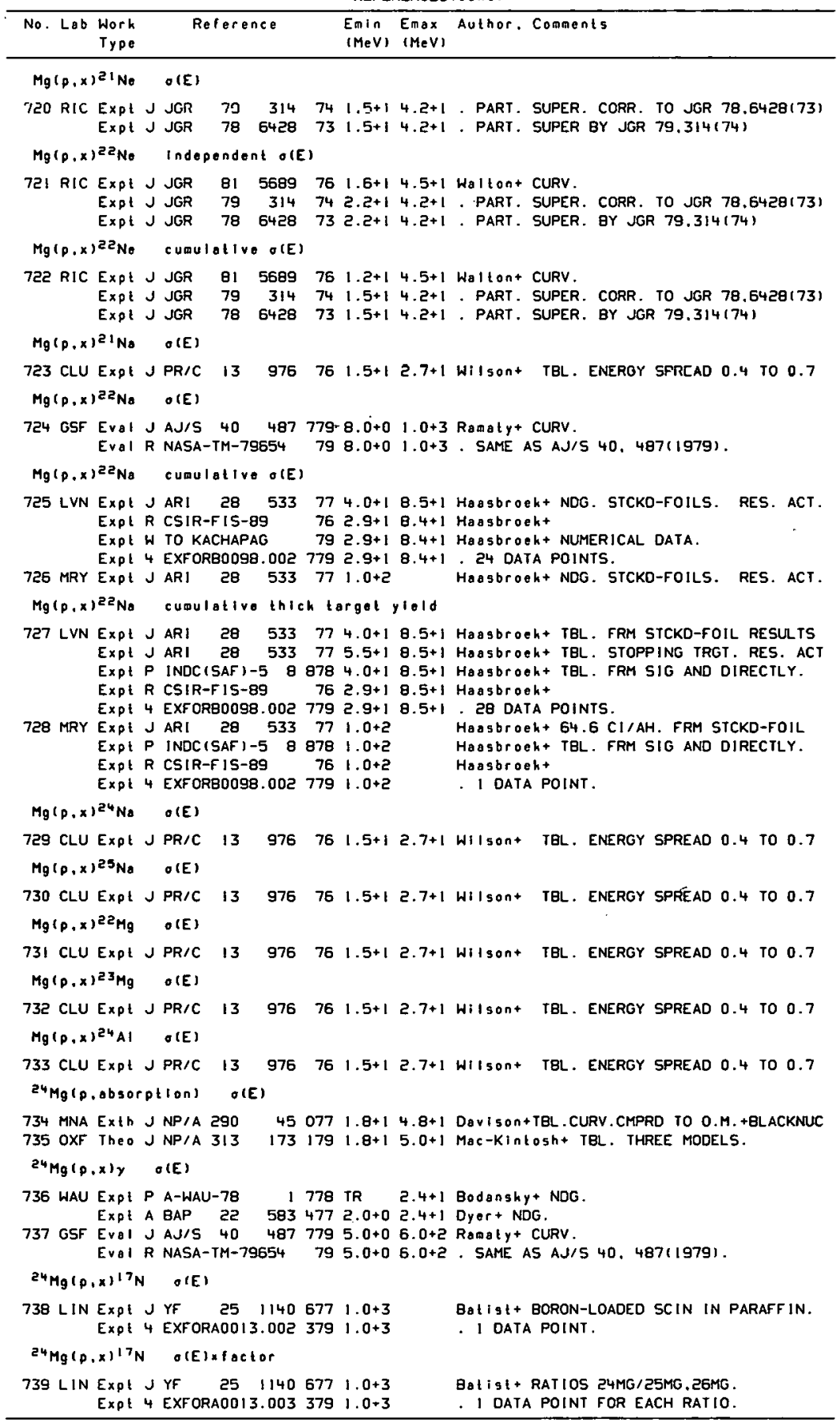


REFERENCES ( c on ()

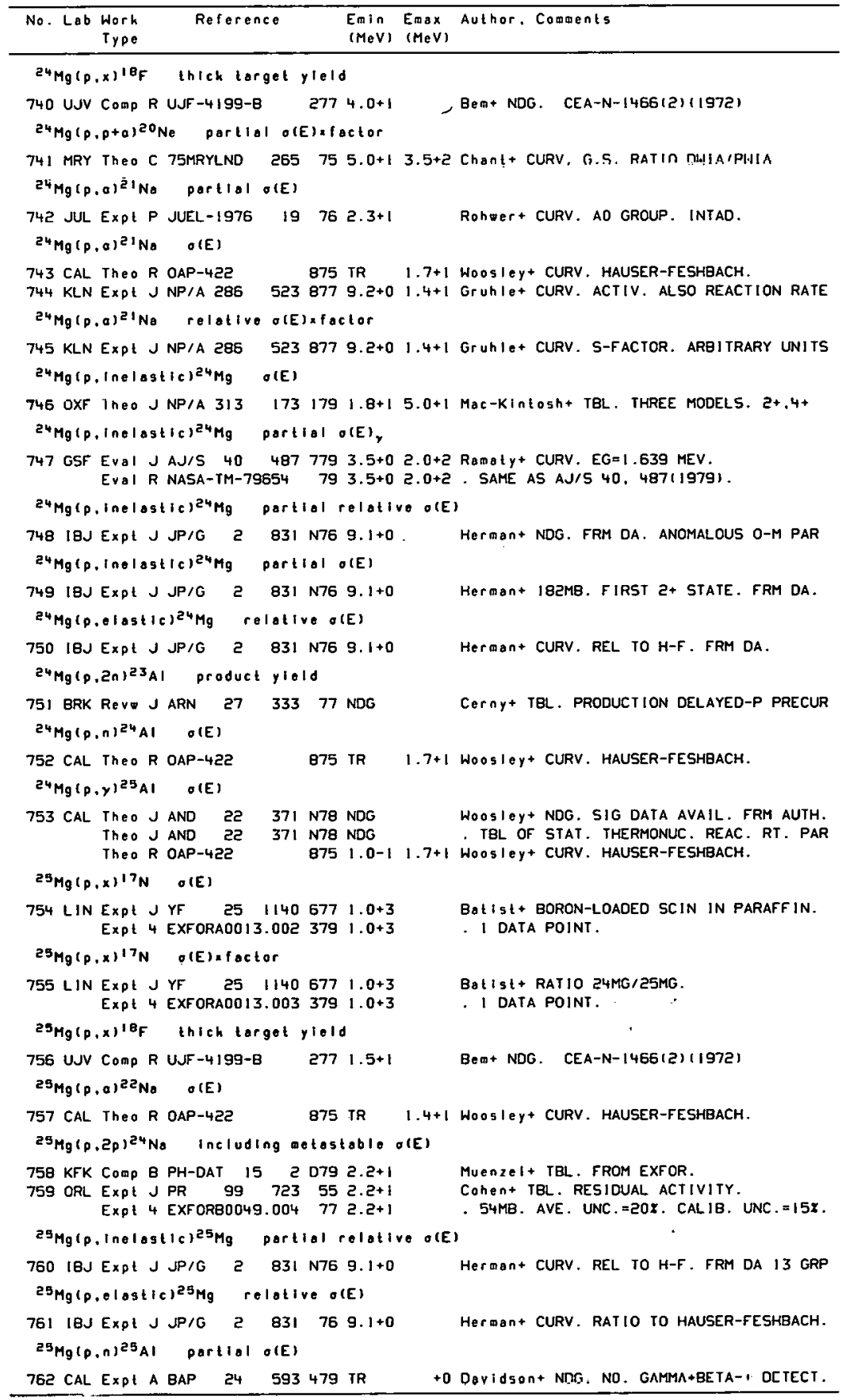




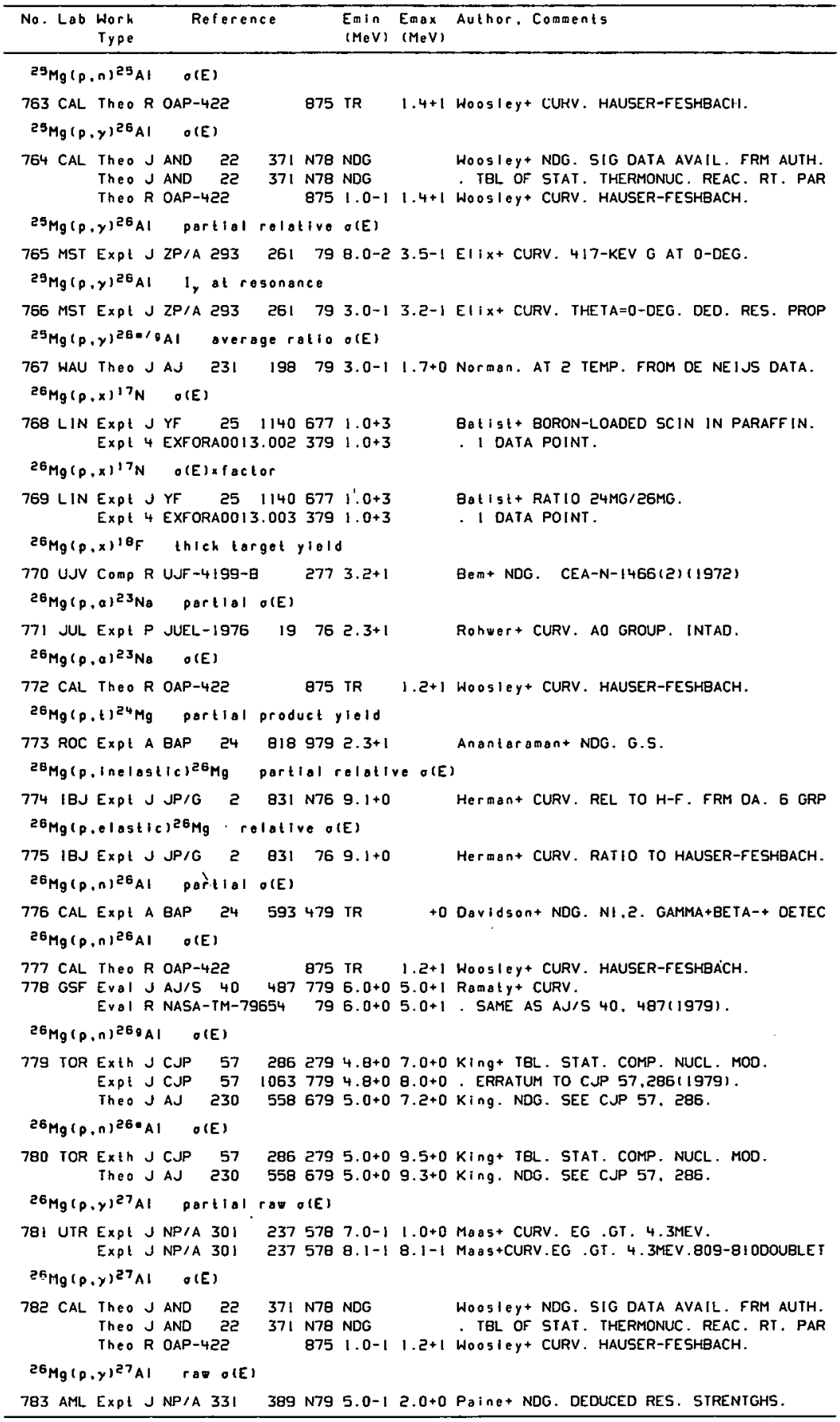


REFERENCES ( 0 ont)

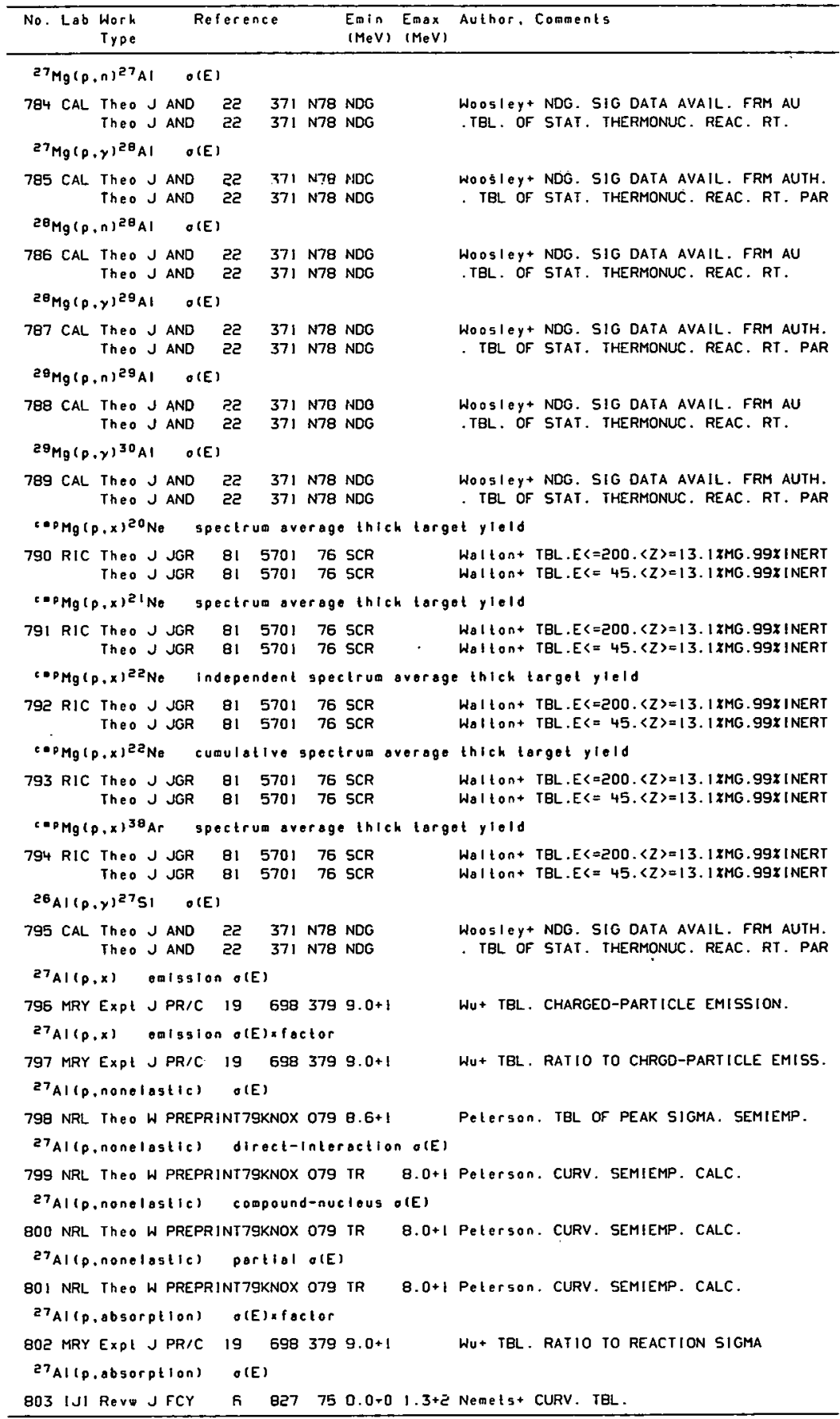




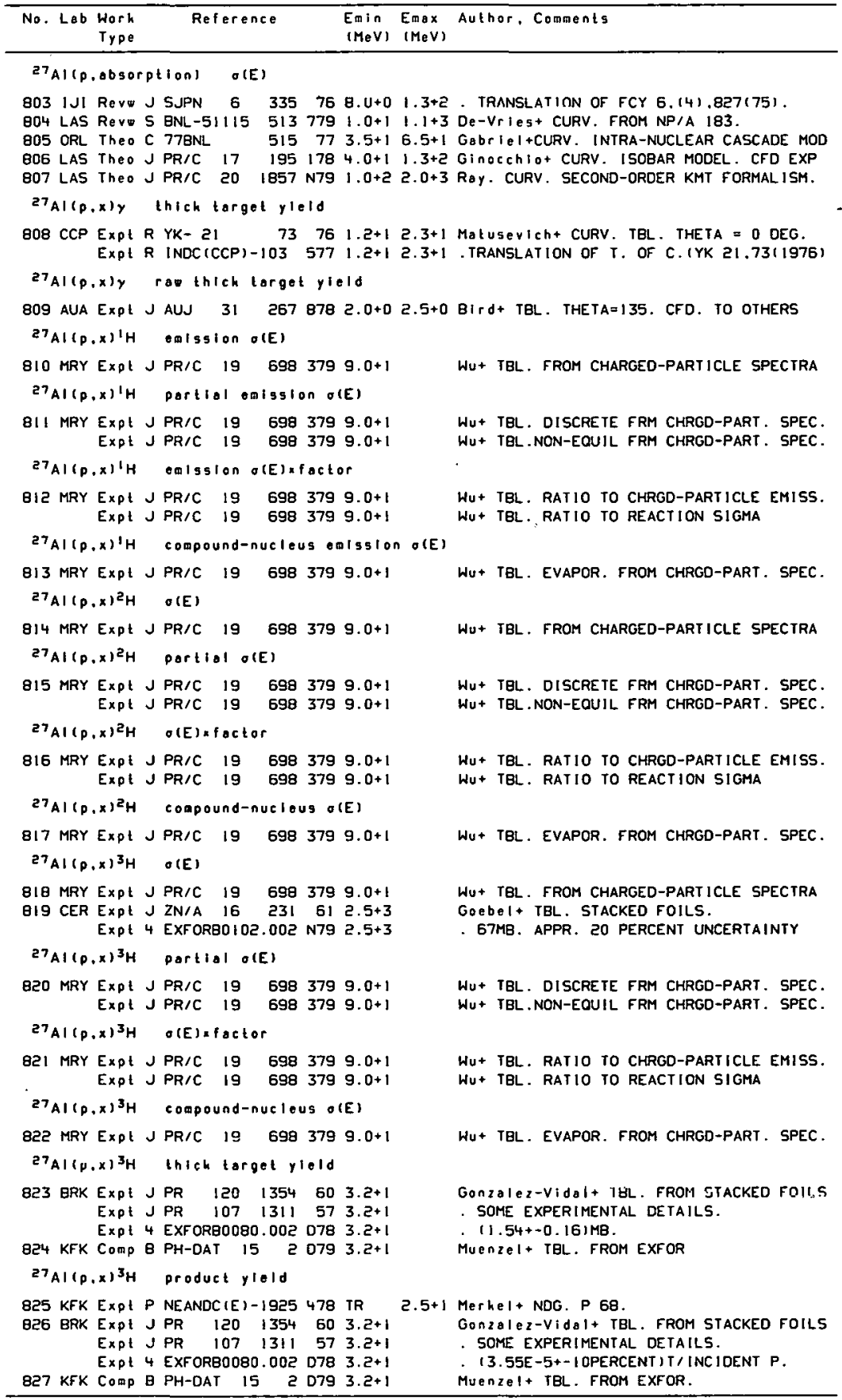


REFERENCES ( $\operatorname{con}$ ()

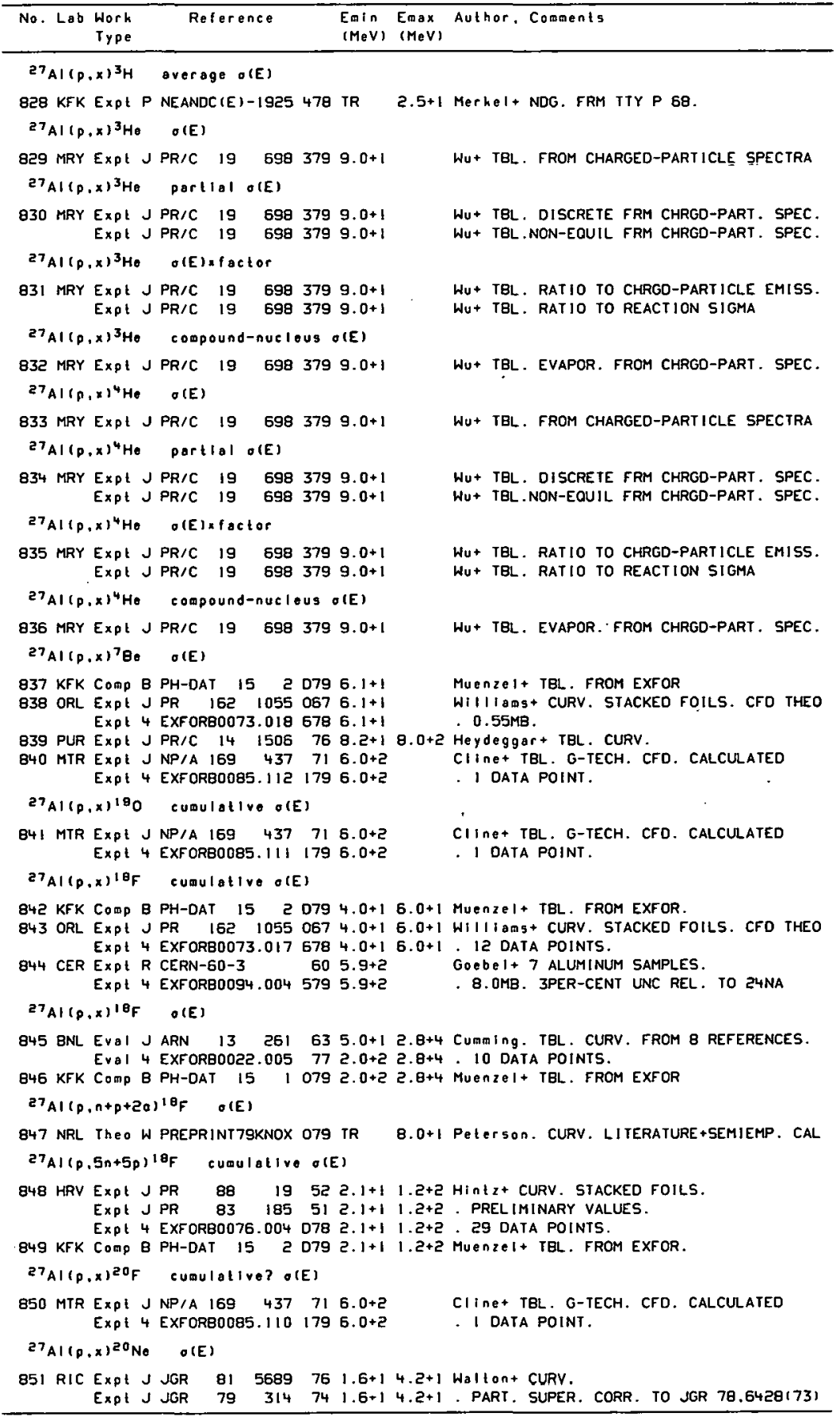


REFERENCES (cont)

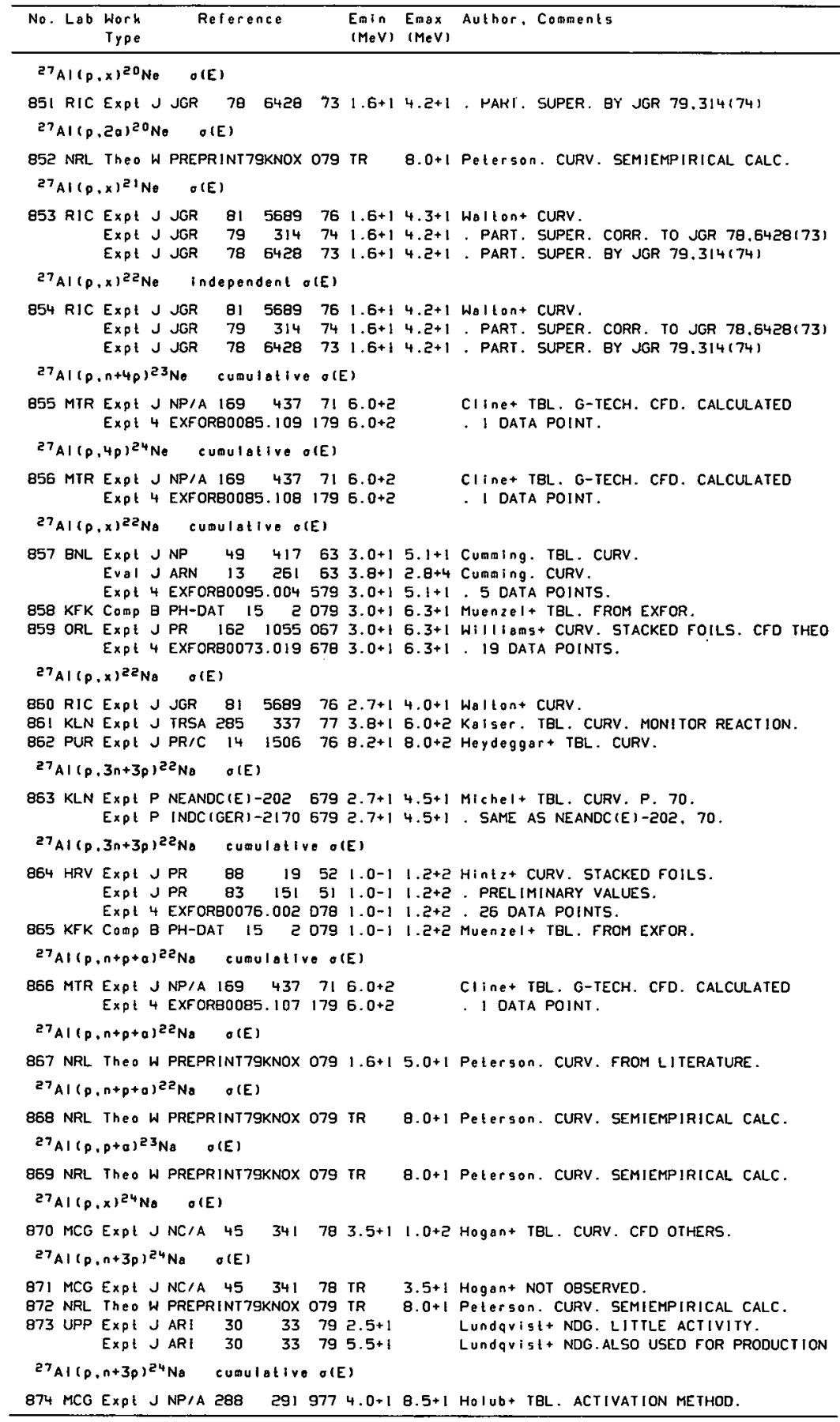


REFERENCES ( cont)

\begin{tabular}{|c|c|c|c|c|c|c|}
\hline No. Lab & $\begin{array}{l}\text { Work } \\
\text { Type }\end{array}$ & Reference & $\begin{array}{l}\text { Emin } \\
\text { (MeV) }\end{array}$ & $\begin{array}{l}\text { Emax } \\
\text { (MeV) }\end{array}$ & Author, & Comments \\
\hline
\end{tabular}

$27 A I(p, n+3 p)^{24} \mathrm{Na}$ cumulative o(E)

874 MCG Expt J NP/A 288 29I $9774.0+11.1+2$ Holub+ CURV.CALC. AND EXPER. Expt 4 EXFORBO082:002 $1794.0+18.5+1$. 12 DATA POINTS.

$27 A \mid(p, n+3 p){ }^{24} \mathrm{Na}$ Including metastable o(E)

975 HRV [MPL J R D $19521.3+1$ l. $5+2$ Hintz+ CURV. STACKED FOILS. EXPt JPR $83 \quad 151511.3+11.2+2$. PRELIMINARY VALUES.

Expt 4 EXFORE0076.003 D78 $1.3+1 \quad 1.2+2.37$ DATA POINTS

876 KFK Comp B PH-DAT I5 2 D79 $1.3+11.2+2$ Muenzel+ TBL. FROM EXFOR. Comp B PH-DAT 15 l $0792.7+12.8+4$ Muenzel+ TBL. FROM EXFOR.

877 MCG Expl T S. MEGHIR+ 62 2.7+1 8.6+1 Meghir. RESIDUAL ACTIVITY. EXPL 4 EXFORBO016.003 N78 $2.7+18.6+1$. 15 DAIA POINIS.

878 BNL Eval J ARN 13 251 $633.2+13.0+4$ Cumming. TBL. CURV. FROM 14 REFERENCES. Eval 4 EXFORBO022.003 $775.0+12.8+4$, I5 DATA POINTS

B79 BNL Expt J NP $49417633.7+15.1+1$ CumIIng. TBL. CURV. Expt 4 EXFORB0095.003 $5793.7+15.1+1.4$ DATA POINTS.

880 BRK Expt JPR $10 ! 329563.5+2 \quad$ Crandall + TBL. STACKED FOILS. Expt 4 EXFORBO101.005 N79 $3.5+2 \quad(11.1+-0.2) \mathrm{MB}$.

881 CER Expt J NP 24 28 $615.9+2$ Goebel+ TBL. 7 ALUMINUM SAMPLES. Expt R CERN-60-3 60 $5.9+2$ Goebe 1+ Expt 4 EXFORBO094.002 $5795.9+2 \quad$. $111.0+-0.51 \mathrm{MB}$.

$27 A \mid(p, n+3 p)^{24} \mathrm{Na}$ Including metastable olE)

882 BNL Eval J ARN $13261633.2+12.8+4$ Cumning.TBL.CURV.RATCIZ(P,NP)/AL (P, 3PN) Eval 4 EXFORBO022.004 $775.0+12.8+4,15$ DATA POINTS.

883 KFK Comp B PH-DAT $1510795.0+12.8+4$ Muenzel+ TBL. FROM EXFOR.

884 CER Expl J NP $24 \quad 28615.9+2$ GoebeI+RATIO ICCIP,NPI/27AL(P,N3P)

${ }^{27} A l(p, n+3 p){ }^{24} N o$ including aetostoble olE

885 KFK COMP B PH-DAT 15 1 $0793.4+16.1+1$ Muenzel+ TBL. FROM EXFOR.

886 ORL EXPi J PR $16210550673.4+16.1+1$ Williams + CURV. STACKED FOILS. CFD THEO Expt 4 EXFORB0073.020 $6783.4+16.1+1$. 12 DATA POINTS.

$27 A \mid(p, n+3 p)^{24} N_{0}$ Including metastable o(E)xfoctor

887 BRK Expl J PR $101329563.4+2 \quad$ Crandall+ C12(P.N+P)/AL27(P.N+3P) EXPl 4 EXFORBO101.009 N79 $3.4+2 \quad 3.21$

$27 \mathrm{~A} I(p, n+3 p)^{24} \mathrm{Na} \quad 0(E)$

688 NRL Theo W PREPRINT79KNOX $0792.9+1$ 8.0+1 Peterson. CURV. FROM LITERATURE.

$27 \mathrm{Al}\left(p, p+{ }^{3} \mathrm{He}\right){ }^{24} \mathrm{No} \quad$ o(E)

889 MCG Expt J NC/A $45 \quad 34178$ TR $3.5+1$ Hogan+ NOT OBSERVEO.

$27 A I(p, 3 p)^{25} N_{0}$ cumulative o(E)

890 MTR EXPt J NP/A $169437716.0+2 \quad$ CIINE+ TBL. G-TECH. CFD. CALCULATED EXPE 4 EXFORB0085.106 $1796.0+2 \quad$. I DATA POINT.

$2{ }^{7} A I(P, X)^{23} \mathrm{Mg} \quad O(E)$

$891 \mathrm{CLU}$ Expt $J$ PR/C $13976761.5+12.7+1$ Wilsont TBL. ENERGY SPREAD 0.4100 .7

$27 \mathrm{~A} 1(p, n+a)^{23} \mathrm{Mg} \quad \sigma(E)$

892 NRL Theo W PREPRINITGKNOX 079 TR 8.0+1 Peterson. CURV. SEMIEMPIRICAL CALC.

${ }^{27} A 1(p, 2 n+2 p)^{24} M g \quad O(E)$

893 NRL Theo W PREPRINT79KNOX 079 TR 8.0+1 Petergon. CURV. SEMIEMPIRICAL CALC.

${ }^{27} A I(0, Q)^{24} \mathrm{Mg}$ partial rao O(E)

694 DEB Expt J IZV 40 2!19 $761.5+01.9+0$ Zolnai+ CURV. A0. Al GRPS. THETA=BO DEG Expt J IZV $402119761.9+02.2+0201$ nait CURV. AO GRP. THETA=90,150 DEG.

EXPt J BAS $4078761.5+02.2+0$. TRANSLATION OF $12 \mathrm{~V} 40,2119$ (1976)

895 ANL Expl J NIM $1405192772.0+02.4+0$ Kennedy+ CURV. Al1) GROUP. MEAS. GAMMAS $27 A I(p, a)^{24} \mathrm{Mg}$ partiol o(E)

896 THU Expl J JP/G $3175377 \quad 1.1+0 \quad 3.1+0$ Hsu+CURV. TBL. AO GRP. LEG. COEF. GIVEN 897 DEB Expt J NIM 147 2B7 N77 1.5+0 $1.9+0$ Somogyi+FBL. NDG.USE OF PLASTICTRACK DET 898 JUL Expi P JUEL- $197619762.3+1 \quad$ Roheer + CURV. AD GROUP. INTAD.

$27 A I(p, a)^{24} \mathrm{Mg}$ oi(E)

899 CAL TheO J AND $22 \quad 371$ N78 NDG WoOSI EY + NDG. SIG DATA AVAIL. FRM AUTH. Theo $J$ AND $22 \quad 371$ N78 NDG . TEL OF STAT. THFRMONUC. REAC. RT. PAR Theo R OAP-422 875 5.2-1 $8.3+0$ WOOSley CURV. HAUSER-FESHBACH. 
REFERENCES ( c on t )

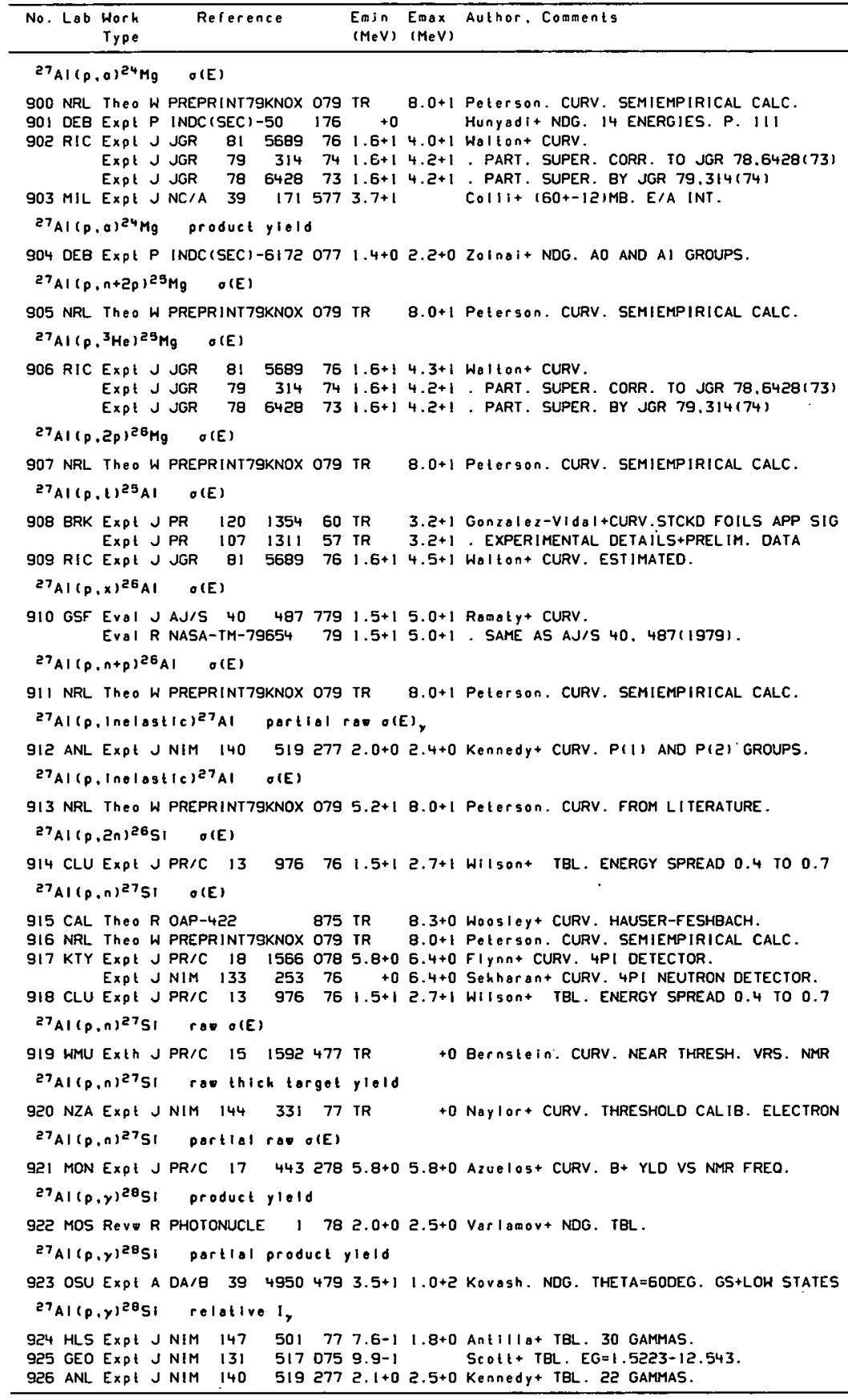




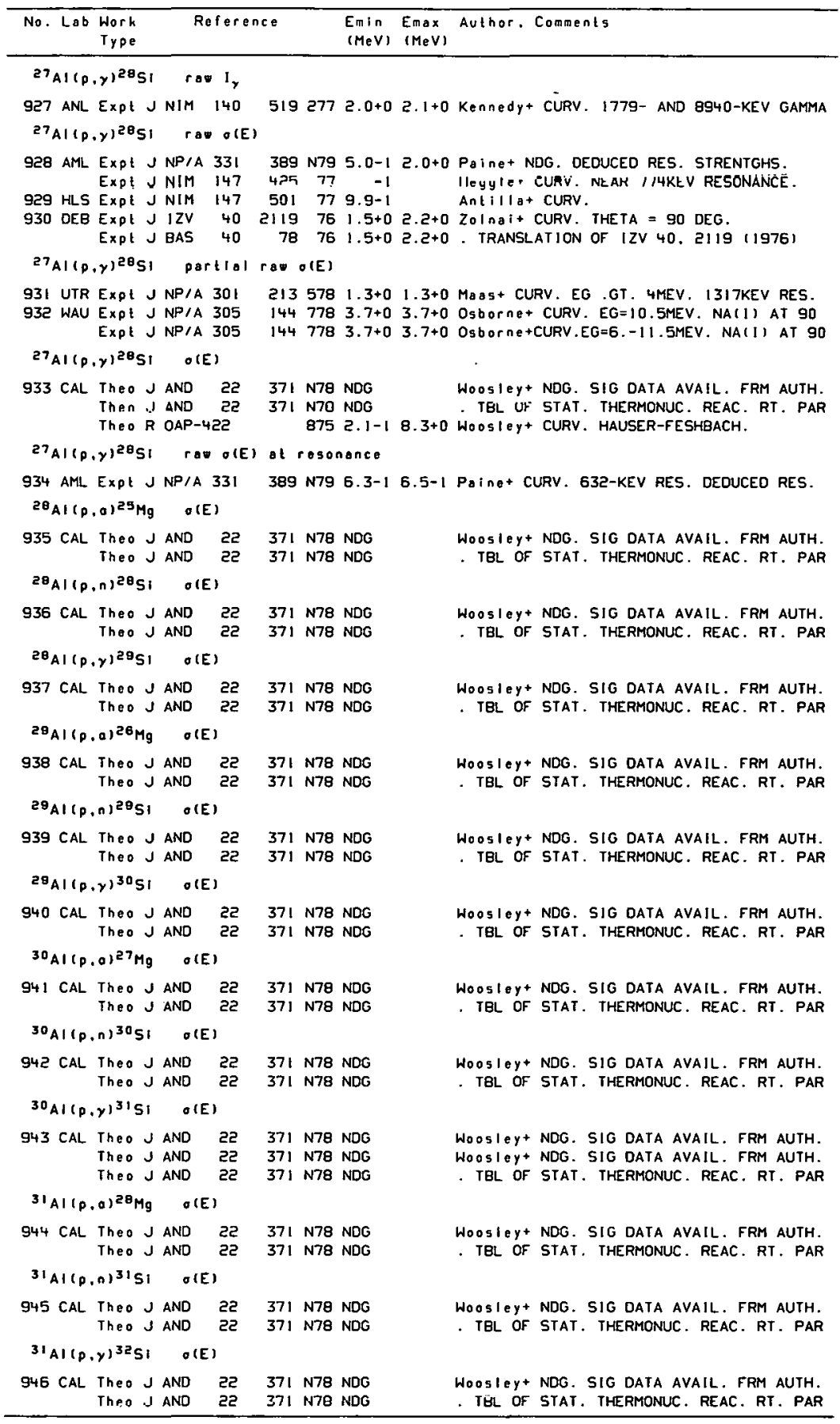




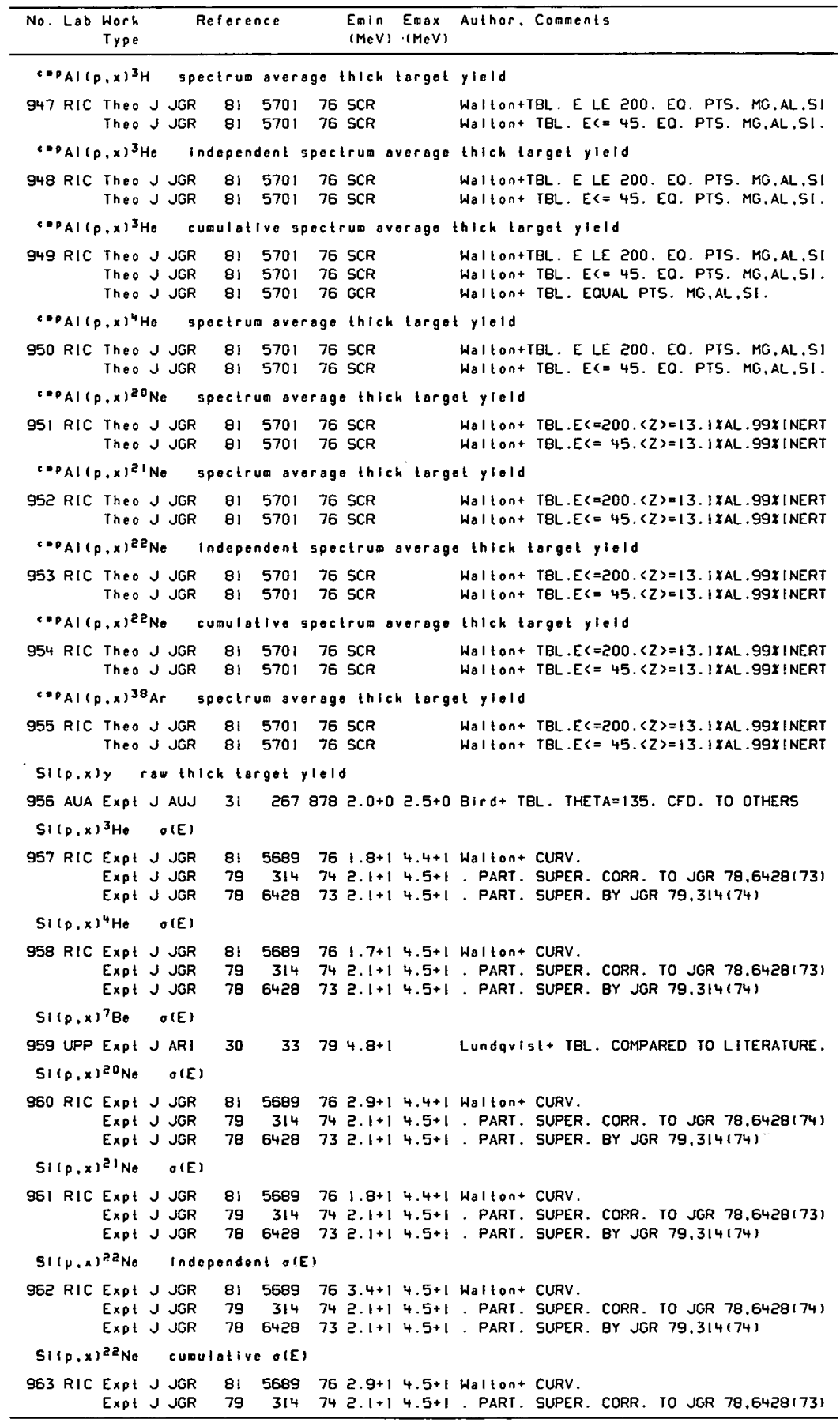


REFERENCES ( c on t )

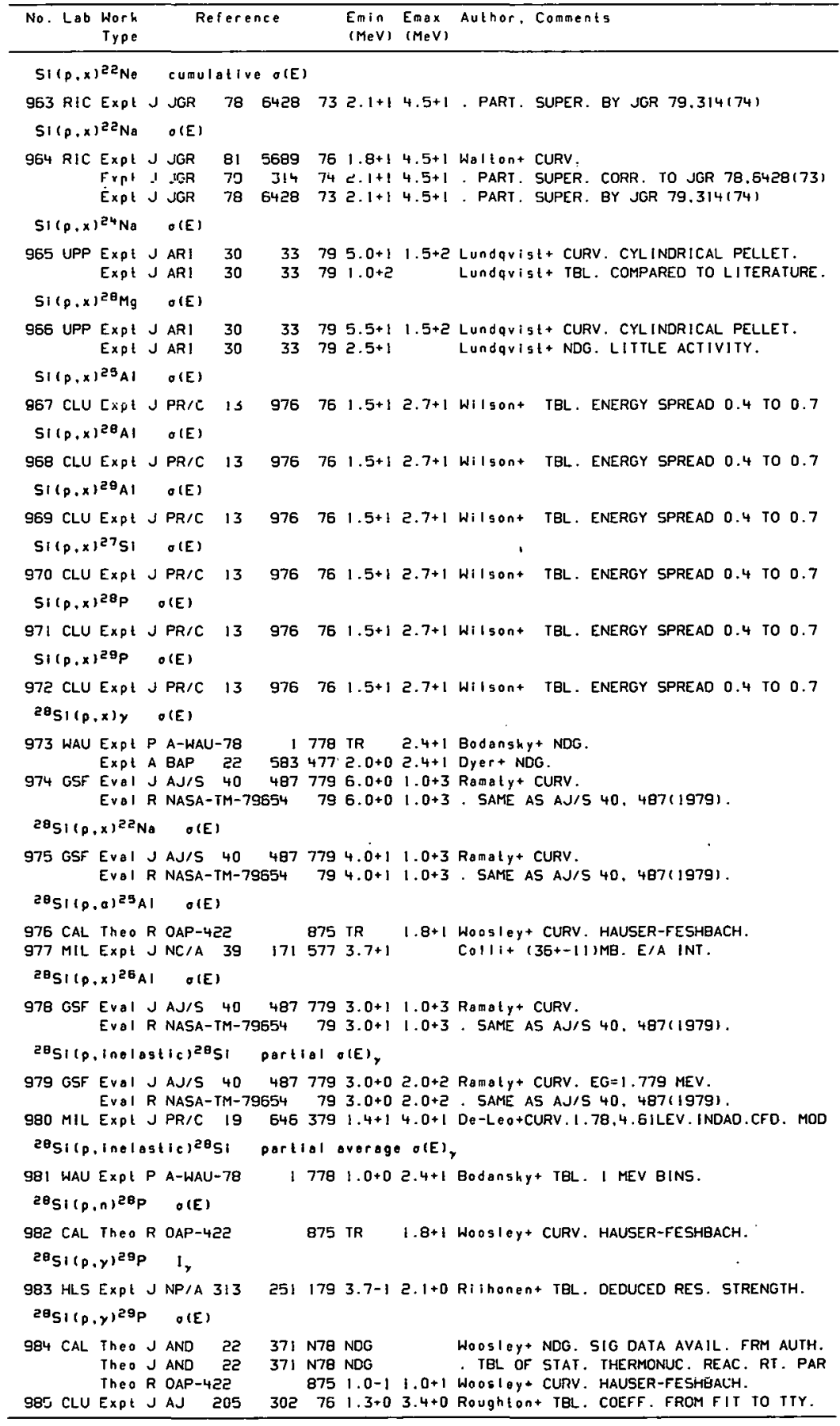




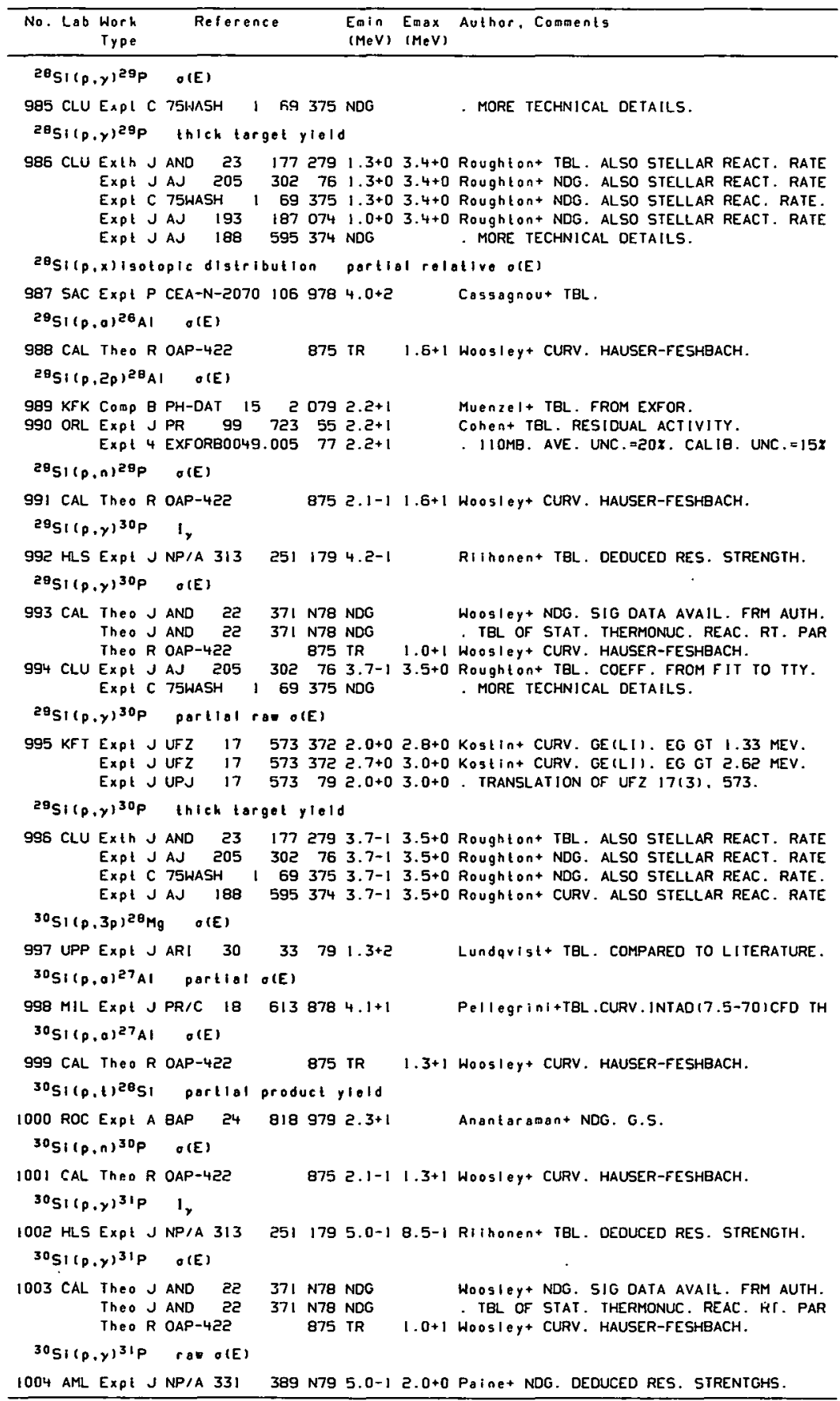


REFERENCES ( $c$ on t )

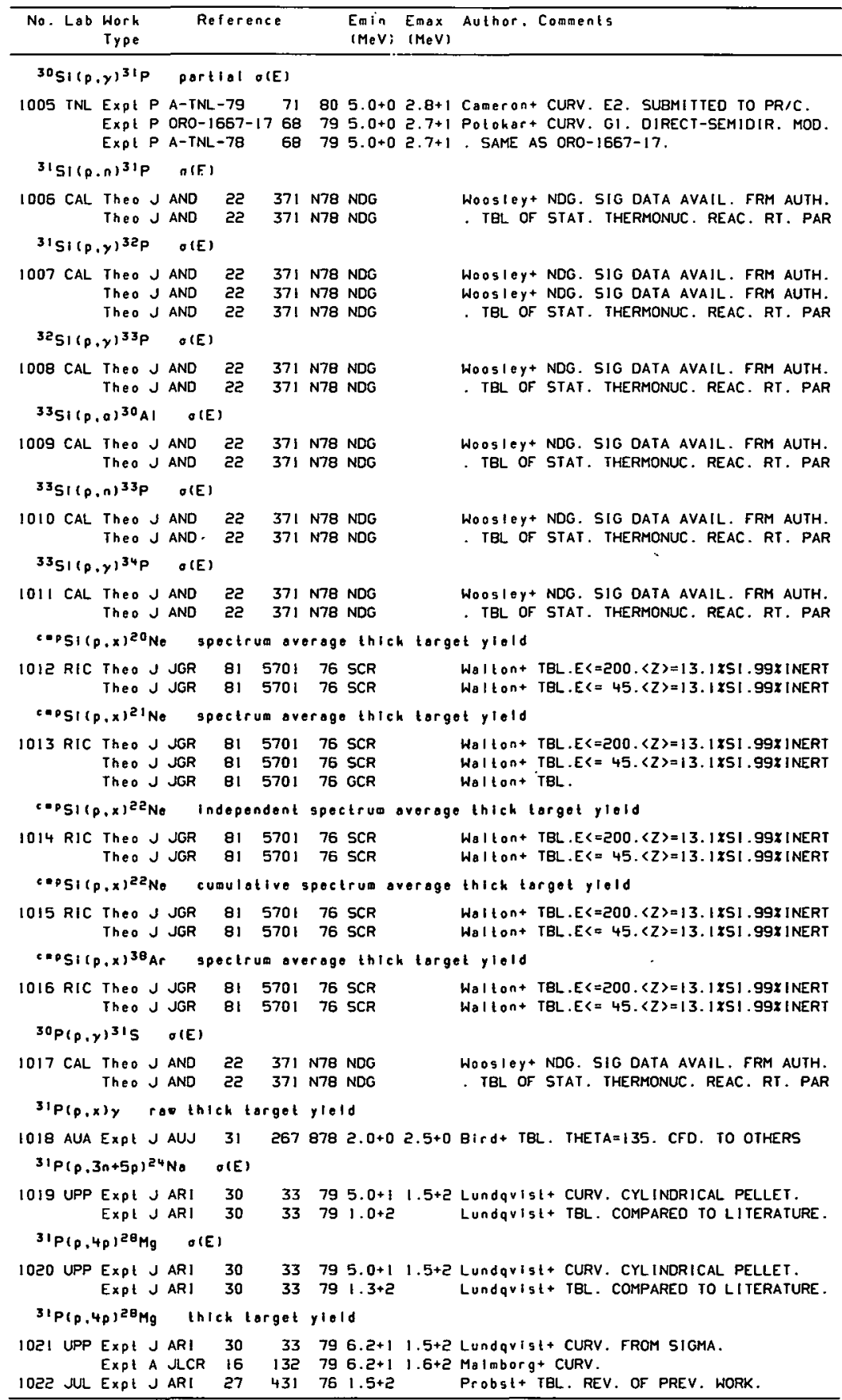




\begin{tabular}{|c|c|c|c|c|c|c|c|c|}
\hline No. Lab & $\begin{array}{l}\text { Work } \\
\text { Type }\end{array}$ & \multicolumn{4}{|c|}{ Reference } & $\begin{array}{l}\text { Emin } \\
(M e V)\end{array}$ & $\begin{array}{l}\text { Emax } \\
(M \in V)\end{array}$ & Author. Comments \\
\hline \multicolumn{2}{|c|}{$31 p(p .4 p)^{28} \mathrm{Mg}$} & \multicolumn{4}{|c|}{ product yield } & & & \\
\hline $\begin{array}{l}1023 \text { UPP } \\
{ }^{31 P 1 P .0}\end{array}$ & $\begin{array}{l}\text { Expt P } \\
\text { Expt P } \\
\text { 20 }{ }^{201}\end{array}$ & $\begin{array}{l}\text { NEAND } \\
\text { NEAND } \\
\text { O(E) }\end{array}$ & $\begin{array}{l}C(O R) \\
C(O R)\end{array}$ & $\begin{array}{l}150 \\
146\end{array}$ & $\begin{array}{l}477 \\
476\end{array}$ & $\begin{array}{l}1.0+2 \\
1.0+2\end{array}$ & & $\begin{array}{l}\text { Lundqrist }+N D G \text {. PROD. LABELLED CMP. P. } 15 \\
\text { Lundqrist+NDG. PROD. LABELLED CMP. P. } 16\end{array}$ \\
\hline $1024 \mathrm{CAL}$ & $\begin{array}{l}\text { Theo J } \\
\text { Theo J } \\
\text { Theo R }\end{array}$ & $\begin{array}{l}\text { AND } \\
\text { AND } \\
\text { OAP-4 }\end{array}$ & $\begin{array}{l}52 \\
52 \\
52\end{array}$ & $\begin{array}{l}37 ! \\
37 !\end{array}$ & $\begin{array}{l}\text { N78 } \\
\text { N78 } \\
875\end{array}$ & $\begin{array}{l}\text { NDG } \\
\text { NDG } \\
\text { TR }\end{array}$ & $8 \cdot 3+0$ & $\begin{array}{l}\text { Woosley+ NDG. SIG DATA AVAIL. FRM AUTH. } \\
\text { TBL OF STAT. THERMONUC. REAC. RT. PAR } \\
\text { Woosley+ CURV. HAUSER-FESHBACH. }\end{array}$ \\
\hline \multicolumn{2}{|c|}{$31 P(p, x)^{30} p$} & \multicolumn{2}{|c|}{ cumulative } & $O(E)$ & & & & \\
\hline $\begin{array}{l}1025 \mathrm{KFK} \\
1026 \mathrm{ORL}\end{array}$ & $\begin{array}{ll}\text { Comp } & B \\
\text { Expl } & J \\
\text { Expt } & 4\end{array}$ & $\begin{array}{l}\text { PH-DA } \\
\text { PR } \\
\text { EXFOR }\end{array}$ & $\cos ^{15}$ & $\begin{array}{r}2 \\
723 \\
3.006\end{array}$ & $\begin{array}{r}\text { D79 } \\
55 \\
77\end{array}$ & $\begin{array}{l}5 \cdot 5+1 \\
5 \cdot 5+1 \\
5 \cdot 5+1\end{array}$ & & $\begin{array}{l}\text { MuenzeI+ TBL. FROM EXFGR. } \\
\text { Cohen+ TBL. ACTIVITY AFTER } 305 \text { DECAY. } \\
\text {. 24OMB. AVE. UNC. }=20 \pi . \text { CAL IB. UNC. }=15 x\end{array}$ \\
\hline \multicolumn{2}{|c|}{${ }^{31} P(p, n+p) 30^{30} p$} & \multicolumn{3}{|c|}{ thich } & $y i \in 1$ & & & \\
\hline \multicolumn{2}{|c|}{$31 p(p, n+p) 30 p$} & AR! & 30 & 3 & 79 & $2 \cdot \theta+1$ & & Sahahundu+ 4,74MCI/MUAMIN. FROM SIGMA. \\
\hline $\begin{array}{l}1028 \text { JUL } \\
31 P(p, n\end{array}$ & $\begin{array}{l}\text { Expl J } \\
\text { Revo } C \\
\operatorname{lis}^{3} \mathbf{S}\end{array}$ & $\begin{array}{l}\text { AR! } \\
\text { 78HAR! } \\
\text { O(E) }\end{array}$ & $\begin{array}{c}30 \\
\text { WEL }\end{array}$ & $\begin{array}{r}3 \\
667\end{array}$ & $\begin{array}{l}79 \\
78\end{array}$ & $\begin{array}{l}1.9+1 \\
2.0+1\end{array}$ & $\begin{array}{l}3 \cdot 5+1 \\
3 \cdot 3+1\end{array}$ & $\begin{array}{l}\text { Sahahundu+ CURV. PCL5 STACKED BAGS. } \\
\text { Slochlin+ CURV. FROM AR! } 28.131 \text {. }\end{array}$ \\
\hline \multicolumn{2}{|c|}{${ }^{31} P(p, y)^{32} s$} & \multicolumn{3}{|c|}{ product ylold } & 875 & $2.1-1$ & $8 \cdot 3+0$ & Woosley+ CURV. HAUSER-FESHBACH. \\
\hline \multicolumn{2}{|c|}{${ }^{31} P(p, y)^{32} s$} & \multicolumn{2}{|c|}{$\begin{array}{l}\text { PHOTONUCLE } \\
\text { O(E) }\end{array}$} & 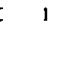 & 78 & $1.4+0$ & $1.5+0$ & Varlamov+ NDG. TBL. FROM 55, 90 DEG DAT \\
\hline $\begin{array}{l}1031 \text { CAL } \\
1032 \text { USA } \\
31 P(P, Y\end{array}$ & $\begin{array}{l}\text { Theo J } \\
\text { Theo J } \\
\text { Theo R } \\
\text { Expt A } \\
\text { Yiss }\end{array}$ & $\begin{array}{l}\text { AND } \\
\text { AND } \\
\text { OAP-4 } \\
\text { BAP } \\
r A=0\end{array}$ & $\begin{array}{l}52 \\
25 \\
25 \\
23 \\
\text { (E) }\end{array}$ & $\begin{array}{l}371 \\
37 ! \\
518\end{array}$ & $\begin{array}{l}N 78 \\
N 78 \\
875 \\
478\end{array}$ & $\begin{array}{l}\text { NDG } \\
\text { NDG } \\
\text { TR } \\
5.0-1\end{array}$ & $\begin{array}{l}1.0+1 \\
2.5+0\end{array}$ & $\begin{array}{l}\text { Woosley+ NDG. SIG DATA AVAIL. FRM AUTH. } \\
\text { TBL OF STAT. IHERMONUC. REAC. RT. PAR } \\
\text { Woosl ey+ CURV. HAUSER-FESHBACH. } \\
\text { No Aulhor-Lab. NDG. DED. STRENGTHS. }\end{array}$ \\
\hline $\begin{array}{l}1033 \mathrm{AML} \\
1034 \mathrm{CCP} \\
32 \mathrm{P}(\mathrm{p} .0\end{array}$ & $\begin{array}{l}\text { Expl J } \\
\text { Expl J } \\
\text { a }^{28} S !\end{array}$ & $\begin{array}{l}\text { PR/C } \\
\text { UF } Z \\
\sigma(E)\end{array}$ & $\begin{array}{l}17 \\
20\end{array}$ & $\begin{array}{l}1550 \\
1787\end{array}$ & $\begin{array}{l}578 \\
N 75\end{array}$ & $\begin{array}{l}8.1-1 \\
1.9+0\end{array}$ & $\begin{array}{l}8.2-1 \\
3.0+0\end{array}$ & $\begin{array}{l}\text { Paine+ CURV. ALSO DEOUCED RES. STRENGT } \\
\text { Kostin+ CURV. }\end{array}$ \\
\hline $\begin{array}{l}1035 \mathrm{CAL} \\
3 \mathrm{eP}_{\mathrm{P}} \mathrm{n}, \mathrm{n}\end{array}$ & $\begin{array}{l}\text { Theo J } \\
\text { Theo J } \\
\text { j25 }\end{array}$ & $\begin{array}{l}\text { AND } \\
\text { AND } \\
\sigma(E)\end{array}$ & $\begin{array}{l}22 \\
22\end{array}$ & $\begin{array}{l}371 \\
371\end{array}$ & $\begin{array}{l}N 78 \\
N 78\end{array}$ & $\begin{array}{l}\text { NDG } \\
\text { NDG }\end{array}$ & & $\begin{array}{l}\text { WoOsley* NDG. SIG DATA AVAIL. FRM AUTH. } \\
\text {. TBL OF STAT. THERMONUC. REAC. RT. PAR }\end{array}$ \\
\hline $\begin{array}{l}1036 \mathrm{CAL} \\
32_{\mathrm{P}}(p, y\end{array}$ & $\begin{array}{l}\text { Theo J } \\
\text { Theo J } \\
\mathrm{J}^{33} \mathrm{~S}\end{array}$ & $\begin{array}{l}\text { AND } \\
\text { AND } \\
\text { O(E) }\end{array}$ & $\begin{array}{l}22 \\
22\end{array}$ & $\begin{array}{l}371 \\
371\end{array}$ & $\begin{array}{l}\text { N7B } \\
\text { N7B }\end{array}$ & $\begin{array}{l}\text { NDG } \\
\text { NDG }\end{array}$ & & $\begin{array}{l}\text { Woosley+ NDG. SIG DATA AVAIL. FRM AUTH. } \\
\text {. TBL OF STAT. THERMONUC. REAC. RT. PAR }\end{array}$ \\
\hline $\begin{array}{l}1037 \mathrm{CAL} \\
{ }^{33} \mathrm{P}(\mathrm{P} .0\end{array}$ & $\begin{array}{l}\text { Theo J } \\
\text { Theo J } \\
\text { Theo J } \\
3^{305}\end{array}$ & $\begin{array}{l}\text { AND } \\
\text { AND } \\
\text { AND } \\
\text { - (E) }\end{array}$ & $\begin{array}{l}22 \\
22 \\
22\end{array}$ & $\begin{array}{l}371 \\
371 \\
371\end{array}$ & $\begin{array}{l}N 78 \\
N 7 B \\
N 78\end{array}$ & $\begin{array}{l}\text { NDG } \\
\text { NDG } \\
\text { NDG }\end{array}$ & & $\begin{array}{l}\text { Woosley+ NDG. SIG DATA AVAIL. FRM AUTH. } \\
\text { Woosley+ NDG. SIG DATA AVAIL. FRM AUTH. } \\
\text {. IBL OF STAT. THERMONUC. REAC. RT. PAR }\end{array}$ \\
\hline $1038 \mathrm{CAL}$ & $\begin{array}{l}\text { Theo J } \\
\text { Theo J }\end{array}$ & $\begin{array}{l}\text { AND } \\
\text { AND }\end{array}$ & $\begin{array}{l}22 \\
22\end{array}$ & $\begin{array}{l}371 \\
371\end{array}$ & $\begin{array}{l}\text { N78 } \\
N 78\end{array}$ & $\begin{array}{l}\text { NDG } \\
\text { NDG }\end{array}$ & & $\begin{array}{l}\text { Woosley+ NDG. SIG DATA AVAIL. FRM AUTH. } \\
\text {. TBL OF STAT. THERMONUC. REAC. RT. PAR }\end{array}$ \\
\hline \multicolumn{2}{|c|}{${ }^{33} P(p, y)^{34} s$} & \multicolumn{2}{|l|}{ o(E) } & & & & & \\
\hline $\begin{array}{l}1039 \mathrm{CAL} \\
{ }^{34} \mathrm{PIP} .0\end{array}$ & $\begin{array}{l}\text { Theo J } \\
\text { Theo J } \\
\text { (I) } 31\end{array}$ & $\begin{array}{l}\text { AND } \\
\text { AND } \\
\text { O(E) }\end{array}$ & $\begin{array}{l}22 \\
22\end{array}$ & $\begin{array}{l}371 \\
371\end{array}$ & $\begin{array}{l}\text { N78 } \\
\text { N78 }\end{array}$ & $\begin{array}{l}\text { NDG } \\
\text { NDG }\end{array}$ & & $\begin{array}{l}\text { Woosley+ NDG. SIG DATA AVAIL. FRM AUTH. } \\
\text {. TBL OF STAT. THERMONUC. REAC. RT. PAR }\end{array}$ \\
\hline $1040 \mathrm{CAL}$ & $\begin{array}{l}\text { Theo J } \\
\text { Theo J }\end{array}$ & $\begin{array}{l}\text { AND } \\
\text { AND }\end{array}$ & $\begin{array}{l}22 \\
22\end{array}$ & $\begin{array}{l}371 \\
371\end{array}$ & $\begin{array}{l}\text { N78 } \\
N 78\end{array}$ & $\begin{array}{l}\text { NDG } \\
\text { NDG }\end{array}$ & & $\begin{array}{l}\text { Woosley + NDG. SIG DATA AVAIL. FRM AUTH. } \\
\text {. IBL OF STAT. THERMONUC. REAC. RT. PAR }\end{array}$ \\
\hline \multicolumn{2}{|c|}{${ }^{34} P(p, n)^{34} s$} & \multicolumn{2}{|l|}{$\sigma(E)$} & & & & & \\
\hline $1041 \mathrm{CAL}$ & $\begin{array}{l}\text { Theo J } \\
\text { Theo J }\end{array}$ & $\begin{array}{l}\text { AND } \\
\text { AND }\end{array}$ & $\begin{array}{l}22 \\
22\end{array}$ & $\begin{array}{l}371 \\
371\end{array}$ & $\begin{array}{l}N 78 \\
N 78\end{array}$ & $\begin{array}{l}\text { NDG } \\
\text { NDG }\end{array}$ & & $\begin{array}{l}\text { Woosley+ NDG. SIG DATA AVAIL. FRM AUTH. } \\
\text {. IBL UF SIAI. IHLKMUNUC. REAC. RT. PAR }\end{array}$ \\
\hline \multicolumn{2}{|c|}{${ }^{34} P(p \cdot y)^{35} S$} & $\sigma(E)$ & & & & & & \\
\hline 1042 CAL & $\begin{array}{l}\text { Theo J } \\
\text { Theo J }\end{array}$ & $\begin{array}{l}\text { AND } \\
\text { AND }\end{array}$ & $\begin{array}{l}22 \\
22\end{array}$ & $\begin{array}{l}371 \\
371\end{array}$ & $\begin{array}{l}\text { N78 } \\
N 78\end{array}$ & $\begin{array}{l}\text { NDG } \\
\text { NDG }\end{array}$ & & $\begin{array}{l}\text { Woosley+ NDG. SIG DATA AVAIL. FRM AUTH. } \\
\text {. IBL OF STAT. THERMONUC. REAC. RT. PAR }\end{array}$ \\
\hline
\end{tabular}


REFERENCES ( c ont )

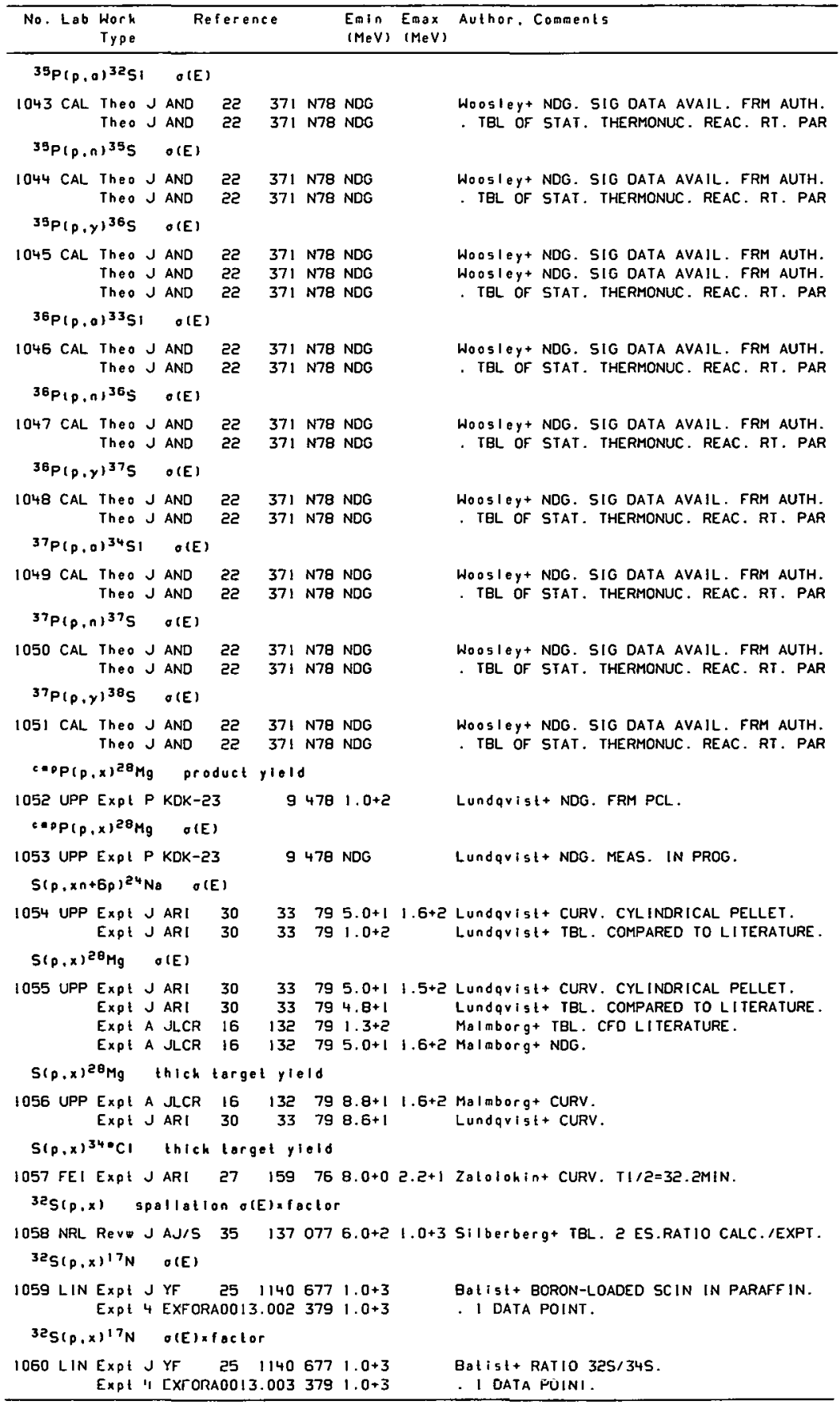




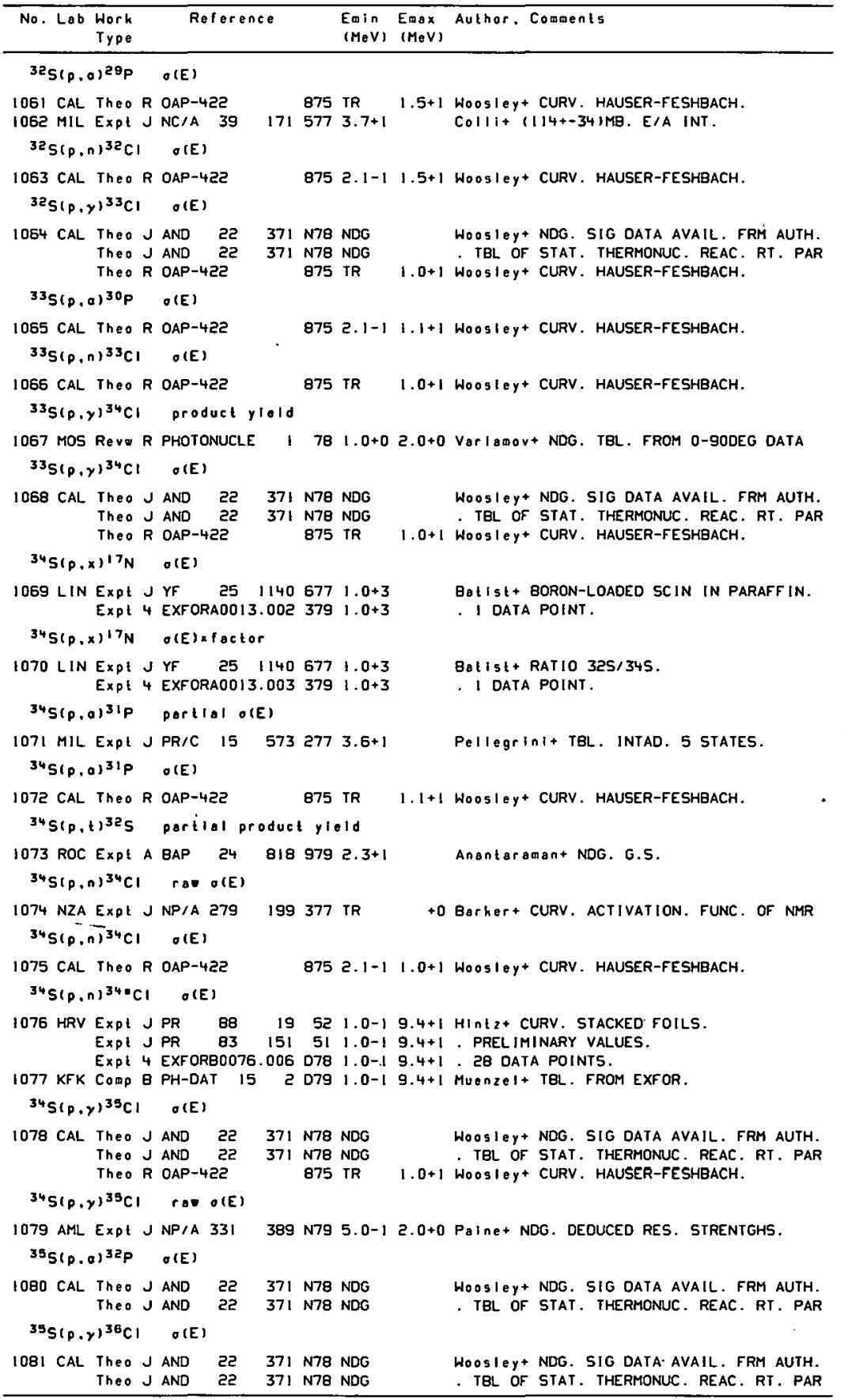




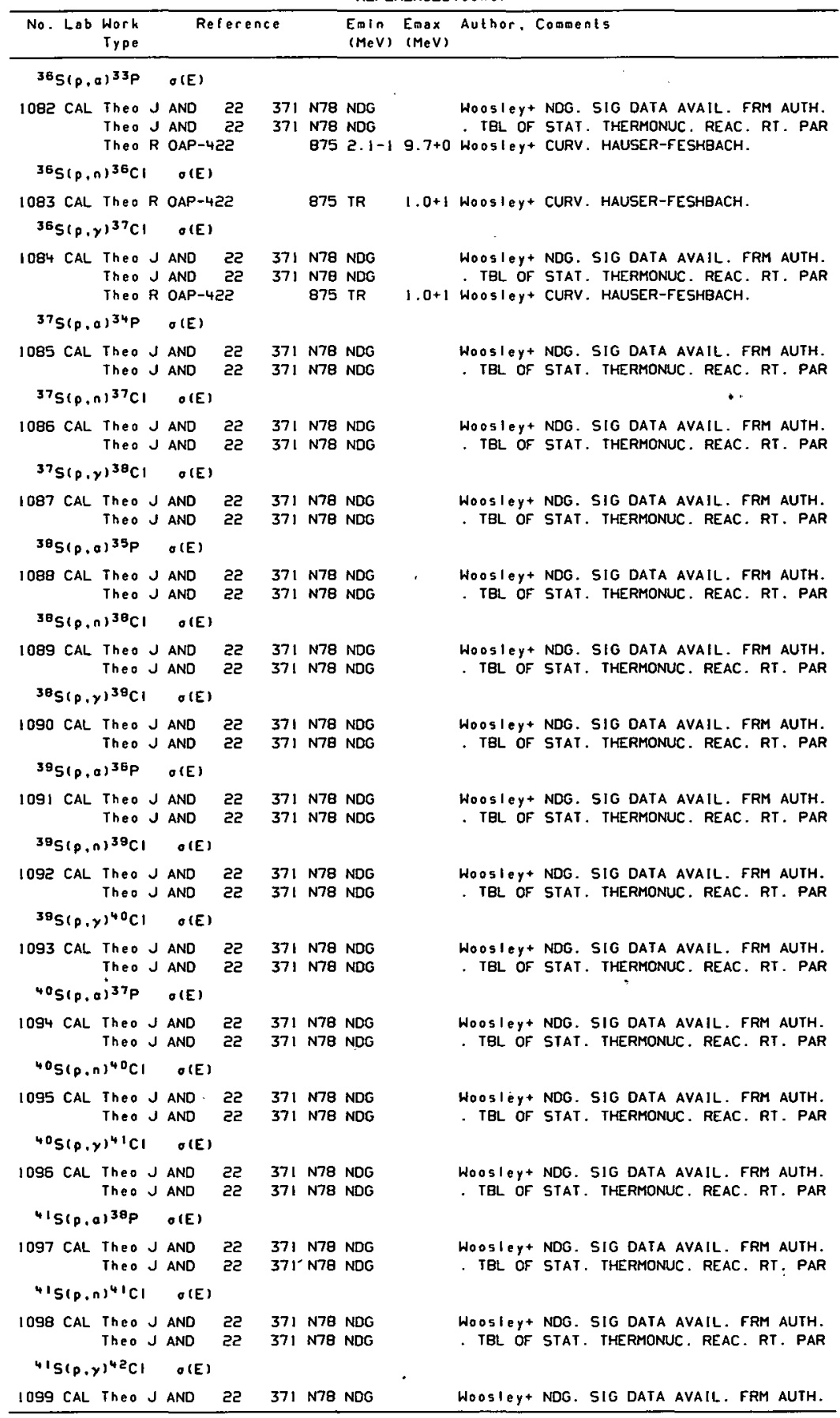


REFERENCES ( c ont )

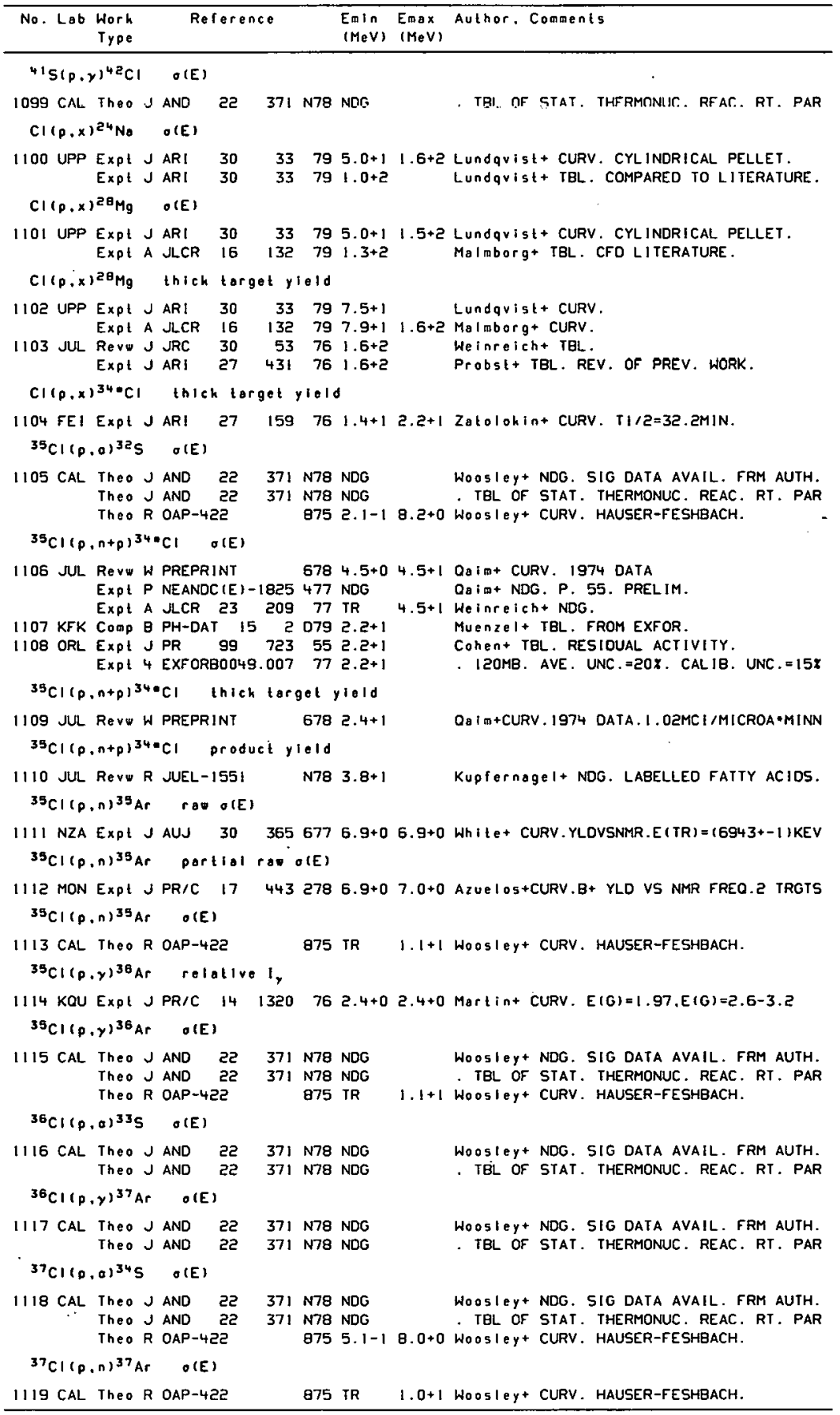




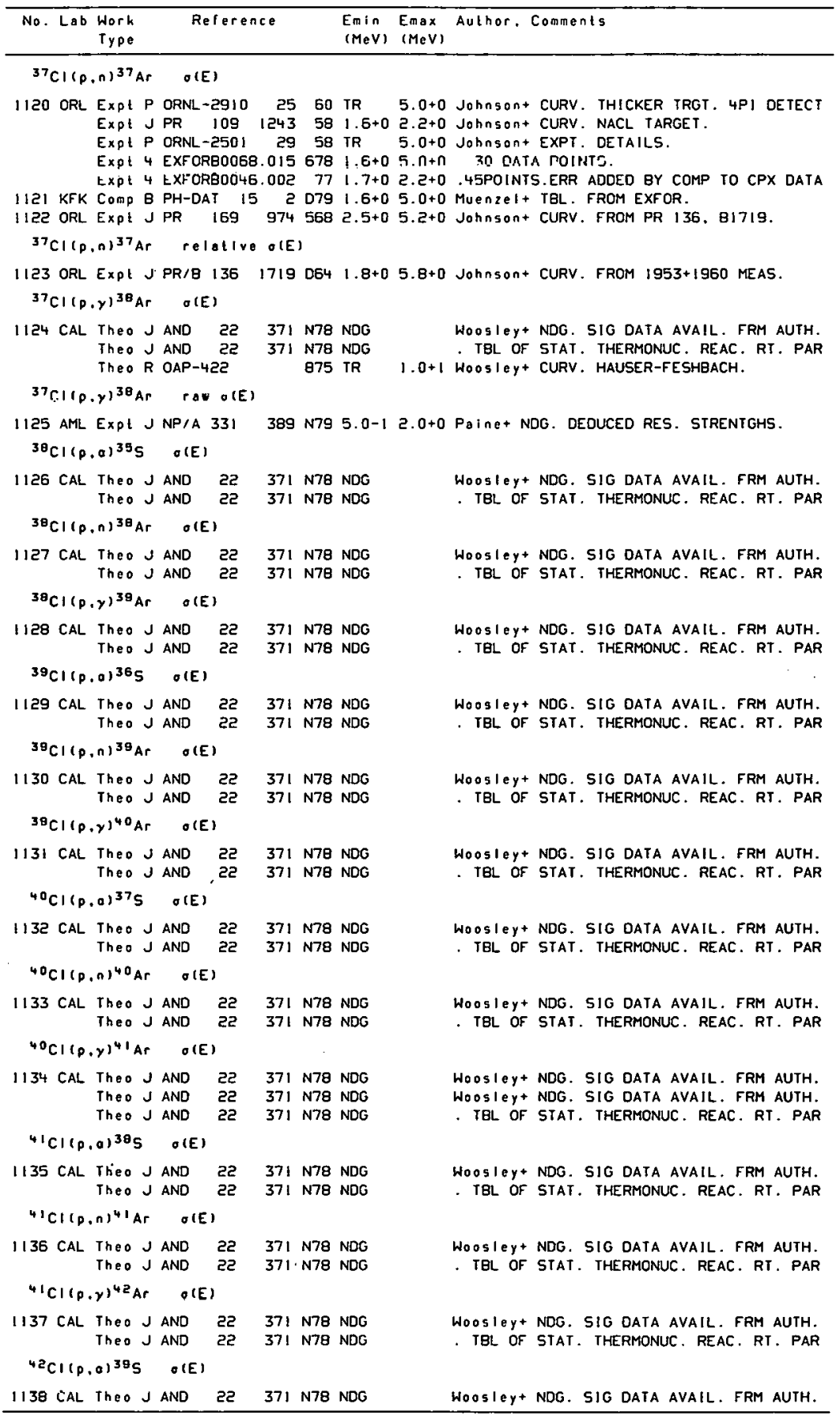




\begin{tabular}{|c|c|c|c|c|c|c|c|c|}
\hline No. Lab & $\begin{array}{l}\text { Work } \\
\text { Type }\end{array}$ & & feren & & & $\begin{array}{l}\text { Emin } \\
(M e V)\end{array}$ & $\begin{array}{l}\text { Emax } \\
(M e V)\end{array}$ & Author. Comments \\
\hline \multicolumn{2}{|c|}{${ }^{42} \mathrm{C} 1(p, 0)^{39} \mathrm{~S}$} & o(E) & & & & & & \\
\hline $\begin{array}{l}1138 \mathrm{CAL} \\
42 \mathrm{CI} / \mathrm{p}\end{array}$ & $\begin{array}{l}\text { Theo J } \\
\text {, n) }{ }^{2} \text { Ar }\end{array}$ & AND & 22 & 371 & N78 & NDG & & . IBL OF STAT. THERMONUC. REAC. RT, PAR \\
\hline $\begin{array}{l}1139 \mathrm{CAL} \\
42 \mathrm{CI} / \mathrm{P}\end{array}$ & $\begin{array}{l}\text { Theo J } \\
\text { Theo J } \\
.2)^{43} \text { Ar }\end{array}$ & $\begin{array}{l}\text { AND } \\
\text { AND } \\
\text { otE }\end{array}$ & $\begin{array}{l}22 \\
22\end{array}$ & $\begin{array}{l}371 \\
371\end{array}$ & $\begin{array}{l}\text { N7B } \\
\text { N78 }\end{array}$ & $\begin{array}{l}\text { NDG } \\
\text { NDG }\end{array}$ & & $\begin{array}{l}\text { WOOSley+ NDG. SIG DATA AVAIL. FRM AUTH. } \\
\text {. TBL OF STAT. THERMONUC. REAC. RT. PAR }\end{array}$ \\
\hline $\begin{array}{l}1140 \mathrm{CAL} \\
{ }^{43} \mathrm{CI} / \mathrm{P}\end{array}$ & $\begin{array}{l}\text { Theo J } \\
\text { Theo J } \\
.0)^{40} \mathrm{~S}\end{array}$ & $\begin{array}{l}\text { AND } \\
\text { AND } \\
\text { O(E) }\end{array}$ & $\begin{array}{l}22 \\
22\end{array}$ & $\begin{array}{l}371 \\
371\end{array}$ & $\begin{array}{l}\text { N78 } \\
\text { N7B }\end{array}$ & $\begin{array}{l}\text { NDG } \\
\text { NDG }\end{array}$ & & $\begin{array}{l}\text { WoOsley+ NDG. SIG DATA AVAIL. FRM AUTH. } \\
\text {. TBL OF STAT. THERMONUC. REAC. RT. PAR }\end{array}$ \\
\hline $\begin{array}{l}1141 \mathrm{CAL} \\
{ }^{43} \mathrm{CI} / \mathrm{P} .\end{array}$ & $\begin{array}{l}\text { Theo J } \\
\text { Theo J } \\
.0)^{43} \text { Ar }\end{array}$ & $\begin{array}{l}\text { AND } \\
\text { AND } \\
\quad \sigma(E\end{array}$ & $\begin{array}{l}22 \\
22\end{array}$ & $\begin{array}{l}371 \\
371\end{array}$ & $\begin{array}{l}\text { N78 } \\
\text { N78 }\end{array}$ & $\begin{array}{l}\text { NDG } \\
\text { NDG }\end{array}$ & & $\begin{array}{l}\text { WoOsley+ NDG. SIG, DATA AV́AIL. FRM AUTH. } \\
\text {. TEL OF STAT. THERMONUC. REAC. RT. PAR }\end{array}$ \\
\hline $\begin{array}{l}1142 \mathrm{CAL} \\
{ }^{43} \mathrm{CICP}\end{array}$ & $\begin{array}{l}\text { Theo J } \\
\text { Theo J } \\
.2)^{44} \text { Ar }\end{array}$ & $\begin{array}{l}\text { AND } \\
\text { AND } \\
\text { OIE }\end{array}$ & $\begin{array}{l}22 \\
22\end{array}$ & $\begin{array}{l}371 \\
371\end{array}$ & $\begin{array}{l}\text { N78 } \\
\text { N78 }\end{array}$ & $\begin{array}{l}\text { NDG } \\
\text { NDG }\end{array}$ & & $\begin{array}{l}\text { Woosley+ NDG. SIG DATA AVAIL. FRM AUTH. } \\
\text { TBL OF STAT. THERMONUC. REAC. RT. PAR }\end{array}$ \\
\hline $\begin{array}{l}1143 \mathrm{CAL} \\
{ }^{44} \mathrm{CI} / \mathrm{P} .\end{array}$ & $\begin{array}{l}\text { Theo J } \\
\text { Theo J } \\
.0)^{4} 15\end{array}$ & $\begin{array}{l}\text { AND } \\
\text { AND } \\
\text { O(E) }\end{array}$ & $\begin{array}{l}22 \\
22\end{array}$ & $\begin{array}{l}371 \\
371\end{array}$ & $\begin{array}{l}\text { N78 } \\
\text { N78 }\end{array}$ & $\begin{array}{l}\text { NDG } \\
\text { NDG }\end{array}$ & & $\begin{array}{l}\text { Woosley+ NDG. SIG DATA AVAIL. FRM AUTH. } \\
\text {. TBL OF STAT. THERMONUC. REAC. RT. PAR }\end{array}$ \\
\hline $\begin{array}{l}1144 \mathrm{CAL} \\
{ }^{44} \mathrm{CI} / \mathrm{P} .\end{array}$ & $\begin{array}{l}\text { Theo J } \\
\text { Theo J } \\
\text {, n }{ }^{44} \text { Ar }\end{array}$ & $\begin{array}{l}\text { AND } \\
\text { AND } \\
\text { OIE }\end{array}$ & $\begin{array}{l}22 \\
22\end{array}$ & $\begin{array}{l}371 \\
371\end{array}$ & $\begin{array}{l}\text { N78 } \\
\text { N79 }\end{array}$ & $\begin{array}{l}\text { NDG } \\
\text { NDG }\end{array}$ & & $\begin{array}{l}\text { WOOSIEY+ NDG. SIG DATA AVAIL. FRM AUTH. } \\
\text {. TBL OF STAT. THERMONUC. REAC. RT. PAR }\end{array}$ \\
\hline $\begin{array}{l}1145 \mathrm{CAL} \\
{ }^{44} \mathrm{CI} / \mathrm{P} .\end{array}$ & $\begin{array}{l}\text { Theo J } \\
\text { Theo J } \\
. y)^{45} \text { Ar }\end{array}$ & $\begin{array}{l}\text { AND } \\
\text { AND } \\
\text { OIE }\end{array}$ & $\begin{array}{l}22 \\
22\end{array}$ & $\begin{array}{l}371 \\
371\end{array}$ & $\begin{array}{l}\text { N7G } \\
\text { N78 }\end{array}$ & $\begin{array}{l}\text { NDG } \\
\text { NDG }\end{array}$ & • & $\begin{array}{l}\text { WOOSley+ NDG. SIG OATA AVAIL: FRM AUTH. } \\
\text {. TBL OF STAT. THERMONUC. REAC. RT. PAR } \\
\text {. }\end{array}$ \\
\hline $\begin{array}{l}1146 \mathrm{CAL} \\
{ }^{45} \mathrm{CICP} .\end{array}$ & $\begin{array}{l}\text { Theo J } \\
\text { Theo J } \\
\text { a) } 4)^{4}\end{array}$ & $\begin{array}{l}\text { AND } \\
\text { AND } \\
\text { o(E) }\end{array}$ & $\begin{array}{l}22 \\
22\end{array}$ & $\begin{array}{l}371 \\
371\end{array}$ & $\begin{array}{l}\text { N78 } \\
\text { N78 }\end{array}$ & $\begin{array}{l}\text { NDG } \\
\text { NDG }\end{array}$ & & $\begin{array}{l}\text { Woosley+ NDG. SIG DATA AVAIL. FRM AUTH. } \\
\text {. TBL OF STAT. THERMONUC. REAC. RT. PAR }\end{array}$ \\
\hline $\begin{array}{l}1147 \mathrm{CAL} \\
{ }^{45} \mathrm{CI} / \mathrm{P} .\end{array}$ & $\begin{array}{l}\text { Theo J } \\
\text { Theo J } \\
\text { (n) })^{45} \text { Ar }\end{array}$ & $\begin{array}{l}\text { AND } \\
\text { AND } \\
\text { OLE }\end{array}$ & $\begin{array}{l}22 \\
22\end{array}$ & $\begin{array}{l}37 ! \\
37 !\end{array}$ & $\begin{array}{l}\text { N78 } \\
N 78\end{array}$ & $\begin{array}{l}\text { NDG } \\
\text { NDG }\end{array}$ & & $\begin{array}{l}\text { WOOSI EY + NDG. SIG DATA AVAIL. FRM ALTH. } \\
\text {. TEL. OF STAT. THERMONUC. REAC. RT. PAR }\end{array}$ \\
\hline $\begin{array}{l}1148 \mathrm{CAL} \\
{ }^{45} \mathrm{CI} 10 .\end{array}$ & $\begin{array}{l}\text { Theo J } \\
\text { Theo J } \\
. \gamma^{46} \mathrm{Ar}\end{array}$ & $\begin{array}{l}\text { AND } \\
\text { AND } \\
. \text { OIE }\end{array}$ & $\begin{array}{l}22 \\
22\end{array}$ & $\begin{array}{l}371 \\
371\end{array}$ & $\begin{array}{l}N 78 \\
N 78\end{array}$ & $\begin{array}{l}\text { NDG } \\
\text { NDG }\end{array}$ & & $\begin{array}{l}\text { WOOSI Ey+ NDG. SIG DATA AVAIL. FRM AUTH. } \\
\text {. TBL OF STAT. THERMONUC. REAC. RT. PAR }\end{array}$ \\
\hline $\begin{array}{l}1149 \mathrm{CAL} \\
\because \because \mathrm{CI}^{\circ}\end{array}$ & $\begin{array}{l}\text { Theo J } \\
\text { Theo J } \\
(, x)^{20} \mathrm{Mg}\end{array}$ & $\begin{array}{l}\text { AND } \\
\text { AND } \\
g \text { pr }\end{array}$ & $\begin{array}{c}22 \\
22 \\
\text { oduct }\end{array}$ & $\begin{array}{l}371 \\
371 \\
y 101\end{array}$ & $\begin{array}{l}\text { N78 } \\
\text { N78 } \\
\text { Id }\end{array}$ & $\begin{array}{l}\text { NDG } \\
\text { NDG }\end{array}$ & & $\begin{array}{l}\text { Woosley+ NDG. SIG DATA AVAIL. FRM AUTH. } \\
\text {. TBL OF STAT. THERMONUC. REAC. RT. PAR }\end{array}$ \\
\hline $\begin{array}{l}1150 \text { UPP } \\
\qquad=P C \mid 1 P\end{array}$ & $\begin{array}{l}\text { Expt } P \\
0, x, 2 \theta_{M g}\end{array}$ & $\begin{aligned} K O K-5 \\
\quad\end{aligned}$ & & 9 & 478 & $1.0+2$ & & Lundquist+ NDG. FRM NACL. \\
\hline $\begin{array}{l}1151 \text { UPP } \\
\operatorname{Ar}(P . x)\end{array}$ & $\begin{array}{l}\text { Expt P } \\
\text {,24 Na }\end{array}$ & $\begin{array}{l}K D K-2 \\
\sigma(E)\end{array}$ & & 9 & 478 & NDG & & Lundquist+ NDG. MEAS. IN PROG. \\
\hline $\begin{array}{l}1152 \text { UPP } \\
\operatorname{Ar}(p, x)\end{array}$ & $\begin{array}{l}\text { Expt J } \\
\text { EबMg }\end{array}$ & $\begin{array}{l}\text { AR1 } \\
\text { O(E) }\end{array}$ & 30 & 33 & 79 & $5.5+1$ & $1.6+2$ & Lundquist+ CURV. CYLINDRICAL PELLET. \\
\hline 1153 UPP & $\begin{array}{l}\text { Expl } J \\
\text { Expl } \\
\text { Expl } A\end{array}$ & $\begin{array}{l}\text { AR I } \\
\text { AR I } \\
\text { 几CR }\end{array}$ & $\begin{array}{l}30 \\
30 \\
16\end{array}$ & $\begin{array}{r}33 \\
33 \\
132\end{array}$ & $\begin{array}{l}79 \\
79 \\
79\end{array}$ & $\begin{array}{l}5 \cdot 5+1 \\
2 \cdot 5+1 \\
1 \cdot 3+2\end{array}$ & $1.7+2$ & $\begin{array}{l}\text { Lundquigl+ CURV. CYLINDRICAL PELLET. } \\
\text { Lundquis }+ \text { NOS. LITTLE ACTIVITY. } \\
\text { Malmborg + TBL. CFD LITERATURE. }\end{array}$ \\
\hline $\operatorname{Ar}(p, x)$ & $1^{20} \mathrm{Mg}$ & thich & $\operatorname{lar}$ & et $y^{\prime}$ & 1. Id & & & \\
\hline $\begin{array}{l}1154 \text { UPP } \\
36 \text { Ar (P. }\end{array}$ & $\begin{array}{l}\text { Expt } \\
\text { Expt } A \\
\operatorname{ar}^{33} \mathrm{Cl}\end{array}$ & $\begin{array}{l}\text { AR! } \\
\text { JCR } \\
\text { OCE }\end{array}$ & $\begin{array}{l}30 \\
15\end{array}$ & $\begin{array}{r}33 \\
132\end{array}$ & $\begin{array}{l}79 \\
79\end{array}$ & $\begin{array}{l}7.8+1 \\
7.5+1\end{array}$ & $1.6+2$ & $\begin{array}{l}\text { Lundquist + CURV. } \\
\text { Malmborg + CURV. }\end{array}$ \\
\hline $\begin{array}{l}1155 \mathrm{CAL} \\
36 \mathrm{Ar}(\mathrm{P} .\end{array}$ & $\begin{array}{l}\text { Theo } R \\
\text { n) }{ }^{36} \mathrm{~K}\end{array}$ & $\begin{array}{l}\text { OAP-4 } \\
\text { O(E) }\end{array}$ & & & 875 & $2.1-1$ & $1.6+1$ & Woosley+ CURY. HAUSER-FESHBACH. \\
\hline $1155 \mathrm{CAL}$ & Theo $\mathbf{R}$ & OAP -4 & & & 875 & TR & $1.0+1$ & Woosley + CURV. HAUSER-FESHBACH. \\
\hline
\end{tabular}


REFERENCES $(c o n t)$

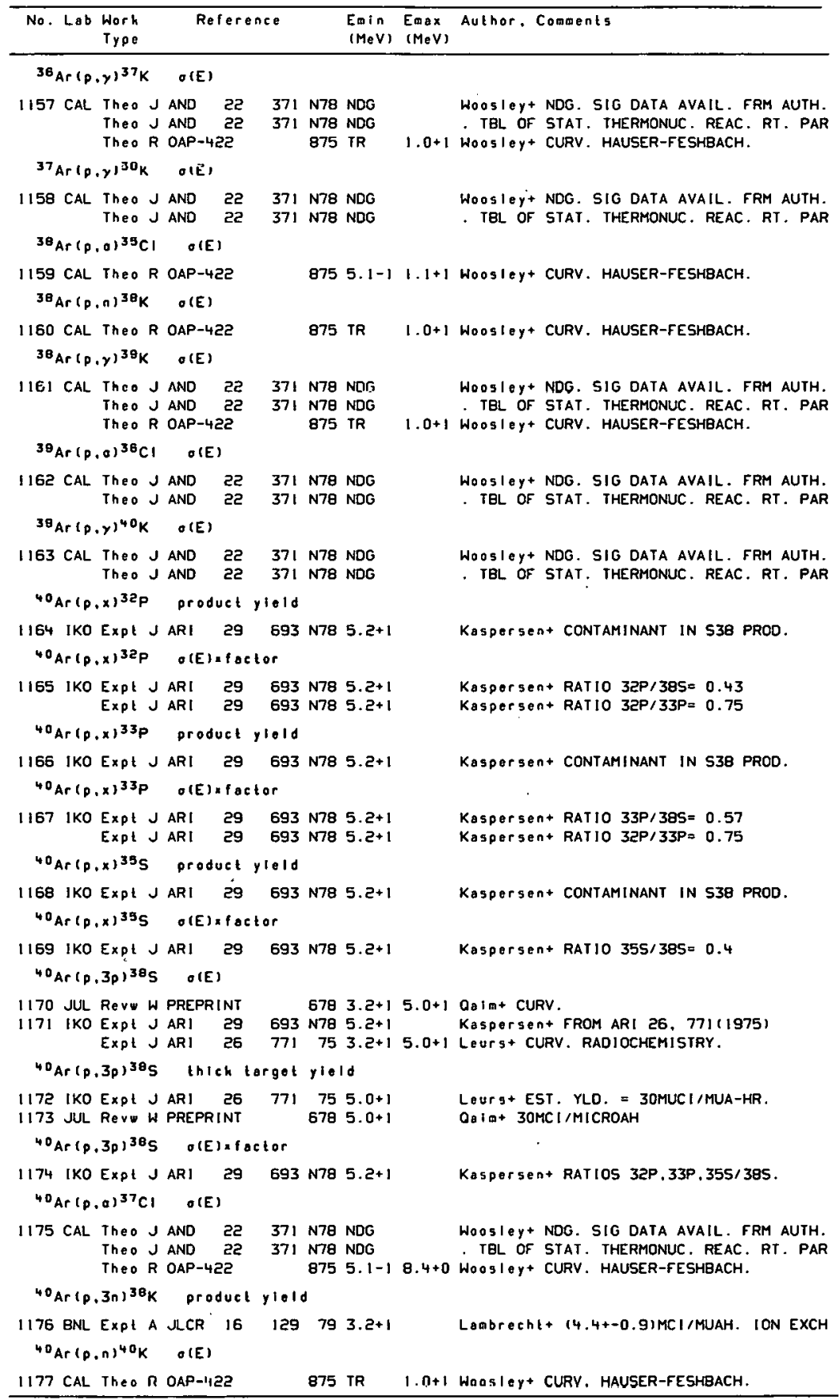


REFERENCES $(c$ ont $)$

\begin{tabular}{|c|c|c|c|c|c|c|c|c|}
\hline No. Lab & $\begin{array}{l}\text { Work } \\
\text { Type }\end{array}$ & & eferenc & & & $\begin{array}{l}\text { Emin } \\
(\operatorname{MeV})\end{array}$ & $\begin{array}{l}\text { Emax } \\
(M e V)\end{array}$ & Author. Comments \\
\hline \multicolumn{2}{|c|}{${ }^{40} \mathrm{Ar}(\mathrm{p}, y)^{4} \mathrm{I} K$} & o(E) & & & & & & \\
\hline $\begin{array}{l}1178 \mathrm{CAL} \\
41 \mathrm{Ar} \text { (P }\end{array}$ & $\begin{array}{l}\text { Theo J } \\
\text { Theo J } \\
\text { Theo R } \\
.0)^{30} \mathrm{Cl}\end{array}$ & $\begin{array}{l}\text { AND } \\
\text { AND } \\
\text { OAP-4 } \\
\text { OIE }\end{array}$ & $\begin{array}{r}E 2 \\
22 \\
425\end{array}$ & $\begin{array}{l}371 \\
371\end{array}$ & $\begin{array}{l}N 78 \\
N 78 \\
875\end{array}$ & $\begin{array}{l}\text { NOG } \\
\text { NDG } \\
\text { TR }\end{array}$ & 1. $0+1$ & $\begin{array}{l}\text { WoOsley+ NDG. SIG DATA AVAIL. FRM AUTH. } \\
\text {. TBL OF STAT. THERMONUC. REAC. RI. PAR } \\
\text { WoOsley+ CURV. HAUSER-FESHBACH. }\end{array}$ \\
\hline $\begin{array}{l}1179 \mathrm{CAL} \\
{ }^{41} \mathrm{Ar}(\mathrm{P}\end{array}$ & $\begin{array}{l}\text { Theo J } \\
\text { Theo J } \\
. \mathrm{nJ}^{4} \mathrm{~K}\end{array}$ & $\begin{array}{l}\text { AND } \\
\text { AND } \\
\sigma(E)\end{array}$ & $\begin{array}{l}22 \\
22\end{array}$ & $\begin{array}{l}37 ! \\
37 !\end{array}$ & $\begin{array}{l}N 78 \\
N 78\end{array}$ & $\begin{array}{l}\text { NDG } \\
\text { NDG }\end{array}$ & & $\begin{array}{l}\text { Woosley+ NDG. SIG DATA AVAIL. FRM AUTH. } \\
\text {. TBL OF STAT. THERMONUC. REAC. RT. PAR }\end{array}$ \\
\hline $\begin{array}{l}180 \mathrm{CAL} \\
41 \mathrm{ArCP}\end{array}$ & $\begin{array}{l}\text { Theo J } \\
\text { Theo J } \\
. \gamma)^{42 K}\end{array}$ & $\begin{array}{l}\text { AND } \\
\text { AND } \\
\sigma(E)\end{array}$ & $\begin{array}{l}22 \\
2 己\end{array}$ & $\begin{array}{l}37 ! \\
37 !\end{array}$ & $\begin{array}{l}\text { N78 } \\
\text { N78 }\end{array}$ & $\begin{array}{l}\text { NDG } \\
\text { NDG }\end{array}$ & & $\begin{array}{l}\text { WOOSley+ NDG. SIG DATA AVAIL. FRM AUTH. } \\
\text { - TBL OF STAT. THERMONUC. REAC. RT. PAR }\end{array}$ \\
\hline $\begin{array}{l}1181 \text { CAL } \\
42 \operatorname{Ar} 1 \mathrm{P}\end{array}$ & $\begin{array}{l}\text { Theo J } \\
\text { Theo J } \\
.0)^{39} \mathrm{Cl}\end{array}$ & $\begin{array}{l}\text { AND } \\
\text { AND } \\
\quad \sigma(E\end{array}$ & $\begin{array}{l}22 \\
22\end{array}$ & $\begin{array}{l}371 \\
371\end{array}$ & $\begin{array}{l}\text { N78 } \\
\text { N78 }\end{array}$ & $\begin{array}{l}\text { NDG } \\
\text { NDG }\end{array}$ & & $\begin{array}{l}\text { WoOsley+ NDG. SIG DATA AVAIL. FRM AUTH. } \\
\text {. TBL OF STAT. THERMONUC. REAC. RT. PAR }\end{array}$ \\
\hline $\begin{array}{l}1182 \mathrm{CAL} \\
42 \mathrm{Ar}(\mathrm{P}\end{array}$ & $\begin{array}{l}\text { Theo J } \\
\text { Theo J } \\
. y^{43} \mathrm{~K}\end{array}$ & $\begin{array}{l}\text { AND } \\
\text { AND } \\
\text { O(E) }\end{array}$ & $\begin{array}{l}22 \\
22\end{array}$ & $\begin{array}{l}371 \\
371\end{array}$ & $\begin{array}{l}\text { N78 } \\
\text { N78 }\end{array}$ & NDG & & $\begin{array}{l}\text { WOOSI ey+ NDG. SIG DATA AVAIL. FRM AUTH. } \\
\text {. TBL OF STAT. THERMONUC. REAC. RT. PAR }\end{array}$ \\
\hline $\begin{array}{l}1183 \mathrm{CAL} \\
43 \mathrm{Ar}(\mathrm{P}\end{array}$ & $\begin{array}{l}\text { Theo J } \\
\text { Theo J } \\
.0)^{40} \mathrm{CI}\end{array}$ & $\begin{array}{l}\text { AND } \\
\text { AND } \\
\quad \text { o } 1 E\end{array}$ & $\begin{array}{l}22 \\
22\end{array}$ & $\begin{array}{l}371 \\
371\end{array}$ & $\begin{array}{l}\text { N78 } \\
N 78\end{array}$ & $\begin{array}{l}\text { NDG } \\
\text { NDG }\end{array}$ & & $\begin{array}{l}\text { WOOSley+ NDG. SIG DATA AVAIL. FRM AUTH. } \\
\text {. TBL OF STAT. THERMONUC. REAC. RT. PAR }\end{array}$ \\
\hline $\begin{array}{l}1184 \mathrm{CAL} \\
43 \mathrm{Ar} 1 \mathrm{P}\end{array}$ & $\begin{array}{l}\text { Theo J } \\
\text { Theo J } \\
. n)^{43} \mathrm{~K}\end{array}$ & $\begin{array}{l}\text { AND } \\
\text { AND } \\
\text { O(E) }\end{array}$ & $\begin{array}{l}22 \\
22\end{array}$ & $\begin{array}{l}371 \\
371\end{array}$ & $\begin{array}{l}\text { N78 } \\
\text { N78 }\end{array}$ & NOG & & $\begin{array}{l}\text { WOOSIEY+ NDG. SIG DATA AVAIL. FRM AUTH. } \\
\text {. TBL OF STAT. THERMONUC. REAC. RT. PAR }\end{array}$ \\
\hline $\begin{array}{l}1185 \mathrm{CAL} \\
{ }^{43} \mathrm{Ar} . \mathrm{PP}\end{array}$ & $\begin{array}{l}\text { Theo J } \\
\text { Theo } J \\
. \gamma)^{44} \mathrm{~K}\end{array}$ & $\begin{array}{l}\text { AND } \\
\text { AND } \\
\text { O(E) }\end{array}$ & $\begin{array}{l}22 \\
25\end{array}$ & $\begin{array}{l}371 \\
371\end{array}$ & $\begin{array}{l}\text { N78 } \\
\text { N78 }\end{array}$ & $\begin{array}{l}\text { NDG } \\
\text { NDG }\end{array}$ & & $\begin{array}{l}\text { WoOsley+ NDG. SIG DATA AVAIL. FRM AUTH. } \\
\text {. TBL OF STAT. THERMONUC. REAC. RT. PAR }\end{array}$ \\
\hline $\begin{array}{l}1186 \mathrm{CAL} \\
{ }^{44} \mathrm{Ar}(\mathrm{P} .\end{array}$ & $\begin{array}{l}\text { Theo J } \\
\text { Theo J } \\
. n J^{44} \mathrm{~K}\end{array}$ & $\begin{array}{l}\text { AND } \\
\text { AND } \\
\sigma(E)\end{array}$ & $\begin{array}{l}22 \\
22\end{array}$ & $\begin{array}{l}371 \\
371\end{array}$ & $\begin{array}{l}\text { N78 } \\
\text { N78 }\end{array}$ & $\begin{array}{l}\text { NDG } \\
\text { NDG }\end{array}$ & · & $\begin{array}{l}\text { Woosley+ NDG. SIG DATA AVAIL. FRM AUTH. } \\
\text {. TBL OF STAT. THERMONUC. REAC. RT. PAR }\end{array}$ \\
\hline $\begin{array}{l}1187 \mathrm{CAL} \\
{ }^{44} \mathrm{Ar}(\mathrm{P} \text {. }\end{array}$ & $\begin{array}{l}\text { Theo J } \\
\text { Theo J } \\
. y)^{45} \mathrm{~K}\end{array}$ & $\begin{array}{l}\text { AND } \\
\text { AND } \\
\text { O(E) }\end{array}$ & $\begin{array}{l}22 \\
22\end{array}$ & $\begin{array}{l}371 \\
371\end{array}$ & $\begin{array}{l}\text { N78 } \\
\text { N78 }\end{array}$ & $\begin{array}{l}\text { NDG } \\
\text { NDG }\end{array}$ & & $\begin{array}{l}\text { Woosley+ NDG. SIG DATA AVAIL. FRM AUTH. } \\
\text {. TBL OF STAT. THERMONUC. REAC. RT. PAR }\end{array}$ \\
\hline $\begin{array}{l}1188 \mathrm{CAL} \\
{ }^{45} \mathrm{Ar}(\mathrm{P} .\end{array}$ & $\begin{array}{l}\text { Theo J } \\
\text { Theo J } \\
\text { n })^{45 K}\end{array}$ & $\begin{array}{l}\text { AND } \\
\text { AND } \\
\text { (E) }\end{array}$ & $\begin{array}{l}22 \\
22\end{array}$ & $\begin{array}{l}371 \\
371\end{array}$ & $\begin{array}{l}\text { N78 } \\
\text { N78 }\end{array}$ & $\begin{array}{l}\text { NDG } \\
\text { NDG }\end{array}$ & & $\begin{array}{l}\text { Woos ley+ NDG. SIG DATA AVAIL. FRM AUTH. } \\
\text {. TBL OF STAT. THERMONUC. REAC. RT. PAR }\end{array}$ \\
\hline $\begin{array}{l}1189 \mathrm{CAL} \\
{ }^{45 \mathrm{AC}} \mathrm{CP} .\end{array}$ & $\begin{array}{l}\text { Theo J } \\
\text { Theo J } \\
+\gamma^{46} \mathrm{~K}\end{array}$ & $\begin{array}{l}\text { AND } \\
\text { AND } \\
\text { o(E) }\end{array}$ & $\begin{array}{l}22 \\
22\end{array}$ & $\begin{array}{l}371 \\
371\end{array}$ & $\begin{array}{l}\text { N78 } \\
\text { N78 }\end{array}$ & $\begin{array}{l}\text { NDG } \\
\text { NDG }\end{array}$ & & $\begin{array}{l}\text { Woosley + NDG. SIG DATA AVAIL. FRM AUTH. } \\
\text {. TBL OF STAT. THERMONUC. REAC. RT. PAR }\end{array}$ \\
\hline $\begin{array}{l}1190 \mathrm{CAL} \\
K(p, x)^{2}\end{array}$ & $\begin{array}{l}\text { Theo J } \\
\text { Theo J } \\
2{ }^{4} \mathrm{No}\end{array}$ & $\begin{array}{l}\text { AND } \\
\text { AND } \\
\text { O(E) }\end{array}$ & $\begin{array}{l}22 \\
22\end{array}$ & $\begin{array}{l}371 \\
371\end{array}$ & $\begin{array}{l}\text { N78 } \\
\text { N78 }\end{array}$ & $\begin{array}{l}\text { NDG } \\
\text { NDG }\end{array}$ & & $\begin{array}{l}\text { Woosley+ NDG. SIG DATA AVAIL. FRM AUTH. } \\
\text {. TBL OF STAT. THERMONUC. REAC. RT. PAR }\end{array}$ \\
\hline $\begin{array}{l}1191 \text { UPP } \\
K(p, x)^{2}\end{array}$ & $\begin{array}{l}\text { Expt J } \\
\text { Expt J } \\
2 \mathbf{B}_{\mathrm{Mg}}\end{array}$ & $\begin{array}{l}\text { AR I } \\
\text { ARI } \\
\text { O(E) }\end{array}$ & $\begin{array}{l}30 \\
30\end{array}$ & $\begin{array}{l}33 \\
33\end{array}$ & $\begin{array}{l}79 \\
79\end{array}$ & $\begin{array}{l}5 \cdot 0+1 \\
1 \cdot 0+2\end{array}$ & $1.6+2$ & $\begin{array}{l}\text { Lundquist+ CURV. CYLINDRICAL PELLET. } \\
\text { Lundquist+ TEL. COMPARED TO LITERATURE. }\end{array}$ \\
\hline $\begin{array}{l}1192 \text { UPP } \\
K(p, x)^{2}\end{array}$ & $\begin{array}{l}\text { Exp: J } \\
20 \mathrm{Mg}\end{array}$ & $\begin{array}{l}\text { ARI } \\
\text { thick }\end{array}$ & $\begin{array}{l}30 \\
\text { larget }\end{array}$ & $\begin{array}{l}33 \\
y 10\end{array}$ & $\begin{array}{r}79 \\
\text { eId }\end{array}$ & $8.0+1$ & $1.5+2$ & Lundquist + CURV. CYLINDRICAL PELLET. \\
\hline $\begin{array}{l}1193 \text { UPP } \\
{ }^{39} \mathrm{~K} / \mathrm{P}, 0\end{array}$ & $\begin{array}{l}\text { Expt J } \\
\text { Expt J } \\
\text { Expt A } \\
\sigma^{36} \mathrm{Ar}\end{array}$ & $\begin{array}{l}\text { ARI } \\
\text { ARI } \\
\text { JLCR } \\
\text { o(E) }\end{array}$ & $\begin{array}{l}30 \\
30 \\
16\end{array}$ & $\begin{array}{r}33 \\
33 \\
132\end{array}$ & $\begin{array}{l}79 \\
79 \\
79\end{array}$ & $\begin{array}{l}1 \cdot 2+5 \\
1 \cdot 3+5 \\
1 \cdot 2+5\end{array}$ & $1.6+2$ & $\begin{array}{l}\text { Lundquist+ CURV. } \\
\text { Lundquist+ TBL. COMPARED TO LITERATURE. } \\
\text { Malmborg + CURV. }\end{array}$ \\
\hline $1194 \mathrm{CAL}$ & $\begin{array}{l}\text { Theo J } \\
\text { Theo J } \\
\text { Theo R }\end{array}$ & $\begin{array}{l}\text { AND } \\
\text { AND } \\
\text { OAP-4 }\end{array}$ & $\begin{array}{l}22 \\
25 \\
425\end{array}$ & $\begin{array}{l}371 \\
371\end{array}$ & $\begin{array}{l}\text { N78 } \\
\text { N78 } \\
875\end{array}$ & $\begin{array}{l}\text { NDG } \\
\text { NDG } \\
5.1-1\end{array}$ & $9.2+0$ & $\begin{array}{l}\text { Woosley+ NDG. SIG DATA AVAIL. FRM AUTH. } \\
\text {. TBL OF STAT. THERMONUC. REAC. RT. PAR } \\
\text { Woosley+ CURV. HAUSER-FESHBACH. }\end{array}$ \\
\hline
\end{tabular}


REFERENCES ( cont)

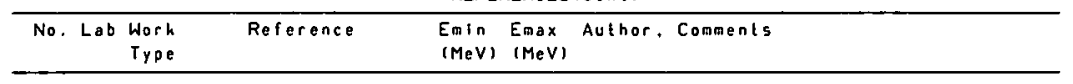

$39 K(p, n+p) 369 K \quad O(E)$

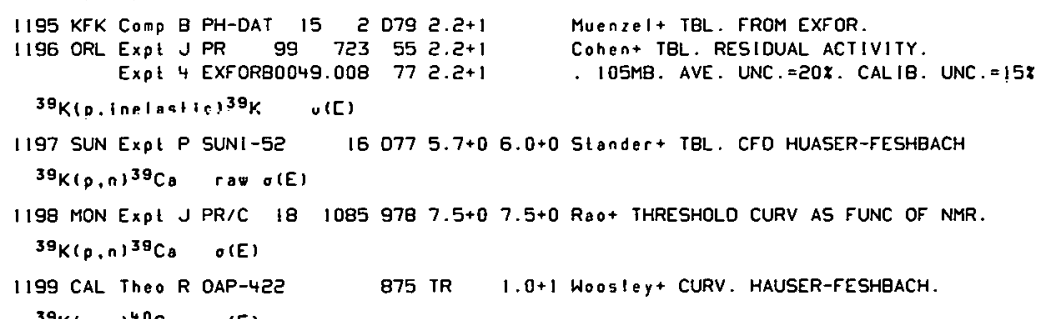

371 N78 NDG Woosley+ NDG. SIG DATA AVAIL. FRM AUTH. $29581.1+0$ 6.0+0 Johnsont EXPT. DETAlLS.

389 N79 5.0-1 2.0+0 Paine+ NDG. DEDUCED RES. STRENTGHS.

371 N7B NDG WODSley+ NDG. SIG DATA AVAIL. FRM AUTH. 371 N78 NDG TBL OF STAT. THERMONUC. REAC. RT. PAR 875 5.1-1 8.0+0 WoOSley+ CURV. HAUSER-FESHBACH.

371 N78 NDG WoOsley + NDG. SIG DATA AVAIL. FRM AUTH. 371 N7B NDG TBL OF STAT. THERMONUC. REAC. RT. PAR 875 TR 1.0+1 Woosley+ CURV. HAUSER-FESHBACH.

371 N78 NDG Woosley + NDG. SIG DATA AVAIL. FRM AUTH. 371 N78 NDG . TBL OF STAT. THERMONUC. REAC. RT. PAR 875 TR 1.0+1 WoOSley+ CURV. HAUSER-FESHBACH.

TBL OF STAT. THERMONUC. REAC. RT. PAR (E)

$160775.7+06.0+0$ Stander+ NDG. CFO HUASER-FESHBACH

875 TR $1.0+1$ WOOSley+ CURV. HAUSER-FESHBACH.

$20791.1+0$ 6.0+0 Muenzel+ TBL. FROM EXFOR.

$25601.1+06.0+0$ Johnson+ CURV. THICKER TRGT. 4PI DETECT

EXpt 4 EXFORBO068.002 $6781.1+06.0+0$. 45 DATA POINTS.

$$
4 I_{K}(p, n)^{4} \mathrm{ICa}_{\mathrm{C}} \text { average o(E) }
$$

1210 ORL Expt J PR/B $13617190641.2+01.3+0$ Johnson+ CURV. FROM I952 MEASURMENT.

$$
4 I_{K}(p, y)^{4} \mathrm{C}_{\mathrm{C}} \quad O(E)
$$

1211 CAL The J AND 22

Theo I AND 2 ?
The $R$ OAP-425

$4 E_{K}(p, 0)^{39_{A R}}$ o(E)

III CAL TheO J AND 22

Theo J AND 22

$4{ }^{2} K(p, n)^{42} C_{\theta} \quad \sigma(E)$

1213 CAL ThEO J AND 22 Theo J AND $22 \quad 371$ N78 NDG

${ }^{4} e_{K}(p, y){ }^{43} \mathrm{C}_{8} \quad \sigma(E)$

1214 CAL Theo J AND 22371 N78 NOG Theo $J$ AND $22 \quad 371$ N78 NDG

371 N78 NDG 1 N7B NDG

371 N78 NOG 371 N79 NDG
Woostey+ NDG. SIG DATA AVAIL. FRM AUTH. - TBL OF STAT. THERMONUC. REAC. RT. PAR $1.0+1$ Woosley+ CURV. HAUSER-FESHBACH.

WoOsléy+ NDG. SIG DATA AVAIL. FRM AUTH. - TBL OF STAT. THERMONUC. REAC. RT. PAR

Woosley+ NDG. SIG DATA AVAIL. FRM AUTH. - TBL OF STAT. THERMONUC. REAC. RT. PAR

Woosiey+ NDG. SIG DATA AVAIL. FRM AUTH. - TBL OF STAT. THERMONUC. REAC. RT. PAR 


\begin{tabular}{|c|c|c|c|c|c|c|c|c|c|}
\hline \multirow{3}{*}{$\begin{array}{l}\text { No. Lab } \\
43 \mathrm{~K}(0 . \\
1215 \mathrm{CAL}\end{array}$} & \multirow{2}{*}{$\begin{array}{l}\text { Worh } \\
\text { Type } \\
a)^{40} \text { Ar }\end{array}$} & \multicolumn{3}{|c|}{ Reference } & & \multirow[t]{2}{*}{$\begin{array}{l}\text { EmIn } \\
(M \circ V)\end{array}$} & \multirow[t]{2}{*}{$\begin{array}{l}\text { Emax } \\
(M e V)\end{array}$} & \multirow[t]{2}{*}{ Author. } & \multirow[t]{2}{*}{ Comments } \\
\hline & & $\sigma(E)$ & & & & & & & \\
\hline & $\begin{array}{l}\text { Theo J } \\
\text { Theo J } \\
)^{43} \mathrm{Ca}\end{array}$ & $\begin{array}{l}\text { AND } \\
\text { AND } \\
\text { O(E) }\end{array}$ & $\begin{array}{l}22 \\
22\end{array}$ & $\begin{array}{l}371 \\
371\end{array}$ & $\begin{array}{l}\text { N7g } \\
\text { N7g }\end{array}$ & $\begin{array}{l}\text { NDG } \\
\text { NDG }\end{array}$ & & $\begin{array}{l}\text { Woosleyt } \\
\text { TBL OF }\end{array}$ & $\begin{array}{l}\text { NDG. SIG DATA AVAIL. FRM NUTH. } \\
\text { STAT. THERMONUC. REAC. RT. PAR }\end{array}$ \\
\hline $\begin{array}{l}1216 \mathrm{CAL} \\
4{ }^{3} \mathrm{~K}(0, y)\end{array}$ & $\begin{array}{l}\text { Theo J } \\
\text { Theo J } \\
J^{44} \mathrm{Ca}\end{array}$ & $\begin{array}{l}\text { AND } \\
\text { AND } \\
\text { O(E) }\end{array}$ & $\begin{array}{l}22 \\
22\end{array}$ & $\begin{array}{l}371 \\
371\end{array}$ & $\begin{array}{l}\text { N78 } \\
\text { N78 }\end{array}$ & $\begin{array}{l}\text { NDG } \\
\text { NDG }\end{array}$ & & $\begin{array}{l}\text { Woosley+ } \\
\text { TBL OF }\end{array}$ & $\begin{array}{l}\text { NDG. SIG DATA AVAIL. FRM AUTH. } \\
\text { STAT. THERMONUC. REAC. RT. PAR }\end{array}$ \\
\hline $\begin{array}{l}1217 \mathrm{CAL} \\
{ }^{44} \mathrm{~K}(\mathrm{p}, 0\end{array}$ & $\begin{array}{l}\text { Theo J } \\
\text { Theo J } \\
\text { J Ar }\end{array}$ & $\begin{array}{l}\text { AND } \\
\text { AND } \\
\text { O(E) }\end{array}$ & $\begin{array}{l}22 \\
22\end{array}$ & $\begin{array}{l}371 \\
371\end{array}$ & $\begin{array}{l}\text { N78 } \\
\text { N78 }\end{array}$ & $\begin{array}{l}\text { NDG } \\
\text { NDG }\end{array}$ & & $\begin{array}{l}\text { Woostey* } \\
\text { TBL OF }\end{array}$ & $\begin{array}{l}\text { NDG. SIG DATA AVAIL. FRM AUTH. } \\
\text { STAT. THERMONUC. REAC. RT. PAR }\end{array}$ \\
\hline $\begin{array}{l}1218 \mathrm{CAL} \\
{ }^{44} \mathrm{~K} / \mathrm{p} .0\end{array}$ & $\begin{array}{l}\text { Theo J } \\
\text { Theo J } \\
{ }^{44} \mathrm{Ca}\end{array}$ & $\begin{array}{l}\text { AND } \\
\text { AND } \\
\sigma(E)\end{array}$ & $\begin{array}{l}22 \\
22\end{array}$ & $\begin{array}{l}371 \\
371\end{array}$ & $\begin{array}{l}\text { N78 } \\
\text { N78 }\end{array}$ & $\begin{array}{l}\text { NDG } \\
\text { NDG }\end{array}$ & & $\begin{array}{l}\text { Woosley+ } \\
\text { TBL OF }\end{array}$ & $\begin{array}{l}\text { NDG. SIG DATA AVAIL. FRM AUTH. } \\
\text { STAT. THERMONUC. REAC. RT. PAR }\end{array}$ \\
\hline $\begin{array}{l}1219 \mathrm{CAL} \\
{ }^{44} \mathrm{~K}(\mathrm{p}, \mathrm{y}\end{array}$ & $\begin{array}{l}\text { Theo J } \\
\text { Theo J } \\
{ }^{45} \mathrm{Ca}\end{array}$ & $\begin{array}{l}\text { AND } \\
\text { AND } \\
\text { o(E) }\end{array}$ & $\begin{array}{l}22 \\
22\end{array}$ & $\begin{array}{l}371 \\
371\end{array}$ & $\begin{array}{l}\text { N78 } \\
\text { N78 }\end{array}$ & $\begin{array}{l}\text { NDG } \\
\text { NDG }\end{array}$ & & $\begin{array}{l}\text { Woosley* } \\
\text {. TBL OF }\end{array}$ & $\begin{array}{l}\text { NDG. SIG DATA AVAIL. FRM AUTH. } \\
\text { STAT. THERMONUC. REAC. RT. PAR }\end{array}$ \\
\hline $\begin{array}{l}1220 \mathrm{CAL} \\
\quad 45 \mathrm{~K}(\mathrm{p}, 0\end{array}$ & $\begin{array}{l}\text { Theo J } \\
\text { Theo J } \\
J^{42_{A r}}\end{array}$ & $\begin{array}{l}\text { AND } \\
\text { AND } \\
\text { O(E) }\end{array}$ & $\begin{array}{l}22 \\
22\end{array}$ & $\begin{array}{l}371 \\
371\end{array}$ & $\begin{array}{l}\text { N7G } \\
\text { N7G }\end{array}$ & $\begin{array}{l}\text { NDG } \\
\text { NDG }\end{array}$ & & $\begin{array}{l}\text { Woosley* } \\
\text { TBL OF }\end{array}$ & $\begin{array}{l}\text { NDG. SIG DATA AVAIL. FRM AUTH. } \\
\text { STAT. THERMONUC. REAC. RI. PAR }\end{array}$ \\
\hline $\begin{array}{l}1221 \mathrm{CAL} \\
{ }^{45} \mathrm{~K}(p, n\end{array}$ & $\begin{array}{l}\text { Theo J } \\
\text { Theo J } \\
{ }^{45} \mathrm{Ca}\end{array}$ & $\begin{array}{l}\text { AND } \\
\text { AND } \\
\text { O(E) }\end{array}$ & $\begin{array}{l}22 \\
2 己\end{array}$ & $\begin{array}{l}371 \\
371\end{array}$ & $\begin{array}{l}\text { N78 } \\
\text { N78 }\end{array}$ & $\begin{array}{l}\text { NDG } \\
\text { NDG }\end{array}$ & & $\begin{array}{l}\text { Woosleyt } \\
\text { TBL OF }\end{array}$ & $\begin{array}{l}\text { NDG. SIG DATA AVAIL. FRM AUTH. } \\
\text { STAT. THERMONUC. REAC. RT. PAR }\end{array}$ \\
\hline $\begin{array}{l}1222 \mathrm{CAL} \\
{ }^{45} \mathrm{~K}(0, \gamma\end{array}$ & $\begin{array}{l}\text { Theo J } \\
\text { Theo J } \\
{ }^{46} \mathrm{C} \text {. }\end{array}$ & $\begin{array}{l}\text { AND } \\
\text { AND } \\
\sigma(E)\end{array}$ & $\begin{array}{l}22 \\
22\end{array}$ & $\begin{array}{l}37 ! \\
37 !\end{array}$ & $\begin{array}{l}\text { N78 } \\
\text { N78 }\end{array}$ & $\begin{array}{l}\text { NDG } \\
\text { NDG }\end{array}$ & & $\begin{array}{l}\text { Woosley+ } \\
\text {. TBL OF }\end{array}$ & $\begin{array}{l}\text { NDG. SIG DATA AVAIL. FRM AUTH. } \\
\text { STAT. THERMONUC. REAC. RT. PAR }\end{array}$ \\
\hline $\begin{array}{l}1223 \mathrm{CAL} \\
{ }^{48} \mathrm{~K} 1 \mathrm{p} .\end{array}$ & $\begin{array}{l}\text { Theo J } \\
\text { Theo J } \\
)^{43} \mathrm{Ar}\end{array}$ & $\begin{array}{l}\text { AND } \\
\text { AND } \\
\text { o(E) }\end{array}$ & $\begin{array}{l}22 \\
22\end{array}$ & $\begin{array}{l}371 \\
371\end{array}$ & $\begin{array}{l}\text { N78 } \\
\text { N78 }\end{array}$ & $\begin{array}{l}\text { NDG } \\
\text { NDG }\end{array}$ & & $\begin{array}{l}\text { Woosleyt } \\
\text { TBL OF }\end{array}$ & $\begin{array}{l}\text { NDG. SIG DATA AVAIL. FRM AUTH. } \\
\text { STAT. THERMONUC. REAC. RT. PAR }\end{array}$ \\
\hline $\begin{array}{l}1224 \text { CAL } \\
{ }^{46} \mathrm{~K} 60,0\end{array}$ & $\begin{array}{l}\text { Theo J } \\
\text { Theo J } \\
J^{46} \mathrm{Ca}\end{array}$ & $\begin{array}{l}\text { AND } \\
\text { AND } \\
\text { o(E) }\end{array}$ & $\begin{array}{l}22 \\
22\end{array}$ & $\begin{array}{l}371 \\
371\end{array}$ & $\begin{array}{l}\text { N7B } \\
\text { N78 }\end{array}$ & $\begin{array}{l}\text { NDG } \\
\text { NDG }\end{array}$ & & $\begin{array}{l}\text { Woosleyt } \\
\text { TBL OF }\end{array}$ & $\begin{array}{l}\text { NDG. SIG DATA AVAIL. FRM AUTH. } \\
\text { STAT. THERMONUC. REAC. RT. PAR }\end{array}$ \\
\hline $\begin{array}{l}\text { I } 225 \mathrm{CAL} \\
{ }^{46} \mathrm{~K}(\mathrm{p}, 2\end{array}$ & $\begin{array}{l}\text { Theo J } \\
\text { Theo J } \\
{ }^{47} \mathrm{Co}\end{array}$ & $\begin{array}{l}\text { AND } \\
\text { AND } \\
\text { o(E) }\end{array}$ & $\begin{array}{l}22 \\
22\end{array}$ & $\begin{array}{l}371 \\
371\end{array}$ & $\begin{array}{l}\text { N7B } \\
\text { N78 }\end{array}$ & $\begin{array}{l}\text { NDG } \\
\text { NDG }\end{array}$ & & $\begin{array}{l}\text { Woosleyt } \\
\text {. TBL OF }\end{array}$ & $\begin{array}{l}\text { NDG. SIG DATA AVAIL. FRM AUTH. } \\
\text { STAT. THERMONUC. REAC. RT. PAR }\end{array}$ \\
\hline $\begin{array}{l}1226 \mathrm{CAL} \\
{ }^{47} \mathrm{~K}(\mathrm{p}, 0\end{array}$ & $\begin{array}{l}\text { Theo J } \\
\text { Theo J } \\
3^{44} \mathrm{Ar}\end{array}$ & $\begin{array}{l}\text { AND } \\
\text { AND } \\
\text { O(E) }\end{array}$ & $\begin{array}{l}22 \\
22\end{array}$ & $\begin{array}{l}371 \\
371\end{array}$ & $\begin{array}{l}\text { N78 } \\
\text { N78 }\end{array}$ & $\begin{array}{l}\text { NDG } \\
\text { NDG }\end{array}$ & & $\begin{array}{l}\text { Woosley+ } \\
\text { TBL of }\end{array}$ & $\begin{array}{l}\text { NOG. SIG DATA AVAIL. FRM AUTH. } \\
\text { STAT. THERMONUC. REAC. RT. PAR }\end{array}$ \\
\hline $\begin{array}{l}1227 \text { CAL } \\
47 \mathrm{~K}(p, n\end{array}$ & $\begin{array}{l}\text { Theo J } \\
\text { Theo J } \\
J^{47} \mathrm{Ca}\end{array}$ & $\begin{array}{l}\text { AND } \\
\text { AND } \\
\text { o(E) }\end{array}$ & $\begin{array}{l}22 \\
22\end{array}$ & $\begin{array}{l}371 \\
371\end{array}$ & $\begin{array}{l}\text { N78 } \\
\text { N78 }\end{array}$ & $\begin{array}{l}\text { NDG } \\
\text { NDG }\end{array}$ & & $\begin{array}{l}\text { Woosley+ } \\
\text { TBL OF }\end{array}$ & $\begin{array}{l}\text { NDG. SIG DATA AVAIL. FRM AUTH. } \\
\text { STAT. THERMONUC. REAC. RT. PAR }\end{array}$ \\
\hline $\begin{array}{l}1228 \mathrm{CAL} \\
{ }^{47} \mathrm{~K}(\mathrm{p}, \gamma\end{array}$ & $\begin{array}{l}\text { Theo J } \\
\text { Theo J } \\
{ }^{48} \mathrm{Ca}\end{array}$ & $\begin{array}{l}\text { AND } \\
\text { AND } \\
O(E)\end{array}$ & $\begin{array}{l}22 \\
22\end{array}$ & $\begin{array}{l}37 ! \\
37 !\end{array}$ & $\begin{array}{l}\text { N78 } \\
\text { N78 }\end{array}$ & $\begin{array}{l}\text { NDG } \\
\text { NDG }\end{array}$ & & $\begin{array}{l}\text { Woosley+ } \\
\text { TBL OF }\end{array}$ & $\begin{array}{l}\text { NDG. SIG DATA AVAIL. FRM AUTH. } \\
\text { STAT. THERMONUC, REAC. RT. PAR }\end{array}$ \\
\hline $\begin{array}{l}1229 \text { CAL } \\
{ }^{4 \theta_{K}}(p, 0\end{array}$ & $\begin{array}{l}\text { Then J } \\
\text { Theo J } \\
\text { J }^{4} \mathrm{Ar}\end{array}$ & $\begin{array}{l}\text { AND } \\
\text { AND } \\
\text { O(E) }\end{array}$ & $\begin{array}{l}22 \\
22\end{array}$ & $\begin{array}{l}371 \\
371\end{array}$ & $\begin{array}{l}\text { N7G } \\
\text { N78 }\end{array}$ & $\begin{array}{l}\text { NDG } \\
\text { NDG }\end{array}$ & & $\begin{array}{l}\text { Woosley+ } \\
\text { TBL OF }\end{array}$ & $\begin{array}{l}\text { NDG. SIG DATA AVAIL. FRM AUTH. } \\
\text { STAT. THERMONUC. REAC. RT. PAR }\end{array}$ \\
\hline $\begin{array}{l}1230 \mathrm{CAL} \\
{ }^{40} \mathrm{~K}(\mathrm{p}, \mathrm{n}\end{array}$ & $\begin{array}{l}\text { Theo J } \\
\text { Theo J } \\
{ }^{40} \mathrm{Ca}\end{array}$ & $\begin{array}{l}\text { AND } \\
\text { AND } \\
\text { O(E) }\end{array}$ & $\begin{array}{l}22 \\
22\end{array}$ & $\begin{array}{l}371 \\
371\end{array}$ & $\begin{array}{l}\text { N78 } \\
\text { N78 }\end{array}$ & $\begin{array}{l}\text { NDG } \\
\text { NDG }\end{array}$ & & $\begin{array}{l}\text { Woostey+ } \\
\text {. TBL OF }\end{array}$ & $\begin{array}{l}\text { NDG. SIG DATA AVAIL. FRM AUTH. } \\
\text { STAT. THERMONUC. REAC. RT. PAR }\end{array}$ \\
\hline $1231 \mathrm{CAL}$ & $\begin{array}{l}\text { Theo J } \\
\text { Theo J }\end{array}$ & $\begin{array}{l}\text { AND } \\
\text { AND }\end{array}$ & $\begin{array}{l}22 \\
22\end{array}$ & $\begin{array}{l}371 \\
371\end{array}$ & $\begin{array}{l}\text { N78 } \\
\text { N78 }\end{array}$ & $\begin{array}{l}\text { NDG } \\
\text { NDG }\end{array}$ & & $\begin{array}{l}\text { Woosley+ } \\
\text { TBL OF }\end{array}$ & $\begin{array}{l}\text { NDG. SIG DATA AVAIL. FRM AUTH. } \\
\text { STAT. THERMONUC, REAC. RT. PAR }\end{array}$ \\
\hline${ }^{4} \theta_{K}(p)$, & & & & & & & & & \\
\hline $1232 \mathrm{CAL}$ & $\begin{array}{l}\text { Theo J } \\
\text { Theo J }\end{array}$ & $\begin{array}{l}\text { AND } \\
\text { AND }\end{array}$ & $\begin{array}{l}22 \\
22\end{array}$ & $\begin{array}{l}371 \\
371\end{array}$ & $\begin{array}{l}\text { N78 } \\
\text { N78 }\end{array}$ & $\begin{array}{l}\text { NDG } \\
\text { NDG }\end{array}$ & & $\begin{array}{l}\text { Woosley* } \\
\text { TBL OF }\end{array}$ & $\begin{array}{l}\text { NDG. SIG DATA AVAIL. FRM AUTH. } \\
\text { STAT. THERMONUC. REAC. RT. PAR }\end{array}$ \\
\hline
\end{tabular}


REFERENCES ( c on t)

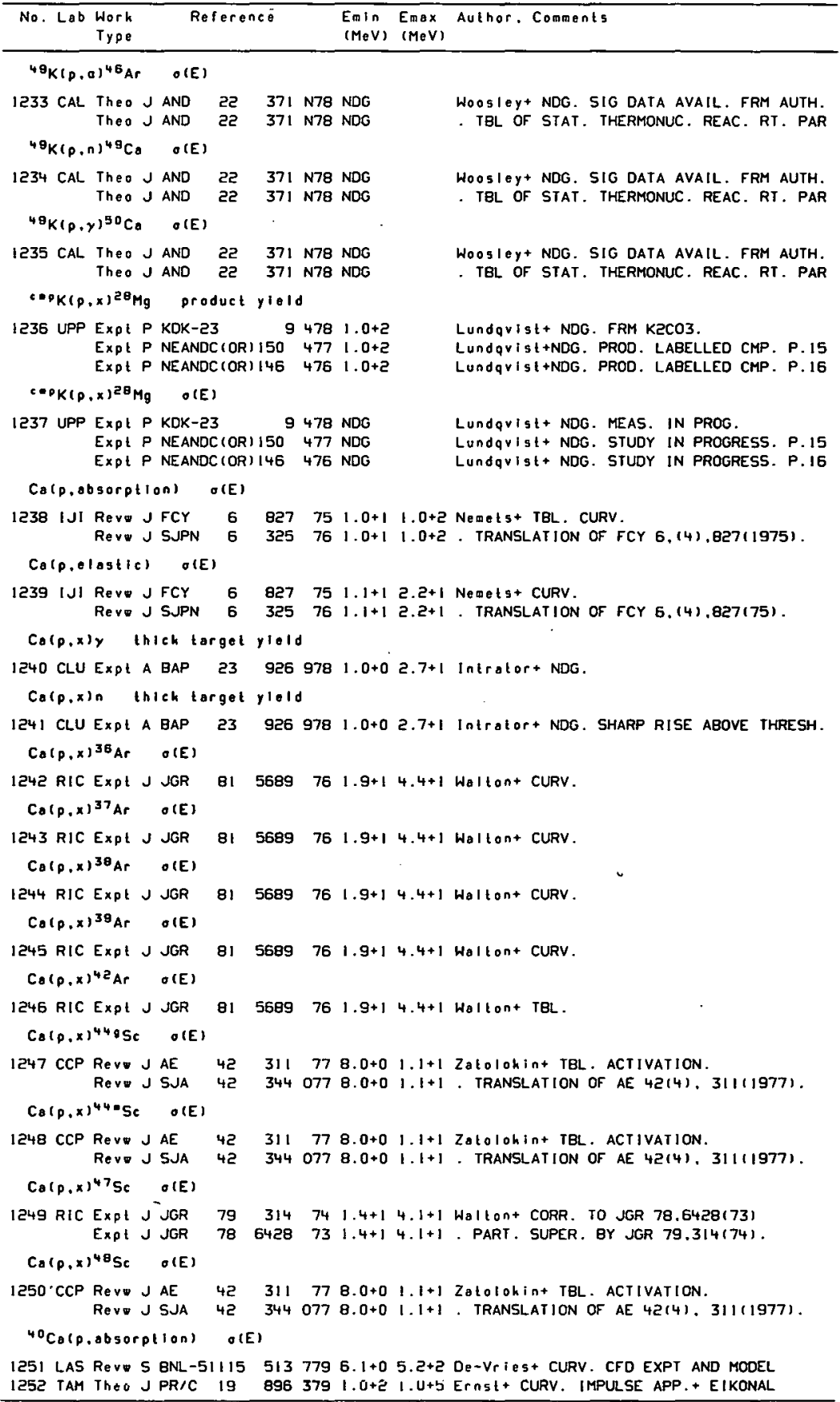


REFERENCES ( $c$ on t)

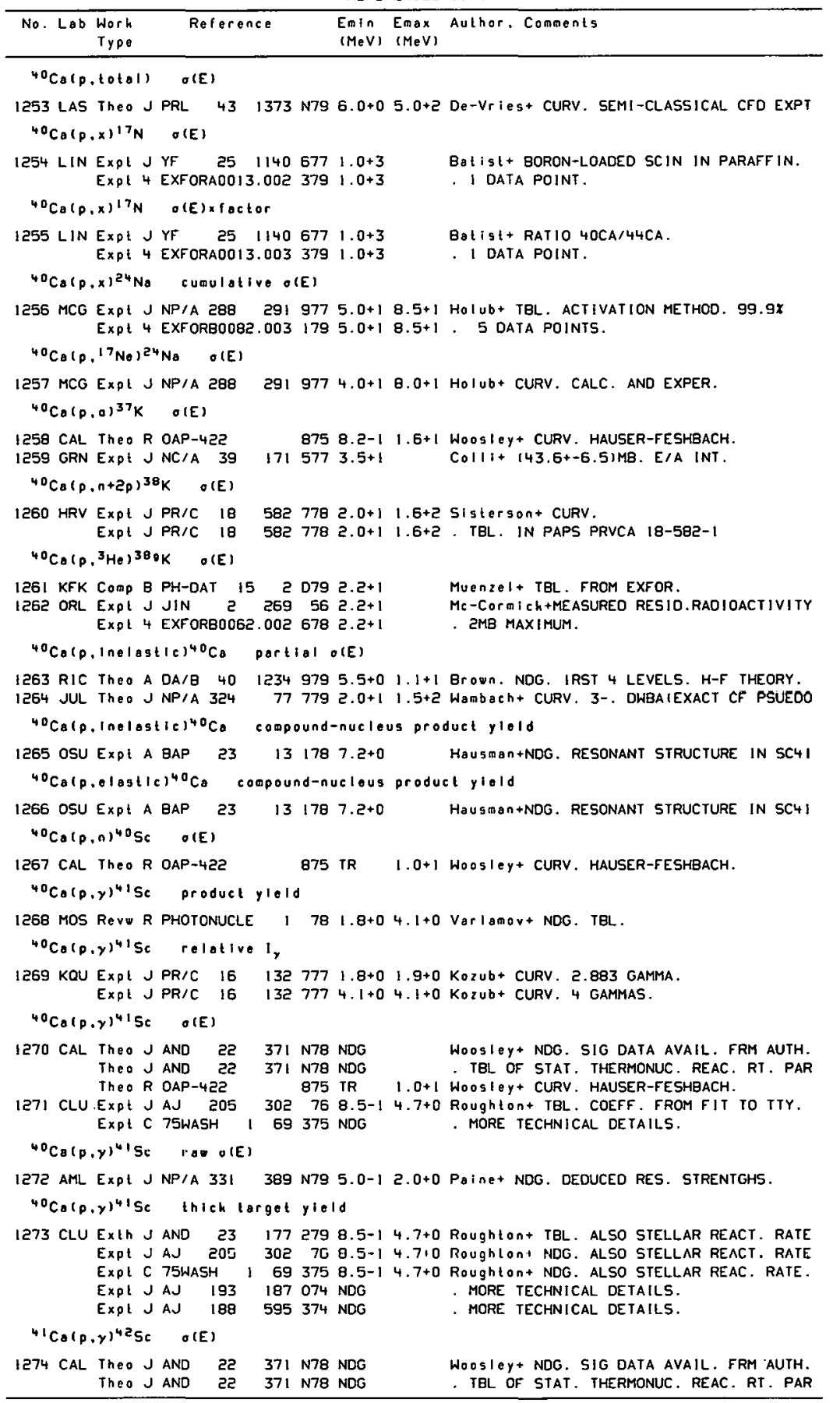


REFERENCES ( c on t)

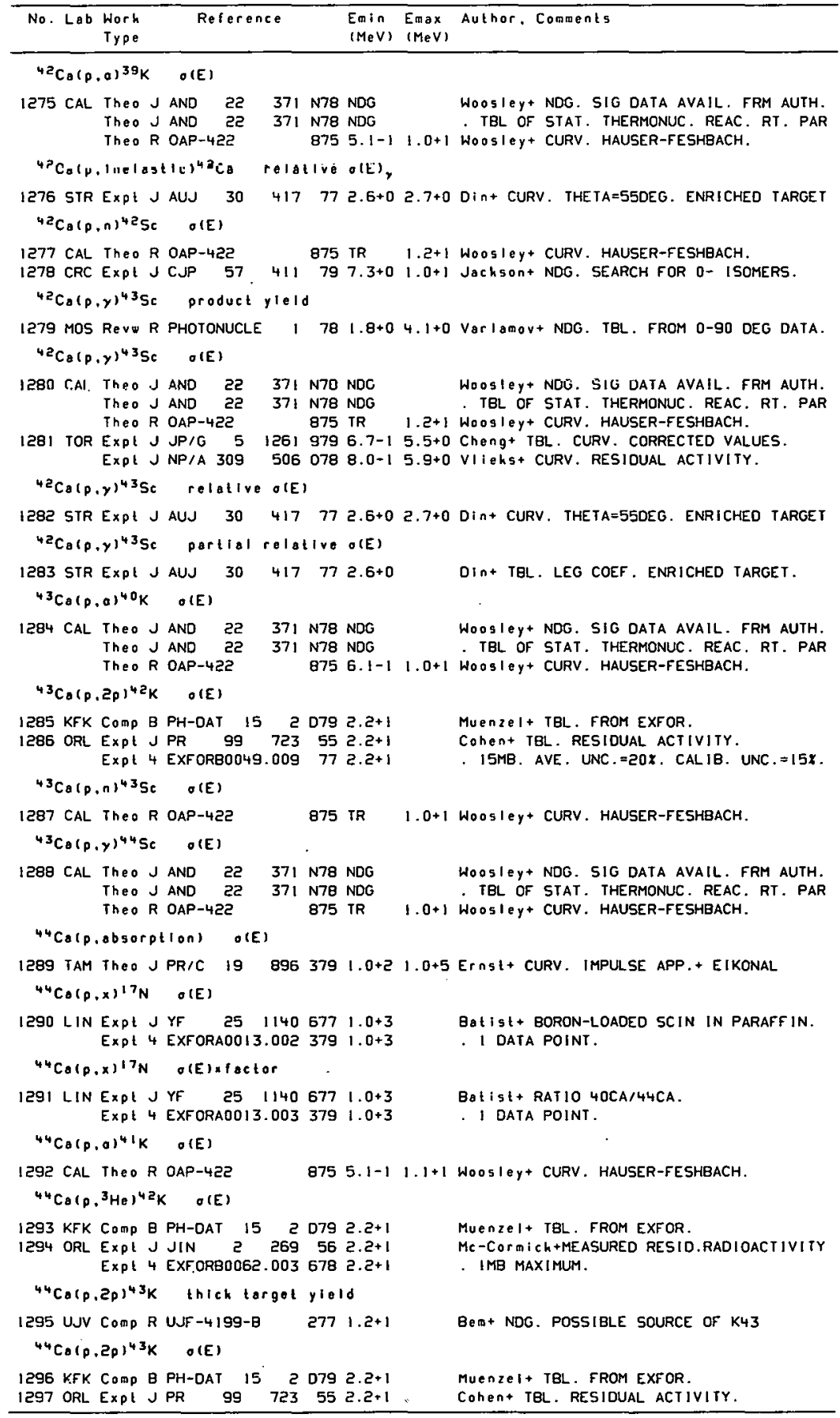


REFERENCES (cont)

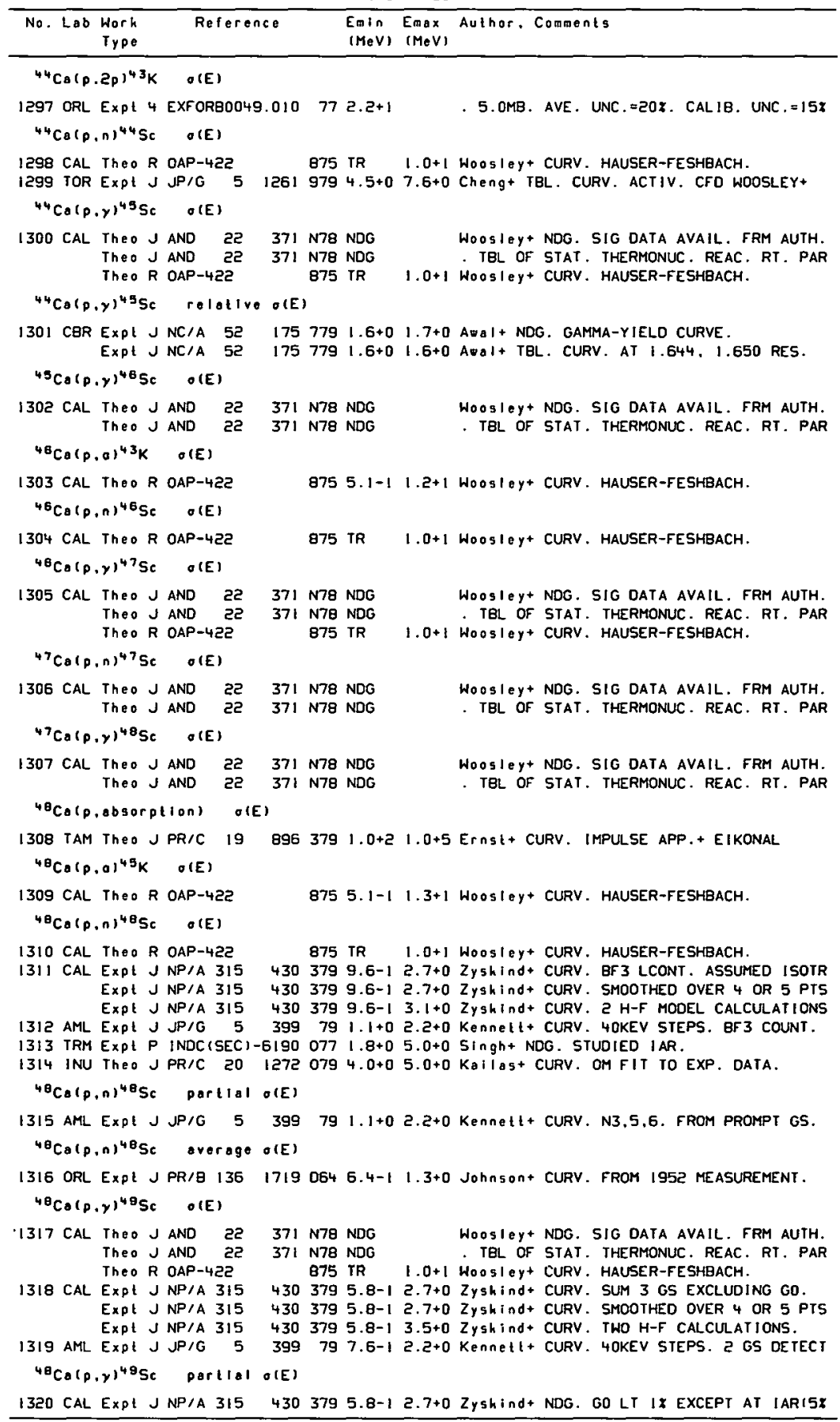




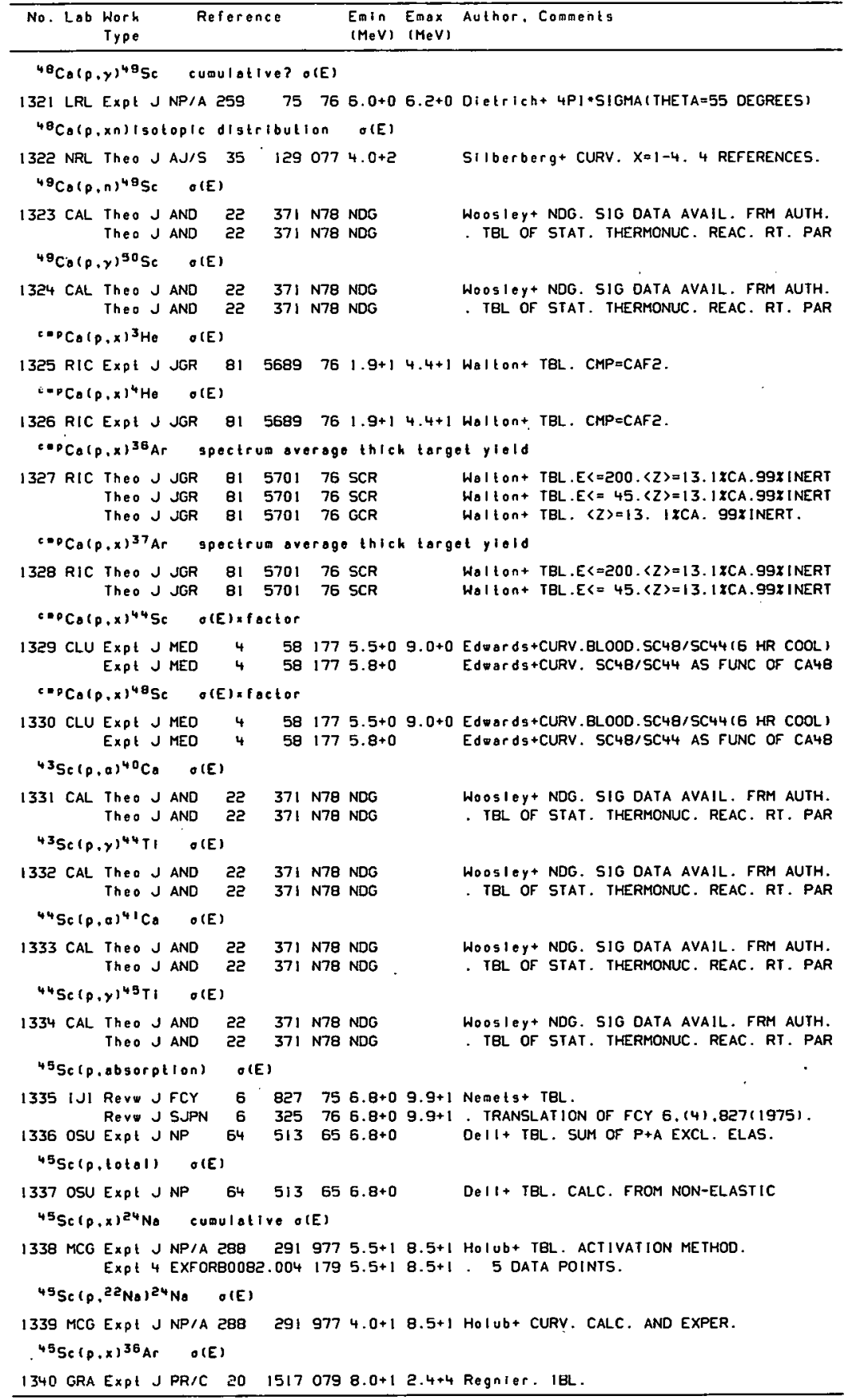


REFERENCES ( c on ()

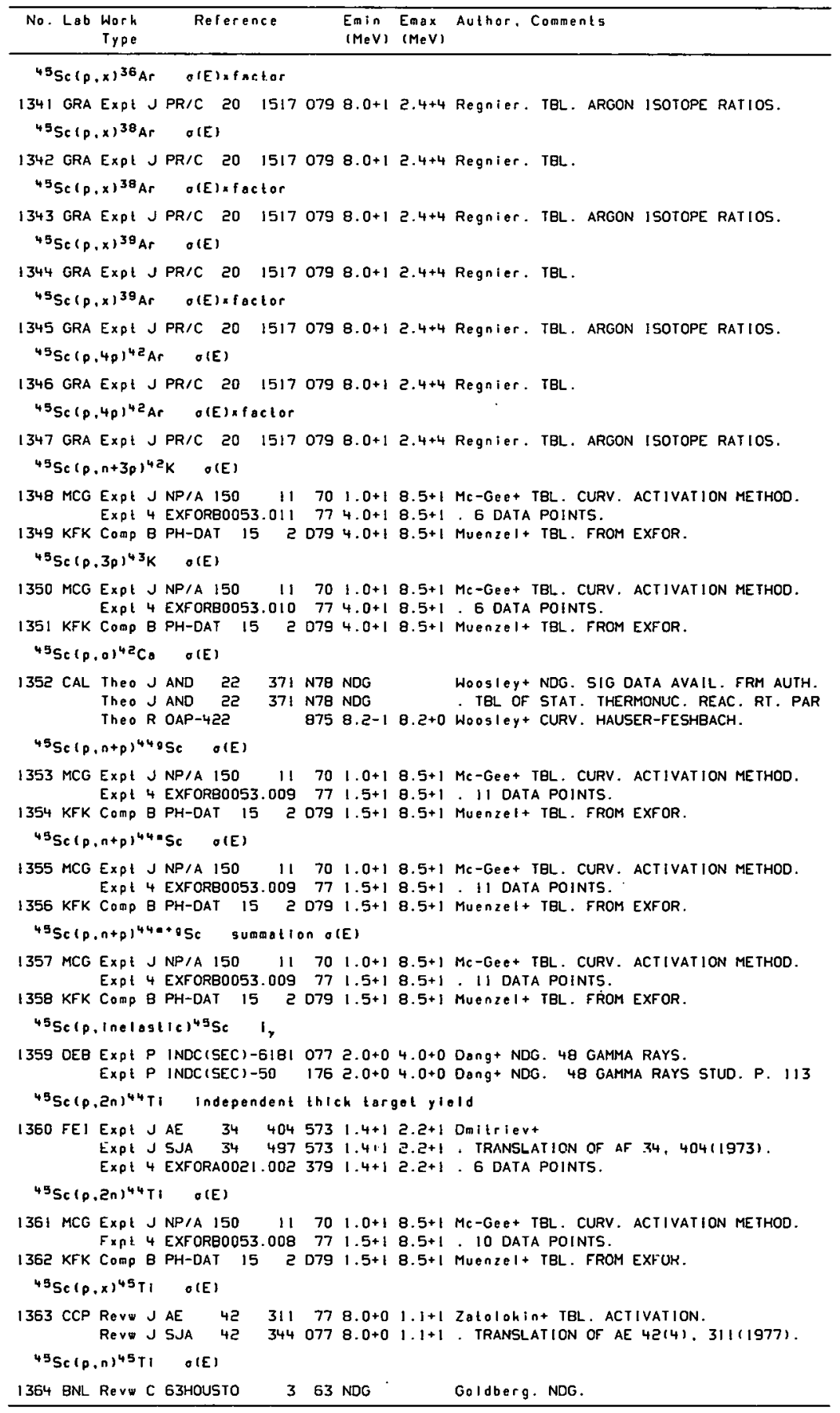




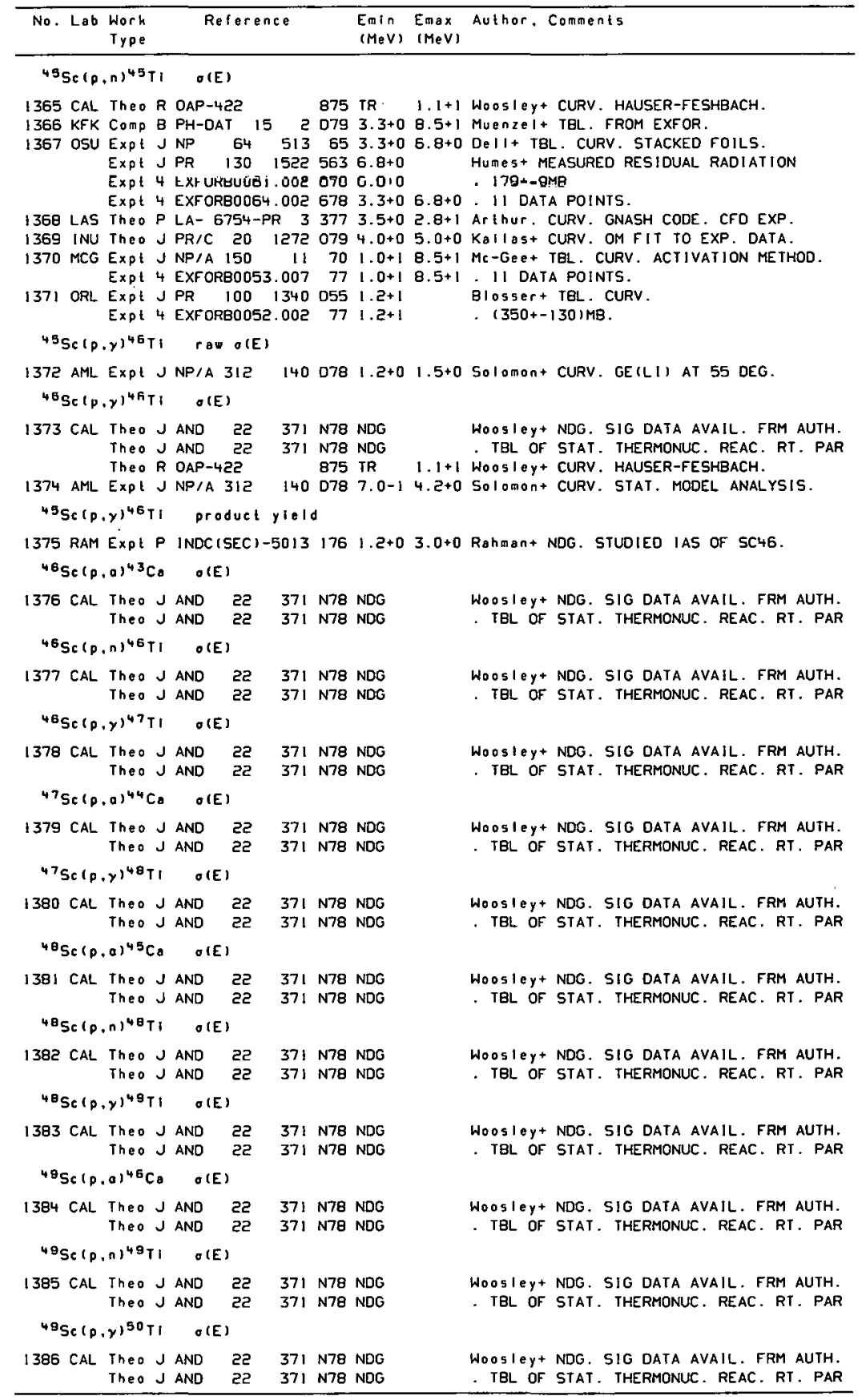




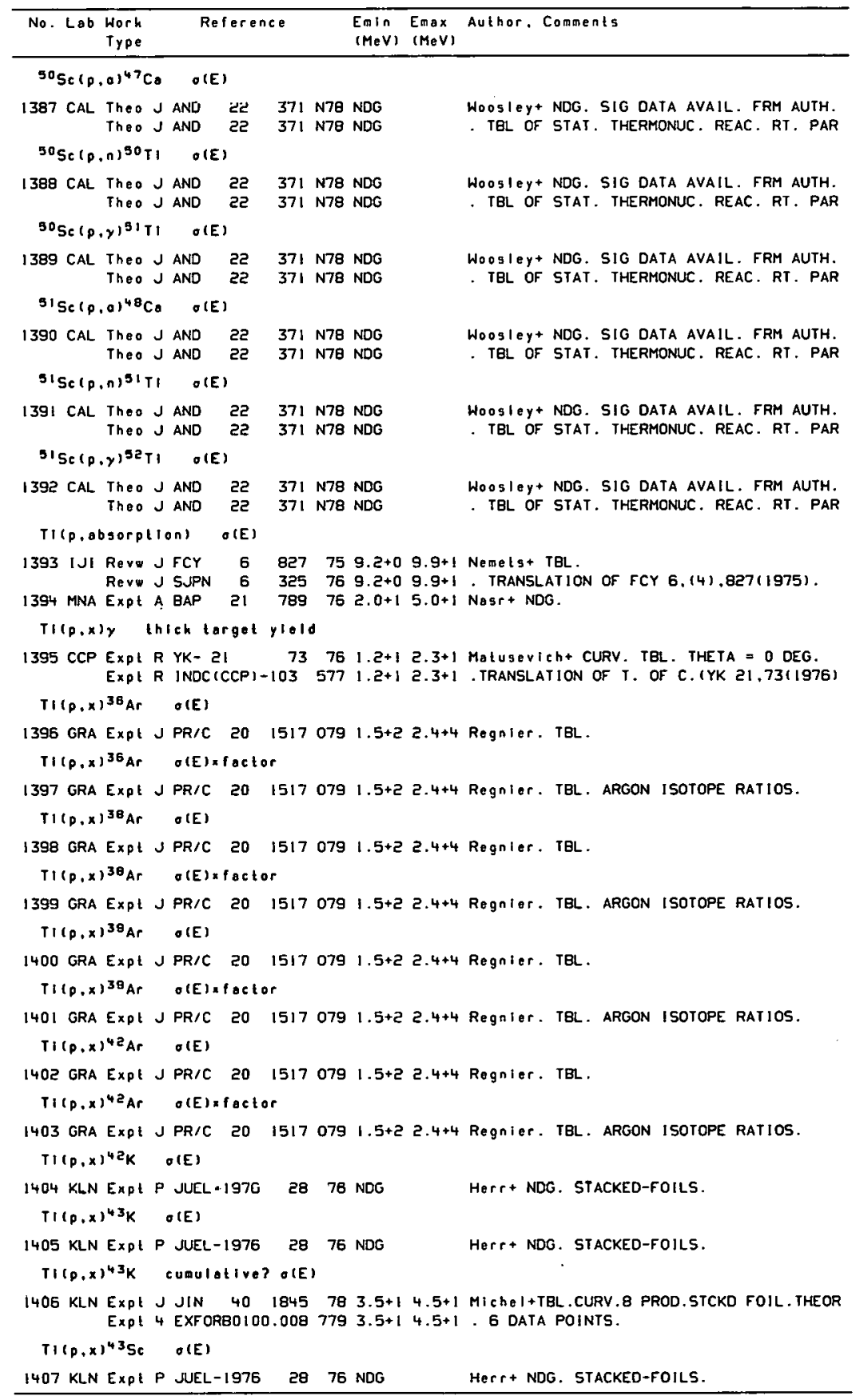




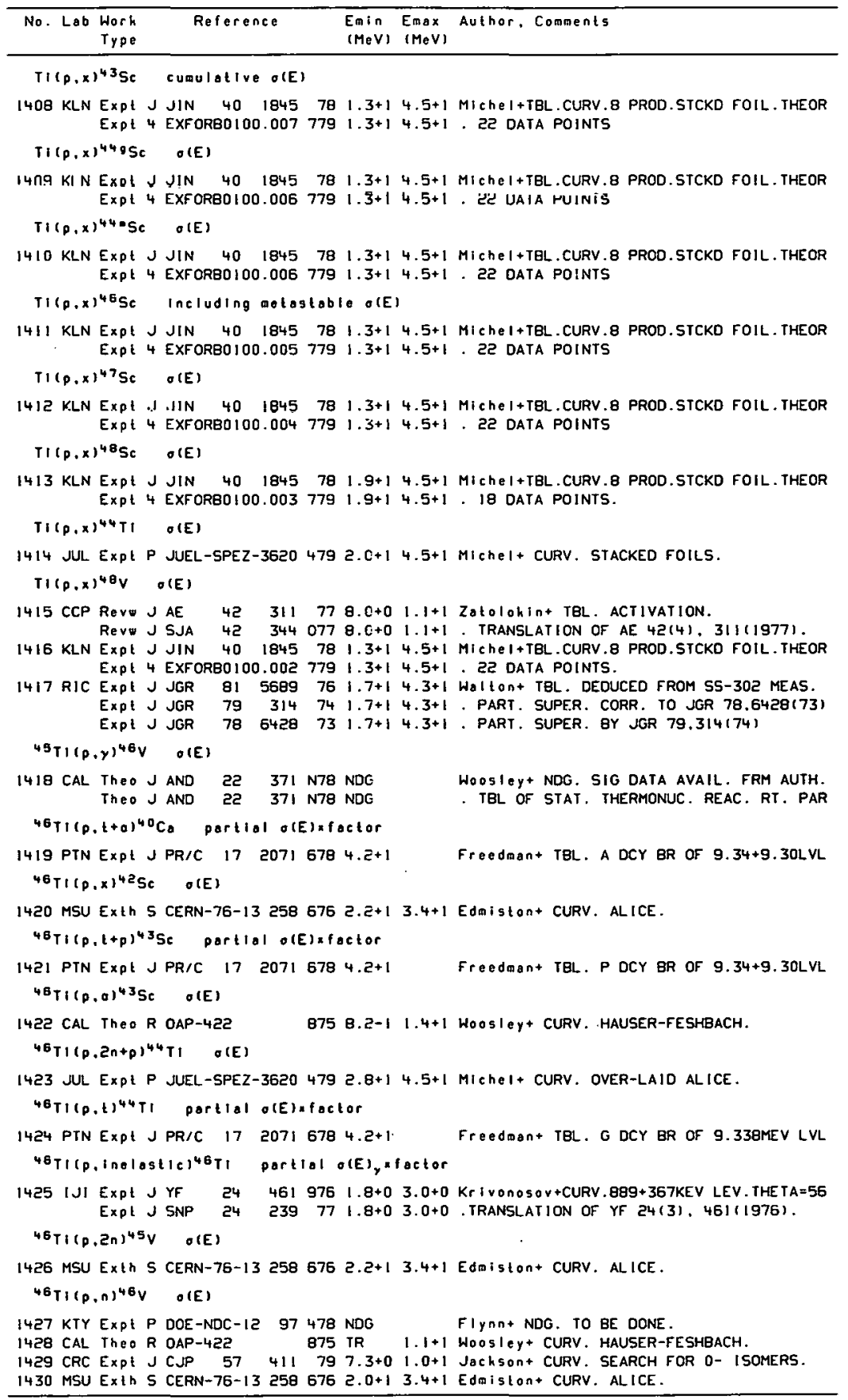




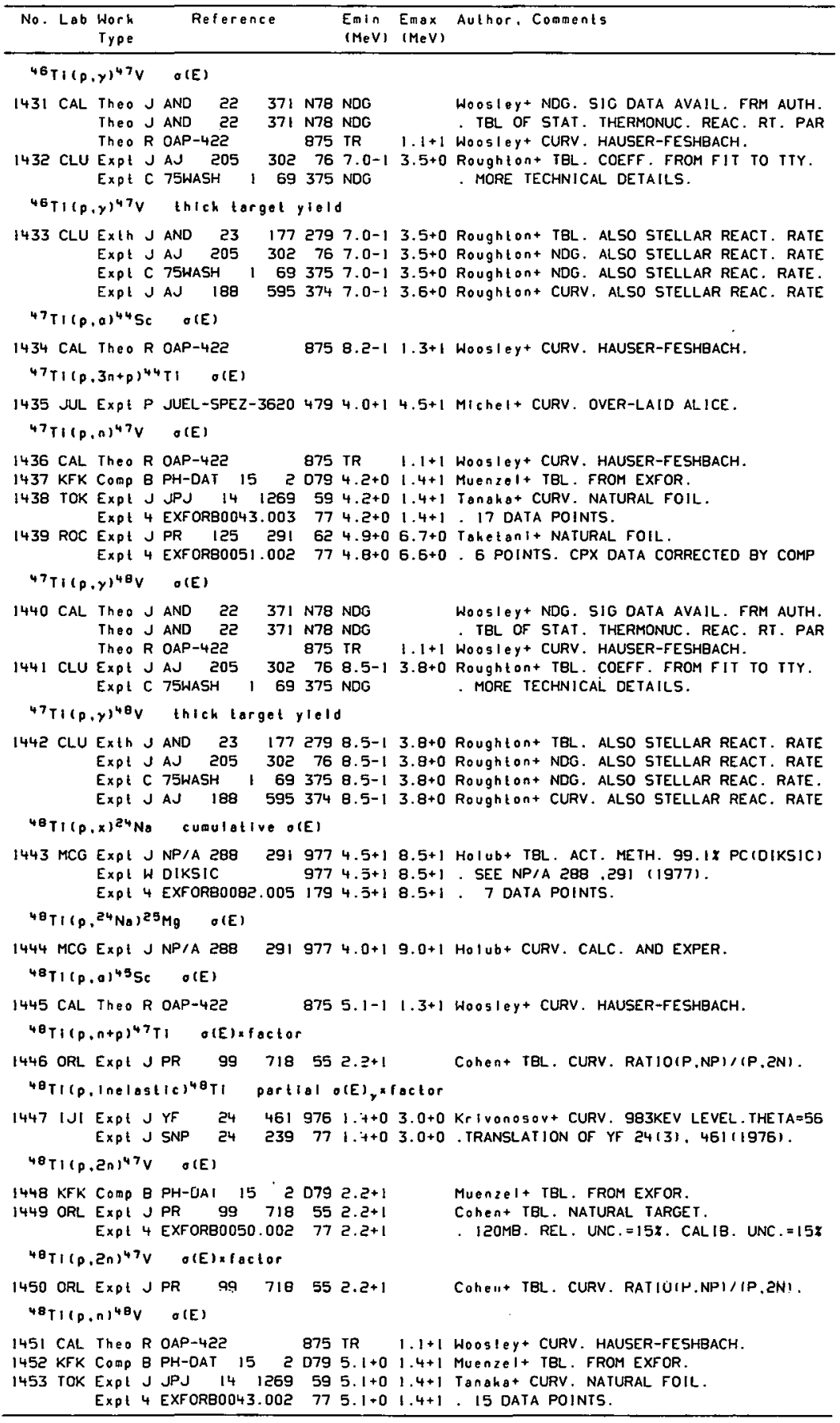




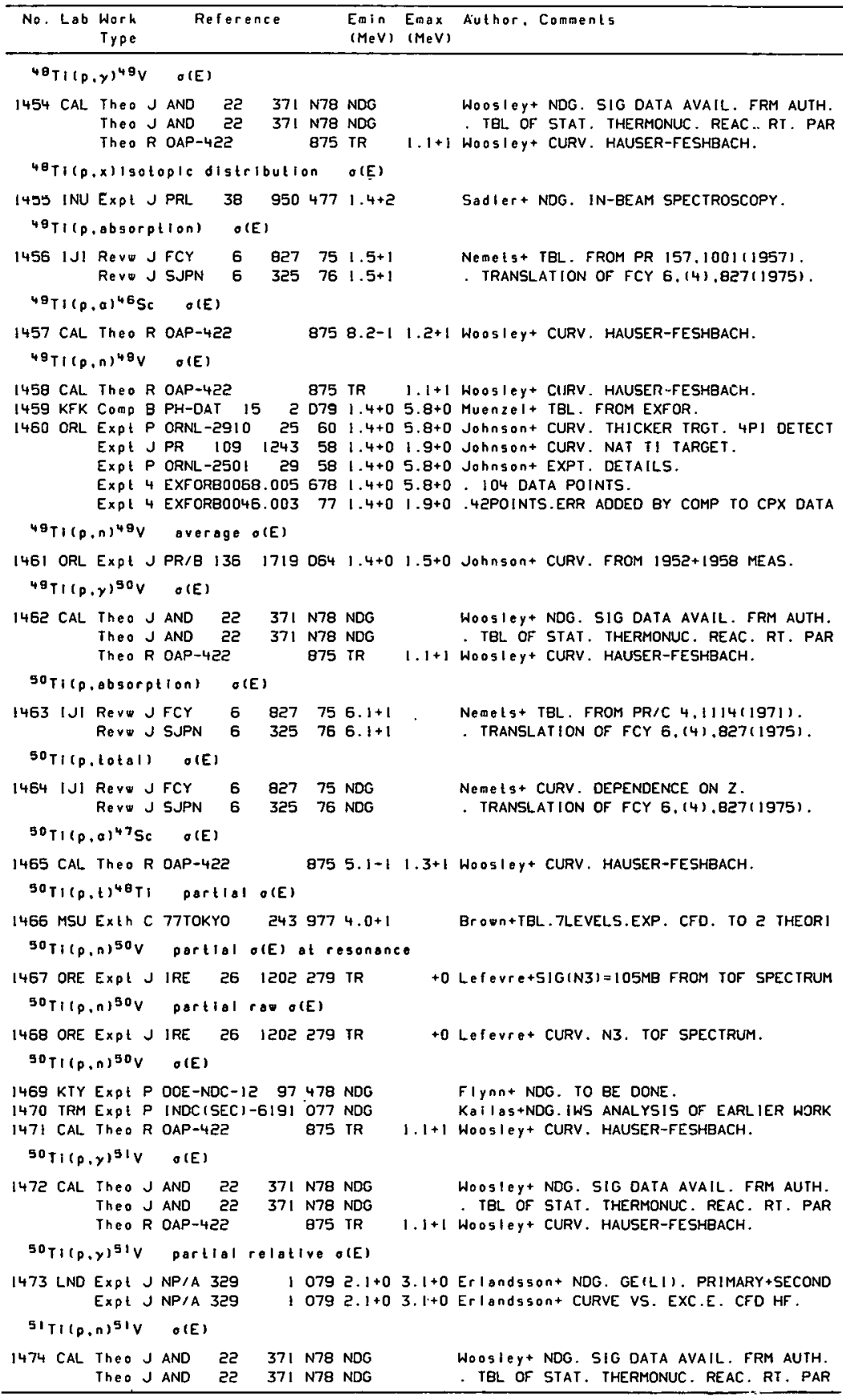


REFERENCES ( c on t )

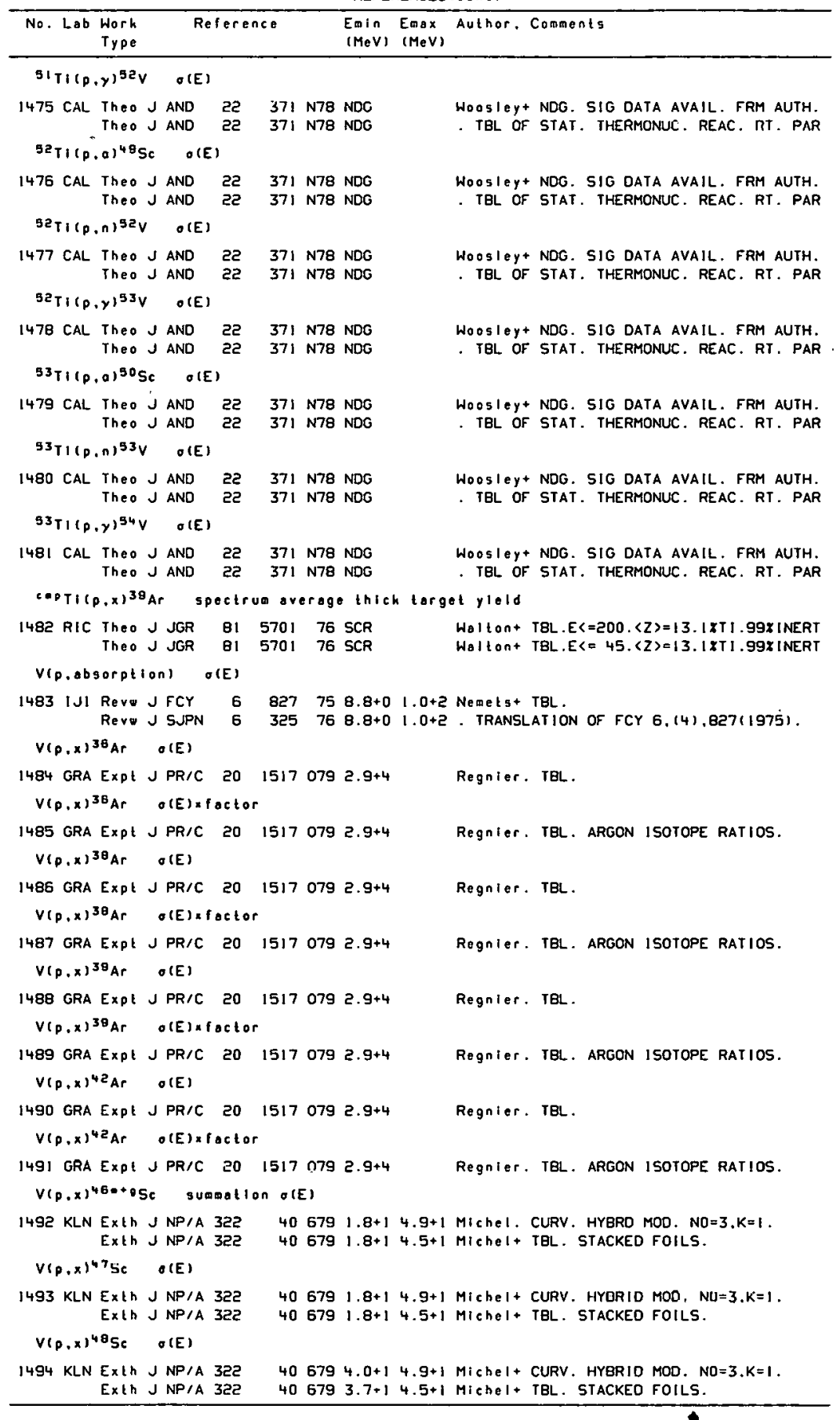


REFERENCES ( c on t)

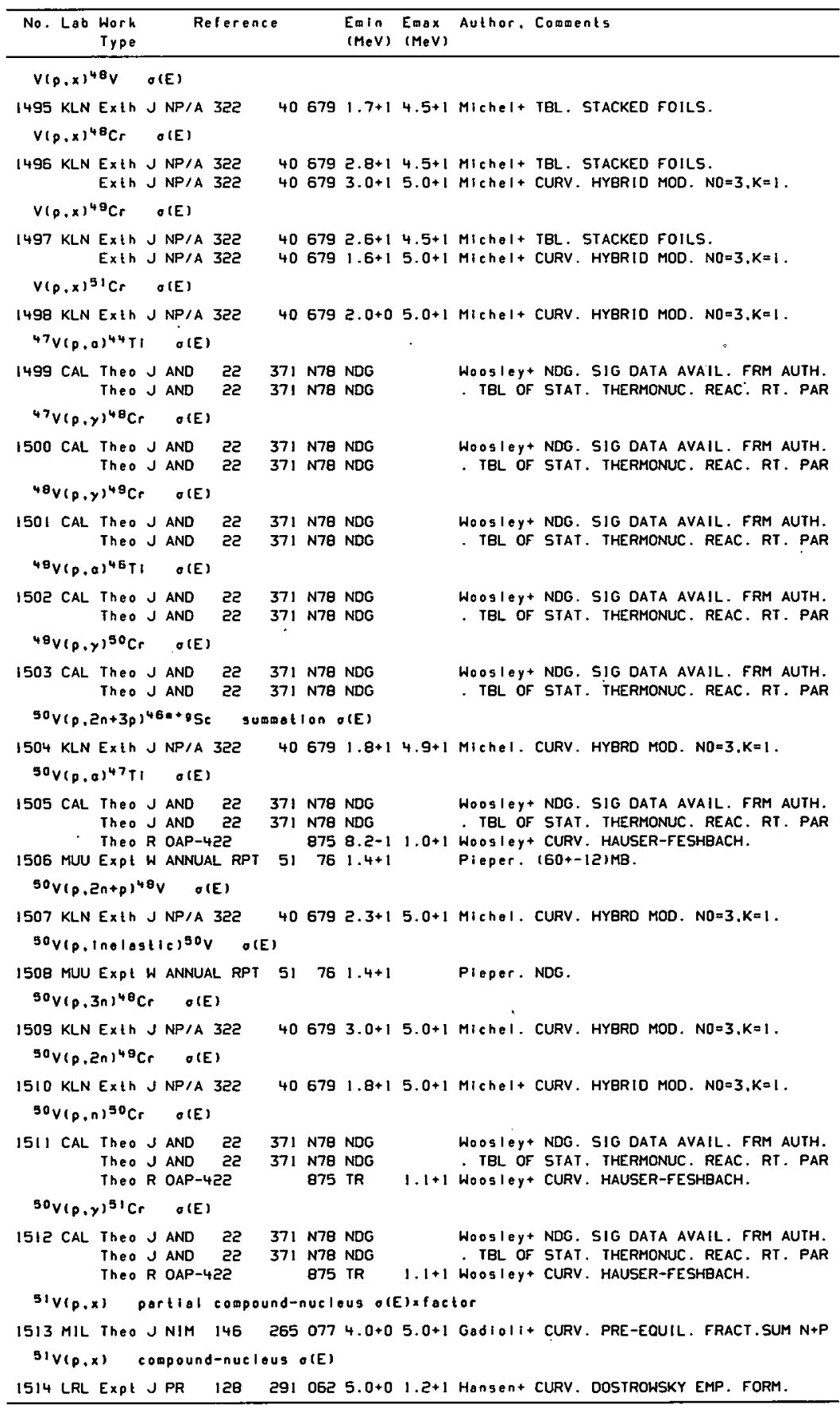


REFERENCES ( $c$ on $t$

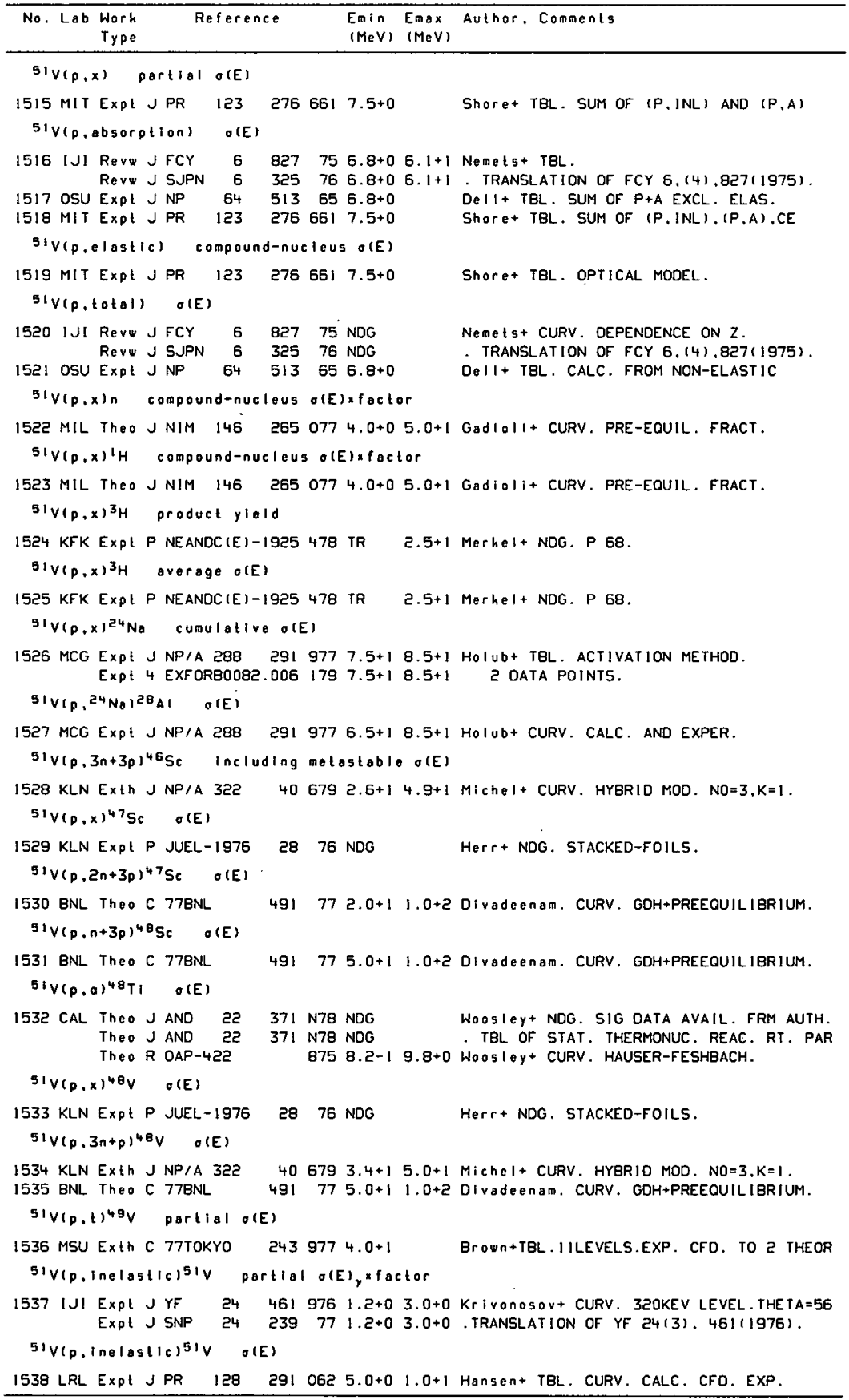


REFERENCES $(\operatorname{con} t)$

\begin{tabular}{ccc}
\hline No. Lab Work & Reference & $\begin{array}{l}\text { Enin Emax Author. Comments } \\
\text { TMeV) (MeV) }\end{array}$ \\
\hline
\end{tabular}

$51 \mathrm{~V}(p, 4 n)^{48} \mathrm{Cr} \quad$ (E)

1539 KLN Exth J NP/A 322

$406794 \cdot 0+15 \cdot 0+1$ Michel + CURV. HYBRID MOD. NO=3.K=1.

$51 \mathrm{~V}(\mathrm{p} .3 n)+9 \mathrm{Cr} \quad \sigma(\mathrm{E})$

1540 KLN Expt P JUEL-1976 2876 NDG Herr+ NDG. STACKFD-FQ!Ls.

154! GNIL Thes C 77BIdL 4y/ $172.5+11.0+2$ Divade 2 nam. CURV. GDH+PREEOUILIBRIUM.

$$
\text { Siv(p.n)sicr o(E) }
$$

1542 KTY Expt P ERDA-NDC-2 68575 NDG

1543 CAL ThEO R OAP-425

1543 CAL Theo R OAP-422
1544 ORL Expl P ORNL-2910 25 60 TR TR $1.1+1$ Woosley+ CURV. HAUSER-FESHBACH.

Sekharan+ NDG. $4 P I$ DET. AND ACTIVATION.

Expt P ORNL-2501 2958 TR $5.7+0$ Johnsont EXPT. OETAILS.

EXpt 4 EXFORB0068.024 678 1.6+0 5.4+0. 115 DATA POINTS.

Expt 4 EXFORB0046.004 77 1.6+0 $2.3+0$. 59 DATA POINTS.

1545 KFK Comp B PH-DAT is $20791.6+01.3+1$ Muenze $1+$ TBL. FROM EXFOR. COmp B PH-DAT $1510791.1+14.5+1$ Muenzel+ TBL. FROM EXFOR

$154 \mathrm{C}$ BNL ReVW C BSHOUSTO $3631.6+02.3+0$ Goldberg. NDG.

1547 CAL Expt A DA/8 $4012379791.6+04.5+0$ Zyshind. SIGNIFICANT N COMPETITION CUSP

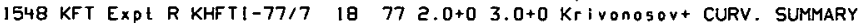

1549 INU Theo J PR/C 20 $12720793.0+05.0+0 \mathrm{KaII}$ as + CURV. OM FIT TO EXP. DATA.

1550 ANL Expt $J$ PR 128 280 OS2 $3.1+01.0+1$ WIIng TBL. CURV. RESIO. RADIOACTIVITY. Expt R TID-12696 $613.1+01.0+1$. SUPERSEDEO

Expt 4 EXFORBO065.002 $6783.1+01.0+1$. 16 DATA PTS.

1551 OSU Expt J NP $64513653.7+06.7+0$ Dell+ TBL. CURV. STACKEO FOILS.

Exp: J PR $13015225636.8+0$ Humes+ MEASURED RESIDUAL RADIATION

EXPt 4 EXFORBO061.003 678 6.8+0 . 480+-31MB

EXPt 4 EXFORBD064.003 678 3.7+0 6.7+0: 10 DATA POINTS.

$1552 \mathrm{KLN}$ Exth J NPIA $322 \quad 406794.0+05.0+1$ Michel+ CURV. HYBRID MOD. $K=1-3$. CFD EX

1553 LRL Expt J PR $115925594.0+0$ 5.5+0 Albert. CURV. EXPt W TO ORNL-CPX $643.6+0$ 8.1+0 Albert.

Expt 4 EXFORB0093.002 $5793.6+0$ 8.1+0 . 7 DATA POINTS

1554 ROC Expt J PR 125 29I 62 4.5+0 6.7+0 Tahetani+ NATURAL FOIL. EXP1 4 EXFORBO051:005 $774,4+0$ 6.5+0 . 4 POINTS. CPX OATA CORRECTEO BY COMP

1555 LRL EXPt JPR $128 \quad 2910625.0+0 \quad 1.1+1$ Hansen+ CURV. LONG COUNTER. CFO. THEORY Exp! J PRL $6 \quad 136 ! 5.0+01.1+1$. PRELIMINARY. Expt 4 EXFORB0065.002 678 5.0+0 1.1+1. 7 DATA POINTS.

1556 TOK Expt J JPJ $141269596.7+01.3+1$ Tanaka+ CURV. NATURAL FOIL. Expt 4 EXFORB0043.004 $776.7+0 \quad 1.3+1$. 8 DATA POINTS.

1557 MIT EXPI JPR $1232766517.5+0$ Shore+ MEAS. RESIDUAL ACTIVITY. Expt 4 EXFOR日0067.002 $6787.5+0$. 555+-30MB.

1558 CCP Revw J AE 4 ᄅ $311778.0+01.1+1$ Zatolohint TBL. ACTIVATION. Reve J SJA $42 \quad 3440779.0+01.1+1$. TRAN5LATION OF AE $42(4), 311(1977)$

$1559 \mathrm{BNL}$ Theo $\mathrm{C} 77 \mathrm{BNL} \quad 491771.0+1 \quad 1.2+2$ 0ivadeenam. CURV. GDH+PREEOUILIERIUM.

$1560 \mathrm{MIL}$ EXPt J NC/A $225478741.1+14.5+1$ Godioli+ TBL. CURV. NAT. V. EXCITON MOD Expl 4 EXFORBD027.002 $771.1+14.5+1.17$ DATA POINTS.

1561 PAR Expl J JPR 231000 62 $3.2+1 \quad 1.5+2$ Albouy+ CURV. CFD CASCADE MODEL. Expl 4 EXFORBO106.003 N79 $3.2+1 \quad 1.5+2$. 9 OATA POINTS.

SIV(p,n)sicr olE)abundonce

$1562 \mathrm{KLN}$ Exth J NP/A $322406791.3+14.5+1$ Michel + TBL. STACKED FOILS.

Sivip,n)Sicr product yield

1563 CAL Expt A BAP 22542477 NDG Zyshind+ NDG.

$51 \mathrm{~V}(p, n)^{51} \mathrm{Cr} \quad I_{y}$

1564 TOK Expt J JPJ $4510990784.0+04.6+0 \mathrm{Kasagi+NDG.} \mathrm{G} \mathrm{EXCIT.} \mathrm{FUNC.} \mathrm{AT} \mathrm{550EG.}$

SIV(p,n)SICr partiol o(E)

1565 KTY Expl A DA/B 372347 N76 4.0+0 4.4+0 Sehharan. NDG.

$51 V(p, n)^{51} \mathrm{Cr}$ avorage o(E)

1566 ORL Expl J PR/B $13517190641.6+01.6+0$ Johnson+ CURV. FROM 1952 MEASUREMENT

$51 \mathrm{~V}(\rho, Y) 52 \mathrm{Cr} \quad \sigma(E) \times$ factor

1567 CAL Expt C 77TOKYO 846977 NOG ZyShINd+ IBL. WIGNER CUSPIMINIMUM RATIO

$51 \mathrm{~V}(\mathrm{p}, y)^{52} \mathrm{Cr} \quad$ o(E)

1568 CAL Theo J AND 22 37! N7B NDG WOOSIey+ NDG. SIG DATA AVAIL. FRM AUTH. Theo $\checkmark$ AND 22 37! N78 NDG IBL OT STAT. THERMŪNUL: REAC. RT. PAR Ihe R OAP-422 875 TR $1.1+1$ WoOsley+ CURV. HAUSER-FESHBACH. 
REFERENCES (cont)

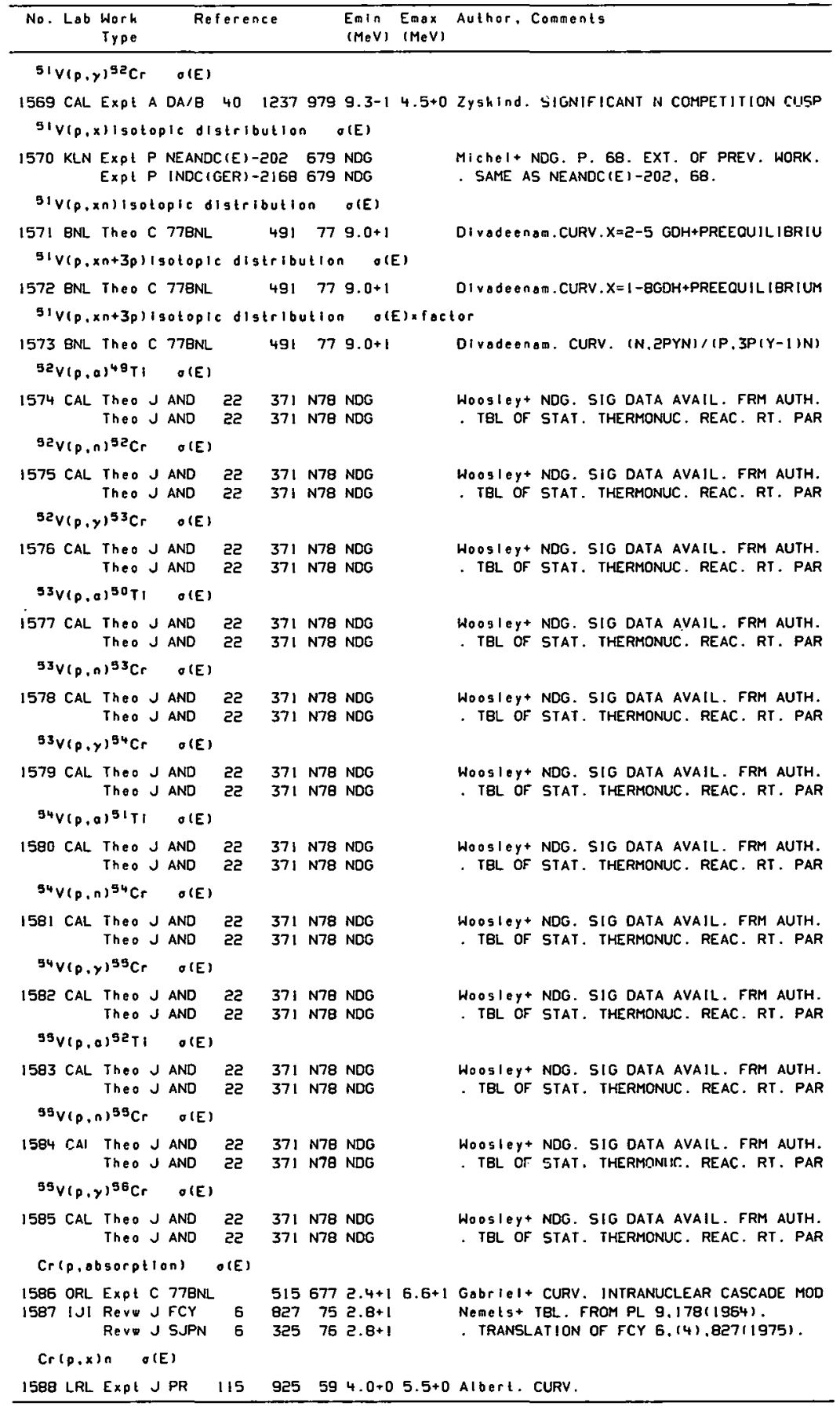




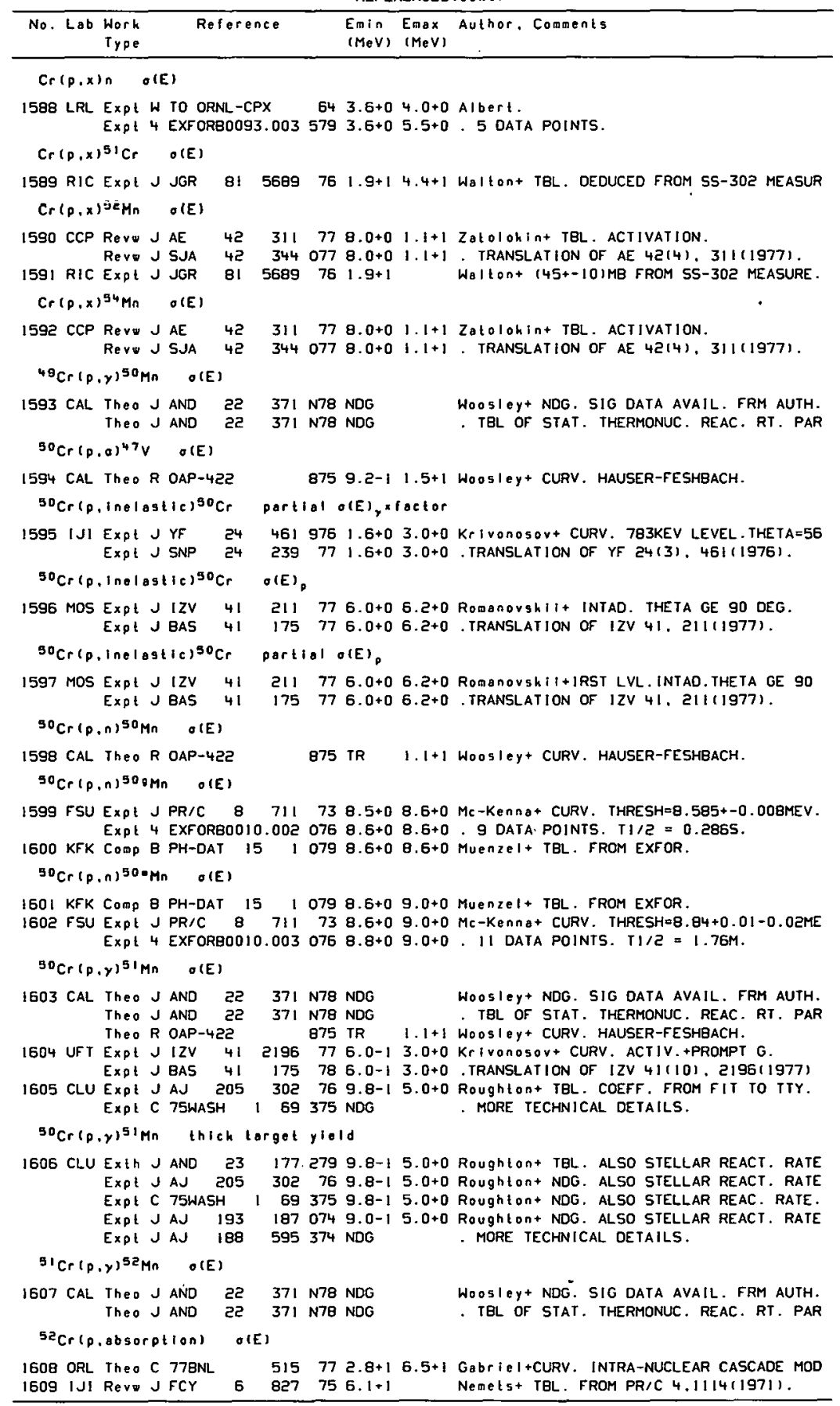




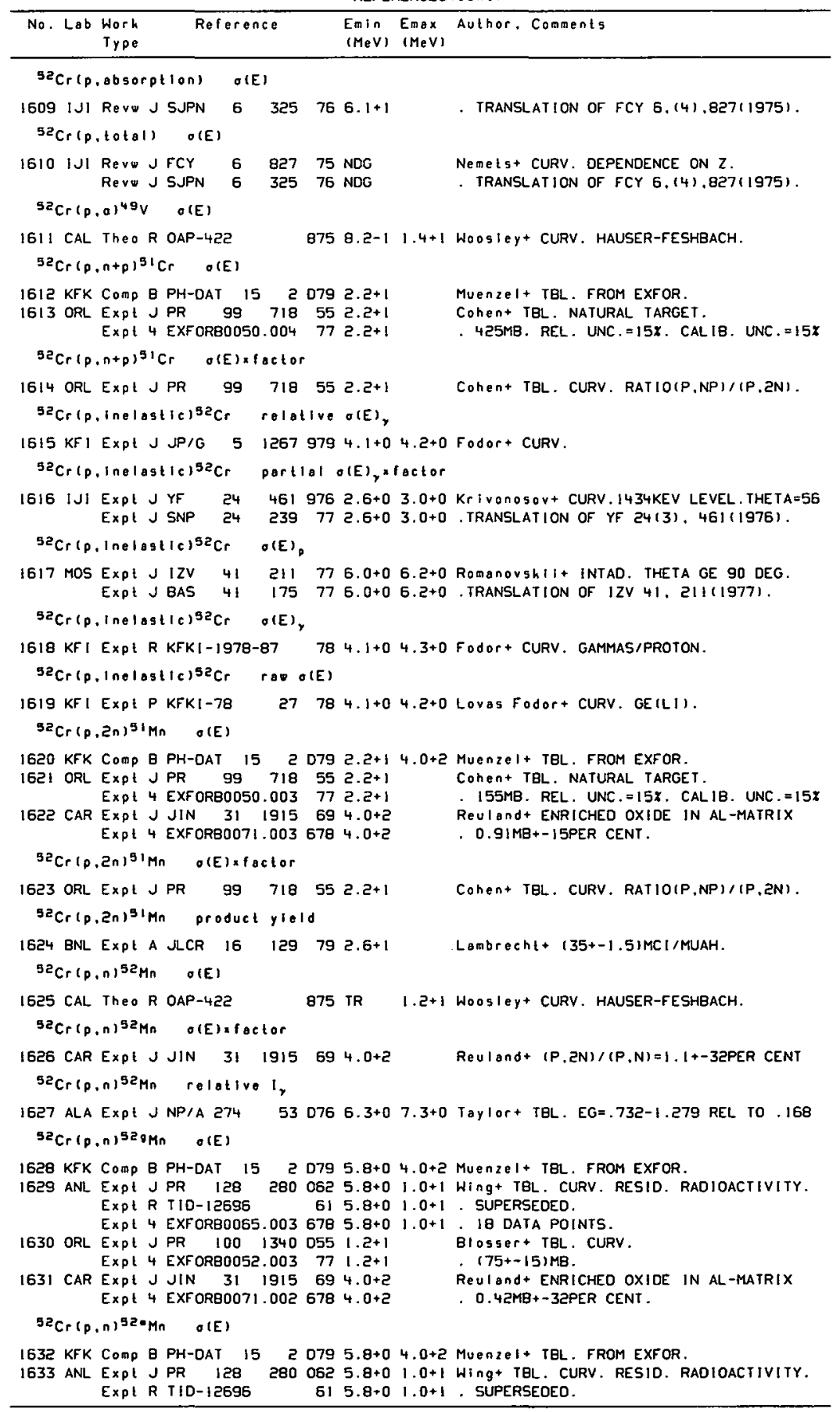


REFERENCES ( cont)

\begin{tabular}{|c|c|c|c|c|c|}
\hline No. Lab & $\begin{array}{l}\text { Work } \\
\text { Type }\end{array}$ & Refereace & $\begin{array}{l}\text { Enin } \\
\text { (MeV) }\end{array}$ & $\begin{array}{l}\text { Emax } \\
(M e V)\end{array}$ & Author, Comments \\
\hline
\end{tabular}

${ }^{52} \operatorname{Cr}(p, n) 52 \cdot M n \quad O(E)$

1633 ANL EXPt 4 EXFORB0065.003 678 5.8+0 1.0+1. 18 DATA POINTS.

1634 ROC Expt J PR 125 291 62 $6.2+0$ 6.7+0 Tahelani+ NATURAL FOIL.

Expt 4 EXFORB0051.003 $776.0+06.6+0.3$ POINTS. CPX DATA CORRECTED BY COMP

1635 ORL Expt J PR 1001340 DS5 $1.2+1$

EXpt 4 EXFORB0052.003 $77 \quad 1.5+1$ Blosser+ TBL. CURV.

$1310+-1,0) M B$

ITZ6 CAR EXPL J JIN BI ISI5 $694.0+2$

Reuland+ ENRICHED OXIDE IN AL-MATRIX

Expt 4 EXFORB0071.002 $678+.0+2$

. $0.36 M B+-32 P E R$ CENT

sacr(p,n)5eam product yleld

1637 BNL Expt A JLCR $16129791.5+1 \quad$ Lambrecht+ 50MC1/MUAH.

${ }^{52} \mathrm{Cr}(p, n) 52=+9 M n$ sumation $\sigma(E)$

1638 KFK Comp B PH-OAT $1520795.8+04.0+2$ Muenzel+ TBL. FROM EXFOR.

1639 ANL Expl J PR 128 280 052 5.8+0 1.0+1 Wing+ TBL. CURV. RESID. RADIOACTIVITY. ExpL R T1D-12695 $6 ! 5.8+01.0+1$. SUPERSEDED.

Expl 4 EXFORBO065.003 $6785.8+01.0+1$. 18 DATA POINTS

1640 TOK Expl J JPJ $141269596.4+01.4+1$ Tanaka+ CURV. NATURAL FOIL.

Expt 4 EXFORBO043.005 $776.4+0 \quad 1.4+1$. 11 DATA POINTS

1641 CAR Expl J JIN $311915694.0+2$ ReuIand+ ENRICHED OXIDE IN AL-MATRIX Expt 4 EXFORB0071.002 $6784.0+2$. $0.8 M B+-45 P E R$ CENT

$32 \mathrm{Cr}(p . n) 52=+9 \mathrm{Mn} \quad$ o(E) af actor

1642 CAR Expt J JIN $3 ! 1915694.0+2 \quad$ Reuland+ (P, 2N)/(P.N) $=1,1+-32 P E R$ CENT

$32 \mathrm{Cr}(p, \gamma)^{53} \mathrm{Mn} \quad r a \otimes \sigma(E)$

1643 AML Expt J NIM $159407 \quad 791.0+0 \quad 1.0+0$ SwitkooshitCURV. VS. NMR FREO. 1005. 2RES

${ }^{52} \mathrm{Cr}(p, \gamma)^{53} \mathrm{Mn}$ partlal raw o(E)

1644 AML Expl J NIM $159407791.0+0$ 1.0+0 Svitkowshi+ CURV. METAL. 378-KEV G.

${ }^{52} \mathrm{Cr}(p, y)^{53} \mathrm{Mn}$ partial relotive olE

$1645 \mathrm{KFJ}$ Expt J JP/G $512679794.1+04.3+0$ Fodor+ CURV. EG .GT. 9 MEV. ALSO AR. A4, Expt R KFKI-1978-87 $784.1+04.3+0$ Fodor + CURV. EG .GT. 9 MEV.

${ }^{52} \mathrm{Cr}(\rho, \gamma)^{53} \mathrm{Mn} \quad \mathrm{O}(\mathrm{E})$

1646 CAL Theo J AND 22371 N78 NDG

Theo $J$ AND $22 \quad 371$ N78 NDG

The O R OAP-422 875 TR

Woosiey + NDG. SIG DATA AVAIL. FRM AUTH.

- TBL OF STAT. THERMONUC. REAC. RT. PAR

$52 \mathrm{Cr}(p, y)^{33} \mathrm{Mn}$ parllal o(E)

1647 KFI EXPI R KFKI-1978-87 78 4.1+0 4,3+0 Fodor+ CURV. 4 PRIMARY GAMMAS. GAMMA/P

${ }^{53} \mathrm{Cr}(0,0)^{50 \mathrm{~V}}$ O(E)

1648 CAL TheO R OAP-422

975 8.2-1 $1.2+1$ WoOSley+ CURV. HAUSER-FESHBACH.

${ }^{53} \mathrm{Cr}(\mathrm{p}, \mathrm{inelagtic})^{53} \mathrm{Cr}$

1649 IJIExpl J YF 24 Expt J SNP 54

partial o[E], wactor

${ }^{53} \mathrm{Cr}(\mathrm{p}, \mathrm{inelagt} \text { I })^{53} \mathrm{Cr}$

$4619761.8+03.0+0 \mathrm{Kr} 1$ YONOSOY+ CURV. 564KEV LEVEL. THETA=55 $239771.8+03.0+0$. TRANSLATION OF YF $24(3), 46111976)$.

partlat thlek torget yieldafoctor

1650 TRM Expt J JP/G $312469772.5+03.5+0$ Patrawale+ NDG. 10 G.5. B(E2) + B(M) $).$ 1651 VIP Expl J JP/G 3 l246 977 2.5+0 3.5+0 Patrawale+ NDG. 10 G.S. B(EZ) + B(M) I.

${ }^{53} \mathrm{Cr}(p, n)^{53} \mathrm{Mn} \quad O(E)$

1652 CAL TheO R OAP-422

B75 TR 1.2+1 WoOsley+ CURV. HAUSER-FESHBACH.

1653 ORL Expt P ORNL-2910 25 60 TR 5.9+0 Johnson+ CURV. THICKER IRGT. 4PI OETECT Expt J PR $1091243581.4+01.9+0$ Johnsont CURV. NAT CR TARGET.

Expl P ORNL-2501 2958 IR 5.9+0 Johnsont EXPT. DETAlLS.

Expt 4 EXFORB0068.010 $6781.5+05.9+0$. 22 OATA POINTS.

Expl 4 EXFORBO046.005 77 $1.4+0 \quad 1.9+0.4$ IPOINTS.ERR ADOED GY COMP TO CPX DATA

1654 KFK Comp B PH-DAT 15 2 O79 $1.4+05.9+0$ Muenzel+ TBL. FROM EXFOR.

1655 KFT Expl R KHFTI-77/7 $18 \quad 772.0+0 \quad 3.0+0 \mathrm{Krivonos}$ (8) + CURV. SUMMARY.

1656 IJIExpl J UFZ $161947 \quad 712.8+03.9+0$ Dryapachenho+CURV.LONG CONT. CRYS. SPEC Expt J UPJ $162340782.8+03.9+0$. TRANSLATION OF UFZ 161121, 1947119711

${ }^{53} \mathrm{Cr}(p, y)^{54} \mathrm{Mn}$ o(E)

1657 CAL Theo J AND ट2 37! N78 NDG Theo $\checkmark$ AND $22 \quad 371$ N79 NDG The 0 R OAP-422 875 TR

Woosley+ NDG. SIG DATA AVAIL. FRM AUTH. . TBL OF STAT. THERMONUC. REAC. RT. PAR $1.2+1$ Woosley+ CURV. HAUSER-FESHBACH. 
REFERENCES (c ont)

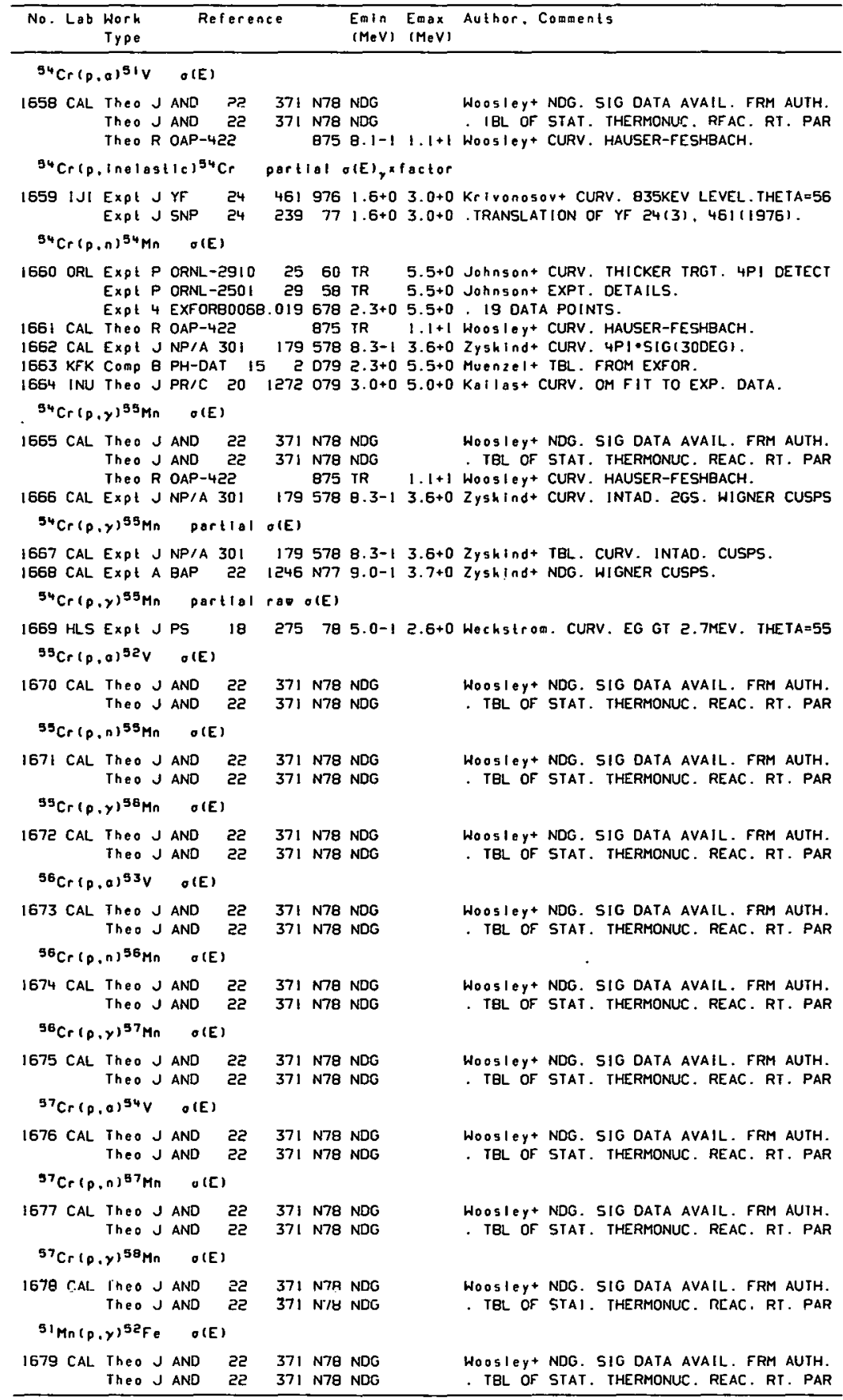


REFERENCES ( $c$ on ( )

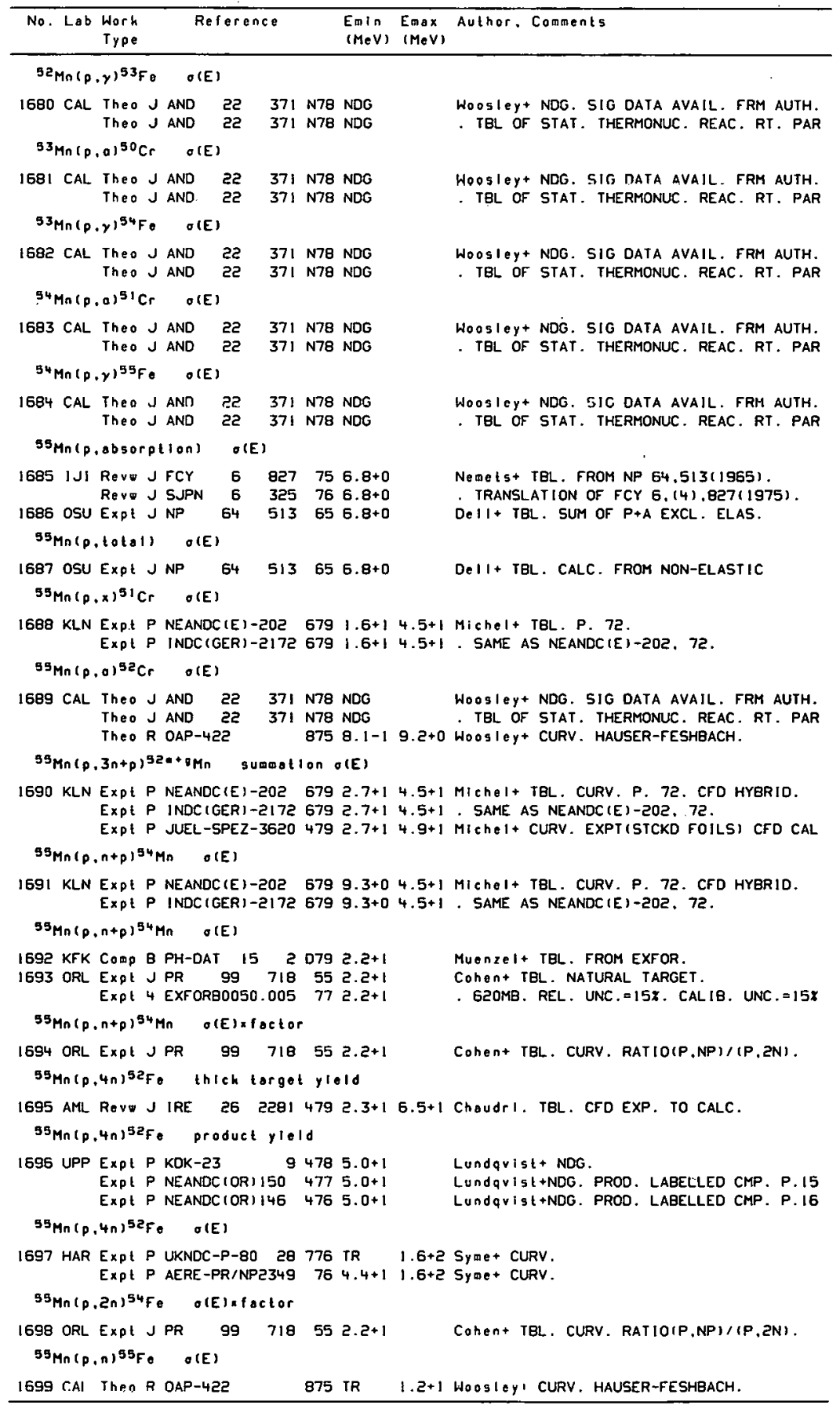


REFERENCES (cont)

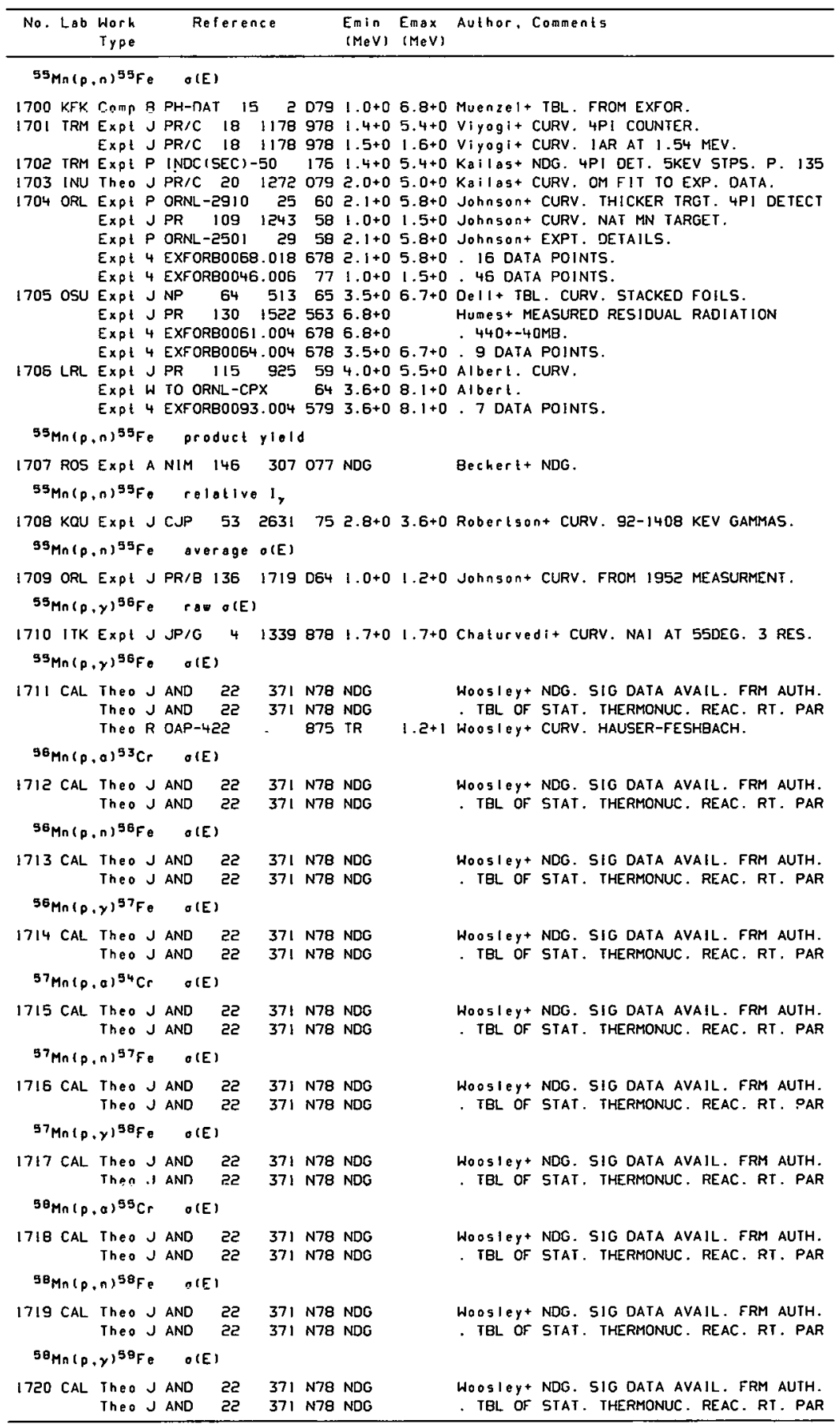




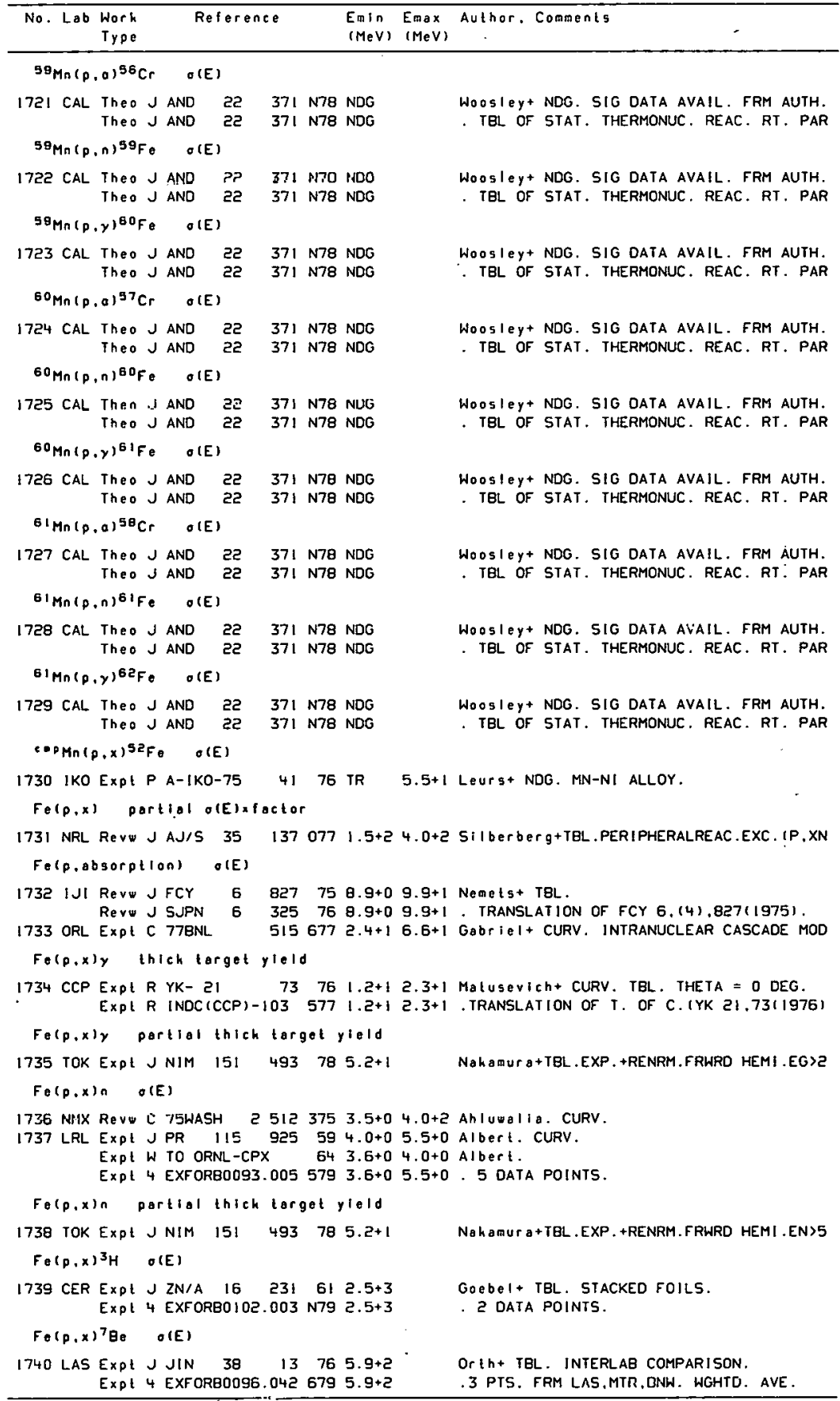




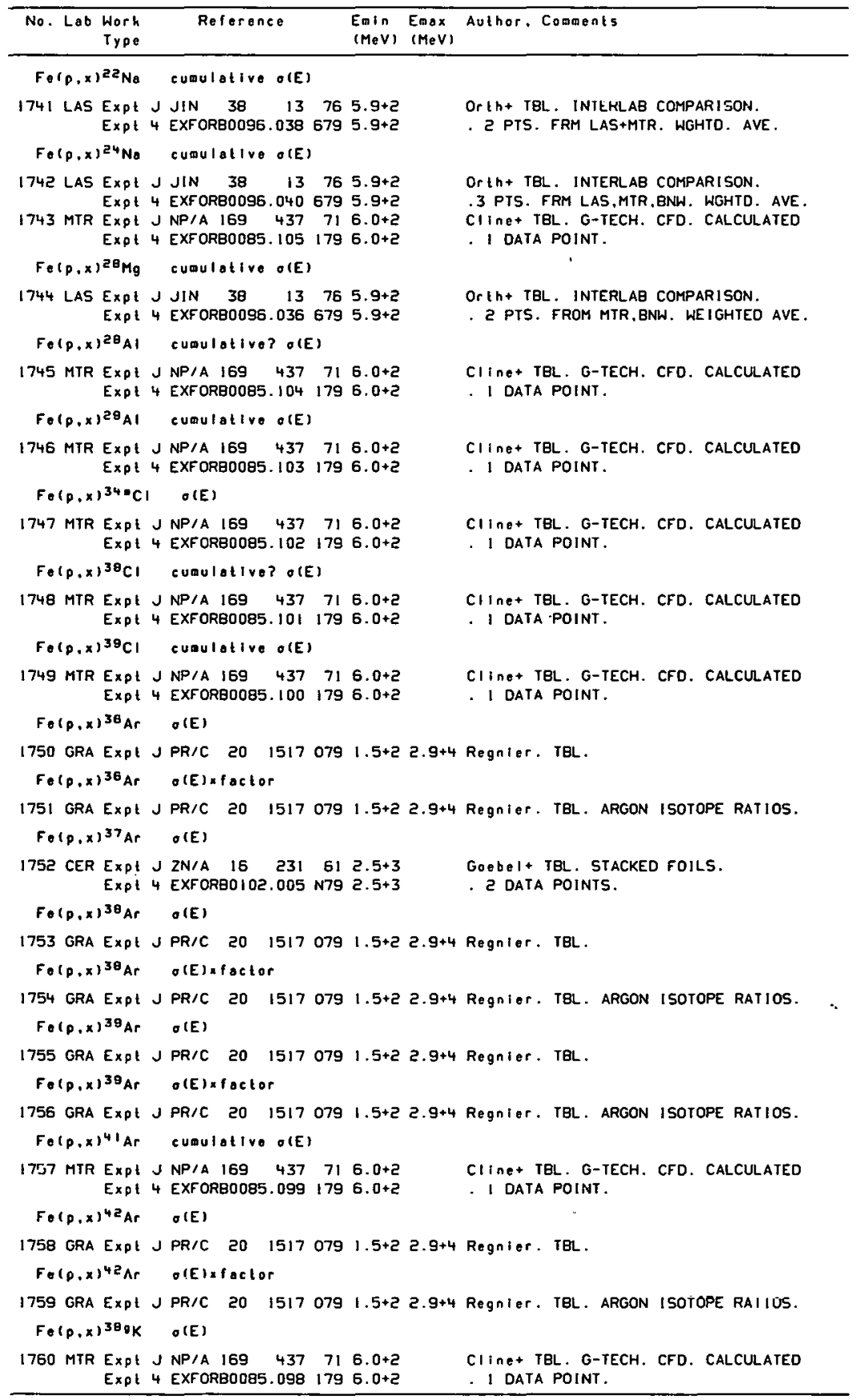




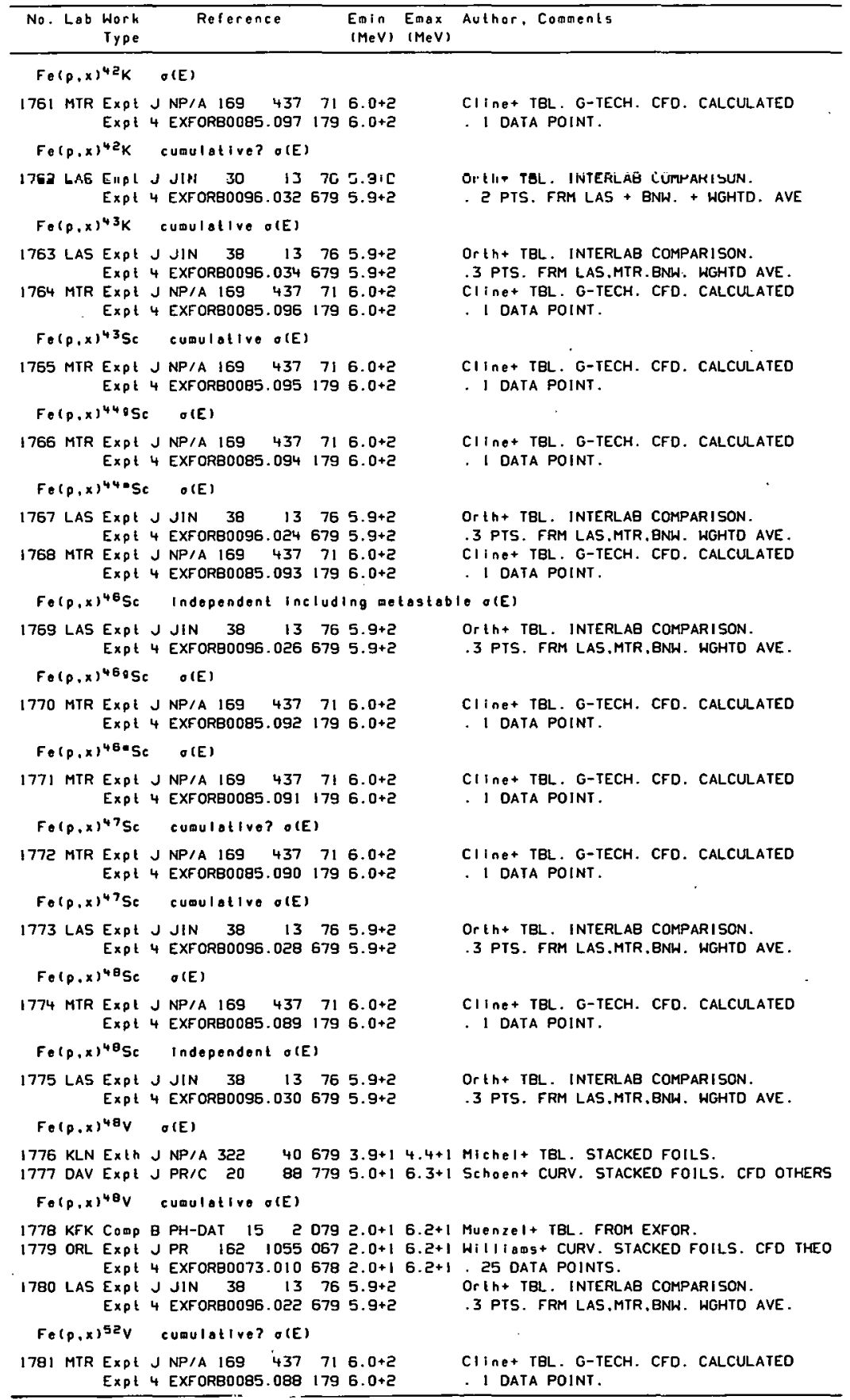




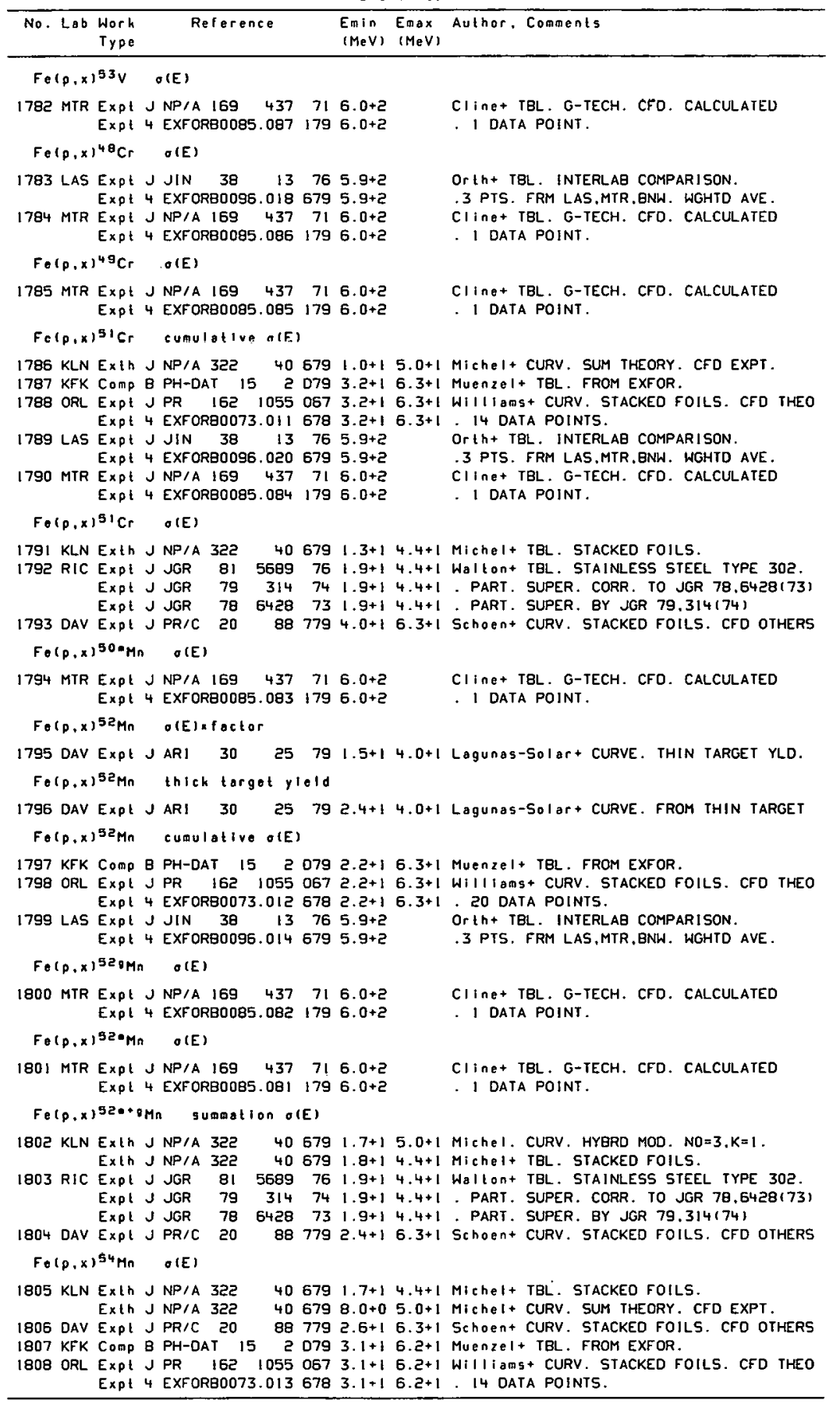




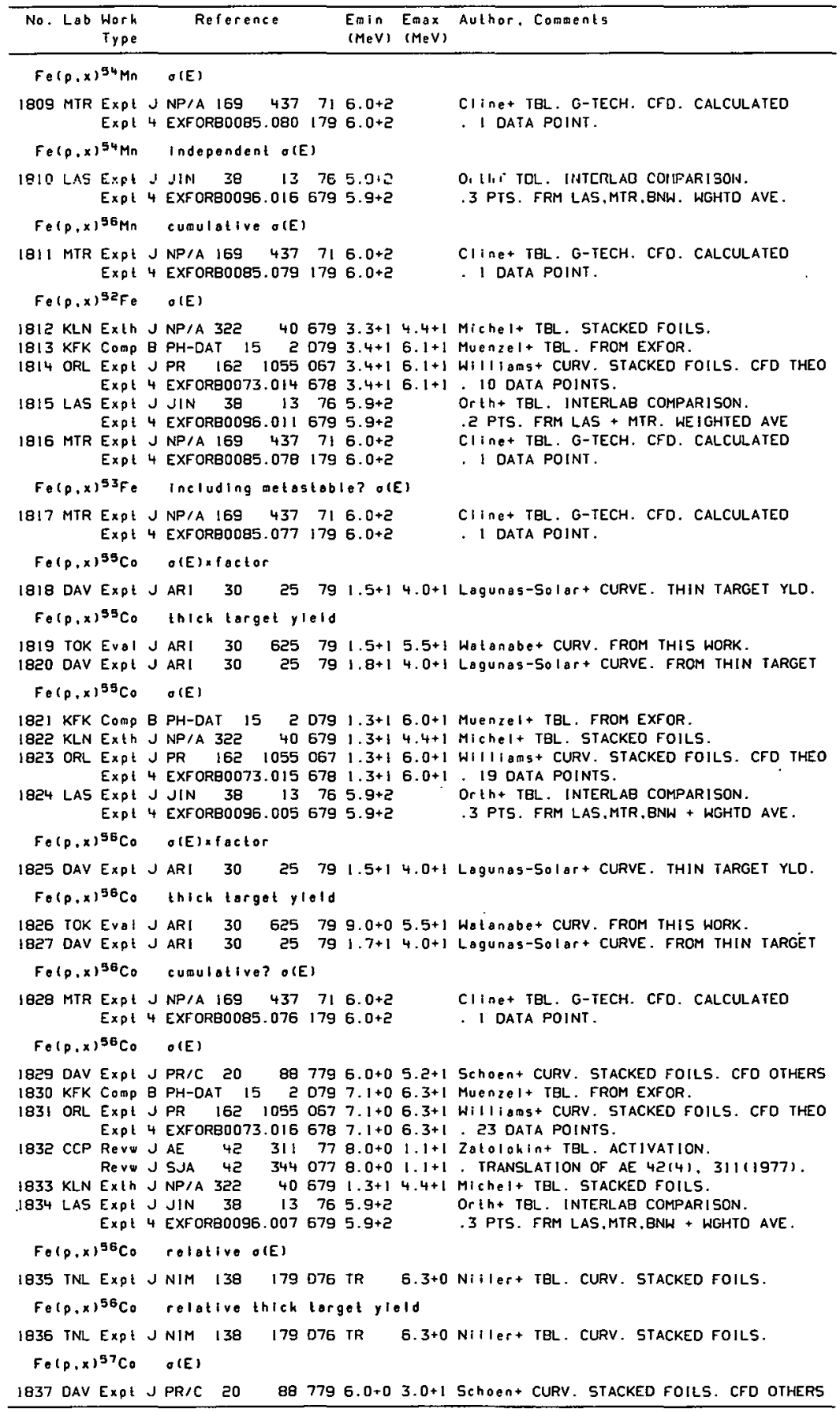




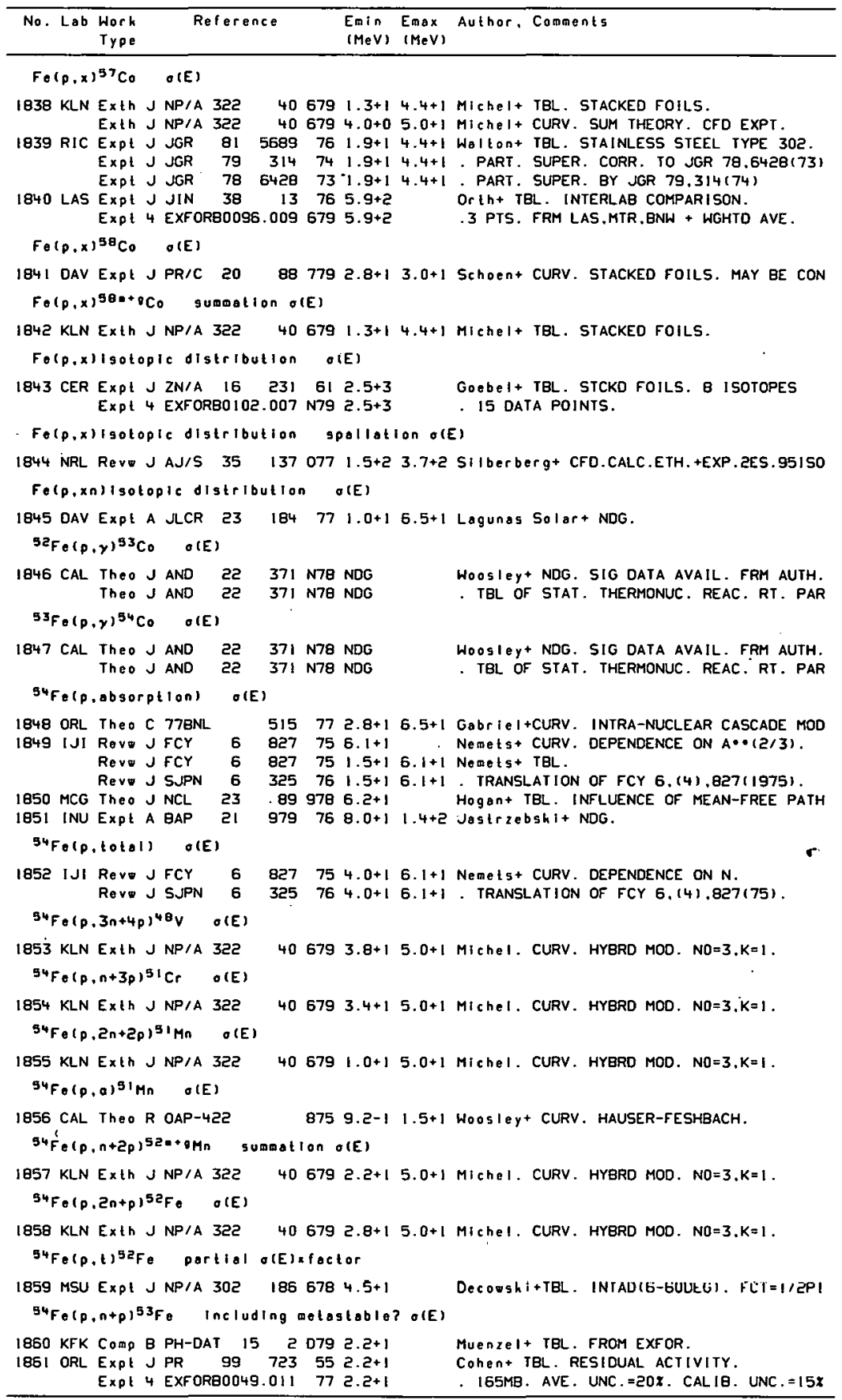


REFERENCES ( c on l)

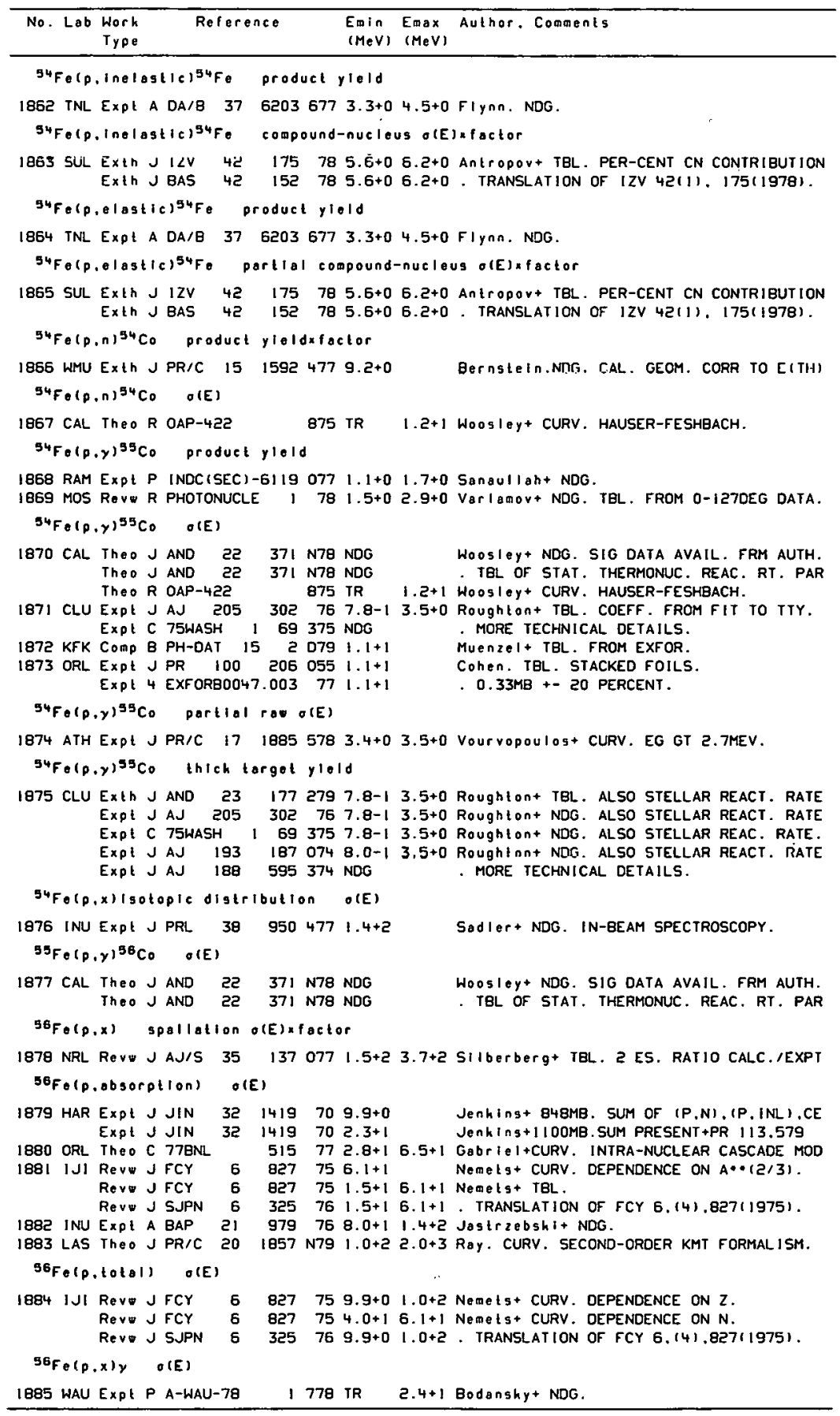


REFERENCES ( c on t)

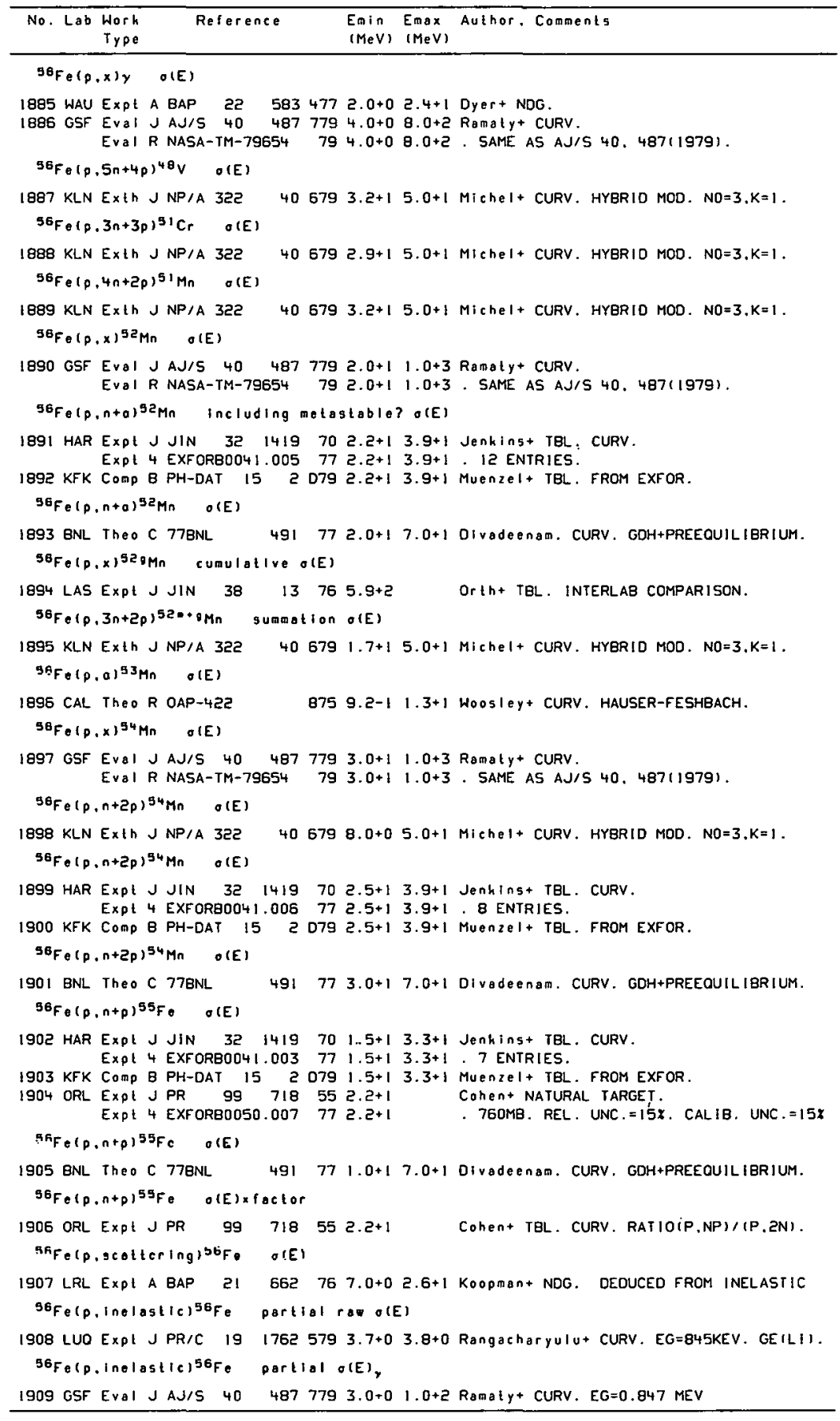




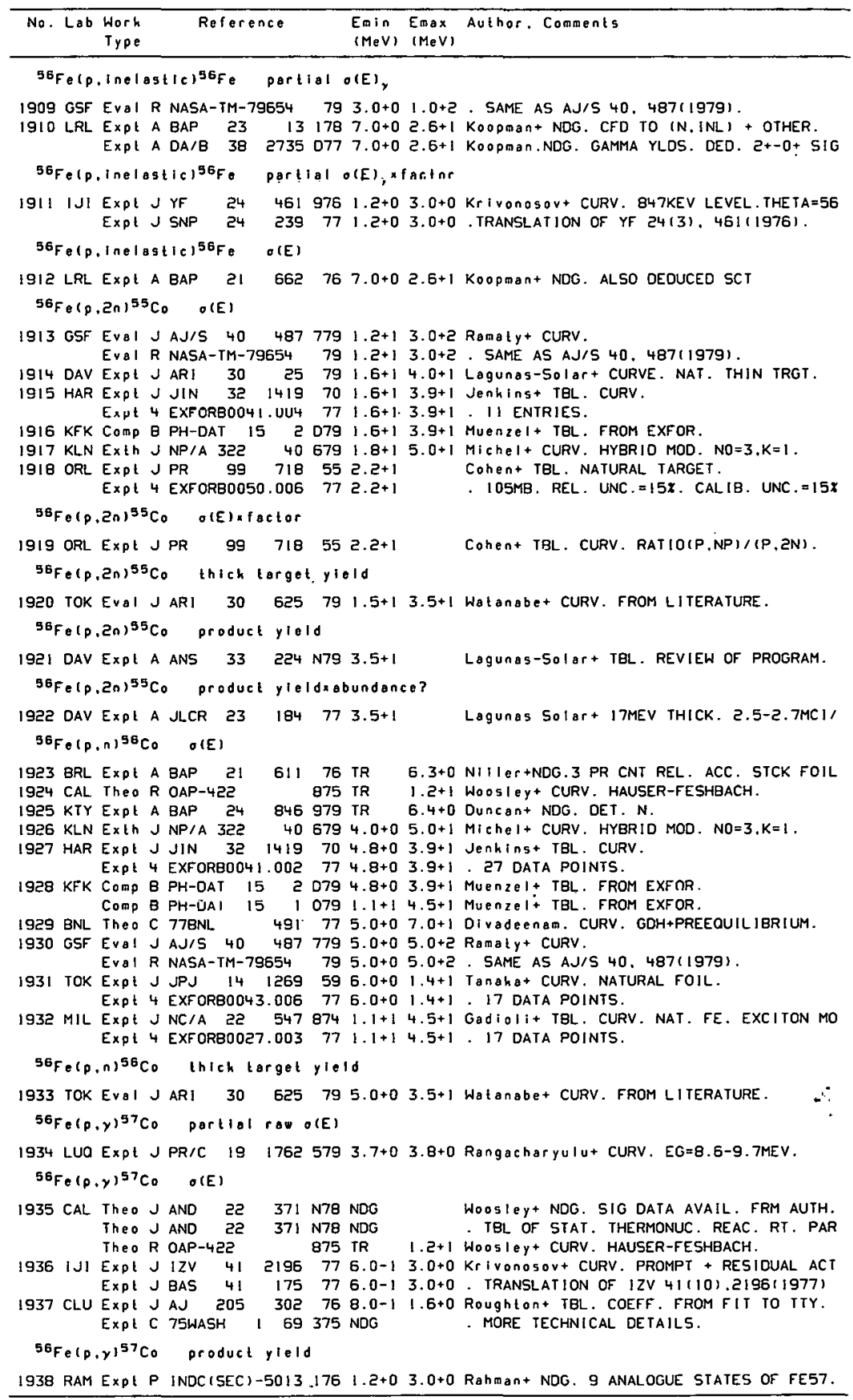


REFERENCES (cont)

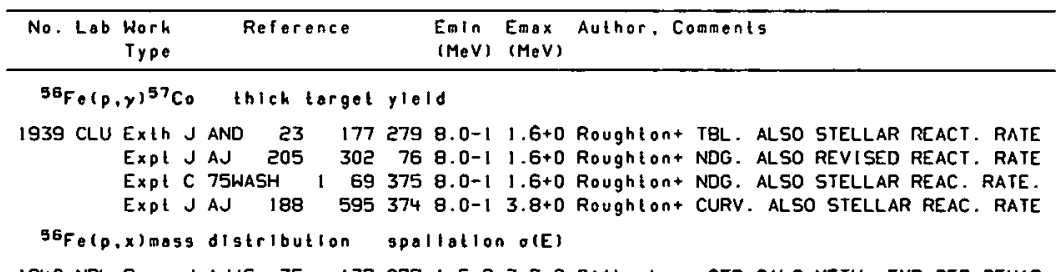

1940 NRL Revw J AJ/S $351370771.5+23.7+2511 b e r b e r g+C F D . C A L C . M E T H .+E X P .2 E S .25 M A S$ 56 Fo $(p, x)$ isotople distribution $\sigma(E)$

1941 INU Expt J PRL $38 \quad 9504771.4+2 \quad$ Sadler+ NDG. IN-BEAM SPECIROSCOPY.

$57 F \cdot(p, a b s o r p t i o n) \quad O(E)$

I942 1JI Revo J FCY $6 \quad 827751.5+16.1+1$ Nemets + TBL.

Revw J SJPN $6 \quad 325761.5+16.1+1$. IRANSLATION OF FCY 6.14),827(1975).

$57 F_{0}\left(D, t \circ t_{01}\right) \quad O(E)$

1943 lJl ReV J FCY 6 ge7 $754.0+16.1+1$ Nemels + CURV. DEPENDENCE ON N.

Rev J SJPN $6325764.0+16.1+1$. TRANSLATION OF FCY $6,141,827(75)$.

$57 F_{0}(p, 6 n+4 p) 48_{V} \quad O(E)$

$1944 \mathrm{KLN}$ Exth J NP/A $3224406794.7+15.0+1$ Michels + CURV. HYBRID MOD. NO=3,K=1.

$\left.{ }^{57} \mathrm{~F} \cdot(p, 4 n+3 p)\right)^{51} \mathrm{Cr} \quad \sigma(E)$

$1945 \mathrm{KLN}$ Exth J NP/A $322406794.1+15.0+1$ MIchel+ CURV. HYBRID MOD, NO, $K=1$.

$57^{7} \cdot(p, 4 n+2 p) 5 z+9 M n \quad$ summotion $\sigma(E)$

1946 KLN Exth J NP/A $322406793.0+15.0+1$ Michel+ CURV. HYBRID MOO. NO=3. $K=1$.

$57 F \circ(p, 2 n+2 p) 54 M n \quad O(E)$

$1947 \mathrm{KLN}$ Exth J NP/A $322 \quad 406793.5+15.0+1 \mathrm{Michel}+$ CURV. HYBRID MOO. NO=3, K=1.

${ }^{57} \mathrm{Fe}(p, Q)^{94} \mathrm{Mn} \quad O(E)$

1948 CAL Theo J AND 22371 N78 NDG WOOSIEy+ NOG. SIG DATA AVAIL. FRM AUTH. Theo J AND 22 371 N78 NDG TEL OF STAT. THERMONUC REAC. RT. PAR Theo R OAP-422 $8751.0+01.2+1$ Woosley+ CURV. HAUSER-FESHBACH.

$57 F=(p, 2 p)^{56} \mathrm{Mn} \quad O(E)$

1949 KFK Comp B PH-DAT 15 2 D79 $2.2+1$ Muenzel+ TBL. FROM EXFOR.

1950 ORL Expt J PR $99723552.2+1$ Cohent TBL. RESIDUAL ACTIVITY.

Expt 4 EXFORE0049.012 $772.2+1 \quad$. 12.5MB. AVE.UNC. $=20 x$. CALIB. UNC. $=15 x$

57Fetp,inelastici57Fe portlal o(E), foctor

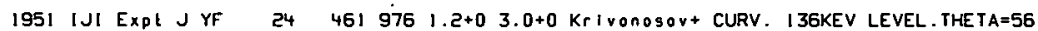
Expt J SNP $24 \quad 239771.2+03.0+0$. TRANSLATION OF YF $24(3), 461(1976)$.

${ }^{57} \mathrm{~F} \cdot(\mathrm{P}, \mathrm{n})^{57} \mathrm{CO} \quad 0(\mathrm{E})$

1952 KTY Expl P ERDA-NDC-2 68575 NDG Sokharant NDG. 4PI DET. AND ACTIVATION. 1953 CAL ThEO R OAP-422 875 IR $1.2+1$ Woosley+ CURV. HAUSER-FESHBACH.

1954 ORL Expt P ORNL-2910 25 G0 TR $5.5+0$ Johnsont CURV. 4PI DETECTOR. Expl P ORNL-2501 2958 TR $5.5+0$ Johngon+ EXPT. DETAILS.

Expl 4 EXFORBO068.016 $6781.7+05.5+0.67$ DATA POINTS.

1955 KFK Comp B PH-DAT 152 $0791.7+01.4+1$ Muenzel+ TBL. FROM EXFOR.

$1956 \mathrm{KLN}$ Exth J NP/A 322 $406792.0+05.0+1$ Michel+CURV. HYBRID MOD. ND=3.K=1.

1957 TOK Expl J JPJ $141269593.7+01.4+1$ Tanaha+ CURV. NATURAL FOlL.

Expl 4 EXFORB0043.007 $773.7+01.4+1$. I3 DATA POINTS.

${ }^{57} F_{0}(p . n)^{57} \mathrm{Co} \quad$ overago $0(E)$

I958 ORL Expl J PR/B 1361719 D64 1.6+0 1.9+0 Johnson+ CURV. FROM 1953+1960 MEAS.

${ }^{57} F_{0}(P, y)^{50} C_{0} \quad O(E)$

1959 CAL Theo $J$ AND $22 \quad 371$ N79 NDG Woosley+ NDG. SIG DATA AVAIL. FRM AUTH. Theo $J$ AND 22 371 N79 NDG Theo R OAP-422 R75 IR I.2+1 WoOSley+ CURV. HAUSER-FESHBACH.

$57_{F}$ OP.Xnlisotoplc distribution o(E)

1960 NRL Theo J AJ/S $351290774.0+2 \quad$ SIIberberg+ CURV. $X=1-4.4$ REFERENCES.

50Fo(p,absorption) o(E)

196। JJ! Revw J FCY 6 B $827751.5+16.1+1$ Nemets+ TBL.

Revw J SJPN $6 \quad 325761.5+16.1+1$. TRANSLATION OF FCY $6.141 .827(1975)$. 


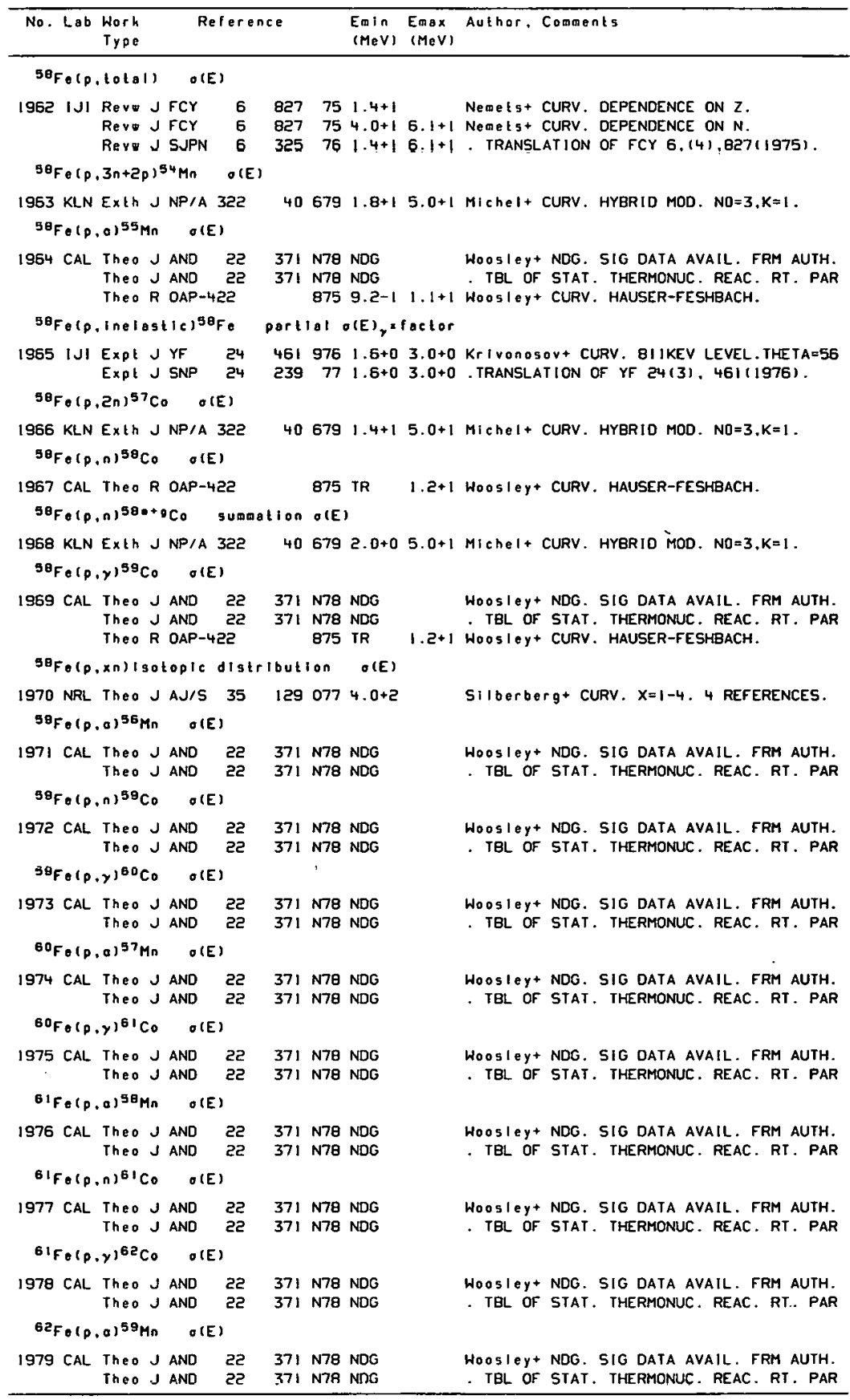




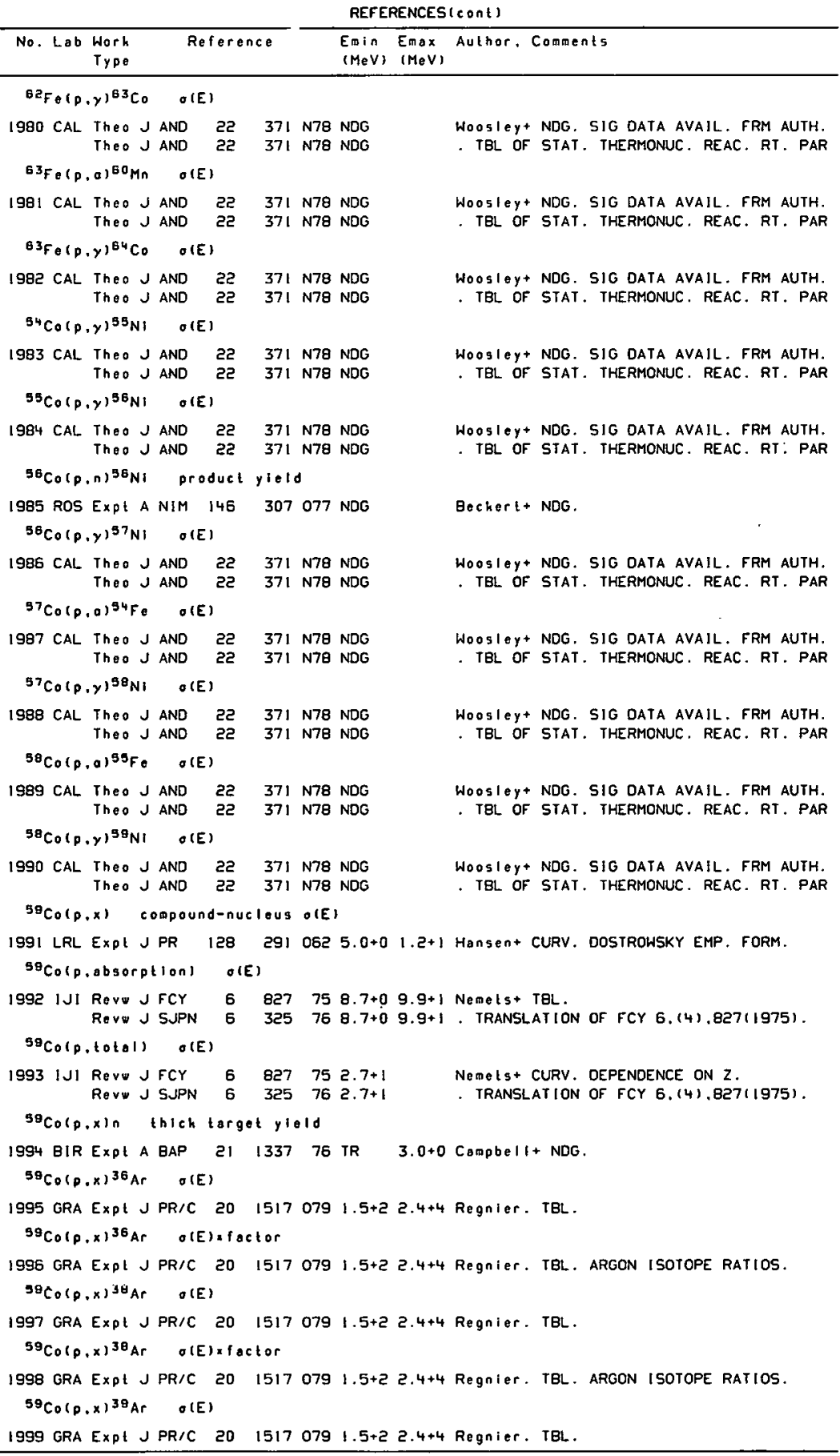


REFERENCES (cont)

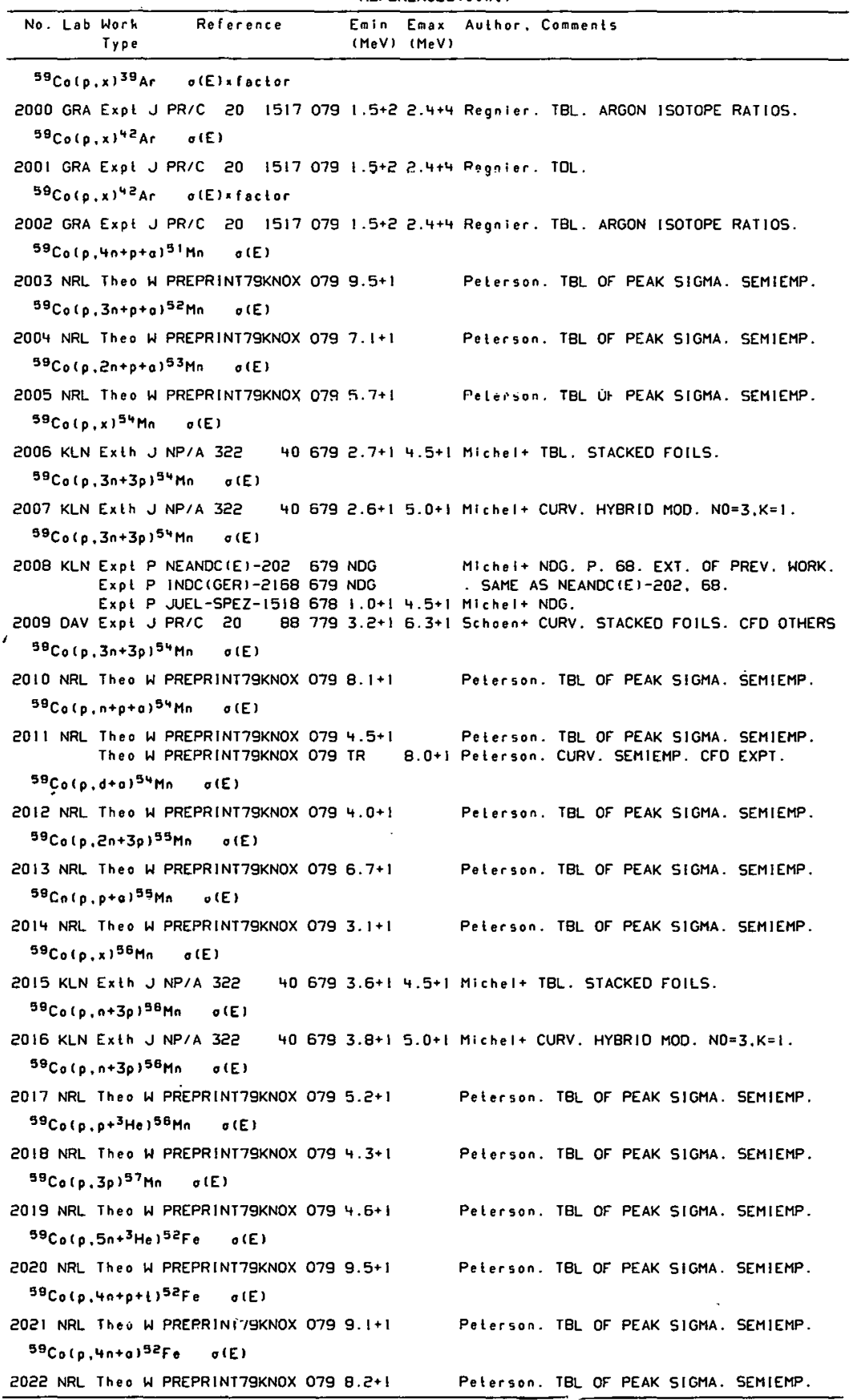


REFERENCES (cont)

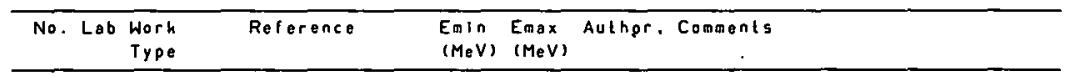

$\left.{ }^{59} C_{0}(p, 4 n+p+d)\right)^{33} F_{0} \quad O$ (E)

2023 NRL Theo $W$ PREPRINT79KNOX $0798.4+1$

Peterson. IGL OF PEAK SIGMA. SEMIEMP.

${ }^{9} \mathrm{Co}_{0}\left(p, 4 n+{ }^{3} \mathrm{He}\right)^{53} \mathrm{Fe} \quad$ O(E)

2024 NRL Theo W PREPRINT79KNOX 079 8.1+1

Peterson. TBL OF PEAK SIGMA. SEMIEMP.

${ }^{59} \mathrm{Co}(p, 3 n+p+t)^{53} \mathrm{Fe} \quad$ o(E)

2025 NRL Theo $W$ PREPRINT79KNOX $0797.5+1$

Peterson. TBL OF PEAK SIGMA. SEMIEMP.

${ }^{59} \mathrm{Co}(0,3 n+a) 53 \mathrm{Fo} \quad$ O(E)

2026 NRL Theo W PREPRINT79KNOX $0796.6+1$

Peterson. TBL OF PEAK SIGMA. SEMIEMP.

${ }^{59} \mathrm{Co}(p, 3 n+p+d)^{54} \mathrm{Fe} \quad$ of $(\mathrm{E})$

2ח?77 NRL Theo W PREPRINT79KNOX $0797.0+1$

${ }^{59} \mathrm{Co}\left(p, 3 n+{ }^{3} \mathrm{H}_{0}\right){ }^{54} \mathrm{Fe}_{0} \quad \sigma(E)$

2028 NRL Theo W PREPRINT79KNOX $0796.5+1$

${ }^{50} \mathrm{Co}\left(p, 2_{n+p+t}\right)^{54} \mathrm{~F}_{0} \quad \sigma(E)$

2029 NRL Theo $W$ PREPRINT79KNOX $0796.2+1$

${ }^{59} \mathrm{Co}\left(p, \mathrm{Zn}_{0}\right)^{54} \mathrm{Fe}$ a(E)

$2030 \mathrm{NRL}$ Theo W PREPRINT79KNOX $0794.2+1$

${ }^{59} \mathrm{Co}\left(p, 4 n+2_{p}\right){ }^{54} \mathrm{Fe} \quad O(E)$

2031 NRL Theo W PREPRINT79KNOX 079 8. $1+1$

${ }^{59} \mathrm{Co}(p, 2 n+p+d)^{55} \mathrm{~F}_{0} \quad$ O(E)

2032 NRL Theo W PREPRINT79KNOX $0795.9+1$

${ }^{50} \mathrm{Co}_{0}\left(p_{1} \mathrm{Zn}{ }^{3} \mathrm{He}_{\theta}\right)^{55} \mathrm{Fe} \quad \sigma(E)$

2033 NRL Theo W PREPRINT79KNOX $0795.3+1$

${ }^{59} \mathrm{Co}(p, n+p+t){ }^{55} F_{0} \quad \sigma(E)$

2034 NRL Theo W PREPRINT79KNOX $0795.2+1$

${ }^{59} C_{0}(p .3 n+2 p) 55 F_{e}$ o(E)

2035 NRL ThEO W PREPRINT79KNOX $0796.5+1$ The $W$ PREPRINT79KNOX O79 TR

${ }^{59} C_{0}(p, n+q){ }^{52} F_{\theta} \quad \sigma(E)$

2036 LVN Expl J ARI $28 \quad 533 \quad 772.4+17.6+1$ Hasbroek+ CURV. STCKD-FOILS. RES. ACT. Exp! R CSIR-F15-89 76 2.2+1 $8.4+1$ Hassbroek+

Expt W TO KACHAPAG $792.2+18.4+1$ Has as oeht NUMERICAL DATA.

Expt 4 EXFORB0098.003 $7792.2+1$ 9.4+1. 27 DATA POINTS.

$\left.{ }^{59} \mathrm{Co} p, n+a\right)^{55} F_{0}$ thick torget yleld

2037 LVN Expl J ARI $2 B \quad 533772.5+18.5+1$ HaAsbroeht IBL. CURV. FRM STCKD-FOILS. Expt J ARI $28533778.5+1$ HaAsbroek+(31.9+-1.6)C1/AH. STPPNG TRGT Expt P INDC(SAF)-5 8 878 2.5+1 $9.5+1$ Hasbroek+ TBL. FRM $51 G$ AND DIRECTLY. Expl R CSIR-FIS-89 $76 \mathrm{2.2+1} 8.5+1$ Habbroeht

Expt 4 EXFORBO098.003 $7792.2+18.5+1$. 32 DATA POINTS.

${ }^{59} \mathrm{Co}(p, n+a){ }^{55} \mathrm{Fe} \quad \sigma(E)$

2038 NRL Theo $W$ PREPRINT79KNOX $0792.8+1$ Peterson. TBL OF PEAK SIGMA. SEMIEMP. Theo W PREPRINT79KNOX 079 TR 8.0+1 Peterson. CURV. SEMIEMPIRICAL CALC.

${ }^{59} \mathrm{Co}(p, n+p+d)^{56} F$ e o(E)

2039 NRL The $W$ PREPRINT79KNOX 079 $4.7+1$ Peterson. TBL OF PEAK SIGMA. SEMIEMP.

${ }^{59} \mathrm{Co}_{0}\left(p_{0}{ }^{n+{ }^{3}} \mathrm{He}^{56} \mathrm{~F}_{\mathrm{O}} \quad\right.$ O(E)

2040 NRL Theo W PREPRINT79KNOX 079 3.8+1 Peterson. TBL OF PEAK SIGMA. SEMIEMP.

${ }^{59} \mathrm{Co}(p, 2 \mathrm{P}+2 p){ }^{56} \mathrm{Fe} \quad$ O(E)

2041 NRL The0 W PREPRINT79KNOX 079 $5.1+1$ Pelerson. TEL OF PEAK SIGMA. SEM!EMP. Theo W PREPRINT79KNOX O79 TR B.D+I Peterson. CURV. SEMIEMPIRICAL CALC.

${ }^{59} \mathrm{Co}\left(\mathrm{P}_{0} \mathrm{D+t}\right)^{56} \mathrm{Fe} \quad O(\mathrm{E})$

2042 NRL ThEO $\mathrm{H}$ PREPRINT79KNOX $0793.7+1$

Peterson. IBL OF PEAK SIGMA. SEMIEMP. 


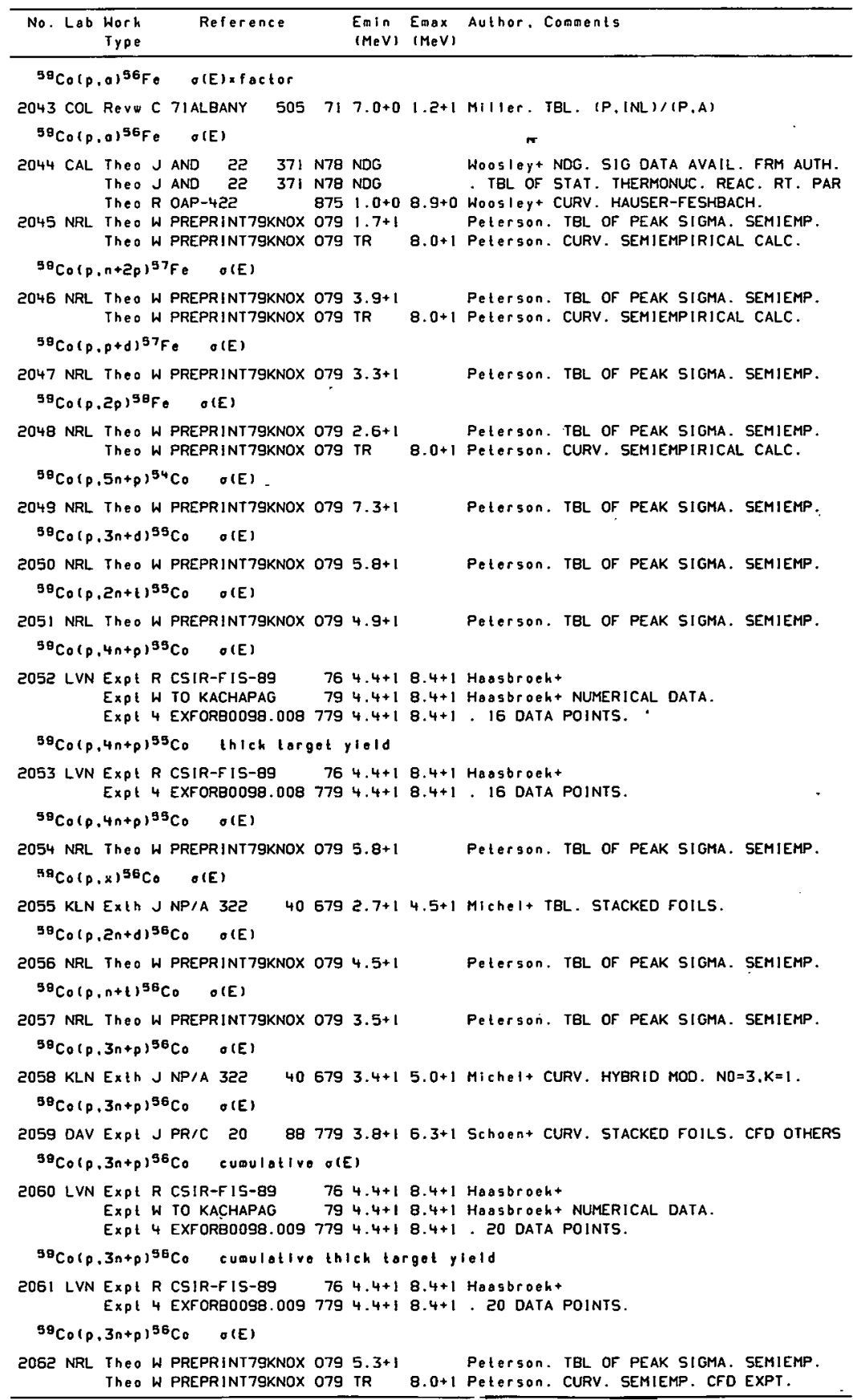


REFERENCES i c ont $)$

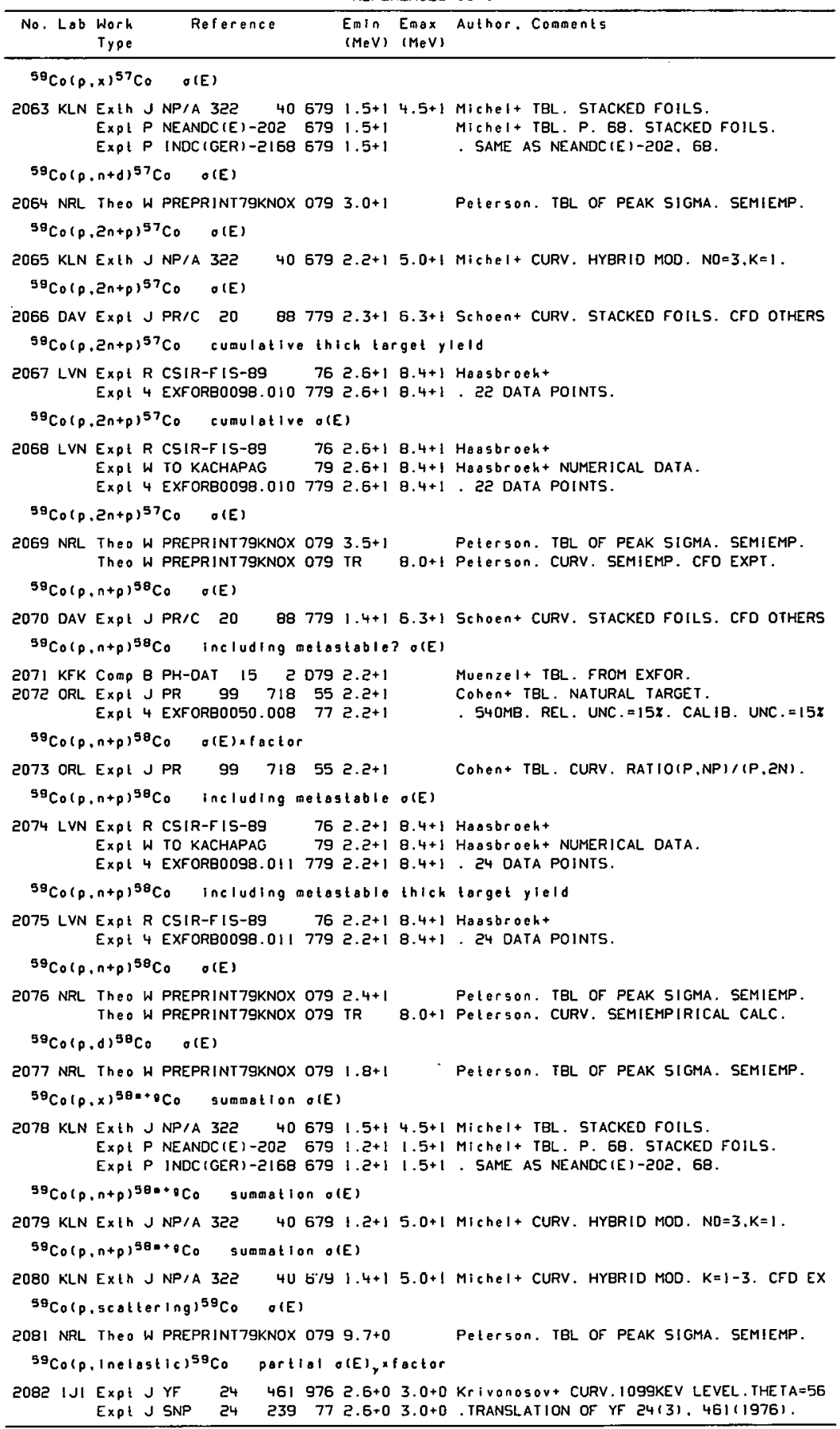


REFERENCES ( c on t)

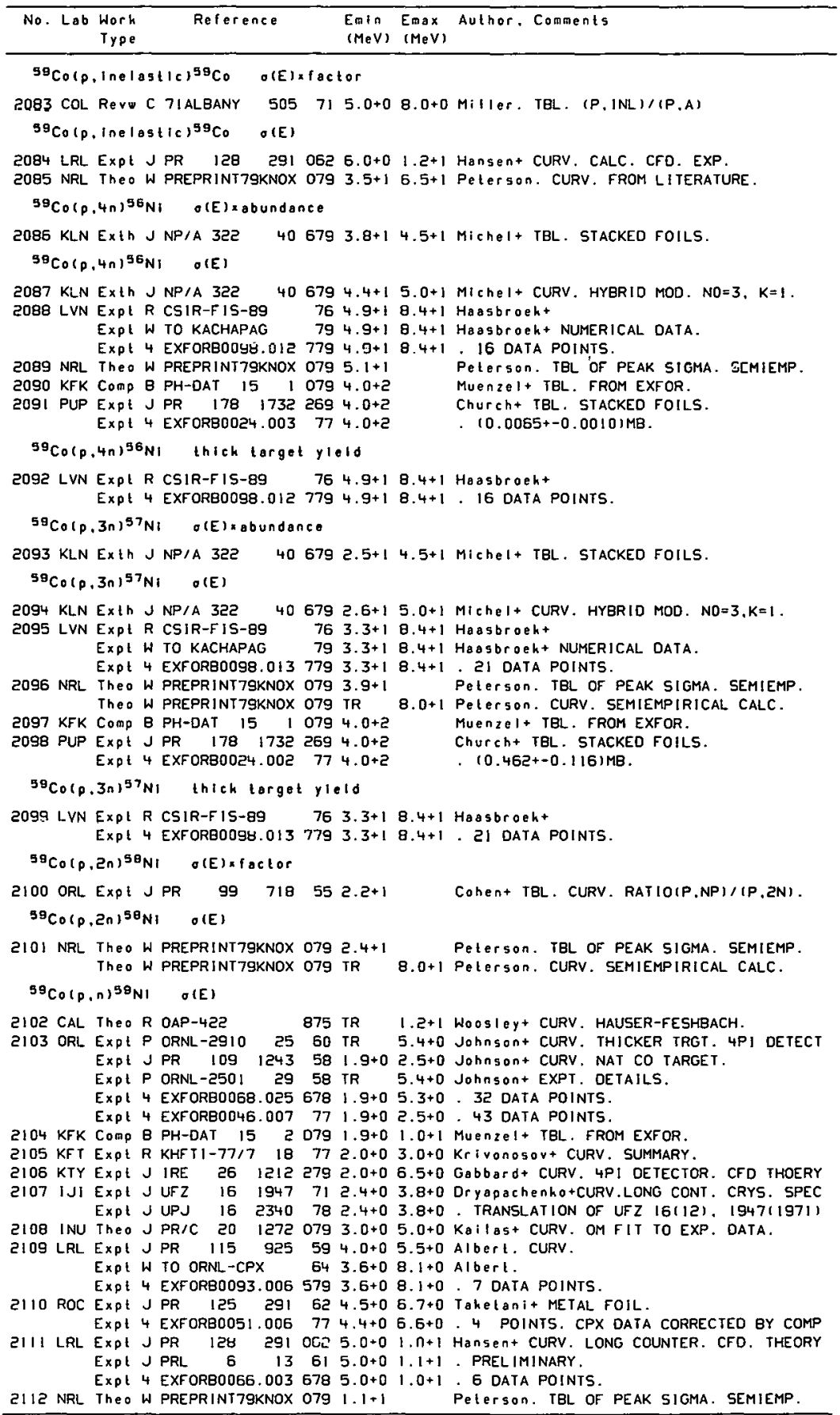




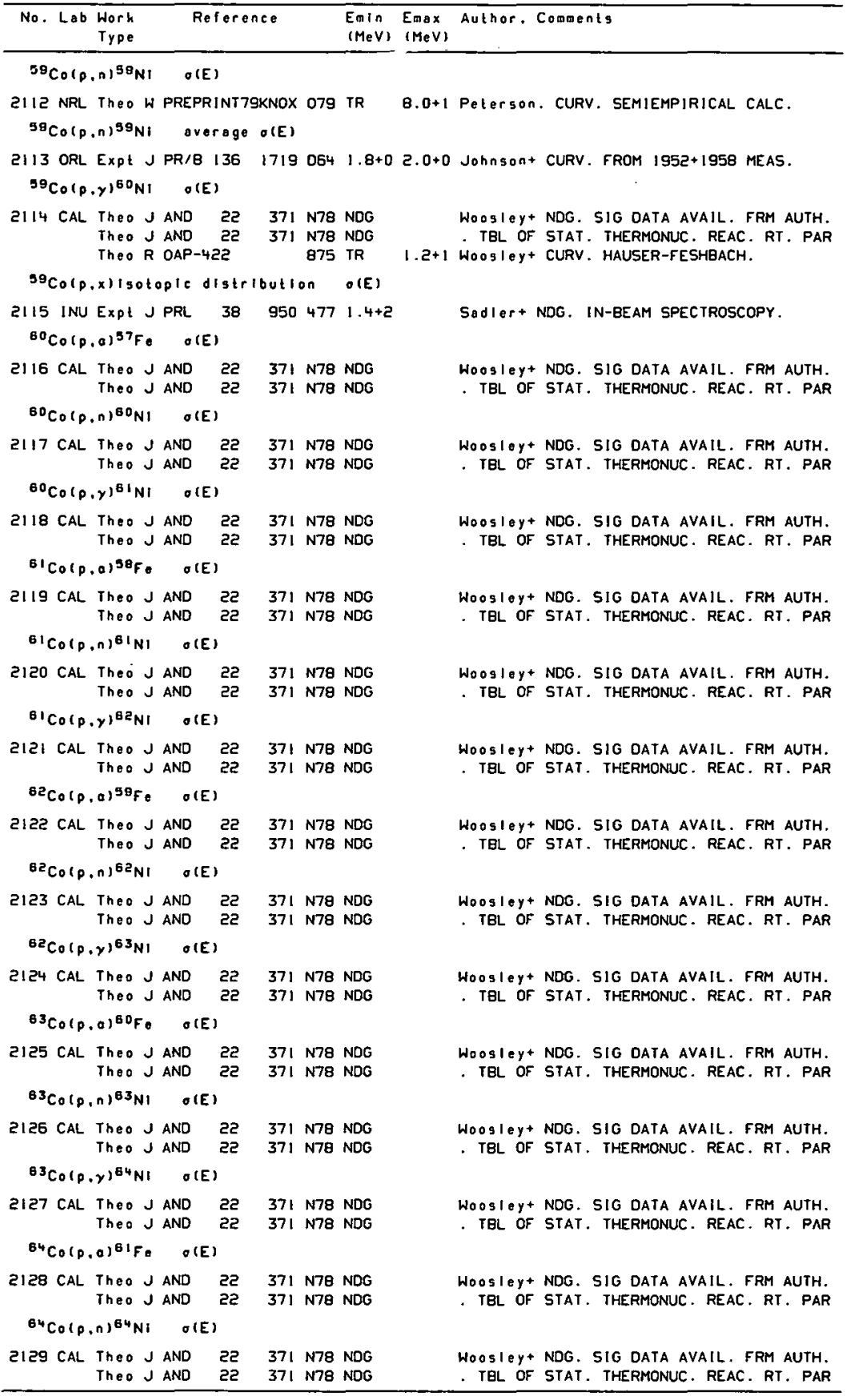


REFERENCES ( con ( )

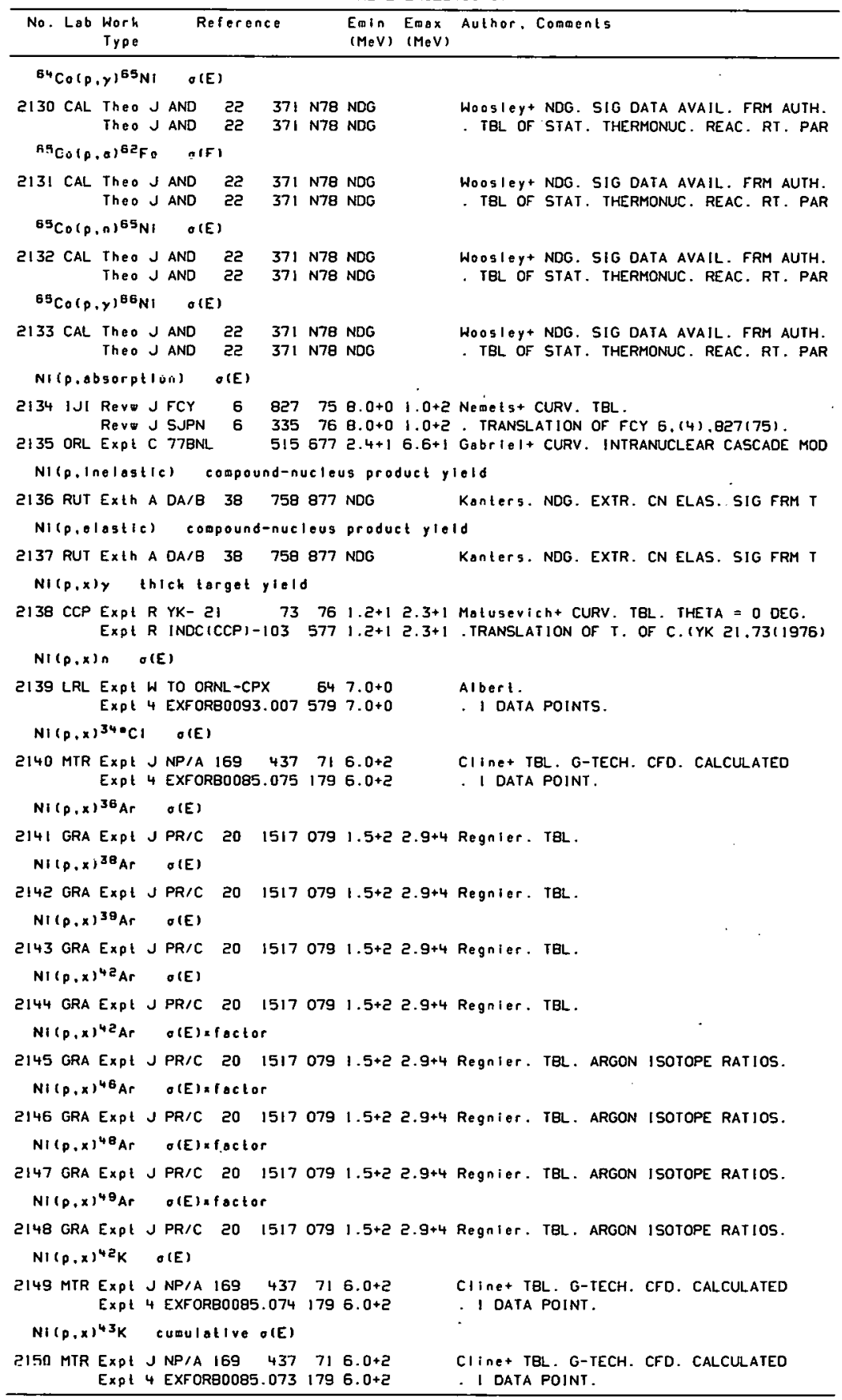




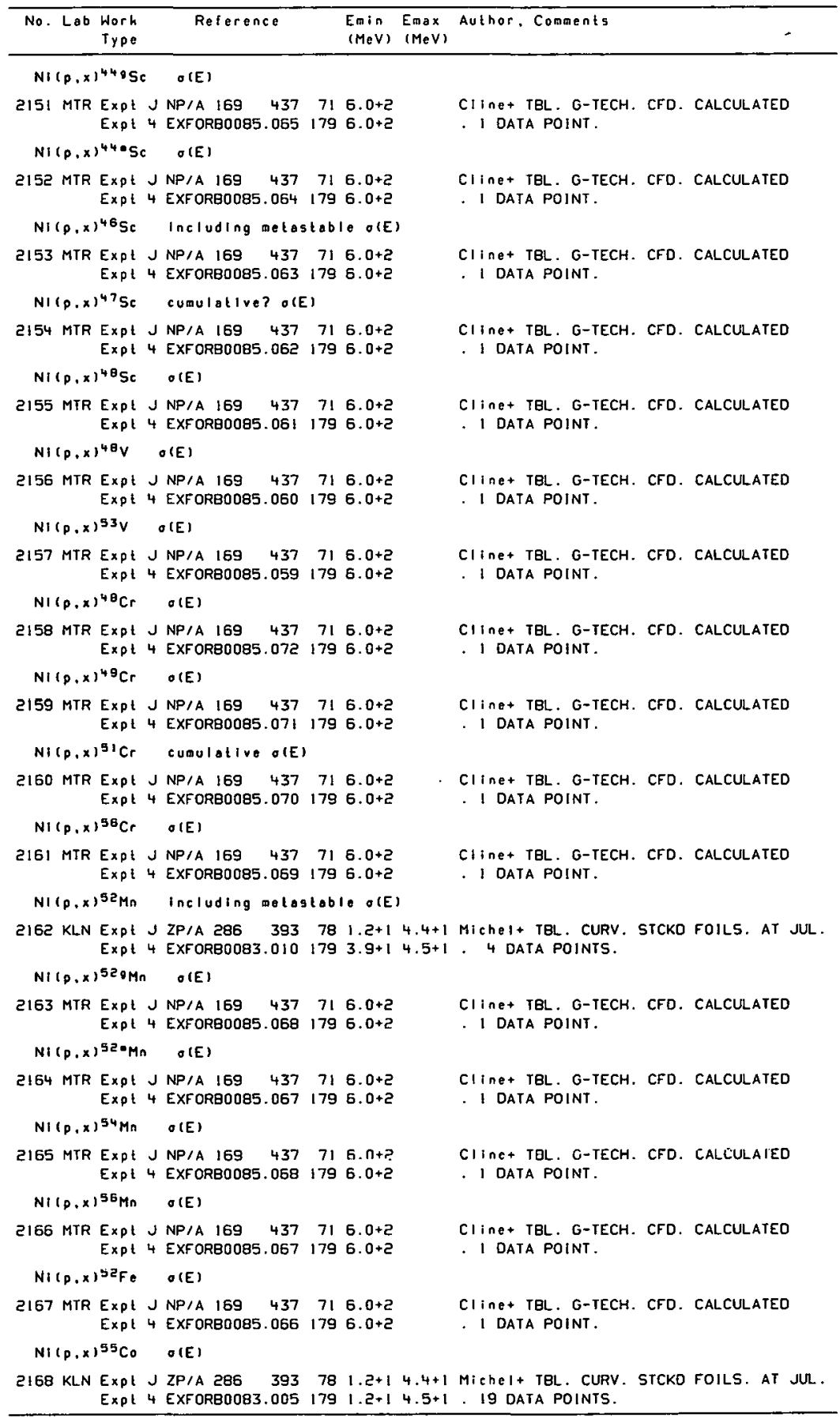




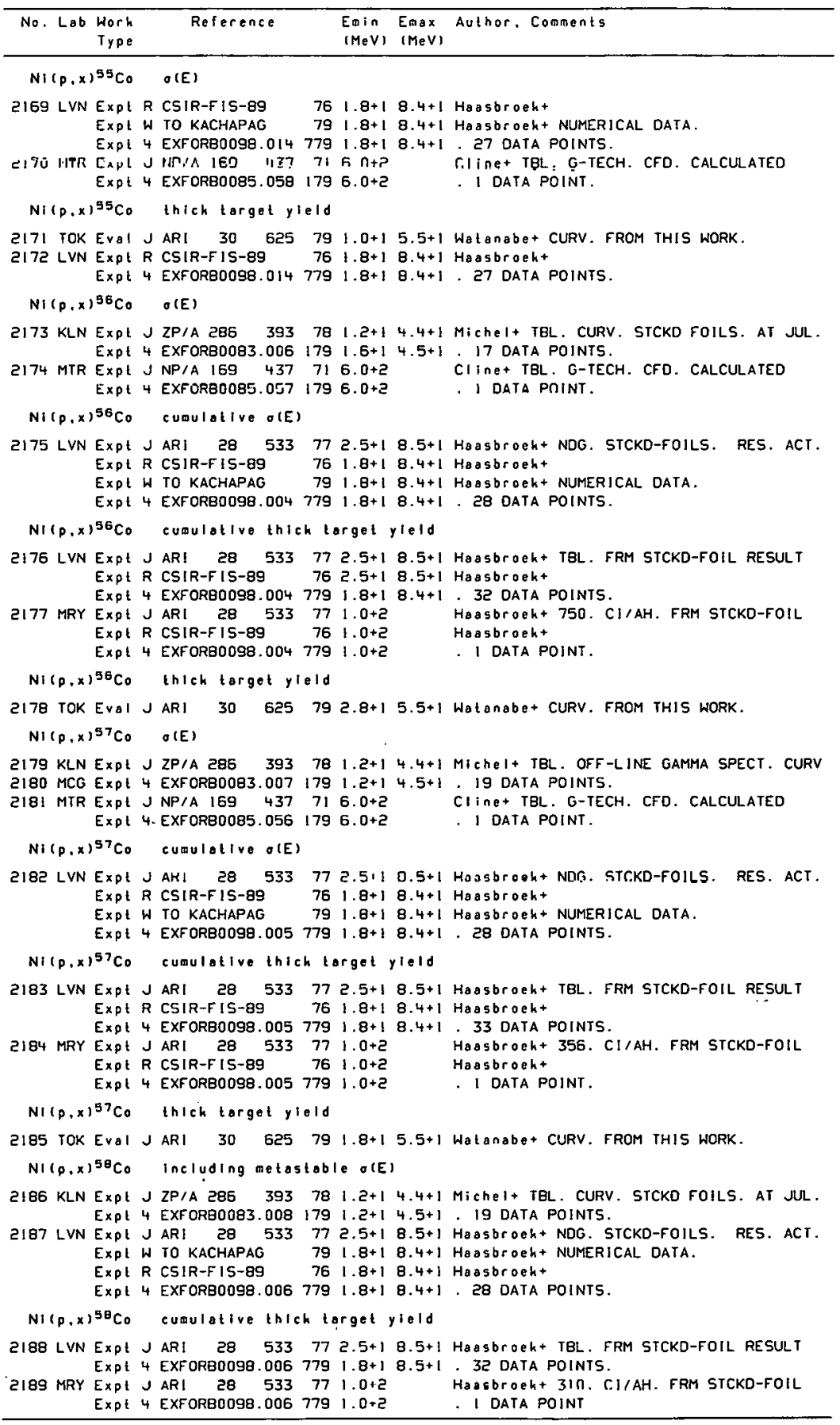




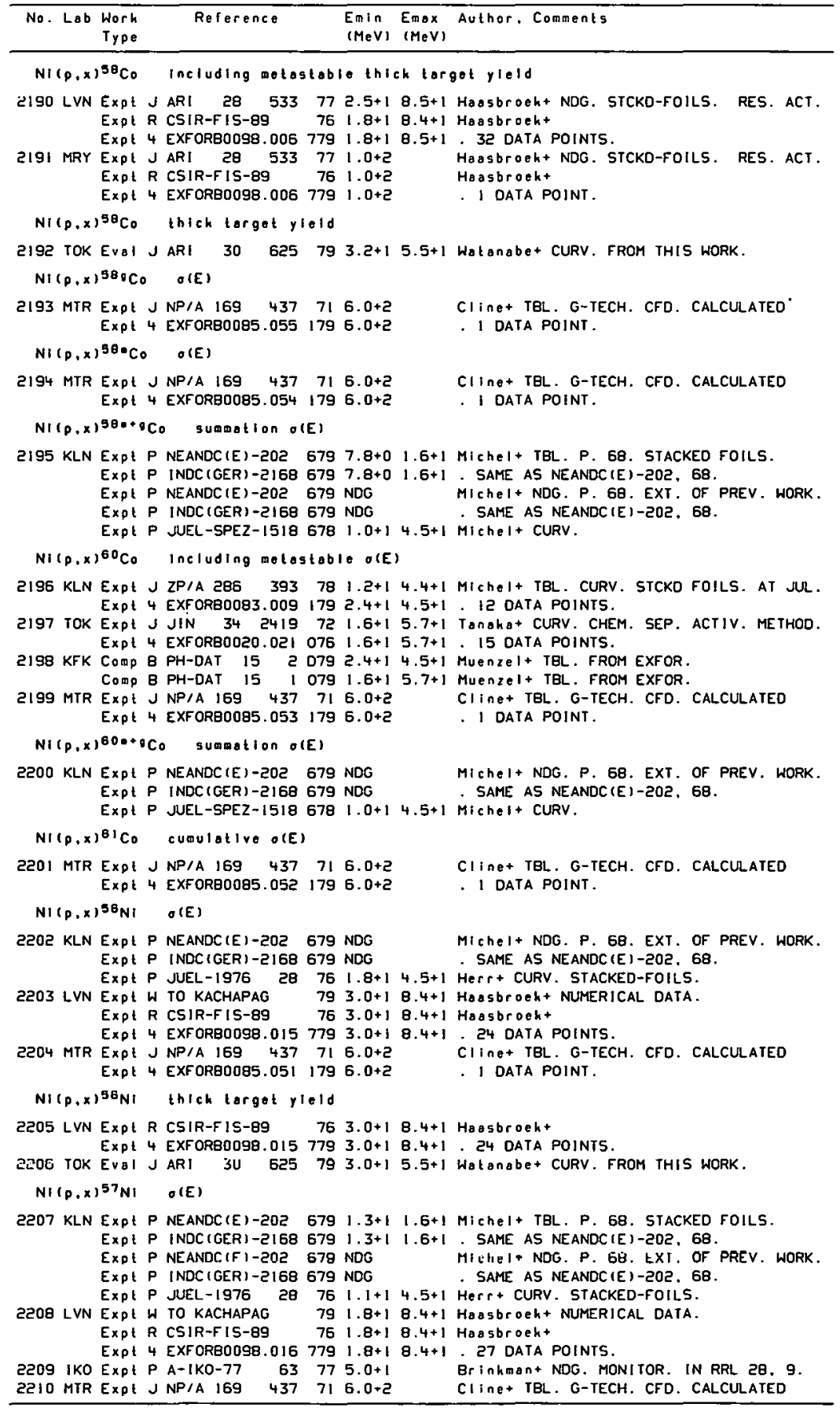


REFERENCES ( $c$ on ()

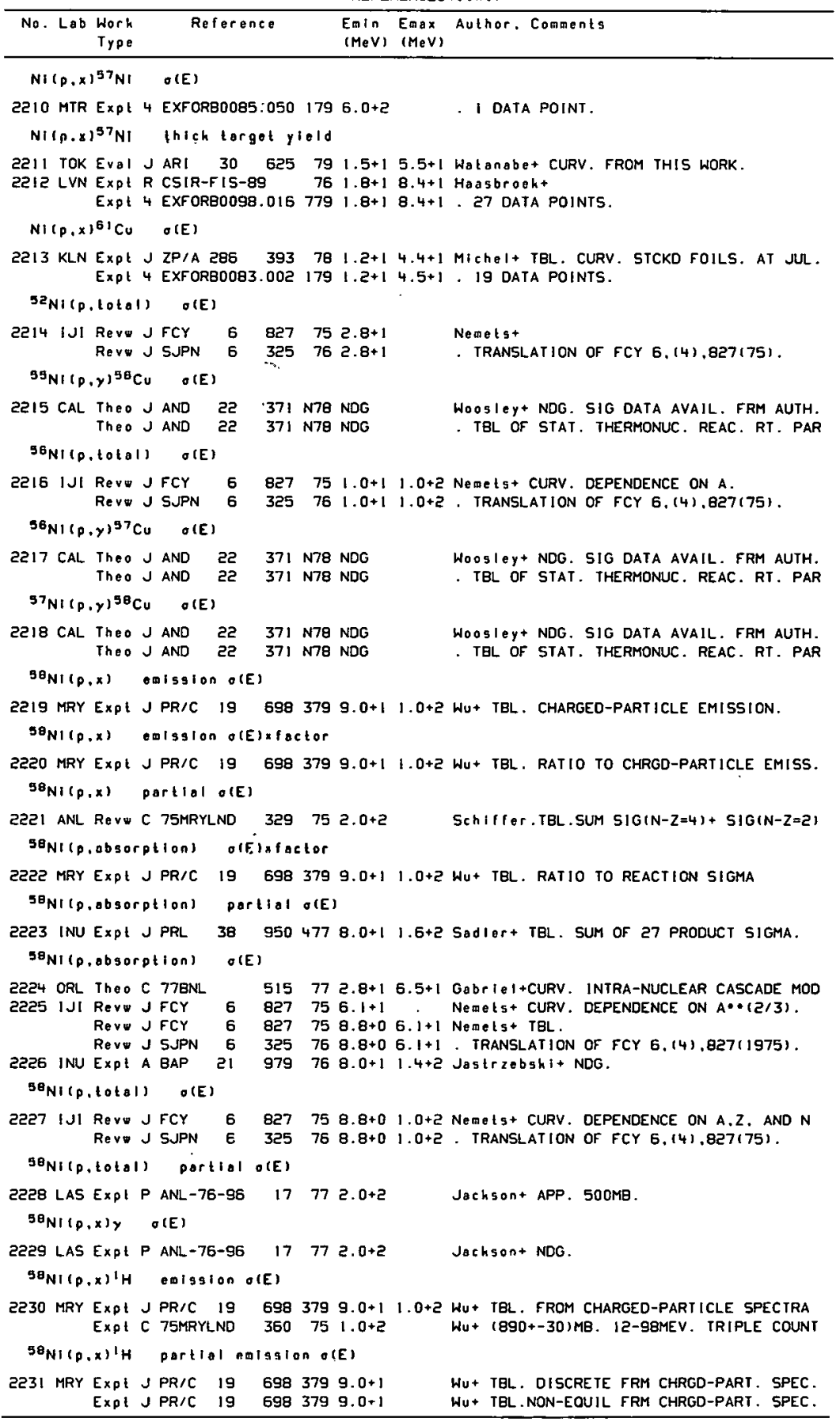


REFERENCES ( c on ()

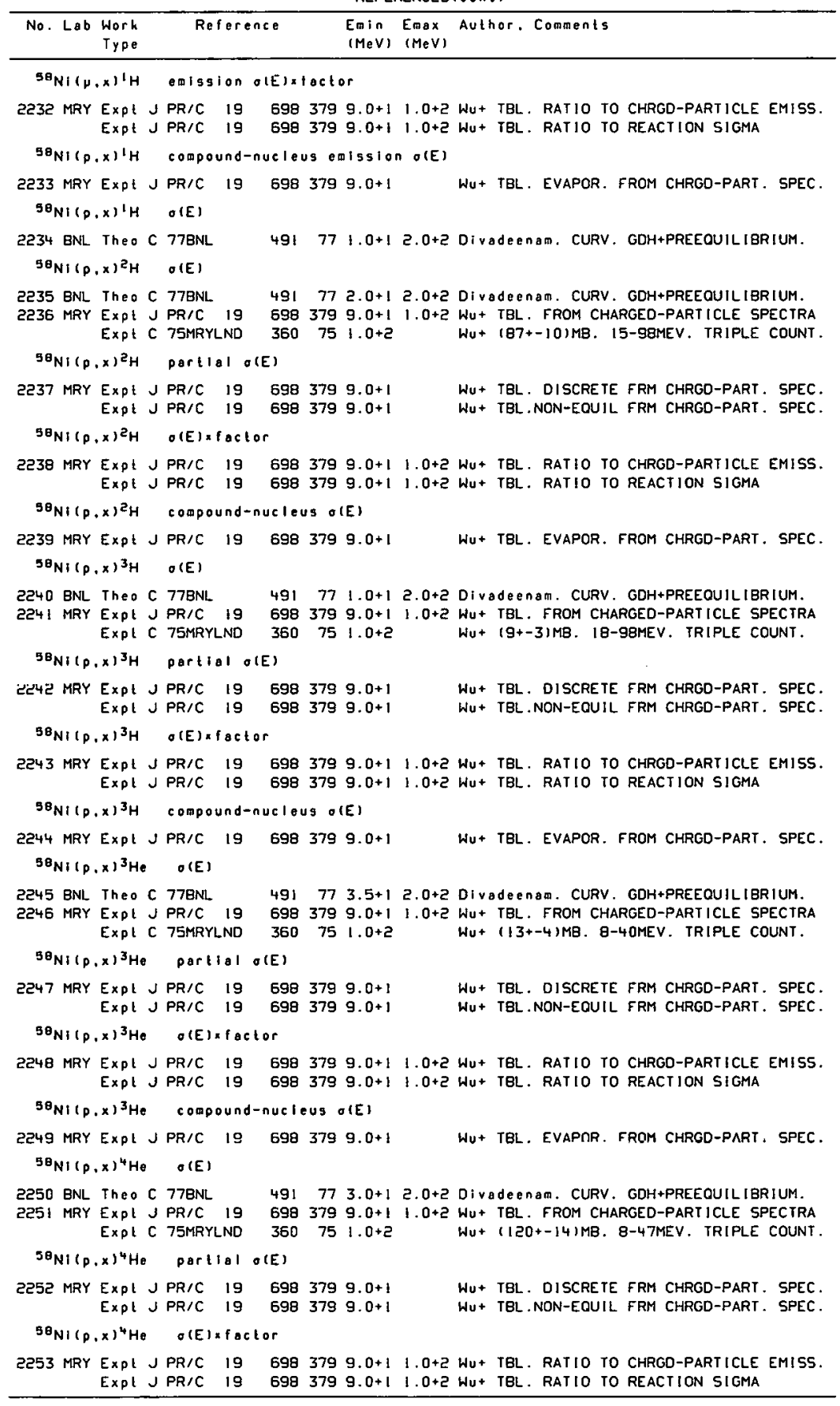


REFERENCES ( c ont)

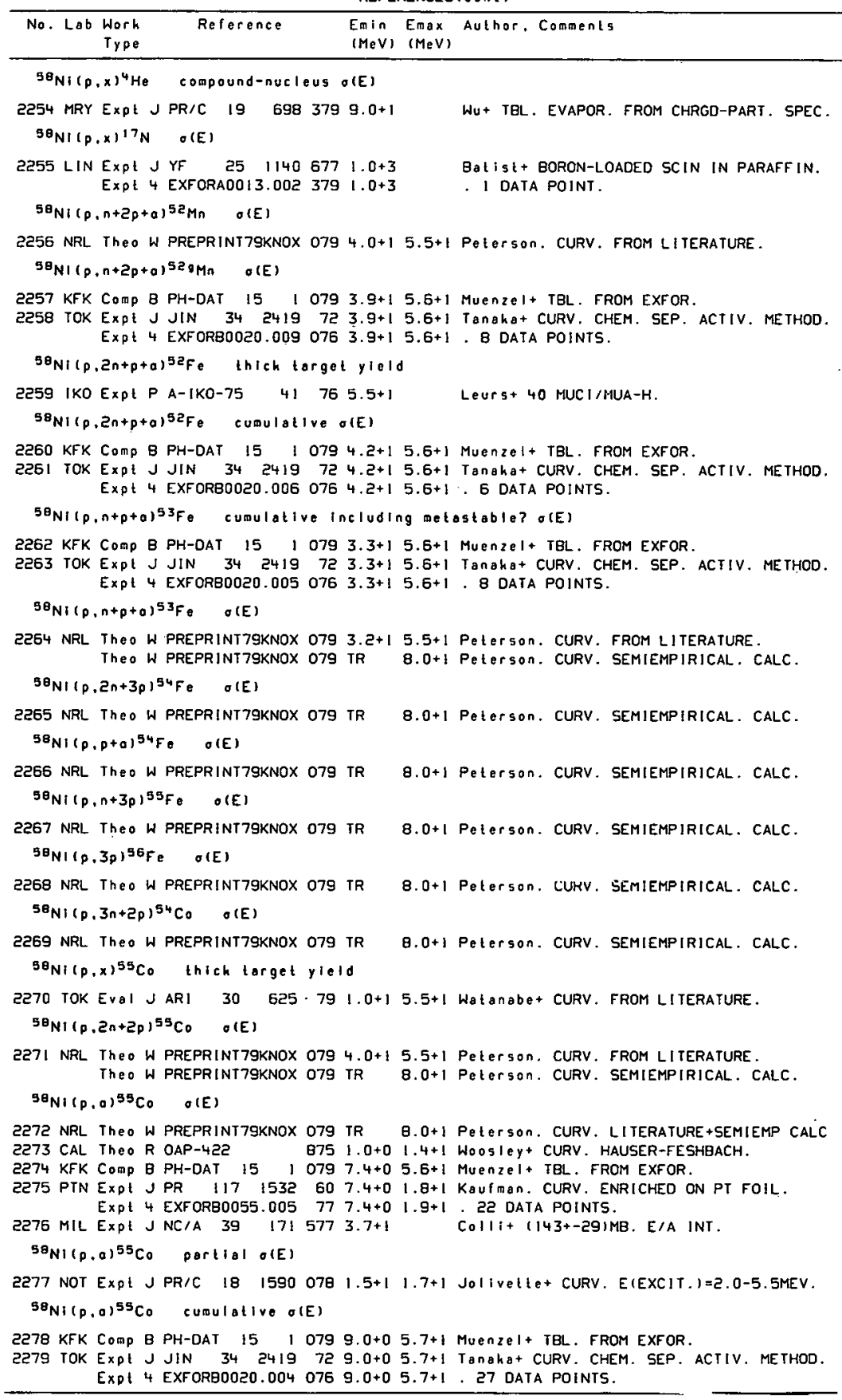




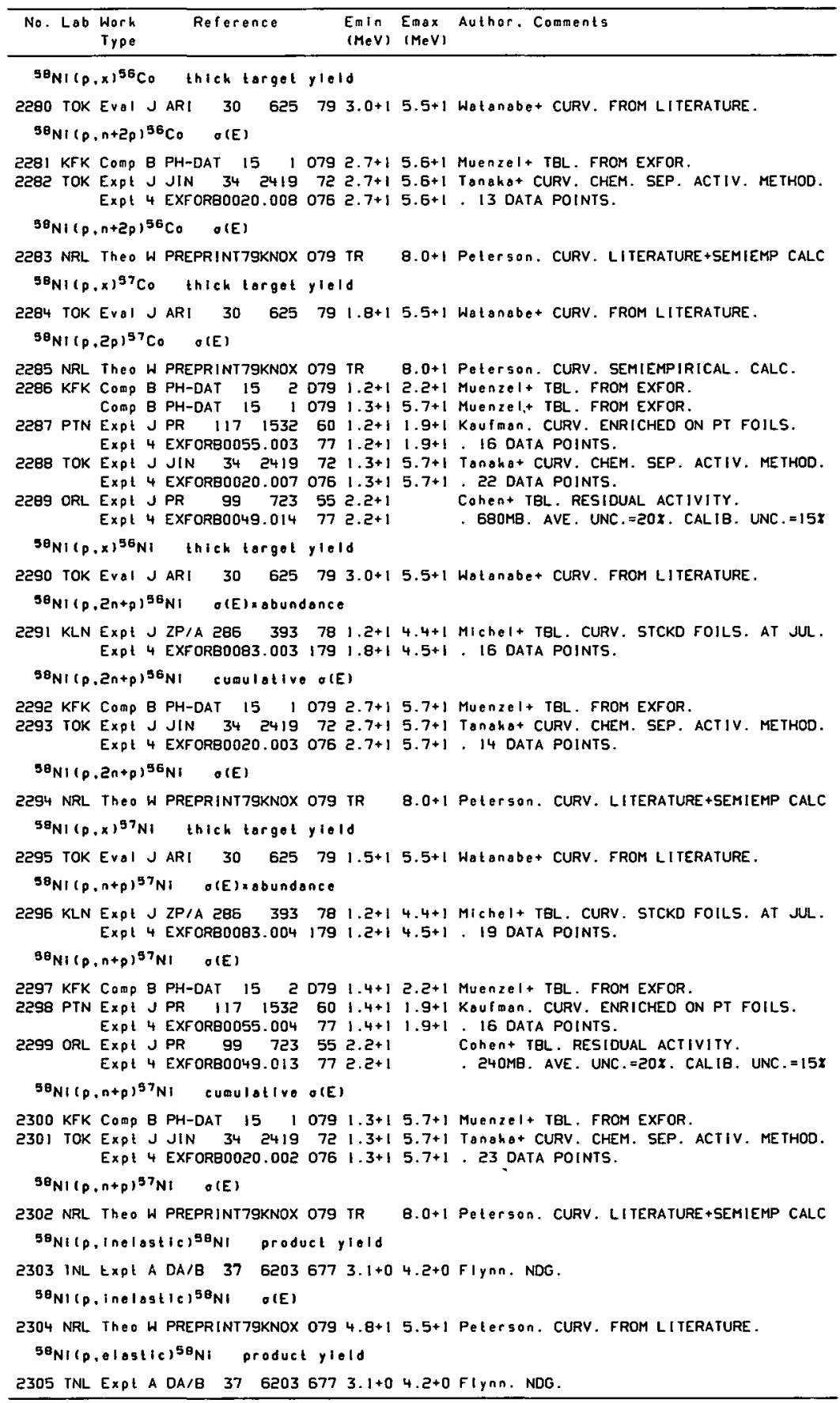


REFERENCES $(c$ ont $)$

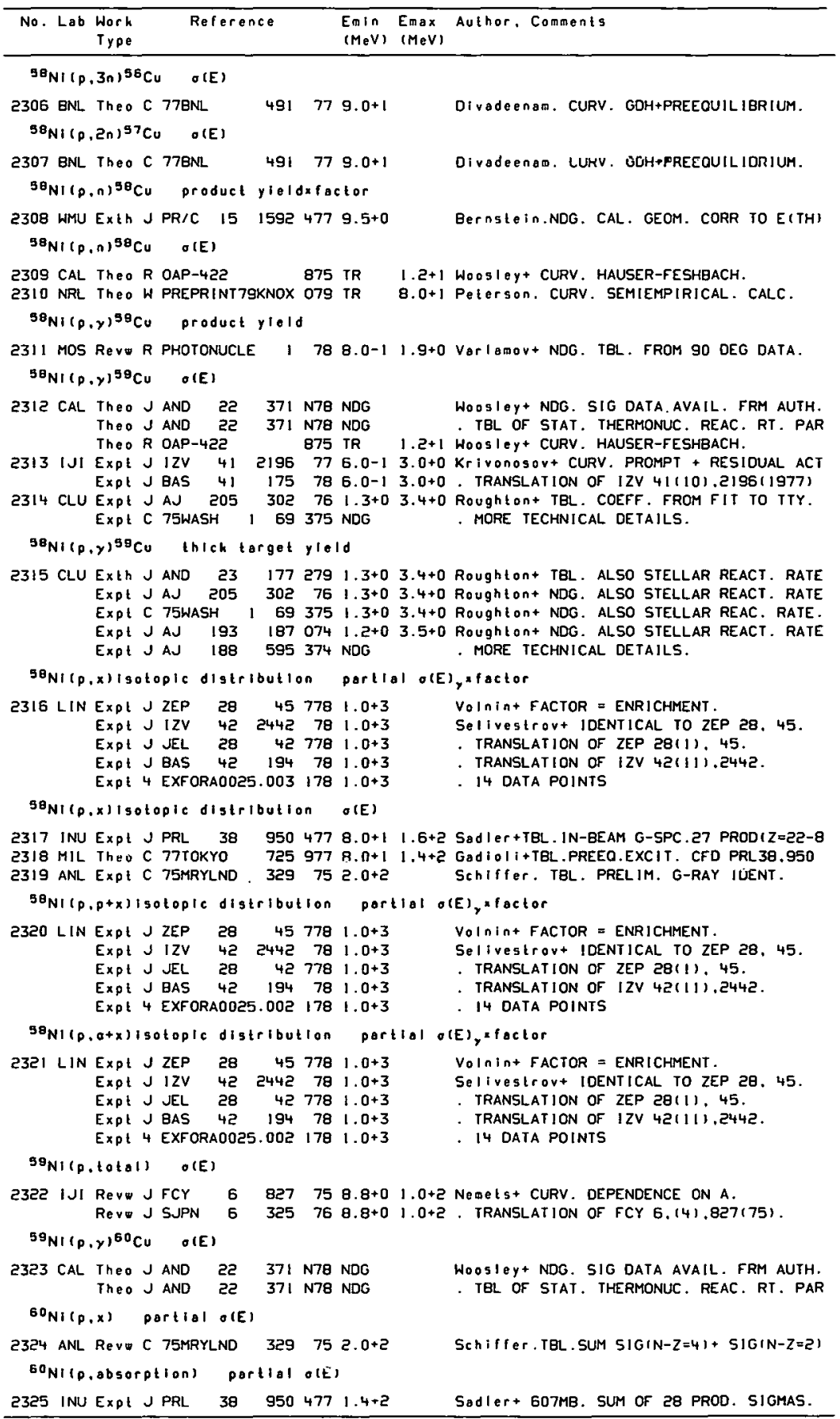


REFERENCES $(\mathrm{c}$ on $t)$

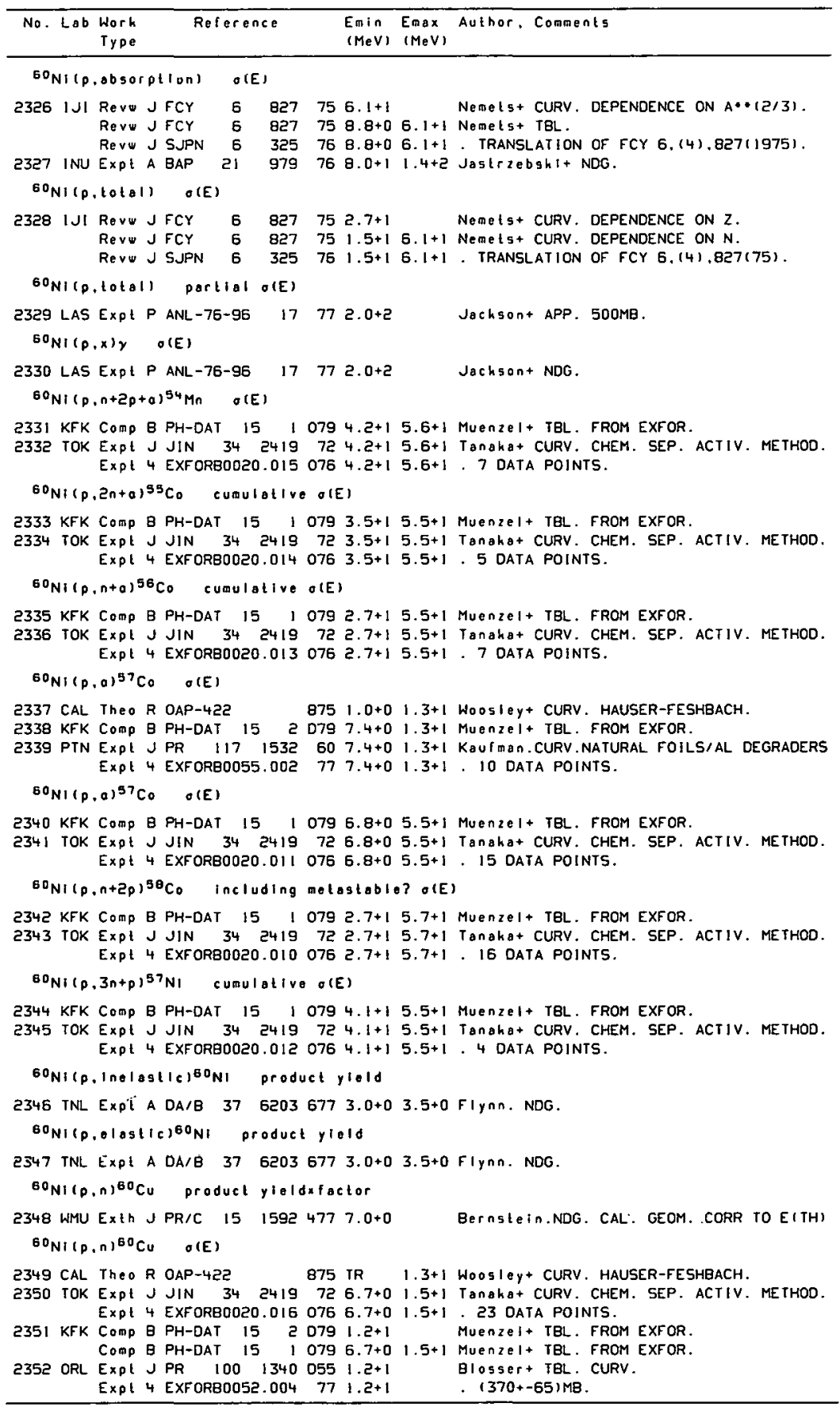


REFERENCES ( c on I)

\begin{tabular}{|c|c|c|c|c|c|}
\hline No. Lob & $\begin{array}{l}\text { Work } \\
\text { Type }\end{array}$ & Reference & $\begin{array}{l}\text { Emin } \\
\text { (MeV) }\end{array}$ & $\begin{array}{l}\text { Emax } \\
\text { (MeV) }\end{array}$ & Author. Comments \\
\hline
\end{tabular}

$60^{2}$ i (p.y)6iCu partlat relative o(E)

2353 ALB Expt J NP/A $3203855791.8+12.9+1$ Bohharee+ CURV. EG .GT. 2.7MEV. RED.WID

$60 \mathrm{Ni}(\rho, \gamma)^{61} \mathrm{Cu} \quad O(\mathrm{E})$

2354 CAL Theo J AND 22 371 N7R NDG Woosley+ NDG. SIG DATA AVAIL. FRM AUTH.

Theo J AND $22 \quad 371$ N78 NDG . TBL OF STAT. THERMONIUC. REAC. RT. PAR

Theo R OAP-422 875 IR $1.3+1$ Woosley + CURV. HAUSER-FESHBACH.

2355 IJI Expt J IZV 41 2196 77 6.0-1 3.0+0 Krivonosov+ CURV. PROMPT + RESIOUAL ACT Expt J BAS $41 \quad 175 \quad 786.0-13.0+0$. TRANSLATION OF 12V 41110$) .2195(1977)$

$2356 \mathrm{CLU}$ Expt J AJ 20530276 9.8-1 3.0+0 Roughtont TBL. COEFF. FROM FIT TO TTY. EXPt C 75WASH 169375 NDG MORE TECHNICAL DETAILS.

2357 KFK Comp B PH-DAT I5 $20791.0+12.3+1$ Muenzel+ IBL. FROM EXFOR.

2358 ORL Expt J PR $100 \quad 2060551.0+12.2+1$ Cohen. TBL. STACKED FOILS. EXPl 4 EXFORB0047.004 $771.0+12.3+1$. 4 DATA POINTS.

$60 \mathrm{NI}(p, y)^{61} \mathrm{Cu}$ partial ray o(E)

2359 DUB Expl J YF $219195791.7+01.9+0 \mathrm{Kraft+}$ CURV. P1/2+F5/2 ANALOG RESONANCE Expt J YF $2010810741.6+01.6+0 \mathrm{Kraft}+$ CURV. P3/2 ANALUO STATE.

Expt J SNP $21 \quad 4725753.1+03.3+0$. TRANSLATION OF YF $21.919(1975)$.

Expt J SNP $20 \quad 5670741.6+01.6+0$. TRANSLAT1ON OF YF 20.1081(1974).

$60 \mathrm{Ni}(p, y)^{6} \mathrm{Cu}$ thick torget yleto

2360 CLU Exth J AND $23 \quad 177279$ 9.8-1 3.0+0 Roughtont TBL. ALSO STELLAR REACT. RATE Expl J AJ 20530276 9.8-1 3.0+0 Roughton+ NDG. ALSO STELLAR REACT. RATE Expl C 75WASH 169375 9.8-1 3.0+0 Roughton+ NDG. ALSO STELLAR REAC. RATE. Expl J AJ $1931870741.0+03.0+0$ Roughton+ NDG. ALSO STELLAR REACT. RATE Expl J AJ 189595374 NOG . MORE TECHNICAL DETAILS.

60Nit(p,x)isotopic distribution o(E)

236I INU Expt J PRL $38 \quad 950477 \quad 1.4+2$ 2362 MIL Theo C 77TOKYO $7259771.4+2$ 2363 ANL Expt C 75MRYLND $329752.0+2$

Sad l er+TBL . IN-BEAM G-SPC . $28 P R O D(Z=23-28$ GadiolitTBL. PREEO.EXCIT. CFD PRL 38,950

${ }^{6}{ }^{N} i(p, a){ }^{50} C_{0}$ including detastable? o(E)

2364 KFK COmp B PH-DAT $15 \quad 10797.0+0 \quad 1,4+1$ Muenzel+ TBL. FROM EXFOR.

2365 TOK Expt J JIN $342419727.0+01.4+1$ Tanaha+ CURV. CHEM. SEP. ACTIV. METHOD Expt 4 EXFORBO020.017 076 $7.0+01.4+1$. IS OATA POINTS.

$B{ }^{\prime} N I(P, 0)^{58} \mathrm{CO} \quad$ OIE)

2366 CAL Theo J AND $22 \quad 37 !$ N78 NDG Woosley+ NDG. SIG DATA AVAIL. FRM AUTH. Theo J AND 22371 N78 NDG . TBL OF STAT. THERMONUC. REAC. RT. PAR Theo R OAP-422 $8751.0+01.2+1$ Woosley+ CURV. HAUSER-FESHBACH.

GINICP.Inelagtic)6INi partIal O(E), factor

2367 IJl Expt J YF $24 \quad 4619761.6+03.0+0 \mathrm{KrivonOSOV+} \mathrm{CURV.} \mathrm{283KEV} \mathrm{LEVEL.} \mathrm{THETA=56}$ Expt J SNP $24 \quad 23977 \quad 1.6+03.0+0$. TRANSLATION OF YF $24(3), 461(1976)$.

$6 I^{N} I(P, n)^{6} \mathrm{CU} \quad O(E)$

2368 CAL TheO R OAP-42Z 875 TR $1.3+1$ WoOSIEY + CURV. HAUSER-FESHBACH.

2369 TOK Expl J JIN $342419722.7+01.4+1$ Tanaka+ CURV. CHEM. SEP. ACTIV. METHOD. Expt 4 EXFORBO020.018 $0762.7+01.4+1$. 17 DATA POINTS.

2370 INU Theo J PR/C $2012720793.0+05.0+0 \mathrm{Kallas}+$ CURV. OM FIT TO EXP. DATA.

2371 KFK Comp B PH-DAT 152 D79 3.1+0 1.4+1 Muenze1+ TBL. FROM EXFOR.

Comp B PH-DAT $1510792.7+01.4+1$ Muenze 1+ TBL. FROM EXFOR.

2372 ORL Expt P ORNL-2910 $25603.1+0$ 6.0+0 Johnsont CURV. THICKER TRGT. 4PI DETECT Expt P ORNL-2501 $29583.1+0$ 6.0+0 Johnsont EXPT. DETAILS.

EXPl 4 EXFORBO068.007 $6783.1+06,0+0$. 20 DATA POINTS.

2373 TOK EXPL J JPJ $141269594.8+01.4+1$ Tanaha+ CURV. NATURAL FOIL.

EXPt 4 EXFORBO0 $43.008774 .8+01.4+1$. 14 DATA POINTS.

2374 ORL EXPL J PR $100 \quad 1340$ D55 $1.2+1$ BIOSser+ TBL. CURV. Expl 4 EXFORBO052.005 $771.2+1 \quad$. (590+-160)MB.

${ }^{1} N i(p, n)^{6} \mathrm{Cu}$ partialo(E)

2375 WAS Expl A DA/B $36 \quad 4473764.1+0 \quad 5.3+0$ Braga. NDG. PARTIALS TO FIRST 5 EXC. ST

$61 N I(P, n)^{61 C U}$ COU O(E)

2376 ORL Expt J PR/B $136 \quad 1719$ DG4 2.9+0 3.4+0 Johnson+ CURV. FROM 1953+1960 MEAS.

$6 I N I(P, y)^{62} \mathrm{Cu}$ o(E)

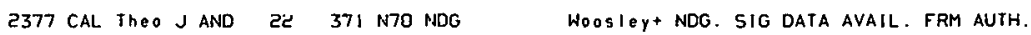
Theo J AND 22 371 N78 NDG . TBL OF STAT. THEKMUNUUC. REAC. RT. PAR 


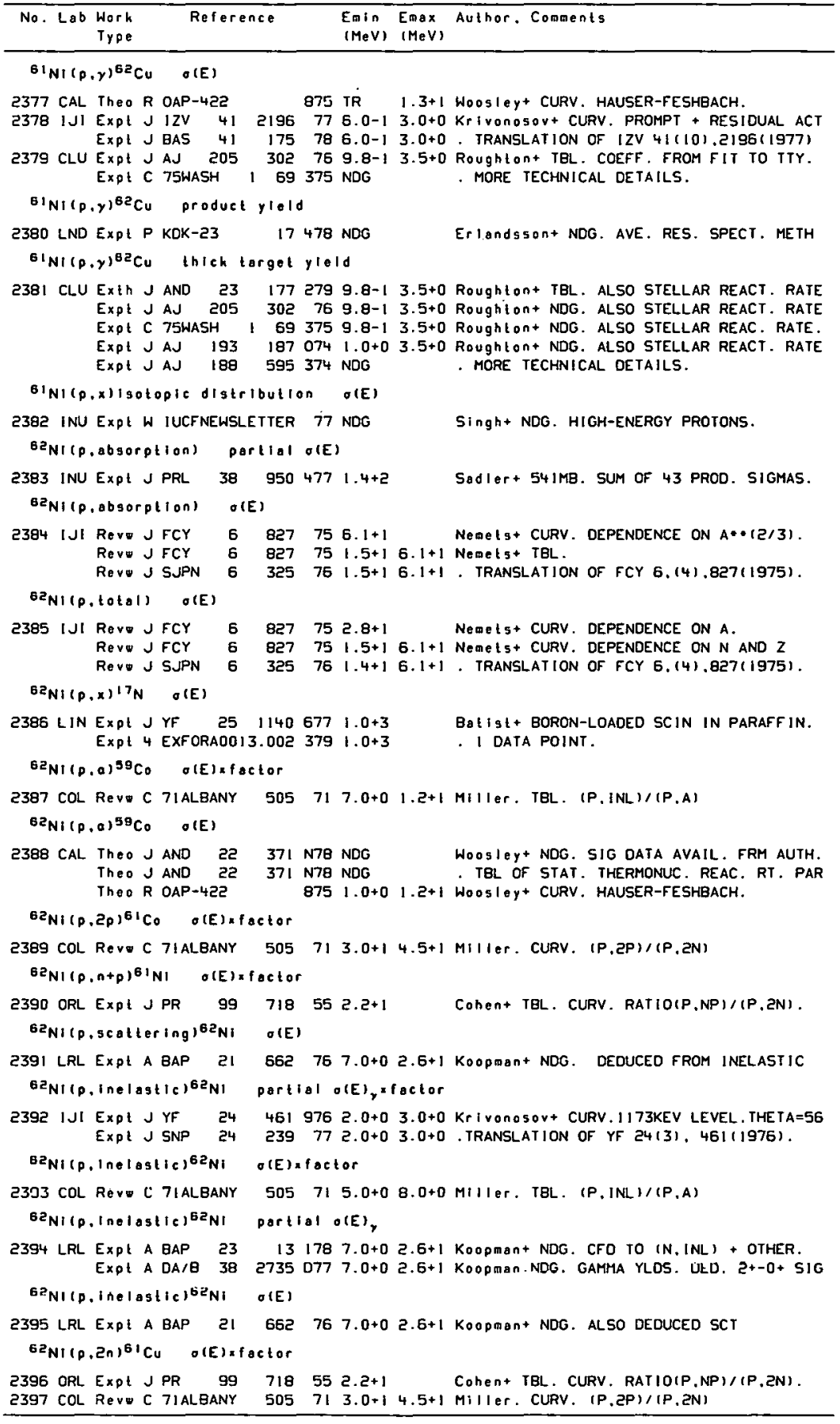


REFERENCES ( con ( )

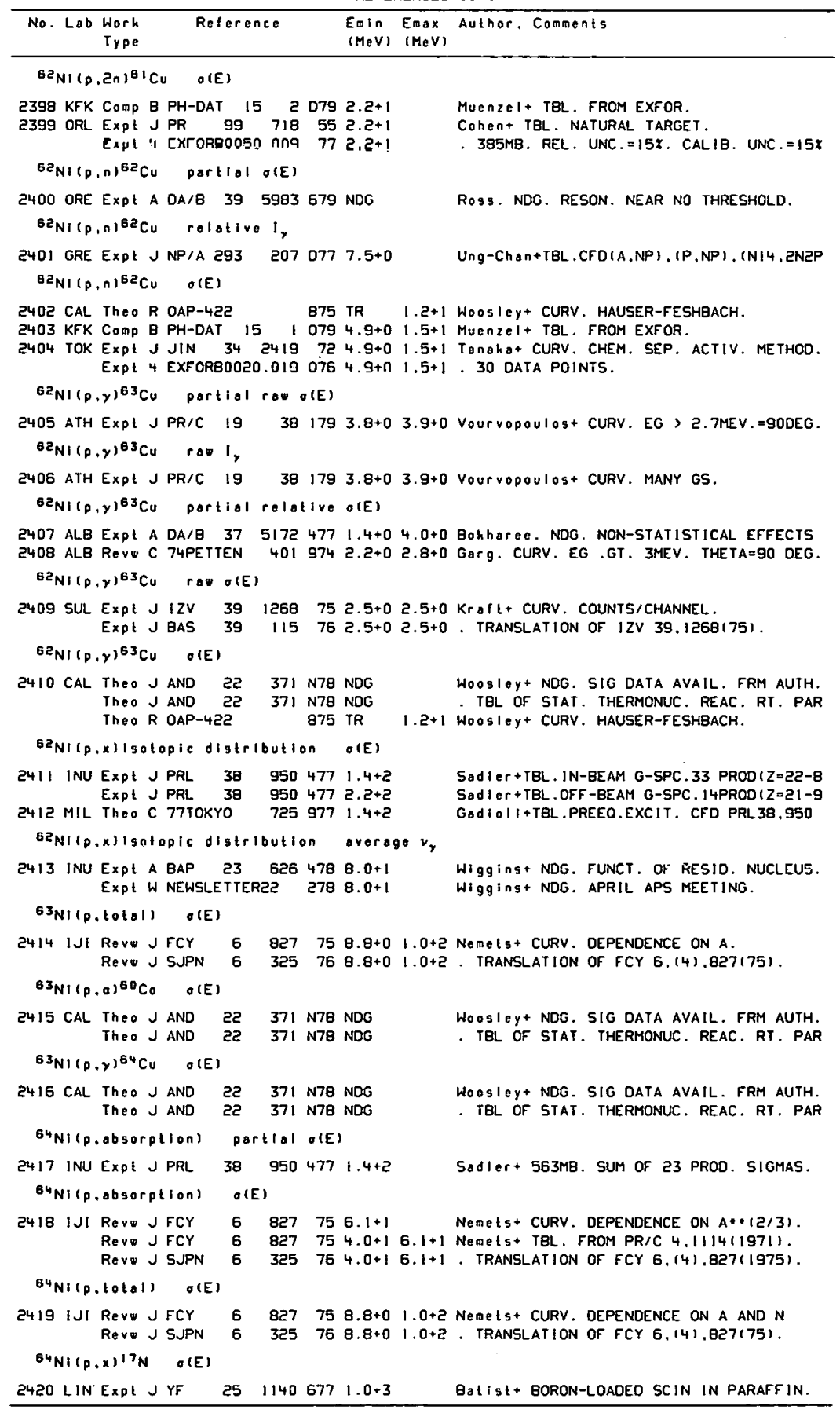




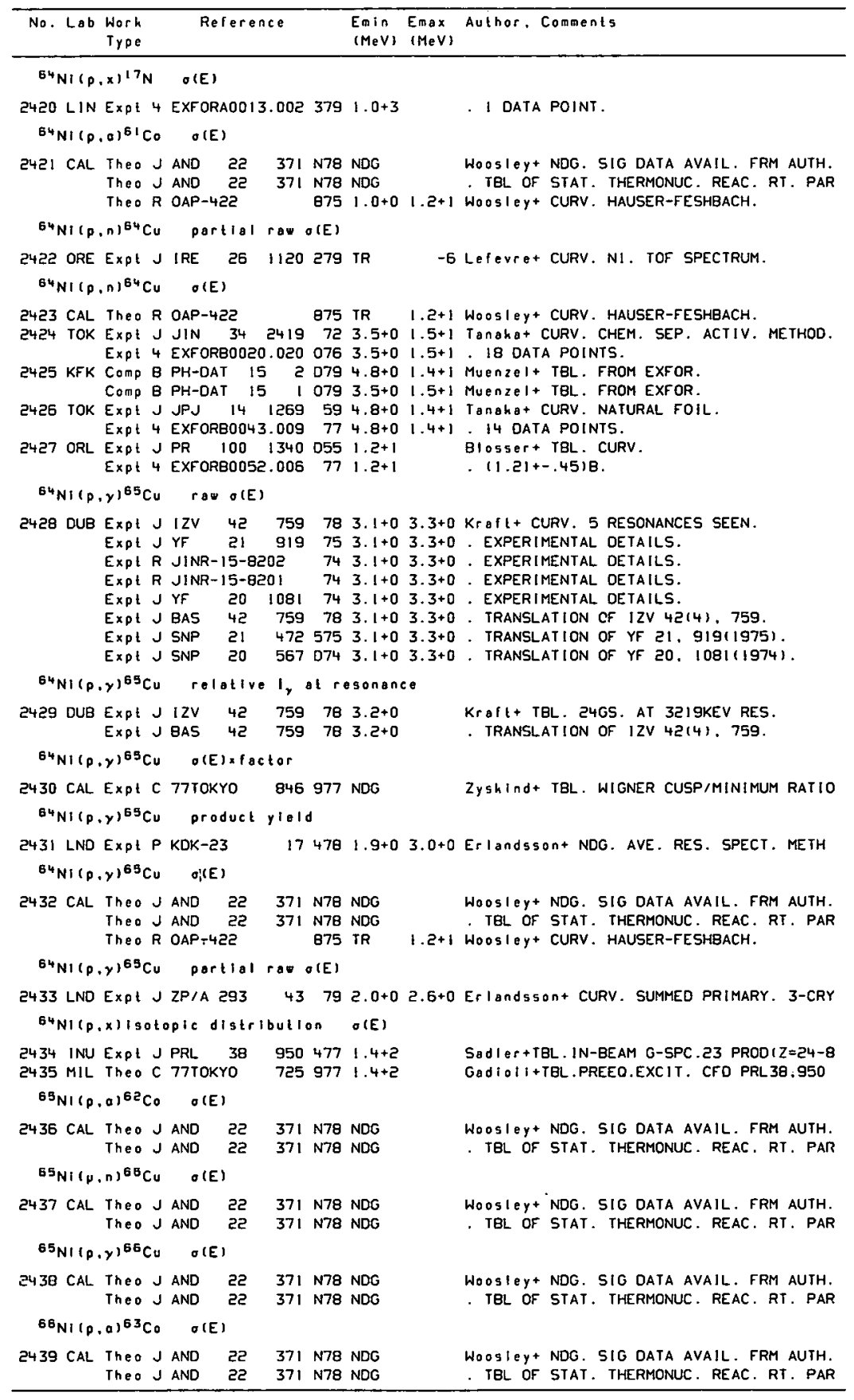


REFERENCES $(\operatorname{con}()$

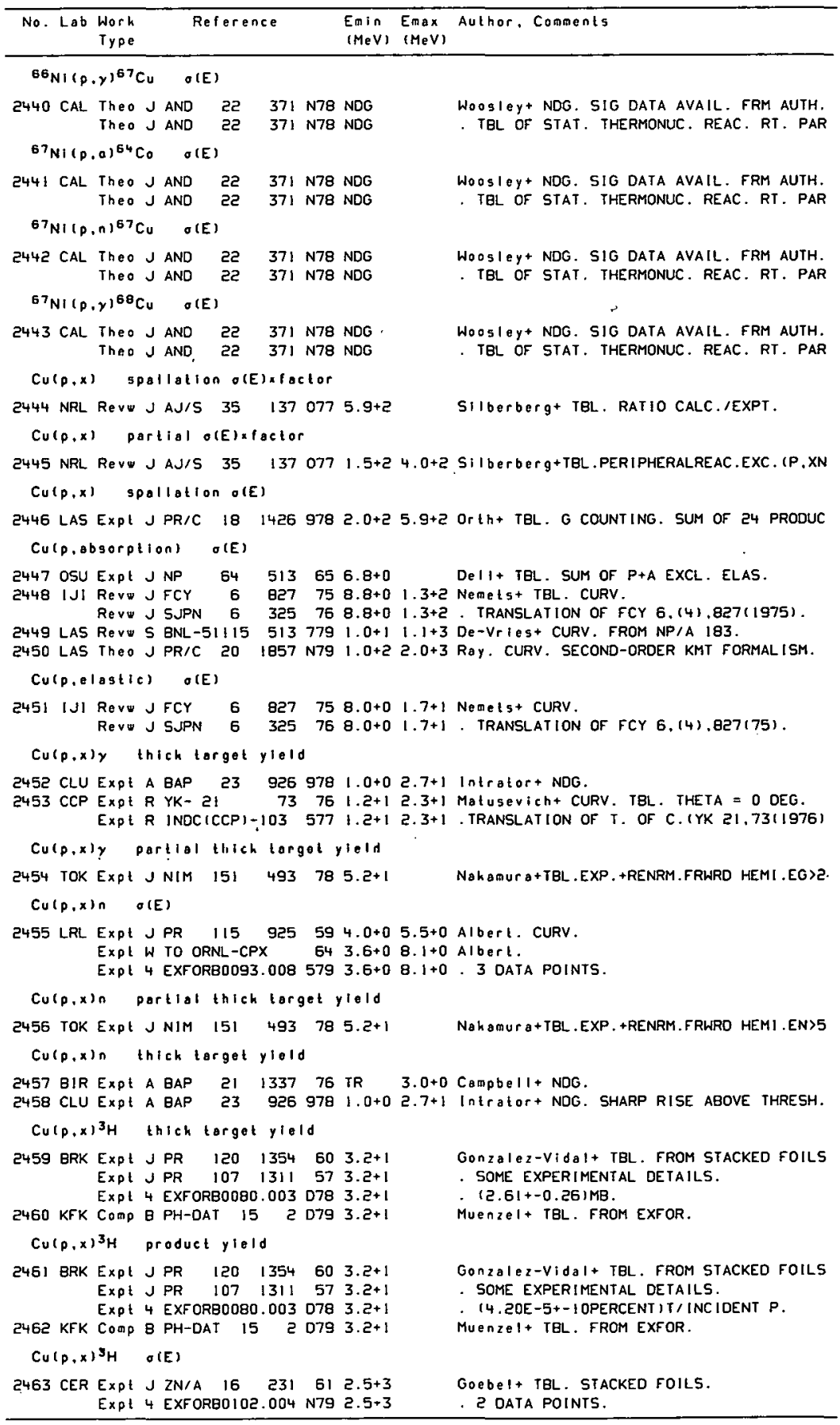




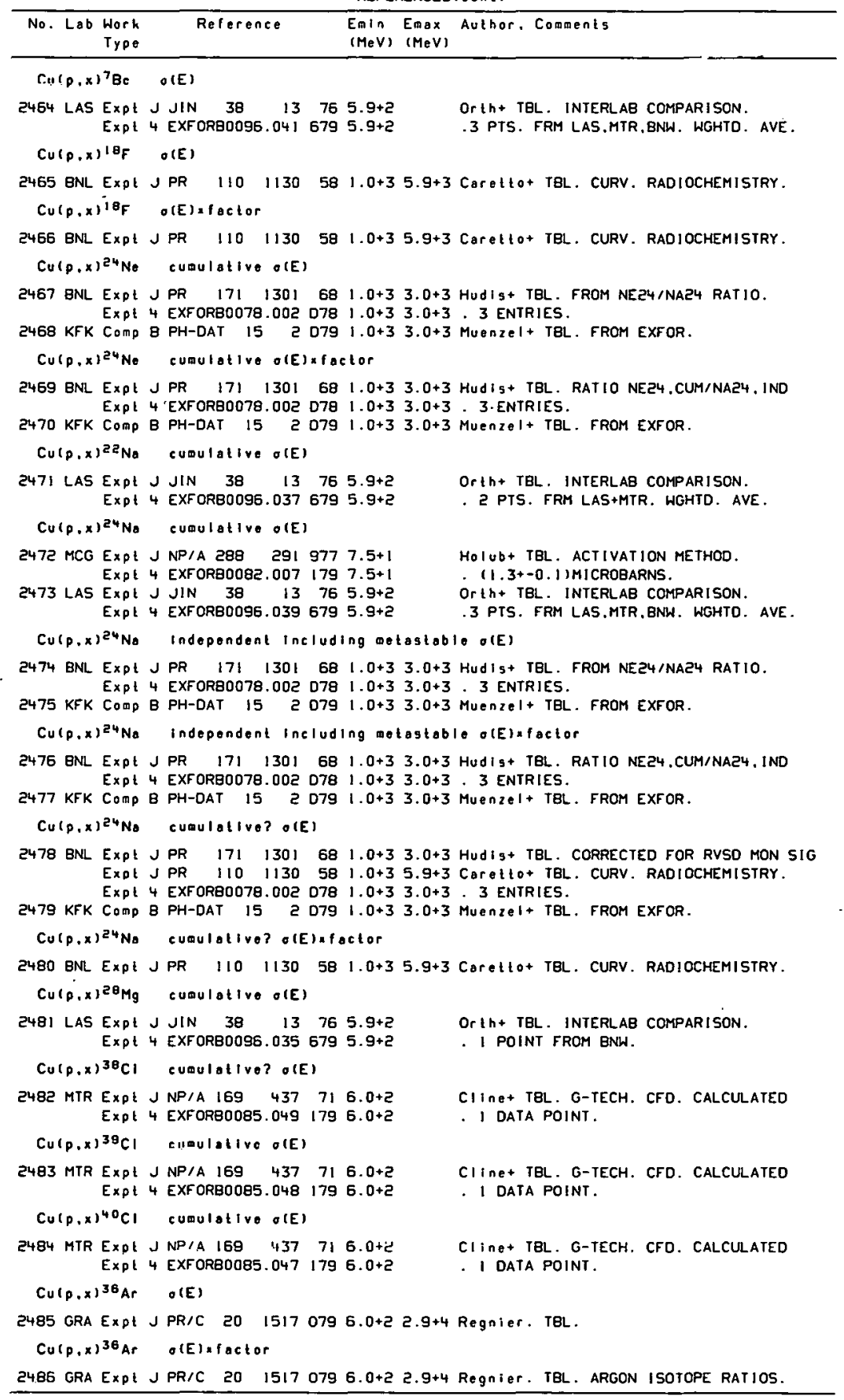


REFERENCES ( $c$ on ()

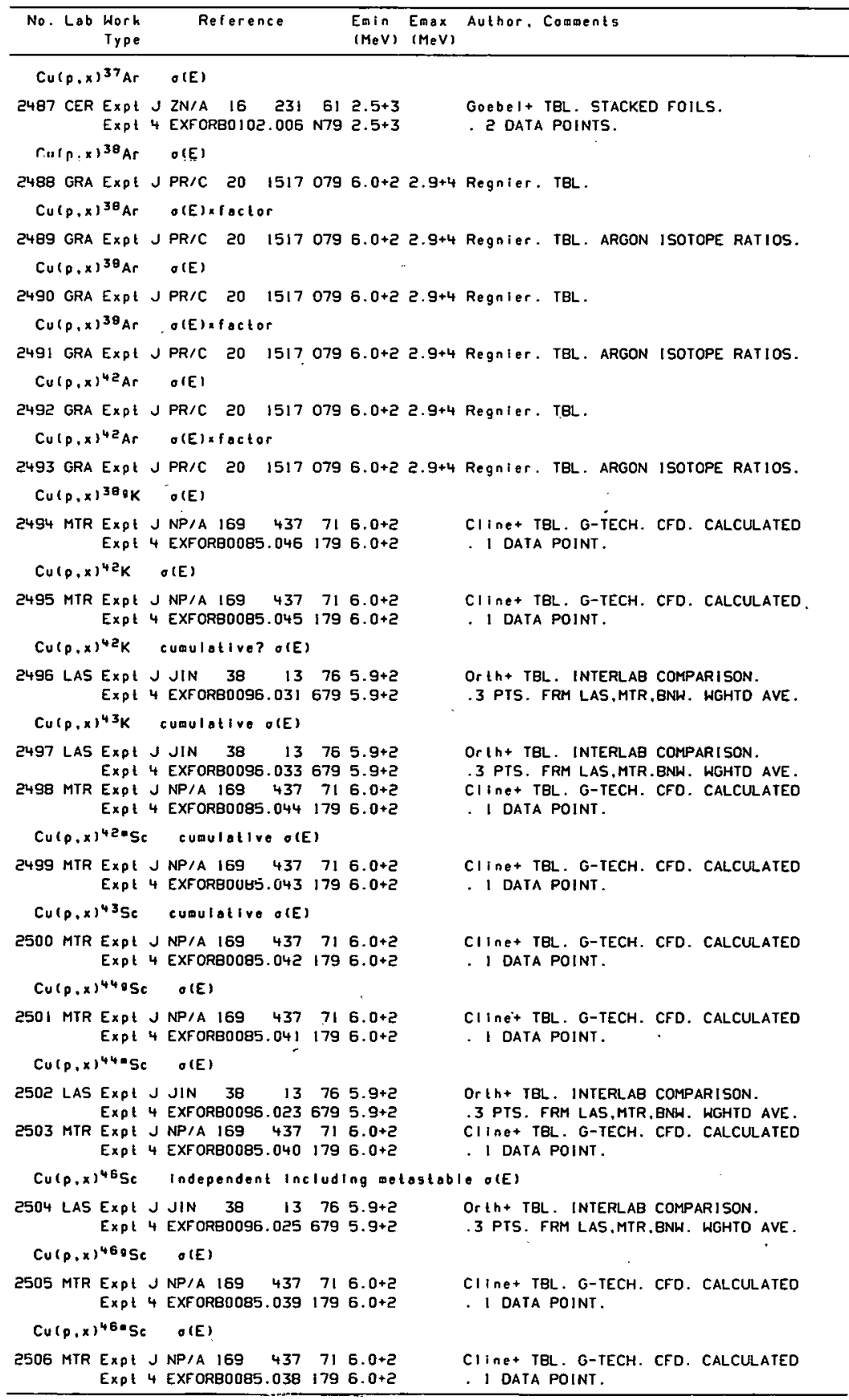


REFERENCES ( c on t)

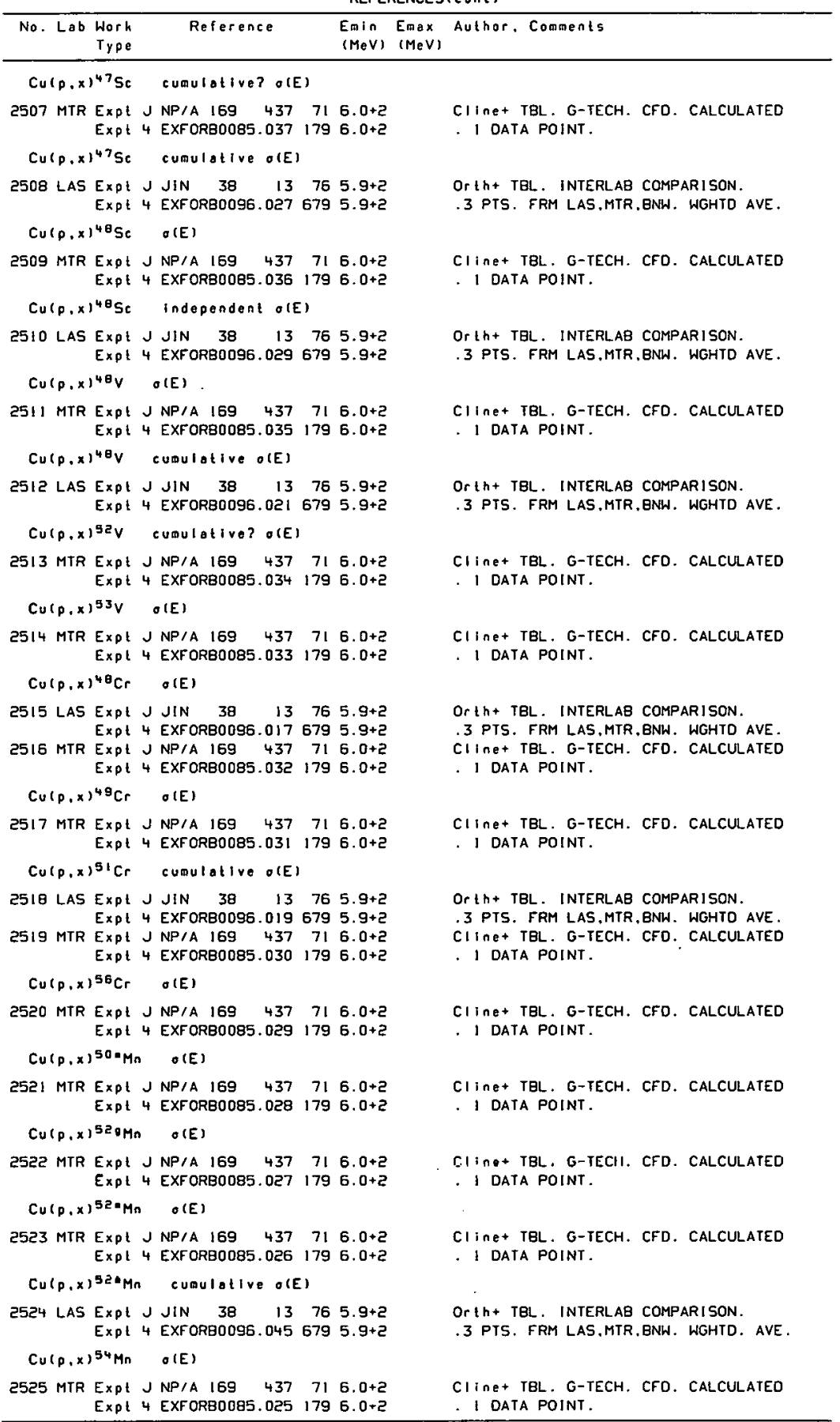




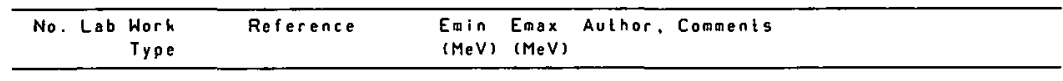

Cu $(p, x)^{54} \mathrm{Mn}$ independent o(E)

2526 LAS Expl J JIN $38 \quad 13765.9+2$ Expl 4 EXFORB0096.015 $6795.9+2$

$$
\text { Cu(p, } x, 56 H_{11} \quad u([)
$$

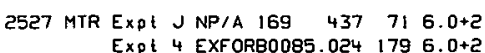

$$
\operatorname{Cu}(\rho, x)^{57} \mathrm{Mn} \text { O(E) }
$$

2528 MTR Expt J NP/A $169 \quad 437 \quad 716.0+2$ Expt 4 EXFORB0085.023 $1796.0+2$

$$
\text { Cu }(p, x)^{38} M n \text { portlal o(E) }
$$

2529 MTR Expt J NP/A $169437 \quad 716.0+2$ EXPL 4 EXFORBO085.022 $1796.0+2$

$$
\text { Cu }(p, x)^{52} F \text { O O(E) }
$$

2530 LAS EXPL J JIN $38 \quad 13 \quad 765.9+2$ Expl 4 EXFORBO096.012 $6795.9+2$

2531 MTR Expt J NP/A $169437 \quad 716.0+2$ Expt 4 EXFORB0085.021 $1796.0+2$

$$
\text { Cu }(p, x)^{53} \mathrm{Fe} \text { Including metastablo? o(E) }
$$

2532 MTR Expt J NP/A $169 \quad 437 \quad 716.0+2$ Expt 4 EXFORBO085.020 $1796.0+2$

$$
\text { Cu }(p, x)^{\text {B9Fe }} \text { o(E) }
$$

2533 LAS Expt J JIN $38 \quad 13 \quad 765.9+2$ Expt 4 EXFORE0096.013 $6795.9+2$ 2534 MTR Expt J NP/A $169 \quad 437 \quad 716.0+2$ Expt 4 EXFORE0085.019 $1796.0+2$

$$
C u(p, x)^{55} C_{0} \quad \sigma(E)
$$

2535 LAS Expt J JIN $38 \quad 13 \quad 75 \quad 5.9+2$ EXPl 4 EXFORE0096.006 $6795.9+2$ 2536 MTR EXPl J NP/A $169 \quad 437 \quad 716.0+2$ Expl 4 EXFORB0085.018 $1796.0+2$

$$
\text { Cu }(p, x)^{56} C_{0} \quad O(E)
$$

2537 MTR EXP! J NP/A $169437 \quad 716.0+2$ Expi 4 EXFORBO085.017 179 6.0+2

$$
\text { Cu(p,x)50Co cumulative o(E) }
$$

2538 KFK Comp B PH-DAT $1520795.2+15.8+1$ Muenzel+ TBL. FROM EXFOR.

2539 ORL Expt J PR 162 I $10550675.2+15.8+1$ Wi 11 ians + CURV. STACKEO FOILS. CFO THEO Exp: 4 EXFORBO073.002 $6785.2+15.8+1$. 7 DATA POINTS.

2540 LAS EXPt J JIN $38 \quad 13 \quad 765.9+2$ Expt 4 EXFORBO096.008 $6795.9+2$

$$
C_{U}(0, x)^{57} C_{0} \quad O(E)
$$

2541 KFK COMp B PH-DAT 15 2 D79 $3.6+15.8+1$ Muenzel+ TBL. FROM EXFOR.

2542 ORL Expt J PR $16210550673.6+15.8+1$ WIIIIans+ CURV. STACKEO FOILS. CFO THEO Expt 4 EXFORBO073.003 $6783.6+15.8+1$. 14 DATA POINTS.

2543 MTR Expt J NP/A $169437716.0+2$ CI 4 ine+ TBL. G-TECH. CFD CALCULATED Expt 4 EXFORBO085.016 $1796.0+2$ I OATA POINT.

$$
\text { Cu }(p, x)^{57} C_{0} \text { cumulative o(E) }
$$

2544 LAS Expt J JIN $38 \quad 13 \quad 76 \quad 5.9+2$ Expt 4 EXFORBO096.010 $6795.9+2$

$$
\text { Culp,x }{ }^{50} \mathrm{Co}_{0} \text { Including melastable ofE) }
$$

Or tht TBL. INTERLAB COMPARISON.

.3 PTS. FRM LAS.MTR, BNW + WGHTD AVE.

2545 KFK COMp B PH-DAT 15 2 D79 $2.7+15.9+1$ Muenzel+ TBL. FROM EXFOR.

2546 ORL Expl J PR $16210550672.7+15.9+1$ WIIIiams+ CURV. 5TACKEO FOILS. CFO THEO Expt 4 EXFOR日0073.009 $6782.7+15.9+1$, 17 DATA POINTS.

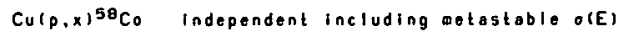


REFERENCES (cont)

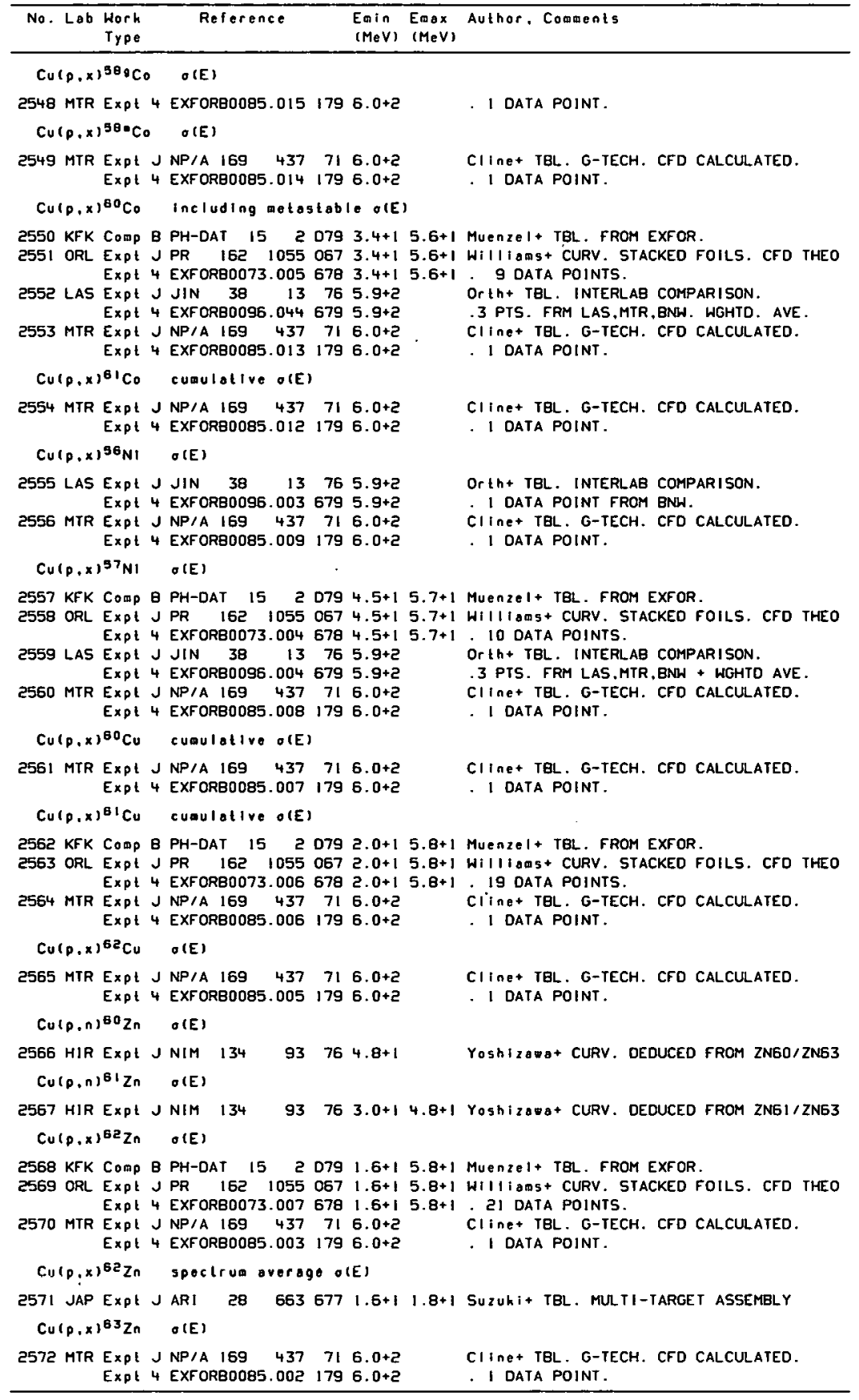


REFERENCES (cont)

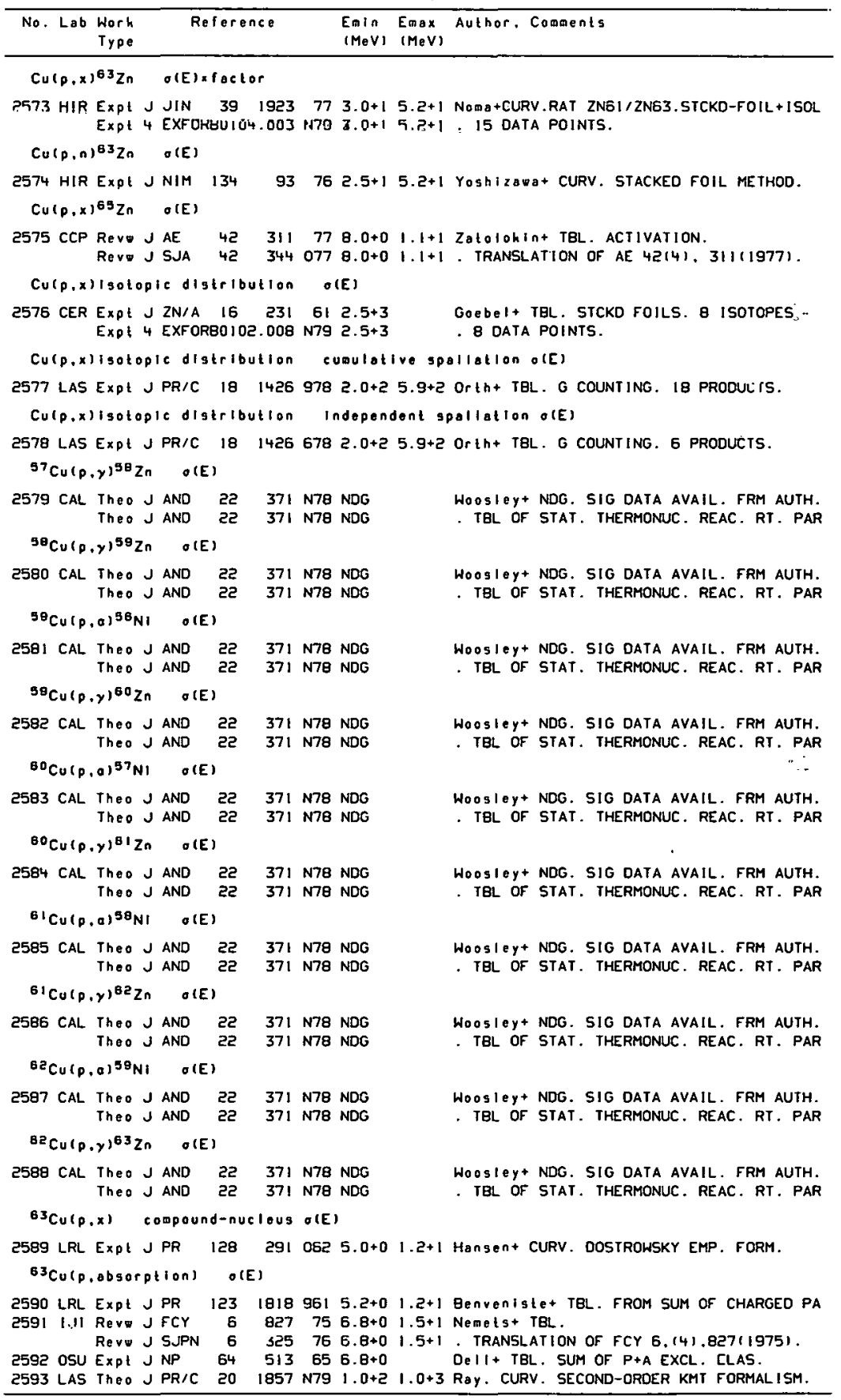


REFERENCES ( con t )

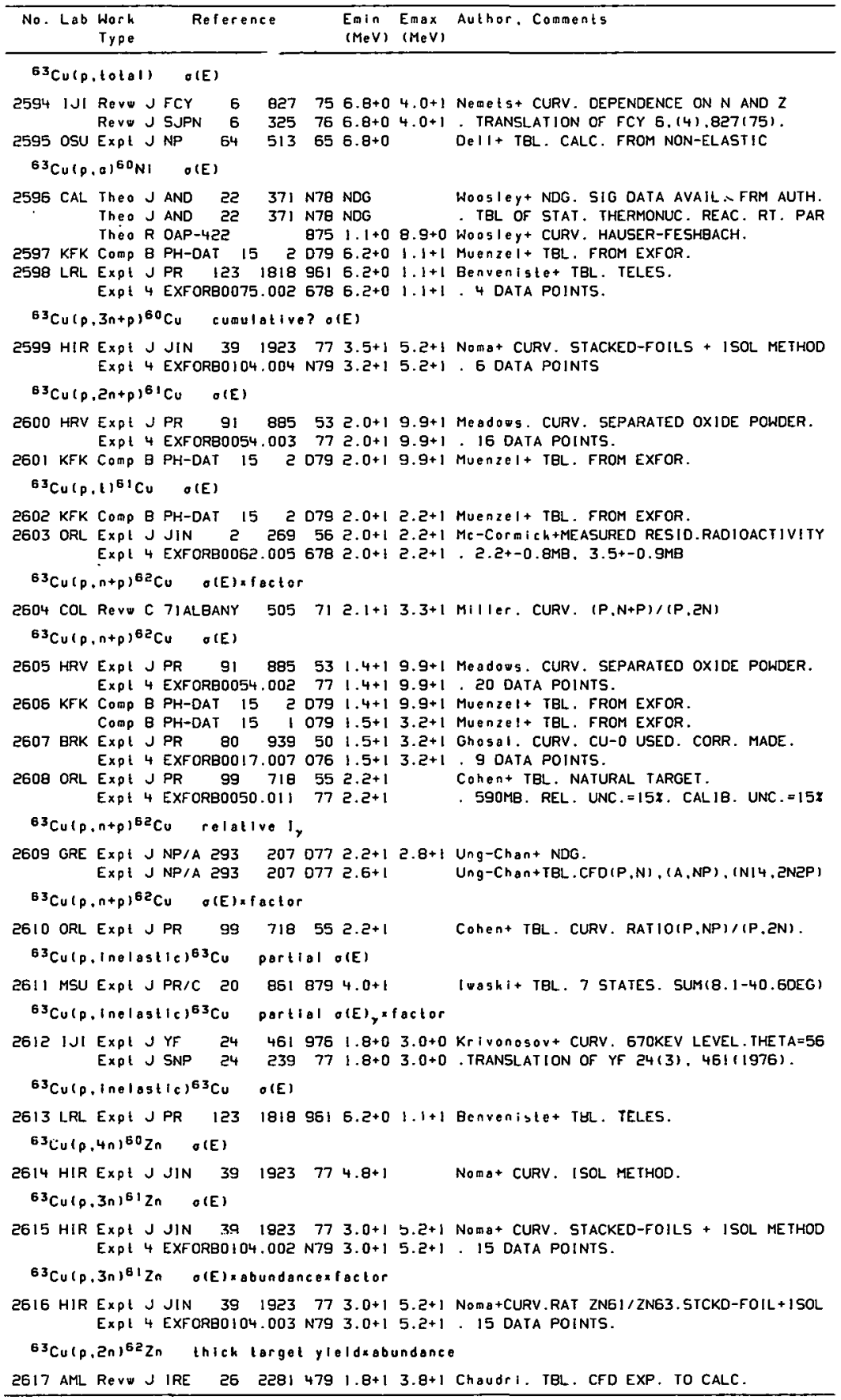


REFERENCES(cont)

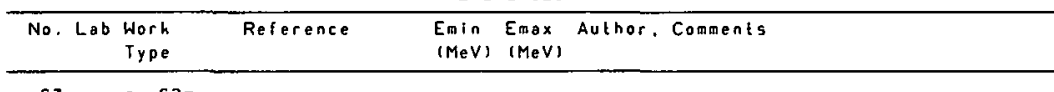

${ }^{63} \mathrm{Cu}\left(p, \mathrm{2n}^{\mathrm{s}}\right)^{62} \mathrm{Zn} \quad$ o(E)

2618 BRK Expt I PR $80 \quad 939501.5+13.2+1$ Ghosal. CURV. CU-0 USEO. CORR. MADE. EXPt 4 EXFORBO017.005 $076 \quad 1.5+1 \quad 3.2+1$. 9 DATA POINTS.

2619 KFK Comp B PH-DAT $1520791.6+14.0+2$ Muenzel+ TBL. FROM EXFOR. Comp B PH-DAT $15,0791.5+11.2+4$ Muenzel+ TBL. FROM EXFOR.

2620 BNL Expl J ARI $23342721.6+13.3+1$ Greene+ TBL. CURV. RESIDUAL ACTIVITY. EXPt 4 EXFORB0074.002 $6781.6+13.3+1$, 17 DATA POINTS.

262) HRV Expt J PR 91 $88553 \quad 1.7+19.9+1$ Meadows. CURV. SEPARATED OXIDE POWDER. Expt 4 EXFORB0054.005 $77 \quad 1.7+19.9+1$ - 18 DATA POINTS.

2622 ORL Expt J PR $99719552.2+1$ CohEn+ TBL. NATURAL TARGET. EXpt 4 EXFORB0050.010 $772.2+1 \quad$. 100MB. REL. UNC. $=15 \pi$. CAL IB. UNC. $=15 \pi$ 2623 CAR Expt J JIN 31 19I5 $694.0+2$ Reuland+ ENRICHED OXIOE-BORIC ACID MATR Expt 4 EXFORB0071.005 $6784.0+2$. 0.8MB+-19 PER CENT.

2624 ANL Expt J PR/C $714104731.5+31.2+4$ Steinberg+ TBL. CURV. RESIDUAL ACTIVITY Exp: 4 EXFORBO031.003 $77 \quad 1.5+3 \quad 1.2+4$. 7 DATA POINTS.

${ }^{63} \mathrm{Cu}\left(p, \mathrm{Zn}^{6}\right)^{62} \mathrm{Zn} \quad$ o(E) $\times$ factor

2625 COL Reve C 7IALBANY $505712.1+13.3+1$ Miller. CURV. (P.N+P)/(P. $2 N)$

2626 ORL Expt JPR $99718552.2+1 \quad$ Cohen+ TQL. CURV. RATIO(P,NP)/(P, $2 N)$.

2627 CAR Expt J JIN $31 \quad 1915694.0+2 \quad$ Reuland+ (P, 2N $) /(P, N)=0.89+-I 6 P E R$ CENT

${ }^{63} \mathrm{Cu}(p, n)^{63} \mathrm{Zn} \quad \mathrm{O}(\mathrm{E})$

2628 BNL ReVW C 63HOUSTO

2629 CAL Theo R OAP-422

363 NDG Goldberg. NDG. EXPI P ORNL-2501 2958 TR $5.8+0$ Johnson+ EXPT. DETAILS.

Expt 4 EXFORBD068.009 $6784.2+05.8+0$. 29 DATA POINTS.

2631 BRK Expt J PR BO $939503.9+0$ 2.2+1 GhOSAI. CURV. CU-0 USED. CORR. MADE. Expt 4 EXFORB0017.005 $0763.9+0$ 2.2+1. 18 DATA POINTS.

2632 BNL Expt J PR/C $918195744.0+02.5+1$ Colle+ TBL. CURV. ACTIVATION METHOD. Expt 4 EXFORB0057.002 $774.0+02.5+1$. 49 DATA POINTS.

2633 ETH Expt J HPA $24 \quad 3 \quad 514.2+0$ 6.3+0 Blaser+ TBL. NATURAL FOIL. Expt 4 EXFORBO0 $48.002774 .2+06.3+0$. 9 DATA POINTS.

2634 KFK Comp B PH-DAT 152 D79 $4.2+09.9+1$ Muenzel+ TBL. FROM EXFOR. Comp B PH-DAT 15,1 079 $3.9+01.2+4$ Muenzel+ TBL. FROM EXFOR.

2635 ROC Expt J PR I 25 291 62 4.3+0 6.6+0 Tahetani+ NATURAL AND ENRICHED FOILS. EXpl 4 EXFORB0051. $774.2+06.6+0$. 10 POINTS.CPX DATA CORRECTED BY COMP

2636 ANL Expt J PR 128 280 $0624.5+0$ i.0+j Wingt IBL. CURV. RESID. RADIOACTIVITY. Expt R TID-12696 6I 9.9+0 $\quad$. SUPERSEDED BY PR 128.280(1962) EXP! 4 EXFORBO065.004 678 4.5+0 1.0+1. 22 DATA POINTS. EXP1 4 EXFORB0063.004 678 NDG . SUPERSEDED BY 00065.004

2637 CLA Expt $j$ PR $1092003584.9+01.0+1$ Howe. CURV. NATURAL FOILS. Expt 4 EXFORBO060.002 $774.5 * 01.1+1.15$ DATA POINTS.

2638 HRV Expl J PR 91 $885535.0+09.9+1$ Meadows. CURV. SEPARATEO OXIDE POWDER. Expt 4 EXFORB0054.004 $775.0+09.9+1$. 16 DATA POINTS.

2639 LRL Expt J PR 128 291 062 6.0+0 $1.1+1$ Hansen+ CURV. LONG COUNTER. CFD. THEORY Expt 4 EXFORB0066.004 $6786.0+01.1+1$. 6 DATA POINTS.

2640 OSU Expt J NP $64 \quad 513656.8+0 \quad$ De II+ IBL. CURV. STACKED FOILS. Expt J PR $13015225636.8+0$ Humes+ MEASURED RESIOUAL RADIATION EXP1 4 EXFORBO061.005 678 6.8+0 . 239+-13MB. FROM PR :30. 1522(1963) Exp! 4 EXFORB0064.005 678 6.8+0 . 239+-13MB. FROM NP 64, 513119651.

2641 MUN Exp! J NP/A 198 $625708.7+0$ 1.6+1 Hille+ TBL. CURV. STCKD FOILS. Expt 4 EXFORB0058.002 $778.7+01.6+1$. B DATA POINTS.

264? BIR Expl J PPS $80738629.3+0$ Exp! 4 EXFORBO070.002 $6789.3+0$

2643 HAR Expl R TID-12696 $619.9+0$

Chachetl + NATURAL TARGET. $480+-20 M B$.

Jones+SPSSO BY PR 128, 280 EXCEPT LONG C EXpl 4 EXFORBD063.002 $6789.9+0$. 325+-39MB. Expl 4 EXFORB0063.004 678 NDG

2644 CAR EXPI J JIN $31 \quad 1915 \quad 694.0+2$ Expl 4 EXFORBO071.004 $6784.0+2$

SUPERSEDED BY BO065.004

Reuland+ ENRICHED OXIOE-BORIC ACID MATR $0.9 M B+-19 P E R$ CENT.

2645 ANL Expl J PR/C $714104731.5+3 \quad 1.2+4$ Steinberg+ FBL. CURV. RESIDUAL ACTIVITY Exp: 4 EXFORBO031.002 $771.5+31.2+4$, 7 DATA POINTS.

${ }^{63} \mathrm{Cu}(\mathrm{p}, \mathrm{n})^{63} \mathrm{Zn}$ o(E) $\times$ factor

2546 CAR Expl J JIN $31 \quad 1915694.0+2 \quad$ Reuland+ $(P .2 N) /(P . N)=0.89+-16 P E R$ CENT

${ }^{63}$ Cu(p.y) $)^{64} \mathrm{Zn}$ product yield

2647 LND Expl P KDK-23 $174782.1+03.1+0$ Erlandssont NDG. AVE. RES. SPECT. METH

${ }^{63} C_{u}(p, y)^{64} \mathrm{Zn}$ cumulative? relative product ylelo

$2648 \mathrm{KFI}$ Expl J JP/G $2365753.1+0 \quad 3.3+0$ Fodor+ CURV. ZKEV STEPS. GE ILII 


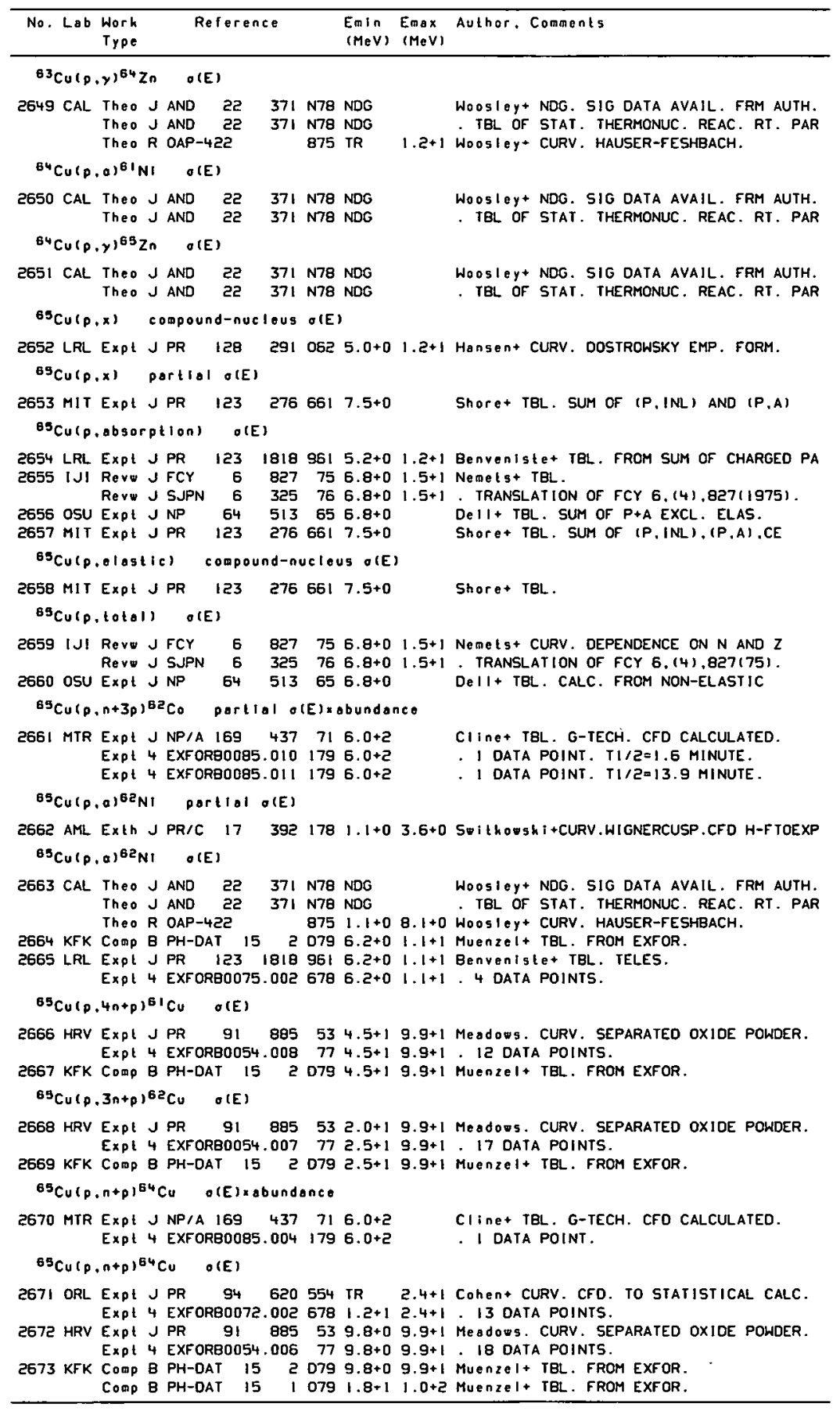


REFERENCES (cont)

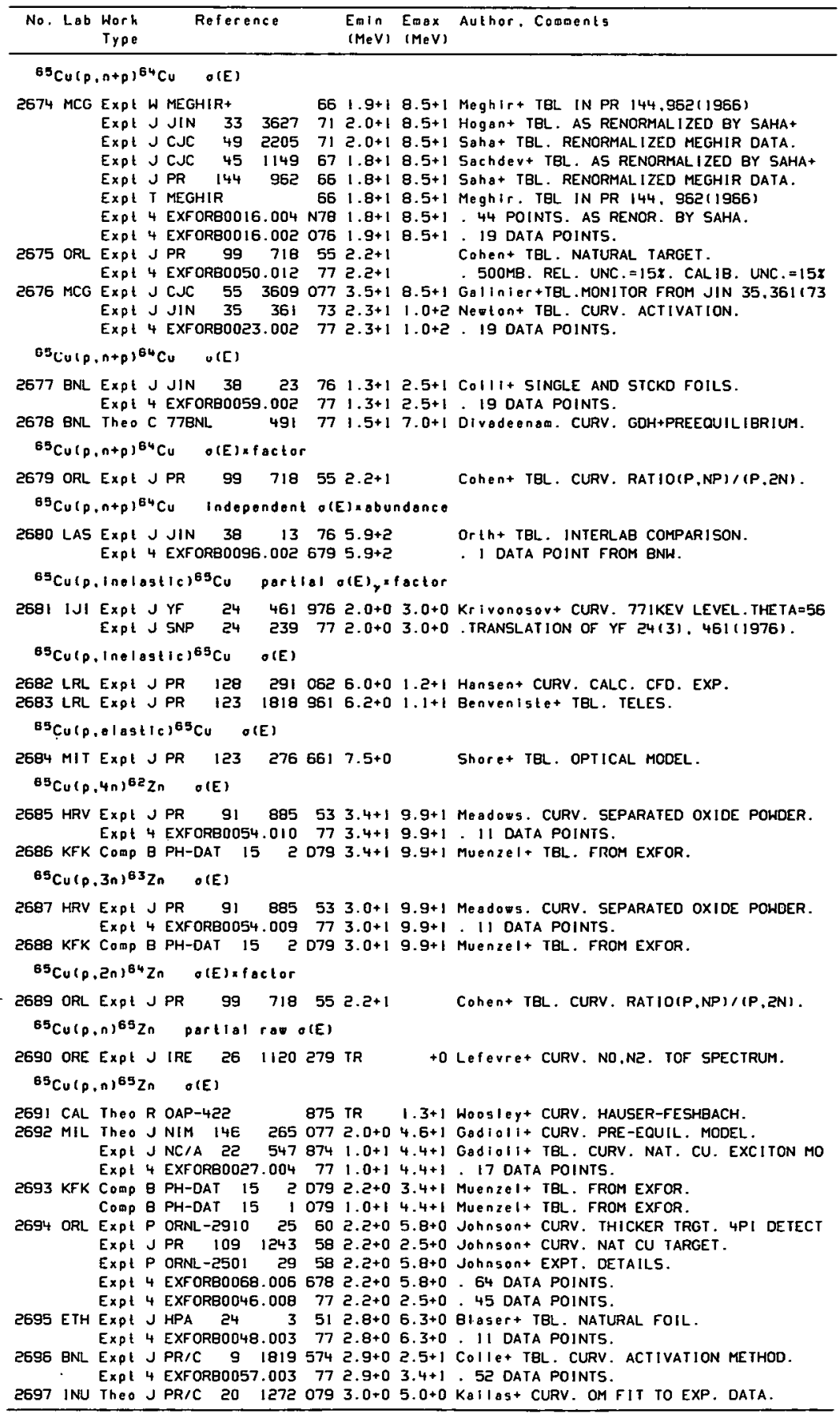


REFERENCES(cont)

\begin{tabular}{|c|c|c|c|c|c|c|}
\hline No. & $L a b$ & $\begin{array}{l}\text { Work } \\
\text { Type }\end{array}$ & Reference & $\begin{array}{l}\text { Emin } \\
\text { (MeV) }\end{array}$ & $\begin{array}{l}\text { Emax } \\
(\operatorname{MeV})\end{array}$ & Author, Comments \\
\hline
\end{tabular}

${ }^{65} \mathrm{Cu}(p, n)^{65} \mathrm{Zn} \quad \mathrm{O}(\mathrm{E})$ Expt R TID-12696 $619.9+0 \quad$ SUPERSEDED BY PR I28.280(1962)

EXPt 4 EXFORBO065.005 $6784.5+01.0+1$. 42 DATA POINTS.

Expt 4 EXFORB0063.005 678 NDG . SUPERSEDED BY B0065.005

$2699 \mathrm{BNL}$ Theo C 77BNL $49 \mathrm{I} 775.0+07.0+1$ Divadeenam. CURV. GDH+PREEQUILIBRIUM.

2700 LRL Expt J PR 128 291 062 5.0+0 1.1+1 Hansen+ CURV. LONG COUNTER. CFD. THEORY Expt J PRL $6 \quad 13161 \quad 1.0+1$ Albert+ PREL IM!NARY VALUES.

EXP! 4 EXFORB0066.005 $6785.0+01.1+1.7$ DATA POINTS.

270 ! CLA Expt J PR $1092083586.4+01.1+1$ Howe. CURV. NATURAL FOILS.

EXpt 4 EXFORBO060.005 $776.4+01.1+1$. 5 DATA POINTS.

2702 OSU EXPt J NP/A $64 \quad 513656.8+0 \quad$ Del + + TBL. CURV. STACKED FOILS.

Expt J PR $13015225636.8+0 \quad$ Humes+ SPSSO BY NP 64, 513119551

EXPt 4 EXFORBO061.006 $5786.8+0$. SUPERSEDED BY B0064.006

EXPt 4 EXFORB0064.006 $6786.8+0 \quad$. $1499+-25) M B$.

2703 MIT Expt J PR 123 275 $6617.5+0$ Shore+ MEAS. RESIDUAL ACTIVITY.

EXpt 4 EXFORB0067.003 $6787.5+0$. 537+-21MB.

2704 HAR Expt J JIN $321419708.3+0$ Jenkinst 624MB.

2705 B!R Expl J PPS $80738629.3+0$ Chachett+ NATURAL TARGET.

Expt 4 EXFORBO070.003 $6789.3+0$. 895+-35M8.

2706 HAR Expt R TID-12696 EI $9.9+0$

Jones+ LONG COUNTER.

Expt 4 EXFORB0063.003 $67899+0 \quad 555+-66 M B$

EXPt 4 EXFORBO063.005 678 NDG

SUPERSEDED BY B0065.005.

2707 BNL EXPt J ARI $23 \quad 342721.6+13.3+1$ Greene+ TBL. CURV. RESIDUAL ACTIVITY. Exp! 4 EXFORB0074.003 678 1.6+1 3.3+1. 16 DATA POINTS.

2708 PAR Expl J JPR $231000624.6+11.4+2$ Albouy + CURV. CFD CASCADE MODEL. Expl 4 EXFORBOI06.004 N79 4.6+1 1.4+2. 9 DATA POINTS.

${ }^{65} \mathrm{Cu}(p, n)^{65} \mathrm{Zn}$ product yieldafoctor

2709 WMU Exth J PR/C 151592477 2.2+0 Bernstein.NDG. CAL. GEOM. CORR TO E(TH)

${ }^{65} \mathrm{Cu}(p, n)^{69} Z_{n} \quad \sigma(E) \times$ factor

2710 ORL Expl JPR I62 $10550671.4+12.2+1$ Willieng+ CURV. STACKED FOILS. CFD THEO Expt 4 EXFORB0073.008 $6781.4+12.2+1$. 8 DATA POINTS.

${ }^{65} \mathrm{Cu}(p, n)^{65} \mathrm{Zn}$ avoráge o(E)

2711 ORL Expt J PR/B 1361719 D64 2.0+0 2.2+0 Johnson+ CURV. FROM 1952+1958 MEAS.

${ }^{65} \mathrm{Cu}(p, y)^{66} \mathrm{Zn} \quad$ o(E)

2712 CAL TheO J AND $22 \quad 371$ N78 NDG WOOSley+ NDG. SIG DATA AVAIL. FRM AUTH. Theo $J$ AND 22371 N78 NDG . TBL OF STAT. THERMONUC. REAC. RT. PAR The $R$ OAP-422 875 TR $1.3+1$ WoOSIEY + CURV. HAUSER-FESHBACH.

2713 AML Exth J PR/C $173921781.1+03.6+0$ Swilkowski+CURV. WIGNERCUSP.CF H-F TOEXP

${ }^{65} \mathrm{Cu}(p, y)^{66} \mathrm{Zn}$ product y101d

2714 LND Exp! P KDK-23 $174782.2+0$ 2.8+0 Erlandsson+ NDG. AVE. RES. SPECT, METH

${ }^{66} \mathrm{Cu}(p, a){ }^{63} \mathrm{Ni} \quad$ o(E) $\begin{array}{rlll}2715 \text { CAL TheO J AND } & 22 & 371 & \text { N78 NDG } \\ \text { TheO J AND } & 22 & 371 & \text { N78 NDG }\end{array}$

${ }^{66} C_{U}(p, n)^{66} Z n$ O(E)

2716 CAL Theo J AND $22 \quad 371$ N78 NDG Theo J AND $22 \quad 371$ N76 NDG

${ }^{66} \mathrm{Cu}(p, y)^{67} \mathrm{Zn}$ o(E)

2717 CAL Theo J AND 2237 ! N78 NDG Theo J AND $22 \quad 371$ N78 NDG

${ }^{67} \mathrm{CU}_{(0.0)^{64} \mathrm{NI}} \mathrm{O(E)}$

$2718 \mathrm{CAL}$ Theo J AND $22 \quad 371$ N78 NDG Theo J AND $22 \quad 371$ NTB NDG

${ }^{67} C_{U}(D, y)^{68} \mathrm{Zn}$ a(E)

$2719 \mathrm{CAL}$ Theo I AND 22 371 N78 NDG Theo $J$ AND 22 37! N78 NDG

${ }^{68} C_{U}(p, a)^{65} \mathrm{~N}$ ( $(E)$

2720 CAL Thoo J AND 22 371 N78 NDG Theo J AND 22 371 N79 NOG

Woosley+ NDG. SIG DATA AVAIL. FRM AUTH. . TBL OF STAT. THERMONUC. REAC. RT. PAR

WoOsley+ NDG. SIG DATA AVAIL. FRM AUTH. - tBl OF STAT. THERMONUC. REAC. RT. PAR

Woosley + NDG. SIG DATA AVAIL. FRM AUTH. - TBL OF STAT. THERMONUC. REAC. RT. PAR

Woosley+ NDG. 5IG DATA AVAIL. FRM AUTH. . TBL OF STAT. THERMONUC. REAC. RI. PAR

Woos ley + NDG. SIG DATA AVAIL. FRM AUTH. - TBL OF STAT. THERMONUC. REAC. RT, PAR

Wooslèy + NDG. SIG DATA AVAIL. FRM AUTH. - TBL OF STAT. THERMONUC. REAC. RT. PAR 


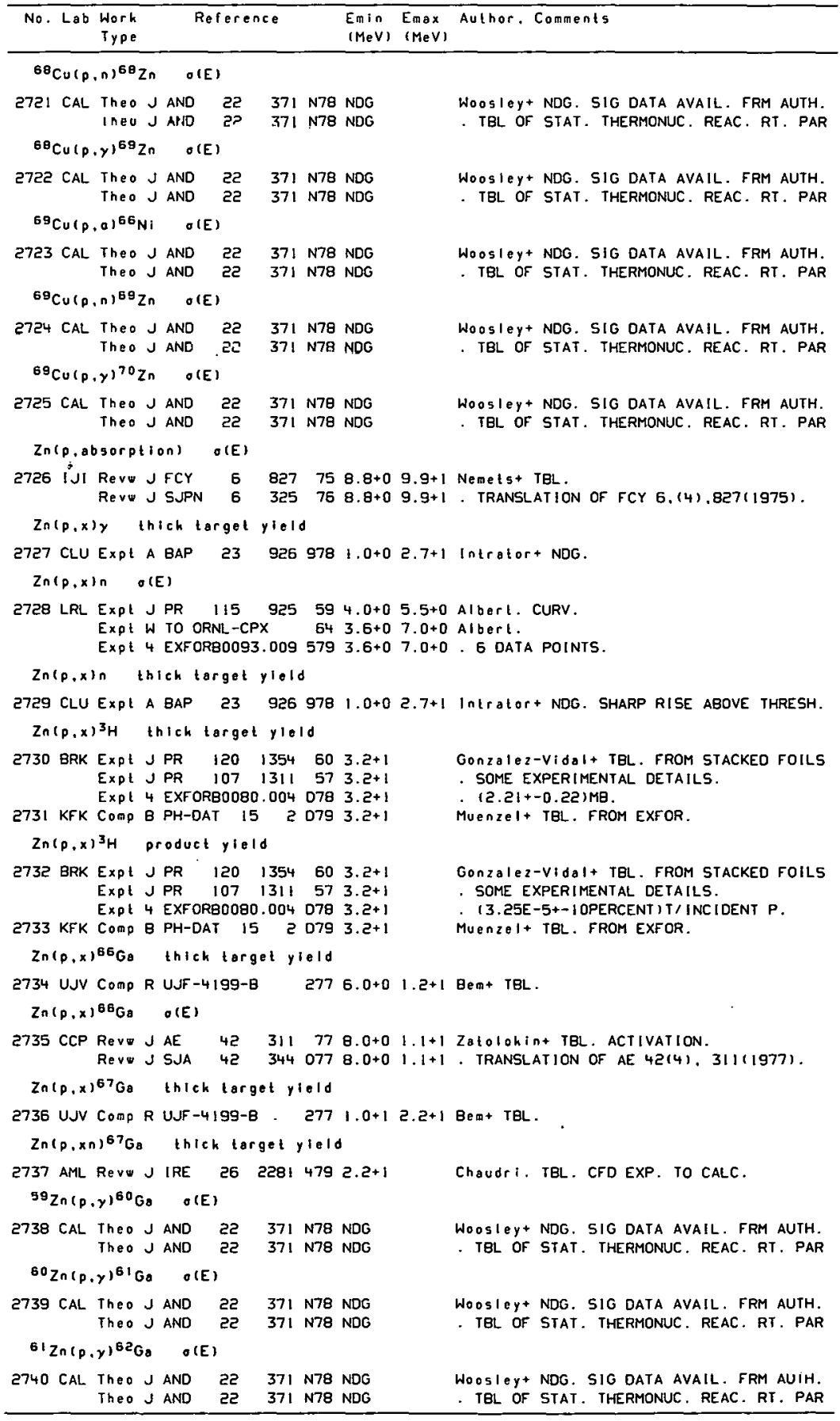




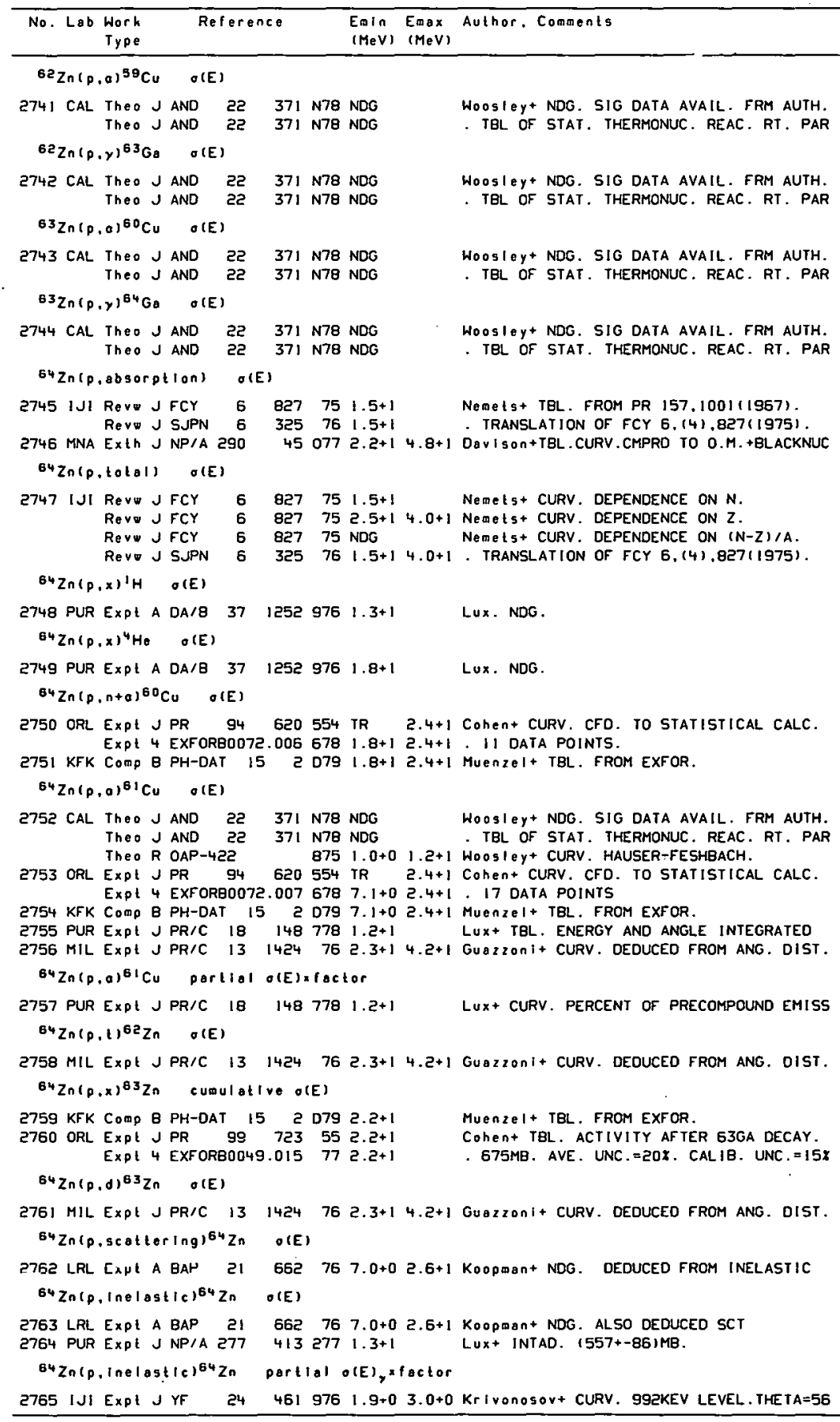




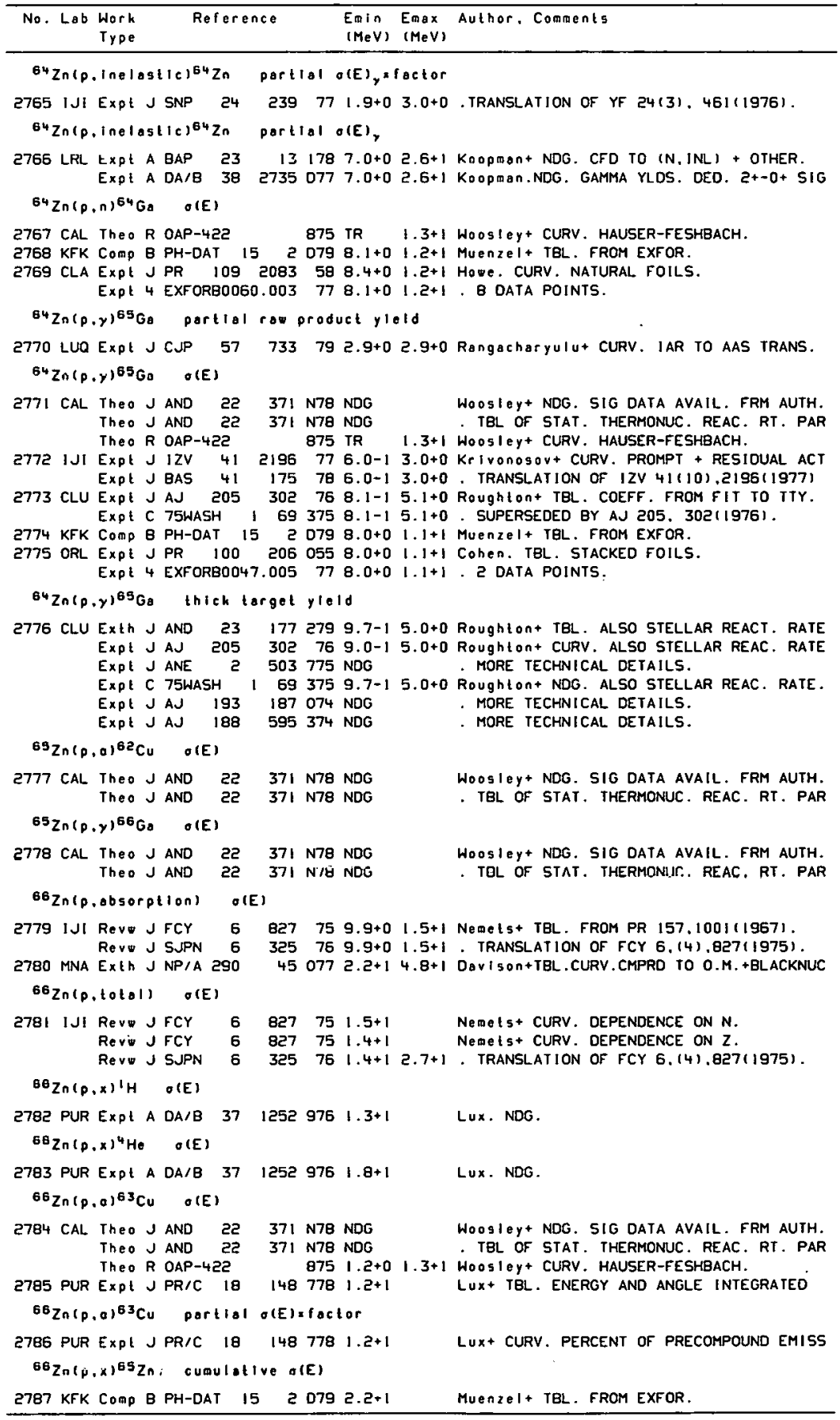


REFERENCES (cont)

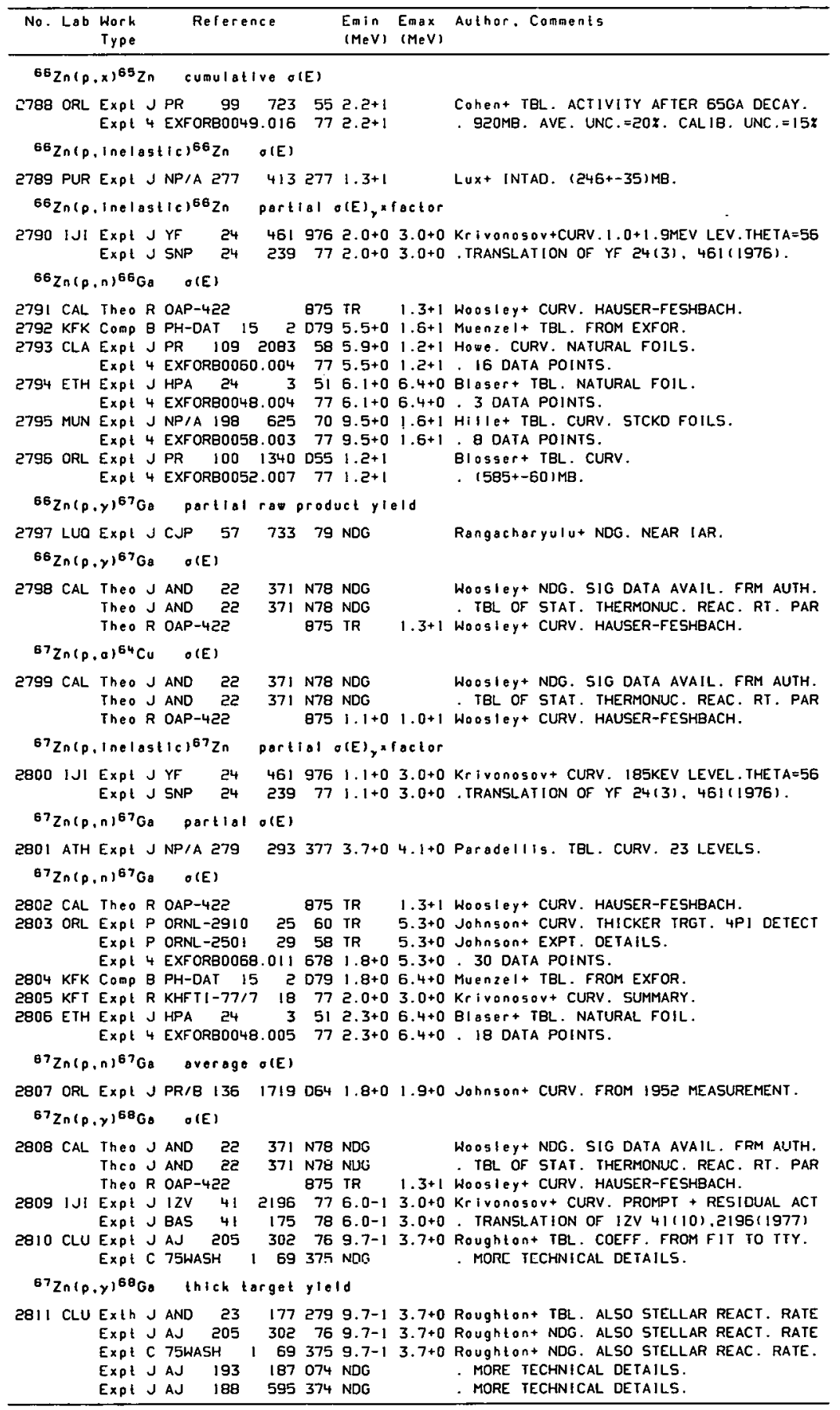




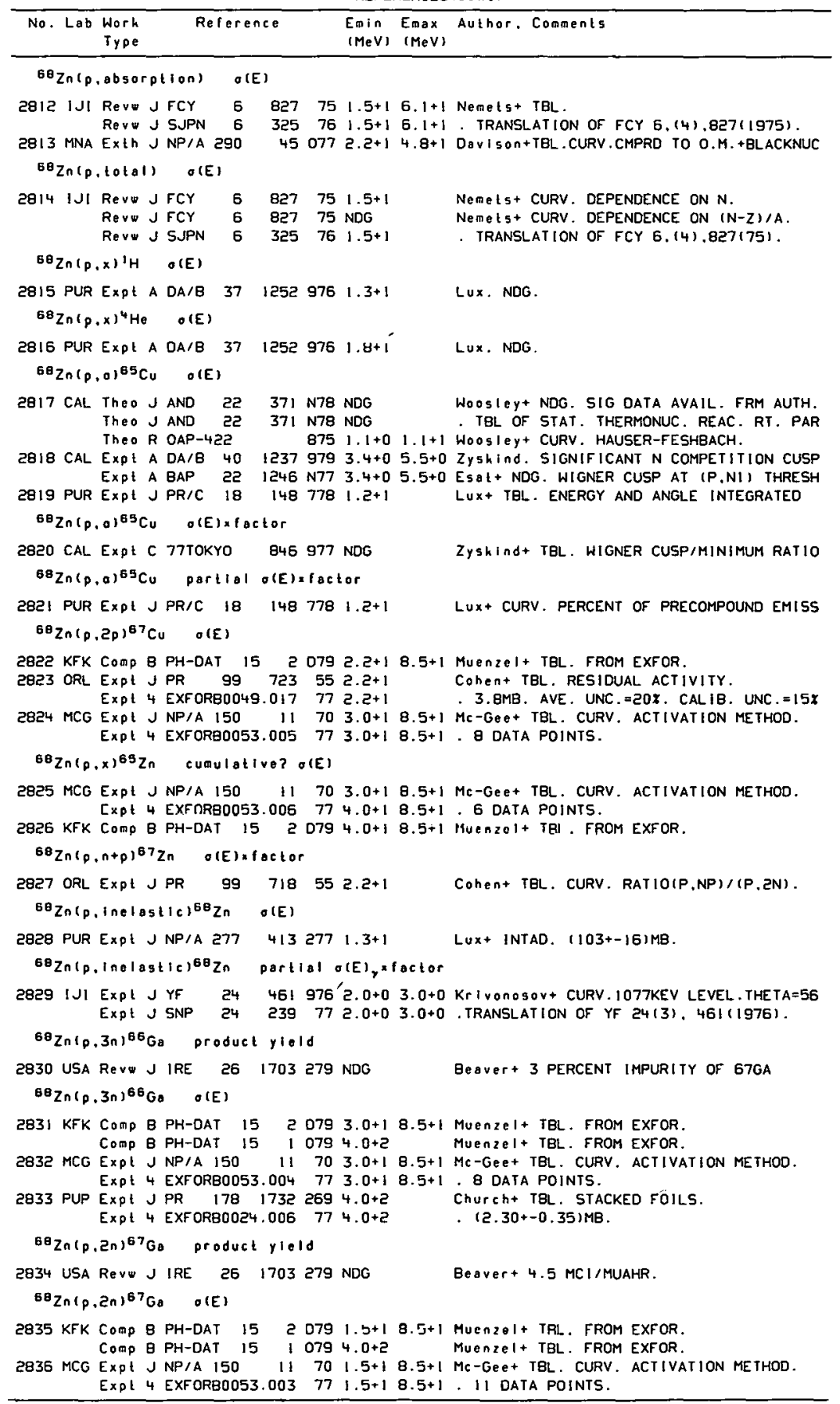




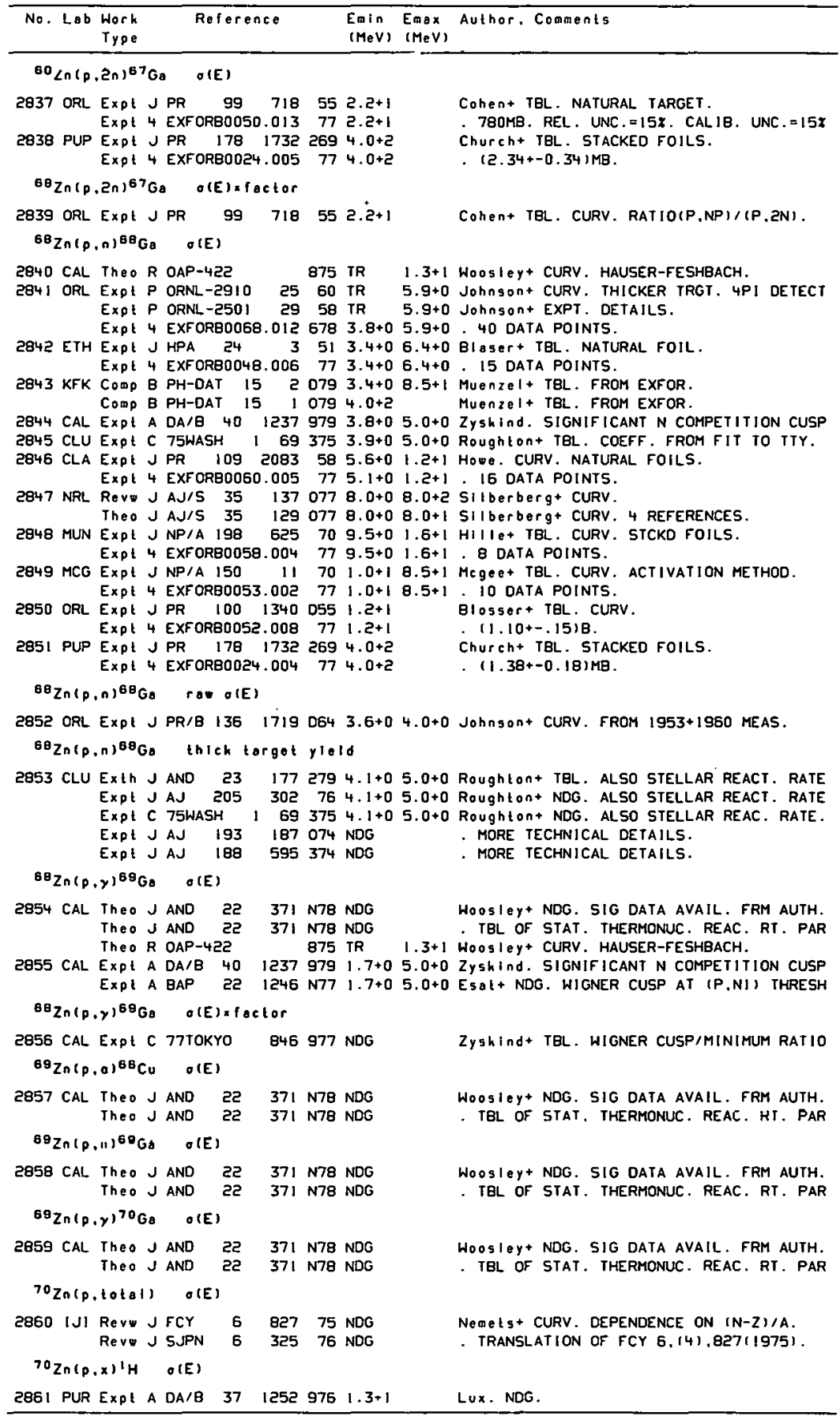




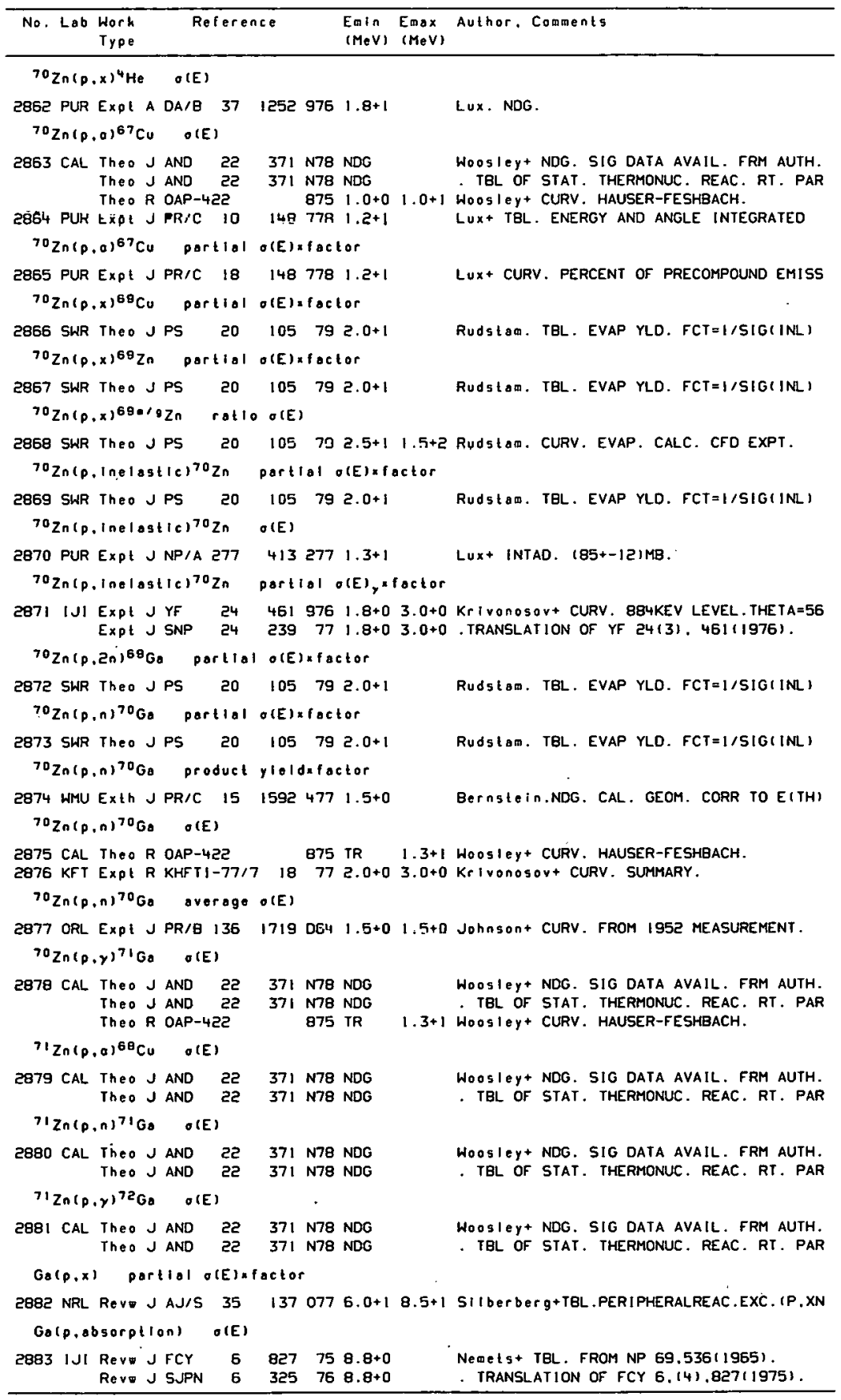


REFERENCES ( cont)

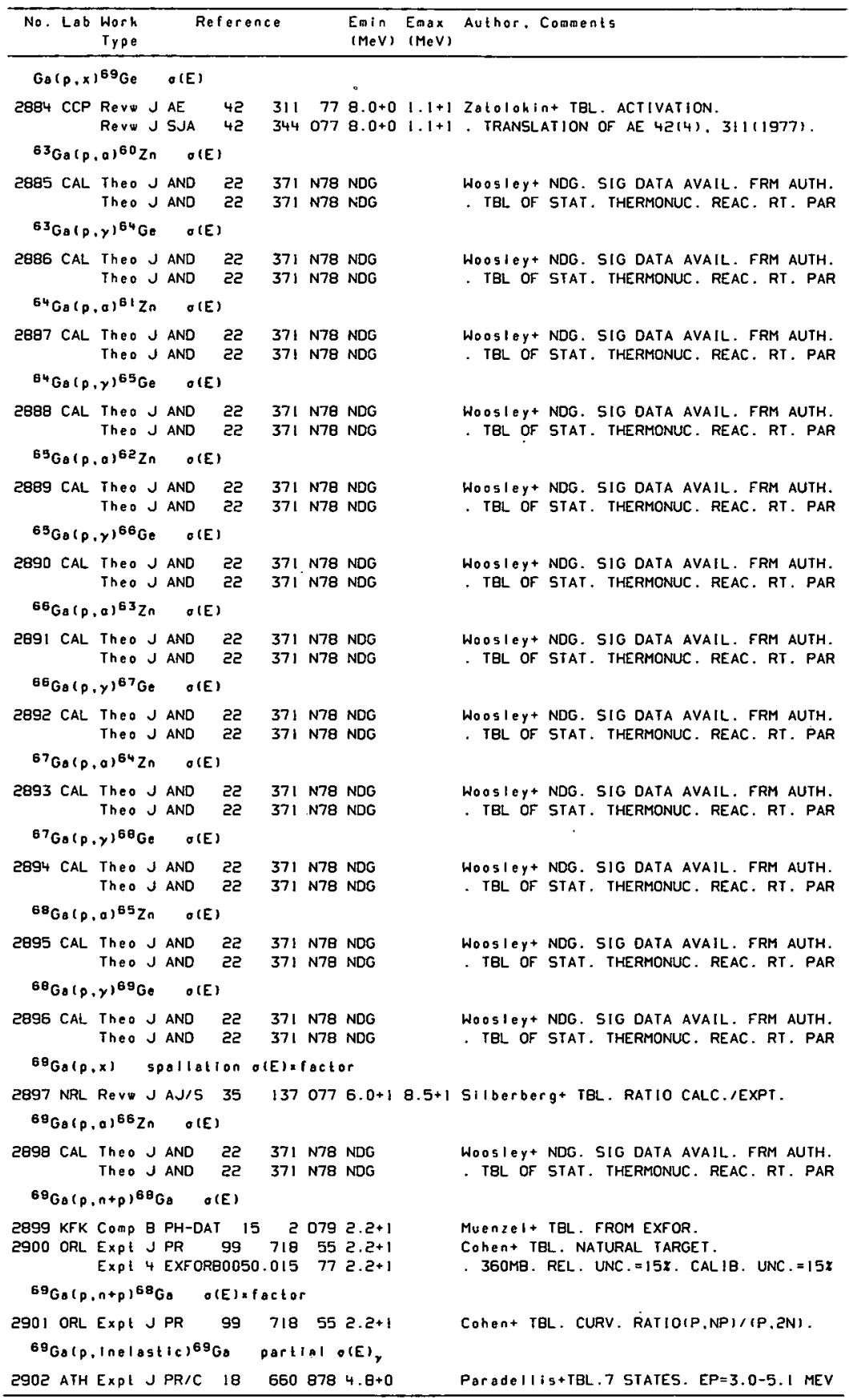




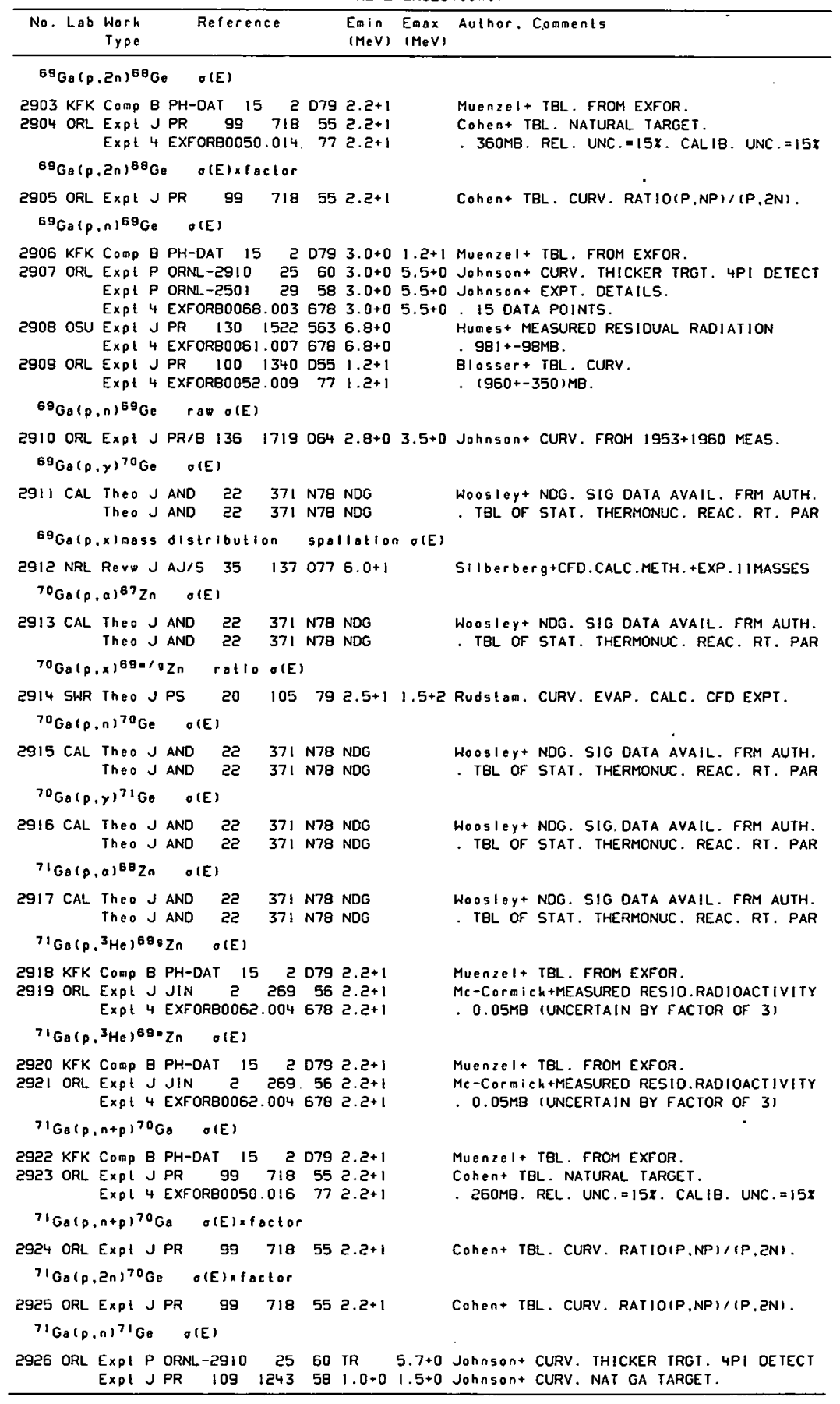




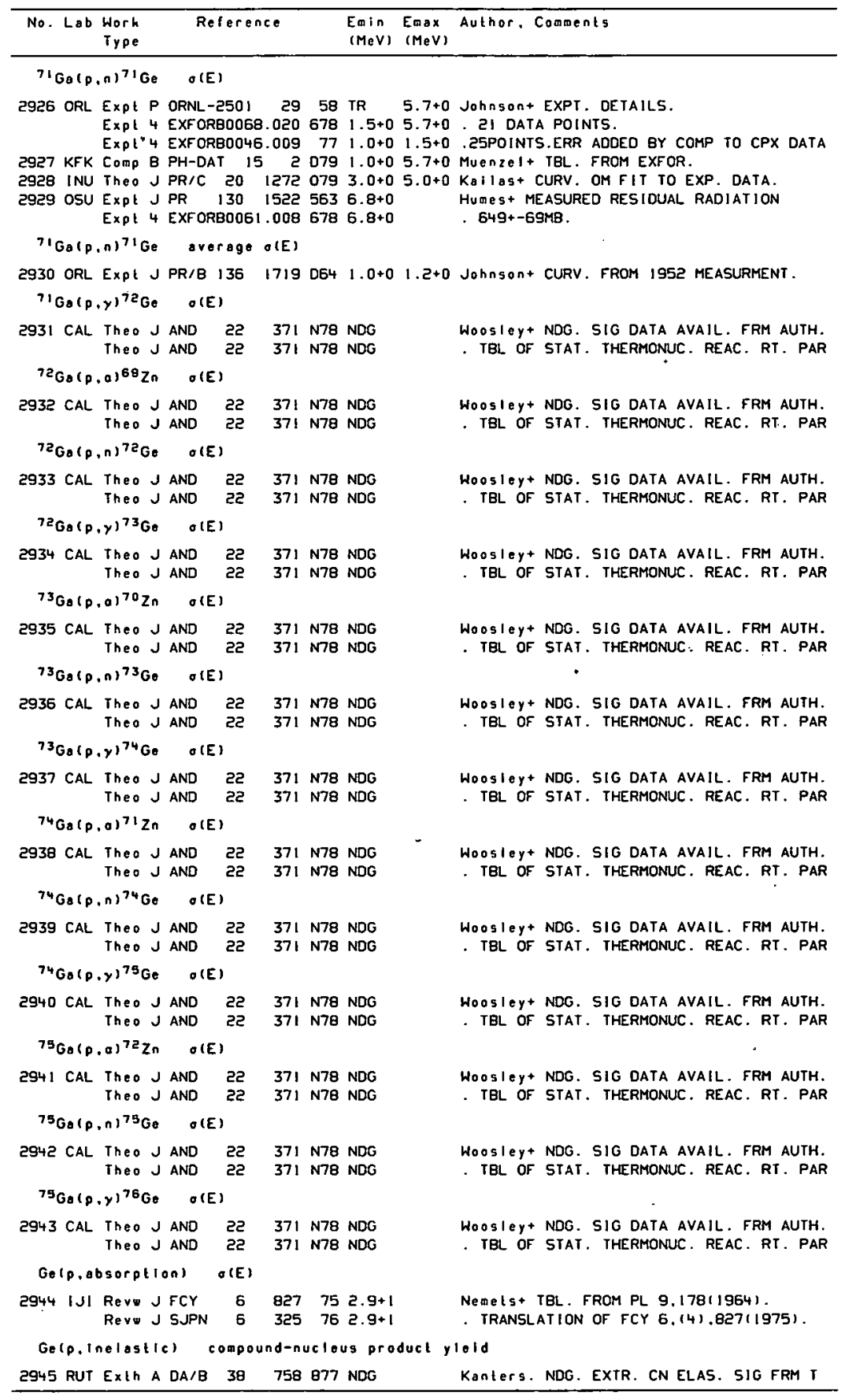


REFERENCES(con)

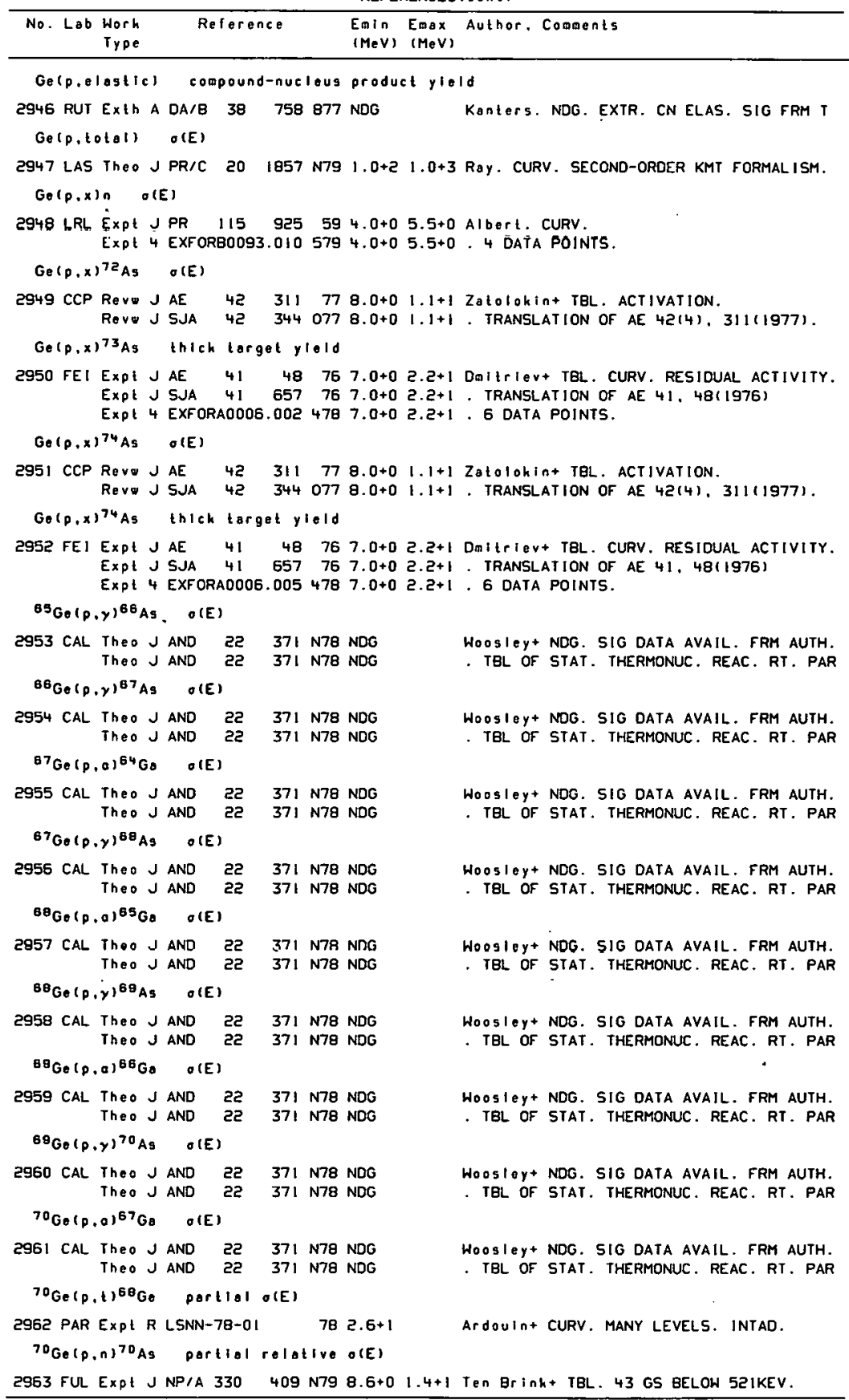


REFERENCES (cont)

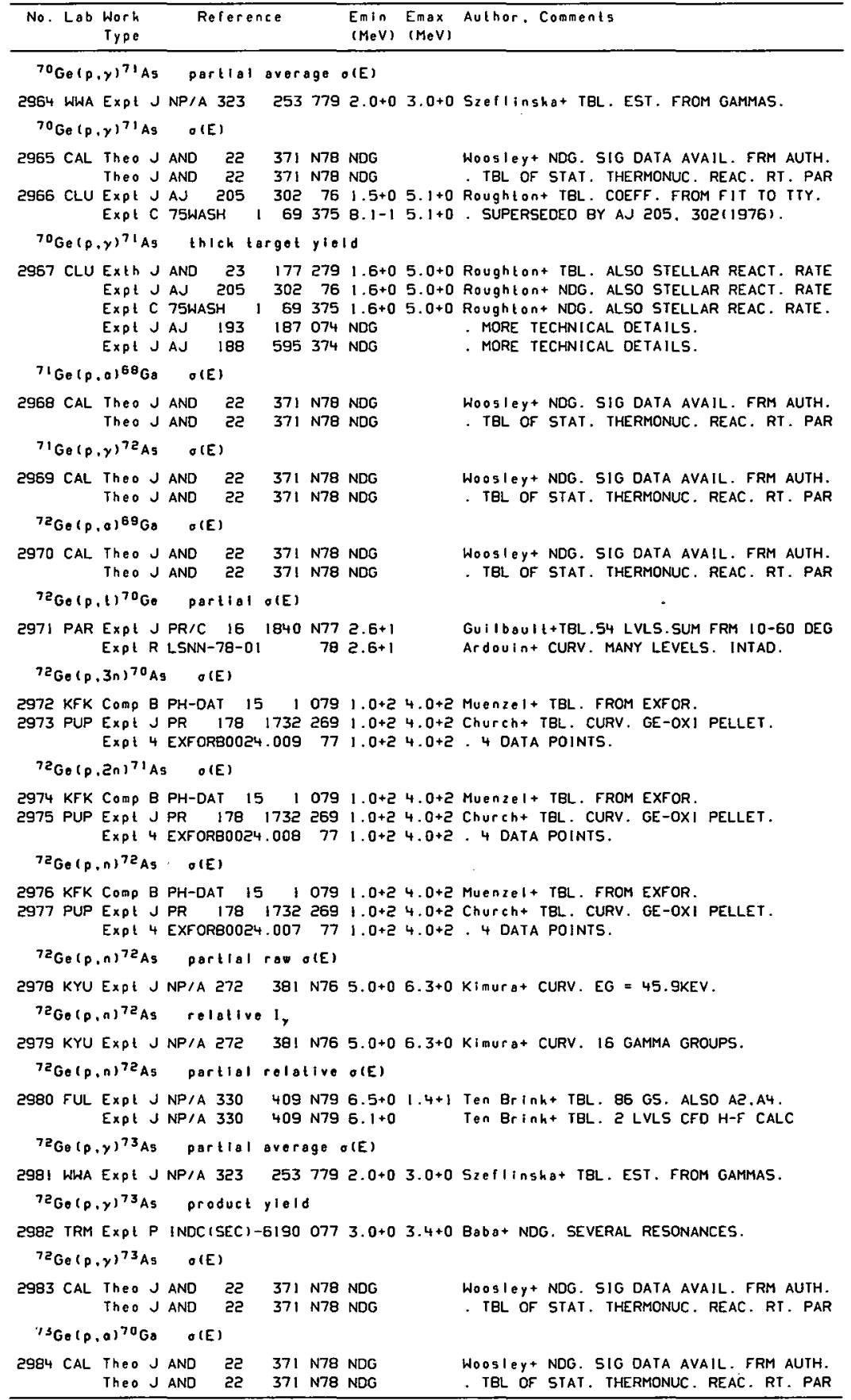




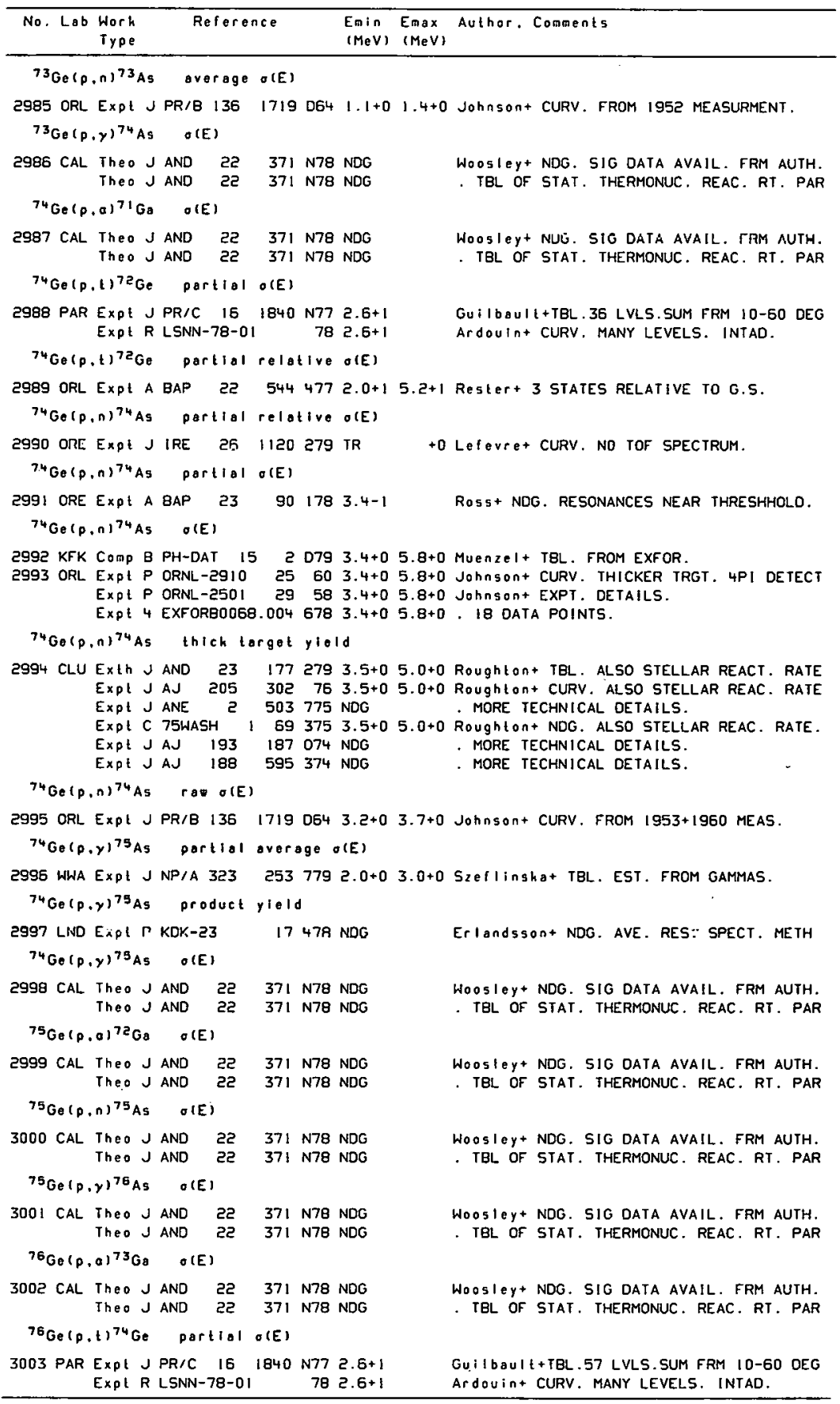


REFERENCES ( $c$ on t)

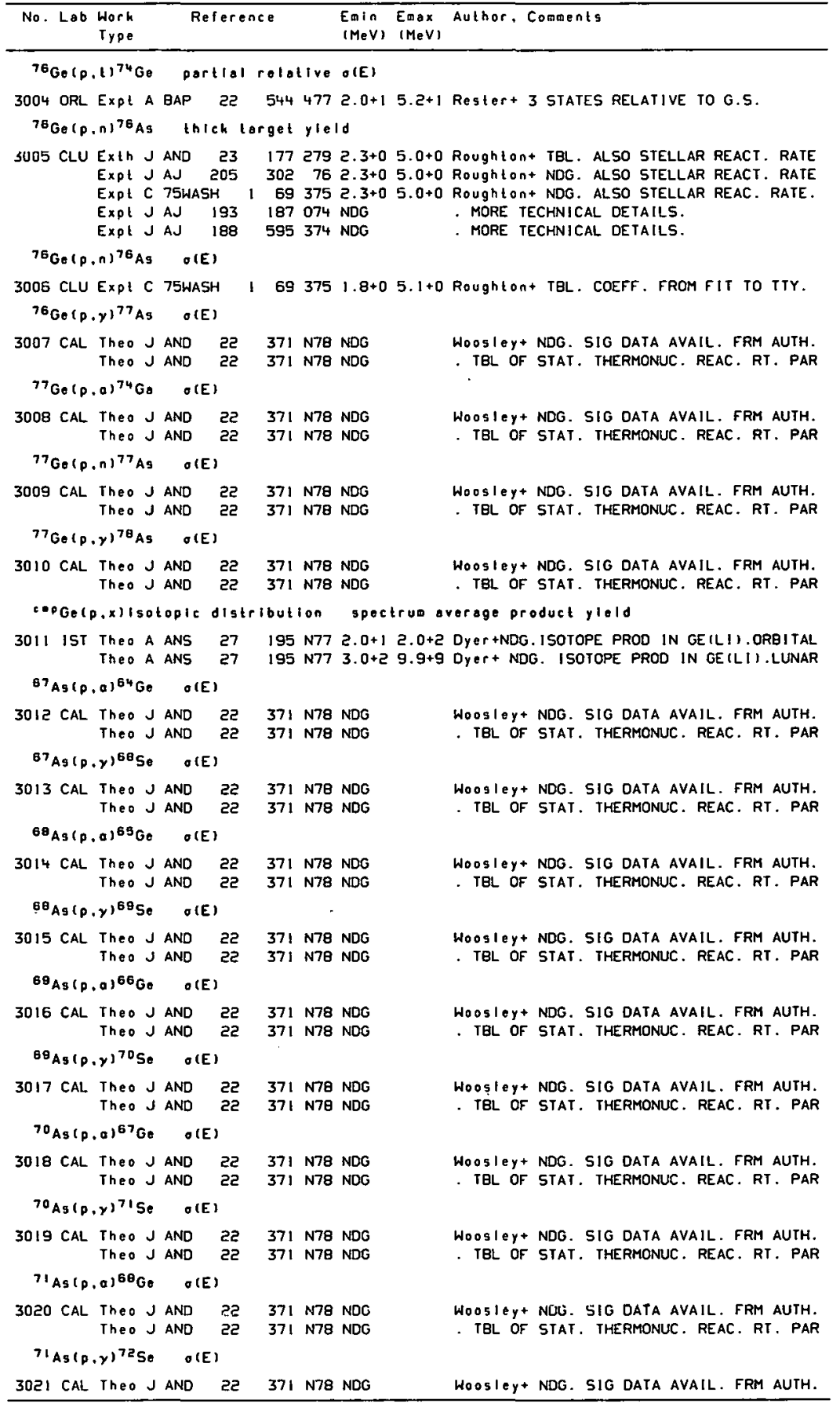




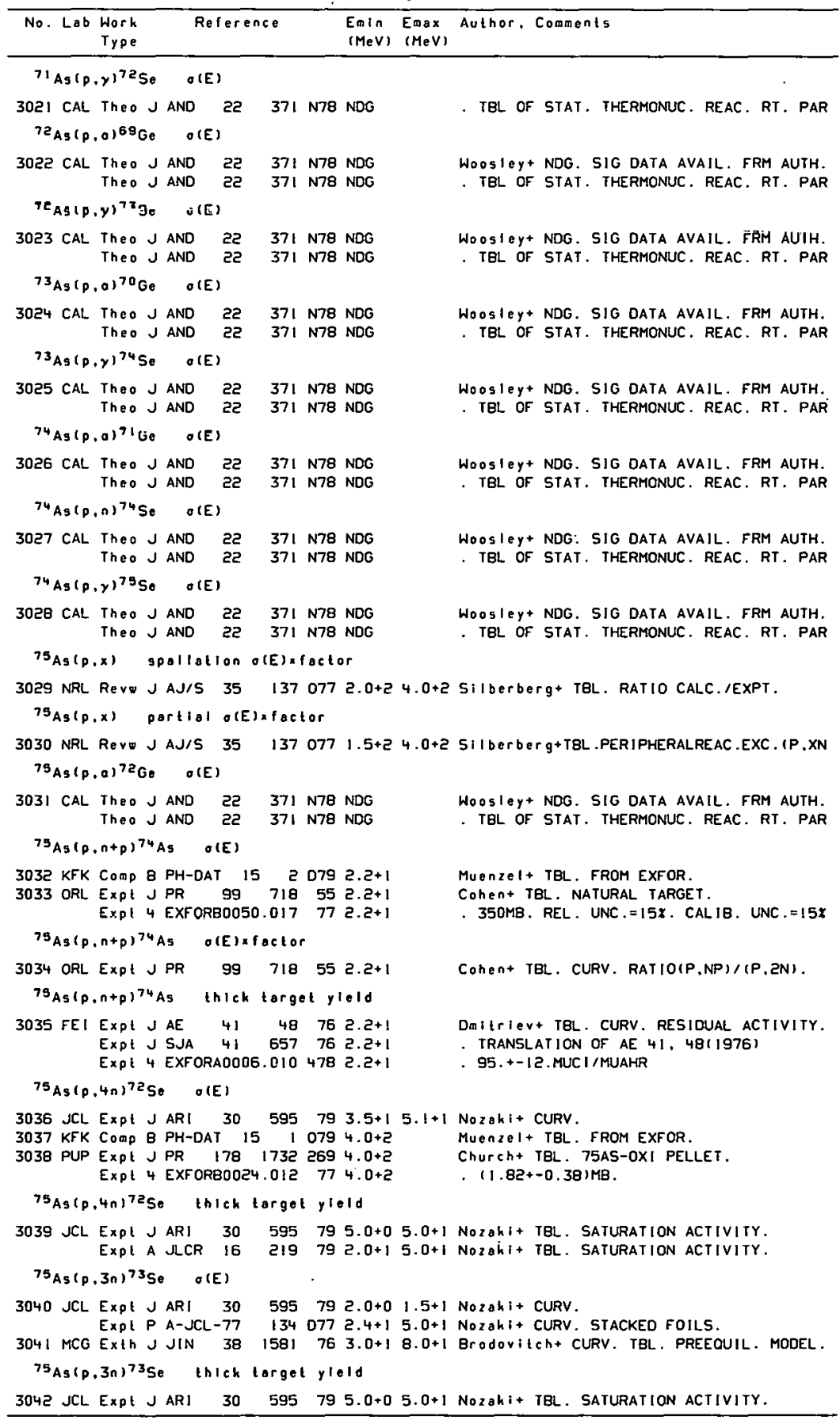


REFERENCES ( $c$ on $($ )

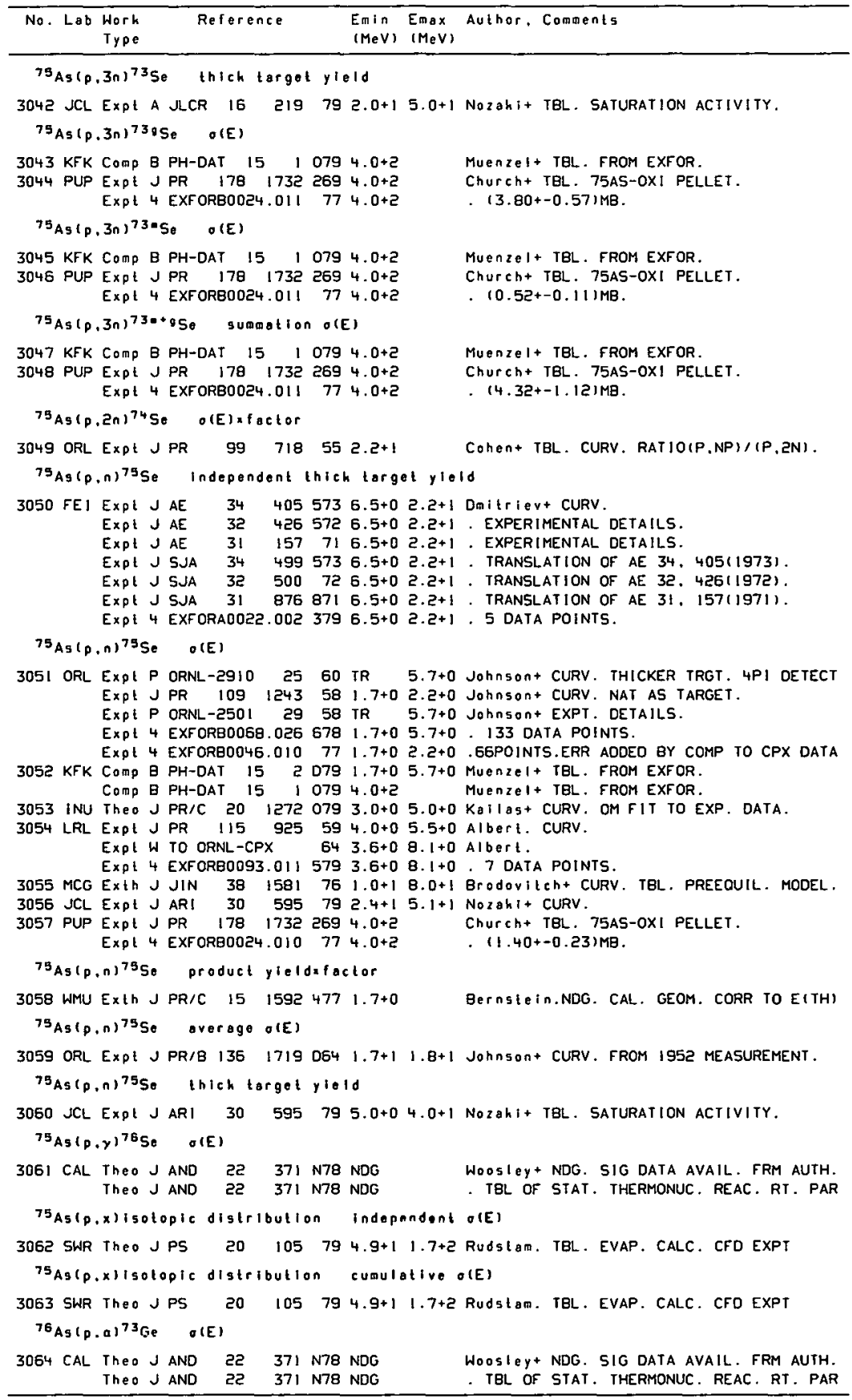


REFERENCES ( cont)

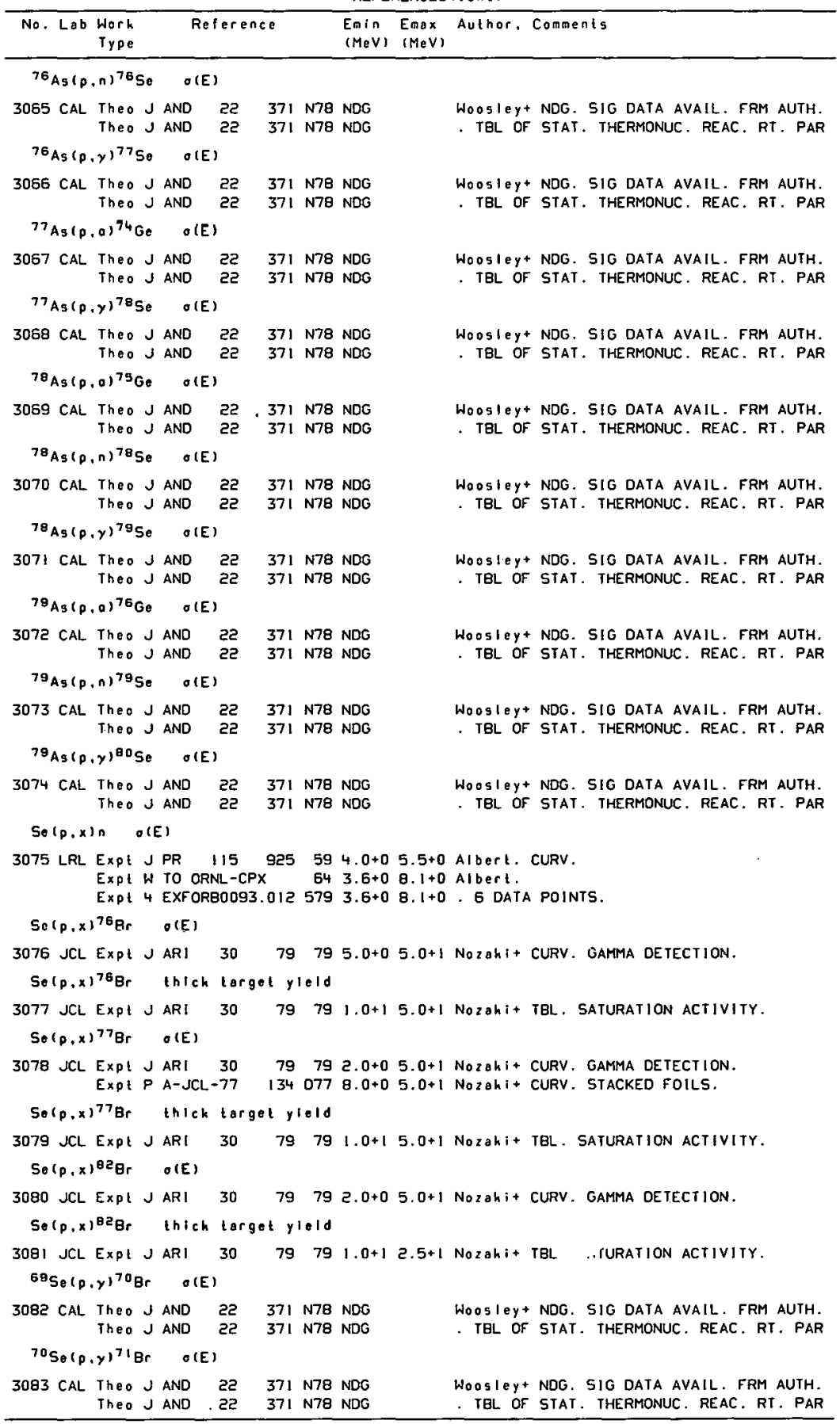


REFERENCES (cont)

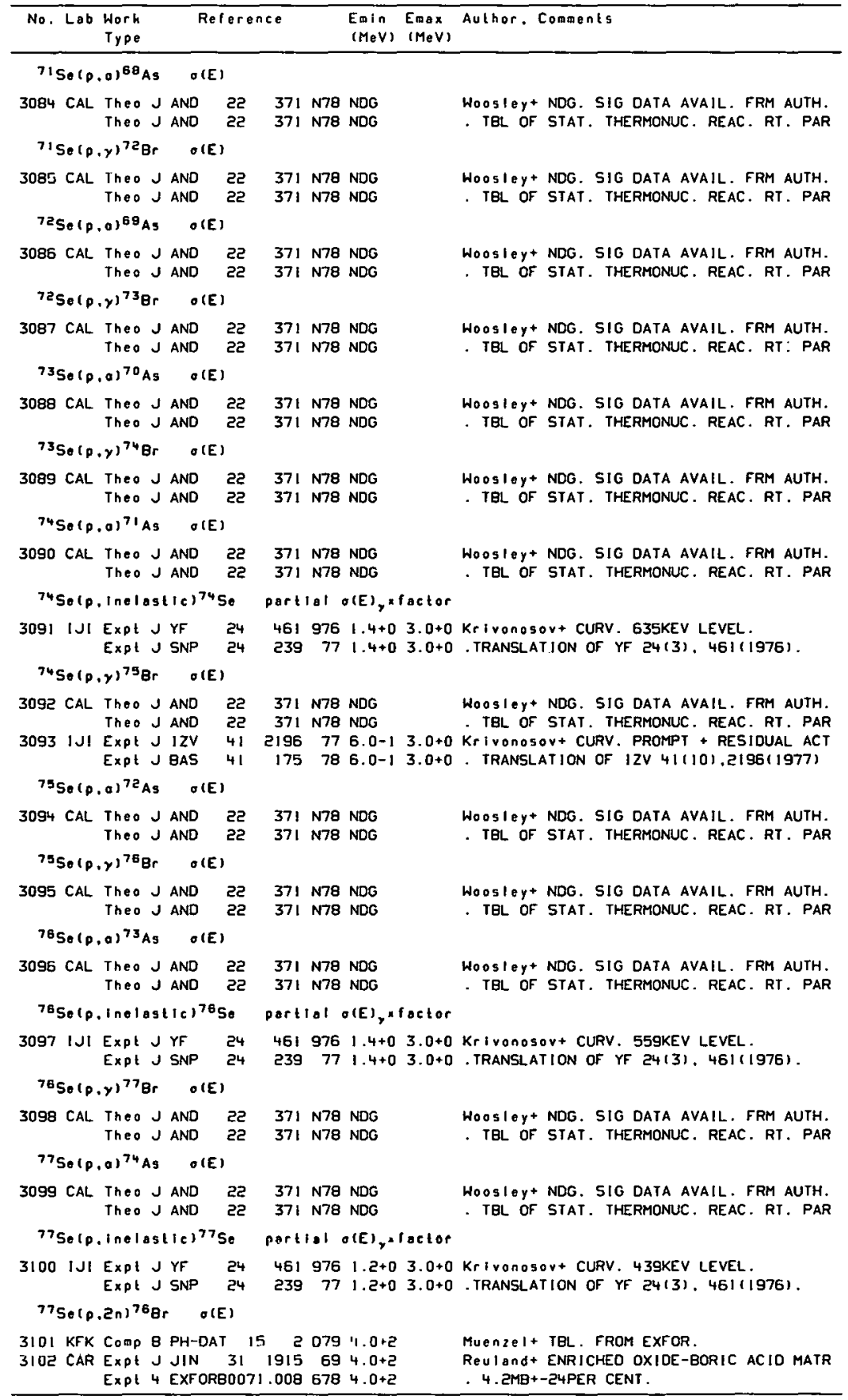




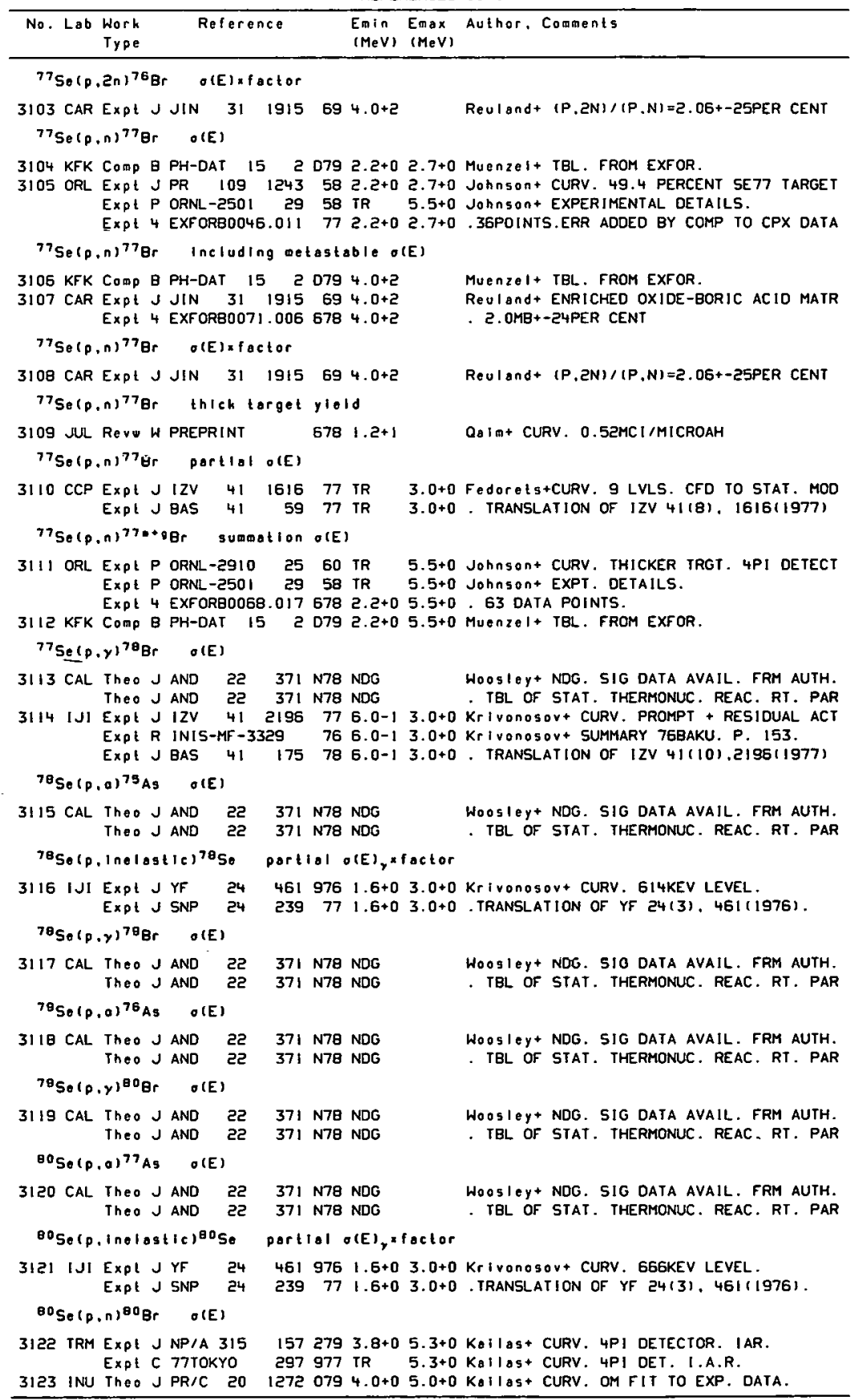




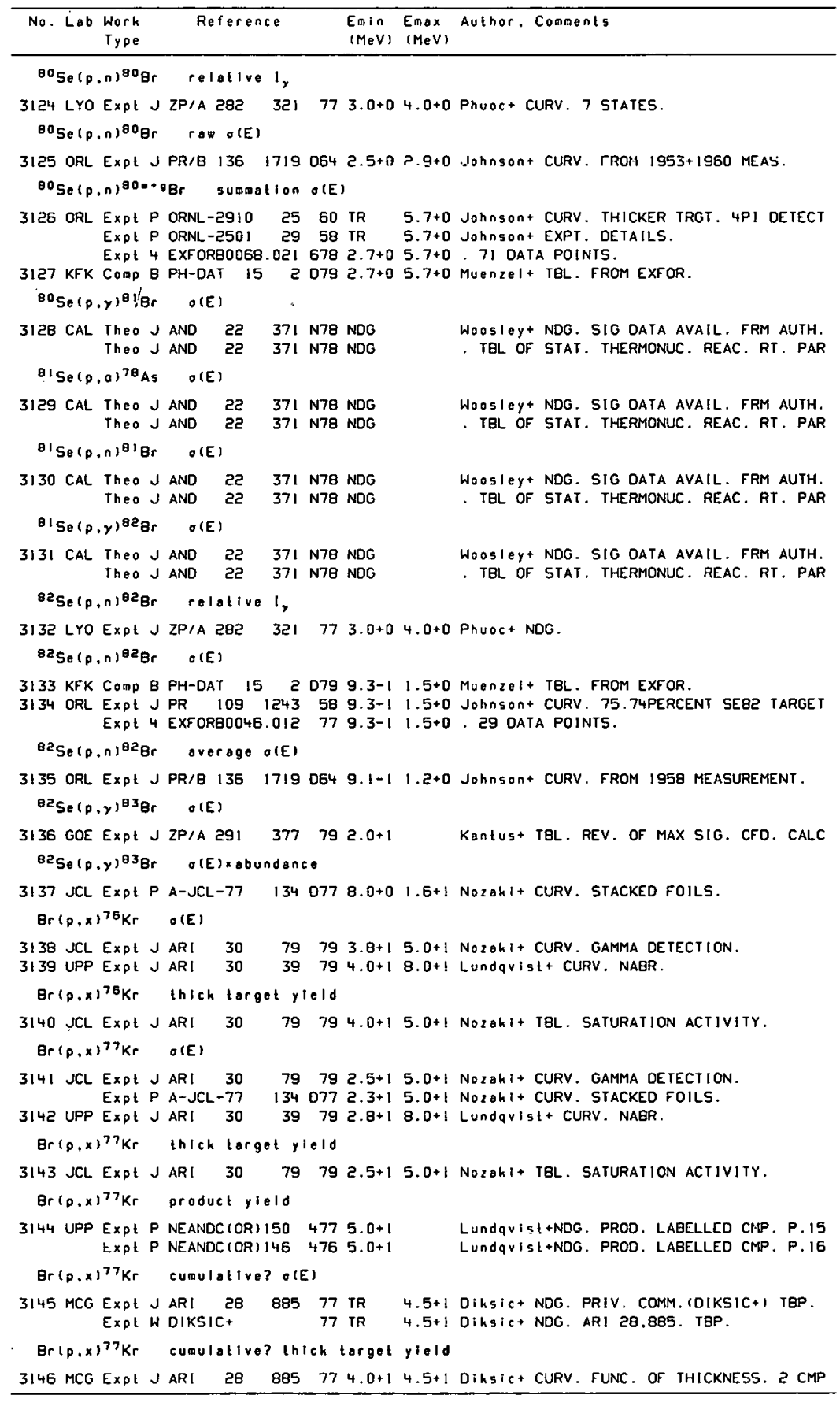




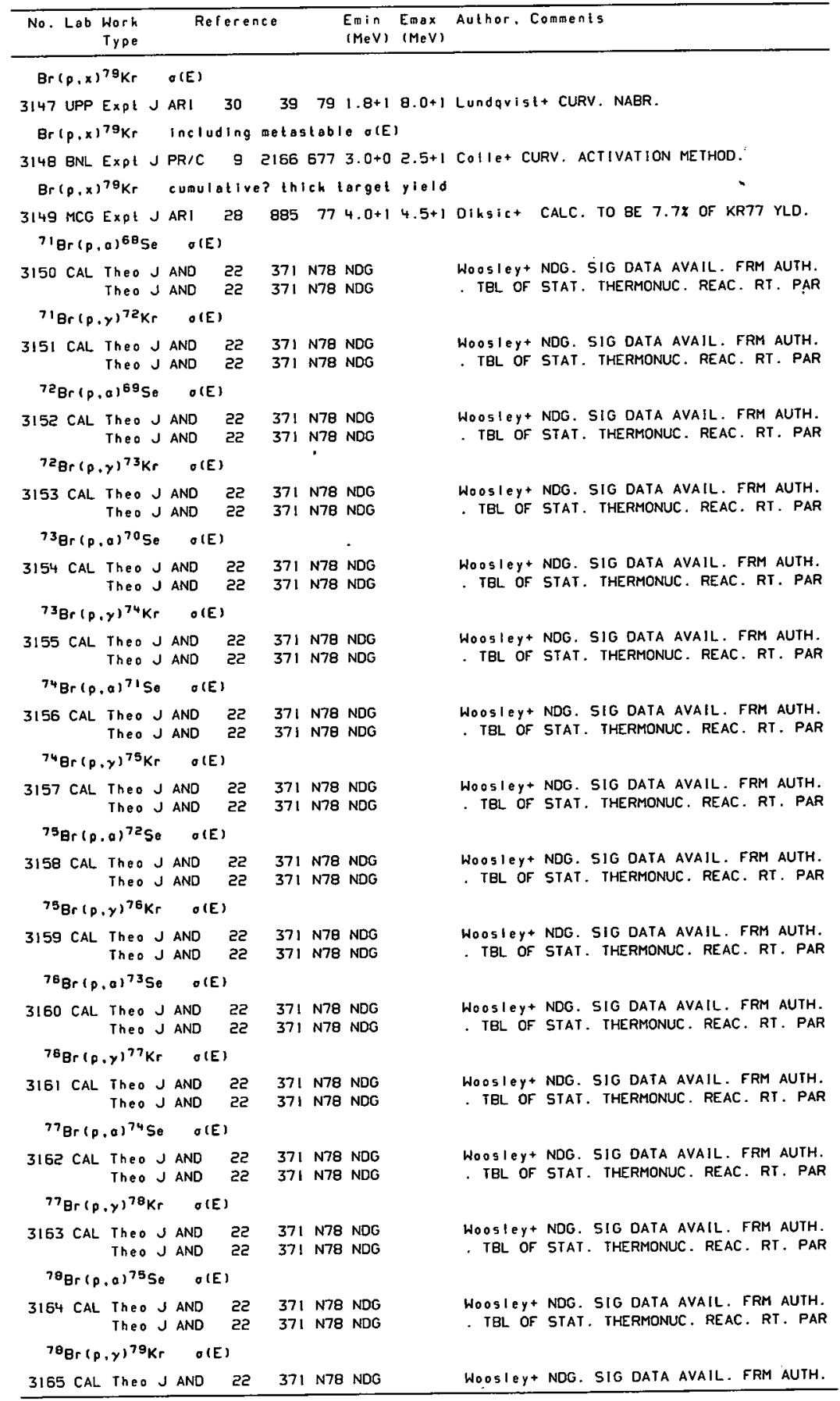




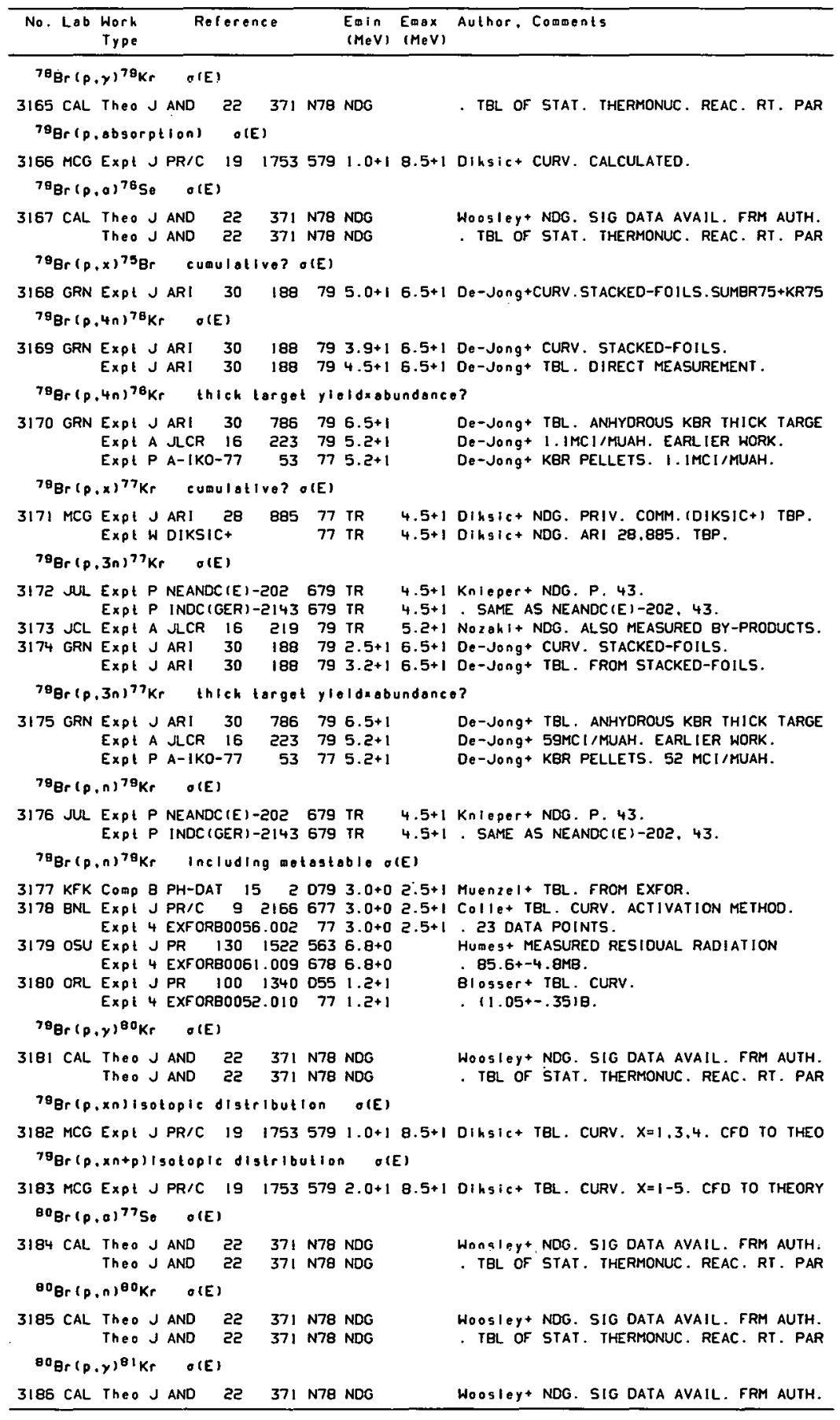


REFERENCES ( con t)

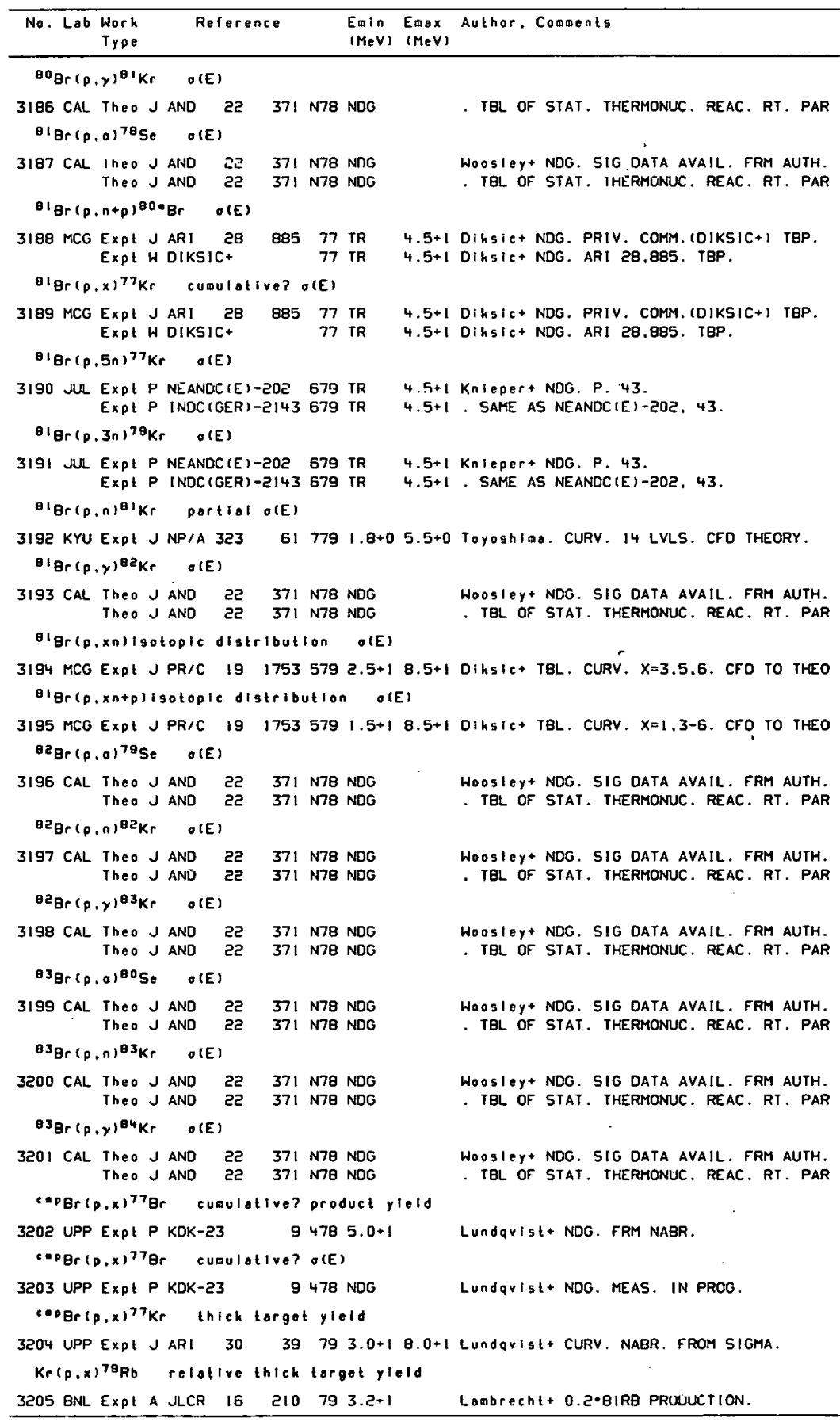


REFERENCES (cont)

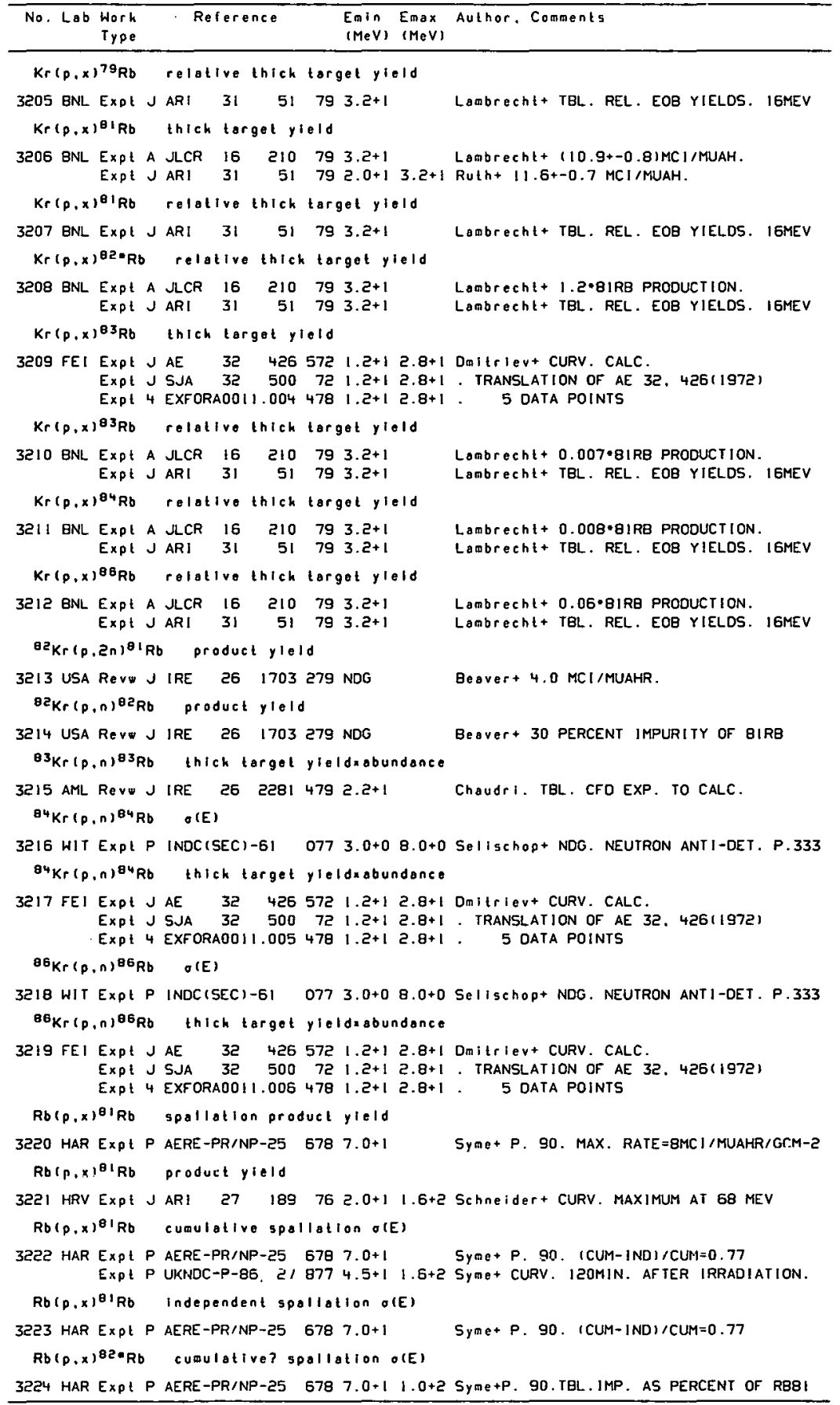




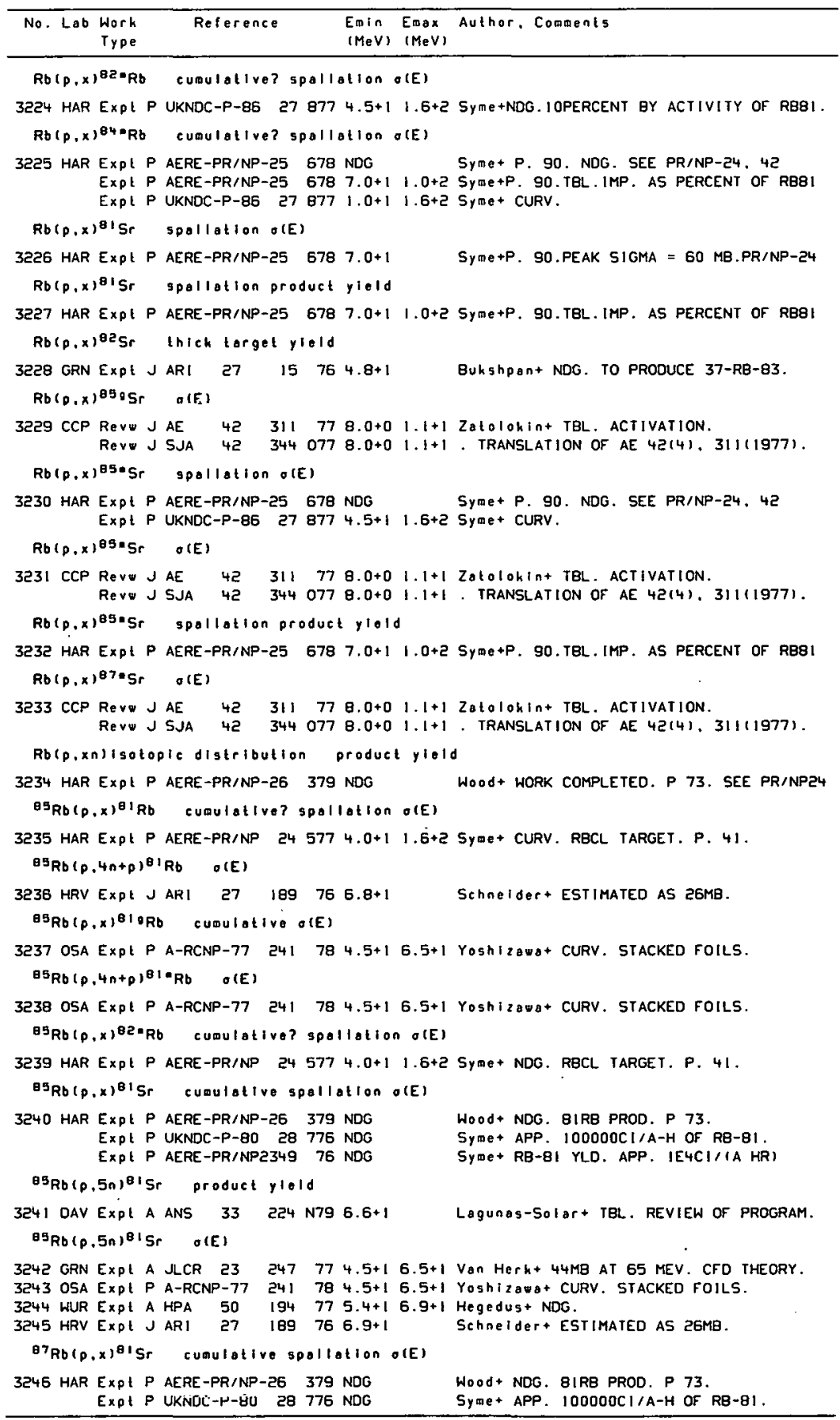


REFERENCES $(\cos t)$

\begin{tabular}{|c|c|c|c|c|c|c|}
\hline No. Lab & $\begin{array}{l}\text { Work } \\
\text { Type }\end{array}$ & Reference & $\begin{array}{l}E m I n \\
(M e V)\end{array}$ & $\begin{array}{l}\text { Emax } \\
\text { (MeV) }\end{array}$ & Author. & Comments \\
\hline
\end{tabular}

${ }^{87} \mathrm{Rb}(p, x)^{\mathrm{Bl}} \mathrm{Sr}$ cumulative spallation o(E)

3246 HAR Expl P AERE-PR/NP-23 76 NUG Syme+ RB-8! YLD. AГT I['ICI/AHR. P. 49

${ }^{67} R b(p, n)^{87 \cdot S r} \quad \sigma(E)$

3247 KFK COMP B PH-DAT $15 \quad 2$ D79 $1.2+1$

3248 ORL Expt J PR 1001340 D55 $1.2+1$

Expt 4 EXFORB0052.011 $771.2+1$

$\operatorname{Sr}(p, x)^{86} Y$ a (E)

3249 CCP Revo J AE 4231177 B.0+0 $1.1+1$ Zatolokint TBL. ACTIVATION.

Reve J SJA 42344077 8.0+0 1.1+1. TRANSLATION OF AE $42(4) .311(1977)$.

${ }^{84} \operatorname{Sr}(p, \gamma)^{85} Y$ partlal averoge o(E)

3250 WWA Expt J NP/A $3232537792.0+03.0+0$ Szeflinsha+ TBL. EST. FROM GAMMAS.

${ }^{86} \operatorname{Sr}(D, 4 n)^{83} y$ thich lorgol yleld

3251 GRN Expi J ARI $27 \quad 15765.5+1 \quad$ Buhshpont NDG. TO PRODUCE 37-RB-83.

${ }^{86} \operatorname{Sr}(p, y)^{87} Y$ partiol overoge O(E)

3252 WWA Expt J NP/A $3232537792.0+03.0+0$ 5zeflingha+ TBL. EST. FROM GAMMAS.

${ }^{87} \operatorname{Sr}(p, n)^{87} Y$ thich torget ylold

$3253 \mathrm{CLU}$ Exth J AND $231772792.6+05.7+0$ Roughton+ TBL. ALSO STELLAR REACT. RATE Expt $J$ AJ 20530276 NDG MORE TECHNICAL DEIALLS.

Expt J AJ 193187074 NDG . MORE TECHNICAL DETAILS.

Expt J AJ 188595374 NDG - MORE TECHNICAL DETAILS.

$07 \mathrm{Sr}(p+\gamma)^{80}$ thich torget yielo

$3254 \mathrm{CLU}$ Exth J AND $23 \quad 1772792.6+04.3+0$ Roughtont TBL. ALSO STELLAR REACT. RATE Expt J AJ 20530276 NDG . MORE TECHNICAL DETAILS.

Expt J AJ 193187074 NDG . MORE TECHNICAL DETAILS.

Expt J AJ 188595374 NDG

${ }^{88} \mathrm{Sr}(p, x) \mathrm{O}$ (E)

3255 LRL Expt P DOE-NDC-15 $1054793.0+0$ + Dietrich+ NDG. GE(LI).NAI. CFD STAT MOD

${ }^{\theta} \mathrm{Sr}(p, x)^{83} \mathrm{Rb} \quad \sigma(E)$

3256 KFK Comp B PH-OAT $1520795.4+18.5+1$ Muenzel+ TBL. FROM EXFOR.

3257 MCG Expt J CJC $451149675.4+18.5+1$ Sachdev+ TBL. CURV. CFD EVAP-CASCADE Expt 4 EXFORB0069.010 678 5.4+1 8.5+1. 6 DATA POINTS.

${ }^{8 B} \operatorname{Sr}\left(p, E_{n+a}\right)^{83} R b \quad o(E)$

3258 KFK Comp 日 PH-DAT 152 D79 3.3+1 4.8+1 Muenzel+ TBL. FROM EXFOR.

3259 MCG Expt J CJC $451149673.3+14.8+1$ Sochdev + TBL. CURV. RESIDUAL ACTIVITY. Expt 4 EXF ORBO069.010 678 3.3+1 $4.8+1$. 5 OATA PO!NTS.

${ }^{\theta B} \operatorname{Sr}(p, 4 n+2 p)^{\theta 3} \mathrm{Rb} \quad \sigma(E)$

3260 MIL Theo J PR/C $1614040773.5+19.5+1$ Gadiolit CURV. PRE-EOUILIBRIUM.

${ }^{88} \mathrm{Sr}(p, x)^{84} \mathrm{Rb}$ lncluding melastable olEs

3261 KFK COMP B PH-DAT 15 2 D79 $4.2+18.5+1$ Muenzel+ TBL. FROM EXFOR.

3262 MCG Expt J CJC $451149674.2+18.5+1$ Sachdev+ TBL. CURV. CFD EVAP-CASCAOE Expt 4 EXFORBO069.009 $6784.2+18.5+1$. 8 DATA POINTS.

${ }^{80} \mathrm{Sr}(p, 3 n+2 p){ }^{84} \mathrm{Rb} \quad O(E)$

$3263 \mathrm{MIL}$ Theo J PR/C $1614040772.8+19.0+1$ Gadioli+ CURV. PRE-EOUILIBRIUM.

Theo J PL/B $653110767.3+1$ Gadioli+ TBL. VAR. IN MEAN-FREE PATH

${ }^{80} \operatorname{Sr}(p, n+a)^{64} R b$ including aelostable o(E)

3264 KFK Comp B PH-OAT 152 D79 2.5+1 4.0+1 Muenzel+ TBL. FROM EXFOR.

3265 MCG Exp!J CJC $451149672.5+14,0+1$ Sachdev+ TBL. CURV. RESIDUAL ACTIVITY. Expt 4 EXFORBO069.009 $6782.5+14.0+1$. 5 DATA POINTS.

${ }^{8} \mathrm{~S} r(p, n+2 p)^{86} R b \quad \sigma(E)$

3266 MIL Theo J PRIC I6 $14040773.8+19.5+1$ Gadioli+ CURV. PKE'-EUUILIBRIUM.

${ }^{8 B} \mathrm{Sr}(p, n+2 p)^{86} \mathrm{Rb}$ Including wetostoble $\sigma(E)$

3267 KFK Comp B PH-DAT $1520793.3+1$ 8.5+1 Muenze1+ TBL. FROM EXFOR.

3268 MCG Expl J CJC $451149673.3+1$ 9.5+1 Sachdev+ TBL. CURV. CFD EVAP-CASCADE Expt 4 EXFORB0069.008 678 $3.3+18.5+1$. II DATA POINTS. 
REFERENCES $(\operatorname{con}()$

\begin{tabular}{|c|c|c|c|c|c|}
\hline No. Lab & $\begin{array}{l}\text { Work } \\
\text { Type }\end{array}$ & Reference & $\begin{array}{l}\text { EnIn } \\
(M e V)\end{array}$ & $\begin{array}{l}\text { Emax } \\
(\operatorname{MeV})\end{array}$ & Author, Comments \\
\hline
\end{tabular}

\footnotetext{
${ }^{\mathrm{e \theta}} \mathrm{Sr}(p, 3 n+p)^{\mathrm{es}} \mathrm{Sr} \quad$ o(E)

3269 MIL theo J PR/C $1614040774.5+1 \quad 1.0+2$ Gadiolit CURV. PRE-EOUILIBRIUM.

Theo J PL/B $653110765.4+1$ Gadiolit TEL. VAR. IN MEAN-FREE PATH

${ }^{88} \mathrm{Sr}(p, 3 n+p)^{85} \mathrm{Sr} \quad$ o(E)
}

$3270 \mathrm{KFK}$ Comp B PH-DAT $15 \quad 20794.2+18.5+1$ Muenzel+ TBL. FROM EXFOR.

3271 MCG Expl J CJC $451149674.2+1$ 8.5+1 Sachdevt TBL. CURV. RESIDUAL ACTIVITY. Expt 4 EXFORB0069.007 $6784.2+18.5+1$. 8 DATA POINTS.

${ }^{00} \mathrm{Sr}(p, 3 n+p)^{050} \mathrm{Sr} \quad$ o(E)

3272 KFK Comp B PH-DAT 152 D79 4.2+1 8.5+1 Muenze1+ TBL. FROM EXFOR.

3273 MCG Expl $J$ CJC $451149674.2+18.5+1$ Sachdev+ TBL. CURV. RESIDUAL ACTIVITY. Expt 4 EXFORBO069.007 $6784.2+18.5+1$. B DATA POINTS.

${ }^{80} \mathrm{Sr}(p, 3 n+p)^{85 n+95 r \quad S u m a t i o n}$ o(E)

3274 KFK COmp B PH-DAT 15 2 D79 $4.2+1$ 8.5+1 Muenzel+ TBL. FROM EXFOR.

3275 MCG Expt J CJC $451149674.2+18.5+1$ Sochdert TBL. CURV. CFD EVAP-CASCADE EXpt 4 EXFORB0069.007 $6784.2+1$ 6.5+1 - 8 DATA POINTS.

${ }^{88} \operatorname{Sr}(p, 5 n)^{84} Y \quad O(E)$

3276 MIL Theo JPR/C $1614040775.0+19.0+1$ Gadiolit CURV. PRE-EQUILIBRIUM. Theo J PL/B 65311076 6.9+1 Godioli+ TBL. VAR. IN MEAN-FREE PATH

${ }^{80} \mathrm{Sr}(p, 5 n)^{84} \mathrm{Y}$ partiol o(E)

3277 KFK COMP B PH-DAT 15 2 D79 6.0+1 8.5+1 Muenzel+ TBL. FROM EXFOR.

3278 MCG Expt J CJC $451149676.0+10.5+1$ Sachdev+ IBL. CURV. CFD EVAP-CASCADE Expt 4 EXFORB0069.006 $6786.0+1$ 8.5+1. 6 OATA POINTS. 4 - HIGH SPIN STATE.

${ }^{80} \mathrm{Sr}(p .4 n)^{85} Y \quad \sigma(E)$

3279 MIL Theo J PR/C $1614040774.0+1 \quad 1.0+2$ Gadiolit CURV, PRE-EQUILIBRIUM.

Theo J PL/B $653110765.4+1$ Gadioli+ TBL. VAR. IN MEAN-FREE PATH

${ }^{\theta} \mathrm{Sr}(0,4 n)^{850 Y} O(E)$

3280 KFK COMP B PH-DAT I5 2 D79 $4,2+18.5+1$ Muenzel+ TBL. FROM EXFOR.

328! MCG Expt J CJC $451149674.2+18.5+1$ Sachdev+ TBL. CURV. RESIDUAL ACTIVITY. EXPt 4 EXFOR日O069.005 678 $4.2+18.5+1$. 9 DATA POINTS.

${ }^{80} \operatorname{Sr}(p .4 n)^{85 * Y} \quad O(E)$

3282 KFK Comp B PH-DAT 15 2 D79 $4.2+1$ 8.5+1 Muenzel+ TBL. FROM EXFOR.

3283 MCG Expt J CJC 45 1149 $674.2+1 \quad 0.5+1$ SechdeV+ TBL. CURV. RESIDUAL ACTIVITY. EXP: 4 EXFORBD069.005 $6784.2+1$ 8.5+1 - 9 DATA POINTS.

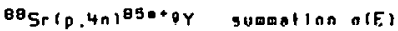

3284 KFK COMP B PH-DAT I5 $20794.2+18.5+1$ Muenzel+ TBL. FROM EXFOR.

3285 MCG Expt J CJC $451149674.2+18.5+1$ Sochdev+ TBL. CURV. CFD EVAP-CASCADE Expt 4 EXFORBO069.005 $6784.2+18.5+1$. 9 DATA POINTS.

${ }^{8 B} \operatorname{Sr}(p, 3 n)^{86} \mathrm{Y} \quad \sigma(E)$

3286 MIL Theo J PR/C $1614040772 . B+19.0+1$ Gadiolit CURV. PRE-EQUILIBRIUM. Theo J PL/B 65 311 $0763.9+1$ Gadiolit TBL. VAR. IN MEAN-FREE PATH

${ }^{88} \mathrm{Sr}(p, 3 n) 809 \mathrm{O} \quad \mathrm{G}(\mathrm{E})$

3287 KFK COMp 8 PH-DAT I5 $20792.9+1$ 日.5+1 Muenzel+ IBL. FROM EXFOR.

3288 MCG Expt J CJC $451149672.9+1$ 8.5+1 Sochdev+ JBL. CURV. RESIDUAL ACTIVITY. Expt 4 EXFORBO069.004 $6782.9+18.5+1$. I2 DATA POINTS

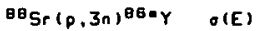

3289 KFK Comp B PH-DAT 152 D79 2.9+1 8.5+1 Muenze1+ TBL. FROM EXFOR.

3290 MCG Expt J CJC $451149673.3+18.5+1$ Sachdev+ TBL. CURV. RESIOUAL ACIIVITY. Expt 4 EXFORBO069.004 $6782.9+18.5+1$. I2 DATA POINTS

${ }^{8 B} \mathrm{Sr}(0.3 n)^{86-+0 Y}$ Sumation o(E)

329! KFK Comp B PH-DAT 15 2 $0792.9+18.5+1$ Muenzel+ IBL. FROM EXFOR.

3292 MCG Expl J CJC $451149672.9+18.5+1$ Sachdev+ IBL. CURV. CFD EVAP-CASCAOE EXPt 4 EXFORB0069.004 678 $2.9+18.5+1$. 12 DATA POINTS

${ }^{80} \mathrm{Sr}(\mathrm{D}, \mathrm{Sn})^{87} \mathrm{OY} \quad \mathrm{Q}(\mathrm{E})$

3293 KFK COMp B PH-DAT 152 D D79 $1.5+13.3+1$ Muenzel+ TBL. FROM EXFOR.

3294 MCG Expt J CJC $451149671.5+18.5+1$ 5achder+ TBL. CURV. RESIDUAL ACTIVITY. EXP: 4 EXFORBOUSY. UUS $6 \% 181.5+13.3+1$. 8 DATA FOINTS. 


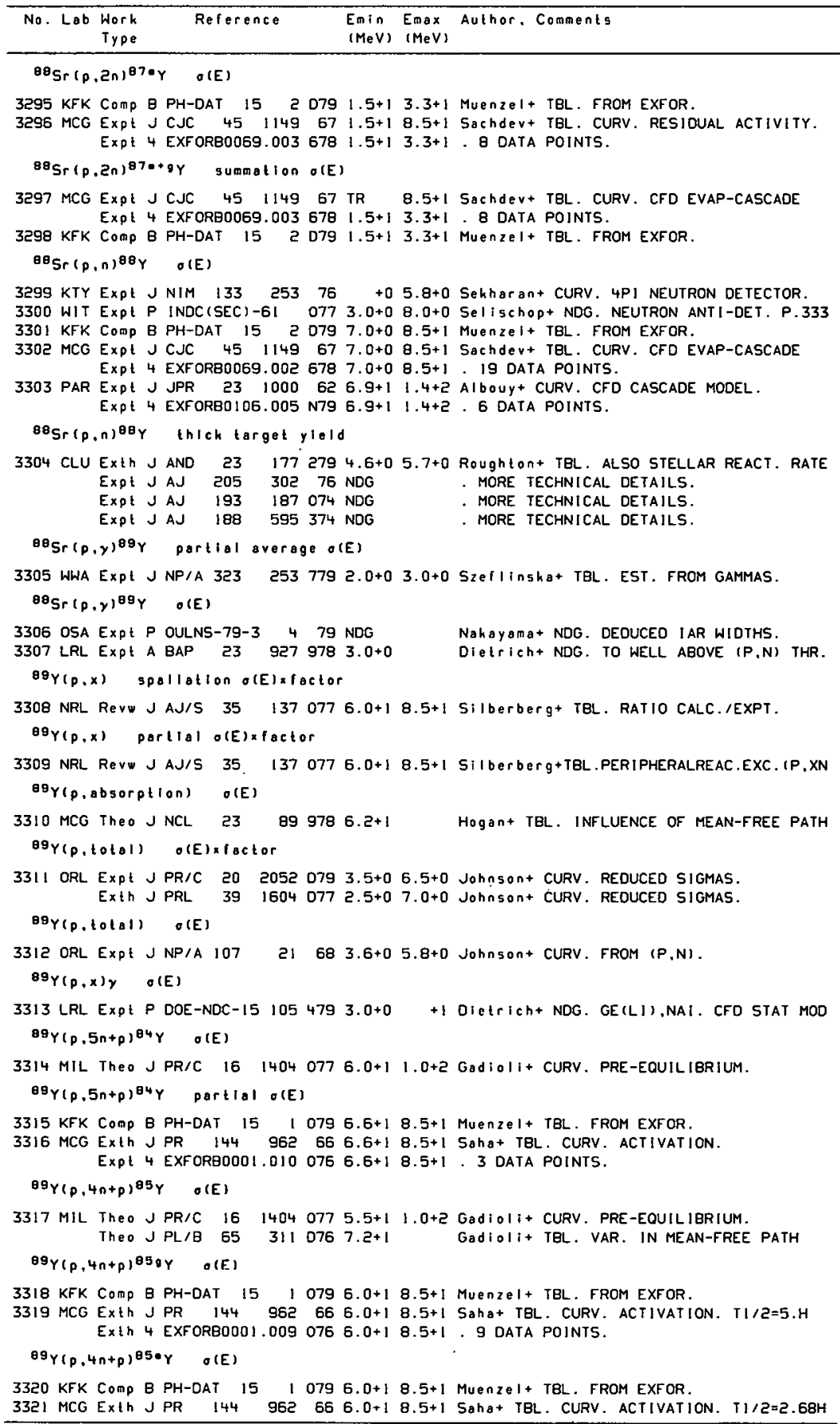


REFERENCES $(c$ ont)

\begin{tabular}{|c|c|c|c|c|c|c|}
\hline No. Lab & $\begin{array}{l}\text { Work } \\
\text { Type }\end{array}$ & Reference & $\begin{array}{l}\text { Emin } \\
(M e V)\end{array}$ & $\begin{array}{l}\text { Emax } \\
(M e V)\end{array}$ & Author. & Comments \\
\hline
\end{tabular}

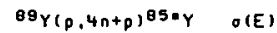

3321 MCG Exth 4 EXFORBDO0! 1.009076 6.0+1 8.5+1, 9 DATA POINTS.

89Y $(p, 4 n+p)$ B5n+oY sumation o(E)

3322 V.FY, COPP R PH-חAT $1510796.0+18.5+1$ Muenzel+ TBL. FROM EXFOR.

3323 MCG Exth J PR $144962666.0+18.5+1$ bahat 1 GL. CURV. DCDUCLD.

Exth 4 EXFORB0001.009 076 6.0+1 8.5+1. 9 DATA POINTS.

$09 Y(p, 4 n+p) 85,10 Y \quad r a t 10$ O(E)

3324 KFK Comp B PH-DAT $1510796.0+18.5+1$ Muenze + + TBL. FROM EXFOR.

3325 MCG Exth J PR $144962666.0+18.5+15 a h a+$ TBL. CURV. DEDUCED. Expt 4 EXFORBO001.009 $0766.0+18.5+1$. 9 DATA POINTS.

${ }^{89} Y(p, 3 n+p)^{86} Y \quad O(E)$

3326 MIL Theo J PR/C $1614040774.5+19.0+1$ Gadioli+ CURV. PRE-EQUILIBR!UM.

Theo J PL/B 65311 D76 $5.8+1$ Gadlali+ TBL. VAR. IN MEAN-FREE PATH

${ }^{60} Y(p, 3 n+p) \theta 6 \% Y \quad \sigma(E)$

3327 KFK COMP B PH-DAT $1510794.5+18.5+1$ Muenzel+ TBL. FROM EXFOR.

3328 MCG Exth J PR $144962664.5+18.5+1$ Saha+ TBL. CURV. ACTIVATION. TII $9=4.9 H$ Exth 4 EXFORBO001.008 $0764.5+18.5+1$. 9 DATA POINTS.

${ }^{89} Y(p, 3 n+p) \operatorname{Be} \cdot Y \quad O(E)$

3329 KFK COMP B PH-DAT $1510794.5+18.5+1$ Muenzel+ TBL. FROM EXFOR.

3330 MCG Exth J PR $144962664.5+18.5+1$ Saha+ TBL. CURV. ACTIVATION. TI/2=48.5M Exth 4 EXFORB0001.008 $0764.5+18.5+1$. 9 DATA POINTS.

$89 Y(p, 3 n+p) 06 \cdots+0 Y$ sumation $O(E)$

3331 KFK COMP B PH-DAT $1510793.7+18.5+1$ Muenze $1+$ TBL. FROM EXFOR.

3332 MCG Exth J PR 144 S62 $663.7+18.5+1$ Saha+ TBL. CURV. DEDUCED.

Exth JPR 144 S62 $664.5+18.5+1$ Sahat TBL. CURV. ACTIVAIION. TI/Z=14.7H Expt 4 EXFORBO001.008 $0763.7+18.5+1$. 1! DATA POINTS.

${ }^{80} Y(p, 3 n+p) 860 / 8 Y \quad r a t i o \sigma(E)$

3333 KFK COmp B PH-DAT $1510794.5+18.5+1$ Muenzel+ TBL. FROM EXFOR.

3334 MCG Exth J PR $144962664.5+18.5+1$ Sahat TBL. CURV. DEDUCED.

Exth 4 EXFORBODOI.008 $0764.5+18.5+1$. 9 DATA POINTS.

$80_{Y}\left(p, 2_{n+p} 07 Y\right.$ o(E)

3335 LAS Theo $C 778 N L \quad 467772.5+14.0+1$ Arthur + CURV. GNASH CODE.

3336 MIL Theo J PL/8 $653110764.3+1 \quad$ Gadioli+ TBL. VAR. IN MEAN-FREE PATH

${ }^{89} Y\left(p, z_{n+p)} 07, y \quad \sigma(E)\right.$

3337 KFK COmp B PH-DAT I5 1 $0792.5+18.5+1$ Muenze1+ TBL. FROM EXFUK.

3338 MCG Exth J PR 144 S62 $662.5+18.5+1$ Saho+ TEL. CURV. ACTIVATION. TI/2=80.H EXPl 4 EXFORBO001.003 $0762.5+18.5+1$. 16 DATA POINTS.

${ }^{8 Q} Y(p, 2 n+p) B 7 \cdot Y \quad \sigma(E)$

3339 KFK Comp B PH-DAT 15 , $0792.5+18.5+1$ Muenzel+ TBL. FROM EXFOR.

3340 MCG Exth JPR $144962662.5+18.5+1$ Saha+ TBL. CURV. ACTIVATION. TI/2=13.2H Expt 4 EXFORBO001.003 $0762.5+18.5+1$. 16 DATA POINTS.

89Y $(p, 2 n+p) 97 a+g Y$ sumation o(E)

3341 KFK COmp B PH-DAT 15 l $0792.5+18.5+1$ Muenzel+ TBL. FROM EXFOR.

3342 MCG Exth J PR $144962662.5+18.5+1$ Saha+ TBL. CURV. DEDUCED.

Ex:h 4 EXFORBO001.003 $0762.5+10.5+1$. 16 DATA POINTS.

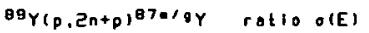

$3343 \mathrm{KFK}$ Comp B PH-DAT $1510792.5+1$ B.5+1 Muenzel+ TBL. FROM EXFOR.

3344 MCG Exth J PR $144962662.5+18.5+1$ Saha+ TBL. CURV. OEDUCED. Expl 4 EXFORBO001.003 $0762.5+1$ 8.5+1. I6 DATA POINTS.

$89 Y(p, n+p)^{88 Y} \quad \sigma(E)$

3345 LAS Theo C 77BNL $467771.7+14.0+1$ Arthurt CURV. GNASH CODE

3346 MIL Then J PR/C $1614040773.0+19.0+1$ Gadiolit CURV. PRE-EOUILIBRIUM.

${ }^{89} Y(p, n+p)^{88} Y \quad \sigma(E)$

3347 KFK Comp B PH-DAT $1510791.5+18.5+1$ Muenze 1+ TBL. FROM EXFOR.

3348 MCG Exth J PR 144 g62 $661.5+18.5+1$ Sahat TBL. CURV. ACIIVATION.

Expt 4 EXTORBO001.007 D7א 1.5+1 8.5+1. I6 DATA POINTS. 
REFERENCES (cont)

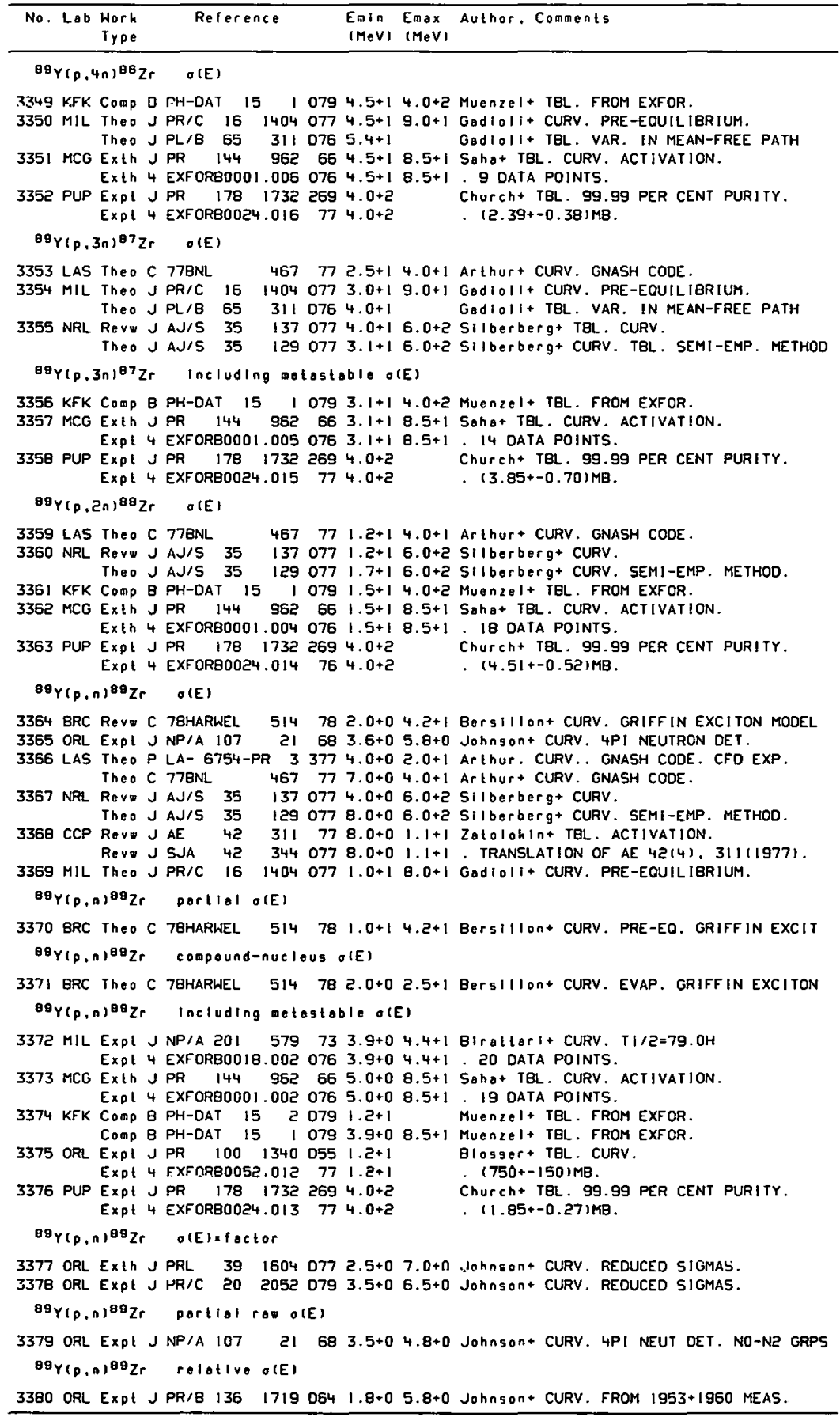


REFERENCES (con t)

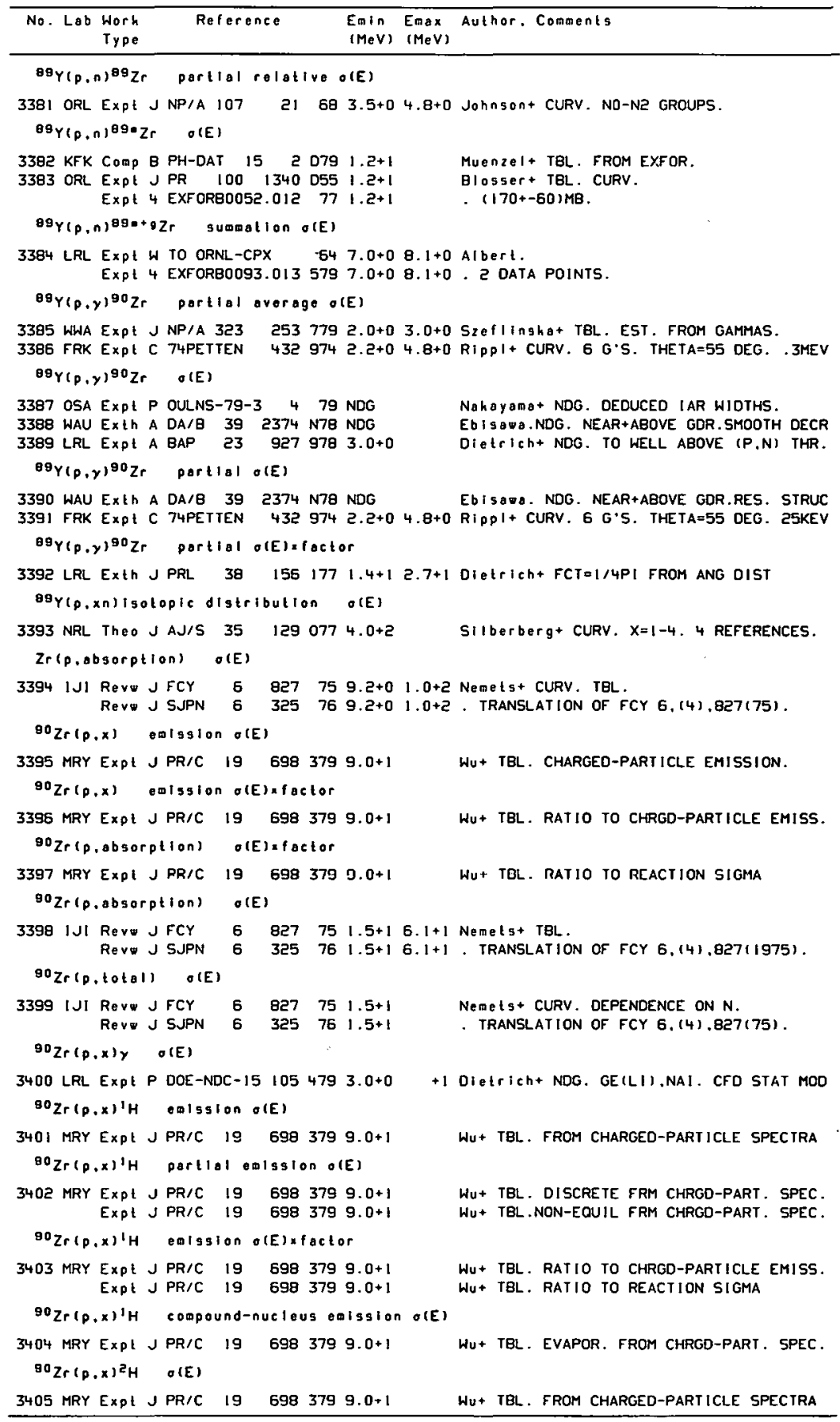




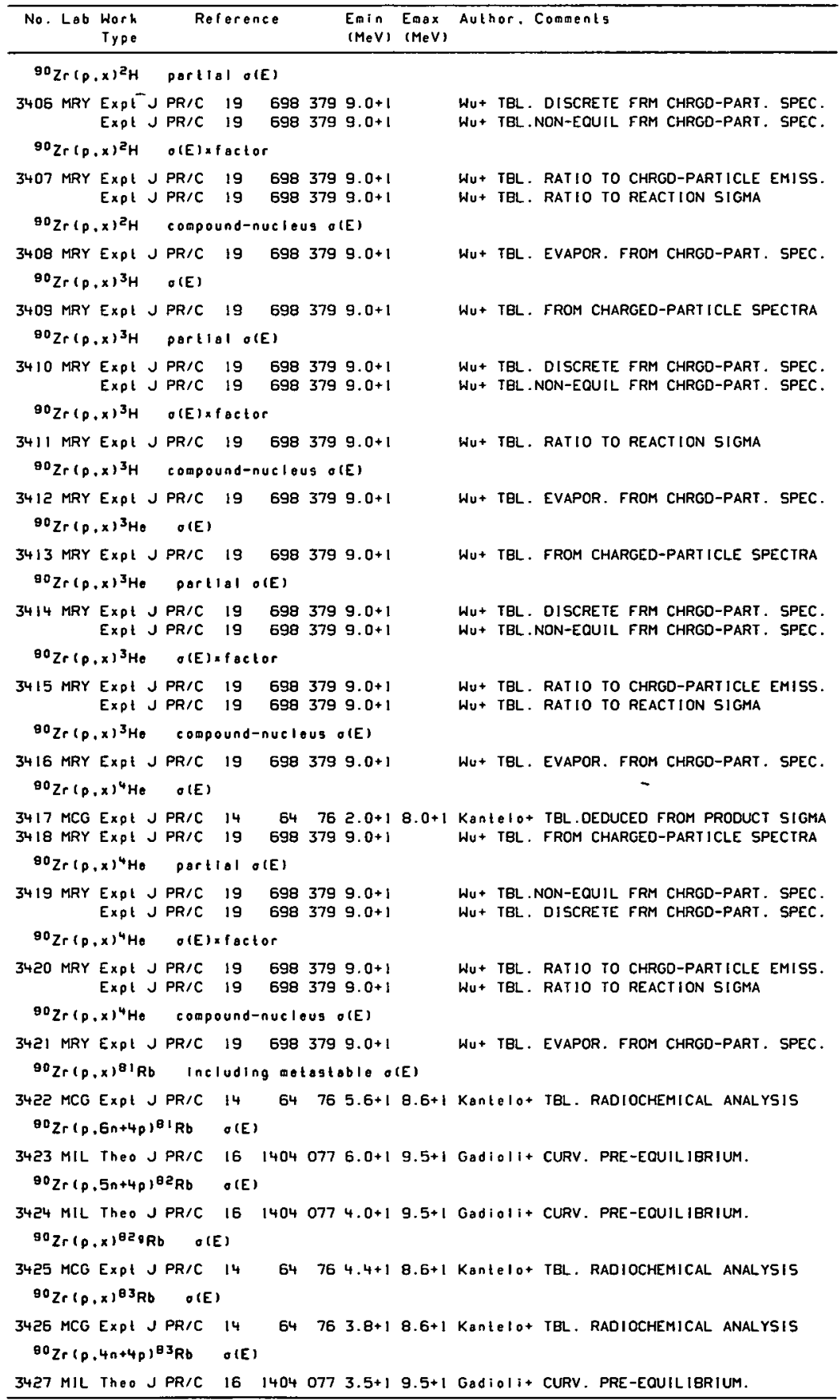




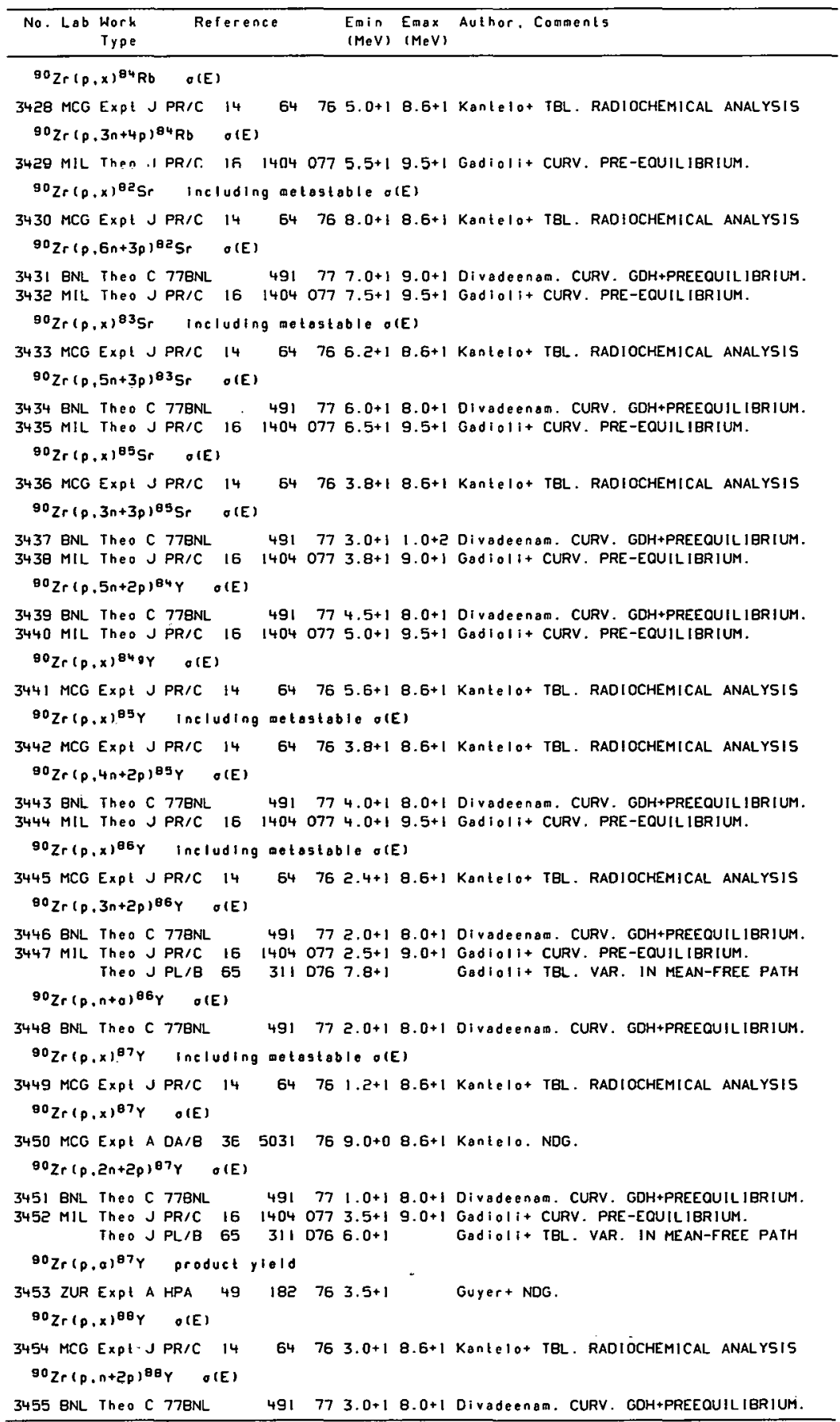


REFERENCES ( c ont)

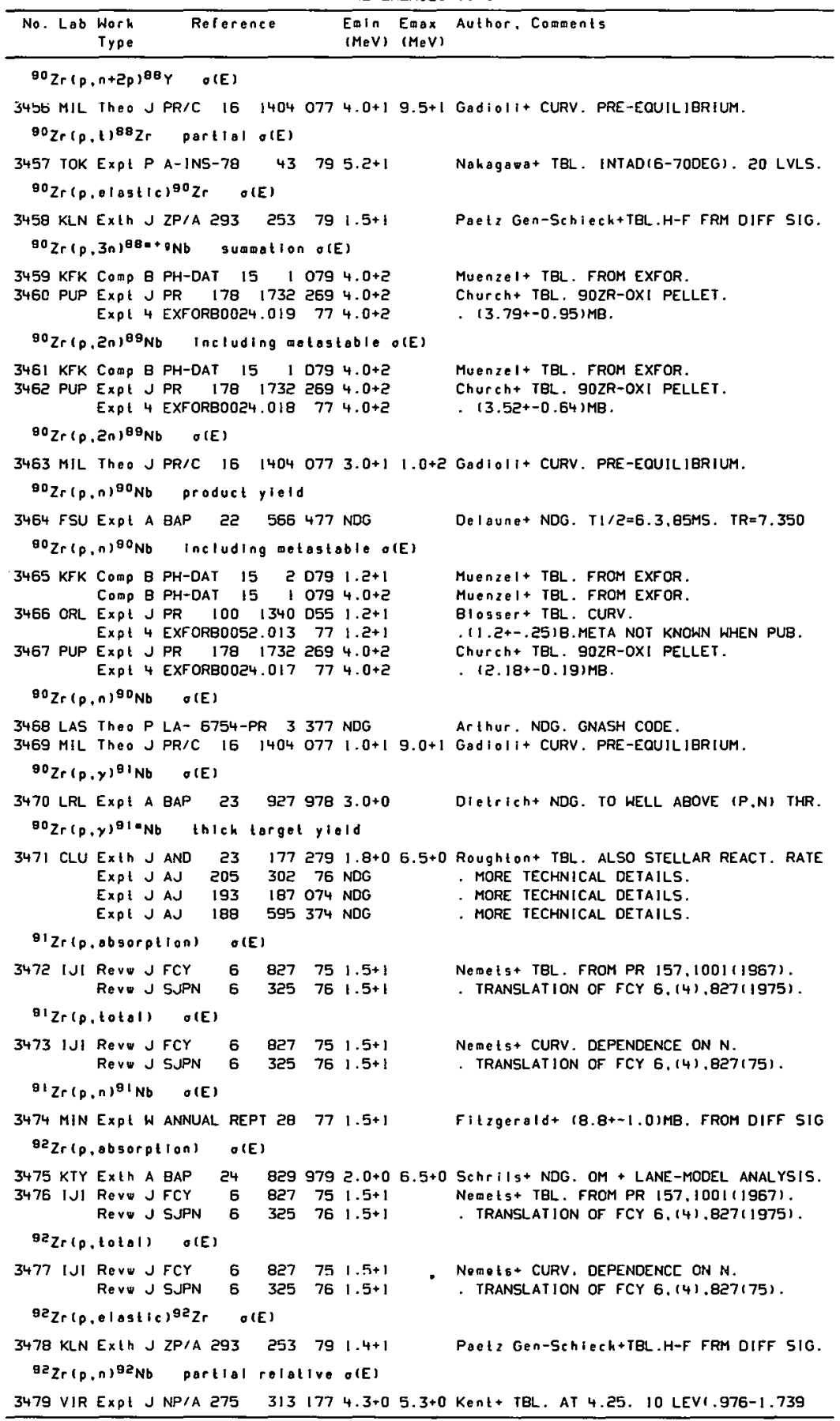


REFERENCES (cont)

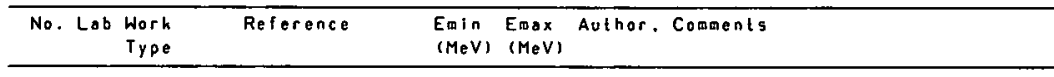

${ }^{82} \mathrm{Zr}(p, n)^{92} \mathrm{Nb} \quad \sigma(E)$

3480 KTY EXPt J PR/C 201700 N79 TR 6.7+0 FIYnn+ NDG. 4PI DETECTOR. DEDUCED SF. 3481 WIT Expl $P$ INDC(SEC)-61 $0773.0+0$ 8.0+0 Sel ischop+ NDG. NEUTRON ANTI-DET. P.333

$$
92 \mathrm{Zr}(\mathrm{p}, n)^{92} \mathrm{Nb} \quad O(E) \times f a c t o r
$$

3482 UEN EXpt C 77TOKYO $4989773.5+07.5+0$ Feigel+ NDG. REDUCED SIGMA.

$$
{ }^{94} \mathrm{Zr}(\mathrm{p} . \mathrm{Qbs} \text { orptIon) O(E) }
$$

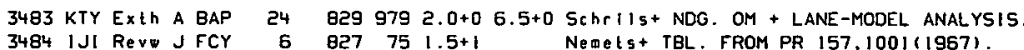

3484 IJI Revw J FCY 5 627 $751.5+1 \quad$ Nemets+ TBL. FROM PR 157,1001(1967).

$$
{ }^{84} \operatorname{Zr}(p, 10(0) \text { O(E) }
$$

3485 iJl Revw J FCY $6827751.5+1 \quad$ Nemets + CURV. DEPENDENCE ON N.

$$
\text { Rev J SJPN } 6 \quad 325761.5+1 \quad \text { TRANSLATION OF FCY } 6 .(4) .827(75) \text {. }
$$

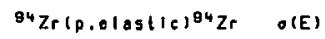

3486 KLN Exth J ZP/A $293253791.1+1 \quad 1.3+1$ Paetz Gen-Schiech+TBL.H-F FRM DIFF SIG.

$$
{ }^{94} \mathrm{Zr}(\mathrm{P}, \mathrm{n})^{94} \mathrm{Nb} \quad O(E)
$$

3487 KIY Expl J PR/C 201700 N79 TR 6.7+0 FIynn+ NDG. 4PI DETECTOR. OEDUCED SF. Expl J IRE 26 $12122792.0+06.5+0$ Gabberd+ CURV. 4PI DETECAOR. CFD THEORY

$$
{ }^{4} \mathrm{Zr}(p, n)^{94} \mathrm{NB} \text { portialo(E) }
$$

3488 CCP Expt J IZV $411665771.7+03.0+0$ Fedorets+ CURV. I2 LVLS FRM G ANG. DIST EXPt J BAS $41 \quad 110 \quad 77 \quad 1.7+0 \quad 3.0+0$. TRANSLATION OF $12 \mathrm{ZV} 41(8), 1665(1977)$

3489 KTY Expt A BAP $23 \quad 5264783.0+07.0+0$ Hershberger+ NDG. 4 PI DETECTOR. DED STR Expt P DOE-NDC-12 $994783.0+0 \quad 7.0+0$ Hershberger+ NDG. 4PI N DETECTOR. EXth P U/KTY-77 $47772.2+02.5+0$ Hagen+CURV.4 LEVELS.CFD HAUSER-FESHBACH Expt A BAP 2166376 NDG Hagen+ NDG. 4PI NEUTRON DETECTOR.

$$
{ }^{94} \mathrm{Zr}(P, n)^{84} \mathrm{NO} \text { relative I, }
$$

3490 CCP EXpi J IZV $401260761.7+03.0+0$ Fedoreis+ CURV. 5 GAMMA EXCIIATION FUNC Expl J BAS $40 \quad 134 \quad 761.7+03.0+0$. TRANS. OF $12 \mathrm{~V} 40(6), 1260(1976)$

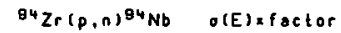

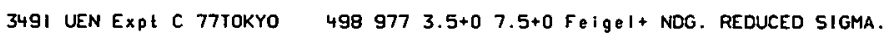

${ }^{8 *} \mathrm{Zr}(p, n)^{940 \mathrm{Nb}}$ thleh target yleld

3492 CLU Exth J AND $23 \quad 1772793.1+0$ 6.0+0 Roughtont TBL. ALSO SIELLAR REACT. RATE Expt J AJ 20530276 NDG Expt J.AJ $193 \quad 187074$ NDG Expt J AJ $188 \quad 595374$ NDC

. MORE TECHNICAL DETAILS.

- MORE techNical details.

$96 \mathrm{Zr}(0, a b s o r p t 10 n) \quad$ (E)

$$
\begin{aligned}
& 3493 \text { iJI Revw J FCY } 6 \quad 827 \quad 756.1+1 \\
& \text { Revw J SJPN } 6 \quad 32576 \text { G. } 1+1 \\
& 96 \operatorname{Zr}(p, \operatorname{tot} 01) \text { o(E) }
\end{aligned}
$$

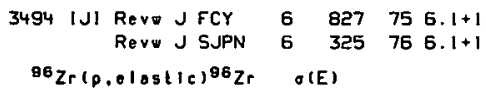

3495 KLN Exth J ZP/A $293 \quad 253 \quad 79 \quad 1.3+1$

${ }^{96} \mathrm{Zr}\left(0,2_{n}\right)^{95_{\mathrm{Nb}}}$ including ootagtable o(E)

3496 KFK Comp B PH-DAT $15,0794.0+2$ 3497 PUP Expt J NP/A $162 \quad 643 \quad 714.0+2$ Expt J PR $178 \quad 17322694.0+2$ Expl 4 EXFORBO024.021 $774.0+2$

$$
{ }^{96} \mathrm{Zr}\left(p, Z_{n}\right) 950 \mathrm{Nb} \quad \text { (E) }
$$

3498 KFK Comp B PH-DAT $15 \quad 10794.0+2$ 3499 PUP Exp! J NP/A $162 \quad 643 \quad 714.0+2$ Expt J PR $178 \quad 1732 \quad 2694.0+2$ Expt 4 EXFORBO024.021 .774.0+2

${ }^{96} Z r\left(p, Z_{n}\right)^{05}$ 'IONb ratio o(E)
Nemets + TBL. FROM PR/C 4.1114(1971).

. IRANSLATION OF FCY 6, (4).827(1975).

Neme 15 + CURV. DEPENDENCE ON N.

- IRANSLATION OF FCY 6.14).827(75).

Paetz Gen-Schieck+TBL.H-F FRM DIFF SIG.

Muenze1+TBL. FROM EXFOR.

Church. TBL- 96ZR-OXI PELLET.

Chureh+ SUPERCEDEO BY NP/A I62,6431711, . $14.45+-0.611 \mathrm{MB}$.

Muenzel+ TEL. FROM EXFOR

Church. TBL. Ti/2=90H.96ZR-OXI PELLET Church + SUPERCEDED BY NP/A $162.643(7)$ ). . $11.06+-0.181 \mathrm{Mg}$. 


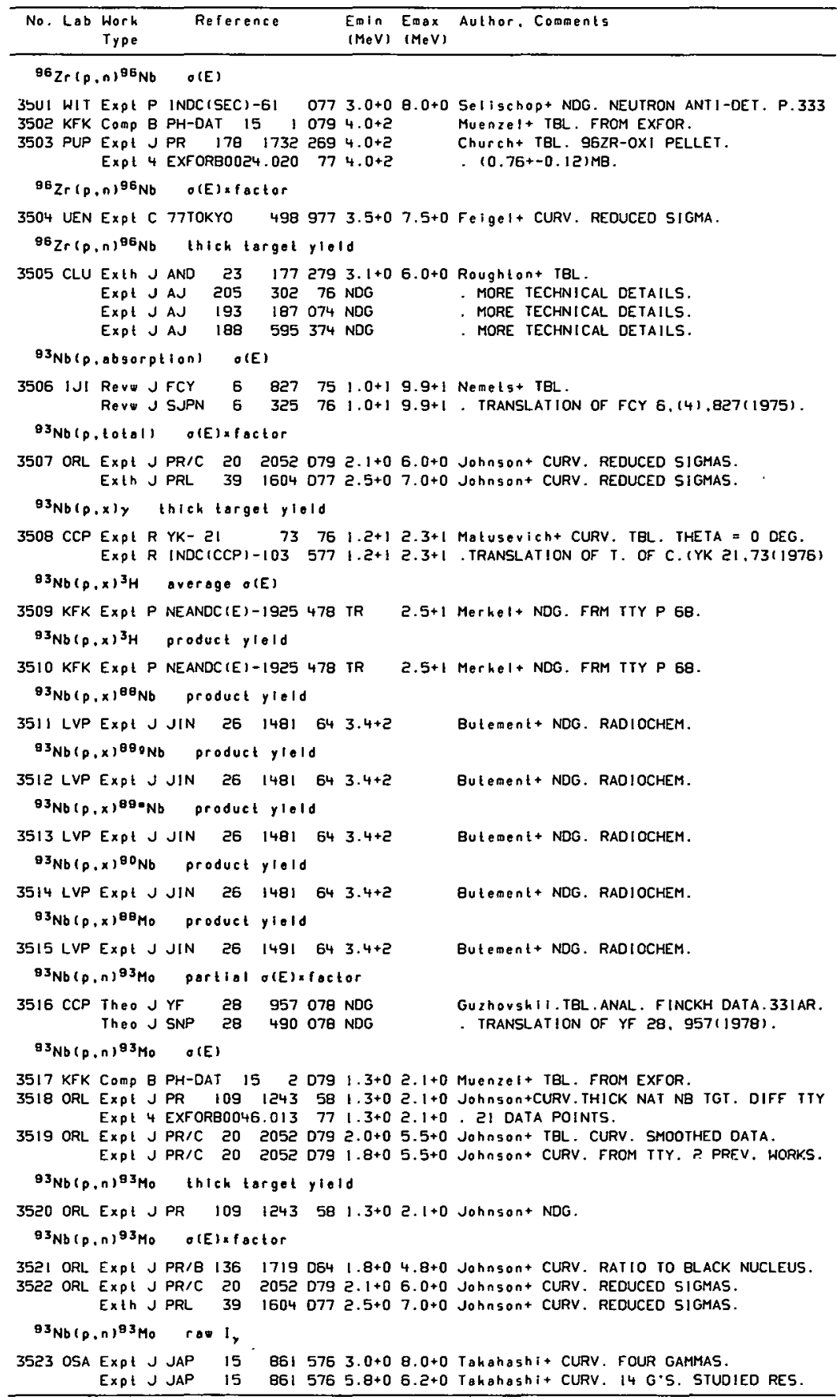




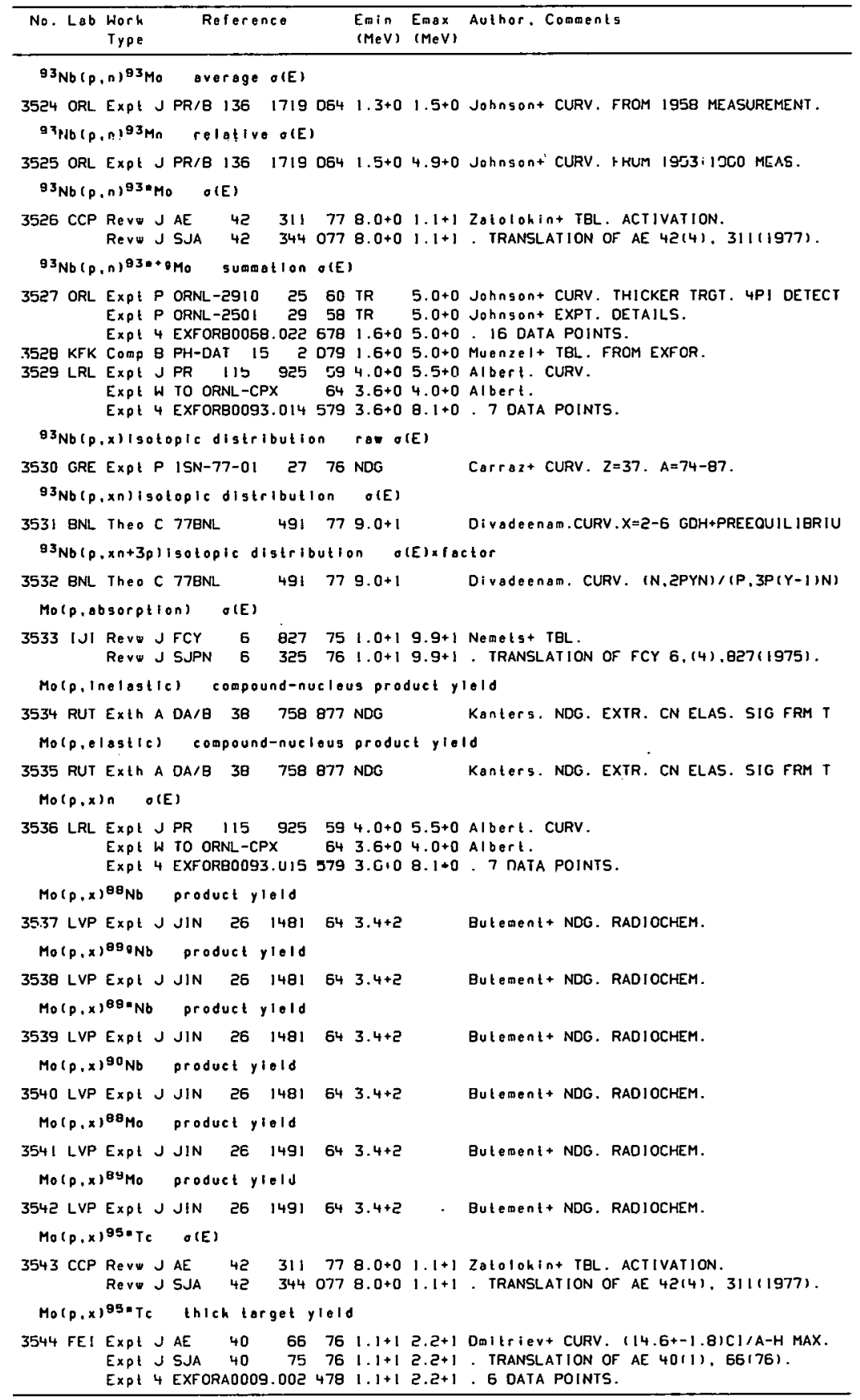


REFERENCES $(\operatorname{con}$ )

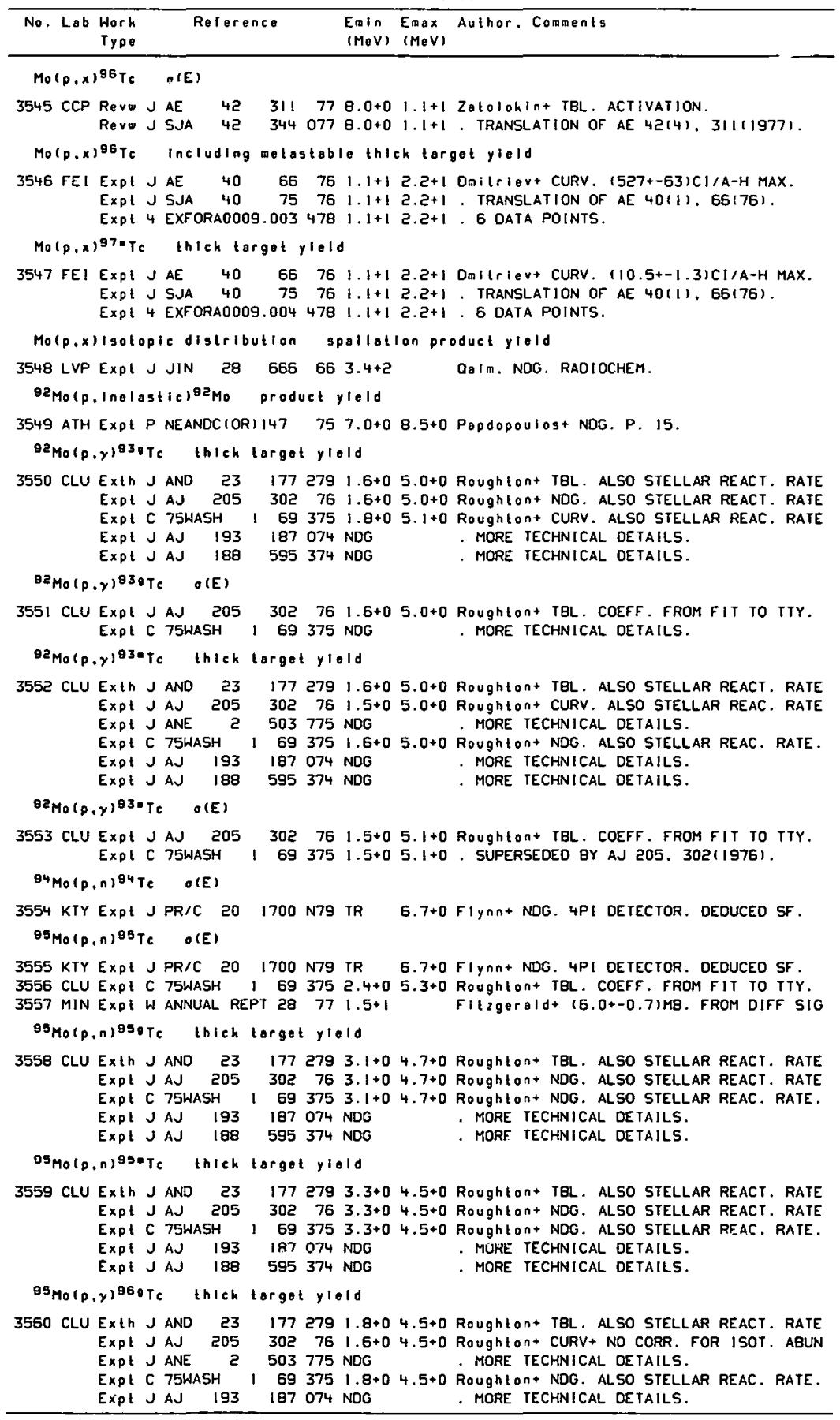


REFERENCES (cont)

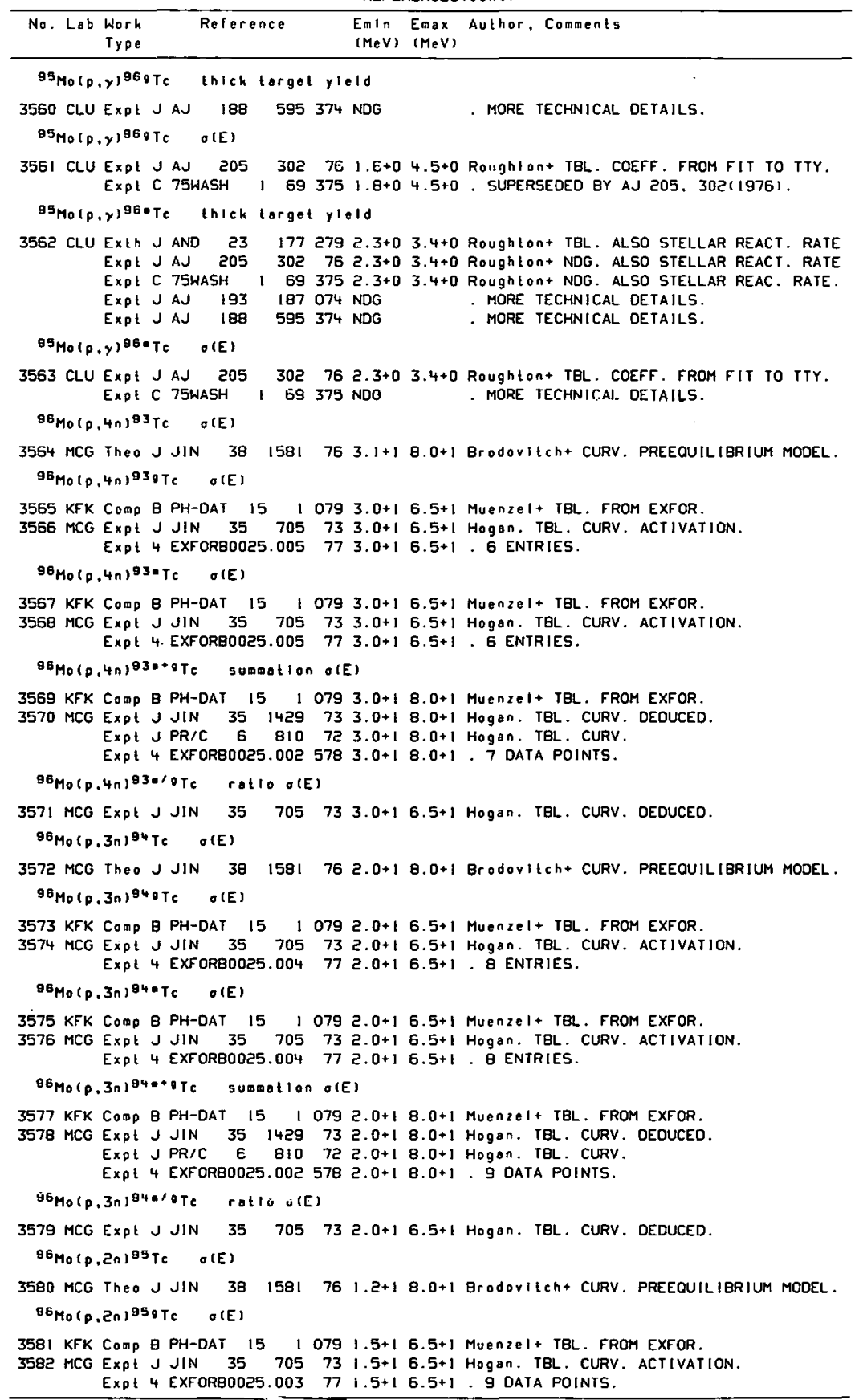


REFERENCES( $\operatorname{con}$ ()

\begin{tabular}{|c|c|c|c|c|c|c|c|}
\hline No. & $\mathrm{Lab}$ & $\begin{array}{l}\text { Work } \\
\text { Type }\end{array}$ & Reference & $\begin{array}{l}\text { Enin } \\
\text { (MeV) }\end{array}$ & $\begin{array}{l}E_{\text {max }} \\
(\mathrm{MeV})\end{array}$ & Author. & Comatents \\
\hline
\end{tabular}

$96 M_{0}(p, 2 n)^{95} \cdot T C \quad \sigma(E)$

3583 KFK COMp B PH-DAT $1510791.5+16.5+1$ Muenze $1+$ TBL. FROM EXFOR.

3584 MCG Expt J JIN $35705731.5+16.5+1$ Hogan. TBL. CURV. ACTIVATION.

Expt 4 EXFORBO025.003 77 1.5+1 6.5+1 - 9 DATA POINTS.

$96 M_{0}\left(p, 2_{n}\right) 95 \bullet+0 T c \quad$ sumation $O(E)$

3585 KFK COmp B PH-DAT $1510791.5+1$ 8.0+1 Muenze1+ TBL. FROM EXFOR.

3586 MCG Expt J JIN $351429731.5+1$ 8.0+1 Hogan. TBL. CURV. DEDUCED.

Expt J PR/C 6 810 72 $1.5+18.0+1$ Hogan. TBL. CURV.

Expt 4 EXFORBO025.002 $5781.5+18.0+1$. 10 DATA POINTS.

96mo(p.en) $93.10 T C$ ratlo o(E)

3587 MCG Expt J JIN $35705731.5+16.5+1$ Hogan. TBL. CURV. DEDUCED.

g8 Mo $(p, n)^{96} T_{C} \quad O(E)$

3588 KTY Expt J PR/C 201700 N79 TR 6.7+0 FIynn+ NOG. 4PI OETECTOR. DEDUCEO SF. 3589 MCG Theo J JIN $381581763.0+0$ 8. $2+1$ Brodovitch+ CURV. PREEQUILIBRIUM MODEL.

$86 M_{0}(p, n)^{860 T C}, O(E)$

3590 KFK COmp B PH-OAT 151 079 1.0+1 6.5+1 Muenzel+ TBL. FROM EXFOR.

359! MCG Expl J JIN $35142973 \quad 1.0+16.5+1$ Hogan. TBL. CURV. ACTIVATION.

Expt 4 EXFORBO025.002 $771.0+16.5+1$. 10 DATA POINTS.

${ }^{86} M_{0}(p, n)^{860 T C}$ thlck targot $y l e l d$

$3592 \mathrm{CLU}$ Exth $\checkmark$ AND $23 \quad 1772793.8+0$ 5.0 3 0 Roughton+ TBL. ALSO STELLAR REACT. RATE Expt J AJ 20530276 TR 5.0+0 Roughton+ CURV+ NO CORR. FOR 1SOT. ABUN Expt J ANE 2503775 NDG - MORE TECHNICAL DETAILS.

Expt C 75WASH, $693753.8+0$ 5.0+0 Roughton+ NDG. ALSO STELLAR REAC. RATE.

Exp! J AJ 193187074 NDG . MORE TECHNICAL DETAILS.

Expl $J$ AJ 188595374 NDG . MORE TECHNICAL DETAILS.

$9 \theta_{0}(p, n)^{96 \bullet T c} \sigma(E)$

3593 KFK COMp B PH-DAT $1510791.0+16.5+1$ Muenzel+ TBL. FROM EXFOR.

3594 MCG Expt J JIN $351429731.0+16.5+1$ Hogan. THL. CURV. ACTIVATION.

Expt 4 EXFORBO025.002 $771.0+16.5+1$. IO DATA POINTS.

g8mo(p,n)980Te thich target yleld

3595 CLU Exth J AND $23 \quad 1772793.8+0$ 5.0+0 Roughtoft TBL. ALSO STELLAR REACT. RATE Expt J AJ $205302763.8+0$ 5.0+0 Roughton+ NDG. ALSO STELLAR REACT. RATE Expt $C$ 75WASH, $693753.8+0$ 5.0+0 Roughton+ NDG. ALSO STELLAR REAC. RATE. Expt J AJ 193187074 NDG . MORE TECHNICAL DETAILS.

Expt J AJ 188595374 NDG - MORE TECHNICAL DETAILS.

$9 M_{0}(\rho, n) 98 \cdot+9 T$ Sumation $O(E)$

3596 KFK COmp B PH-DAT 15 O 079 1.D+1 8.D+1 Muenzel+ TBL. FROM EXFOR.

3597 MCG Expt J JIN $351429731.0+1$ 8.0+1 Hogan. TBL. CURV. DEDUCED

Expt J PR/C $6810721.0+18.0+1$ Hogan. TBL. CURV.

EXDt 4 EXFOREO025.002 $578 \quad 1.0+18.0+1.10$ DATA POINTS.

$86 M_{0}(p, n)^{96010 T C}$ rollo o(E)

3599 MCG Expt J JIN $351429731.0+16.5+1$ Hogan. TBL. CURV. DEDUCED.

$97 M_{0}(p . n)^{97} T C$ partlal relallve o(E)xobundance

3599 ATH Expt J PR/C $201457791.5+0$ 8.0+0 Xenoulis+ CuRV. 3 GAMMAS.

$90_{\text {MO }}(p, n)^{00}$ TC POE $t$,

3600 KTY Expt J PR/C IE 111777 TR $3.1+0$ Mirocle+ CURV. 4 GaMmas.

${ }^{B \theta} M_{0}(p, n)^{g \theta} T e, o(E)$

3601 KTY EXPt J PR/C 201700 N79 TR 6.7+0 FIynot CURV. 4PI DETECTOR. DEOUCED SF.

${ }^{80} \mathrm{MO}_{0}(\mathrm{P},)^{\mathrm{99}} \mathrm{TC}$ O(E)

3602 CLU EXPt C 75WASH $1693751.4+05.3+0$ Roughtont IBL. COEFF. FROM FII TO TTY.

90Molp.yig9-Ts thich torget ylold

$3603 \mathrm{CLU}$ Exth J AND $231772791.6+04.7+0$ Roughton+ TBL. ALSO STELLAR REACT. RATE Exp: J AJ $205302761.6+04.7+0$ Roughton+ NDG. ALSO STELLAR REACI. RATE

Exp! C 75WASH $1693751.6+04.7+0$ Roughton+ NDG. ALSO STELLAR REAC. RATE

Expt J AJ $193 \quad 187074$ NDG . MORE TECHNICAL DETAILS.

Expl J AJ 189595374 NDG . MORE TECHNICAL DEIAILS. 


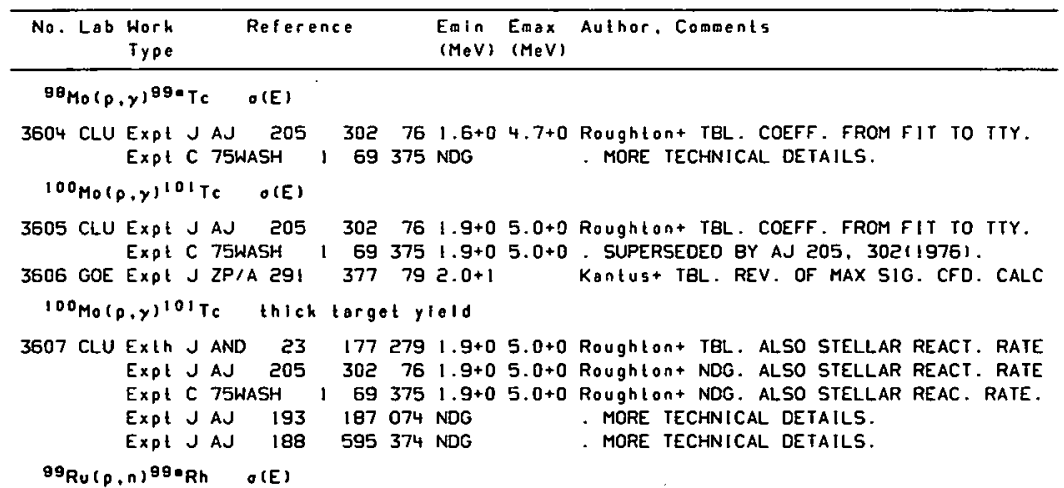

3608 KFK COMP B PH-DAT 15 2 $0741.2+1$ 3609 ORL Expl J PR $100 \quad 1340 \quad 055 \quad 1.2+1$ Expl 4 EXFOREO052.014 $77 \quad 1.2+1$ $101 R_{U}(p, n) 101-R h \quad O(E)$

$3610 \mathrm{KFK}$ Comp B PH-DAT 15 2 D79 $1.2+1$ $36 ! 1$ ORL Exp! J PR $1001340055 \quad 1.2+1$ Expl 4 EXFORBO052.015 $771.2+1$

$$
\text { 103Rh }(p, x) \text { compound-nucleus o(E) }
$$

\author{
Muenzel+ TBL. FROM EXFOR. \\ Blosser TBL. CURV. II/2=4.5 HR. \\ . $(420+-120) \mathrm{MB}$.
}

Muenze I+ TBL. FROM EXFOR

BIosser + TBL. CURV. TI/Z=4.5 DAY. $(365+-100) \mathrm{MB}$.
3612 LRL Expt J PR 128 29I 062 5.0+0 $1.2+1$ Hansen+ CURV. OPTICAL MODEL
103Rh(p.absorption) O(E) $\begin{array}{rllllll}3613 \text { IJI Revw J FCY } & 6 & 827 & 75 & 1.0+1 \\ \text { Rev J SJPN } & 6 & 325 & 76 & 1.0+1\end{array}$
Nenets+ TBL. FROM PR 129,21981(963).
TRANSLATION OF FCY $6,(4), 827(1975)$

103 Rh(p.total) o(E)xfactor

3614 ORL Expt J PR/C 202052 D79 2.5+0 6.5+0 Johnsont CURV. REDUCED SIGMAS.

Exth J PRL $3916040772.5+07.0+0$ Johnsont CURV. REDUCED 5IGMAS.

103 Rh(p.3n)loipd product yleld

3615 OAV Expt A ANS 33224 N79 5.0+1 Lagunas-5olar+ TBL. REVIEW OF PROGRAM.

103 Rh $\left(P, Z_{n}\right) 102 P d \quad O(E)$

3616 TOK Expt J NP 74 8) $651.4+1$ Sahai+ TBL. DEDUCED FRM 2+-0+ TRANS.

$$
103 \text { Rh }(p, n) 103 \text { Pd } O(E)
$$

3617 ORL Expt P ORNL-2910 2560 TR $5.7+0$ Johnsont CURV. THICKER IRGT. 4PI DETECT Expt P ORNL-2501 29 58 TR 5.7+0 Johnson+ EXPT. DETAILS.

EXPt 4 EXFORBO068.023 $6782.3+05.7+0$. 27 DATA POINTS.

$3618 \mathrm{KFK}$ COmp \& PH-OAT 152 279 2.3+0 1.0+1 Muenzel+ TBL. FROM EXFOR.

3619 ORL EXPL J PR/C 20 2052 D79 2.5+0 6.0+0 Johnson+ TBL. CURV. SMOOTHED DATA. Expl J PR/C 20 2052 D79 2.5+0 5.6+0 Johnson+ CURV. FROM TTY+SIG.3 PREV. WRK

3620 LRL ExpIJ JR $115925594.0+05.5+0$ AIbert. CURV. EXDE W TO ORNL-CPX $643.6+04.0+0$ Albert.

EXPL 4 EXFORBO093.016 $5793.6+0$ 8.1+0. 7 DATA POINTS

3621 LRL Expt J PR 128 291 062 5.0+0 1.0+1 Hansen+ CURV. LONG COUNTER. CFD. THEORY Expt 4 EXFORBO066.006 $6785.0+01.0+1$. 6 DATA POINTS.

$103^{\mathrm{Rh}}(p, n) 103 \mathrm{Pd} \quad \sigma(E) \times$ foctor

3622 ORL Exth J PRL $3916040772.5+07.0+0$ Johnson+ CURV. REDUCED SIGMAS.

3623 ORL Expt J PR/C 20 2052 079 2.5+0 6.5+0 Johnson+ CURV. REOUCED SIGMAS.

$$
\text { Pd }(p, a b s o r p t i o n) \quad O(E)
$$

$\begin{array}{rllllll}3624 & 1 J 1 \text { Revo J FCY } & 6 & 827 & 75 & 9.9+1 & \text { Nemets+ TBL. FROM CJP } 44,1847(19661 . \\ \text { Revw J SJPN } & 6 & 325 & 76 & 9.9+1 & \text {. TRANSLATION OF FCY } 6.141 .827119751\end{array}$

$P d(D, X) \oplus \quad \sigma(E)$

3625 ORL EXPl J PR/C $2020520792.0+05.6+0$ Johnson+ CURV. RATIO TO SMOOTH. 2 REF 3626 LRL Expt J PR $115925594.0+05.5+0$ Albert. CURV.

EXPl W TO ORNL-CPX $643.6+04.0+0$ Albert.

EXPt 4 EXFORBO093.017 $5793.6+0$ 8.1+0. 6 DATA POINTS. 
REFERENCES ( conl)

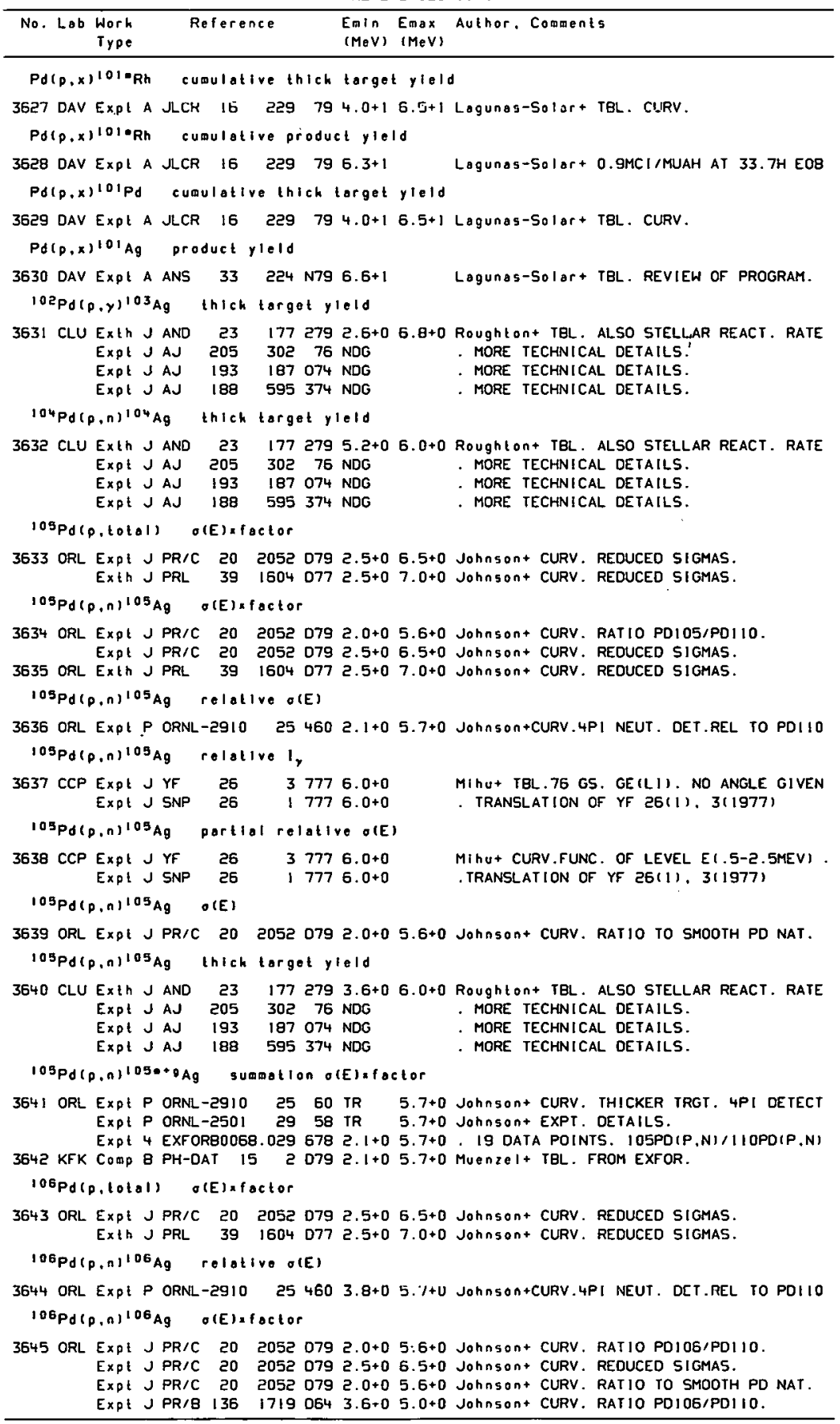


REFERENCES(cont)

\begin{tabular}{|c|c|c|c|c|c|}
\hline No. Lab & $\begin{array}{l}\text { Work } \\
\text { Type }\end{array}$ & Reference & $\begin{array}{l}\text { Emin } \\
(M e V)\end{array}$ & $\begin{array}{l}\text { Emax } \\
(M e V)\end{array}$ & Author. Comments \\
\hline
\end{tabular}

3646 ORL Expt J PR/B $136 \quad 17190643.4+04.1+0$ Johnson+ CURV. FROM 1953+1960 MEAS.

$$
106 P d(p, n) 106 A 9 \text { partial relative o(E) }
$$

3647 OHO Expl J NP/A $3323820796.5+0$

Devant TBL. 18 LEVELS. CFO THEORY.

$$
100 \text { PdP.nII0B,Ag thich target ylold }
$$

3648 CLU Exth J AND $23 \quad 1772794.0+0$ 6.0+0 Roughtont IBL. ALSO STELLAR REACT. RATE Expt J AJ 20530276 NDG MORE TECHNICAL DETAILS.

Expl J AJ $193 \quad 187074$ NDG . MORE TECHINICAL DETAILS.

Expt J AJ 188595374 NDG . MORE TECHNICAL DETAILS.

$$
\text { 106Pd(p.n)106.Ag thich target yield }
$$

3649 CLU Exth J AND $23 \quad 1772794.0+0$ 6.0+0 Roughtont TBL.

Expt J AJ 20530276 NDG . MORE TECHNICAL DETAILS.

Expt J AJ 193187074 NDG . MORE TECHNICAL DETAILS.

Expt J AJ 188595374 NDG . MÜR TECHNICAL DETAILS.

$106 P d(p, n) 1060+9 A g$ sumation olE) a foctor

3650 ORL Expt P ORNL-2910 25 60 TR 5.7+0 Johnson+ CURV. THICKER TRGT. 4PI DETECT Expl P ORNL-2501 29 58 TR $5.7+0$ Johnsont EXPT. DETAILS.

EXPt 4 EXFORB0068.030 678 3.8+0 5.7+0. 19 DATA POINTS. 106PO(P,N)/IIOPD(P,N) 365 I KFK COMP B PH-DAT $1520793.8+0$ 5.7+0 Muenzel+ TBL. FROM EXFOR.

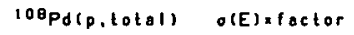

3652 ORL Expl J PR/C $2020520792.5+06.5+0$ Johnson+ CURV. REDUCED SIGMAS. Exth J PRL $3916040772.5+07.0+0$ Johnsont CURV. REDUCED SIGMAS.

100 Pd (p, scattering) 108Pd O(E)

3653 LRL Expt A BAP $21 \quad 662767.0+02.6+1 \mathrm{k} 00 \mathrm{pman+}$ NDG. DEDUCED FROM INELASTIC

100pd(p.InOLOStí)100pd Dartiol O(E),

3654 LRL Expt A BAP $23 \quad 131787.0+02.6+1$ KoOpman+ NDG. CFD TO (N, INL) + OTHER. Expt A DA/B $3827350777.0+02.6+1$ Koopman. NDG. GAMMA YLDS. DED. 2+-0+ 5IG

100 pd (p.inelagtic)100pd o(E)

3655 LRL Expt A BAP 21 G62 $767.0+02.6+1$ Koopman+ NDG. ALSO DEDUCED SCT

108Pd(p,n)100Ag relotive o(E)

3656 ORL Expt P ORNL-2910 25460 2.4+0 5.7+0 Jahnson+CURV. 4PI NEUT. DET. REL TO PO110

100 Pd $(p, n)^{100} \mathrm{Ag}$ part1al o(E)

3657 TIT Expt J JPJ 411830076 2.6+0 4.0+0 Hatlor 1 + CURV. 8 GAMMAS.

100 Pd $(p, n) 100 \mathrm{Ag}$ o(E) * factor

3658 ORL Expl J PR/C 202052079 2.0+0 5.6+0 Johnson+ CURV. RATIO PDI08/PDII0.

Expt J PR/C $2020520792.5+06.5+0$ Johnsont CURV. REDUCED SIGMAS.

Expl J PR/B $13617190642.5+0$ 5.0+0 Johnson+ CURV. RATIO PDIOB/POIIO.

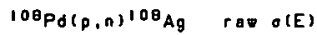

3659 ORL Expt J PR/B 1361719 DG4 2.5+0 3.2+0 Johnson+ CURV. FROM 1953+1960 MEAS.

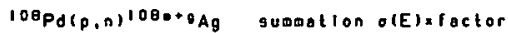

3660 ORL Expt P ORNL-2910 25 60 TR 5.7+0 Johnson+ CURV. THICKER TRGT. 4PI DETECT Expt P ORNL-2501 $2958 \mathrm{TR} 5.7+0$ Johnsont EXPT. DETAILS.

Expt 4 EXFORB0068.031 $6782.4+05.7+0$. 29 DATA POINTS. IOBPD(P.N)/IIOPD(P.N)

3661 KFK Comp B PH-DAT 15 2 D79 2.4+0 5.7+0 Muenzel+ TBL. FROM EXFOR.

110 Pd (p,total) $O(E)=$ factor

3662 ORL Expt J PR/C $2020520792.5+0$ 6.5+0 Johnsont CURV. REDUCED SIGMAS.

Exth J PRL $3916040772.5+07.0+0$ Johnsont CURV. REDUCED SIGMAS.

$110 P d(p, n+p) 100 p d$ including wetaglable o(E)

3663 ORL Expt J PR $94 \quad 620554$ TR $2.4+1$ COhEn+ CURV. CFD. TO STATISTICAL CALC. Expl 4 EXFORBO072.003 $6781.1+12.4+1$. 14 DATA POINTS.

$3654 \mathrm{KFK}$ Comp 8 PH-DAT 15 2 $0791.1+12.4+1$ Muenzel+ TBL. FROM EXFOR.

$110 \mathrm{Pd}(\mathrm{P}, n) 110 \mathrm{Ag}$ O(E) $\times$ factor

3655 ORL Expl J PR/C 20 2052 D79 2.0+0 5.6+D Johnson+ CURV. RATIO PDI09,105,105/110

ExplJ PR/C 20 2052 $0792.5+0$ 6.5+0 Johnsont CURV. RELULED SIGMAS.

Exth J PRL 391604077 2.5+0 7.0+0 Johnson+ CURV. REDUCED SIGMAS. 
REFERENCES (cont)

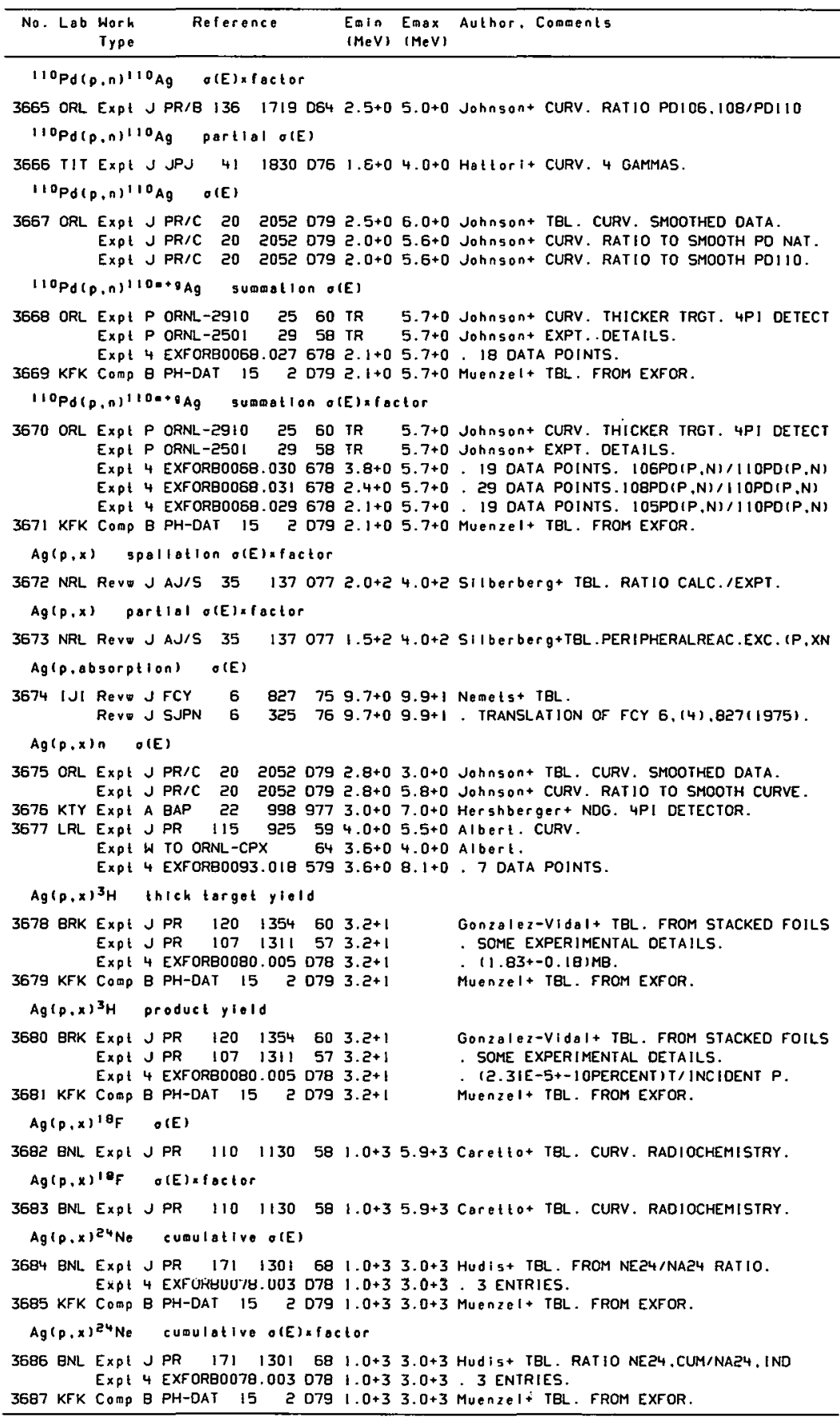




\begin{tabular}{|c|c|c|c|c|c|}
\hline No. Lab & $\begin{array}{l}\text { Work } \\
\text { Type }\end{array}$ & Reference & $\begin{array}{l}\text { Enln. } \\
\text { (MeV) }\end{array}$ & $\begin{array}{l}\text { Emax } \\
(\operatorname{MeV})\end{array}$ & Author. Comments \\
\hline
\end{tabular}

$A g(p, x)^{24} \mathrm{Na}$ independent including metastable o(E)

3688 BNL Expt J PR $17 ! 1301681.0+33.0+3$ Hudis+ TBL. FROM NEZ4/NA24 RATIO. Expt 4 EXFORB0078.003 078 $1.0+33.0+3.3$ ENTRIES.

3689 KFK Comp B PH-DAT 152 D79 $1.0+33.0+3$ Muenzel+ TBL. FROM EXFOR.

Agip,x ev $\mathrm{Na}$ independent including detastable o(E)alallui

3690 ENL EXPl J PR $1711301681.0+33.0+3$ HudIS+ TEL. RAT10 NE24, CUM/NA24, IND EXPt 4 EXFORBO07B.003 D78 $1.0+3 \quad 3.0+3 \cdot 3$ ENTRIES.

3691 KFK Comp a PH-DAT I5 2 D79 $1.0+33.0+3$ Muenzel+ IBL. FROM EXFOR.

$A g(p, x)^{24} N_{0}$ cumulative? O(E)

3692 BNL Expl J PR $171 \quad 1301681.0+3 \quad 3.0+3$ Hudis+ TBL. CORRECTED FOR RVSD MON SIG Expt JPR $1101130581.0+35.9+3$ Caretto+ TBL. CURV. RAOIOCHEMISTRY. EXPt 4 EXFORB0078.003 D78 $1.0+3 \quad 3.0+3.3$ ENTRIES.

3693 KFK COMp B PH-DAT 15 2 D79 $1.0+33.0+3$ Muenzel+ TBL. FROM EXFOR.

$A g(D, x)^{24}{ }^{4}$ cumulative? O(E) a factor

3694 BNL Expt J PR $1101130581.0+35.9+3$ Carellot TBL. CURV. RADIOCHEMISTRY.

$104 \mathrm{Ag}(p, n) 104 \mathrm{Cd}$ partial product yleld

3695 OHO Expt A DA/B 375722577 TR $1.4+0$ Devan. NDG.

$100 \mathrm{AgIP,ON106} \mathrm{Cd}$ partlal product yleld

3696 OHO Expt A DA/B 375722577 TR $1.4+0$ Devan. NDG.

107 Ag(P.QBSOCDtIOO) $\sigma(E)$

3697 KTY EXPl J PR/C $21 \quad 8963803.0+0$ 6.0+0 Hershberger+ NDG. FROM $[P, N)+(P, P)$.

$107 \mathrm{Ag}(p .10 t 01)$ o(E) x foctor

3698 ORL Expt J PR/C $2020520792.5+0$ 6.5+0 Johnsont CURV. REDUCED SIGMAS.

Exth J PRL 391604 D77 $2.5+07.0+0$ Johnson+ CURV. REDUCED SIGMAS.

$107 \mathrm{Ag}(0.0) 104 \mathrm{Pd}$ O(E)

3699 MIL Expt J NC/A $391715772.3+1 \quad$ Coll1+ $(42.7+-8.51 M$ E. E/A INT.

$107 \mathrm{Ag}(p, n+p) 1060 \mathrm{Ag} \quad \sigma(E)$

3700 ORL EXPt JPR 94 G20 554 TR $2.4+1$ Cohent CURV. CFD. TO STATISTICAL CALC. Expt 4 EXFORBD072.004 $678 \quad 1,3+12.4+1$. 12 DATA PO!NTS.

$370 !$ KFK Comp 9 PH-DAT 15 2 D79 $1.3+12.4+1$ Muenzel+ TBL. FROM EXFOR.

$107 \mathrm{Ag}(p, n)^{107} \mathrm{Cd} \quad$ O(E)

3702 KFK COMP B PH-DAT I5 $20791.9+01.0+1$ Muenzel+ TBL. FROM EXFOR.

3703 ANL EXPL J PR 128 280 O62 1.9+0 1.0+1 Wing+ IBL. CURV. RESIO. RADIOACTIVITY, Expl R TID-126S6 $611.9+0 \quad 1.0+1$. SUPERSEDED.

Expt 4 EXFORB0065.006 678 1.9+0 1.0+1. 24 DATA POINTS.

3704 ENL Expt J PRic $918195742.5+02.3+1$ Colle+ TBL. CURV. ACTIVATION METHOD. Expt 4 EXFORB0057.004 $772.5+02.3+1.46$ DATA POINTS.

3705 ORL EXPt J PR/C 202052 D79 2.8+0 5.8+0 Johnson+ CURV. RATIO TO SMOOTH AG NAT.

3706 KTY Expt J PR/C $21 \quad 8963803.0+0$ 6.0+0 Hershberger+ TBL. SMOOTHEO DATA. Exp: J PR/C $21 \quad 8963803.0+06.0+0$. PAPS PRVCA-21-896-S FOR DATA.

3707 ETH Expl J HPA $24 \quad 3 \quad 5 ! 3.3+0$ 6.4+0 BIaser+ TBL. NATURAL FOIL. EXPt 4 EXFORBO048.007 $773.3+06.4+0$. 18 DATA POINTS.

$107 \mathrm{Ag}(P, \cap)^{107} \mathrm{Cd} \quad$ o(E) *factor

3708 ORL Exth J PRL $3916040772.5+07.0+0$ Johnson+ CURV. REDUCED SIGMAS.

3709 ORL Expt J PR/C 202052 D79 2.5+0 6.5+0 Johnsont CURV. REDUCED SIGMAS.

$109_{A g}(p, a b s o r p t i o n) \quad O(E)$

3710 KTY EXPl J PR/C $21 \quad 8963803.0+0$ 6.0+0 Hershberger+ NDG. FROM $(P, N)+(P, P)$.

$\log _{A g}(p, t o t, 1)$ o(E) af actor

3711 ORL Expl J PR/C 202052 D79 2.5+0 6.5+0 Johnson+ CURV. REDUCED SIGMAS.

Expt JPR/C 20 2052 079 2.5+0 6.5+0 Johngont CURV. EFFECI OF PAR VAR.

Exth J PRL 391604 D77 2.5+0 7.0+0 Johnson+ CURV. REDUCEO SIGMAS.

$\log _{A g}(p, a) 106 p d \quad$ of $(E)$

3712 MIL Expt J NC/A $39 \quad 1715772.3+1 \quad$ Collit $126.1+-5.2 I M B$. E/A INT.

${ }^{109} \mathrm{Ag}(0, n)^{109} \mathrm{Cd} \quad \mathrm{O}(\mathrm{E})$

3713 ORL Expl P ORNL-2910 $2560 \mathrm{TR} 5.8+0$ Johnson+ CURV. IHICKER TRGJ, 4PI DETECT Expt P ORNL-2501 2958 TR $5.8+0$ Johnson+ EXPT. OETAILS. 


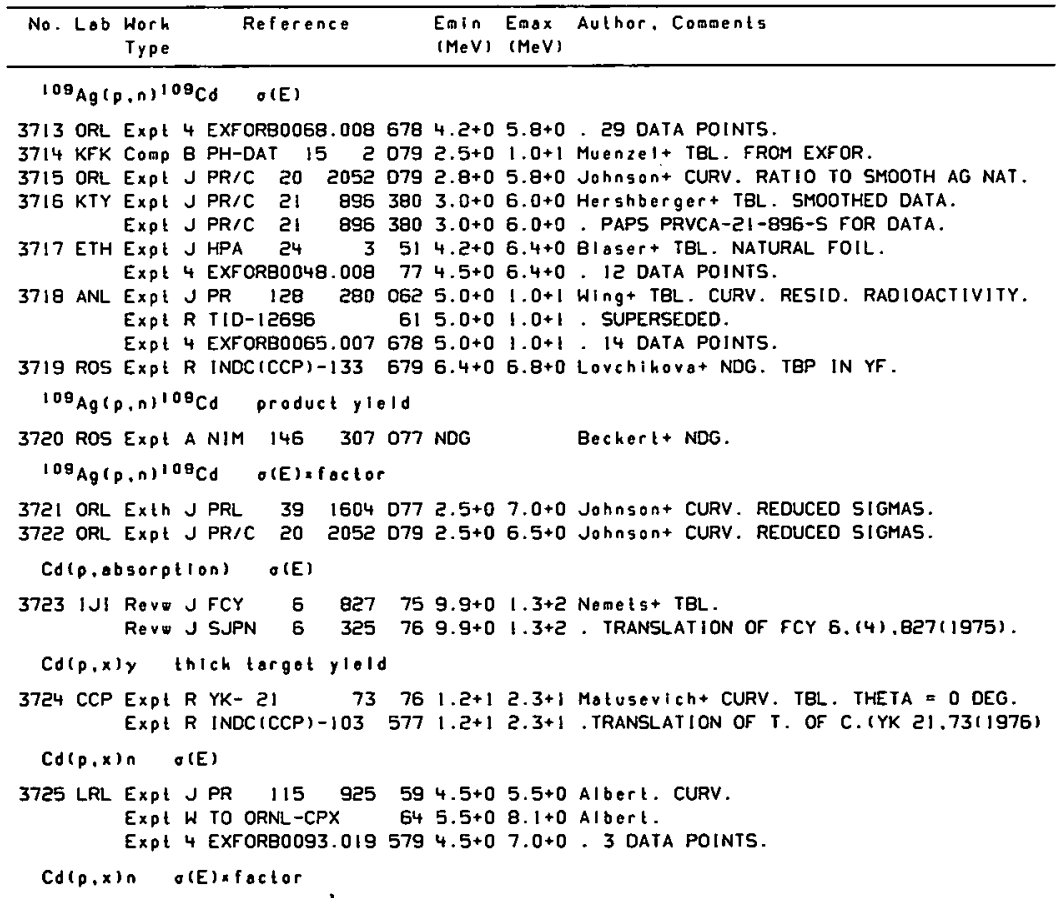

3726 ORL Expt J PR/C 20 2052 D79 2.5+0 5.8+0 Johnsont CURV. RATIO TO SMOOTH CDII6.

Cd $(p, x)^{3} H$ thick torget yield

3727 BRK Expt J PR $120 \quad 1354 \quad 60 \quad 3.2+1$

Expt J PR $107 \quad 1311573.2+1$

Expt 4 EXFORBO080.006 $0783.2+1$

3728 KFK COMp 8 PH-DAT 15 2 D79 $3.2+1$

$\mathrm{Cd}(p, x)^{3} \mathrm{H}$ product yield

3729 BRK Exp: J PR $1201354603.2+$

Exp: J PR $107 \quad 131157 \quad 3.2+1$

EXpt 4 EXFORBO080.006 $0783.2+1$

3730 KFK Comp 9 PH-DAT 152 D79 $3.2+1$

Cd $(p, x)$ llitin product yiold

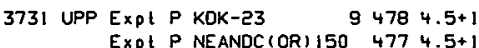

Expt P NEANDC (OR) $150 \quad 4774.5+1$

Expt P NEANDCIORIJ46 $4764.5+1$

Cd $(0, x)^{111}$ In thitk torget yield

3732 UJV Comp R UJF-4199-B $2775.0+02.5+1$ Bem+ IBL.

3733 AML Revw J IRE 26 2281 $4791.5+1$ Chaudri. TBL. CFO EXP. TO CALC.

$\operatorname{Co}(0, x)^{11} \ln$ In $O(E)$

3734 CCP Revid AE $42311778.0+01.1+1$ ZatolohInt TEL. ACTIVATION.

Revw J SJA $42 \quad 3440778.0+01.1+1$. TRANSLATION OF AE $42(4), 311(1977)$.

$C d(p, x)^{114}$ In thick torget yleld

3735 UJV Comp R UJF-4199-B $2776.0+02.2+1$ Bent IBL.

${ }^{106} \mathrm{Cd}(p, y) 107 \mathrm{In}$ thick target yleld

$3736 \mathrm{CLU}$ Exth $J$ AND $23 \quad 1772793.0+0$ 5.0+0 Roughton+ TBR. ALSO STELLAR REACT. RATE Expt $\checkmark$ AJ 20530276 NDG

Expt J AJ $193 \quad 187074$ NDG

Expt $\rfloor$ AJ $188 \quad 595374$ NDG

- more iechinical detaILs.

- MORE TECHNICAL DETAILS.

- more technical detalls.

SOME EXPERIMENTAL OETAILS.

Muenzel+ TBL. FROM EXFOR.

Gonzalez-VIdal+ IBL. FROM STACKED FOILS

DIINCIDENT $P$.

Lundquist + NDG.

Lundquist+NDG. PROD. LABELLED CMP. P. 15 Lundquist+NDG. PROD. LABELLED CMP. P. I6 


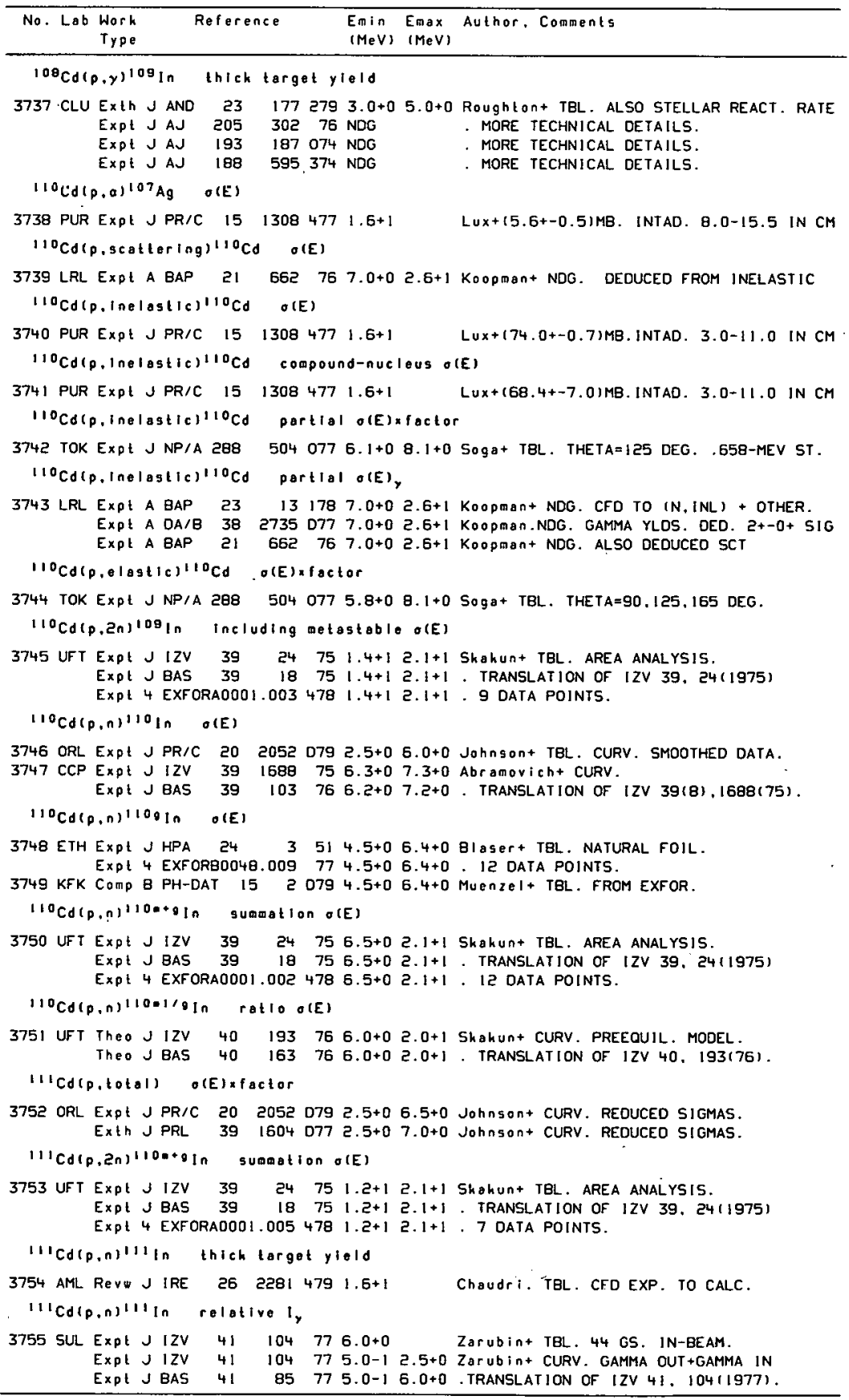


REFERENCES $(c$ on $t)$

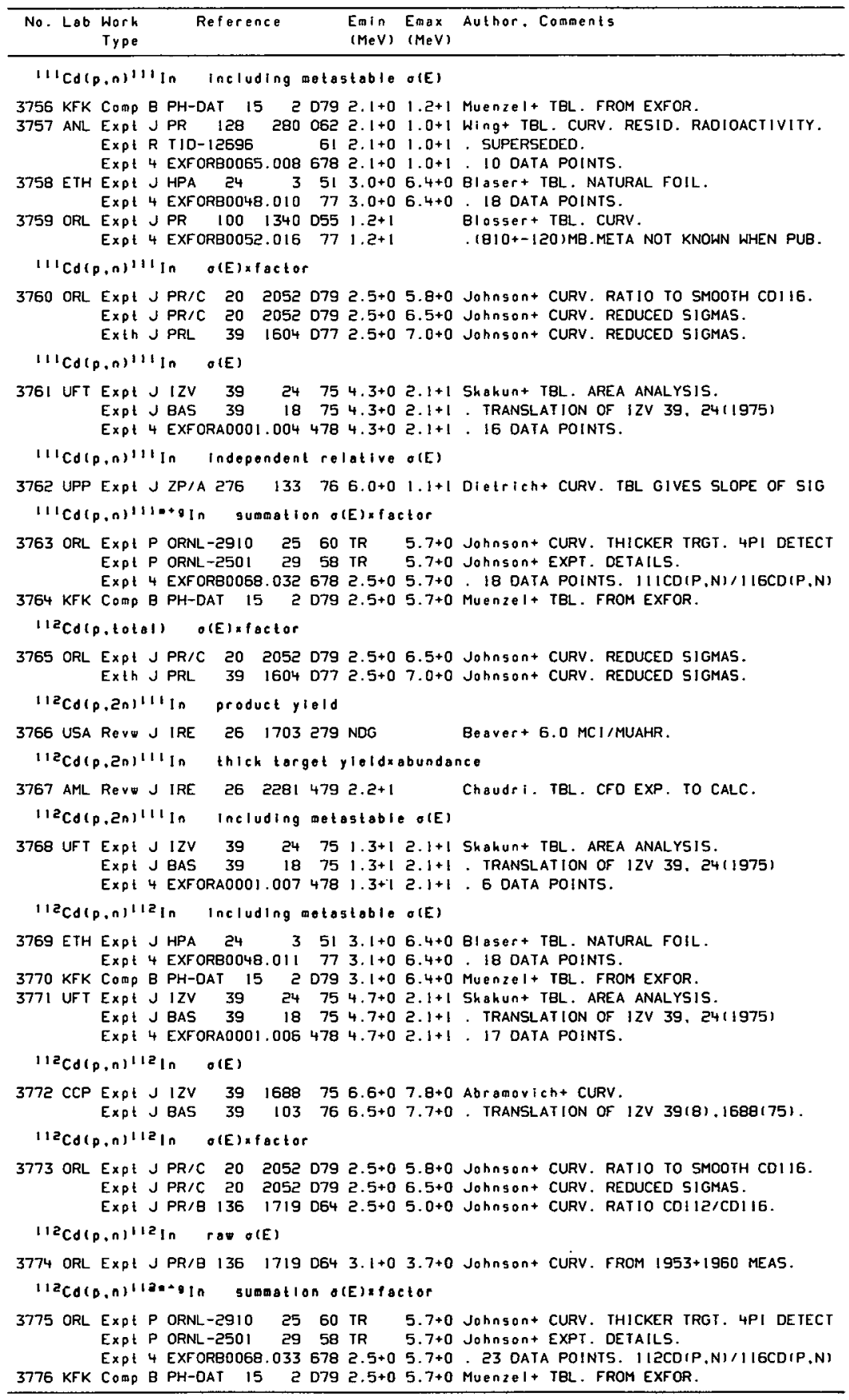


REFERENCES (cont)

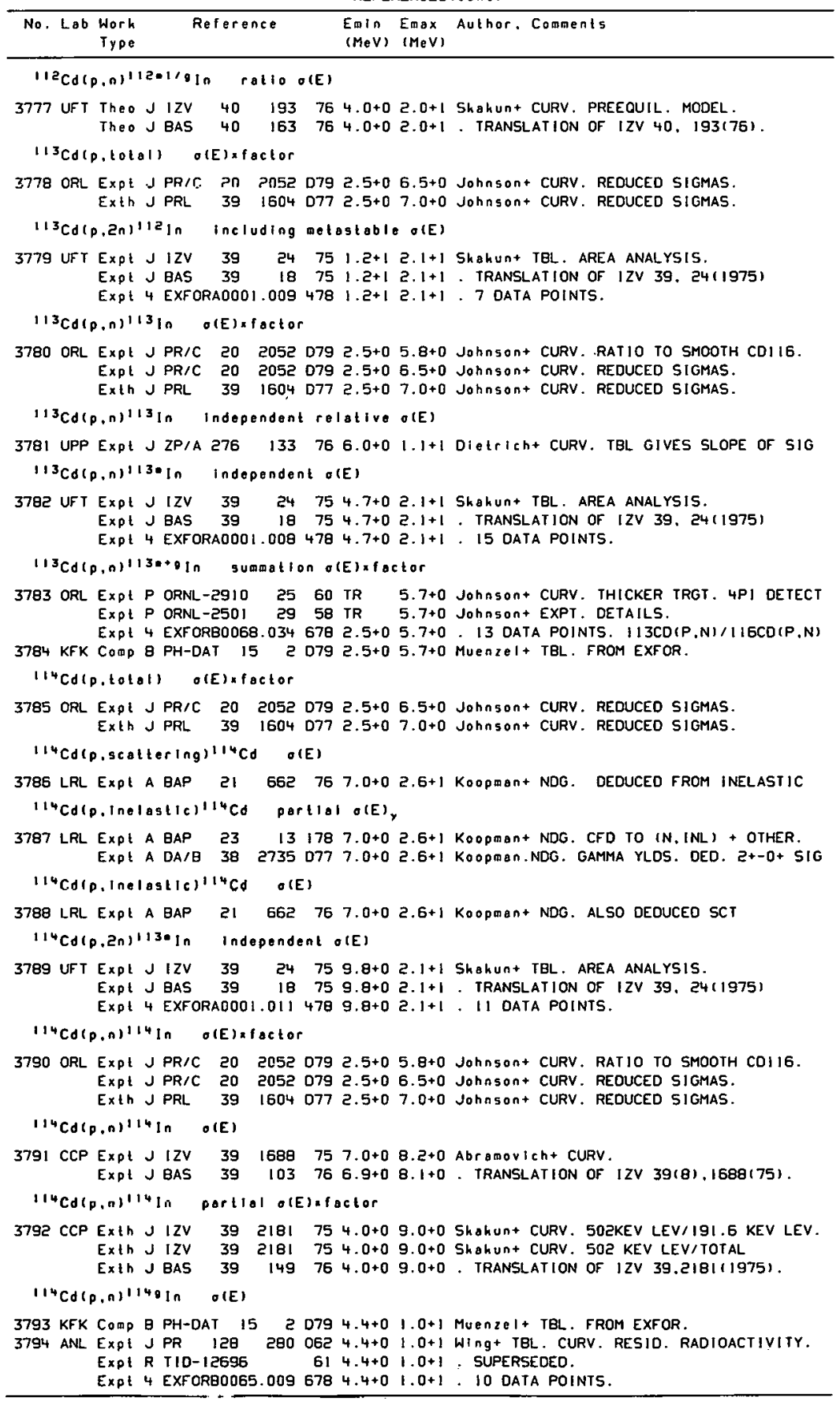




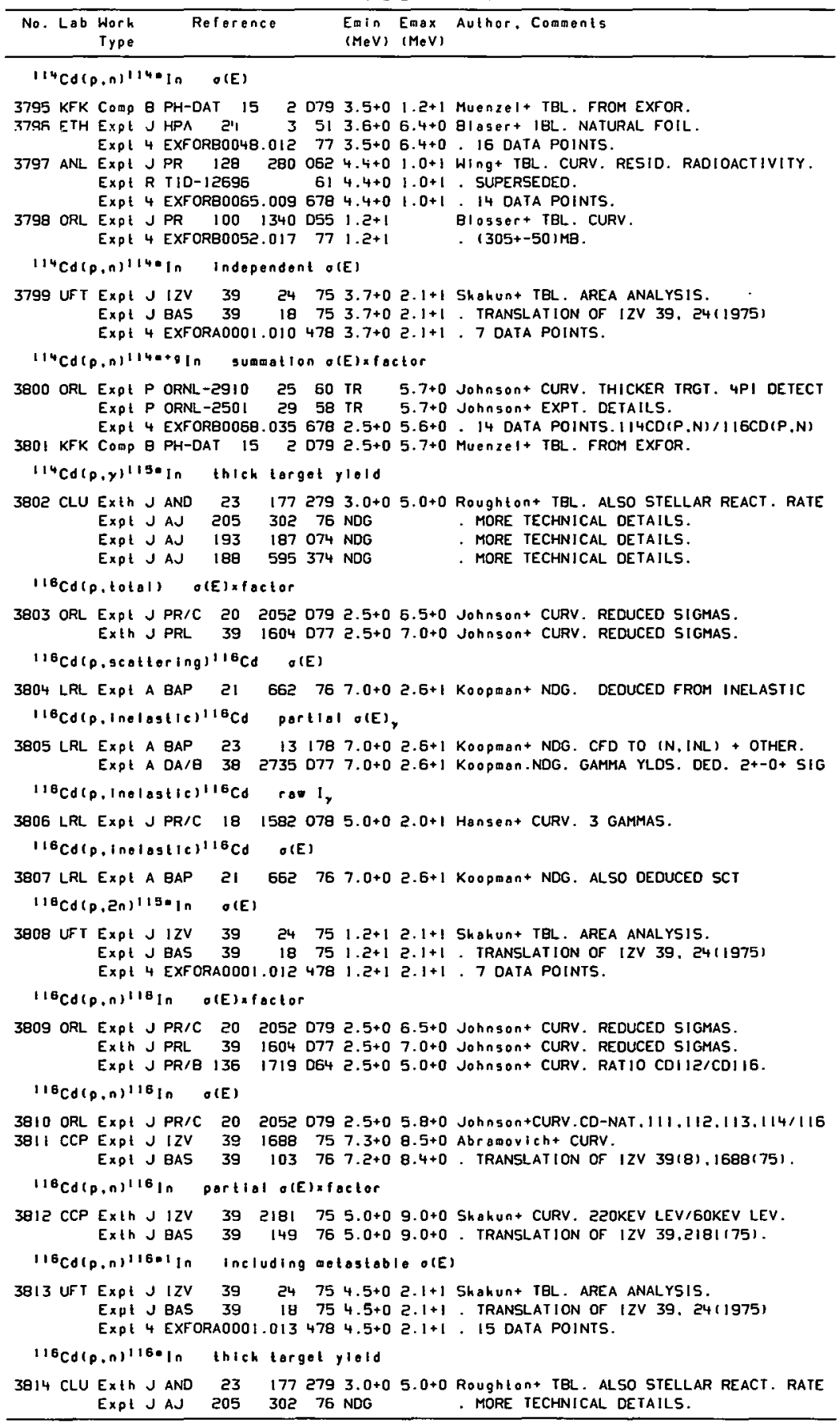


REFERENCES ( c ont)

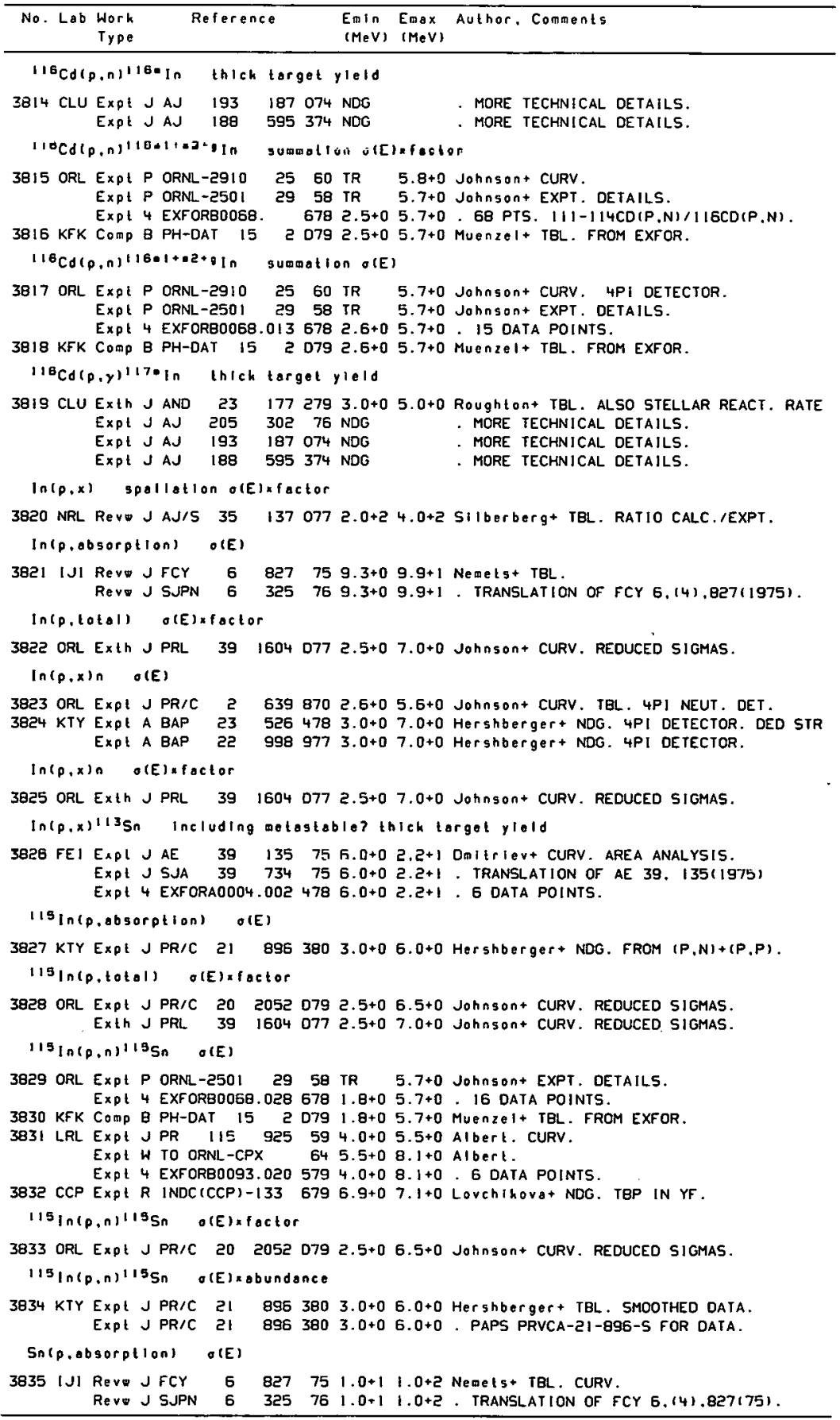


REFERENCES ( c on t)

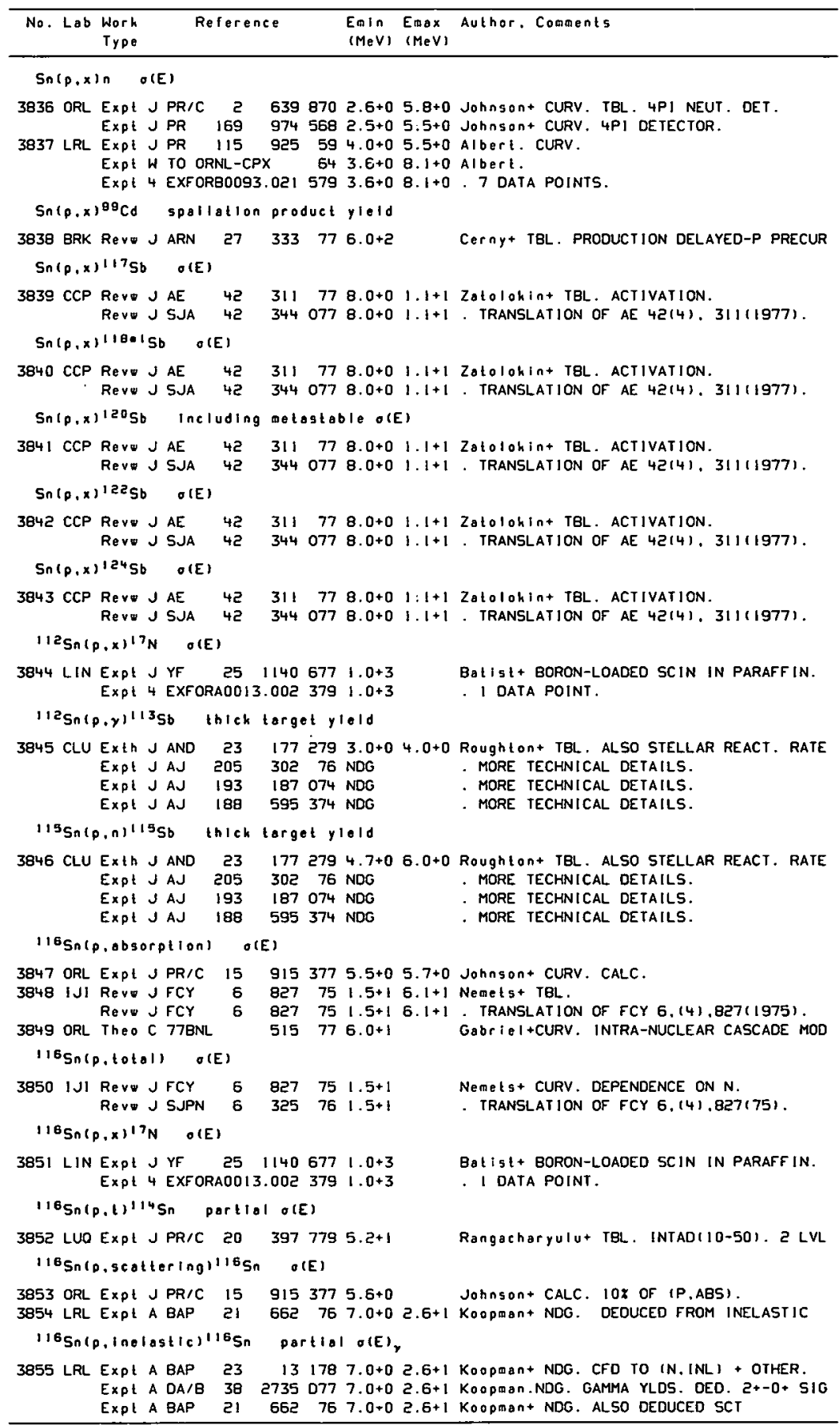


REFERENCES ( c on t)

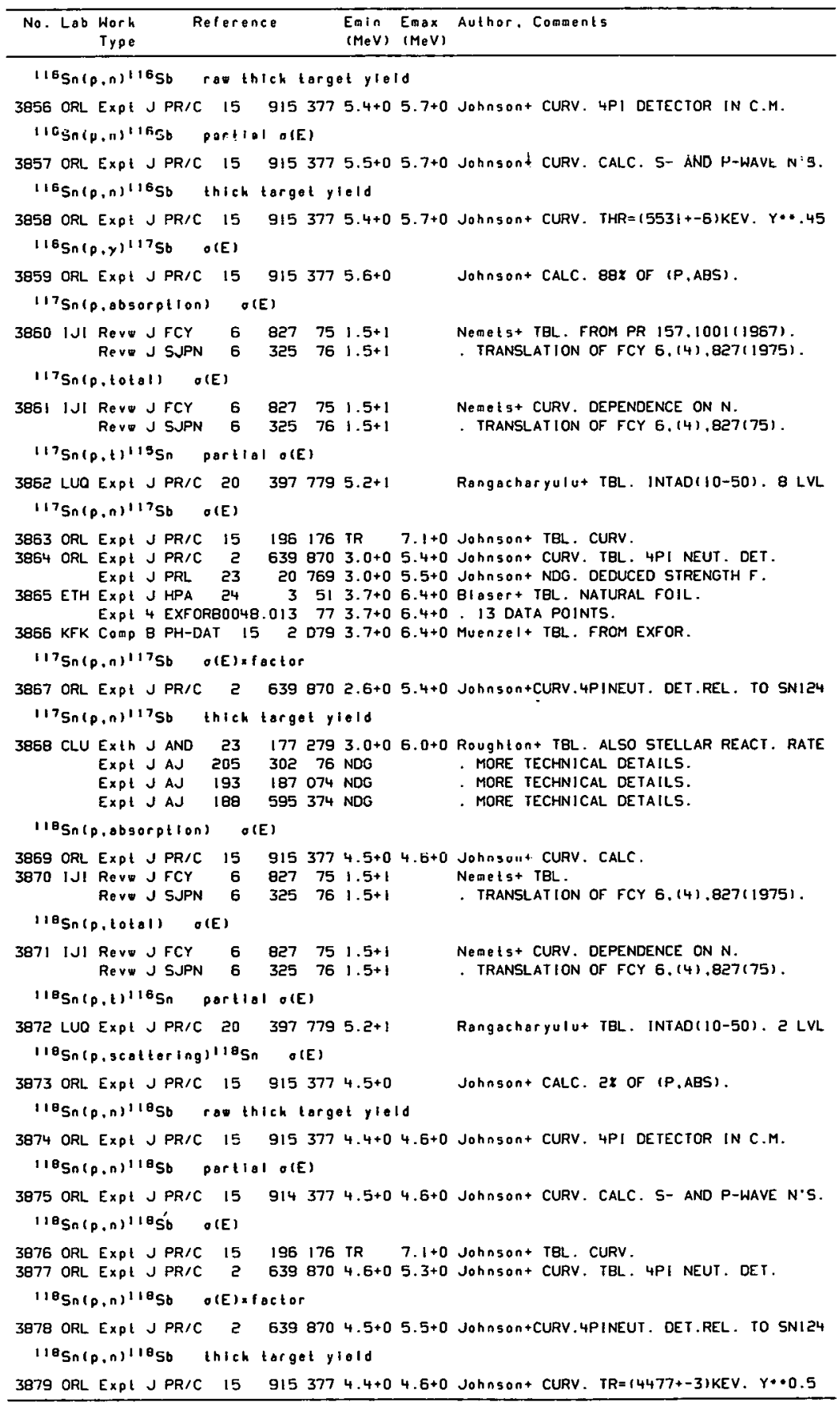


REFERENCES (c ont)

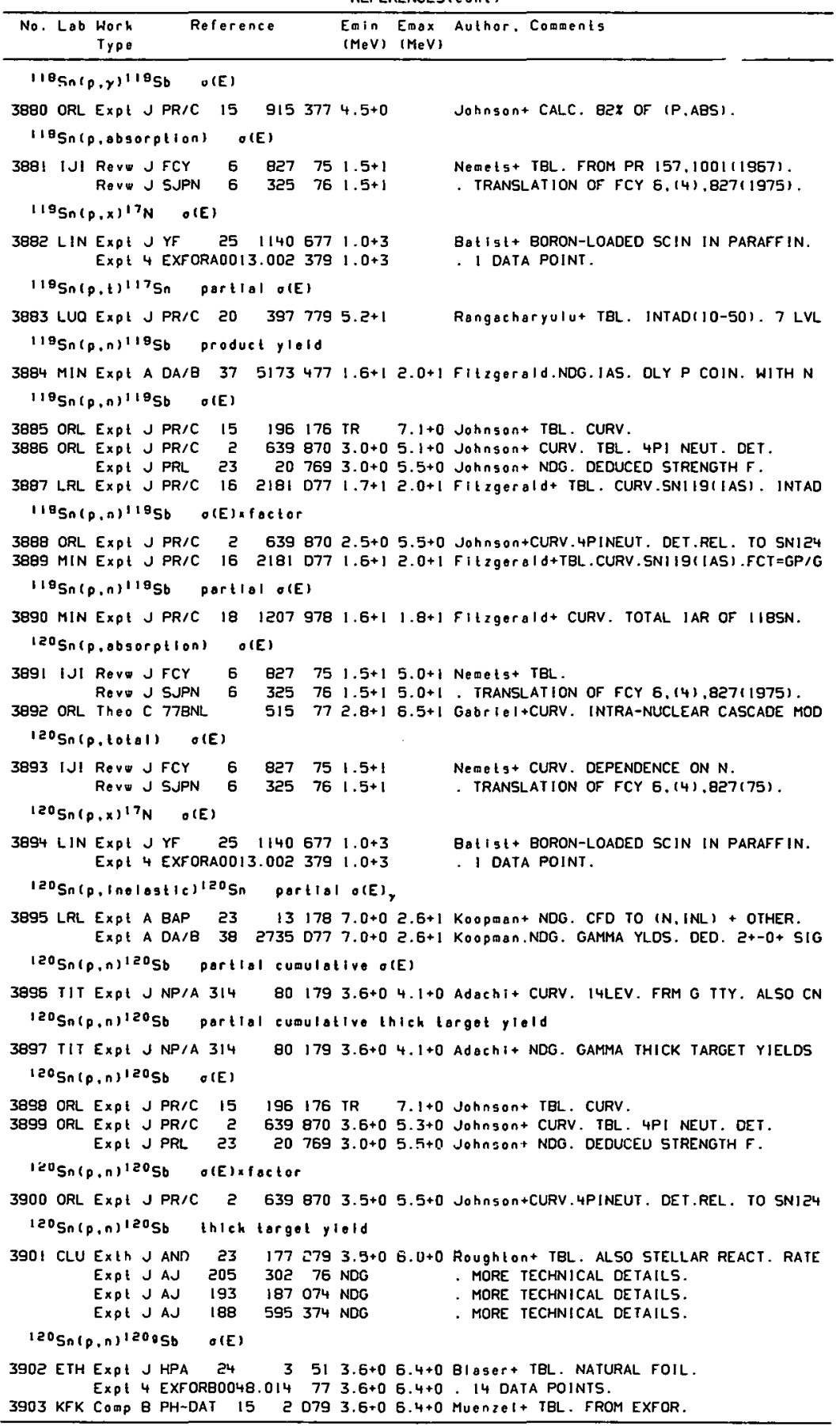


REFERENCES ( cont)

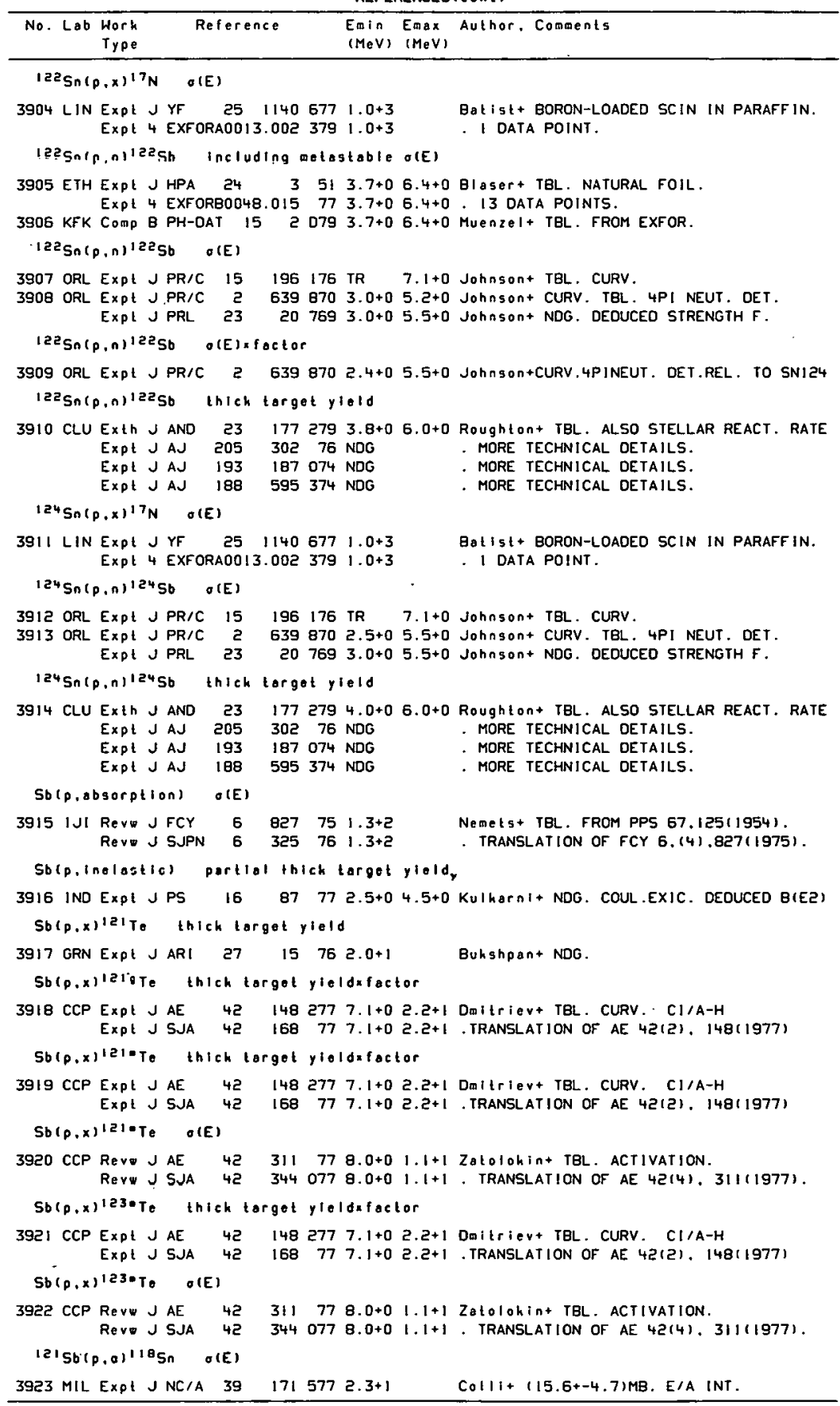


REFERENCES (cont)

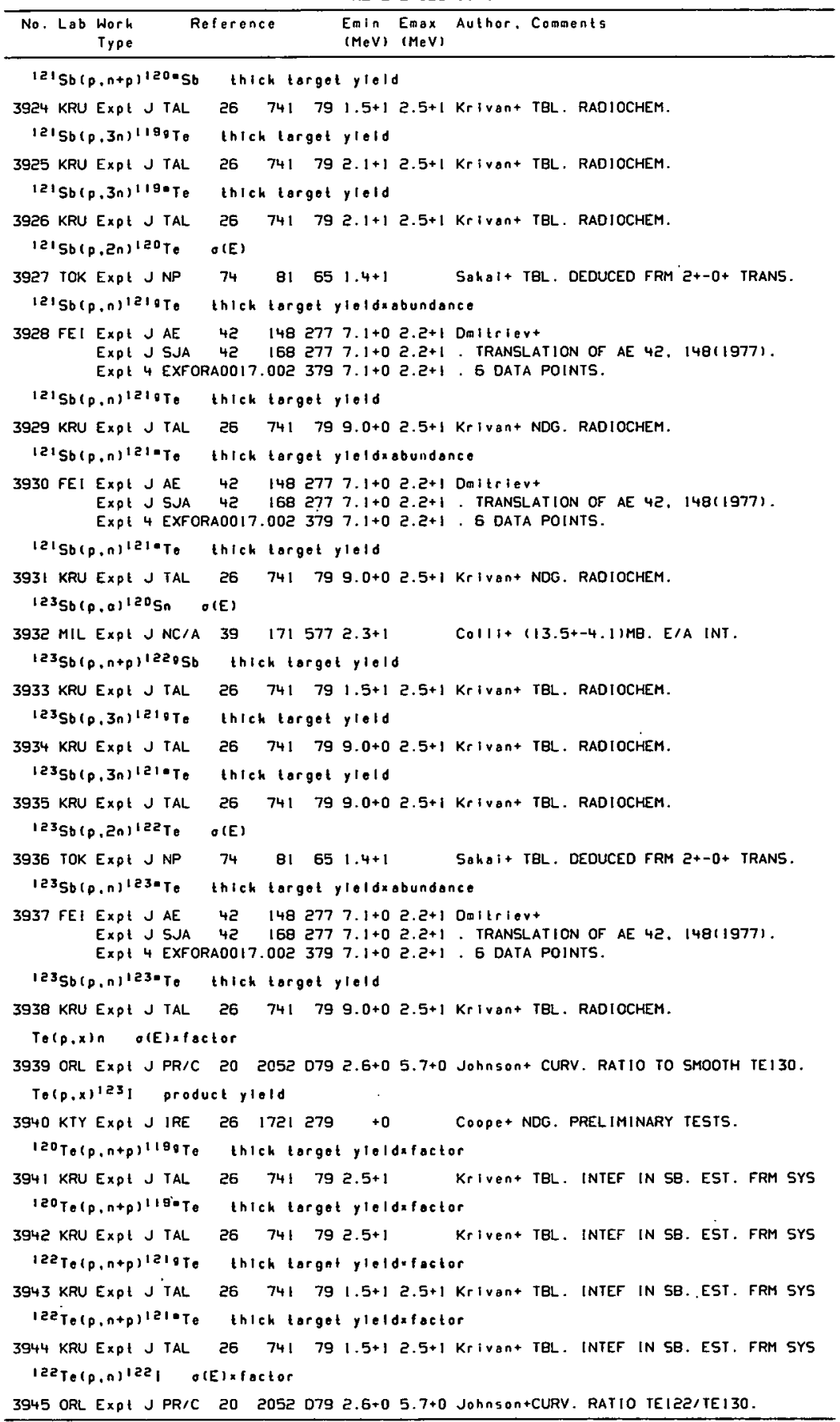




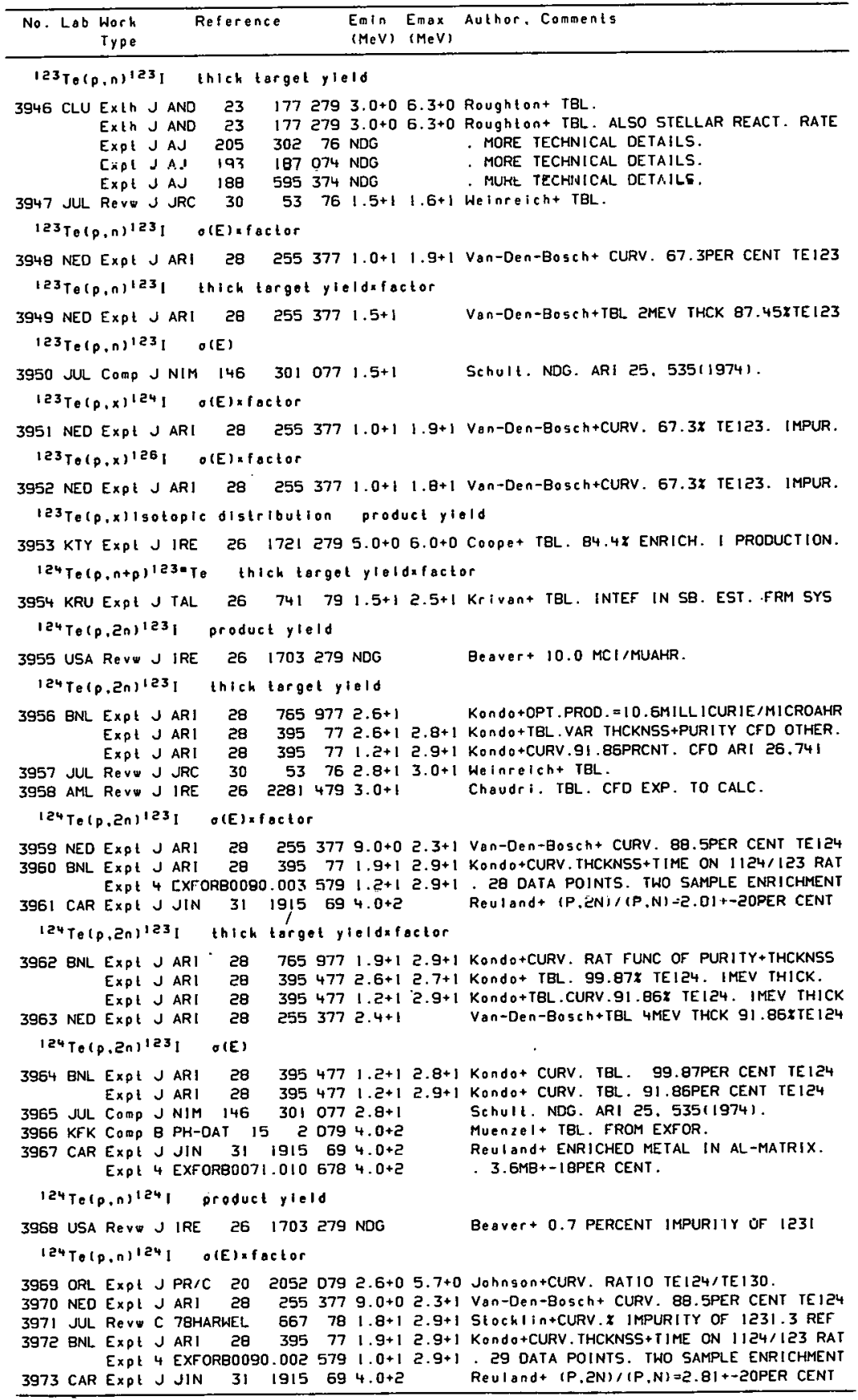




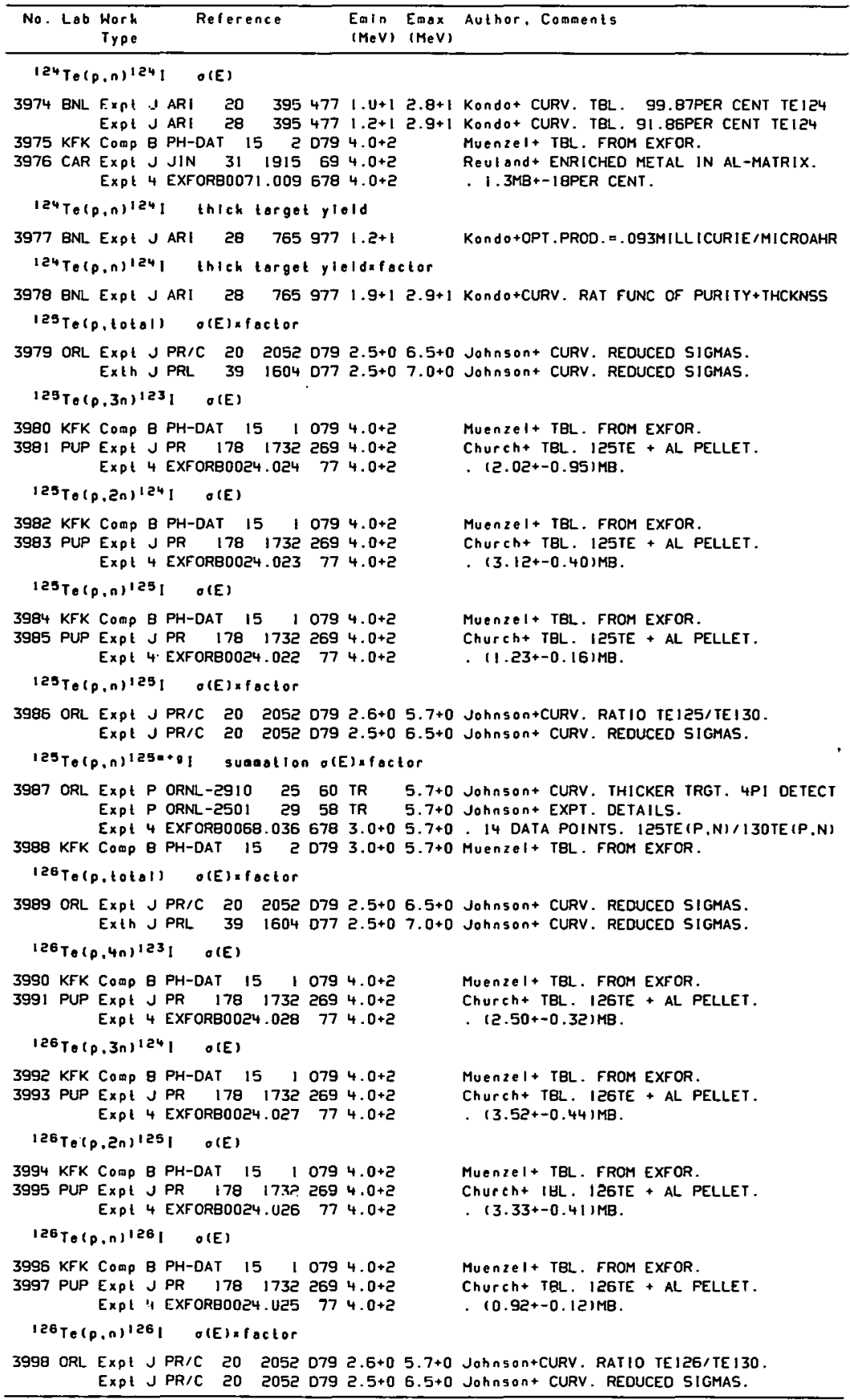


REFERENCES ( con l )

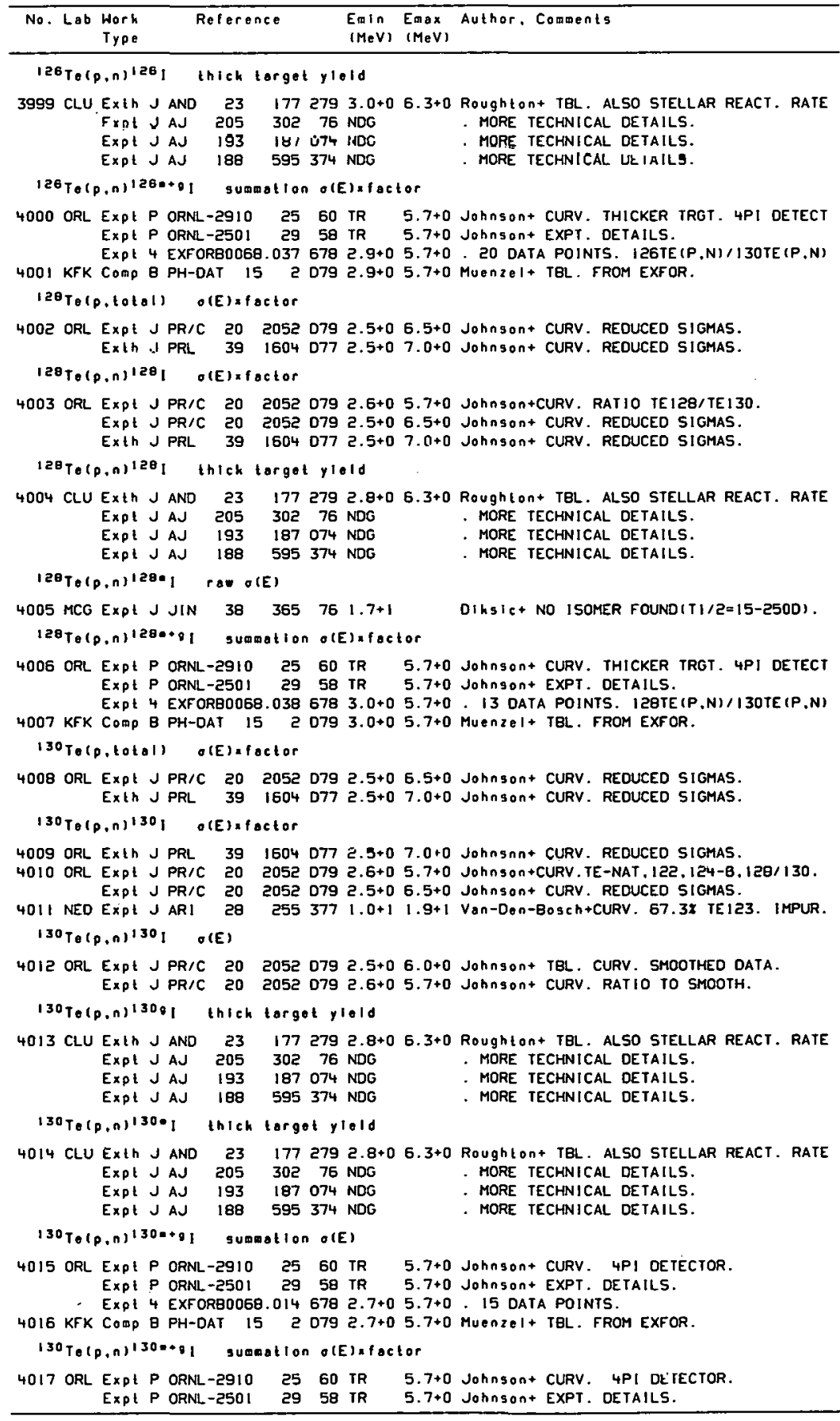




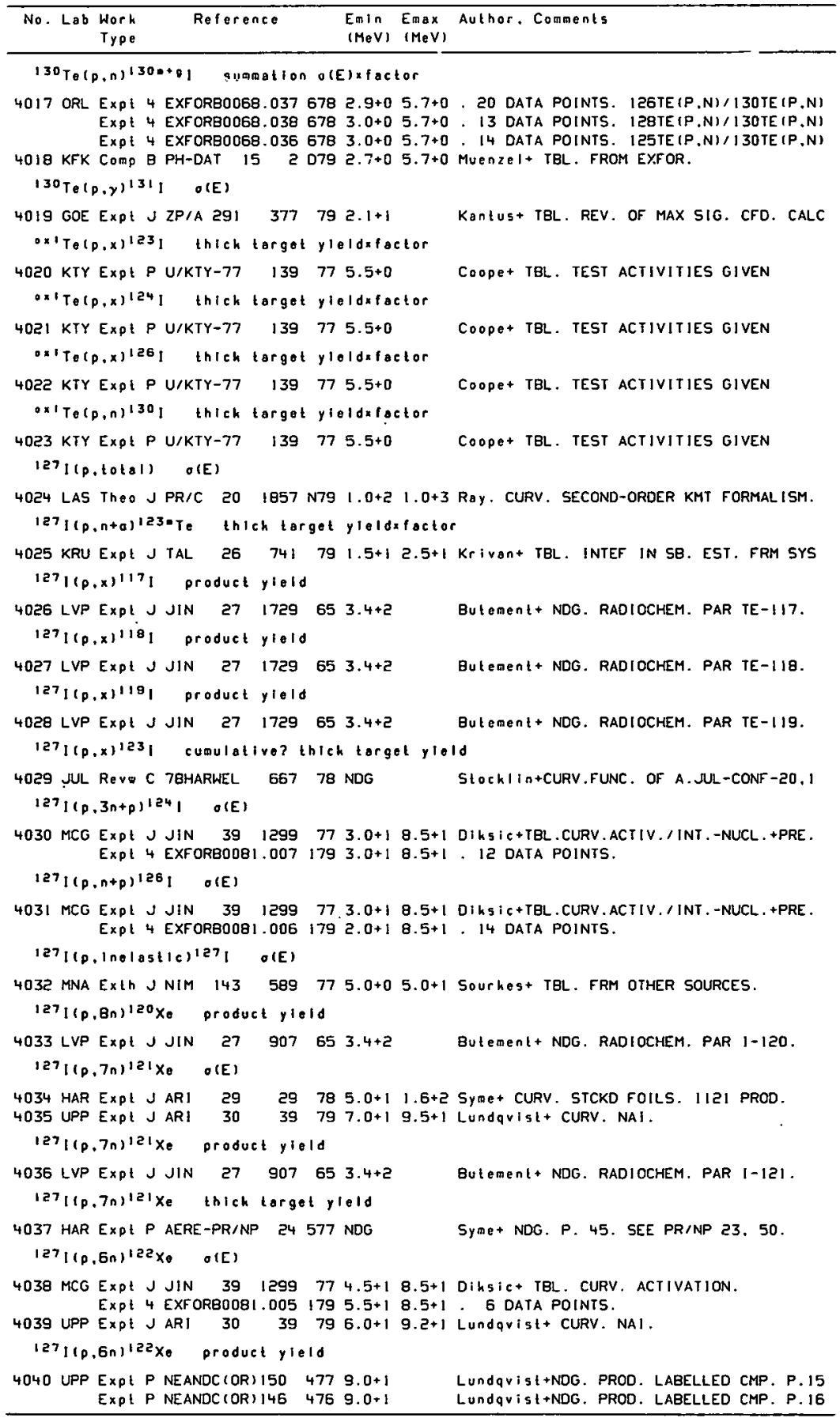




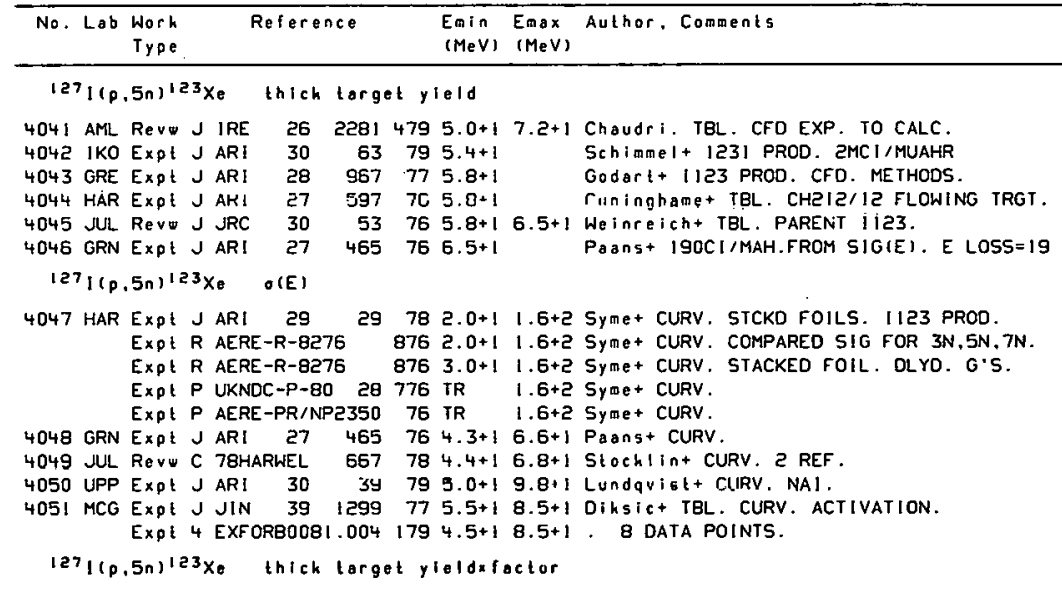

4052 MCG Expt J JIN $391299776.0+16.5+1$ Diksict CURV. FRM ACT. TH=0.4-2.G/CMP

$$
1271(p, 5 n)^{123} x_{e} \text { product yield }
$$

4053 IKO Expt P A-1KO-77 $54 \quad 77 \mathrm{NDO}$

4054 DAV EXPt A ANS 33224 N79 $6.6+1$

4055 UPP Expt $P$ NEANDC $(O R) 150 \quad 4776.8+1$

ExPL $P$ NEANDC(OR) $146 \quad 4766.8+1$

$$
127 \mid(p, x) 124 x \text { Cunulative? o(E) }
$$

4056 MCG Expt J JIN $391299772.0+18.5+1$ Diksic+TBL.CURV.ACTIV. /INT. -NUCL. +PRE.

$$
1271(p, 4 n) 124 \times e \quad O(E)
$$

4057 MCG Expt.J JIN $391299774.5+18.5+1$ 0ihsic+ TBL. CURV. ACTIVATION.

$$
127 I(p, 3 n) 125 \times e \quad O(E)
$$

4058 HAR Expl J ARI 29 2978 2.0+1 1.6+2 SyMe+ CURV. 5TCKD FOILS. 1125 PROD.

EXPI R AERE-R-E276 $8762.0+1 \quad 1.6+2$ Syme+ CURV, COMPARED SIG FOR 3N,5N,7N.

Expt R AERE-R-8276 876 2.0+1 $1.6+2$ Syme+ CURV. SIACKED FOIL. DLYO. G'S.

Expt P UKNDC-P-80 28776 TR $1.6+2$ Syme+ CURV.

ExpI P AERE-PR/NP2350 76 TR $1.6+2$ Syme+ CURV.

4059 UPP Expt J ARI $30 \quad 39794.0+19.8+1$ Lundquist+ CURV. NAI.

4060 GRN Expl J ARI $27465 \%$ 4.3+1 B.6+1 Faons+ CURV

$127(1,0,3 n) 125 x$ e Including metastable $O(E)$

4051 MCG Expl J JIN 39 l299 $772.0+18.5+1$ Diksict IBL. CURV. ACTIVATION. Exp! 4 EXFORB0081.003 $1792.0+18.5+1$. 11 DATA POINTS.

$$
127)(0.3 n) 125 \times \text { product yield }
$$

4062 UPP ExPI P NEANDC (OR) $150 \quad 4774.0+1$ Expt P NEANDC (ORII46 $4764.0+1$ 4063 DAV ExpE A ANS 33224 N79 $4.5+1$

$127,(p, 3 n) 125 x e$ thich target yteld

4064 HAR Expt P AERE-PR/NP 24577 NDG

4065 GRN EXPI J ARI $27 \quad 46576 \quad 6.5+1$

$$
127](0,2 n) 126 x e \text { o(E) }
$$

40GE TOK Expt J NP $74 \quad 8165: 4+1$

$1271(0, n)^{123} x$ e Including metastablo o(E)

Lundquist+NDG. PROD. LABELLED CMP. P. 15 Lundquist+NDG. PROD. LABELLED CMP. P. 16 Lagunas-Solar+ TRL. REVIEW OF PROGRAM.

Syme+ NDG, P. 45. SEE PRINP 23. 50.

Paans+ 7.7CI/MAH.FROM SIG(E), E LOSS $=19$

Sahai+ TBL. DEDUCED FRM 2+-0+ TRANS.

4067 KFK Comp B PH-DAT I5 2 D79 $3.0+02.5+1$ Muenzel+ THL. FROM EXFOR.

4068 BNL Expl J PR/C $921666773.0+0$ 2.5+1 CoIIe+ TBL. CURV. ACTIVATION METHOD. EXPL 4 EXFORBD056.003 $773.0+02.5+1$. 26 DATA POINTS.

4069 MCG EXpt J JIN $391299772.0+18.5+1$ Diksic+ IBL. CURV. ACTIVATION. Expt 4 EXFORBO0BI.002 $1792.0+18.5+1$. 10 DATA POINTS.

$1271(p, n)^{127} x e$ product yleld

4070 UPP Expt P NEANDCIOR)150 477 2.0+1 Lundquist+NDG. PROD. LABELLED CMP. P. 15 Exp! P NEANDC(OR) $146 \quad 4 \% 2.0+1$ 


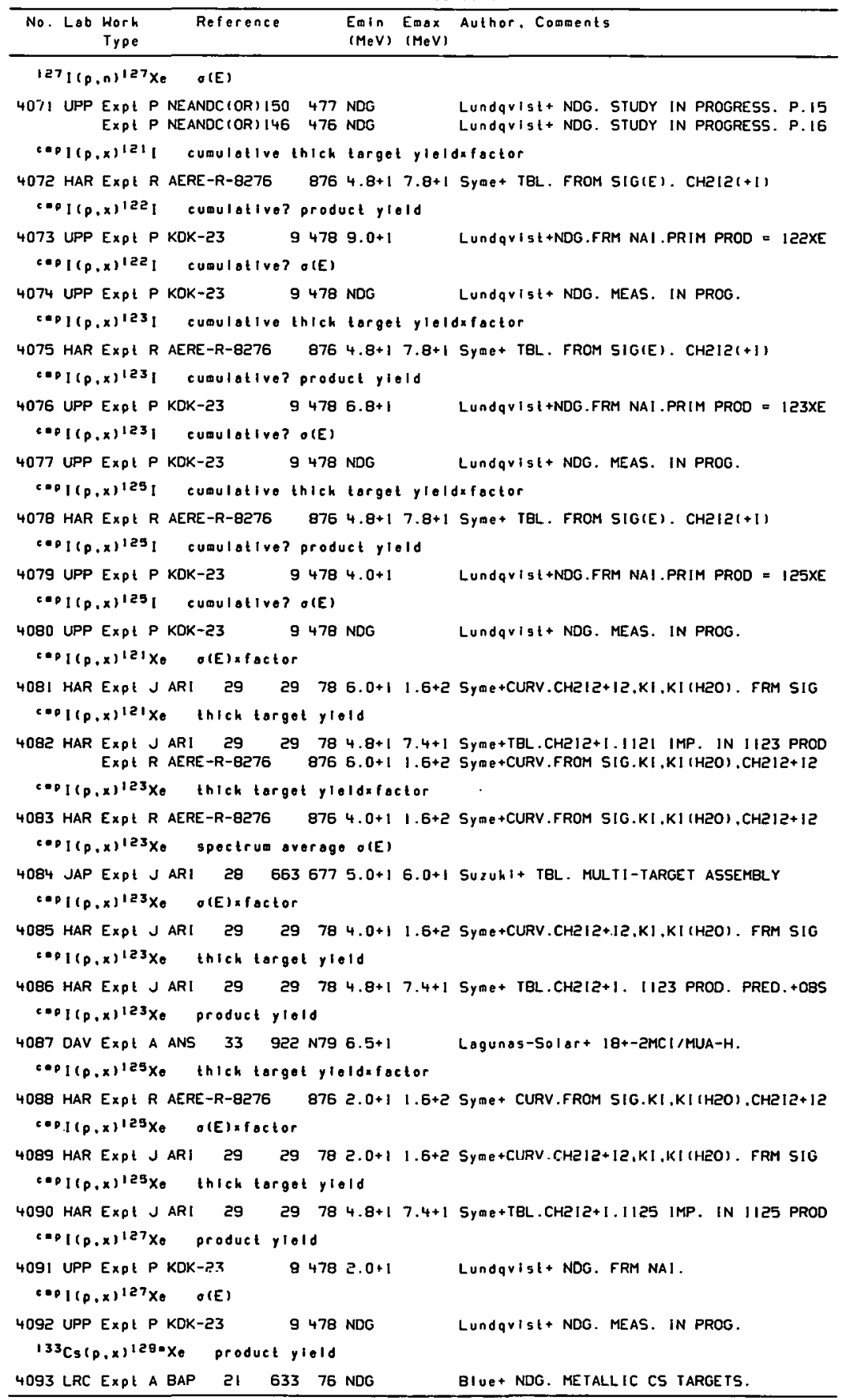


REFERENCES $(\operatorname{con}()$

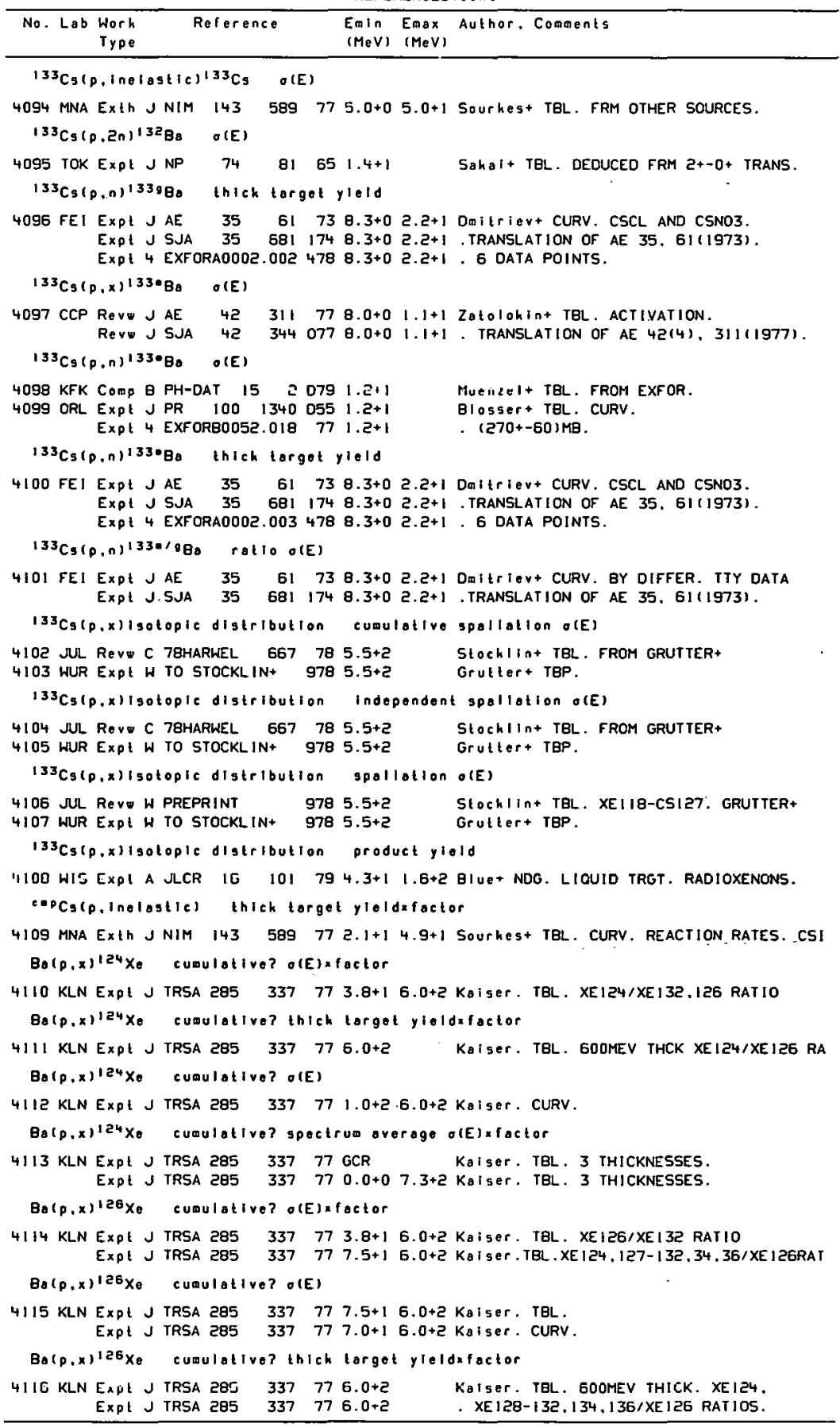


REFERENCES ( c on t)

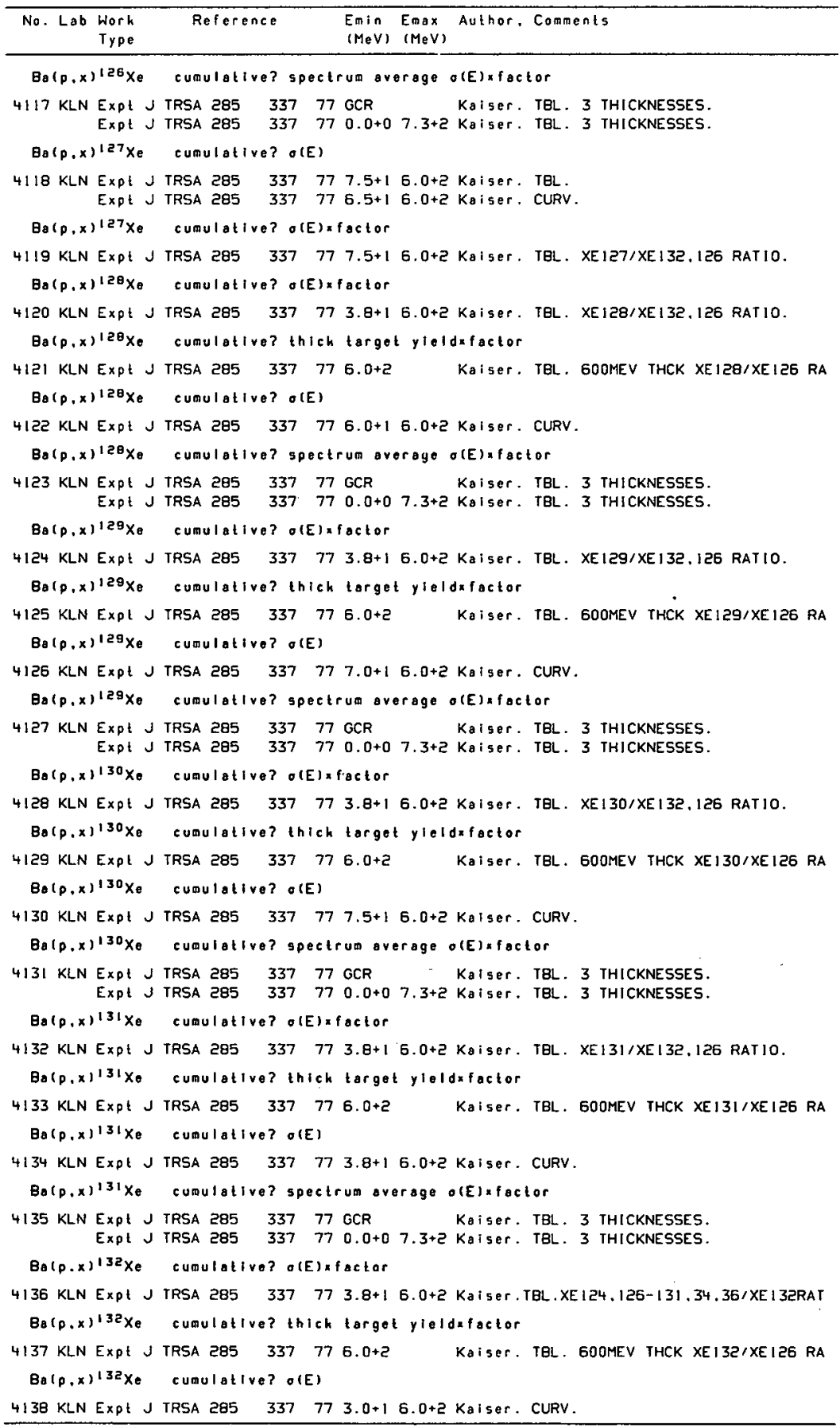


REFERENCES ( c ont)

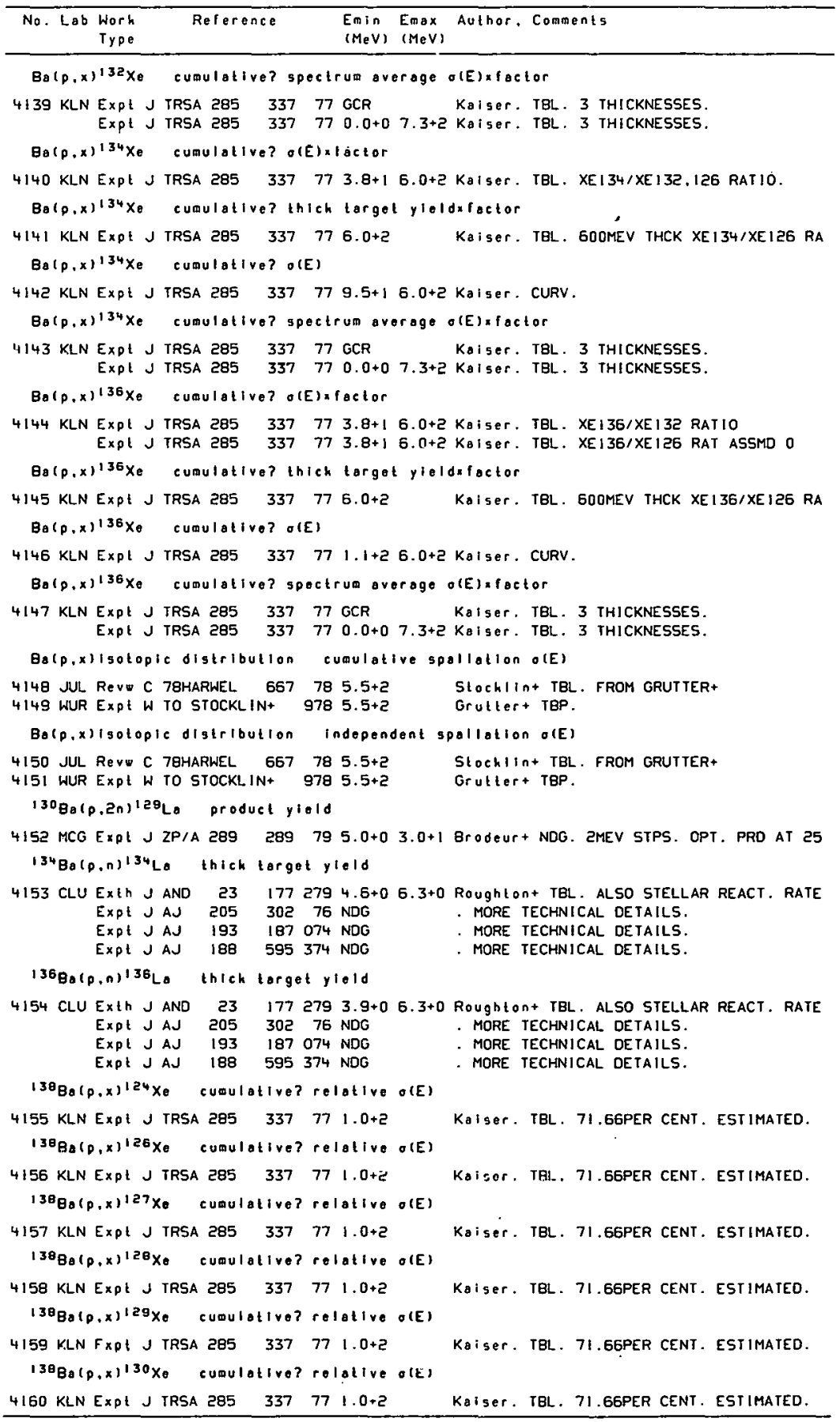


REFERENCES (cont)

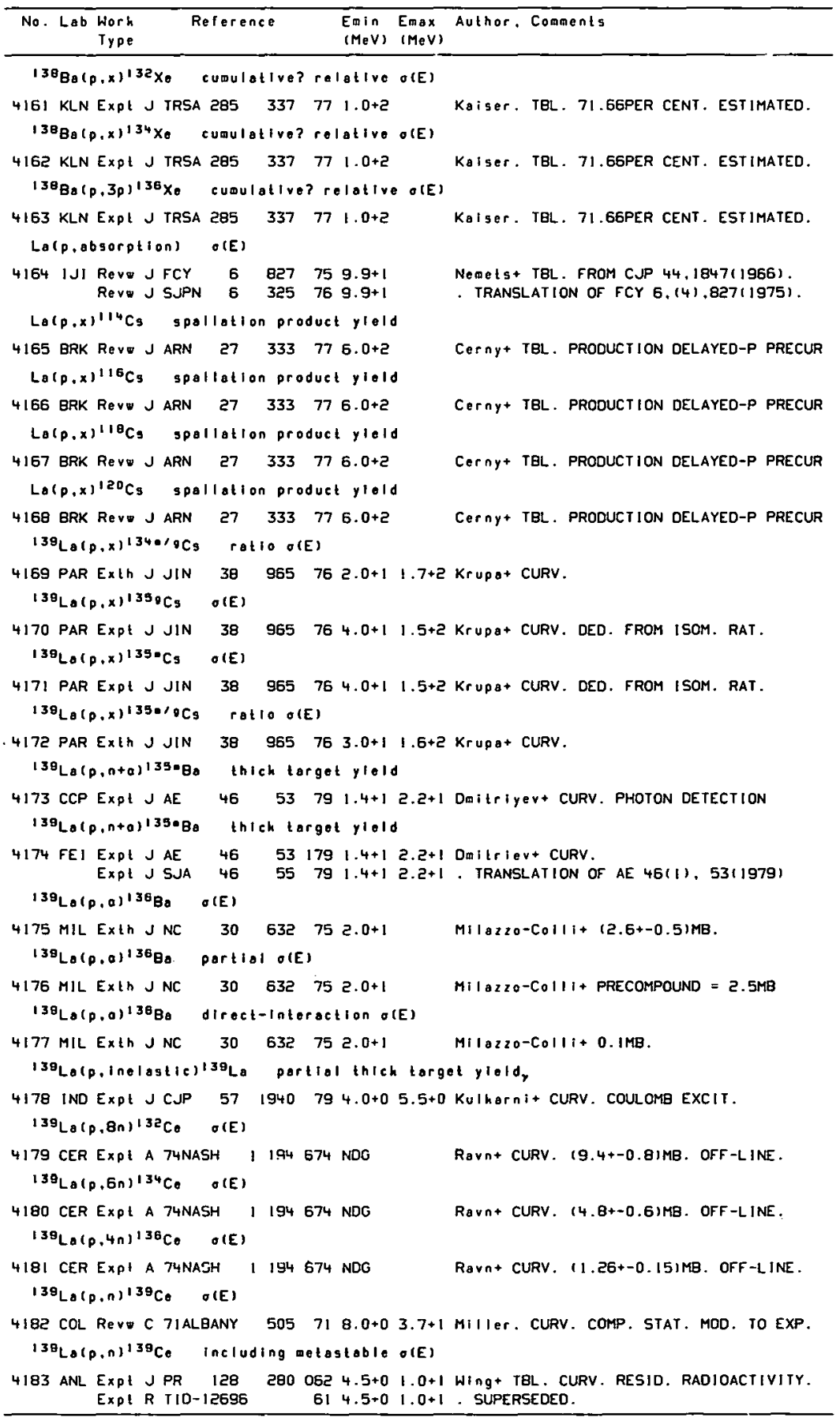




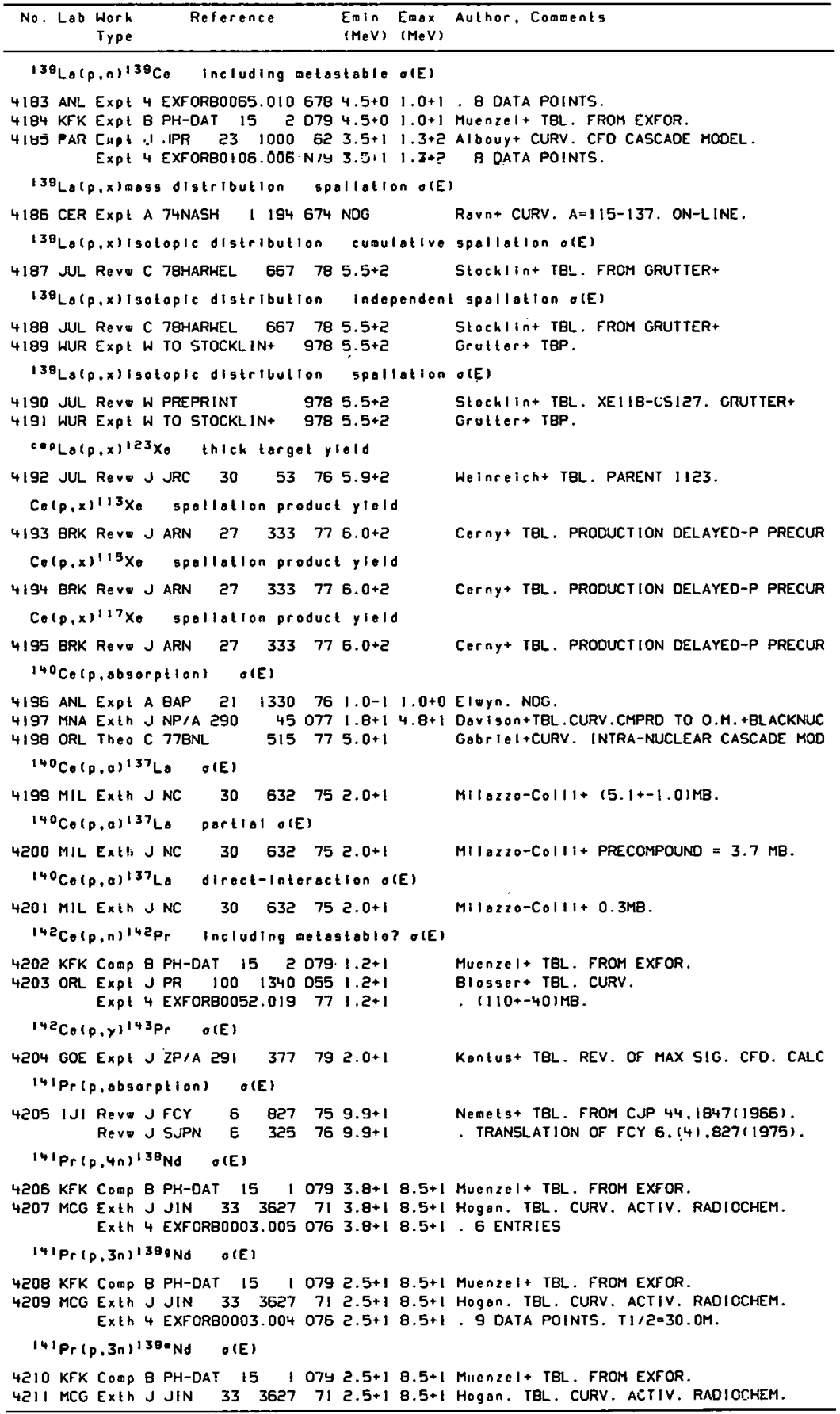


REFERENCES ( c on t)

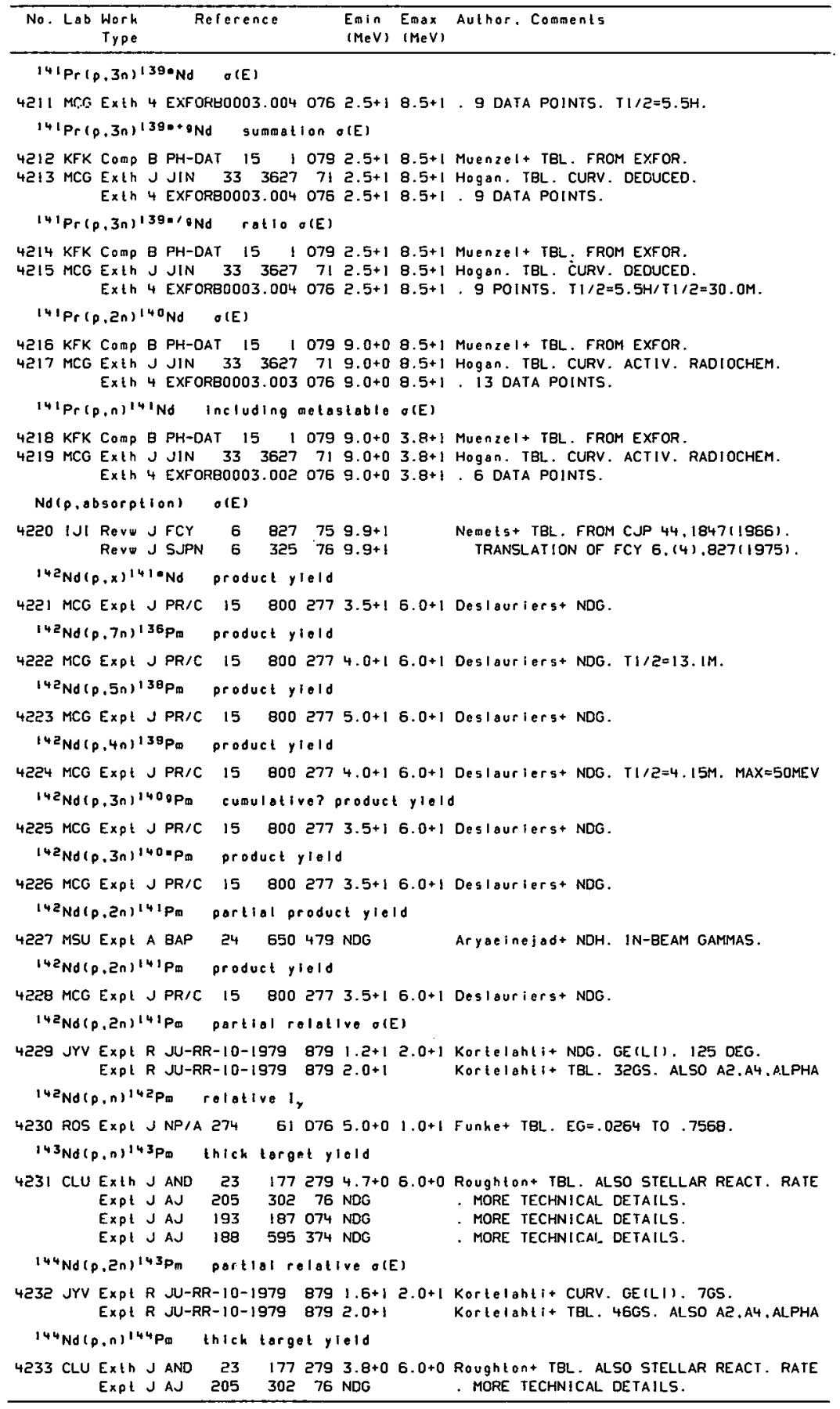


REFERENCES ( c ont

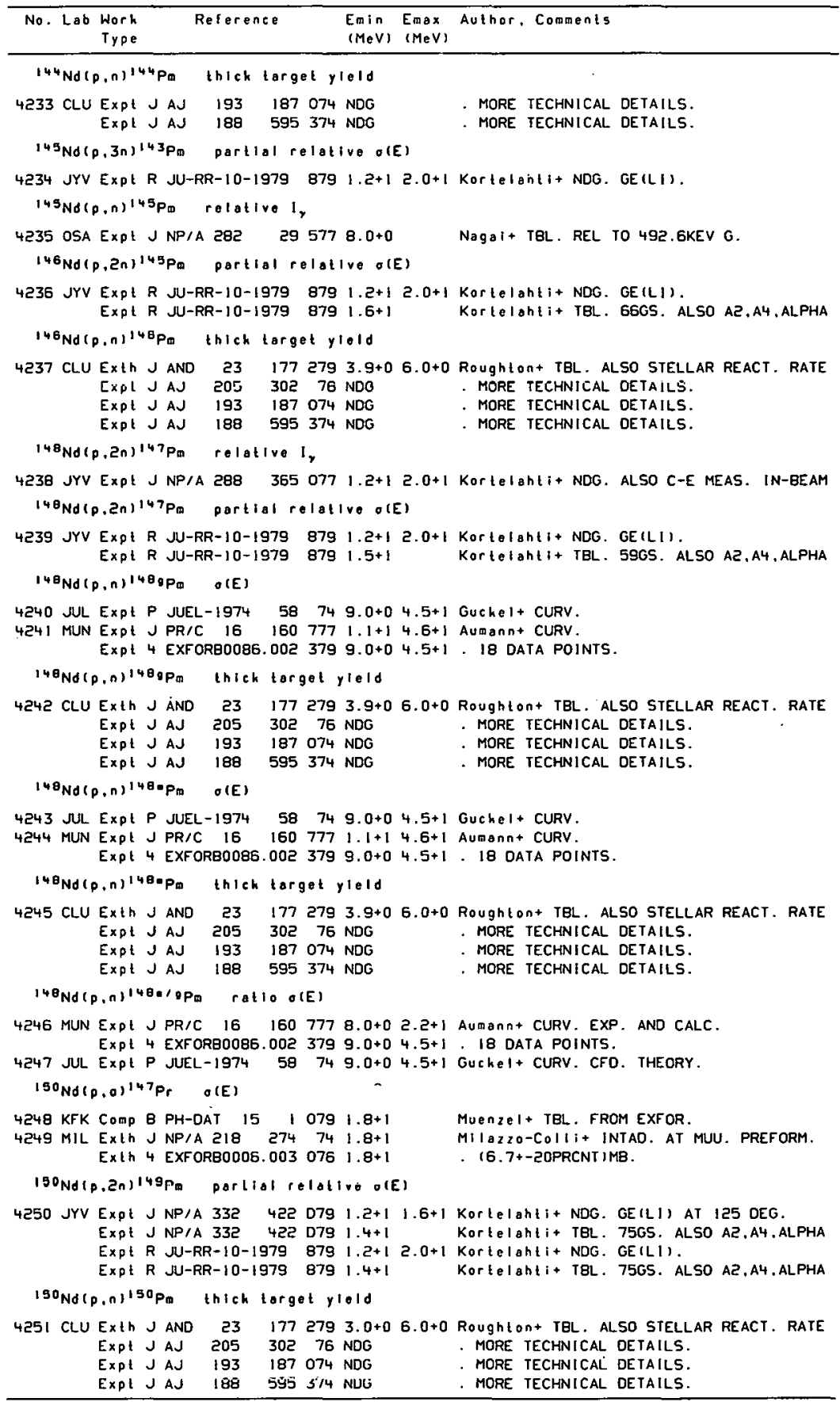


REFERENCES ( cont )

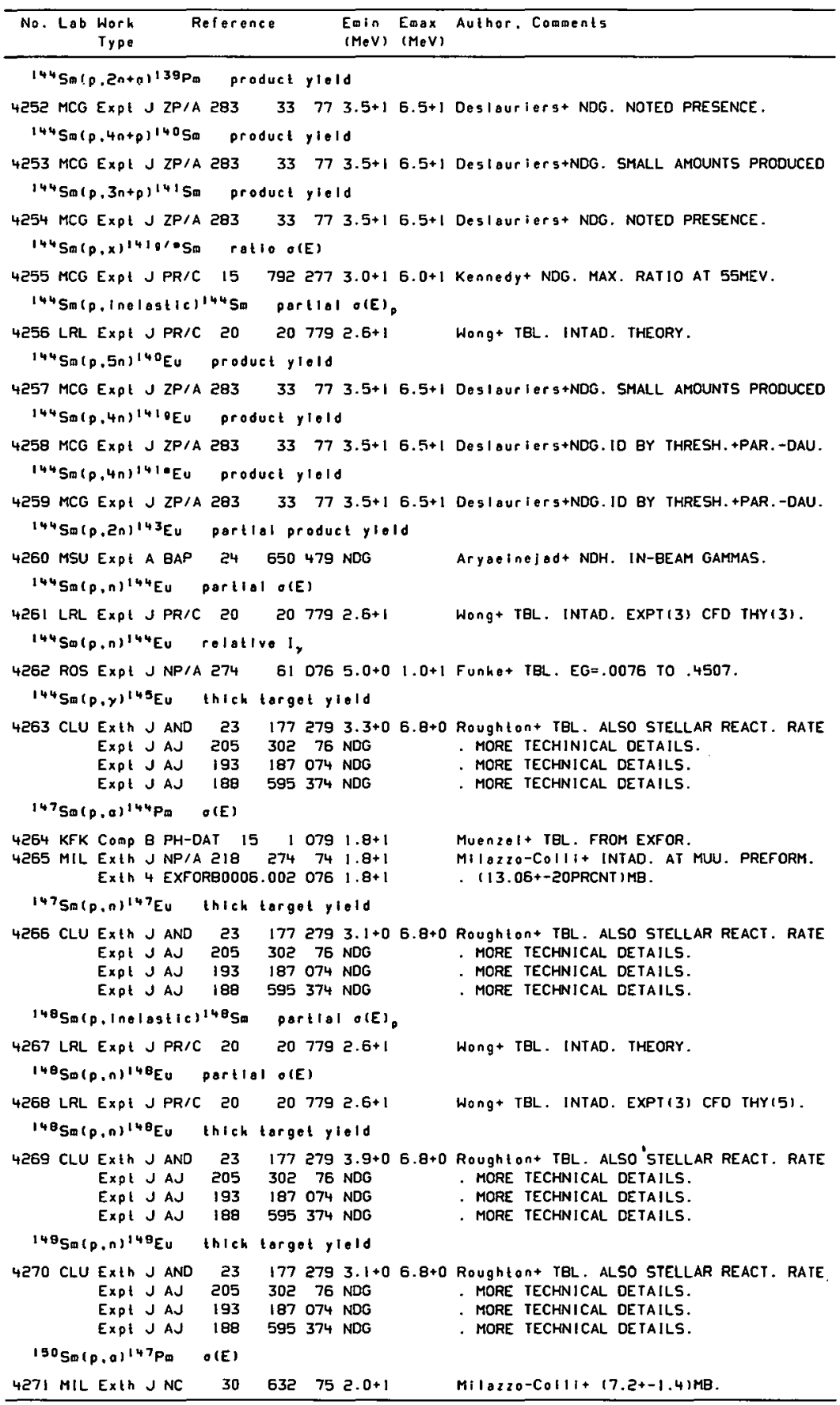




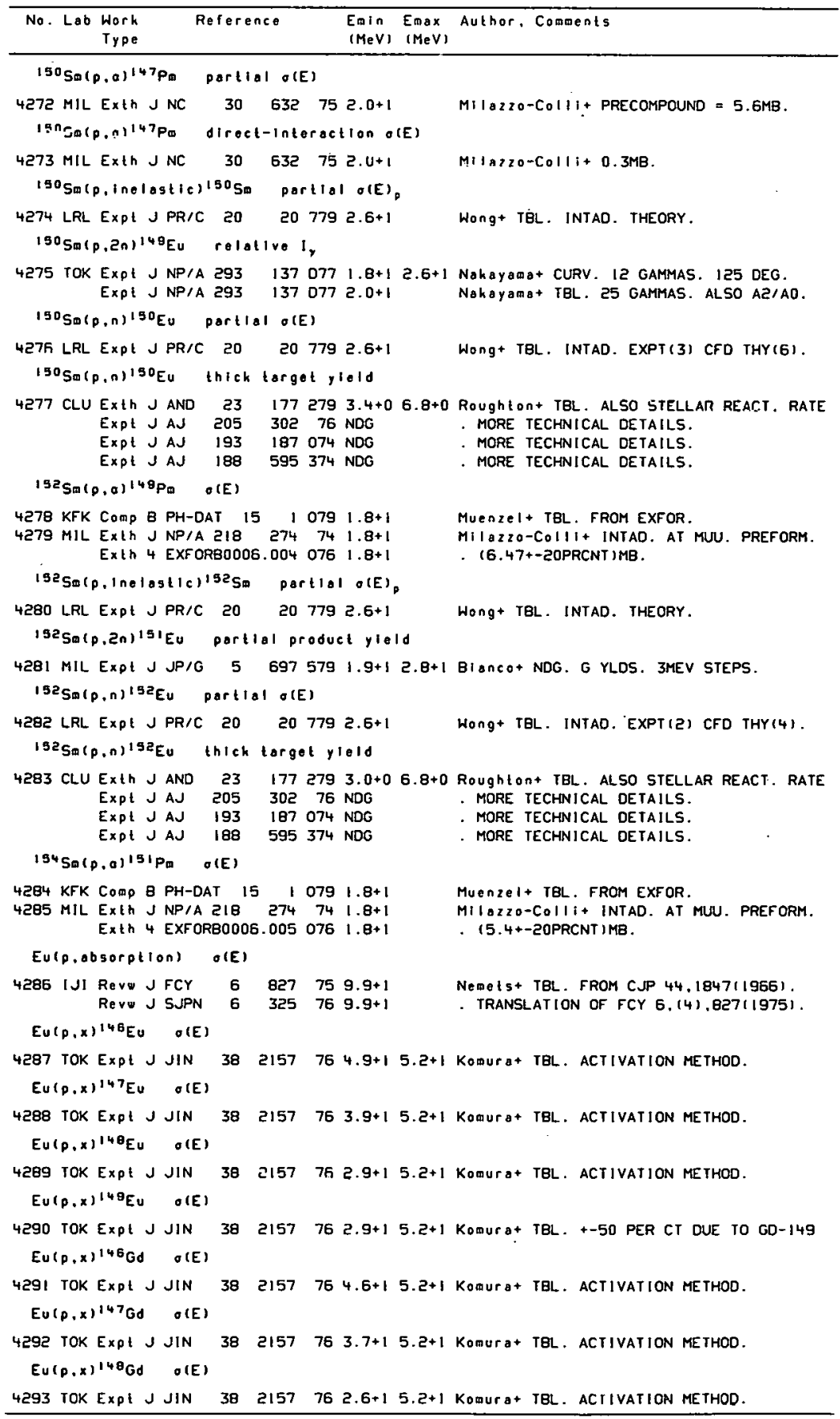




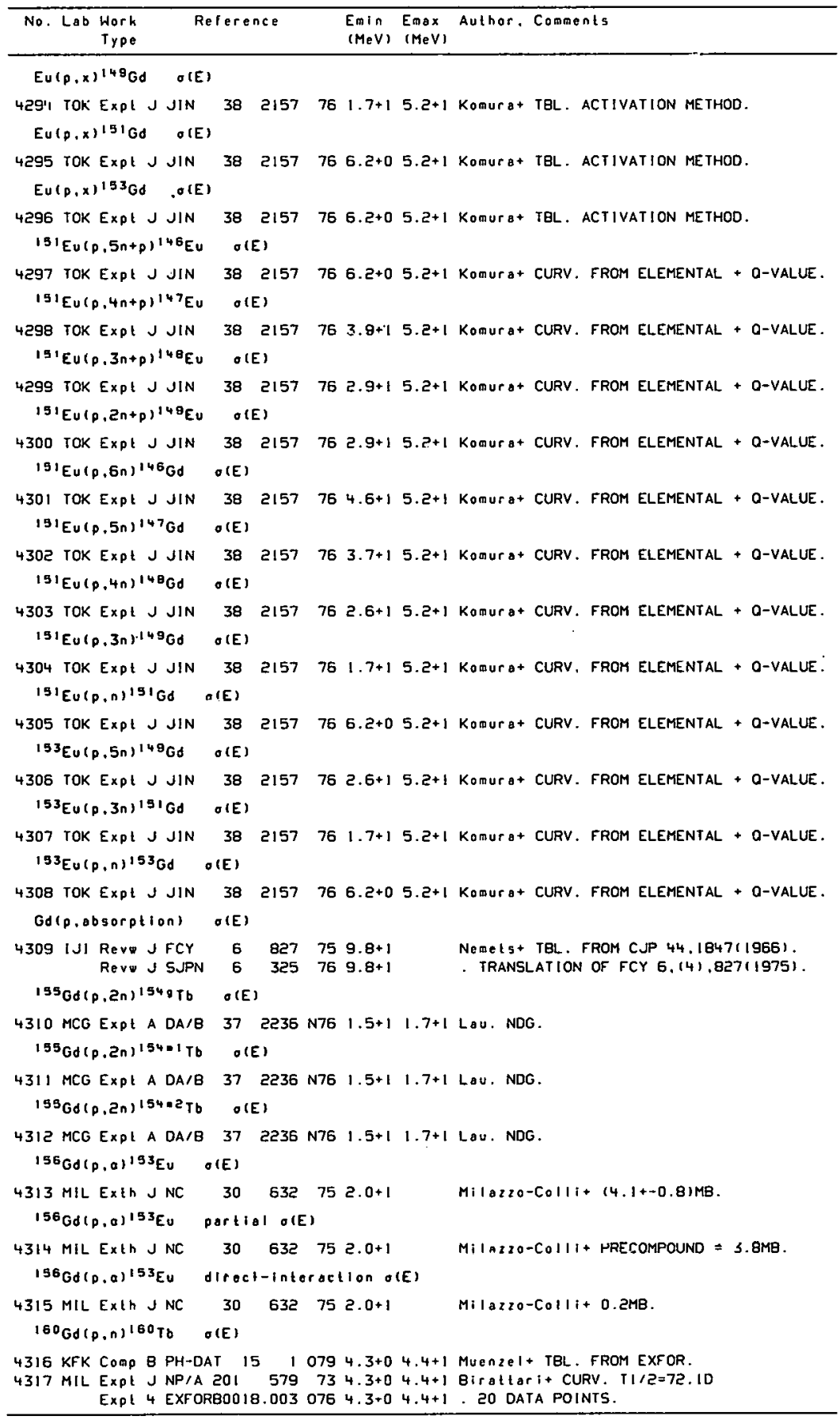


REFERENCES ( c on ()

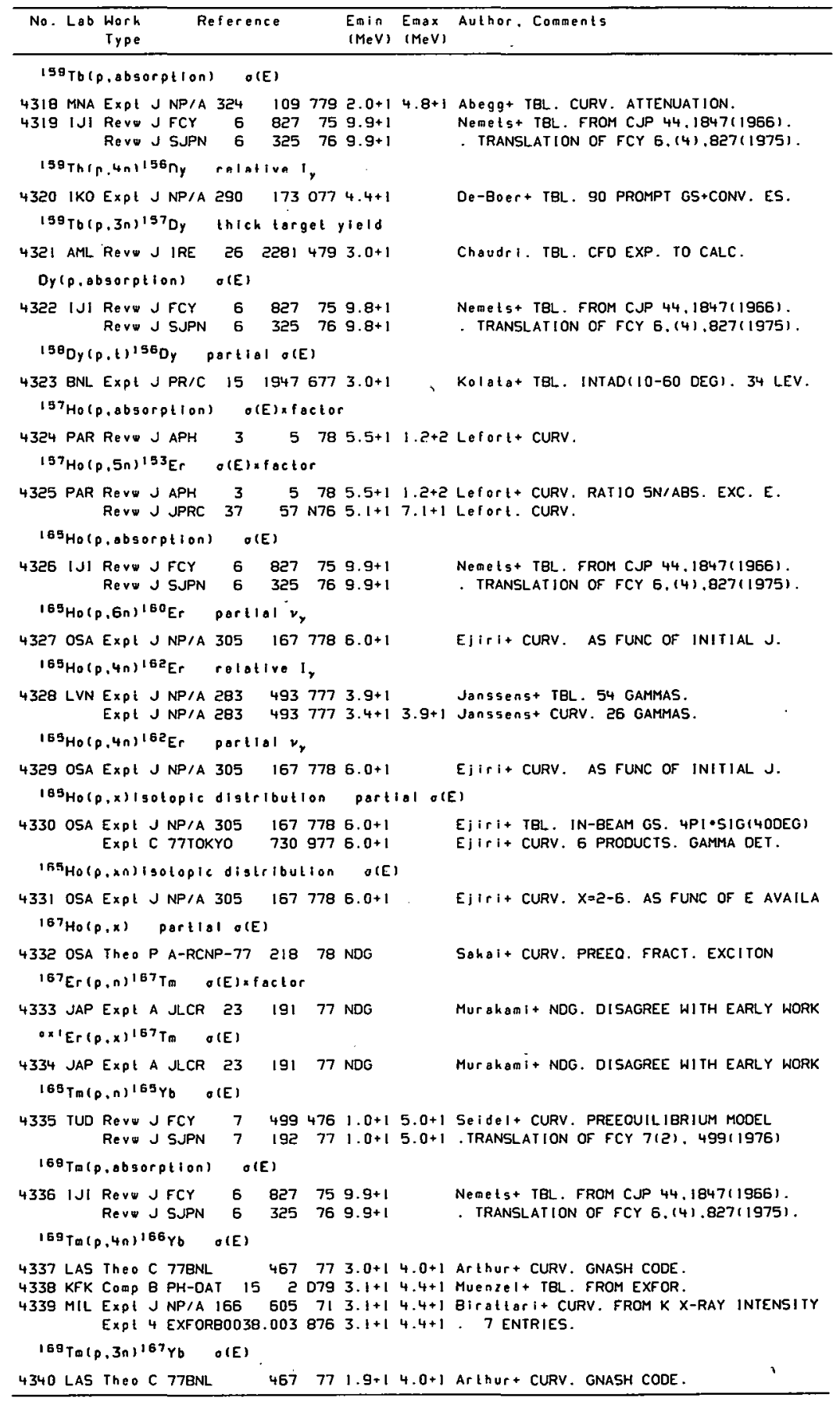




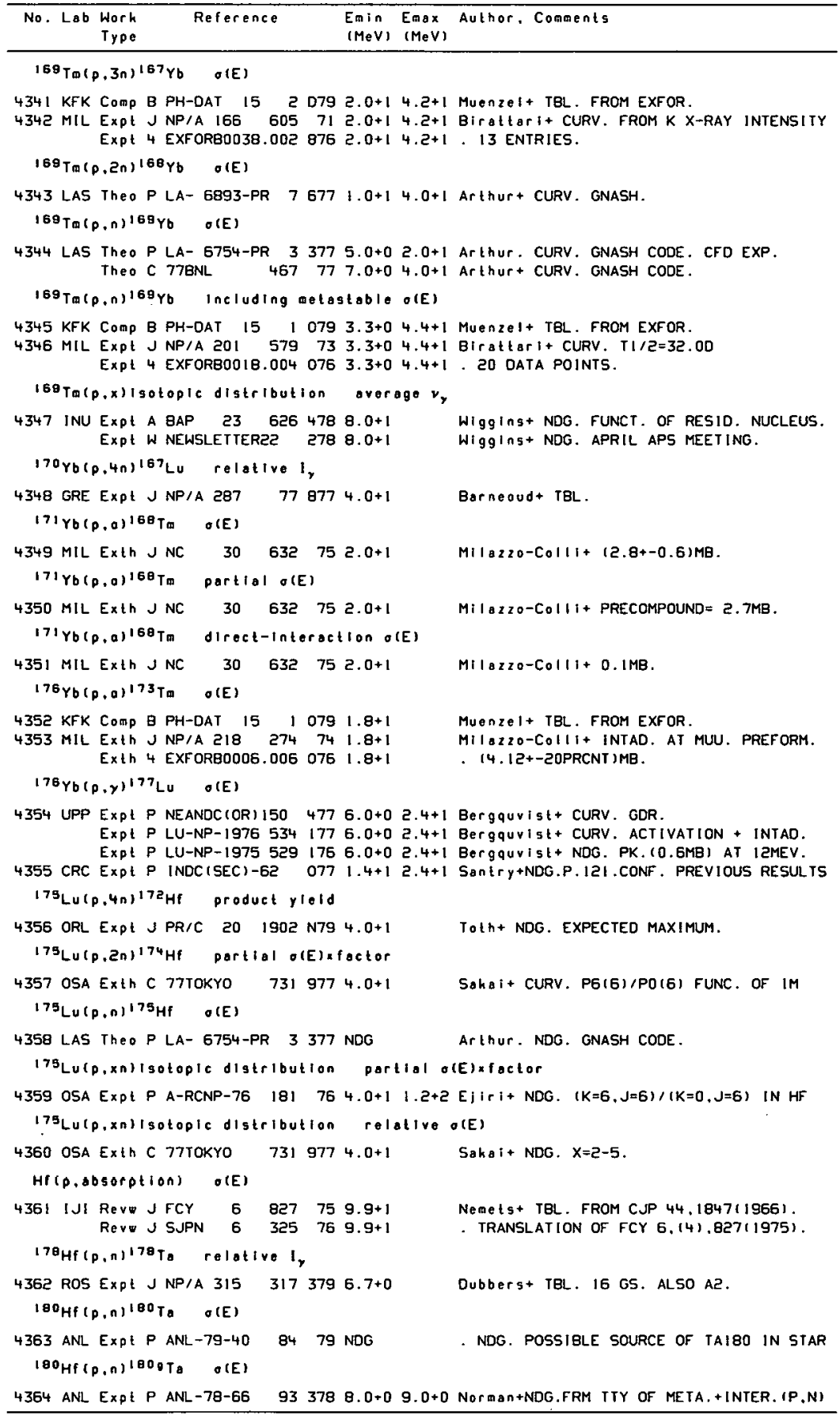


REFERENCES ( c on t)

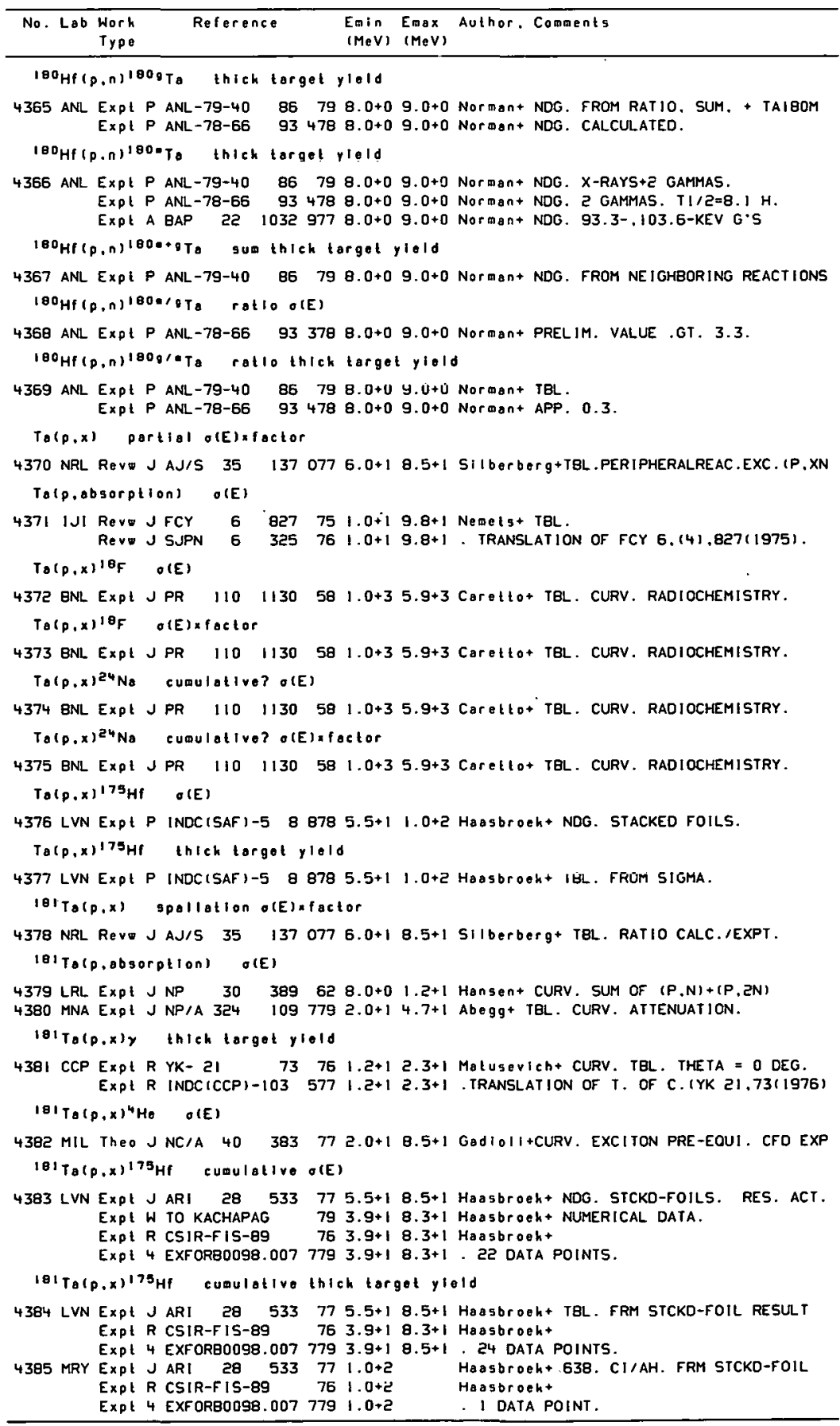




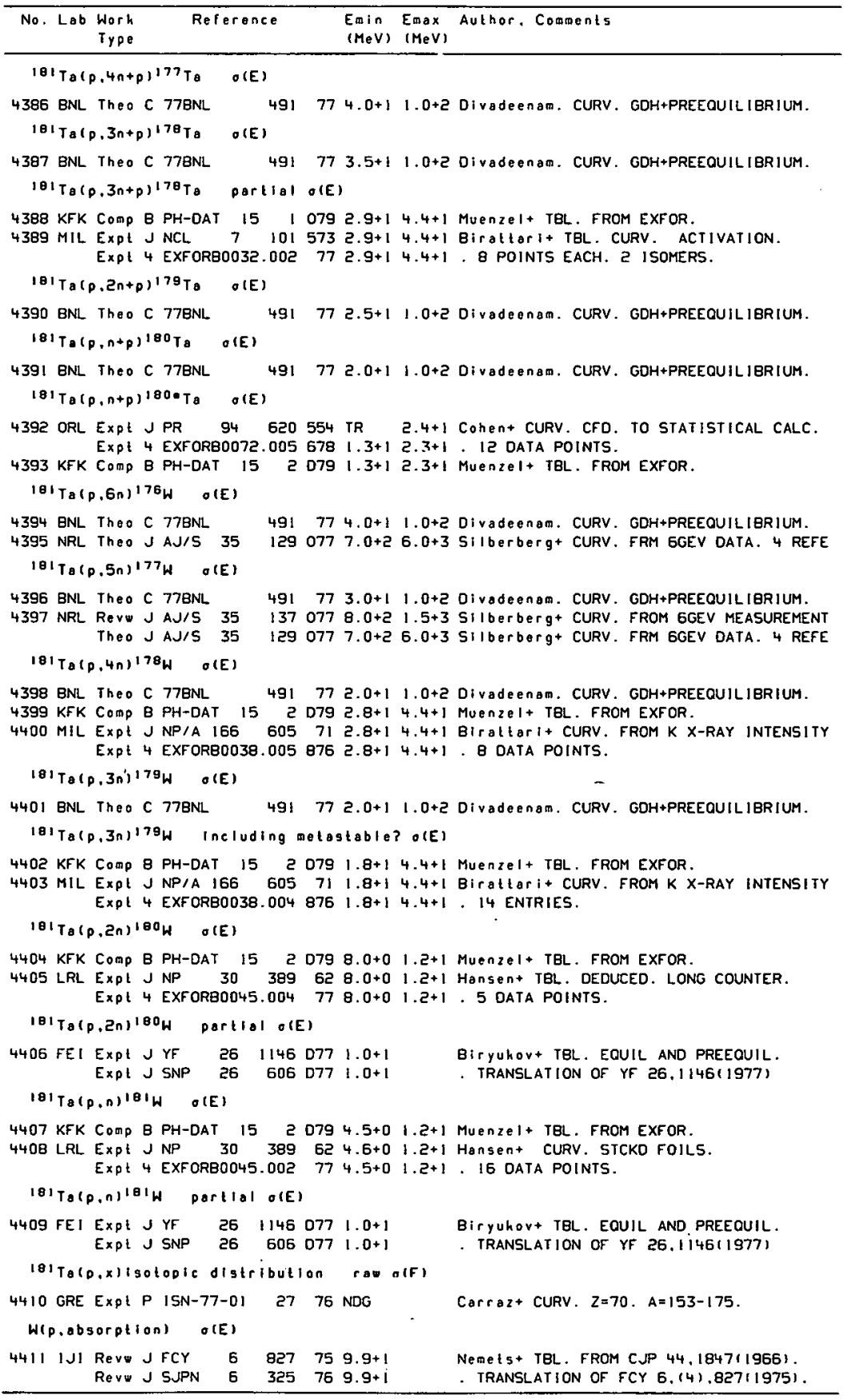




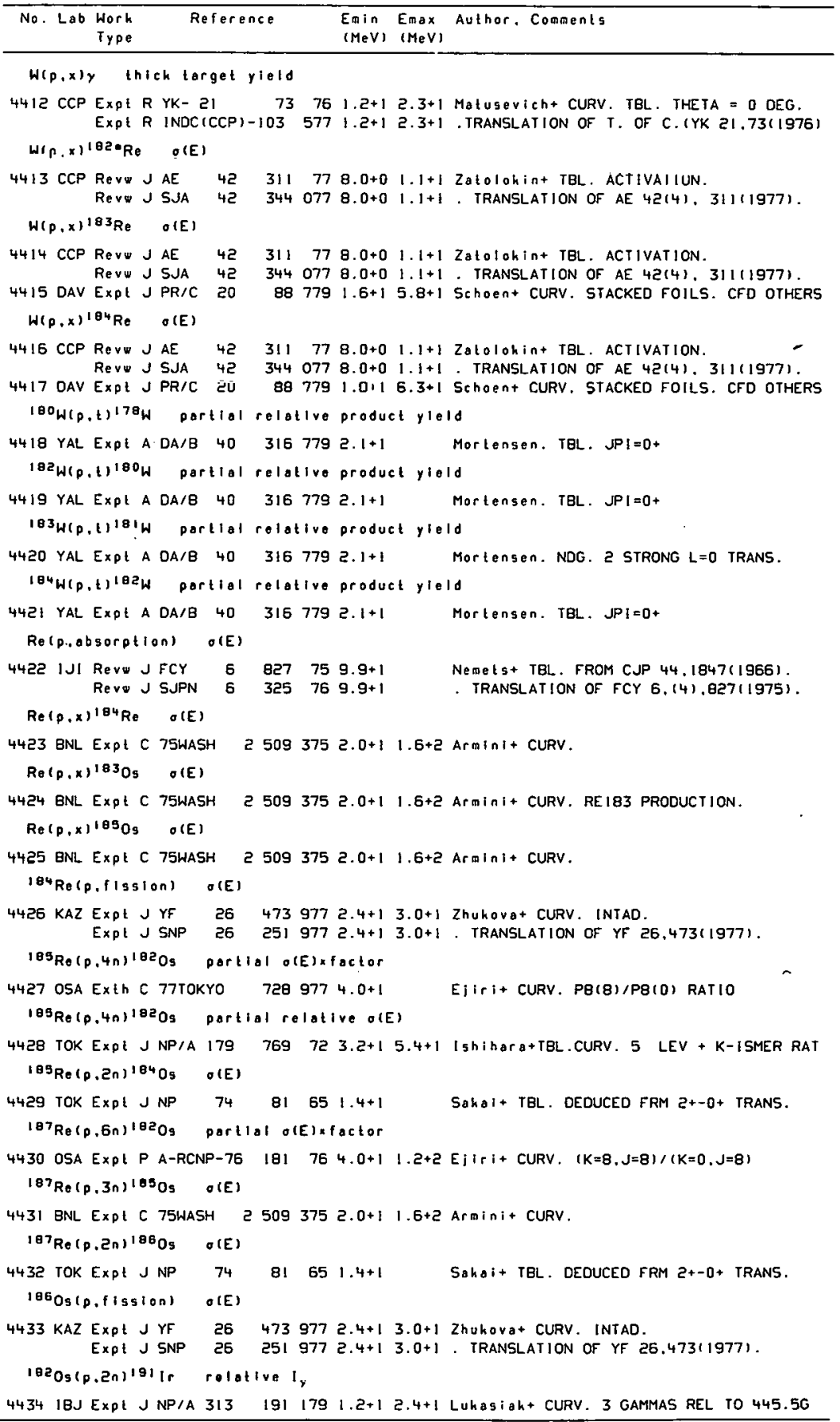




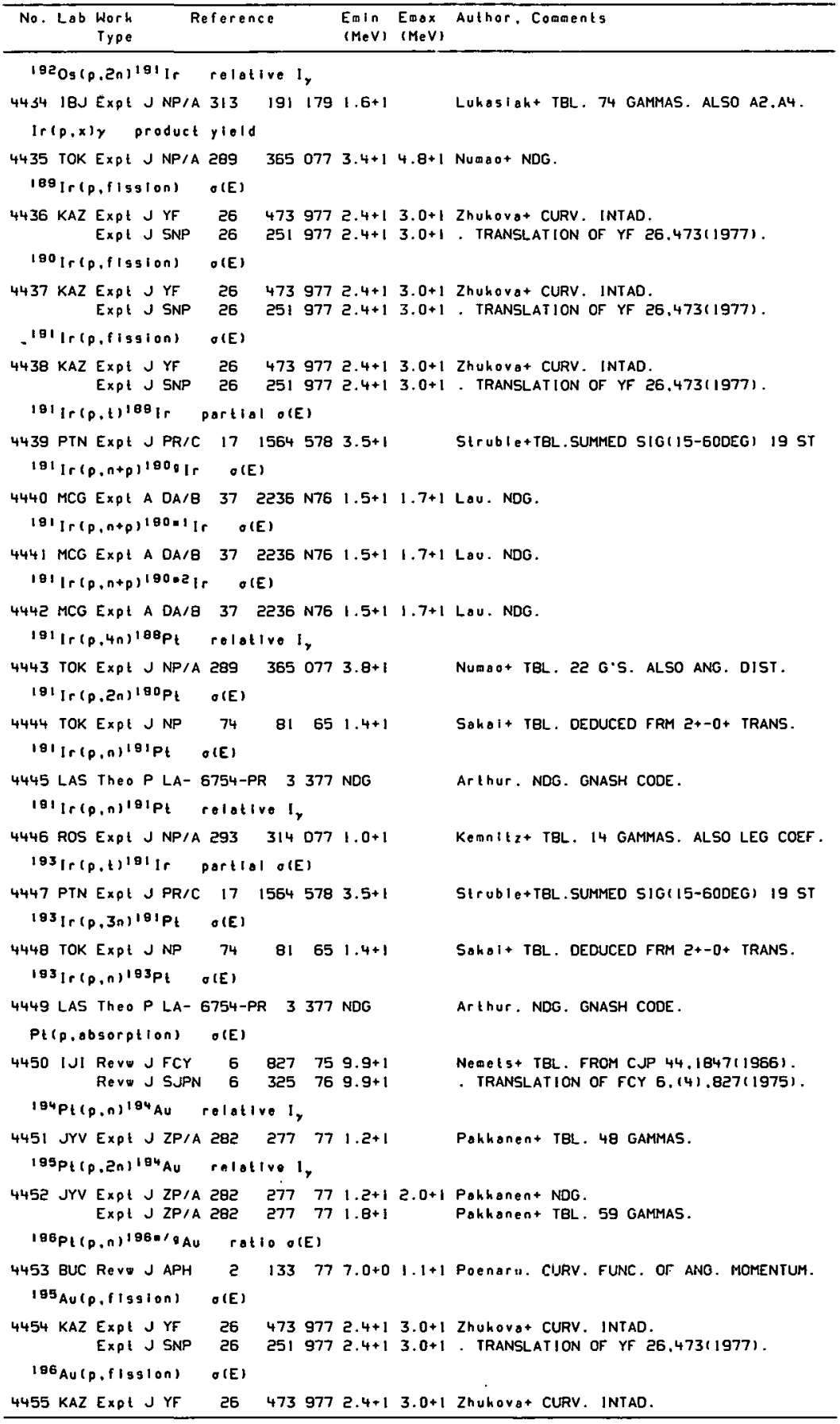


REFERENCES ( $c$ on $t$ )

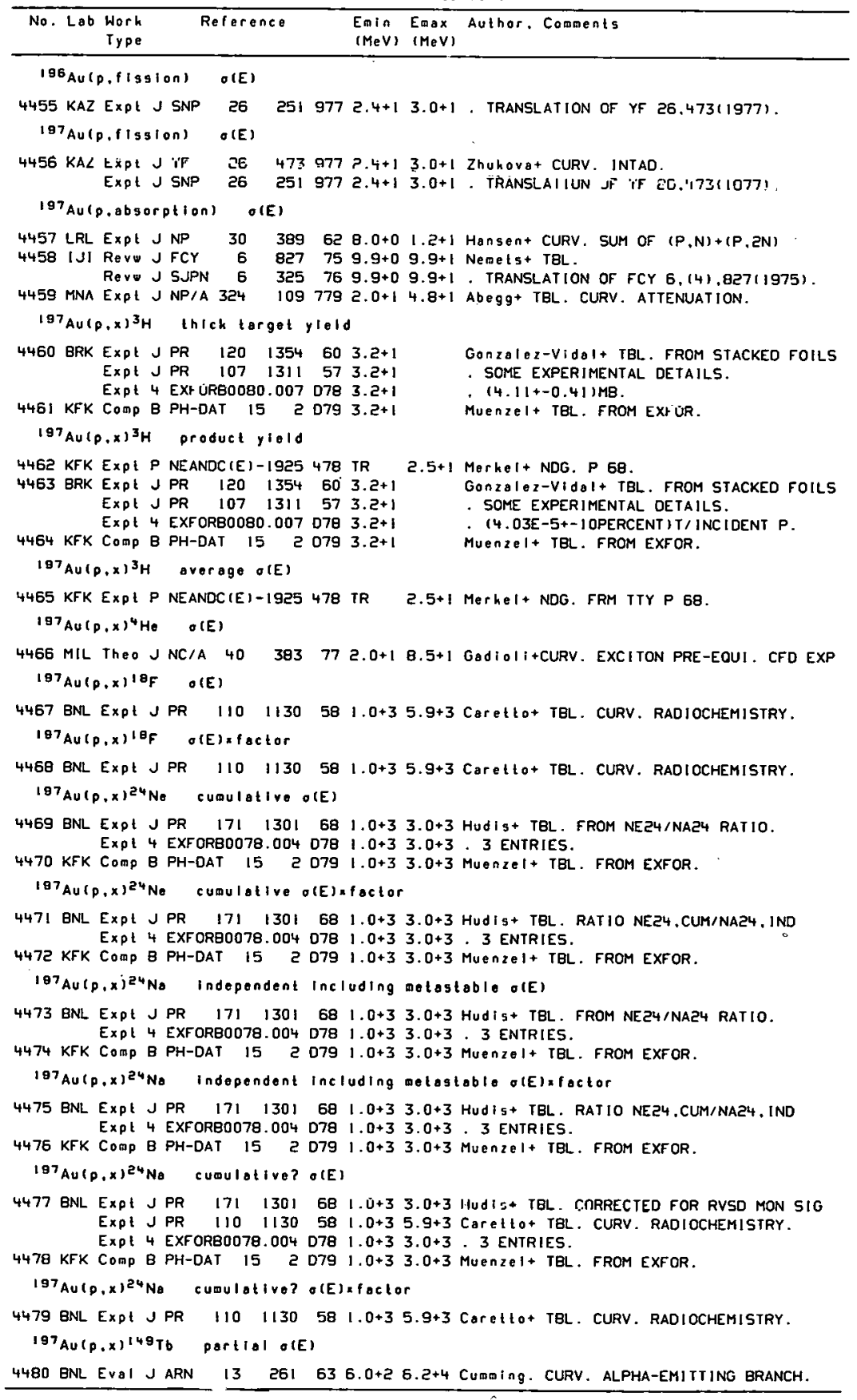


REFERENCES (cont)

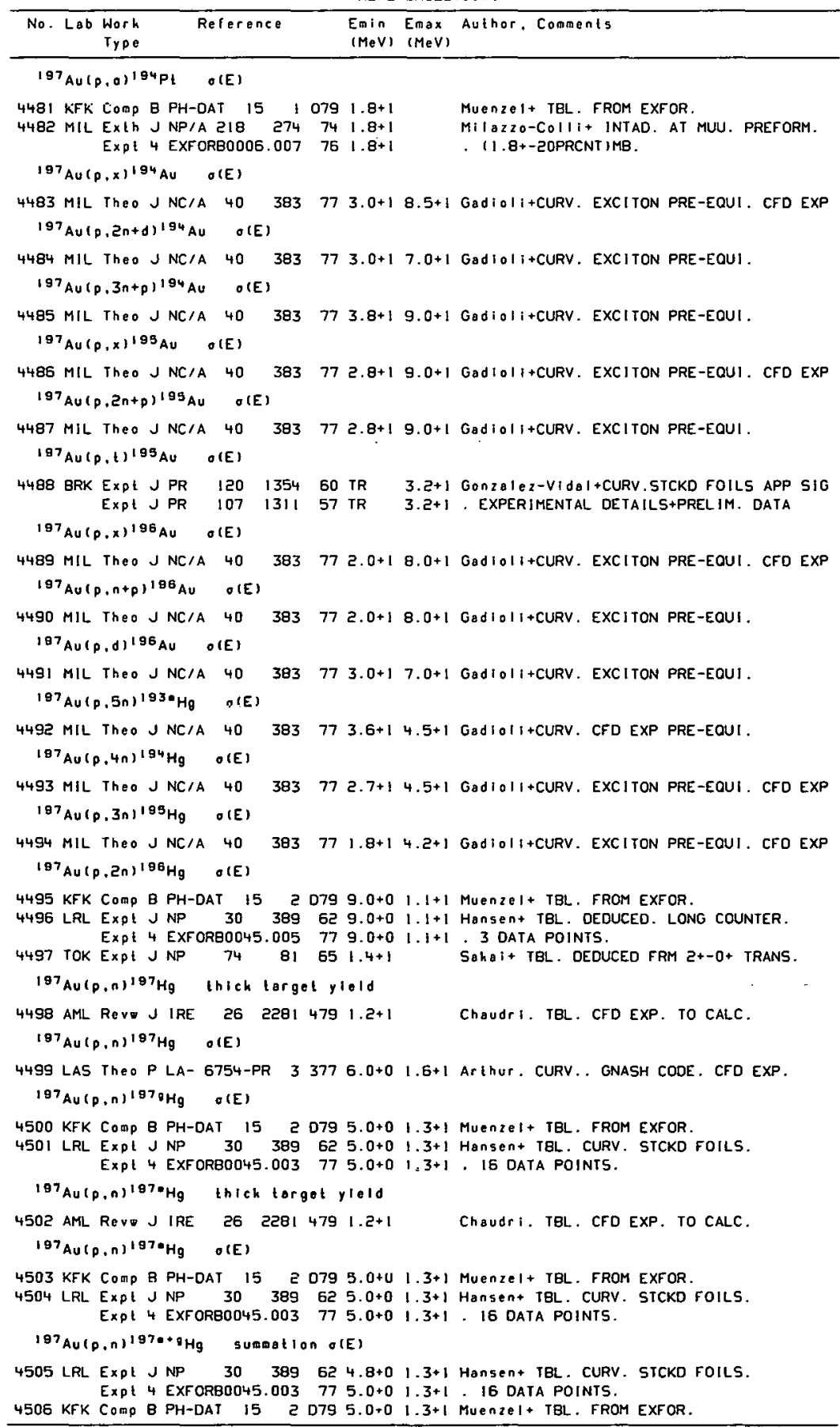




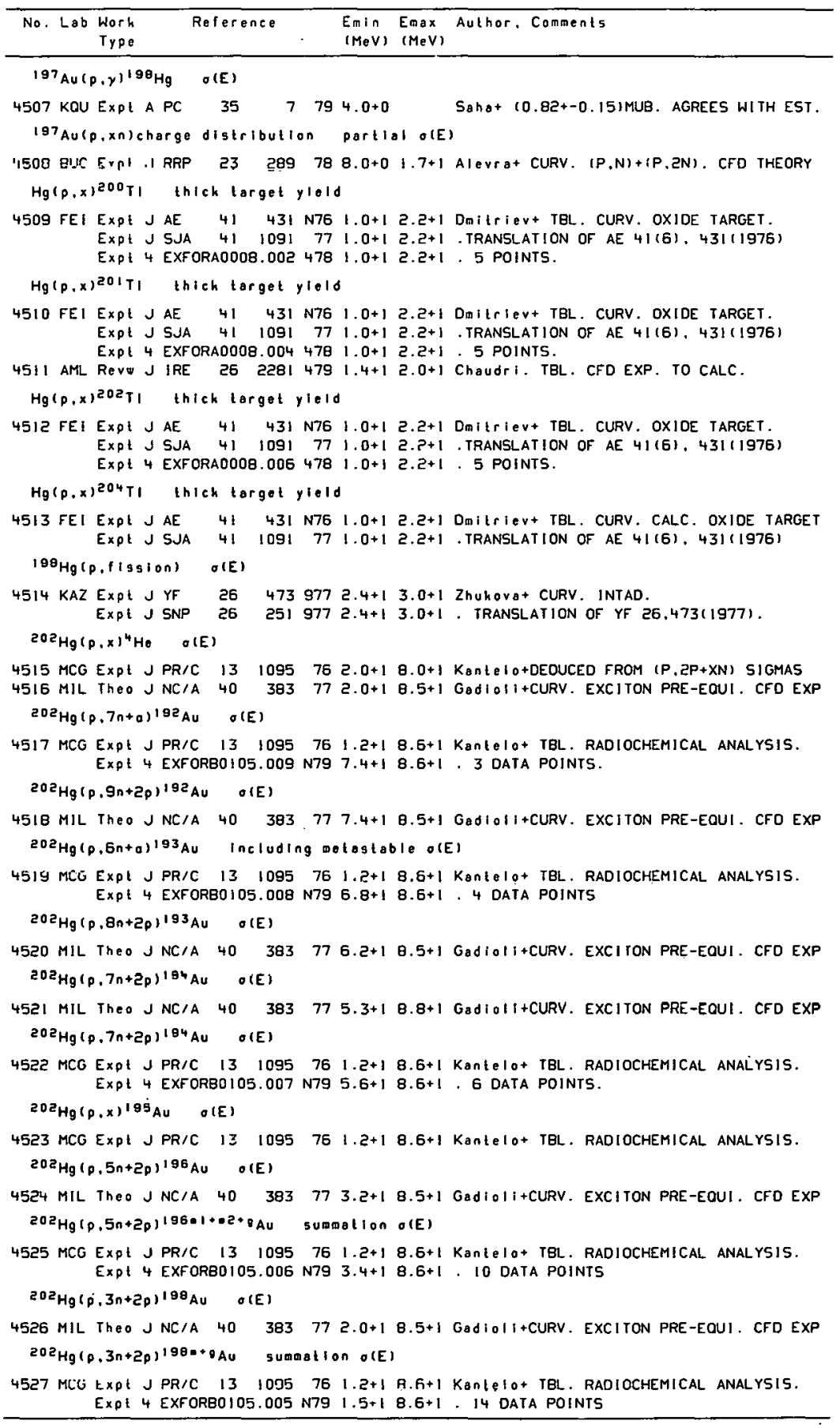




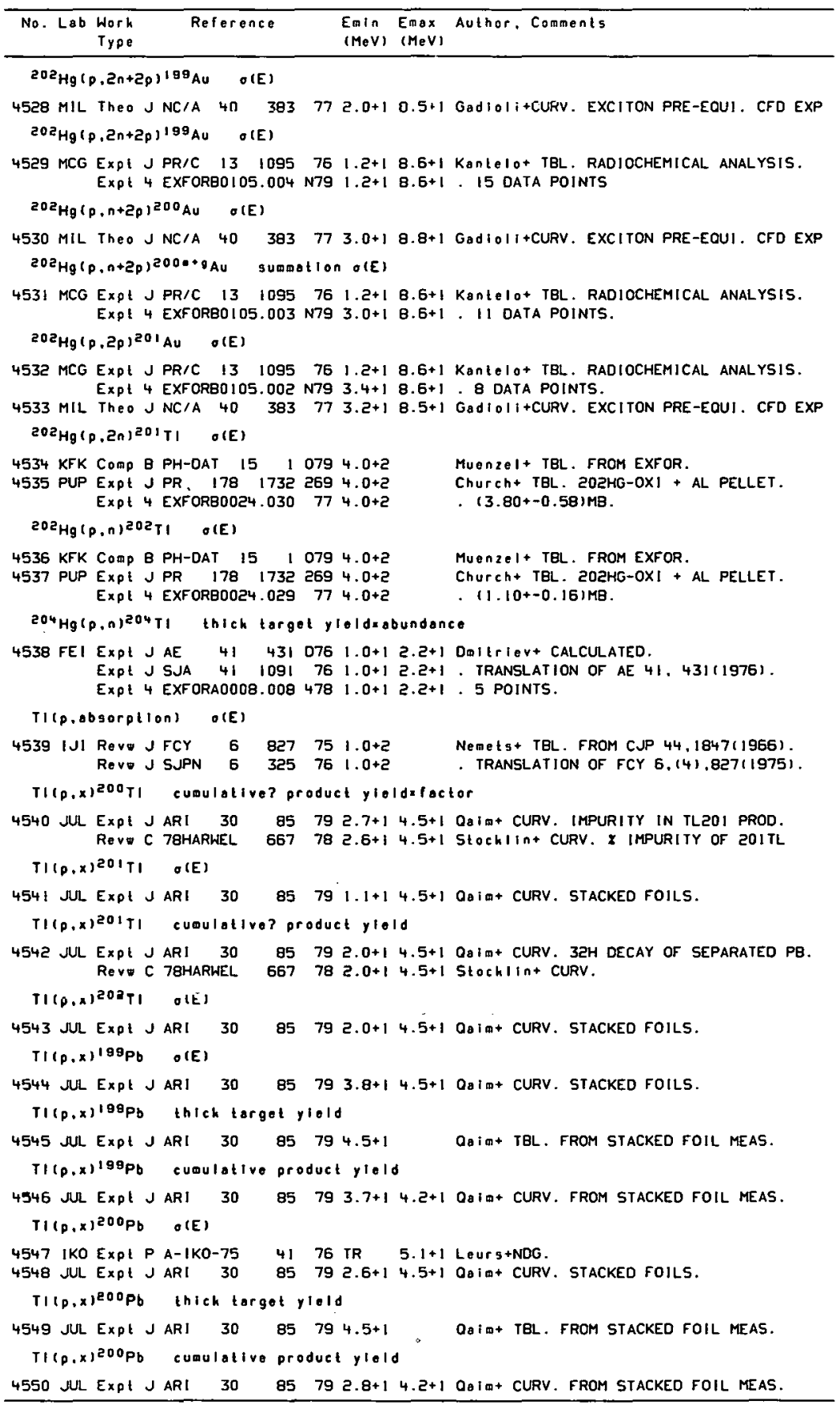


REFERENCES ( c on ( )

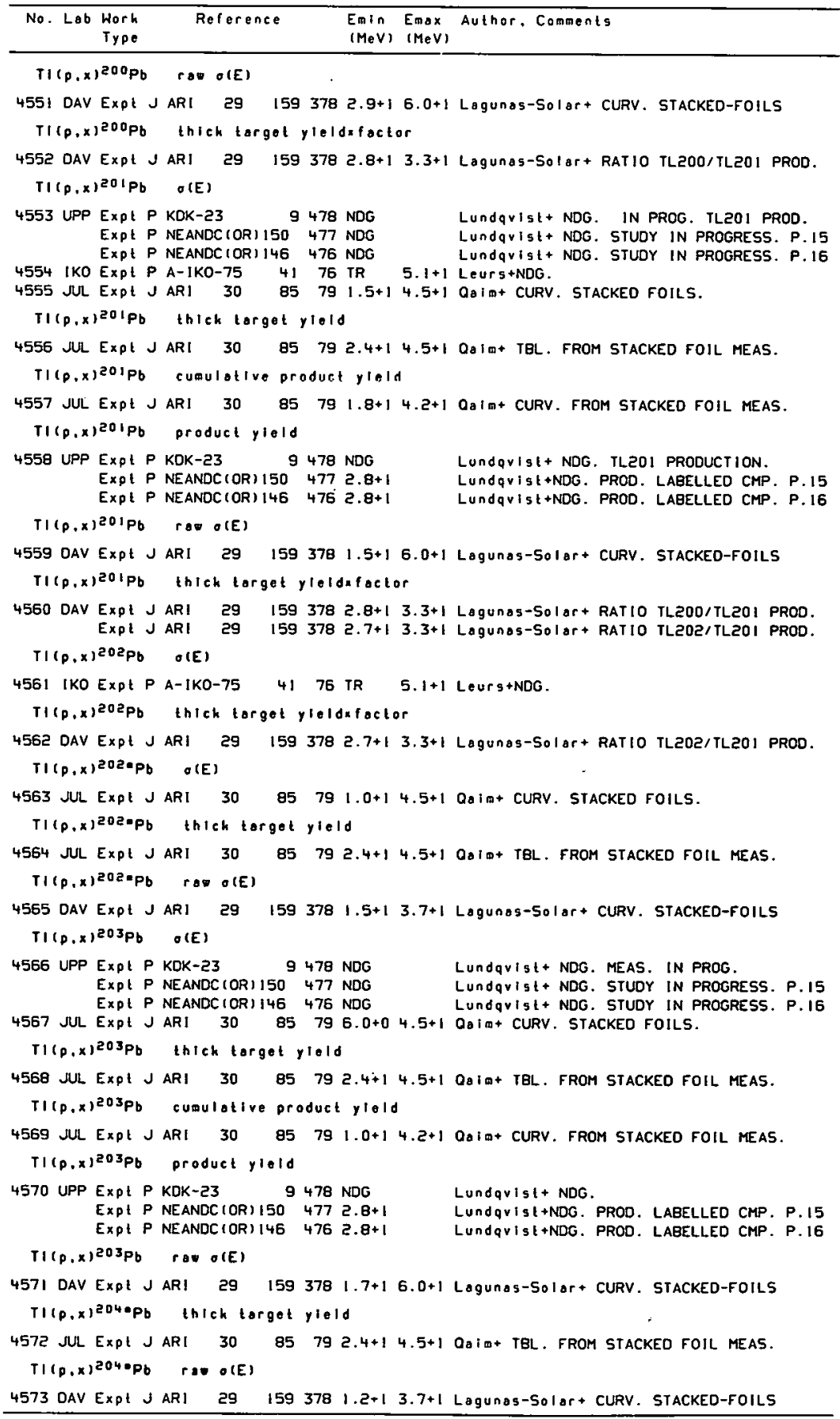


REFERENCES (cont)

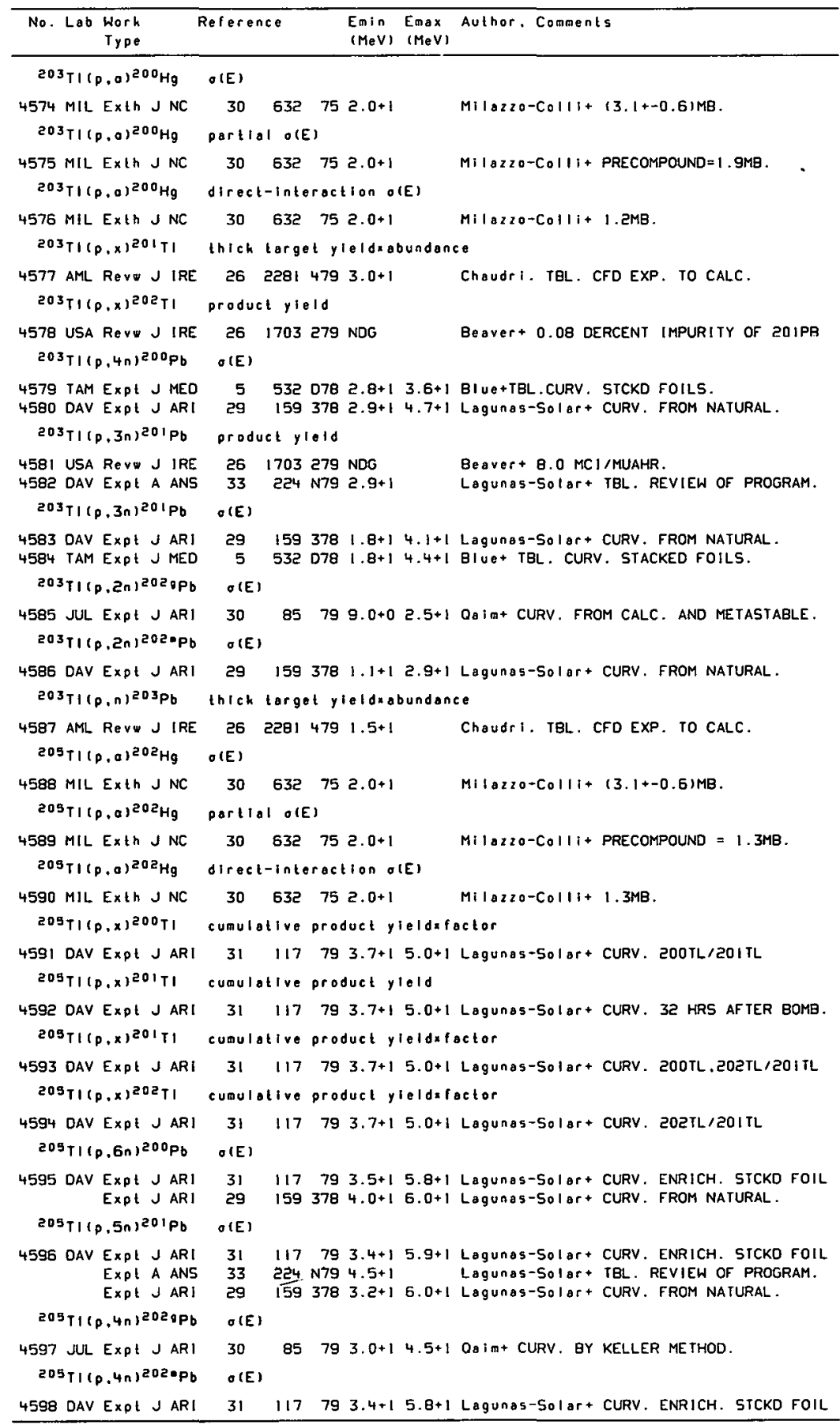




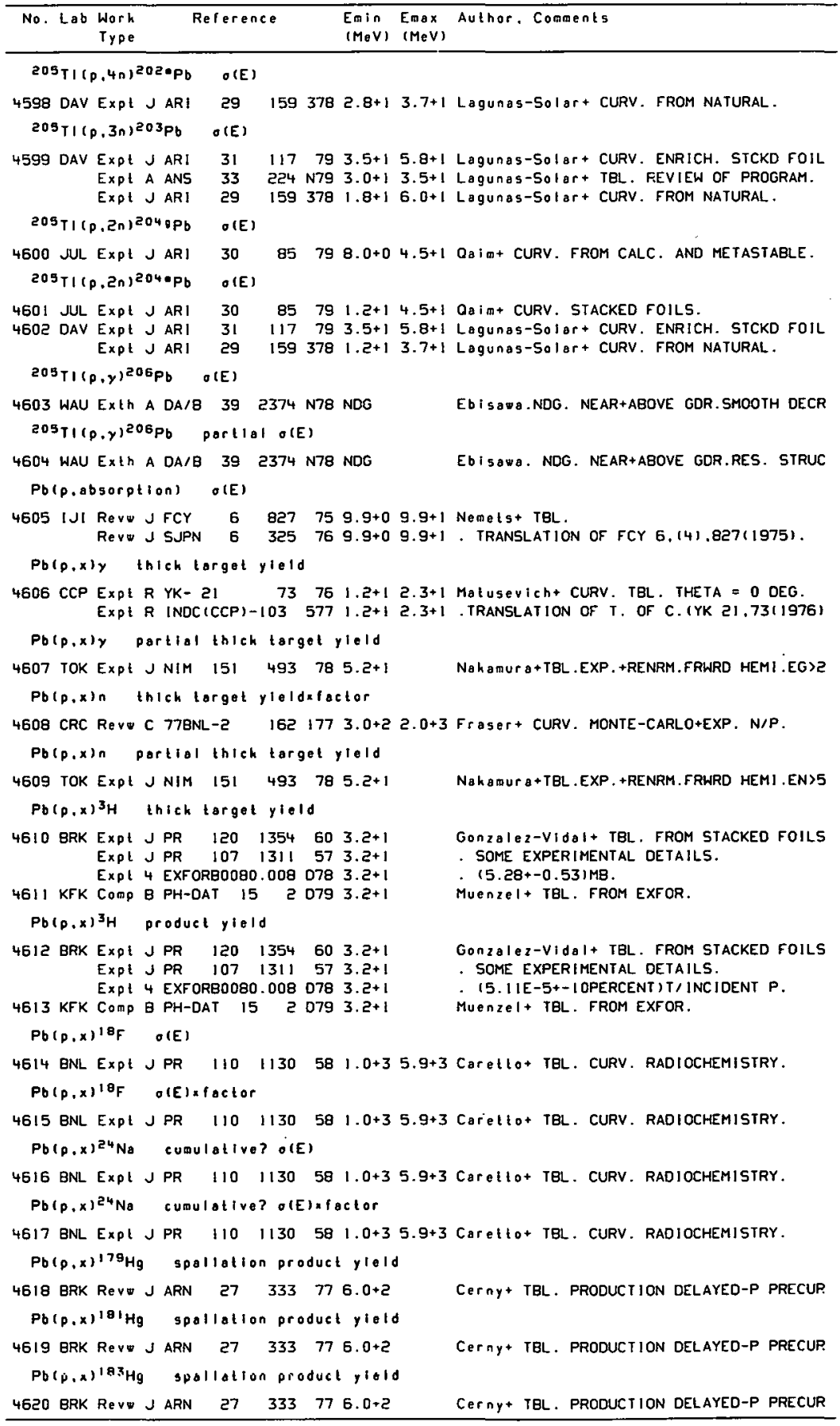


REFERENCES (con ()

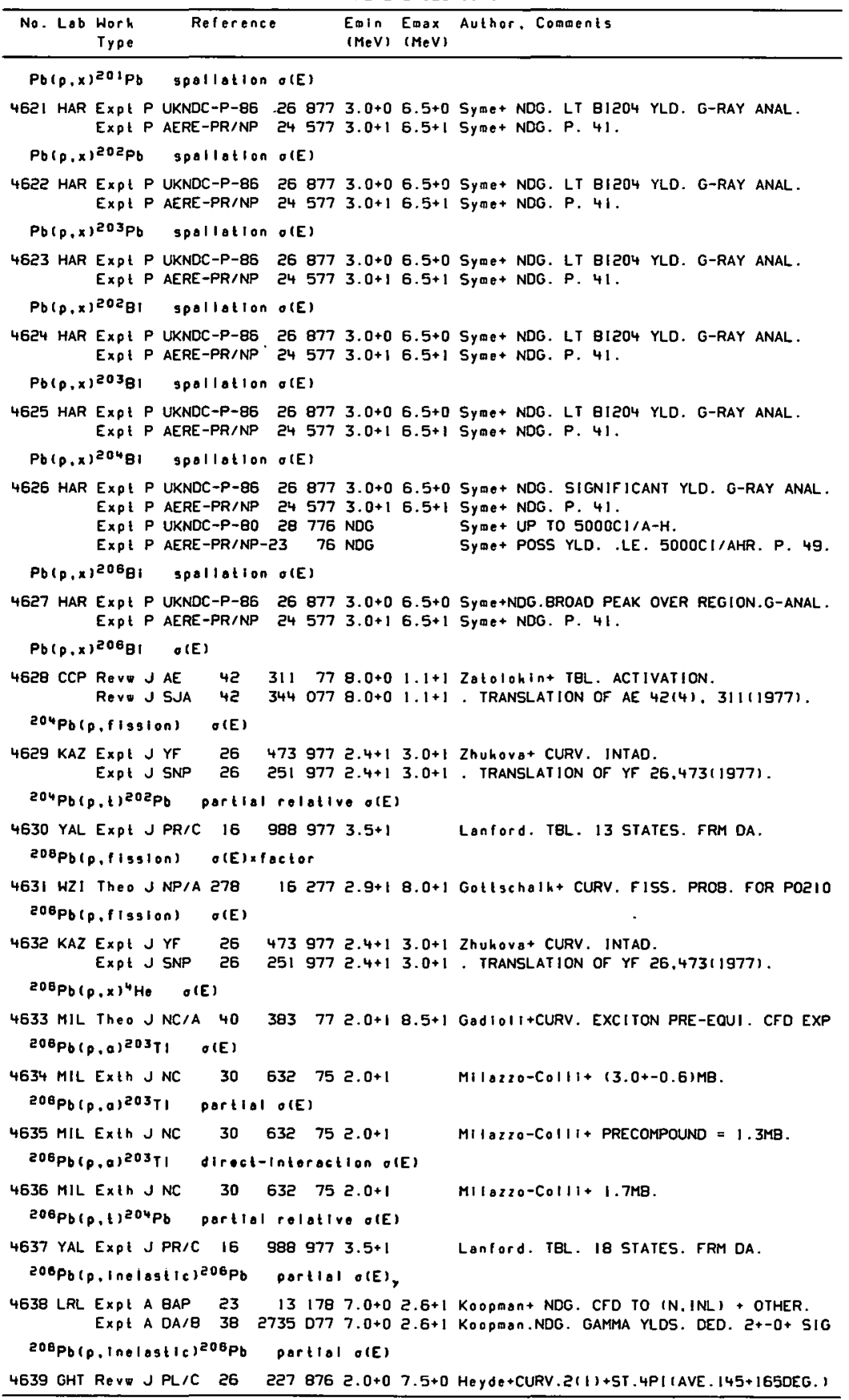




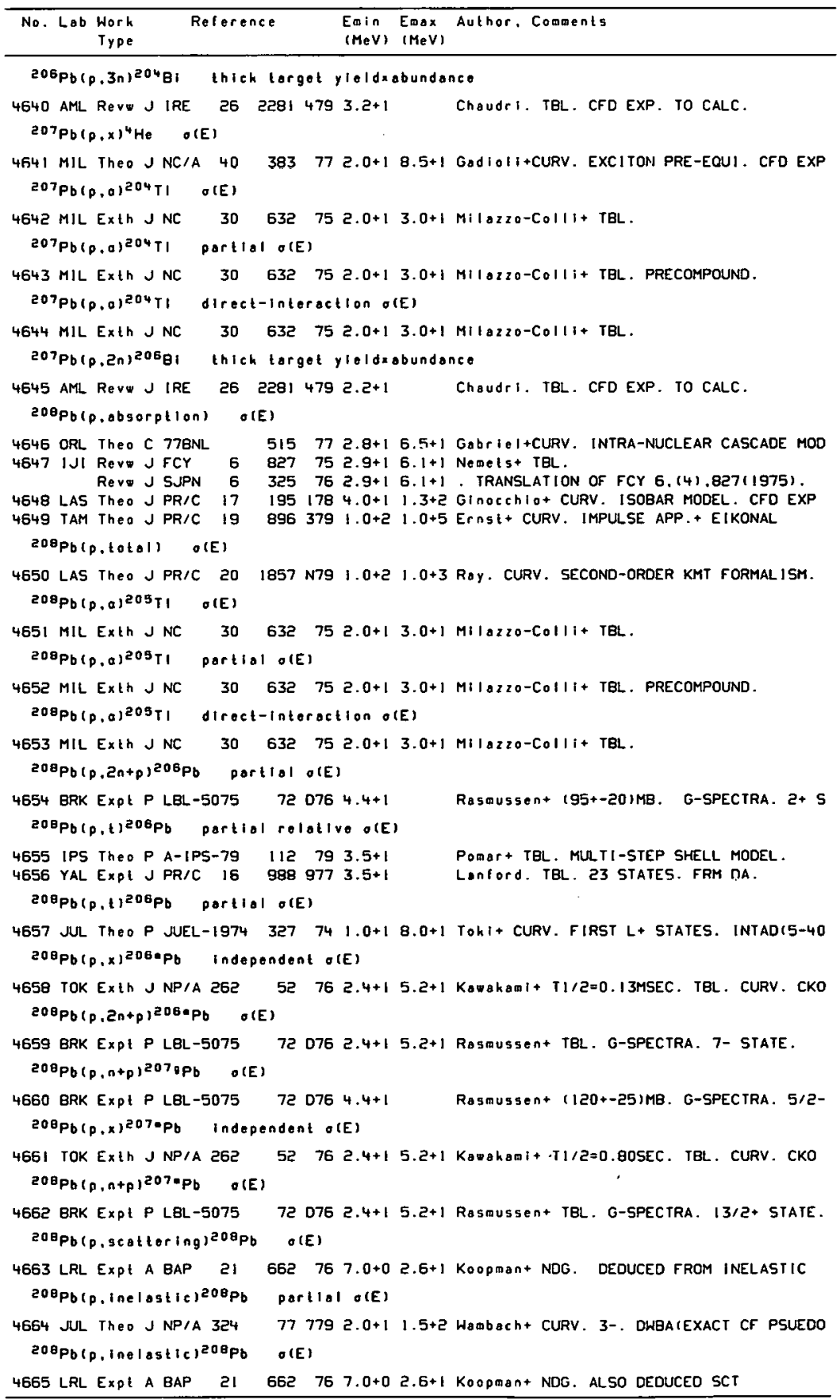




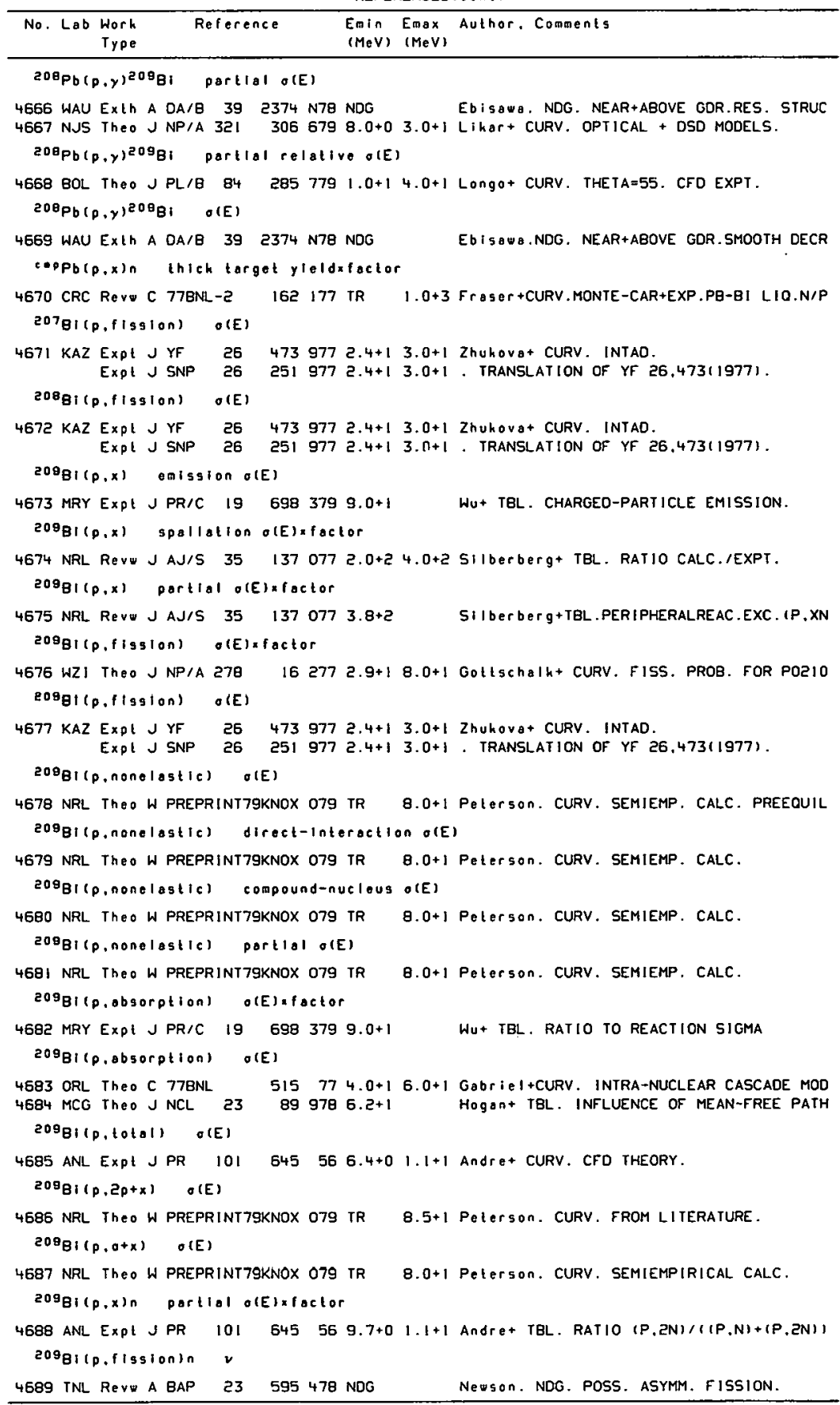




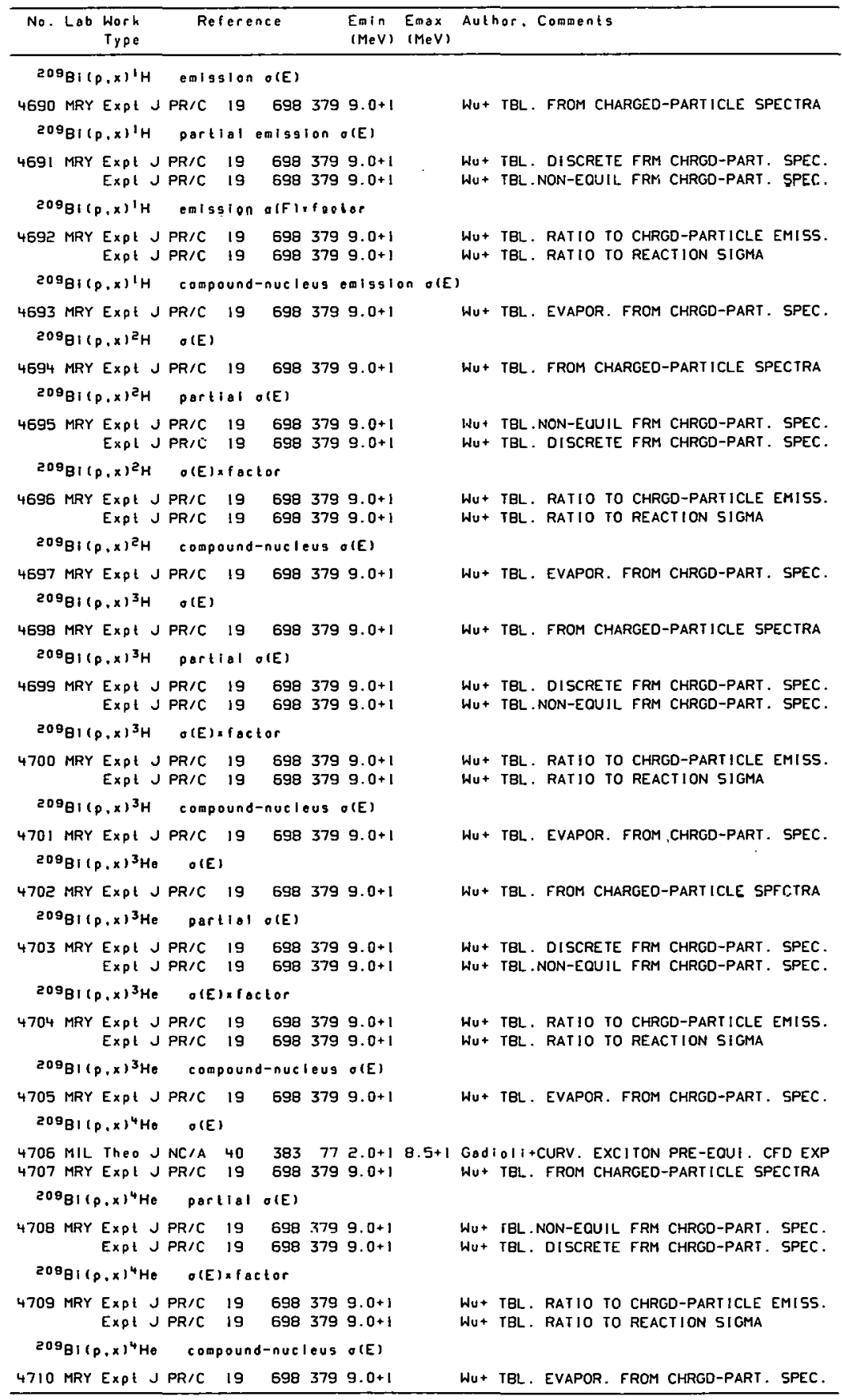


REFERENCES ( c ont)

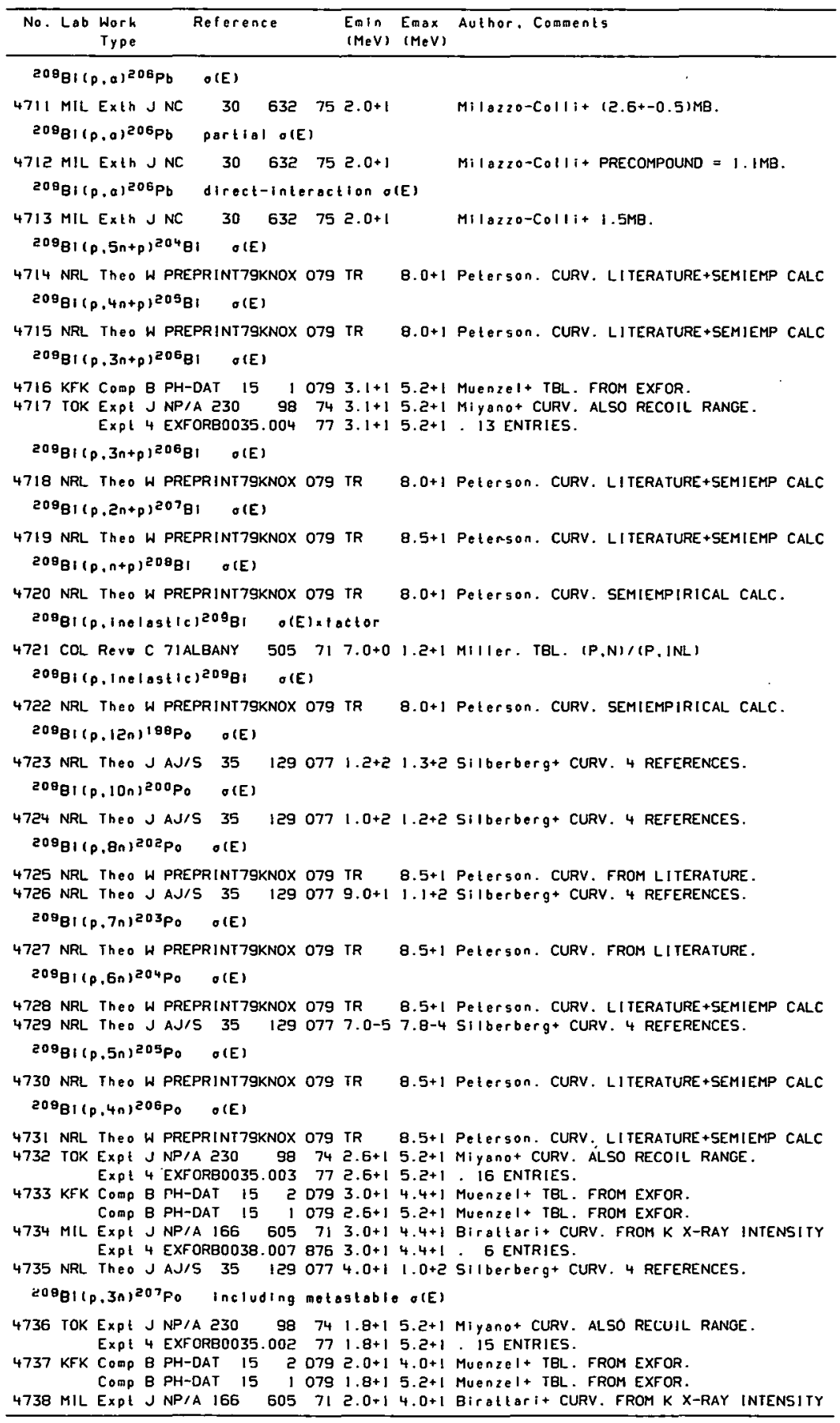




\begin{tabular}{|c|c|c|c|c|c|c|c|}
\hline & Lab & $\begin{array}{l}\text { Work } \\
\text { Type }\end{array}$ & Reference & $\begin{array}{l}\text { Em in } \\
(\mathrm{MeV})\end{array}$ & $\begin{array}{l}\text { Emax } \\
(M e V)\end{array}$ & Author. & Comments \\
\hline
\end{tabular}

$2098(0,3 n)^{207} \mathrm{Po}$ including metastable o(E)

4738 MIL EXPt 4 EXFORB0038.006 $8762.0+14.0+1$. 11 ENTRIES.

$20{ }^{2} \mathrm{BI}(p, 3 n)^{207} \mathrm{PO}_{0} \quad O(E)$

4739 NRL TheO W PREPRINT79KNOX 079 TR $8.5+1$ Peterson. CURV. LITERATURE+SEMIFMP CALC

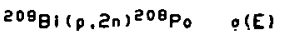

4740 NRL Theo W PREPRINT79KNOX 079 TR 8.0+1 Peterson. CURV. SEMIEMPIRICAL CALC.

4741 ANL Expl JPR $10 ! 645569.7+01.1+1$ Andre+ TBL. CURV. STACKED FOILS.

4742 NRL Theo J AJ/S $351290771.1+19.0+15$ ilberberg+ CURV. 4 REFERENCES.

4743 TOK Exth C 77TOKYO 721 977 1.8+1 6.4+1 Miyano+ CURV. A-COUNTING+ PREEQ. ANALYS

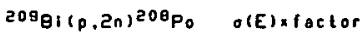

4744 ANL Expl JPR $10 ! 645569.7+01.1+1$ Aadre+ TBL. RATIO $(P, 2 N) /((P, N)+(P, 2 N))$ $200^{2}(p, n)^{209} P_{0} \quad O(E) \times f$ actor

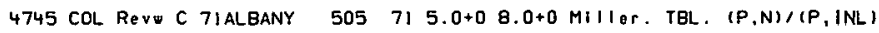
$20{ }^{0} \mathrm{~B}(p, n)^{20 \mathrm{P}^{\circ}}$ o(E)

4746 NRL ThEO W PREPRINT79KNOX O79 TR 8.5+1 Peterson.' CURV. FROM LITERATURE.

4747 KFK Comp B PH-DAT 15 2 $0799.8+01.0+1$ Muenzel+ TBL. FROM EXFOR.

4748 ANL Expl J PR 128 280 o62 9.8+0 $1.0+1$ WINg+ TBL. CURV. RESID. RADIOACTIVITY. Expt R T10-12696 $619.8+01.0+1$. SUPERSEDED.

Expt J PR $101645565.4+01.1+1$ Andre+ TBL. CURV. STACKED FOILS.

EXPt 4 EXFORBO065.0!1 $6789.8+01.0+1$. 2 DATA POINTS. FROM PR 128, 280.

4749 TOK Exth J JPJ $4510710781.8+16.4+1$ MIyano+ CURV. STACKED FOILS. AL DEGRAD. Exth C 77TOKYO $7219771.8+16.4+1$ Miyano+ CURV. A-COUNTING+ PREEO. ANALYS $20{ }^{0} B I(p, n) 20 p_{0}$ partlol o(E)

4750 TOK Exth J JPJ $4510710781.8+16.4+1$ Miyano+CURV. PRE-EOUIL. GDH + STAT. MOD $20{ }^{\circ} B(p, n)^{209} P_{0}$ compound-nucleus otE)

4751 TOK Exth J JPJ $4510710781.8+16.4+1$ Miyano+ CURV. GQH + STAT. MOD.

${ }^{209} B(1 p, n)^{209 P_{0}}$ diract-Interaction $O(E)$

4752 TOK Exth J JPJ $4510710781.8+16.4+1$ Miyano+ CURV. GDH + STAT. MOD.

$20{ }^{B} B(0, \gamma) 210 P_{0} \quad$ O(E)

4753 ANL Expt $J$ PR $101 \quad 645566.4+01.1+1$ And $t$ e + TBL. CURV. STACKED FOILS.

4754 ORL ExPt JPR 100 POS $0551.0+12.2+1$ Cohen. TBL.

4755 PAR Exth P CEA-N-2026 $1589774.0+1 \quad 1.0+2$ Lagarde+ CURV. ALICE CFO TO EXP

4756 INU Expt W NEWSLETTERZ2 $32785.0+11.9+2$ Friese I+ CURV. INCLUDES PIO PRODUCTION.

209 gi (p.x)charge distribution thich terget yleld

4757 LAS EXPI P LA- 7607-PR $471792.0+2 \quad$ Exp. 103(LAS.BNL). AT PRODUCTION.

200 gi(p,xn)charge distribution partlal o(E)

4758 BUC Expt J RRP $23289787.0+01.7+1$ DU⿴囗十 + CURV. (P.N $+(P, 2 N)$. CFD THEORY

209 Bilp,figsionimass distribution fission ylold

4759 PAR theo J JPRL $403187793.6+15.6+1$ Da-5ilveira. CURV. CATASTROPHY CFD EXPT

209 Bito.fissionimass distribution cumulative ligsion yiald

4760 TNL Reva A BAP 23595478 NDG Nenson. NDG. POSS. ASYMM. FISSION.

${ }^{209}$ Bi(p.fission)mass distribution primory figsion yield

4761 TUE Revw J ZP/A $278 \quad 15763.2+1 \quad$ Gonnengeint CURV AS FUNCTION OF FF MASS

$20{ }^{0} B(p, x)$ isolople distribution spallation o(E)

4762 NRL Revw J AJ/S $351370773.8+2 \quad$ Silberberg+CFD.CALC.METH. +EXP. 1461SOTOP

$20{ }^{\circ} B(p, x n)$ Isotoplc digtribution o(E)

4763 NRL REVE J AJ/S $351370771.0+18.0+2$ silberberg+ CURV. $X=2-12$.

Theo J AJ/S $351290774.0+2$ Silberberg+ CURV. $X=1-10.4$ REFERENCES.

4764 PAR Exih P CEA-N-2026 $1589772.0+1$ 1.5+2 Lagardet CURV. $X=2-9$.

Expt T LE BEYEC $4662.0+1 \quad 1.5+2$ Le-Beyec.

$226_{\text {Ratp.fisgionin } v}$

4765 WZI Expt J PR/C $1610669771.2+1 \quad$ Gayer+ CURV. AS FUNC. OF PROMPT F MASS 
REFERENCES (cont)

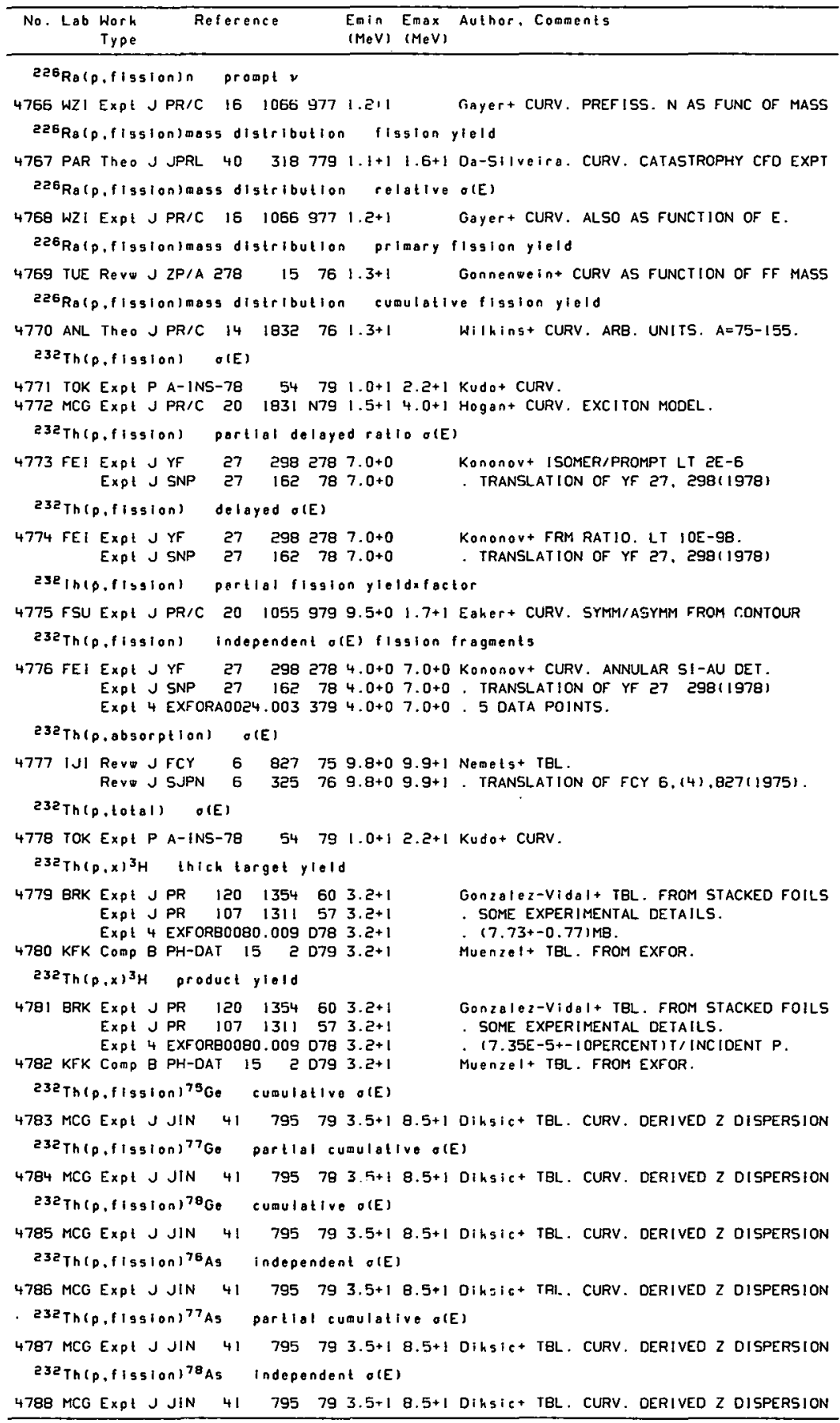




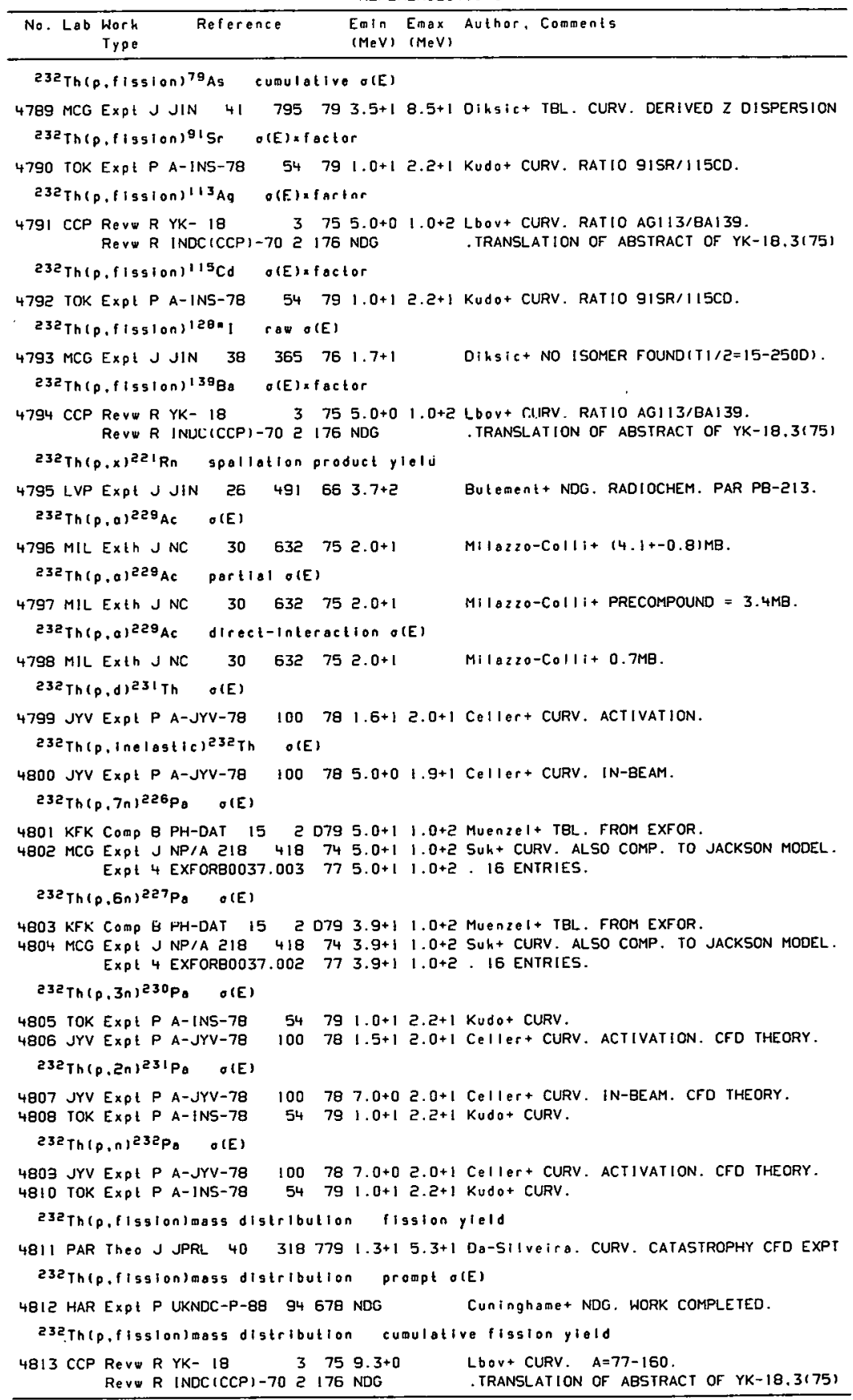


REF ERENCES ( cont)

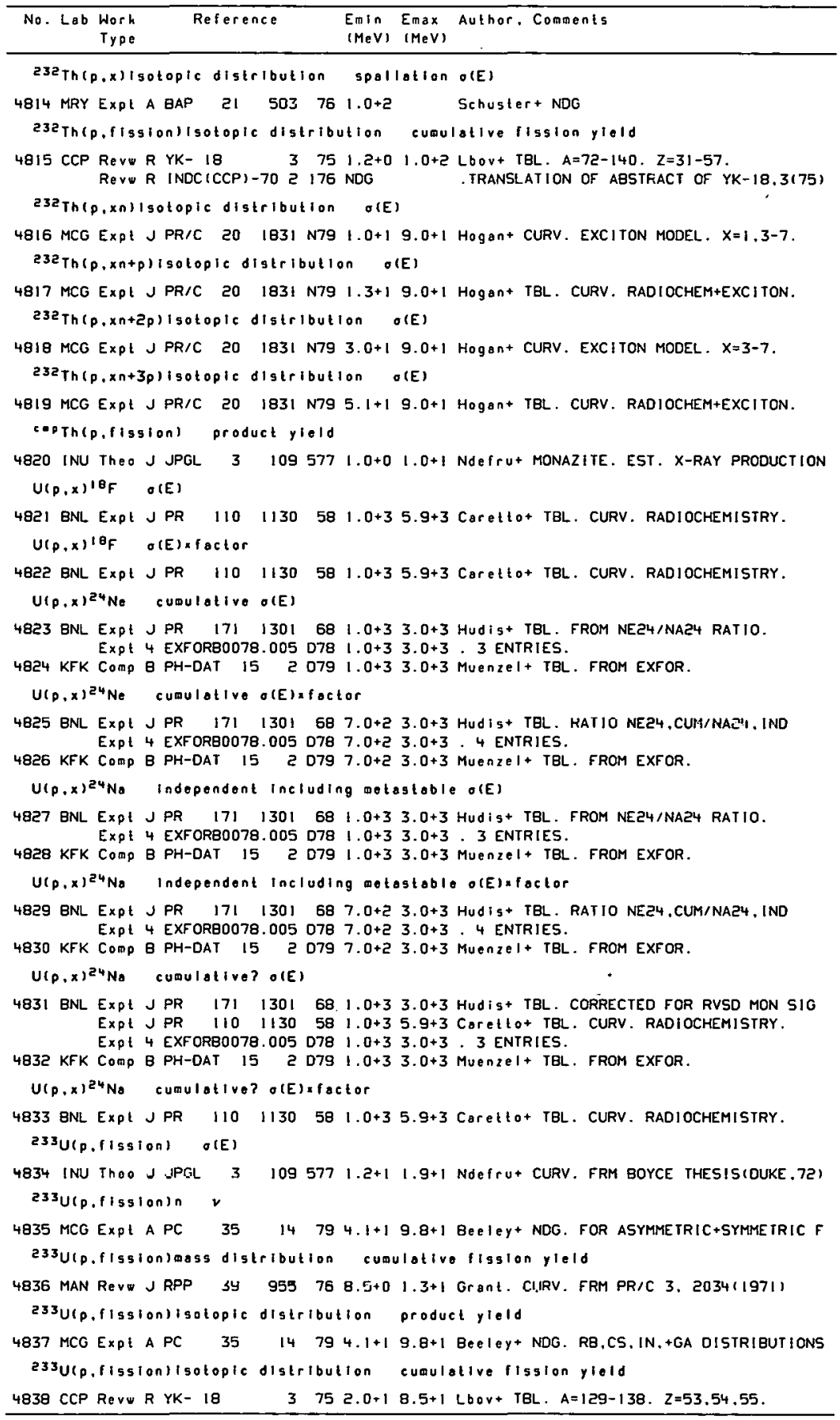


REFERENCES(cont)

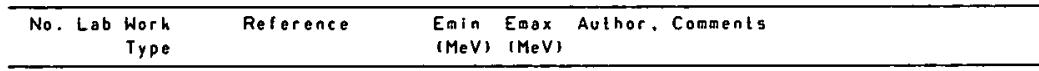

233 (p.lisstonisotopte distribution cumulative fission yield

4838 CCP Rev $R$ (NDC (CCP) $-70 \geq 176$ NDG

. TRANSLATION OF ABSTRACT OF YK-18.3(75)

$234 U(p, f i s s i o n)$ O(E)

4939 INU The J JPGL $31095771.2+1 \quad 1.9+1$ Ndefru+ CURV. FRM BOYCE THESIS(DUKE,72)

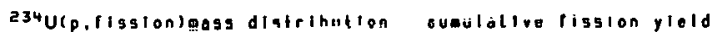

4840 TNL EXPt P DOE-NDC-15 $2194796.5+01.4+1$ Cus 50 + NDG. FROM ENERGY CORRELATIONS.

Expt A DA/B $3944333796.5+01.4+1$ Epperson+ NDG. FROM FF ENERGY-CORR. MEA

Expt P ORO-1667-17 12 $790.0+03.0+1$ Epperson+ NDG. 100 MASSES.ANAL. IN PROG

Theo P ORO-1667-17107 $796.5+01.4+1$ CUS\$ON+ NDG. ANALYSIS OF MASS DIST.

The o P A-TNL-78 $107796.5+01.4+1$. SAME AS ORO-1667-17

Expl P A-TNL-78 12 $790.0+03.0+1$. SAME AS ORO-1667-17

Expt P ERDA-NDC-3 $28 \theta 5766.3+03.0+1$ Purser+ NDG. 100 MASS DISTRIBUTIONS.

$23{ }^{4} U(p, f i s s t o n)$ ass digtribution prompt $\sigma(E)$

4841 TNL Expt A DA/B $3944333796.5+01.4+1$ Epperson+ NDG. FIRST-CHANCE FISS.

The P ORO-1667-17107 $796.5+01.4+1$ CUSBOn+ NDG. TIRST CHANCE-FISSION.

The P A-TNL-78 $107 \quad 796.5+01.4+1$. SAME AS ORO-1657-17

$234 U(p, n+f i s g i o n) m a s s$ distribution fission ylelo

4842 TNL Expl A DA/B $3944333796.5+01.4+1$ Epperson+ NDG. FROM FF ENERGY-CORR. MEA

$234 U(p, n+f i s g i o n) m a s s$ distrtbution prompto(E)

4843 TNL Expl A DA/B $3944333796.5+01.4+1$ Epperson+ NDG. FIRST-CHANCE FISS.

$235 U(p, f i g s i o n)$ O(E)

4844 INU Theo J JPGL $31095771.2+1 \quad 1.9+1$ Ndefru+ CURV. FRM BOYCE THESIS(DUKE.72) 235U(p,figsion) $r a \%$ O(E)

4845 BUC Expl J RRP $22743771.4+11.4+1$ Borcea+ CURV. DEDUCED IAR.

$235 U(p, f 155$ ion) $950 / 9 N b$ ratio OIE)

4846 LAS TheO $J$ ANS $24 \quad 461$ N76 $1.0+1$ MadIand+ CURV. SEMIEMPERICAL.

$235 U(p, t$ lastic) $235 U$ row o(E)

4847 BUC Expt J RRP $22743771.4+11.4+1$ Borgea $\quad$ CURV. DEOUCED IAR.

$235 u(p, f i s s i o n) m a s$ distribution cumulative fission yield

4848 TNL Expt P DOE-NDC-15 $2194796.2+03.0+1$ CUSSON+ NDG. FROM ENERGY CORRELATIONS. Expt A DA/B $3944333796.2+03.0+1$ Eppersont NDG. FROM FF ENERGY-CORR. MEA Expt P ORO-1667-17 I2 $790.0+03.0+1$ Epperson+ NDG. 100 MASSES.ANAL. IN PROG Theo P ORO-1667-17107 $796.2+03.0+1$ Cussont NDG. ANALYSIS OF MASS DIST.

Theo P A-TNL-78 $107 \quad 796.2+0.3 . n+1$. SAME AS ORO-1657-17

Expt P A-TNL-78 ID $790.0+03.0+1$. SAME AS ORO-1667-17

Expt P ERDA-NDC-3 288576 6.3+0 3.0+1 Purser+ NDG. 100 MASS DISTRIBUTIONS.

Expt P ERDA-NDC-2 $1775755.0+01.3+1$ Purser+ NDG. 35 MASS DISTRIBUTIONS.

4849 CCP ReVw R YK- 18 3 $751.2+18.5+1$ Lbov+ TBL. A=75-159. Z $2=34-54$. Rev R INDCICCPI-70 2176 NDG .TRANSLATION OF ABSTRACT OF YK- 18.3175)

$235 U(p, f i s s i o n) m a s s$ distribution prompt o(E)

4850 TNL Expt A DA/B $3944333796.2+03.0+1$ Eppersont NDG. FIRST-CHANCE FISS.

The o P ORO-1667-17107 $796.2+03.0+1$ Cusson+ NDG. FIRST CHANCE-FISSION.

Theo P A-TNL-78 $107796.2+03.0+1$. SAME AS ORO-1667-17

Expt A BAP $23 \quad 5964786.5+03.0+1$ Epperson+NDG.DED. SINGLE FISSIONING YLD

$235 U(p, x)$ isotoplc distribution spollation o(E)

4851 MRY Expt A BAP $21583761.0+2 \quad$ Schuster+ NDG

235U(p.tissionisotopic distribution cumulativelission yield

4852 CCP Revw R YK- $18 \quad 3751.2+18.5+1$ Lbov+ TBL. A=75-159. Z=34-54.

Revw R INDC (CCP)-70 2176 NDG . TRANSLATION OF ABSIRACT OF YK- 18,3175$)$

$236 u(p, f 155 i 0 n) \quad \sigma(E)$

4853 INU Theo J JPGL $31095771.2+11.9+1$ Ndefru+ CURV. FRM BOYCE THESISIDUKE, 72)

236utp.fissionimass distribution cuaulative fisston yield

4854 TNL EXPt P DOE-NDC-15 $2194796.2+03.0+1$ CusSON+ NDG. FROM ENERGY CORRELAT1ONS.

Expl A DA/B $3944333796.2+03.0+1$ Epperson+ NDG. FROM FF ENERGY-CORR. MEA

Expt $P$ ORO-1667-17 I2 79 0.0+0 3.0+1 Epperson+ NDG. 100 MASSES.ANAL. IN PROG The o P ORO-1667-17107 $796.2+0$ 3.0+1 Cussont NDC. ANALYSIS OF MASS UIST. 
REFERENCES (cont)

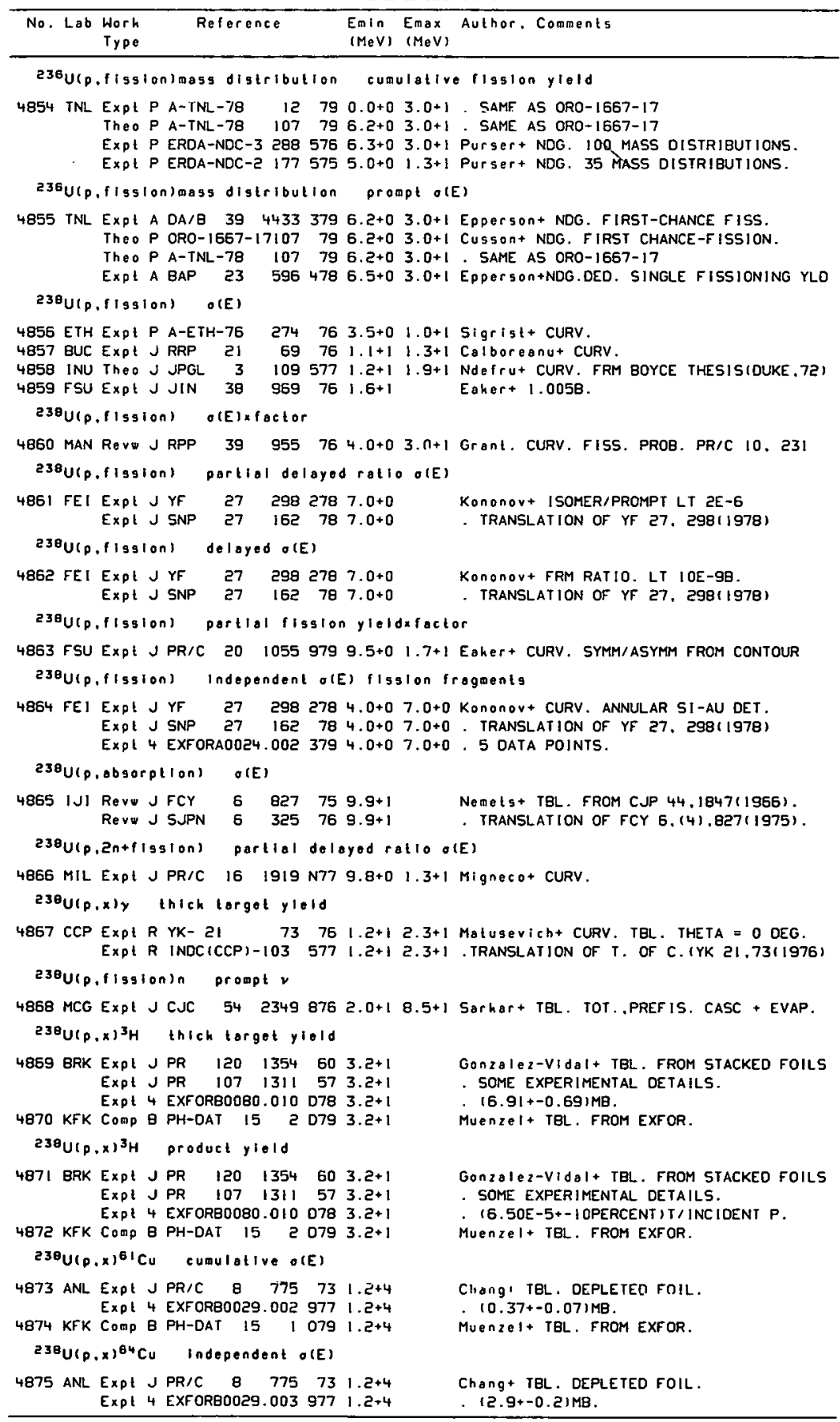


REFERENCES $(\operatorname{con} t)$

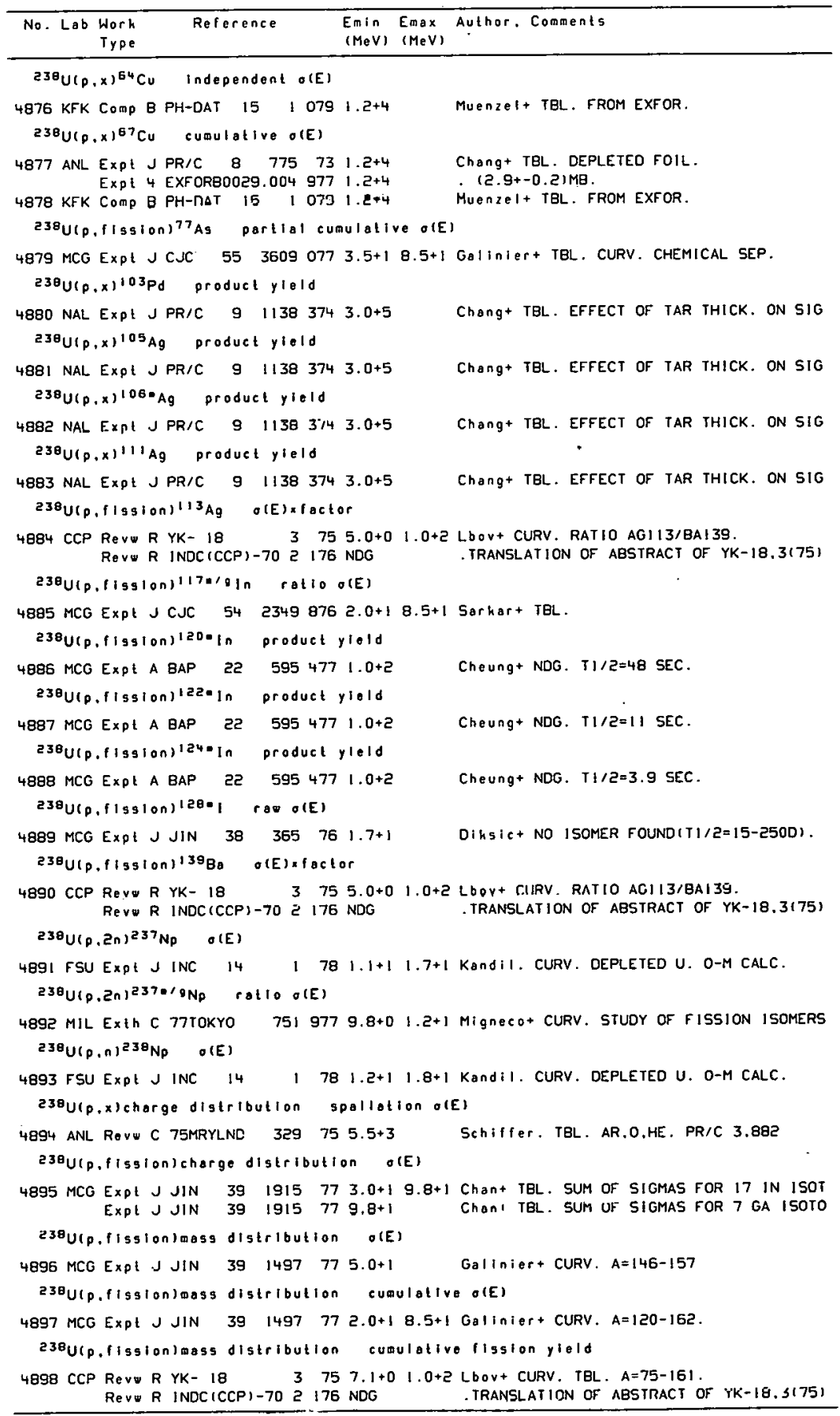


REFERENCES(cont)

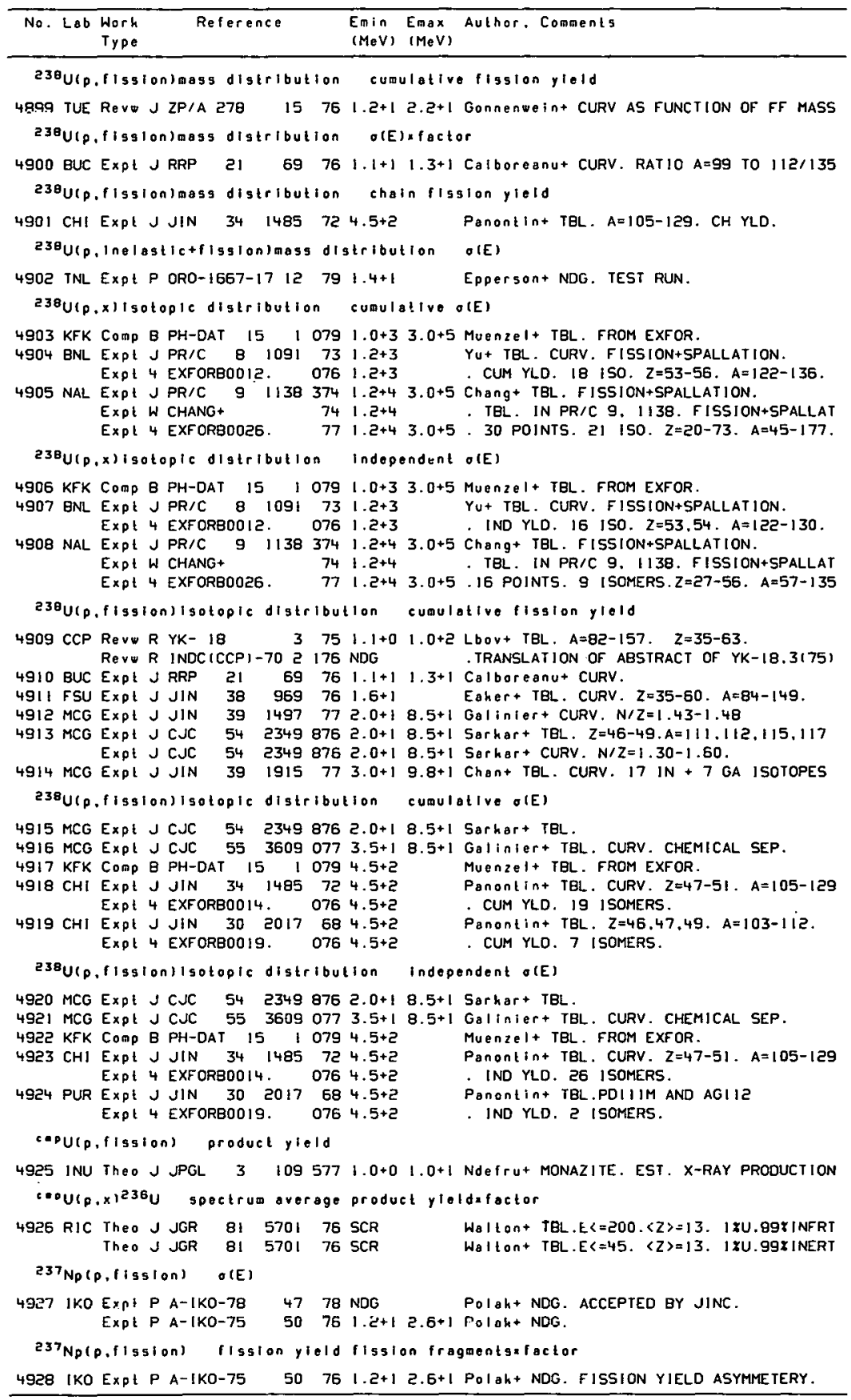




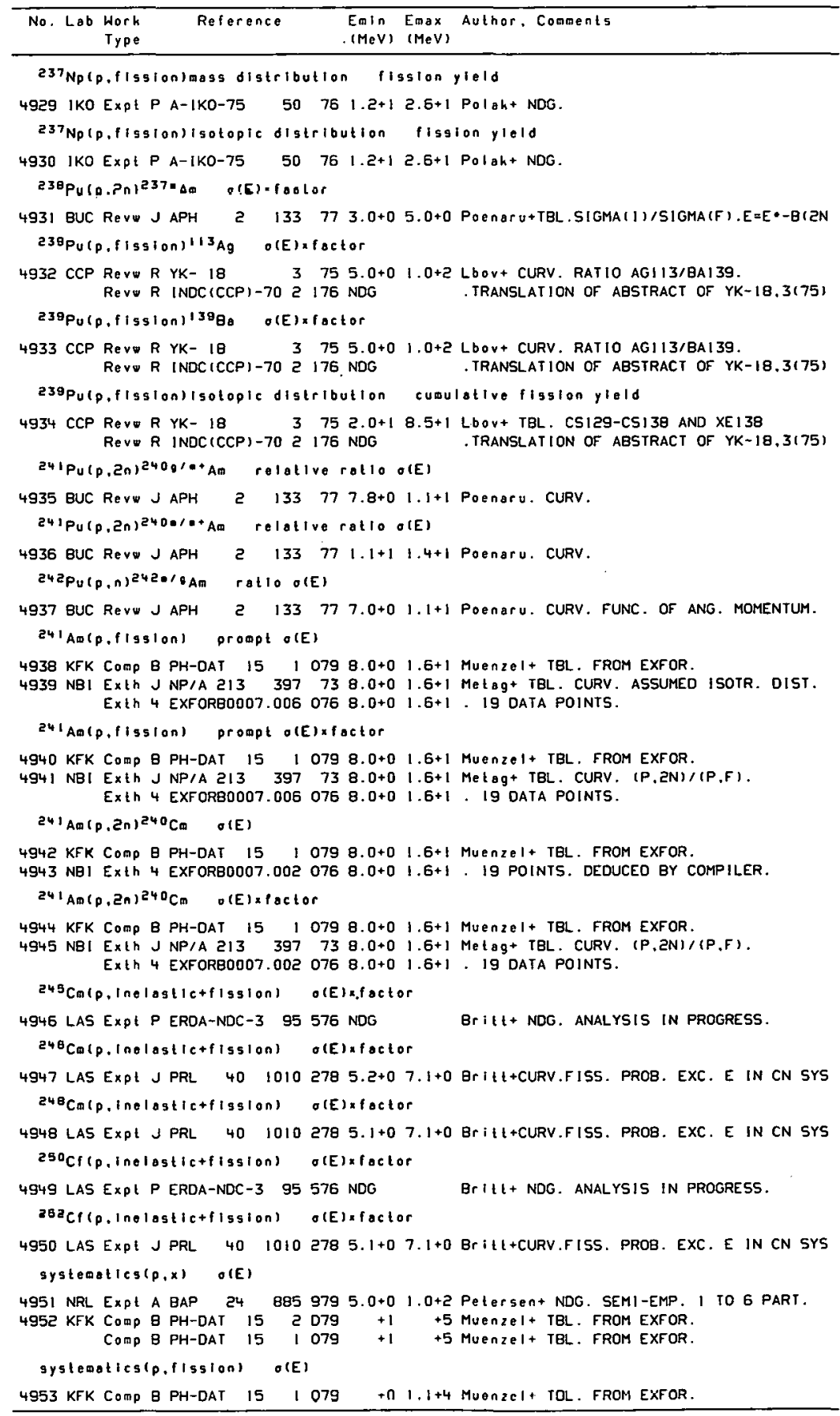




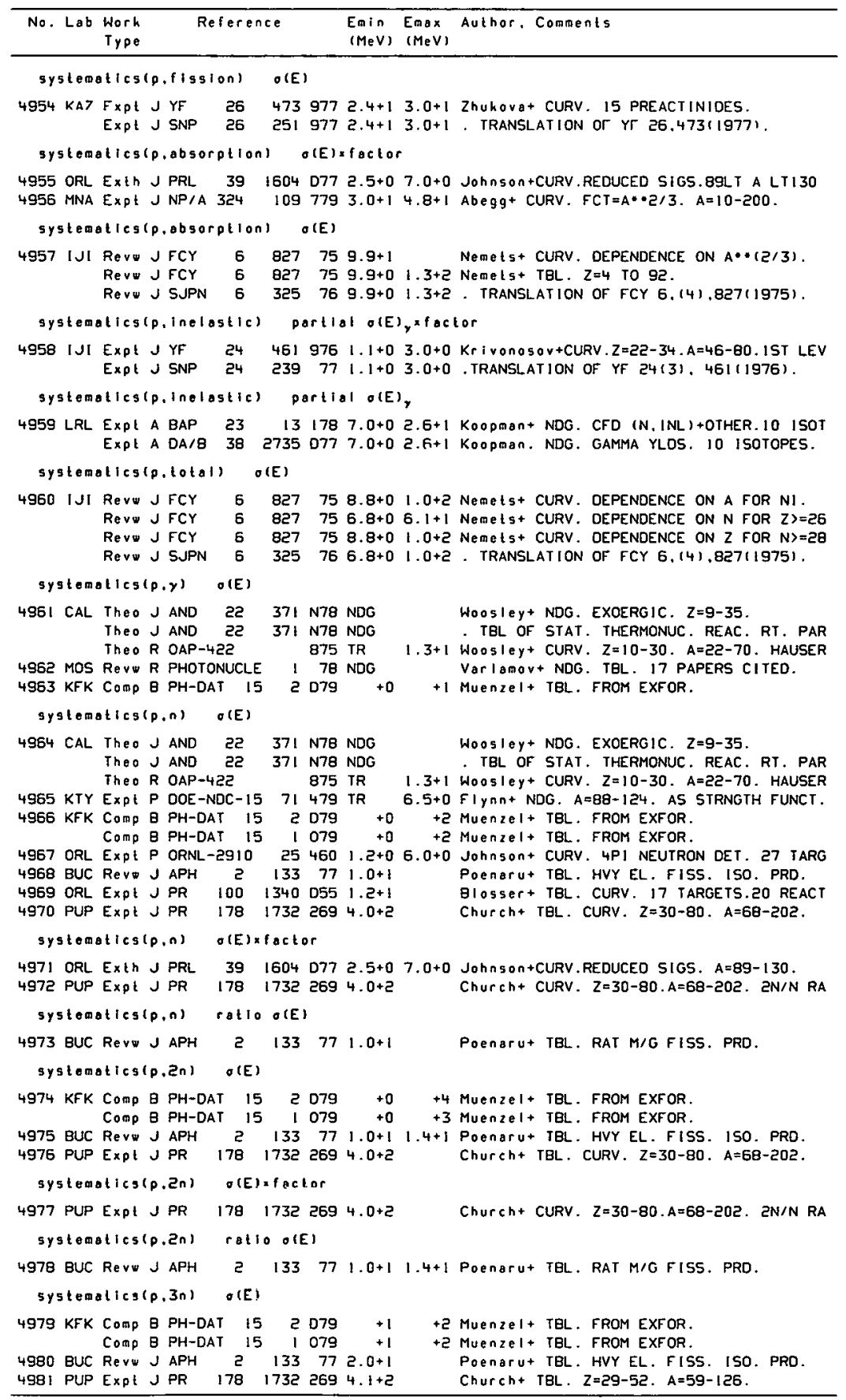


REFERENCES ( c on ()

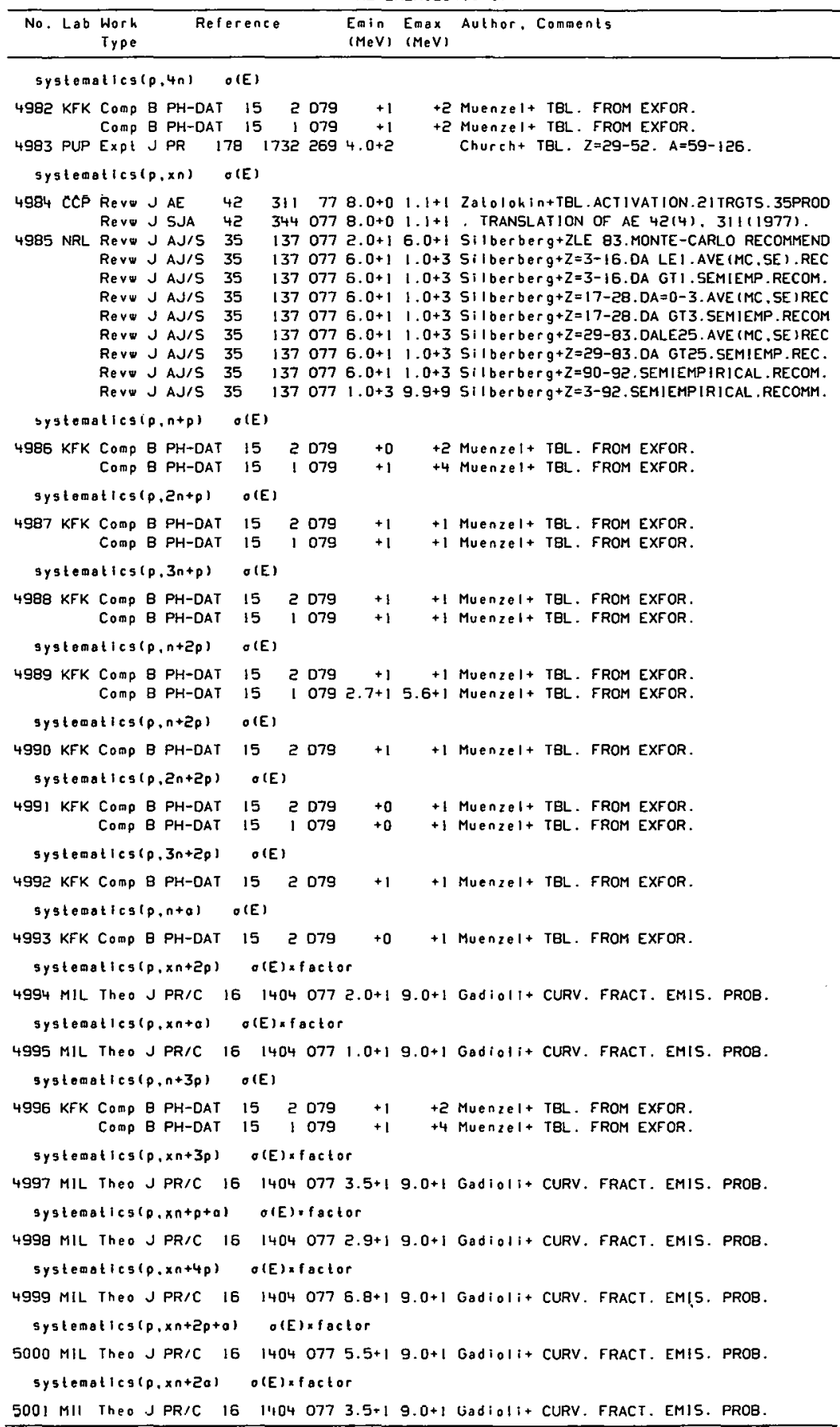




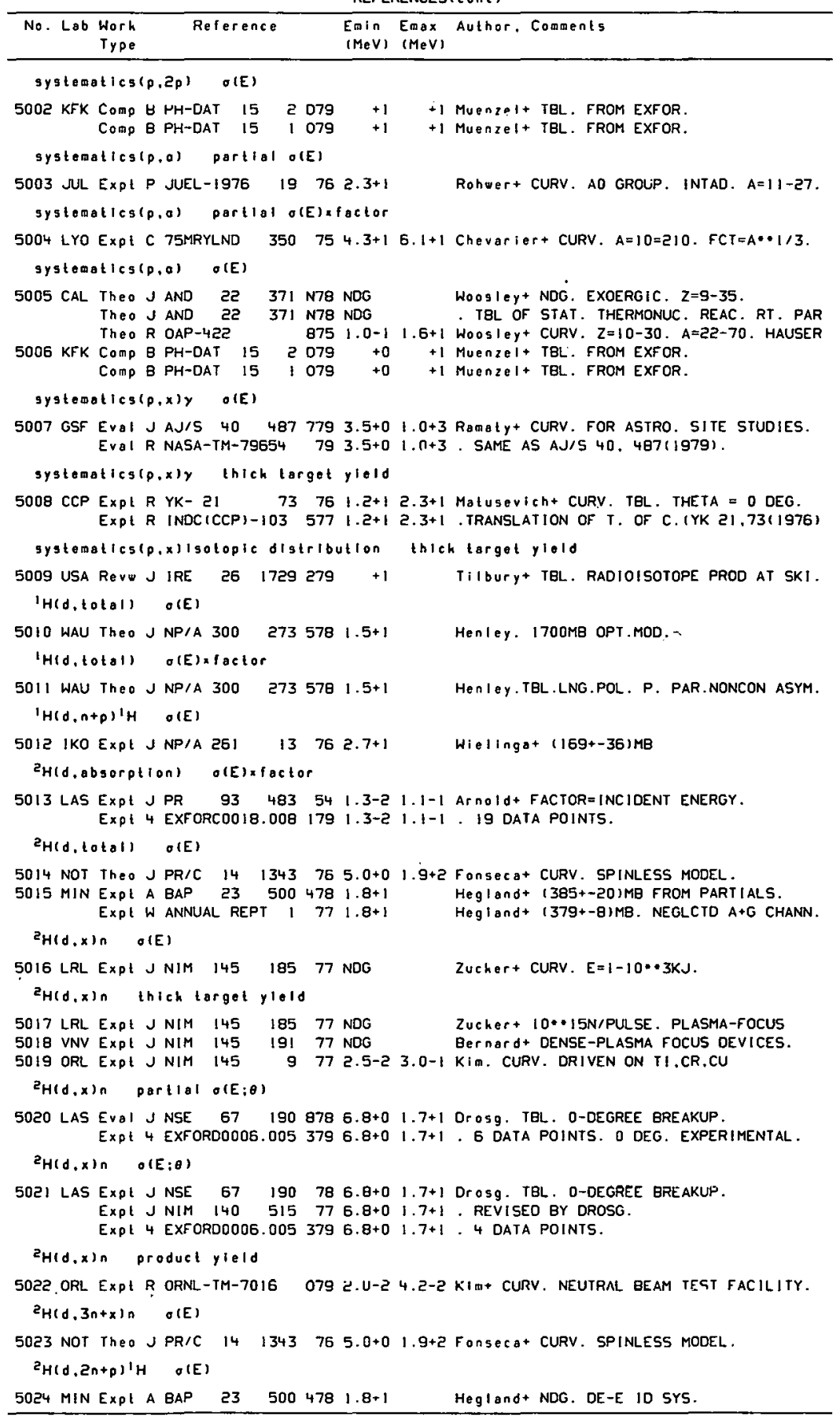




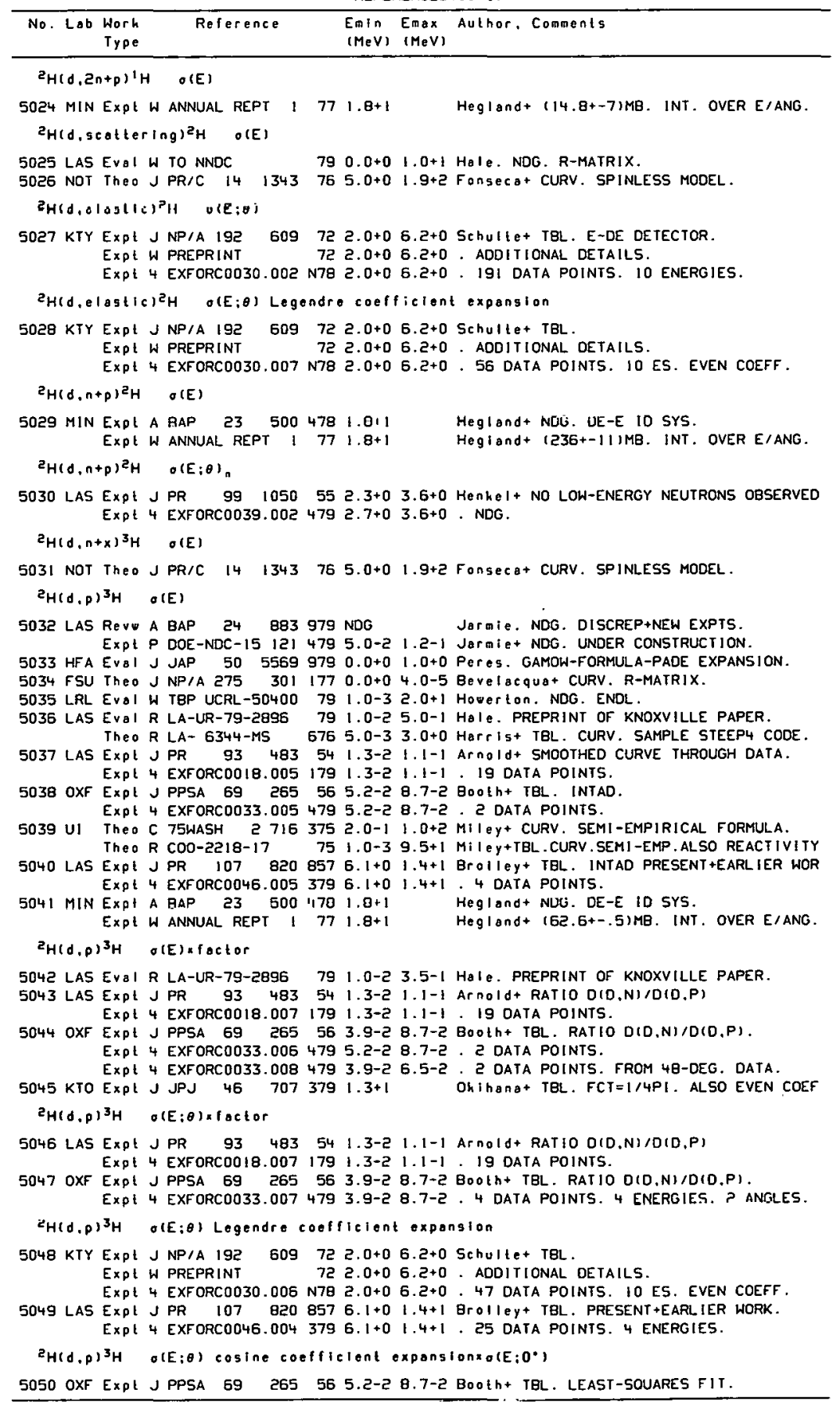


REFEHENLED,..nt)

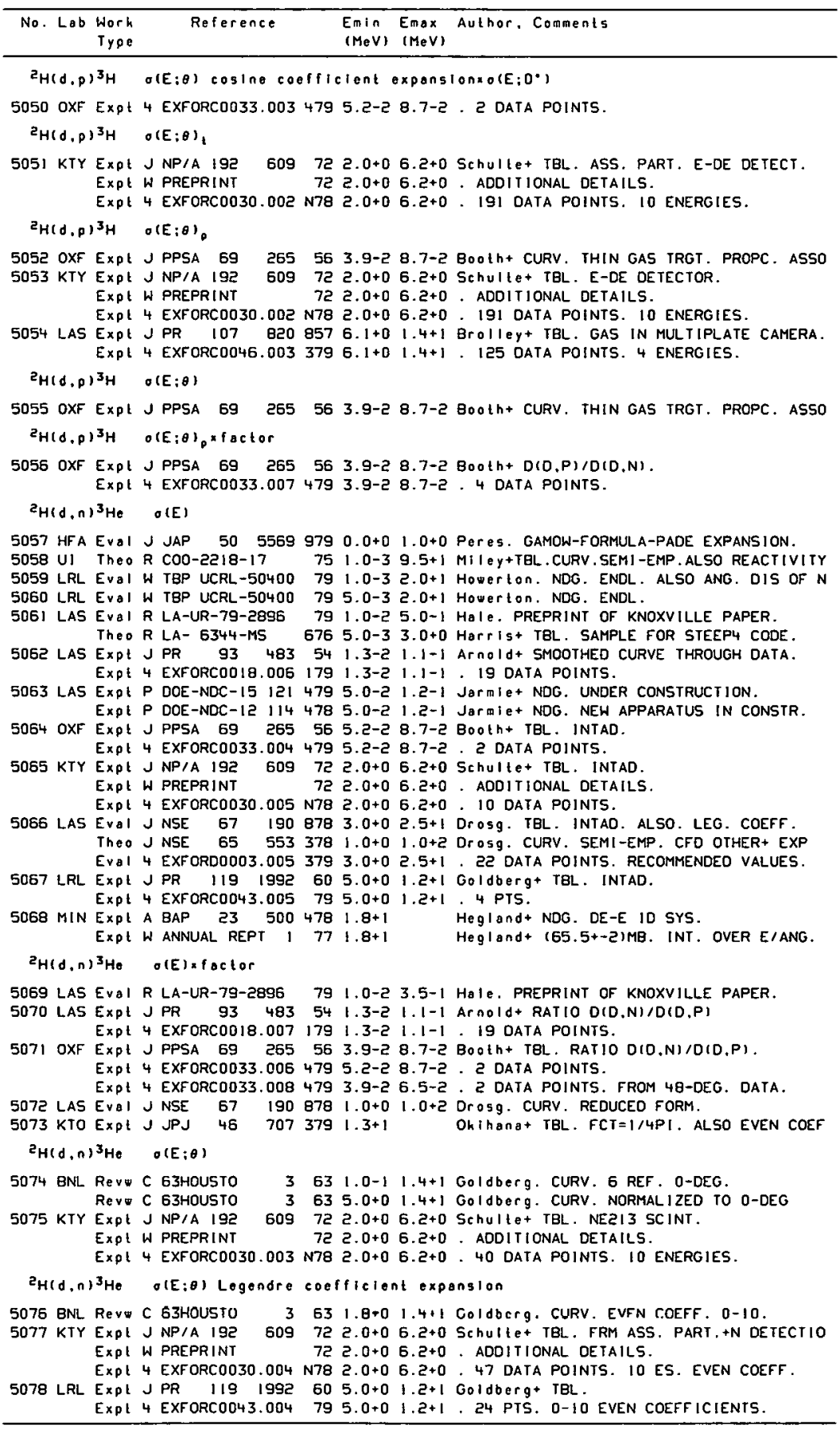




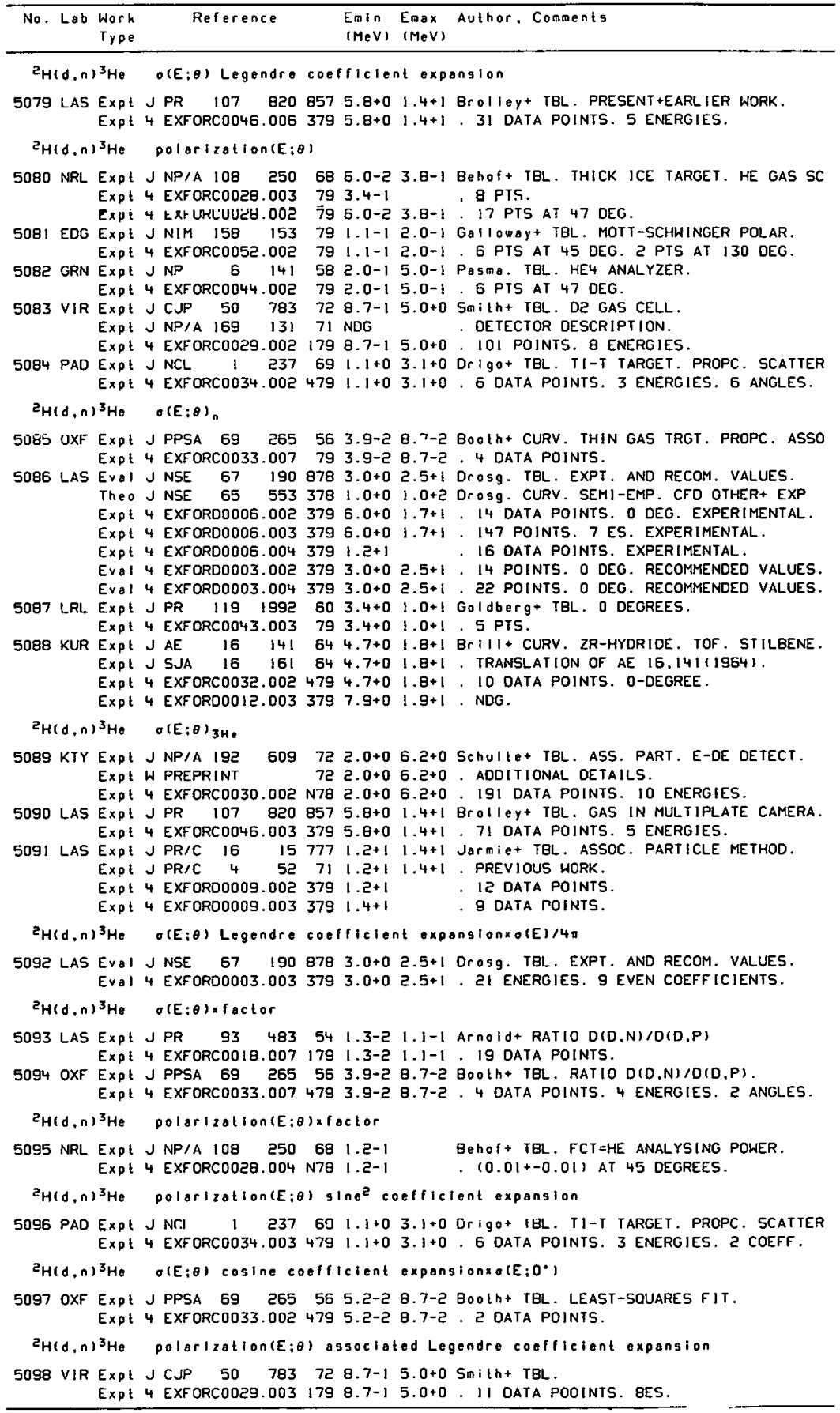


REFERENCES ( con t)

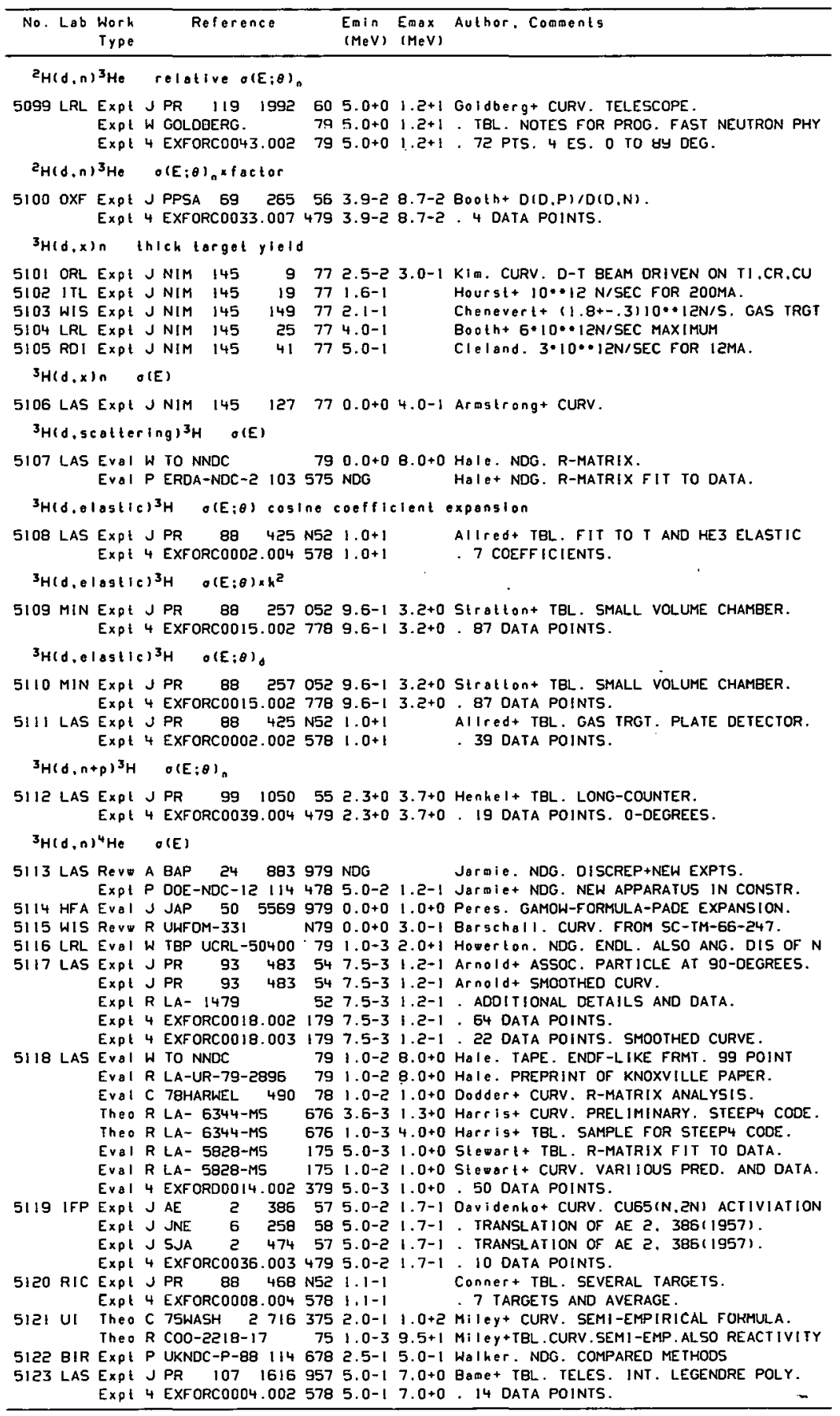


REFERENCES ( c ont $)$

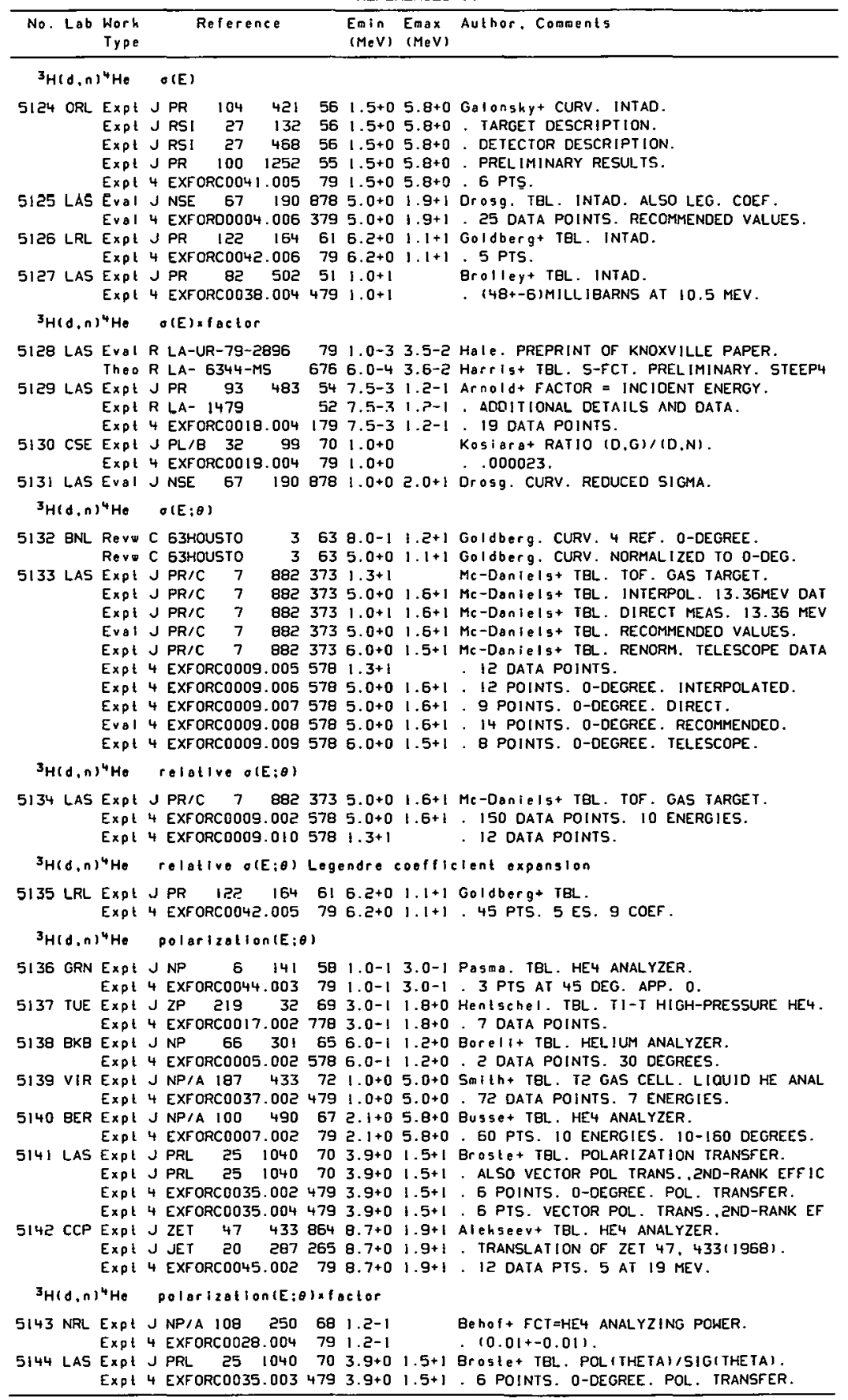




\begin{tabular}{|c|c|c|c|c|c|}
\hline No. Lab & $\begin{array}{l}\text { Work } \\
\text { Type }\end{array}$ & Reference & $\begin{array}{l}\text { En } I n \\
(M e V)\end{array}$ & $\begin{array}{l}E \max \\
(\mathrm{MeV})\end{array}$ & Author, Comments \\
\hline
\end{tabular}

${ }^{3} \mathrm{H}(\mathrm{d}, n)^{4} \mathrm{He}$ product yleld

5145 AUB ExPt A BAP 25 125 PRO NDG AIford+ NDG.

5146 BIR EXPt P NEANDC (E)-2028 479 2.5-1 5.0-1 Scott+ CURV. CONT. FLOW $V$ BATH. P. I06 Expt $P$ UKNDC-P-94 106 479 2.5-1 5.0-1. SAME AS NEANDC(E)-Z02, VOL. 8. Exp: P INDC (UK)-31106 479 2.5-1 5.0-1. SAME AS NEANDC(E)-202, VOL. 8.

${ }^{3} H(d, n)^{4} \mathrm{He} \quad O(E ; \theta)$ Legendro coefficient expansion

5147 LAS Expt JPR 1071616957 5.0-1 7.0+0 Bame+ TBL. FROM LEGENDRE POLY+SIGIO DEG Expt 4 EXFORCO004.003 $5785.0-17.0+0.74$ DATA POINTS. 14 ENERGIES.

5148 VIR Expl J NPIA $187 \quad 433721.0+05.0+0$ 5mith+ TBL.FRM DATA COMPILEO BY BROLLEY Expt 4 EXFORC0037.003 $4791.0+05.0+0$. 29 DATA POINTS. 7 ENERGIES.

5149 GNL Revw $C$ 63HOUSTO $3631.8+0 \quad 1.2+1$ Goldberg. CURV. $0-8$ COEFF.

${ }^{3} \mathrm{H}(d, n)^{4} \mathrm{He} \quad O(E ; \theta)$,

5150 LAS Expt J PR $10716169575.0-17.0+0$ Bame+ TEL. TELES.

EXPt JPR $10716169571.8+02.0+0$ Bame+ TEL. EXTRAPOLATEO LEGENDRE POLY.

EXPt J PR $10716169571.8+02.0+0$ BAme+ TBL. EXTRAPOLATEO LEGENDRE POLY.

Expt 4 EXFORC0004. $5785.0-17.0+0$. I5 DATA POINTS, 0 DEGREES. 2 SUBWORKS

5151 ORL Exp! J PR $104 \quad 421561.1+05.0+0$ Galonsky+ CURV. ABSOLUTE.

Expt J PR $104 \quad 421561.5+05 . R+0$ Galonghy+ CURV. NORMAL 1ZED TO 6-DEG.

Expl J RS! $27 \quad 13256$ NDG . TARGET DESCRIPTION.

ExPL J RSI $27 \quad 468$ 56 NDG DETECTOR DESCRIPTION.

Expl J PR $1001252551.5+05.8+0$. PRELIMINARY RESULTS.

Expt 4 EXFORC0041.004 $791.1+05.0+0$. 48 PTS AT 6 DEG

Expt 4 EXFORCD041.002 79 $1.5+05.8+0$. 63 PTS. 5 ES. FROM GOLDBERG.

Expt 4 EXFORCO041.003 $792.2+0$. 23 PTS. FROM GOLOBERG.

5152 KUR Expt J AE $16 \quad 141644.0+01.9+1$ Brill+ CURV. ZR-HYDRIDE. TOF. 5TILBENE. Exp: J SJA $16 \quad 161644.0+01.9+1$. TRANSLATION OF AE $16,141(1964)$. Expt 4 EXFORC0032.003 479 $4.0+01.9+1$. I1 DATA POINTS. O-DEGREE.

EXPt 4 EXFORDO012.004 $3797.9+01.9+1$. 8 DATA POINTS. FROM NSE 67. 190.

5153 LAS Eval J NSE 67190 B78 $5.0+01.9+1$ Drosg. TBL. EXPT AND RECOM. VALUES. Expt J NIM $105 \quad 573725.0+01.9+1$. EXPERIMENTAL DETAILS.

Expt 4 EXFORO0007.002 $3797.0+01.6+1$. I3 DATA POINTS. O DEG. EXPERIMENTAL.

Eval 4 EXFORD0004.002 $3796.0+01.9+1$. 25 POINTS. O DEO. RECOMMENDEO VALUES. Eval 4 EXFORDO004.003 $3791.3+1$. 22 DATA POINTS. RECOMMENDED VALUES.

Eval 4 EXFORDO004.005 $3795.0+01.9+1$. 50 POINTS. 0.180 DEG. RECOMMENDED VAL Expt 4 EXFORD0007. $3797.0+01.6+1$. 88 POINTS. 4 ES. 4 SUBACCS. EXPT.

5154 LAS Expt JPR/C $6 \quad 159372$ 5.0+0 $1.1+1$ Mac-Danlelst TBL. TOF.

EXPt 4 EXFORDO000.004 $3795.0+01.5+1$. 84 DATA POINTS. 7 ENERGIES.

EXP: 4 EXFORD0008.003 $3796.0+01.1+1$. 5 DATA POINTS. 0 DEGREES.

5155 LAS Exp! J PR $11916499606.1+01.4+1$ Stewarl+ TBL. PLATE DETECTOR. Exp! 4 EXFORCO012.003 $796.1+01.4+1$. 93 PTS IN CM. 4 ES.

5155 LRL Expl J PR 122 $1646 ! 6.4+01.1+1$ Goldberg+ CURV. NORMALIZEO TO BAME+. Expl J PR $122 \quad 164 \quad 616.2+01.1+1$ Goldberg+ CURV. NORMAL IZED TO STEWART + Expt W TO NNDC $796.2+01.1+1$ Goldberg. TBL. FOR 63HOUSTON. EXPt 4 EXFORCDO42. $796.2+01.1+1.9$ DATA POINTS. 0 DEGREES. 2 SUBWORKS.

5157 LAS Expt A BAP $13564687.0+01.9+1$ Simmons+ NDG. TELESCOPE. 0-DEGREES. EXpt 4 EXFORDOO $11.0033797 .0+01.9+1$. I3 POINTS. FROM NSE 67. 190119781.

5158 LAS Expt J PR B2 $50251 \quad 1.0+1 \quad$ Brolley+ GAS CELL. PROPC AND TELESCOPE. Expt 4 EXFORCDO3B.002 $4791.0+1$. I2 DATA POINTS.

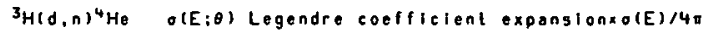

5159 LAS Eval J NSE $671908785.0+0 \quad 1.9+1$ Orosg. TBL. EXPT AND RECOM. VALUES. Eva 14 EXFORDO004.004 $3795.0+01.9+1$. 25 ENERGIES. 15 COEFF ICIENTS.

${ }^{3} \mathrm{H}(d, n)^{4} \mathrm{He} \quad \sigma(E ; \theta)$ Legendre coeffletent expansion/k

5160 LAS Expl J PR/C $78823735.0+01.6+1$ Mc-DanieIs+ TBL. FRM REL DIS+0-DEG 5IG. Exp! 4 EXFORC0009.003 $5785.0+01.6+1$. 90 DATA POINTS. 10 ENERGIES.

${ }^{3} H(d, n)^{4} \mathrm{He} \quad \sigma(E ; \theta) \times f$ actor

5161 LAS Expl A BAP $4367594,7-1 \quad$ COON + PREL. (D,G)/(D,N) THICK T TRGT. Expl 4 EXFORC0020.002 D78 4.7-1 2 DATA POINTS.

5162 LAS Expt J PRL $251040703.9+01.5+1$ Broste + TBL. POL(THETAI/SIGITHETA). Expt 4 EXFORC0035.003 $4793.9+01.5+1$. 6 POINTS. 0-DEGREE. POL. TRANSFER.

5163 LAS Expt J PR/C $78823731.3+1$ Me-Daniels + TBL. RATIO D(T.A)/T(D.N). Expt 4 EXFORCO009.005 $5781.3+1$. 12 DATA POINTS.

${ }^{3} \mathrm{H}(d, n)^{4} \mathrm{He}$ thich target yield

$5 ! 64$ WIS Revo R UWFOM-331 N79 0.0+0 3.0-1 BarschalI. CURV. FROM SC-TM-66-247,

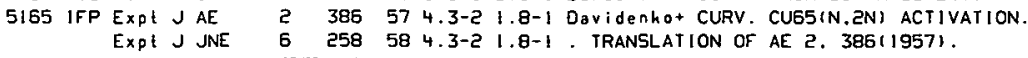


REFERENCES $(c$ on $t)$

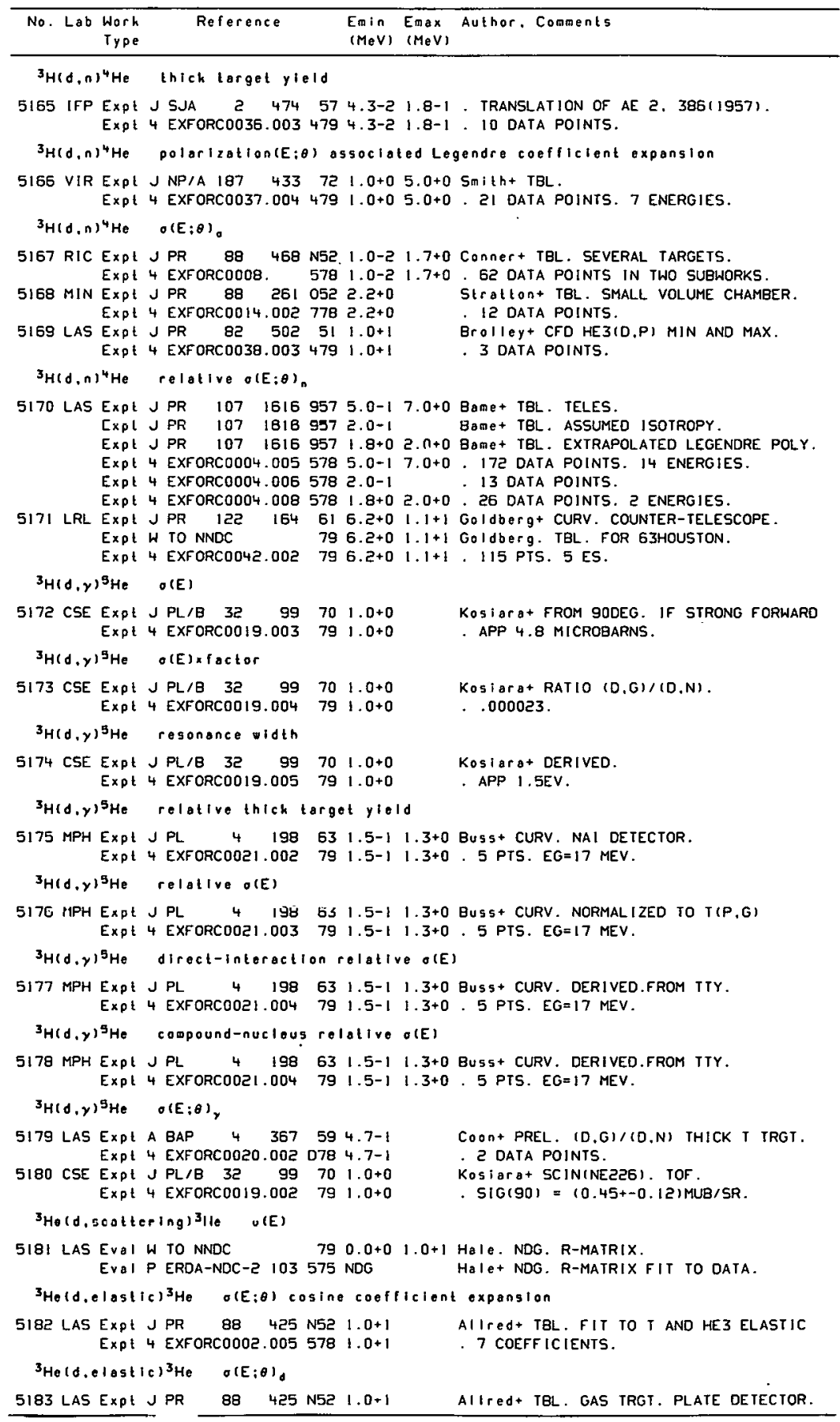


REFERENCES ( con !)

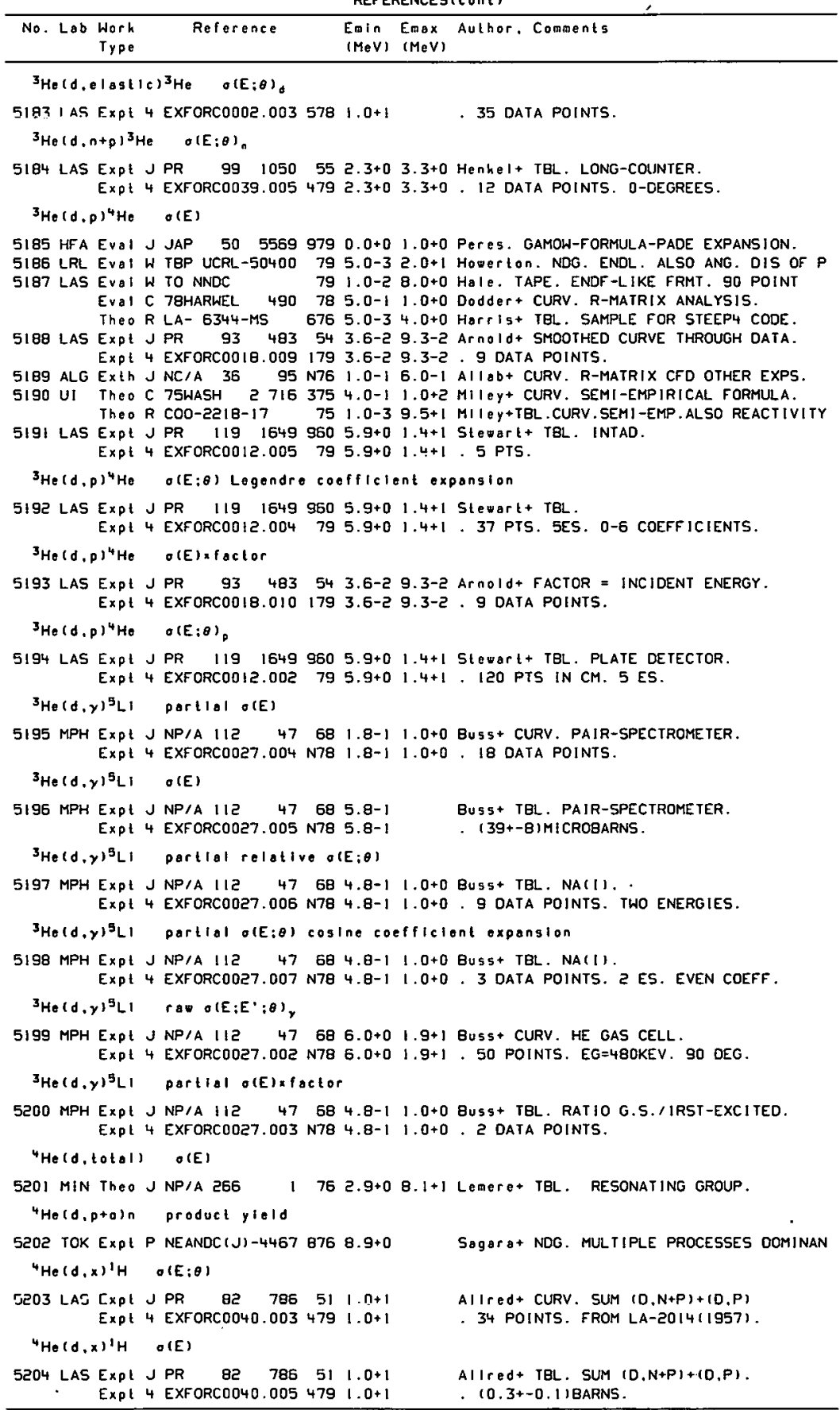


REFERENCE S ( c on l)

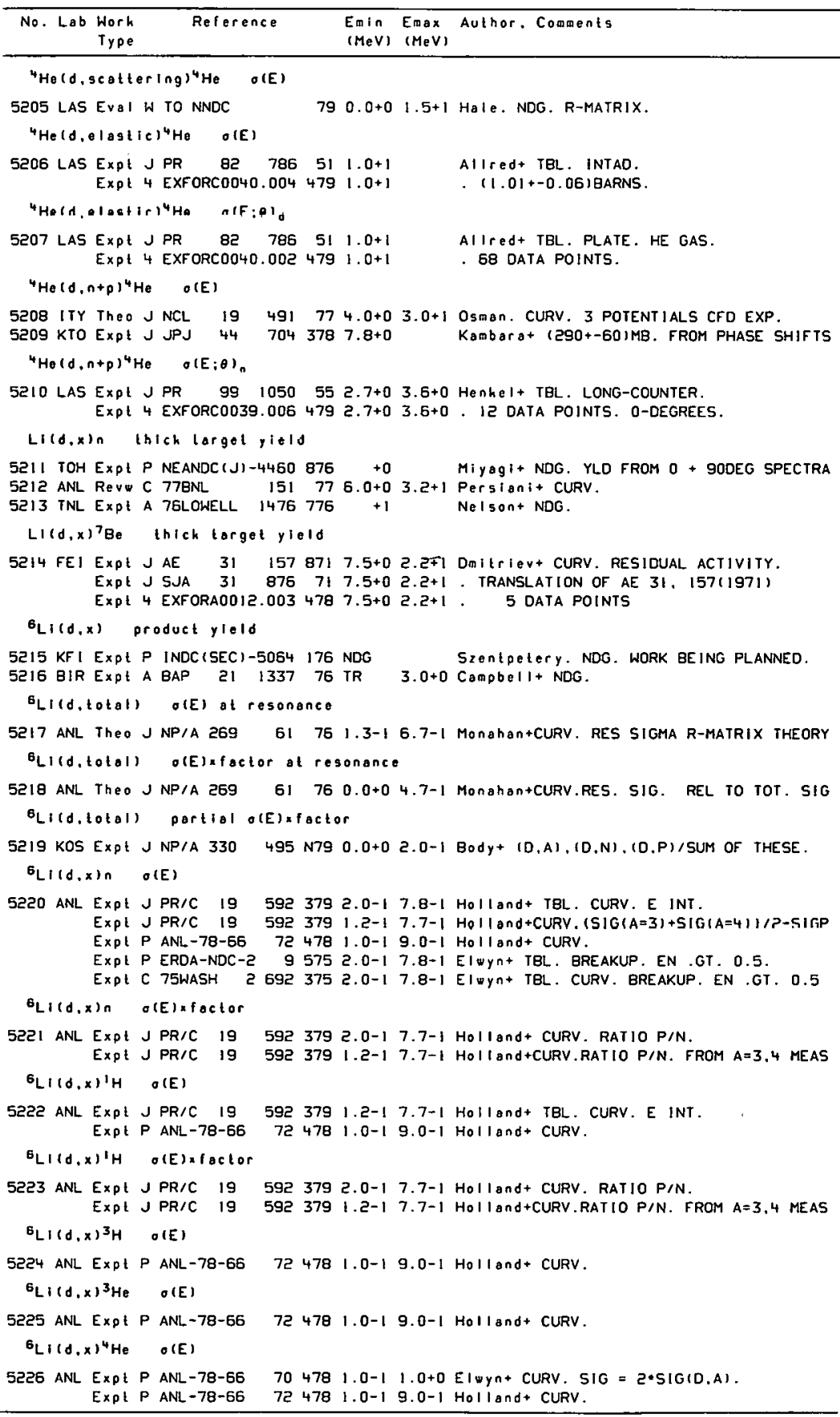


REFERENCES ( c on is )

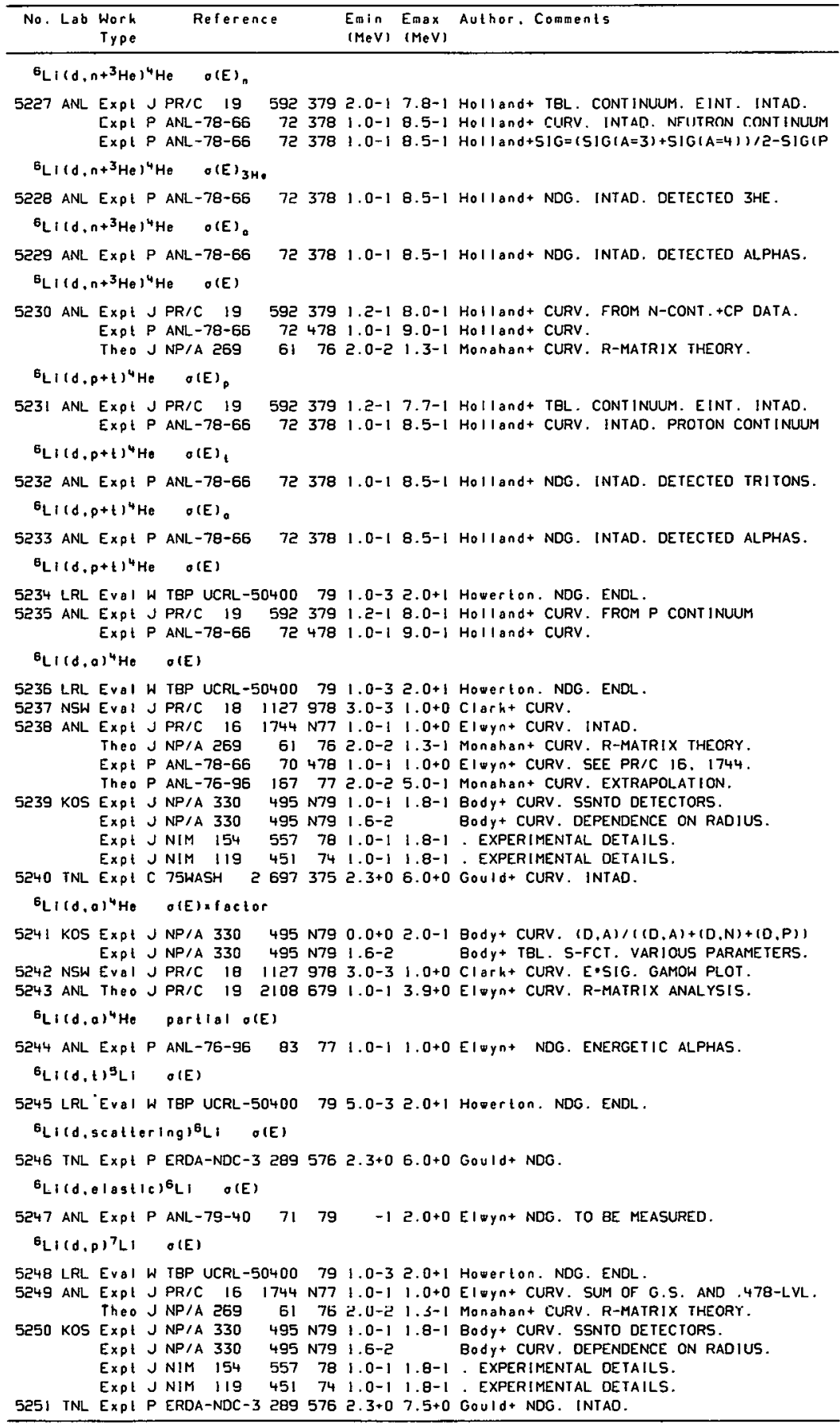


REFERENCES ( con t)

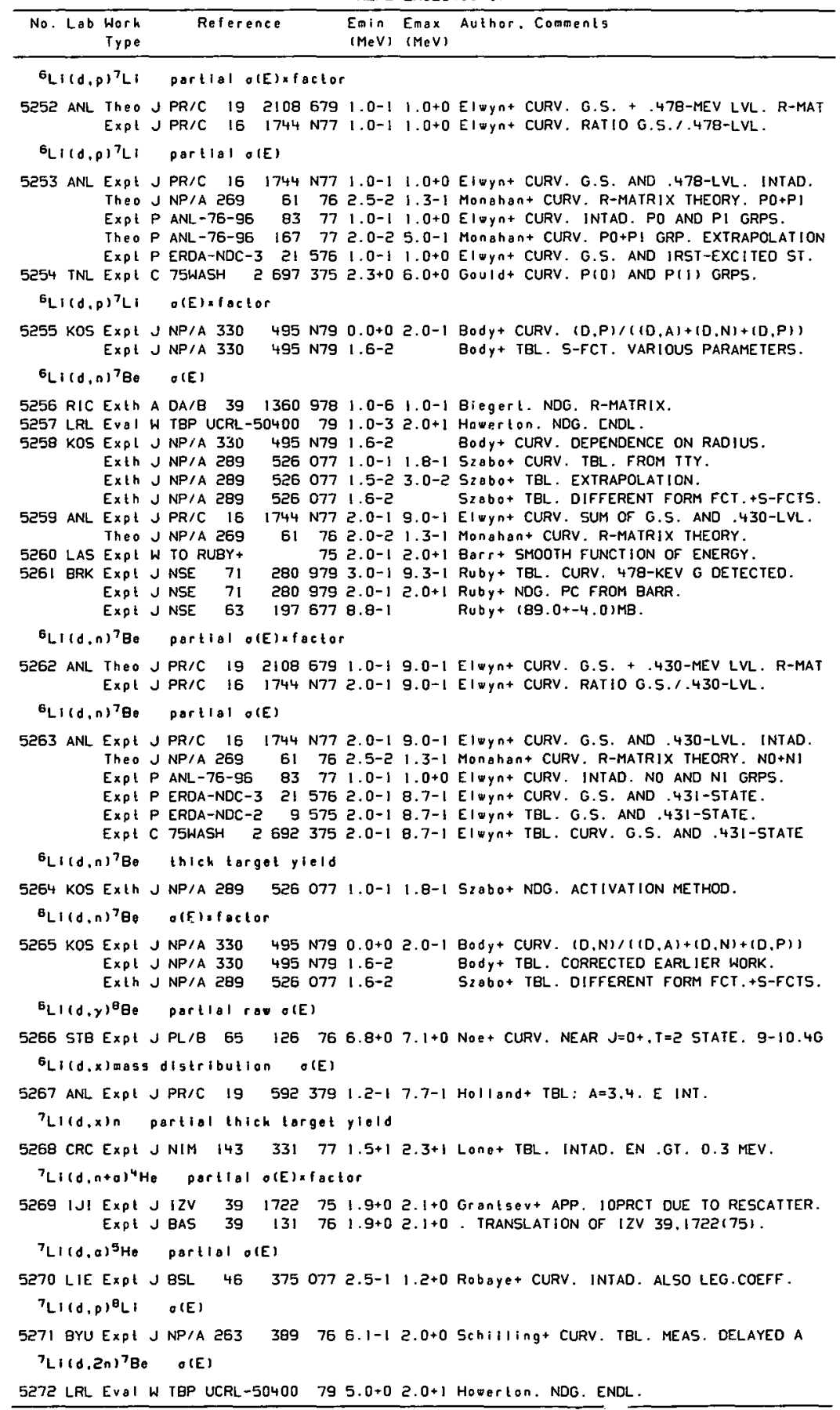




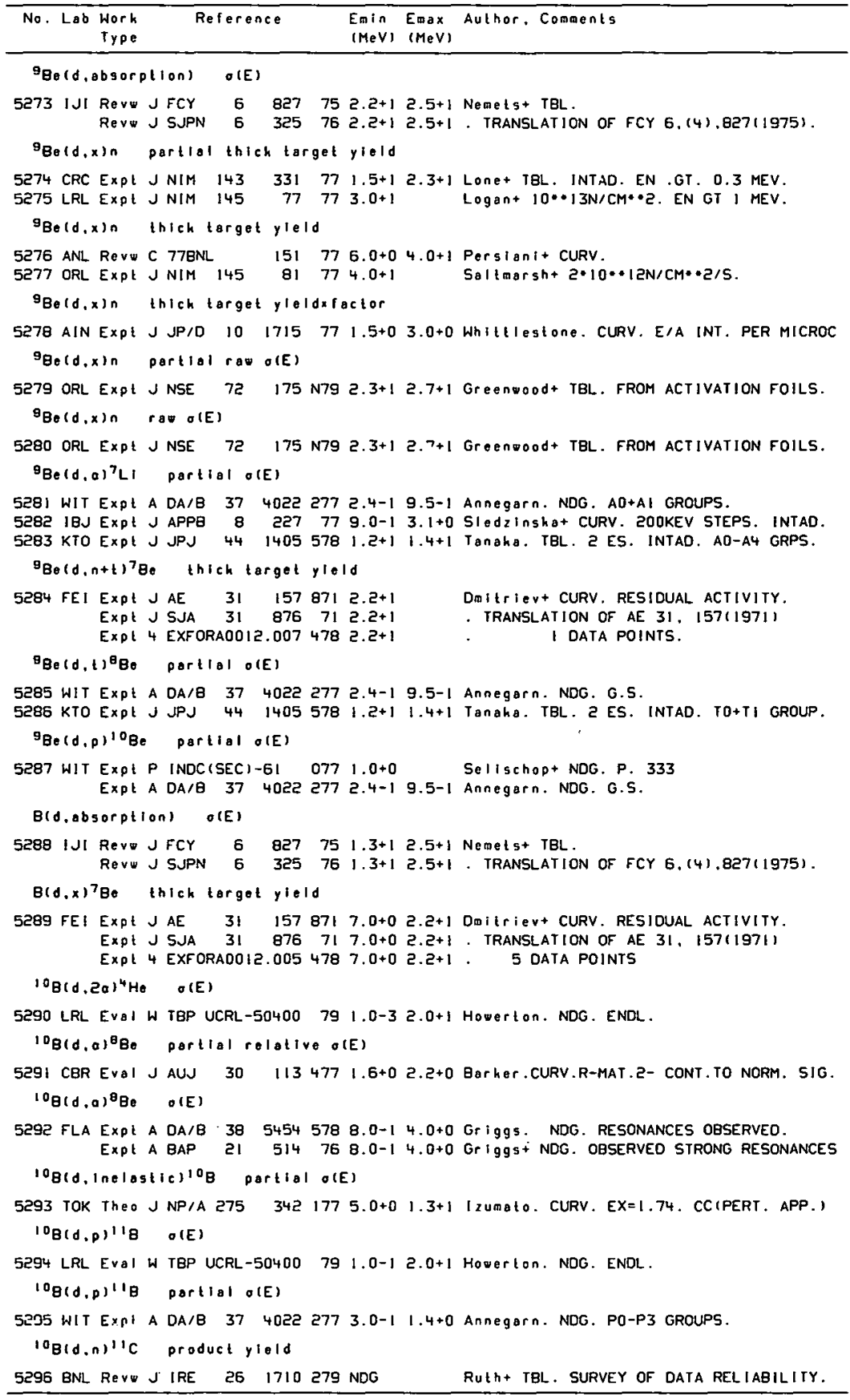


REFERENCES $(c$ on $t)$

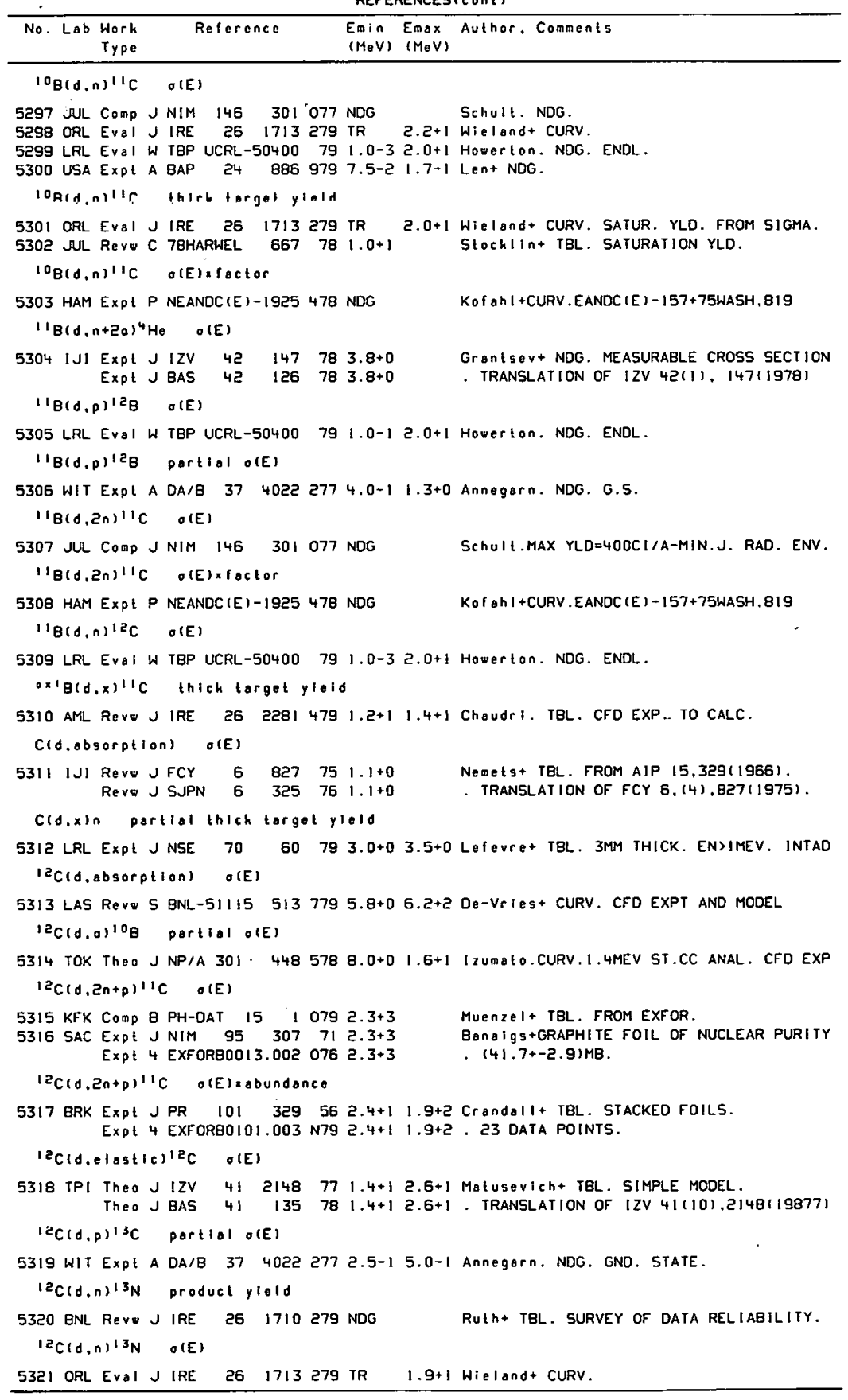


REFERENCES ( cont)

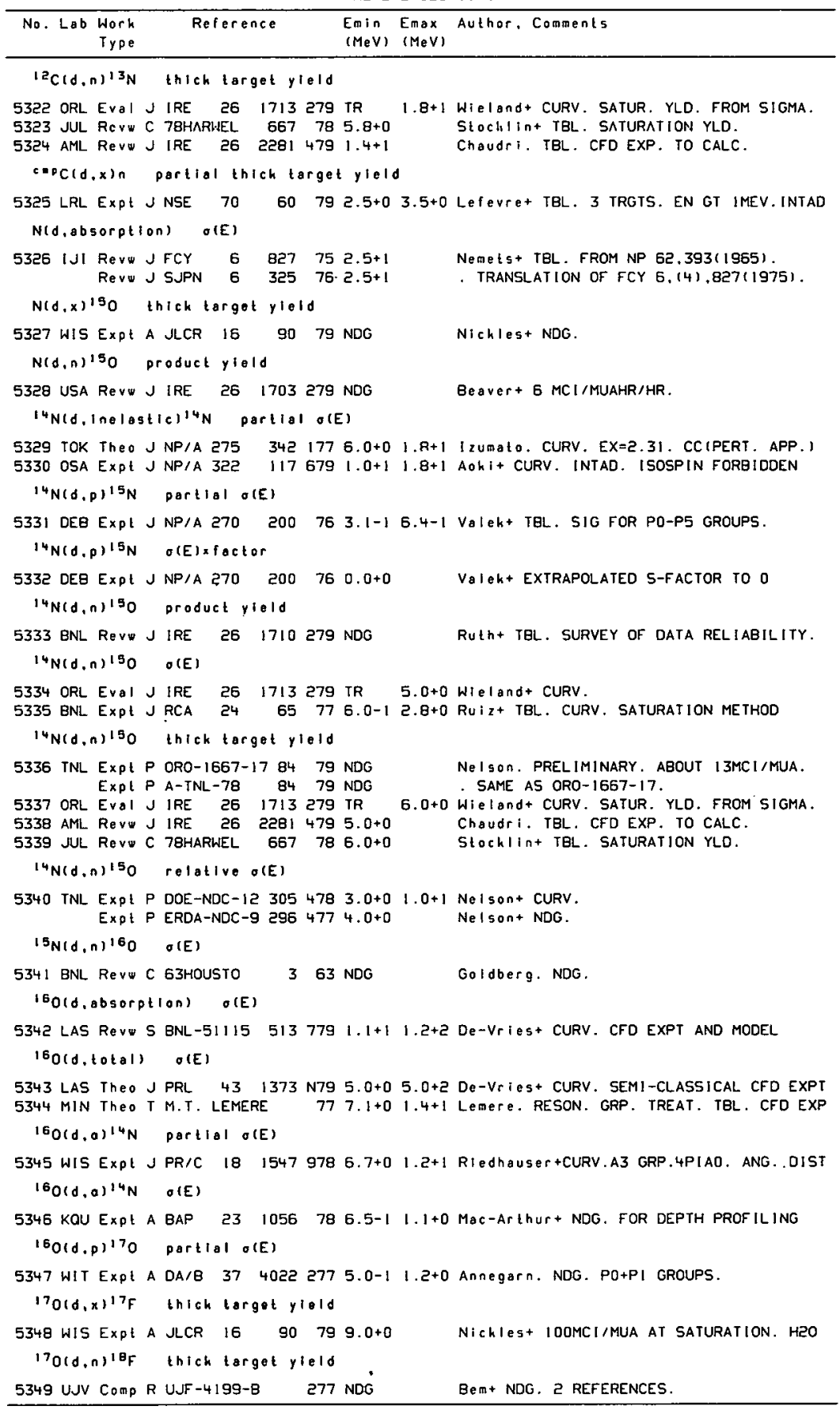


REFERENCES ( c ont)

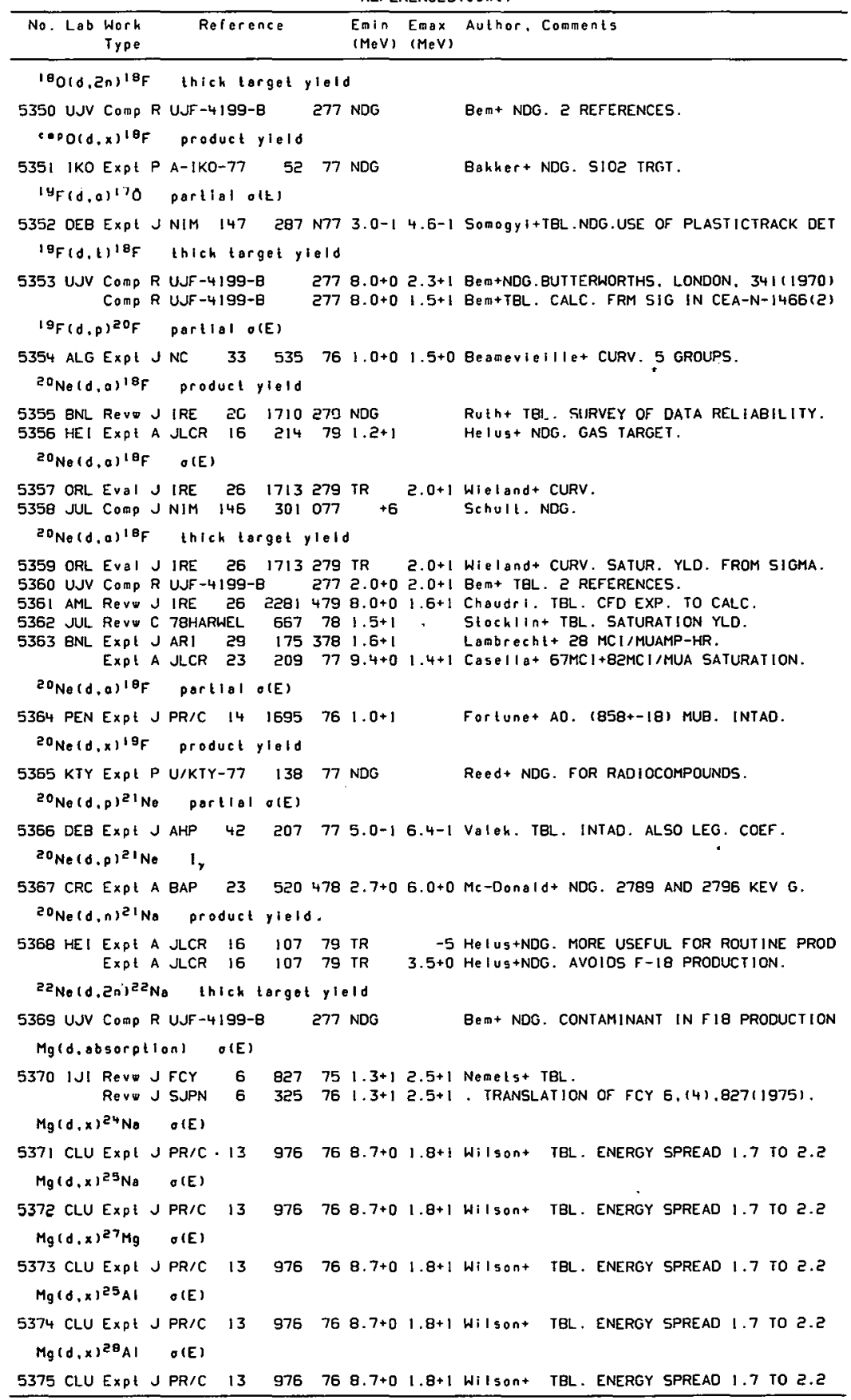


REFERENCES (cont)

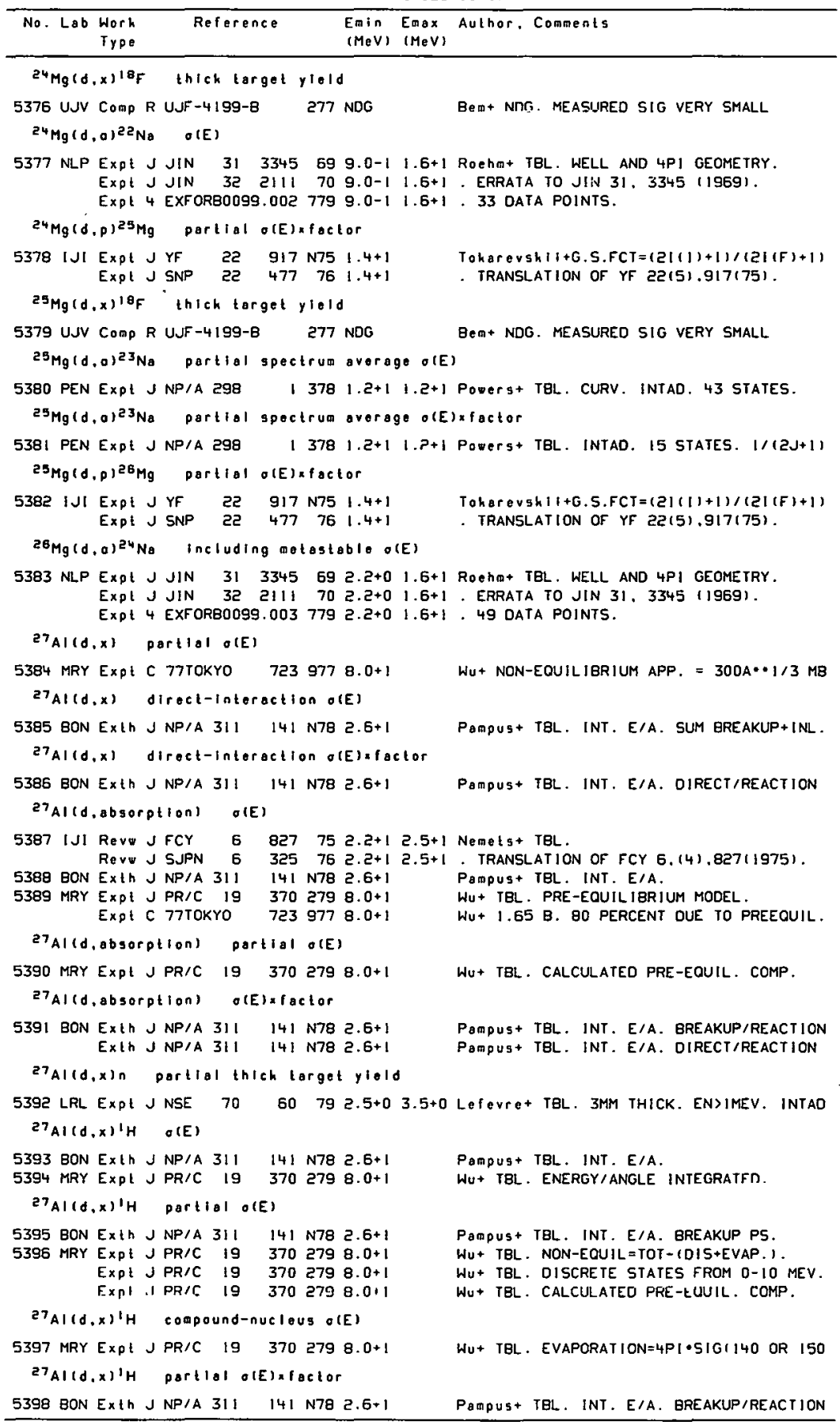




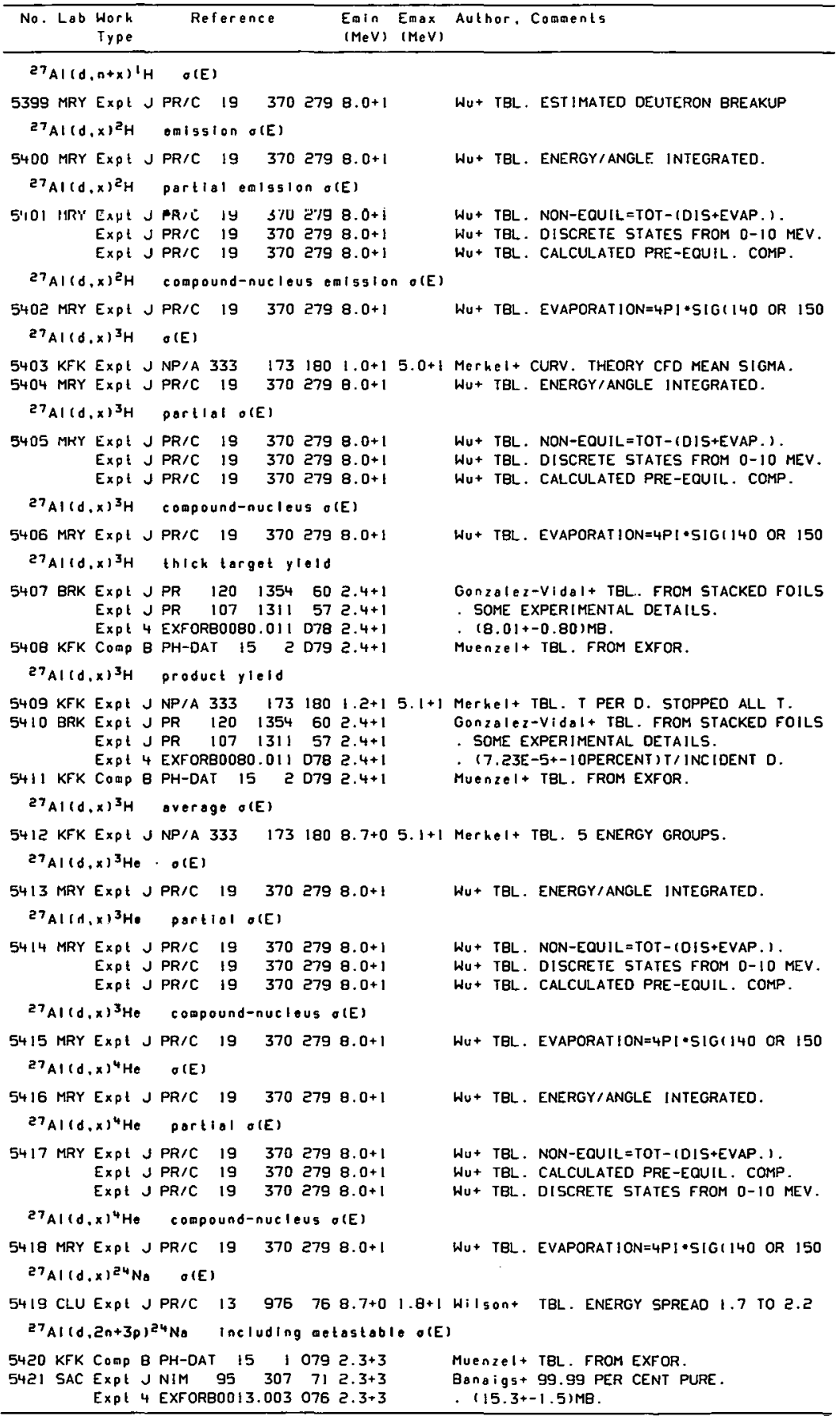


REFERENCES ( cont)

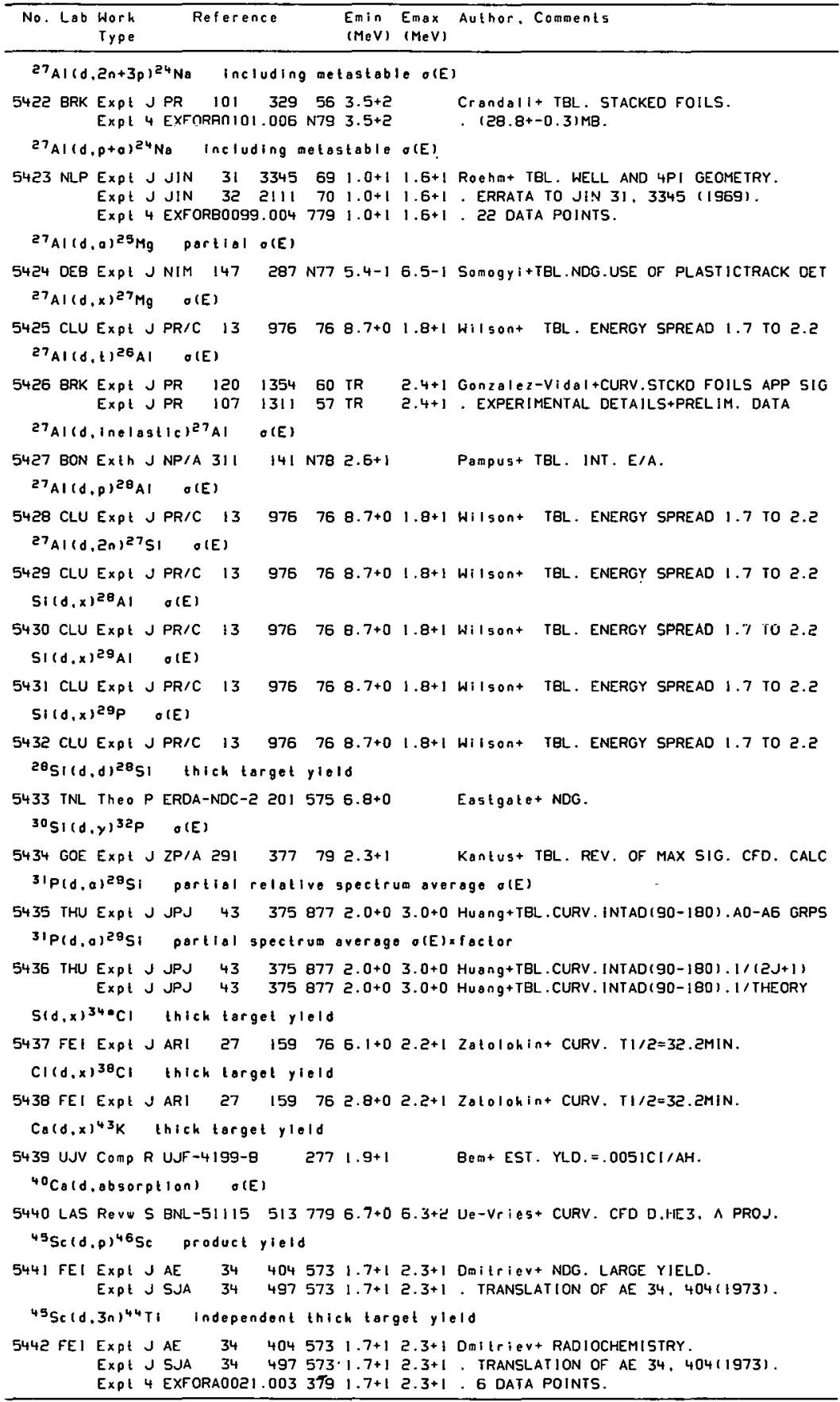




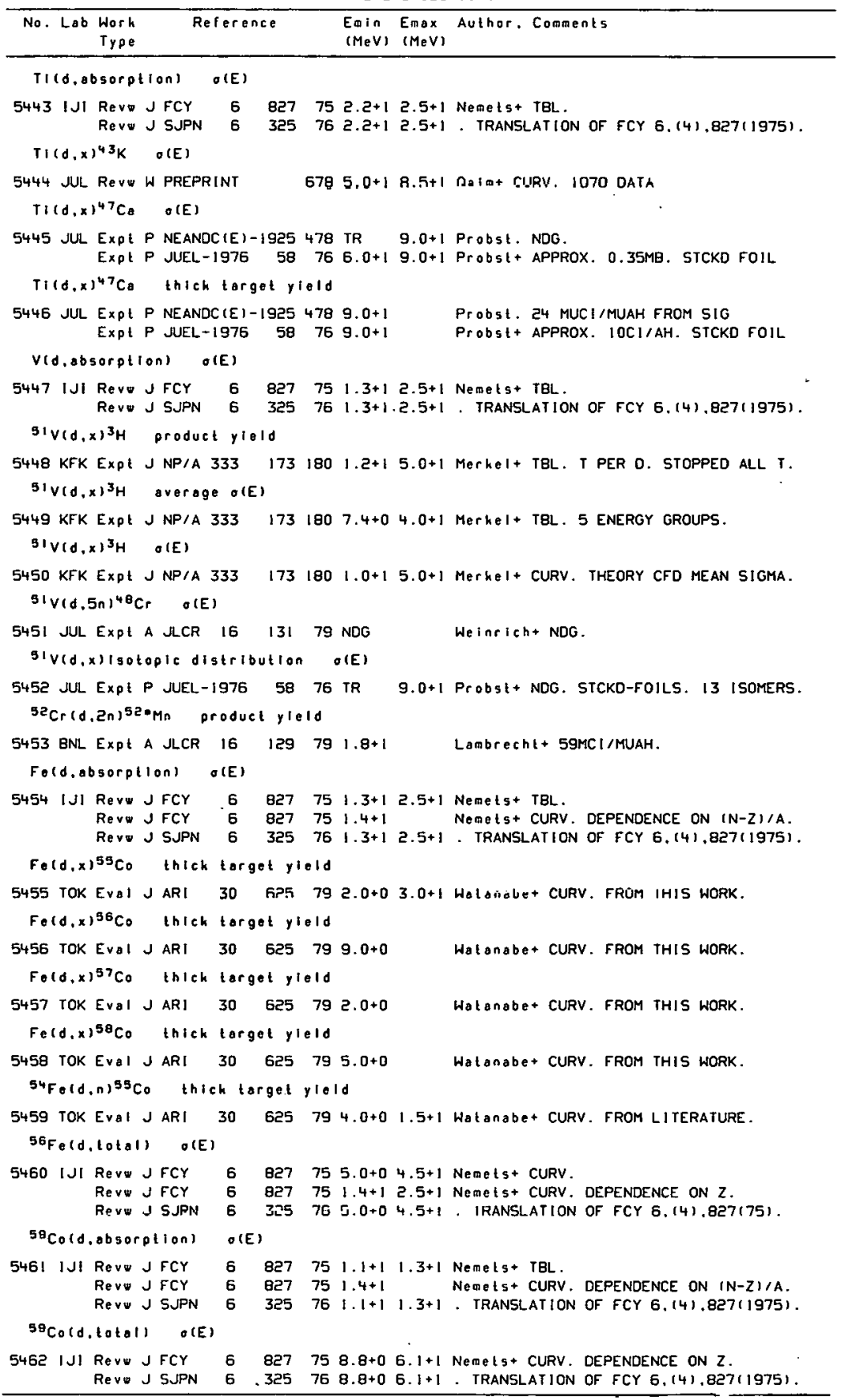




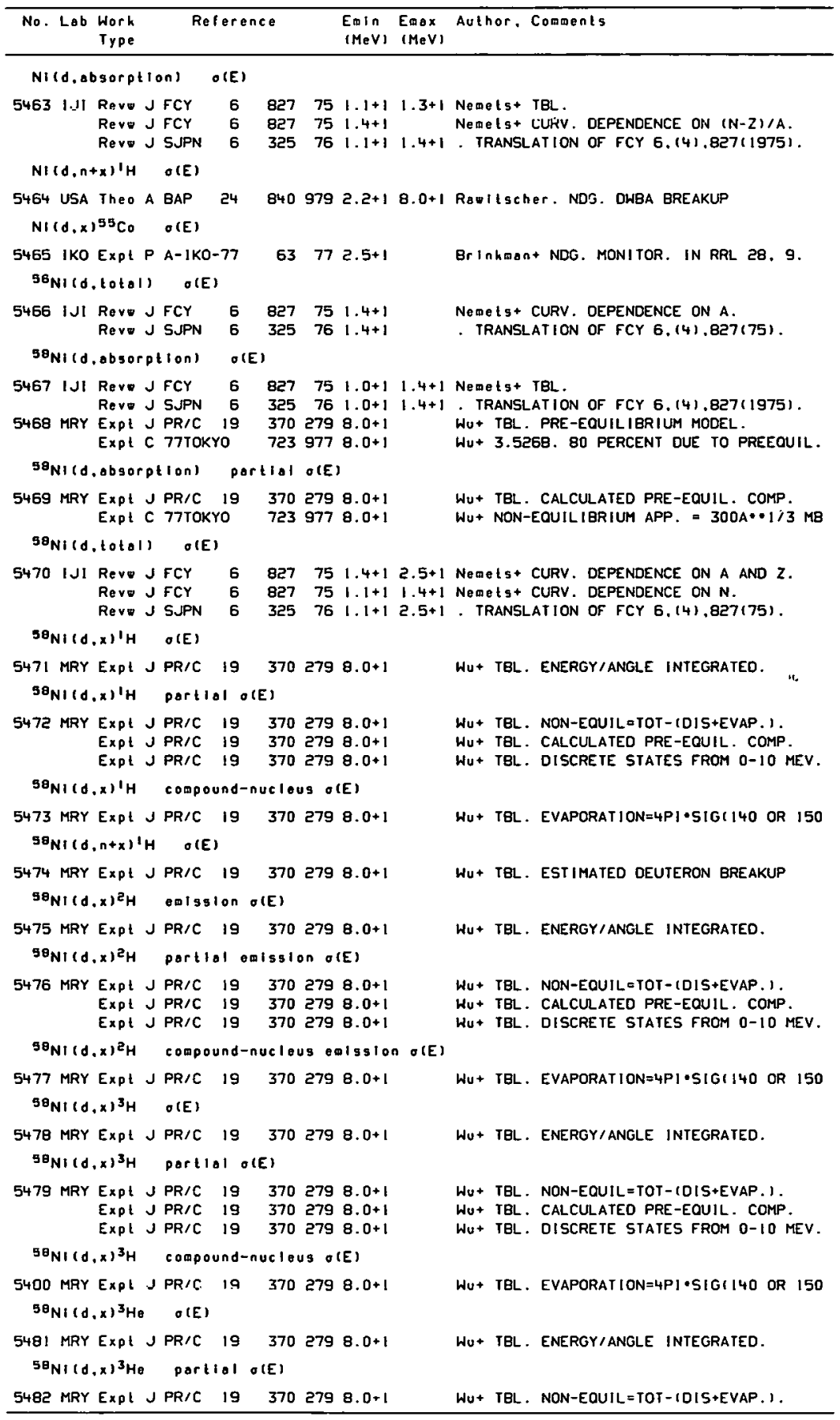


REFERENCES ( c on t )

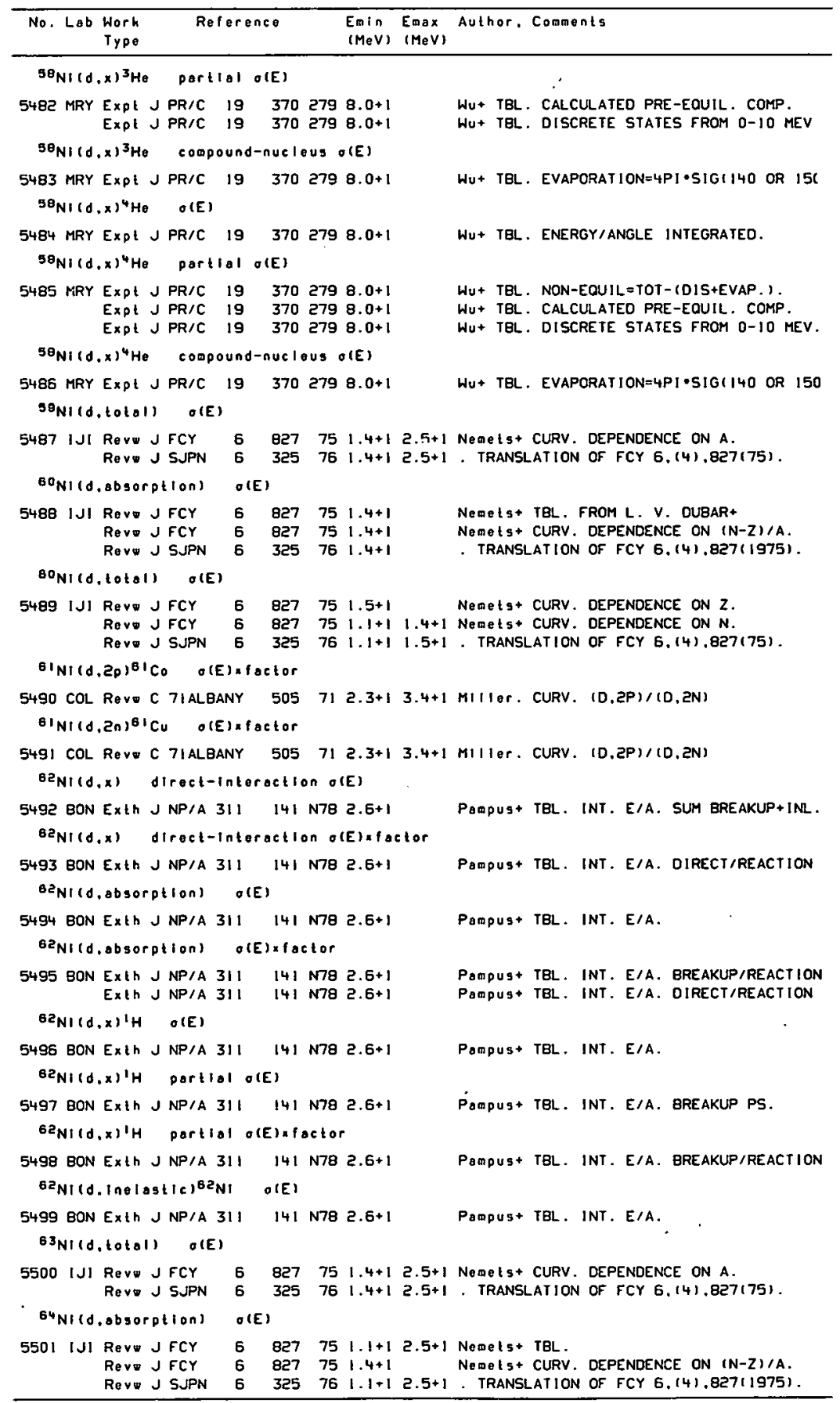


REFERENCES (cont)

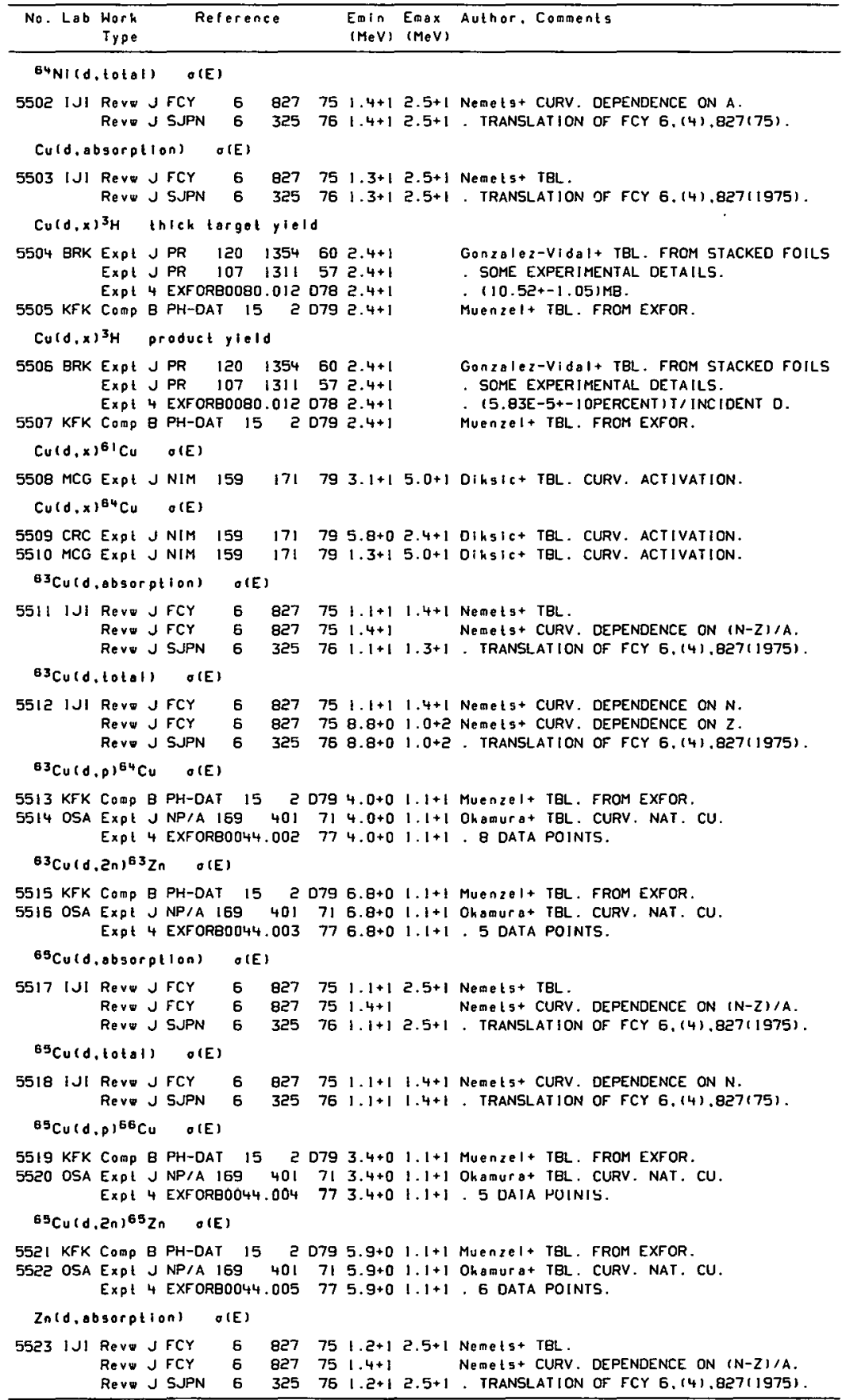




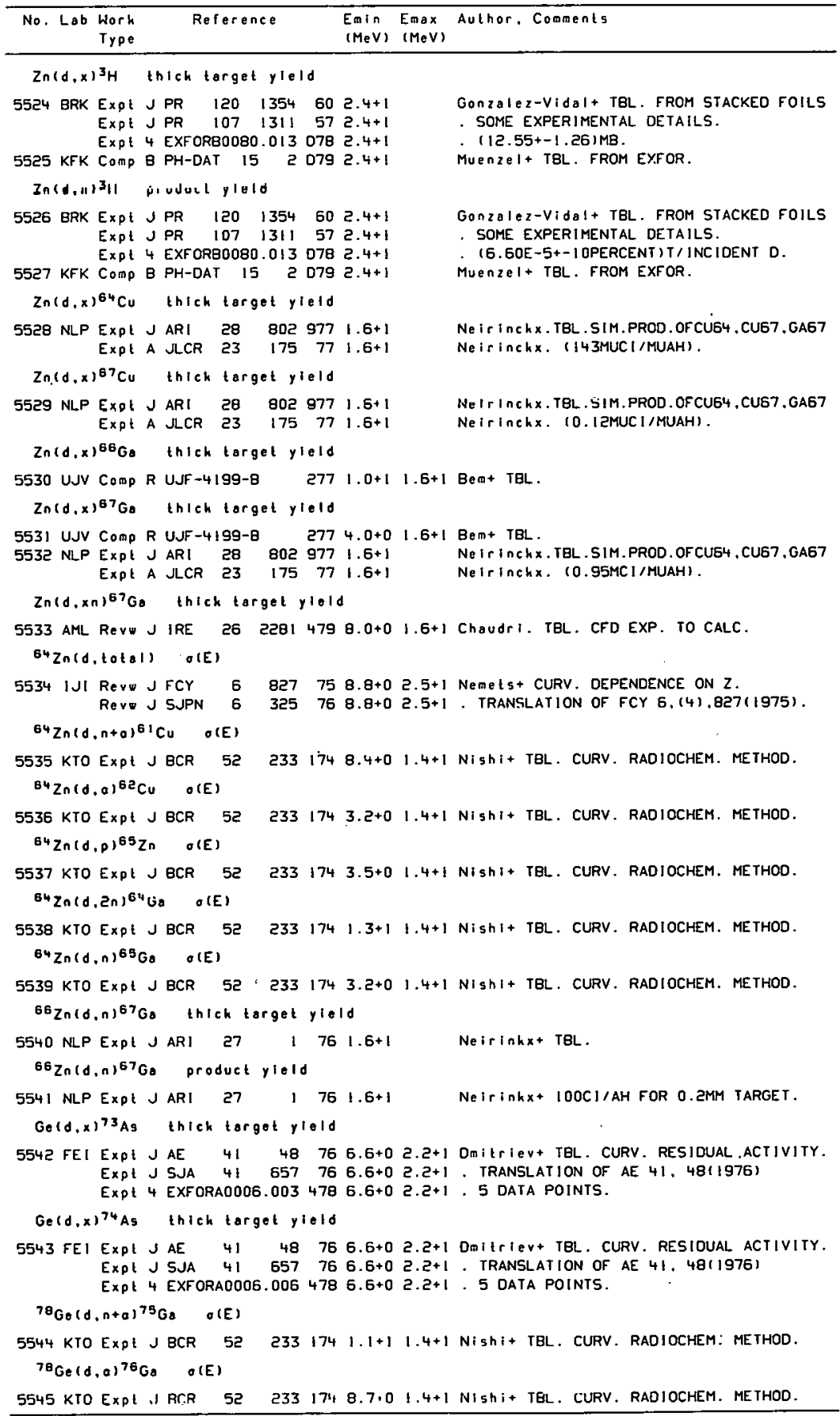




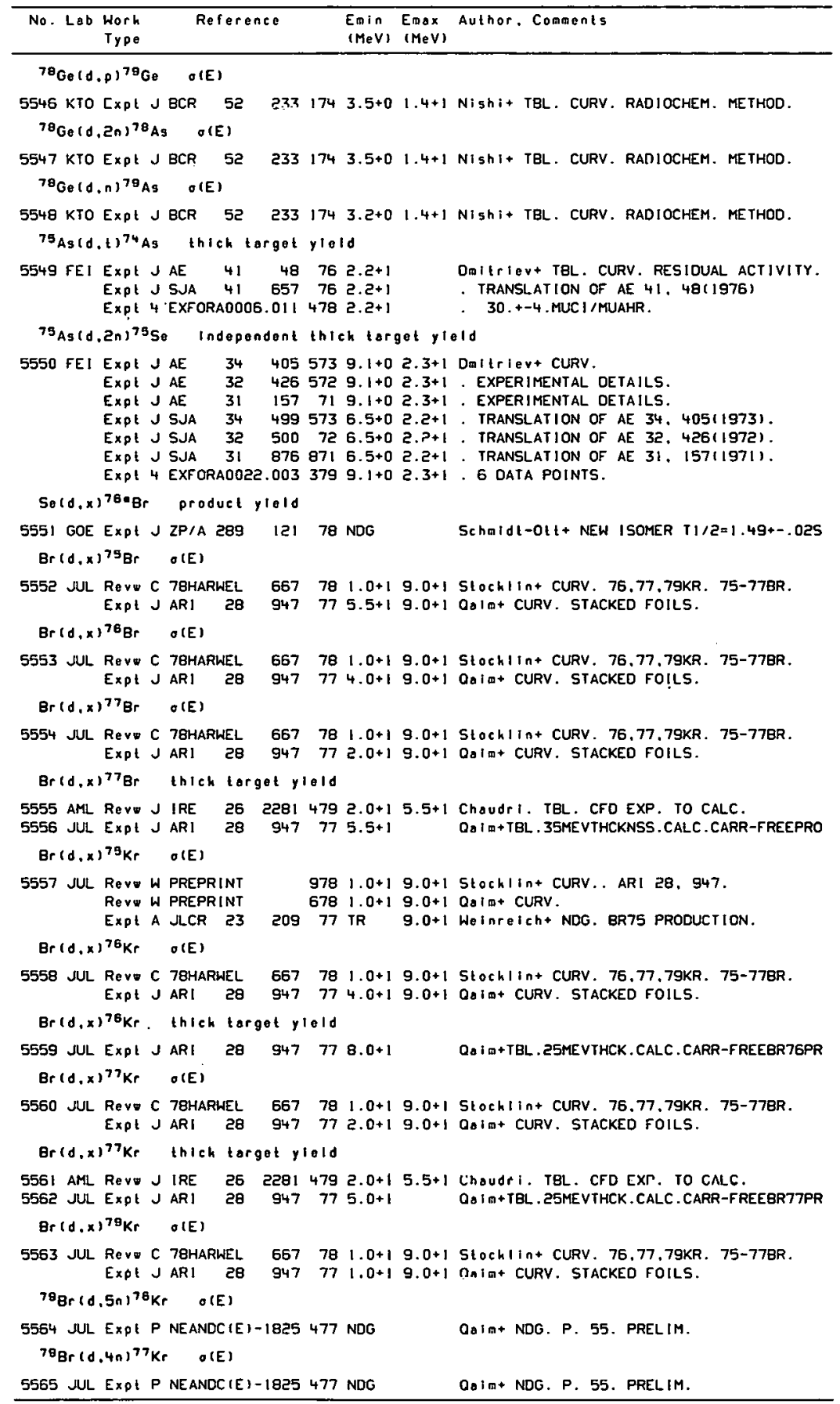


REFERENCES ( c o n t )

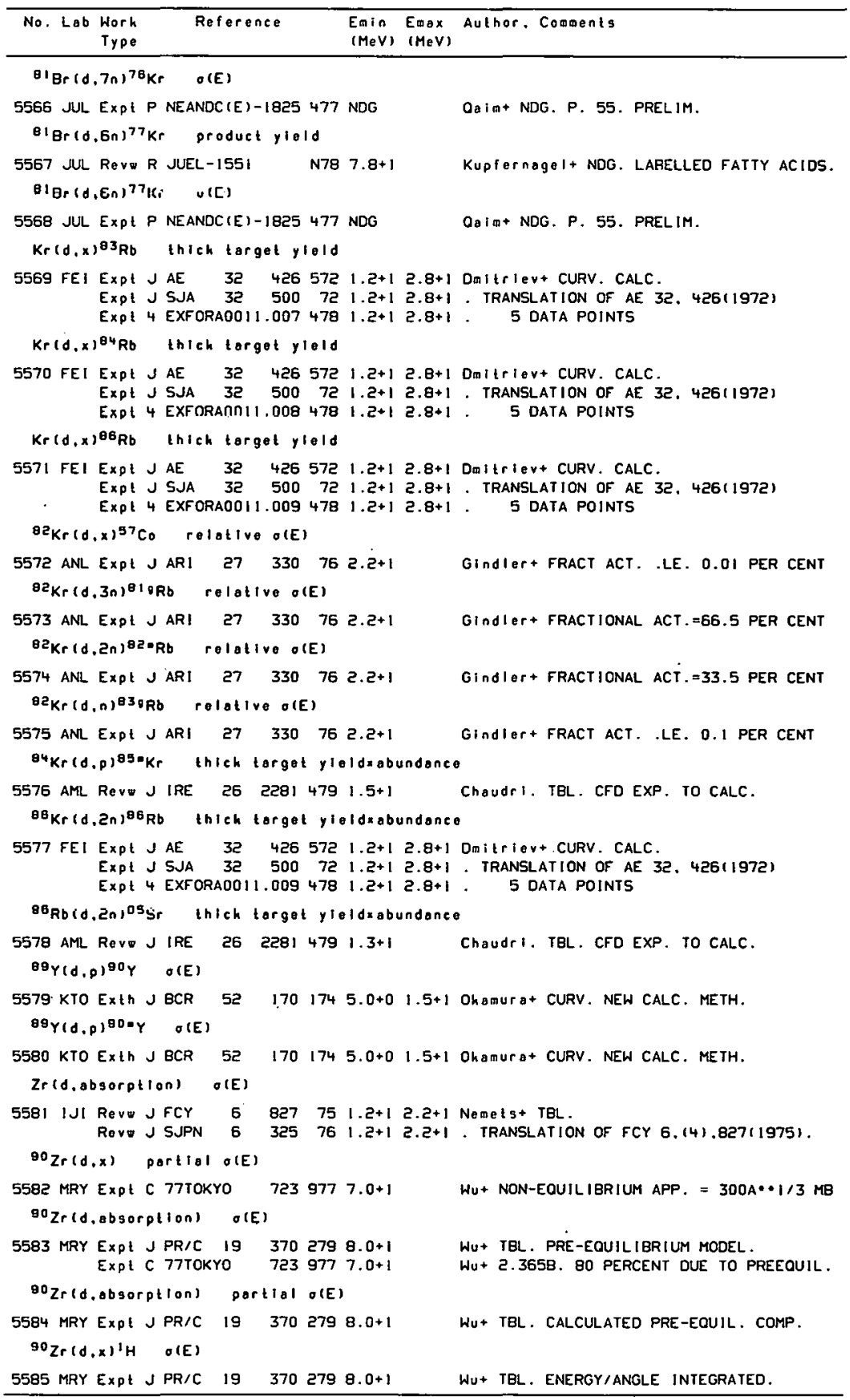


REFERENCES ( cont)

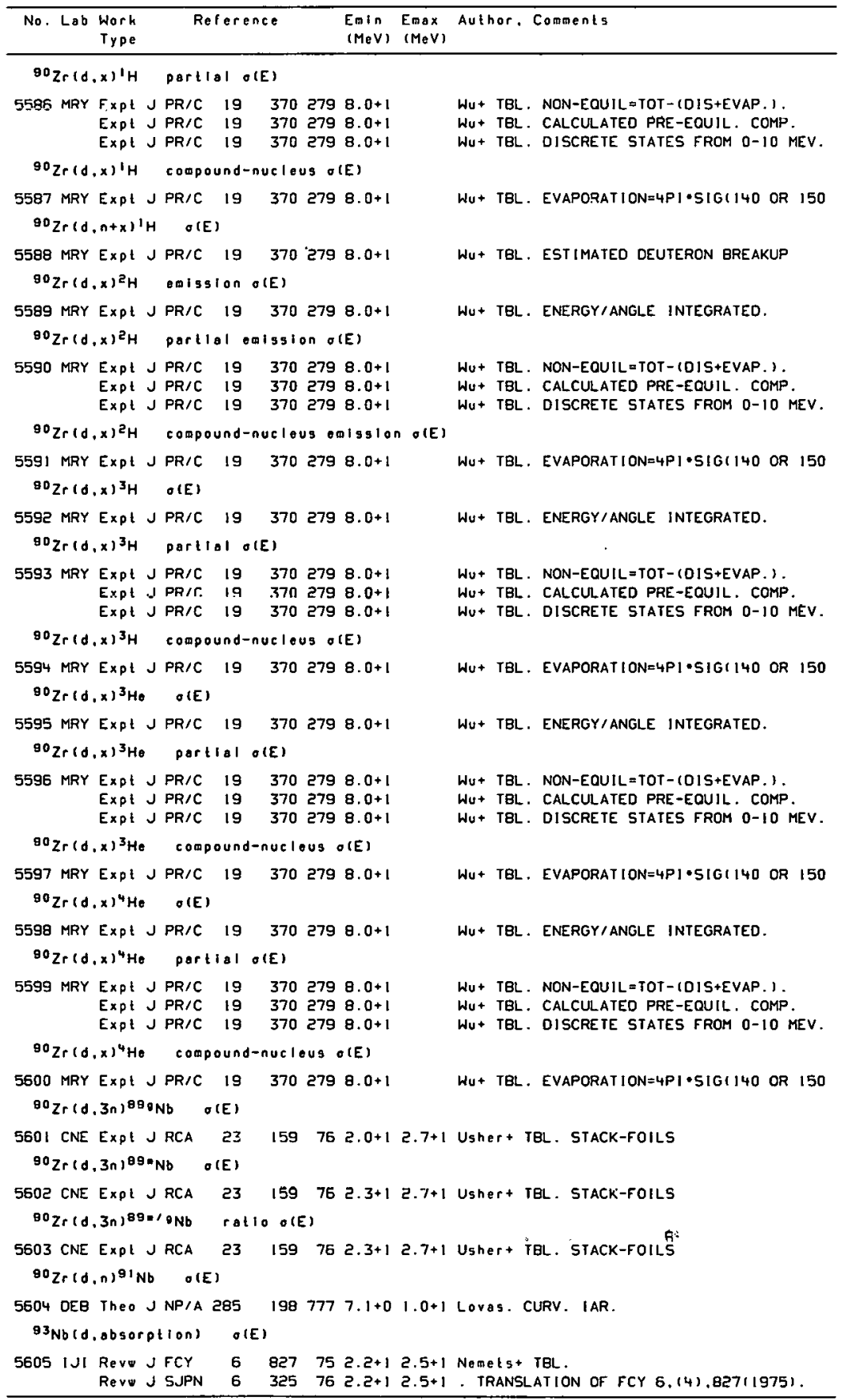


REFERENCES ( c on $t$ )

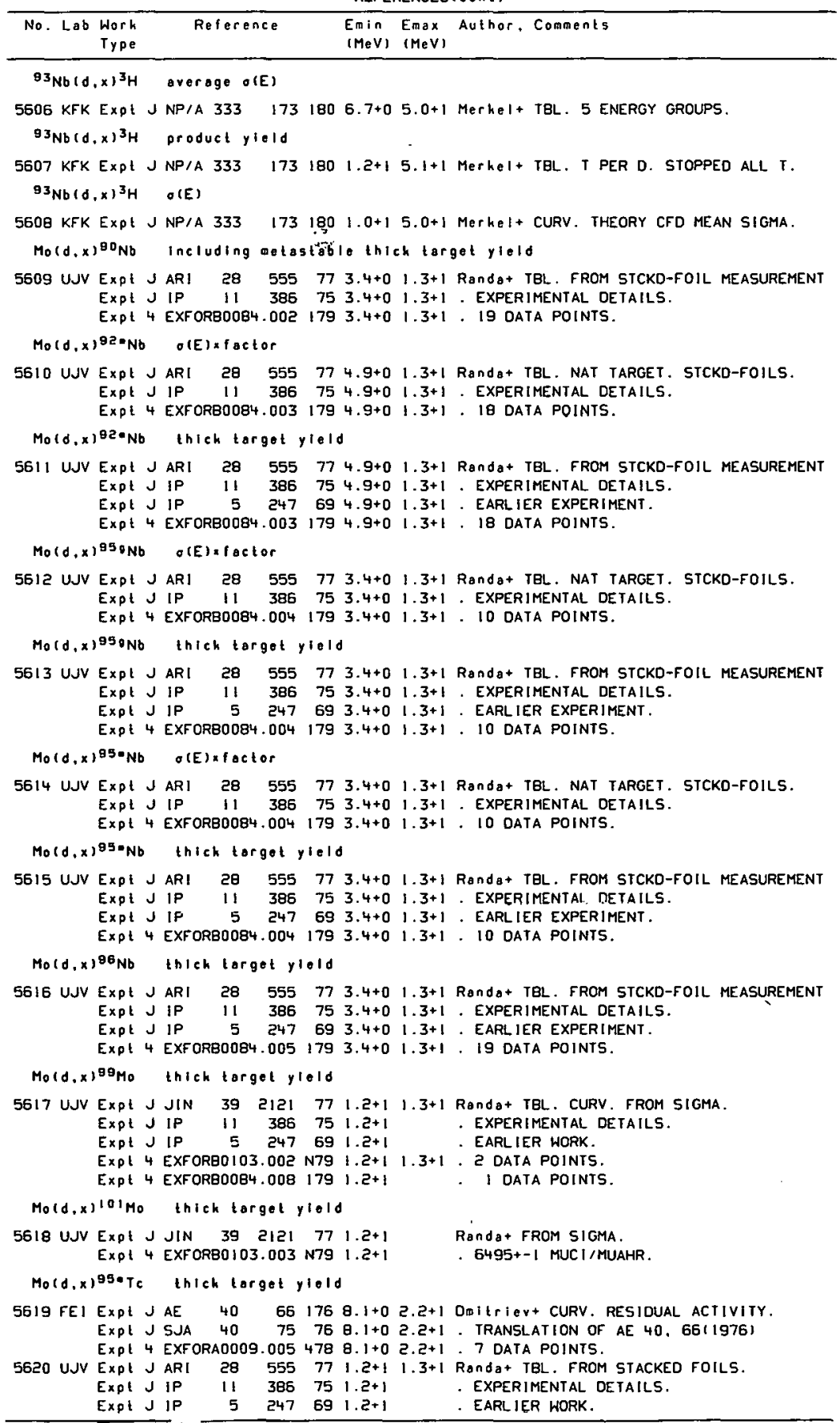


REFERENCES ( c o n t )

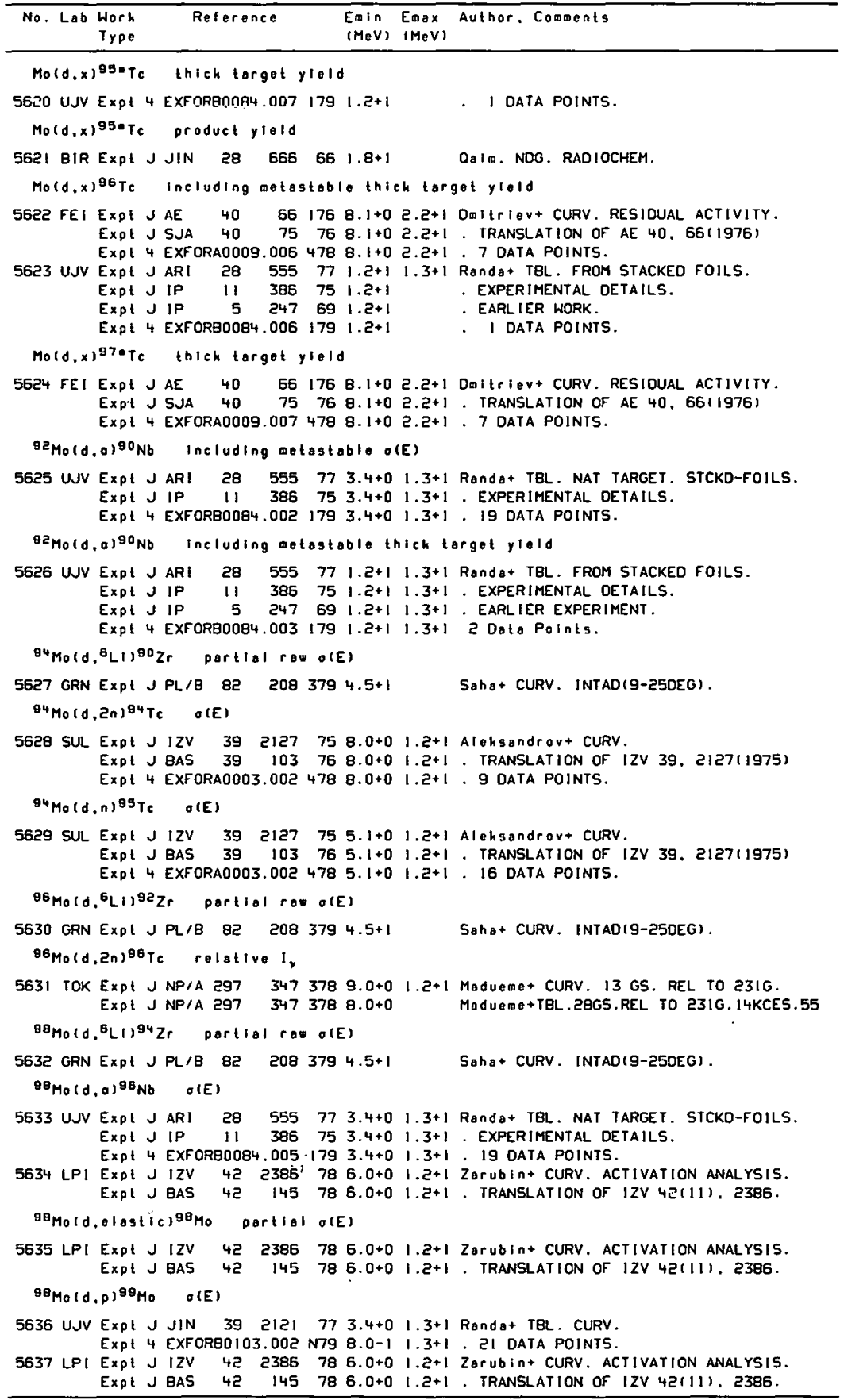




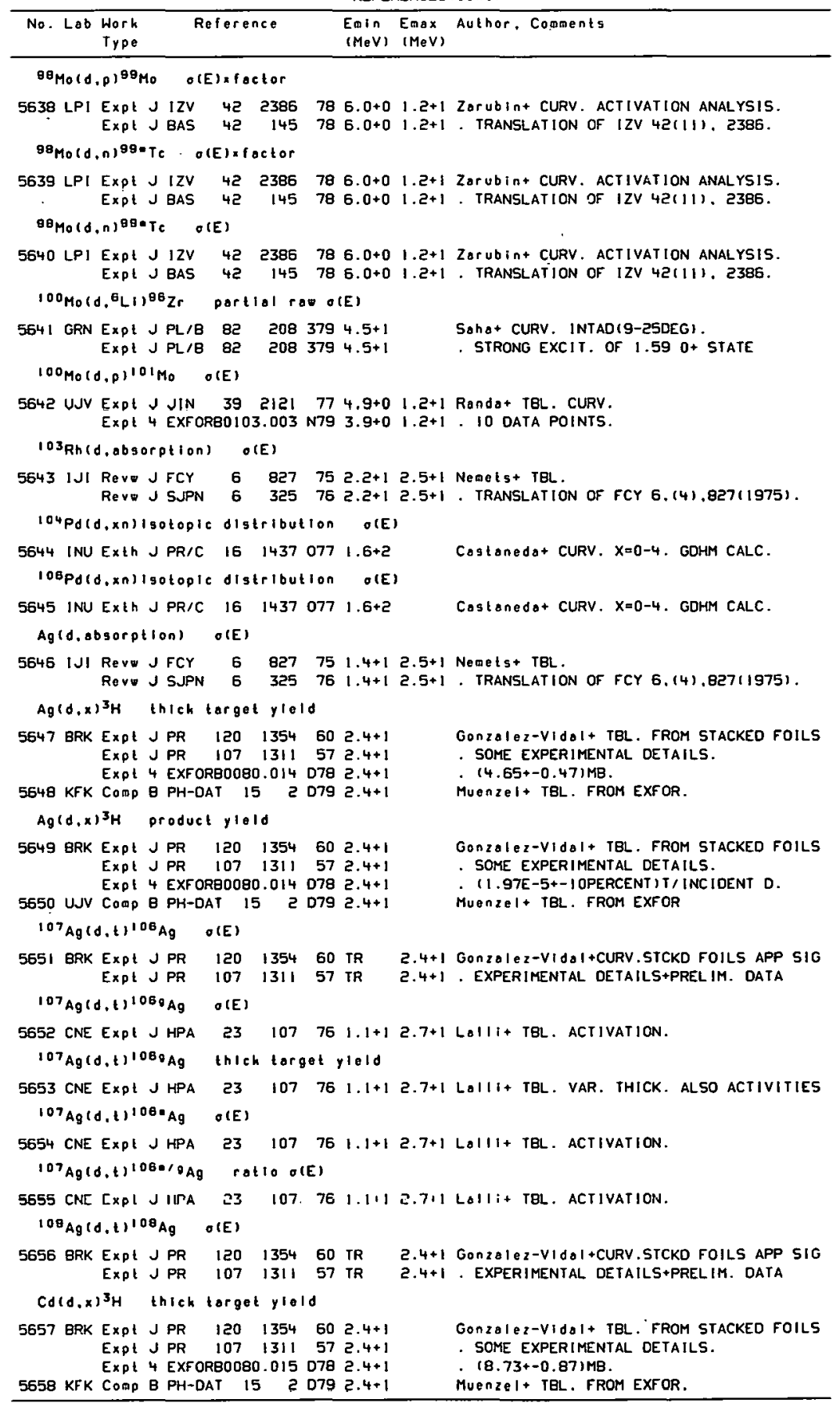


REFERENCES (cont)

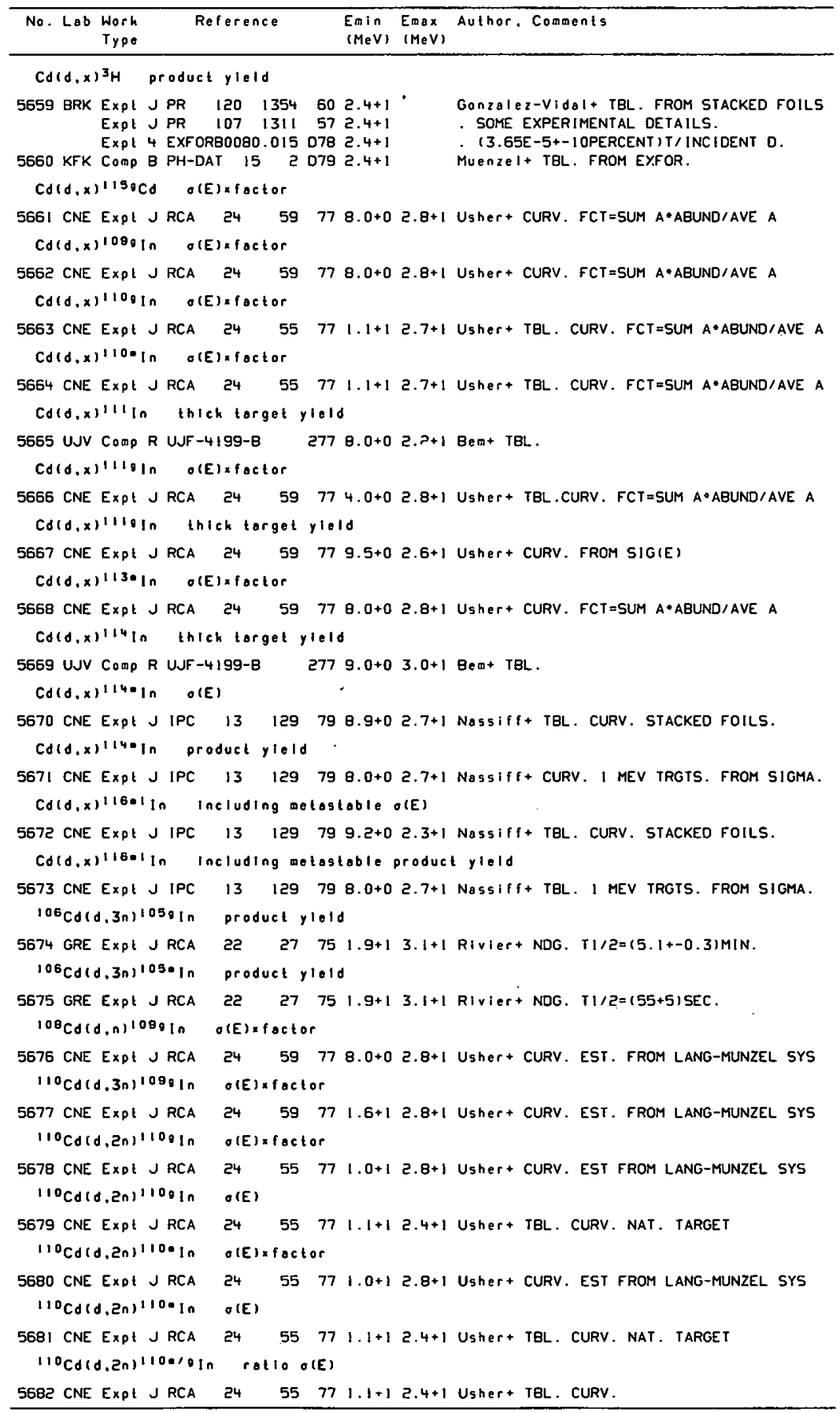


REFERENCES (cont)

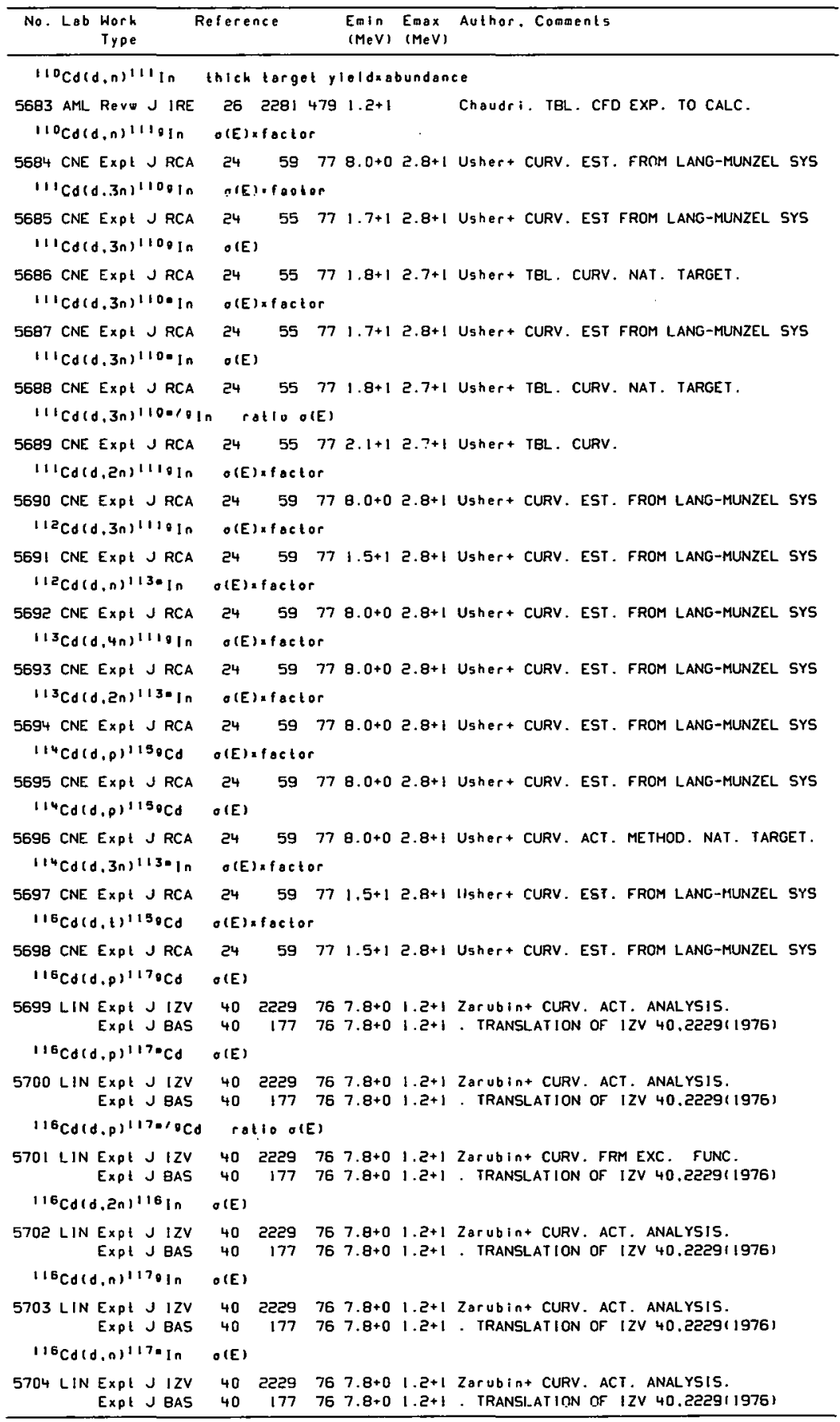


REF ERENCES ( c on ( )

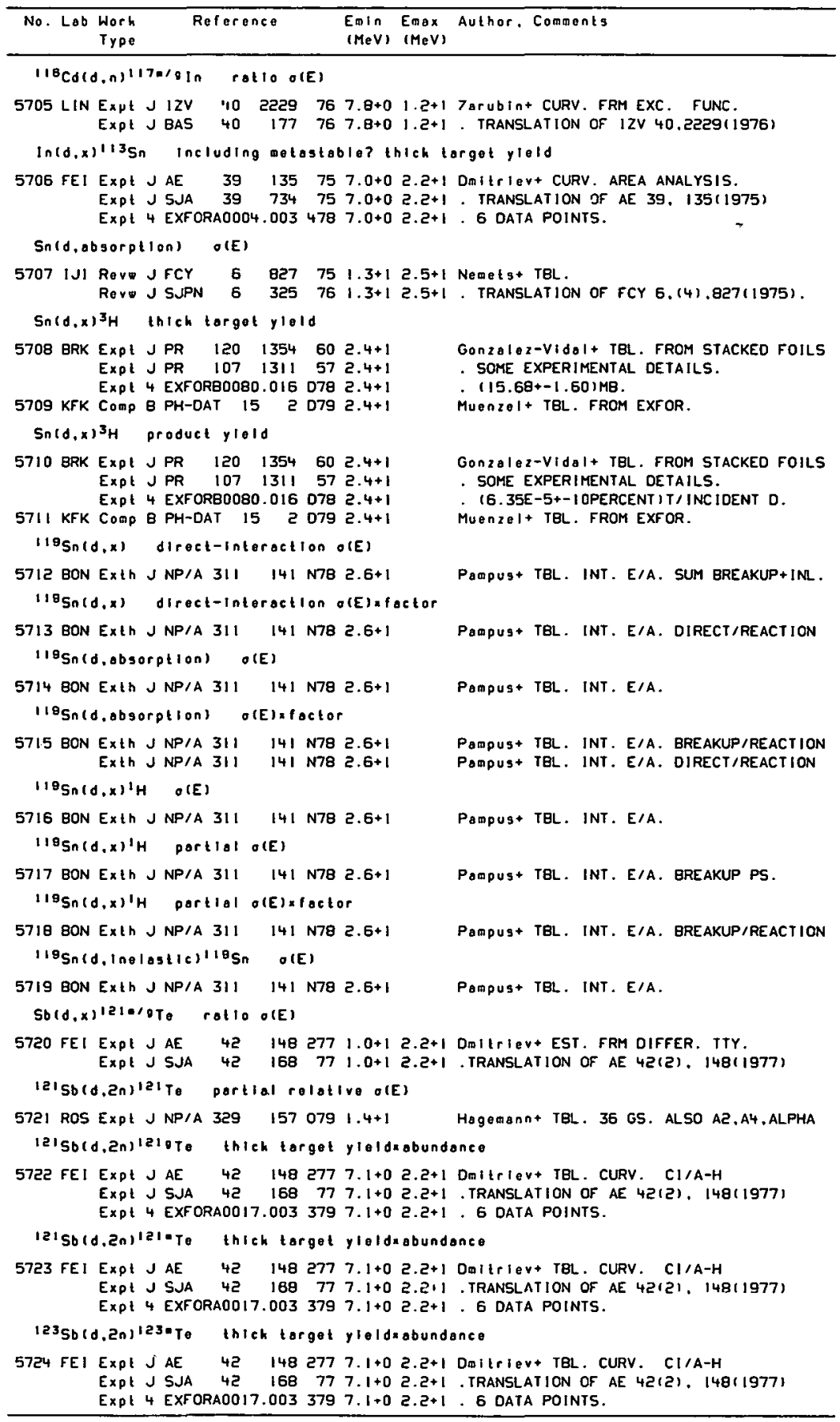




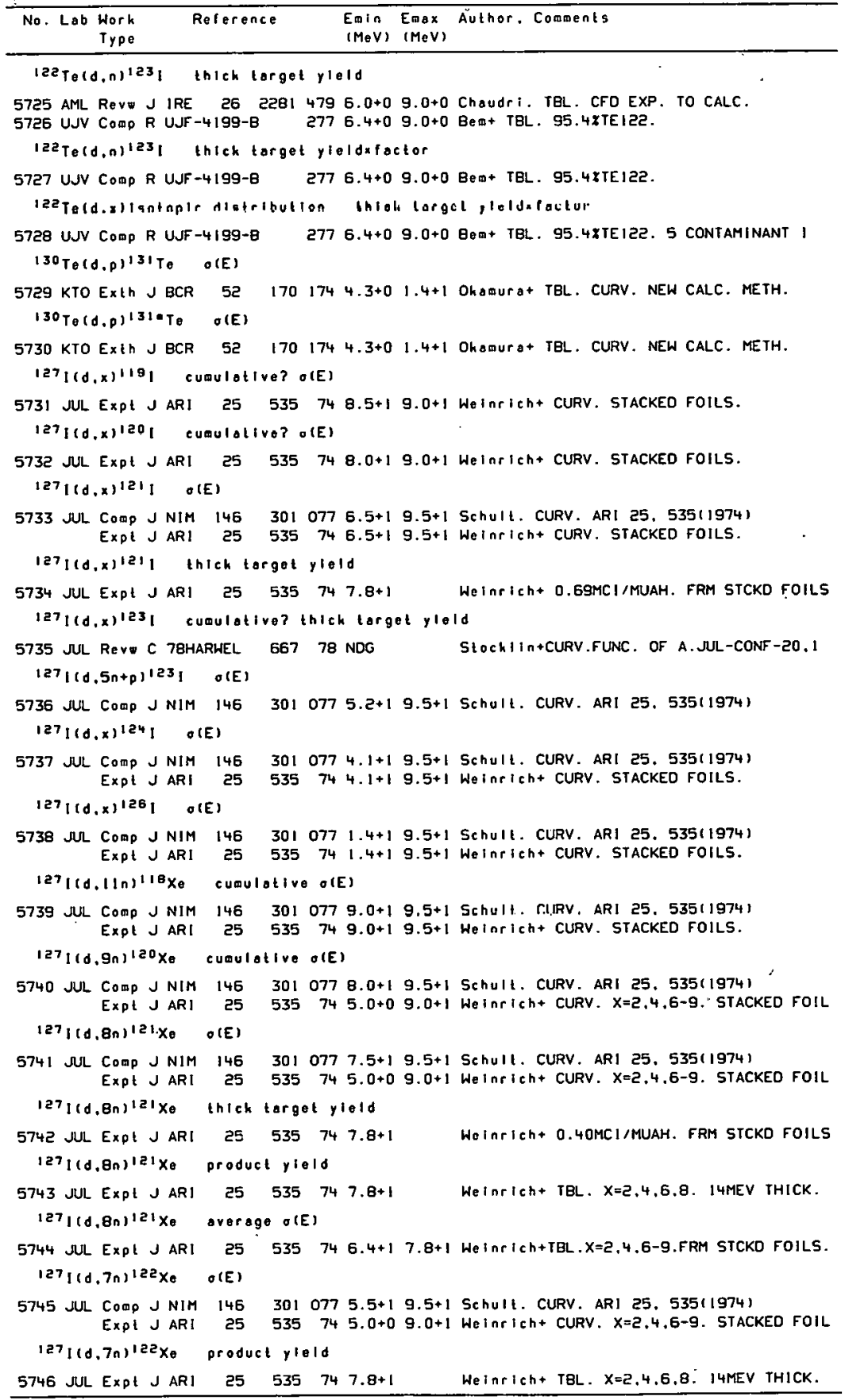


REFERENCES I c ont

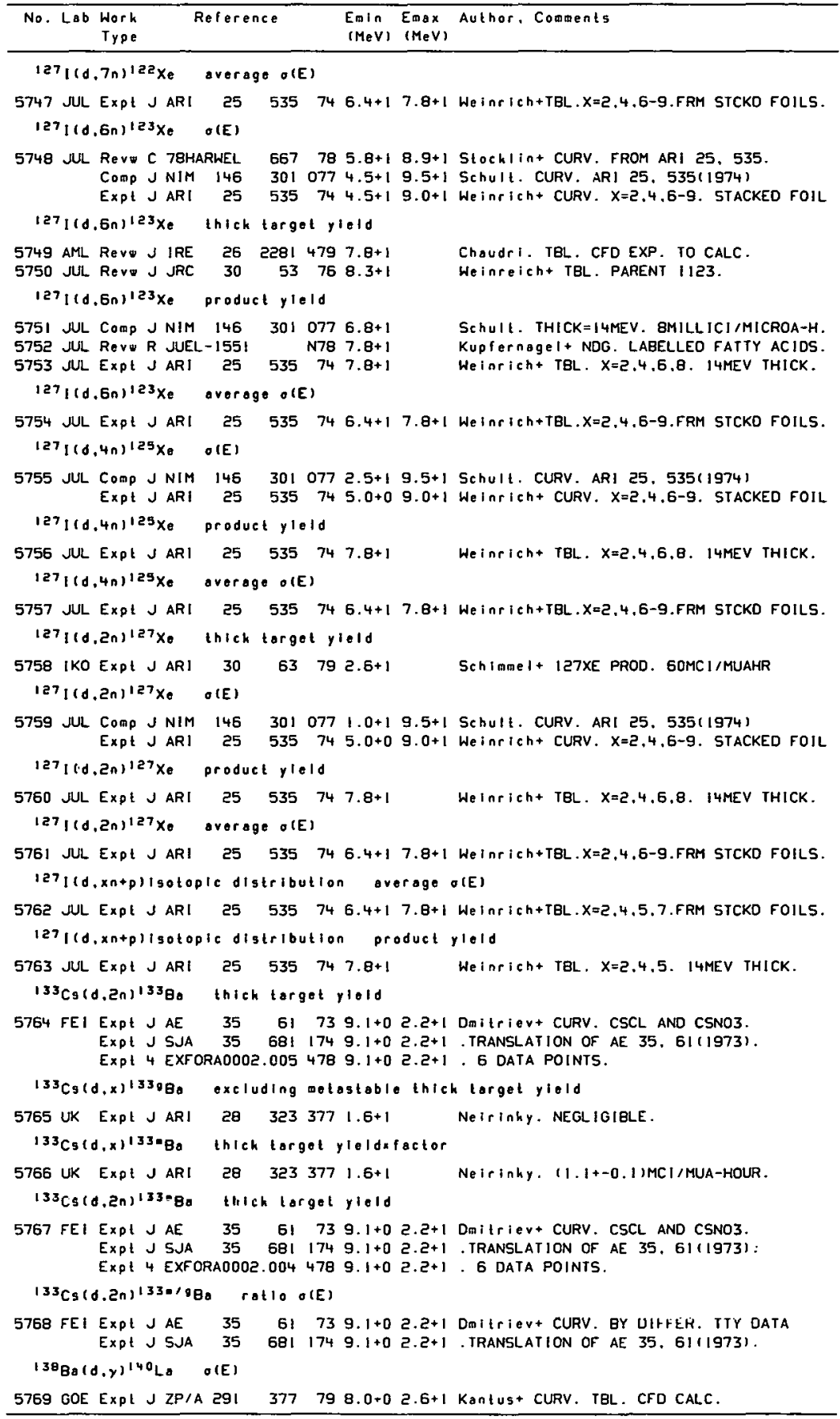


REFERENCES ( c on t)

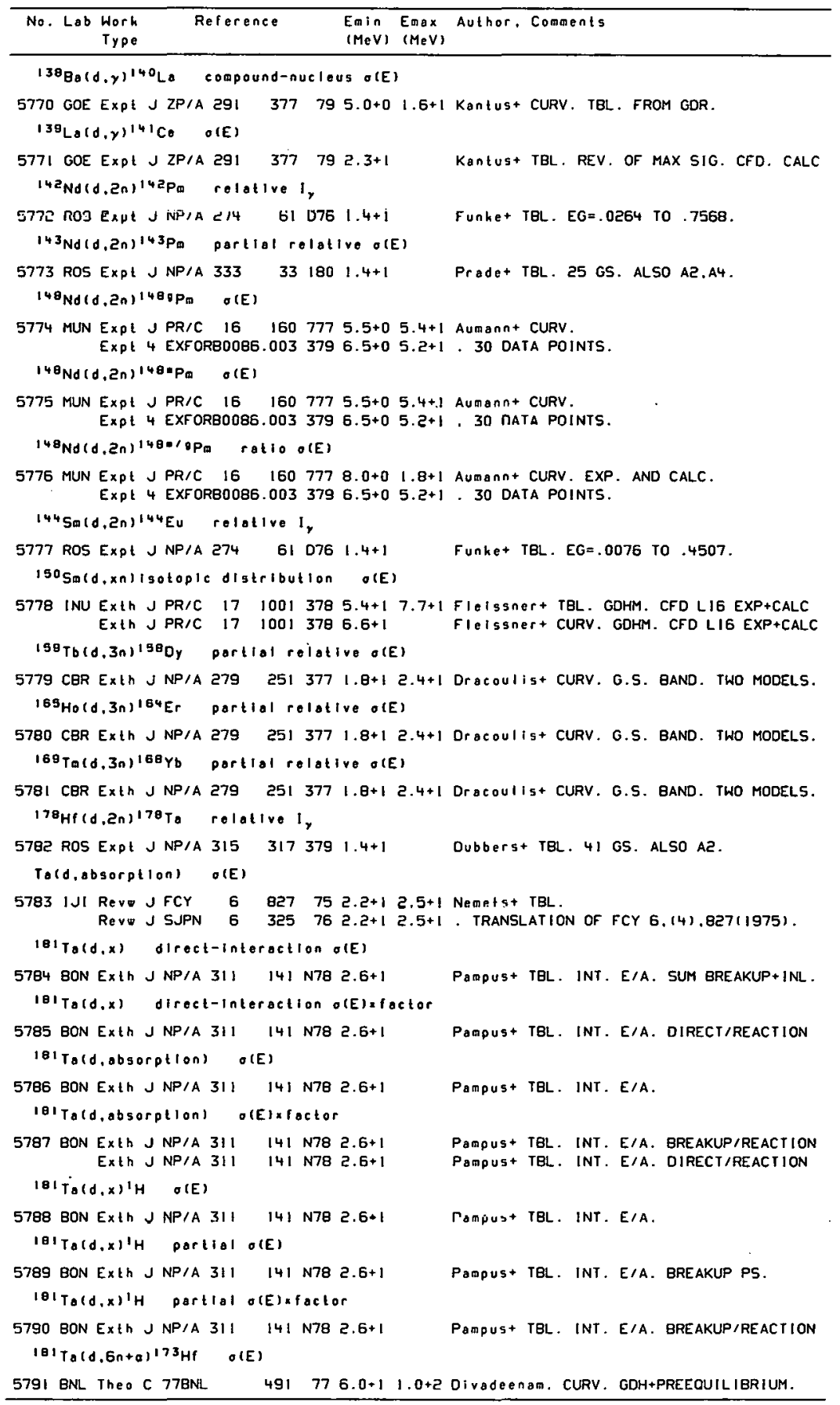


REFERENCES ( c on t)

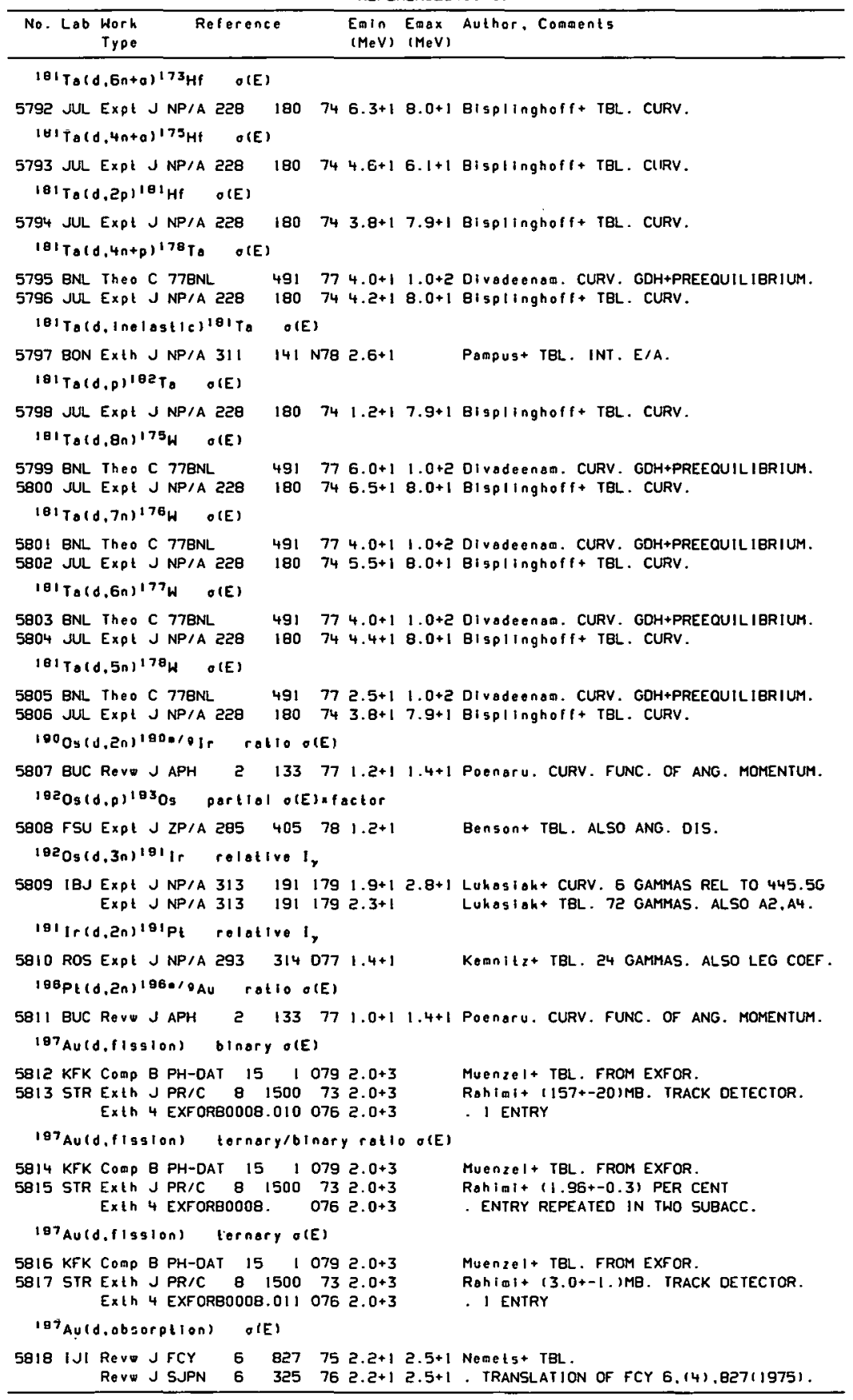


REFERENCES $(c$ on $t)$

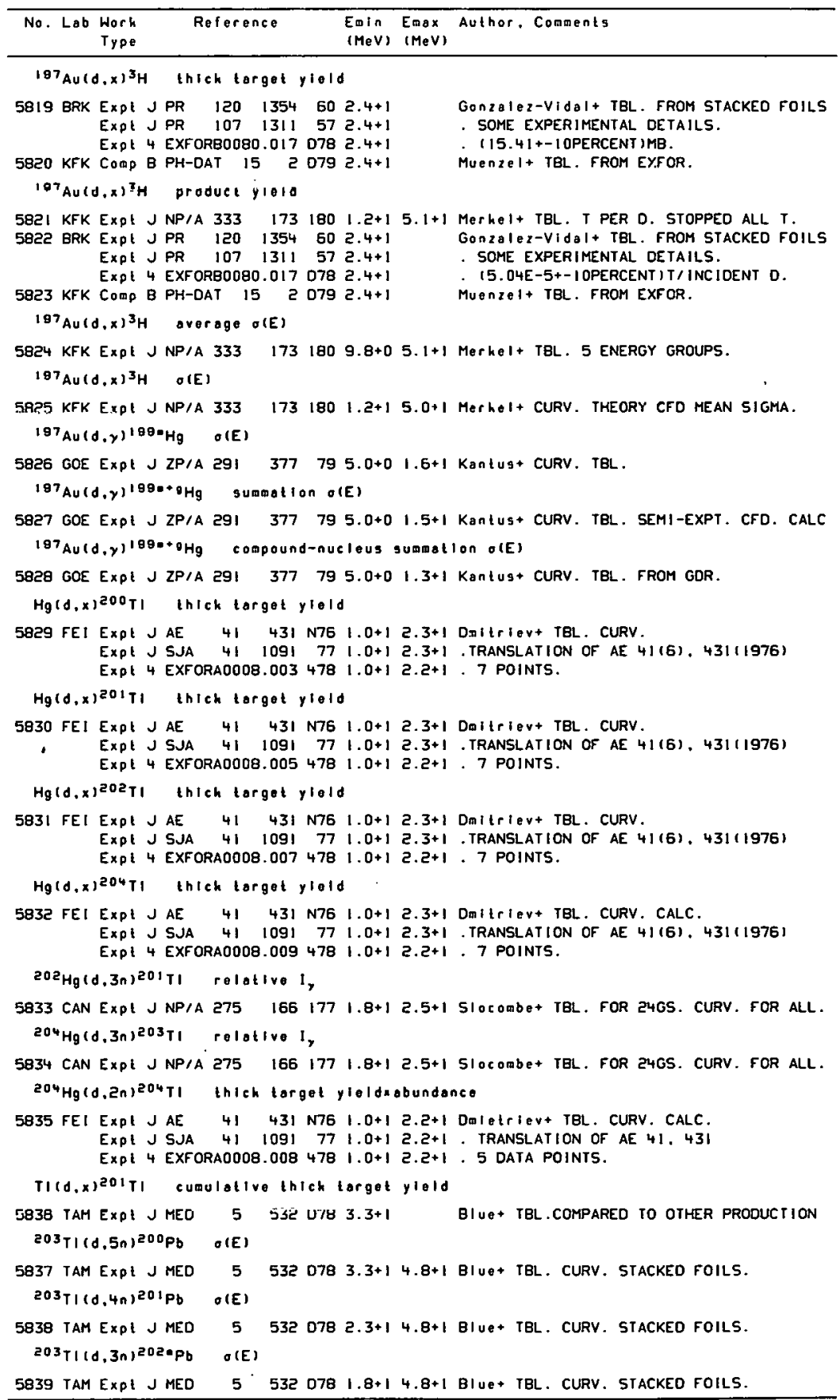


REFERENCES ( c ont )

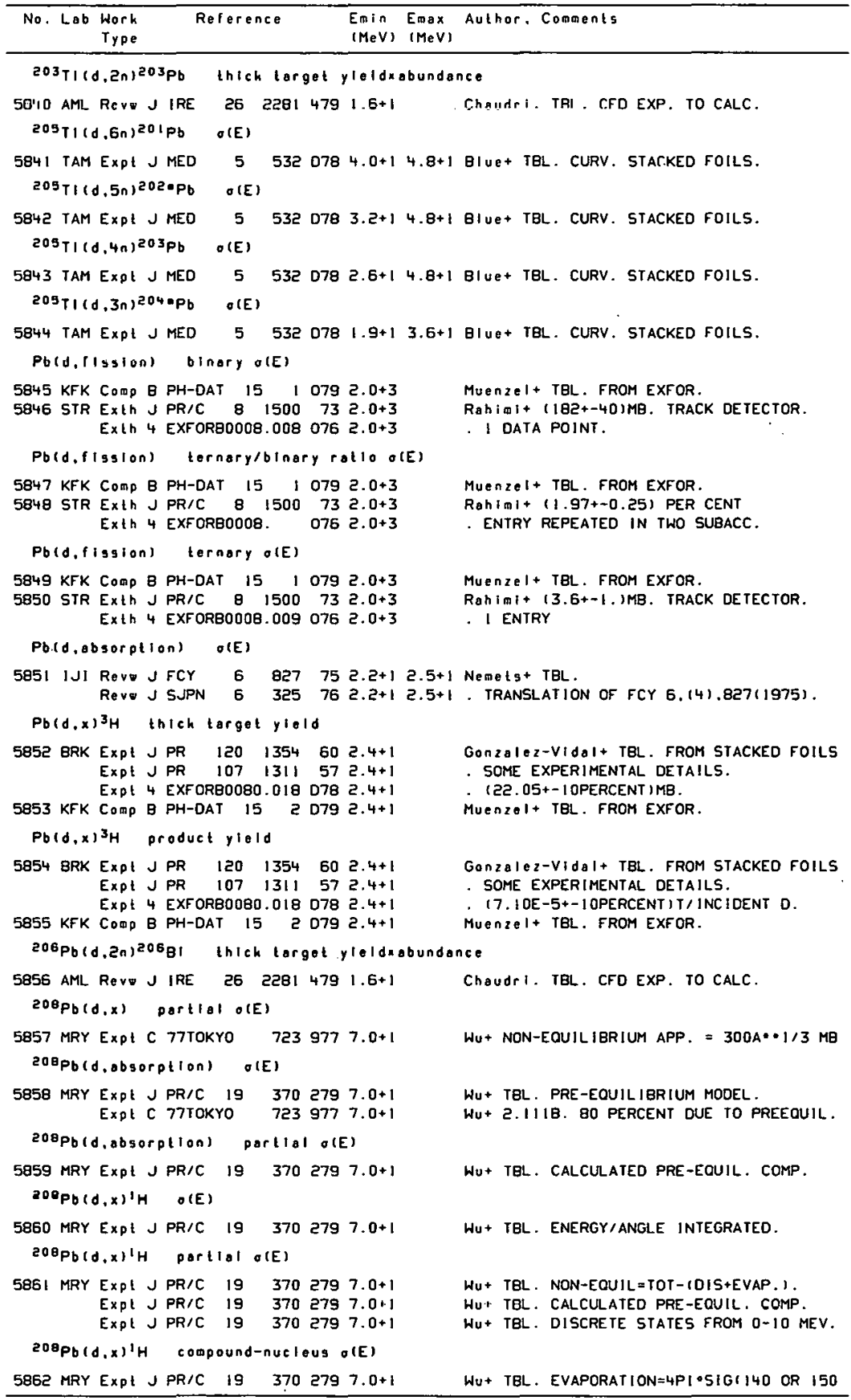




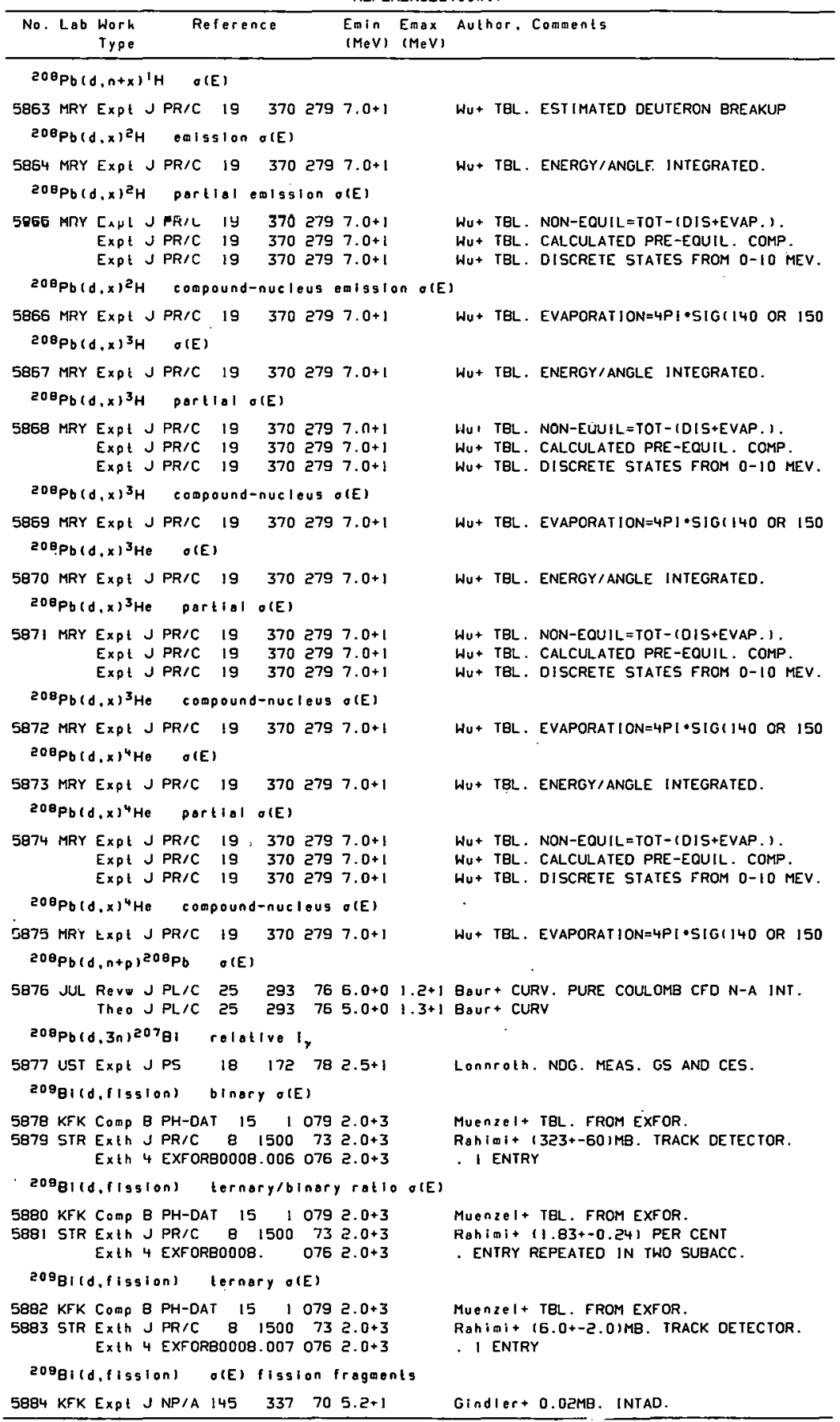




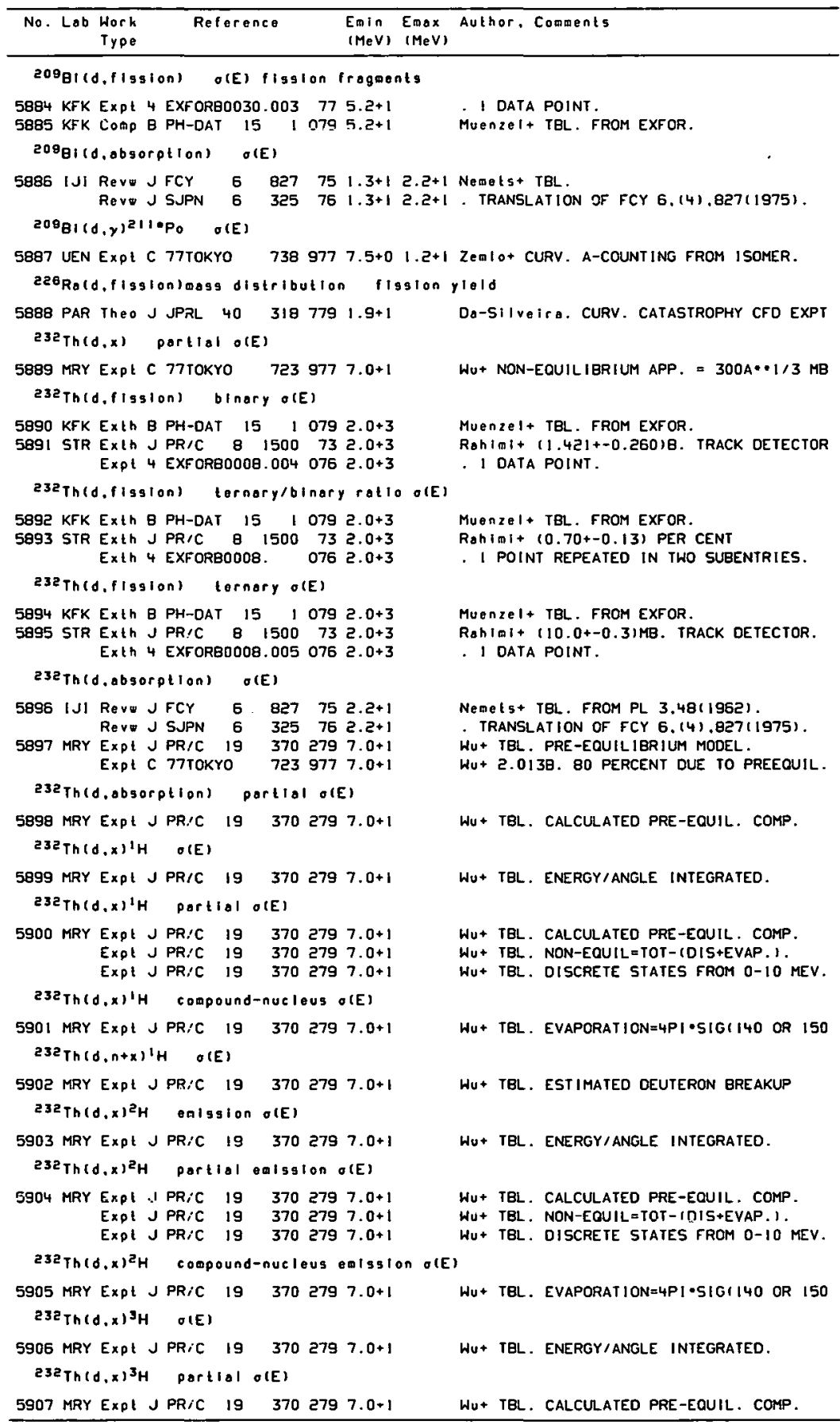


REFERENCES ( $c$ ont)

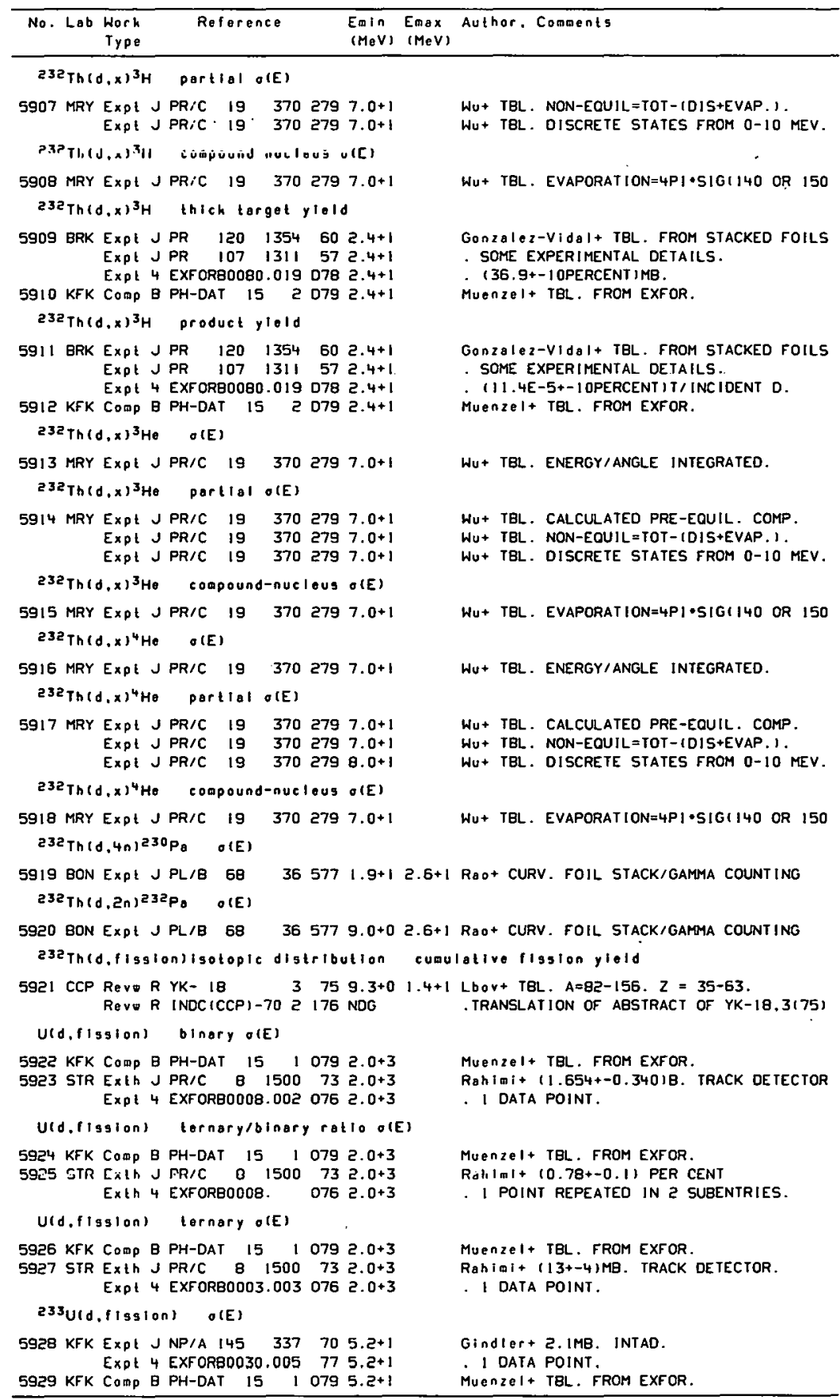


REFERENCES (cont)

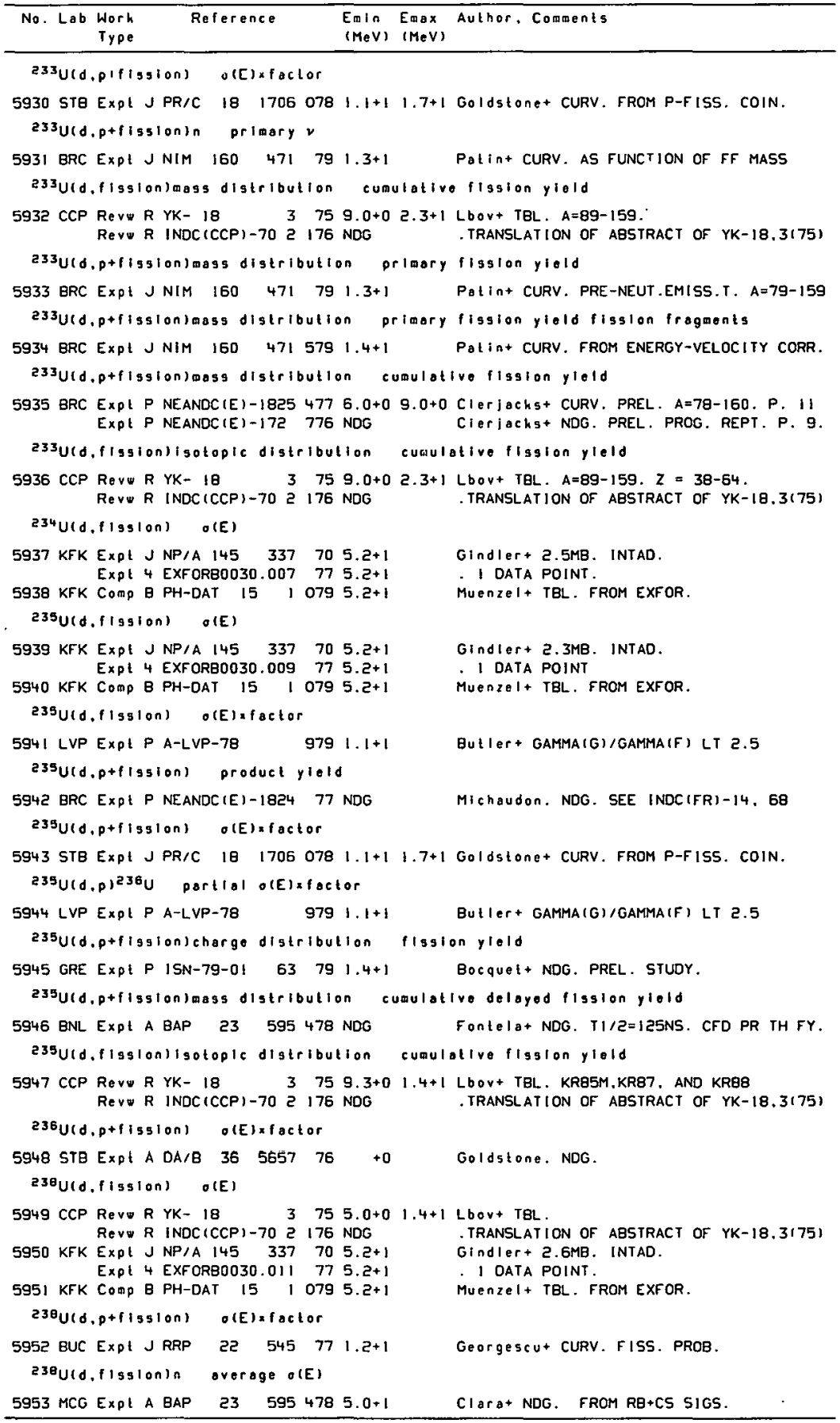


REFERENCES (cont)

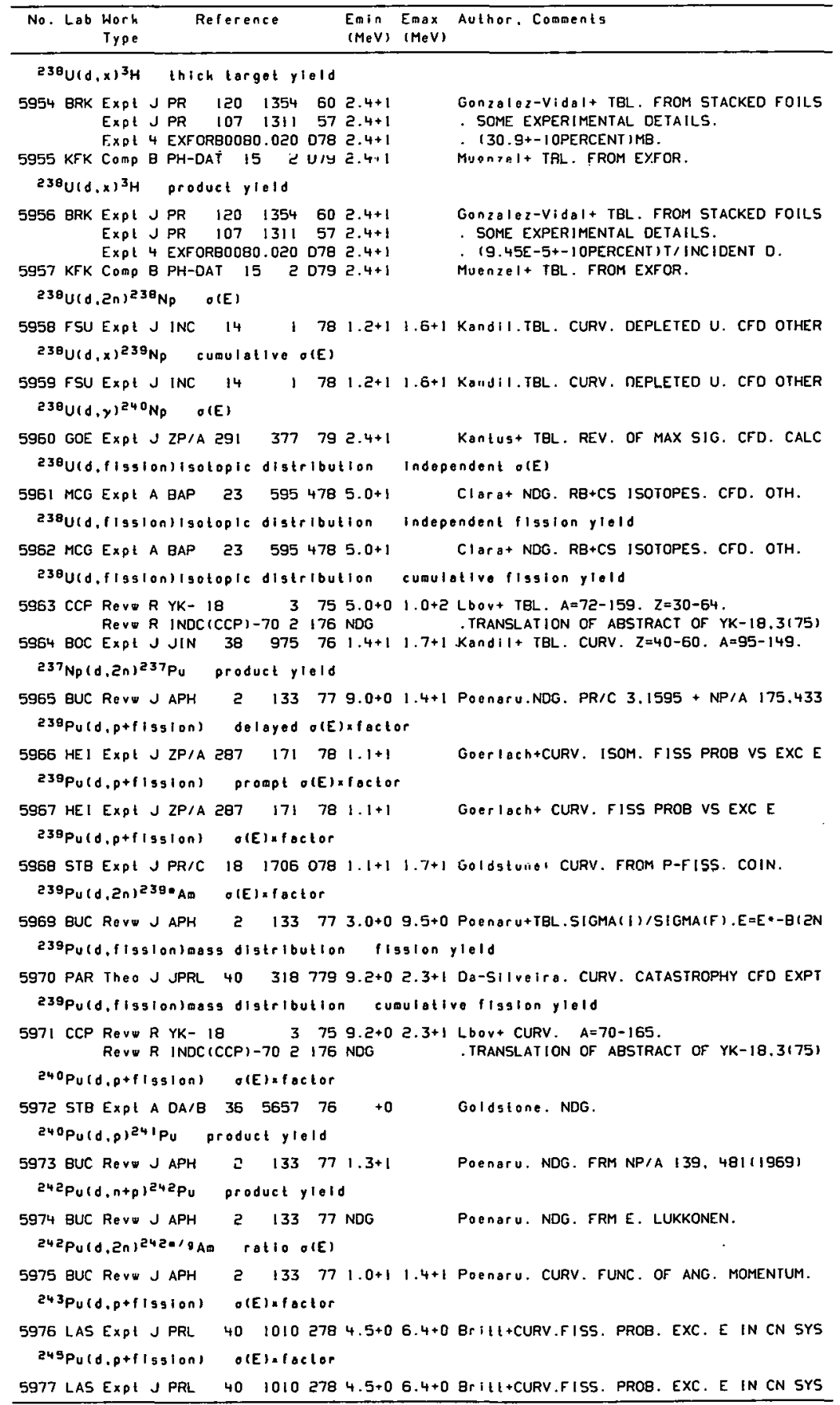




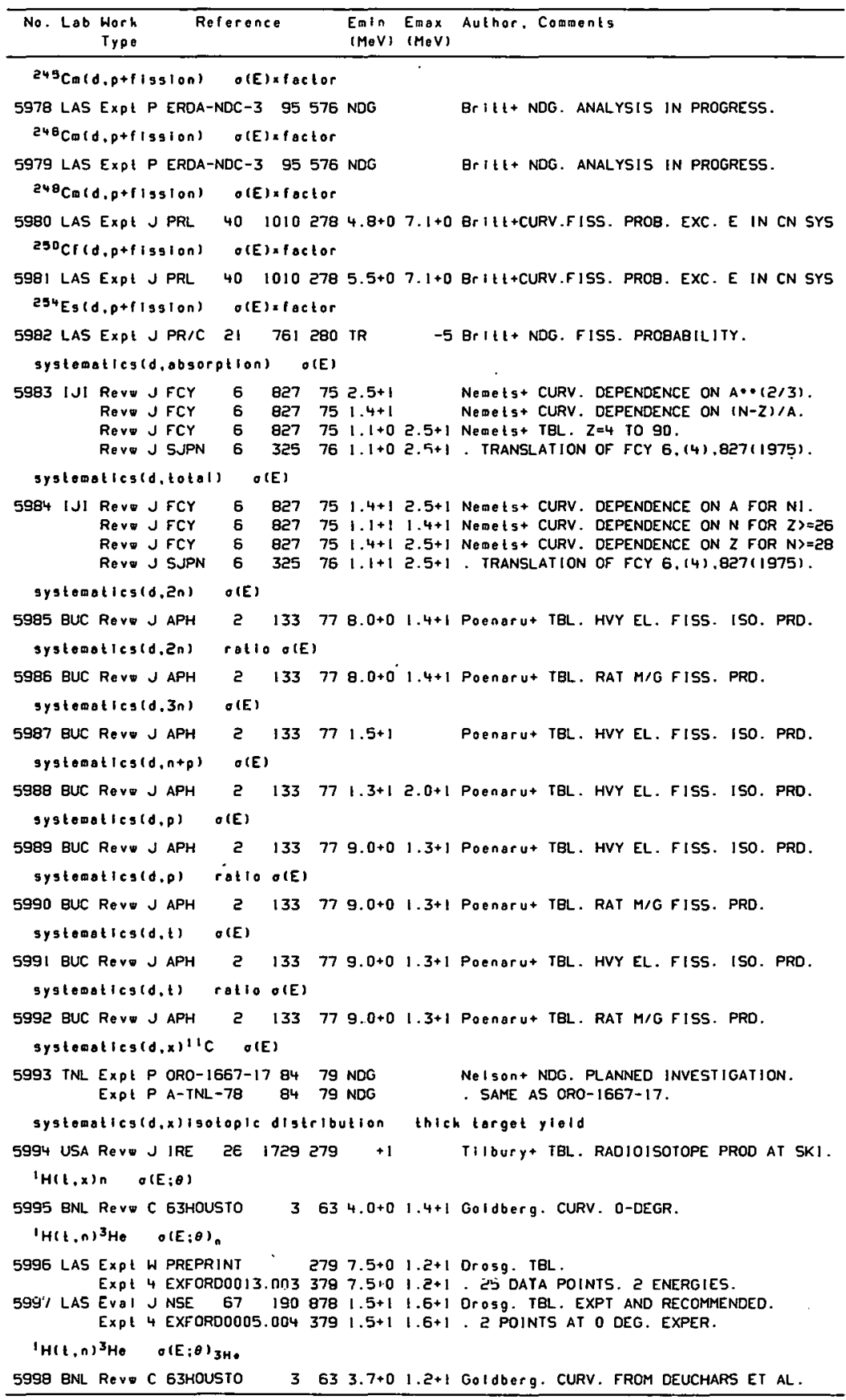


REFERENCES (cont)

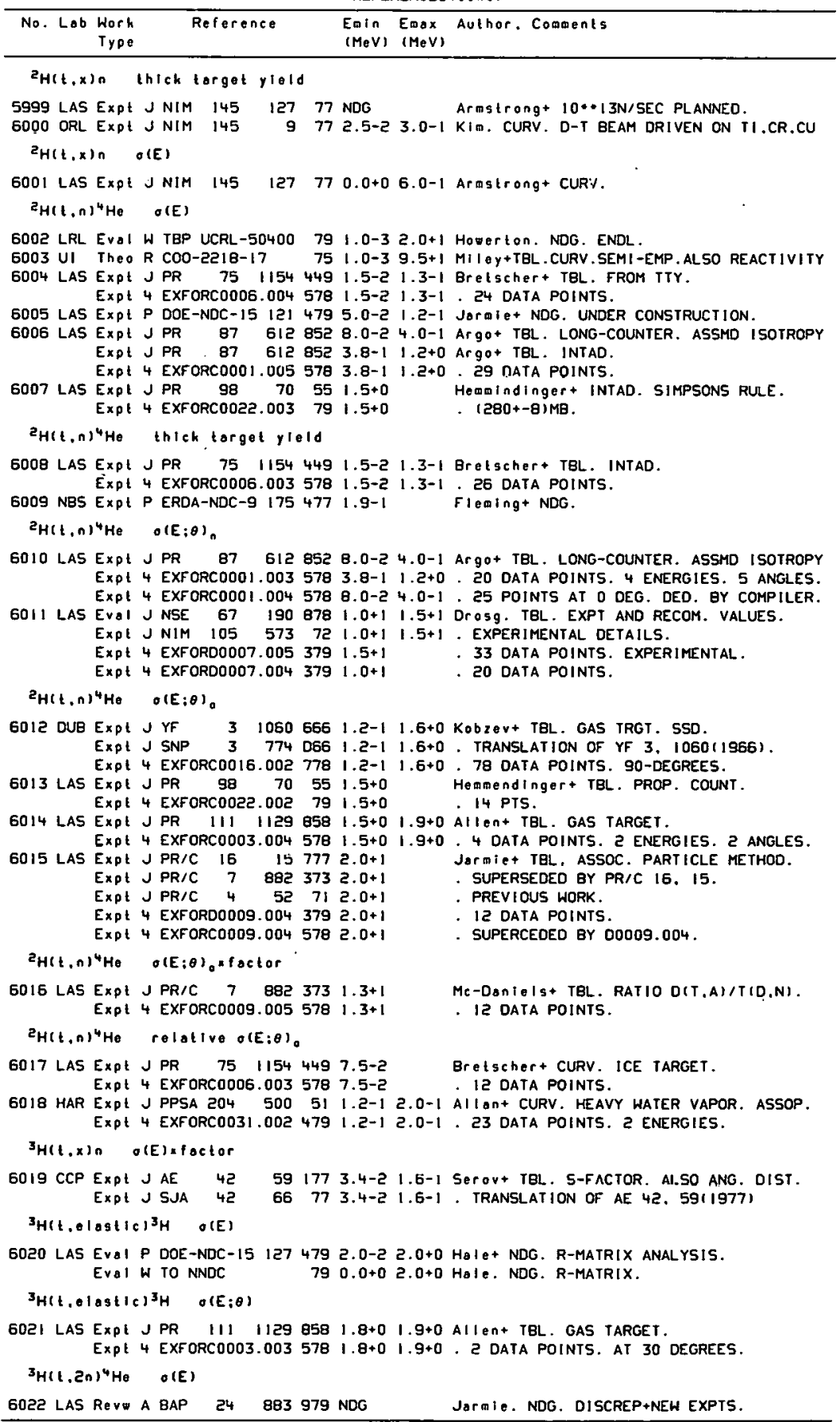




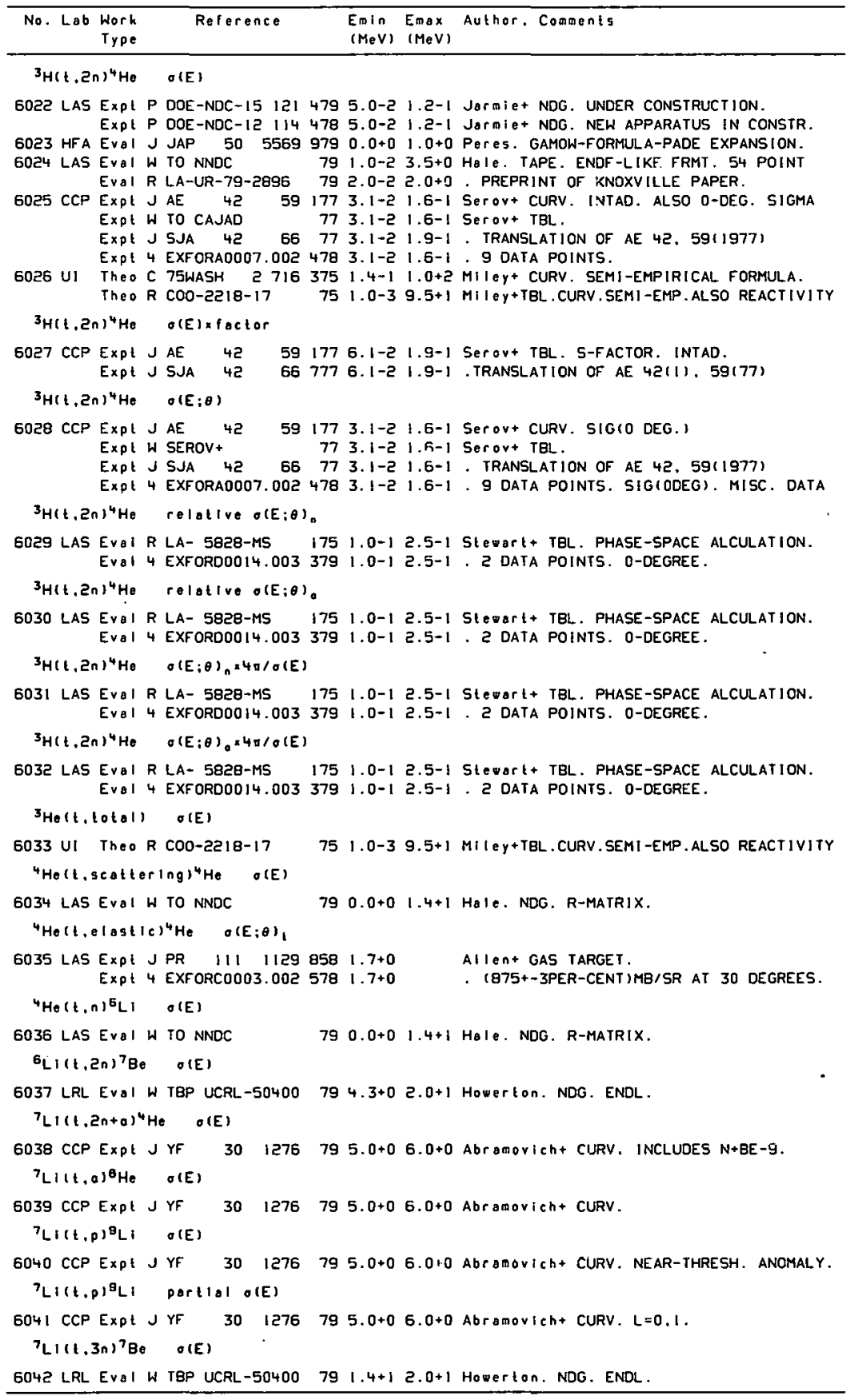


REFERENCES (cont)

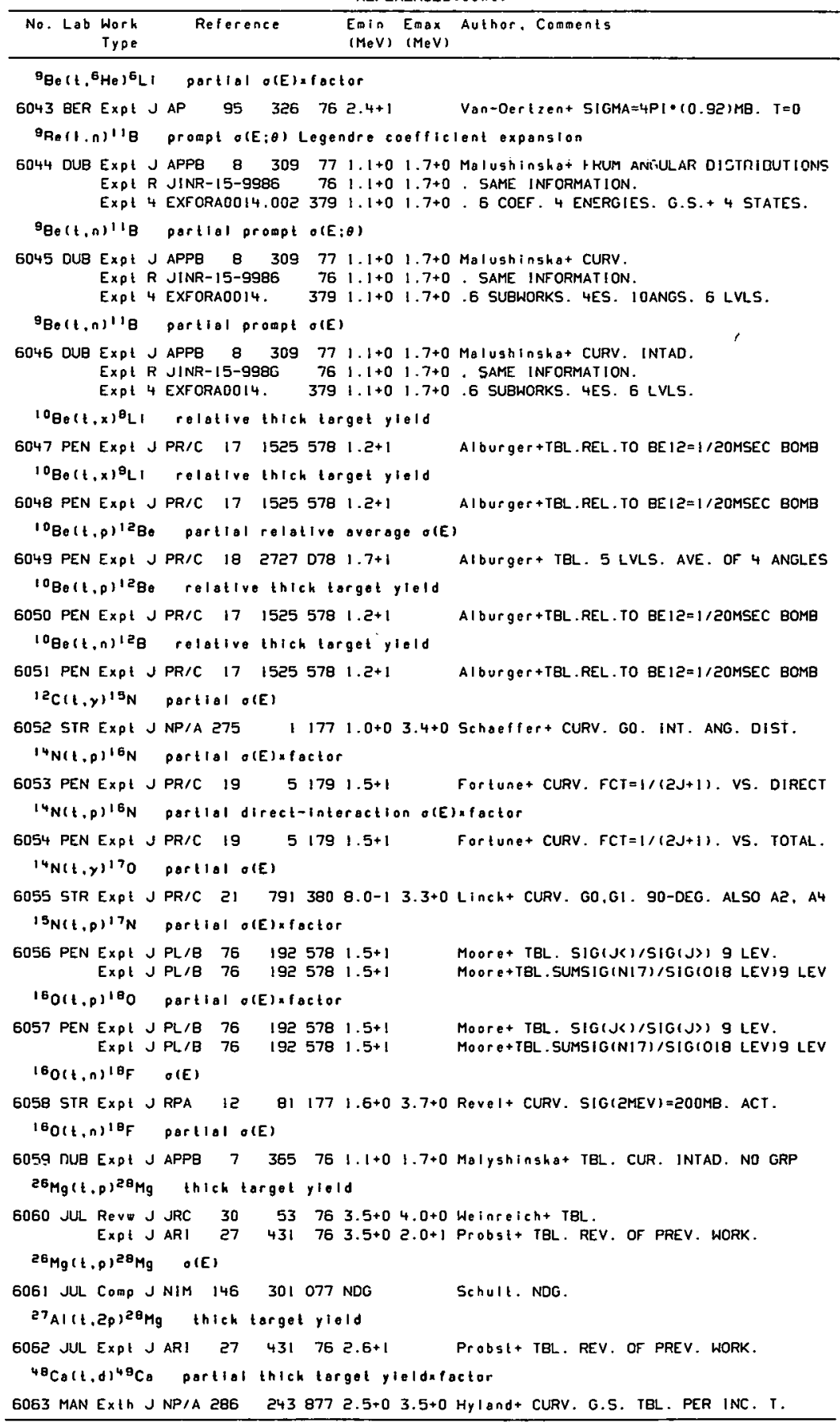




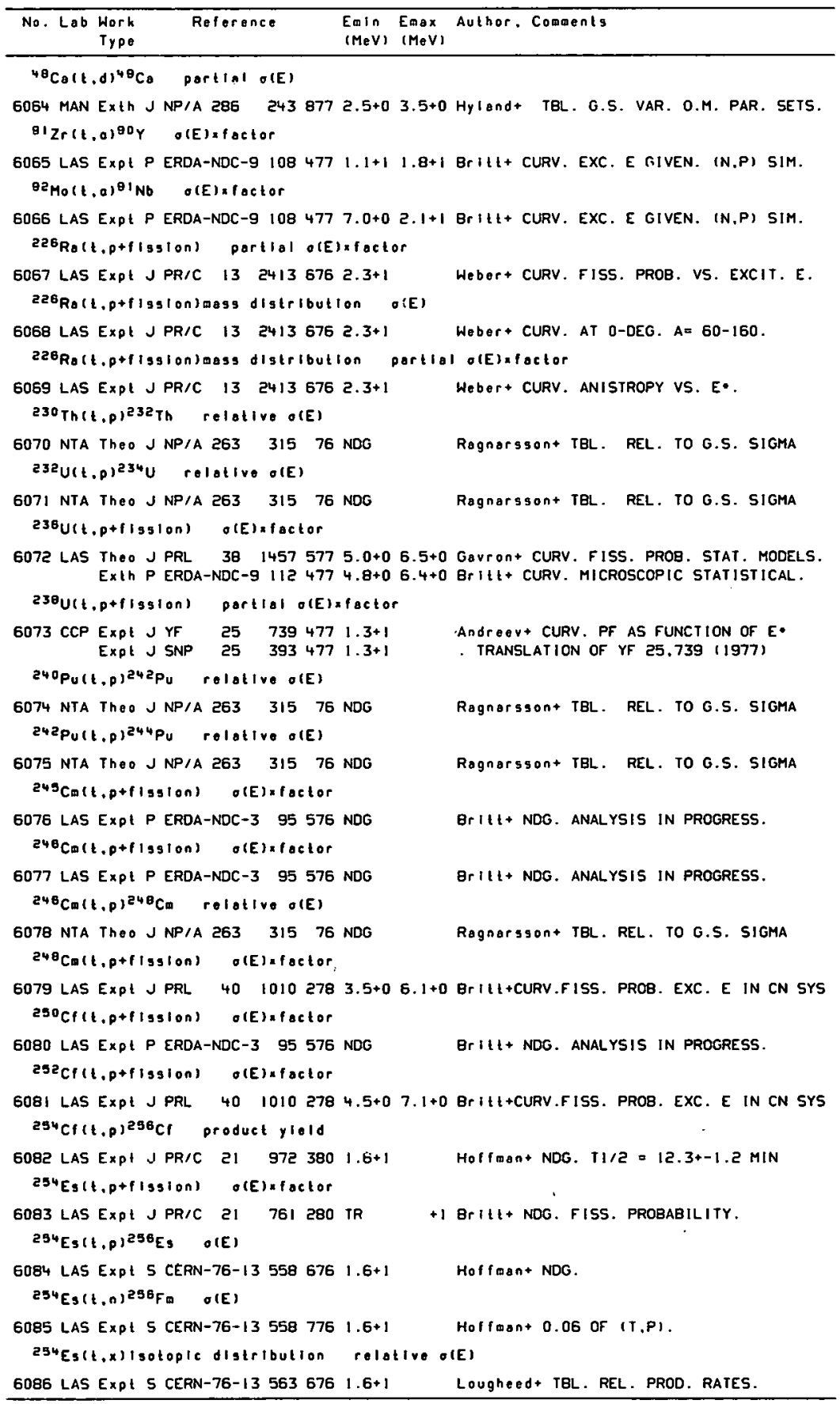




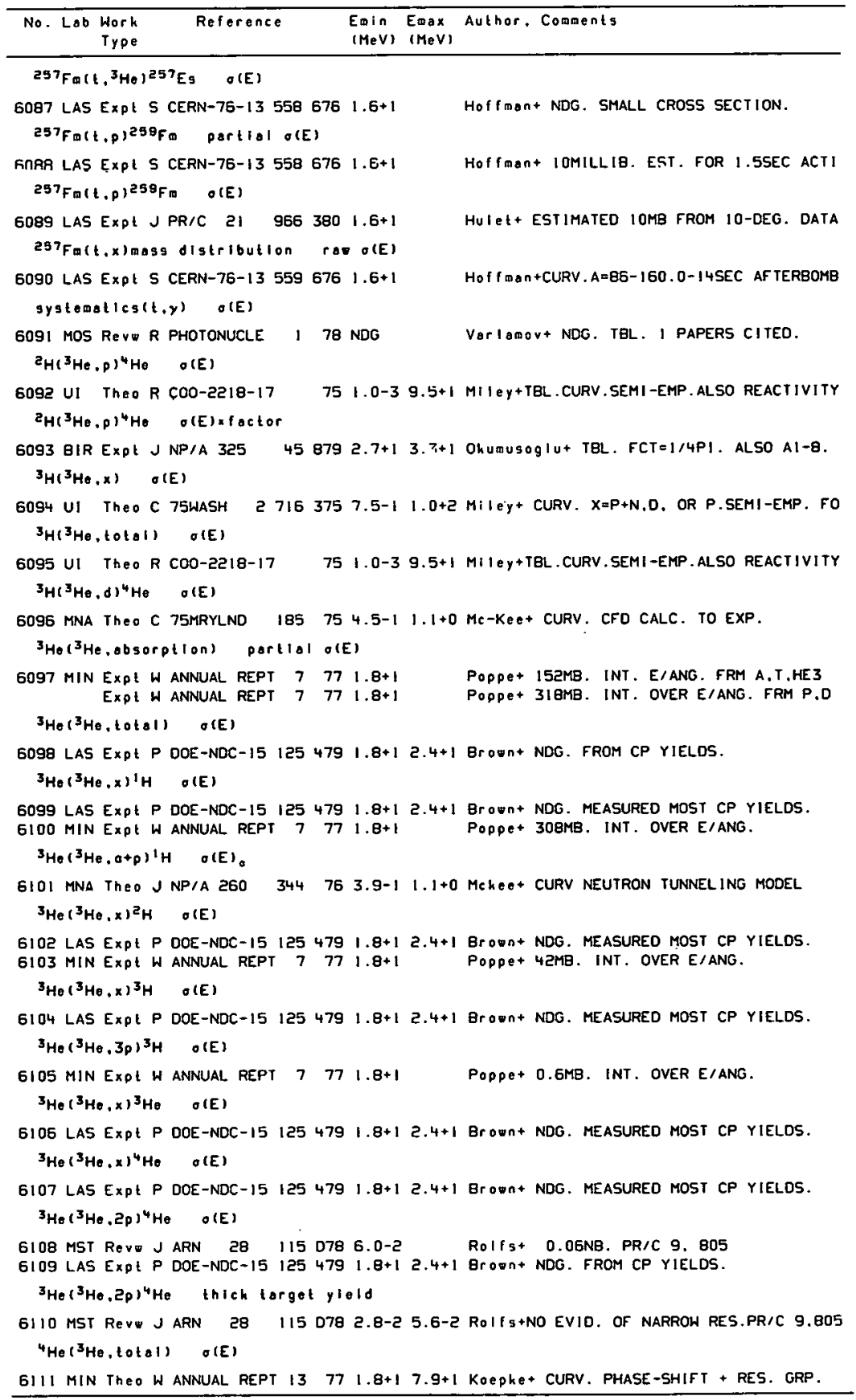




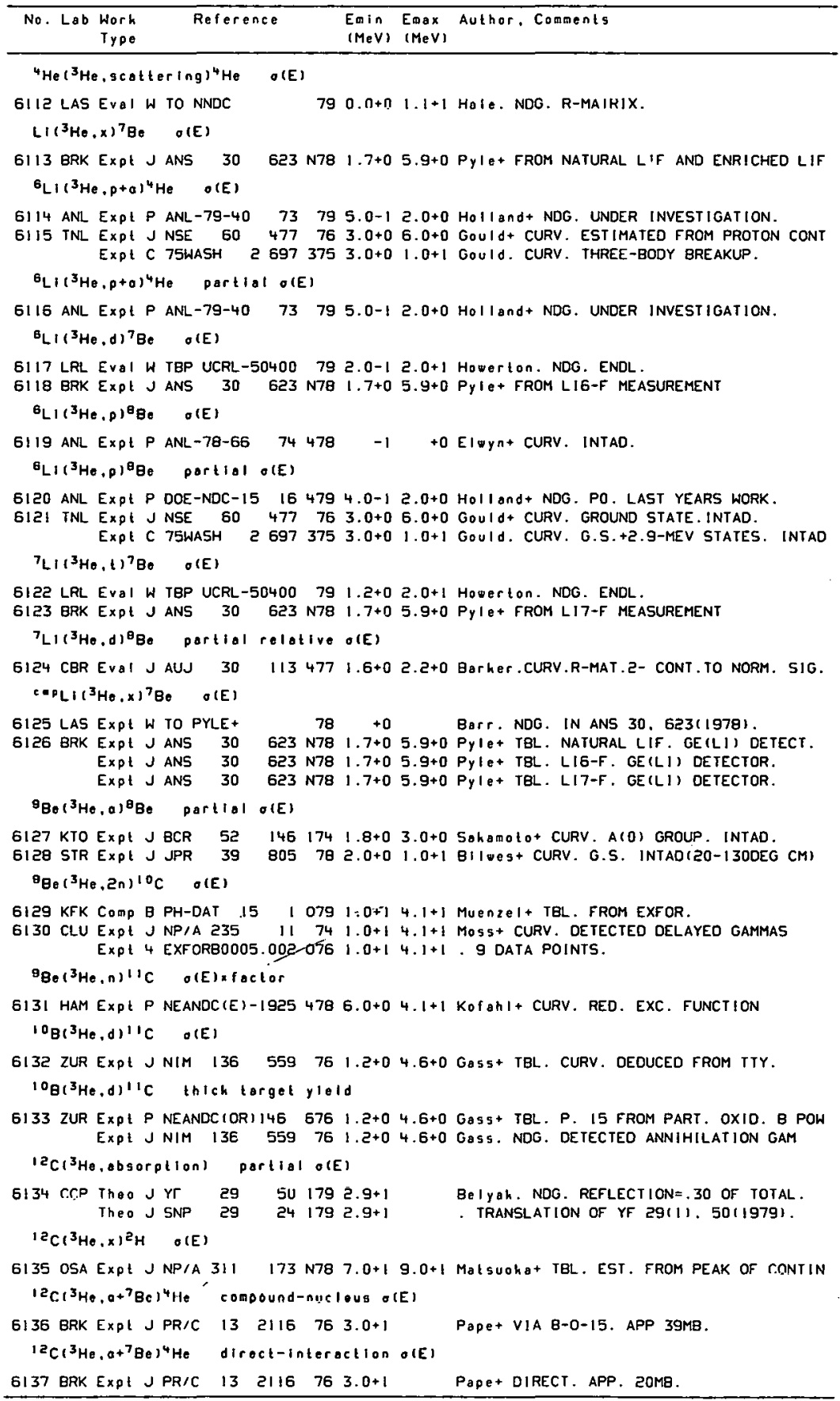


REFERENCES (cont)

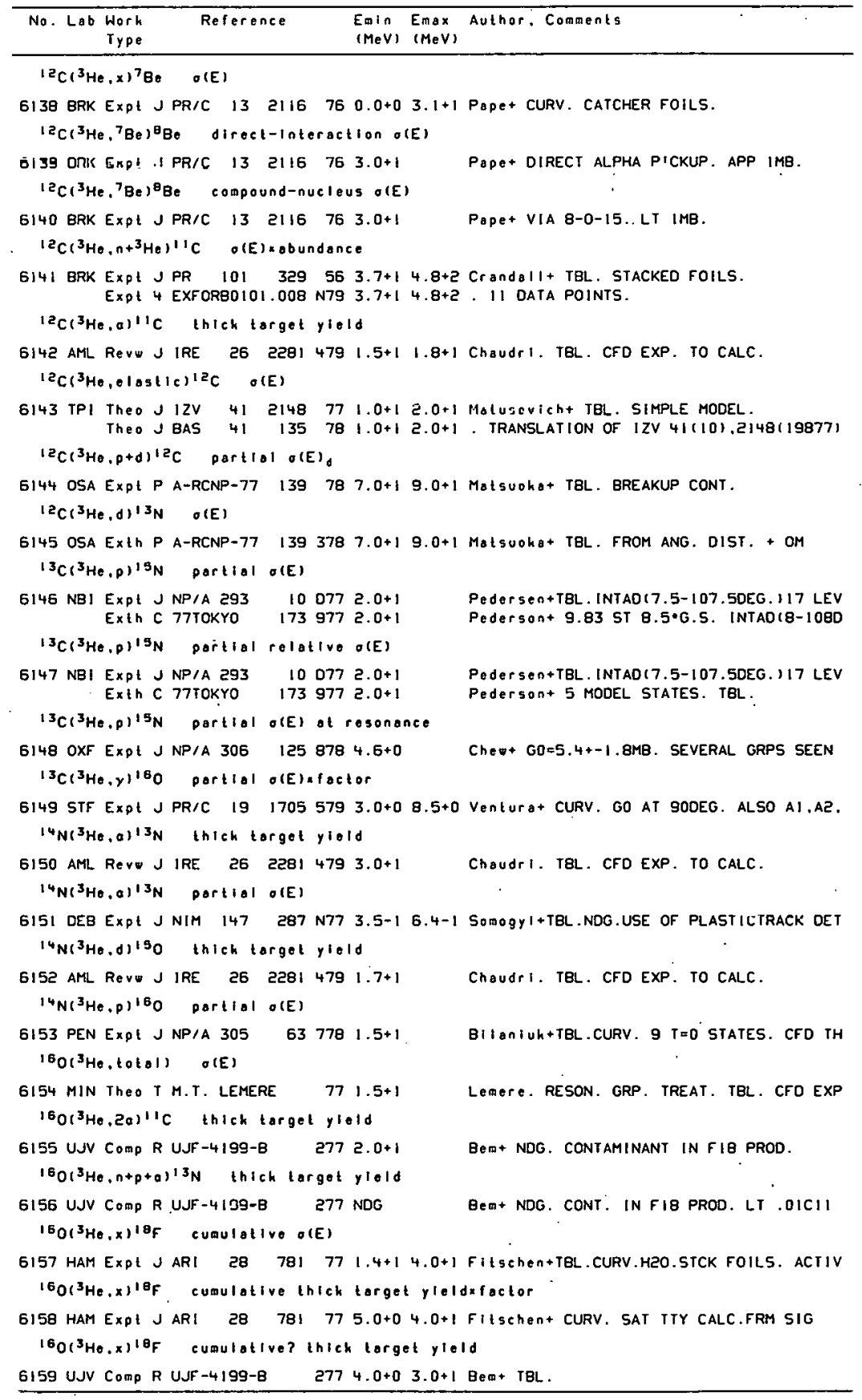


REFERENCES $($ cont $)$

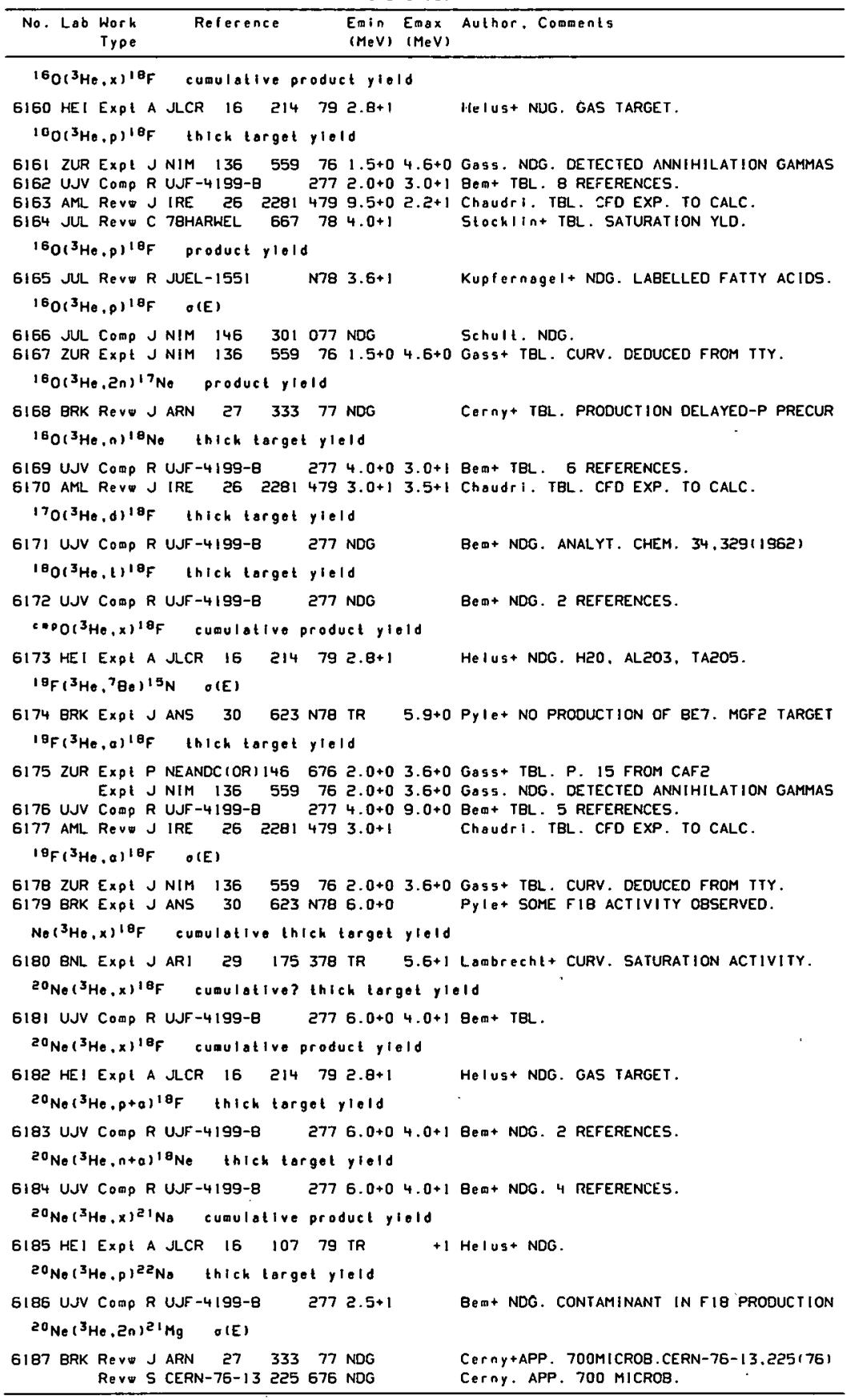


REFERENCES( 0 on t)

\begin{tabular}{|c|c|c|c|c|c|}
\hline No. Lab & $\begin{array}{l}\text { Work } \\
\text { Type }\end{array}$ & Reference & $\begin{array}{l}\text { Emin } \\
(M e V)\end{array}$ & $\begin{array}{l}E \max \\
(M e V)\end{array}$ & Author. Comments \\
\hline
\end{tabular}

$22 \mathrm{Net}^{3} \mathrm{Ho}_{0}, \mathrm{j}^{24} \mathrm{No}$ thick targot yiold

6188 UUV Comp R UJF-4199-B $2771.5+1 \quad$ Bem+SOME ACTIVITY EVIDENT. CONT. IN FIB

$\left.2{ }^{3} \mathrm{Nat}{ }^{3} \mathrm{He}, 20\right)^{18 \mathrm{~F}}$ thich torget yiold

6189 UJV Comp R It. IF -4100 D $2774 . U+U$ 2.8+1 8 eq+ NDG. 3 REFERENCFS.

$M g\left({ }^{3} H e, t o t a l\right) \quad \sigma(E)$

6190 IJ] Revw J FCY 6 827 $752.9+1 \quad$ Nemels+ TBL. FROM PL/B 26.213(1968). Revo J SJPN $6325762.9+1 \quad$. TRANSLATION OF FCY 6. $641.827(1975)$.

$\operatorname{Mg}\left({ }^{3} \mathrm{He}_{0} \times \mathrm{r}^{24} \mathrm{SI}\right.$ product yield

6191 PIN Expl 5 CERN-76-13 $2546765.7+1$ Roberlson+ NDG. PRELIM. SEARCH.

${ }^{24} \mathrm{Mg}\left({ }^{3} \mathrm{He}, x\right)^{2} \mathrm{H} \quad O(E)$

6192 OSA Expt J NP/A 311173 N78 9.0+1 Malsuoha+ IBL. EST. FROM PEAK OF CONTIN

$24 \mathrm{Mg}\left({ }^{3} \mathrm{He}, \mathrm{p}+2 \mathrm{Co}\right)^{10 \mathrm{~F}}$ thick target ylold

6193 UJV COmp R UJF-4199-B $2771.3+12.8+1$ Bem+NDG. BUTTERWORTHS, LONDON.341(1970)

${ }^{24} \mathrm{Mg}\left({ }^{3} \mathrm{He}_{0} \mathrm{p}+d\right)^{24} \mathrm{Mg}$ partlal $\sigma(E) d$

6194 OSA Expt P A-RCNP-77 $139787.0+19.0+1$ Matsuoha+ TBL. BREAKUP CONT.

${ }^{24} \mathrm{Mg}\left({ }^{3} \mathrm{He}, d\right)^{25} \mathrm{Al} \quad O(E)$

6195 OSA Exth P A-RCNP-77 $1393787.0+19.0+1$ Matsuoha+ TBL. FROM ANG. DIST. + OM

${ }^{24} \mathrm{Mg}\left({ }^{3} \mathrm{He}, 3 \mathrm{n}\right)^{24} \mathrm{Si}$ portlal product yleld

6196 MSU Expt P ANL-79-40 8079 NDG BO=I Es+ NDG. T=2 LEVEL.

$24 \mathrm{Mg}\left({ }^{3} \mathrm{He}, \mathrm{Zn}^{2}\right)^{25} \mathrm{SI}$ product yield

6197 BRK Revw J ARN $27 \quad 33377$ NDG Cerny+ TBL. PRODUCTION DELAYED-P PRECUR

${ }^{24} \mathrm{Mg}\left({ }^{3} \mathrm{He}, 2 \mathrm{n}\right)^{25} \mathrm{Si} \quad O(E)$

$6198 \mathrm{CLU}$ EXPt J NP/A $235 \quad 11742.1+14.3+1$ MOS + CURV. DETECTED DELAYED PROTONS. Expt 4 EXFORBO005.004 $0762.5+14.3+1$. 7 DATA POINTS.

6199 KFK Comp B PH-DAT $1510792.5+14.3+1$ Muenzel+ TBL. FROM EXFOR

${ }^{4} \mathrm{Mg}\left({ }^{3} \mathrm{He}, x\right) 1$ sotople distribution rolative o(E)

$6200 \mathrm{NRL}$ Expt J PR/C $141655768.0+1 \quad$ SIngh+ TBL. ALSO PREEO. 20 PROD(Z=8-13)

$25 \mathrm{Mg}\left({ }^{3} \mathrm{He}, a\right)^{24} \mathrm{Mg}$ partiol o(E)

6201 MIL Expt J NC $35 \quad 339769.0+01.3+1$ Notorrigo+ CURV. A(0)-A(5)GRPS. INTAD.

$26 \mathrm{Mg}\left({ }^{3} \mathrm{He}, x\right) i s o t o p l e$ distribution relative o(E)

6202 NRL Expt J PR/C $14165576 \mathrm{~B} .0+1 \quad$ Singht TBL. ALSO PREEQ. 19 PROD $(Z=8-14)$ Expt JPR/C $14 \quad 1655768.0+1 \quad$ Singh+ NOT OBS. PREEO. 8 PROD $(Z=9-14)$.

$27 \mathrm{Al}\left({ }^{3} \mathrm{He}, \mathrm{total}\right)$ O(E)

6203 1Jl Revo J FCY $6827752.9+1 \quad$ Nemets+ TBL. FROM PL/B 26.213(1968). ReVw J SJPN $6325762.9+1 \quad$. IRANSLATION OF FCY 6.14$) .827(1975)$.

${ }^{27} A 1\left({ }^{3} H 0, x\right)^{2} H \quad O(E)$

6204 OSA EXPt J NP/A 311173 N78 7.0+1 9.0+1 Matsuoka+ TBL. EST. FROM PEAK OF CONTIN

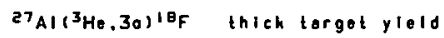

6205 UJV Comp R UJF-4199-B $2771.3+12.9+1$ Bem+NDG. BUTTERWORTHS, LONDON.341(1970)

$27 A !\left({ }^{3} \mathrm{He}, p+d\right)^{27} A l$ partlal o(E)d

6206 OSA EXPL P A-RCNP-77 $139787.0+19.0+1$ Matsuoka+ TBL. BREAKUP CONT.

$27 \mathrm{Al}\left({ }^{3} \mathrm{He}, \mathrm{d}\right)^{28} \mathrm{Si} \quad$ O(E)

6207 OSA Exth P A-RCNP-77 $1393787.0+19.0+1$ Malsuoka+ TBL. FROM ANG. DIST. + OM

$27_{A I}\left({ }^{3} \mathrm{He}_{0}, \mathrm{Zn}^{2}\right)^{2 \theta_{P}} \quad O(E)$

6208 CLU Expt J NP/A $235 \quad 11741.4+14.1+1$ Mos + CURV. DE TECTED DELAYED GAMMAS Expt 4 EXFORBO005.003 $0761.4+14.1+1$. B DATA POINTS.

6209 KFK COMP B PH-DAT 15 ! $0792.0+4$ Muenzel+ TBL. FROM EXFOR.

$27 A\left({ }^{3} \mathrm{He}, x\right) l$ sotopie distribution relative o(E)

6210 NRL Expt J PR/C 14 i655 $768.0+1 \quad$ Singh+ TRI. ALSO PREEO. 14 PROO $12=9-141$ Expt J FR/C $14 \quad 1655768.0+1 \quad$ Singh+NOT OBS.ALSO PREEO.11 PROD $1 Z=8-13$ 
REFERENCES ( c ont)

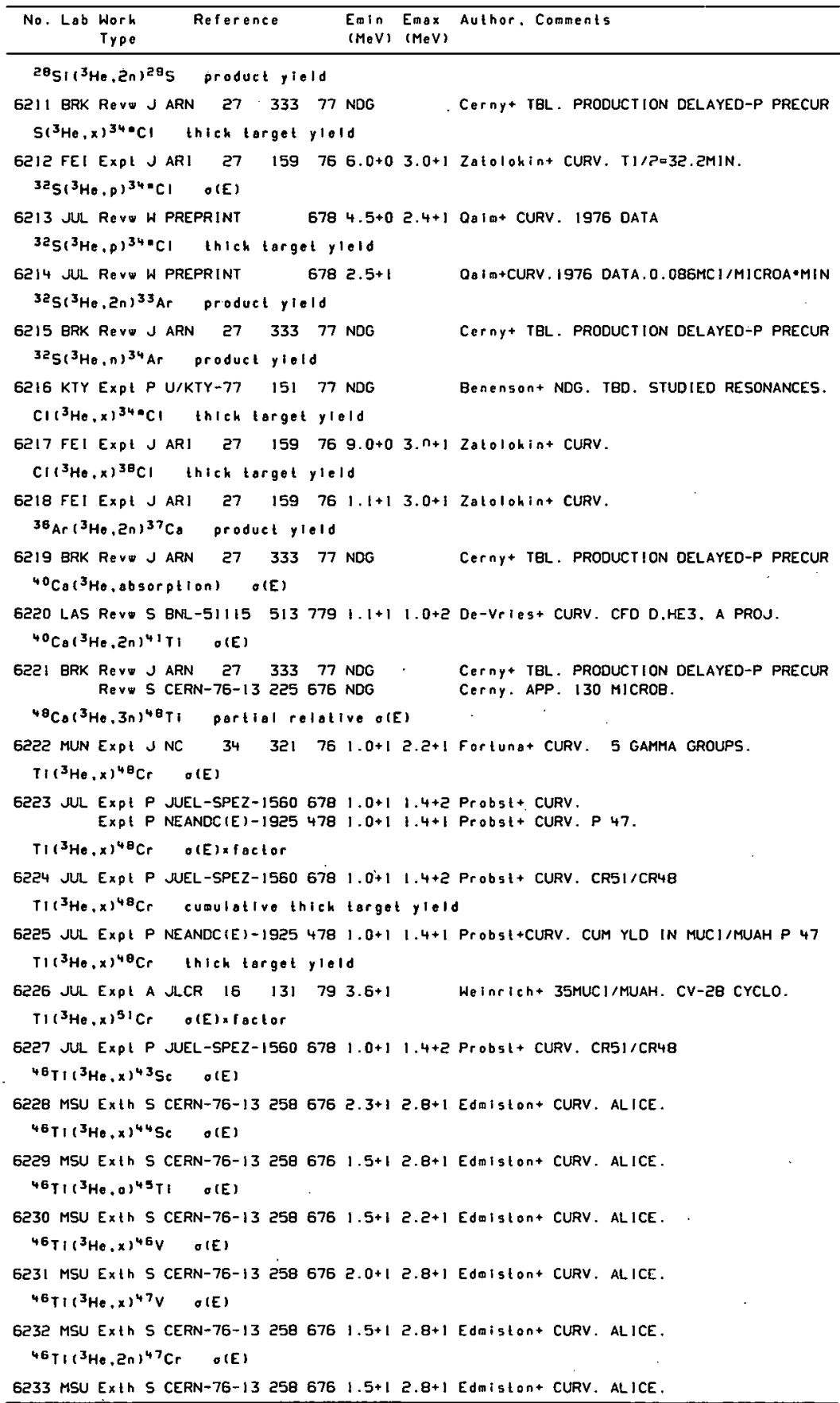


REFERENCES ( c ont

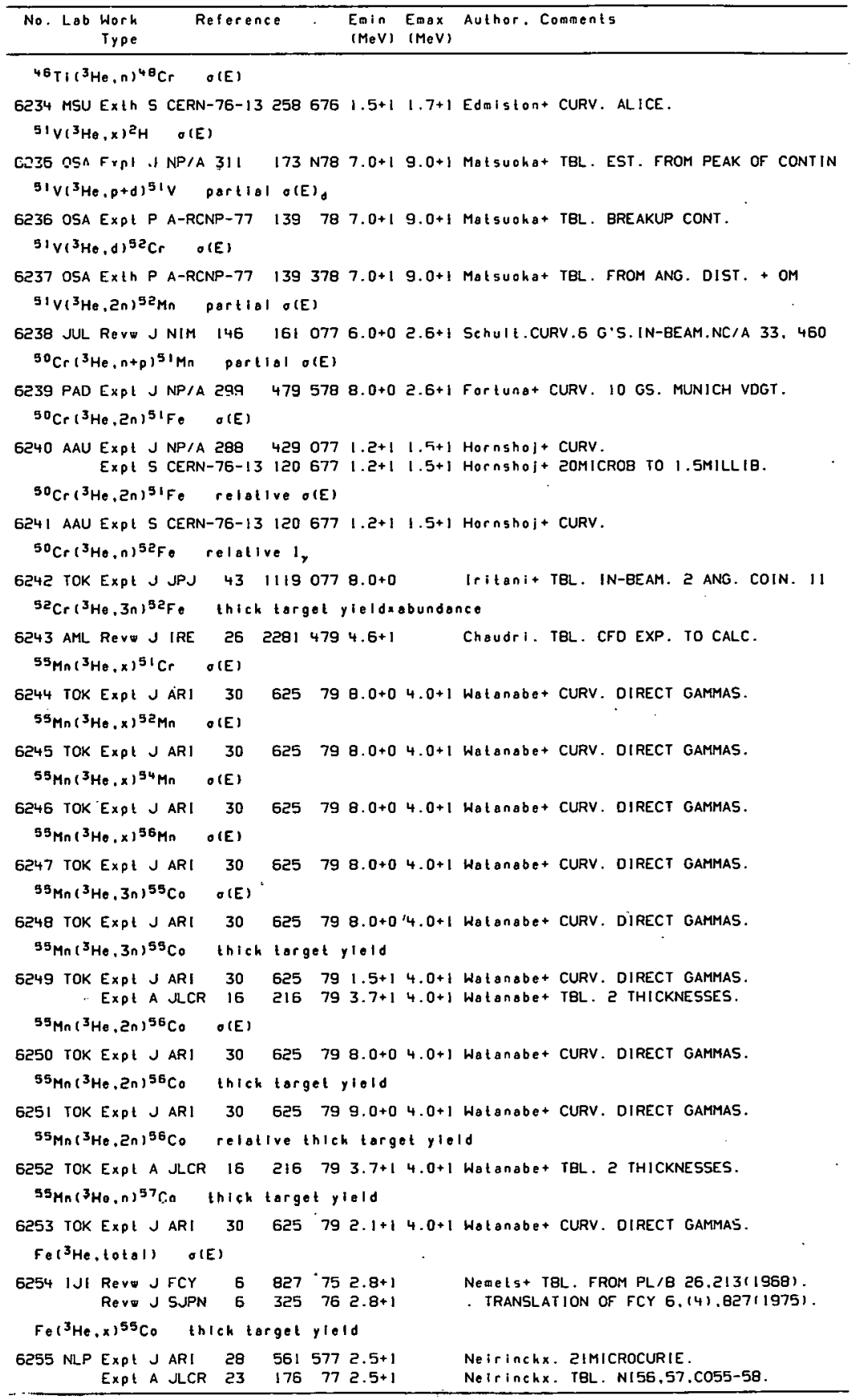


REFERENCES (cont)

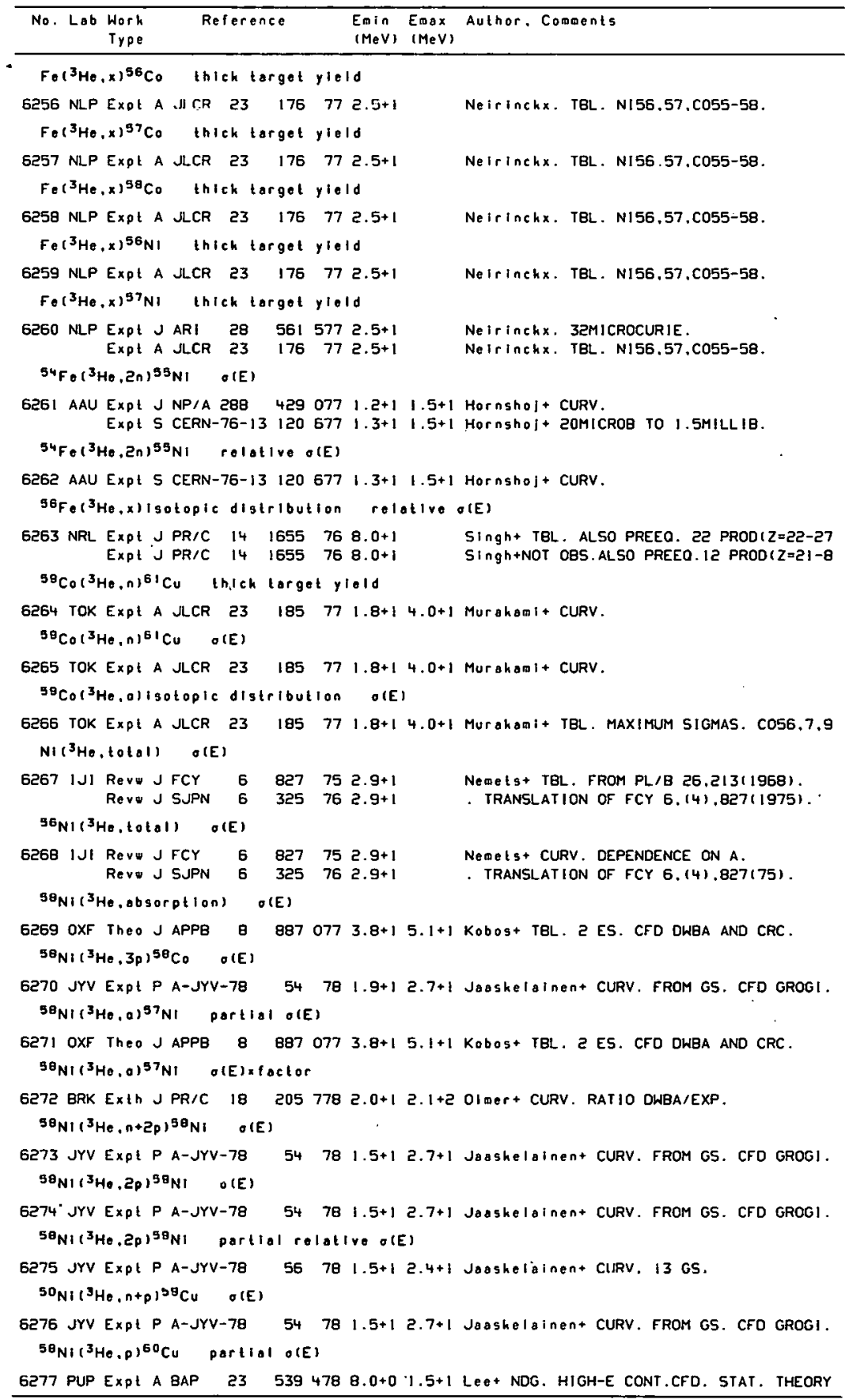


REFERENCES (cont)

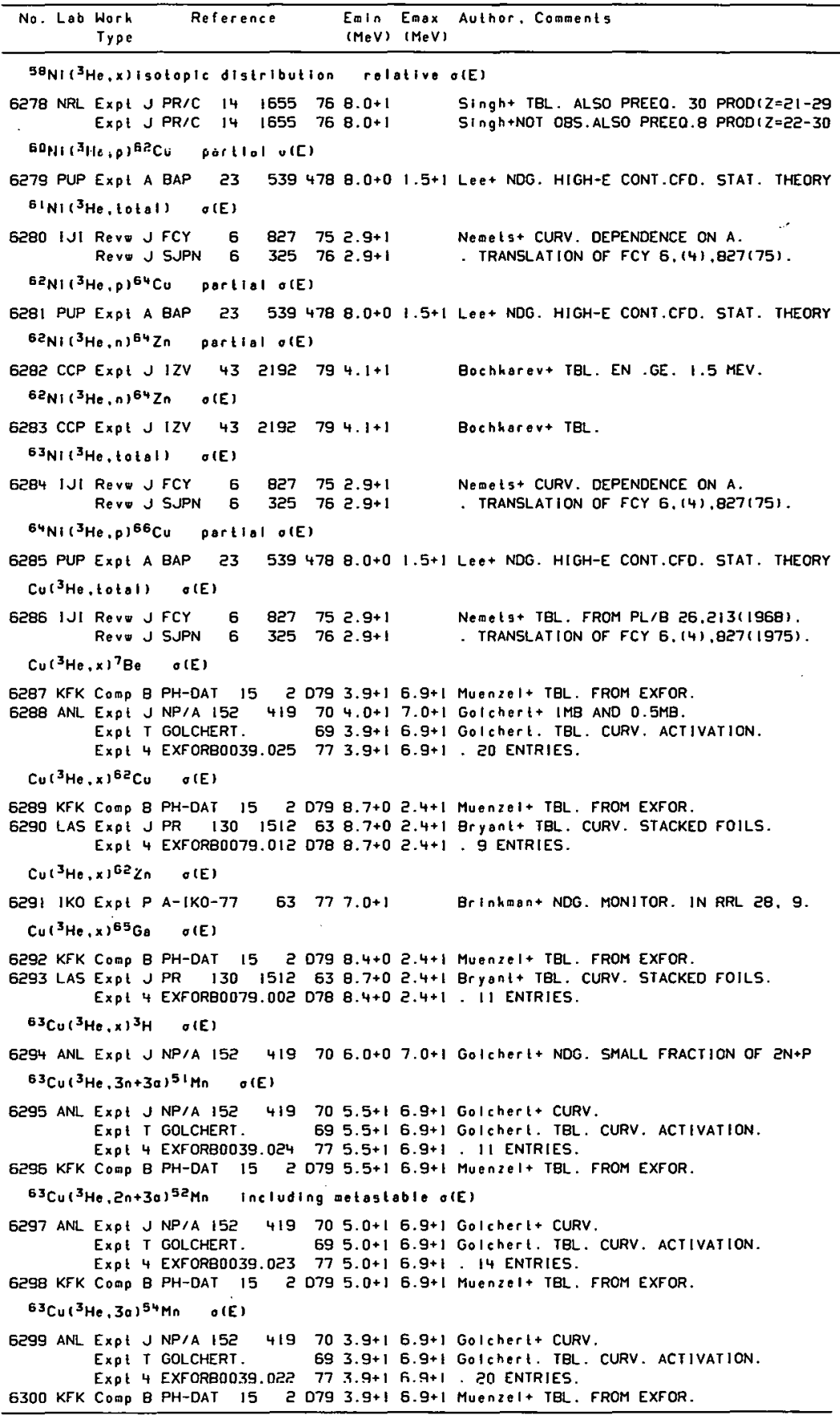




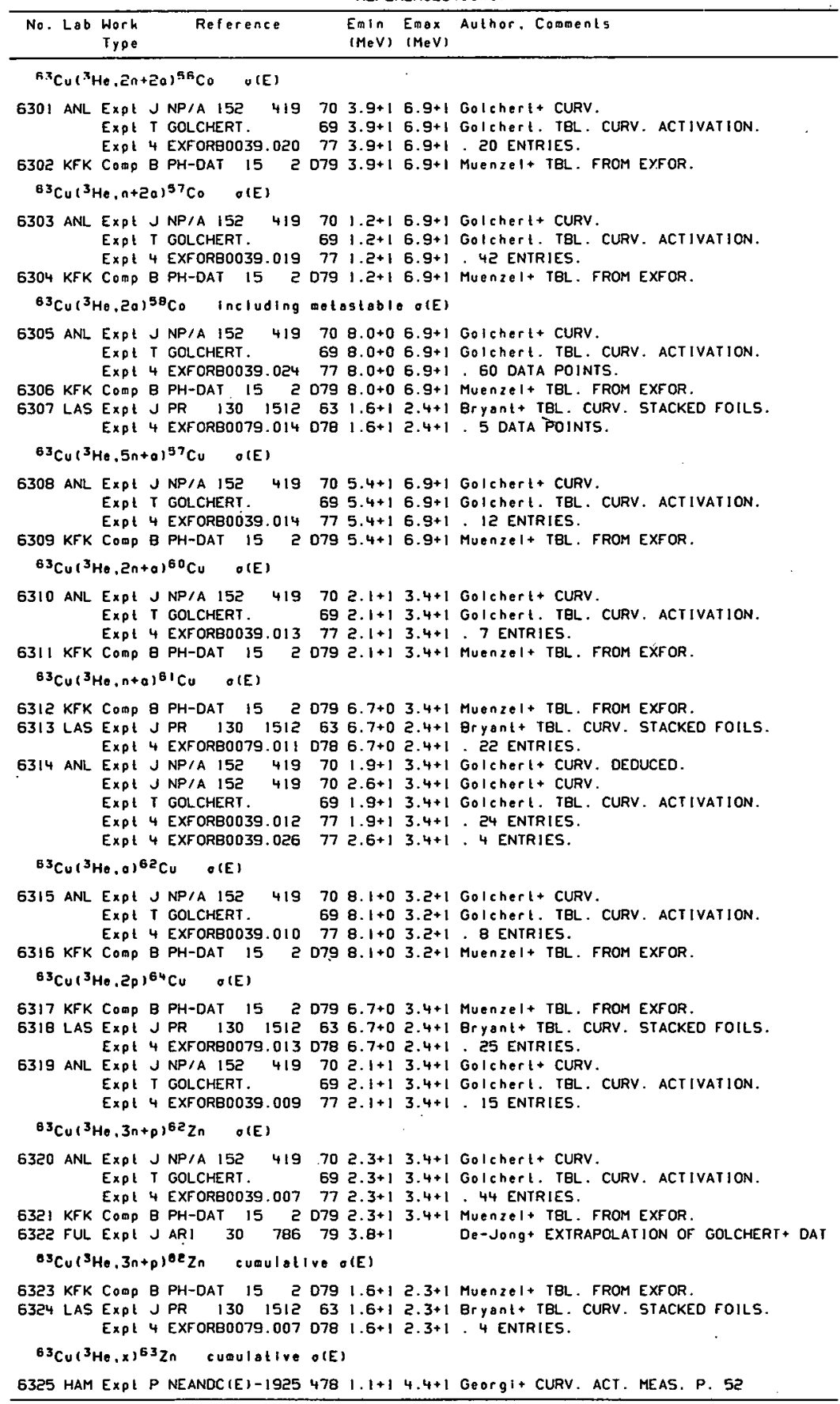




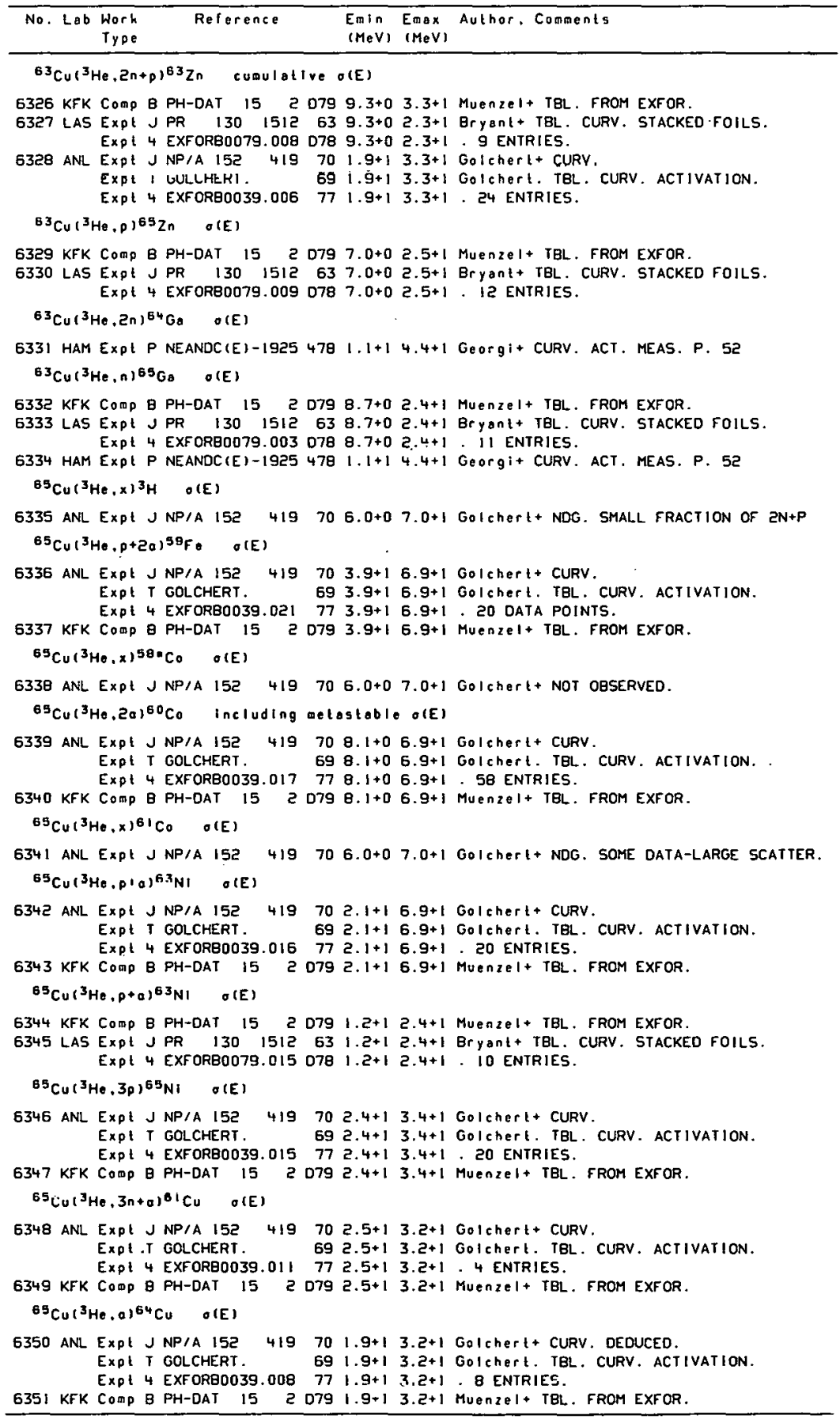


REFERENCES (con ()

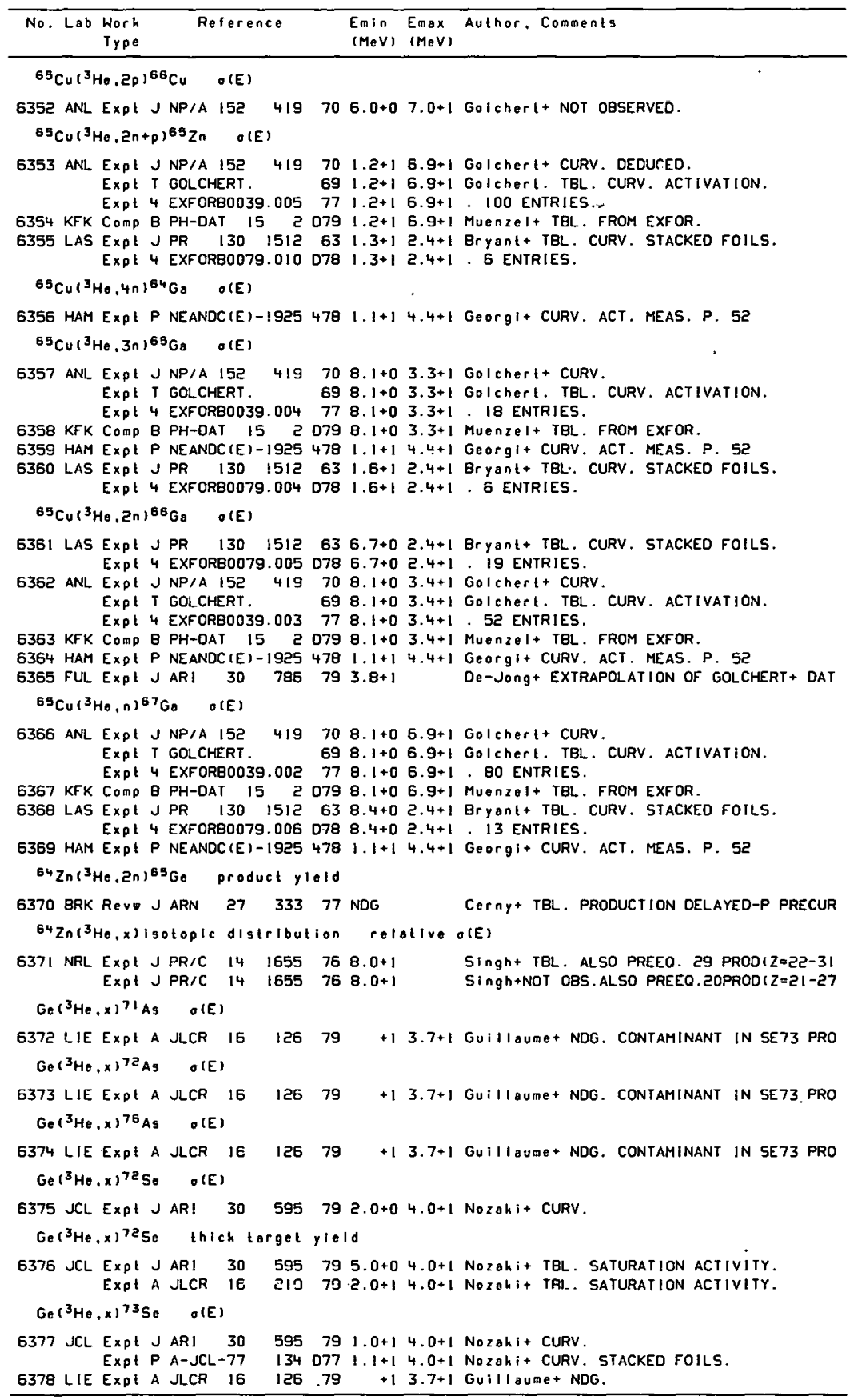




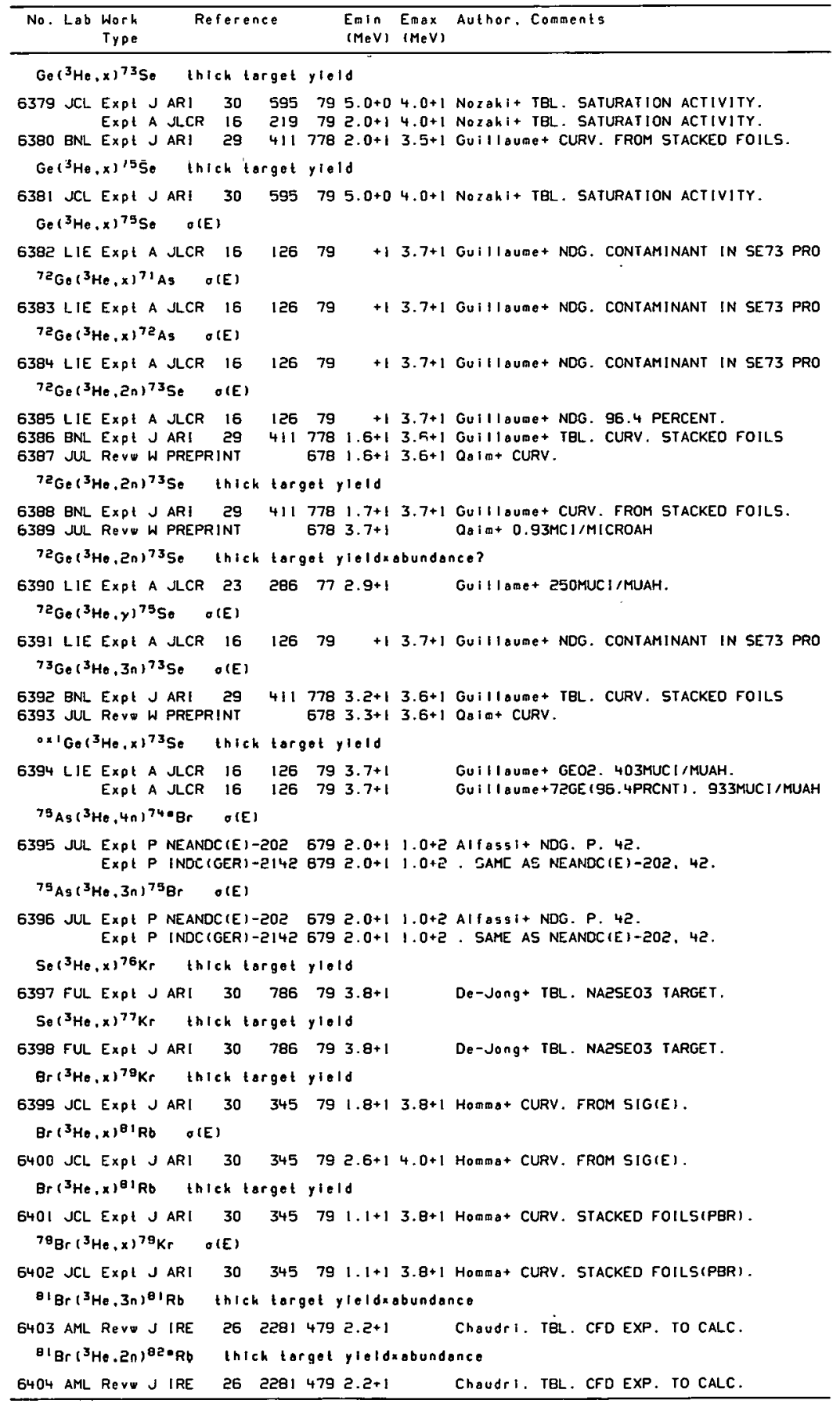


REFERENCES (c ont)

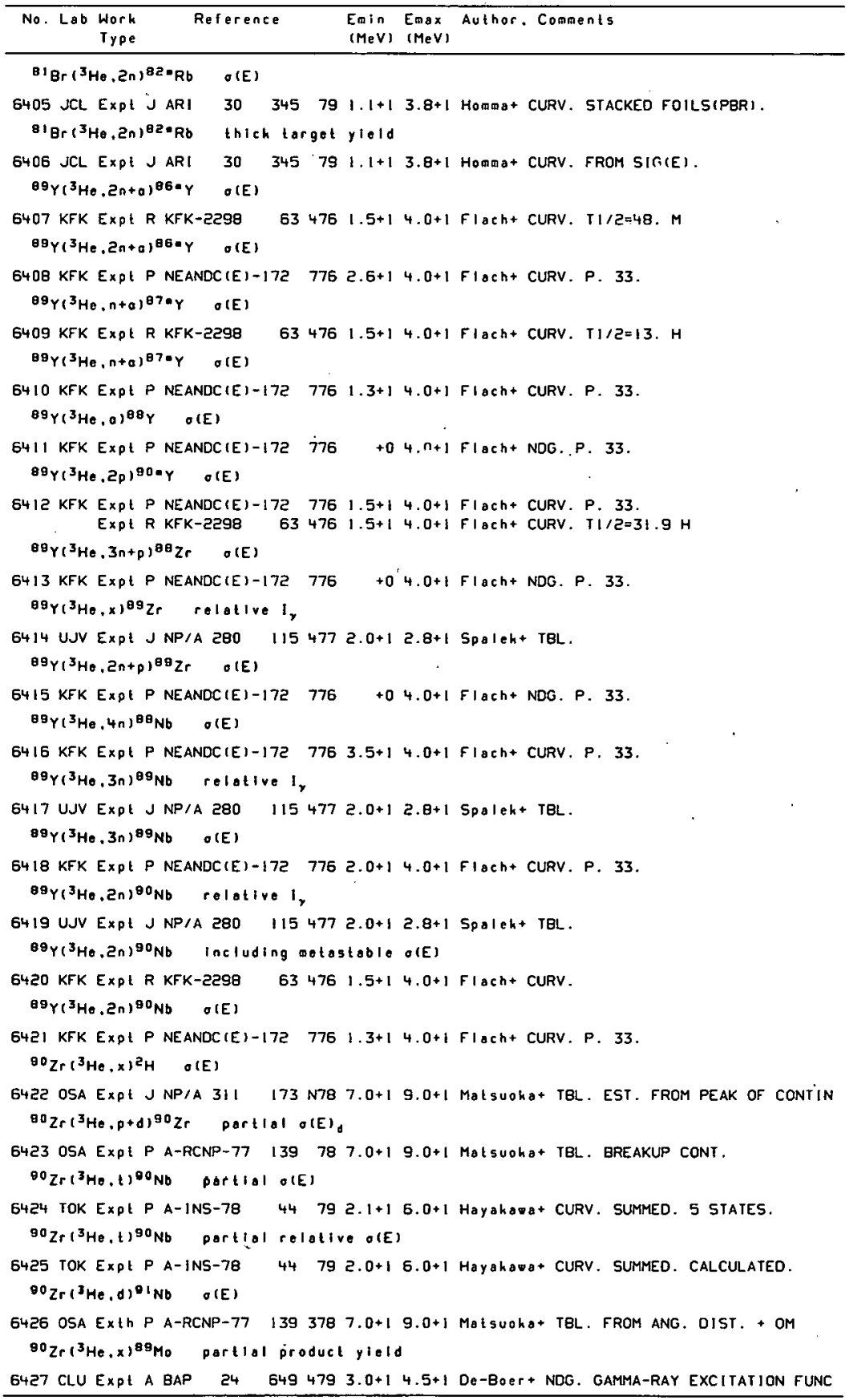


REFERENCES $(\operatorname{con}$ o $)$

\begin{tabular}{|c|c|c|c|c|c|}
\hline No, Lab & $\begin{array}{l}\text { Work } \\
\text { Type }\end{array}$ & Reference & $\begin{array}{l}\text { Emin } \\
(M e V)\end{array}$ & $\begin{array}{l}\text { Emax } \\
(\operatorname{MeV})\end{array}$ & Author. Comments \\
\hline
\end{tabular}

$90 \mathrm{Zr}\left({ }^{3} \mathrm{He}, 3 \mathrm{n}\right)^{90} \mathrm{Mo}$ parllal product yield

6428 CLU Expt A BAP $24 \quad 6494793.0+14.5+1$ De-Boer+ NDG. GAMMA-RAY EXCITATION FUNC $91 \mathrm{Zr}\left({ }^{3} \mathrm{He}, t\right)^{91} \mathrm{Nb} \quad O(E) \times f$ actor

6429 LAS Expl P ERDA-NDC-9 $1084777.0+02.1+1$ Britt+ CURV. EXC. E GIVEN. (N.P) SIM.

${ }^{93} \mathrm{Nb}\left({ }^{3} \mathrm{He}, 2 \mathrm{Cn}+\mathrm{a}\right)^{90} \mathrm{Nb} \quad 0(\mathrm{E})$

6430 KFK Expl P NEANDC (E)-172 776 +0 4.0+1 Flach+NDG. P. 33.

$93^{N b}\left({ }^{3} H_{0}, 2 n+p\right) 93_{M 0} \quad O(E)$

6431 KF́K EXPl P NEANDC(E)-172 776 t0 4.0+1 Flach+ NDG. P. 33.

$9{ }^{3} \mathrm{Nb}\left({ }^{3} \mathrm{He}, 4 \mathrm{n}\right){ }^{92} \mathrm{TC}$ O(E)

6432 KFK Exp! P NEANDC(E)-172 776 +0 4.0+1 Flach+ NDG. P. 33.

6433 HAM ExpI P NEANDC (E)-1925 $4781.1+14.4+1$ Georgi+ CURV. ACT. MEAS. P. 52

$93^{3} \mathrm{~N}\left({ }^{3} \mathrm{He}, 3 \mathrm{n}\right)^{93} \mathrm{TC} \quad \sigma(E)$

6434 KFK Expt P NEANDC(E)-172 776 +0 4.0+1 F lach+ NDG. P. 33.

6435 HAM Expt P NEANDC(E)-1925 $4781.1+14.4+1$ Georgi+ CURV. ACT. MEAS. P. 52

$9{ }^{3} \mathrm{Nb}\left({ }^{3} \mathrm{He}, \mathrm{2n}\right)^{94} \mathrm{TC} \quad \mathrm{O}(\mathrm{E})$

6436 KFK Expt P NEANDC (E)-172 776 +0 $4.0+1$ F I ach+ NDG. P. 33.

6437 HAM Expt P NEANDC (E)-1925 $4781.1+14.4+1$ Georgi+ CURV. ACT. MEAS. P. 52

$93^{\mathrm{Nb}}\left({ }^{3} \mathrm{He}, \mathrm{n}\right) 95 \mathrm{TC} \quad \mathrm{O}(\mathrm{E})$

5438 HAM Expt P NEANDC $(E)-19254781.1+14.4+1$ Georgi+ CURV. ACT. MEAS. P. 52

Mo $\left({ }^{3} \mathrm{HE}, x\right)^{97} \mathrm{Ru}$ product yield

$6439 \mathrm{JUL}$ REvw W PREPRINT $6781.8+13.0+1$ 0aim+ CURV.

$104 \mathrm{Pd}\left({ }^{3} \mathrm{He}, 3 n\right)^{104} \mathrm{Cd}$ partial o(E)

$6440 \mathrm{JCL}$ Expt C 77TOKYO $3359772.6+14.6+1$ Hashizume+ NDG

$A g\left({ }^{3} \mathrm{He}, \mathrm{total}\right) \quad \sigma(E)$

6441 lJl RerQ J FCY $6827752.9+1 \quad$ Nemets + TBL. FROM PL/B 26.213(1968).

Revw S SJPN $6325762.9+1$. TRANSLATION OF FCY 6.14),827(1975).

$106 \mathrm{Cd}\left(3^{3} \mathrm{He}, x\right)^{105} \mathrm{In}$ rov o(E)

$6442 \mathrm{KAZ}$ Expt J ZEP 22 120 $753.2+15.9+1$ Burminshi 3 + CURV. 64XCDI06. DELAYED G'S Expt J JEL $22 \quad 54753.2+15.9+1$.TRANSLATION OF ZEP $22,120(1975)$

$\left.106 \mathrm{Cd} i^{3} \mathrm{H}_{0}, x\right)^{106}[\mathrm{n}$ partial row o(E)

$6443 \mathrm{KAZ}$ Expt J ZEP 22 120 $752.1+15.9+1$ BuraInshil+ CURV. 64XCDI06. OELAYED G'S Expt J JEL $22 \quad 54752.1+15.9+1$. TRANSLATION OF ZEP $22,120(1975)$

$106 \mathrm{Cd}\left({ }^{3} \mathrm{He}, x\right)^{107} \operatorname{In} \quad$ row o(E)

6444 KAZ Expt J ZEP 22 120 $752.1+15.9+1$ Burminshil+ CURV. 64XCDI06. DELAYED G'S Expl J JEL $22 \quad 54752.1+15.9+1$. TRANSLATION OF ZEP 22.120119751

${ }^{106} \mathrm{Cd}\left({ }^{3} \mathrm{He}, x\right)^{107 \cdot I n \quad \operatorname{rag} O(E)}$

$6445 \mathrm{KAZ}$ Expt 3 ZEP 2己. $120752.1+14.5+1$ Burminskit+ CURV. 64XCD106. DELAYED G'S EXPt J JEL 22 $54752.1+14.5+1$. TRANSLATION OF ZEP 22.120(1975)

$105 \mathrm{Cd}\left(3_{\mathrm{He}} .3 n\right)^{108} \mathrm{Sn} \quad \mathrm{raw}$ o(E)

6446 KAZ Expt J ZEP 22 I20 $752.1+15.9+1$ Gurminshi i+ CURV. 64xCDIO6. DELAYED G'S Expt J JEL $22 \quad 54752.1+15.9+1$. TRANSLATION OF ZEP 22.12011975!

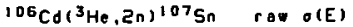

$6447 \mathrm{KAZ}$ Expl J ZEP $22 \quad 120752.1+14.7+1$ Burainsh11+ CURV. B4zCD10G. DCLAYED G'S Expt $J \mathrm{JEL} 2254752.1+14.7+1$. IRANSLATION OF ZEP 22.120(1975)

$117 \mathrm{Sn}\left({ }^{3} \mathrm{He}, 3_{\mathrm{n}}\right) 117 \mathrm{Te}$ partlal product yleld

644 R ROS Expl J NP/A 329157079 NDG Hagemannt NDG.

$118 \mathrm{Sn}\left({ }^{3} \mathrm{He}, x\right)^{2} \mathrm{H}$ O(E)

6449 OSA Expt J NPIA 31 1 173 N78 $7.0+19.0+1$ Matsuoha+ TBL. EST. FROM PEAK OF CONTIN

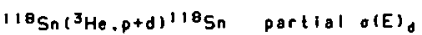

6450 OSA Expt P A-RCNP-77 $139787.0+19.0+1$ Matsuohat TBL. BREAKUP CONT. 


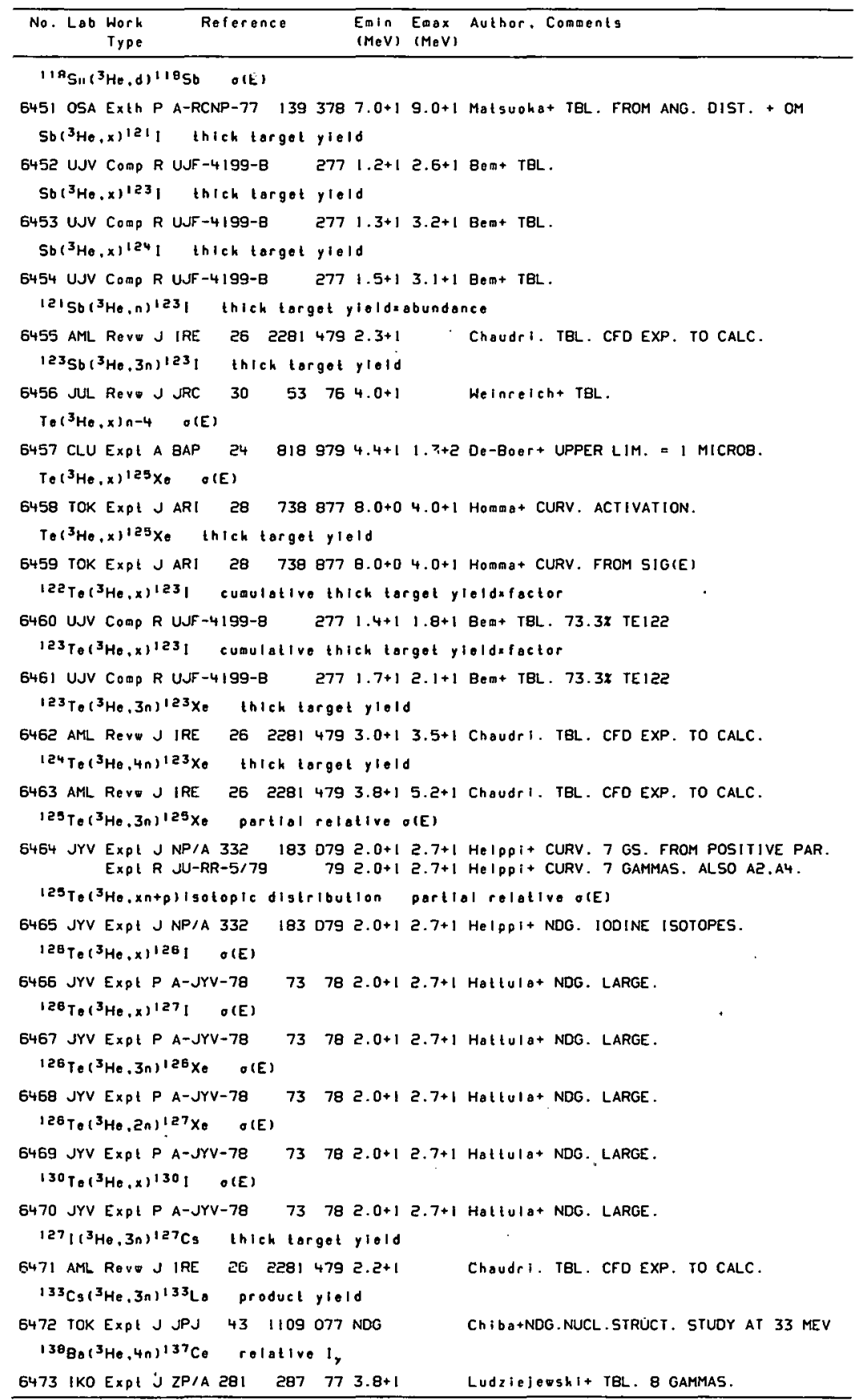


REFERENCES (cont)

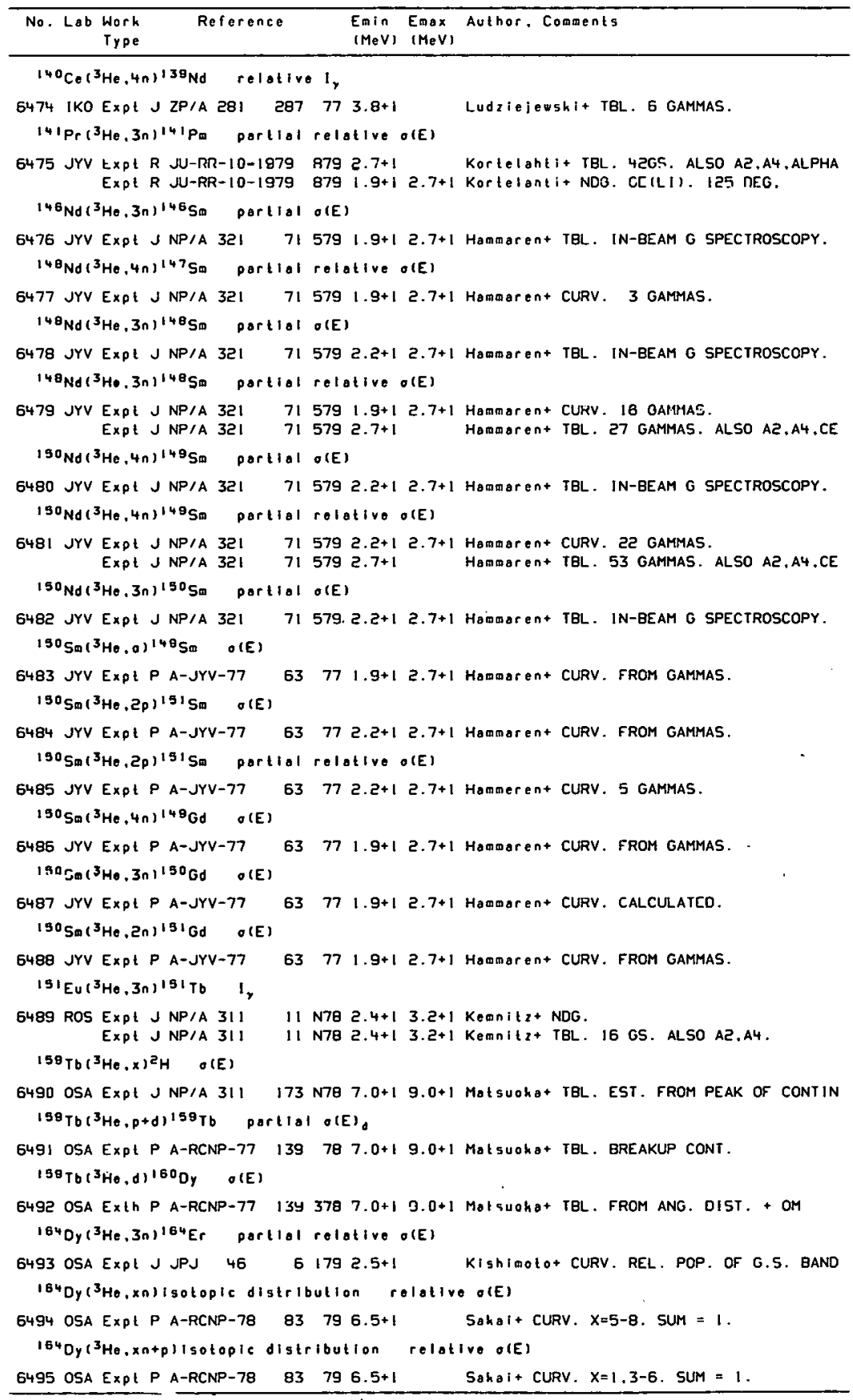


REFERENCES ( $c$ on $t$ )

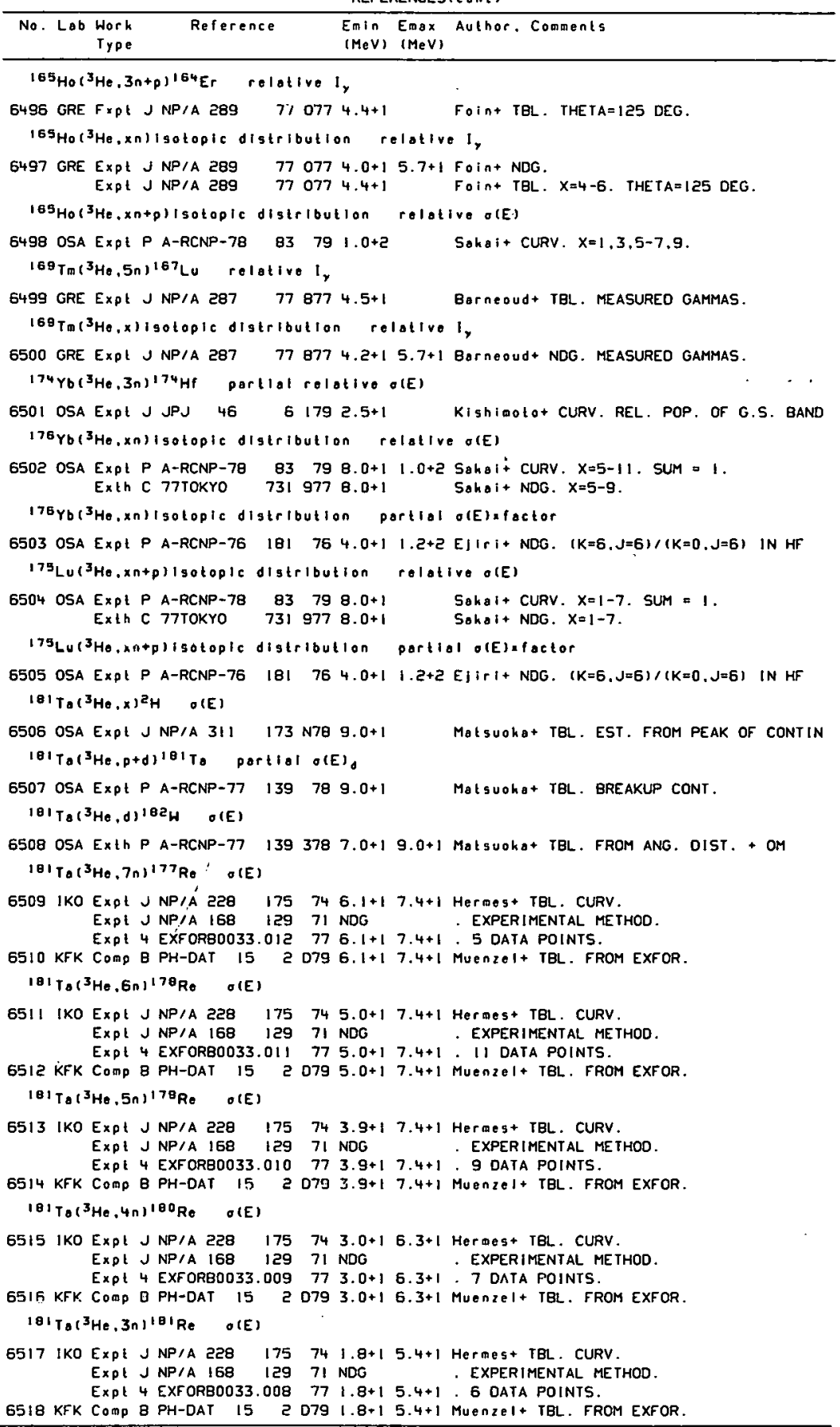




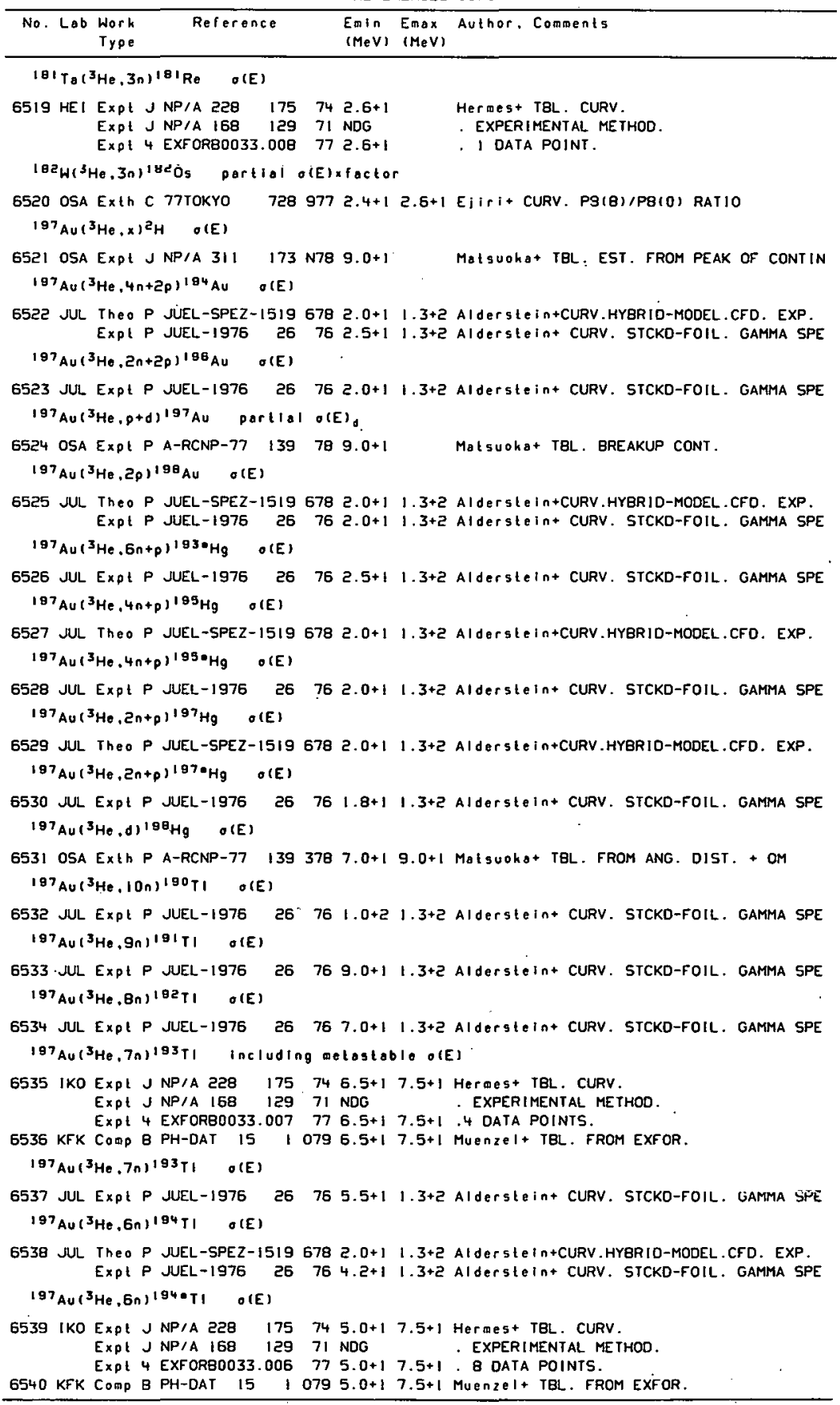


REFERENCES ( cont )

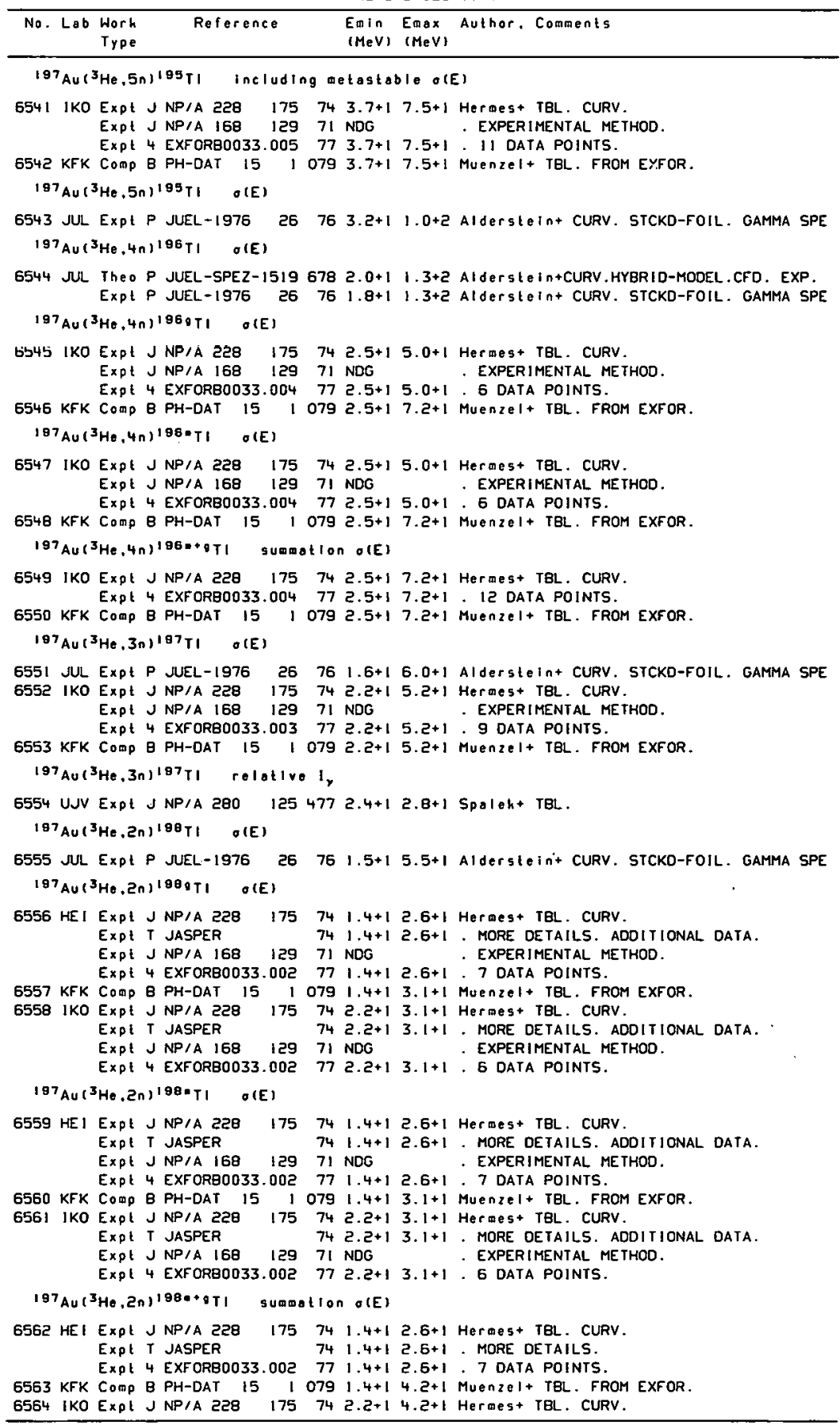


REFERENCES ( $($ on $t)$

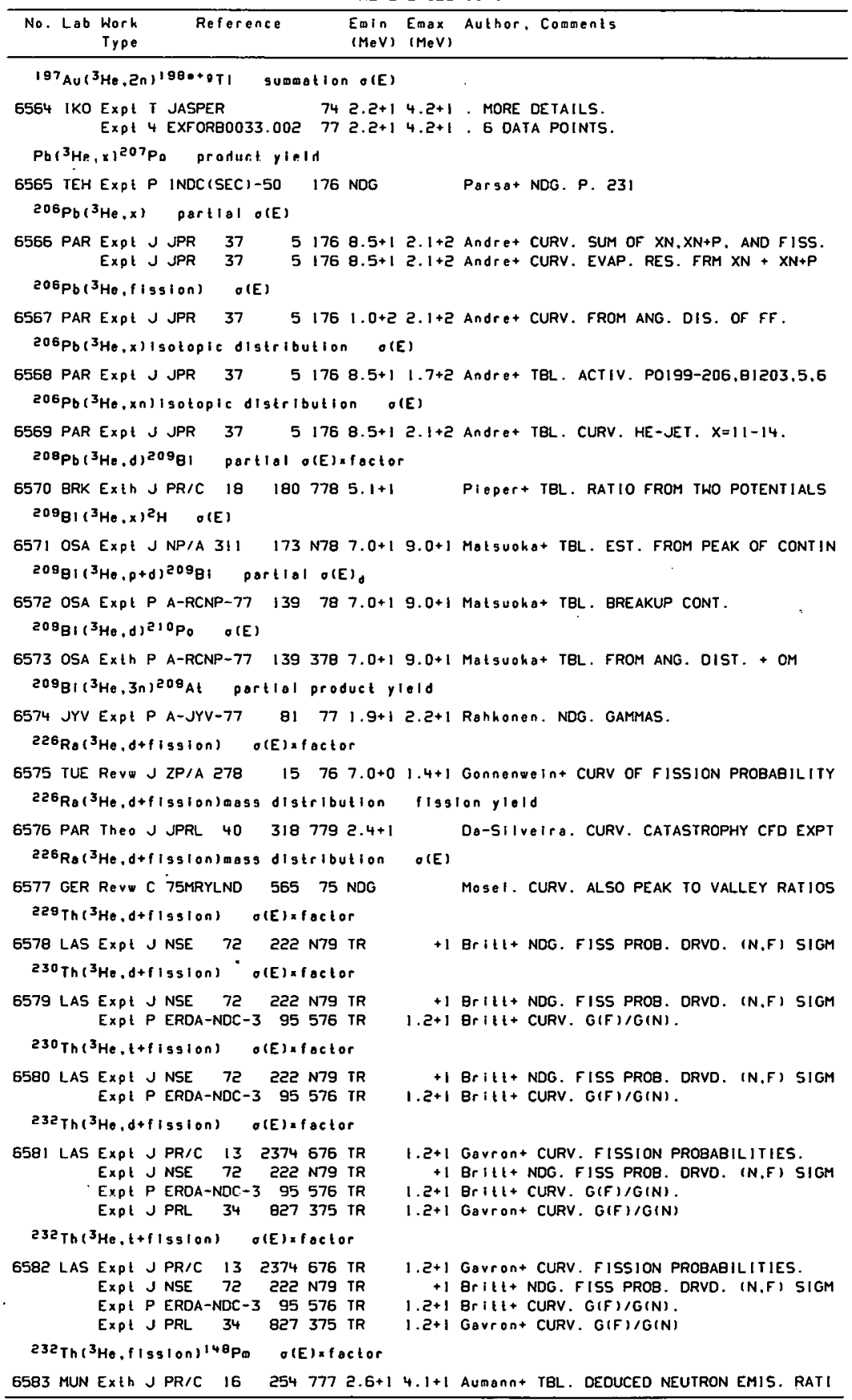


REFERENCES (cont)

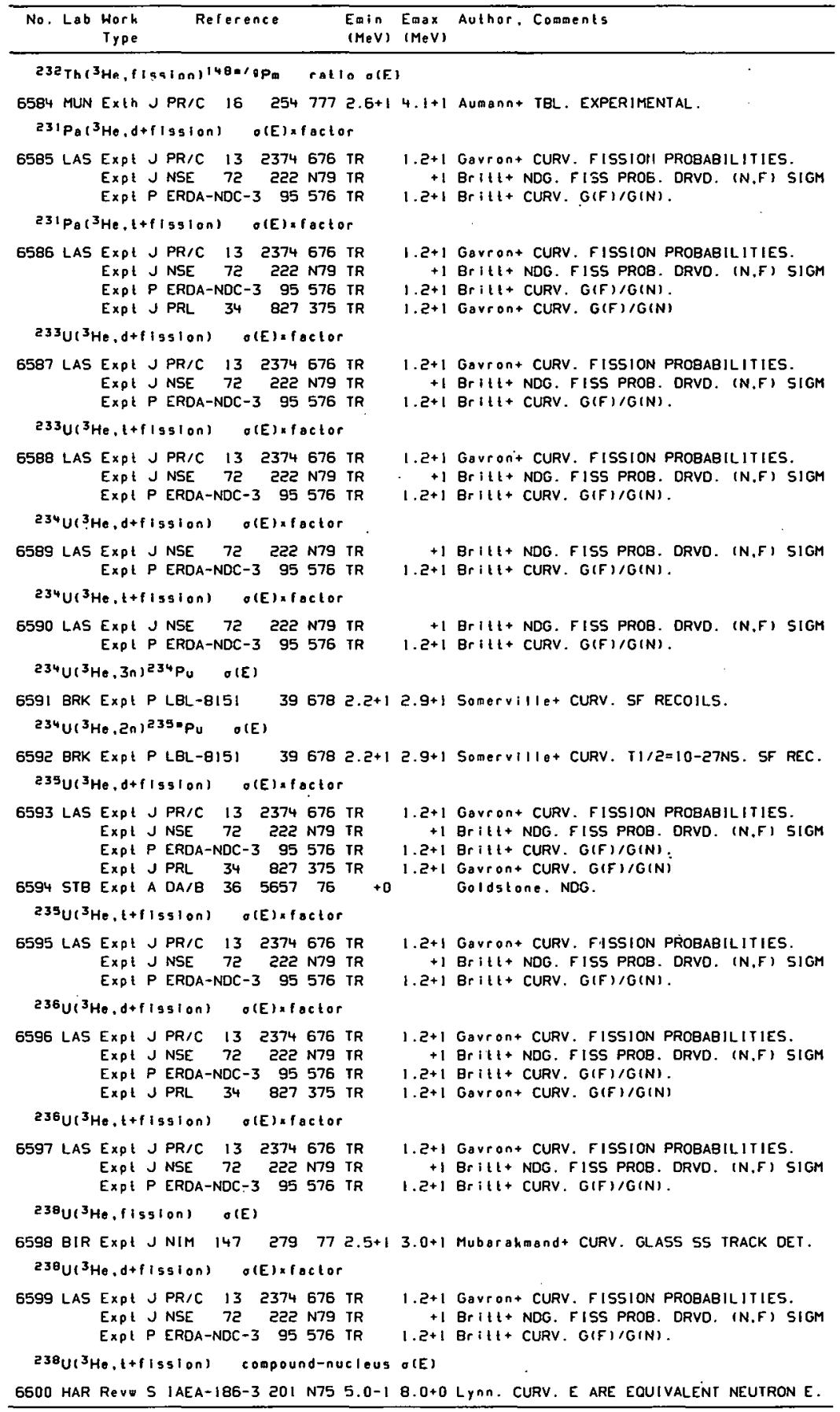


REFERENCES (cont )

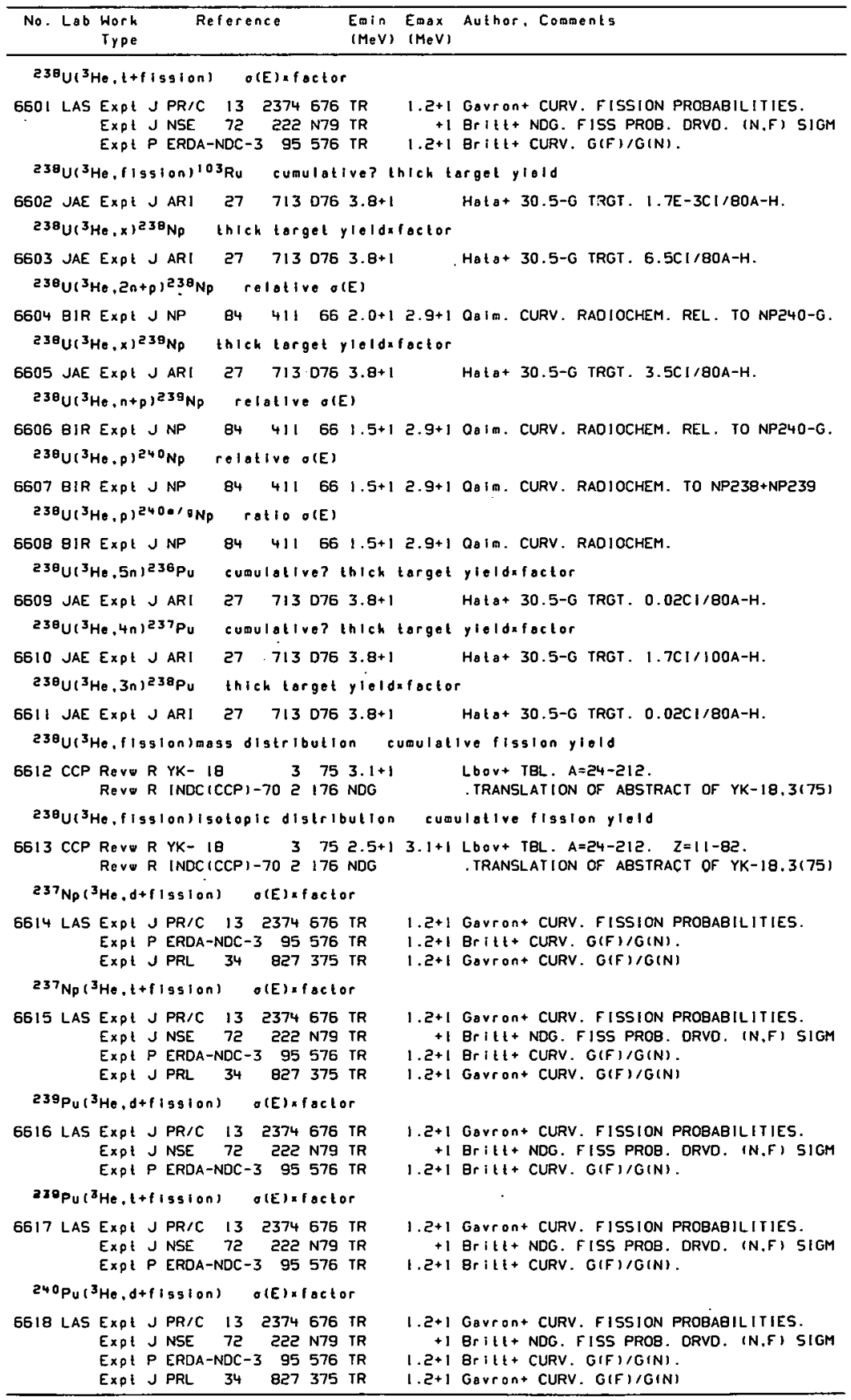


REFERENCES (cont)

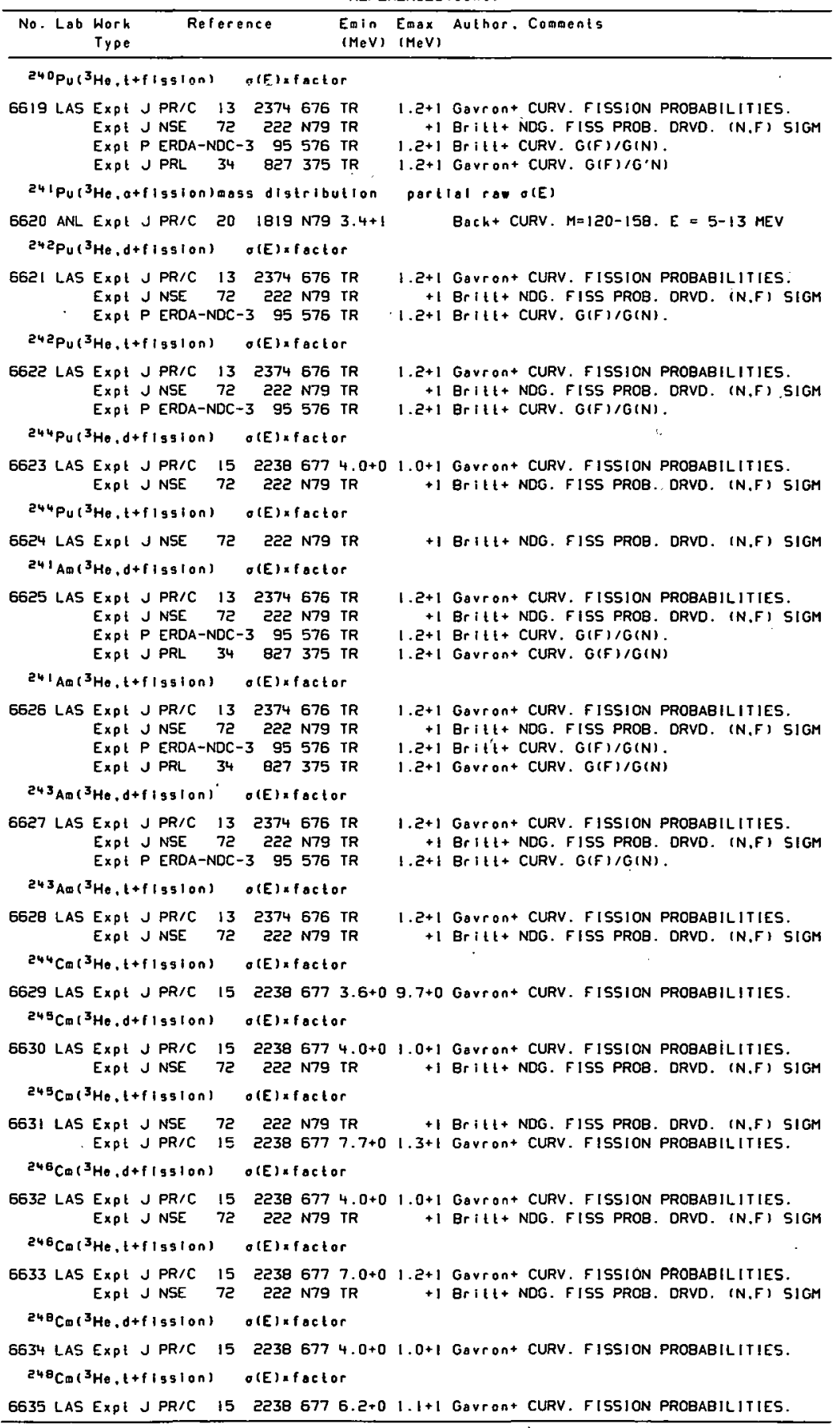


REFERENCES (cont)

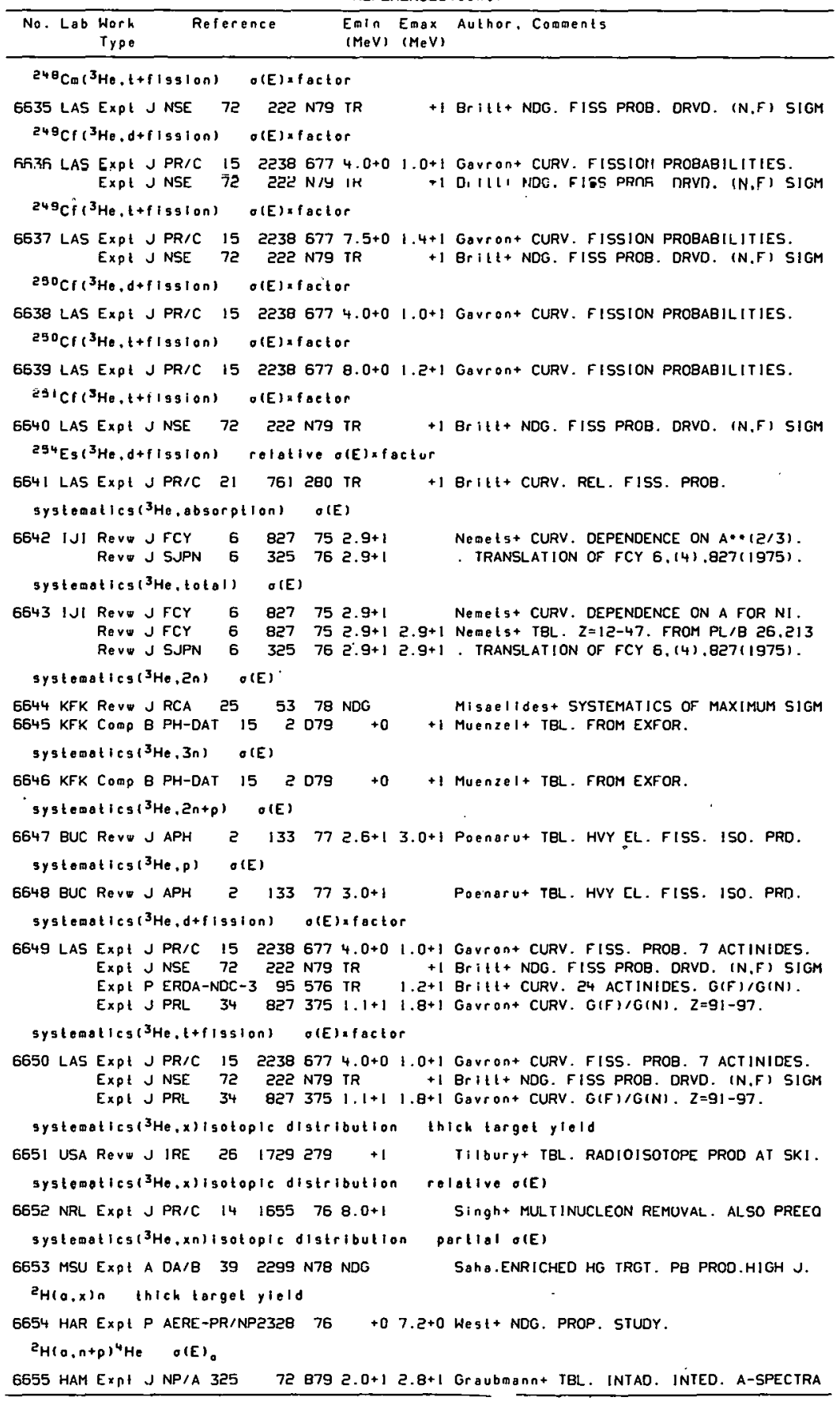




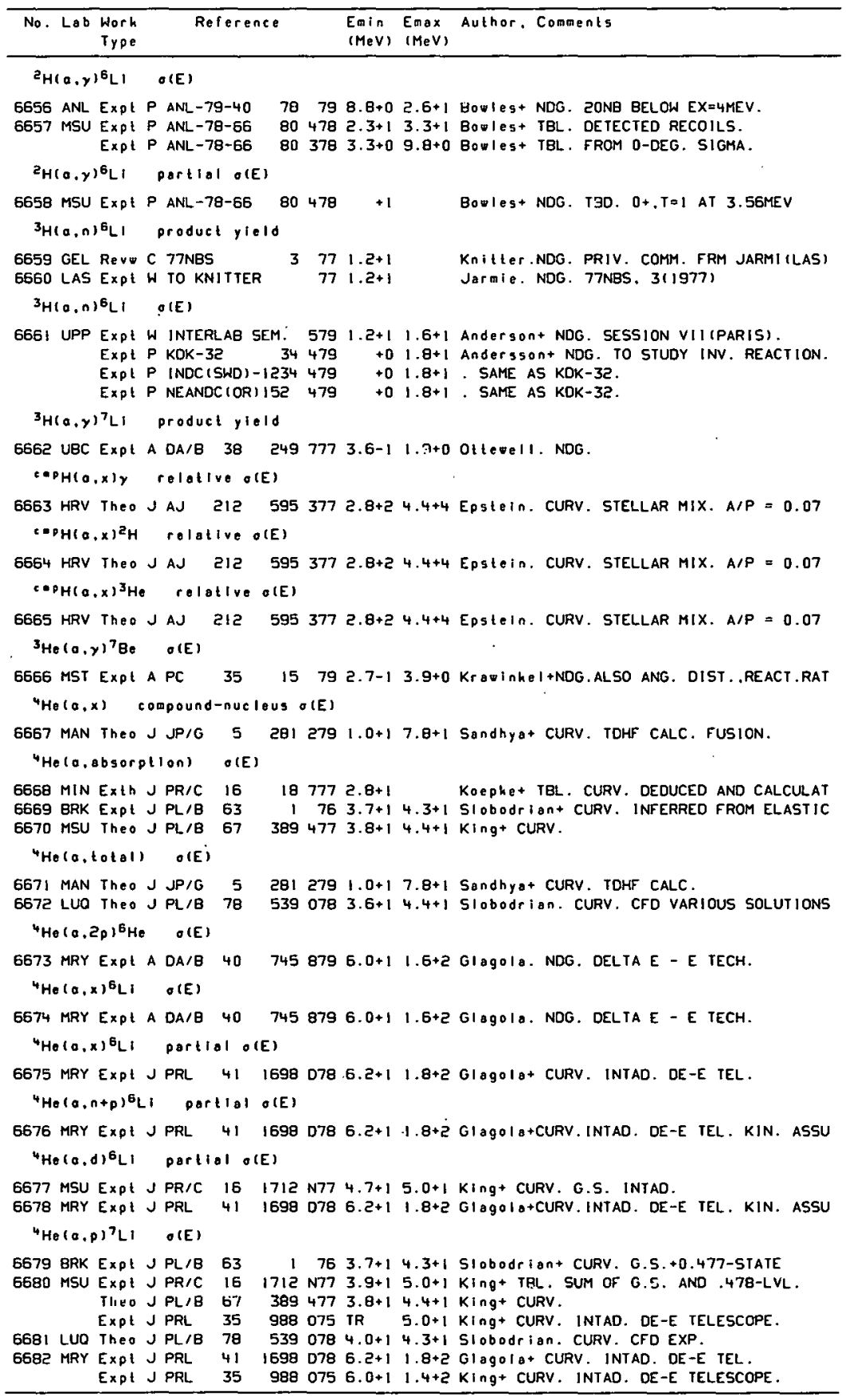


REFERENCES (c on t)

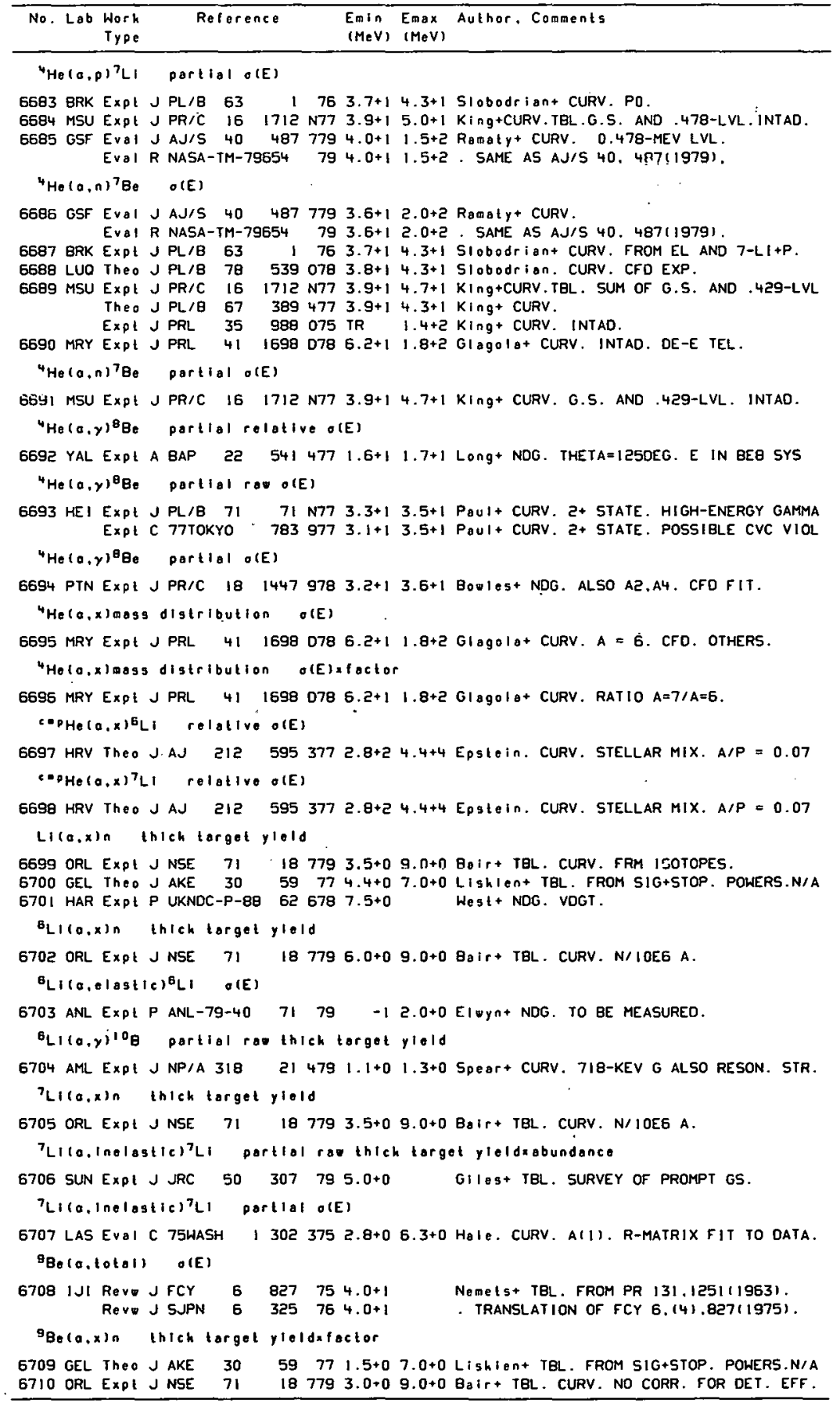


REFERENCES $(\operatorname{ton} t)$

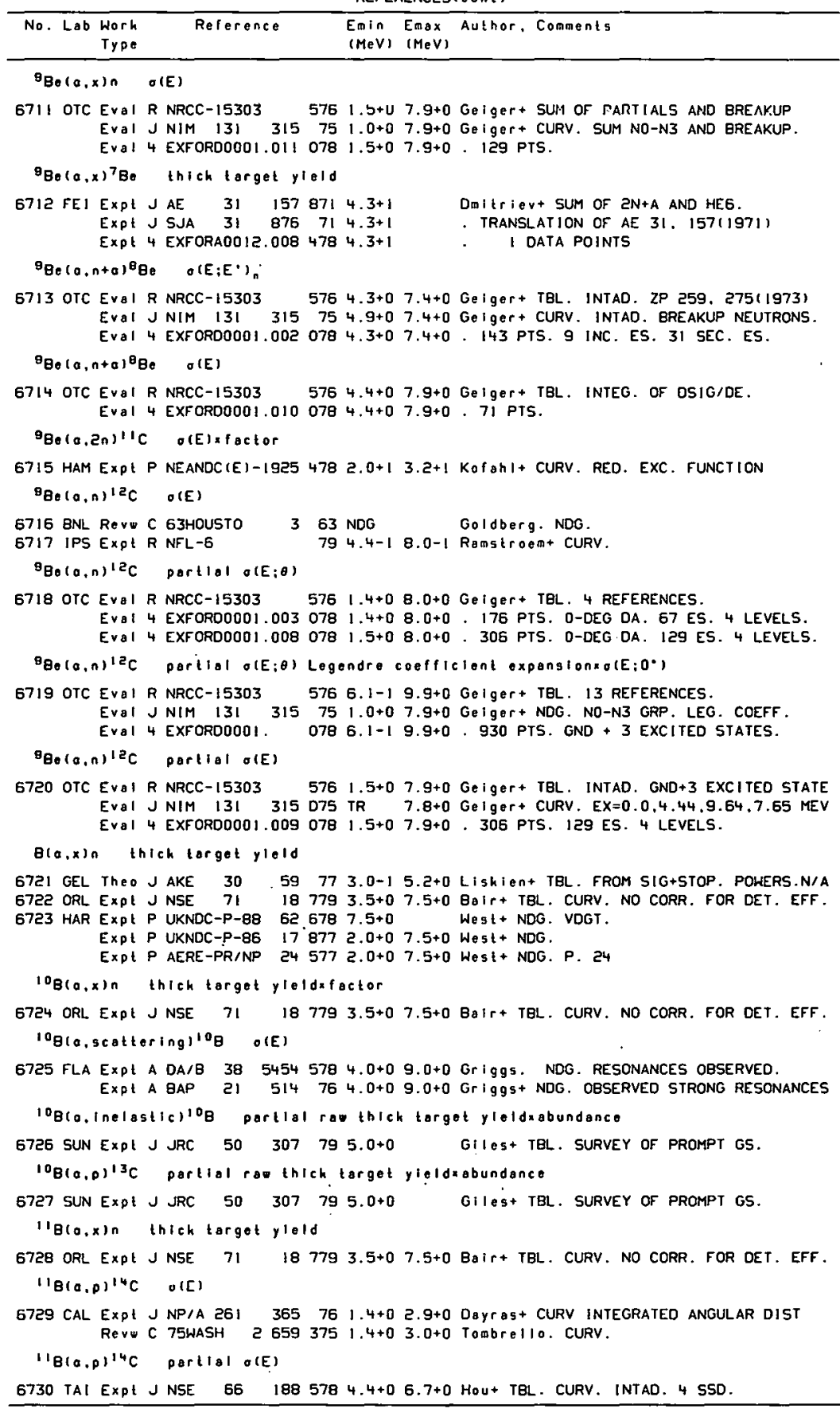


REFERENCES (cont)

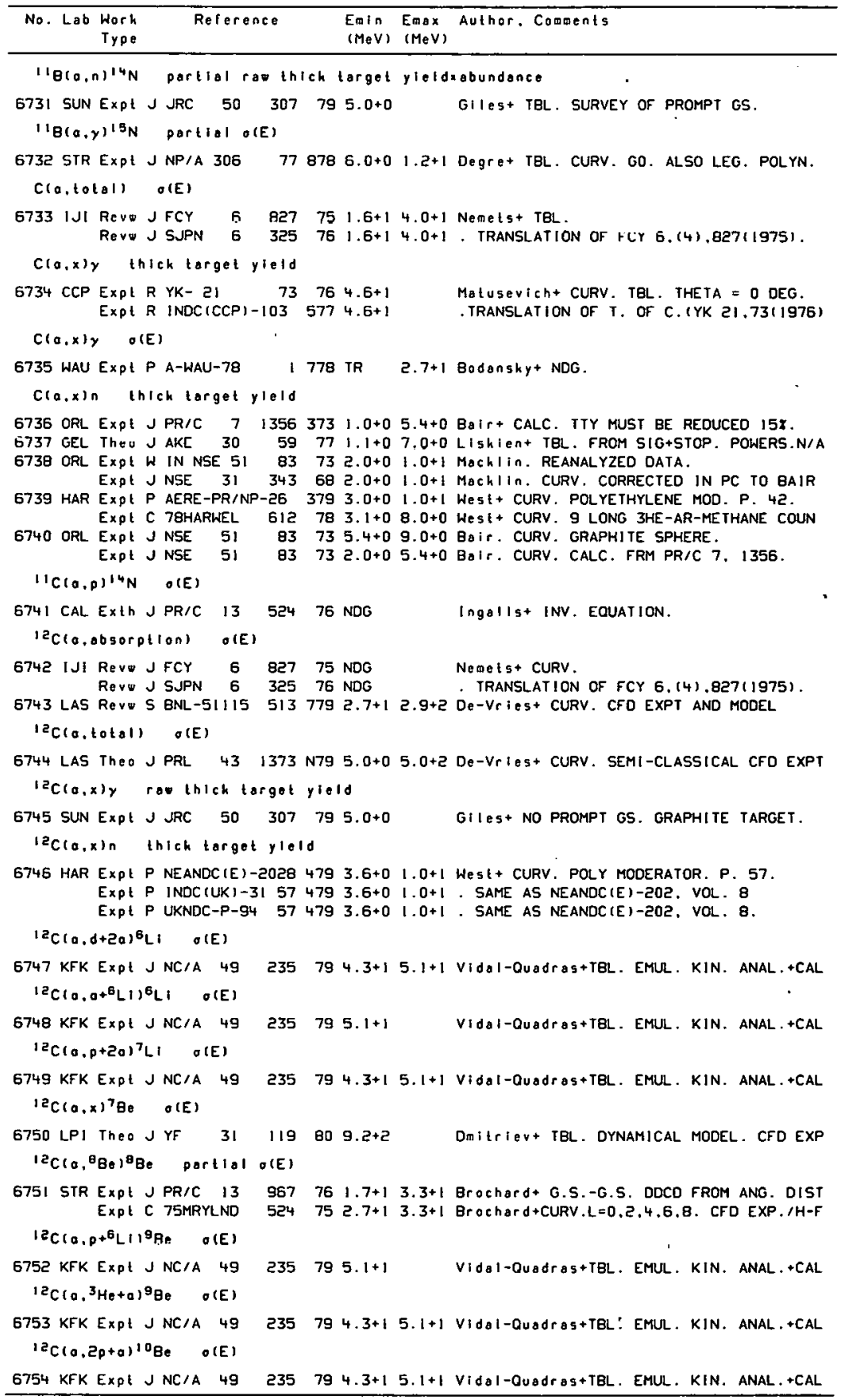


REFERENCES ( cont)

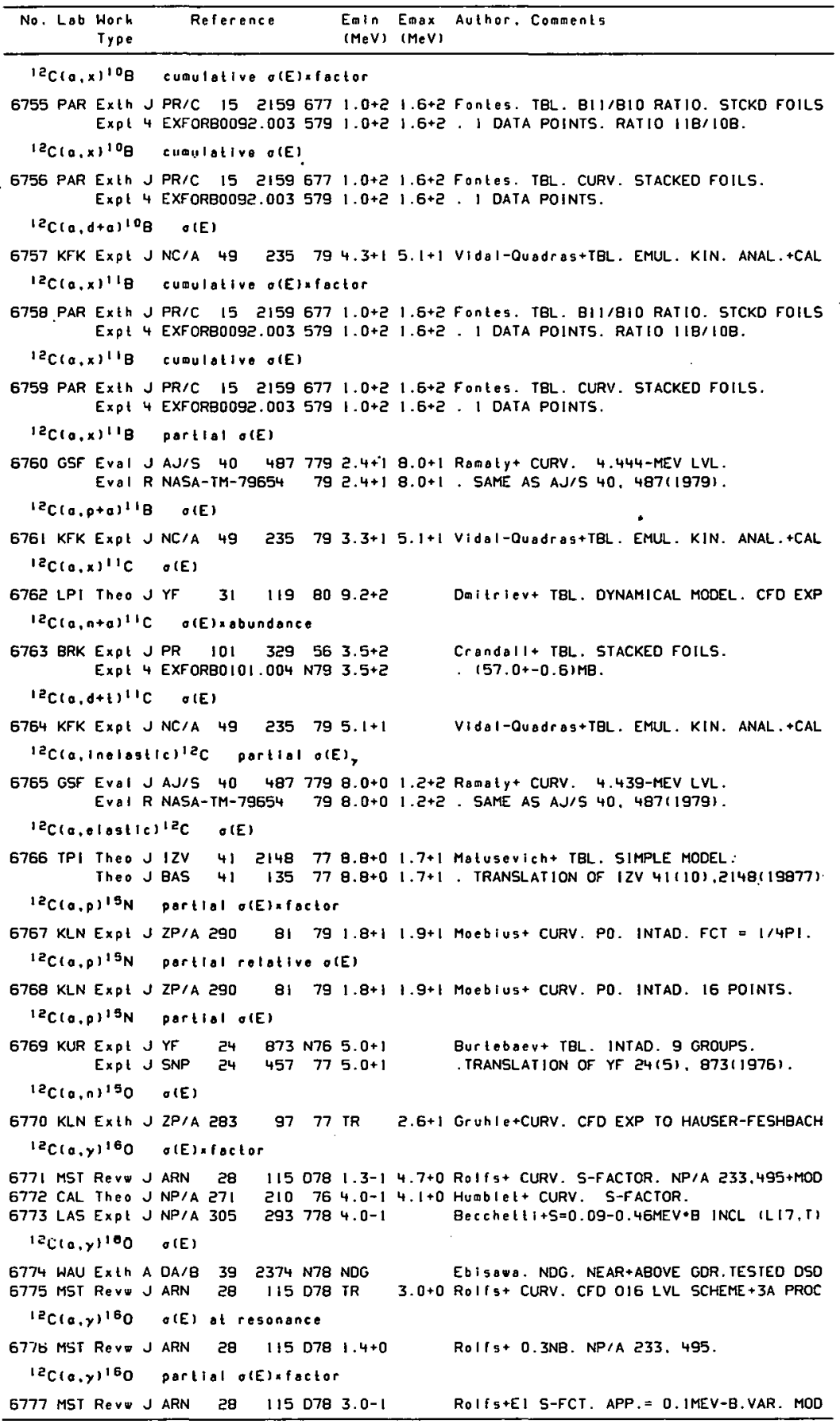




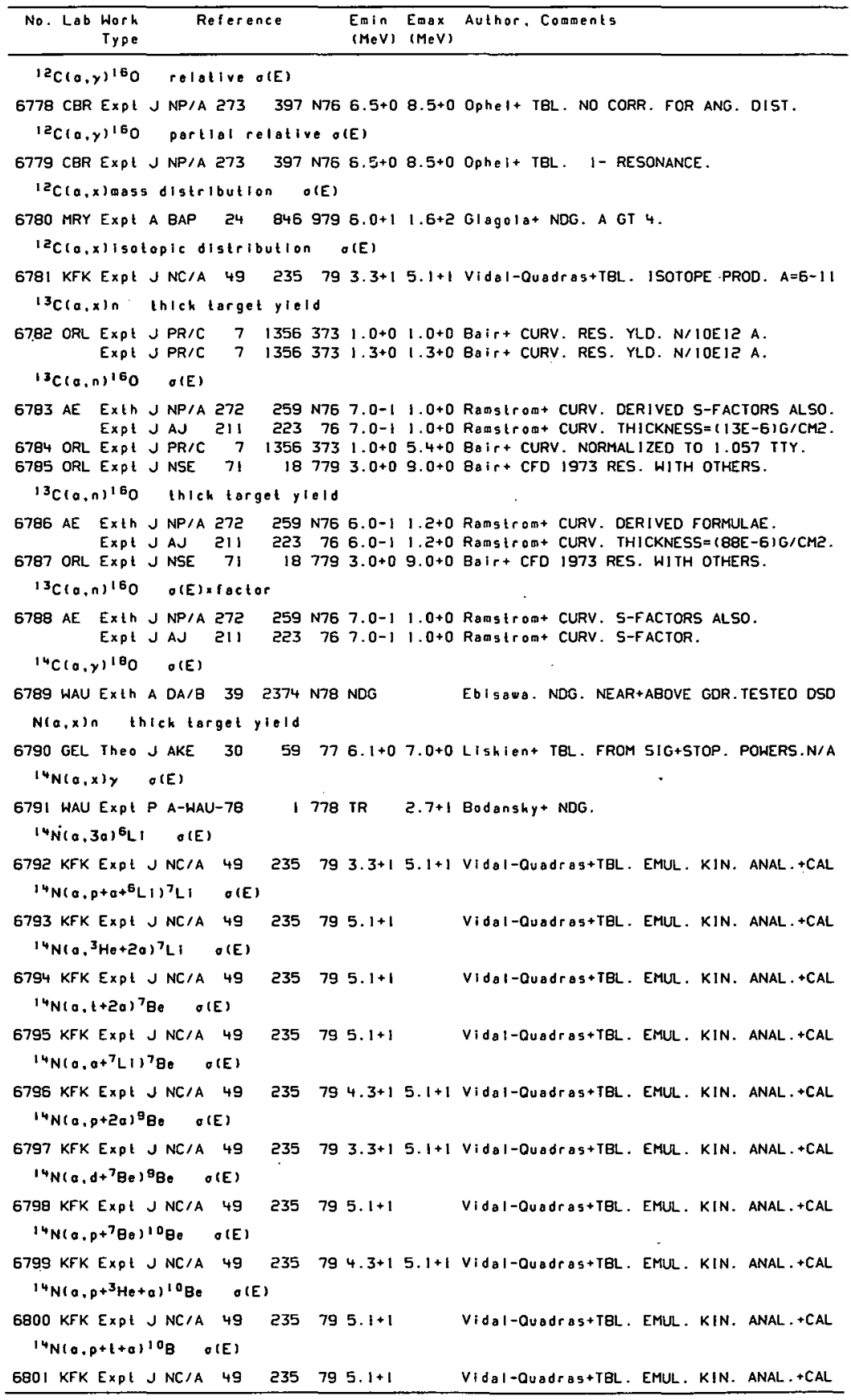




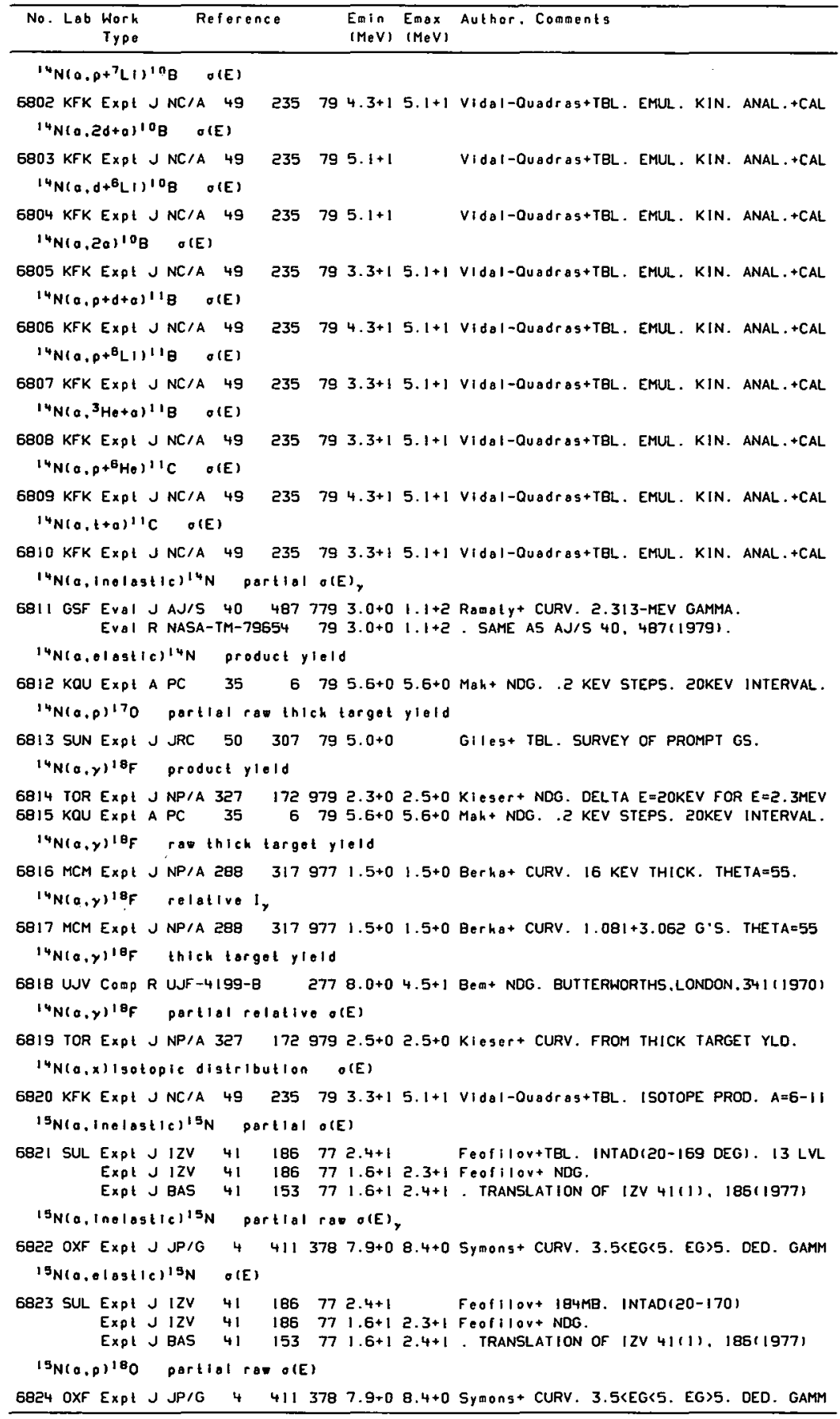




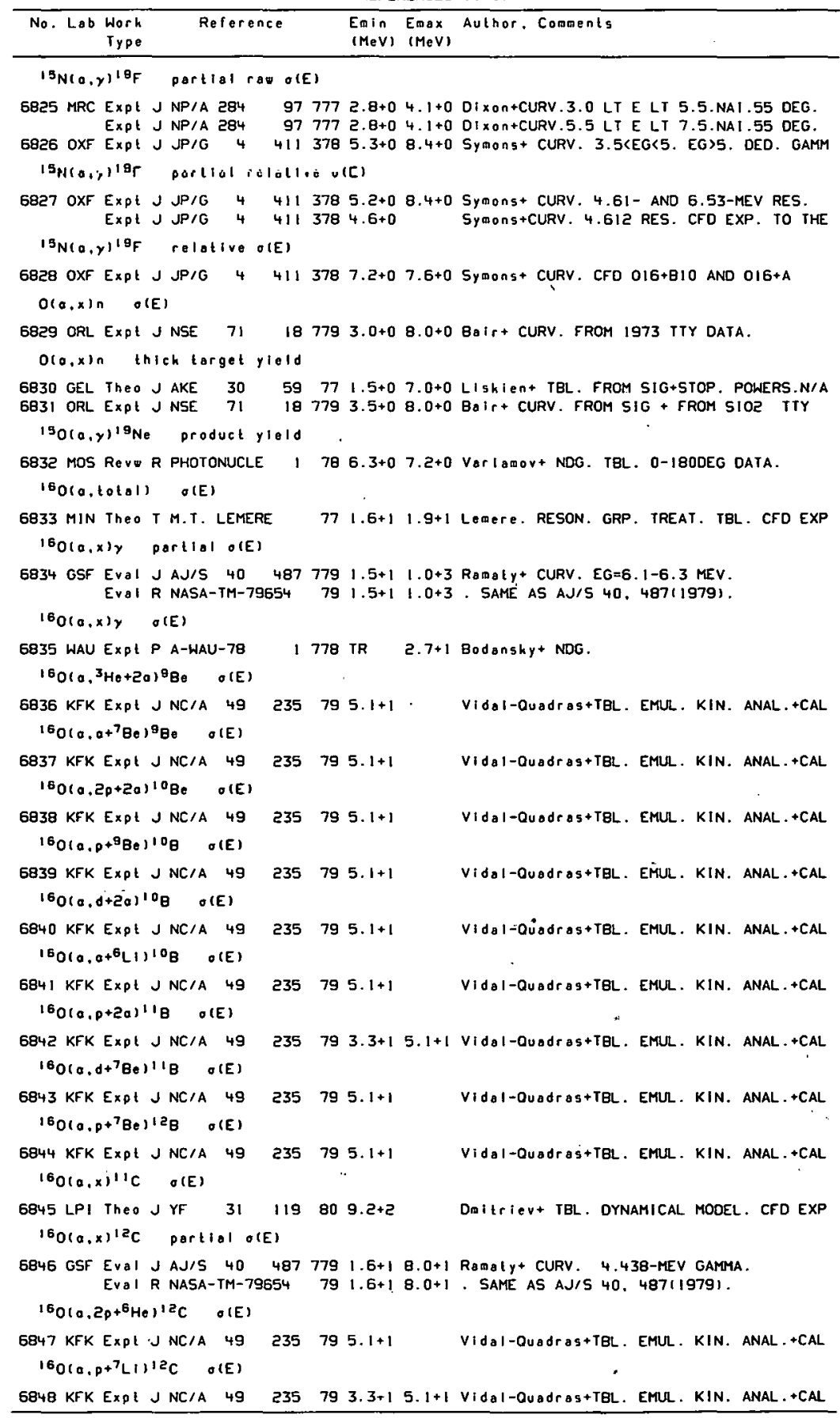


REFERENCES ( cont)

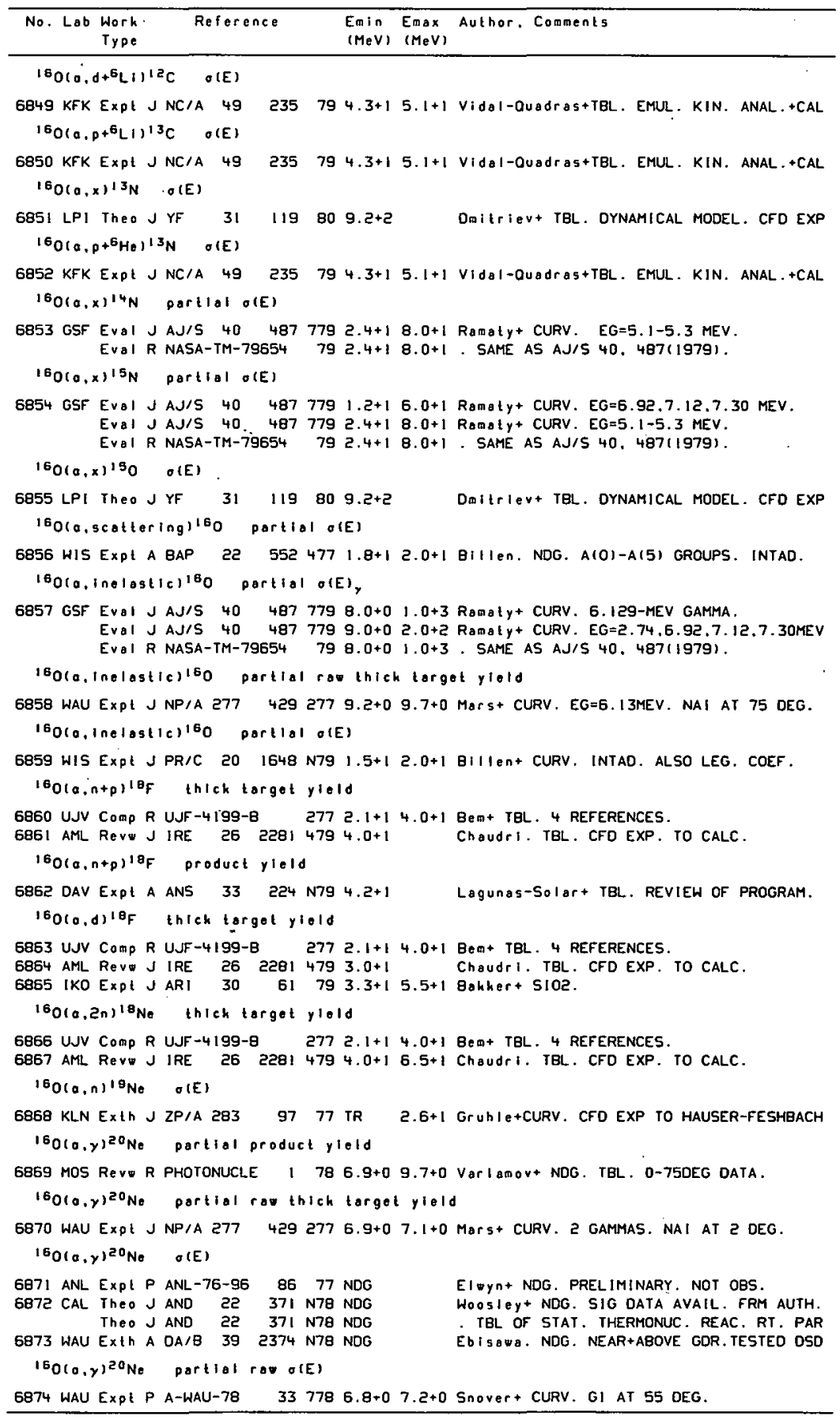


REFERENCES ( CON ( )

\begin{tabular}{|c|c|c|c|c|c|}
\hline No. Lab & $\begin{array}{l}\text { Work } \\
\text { Type }\end{array}$ & Reference & $\begin{array}{l}\text { Enin } \\
(M e V)\end{array}$ & $\begin{array}{l}\text { Emax } \\
(M e V)\end{array}$ & Author. Comments \\
\hline
\end{tabular}

$160(0, y) 20$ Ne partial thich target yield

6875 WAU ExpI A BAP $235014781.6+0$ Snover+1.42E-10+-3.92 G/A. TAZO5 TARGET

$160(a, x) i s o t o p i c$ distribution $O(E)$

6876 KFK Expt J NC/A $49235793.3+15.1+1$ Vidal-Ouadras+TBL. ISOTOPE PROD. A=6-11

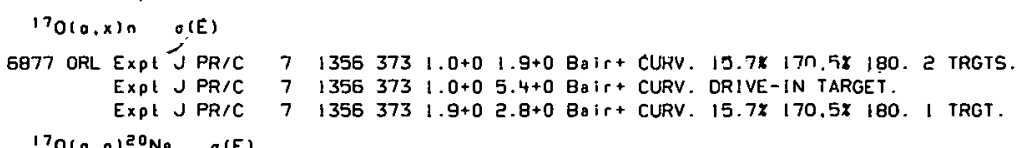

$6878 \mathrm{CAL}$ Theo $J$ AND $22371 \mathrm{N78}$ NDG Woos $10 y+$ NDG. SIG DATA AVAlL. FRM AUTH. Theo $J$ AND $22 \quad 371$ N78 NDG SBL OF STAT. THERMONUC. REAC. RT. PAR 6879 ORL Expt P ORNL-5306 $1056773.0+0$ 9.0+0 Bair+ NDG.

6880 DRL Exp! J NSE 71 18779 4.6+0 4.8+0 Boir+. NEW MEAS. I.35X 1962, 1973 DATA.

$170(a, y)^{21} \mathrm{Ne} \quad O(E)$

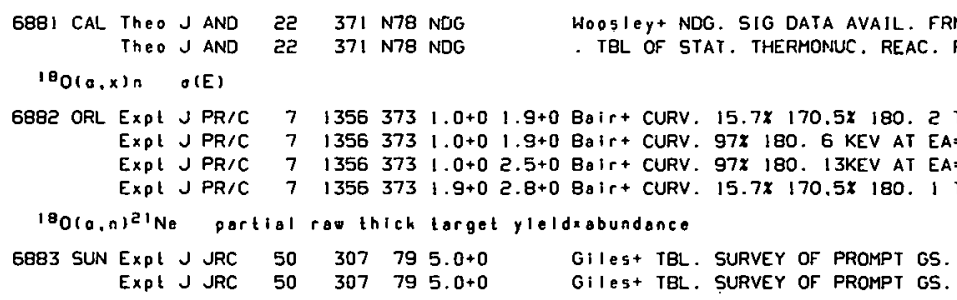

$180(a, n)^{21} \mathrm{Ne} \quad a(E)$

6884 ORL Expt J PR/C $713563731.0+02.5+0$ Bair+ CURV. 97x 180. I3KEV AT EA=2.5MEV ExpE JPR/C $713563731.0+0 \quad 1.9+0$ Bair+ CURV. 978 180. 6 KEV AT EA=2.5MEV 6885 ORL EXPt J PR 128 299 $0522.4+02.8+0 \mathrm{Bair}+\mathrm{CURV}$. 97X 180. 2 KEV AT EA=2.5MEV Expl J PR 128 299 062 $2.4+0$ 5.0+0 Bair+ CURV. 97x 180. 6 KEV AT EA=2.5MEV 6886 ORL EXPL P ORNL-5306 $1056773.0+09.0+0 \mathrm{Bair}+\mathrm{NDG}$.

6887 ORL Expt J NSE 71 19 $7794.6+04.8+0$ Bair+ NEW MEAS. 1.35X 1962.1973 DATA.

$100(0, n)^{2}$ 'Ne portlal o(E)

6888 AML Expt J PR/C 161264977 TR $6.5+0$ Switkowskit CURV. N3.N4 GROUPS. 50* 018

$180(0, n)^{2}{ }^{1} \mathrm{Ne} \quad I_{\text {, }}$

6889 CRC Expl A BAP 23520478 2.7+0 6.0+0 Mc-DonBId+ NDG. 2789-, 2796-KEV LEVELS.

$180(a, y)^{22} \mathrm{Ne}$ partial roo o(E)

6890 IFS Expt J NP/A $2974893786.0-12.3+0$ Traulvellert CURV. ALSO DEO. RES. PROP.

$100(a, y){ }^{22}$ No o(E) foctor

689: IFS Expt J NP/A $297489780.0+02.6+0$ Trautvelter+CURV.S-FCT. FRM DED. STRENG 6892 MST ReVW J ARN 28115 D78 0.0+0 2.5+0 Rolf5+ CURV. S-FACTOR. NPIA 297. 489.

$100(a, y) 2{ }^{2} \mathrm{Ne} \quad O(E)$

6893 CAL TheO $j$ AND 22371 N78 NDG Theo J AND $22 \quad 371$ N78 NDG

$190(0, n) 2{ }^{2} \mathrm{Ne} \quad(E)$

6894 CAL Theo J AND $22 \quad 371$ N78 NDG Theo J AND $22 \quad 371$ N78 NDG

$190(a, y)^{23} \mathrm{Ne}$ o(E)

6895 CAL Theo J AND $22 \quad 371$ N79 NUD Theo J AND $22 \quad 371$ N78 NDG

Woosley+ NDG. SIG DATA AVAIL. FRM AUTH. - TBL OF STAT. THERMONUC. REAC. RT. PAR

Woogley+ NDG. SIG DATA AVAIL. FRM AUTH. . TBL OF STAT. THERMONUC. REAC. RT. PAR

Wnnsley+ NDG. SIG DATA. AVAIL. FRM AUTH. . TEL OF STAT, IHERMOPUC. RE. RT. RT. PAR

copoco, $x$ ) thich target yield

6896 ORL Expt J NSE 71 18 779 3.0+0 8.0+0 Bair+ CURV. SIO2 IRGT. N/10E6 A. Expt J NSE 7I $187792.5+05.2+0$ Bair+ CURV. CALC. FRM REVISED SIGMAS.

6897 HAR Theo P NEANDC (E)-2028 $4794.0+0$ 5.0+0 Hooten+ TBL. 4 CMP. P. 62. Theo P UKNDC-P-94 $624794.0+0$ 5.0+0. SAME AS NEANDC (E)-202, VOL. 8 The o P INDC (UK)-31 62 $4794.0+05.0+0$. SAME AS NEANDC (E)-202, VOL. 8 Theo P AERE-PR/NP-26 $3794.0+0$ 5.0+0 Hootent TBL. EXACT+APP. 4 CMPS. P. 47 
REFERENCES (cont)

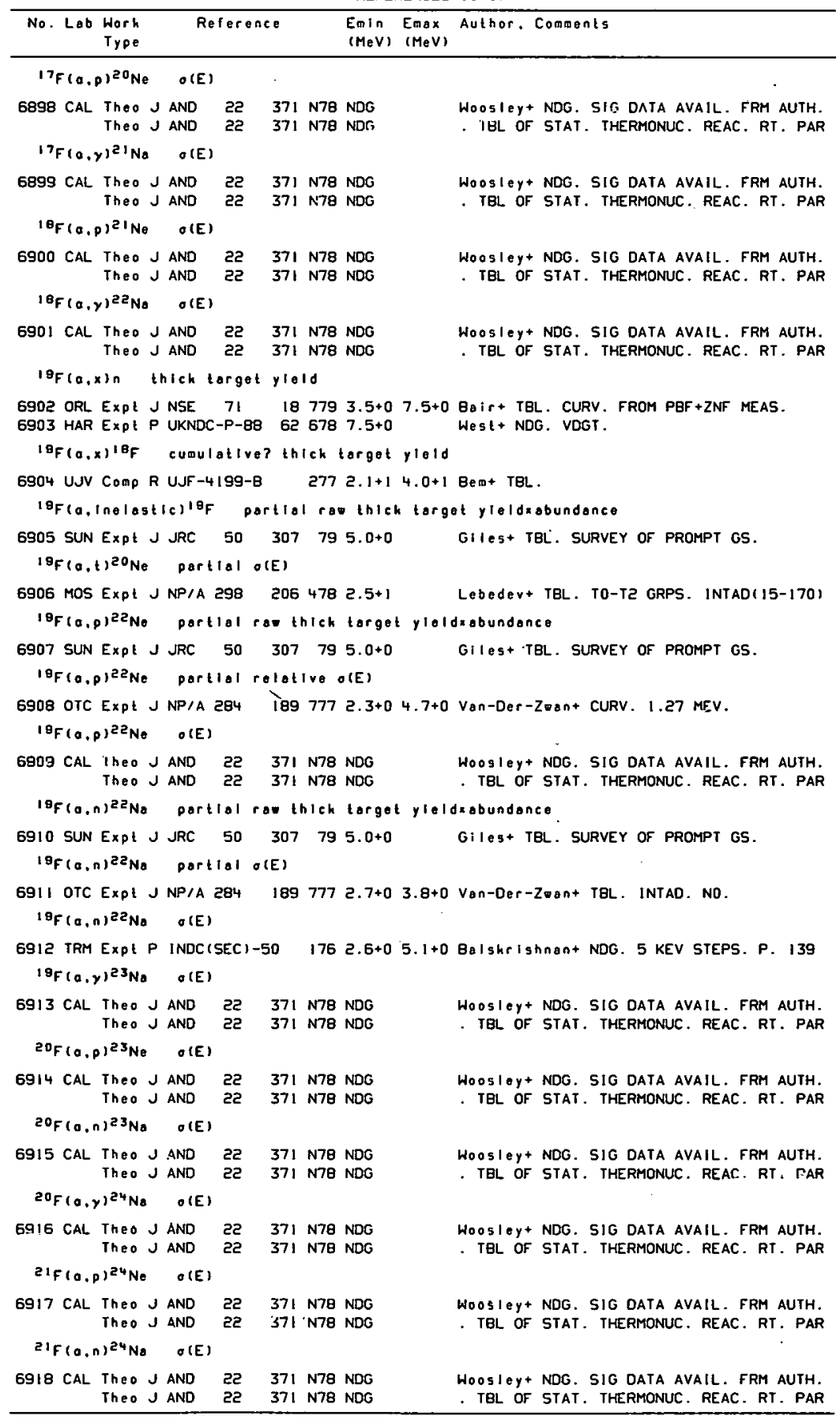


REFERENCES (cont)

\begin{tabular}{|c|c|c|c|c|c|}
\hline No. Lab & $\begin{array}{l}\text { Horh } \\
\text { Type }\end{array}$ & Reference & $\begin{array}{l}\text { Emin } \\
(M e V)\end{array}$ & $\begin{array}{l}\text { Emax } \\
(M e V)\end{array}$ & Author, Comments \\
\hline
\end{tabular}

No(a,x) lhick target ylold

$6919 \mathrm{GEL}$ Theo J AKE $30 \quad 59771.9+05.1+0$ Lishient TBL. FROM SIG+STOP. POWERS.N/A Neta,xiter rumulative thlck larget yield

6920 BNL Expt JARI $29175370 \leftrightarrow, F_{i+1}$ Lambrechi+1.2+-0.1MCI/MUAMP-HR. 23MEV.

$20_{\mathrm{Ne}}(0, \operatorname{total}) \quad \sigma(E)$

6921 IJI Revw J FCY $6 \quad 82775$ 2.0+0 4.5+1 Nemets+ CURV.

Revw J SJPN $6 \quad 325762.0+04.5+1$. TRANSLATION OF FCY $6.141,827(75)$.

$20 \mathrm{Ne}(0, x) \gamma \quad \sigma(E)$

6922 WAU Expl P A-WAU-78 1778 TR $2.7+1$ Bodanghy+ NDG.

6923 GSF Eval J AJ/S 40487779 8.0+0 1.2+3 Ramaty+ CURV.

Eval R NASA-TM-79654 79 9.0+0 1.2+3. SAME AS AJ/S 40. $487(1979)$.

$20 \mathrm{Ne}(0,12 \mathrm{C}) 12 \mathrm{C}$ partial o(E)

6924 WIS Expt A BAP 24 $8179791.3+12.1+1$ Dav1s. NDG. EVEN J+. INTÁD.

$20 \mathrm{Ne}(a, i n e l o s t i c)^{20} \mathrm{Ne}$ portlal o(E),

6925 WIS Expt A GAP $23 \quad 5934785.7+0 \quad 1-1+1$ Dovis+ NDG. LEG. POLY. EXP.

6926 GSF Eval J AJ/S $404877798.0+01.2+2$ Ramaty 4 CURV. EG=1.634 MEV.

Eval R NASA-TM-79554 79 8.0+0 $1.2+2$. SAME AS AJ/S 40. $487(1979)$.

20 Ne $a, 1 n e l a s t l e)^{20} \mathrm{Ne}$ partlal row $O(E)$,

6927 OXF Expt J NP/A $309770782.7+05.8+0$ FIflelo+ CURV.

Expt J NP/A 322 I 679 2.7+0 6.0+0 FIfIeld+ SEE NP/A 309.77(1978I.

$20 \mathrm{Ne}(0,0)^{23} \mathrm{No} \quad 0(E)$

6928 CAL TheO R OAP-422

$20 \mathrm{Ne}(a, n)^{23} \mathrm{Mg} \quad \sigma(E)$

$6929 \mathrm{CAL}$ Theo R OAP-422 $6930 \mathrm{KLN}$ EXth J ZP/A 283

$20 \mathrm{Ne}(a, y){ }^{24} \mathrm{Mg}$ partiol

6931 OXF Expt J NP/A 309 Expt J NP/A 309

Expt J NP/A 322

Expt C 77TOKYO

Expt J PL/B 69

$20 \mathrm{Ne}(0, \gamma)^{24} \mathrm{Mg} \quad \sigma(E)$

6932 CAL TheO J AND 22

Theo J AND 22

Theo $R$ OAP-4Z2

2 Ne (o.p) ${ }^{24} \mathrm{No}$ O(E)

6933 CAL TheO R OAP-4Z2

$21 \mathrm{Ne}(0 . n)^{24} \mathrm{Hg}$ O(E)

6934 CAL Theo J AND 22 Theo $J$ AND 22

Theo R OAP-422

$21 \mathrm{Ne}(0 . y)^{25} \mathrm{Mg} \quad \sigma(E)$

$6935 \mathrm{CAL}$ Theo J AND 22

Theo J AND 25

Theo R OAP- 422

$22_{\mathrm{Ne}}(a, p)^{2 \mathrm{I}_{\mathrm{N}}} \mathrm{O(E)}$

$6936 \mathrm{CAL}$ TheO R OAP-422

$2{ }^{2} \mathrm{Ne}_{0}(0, n)^{25} \mathrm{Mg}$ O(E)

6937 CAL Theo R DAP-42C

$22_{\mathrm{No}}(0 . y)^{26} \mathrm{Mg}$ o(E)

6938 CAL Theo J AND 22

Theo J AND 22

$T t_{1} \in O R$ OAP-42Z

875 TR $1.2+1$ WOOSley+ CURV. HAUSER-FESHBACH.

875 TR 1.2+1 WOOgley+ CURV. HAUSER-FESHBACH.

9777 TR $3.1+1$ Gruhle+CURV. CFO EXP TO HAUSER-FESHEACH

o* O(E)

77078 3.0+0 6.0+0 Fifield+CURV.DIFF. PUMPED TRGT. THET $=125$

$770785.9+0$ 5.8+0 Fifield+ CURV. NEAR $14.153 \mathrm{LVL}$.

$16792.7+06.0+0$ Flfteld+ SEE NP/A 309, 77(1978).

205977 NDG

Fifigld- NDG. DEDUCED WIDTHS

$457775.8+05.8+0$ Fiflelo+ CURV. $3.4-3.7,4.0-4.7,8 \cdot 7-10.5$

371 N78 NDG

371 N78 NDG

875 TR

875 TR

371 N78 NDG

371 N78 NDG

875 TR

371 N78 NDG

371 N78 NDG

875 TR

875 TR

B75 TR

371 N7G NDG

371 N78 NDG 875 TR
Woosley + NDG. SIG DATA AVAIL. FRM AUTH. - TRL OF STAT. THERMONUC. REAC. RT. PAR $1.2+1$ Woosley+ CURV. HAUSER-FESHBACH.

$1.2+1$ WoOsley - CURV. HAUSER-FESHBACH.

Woosley+ NDG. SIG DATA AVAIL. FRM AUTH. - TBL OF STAT. THERMONUC. REAC. RT. PAR $1.2+1$ HoOsley+ CUPV. HAUSER-FESHBACH.

Woosley + NDG. SIG DATA AVAIL. FRM AUTH. - TBL OF STAT. THERMONUC. REAC. RT. PAR $1.2+1$ Woosley+ CURV. HAUSER-FESHaACH.

$1.2+1$ HoOsley+ CURV. HAUSER-FESHBACH.

$1.2+1$ Woos ley+ CURV. HAUSER-FESHBACH.

Woosley + NDG. SIG DATA AVAIL. FRM AUTH. - TBL OF STAT. THERMONUC. REAC. RT. PAR $1.2+1$ Hoosley+ CURV. HAUSER-FESHBACH. 


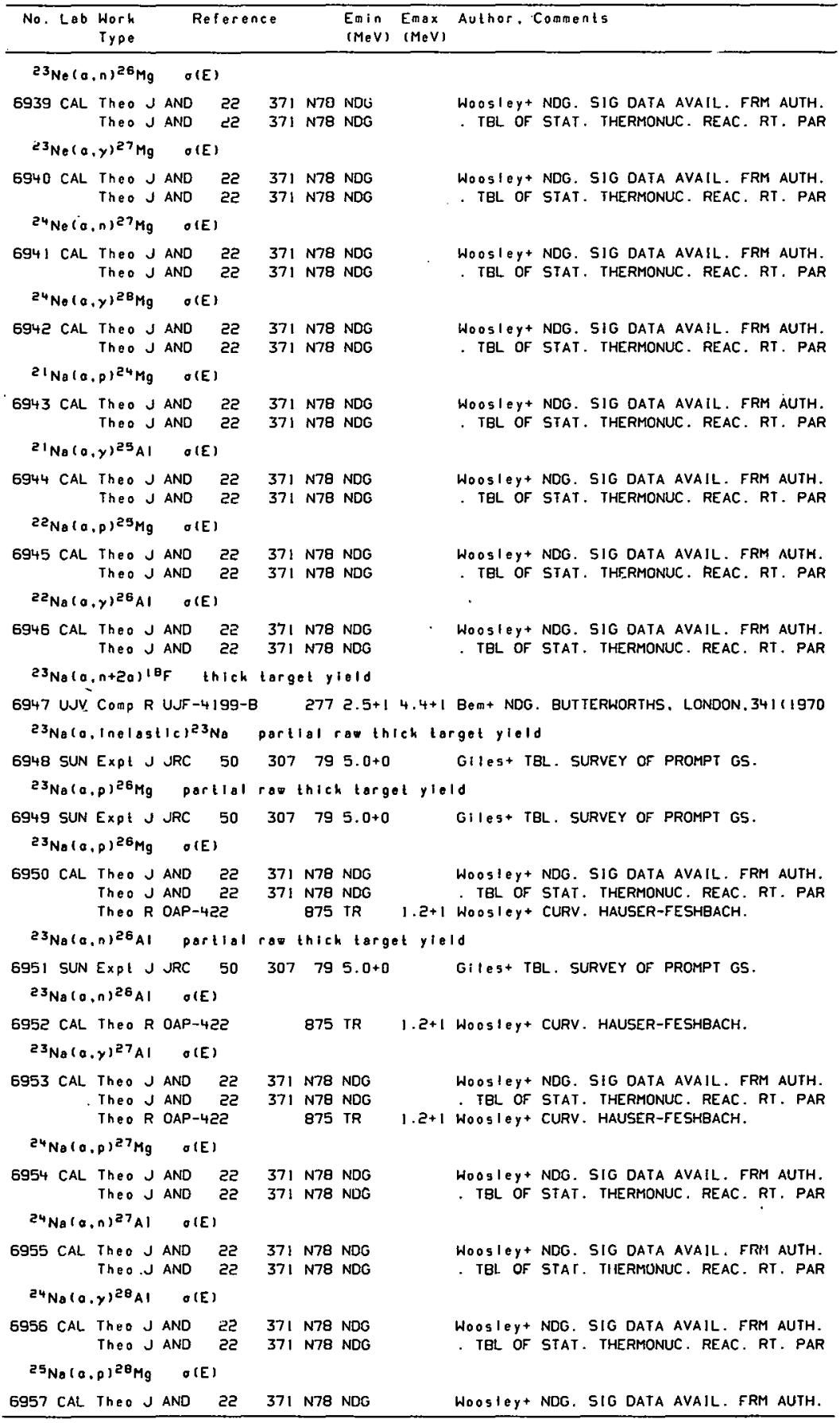




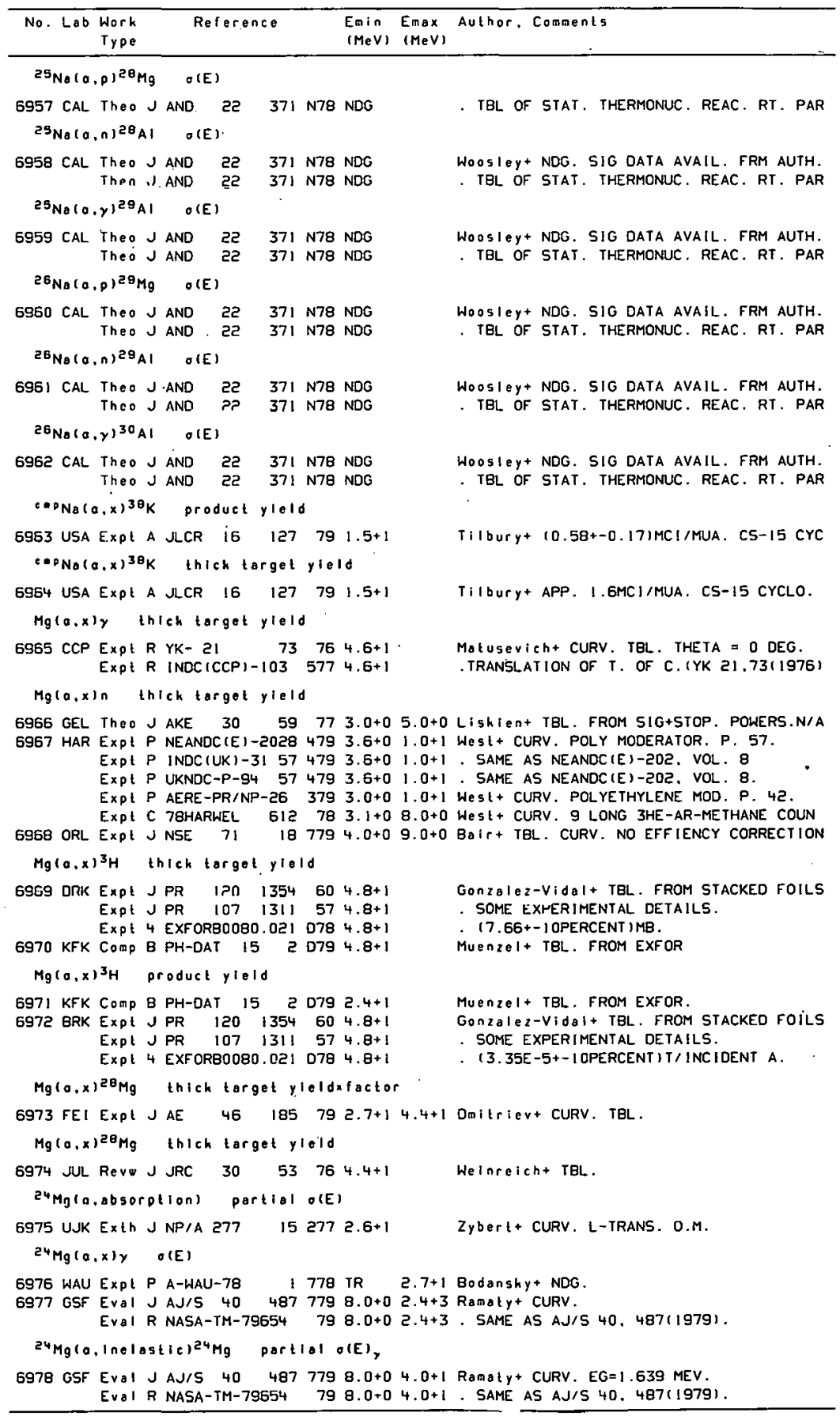


REFERENCES (cont)

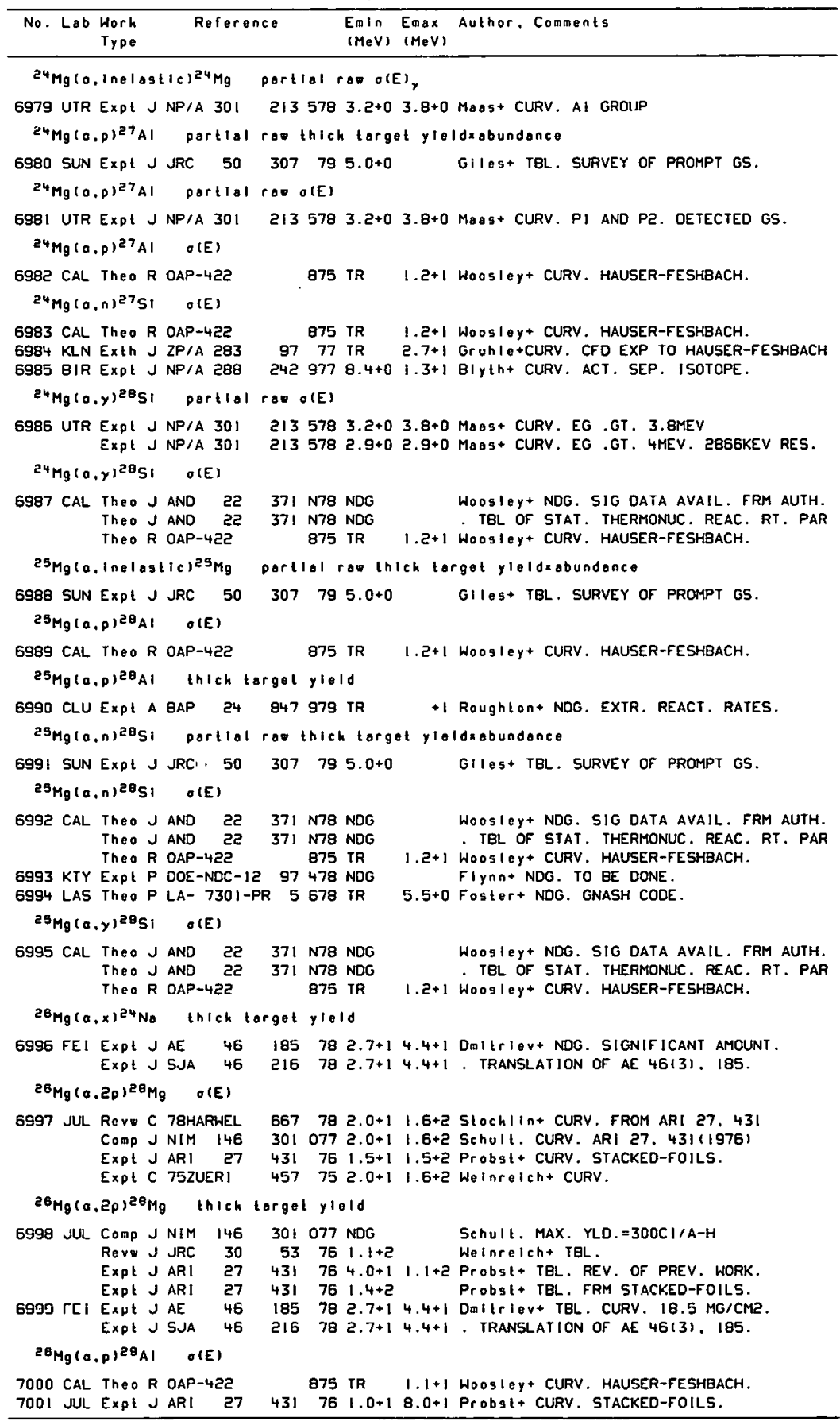


REFERENCES ( cont)

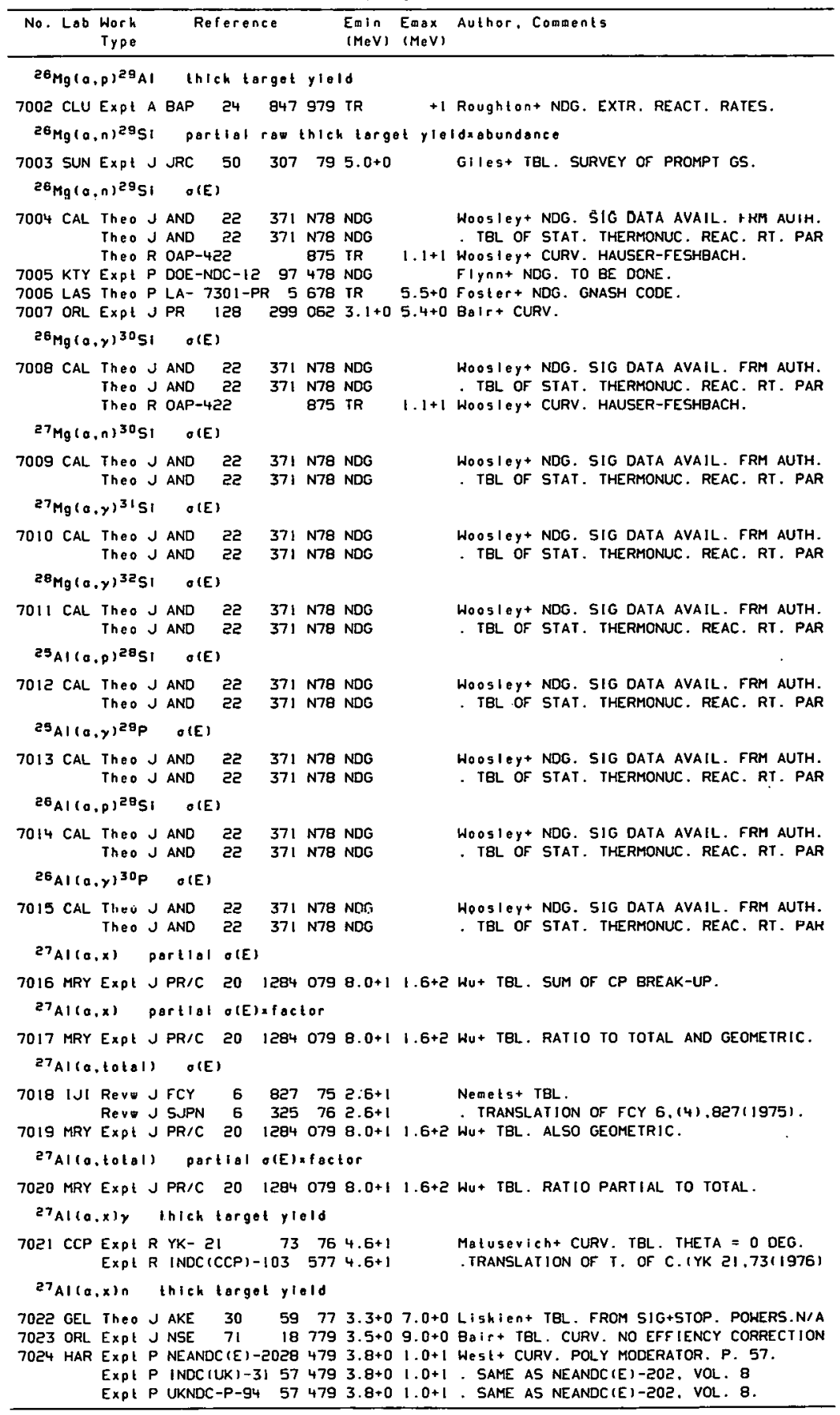




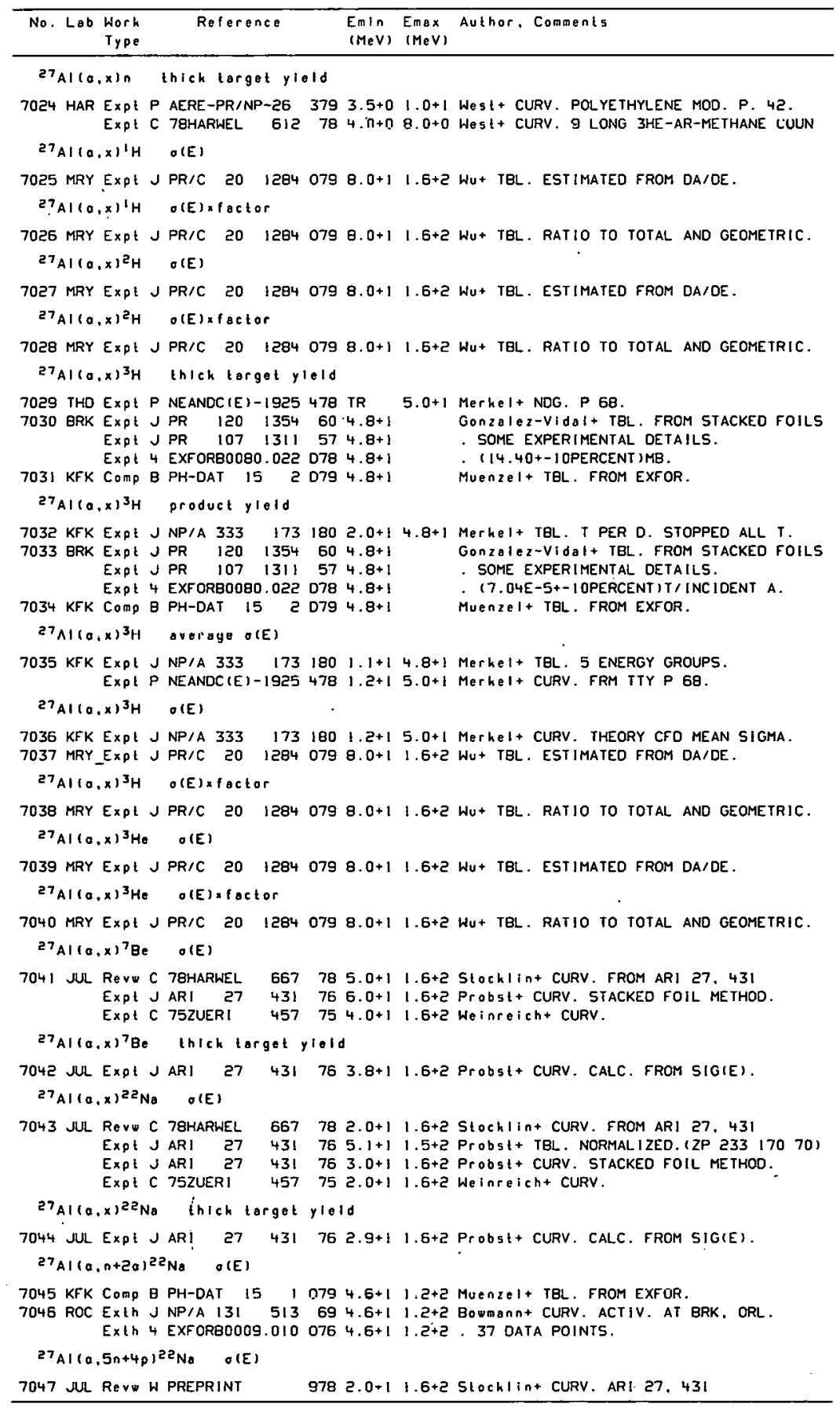


REFERENCES ( cant)

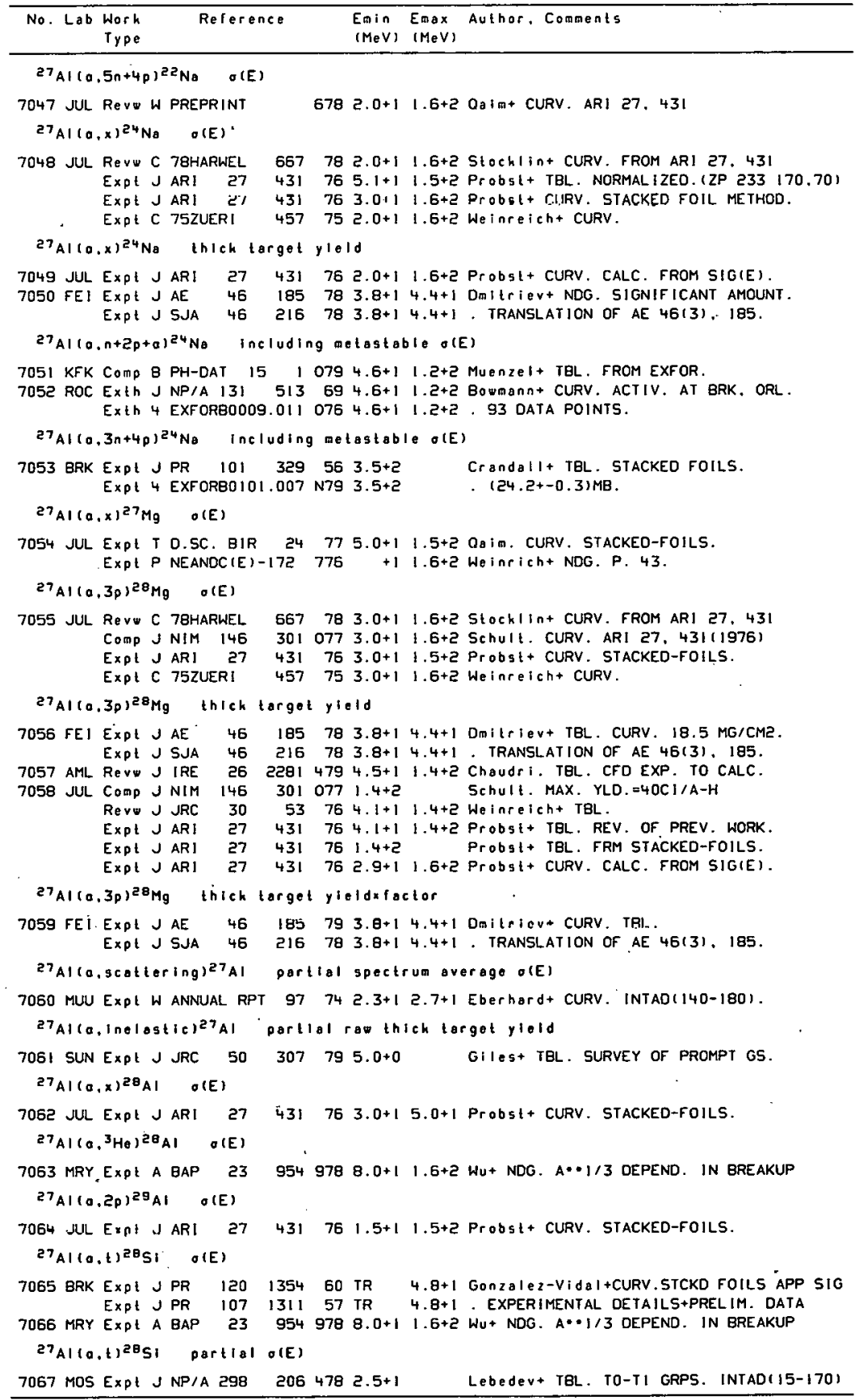




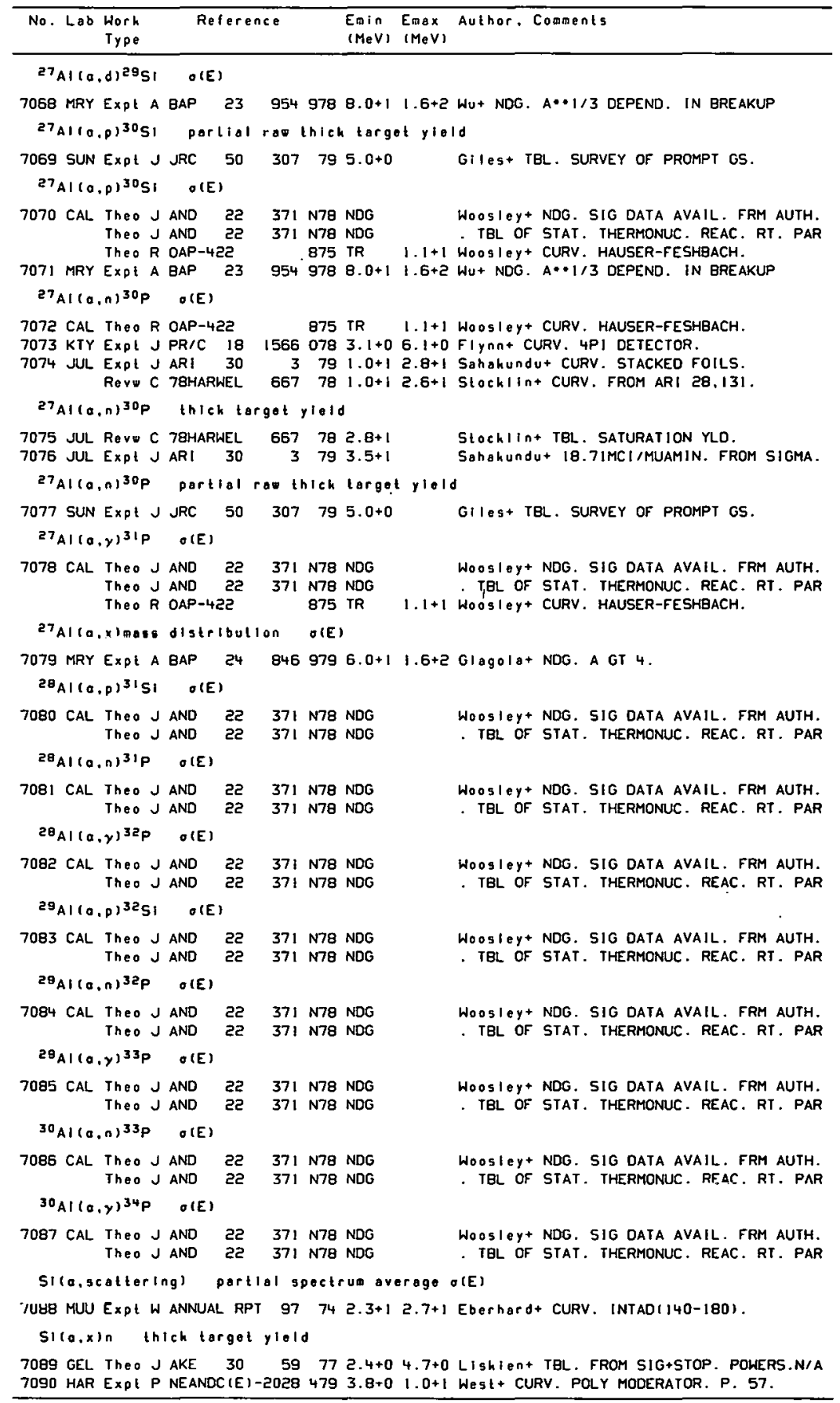


REFERENCES (cont)

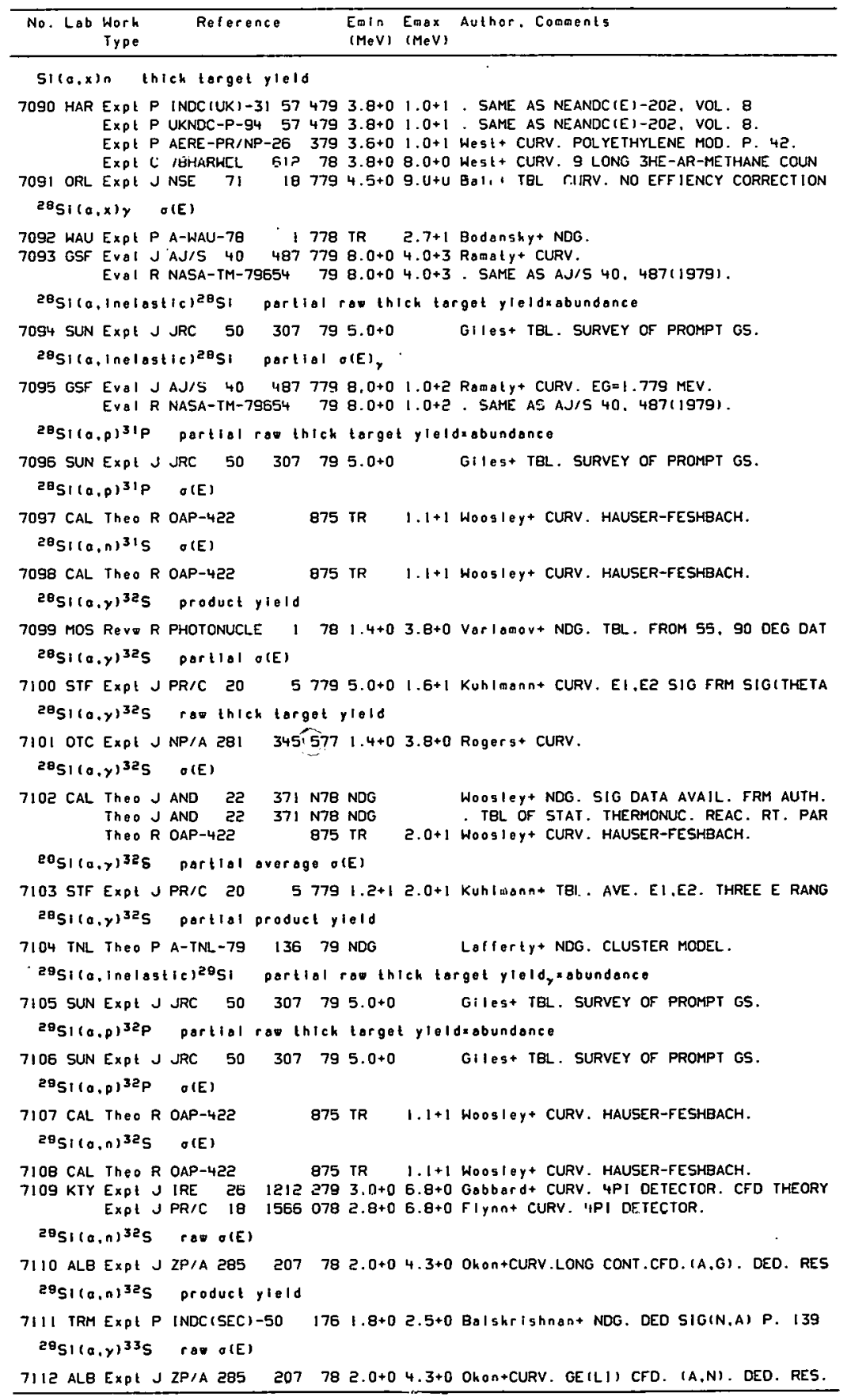


REFERENCES (cont)

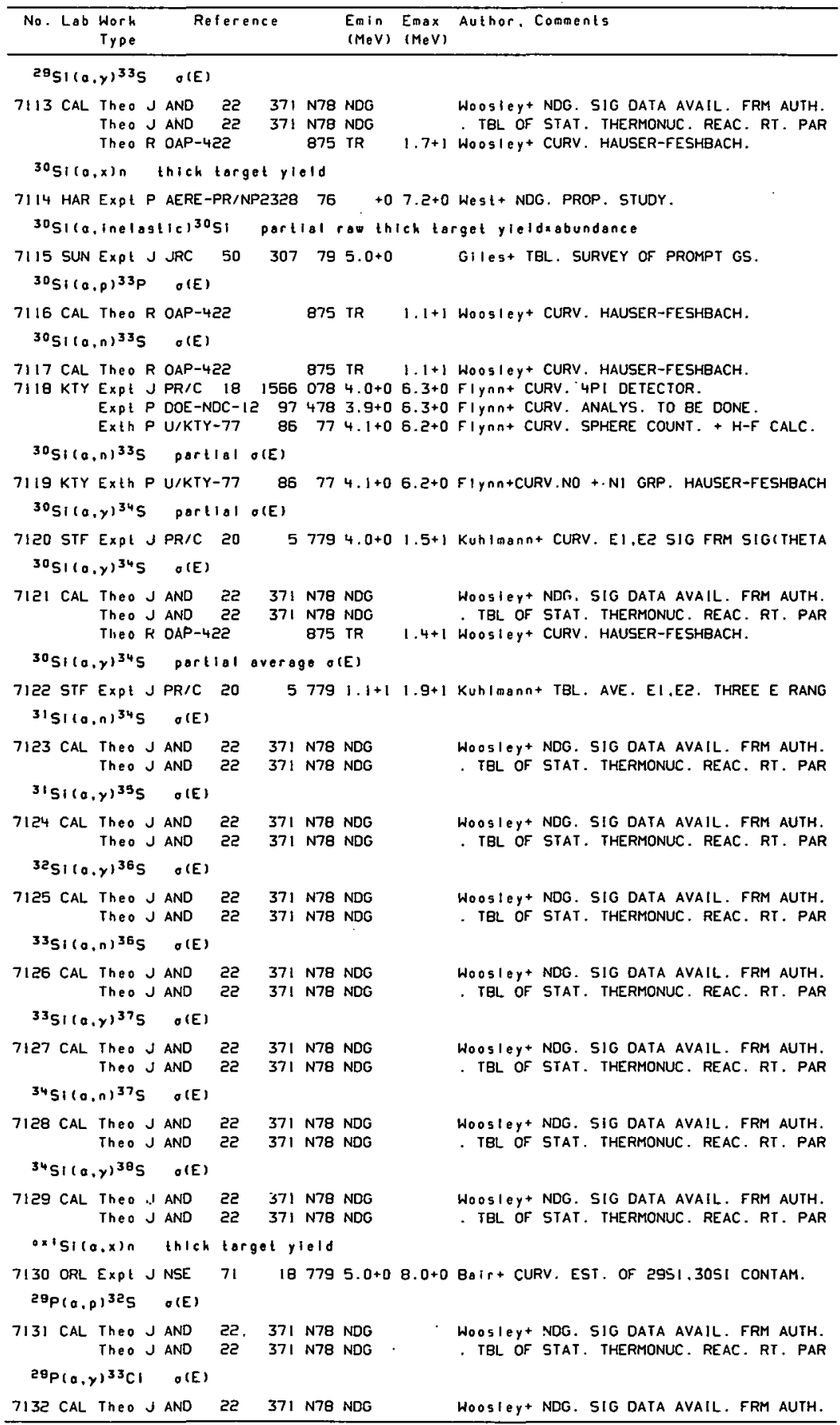


REFERENCES ( con t )

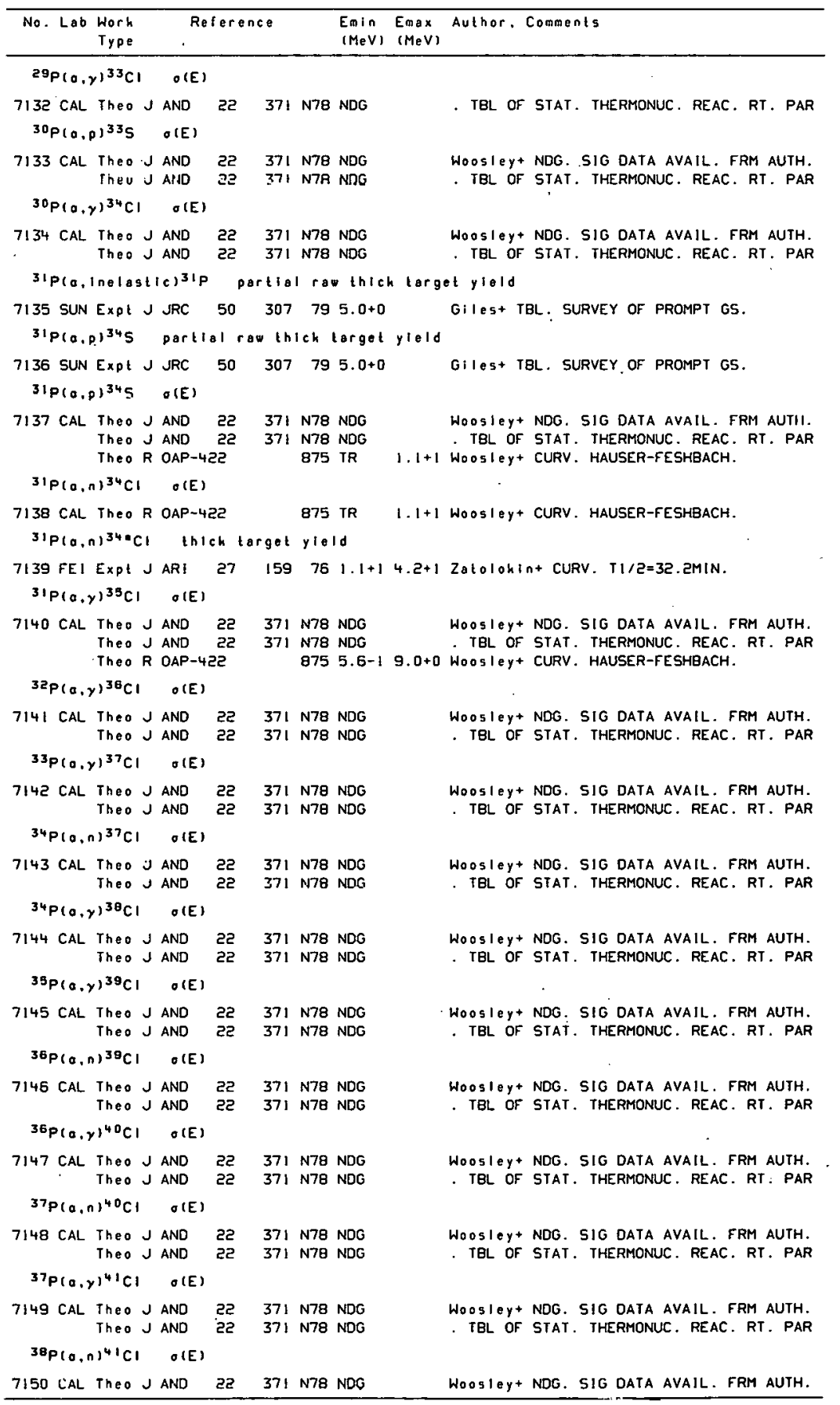


REFERENCES $(\operatorname{con} t)$

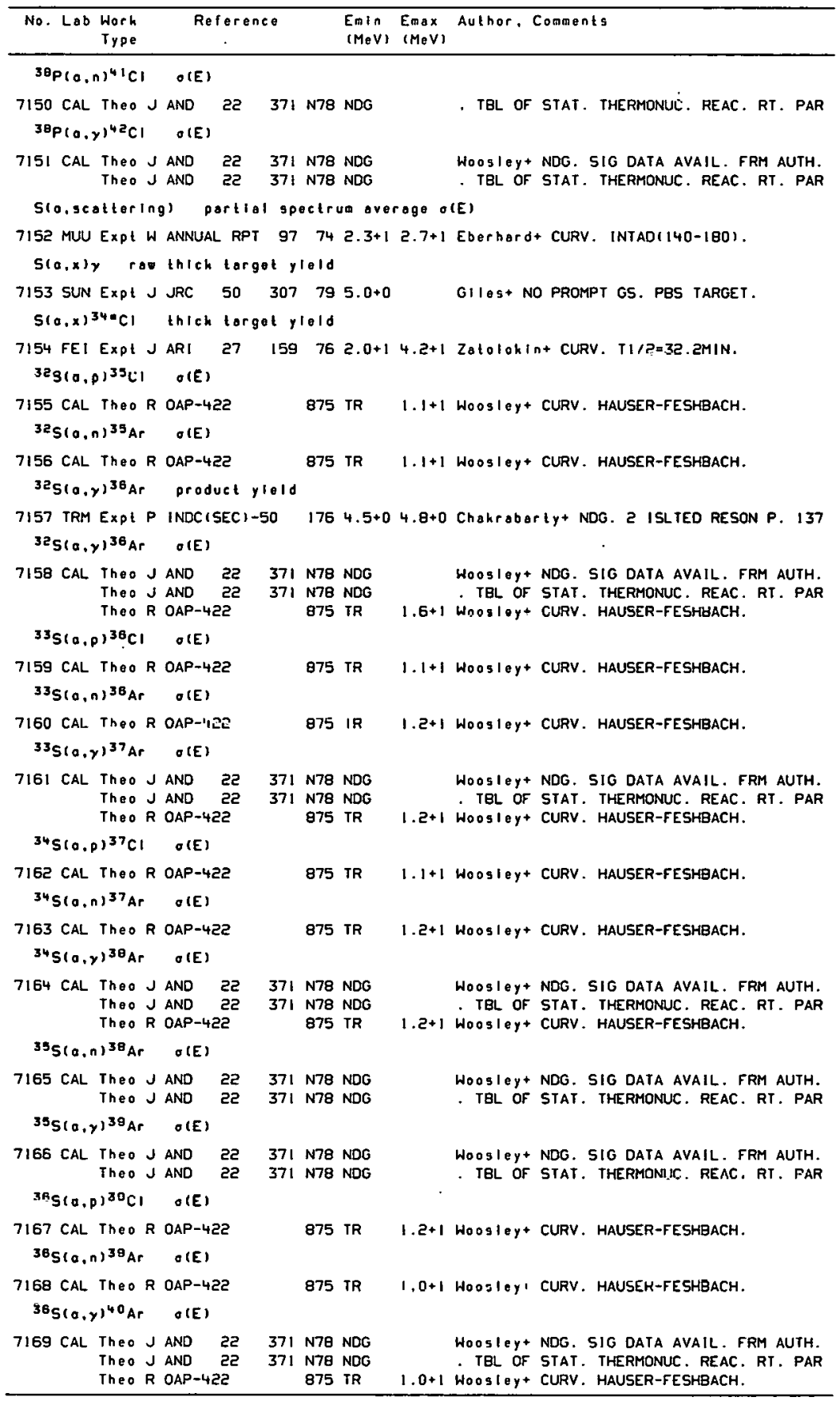


REFERENCES (cont)

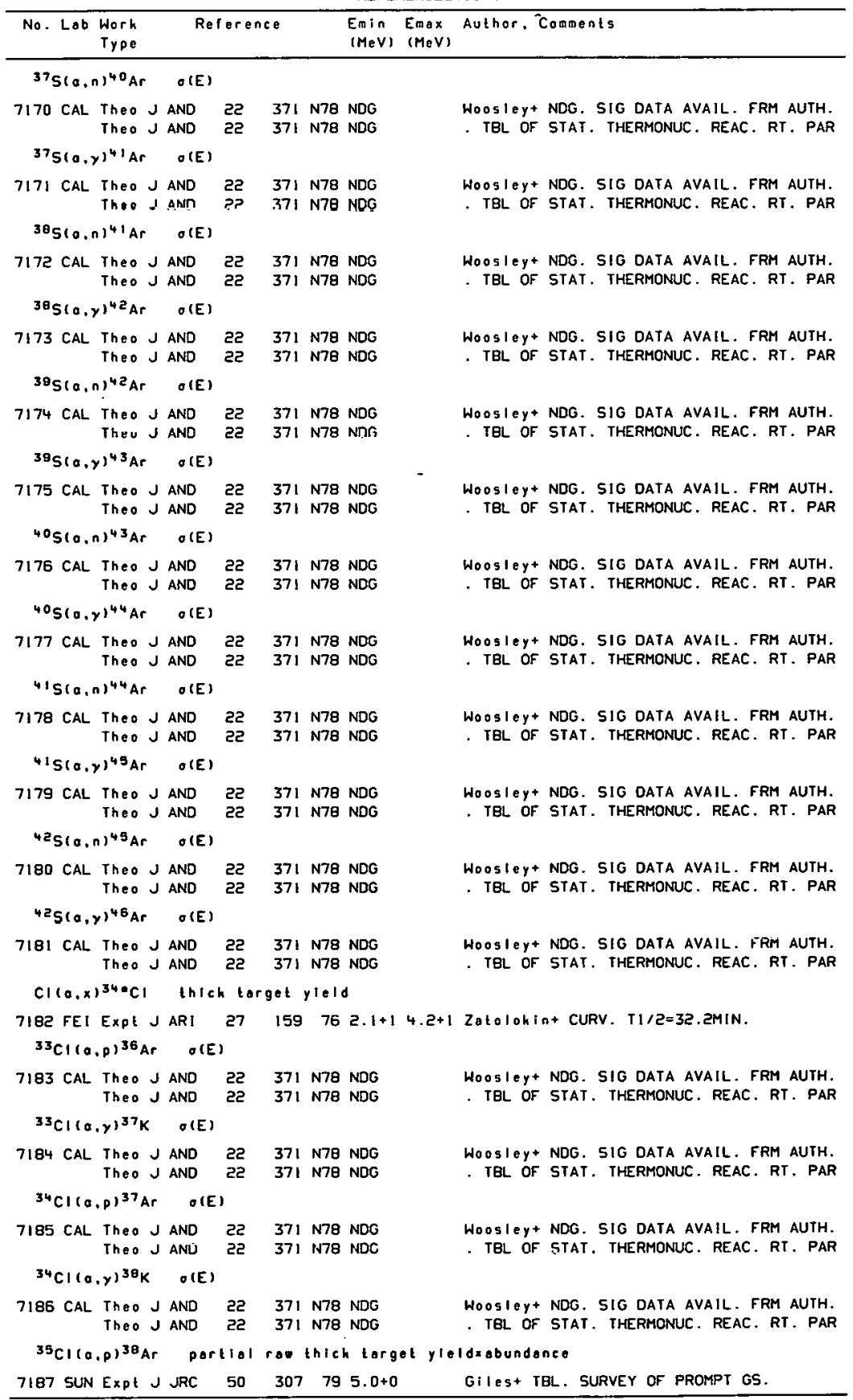




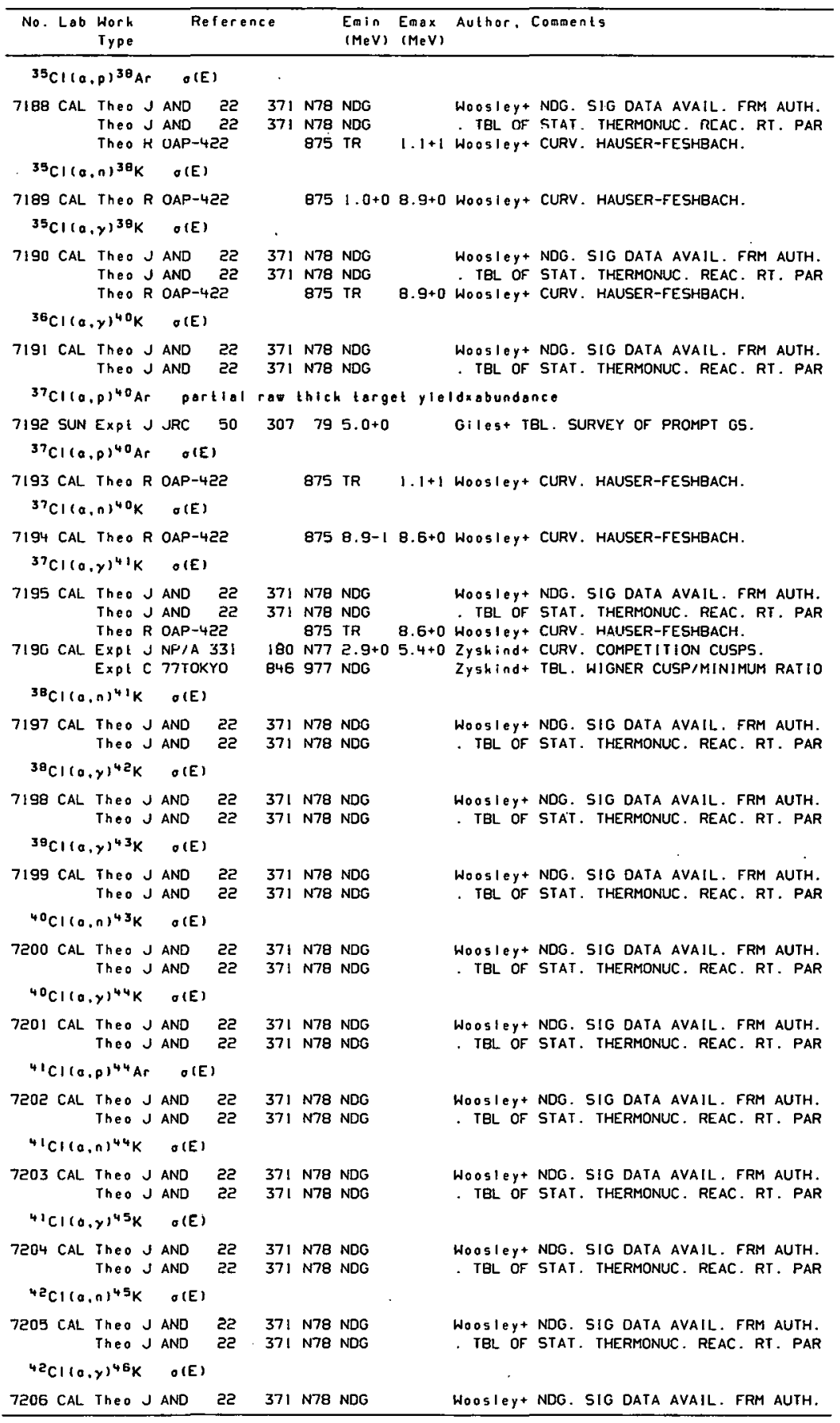


REFERENCES ( $c$ on t)

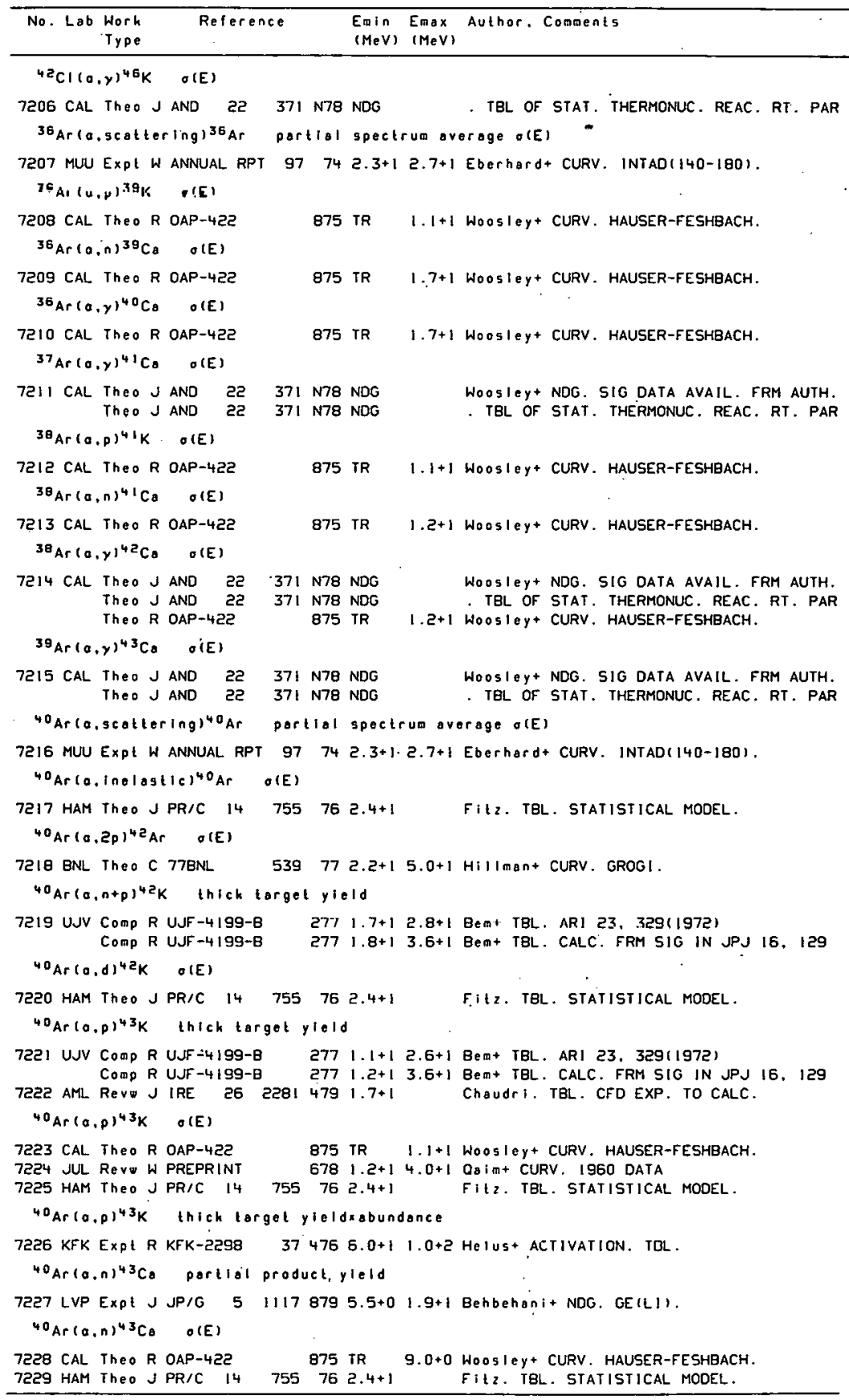




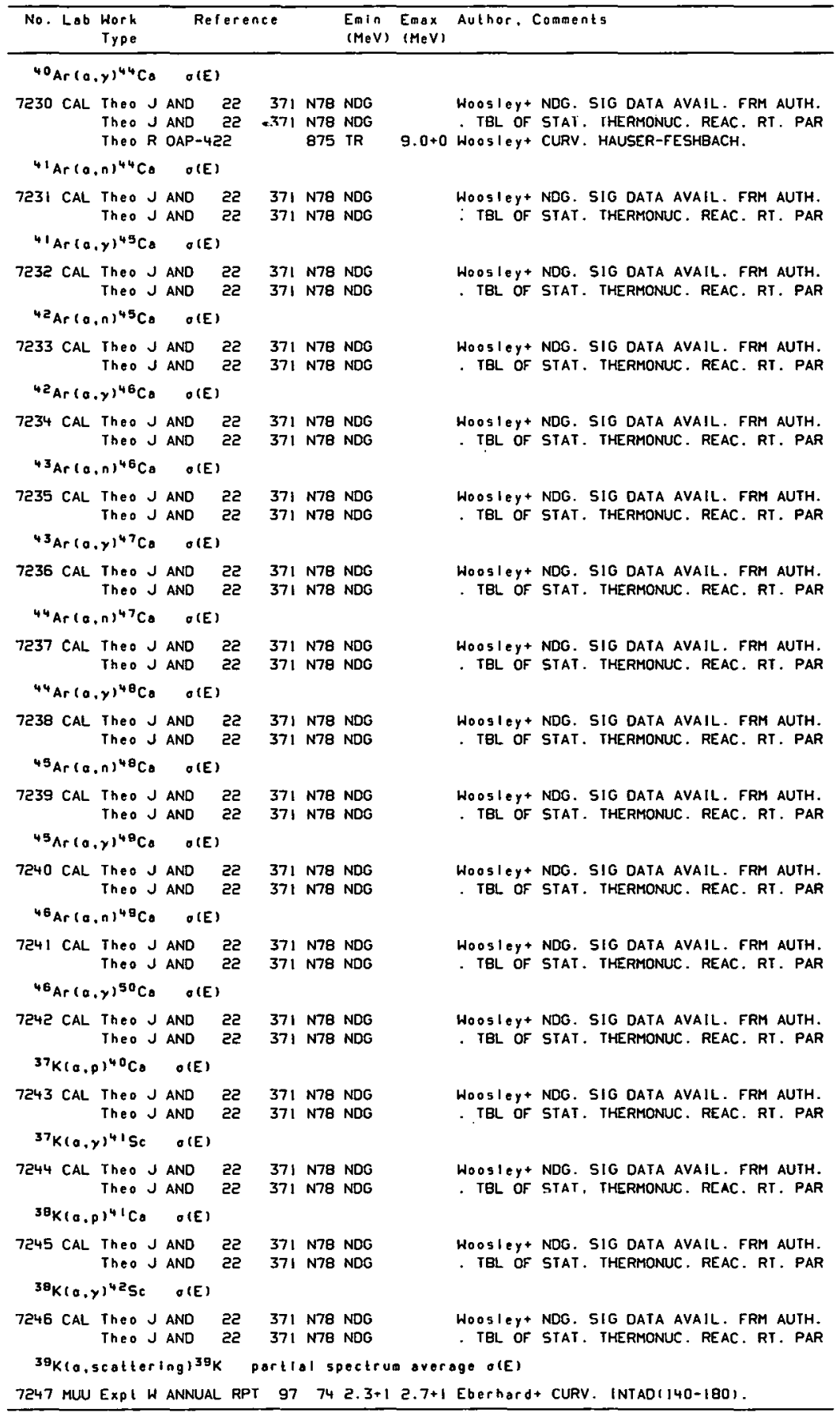


REFERENCES ( c ont)

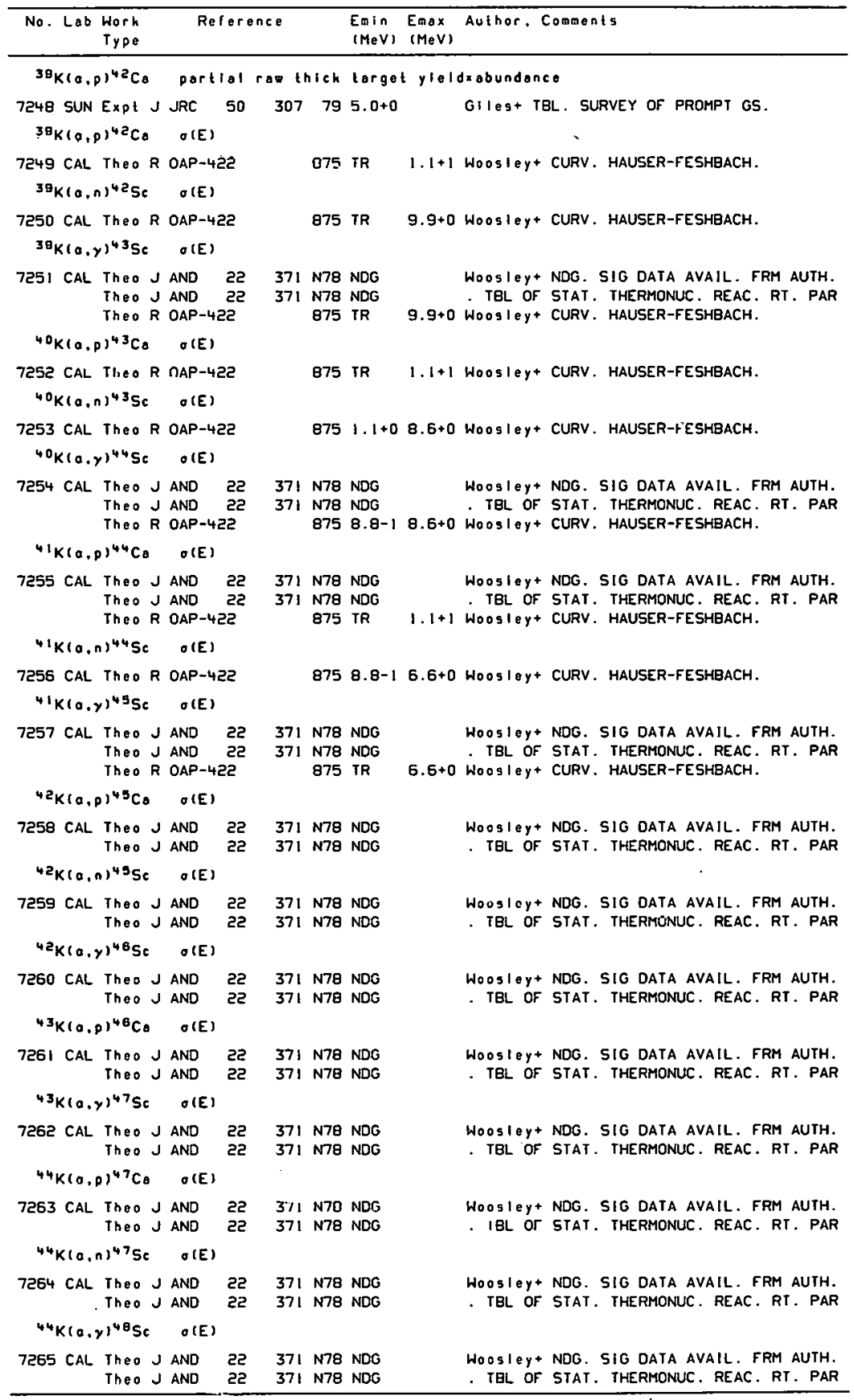


REFERENCES (cont)

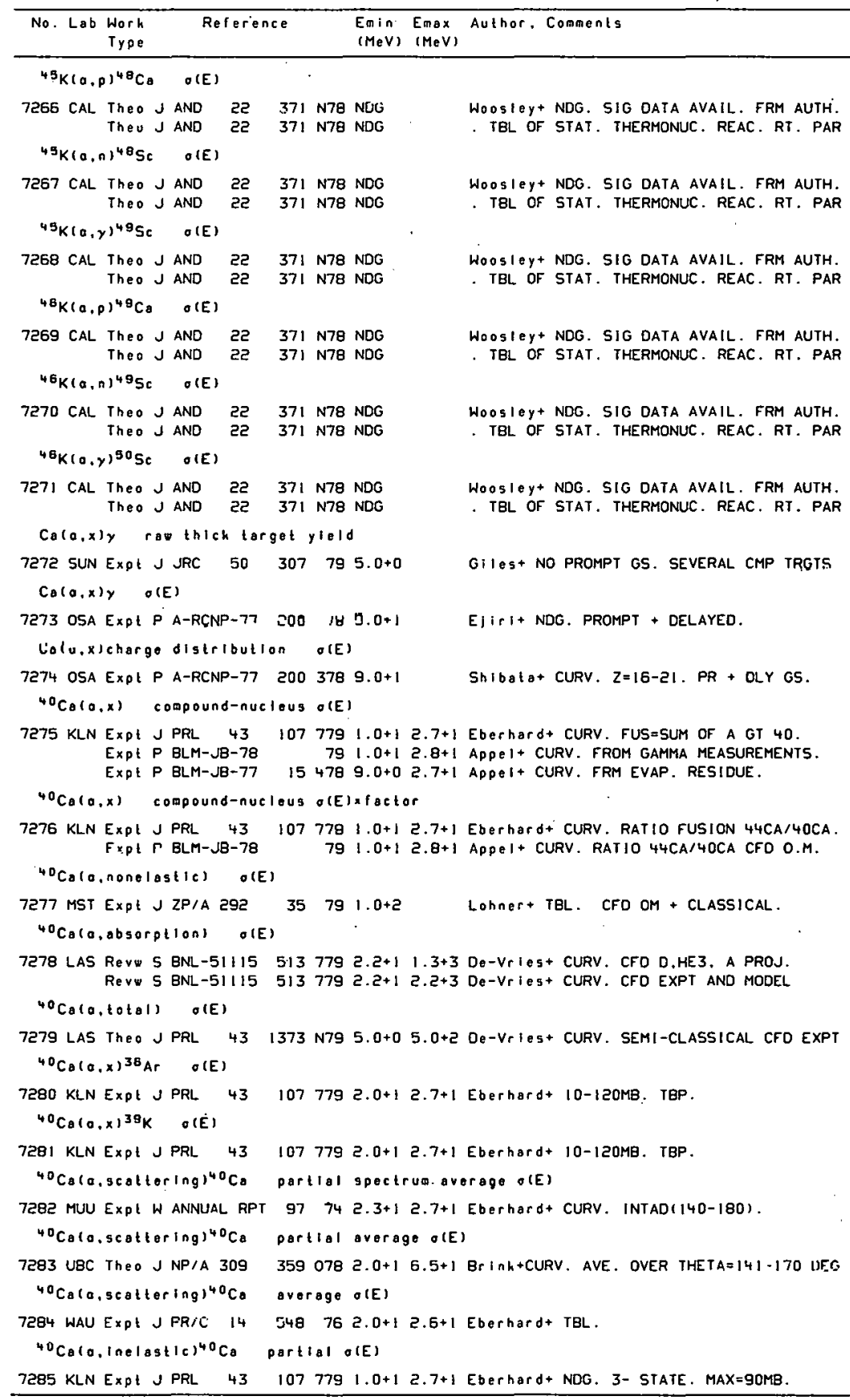




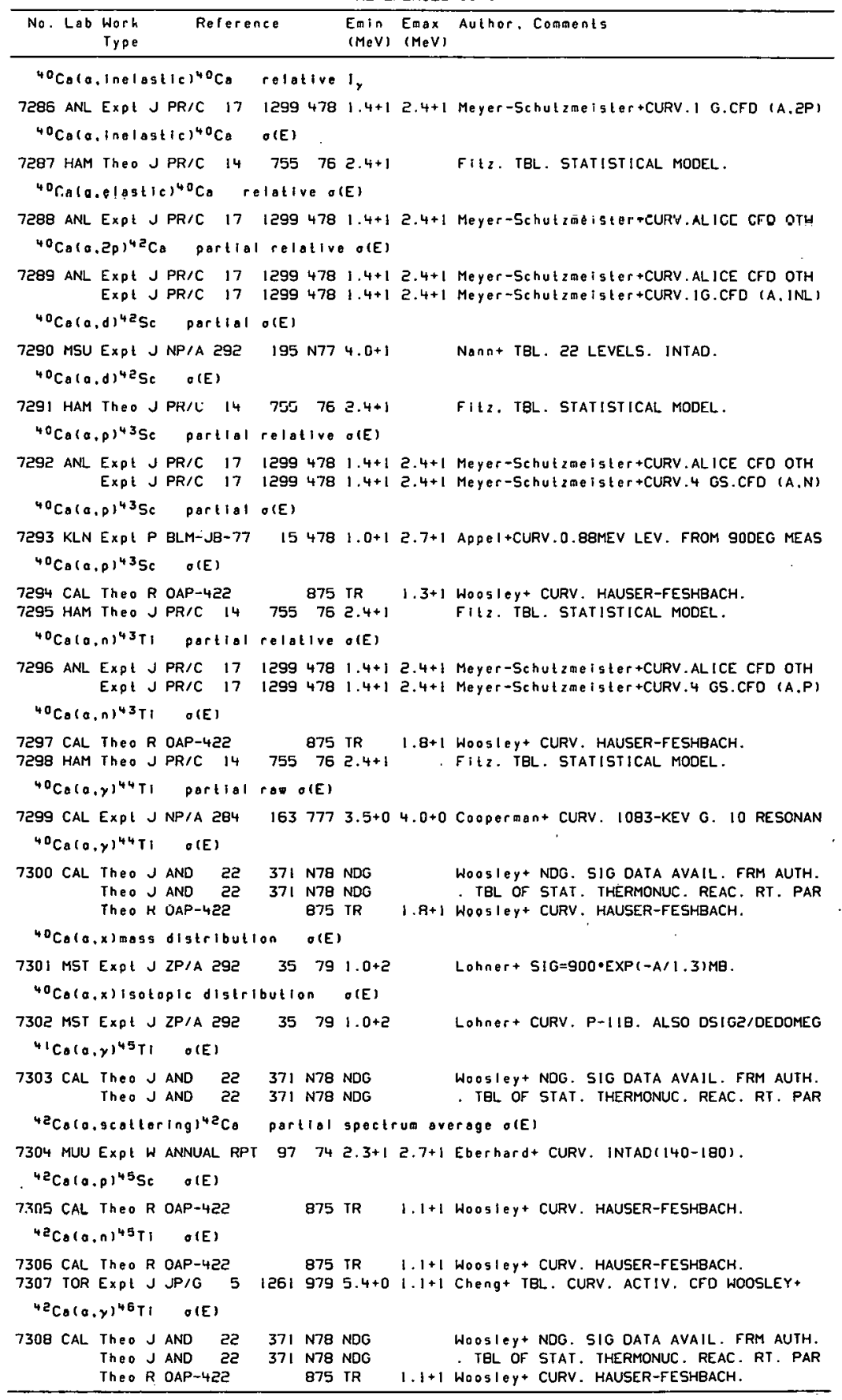




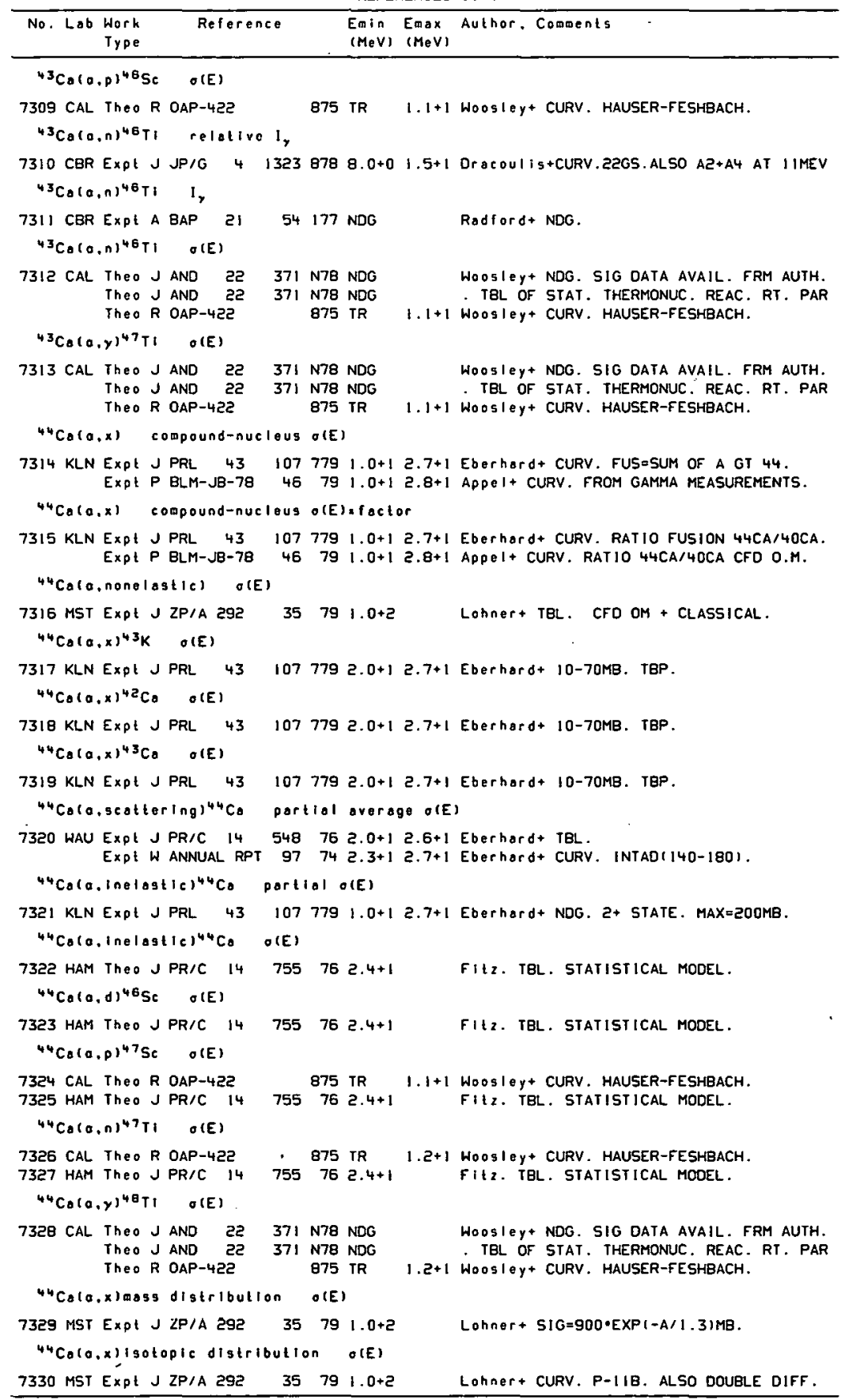


REFERENCES ( c on ()

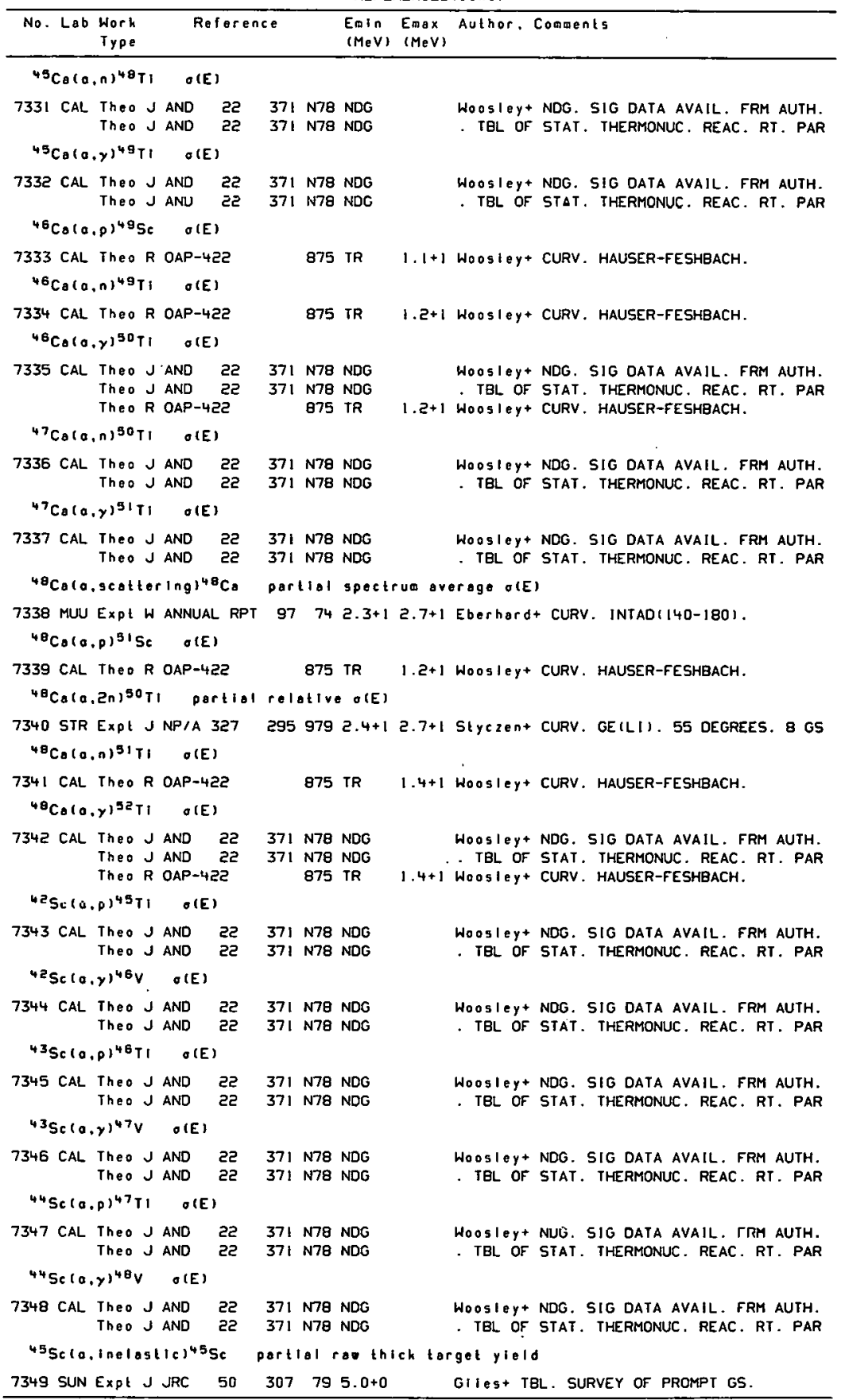


REFERENCES (cont)

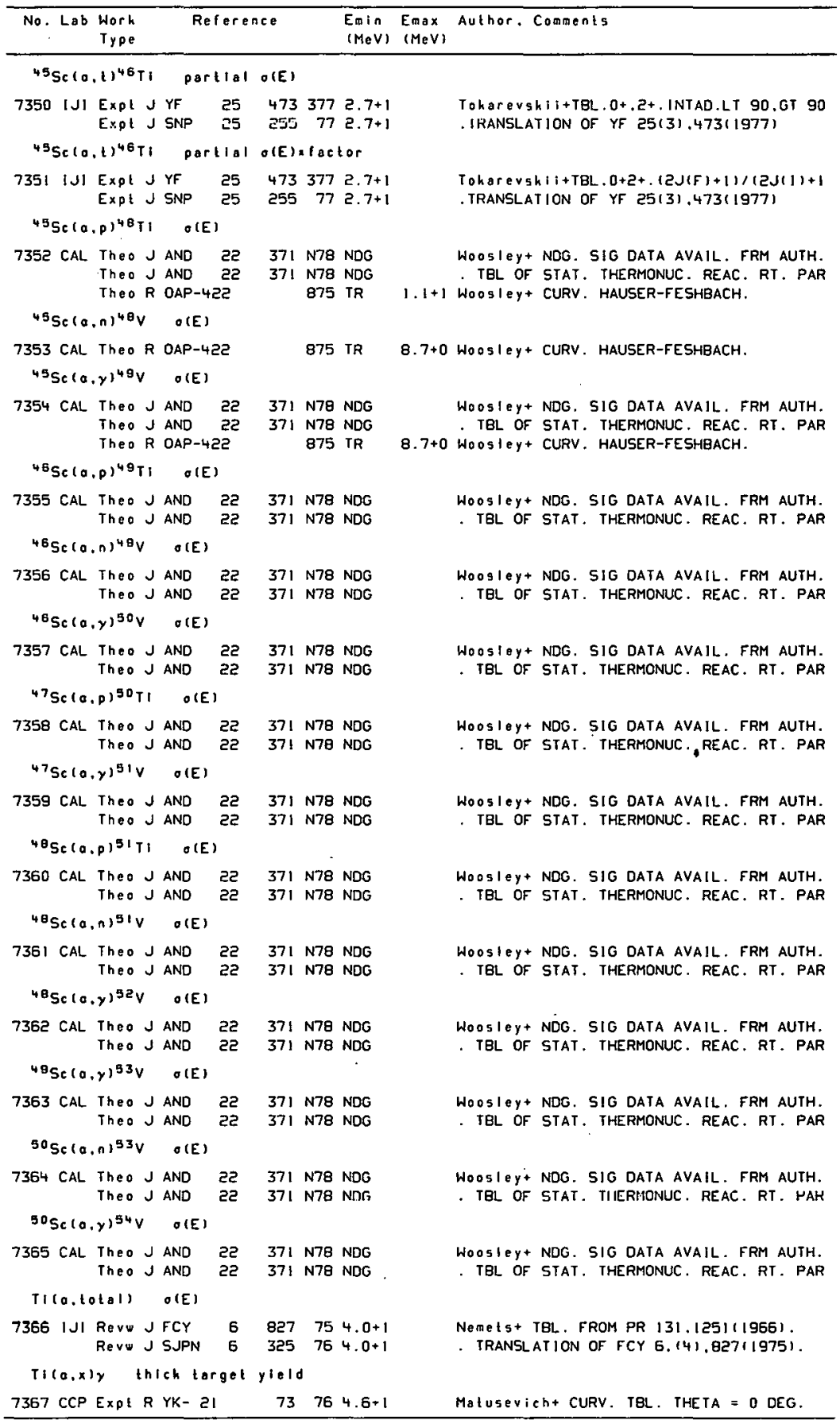




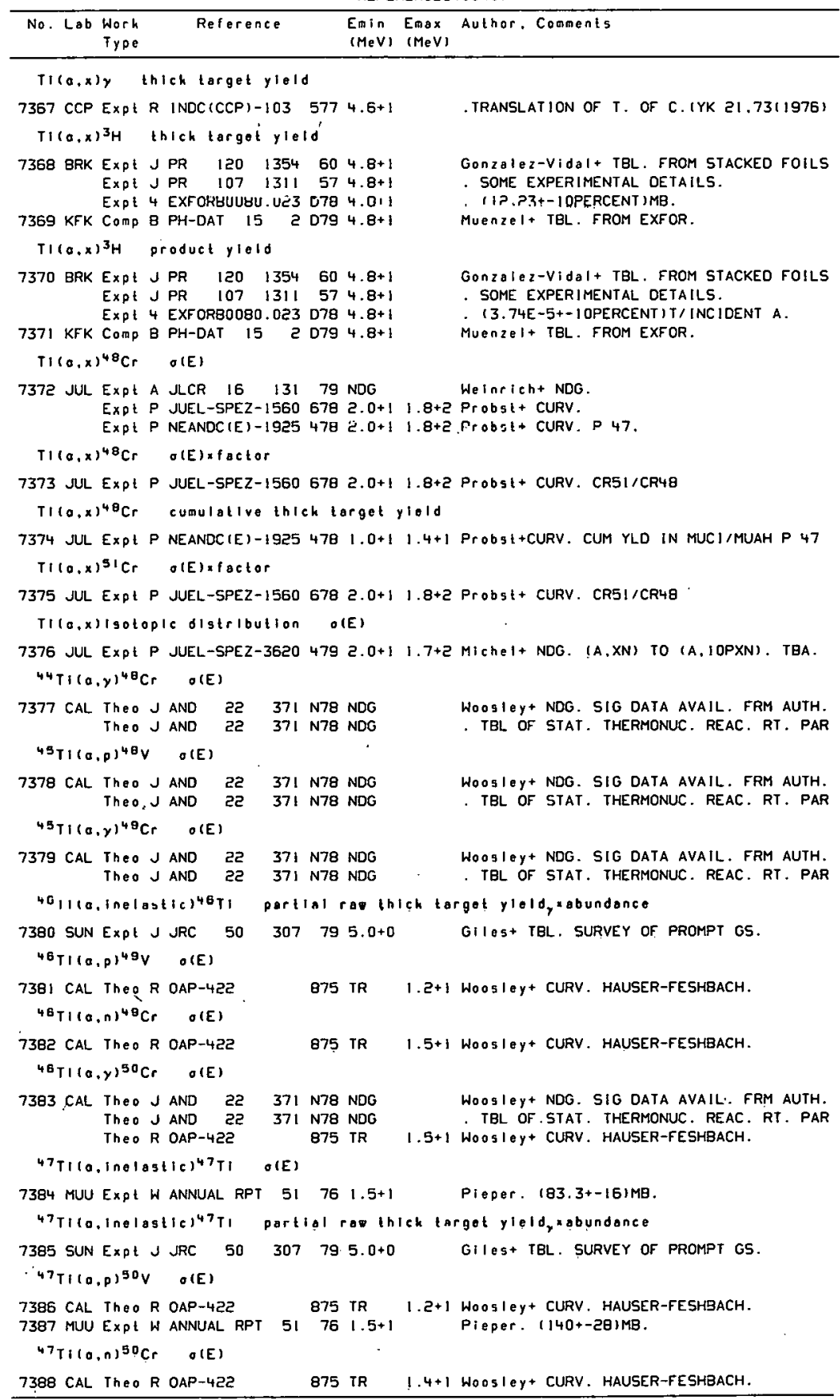




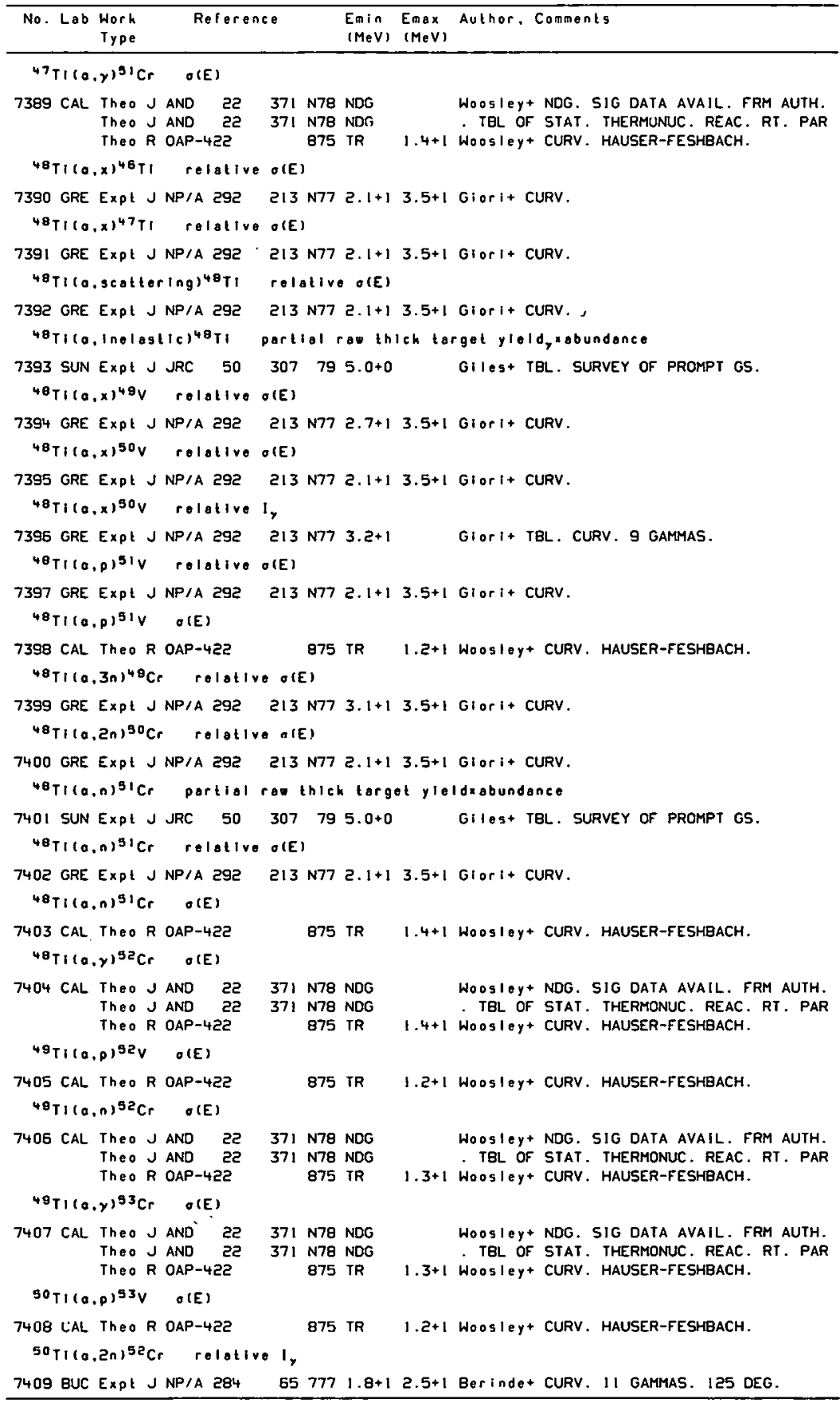


REFERENCES ( c on t)

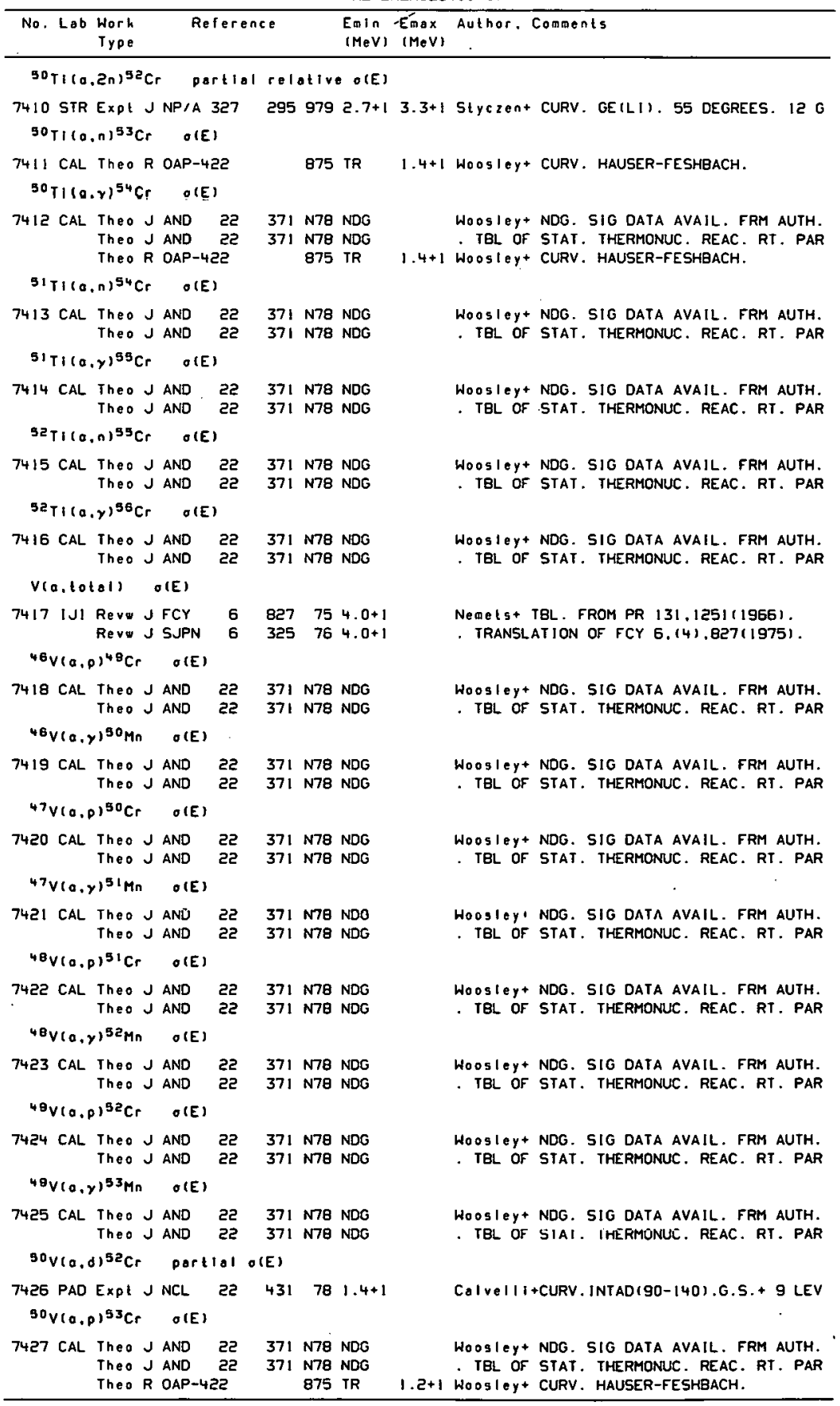


REFERENCES (c on ) )

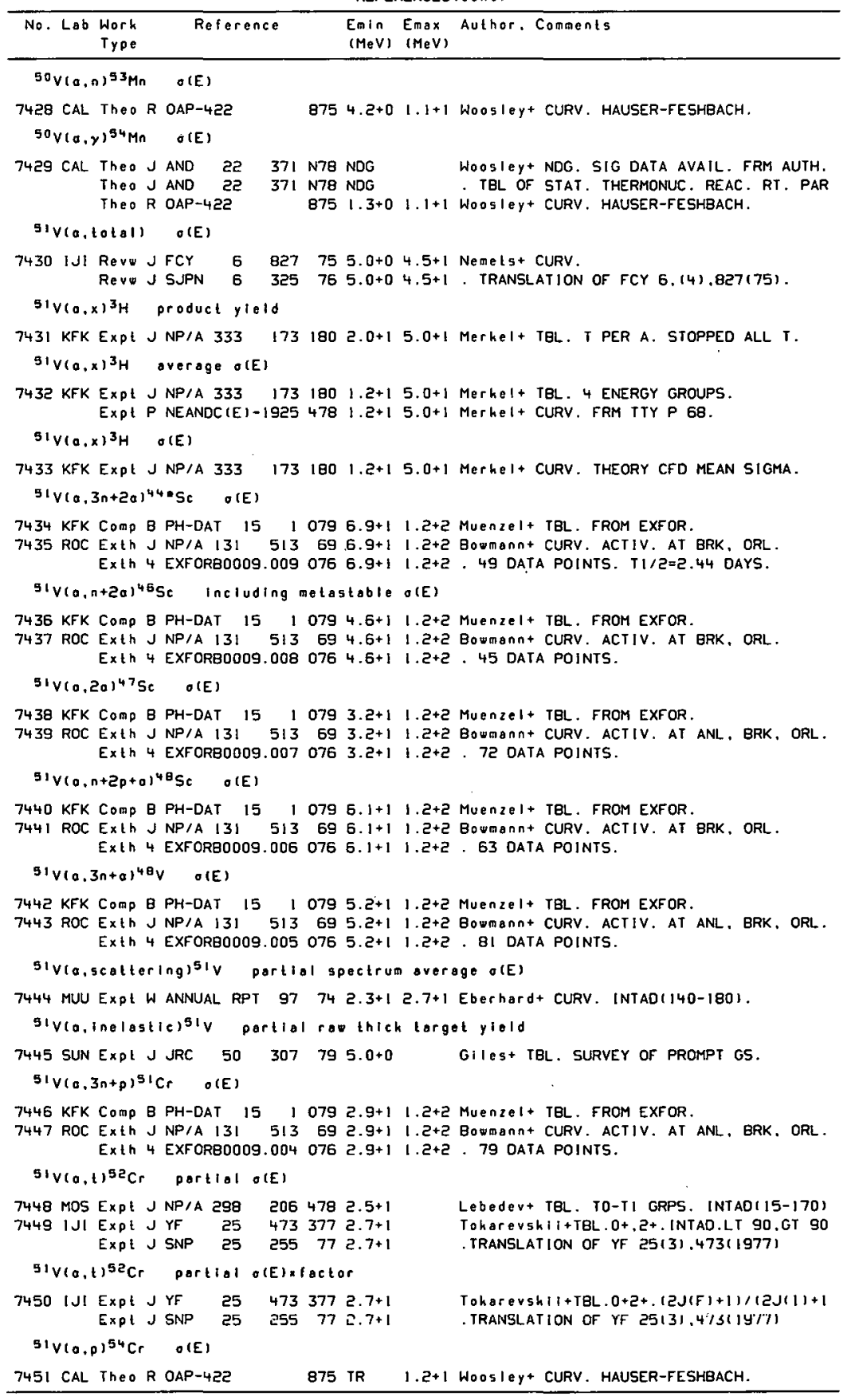


REFERENCES ( cont)

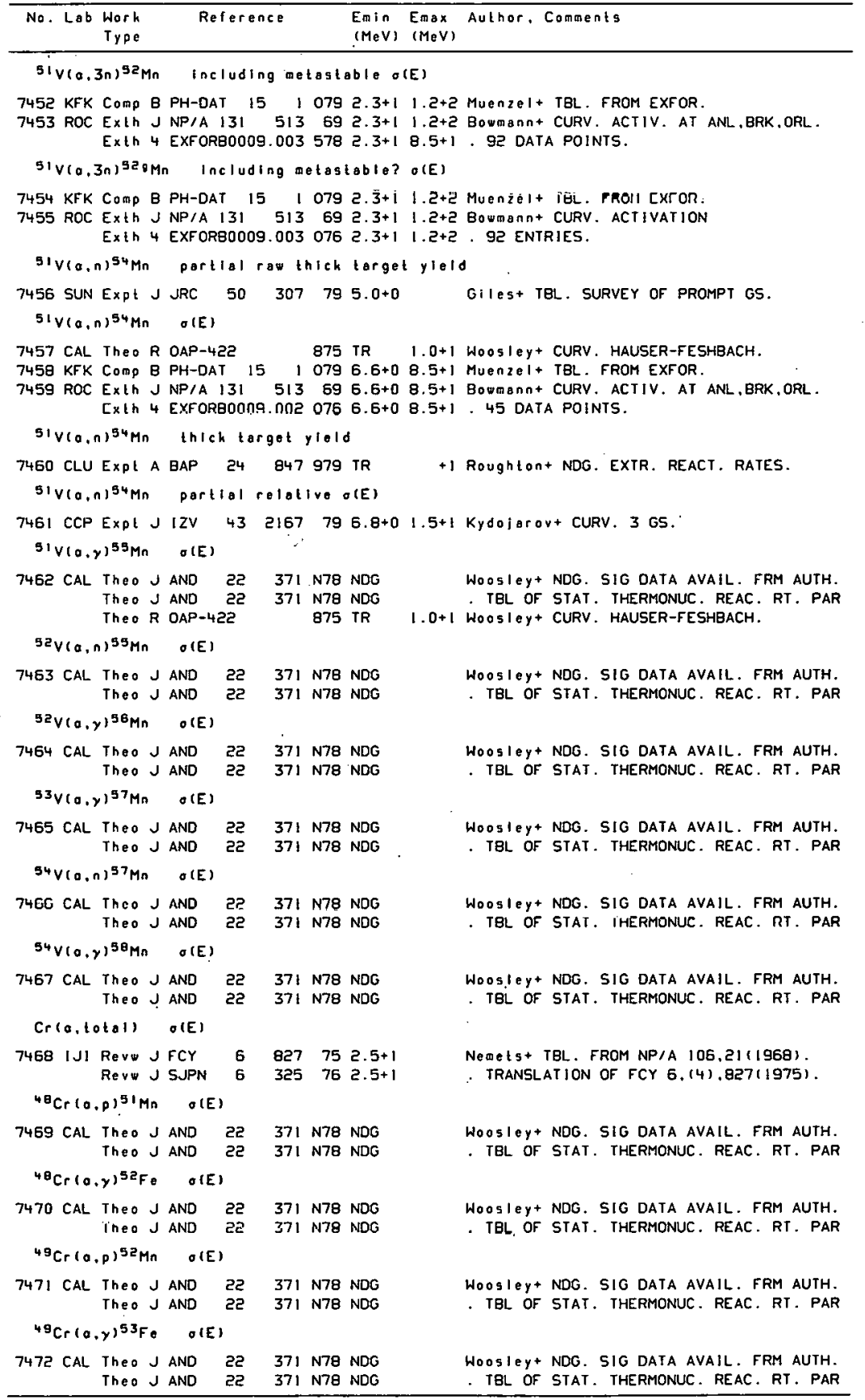


REFERENCES ( c on t)

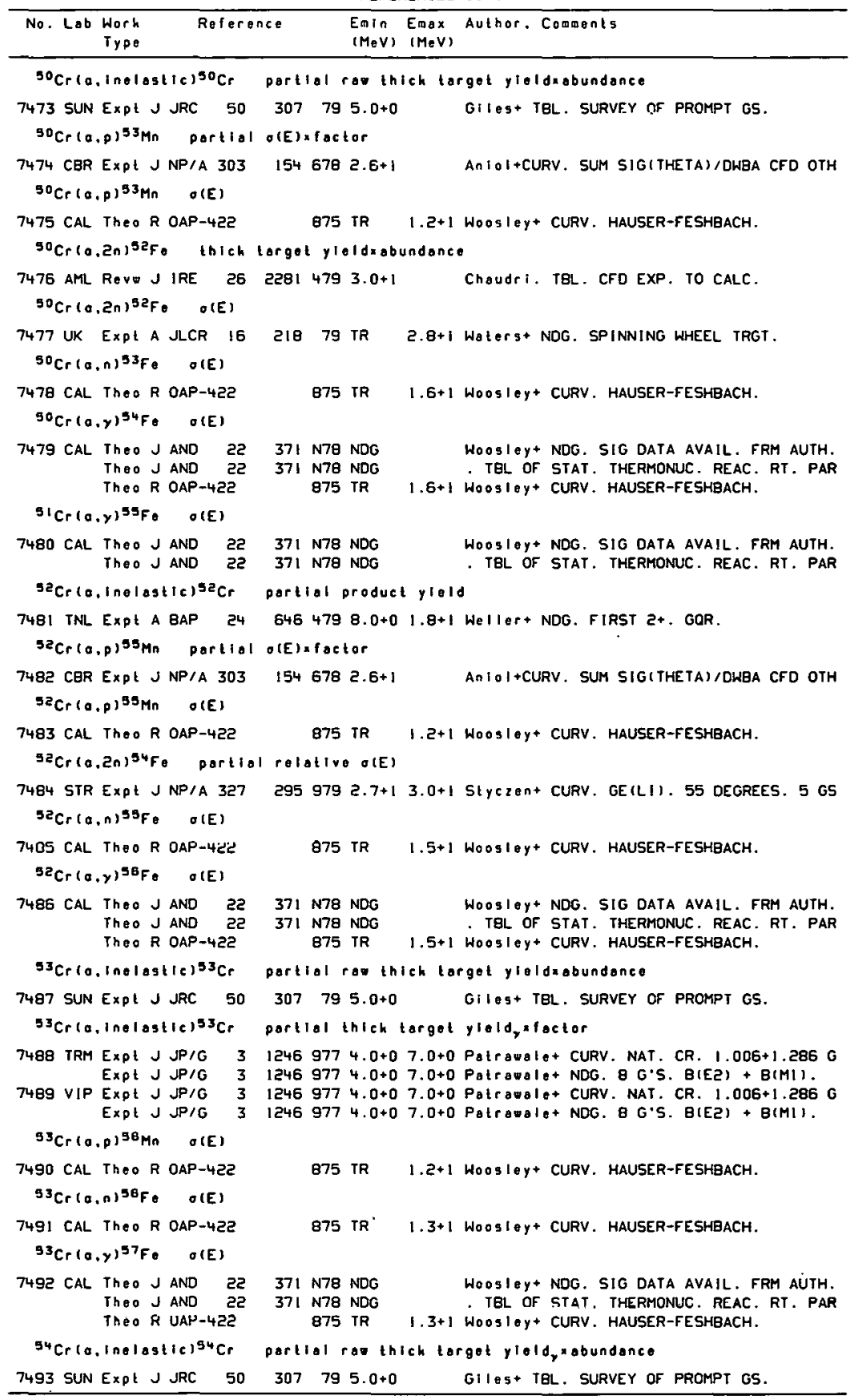




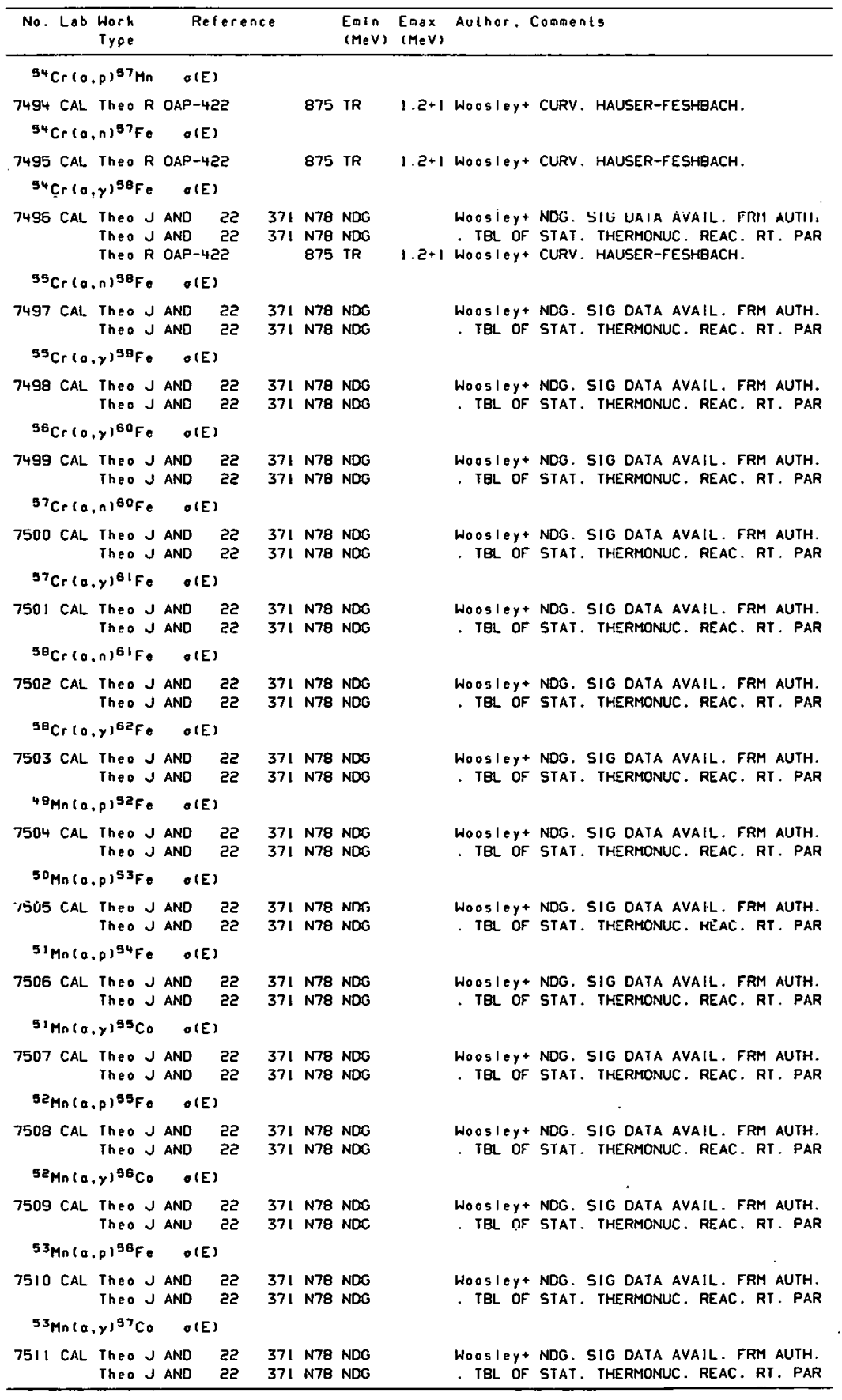




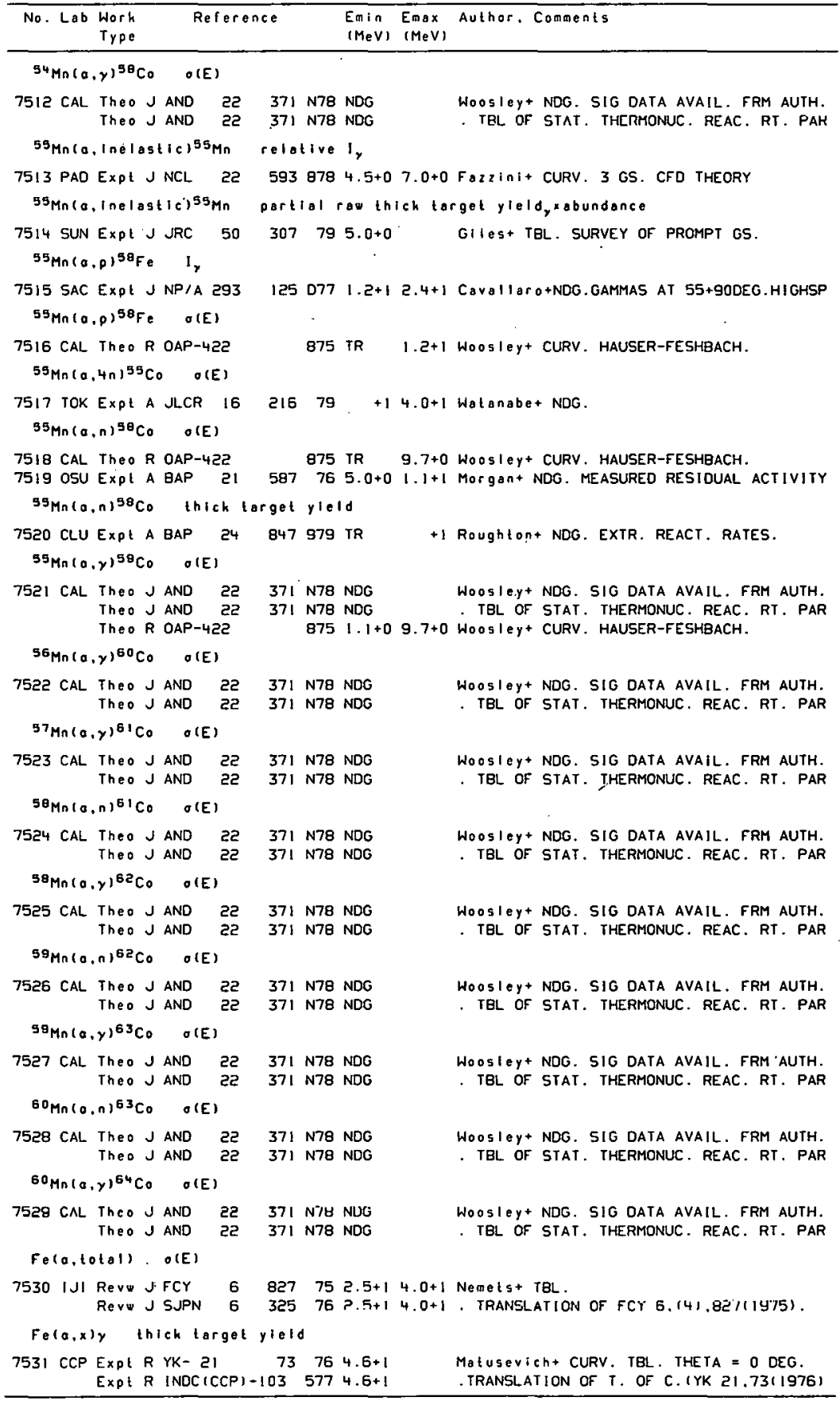




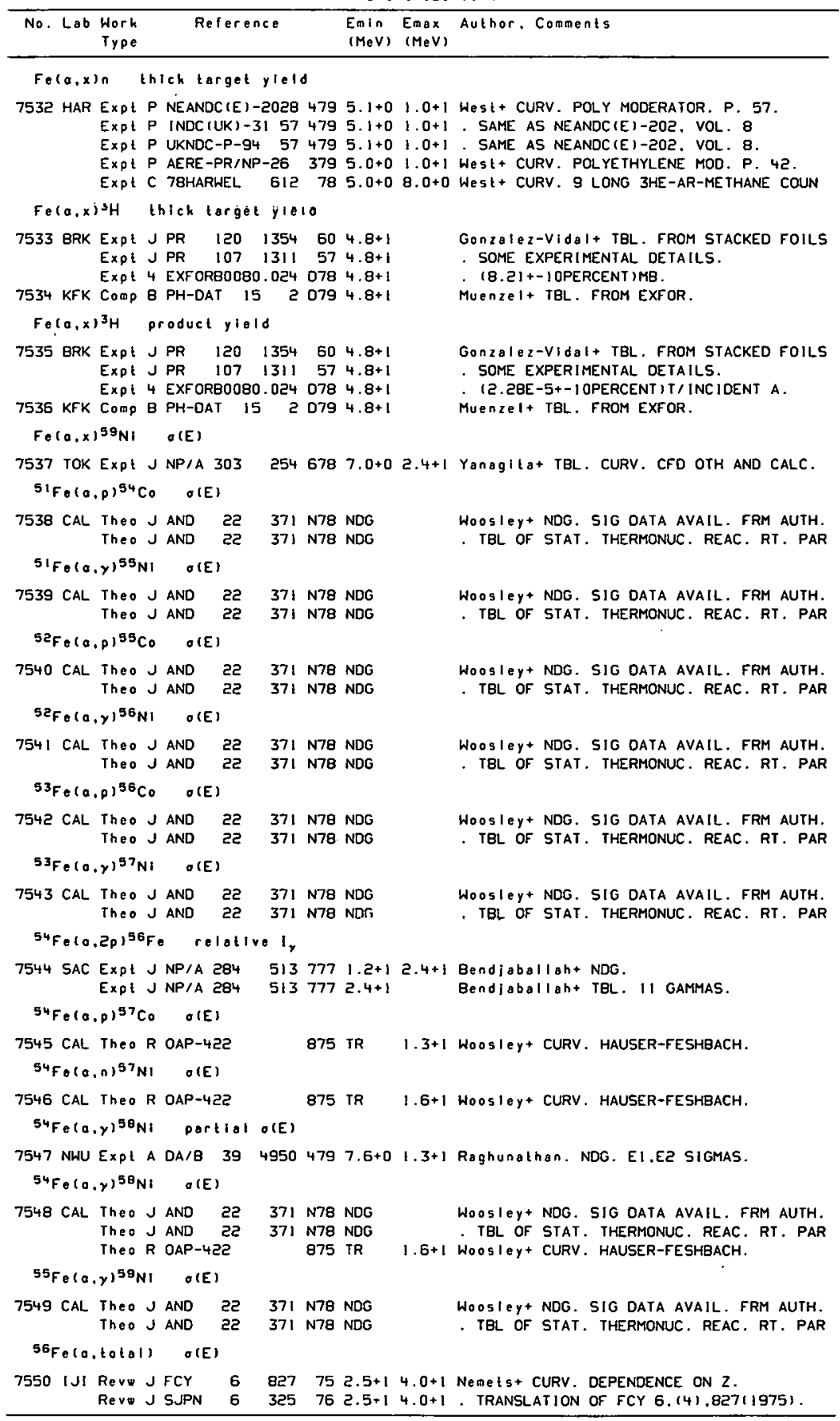


REFERENCES ( c on I )

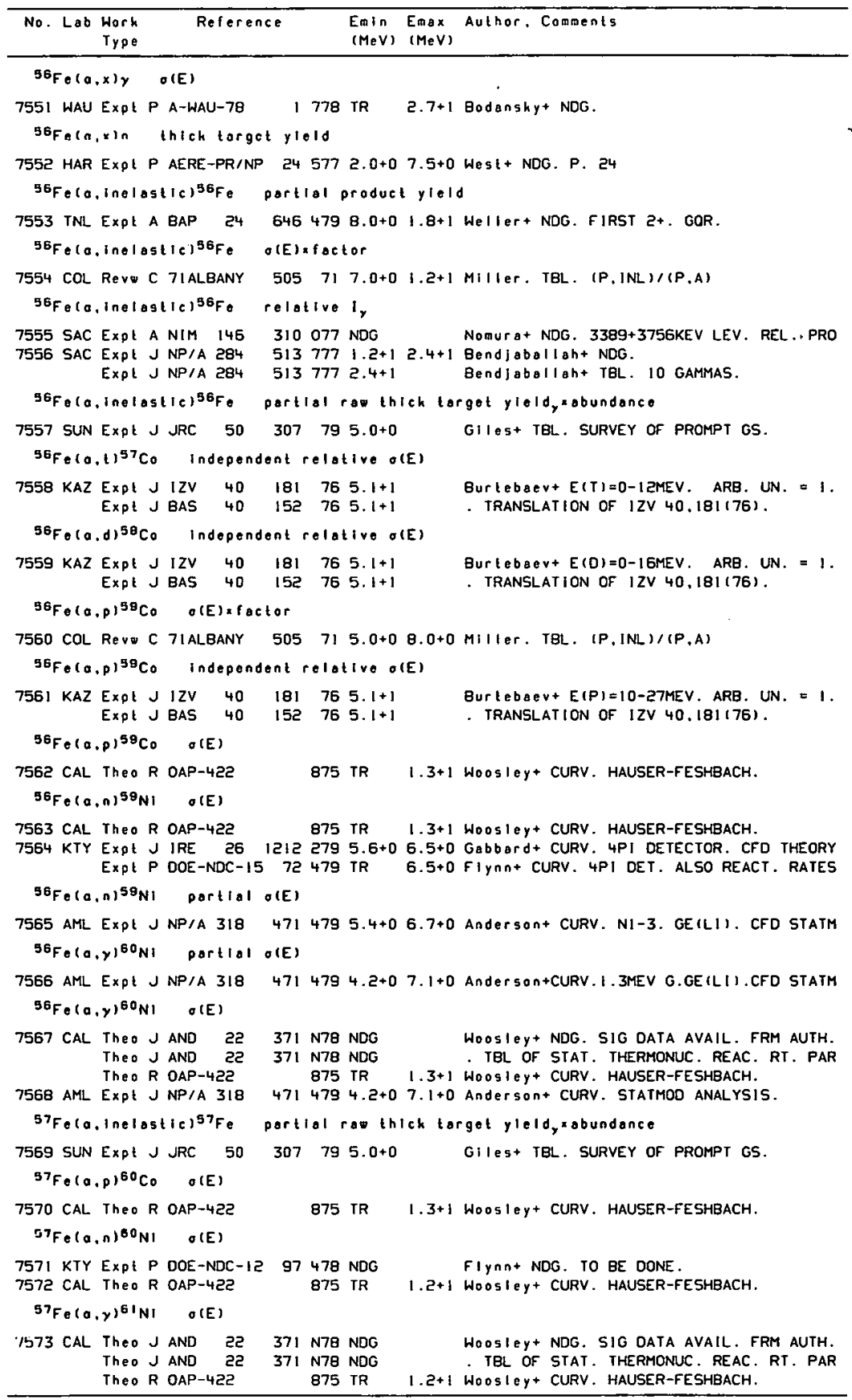


REFERENCES ( cont)

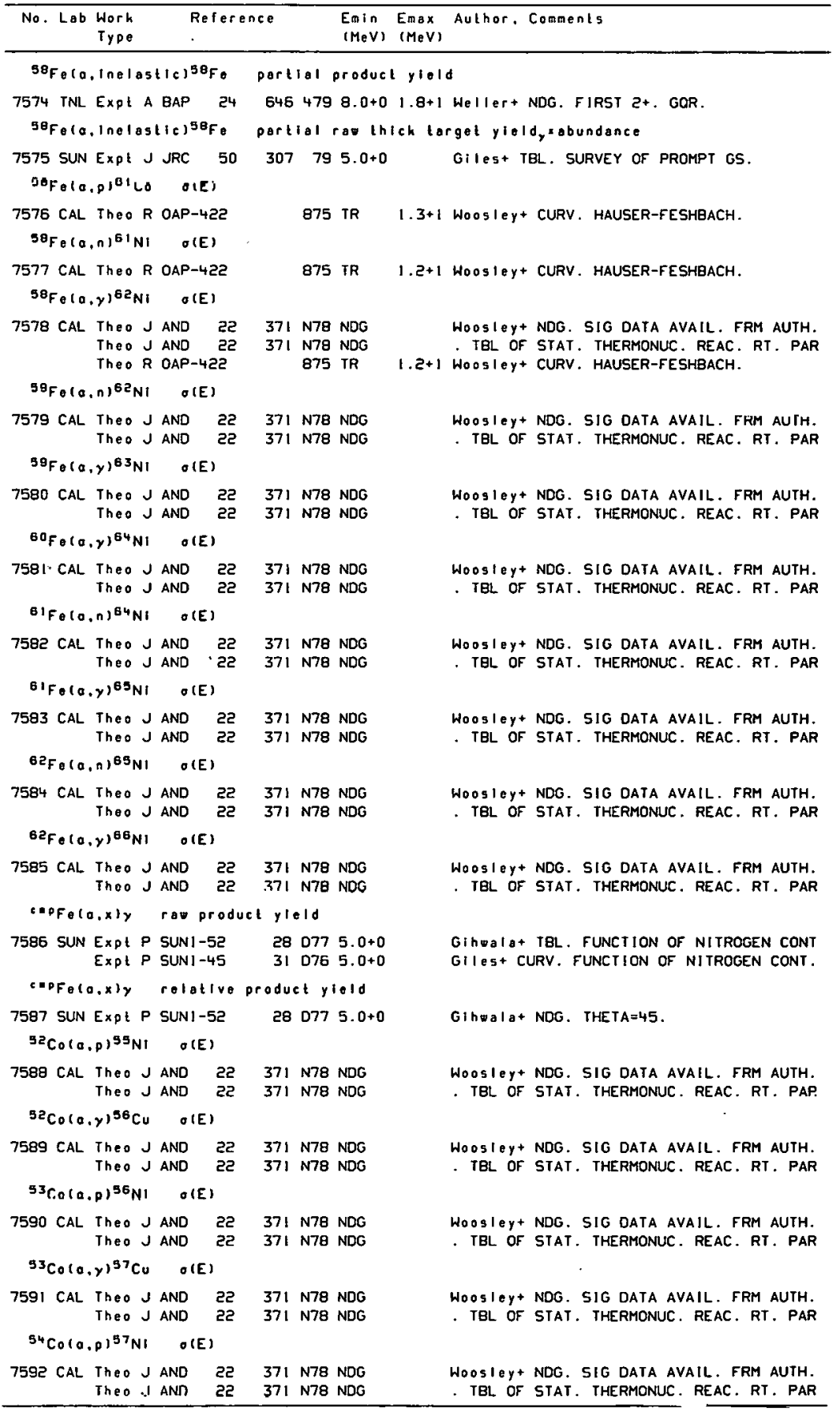




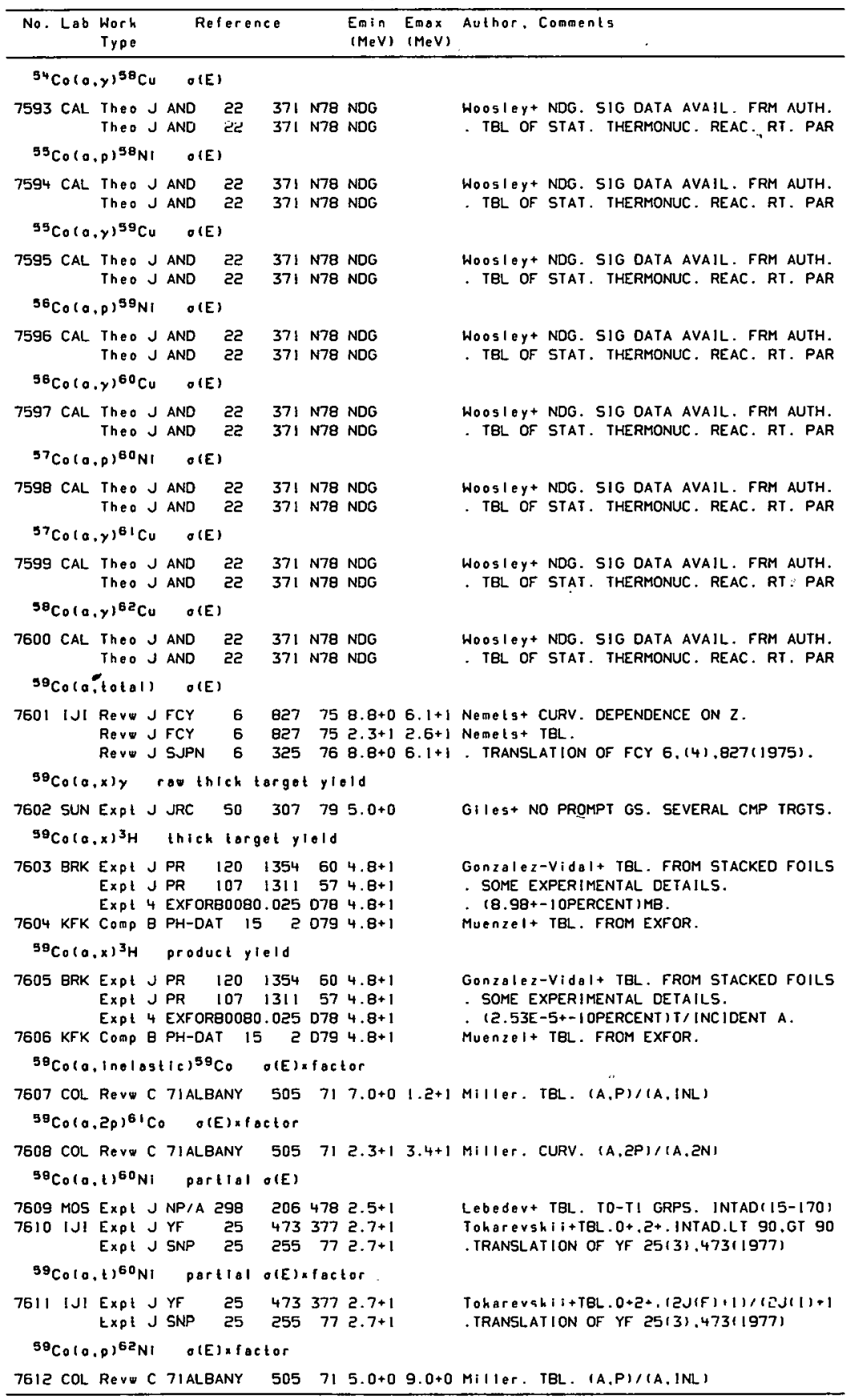


REFERENCES (cont)

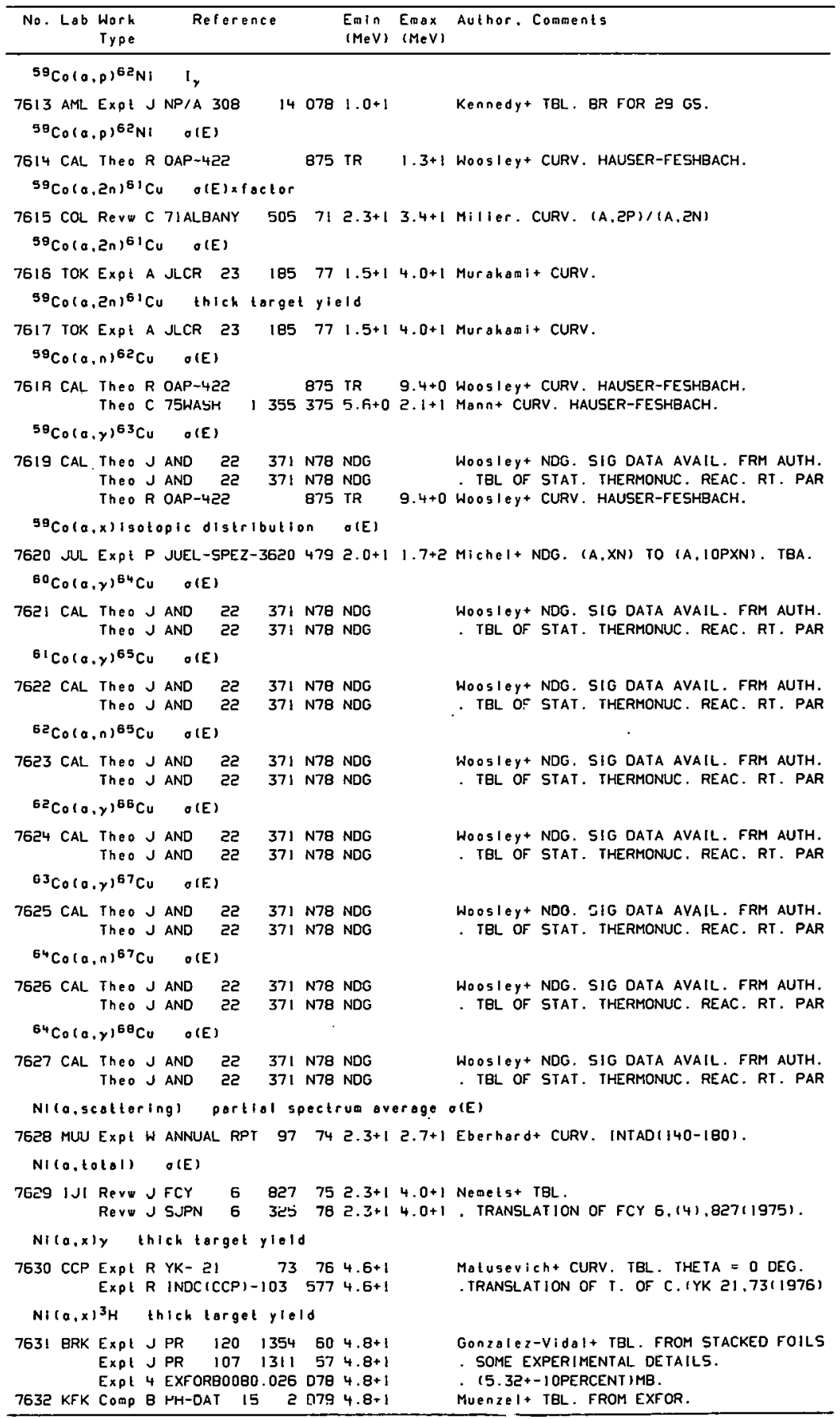


REFERENCES ( cont)

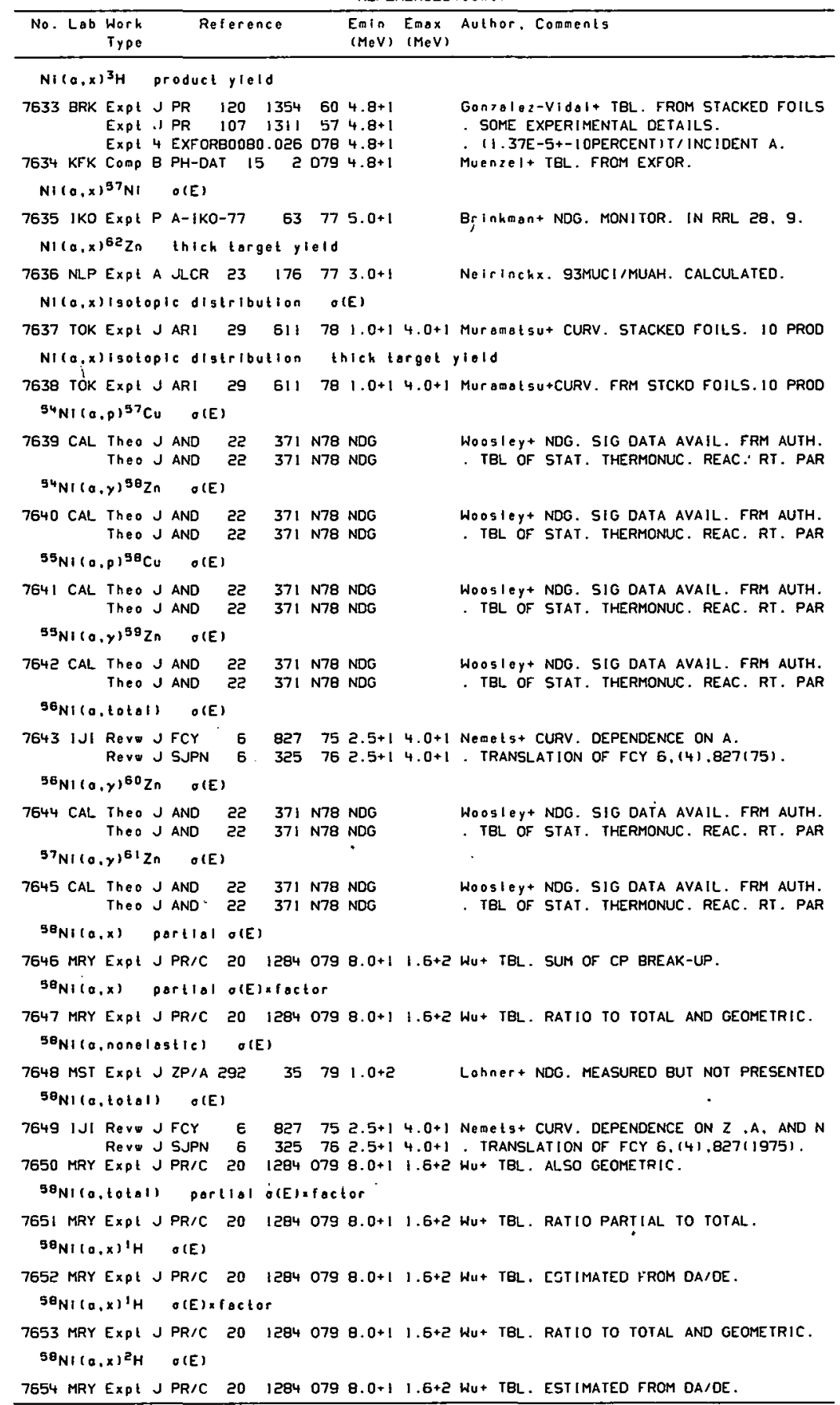




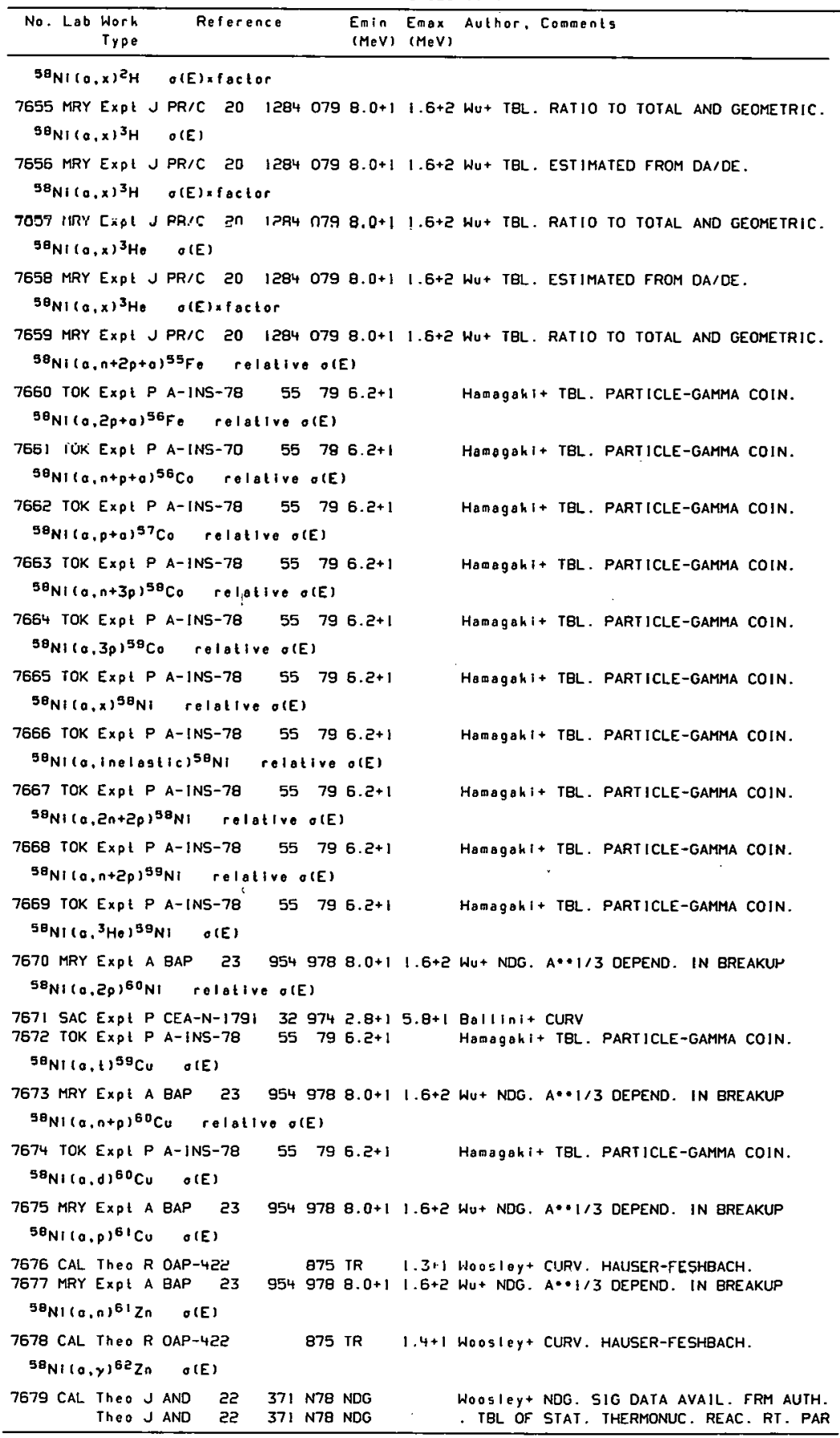




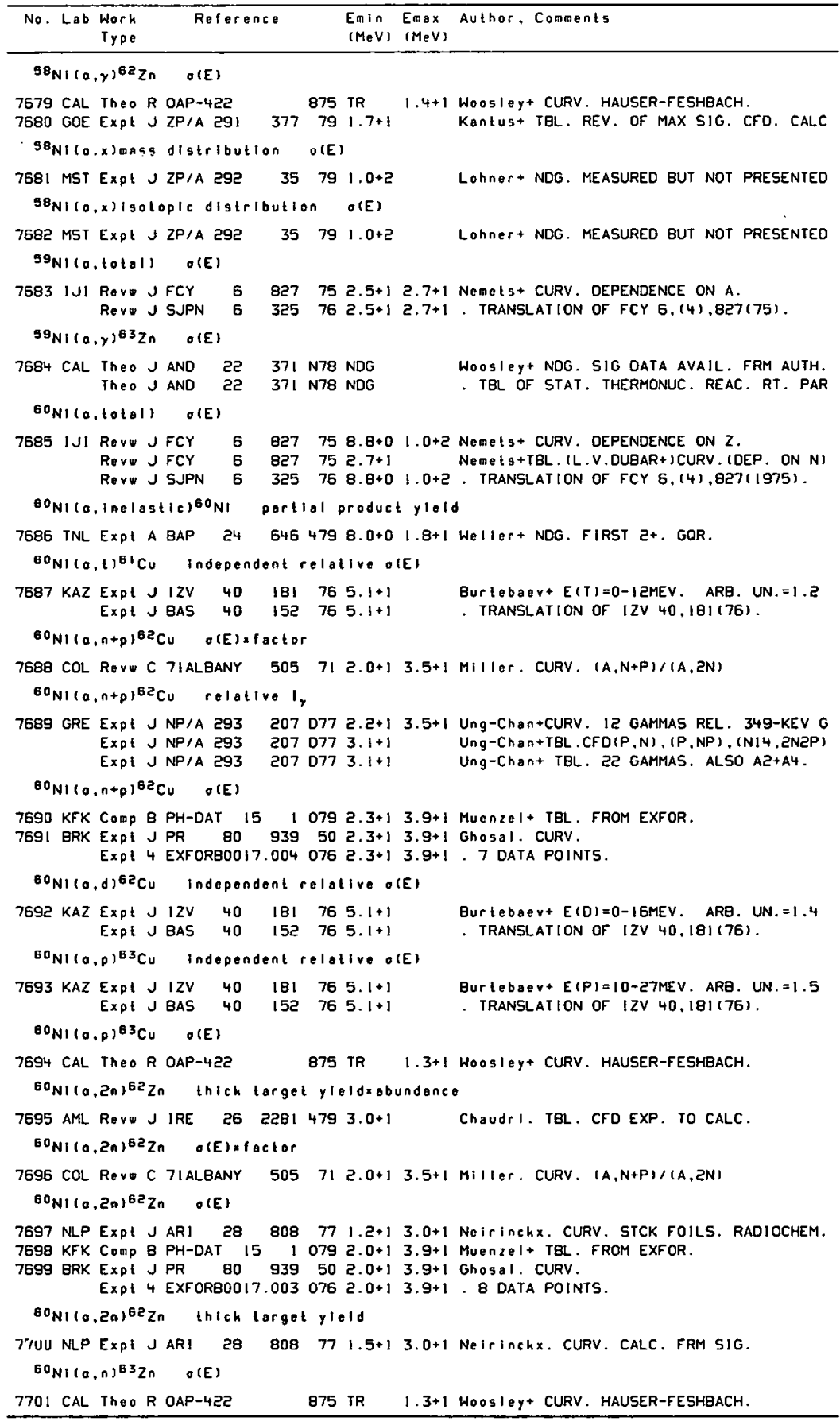




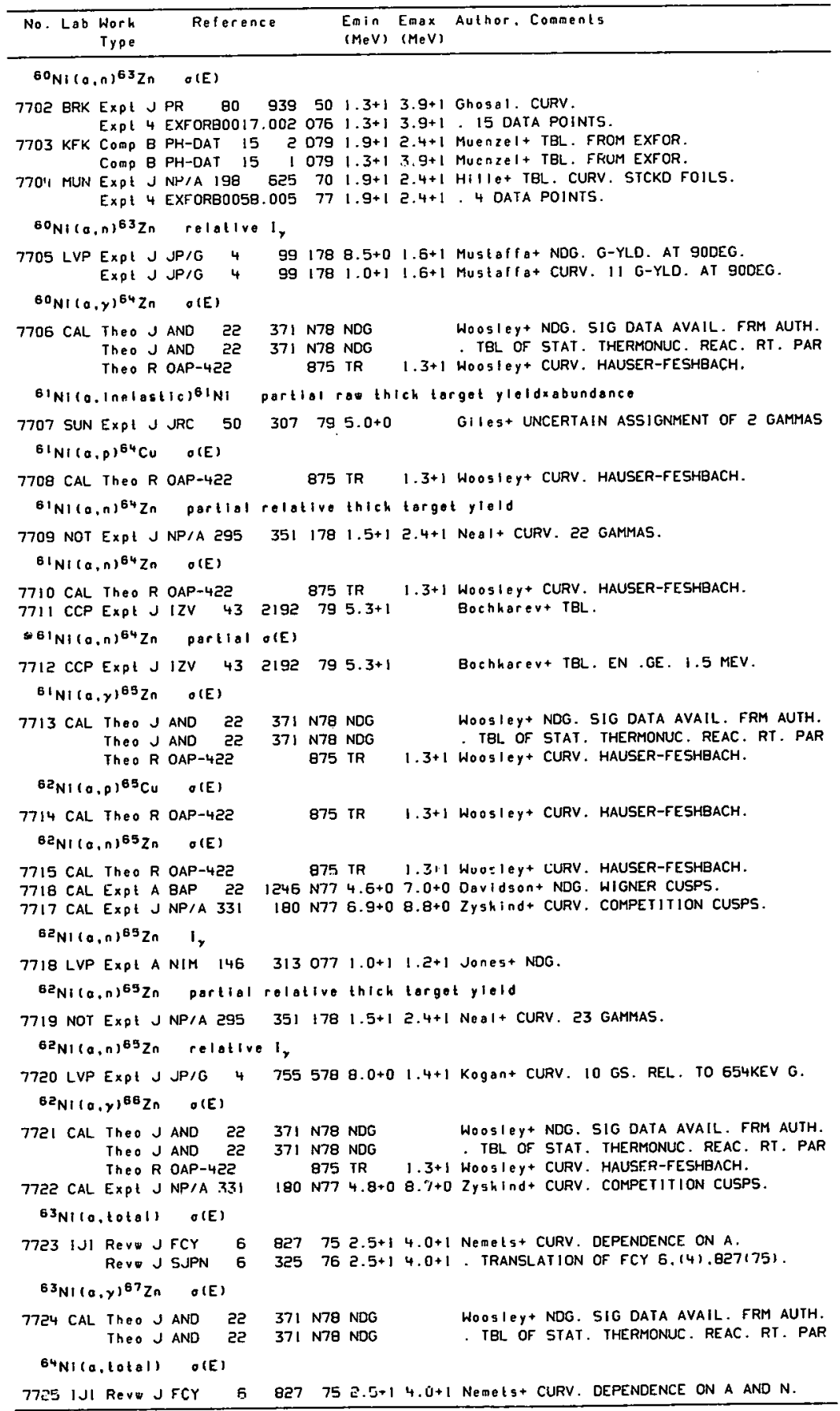


REFERENCES (cont)

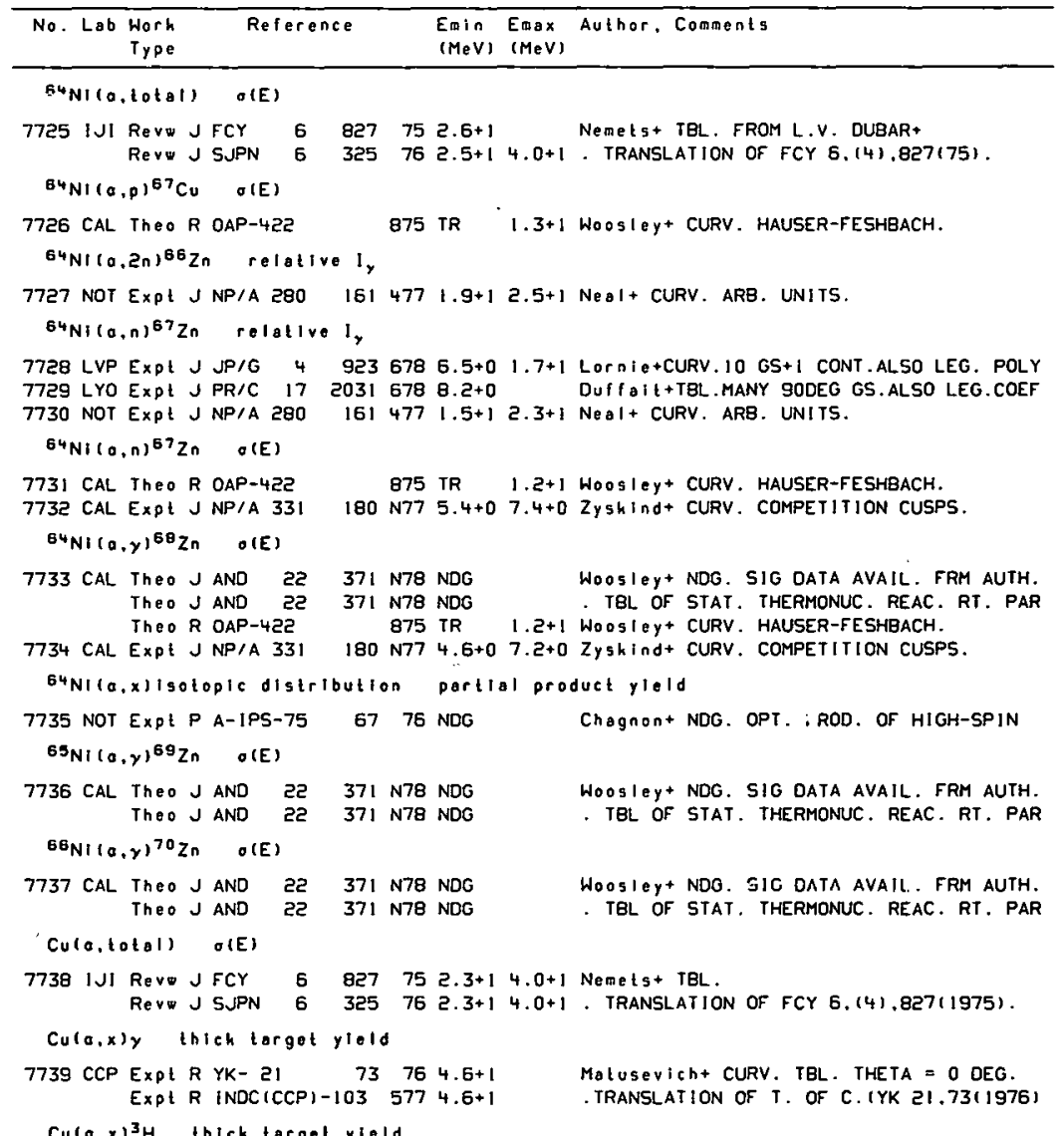

Cu $(0, x)^{3} H$ thick targot yiold

7740 BRK Expt JPR $120 \quad 1354 \quad 604.8+1$ $\begin{array}{llllll}\text { Expt J PR } 107 & 1311 & 57 & 4.8+1\end{array}$ Expt 4 EXFORBO080.027 $0784.8+1$ 7741 KFK Comp B PH-DAT 15 2 D79 $4.8+1$

Gonzalez-Vidal + TBL. FROM STACKED FOILS - SOME EXPERIMENTAL DETAILS.

19.57+- I OPERCENT )MB.

MuenzeI+ TBL. FROM EXFOR.

Cu $(0, x)^{3} H$ product yield

7742 BRK ExPE J PR $120 \quad 1354 \quad 604.8+1$ Exp: J PR $107 \quad 1311 \quad 574.8+1$ EXP ! 4 EXFORBO080.027 D78 $4.8+1$

Gonzalez-Vidal+ TBL. FROM STACKED FOILS . SOME EXPERIMENTAL DETAILS.

. (2.56E-5+-10PERCENT)T/INCIDENT A. 7743 KFK Comp 8 PH-OAT 152 D79 $4.8+1$ Muenzel+ TBL. FROM EXFOR.

Cu $(a, x)^{66}$ Ga thick target yield

7744 UJV Comp R U.JF-4199-8 277 B.0+0 3.0+1 Bem+ TBL.

Cu(o,x)67Ga thick target yield

7745 UJV Comp R UJF-4199-B $2771.6+1 \quad 3.0+1$ Ben+ TBL.

${ }^{56} \mathrm{Cu}(a, p)^{59} \mathrm{Zn} \quad \sigma(E)$

$7745 \mathrm{CAL}$ Theo J AND $22 \quad 371$ N70 NDG Theo J AND $22 \quad 371$ N78 NDG

Woosley + NDG. SIG DATA AVAIL. FRM AUTH.

${ }^{56} \mathrm{Cu}(\mathrm{c} . y)^{60} \mathrm{Ga}$ O(E) $\begin{array}{rlll}7747 \text { CAL Theo J AND } & 22 & 371 \text { N79 NDG } & \text { WOOSI YY NDG. SIG DATA AVAIL. FRM AUTH. } \\ \text { TheO J AND } 22 & 371 \text { N78 NDG } & \text {. TBL OF STAT. THERMONUC. REAC. RI. PAR }\end{array}$ - TBL OF STAT. THERMONUC. REAC. RI. PAR 
REFERENCES (cont)

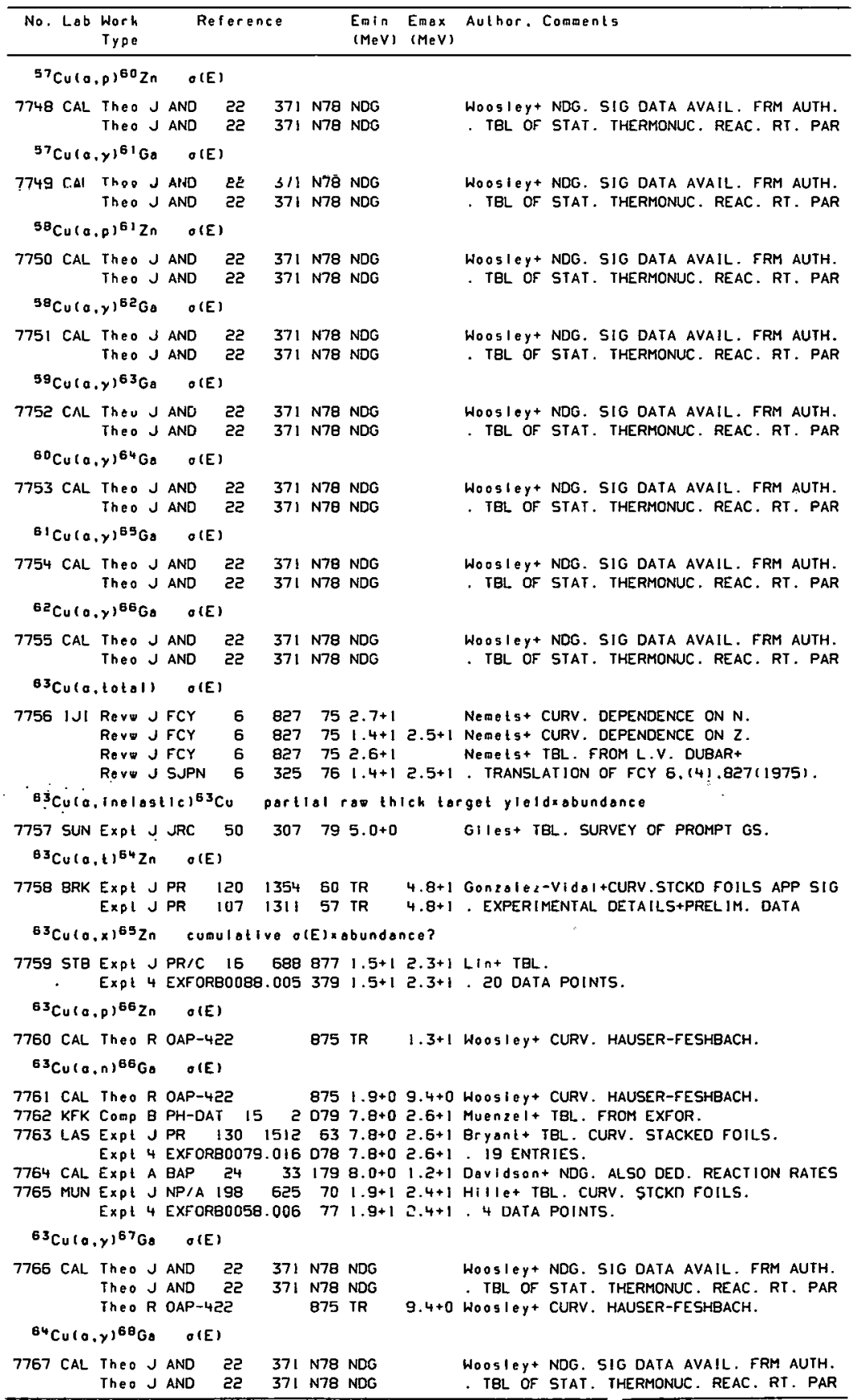


REFERENCES i con ( )

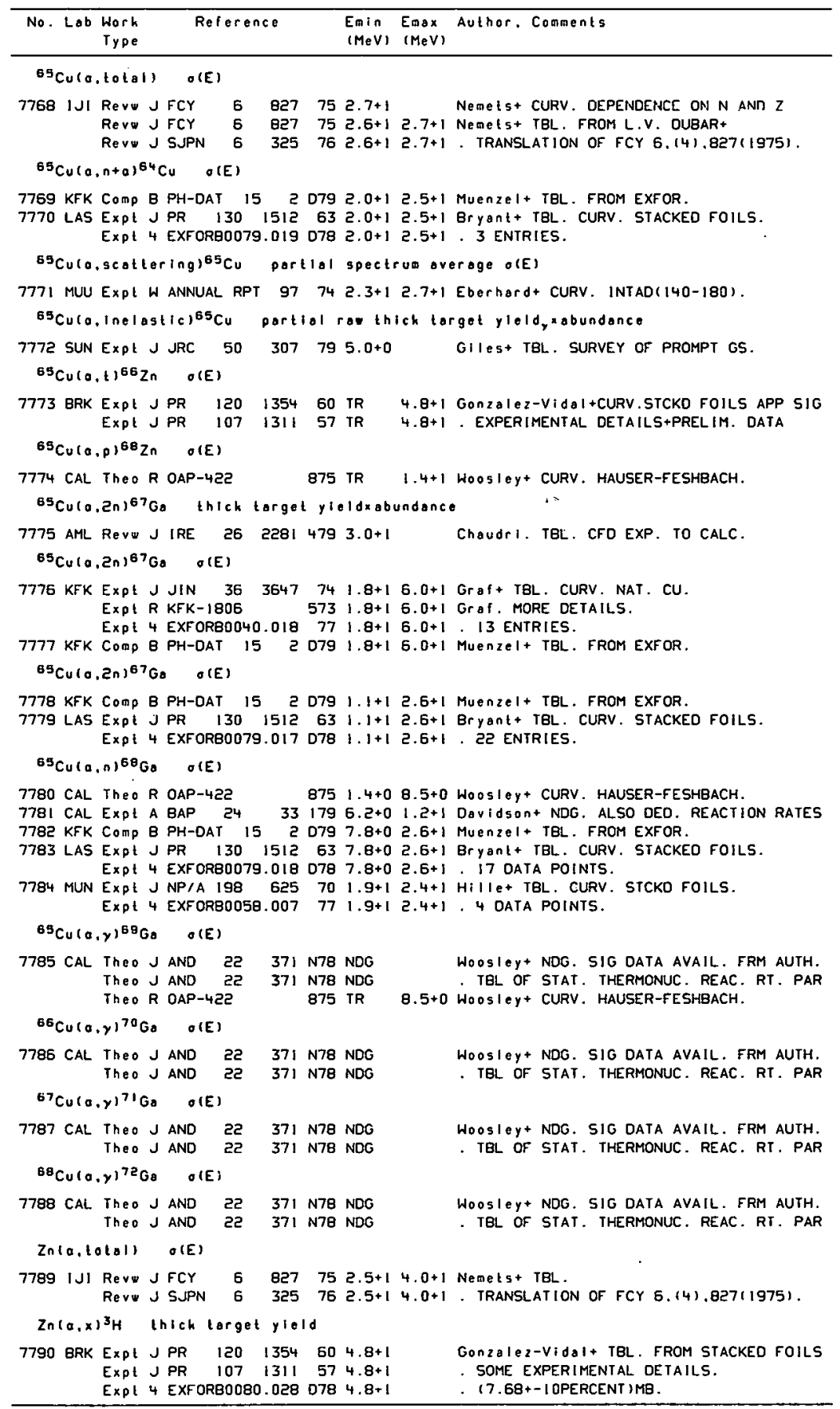


REFERENCES ( c on t)

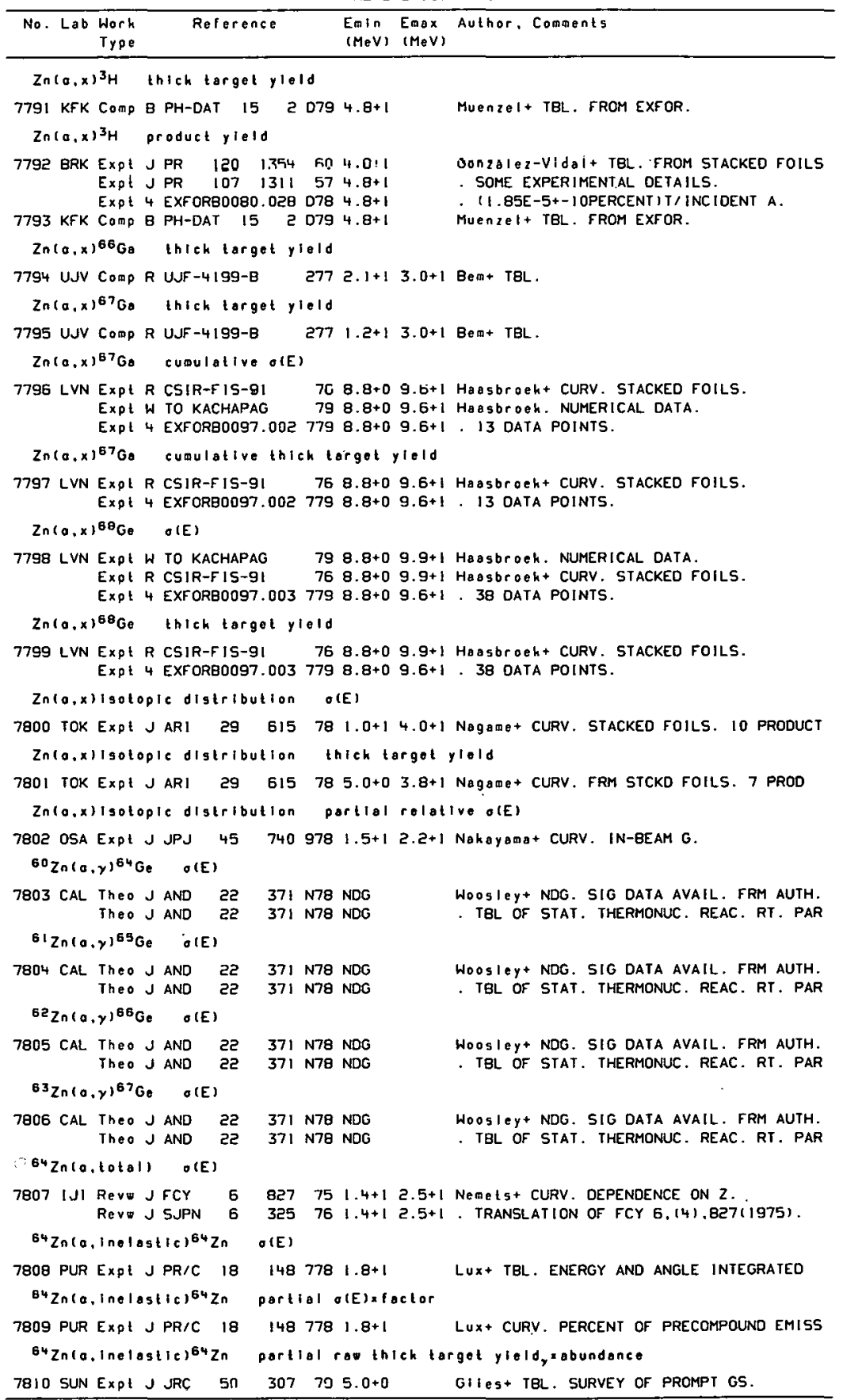


REFERENCES ( c on t )

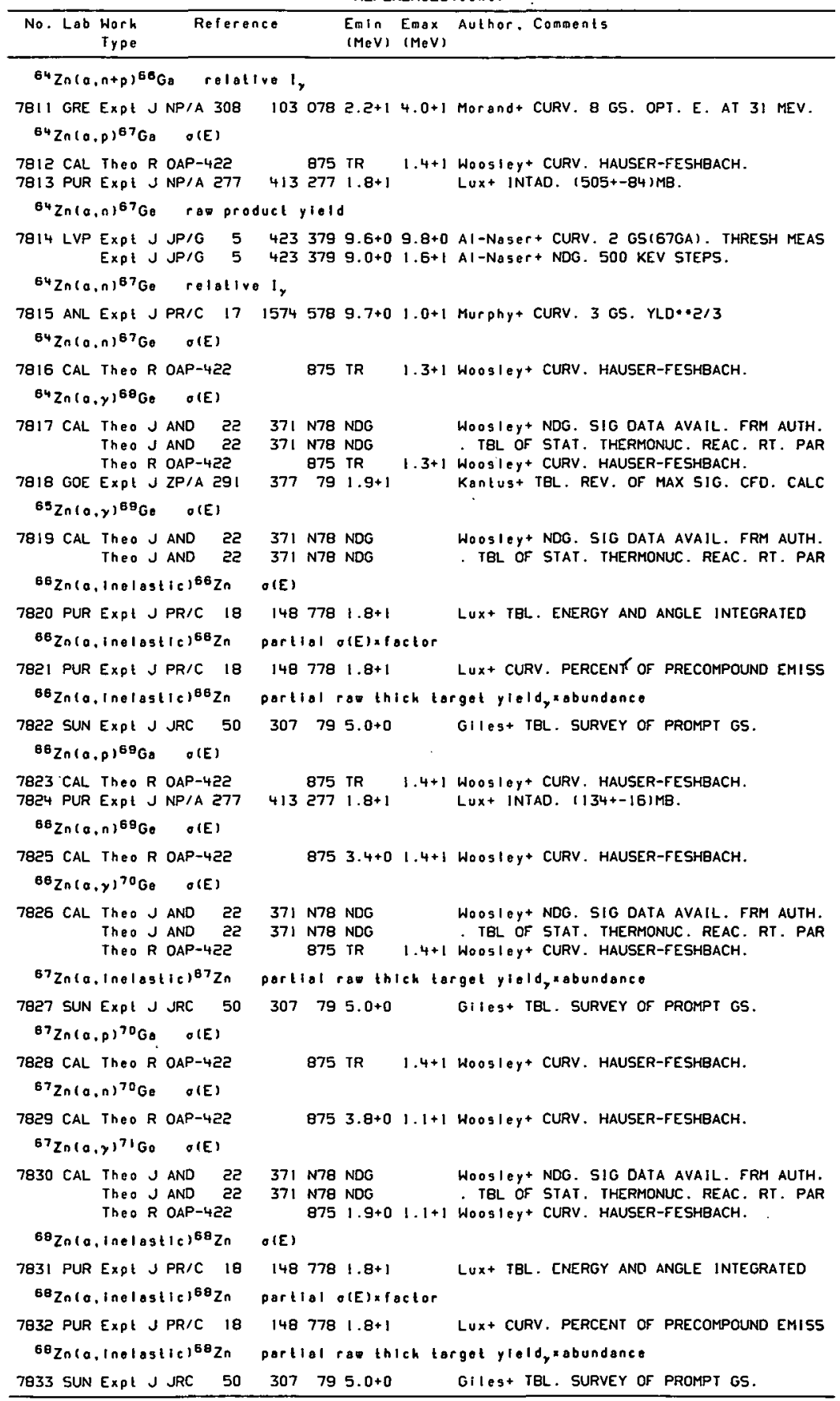


REFERENCES (c ont)

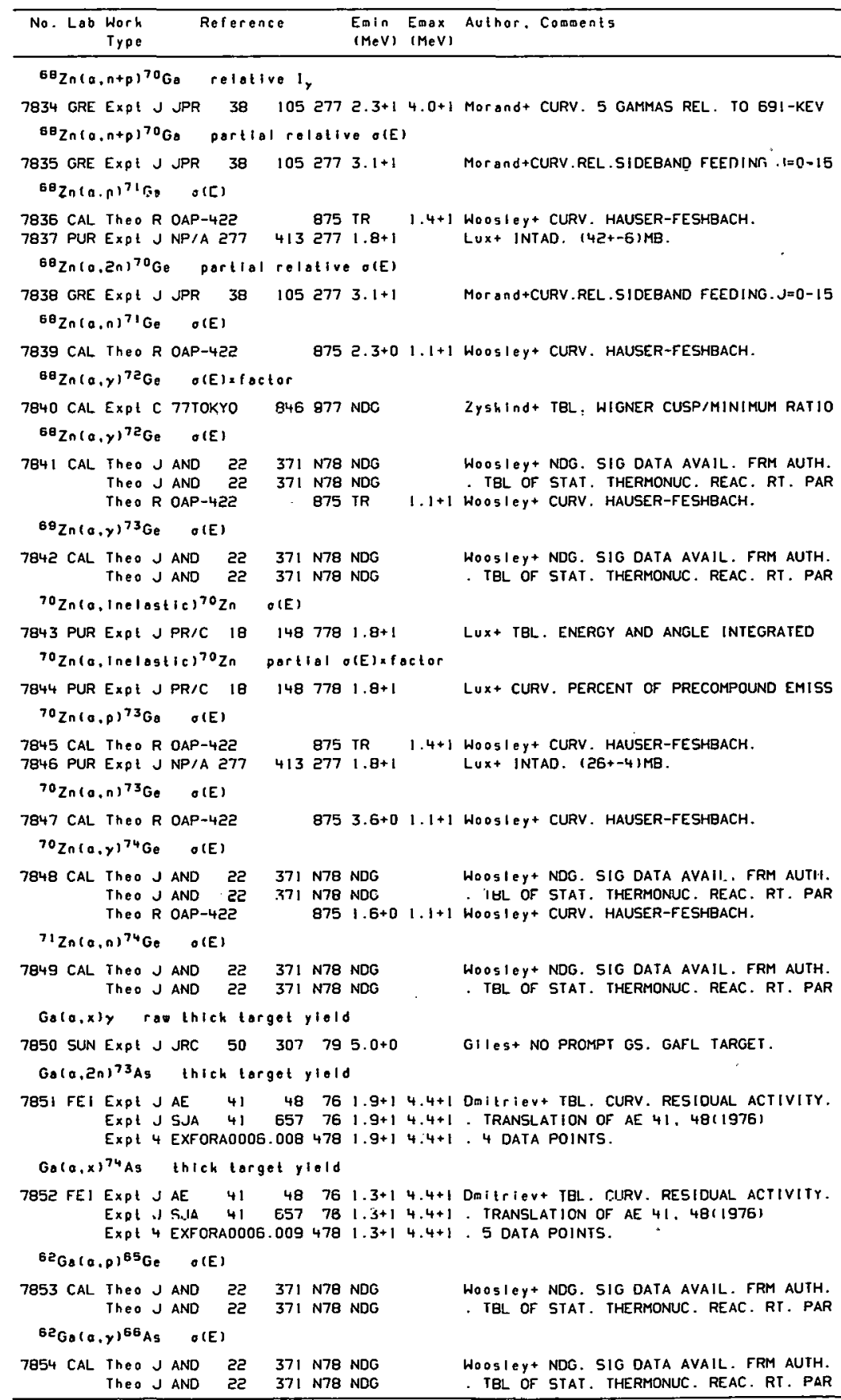


REFERENCES ( cont)

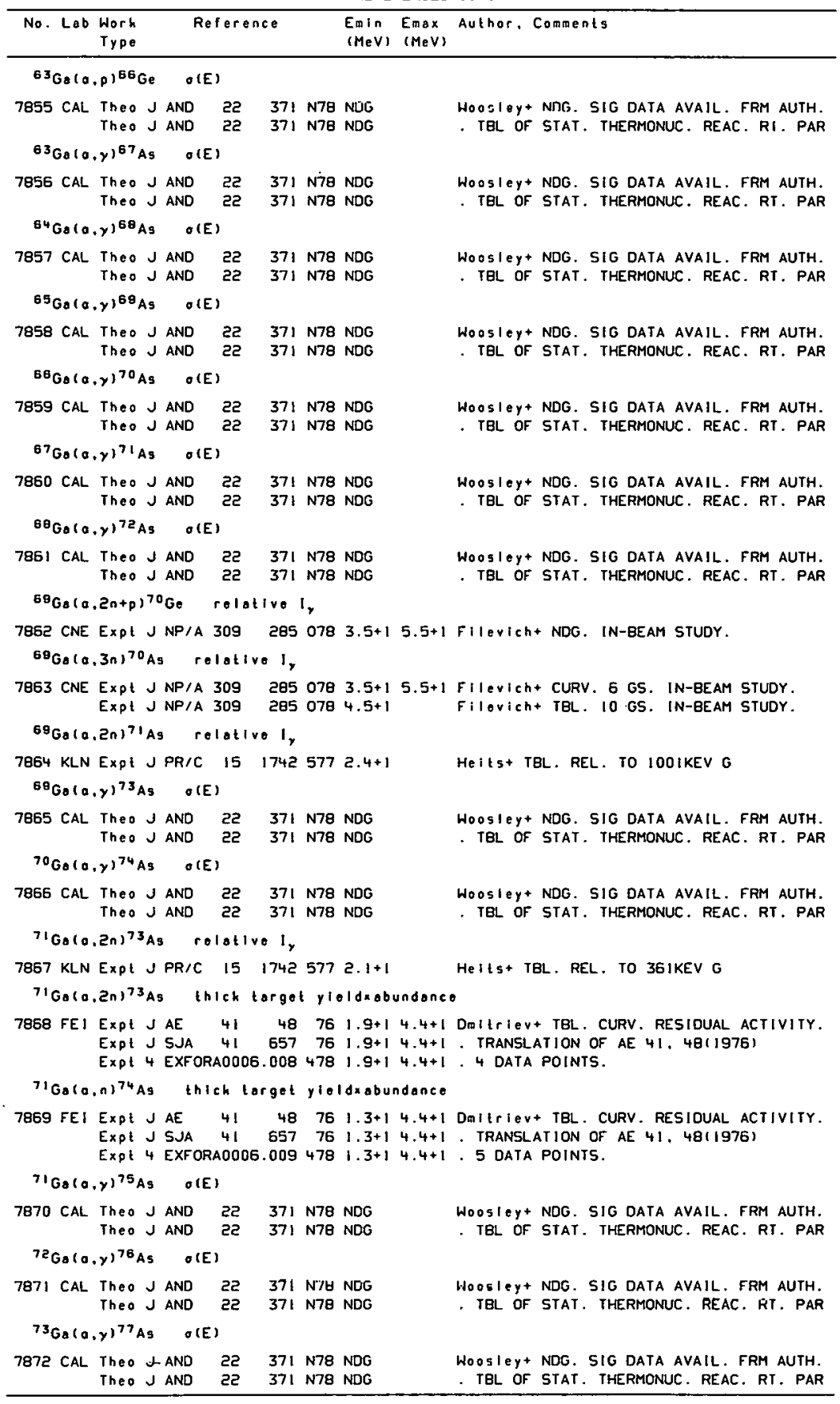




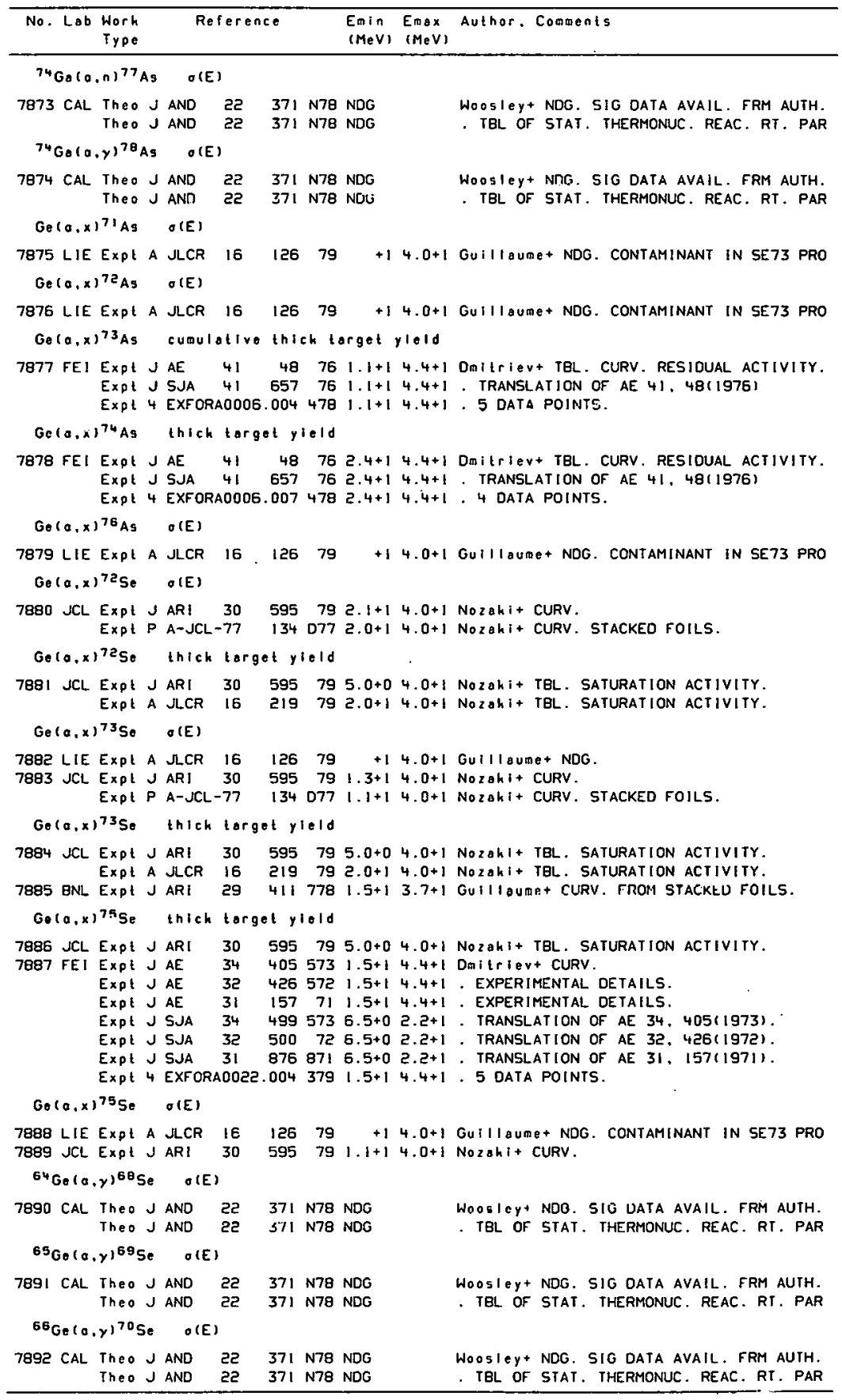


REFERENCES (cont)

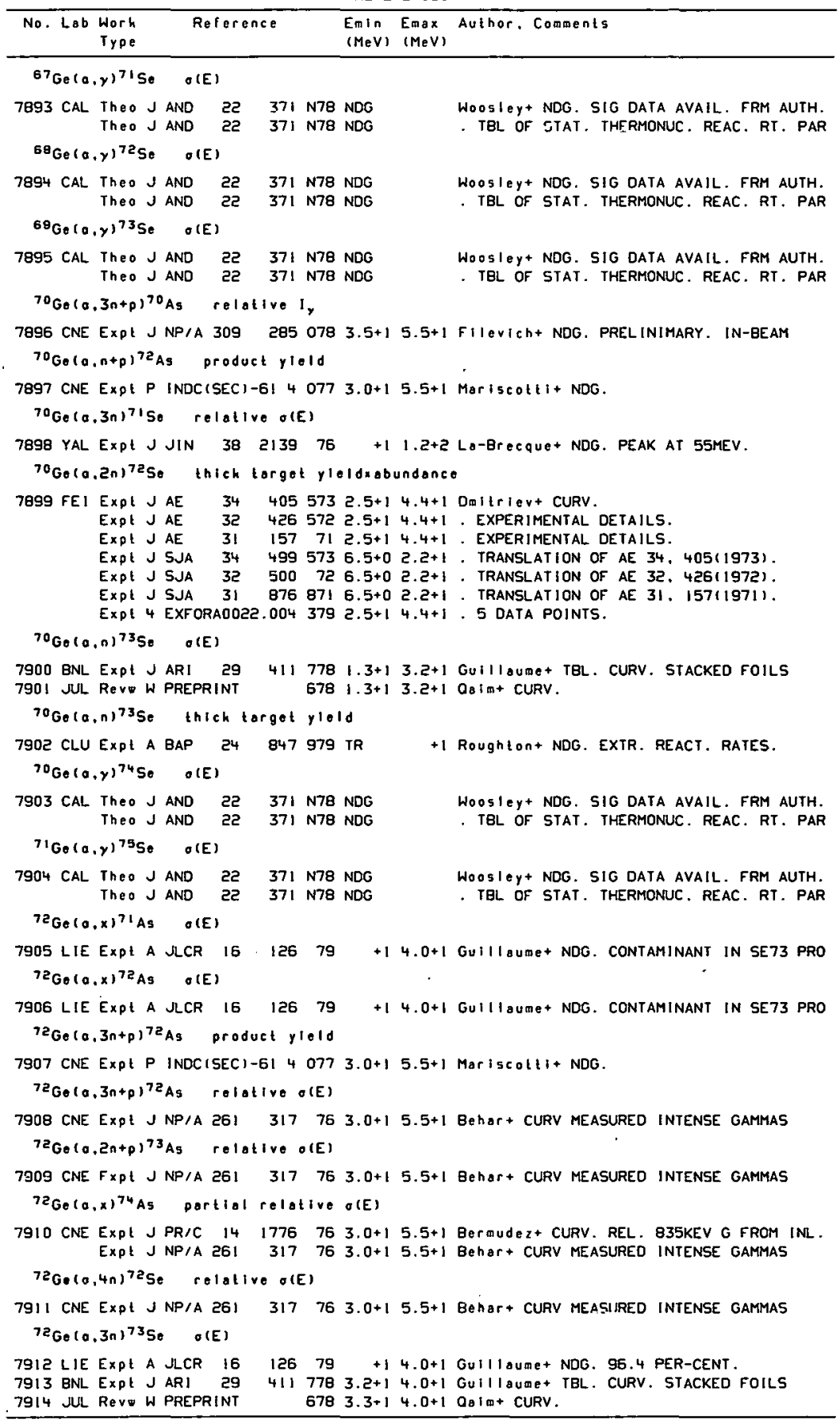




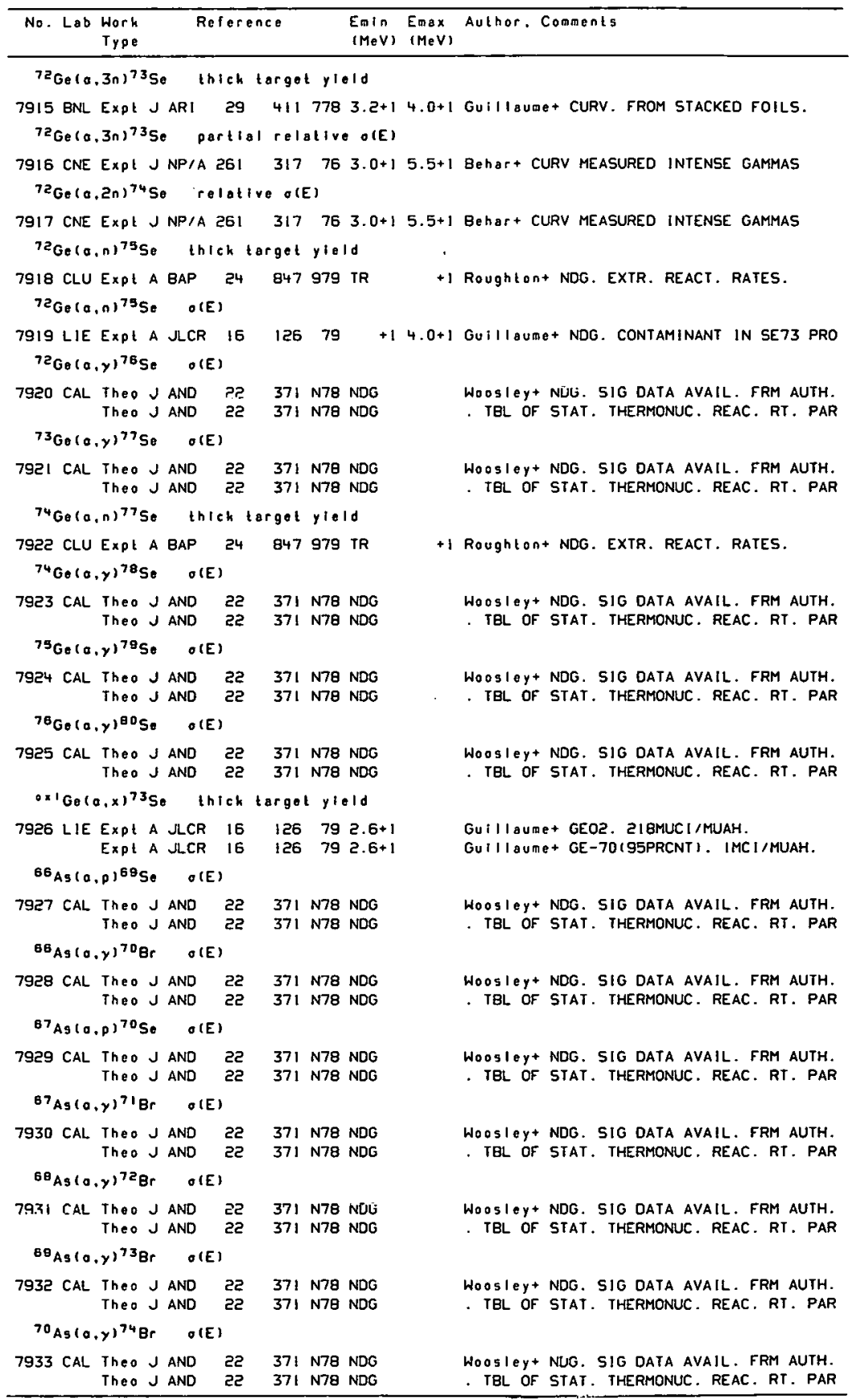


REFERENCES (cont)

\begin{tabular}{|c|c|c|c|c|c|c|}
\hline No. Lab & $\begin{array}{l}\text { Worh } \\
\text { Type }\end{array}$ & Reference & $\begin{array}{l}\text { EnIn } \\
(\mathrm{MeV})\end{array}$ & $\begin{array}{l}\text { Emax } \\
\left(M_{e} V\right)\end{array}$ & Author. & Comments \\
\hline
\end{tabular}

\begin{tabular}{|c|c|c|c|c|c|}
\hline 71 Asto & $\gamma)^{75} \mathrm{gr}$ & & & & \\
\hline $7934 \mathrm{CAL}$ & $\begin{array}{l}\text { Theo J AND } \\
\text { Theo J AND }\end{array}$ & $\begin{array}{l}22 \\
22\end{array}$ & $\begin{array}{l}371 \\
371\end{array}$ & $\begin{array}{l}\text { N78 } \\
\text { N78 }\end{array}$ & $\begin{array}{l}\text { NDC } \\
\text { NDC }\end{array}$ \\
\hline 72 Asta & $\gamma^{76} \mathrm{Br}$ & & & & \\
\hline $935 \mathrm{CAL}$ & $\begin{array}{l}\text { Theo J AND } \\
\text { Theo J AND }\end{array}$ & $\begin{array}{l}22 \\
22\end{array}$ & $\begin{array}{l}371 \\
371\end{array}$ & $\begin{array}{l}\text { N78 } \\
\text { N78 }\end{array}$ & ND \\
\hline${ }^{73} \mathrm{As}(\mathrm{O}$ & $\gamma)^{77} \mathrm{Br}$ & & & & \\
\hline $336 \mathrm{CAL}$ & $\begin{array}{l}\text { Theo J AND } \\
\text { Theo J AND }\end{array}$ & $\begin{array}{l}22 \\
22\end{array}$ & $\begin{array}{l}371 \\
371\end{array}$ & $\begin{array}{l}N 78 \\
N 78\end{array}$ & \\
\hline${ }^{74}$ As 10 & $\gamma)^{78} \mathrm{Br}$ & & & & \\
\hline $1937 \mathrm{CAL}$ & $\begin{array}{l}\text { Theo J AND } \\
\text { Theo J AND }\end{array}$ & $\begin{array}{l}22 \\
22\end{array}$ & $\begin{array}{l}371 \\
371\end{array}$ & $\begin{array}{l}\text { N78 } \\
\text { N78 }\end{array}$ & \\
\hline & 74. & & & et & \\
\hline
\end{tabular}

7930 FEI ExpL J AE Expl J SJA $416577643+1$ Expt 4 EXFORAO006.012 $4784.3+1$

Woosley+ NDG. SIG DATA AVAIL. FRM AUTH. . IBL OF STAT. THERMONUC. REAC. RT. PAR

Woosley+ NDG. SIG DATA AVAIL. FRM AUTH. - IBL OF STAT. THERMONUC. REAC. RT. PAR

Woosley+ NDG. SIG DATA AVAIL. FRM AUTH. . TBL OF STAT. THERMONUC. REAC. RT. PAR

Woosley+ NDG. SIG DATA AVAIL. FRM AUTH. . TBL OF STAT. THERMONUC. REAC. RT. PAR

Dmitrient TEL. CURV. RESIDUAL ACTIVITY. TRANSLATION OF AE 4I, 4811976)

20.+-2. GMUC1/MUAHR

$$
{ }^{79} \text { As }(a .3 n+p) 75 \text { Se relative J, }
$$

7939 CNE Expt J PR/C $175162783.0+15.5+1$ Behor+ CURV. MOST INTENSE GAMMAS.

$7 S_{A s}(a, 2 n+p) 76 s e$ relative I,

7940 CNE Expt J PR/C $175162783.5+15.5+1$ Behar+ CURV. MOST INTENSE GAMMAS.

${ }^{75} \mathrm{As}(a, n+p)^{77}$ So relative $\mathrm{l}_{\text {y }}$

7941 CNE EXPI J PR/C $175162783.0+15.5+1$ Behar+ CURV. MOST INTENSE GAMMAS.

${ }^{75} \mathrm{As}(a, 4 n)^{75} \mathrm{Br}$ relative $\mathrm{I}_{r}$

7942 CNE Exp! J PR/C $175162783.0+15.5+1$ Behar+ CURV. MOST INTENSE GAMMAS. Expt J NP/A $2823314774.5+15.5+1$ Behart CURV. MOST INIENSE GAMMAS.

${ }^{75} \mathrm{As}(0,3 \mathrm{Bn})^{76} \mathrm{Br} \quad \sigma(E)$

7943 JCL Expt J ARI $30 \quad 79792.8+13.8+1$ Nozahl+ CURV. GAMMA DETECTION.

${ }^{75} \mathrm{As}(a, 3 n)^{76} \mathrm{Br}$ thick target yield

$7944 \mathrm{JCL}$ Expl J AR! $30 \quad 79793.0+14.0+1$ Nozah 1+ TBL. SATURATION ACTIVITY.

Expl A JLCR $16 \quad 219792.0+14.0+1$ NozahI+ TBL. SATURATION ACTIVITY.

${ }^{75} \mathrm{As}(a, 3 n) 76 \mathrm{Br}$ relallve $I_{\text {, }}$

7945 CNE EXpt J PR/C 17516278 3.0+1 $5.5+1$ Behar+ CURV. MOST INTENSE GAMMAS. Expt J NP/A $2823314773.5+15.5+1$ Behart CURV. MOST INTENSE GAMMAS.

${ }^{75} \mathrm{As}(\alpha, 3 n)^{76} \mathrm{Br}$ partial o(E)

7946 CNE Expt J NP/A $2823314774.5+1 \quad$ Behor+ TBL. 8 GAMMAS.

$75 \mathrm{As}(a, x)^{76 * \mathrm{Br}}$ product yleld

7947 GOE Expt J ZP/A 289 I2! 78 NDG Schmidt-Ott+ NEW ISOMER TI/2=1.49+-.025

${ }^{75}$ Asta.3n) $760 \mathrm{Br}$ product yield

7948 CNE Expt J PL/B 83 3i 479 NDG Kreiner+ NDG. TI/2=1.32S. 102.6KEV LVL.

${ }^{75} \mathrm{As}(a, 2 n){ }^{77} \mathrm{Br} \quad O(E)$

$7949 \mathrm{JCL}$ Expt J ARI $30 \quad 79791.4+13.8+1$ Nozahi+ CURV. GAMMA DETECTION. Expt P A-JCL-77 $134077 \quad 1.5+1 \quad 3.8+1$ Nozak $1+$ CURV. STACKEO FOILS.

${ }^{75} \mathrm{As}(0,2 \mathrm{n}){ }^{77} \mathrm{Br}$ thlek torget yield

$7950 \mathrm{JCL}$ Expt J AR! $30 \quad 79792.0+14.0+1$ NozahI+ TBL. SATURATION ACTIVITY. Expt A JLCR $16219792.0+14.0+1$ Nozahi+ TBL. SATURATION ACTIVITY.

7951 AML Revw J IRE 26 228! $4792.8+1$ ChBudri. IBL. CFD EXP. TO CALC.

7952 JUL ReVw W PREPRINT 678 2.8+1 Qain+ CURV. 0.4IMCI/MICROAH

${ }^{75} \mathrm{As}(\alpha, 2 \mathrm{n})^{77} \mathrm{Br}$ relalive 1 ,

7953 CNE EXPt J PR/C $175162783.0+15.5+1$ Behor + CURV. MOST INTENSE GAMMAS. Expl J NP/A $2823314773.0+15.5+1$ Behort CURV. MOST INTENSE GAMMAS.

${ }^{75} \mathrm{As}(a, y)^{79} \mathrm{Br} \quad O(E)$

7954 CAL TheO J AND $22 \quad 371$ N78 NDG WOOSIEY+ NDG. SIG DATA AVAIL. FRM AUTH. Theo J AND 22 37 ! N78 NDG . TBL OF STAT. THERMONUC. REAC. RT. PAR 


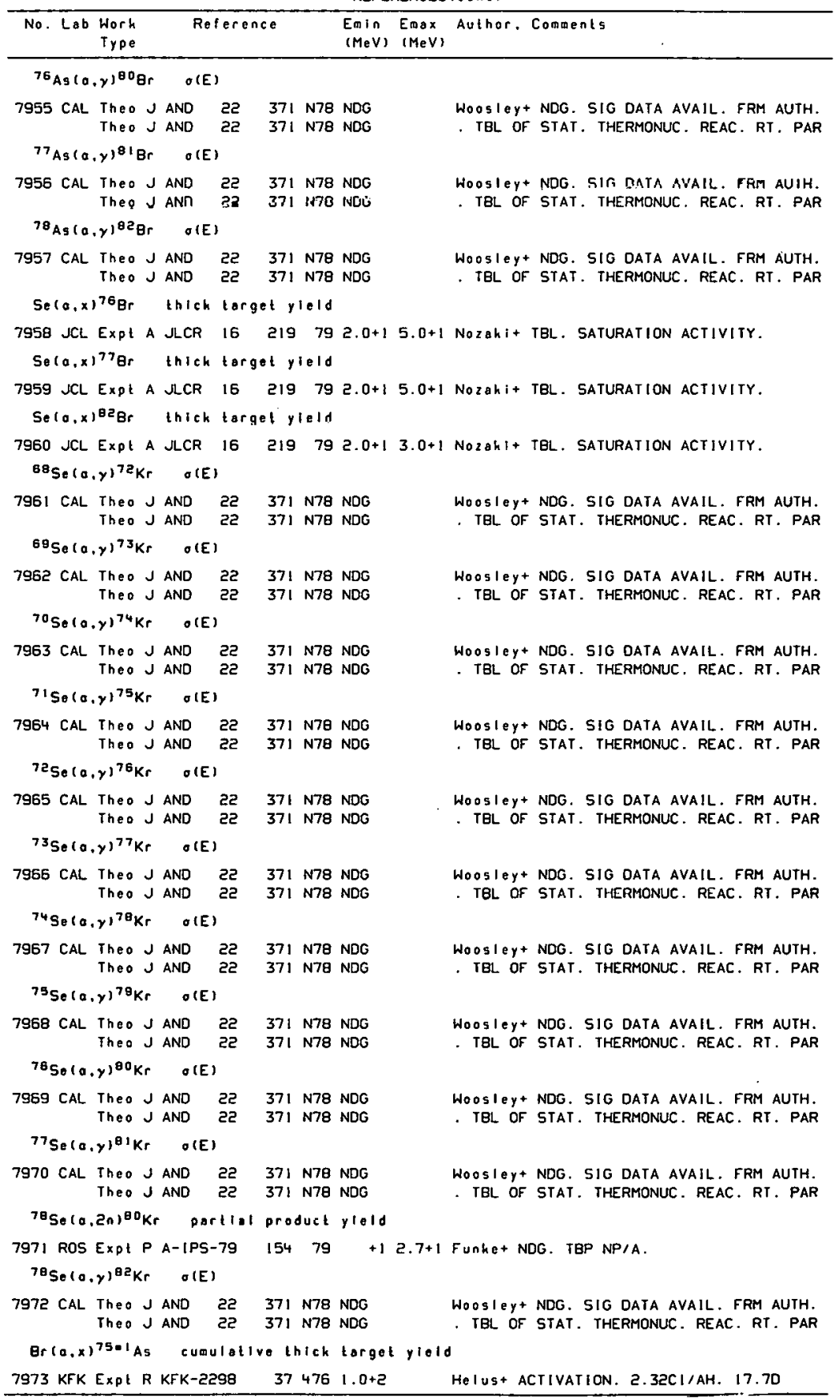


REFERENCES ( c on i)

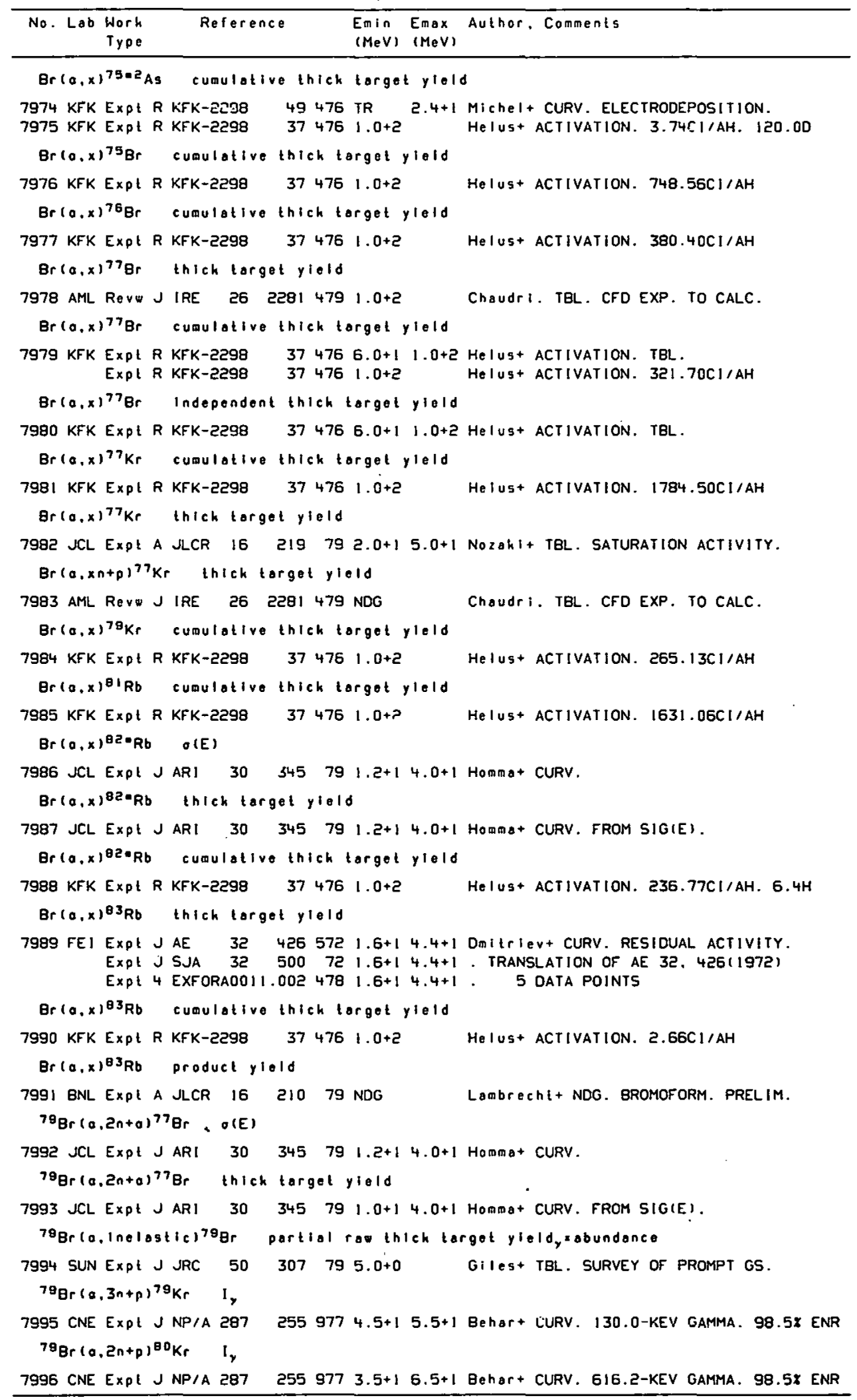


REFERENCES ( c on t)

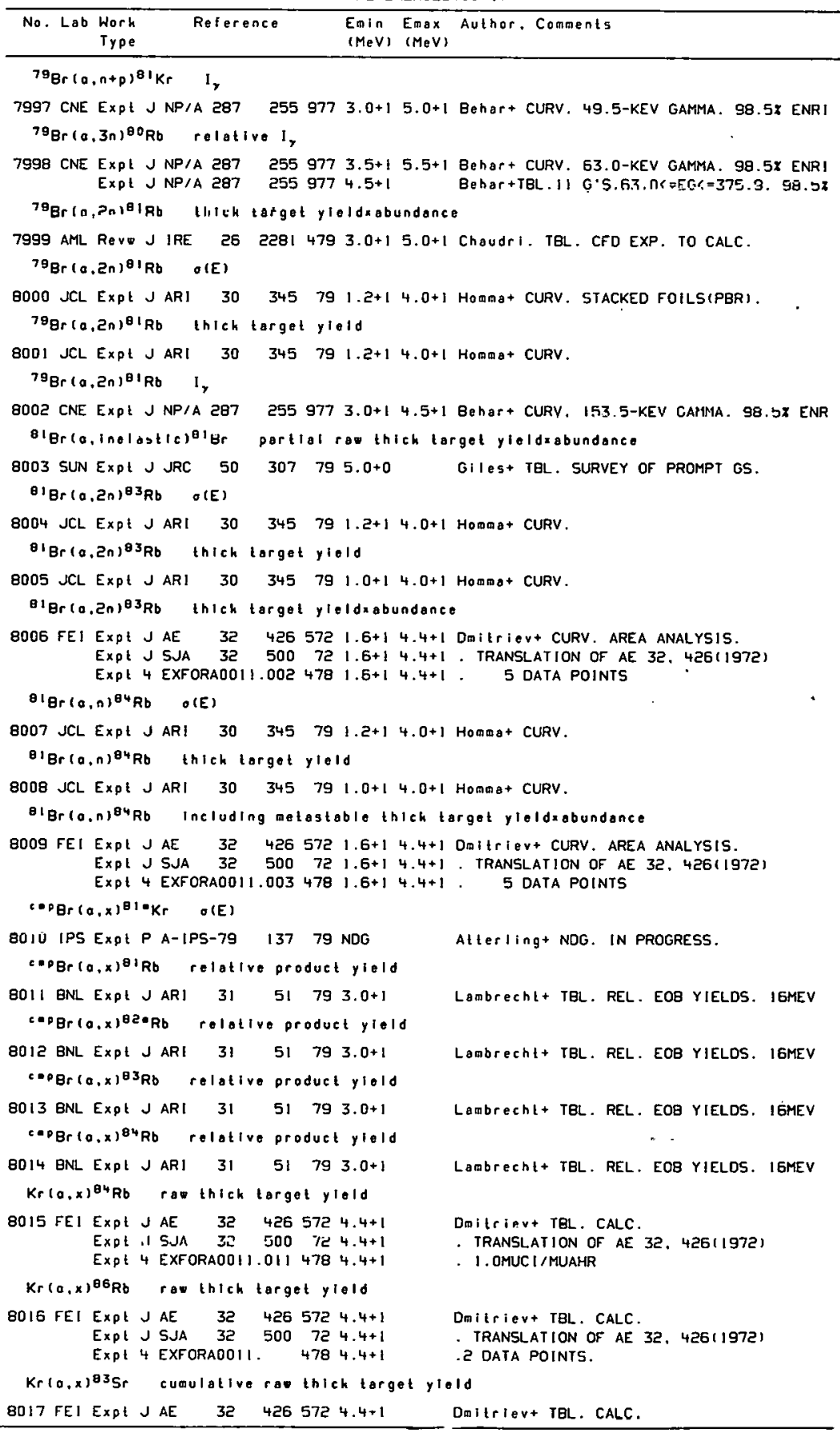


REFERENCES ( $c$ on $t)$

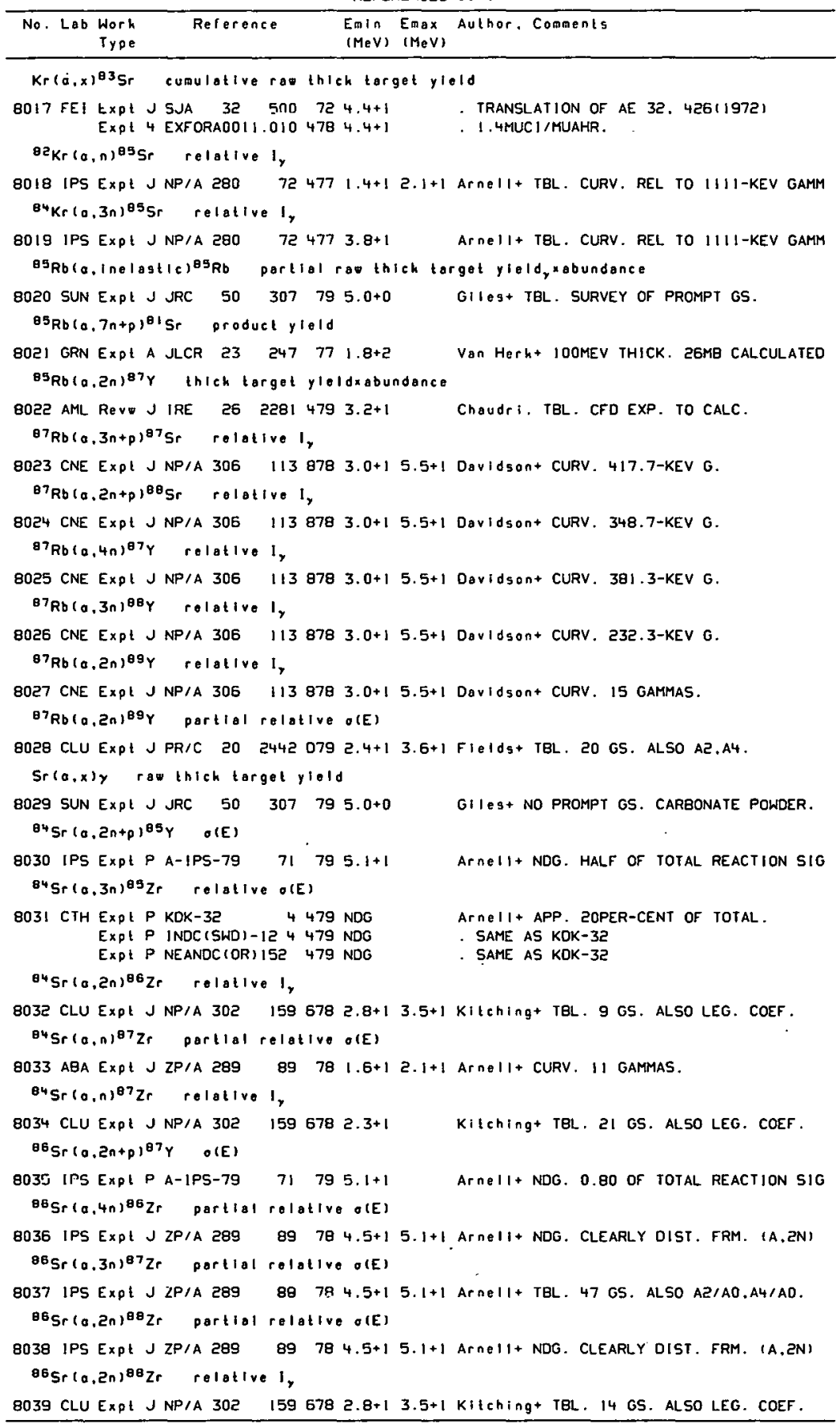


REFERENCES ( cont)

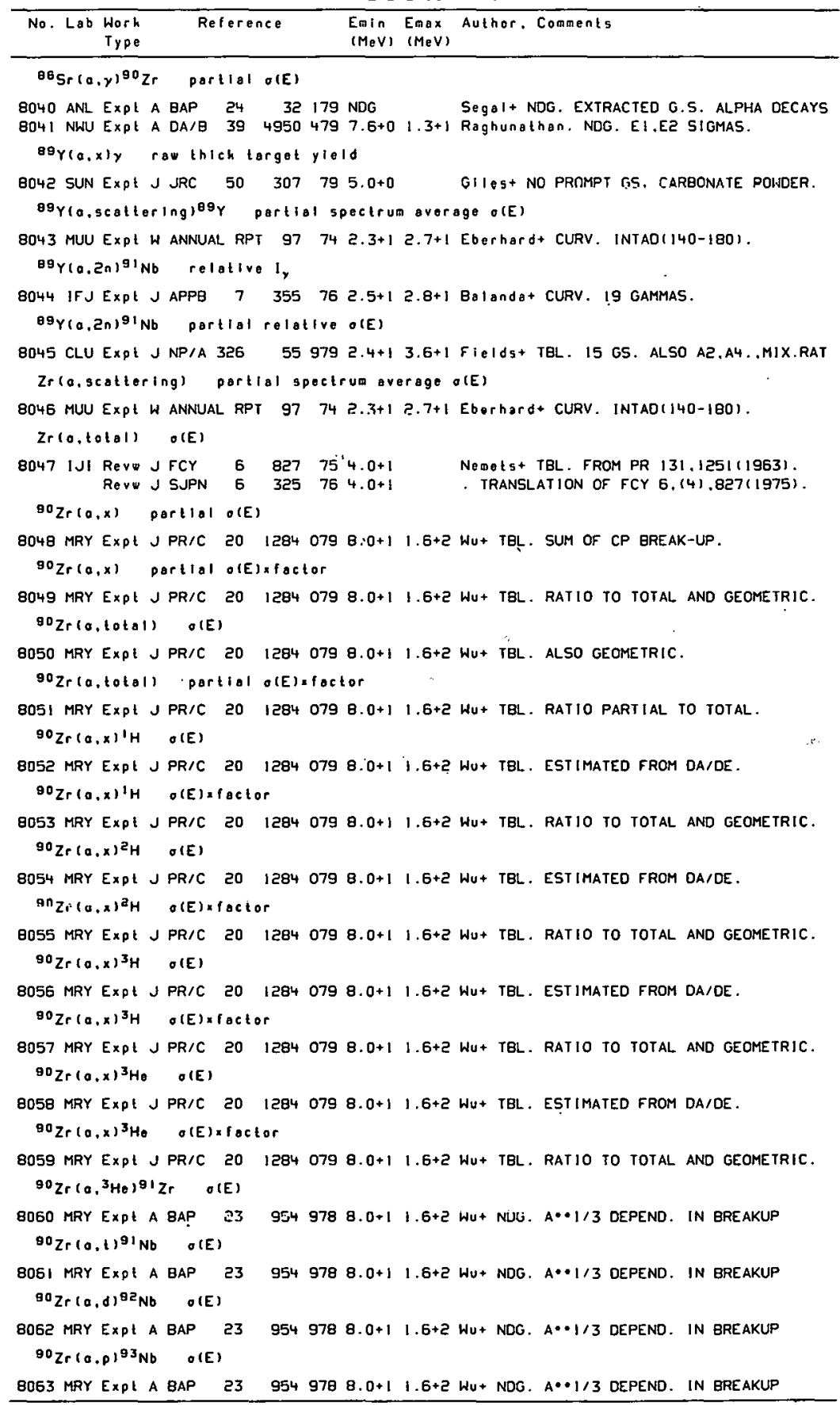


REFERENCES (cont)

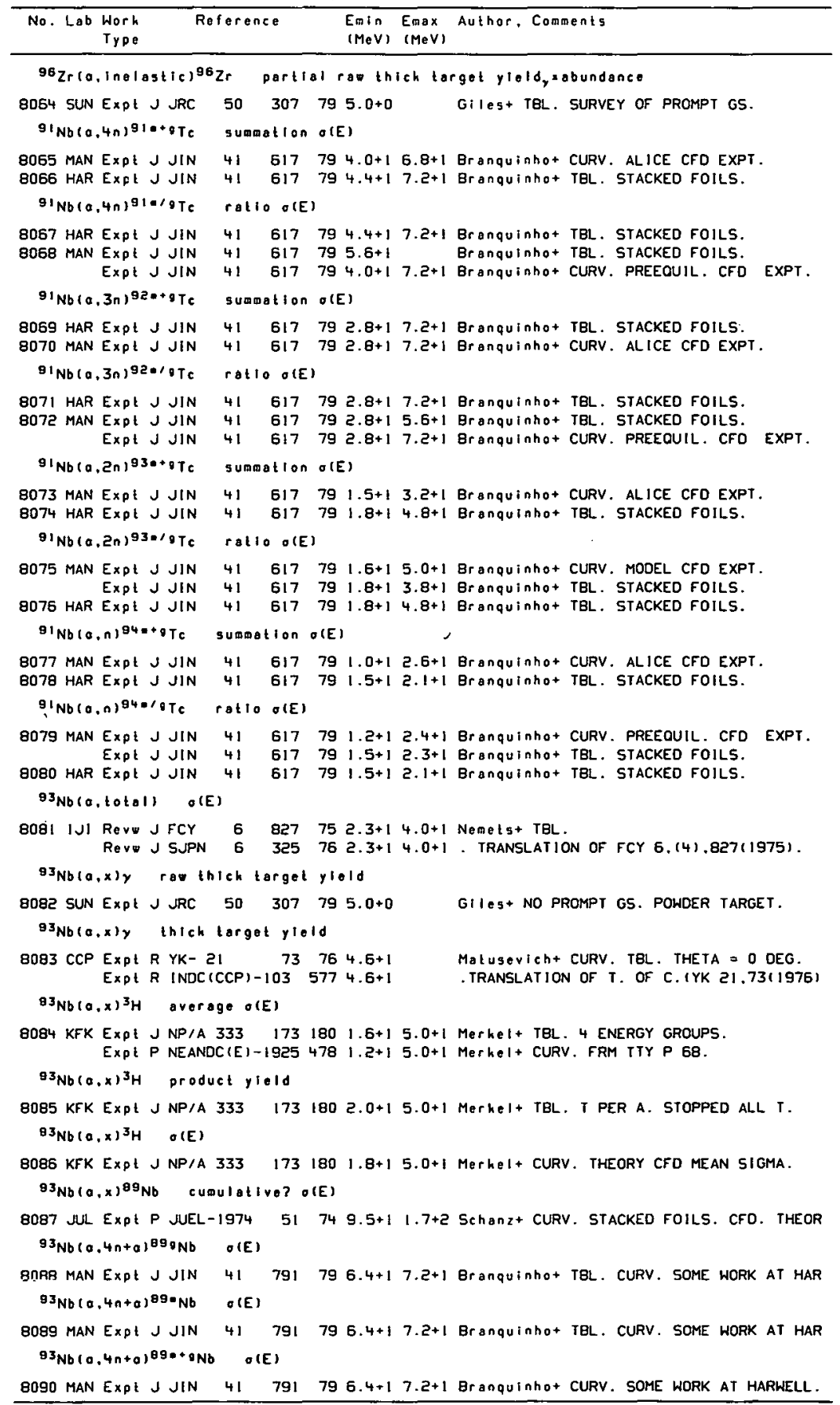




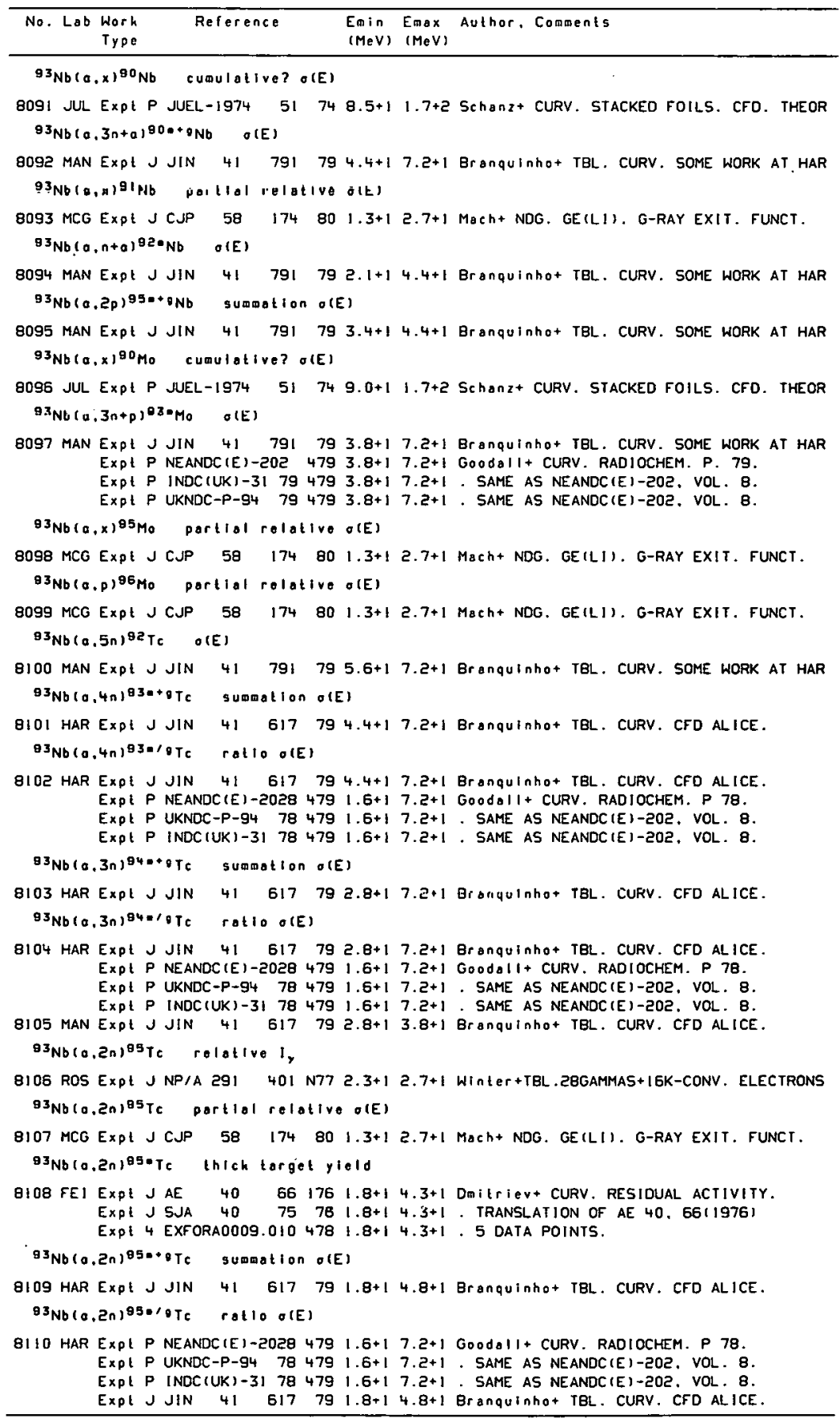


REFERENCES ( cont)

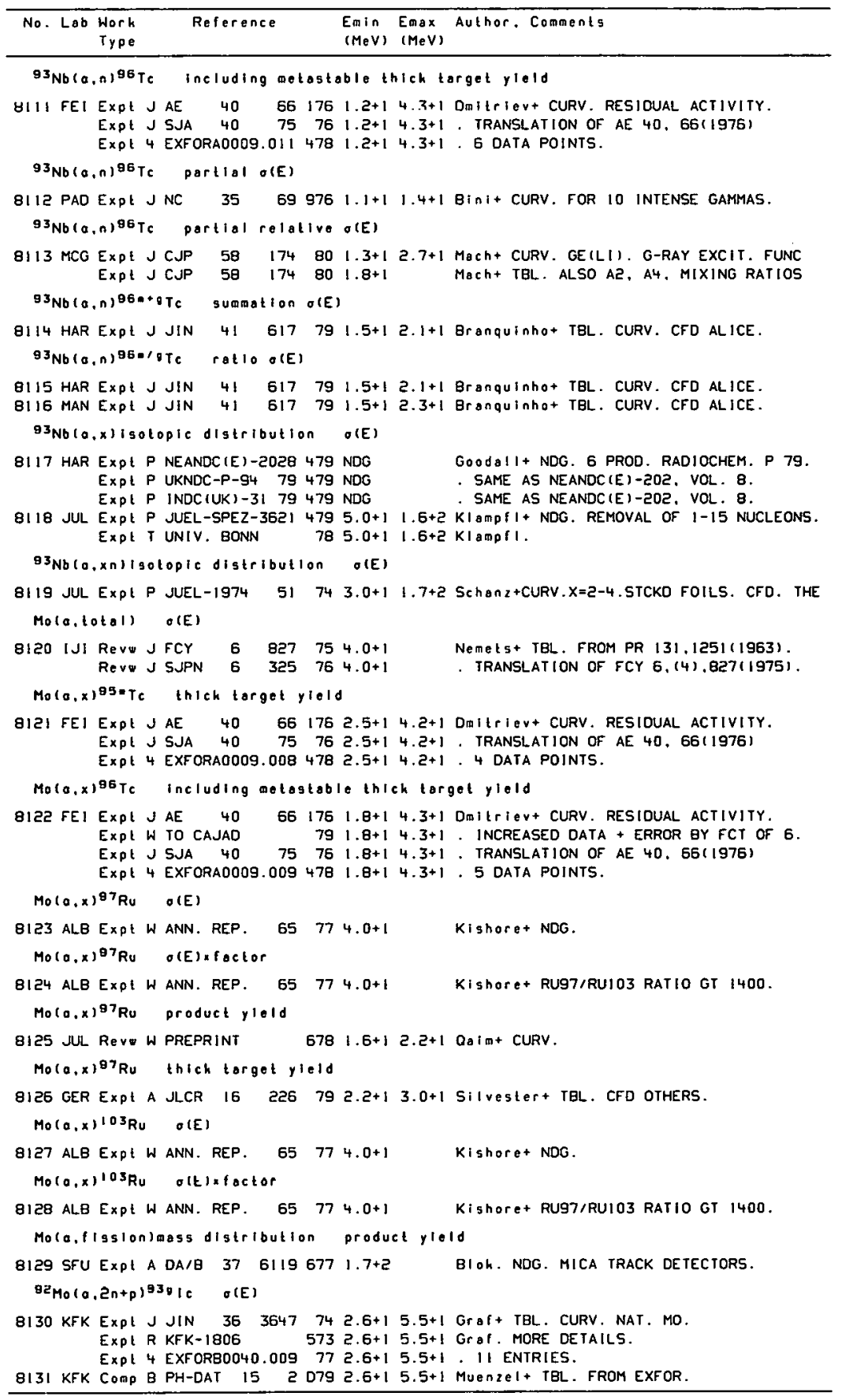


REFERENCES (cont)

\begin{tabular}{cc} 
No. Lob Work Reference & Emin Emax Author. Comments \\
Type & (MeV) (MeV) \\
\hline
\end{tabular}

$92 M_{0}(0,3 n+p) 93 \cdot T c \quad \sigma(E)$

8132 KFK Expt J JIN $36 \quad 3647 \quad 743.0+1 \quad 5.5+1$ Graf+ TBL. CURV. NAT. MO. Expt R KFK- $1806 \quad 5733.0+15.5+1$ Graf. MORE DETAILS. EXPL 4 EXFORBO040.009 $773.0+15.5+1$. 11 ENTRIES.

8133 KFK Comp B PH-DAT 15 2 D79 3.0+! 5.5+1 Muenzel+ TBL. FROM EXFOR.

$92 M a(a . n+n) 94 \% T$ Q $I E)$

8134 KFK Expt J JIN $36 \quad 3647 \quad 741.8+14.2+1$ Graf + TBL. CURV. NAT. MO. Expl R KFK-1806 $573 \quad 1.8+14.2+1$ Graf. MORE DETAILS.

EXPt 4 EXFORBD040.005 $771.8+14.2+1$. 14 ENTRIES.

8135 KFK Comp B PH-DAT 152 D79 1.8+1 $4.2+1$ Muenzel+ TBL. FROM EXFOR.

$92 M_{0}(a, n+p) 94 \cdot T C \quad O(E)$

8136 KFK Expt J JIN $363647 \quad 742.0+13.7+1$ Graf+ TBL. CURV. NAT. MO. OEDUCED. Expt R KFK-1806 $5732.0+1 \quad 3.7+1$ Gr af. MORE DETAILS.

EXPt 4 EXFORB0040.005 $772.0+13.7+1$. G ENTRIES.

8137 KFK COmp B PH-DAT is $20792.0+13.7+1$ Muenzel+ TBL. FROM EXFOR.

$82 M_{0}(0.0) 950 T$ TE $O(E)$

8138 KFK Expl J JIN $36 \quad 3647 \quad 74 \quad 1.2+12.7+1$ Graf+ TEL. CURV. NAT. MO. EXpl R KFK-1806 $573 \quad 1.2+12.7+1$ Graf. MORE DETAILS.

Expt 4 EXFORB0040.007 $77 \quad 1.2+12.7+1.7$ ENTRIES.

8I39 KFK Comp 8 PH-DAT 15 2 $0791.2+12.7+1$ Muenzel+ TBL. FROM EXFOR.

$92 M_{0}(a, p)^{950} T_{c}$ O(E)

8140 KFK EXpt J JIN $36 \quad 3647 \quad 741.3+13.2+1$ Graft TBL. CURV. NAT. MO. EXPl R KFK-1805 $573 \quad 1.3+1 \quad 3.2+1$ Graf. MORE DETAILS.

Expl 4 EXFORB0040.007 $77 \quad 1.3+13.2+1$. I2 ENIRIES.

8141 KFK COMP B PH-DAT I5 $2079: 3+13.2+1$ Muenzel+ TBL. FROM EXFOR.

$82 \operatorname{Mo}\left(a, Z_{n}\right)^{84} R u \quad O(E)$

8142 KFK Expt J JIN $363647741.9+14 \cdot 1+1$ Graf+ IBL. CURV. NAT. MO. Expt R KFK-1805 $573 \quad 1.9+14.1+1$ Graf. MORE OETAILS.

Expt 4 EXFORBO040.004 $771.9+14.1+1.17$ ENTRIES.

8143 KFK COMP B PH-DAT 15 2 079 $1.9+14.1+1$ Muenzel+ TBL. FROM EXFOR.

$92_{M 0}(a, n)^{95} R u \quad O(E)$

B144 KFK Expl J JIN $36 \quad 3647 \quad 74 \quad 1.2+1 \quad 3.0+1$ Grat+ TBL. CURV. NAT. MO. Expt R KFK-1806 $5731.2+13.0+1$ Graf. MORE DETAILS.

EXpl 4 EXFORBO040.002 $771.2+13.0+1$. I2 ENTRIES.

8145 KFK COMp B PH-DAT 15 2 D79 $1.2+13.0+1$ Muenzel+ TBL. FROM EXFOR.

$92_{\text {Mo }}(0 . n)^{95} R u$ thick target y101d

$8146 \mathrm{CLU}$ Expt A BAP $24 \quad 847979$ TR +1 Roughton+ NDG. EXTR. REACT. RATES.

${ }^{94} M_{0}(a, i n e l a s t t c)^{94} M_{0}$ partial rao thlek torget yleldy abundance

8147 SUN Expt J JRC $50 \quad 307 \quad 795.0+0 \quad$ GIIEs+ TBL. SURVEY OF PROMPT GS.

$94 \mathrm{Ma}(a, 2 n+p) 959 T C \quad O(E)$

8148 KFK Expt J JIN $36 \quad 3647743.0+15.5+1$ Graf+ TBL. CURV.93.9x. METAL + CU-MO-O4 Expt R KFK-1806 $5733.0+15.5+1$ Graf. MORE OETA!LS.

Expl 4 EXFORBO040.011 $773.0+15.5+1$. 9 ENIRIES.

B!49 KFK Comp B PH-OAT $1520793.0+15.5+1$ Muenzel+ TBL. FROM EXFOR.

$94 M_{0}(a, 2 n+p)^{950} T C \quad O(E)$

$8150 \mathrm{KFK}$ Expl J JIN $363647743.4+15.6+1$ Graf+ IBL. CURV.93.97. METAL + CU-MO-04 Expt R KFK-1806 $5733.4+15.6+1$ Gr af. MORE DETAILS.

EXPt 4 EXFORBO040.011 $773.4+15.6+1$. 7 ENTRIES.

8151 KFK COMP B PH-DAT 152 D79 $3.4+15.6+1$ Muenzel+ TBL. FROM EXFOR.

$94 M_{0}(0, n+p) 96 T e$ Including metaslablo o(E)

Q152 KFK Expl J JIN 36364774 2, 1+1 5.9+1 Graf+ TBL. CURV, 93.92. CU-MO-04 POWDER Expt R KFK-1806 $5732.1+15.9+1$ Graf. MORE DETALLS.

EXPL 4 EXFORBO040.0I2 $772.1+15.9+1$. I2 ENTRIES.

8153 KFK COMP B PH-OAT 15 2 $0792.1+15.9+1$ Muenzel+ TQL. FROM EXFOR.

$94 M_{0}(a .3 n)^{95} R_{U} \quad O(E)$

8154 KFK Expl J JIN $363647743.0+15.9+1$ Graf+ TBL. CURV. 93.9 PER CENT. EXPt R KFK-1806 $5733.0+15.9+1$ GraI. MORE DETAILS.

Expt 4 EXFORBO040.017 $773.0+15.9+1$ - 10 ENTRIES.

BI55 KFK COMP B PH-DAT 15 2 D79 3.0+1 5.9+1 MUenzel+ IAI. FROM EXFOR. 


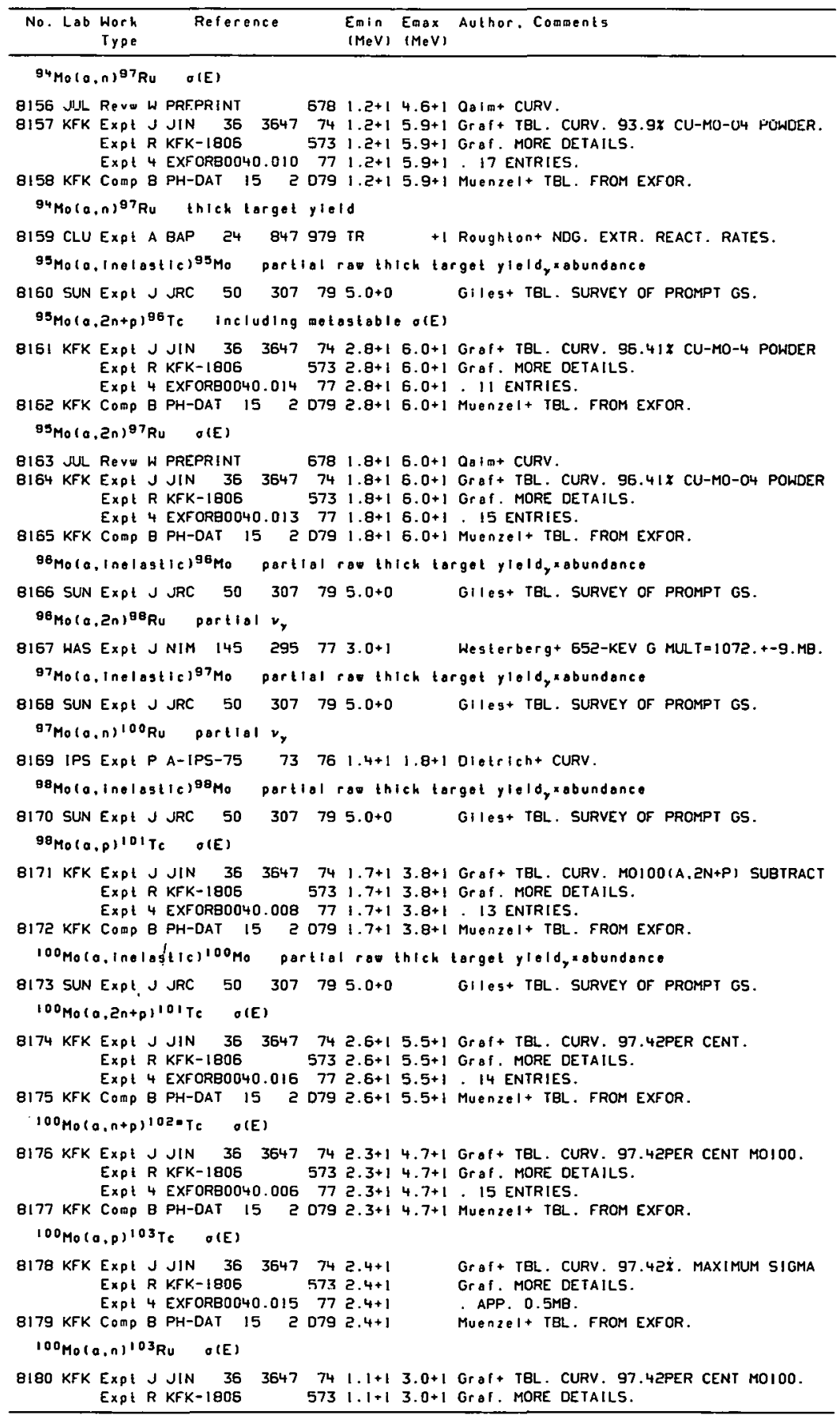




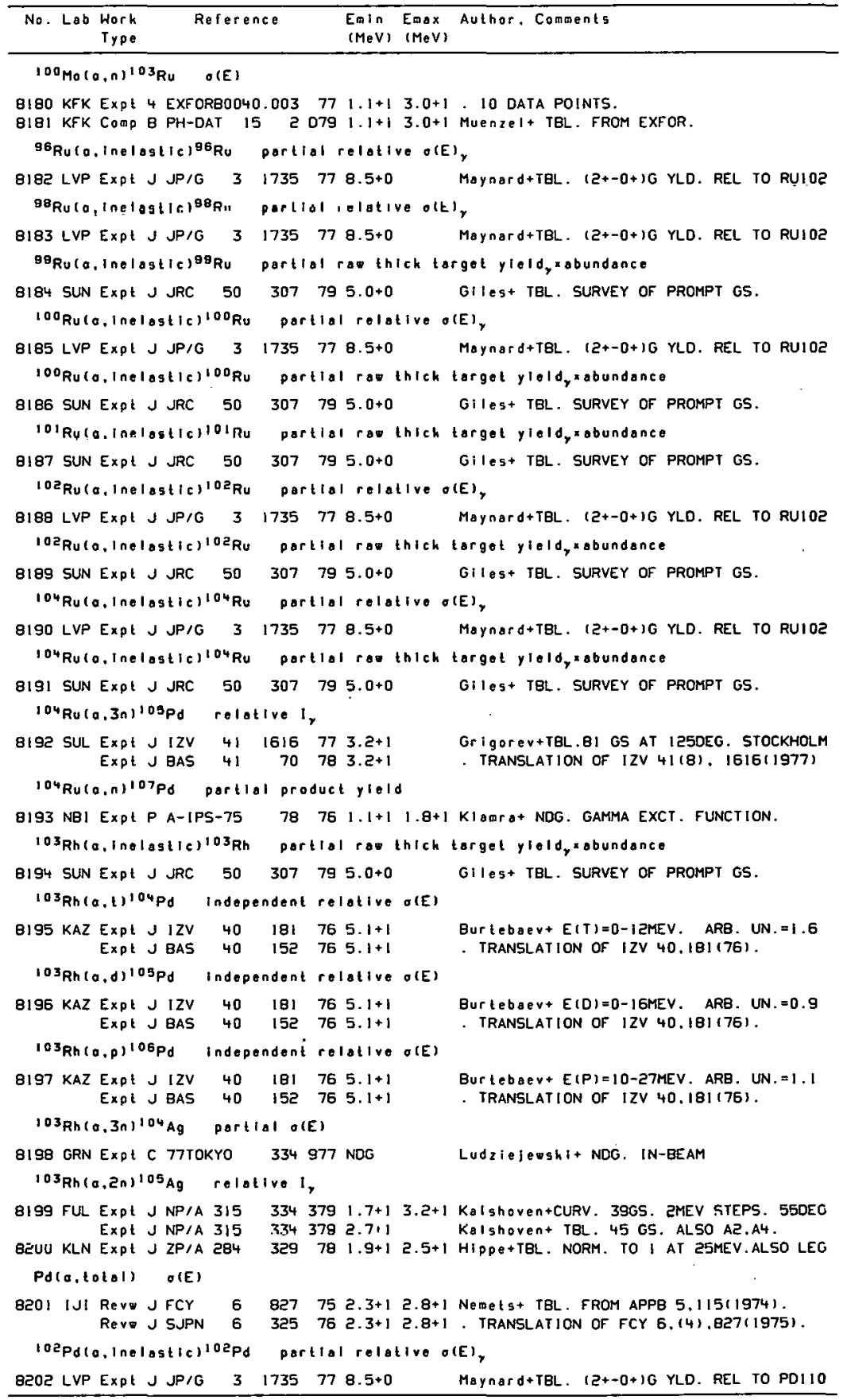




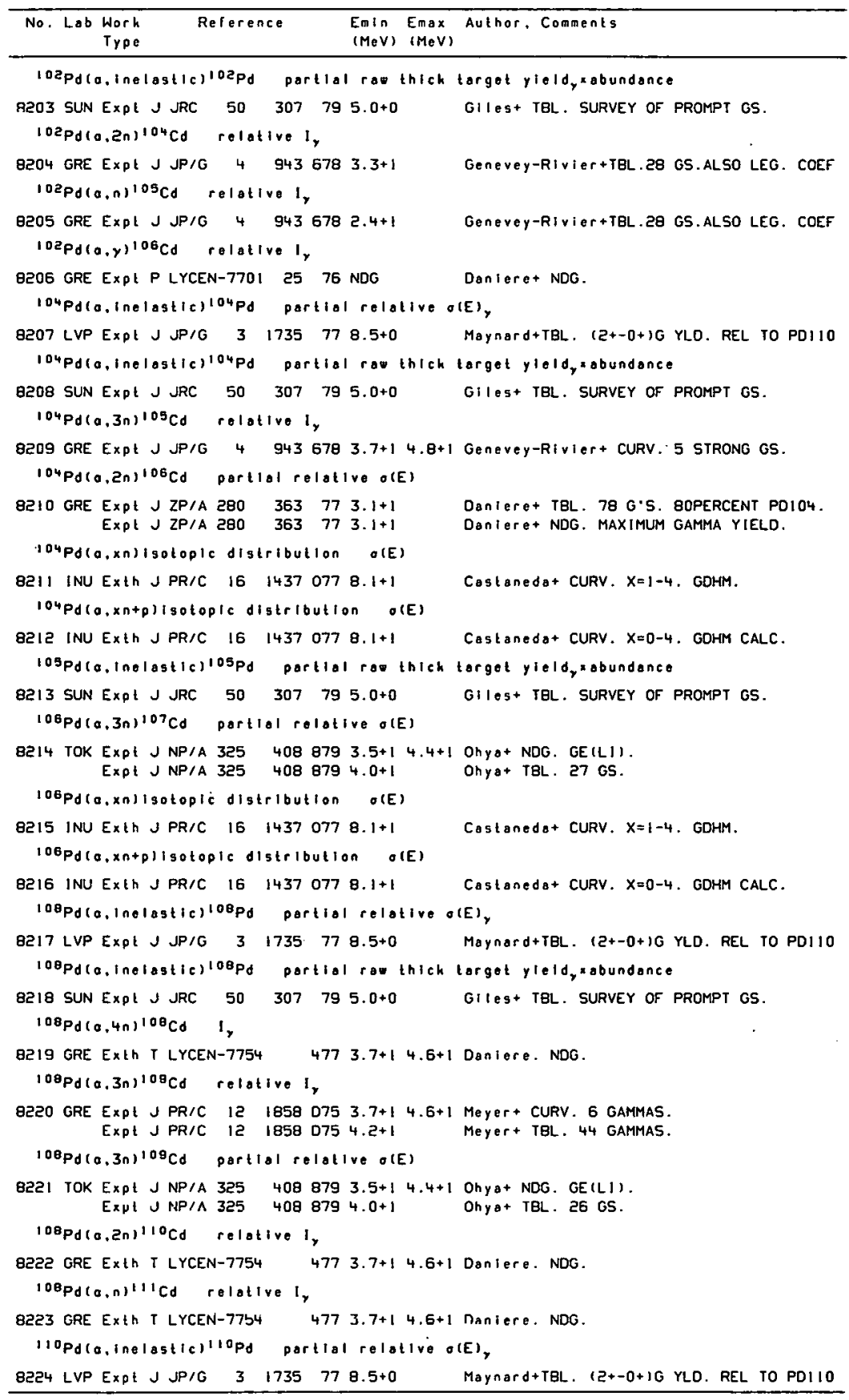


REFERENCES (cont)

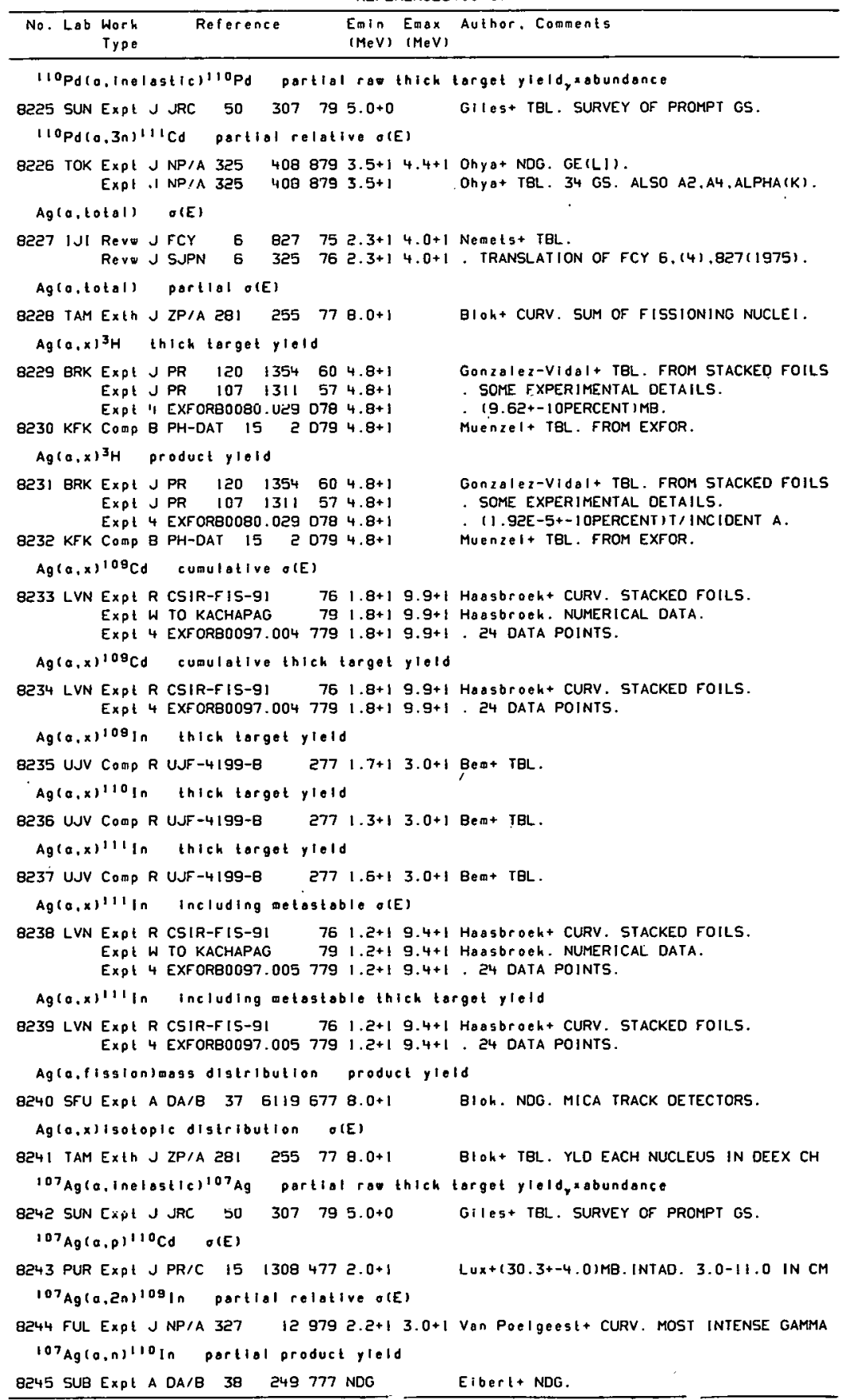


REFERENCES (cont)

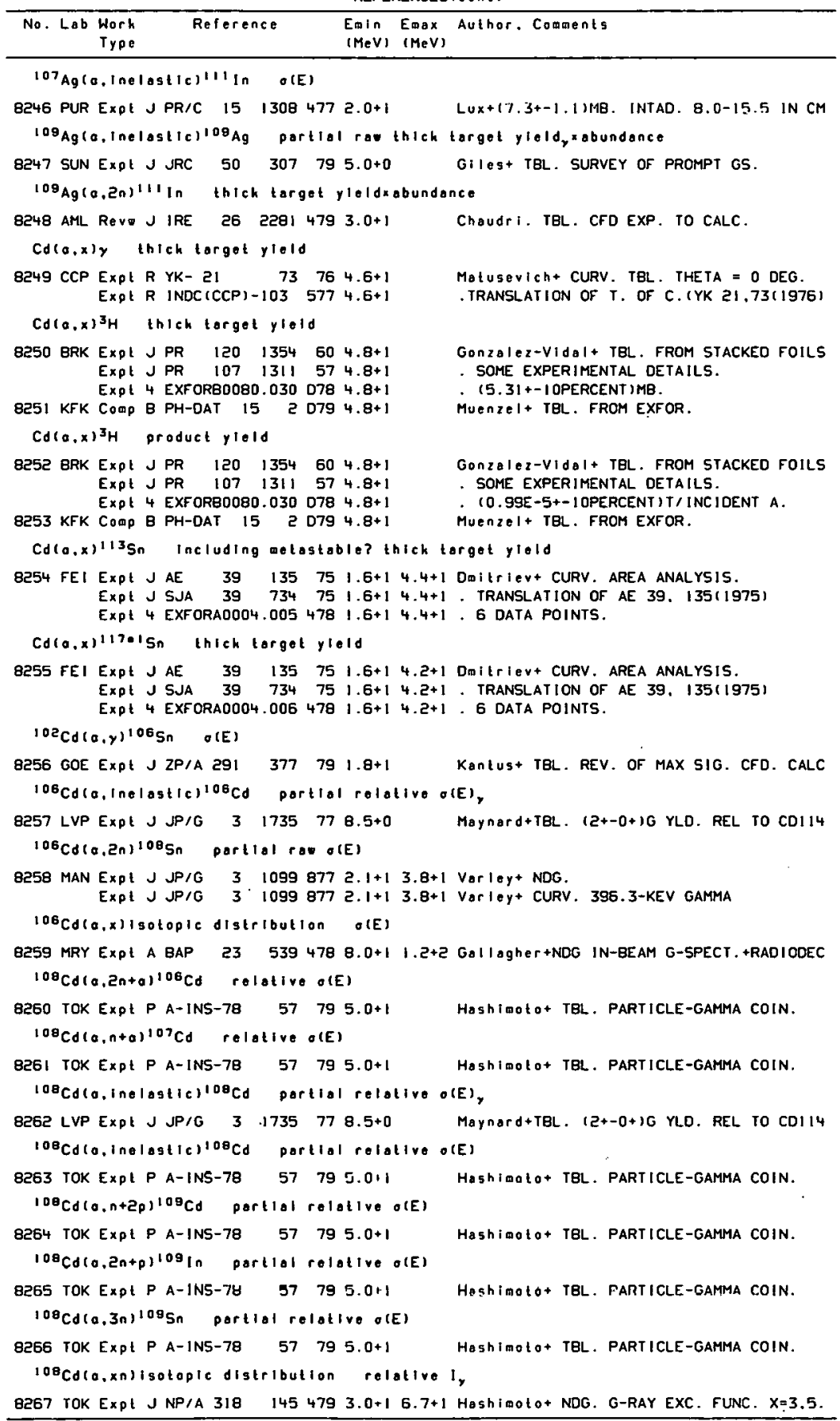




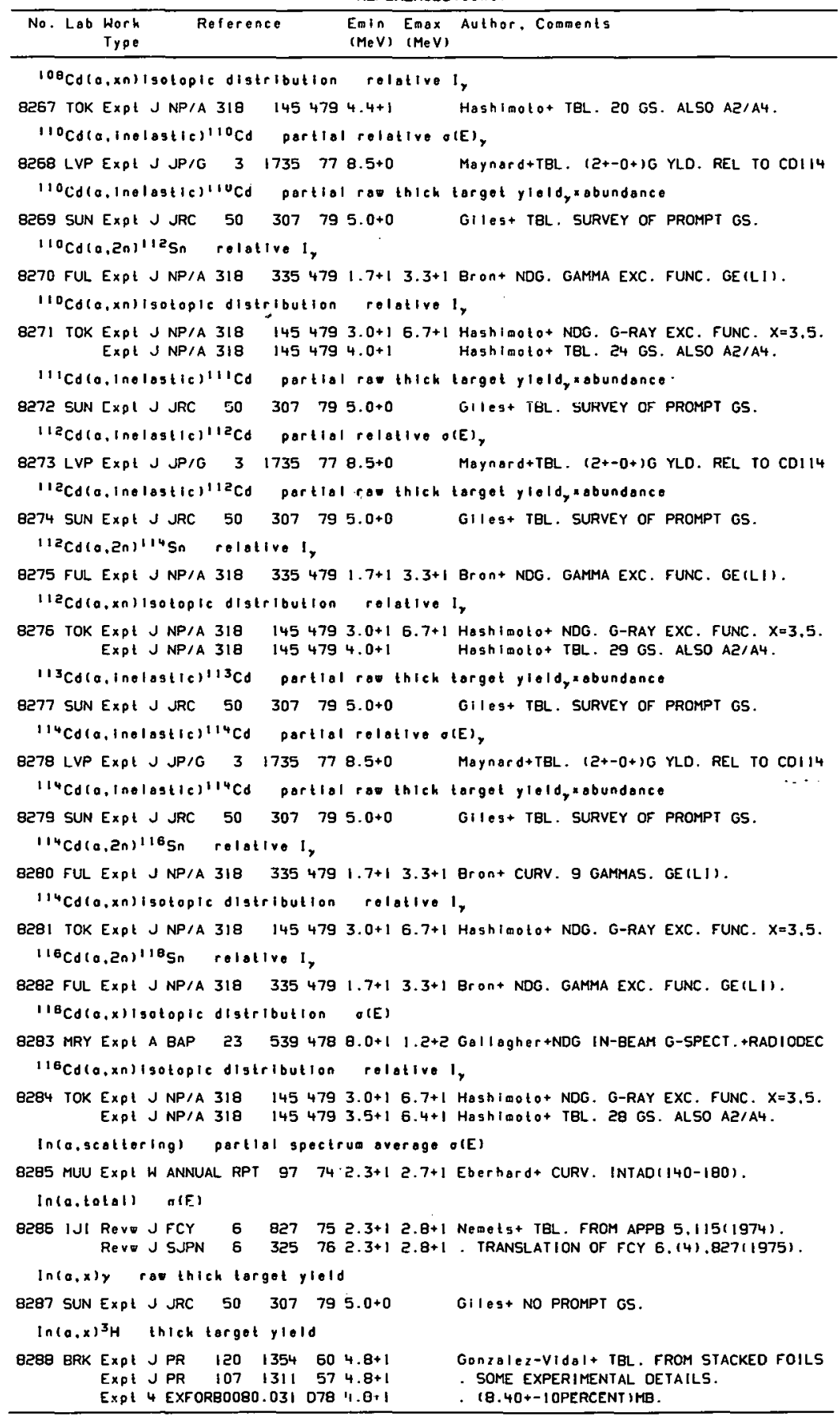




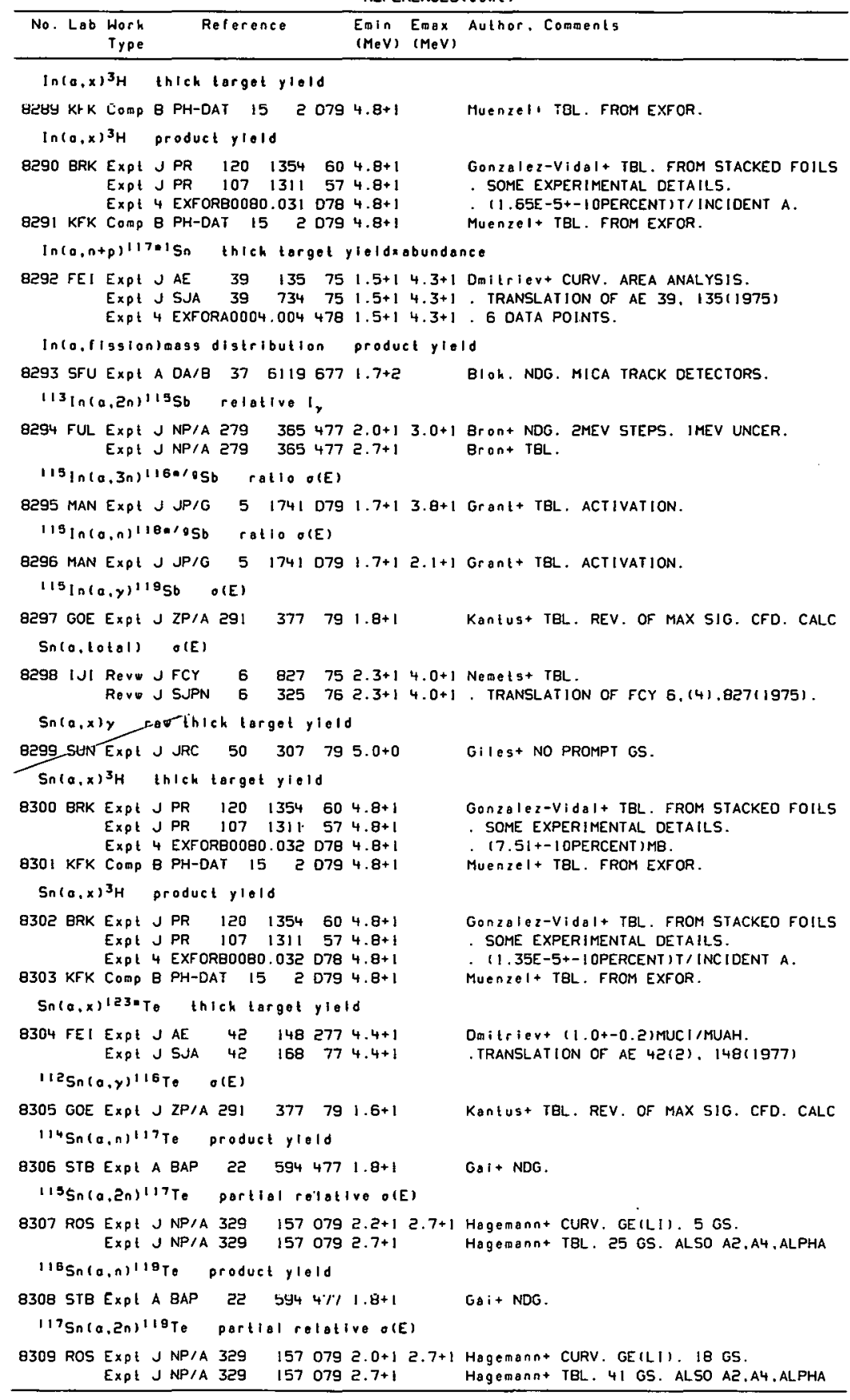




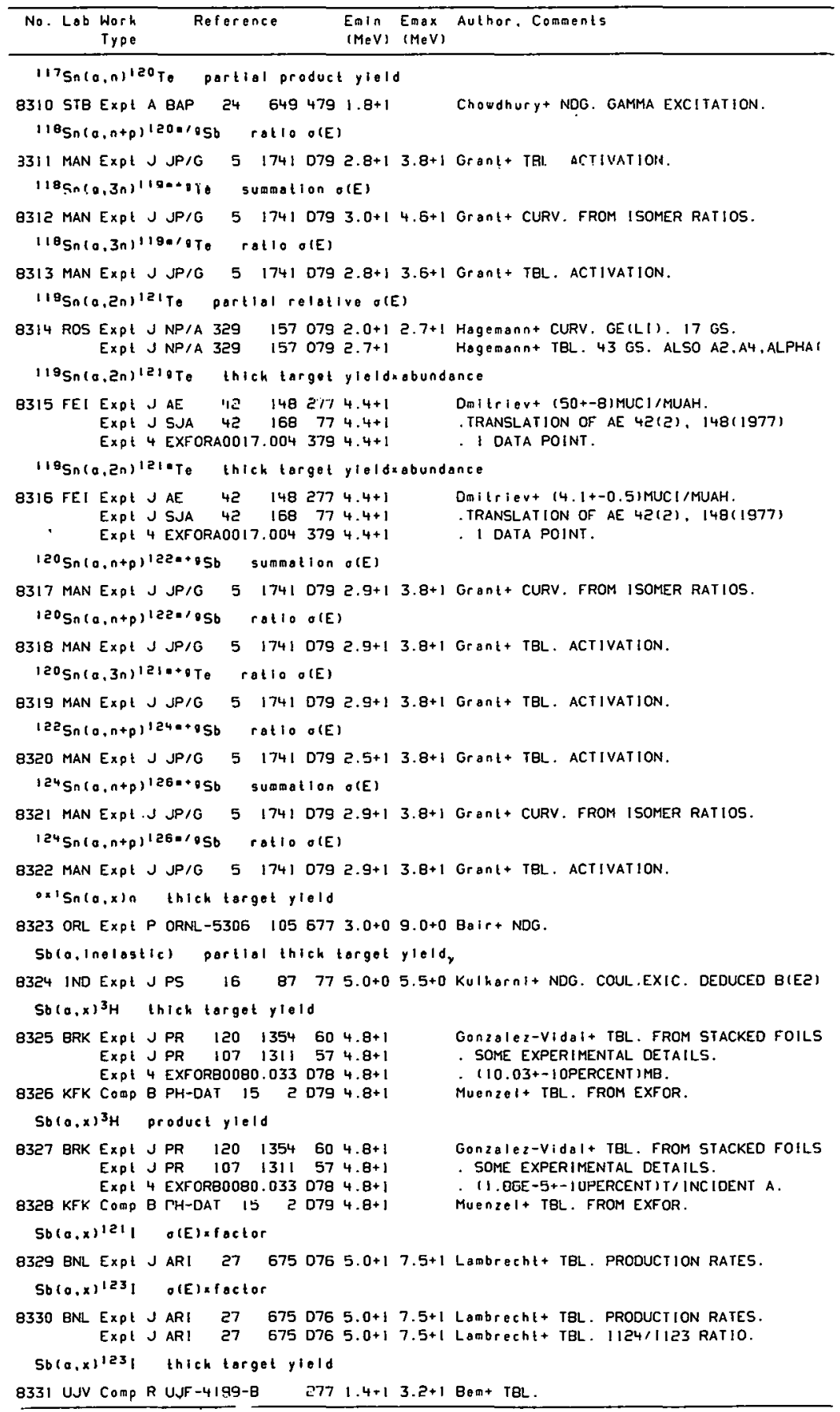




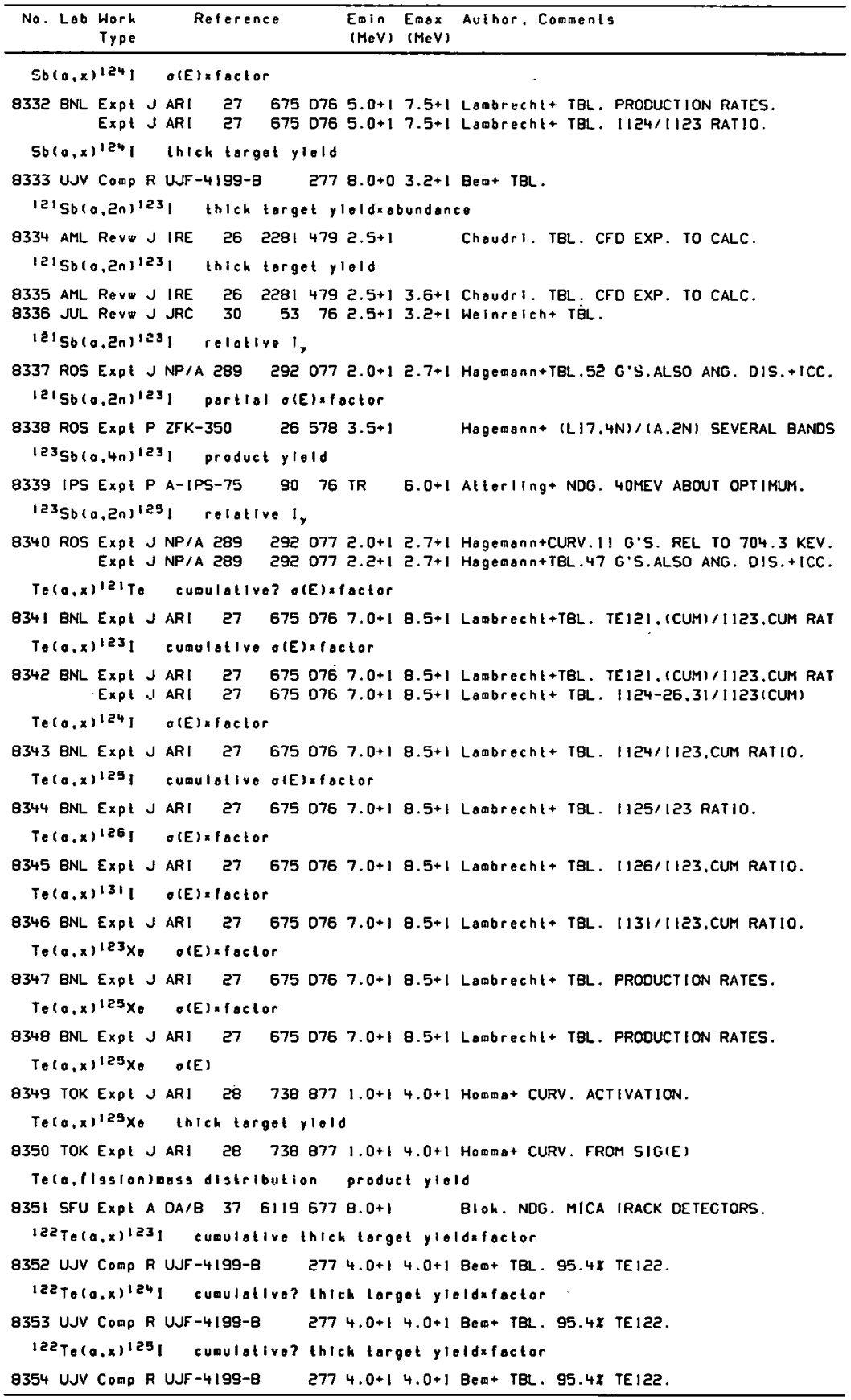


REFERENCES ( c on I )

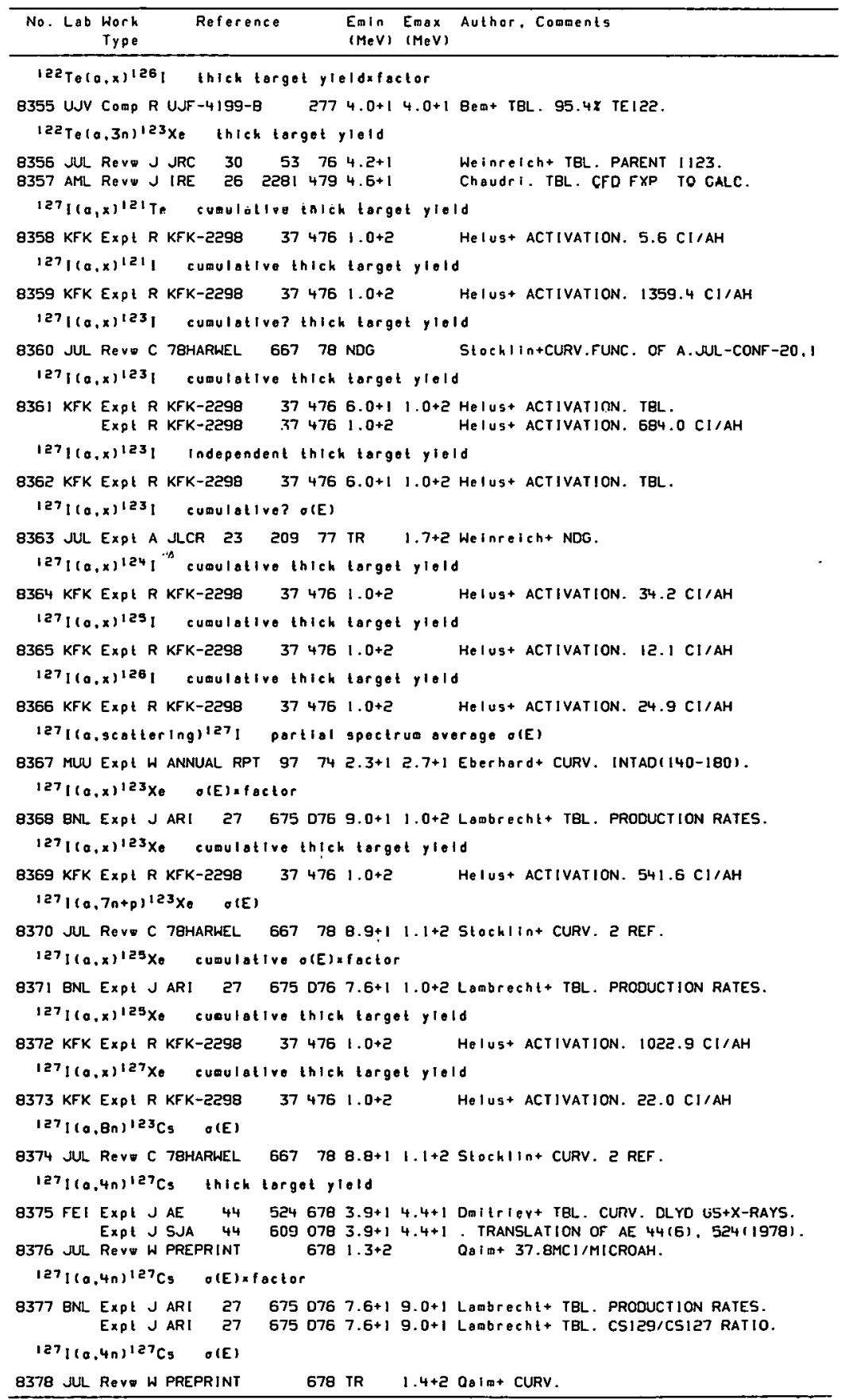




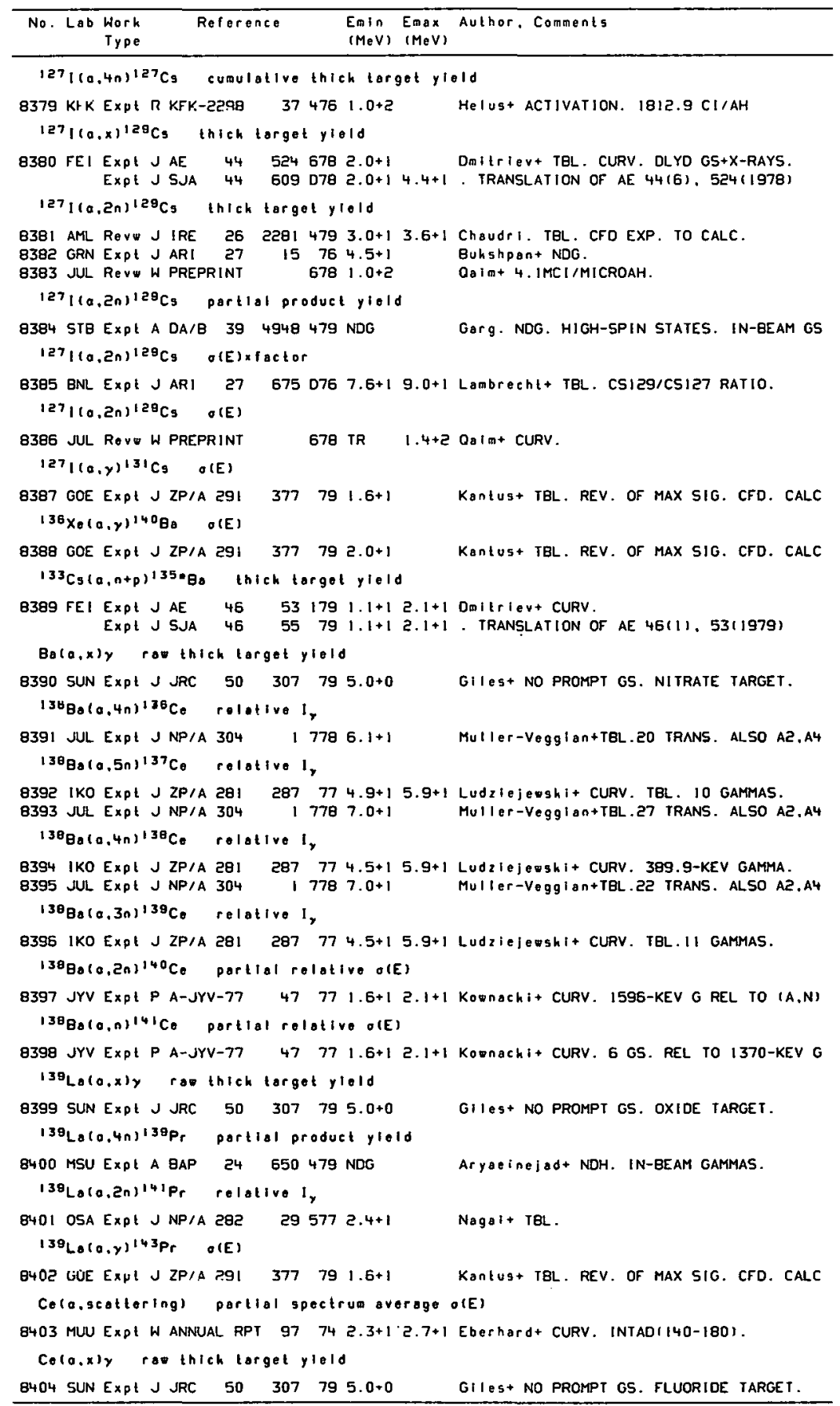




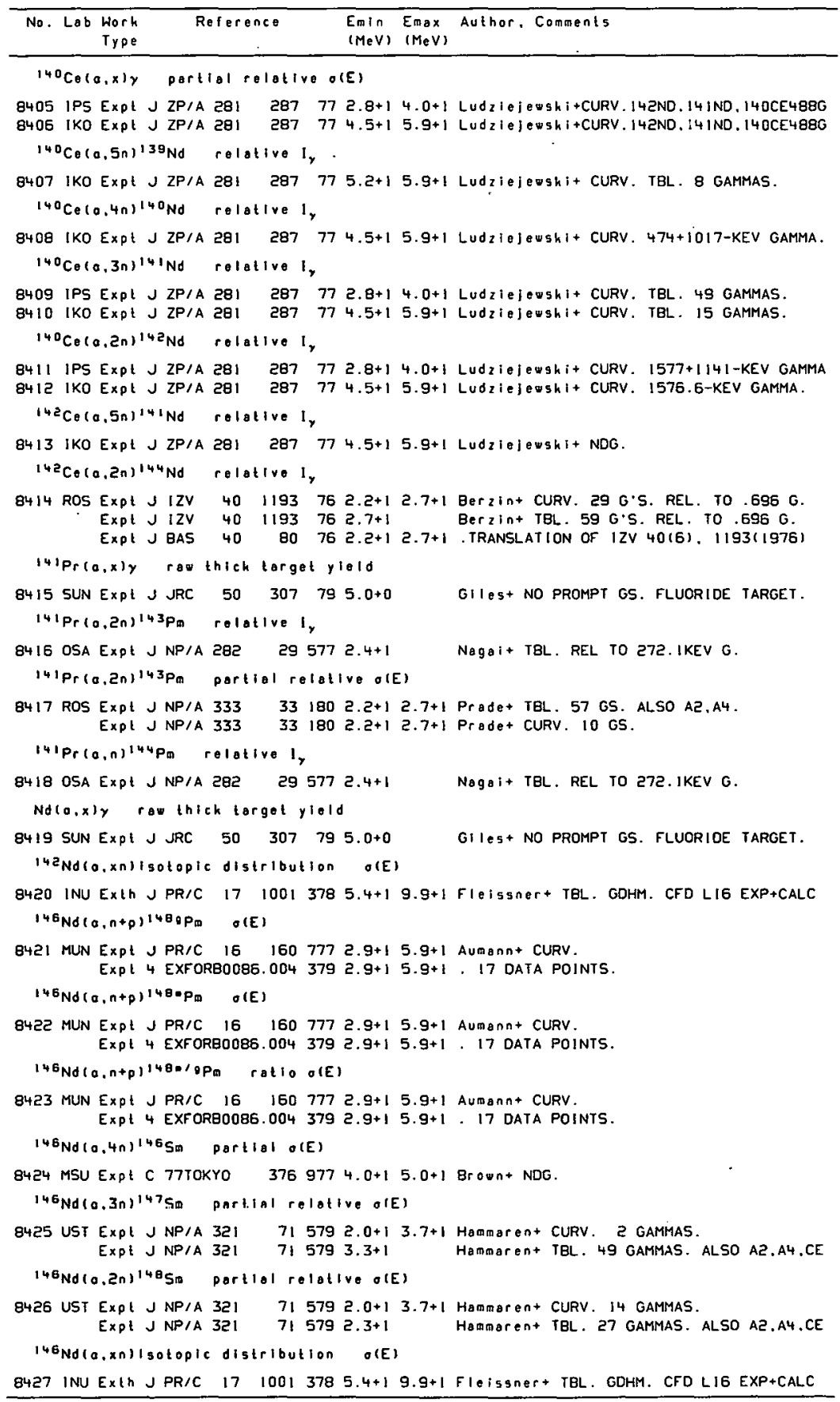




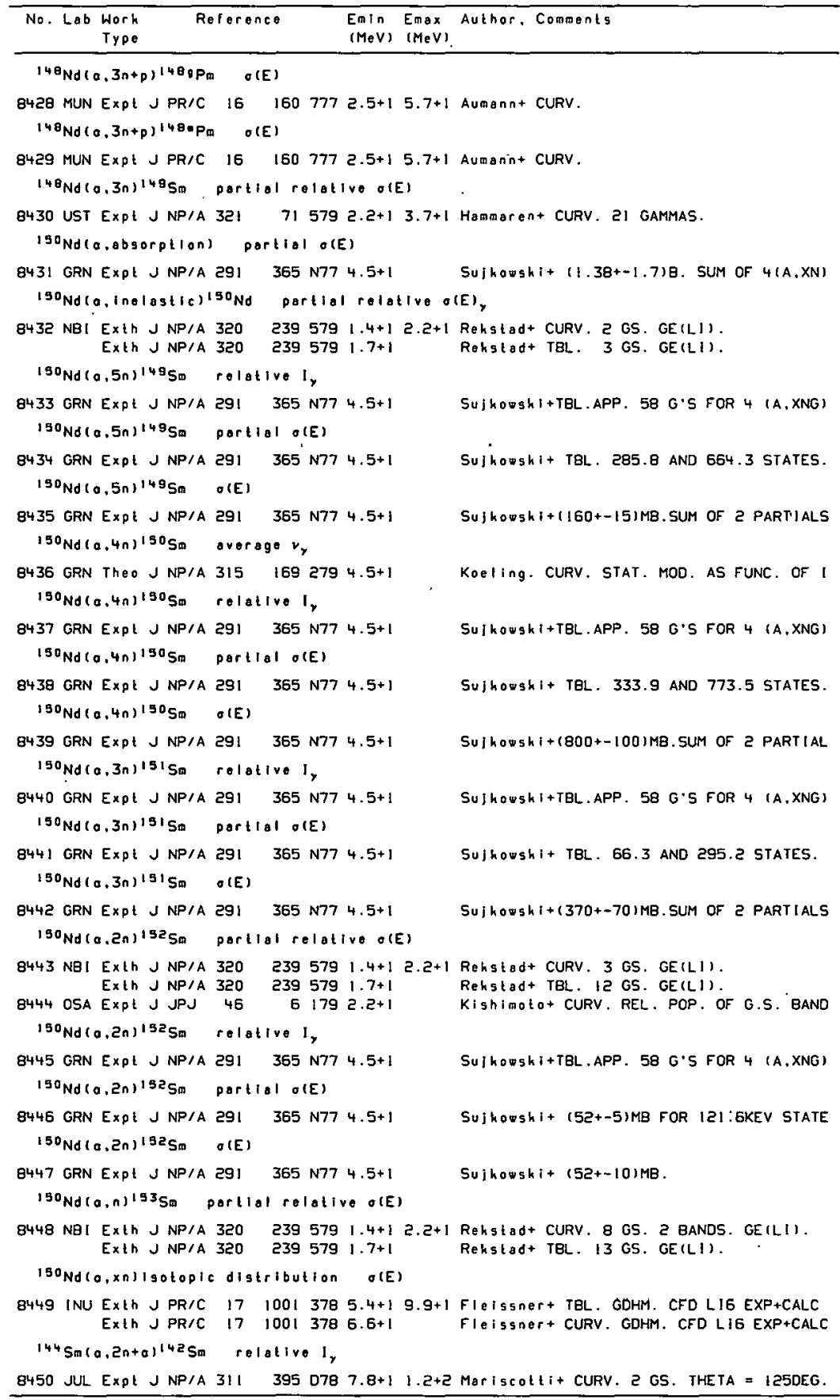




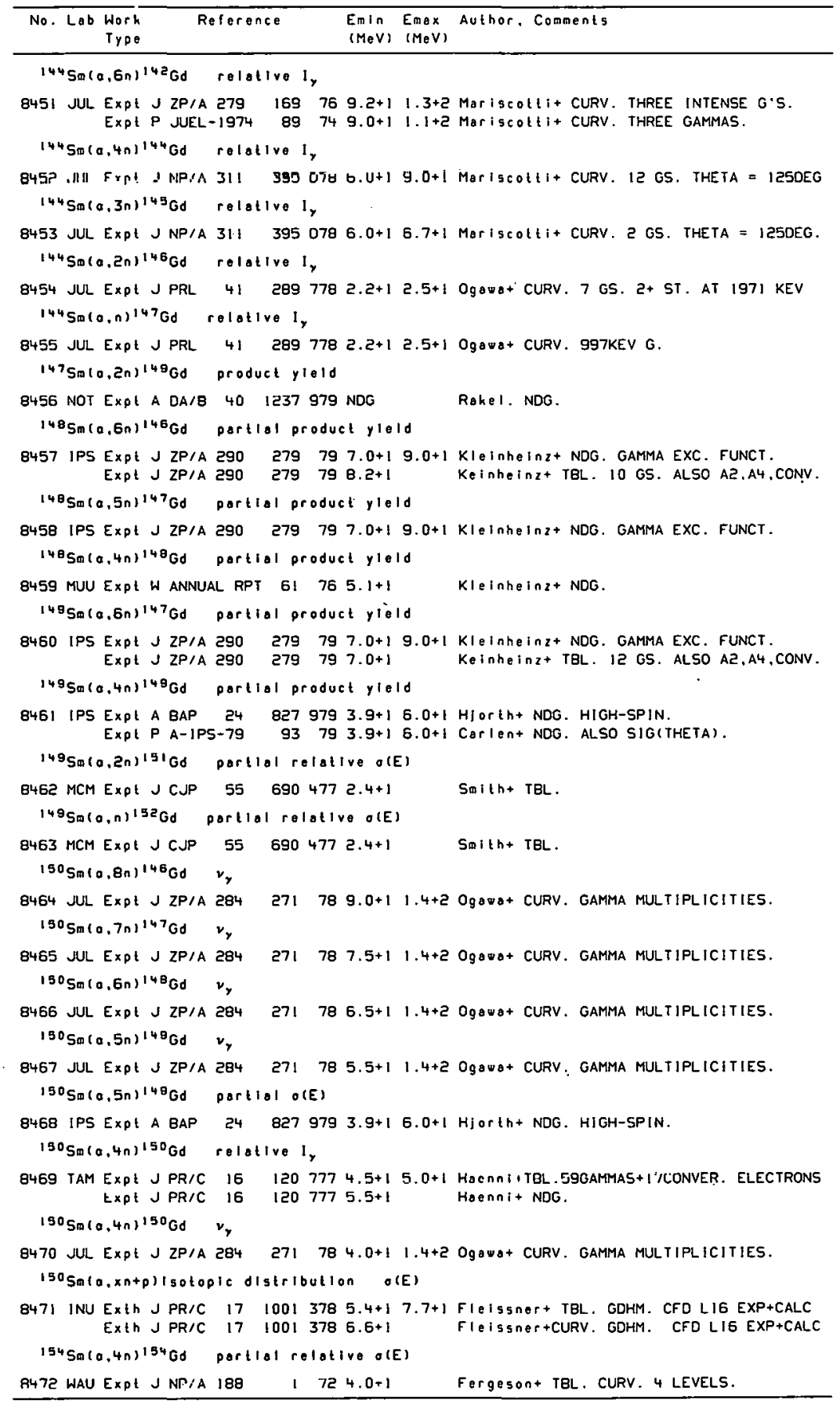




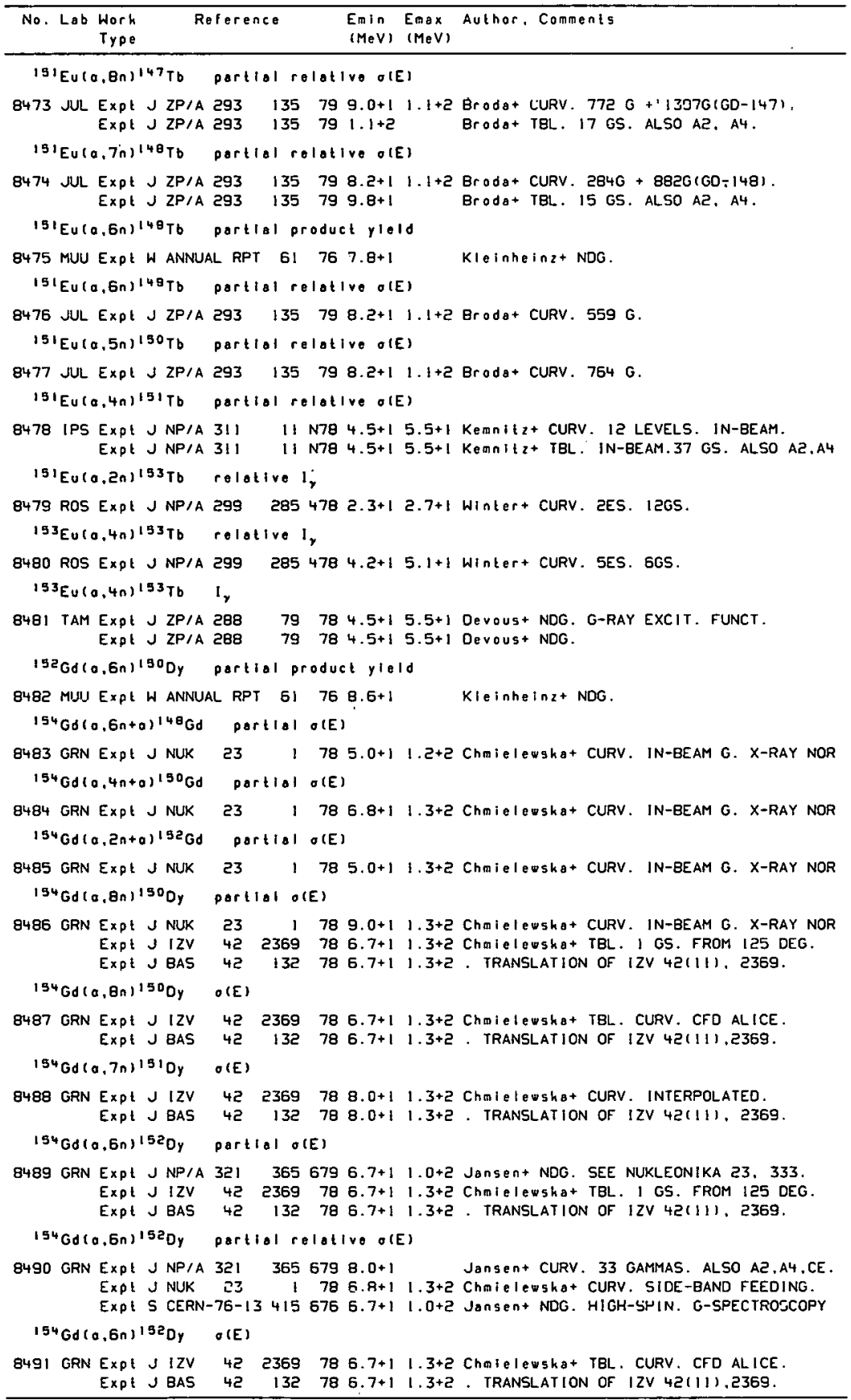


REFERENCES (con $t$ )

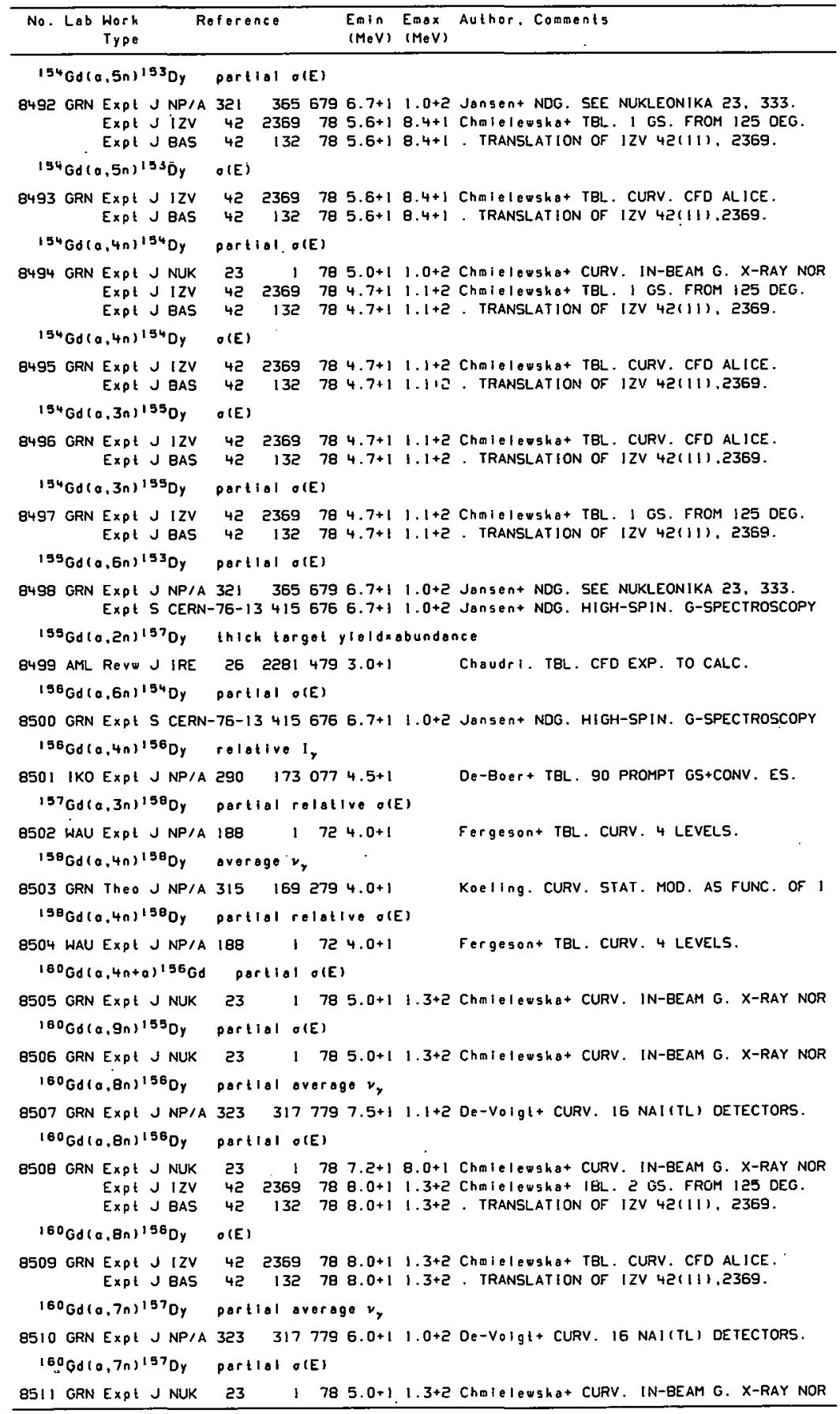




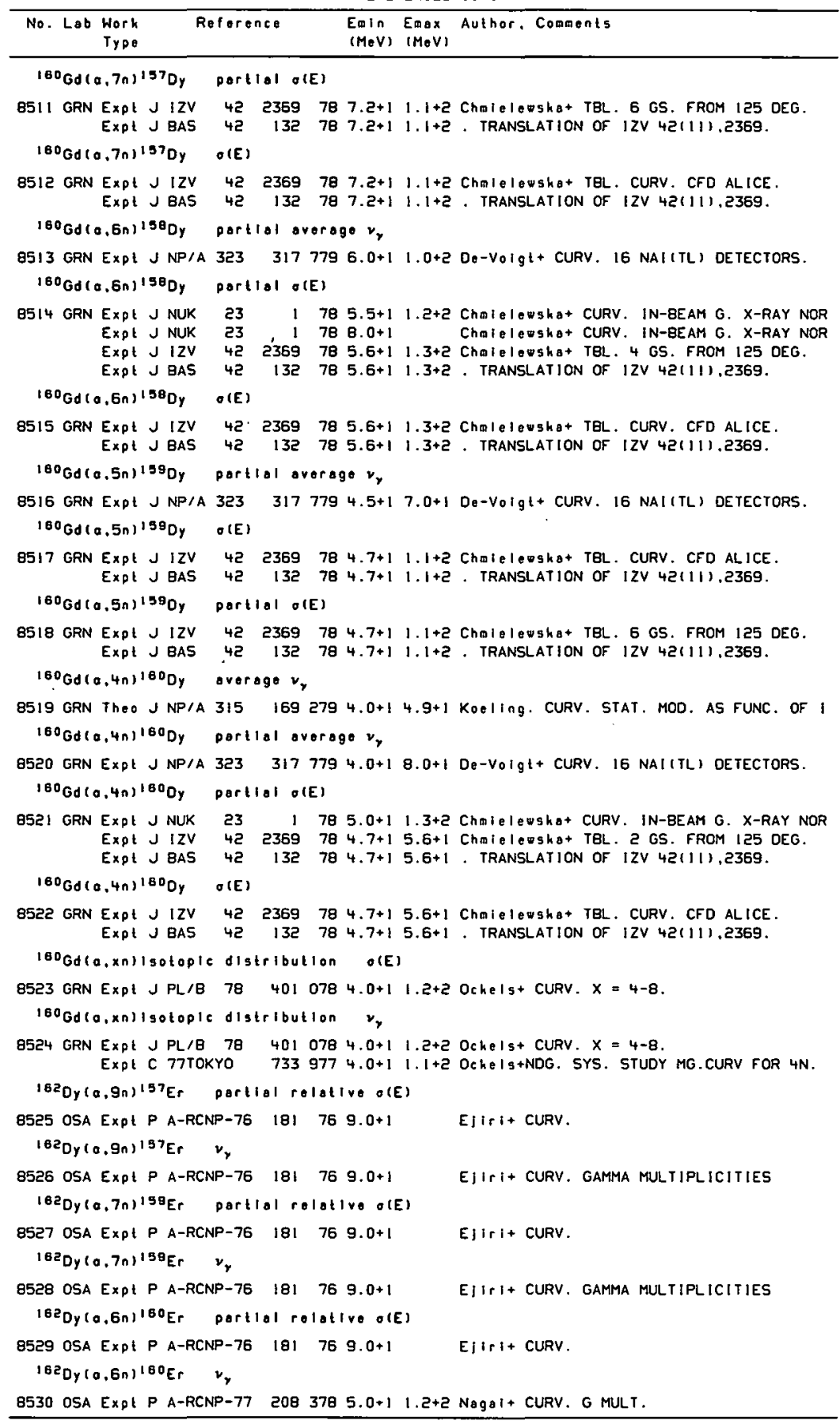




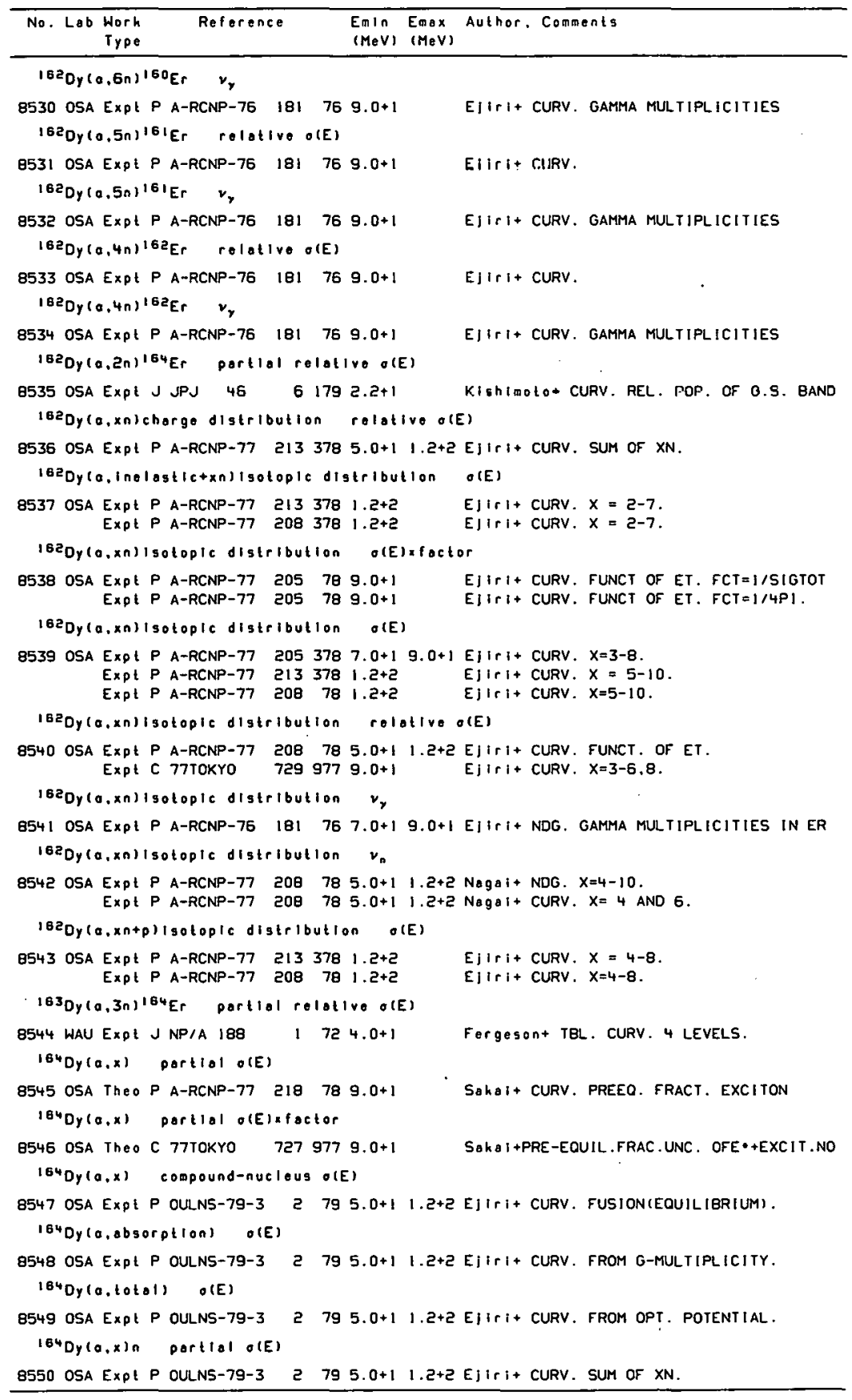




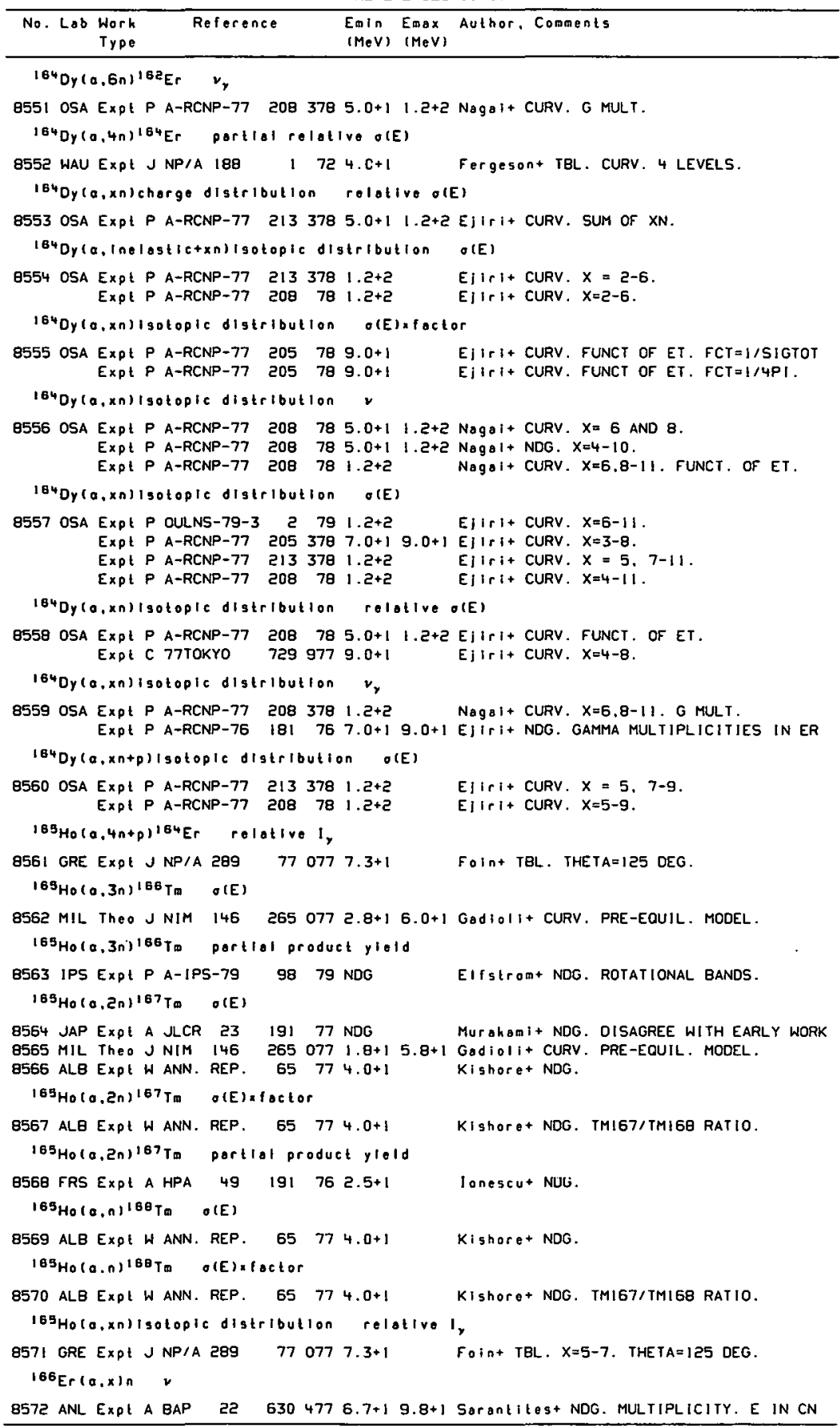


REFERENCES ( $c$ on ()

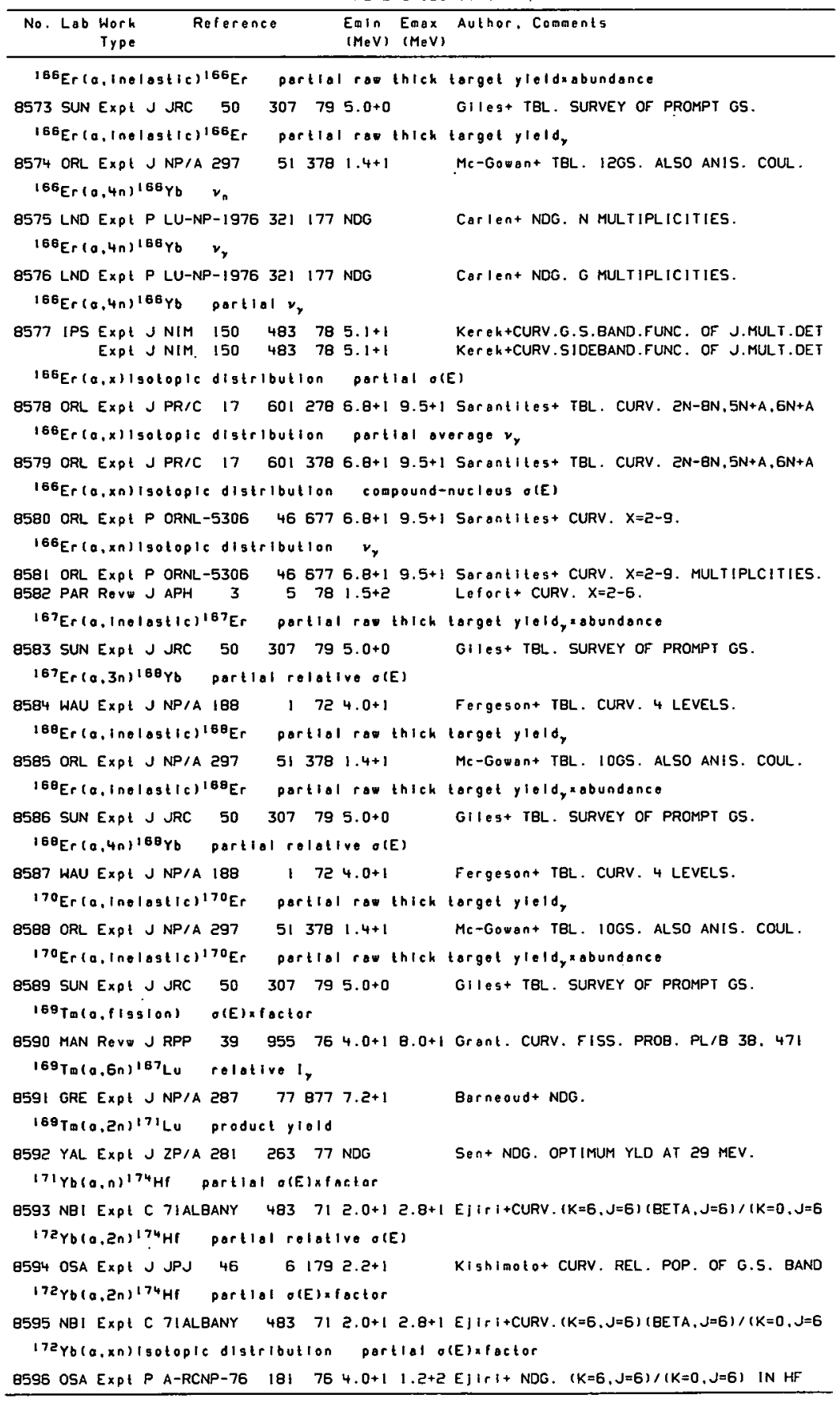




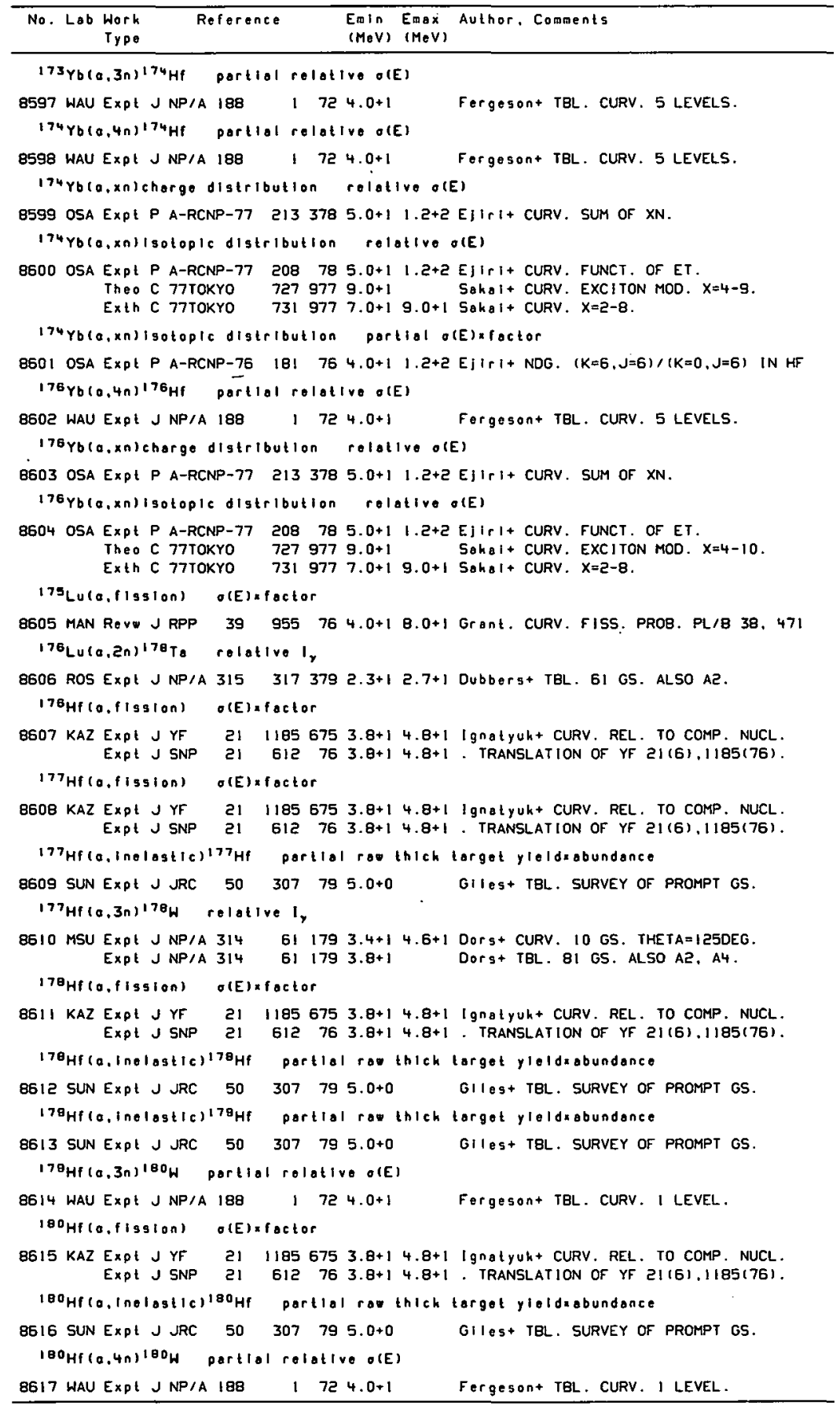


REFERENCES (cont)

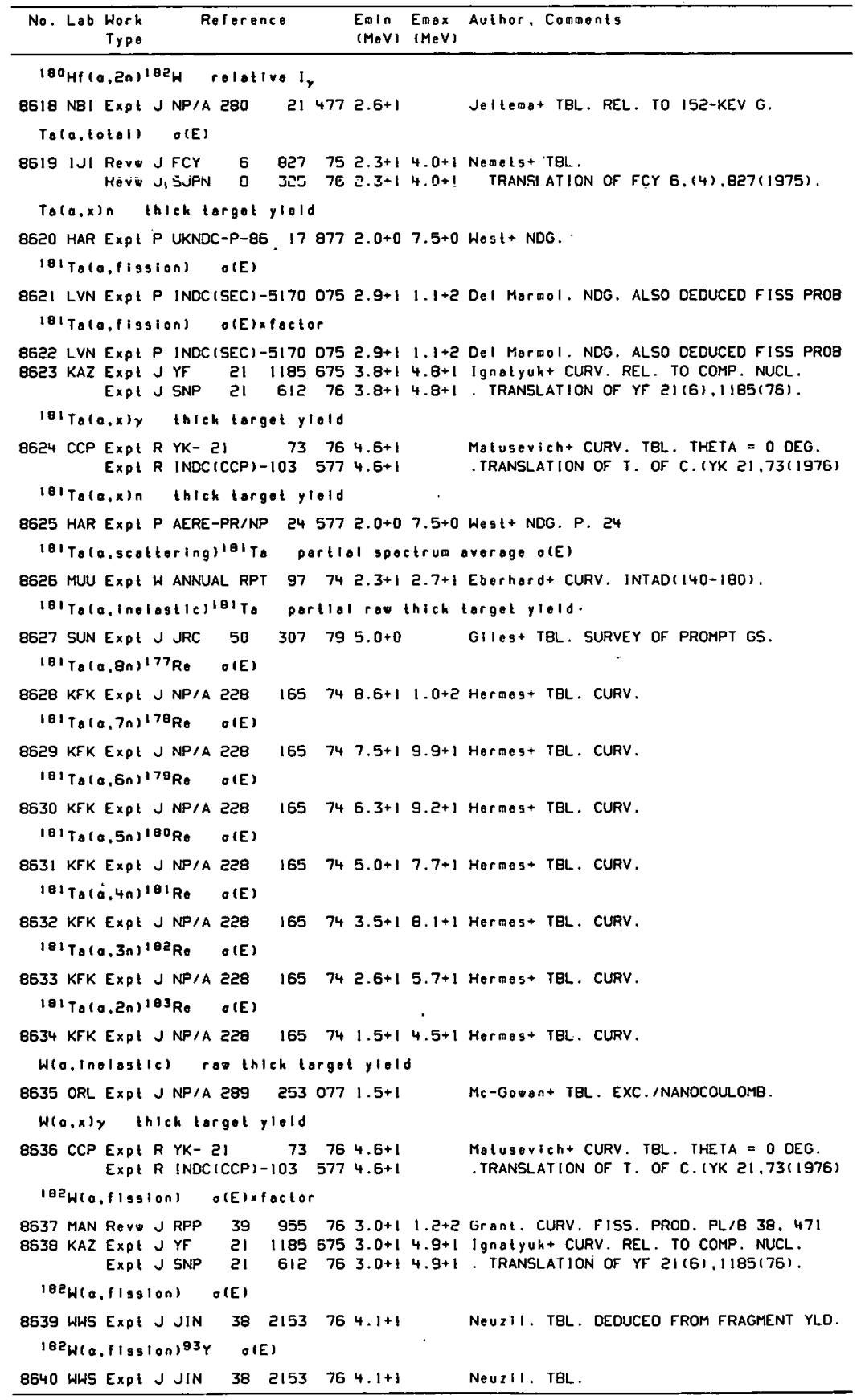




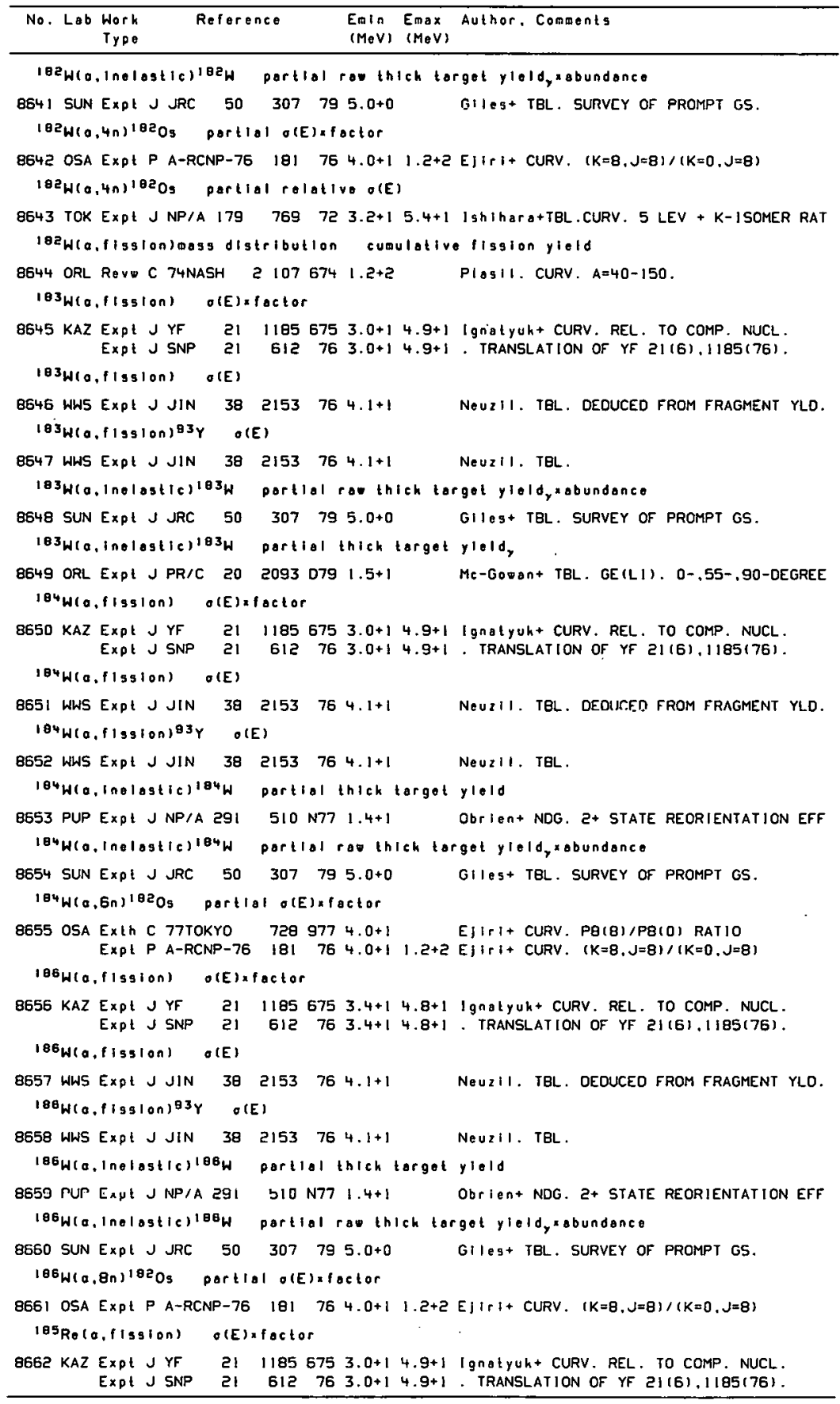




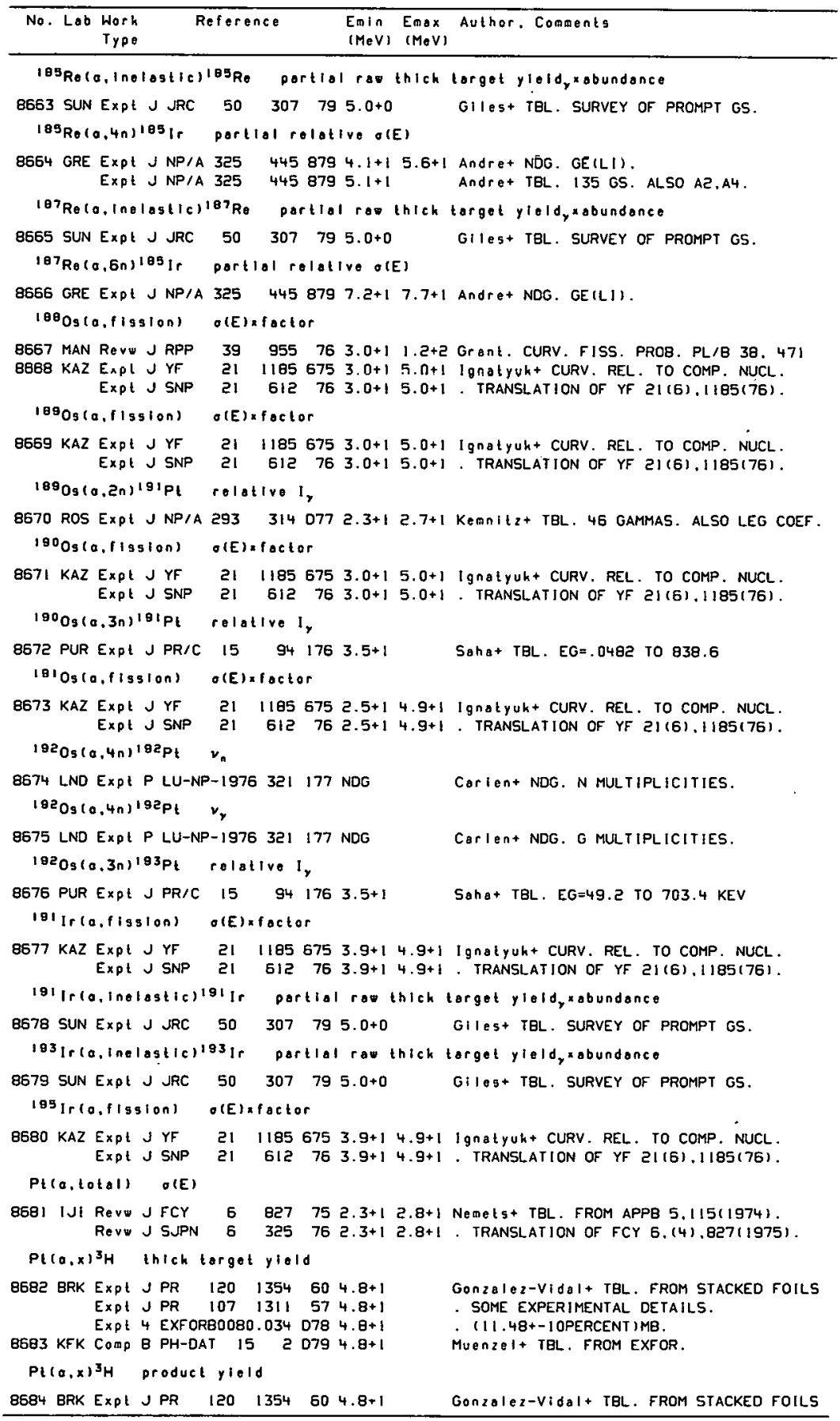




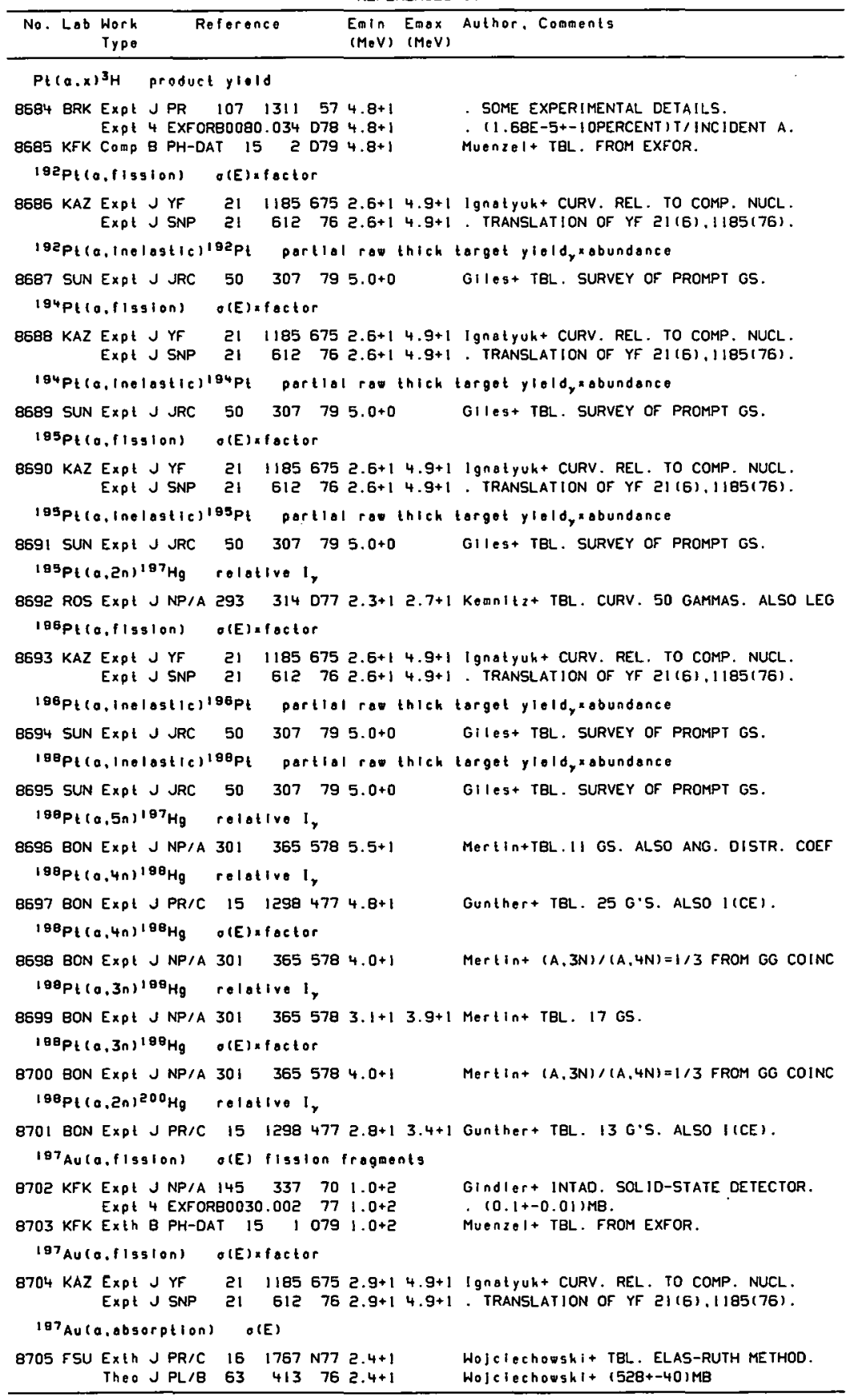


REFERENCES ( c on l )

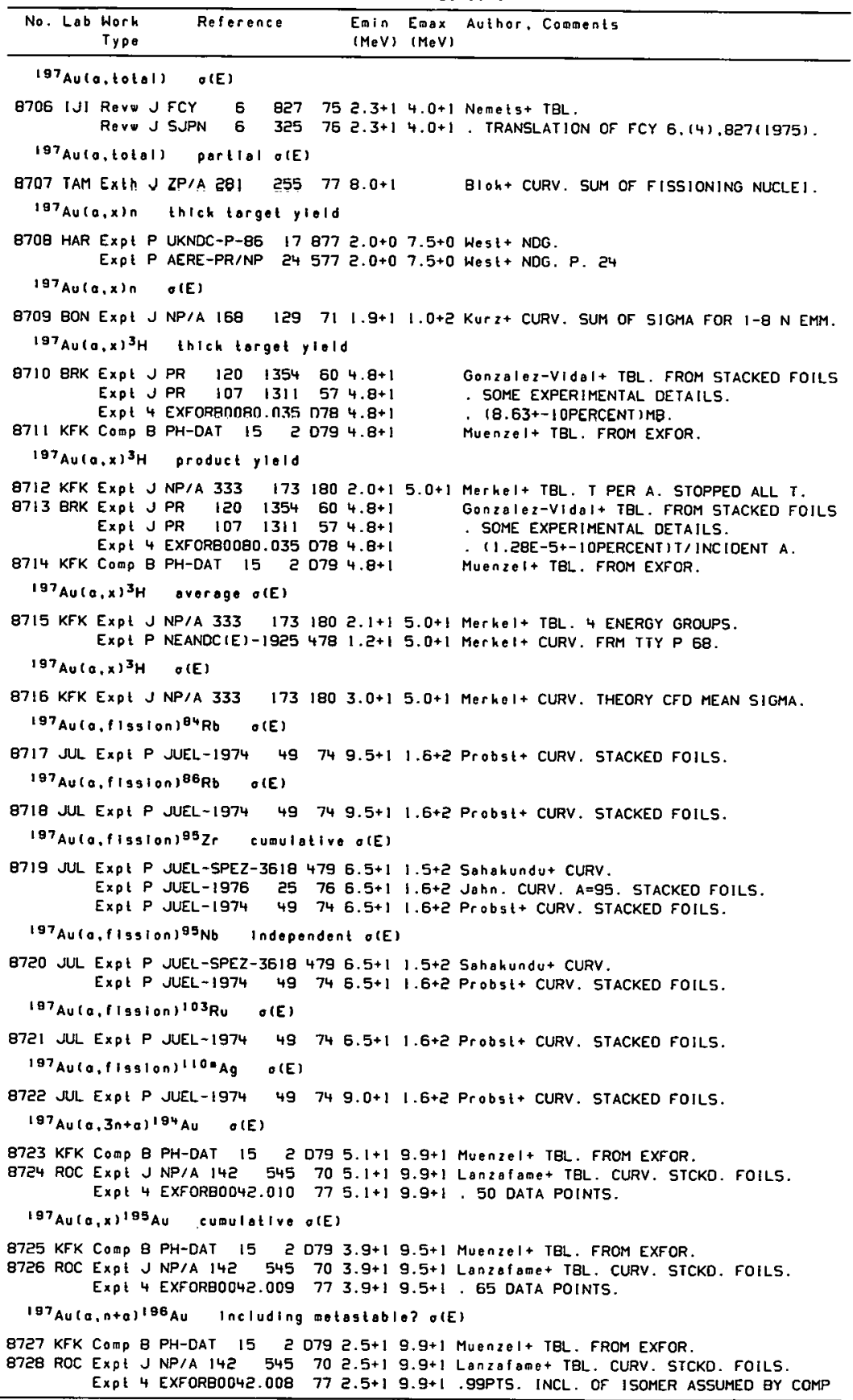


REFERENCES ( c on t)

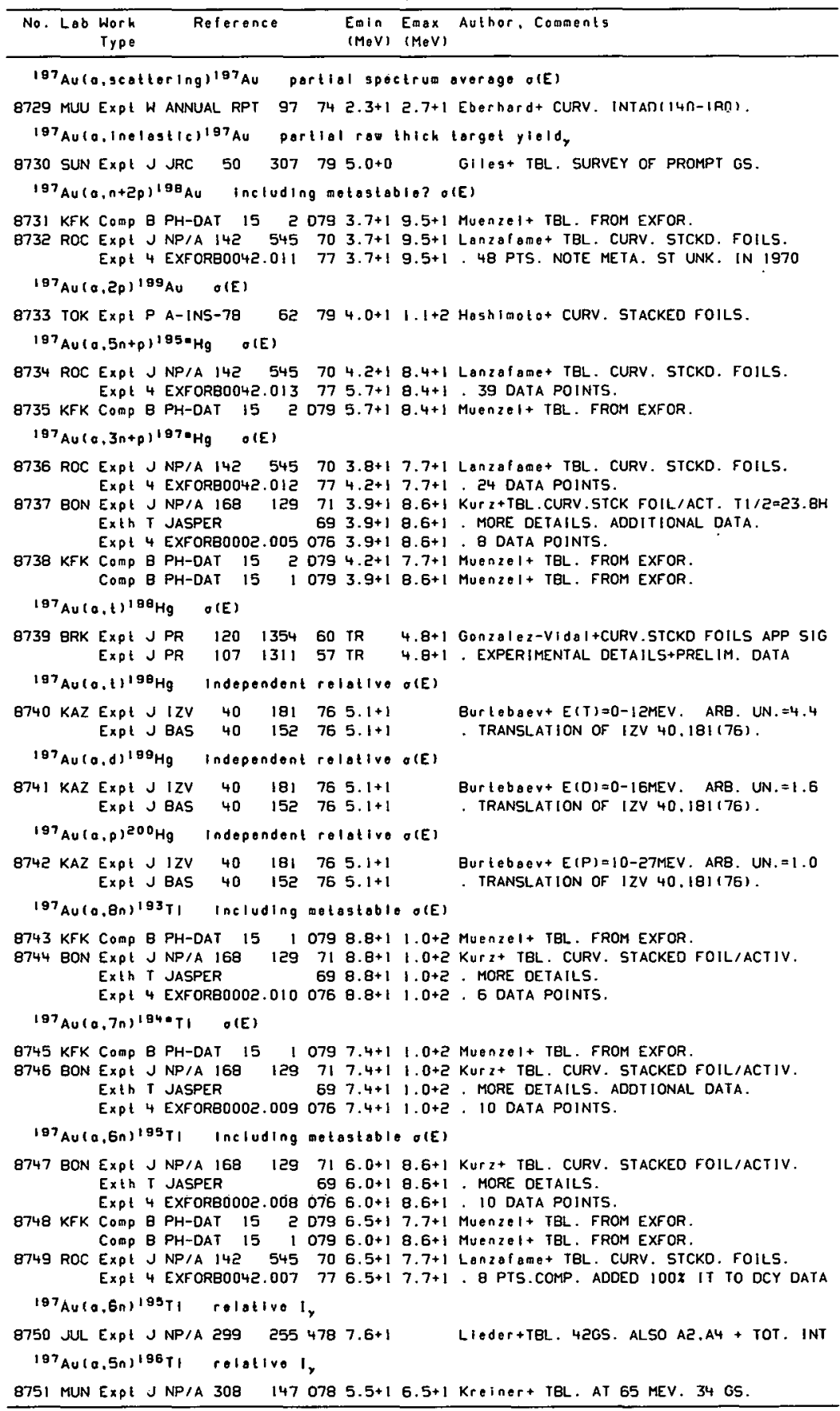




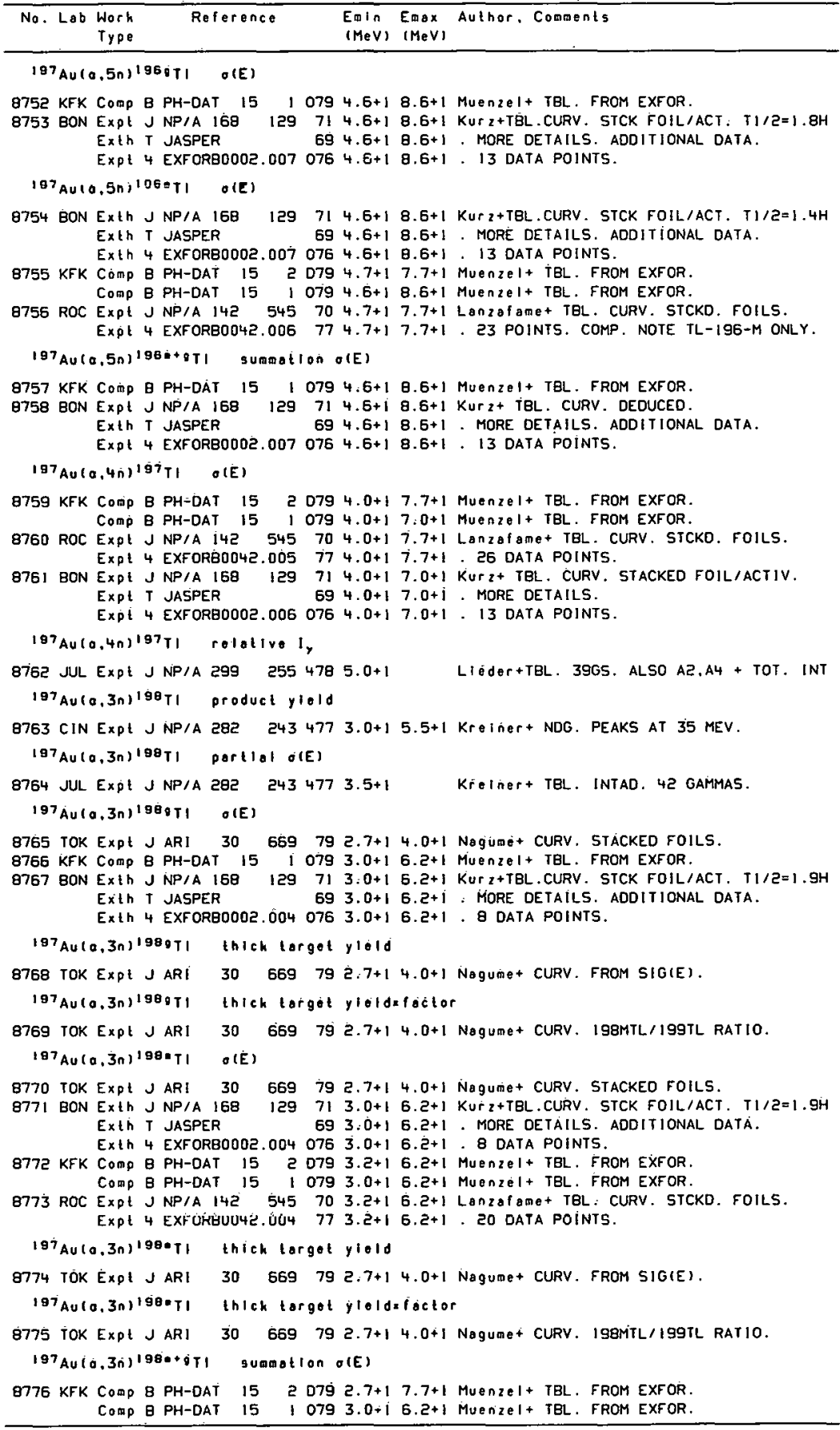




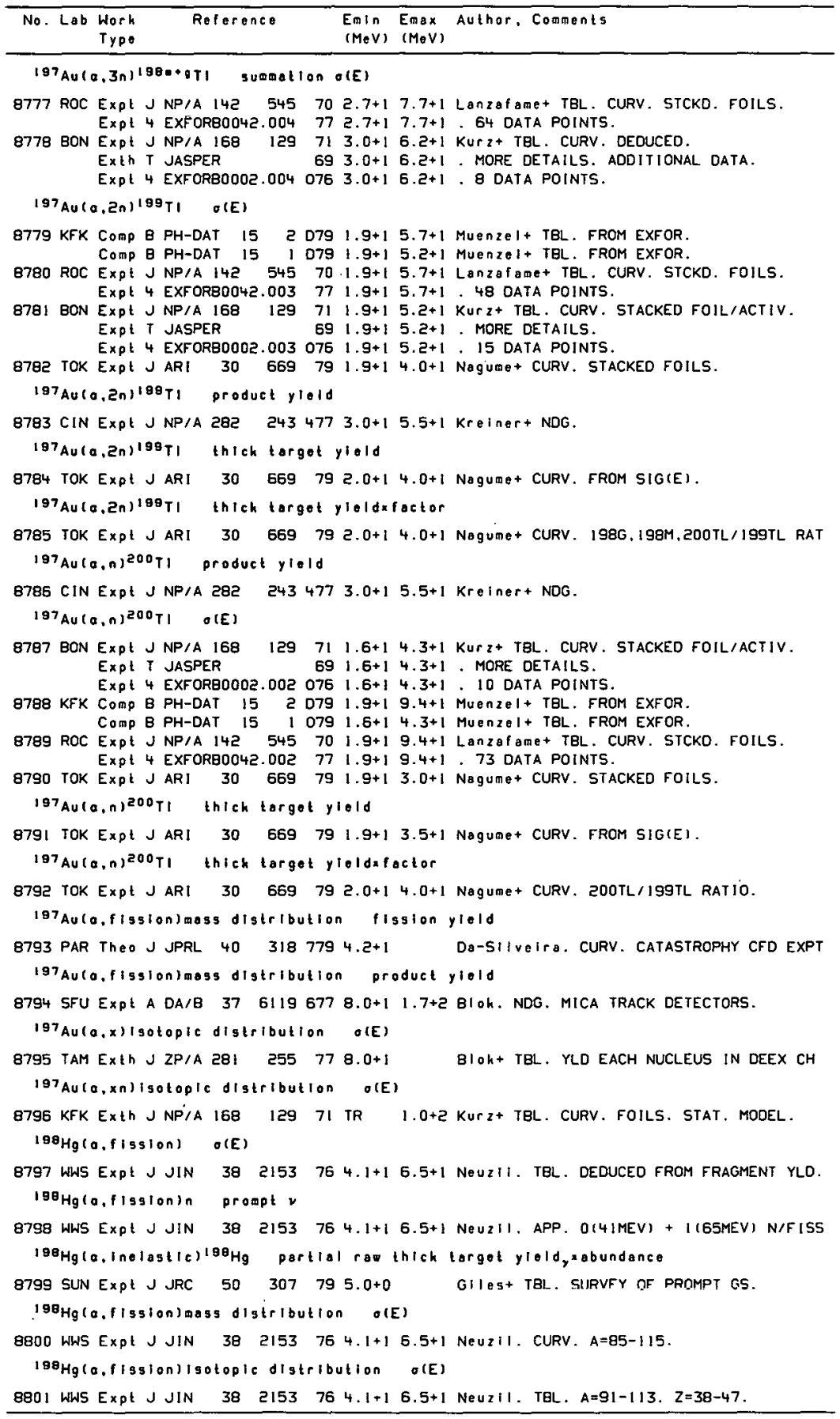




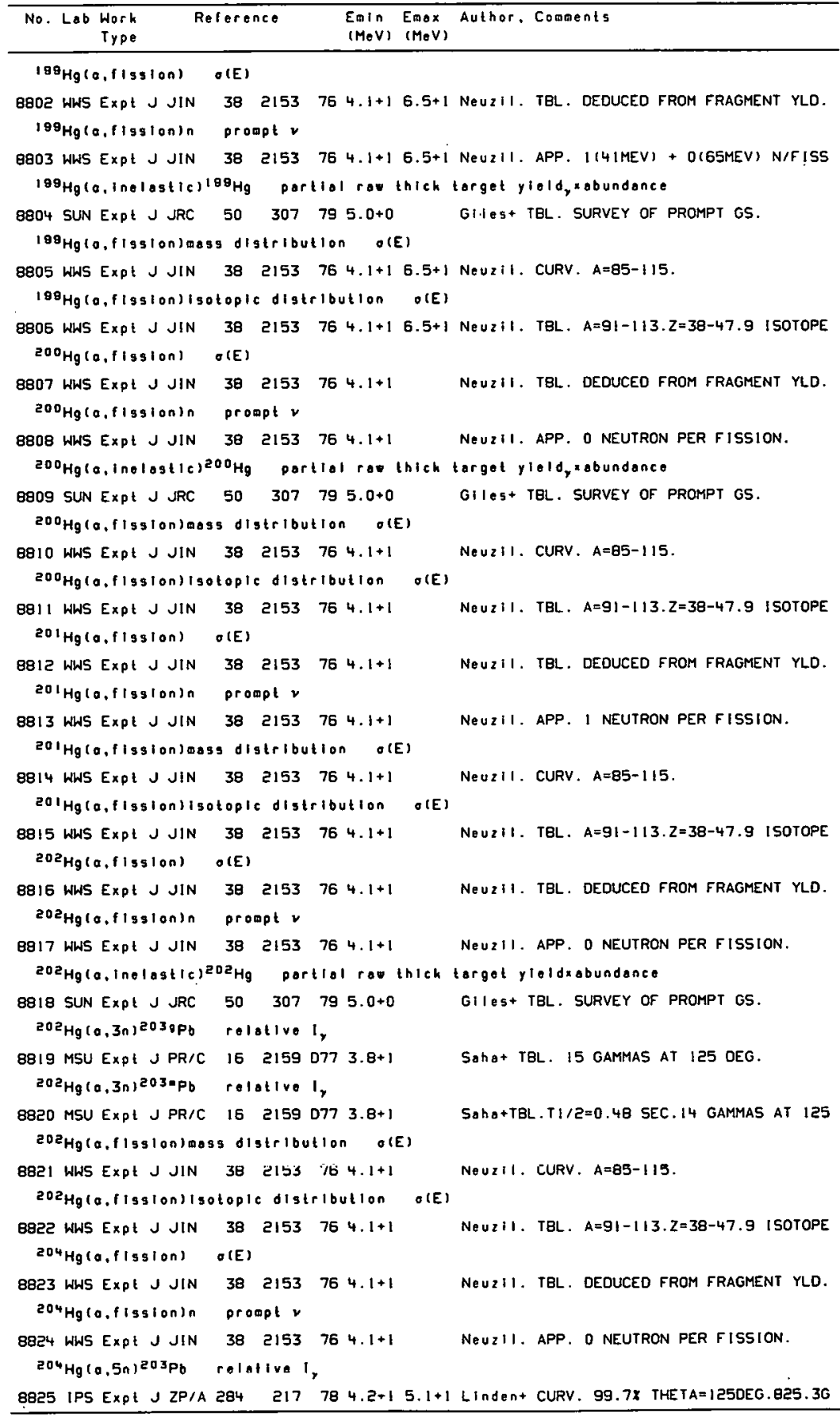




\begin{tabular}{ccc} 
No. Lob Work & Reforence & EmIn Emax Author. Coments \\
Type & & $(M e V)$ (MeV) \\
\hline
\end{tabular}

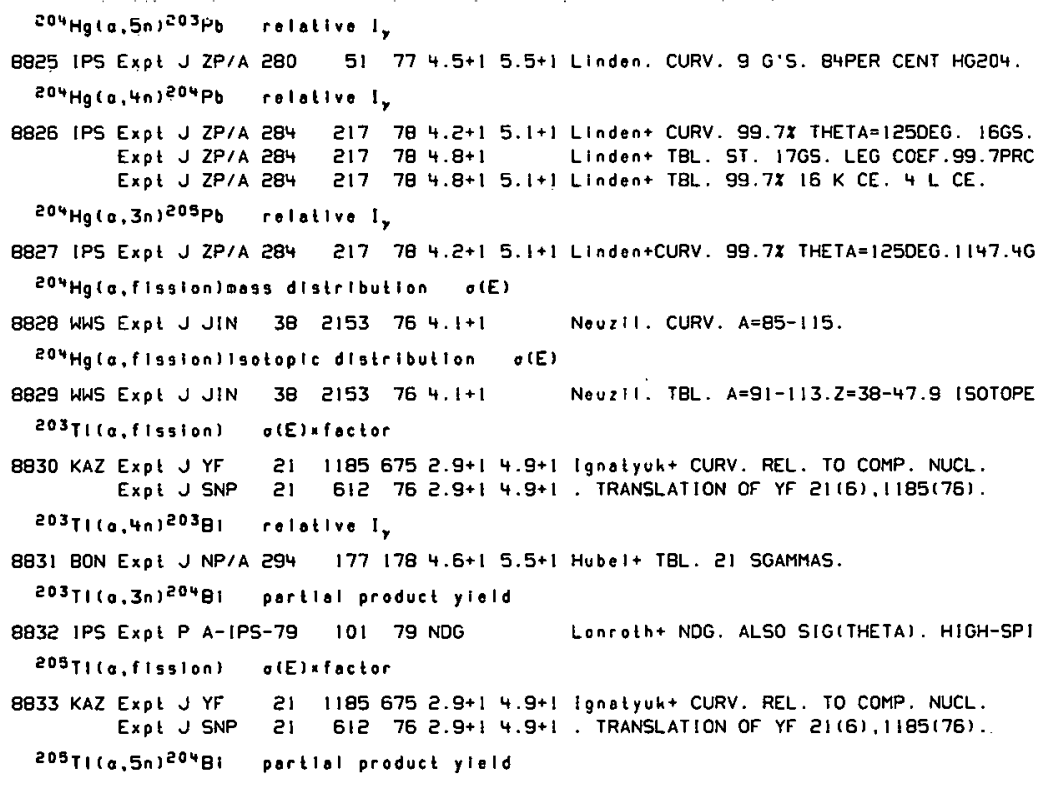




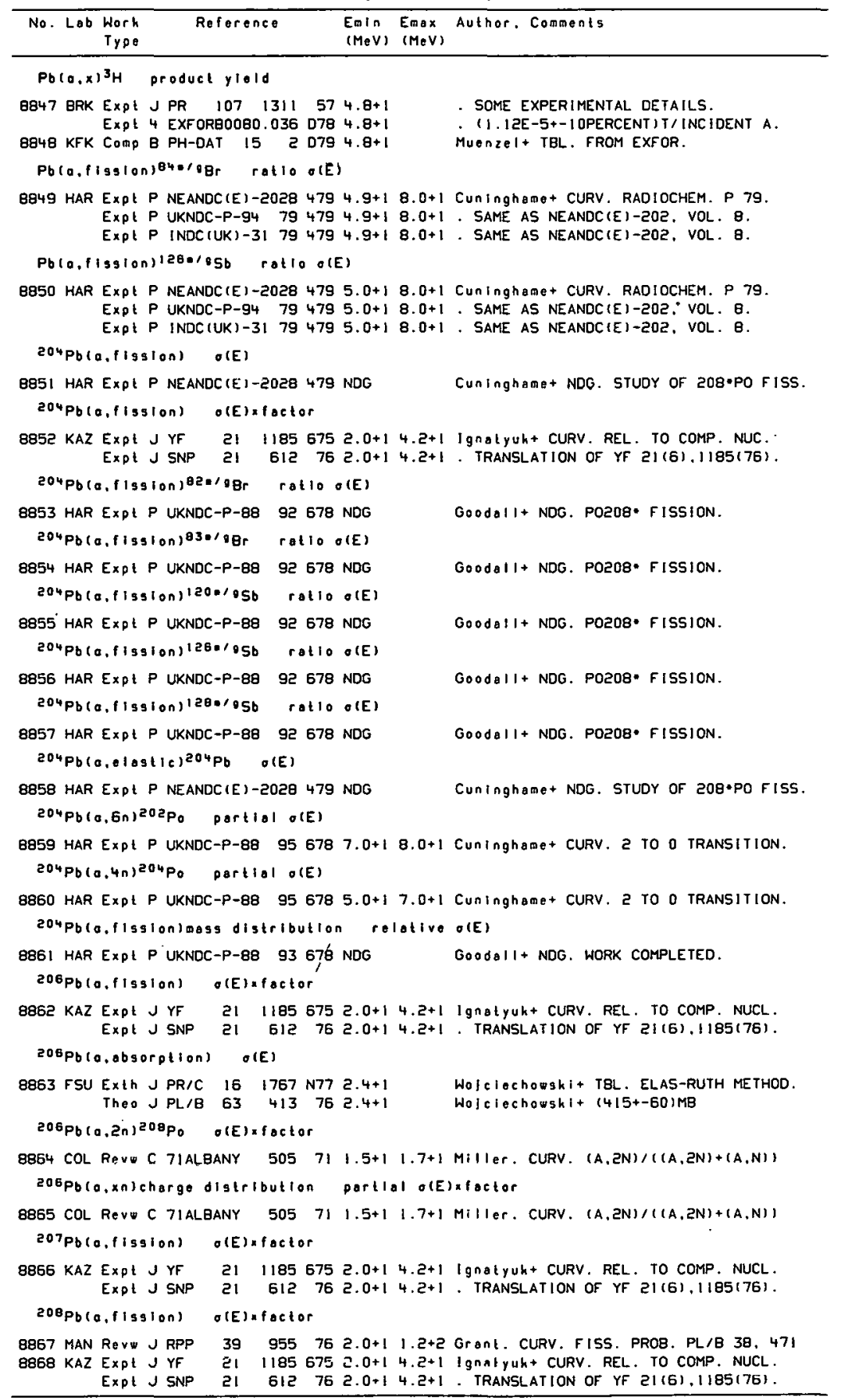


REFERENCES $(\mathrm{c}$ on $\mathrm{t}$ )

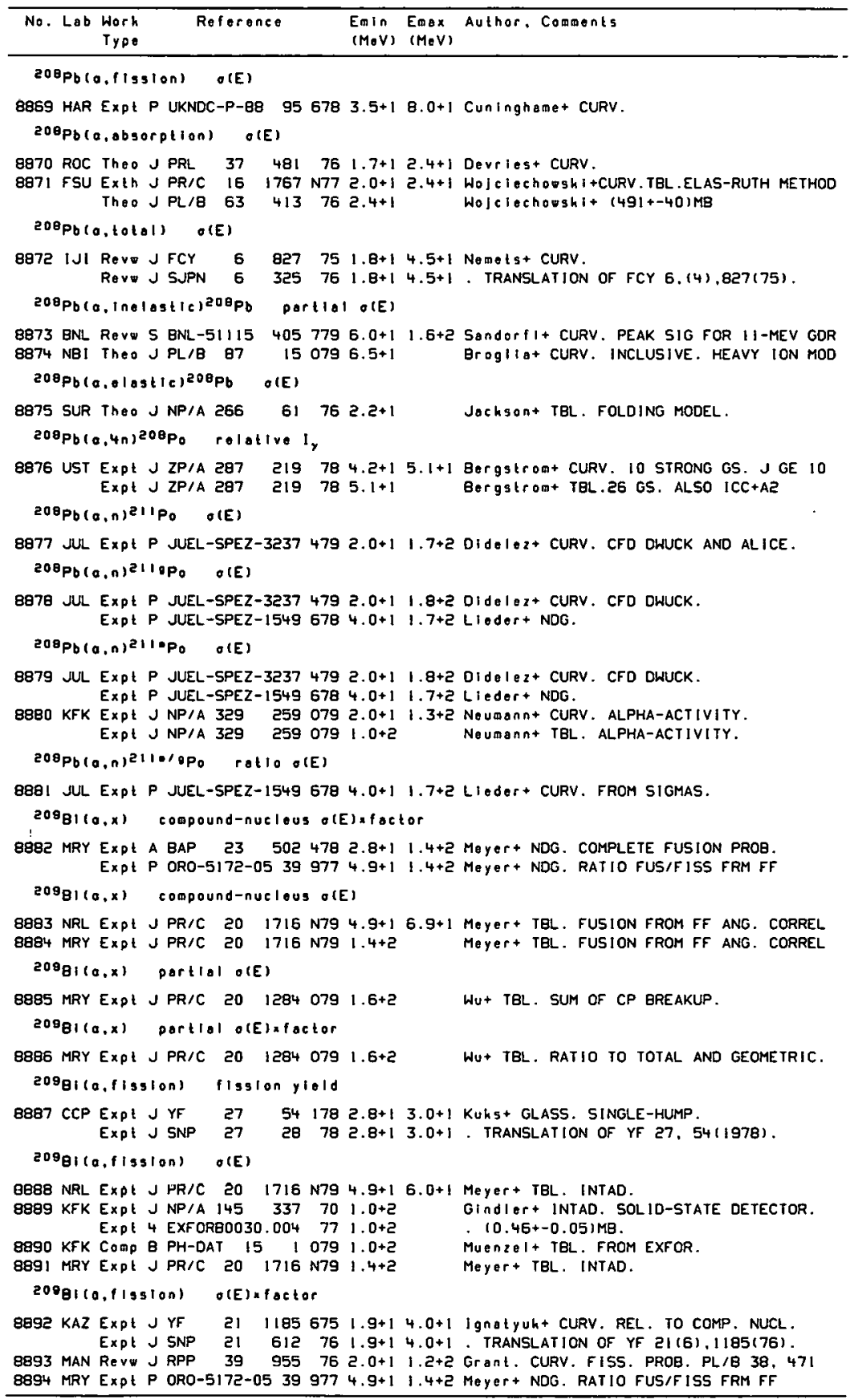


REFERENCES (cont)

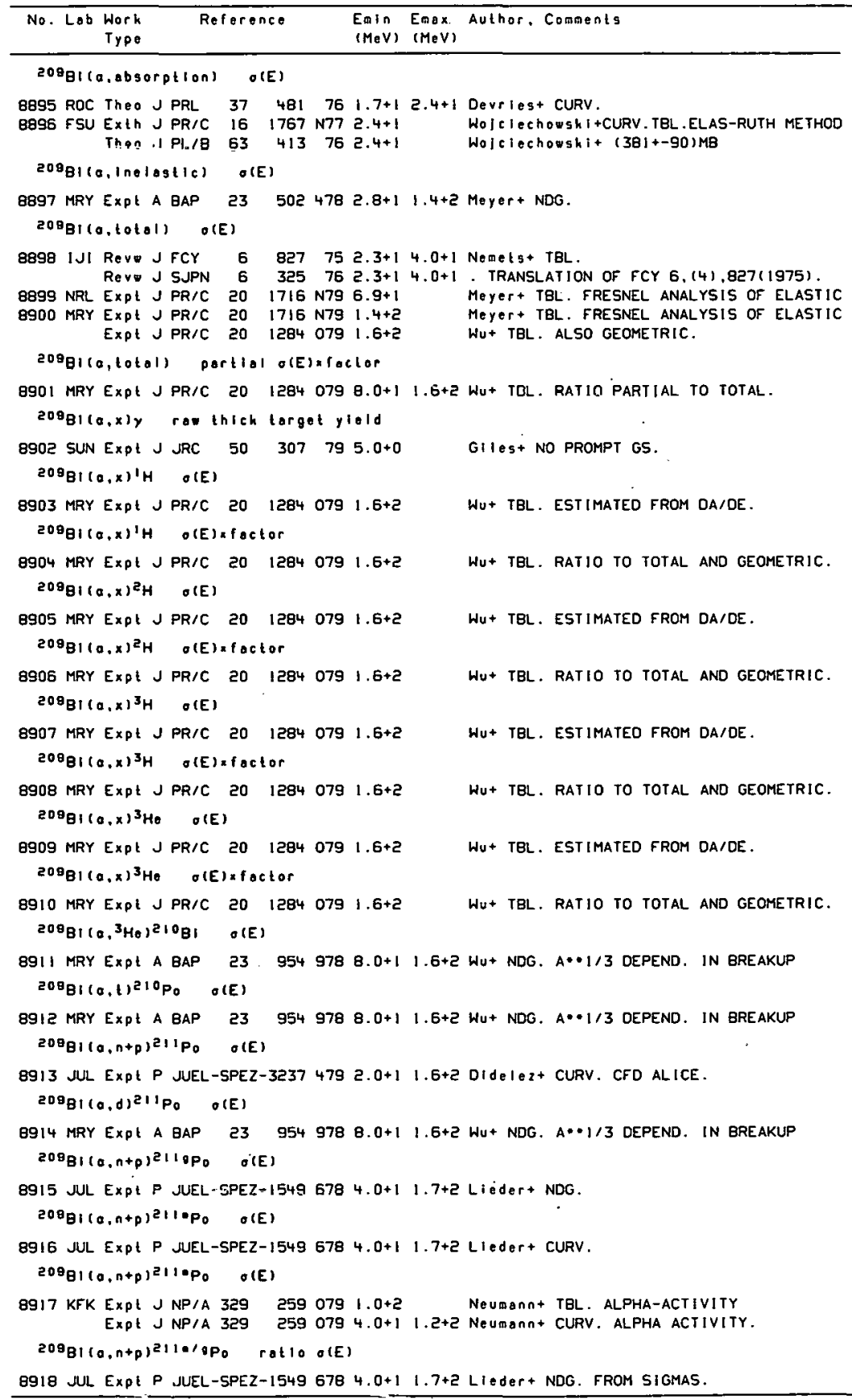


REFERENCES ( c on l)

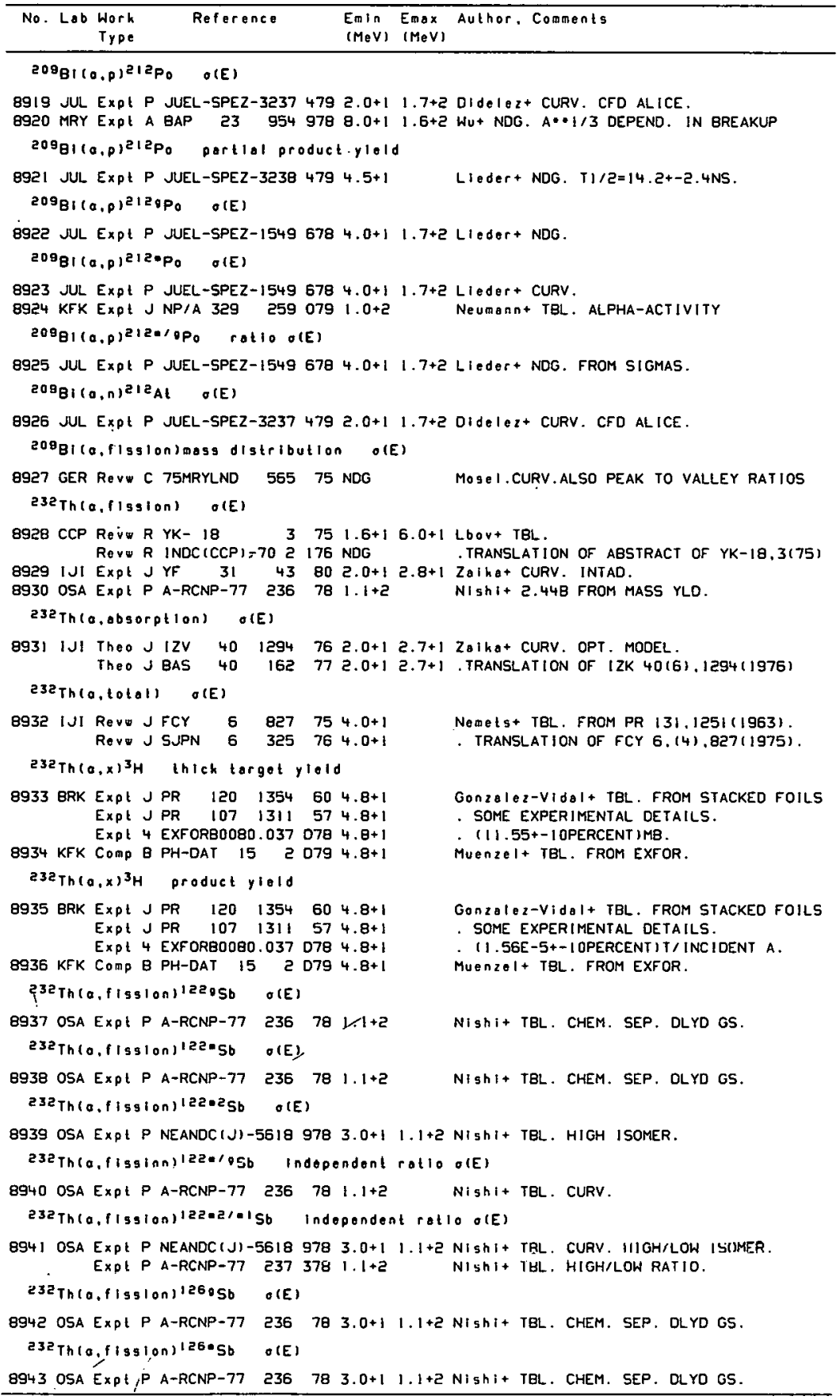


REFERENCES ( cont)

\begin{tabular}{|c|c|c|c|c|c|}
\hline No. Lab & $\begin{array}{l}\text { Work } \\
\text { Type }\end{array}$ & Reference & $\begin{array}{l}\text { Enin } \\
(M e V)\end{array}$ & $\begin{array}{l}\text { Emax } \\
(\operatorname{MeV})\end{array}$ & Author. Comments \\
\hline
\end{tabular}

8944 OSA EXP! P NEANDC (J)-5518 978 3.0+1 $1.1+2$ NIshi+ TBL. HIGH 1SOMER.

azeTh(a,fission)li26orgs . Independent ratio o(E)

Q945 nSA Fxpt P A-RCNP-77 $236783.0+1 \quad 1.1+2 \mathrm{NISh1+}$ TEL. CURV.

azeTh(a,fission)lesezielsb Indopendent ratlo o(E)

8946 05A Expt P NEANDC(J)-561B $9783.0+11.1+2 \mathrm{Nish} 1+$ TEL. CURV. HIGH/LOW ISOMER. Expt P A-RCNP-77 $2373783.0+1 \quad 1.1+2 \mathrm{Nishl+}$ TEL. HIGH/LOW RATIO.

$232 T h(0, f 15 s i o n) 12095 b \quad \sigma(E)$

8947 OSA Expt P A-RCNP-77 $236783.0+1 \quad 1.1+2$ Nishi+ TEL. CHEM. SEP. DLYD GS.

$232 \mathrm{Th}(0, f 15 \mathrm{~s}$ IOn) $120 \times \mathrm{sb} \quad$ o(E)

8949 OSA Expt P A-RCNP-77 236 $783.0+1 \quad 1.1+2$ NIShi+ TBL. CHEM. SEP. DLYD GS.

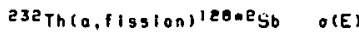

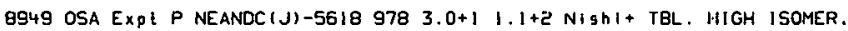

232 Th(a,fission) $1280105 b$ Indepondont rot to o(E)

8950 OSA Expt P A-RCNP-78 $128793.0+11.1+2$ lanishit CURV. RADIOCHEM. DEDUCED L. ExpI P A-RCNP-77 $236783.0+1 \quad 1.1+2 \mathrm{Nish} 1+\mathrm{TBL}$. CURV.

232 Thlo.fisslonilaesils indopendent ratlo o(E)

B951 OSA EXPt P NEANDC(J)-5618 978 3.0+1 1.1+2 NIShI+ TBL. CURV. HIGH/LOW ISOMER. Expl P A-RCNP-77 $2373783.0+11.1+2 \mathrm{NIShI+TBL.} \mathrm{HIGH/LOW} \mathrm{RATIO.}$

$232 \mathrm{Th}(0, \mathrm{flg} 910 \mathrm{O}) 13005 \mathrm{Ob}$ O(E)

8952 OSA EXPt P A-RCNP-77 $236793.0+1 \quad 1.1+2 \mathrm{NiSh}+\mathrm{T} \quad \mathrm{TBL}$. CHEM. SEP. OLYD GS.

232 Th(a,fission)13005b o(E)

8953 OSA Expl P A-RCNP-77 $236783.0+1 \quad 1.1+2 \mathrm{Nishi+}$ TEL. CHEM. SEP. OLYD GS

232 Th $(0, f$ isston) 13002 Sb $O(E)$

8954 OSA Expl P NEANDC(J)-5618 978 3.0+1 1.1+2 Nishi+ TEL. HIGH ISOMER.

232 Th $(0, f$ IssIOn) $1300 / 95 b$ ratio o(E)

8955 OSA Expl P A-RCNP-78 I28 $793.0+11.1+2$ ImBnishi+ CURV. RADIOCHEM. DEDUCED L. ExpI P A-RCNP-77 $236783.0+1 \quad 1.1+2 \mathrm{NIShI+TBL.} \mathrm{CURV.}$

$232 \mathrm{Th}(0, \mathrm{fIs} 1 \mathrm{ION}) 130-2 / 0 \mathrm{ISb}$ rotlo O(E)

B956 OSA Expt P NEANDC (J)-56I8 978 3.0+1 $1.1+2$ NIShi+ TBL. CURV. HIGH/LOW ISOMER. Expl P A-RCNP-77 $2373783.0+11.1+2 \mathrm{NIShi+TBL.} \mathrm{HIGH/LOW} \mathrm{RAT:0.}$

232 Thla,fission)131alote independent rotlo olE

8957 OSA Expt P A-RCNP-78 $128793.0+11.1+21 \mathrm{manish}+$ + CURV. RADIOCHEM. DEDUCED L.

$232 T h(0, f$ isston) $133019 T$ o Independont ratlo o(E)

8958 OSA Expl P A-RCNP-78 $128793.0+1$ 1.1+2 Imonishit CURV. RADIOCHEM. DEDUCED L.

$232 T h(a, f i s s i o n) 132-10$ I Indopondont rotlo o(E)

8959 OSA Expt P A-RCNP-78 $128793.0+1 \quad 1.1+21 \mathrm{manishi+CURV.} \mathrm{RADIOCHEM.} \mathrm{DEOUCED} \mathrm{L.}$

$232 T h(a, f 1 s 510 n) 134+101$ Independent ratlo $O(E)$

B960 OSA Expt P A-RCNP-78 $128793.0+11.1+2$ Imanishi+ CURV. RADIOCHEM. DEDUCEO L.

23aTh(a.tission)1480Pa Independent $\sigma(E)$

8961 MUN Expl J JIN $40 \quad 1782.6+14.1+1$ Aumann+ CURV.

Expl J PR/C $16 \quad 1607772.6+14.1+1$ Aumannt CURV.

Expt R KFK-2298 $614762.7+14.5+1$ Aumonnt CURV. RADIOCHEM.

EXPt 4 EXFORBO0B7.002 $3792.6+14.1+1$. 5 DATA PUINTS

e3eTh(a,tission)1480pa Independent o(E)

8962 MUN Expl J JIN 40 I $782.6+14.1+1$ Aumann+ CURV.

Expt J PR/C $16 \quad 1607772.6+14.1+1$ Aumann+ CURV.

Exp! R KFK-2298 $614762.7+14.5+1$ AunOnn+ CURV. RADIOCHEM.

Exp: 4 EXFORB0087.002 $3792.6+14.1+1$. 5 DATA POINTS.

232 Th(a,fIgsion)148. OPa Independent rollo olE)

8963 MIIN EXPt J PR/C $161607772.6+14.1+1$ Aumann+ CURV.

Expt R KFK-2298 61476 ट. 7+1 4.5+1 Aumannt CURV. RAnIOCHEM. 
REFERENCES (cont)

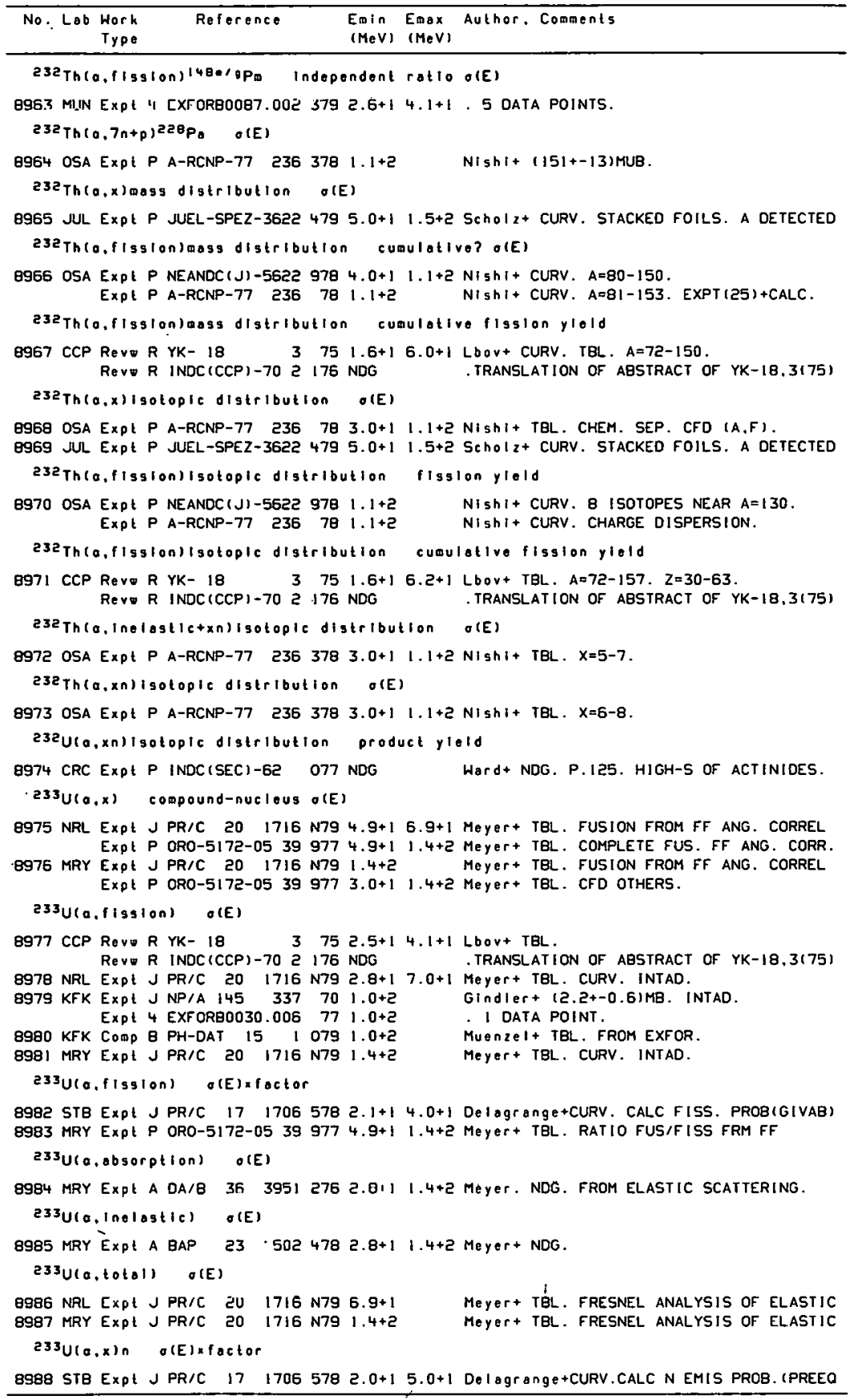


REFERENCES (cont)

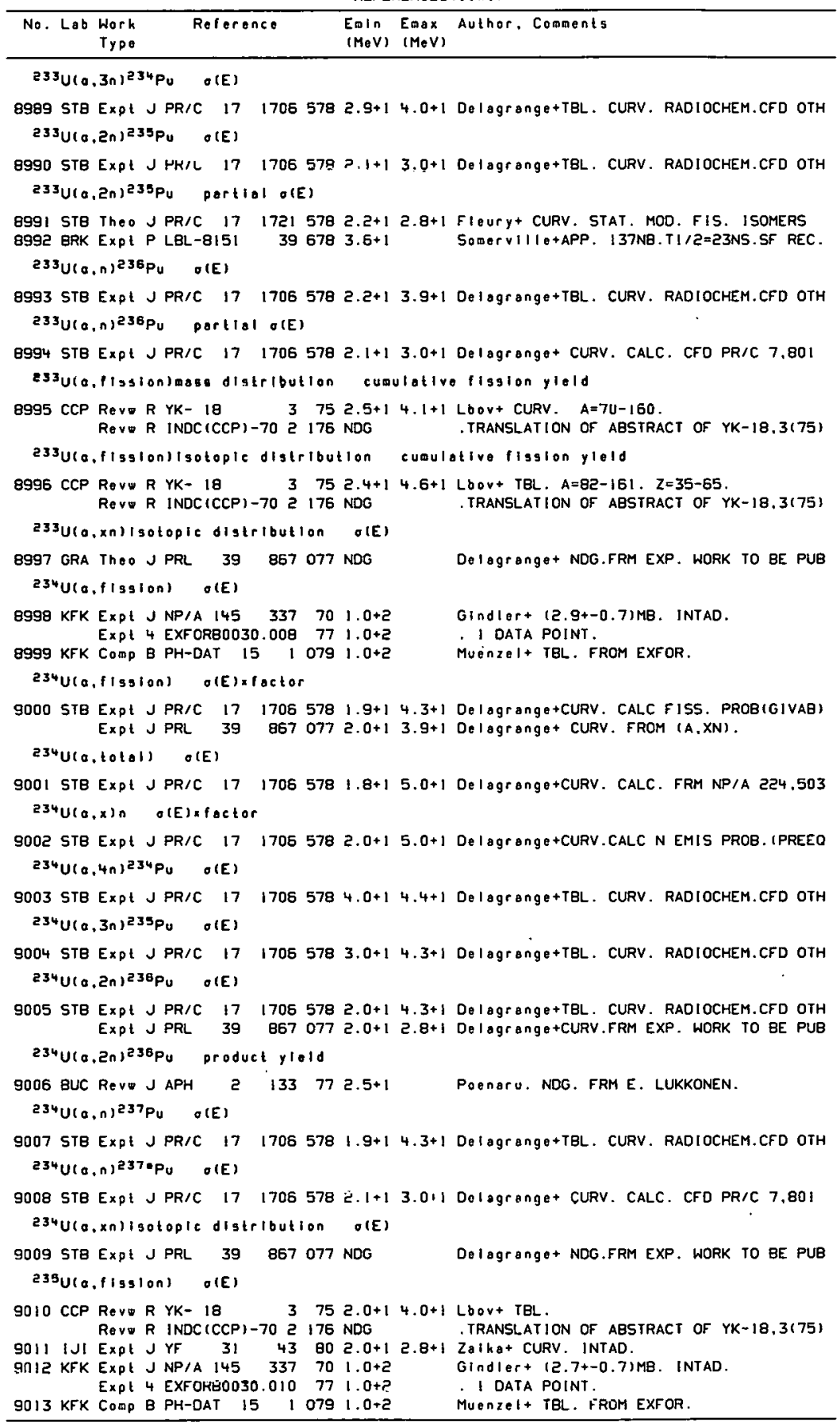




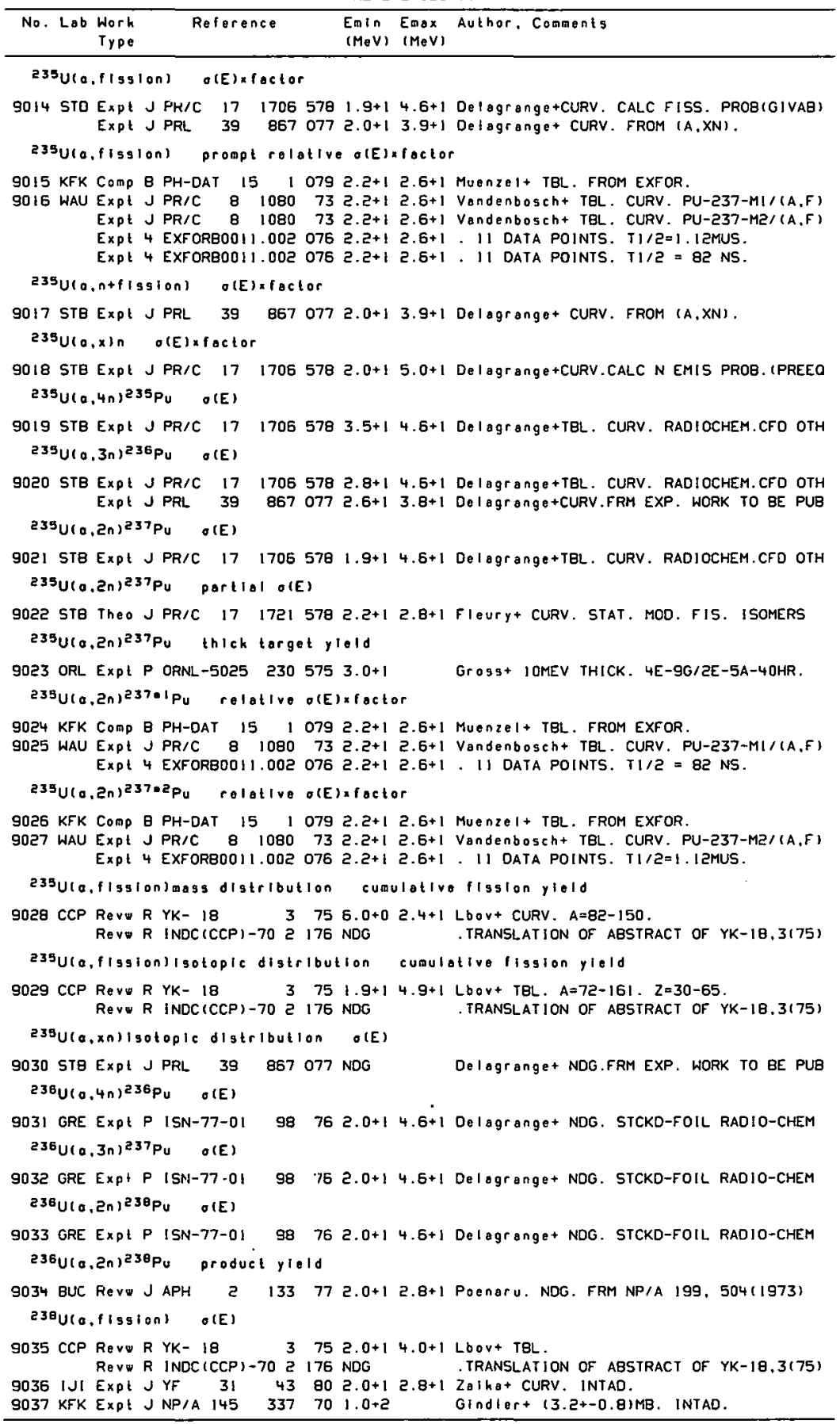




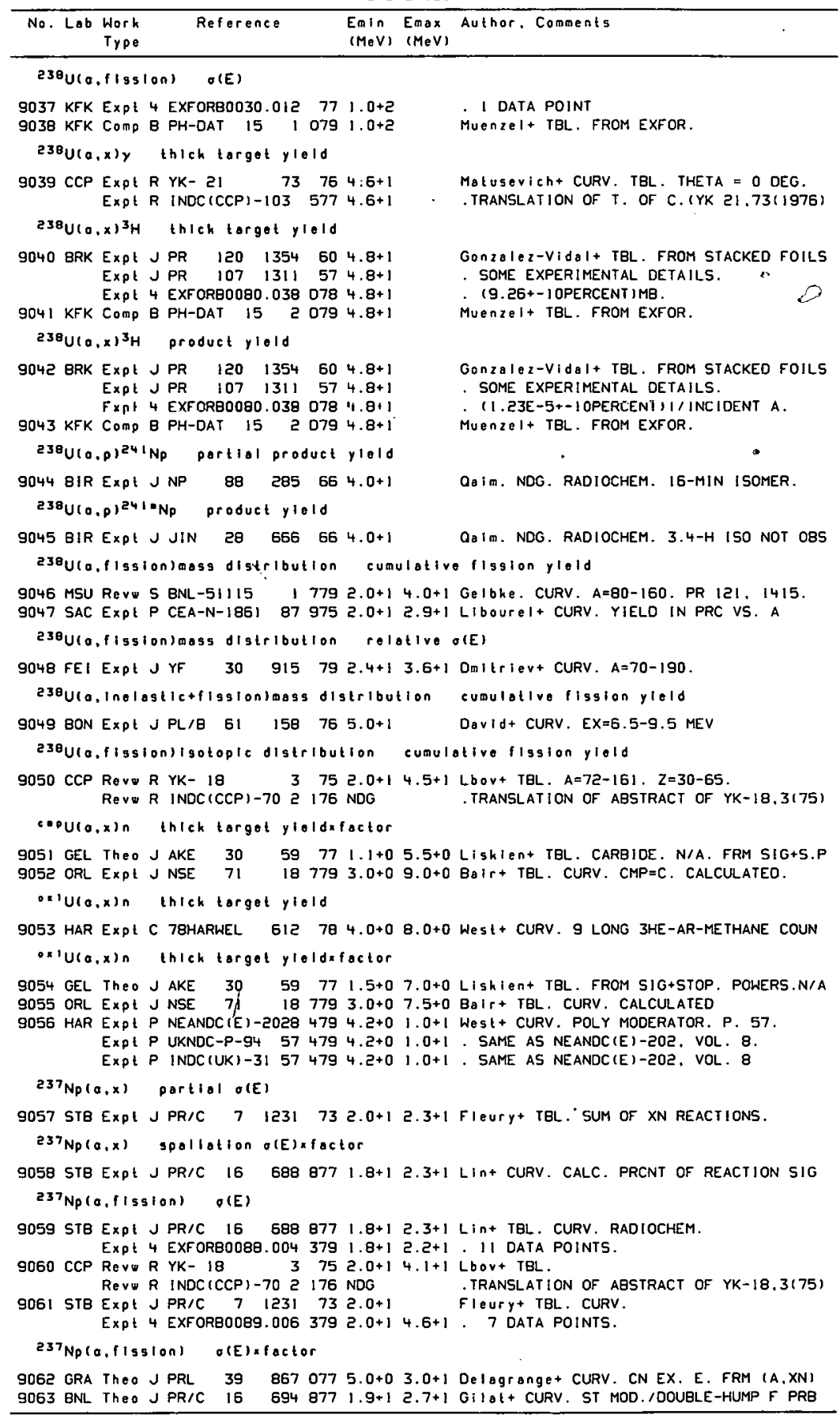




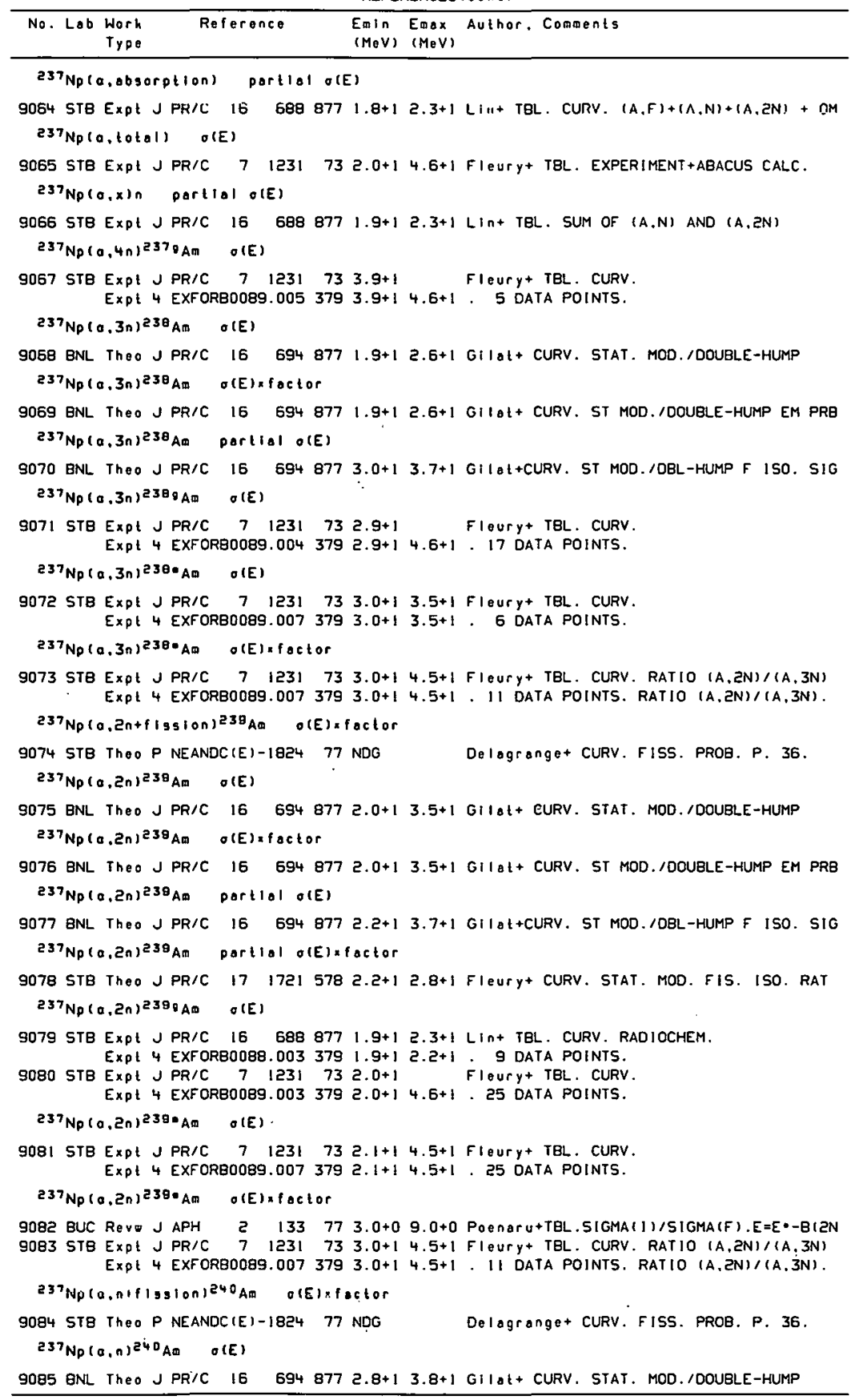




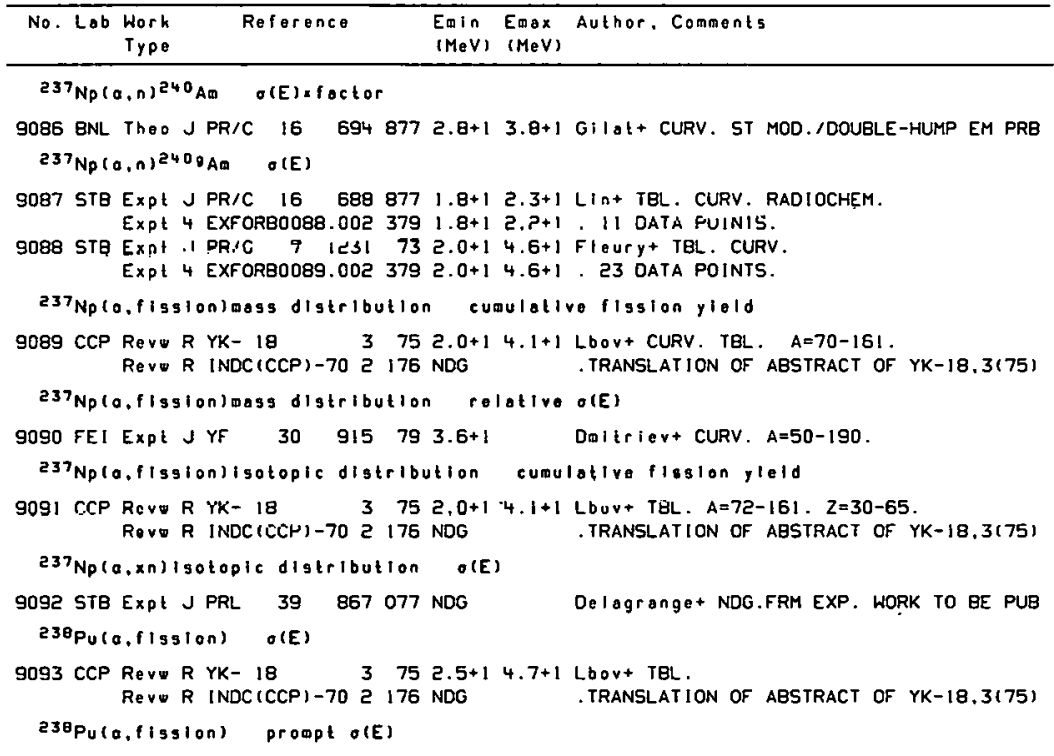

9094 KFK Comp g PH-DAT $1510792.0+12.9+1$ Muenzel+ TBL. FROM EXFOR.

9095 NBI Exth J NP/A $213 \quad 39773$ 2.0+1 2.9+1 Melog+ TQL. CURV. ASSUMEO ISOTR. DIST. Exth 4 EXFORB0007.005 076 2.0+1 2.9+1. 23 DATA POINTS.

$$
230 p u(a, f(s) i o n) \text { prompt o(E), foctor }
$$

9095 KFK Comp B PH-DAT $1510792.0+12.9+1$ Muenze1+ TEL. FROM EXFOR.

9097 NBI Exth J NP/A $213397732.0+12.9+1$ Metag+ TBL. CURV, (A,N), (A, $2 N) /(A, F)$. Exth 4 EXFORB0007.003 $0762.0+12.9+1$. 23 DATA POINTS. (A, EN)/(A,F) Exth 4 EXFORB0007.004 $0762.0+12.4+1$. 12 DATA POINTS. $(A, N) /(A, F)$.

$23 \mathrm{Pu}\left(\alpha, \mathrm{Zn}_{\mathrm{n}}\right)^{240 \mathrm{Cn}} \sigma(\mathrm{E})$

9098 KFK COMp B PH-DAT $15 \quad 10792.0+12.9+1$ Muenzel+ TEL. FROM EXFOR. $9099 \mathrm{NgI}$ Exth 4 EXFORB0007.003 $0762.0+12.9+1.10$ POINTS. DEDUCED BY COMPILER.

$230^{P u}(0,2 n) 240 \mathrm{CD} \quad \sigma(E) \times$ factor

9100 KFK COMp a PH-DAT $1510792.0+12.9+1$ Muenzel+ TBL. FROM EXFOR.

$9101 \mathrm{NB}$ ! Exth J NP/A $213397732.0+12.9+1$ Metag+ TEL. CURV. $(A, 2 N) /(A, F)$ Exth 4 EXFORBD007.003 $0762.0+12.9+1$. 23 DATA POINTS.

$23 \mathrm{Pu}(0, n)^{241} \mathrm{Cm} \quad O(E) \times f a c t o r$

9102 KFK COMP B PH-DAT $1510792.0+12.9+1$ Muenzel+ TBL. FROM EXFOR.

9103 NB! Exlh J NP/A $213 \quad 397732.0+12.9+1$ Melag+ TEL. CURV. (A,N)/(A,F) Exth 4 EXFORB0007.004076 2.0+1 2.4+1. I2 DATA POINTS. (A.N)/(A.F).

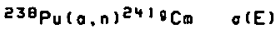

9104 ORL ExPI P ORNL-5306 $1056773.0+09.0+0$ 80ir+ NDG.

9105 KFK Comp B PH-DAT $1510792.0+12.4+1$ Muenzel+ TBL. FROM EXFOR.

9106 NBI Exth 4 EXFORBD007.004 $0762.0+12.4+1$. 12 POINTS. DEDUCED BY COMPILER.

238Pu $(a, n)^{2419} \mathrm{C}_{\mathrm{n}} \quad \sigma(E) \times$ foctor

9107 KFK COMp B PH-DAT 15 I $0792.0+12.4+1$ Muenzel+ TBL. FROM EXFOR.

9108 NBI Exth J NP/A $213397732.0+12.4+1$ Melag+ TBL. CURV. (A,N)/(A,F) Exth 4 EXFORB0007.004 075 2.0+1 2.4+1. 12/DATA POINTS.

ezBpuca,fissionisotople distribution cumulative fission yield

9109 CCP Revw R YK- $18 \quad 3752.5+1 \quad 4.7+1$ Lbov+ TBL. $Z=38-65 . A=91-161.7$ 1SOTOPES. Reve R INDC(CCP) -702176 NDG .TRANSLATION OF ABSTRACT OF YK- $18,3(75)$

239pu(a.f1ssion) o(E)

9110 CCP Reve R YK- $18 \quad 3752.0+14.8+1$ Lbov+ TBL.

Revo $R$ INDC (CCP) -702176 NDG 2 . TRANSLATION OF ABSTRACT OF YK- $18,3(75)$

9111 IJI Expt J YF $31 \quad 43802.0+12.8+1 \mathrm{Zaikg}+$ CURV. INTAD. 
REFERENCES ( c on l )

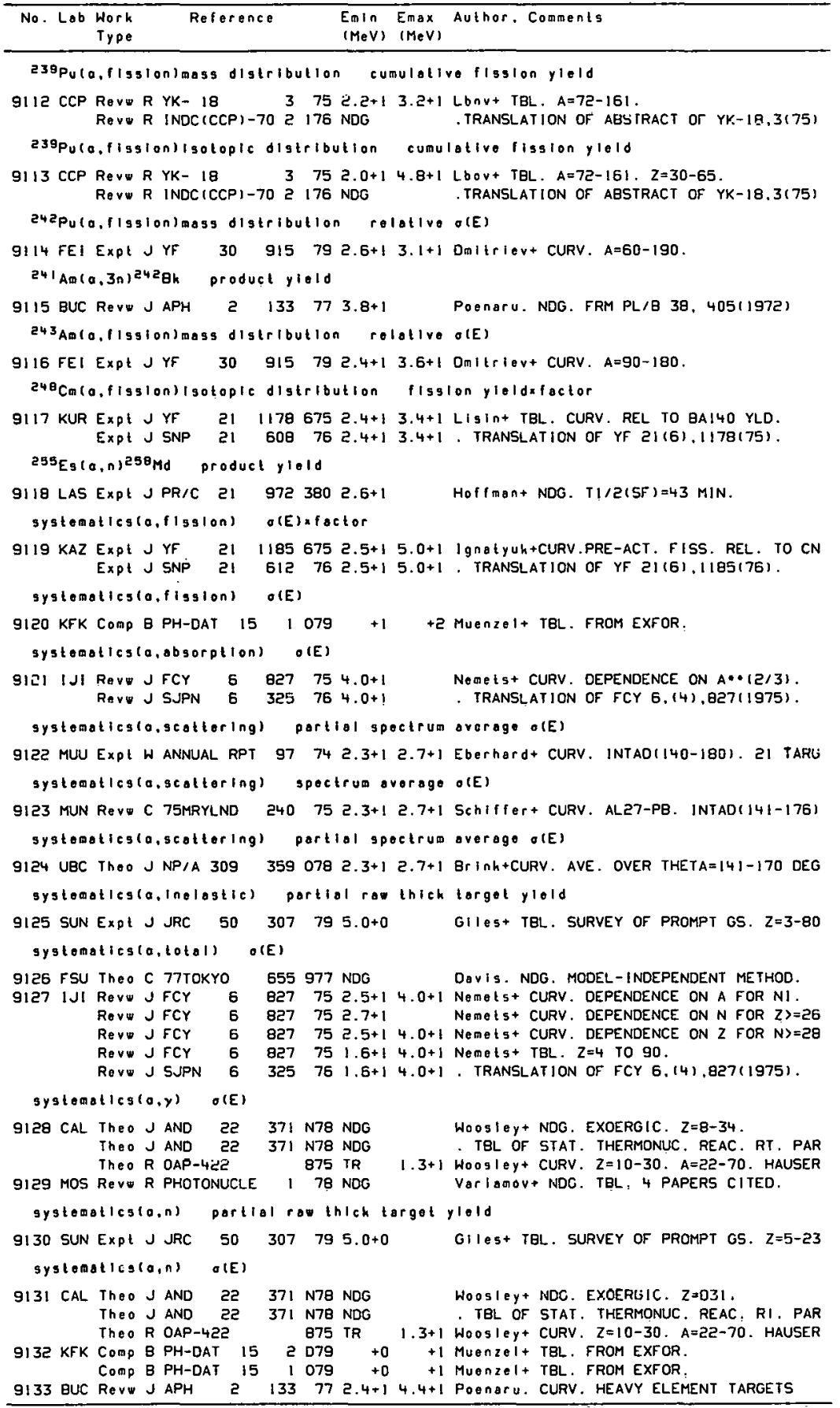




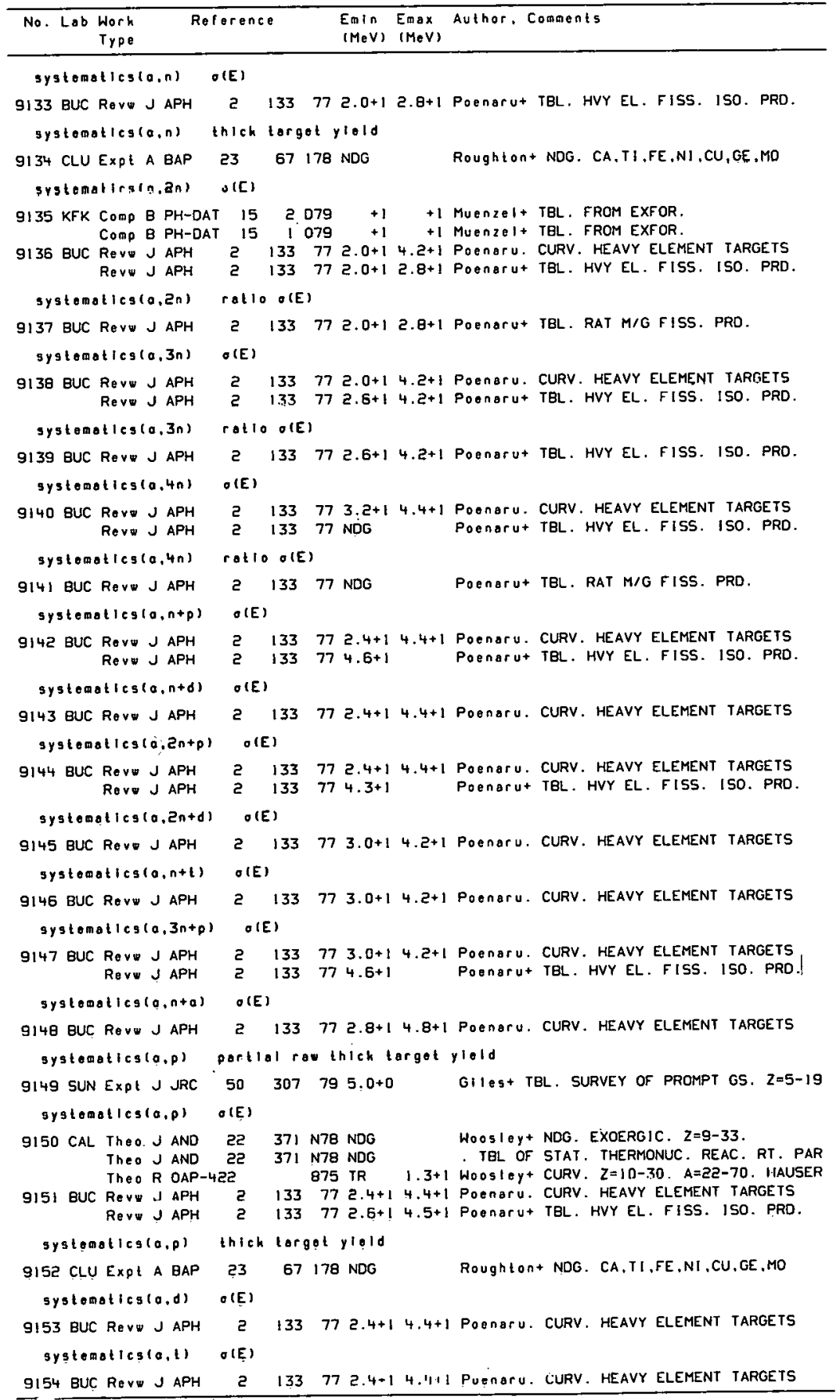




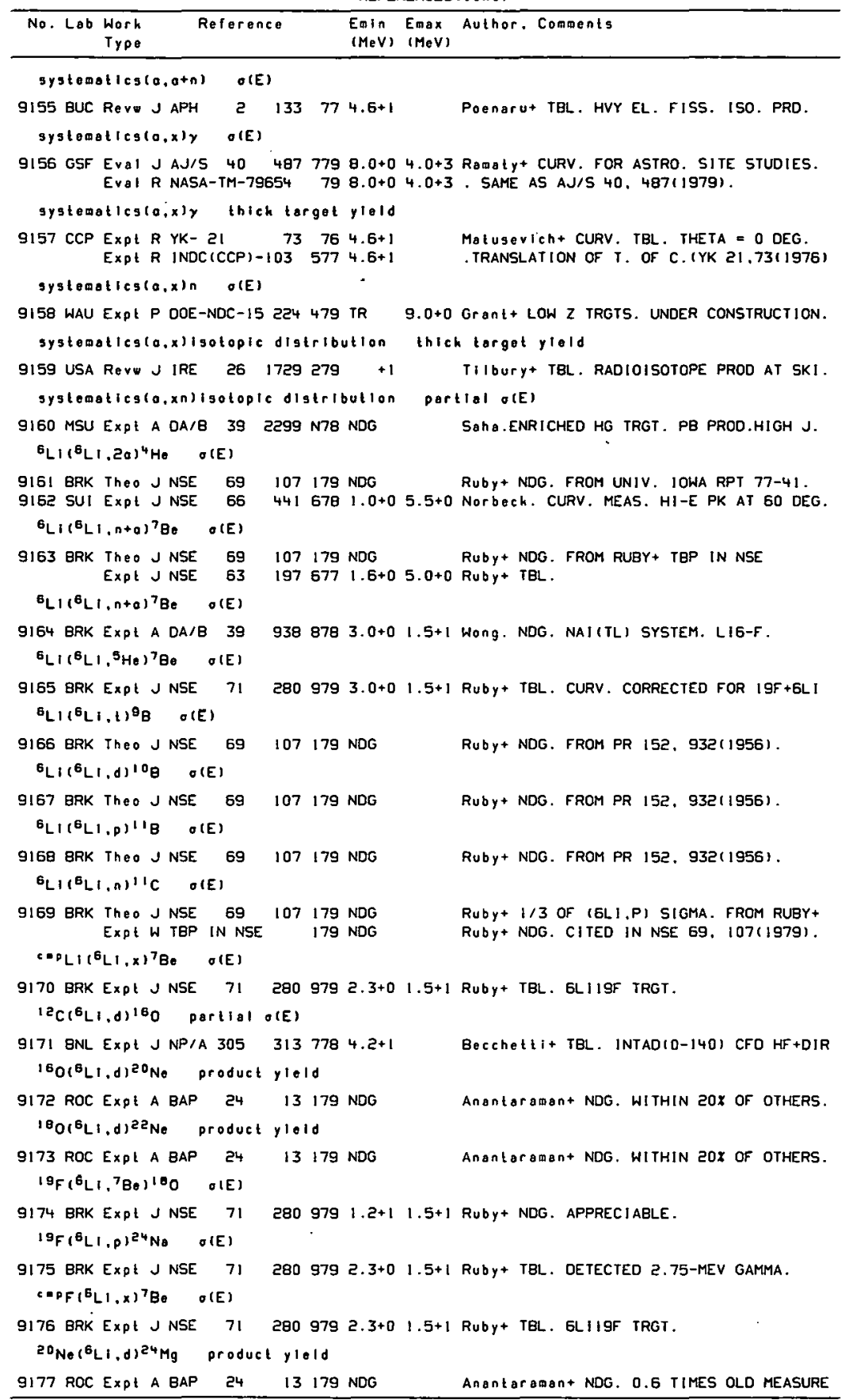




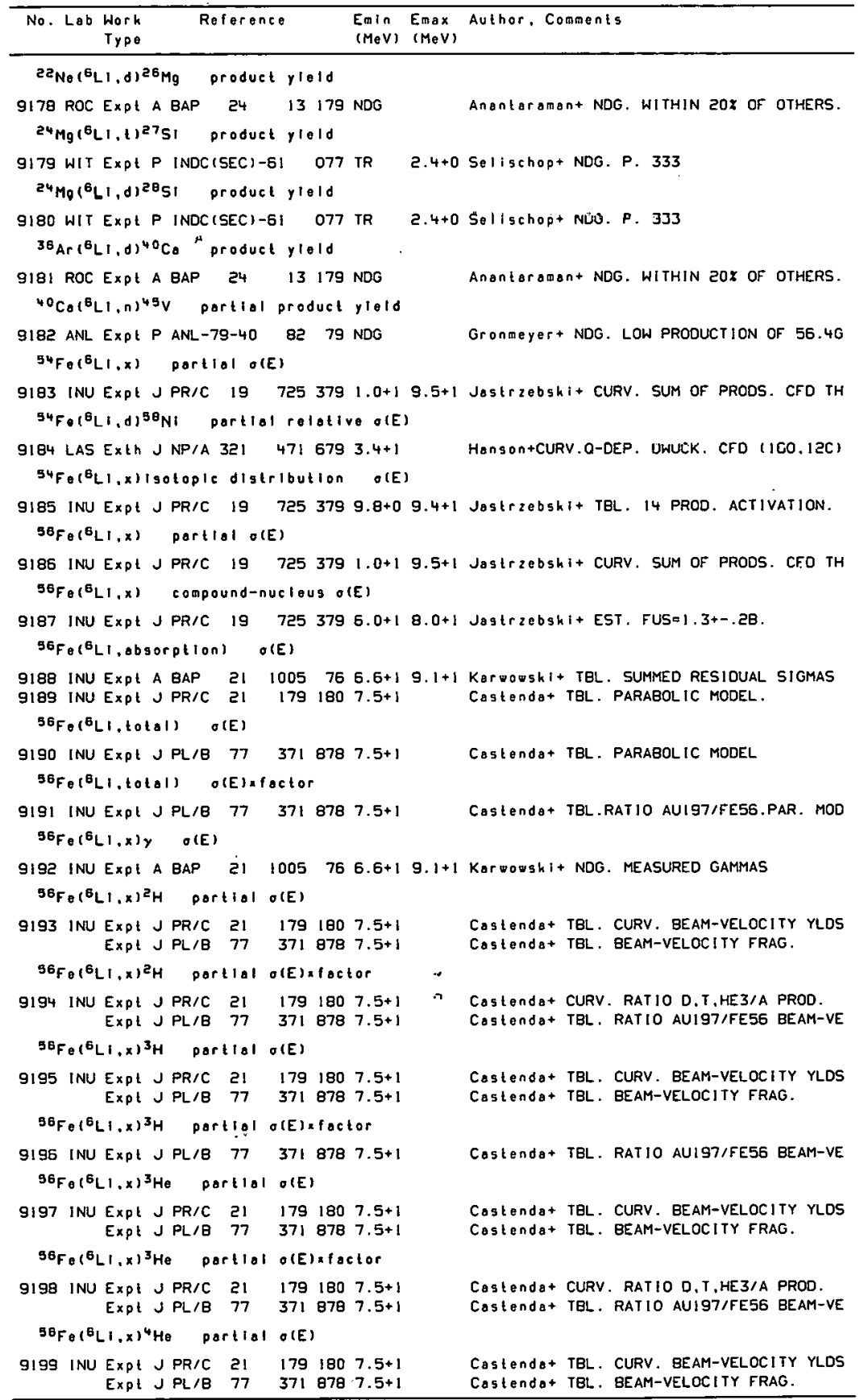


REFERENCES (cont)

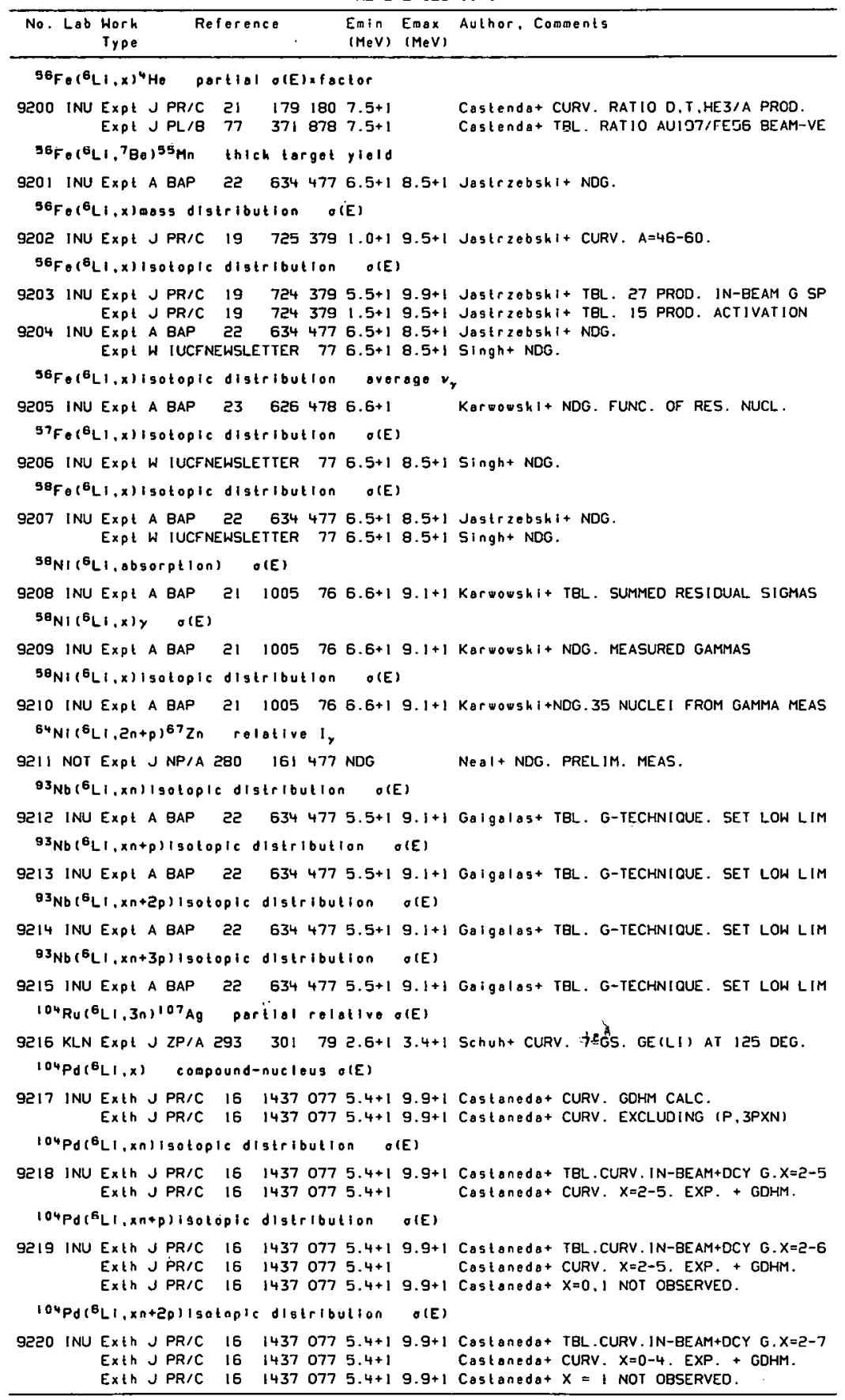




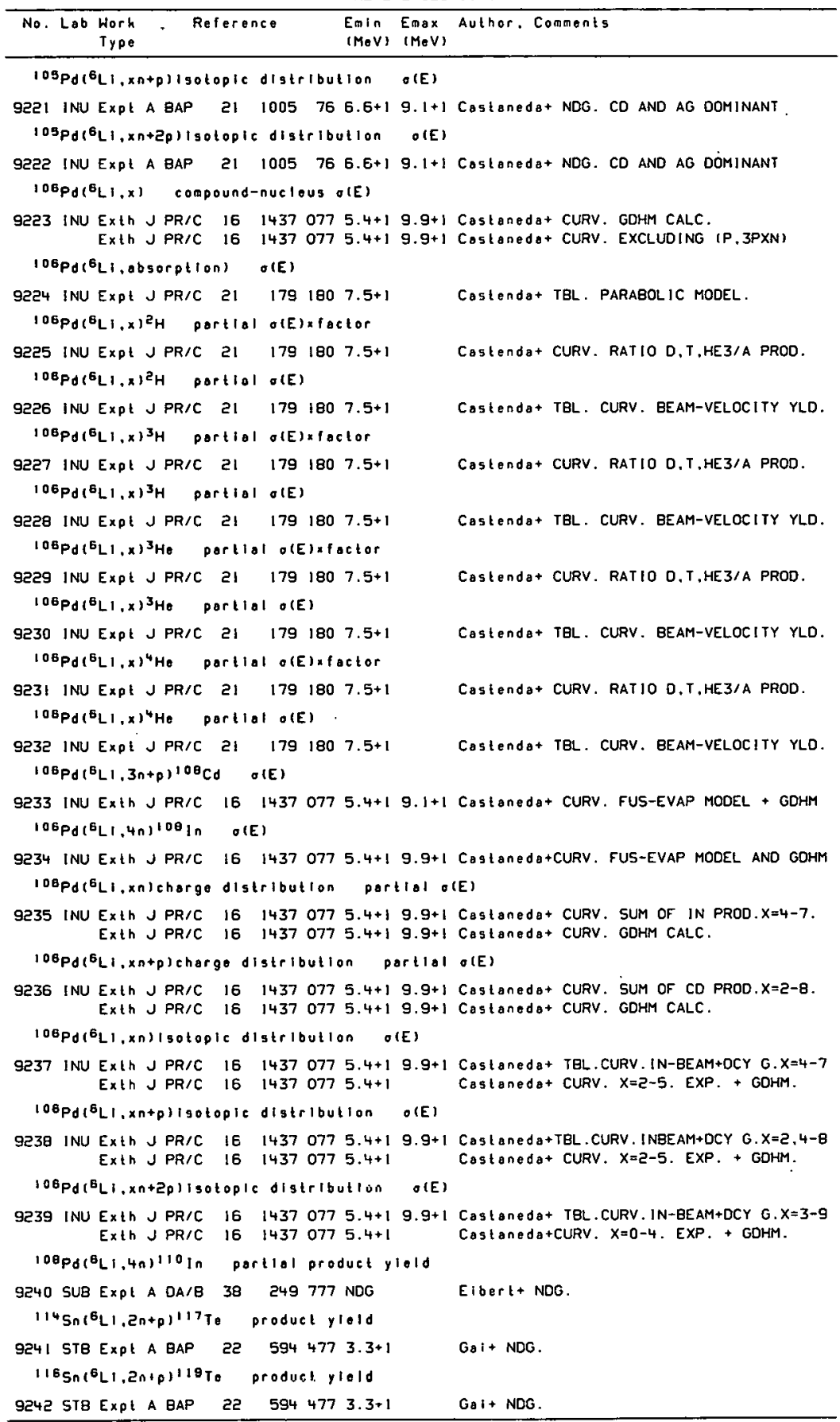


REFERENCES (cont)

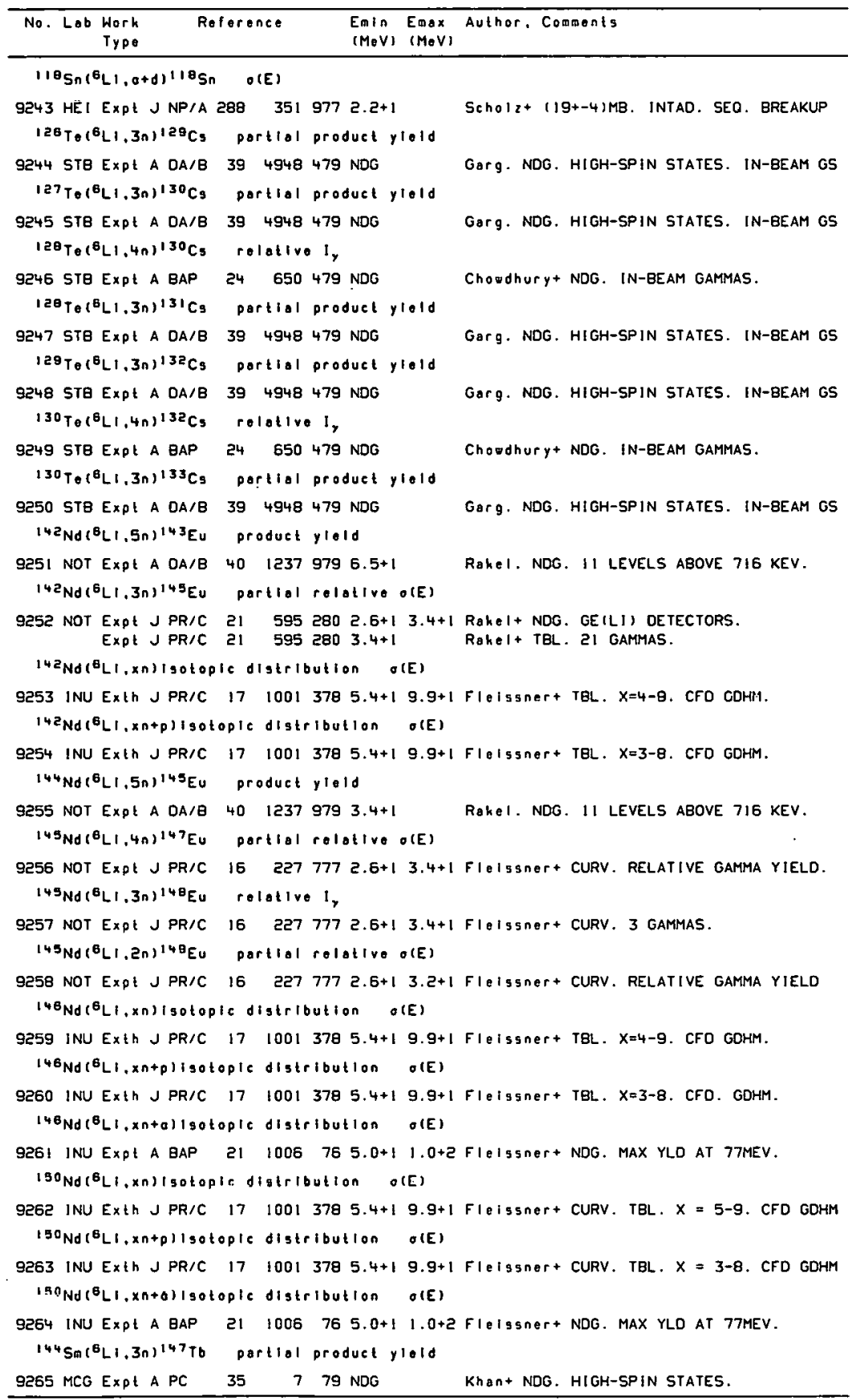




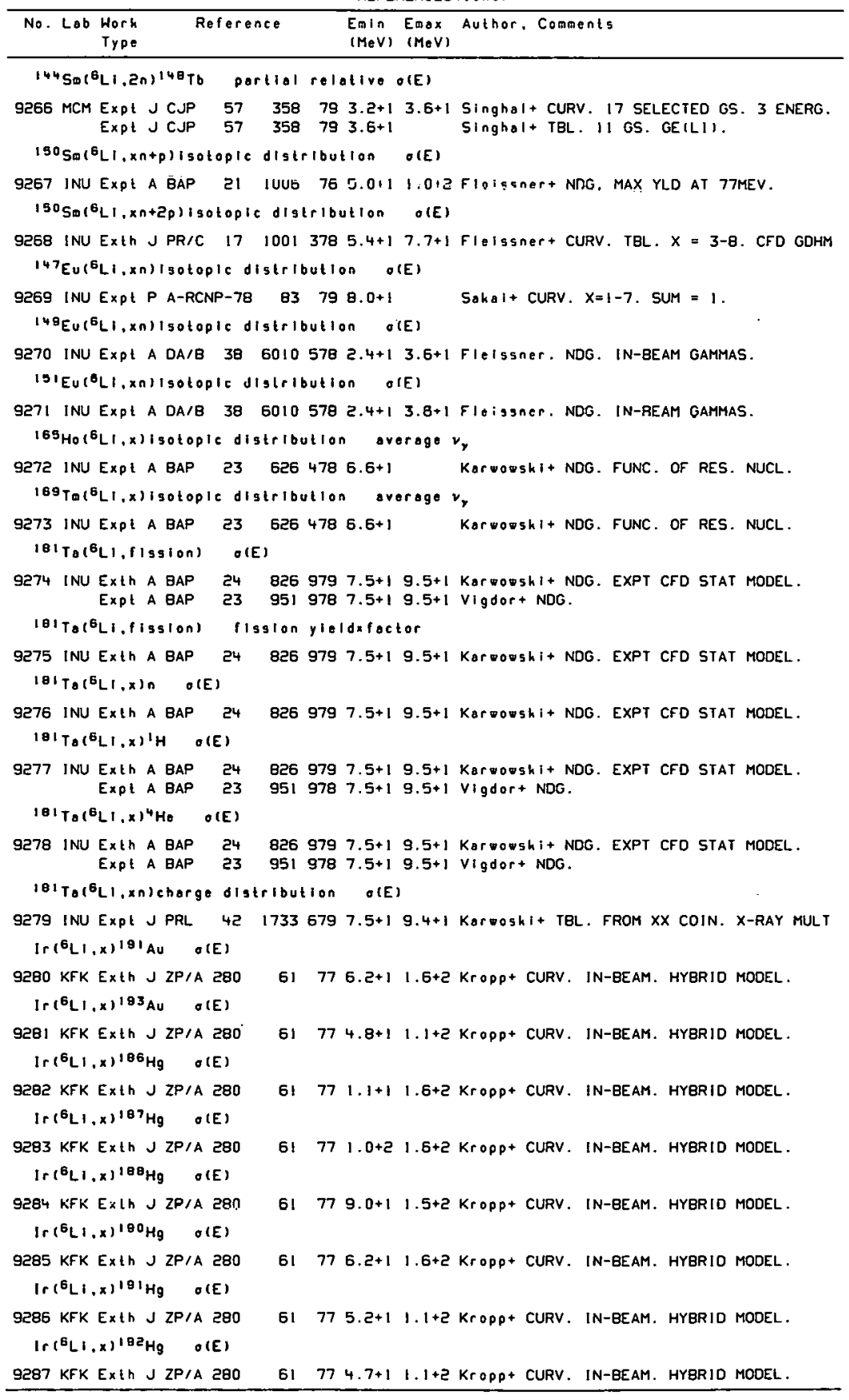


REFERENCES (cont)

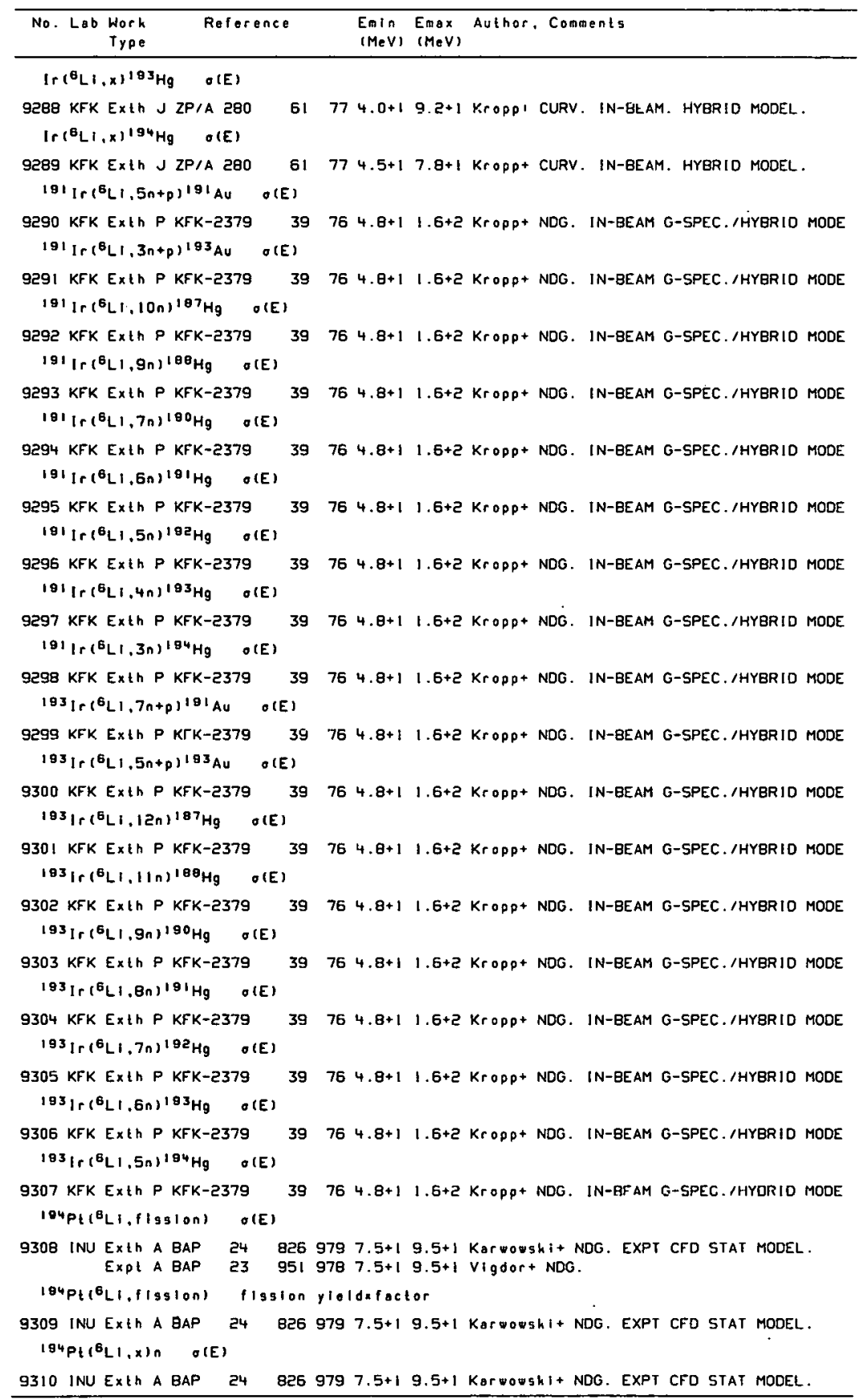




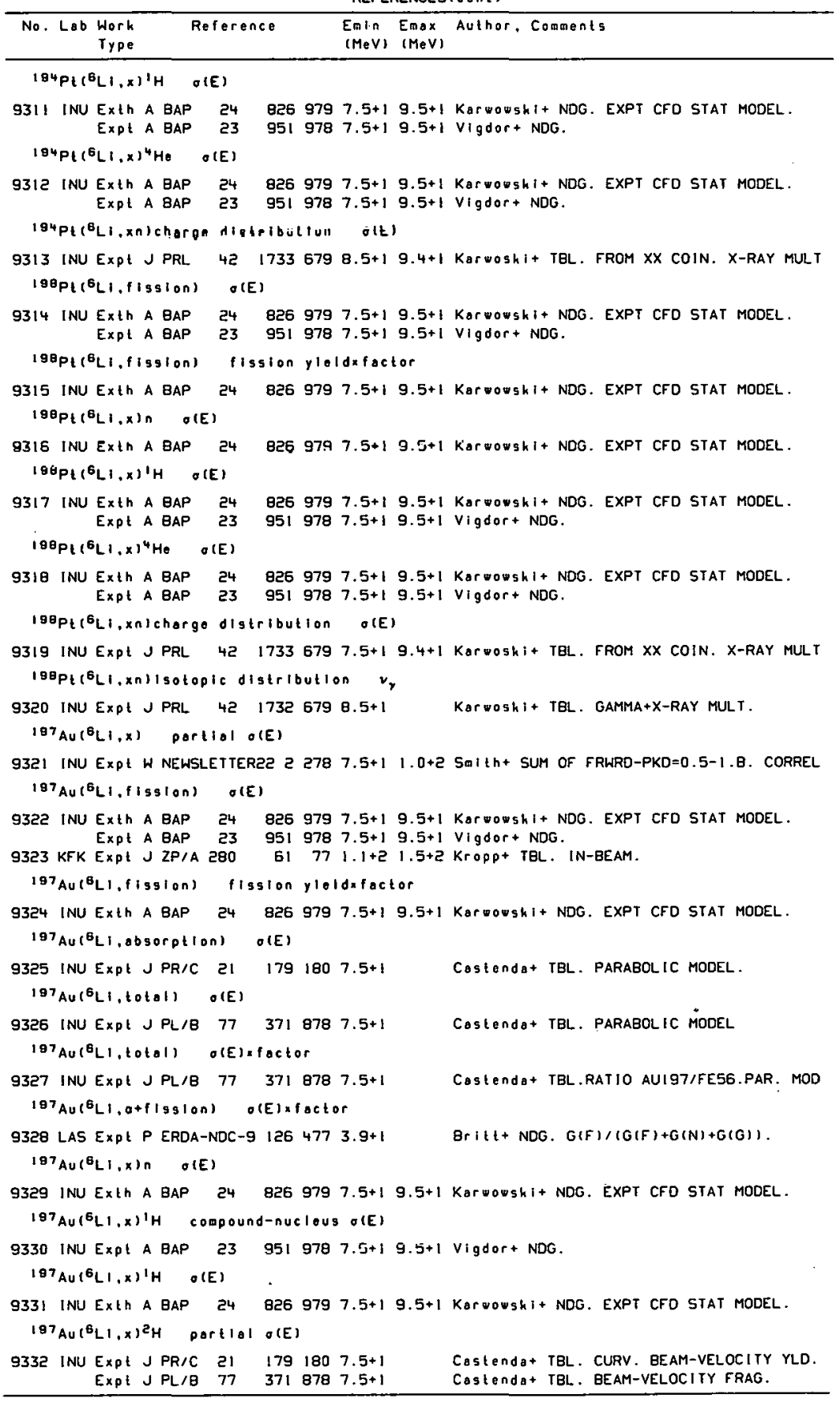




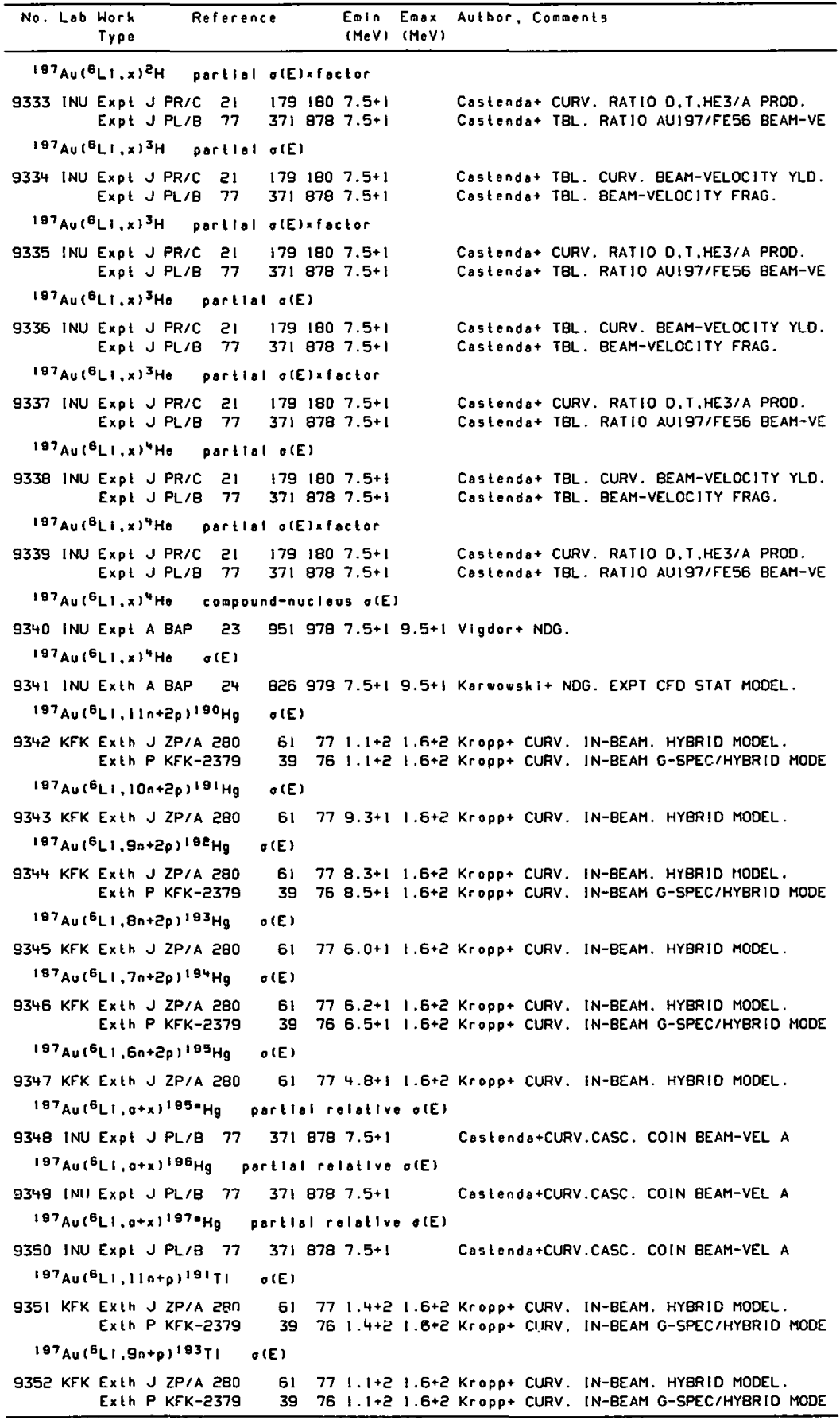


REFERENCES (cont)

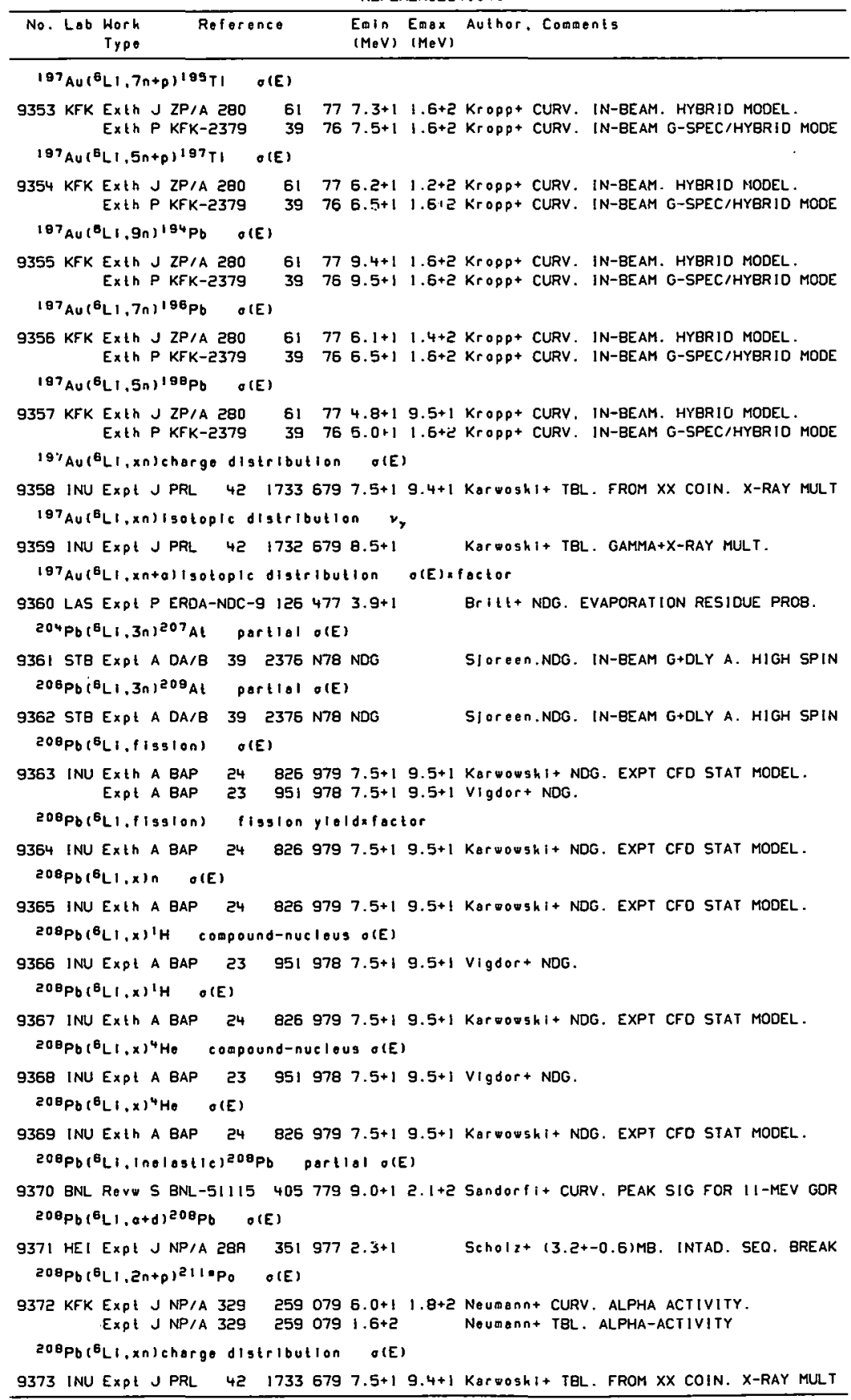




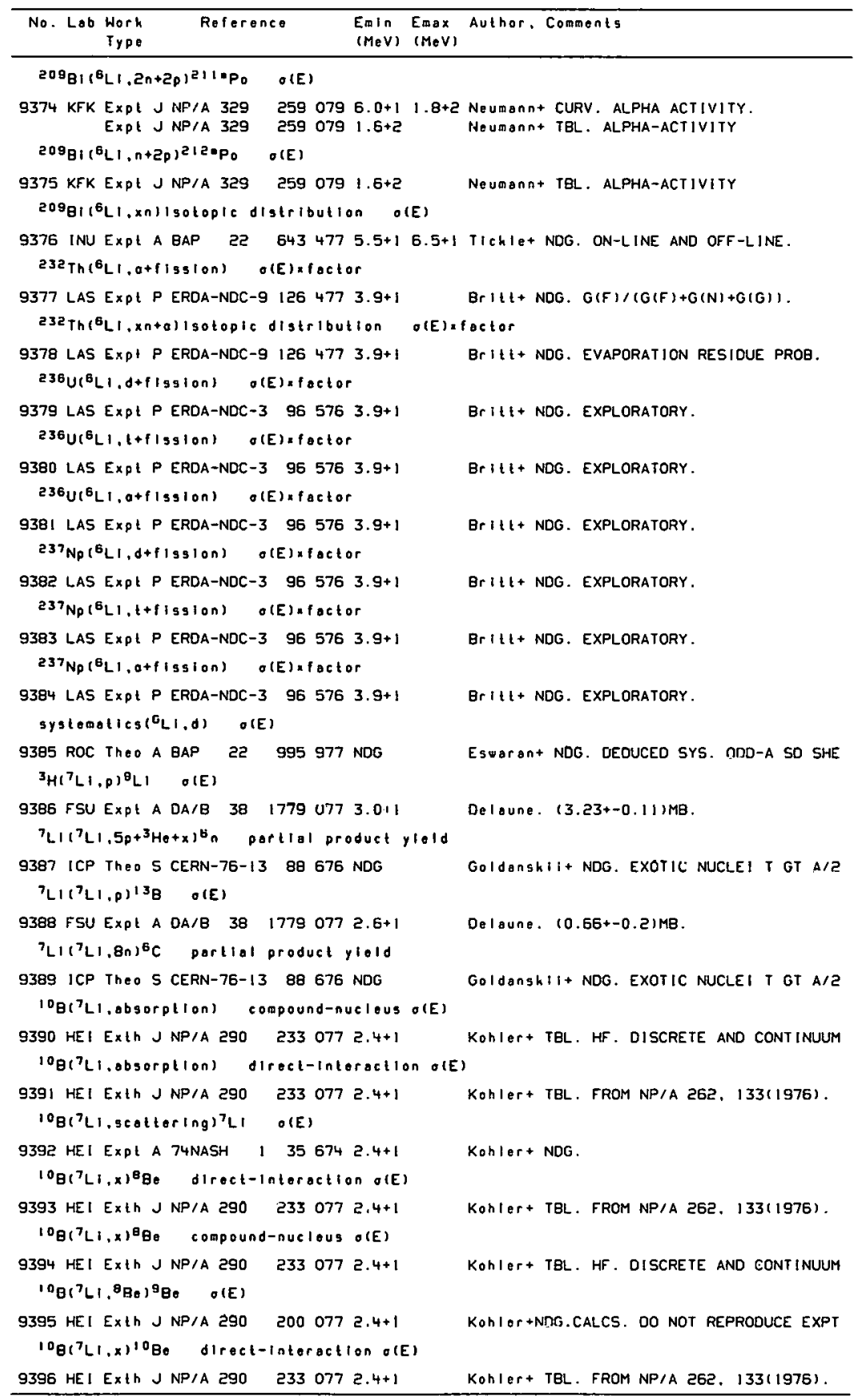




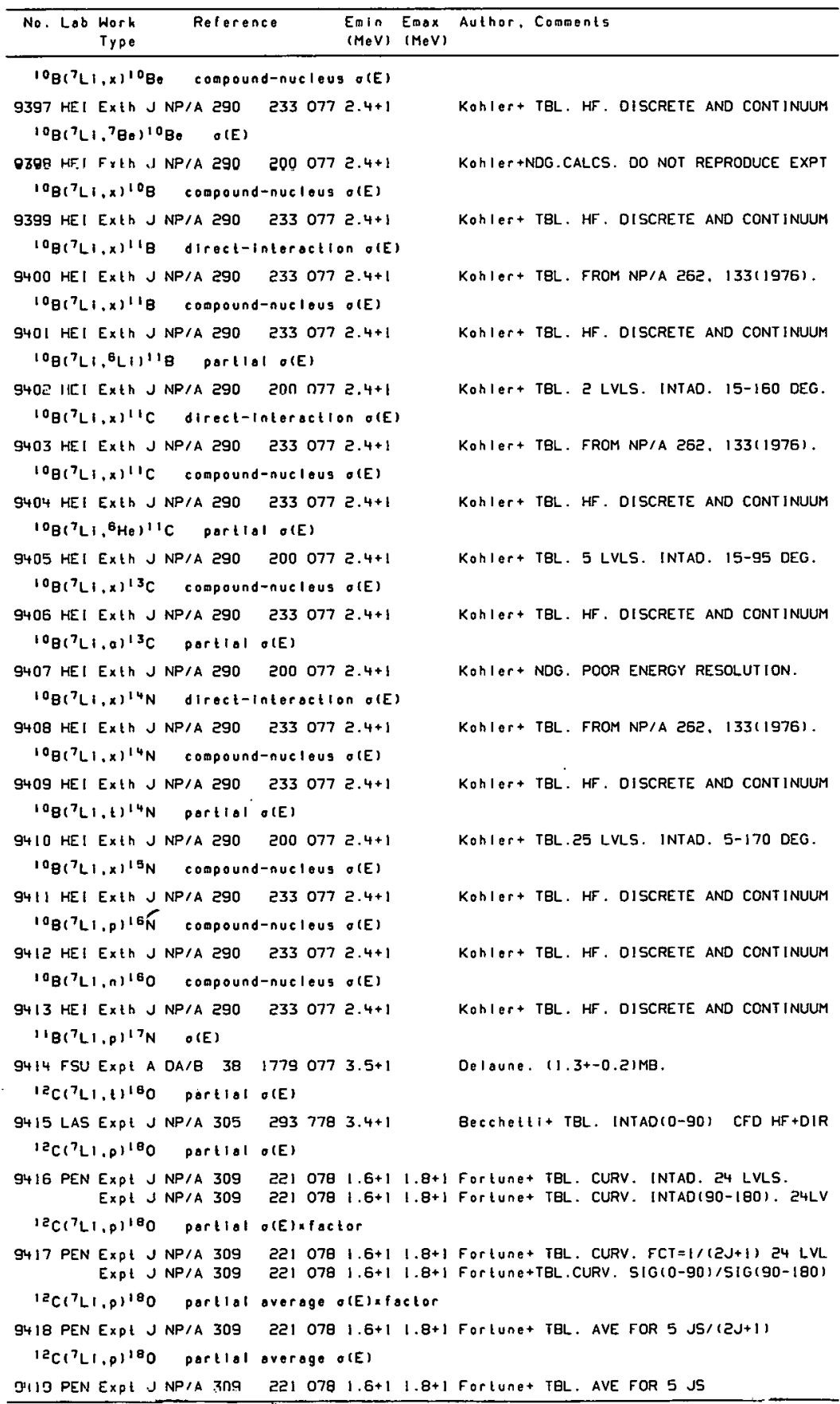




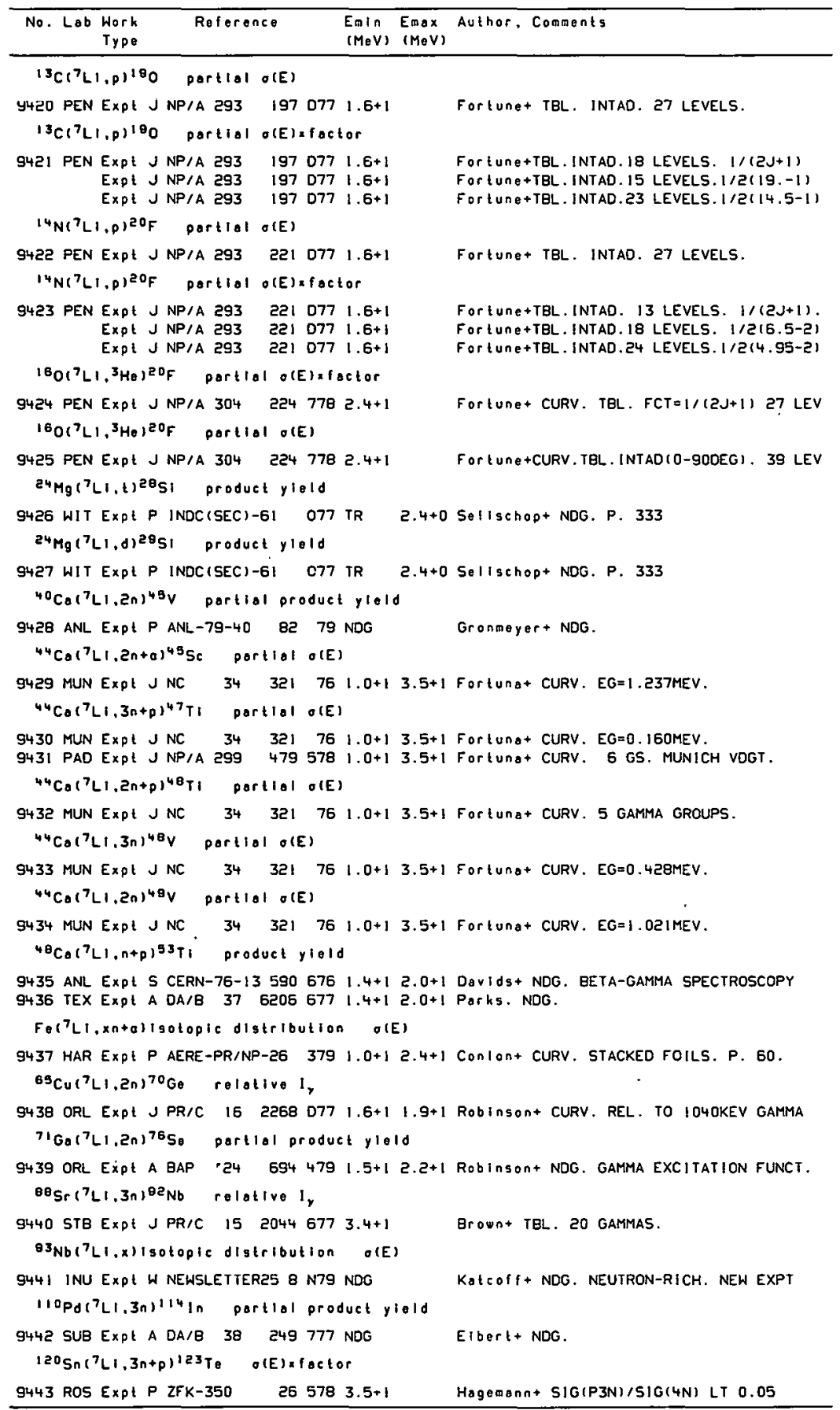


REFERENCES (cont)

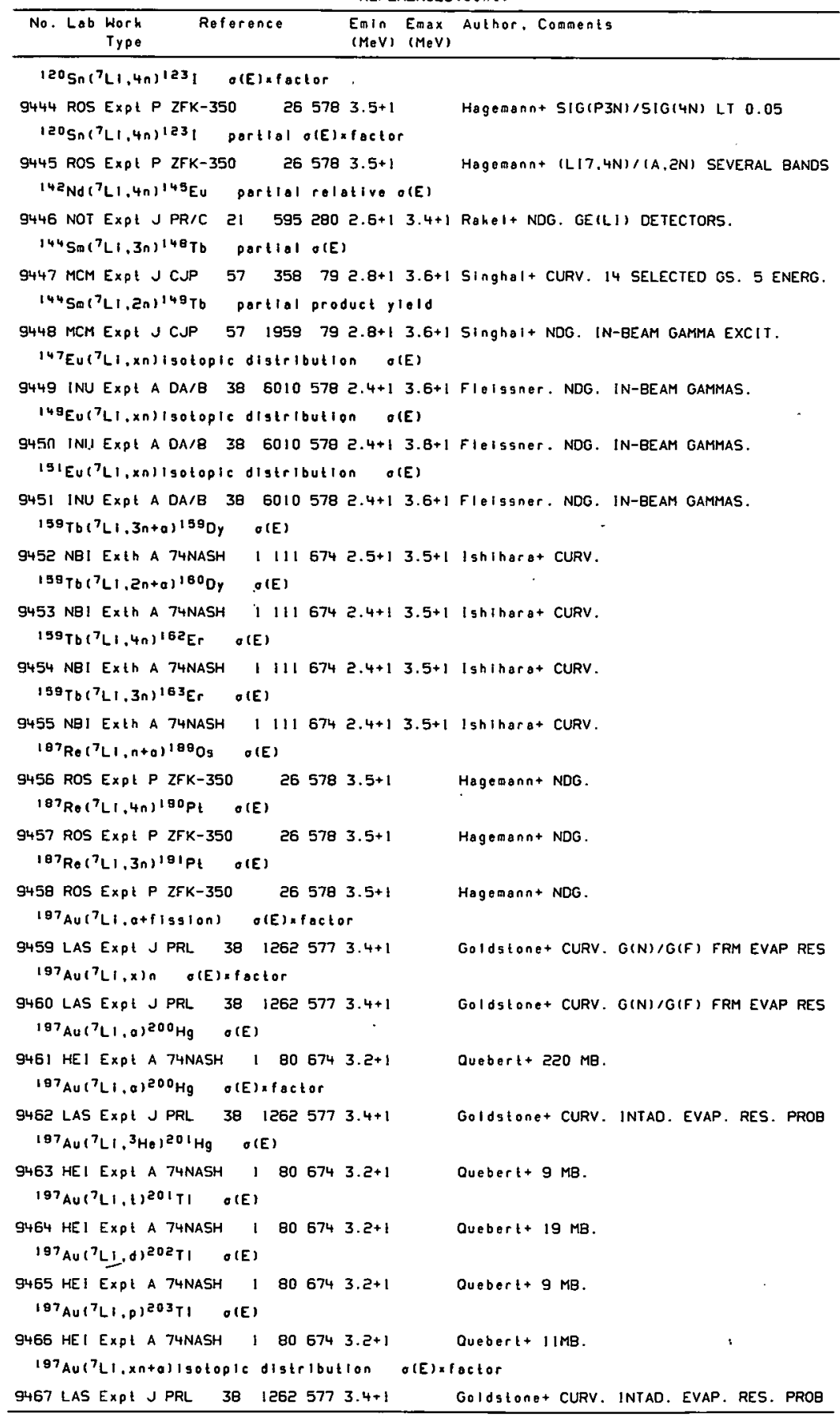




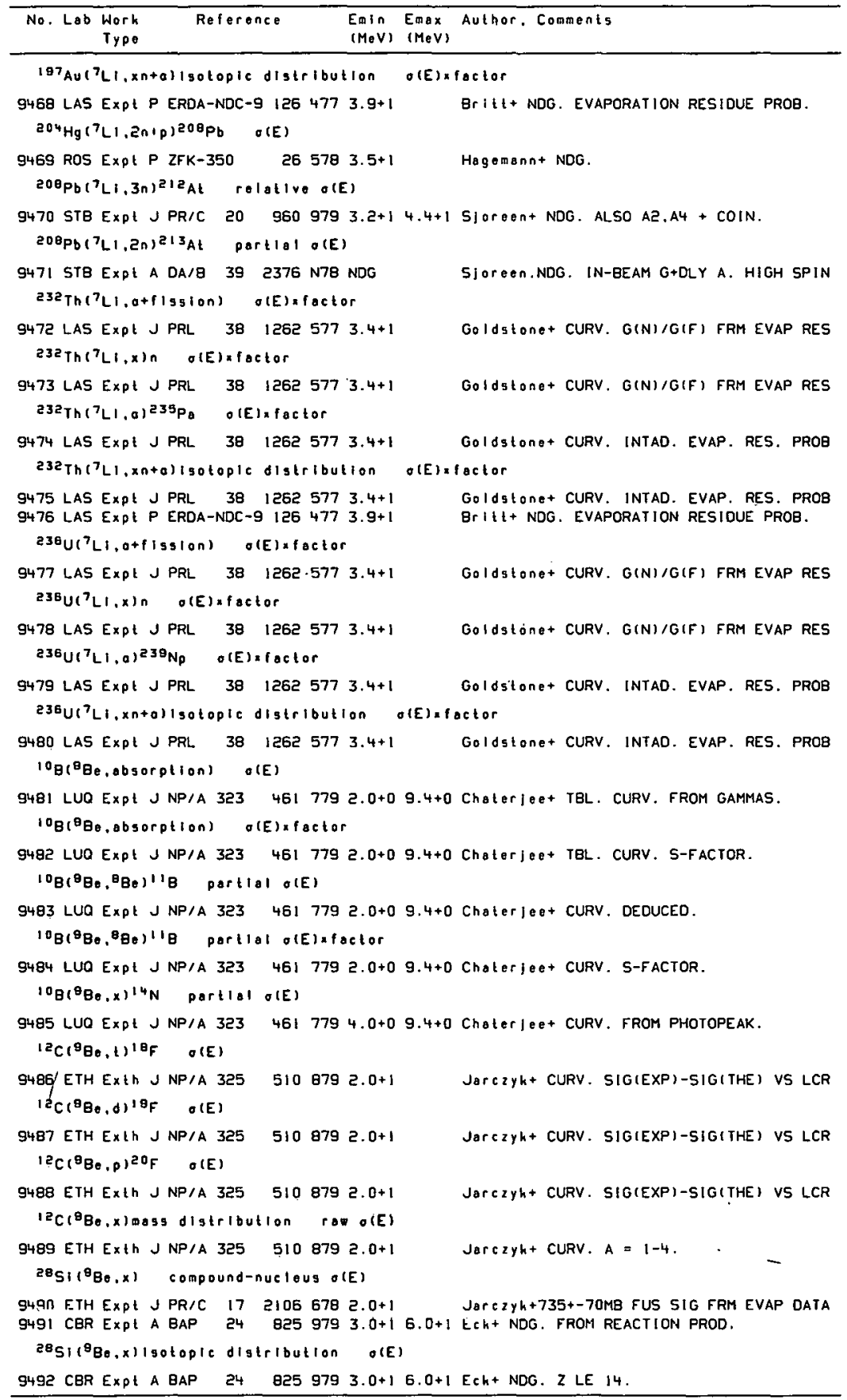


REFERENCES $($ cont $)$

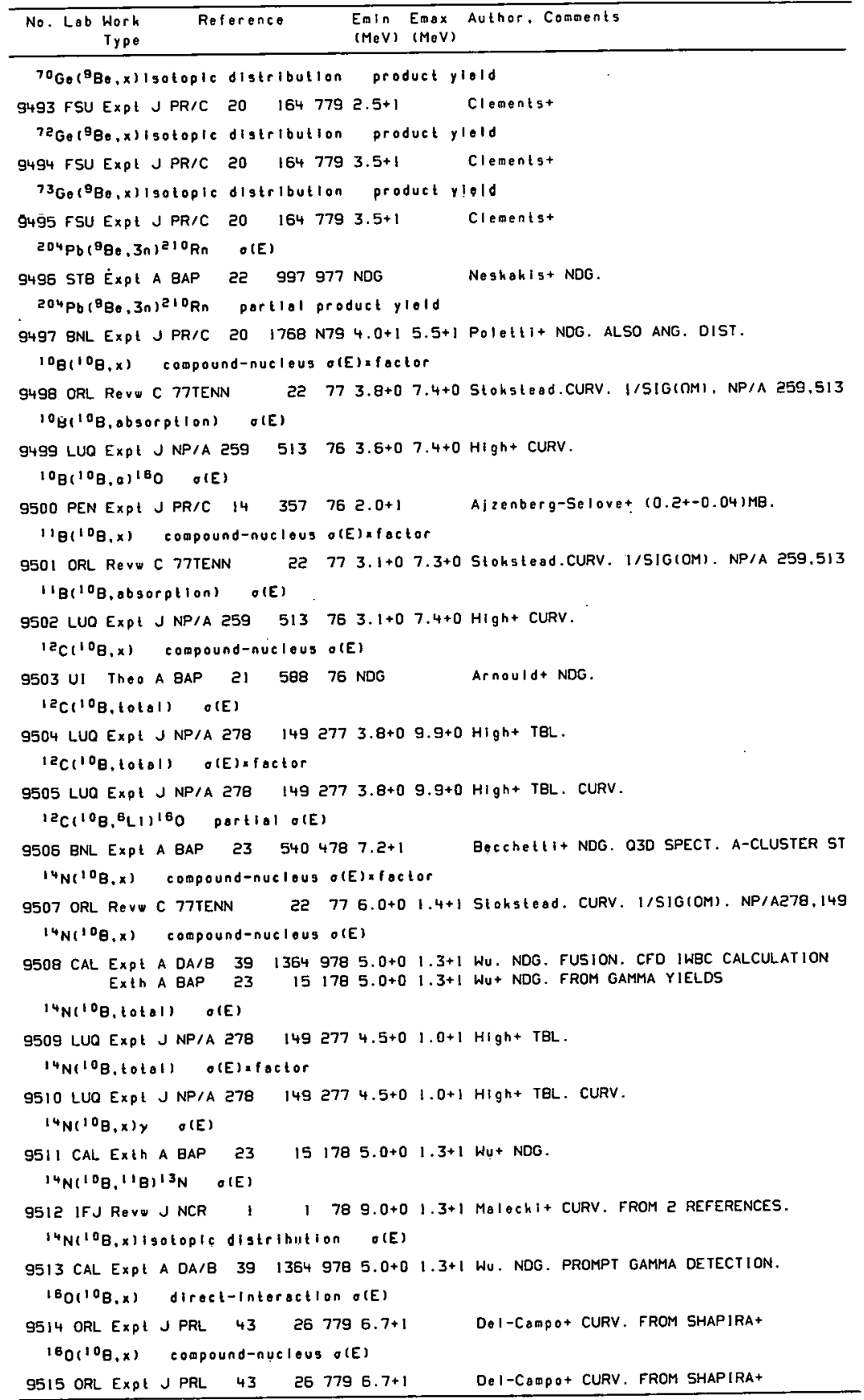




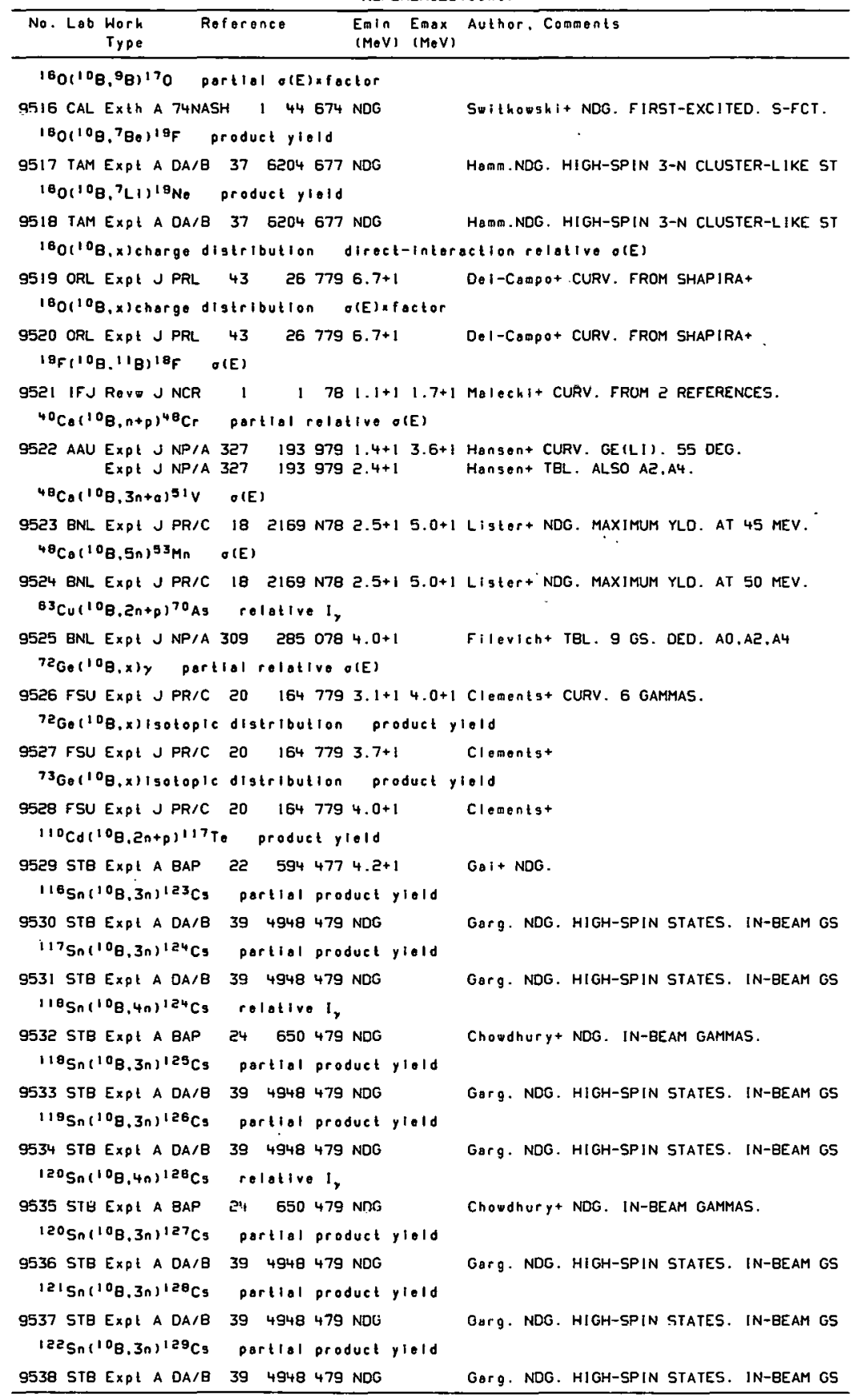


REFERENCES (cont)

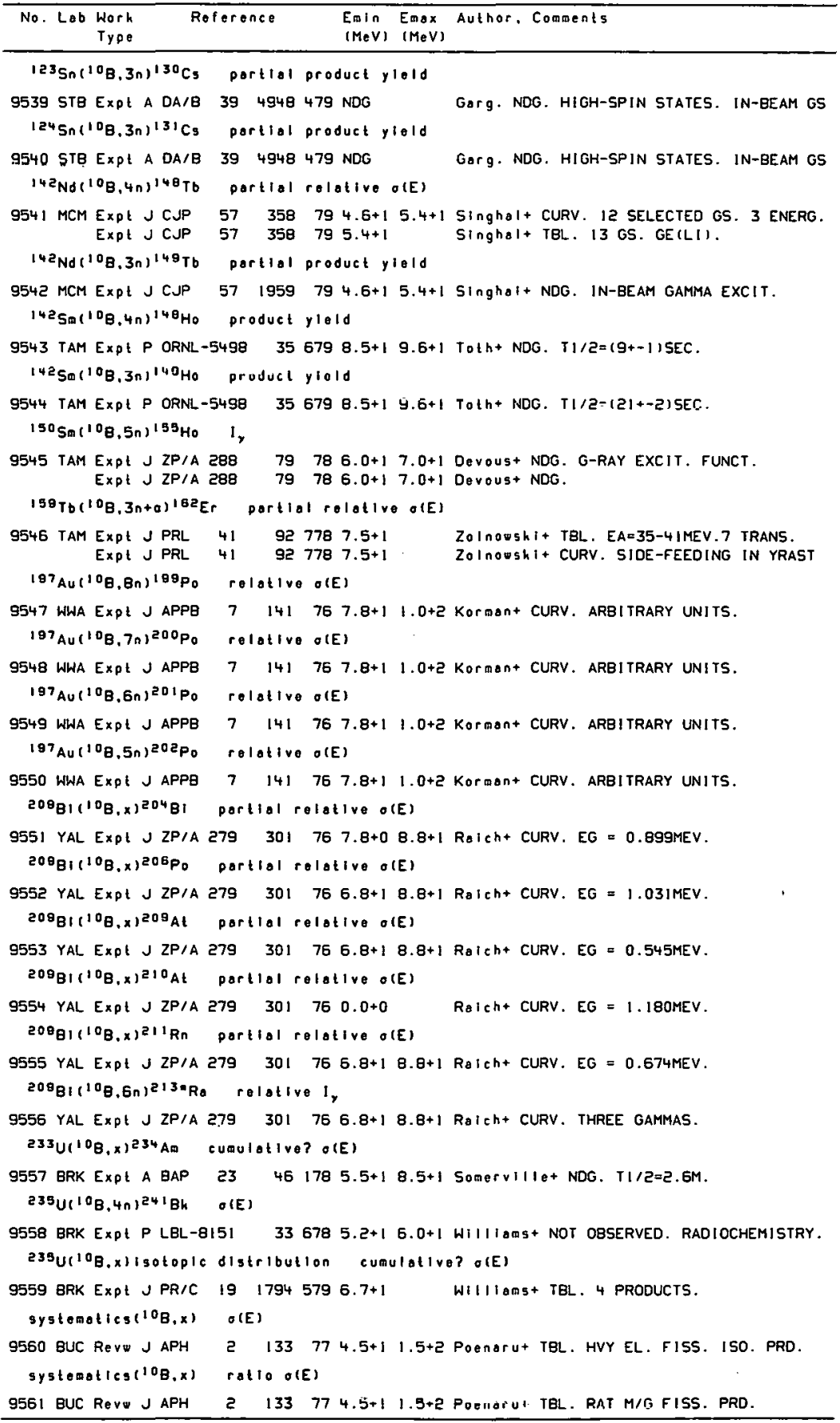




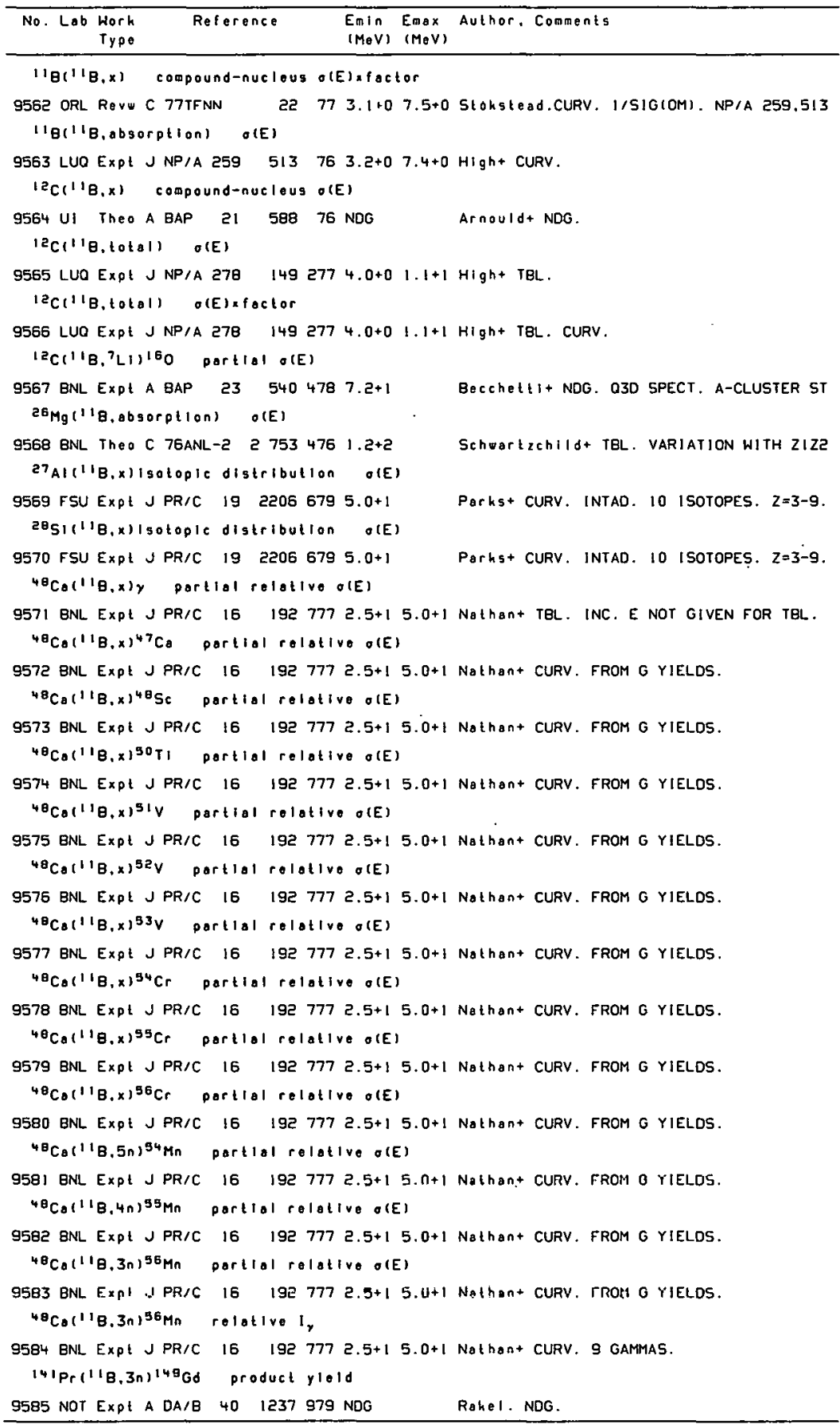


REFERENCES(cont)

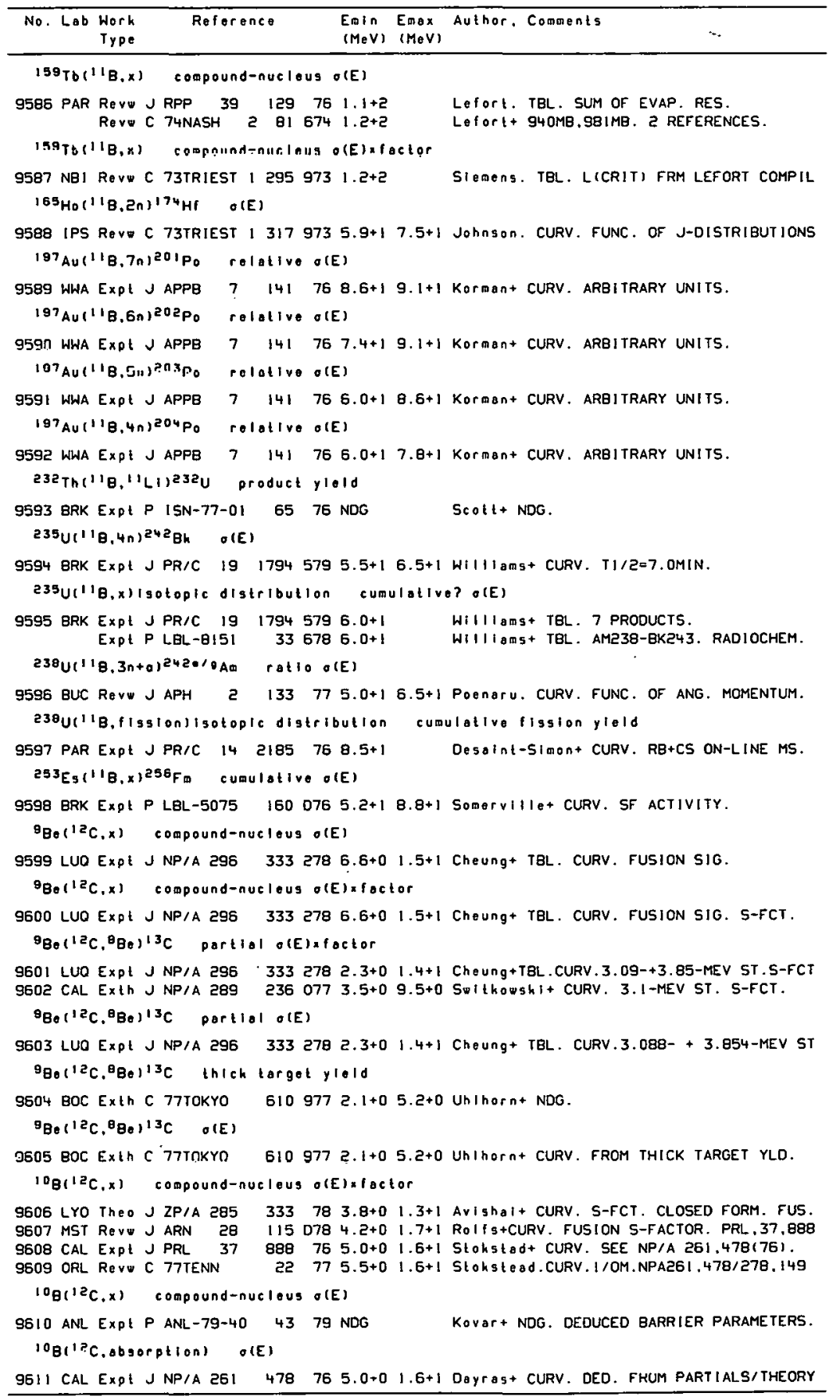


REFERENCES ( cont)

\begin{tabular}{|c|c|c|c|c|c|c|}
\hline No. Lab & $\begin{array}{l}\text { Worh } \\
\text { Typo }\end{array}$ & Reforence & $\begin{array}{l}\text { Emin } \\
(M \in V)\end{array}$ & $\begin{array}{l}E \max \\
(M \in V)\end{array}$ & Author. & Comments \\
\hline
\end{tabular}

9612 CAL Expt J NP/A $261478765.0+01.6+1$ Doyras+ CURV MEASURED GAMMAS

${ }^{10} \mathrm{~B}\left({ }^{12} \mathrm{C}, \mathrm{D}\right) \mathrm{Cl}^{\mathrm{N}} \mathrm{O}$ O(E)

9613 CAL EXPt J NPIA $261478765.0+01.6+1$ Doyras+ CURV MEASURED GAMMAS

$10 \mathrm{~B}\left({ }^{2} \mathrm{C}, n\right)^{21} \mathrm{~N}_{0}$ o(E)

9614 CAL EXPL J NP/A $261478765.0+01.6+1$ Doyras + CURV MEASURED GAMMAS

I'B(IEC, $x)$ compound-nuclous $O(E) x$ factor

9615 LYO Theo J ZP/A $295333783.4+01.2+1$ AYishal+ CURV. S-FCT. CLOSED FORM. FUS. 9616 MST Revw J ARN $28 \quad 1150783.6+01.6+1$ Rolfs+CURV. FUSION S-FACTOR. PRL 37.88B 9617 CAL Expl J PRL $37 \quad 888765.0+01.6+1$ Stokstod+CURV. SEE NP/A 261,4781761.

9618 ORL Rev C 77TENN $22775.5+01.5+1$ Slokstead.CURV.1/OM.NPA261.478/278,149

"IB(IaC, $x$ ) compound-nucleus o(E)

9619 ANL Expt P ANL-79-40 4379 NDG Kovar+ NDG. DEDUCED GARRIER PARAMETERS.

'BB('ac,obsorption) O(E)

9620 CAL Expl J NP/A $261478765.0+01.6+1$ Dayrog+ CURV. DED. FROM PARTIALS/THEORY

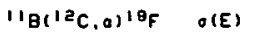

9621 CAL Expl J NPIA $261478765.0+01.6+1$ Dayras + CURV MEASURED gaMMAS

I'B(IEC,p) $2 \mathrm{E}_{\mathrm{Ne}} \quad O(\mathrm{E})$

9622 CAL Expt J NP/A $261478765.0+0 \quad 1.6+1$ Dayras+ CURV MEASURED GAMMAS

IB(I2C,n) 25 Ne $O(E)$

9623 CAL EXPt J NP/A $261478765.0+0 \quad 1.6+1$ Dayras + CURV MEASURED GAMMAS

${ }^{12} \mathrm{C}\left({ }^{2} \mathrm{C}, \mathrm{x}\right)$ compound-nuclous o(E)

9624 BRK Rev» 5 BNL-51115 905779 NDG

9625 ROC Revw S BNL-51115 235779 NDG

9626 TNL Theo P ORO-1667-17109 79 NDG

Theo P A-TNL-78 $107 \quad 79$ NDG

9627 UI Theo A BAP 2158876 NDG

9628 IPN THEO C 75MRYLND

9629 LUO ExPl J NP/A 282

$9630 \mathrm{BZL}$ TheO J PR/C 18

9631 PAR TheO J NP/A 331

g632 SAC Theo J PR/C I9

Expl J NP/A 309

$\begin{array}{lllllll}\text { Theo C 77TOKYO } & 659 & 977 & 1.8+1 & 6.2+1 & \text { Nagatanit FUS. SIG. CALC. CFD } \\ \text { Expt J BIS } 224 & 134 & 777 & 2.8+1 & 5.1+1 & \text { Harar. CURV. COMPLETE FUSION. }\end{array}$

$526752.5+09.0+0$ Arnould + NDG

$1815776.5+01.2+1 \mathrm{H} 1 \mathrm{gh}+$ CURV. FRM (CI2,A) + (CI2,P)

$2152 N 781.0+15.5+1$ Vozt CURV. SYS. SEMIEMP. ANALYS. OF FUS

$213 N 771.0+17.0+1$ Cugnon+ CURV. FUSION. NUCL. MOLECULES.

$7473791.0+13.0+1$ Nagakani+ CURV. ANAL. FUSION. VAR. POTEN

$5150782.8+15.6+1$ Conjeaud+CURV. COMPL. FUS. FRM EVAP. RES

Expt C 76ANL-2 $24994762.4+15.8+1$ Conloaud+ NDG. FUS = SUM OF EVAP. RES.

9633 YAL EXPt J PR/C I9 $13654791.2+16.0+1$ Abe+ CURV. FUSION. SMOOTH-CUTOFF MODEL

9634 JLU REV C 76ANL-2 $13414761.3+16.7+1$ Mogel. CURV.

9635 TOK Theo J NP/A $3092330781.4+16.0+1$ Tanimurat CURV. FUSION J DEP. 2 MODELS. Theo $J \mathrm{NCL} 23 \quad 709781.4+16.4+1$ Tan 1 murat CURV. FUS 516 . 2 MOD CFD EXP. Theo J NCL $23 \quad 709781.4+16.4+1$ taninurat CURV. J OECOMPOSITION OF FUS Theo C 77TOKYO $6349771.4+16.2+1$ Tanimara. CURV.FOLDING MODEL CFD TO EXP

9636 ANL EXPL J PR/C $2013050791.5+16.2+1$ Kovar + TBL. CURV. FUSION. CFD OTHERS. Expl C 77TOKYO 660977 NDG Kovar+ CURV. MAX. FUSION SIGMA.

ExpI P ANL-76-96 $3577 \quad 1.5+16.4+1$ Sper $r+$ CURV. SUM OF EVAP. RES

Exp: $P$ ANL-76-96 3577 NDG Spert $r$ MAX. S1G $=(940+-30) M B$.

Expl C 7GANR-2 $27834761.6+14.2+1$ Sperr+ CURV. FUS = SUM OF EVAP. RES.

Expt J PRL $3732176 \quad 1.5+17.2+1$ Spert+CURV. INTAD. EVAP. RESID.

9637 WAU Theo J PL/B 87183 N79 $1.6+14.8+1$ Vandenbosch. CURV. FUSION.

9538 ENL Expt J PRL 40 924 $4782.0+16.0+1$ Cormior+CURV. FUSION. SPERR+

9639 MBG REV C 78GRAZ $2414782.0+16.0+1 \mathrm{~F} i \mathrm{ck}$. CURV. NARROW RES IN CONIINUUM.

9840 MIt Revw C 77TENN $73772.3+14.7+1$ Negele. CURV. FRM PRL 37. 321(1976)

964 ! STB ReV C 77TOKYO $6569772.4+19.0+1$ Cormler+ CURV. CFD INL.,FU5, +2A SIGMAS

9642 STR Expt J PR/C $215792802.8+16.2+1 \mathrm{Kolata}+$ CURV. FUSION. SUM OF RESID.

9543 IRE Exp! P ISN-75-0! 38 79 $2.9+1$ 2.U+2 Conjeaud+ CURV. GLA-MOSEL MODEL. FUSION Expt P CEA-N-2070 $1999782.9+12.0+2$ Conjeaud+ CURV. GLAS-MODEL CFD EXP.

$12 C\left({ }^{2} C, x\right)$ compound-nuclous o(E)xiactor

9644 NBI Theo I NP/A $2802054774,4+01.8+1$ Christengon+ CURV, S-FCT.

9645 ORL Revw C 77TENN $22775.1+01.6+1$ Stokstead. CURV, 1/S!G(OM). PR/C 7,1280

9646 LYO Theo J ZP/A $285333785.3+01.3+1$ AVIShal+ CURV. S-FCT. CLOSEO FORM. FUS.

9647 CAL EXPt C 77TOKYO 661977 6.0+0 1.8+1 Wu+ CURV.FUS SIG.5-FCT. FROM AAA 13. 69 
REFERENCES (cont)

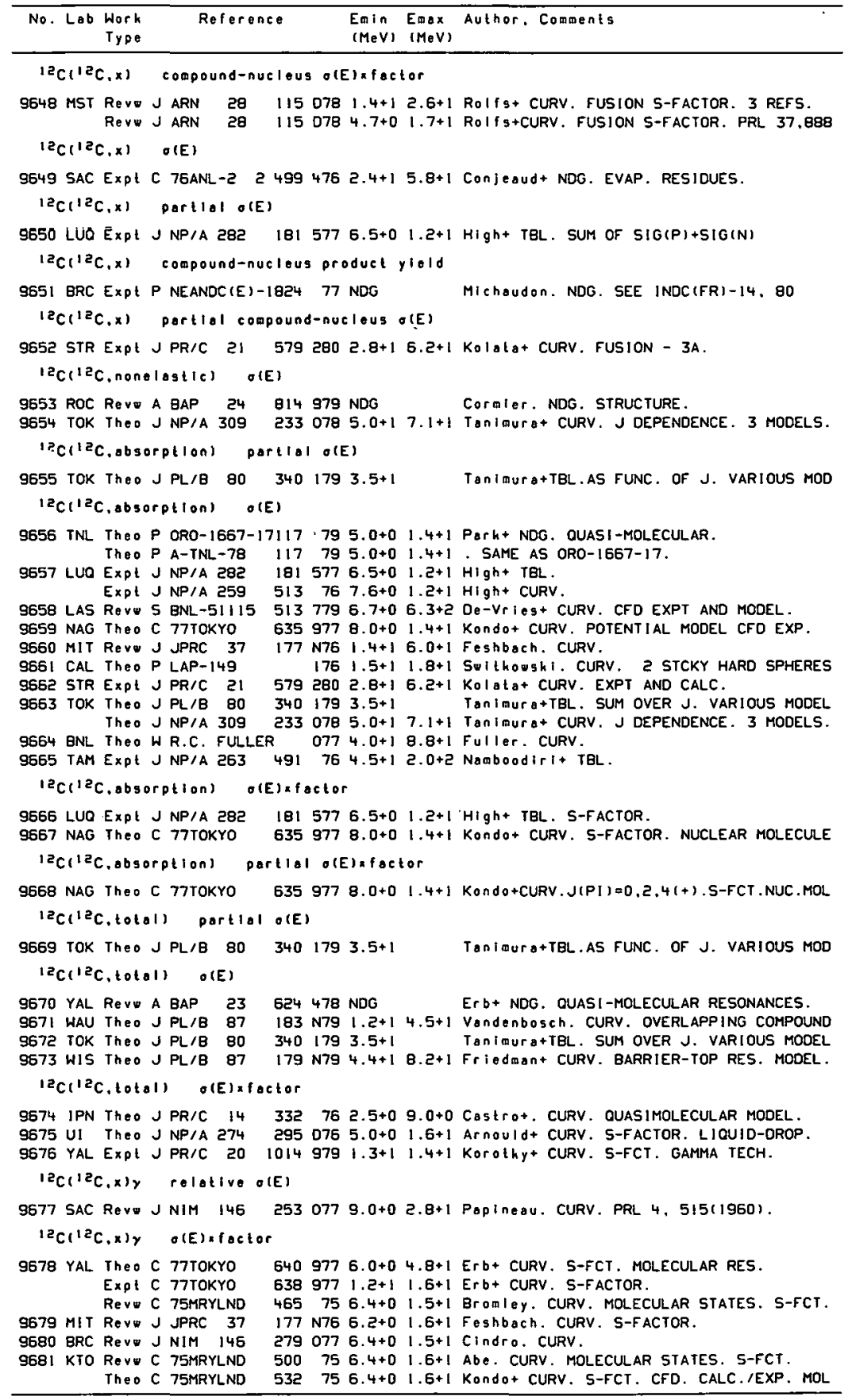




\begin{tabular}{|c|c|c|c|c|c|}
\hline No, Lab & $\begin{array}{l}\text { Work } \\
\text { Type }\end{array}$ & Reforenco & $\begin{array}{l}\text { Emin } \\
(M e V)\end{array}$ & $\begin{array}{l}\text { Emax } \\
(\operatorname{MeV})\end{array}$ & Author, Comments \\
\hline
\end{tabular}

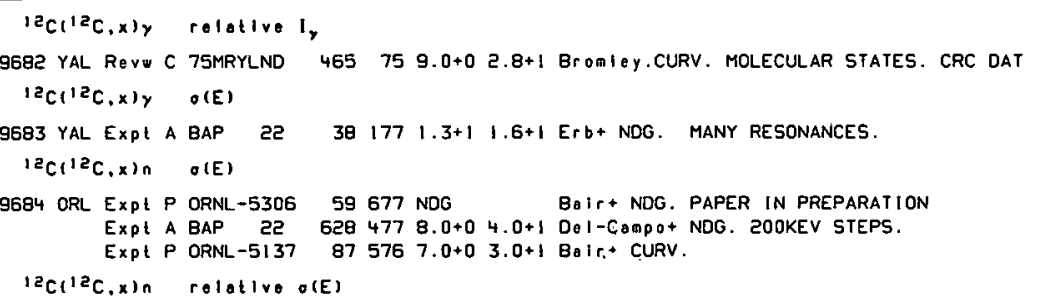

9685 SAC Revw J NIM 146253077 9.0+0 2.8+1 Papinesur CURV. PRL 4, 515(1960).

9686 YAL Rev C 75MRYLND $465759.0+02.8+1$ Bromley.CURV. MOLECULAR STATES. CRC DAT

${ }^{12} \mathrm{C}\left({ }^{12} \mathrm{C}, x\right)^{1} \mathrm{H}$ rolative o(E)

9687 SAC Revw J NIM 146253077 9.0+0 2.8+1 PBpineBu. CURV. PRL 4. 515(1960).

9688 YAL REVU C 75MRYLND $465759.0+02.8+1$ Bromley.CURV. MOLECULAR STATES. CRC DAT

$I^{2} C\left(1^{2} C, x\right)^{4} H$ relative o(E)

9689 SAC Revu J N!M 146253077 9.0+0 2.8+1 Popineau. CURV. PRL 4, 515(1960).

9690 YAL ReVw C 75MRYLND $465759.0+0$ 2.8+1 Bromloy.CURV. MOLECULAR STATES. CRC DAT

$\left.{ }^{l a C t I e C}, x\right)^{4}$ He partial product yleld

9691 KSU Expl A BAP $23 \quad 6164781.8+13.2+1$ Le99+NDG.A-REACTION CHANNELS.R-MAT. ANA

$\left(\mathrm{C}^{2} \mathrm{C}\left(\mathrm{I}^{2} \mathrm{C}, \mathrm{x}\right)^{4} \mathrm{He} \quad \mathrm{O}(\mathrm{E})\right.$

9692 NOT Expt J PR/C $217762802.4+16.3+1 \mathrm{Kolata+} \mathrm{CURV.} \mathrm{INCLUSIVE} \mathrm{PRODUCTION.}$

9693 STR Expl J PR/C $21 \quad 5792802.8+16.2+1 \mathrm{Kolata}$ + CURV. INCLUSIVE PRODUCTION.

${ }^{12} \mathrm{C}\left({ }^{12} \mathrm{C}, x\right)^{4} \mathrm{He}$ portlal o(E)

9694 NOT EXPt J PR/C $21 \quad 7762802.4+16.3+1 \mathrm{Kol}$ ato+ CURV. ANOMALOUS YIELO.

9695 STR Expt J PR/C $215792802.8+16.2+1 \mathrm{Kol}$ eta+ CURV. ANOMALOUS YIELD.

$I^{2} \mathrm{C}\left({ }^{2} \mathrm{C}, 2 \mathrm{a}+\mathrm{x}\right)^{4} \mathrm{He} \quad \sigma(\mathrm{E})$

9696 NOT Expl J PR/C $217762802.4+16.3+1 \mathrm{KolBta+}$ CURV. EFFECTIVE 3A-EMISSION.

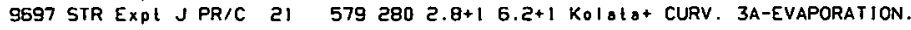

${ }^{12} \mathrm{C}\left({ }^{12} \mathrm{C}, x\right)^{12} \mathrm{C}$ portlal direct-interaction o(E)

9698 STR Expl J PR/C $21-5792802 . \theta+16.2+1 \mathrm{Kol}$ ata+ CURV. TOTAL DIRECT YIELD OF $2+$

${ }^{2} \mathrm{C}\left(\mathrm{I}^{2} \mathrm{C}\right.$,seatlering ${ }^{2} \mathrm{C}$ o(E) ot resonance

9699 ROC Expl J PRL $432567796.2+1 \quad$ Clover+ 63.3M9 FROM PRL 40, 924.

$12 C\left(15 C, s c a t t e r \operatorname{lng} 1{ }^{2} \mathrm{C}\right.$ ole

9700 ROC Revu A BAP $24 \quad 814979$ NDG Cormier. NDG. STRUCTURE.

$12 C\left(12 C, I n \in I O S(1 C)^{12} \mathrm{C}\right.$ port $(a)$ o(E)

9701 NAG Theo C 77TOKYO $6369771.5+14.5+1$ Kondo+CURV. $2+, 2+5 T S . P R L 38,940.17 \times U N 1 T$.

9702 YAL Expl J PR/C $1913564792.0+18.0+1$ Kondo+CURV. SINGLE 2+ BAND-CROSSING MOD

Expt J PR/C $1913564792.0+1$ 8.0+1 Kondo+CURV. MUTUAL 2+ BAND-CROSSING MOD Expi J PR/C $19 \quad 13564792.0+18.0+1$ Kondo+ CURV. J(PI $i=14+$. IRUNCATED MODEL The0 J PRL 42 $5662792.4+16 . \theta+1$ PhIIIIPs+ CURV. OR'IGIN OF GROSS STRUCT.

9703 WIS Theo J PL/B $87 \quad 179$ N79 $2.2+18.0+1$ Frledmant CURV. BARRIER-TOP RES. MODEL.

9704 SAC EXpl J BIS $224 \quad 1347772.4+18.8+1$ Harar. CURV. 2+ TO G.S. CFD. FUS.

9705 STB Revo C 77TOKYO $6569772.4+19.0+1$ Cormier+ CURV. CFD INL.,FUS, +2A SIGMAS

9706 PEN Expl J PRL 43 a37 979 2.9+1 6.4+1 Cannell+ CURV. FROM 'DWBA FIT TO DA.

9707 BNL Expt J PRL $40 \quad 9244783.0+19.0+1$ Cormier+ CURV. TOTAL $2+(1)$ INL. CFD OTHER

9708 TOK Theo J NP/A $3092330783.2+18.0+1$ Tanimura+ CURV. SINGLE+MUTUAL 2+ EXCIT.

9709 ROC Expt J PR/C $21 \quad 1981804.4+1$ 8.0+1 Fulton+ CURV. 3- TO $0+$. FROM DIFF. SIGM Expt J PRL 43 $2567794.4+1$ 8.0+1 Clover+ CURV. INTAD. 2+ STATE FOR EACH.

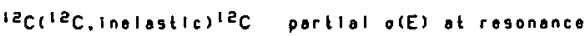

9710 ROC Expt J PRL $432567796.2+1 \quad$ Clover+ 35.3MB FROM PRL 40.924.

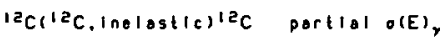

9711 STR Expt JPR/C $215792802.8+16.2+1 \mathrm{Kolata}+$ CURV. G-METHOD. 2+ STATE. Revo 5 LYCEN-7902 379 2.8+1 6.0+1 Ha as+ P. 5.6.1. CURV. G-METH. 2+ TO $0+$

9712 BNL Expt A BAP 23 516 $4783.0+17.0+1$ JacheInsh1+NDG.4.43MEV ST.DED. PRT. WID

9713 5TB Expt J PRL $389404773.0+19.0+1$ Cormier+ CURV. INTAD. 2+ ST OUT + RES

Expt J PRL $38 \quad 9404773.0+19.0+1$ Cormior+ CURV. INTAD. 2+ TO O+ TRANSITI 


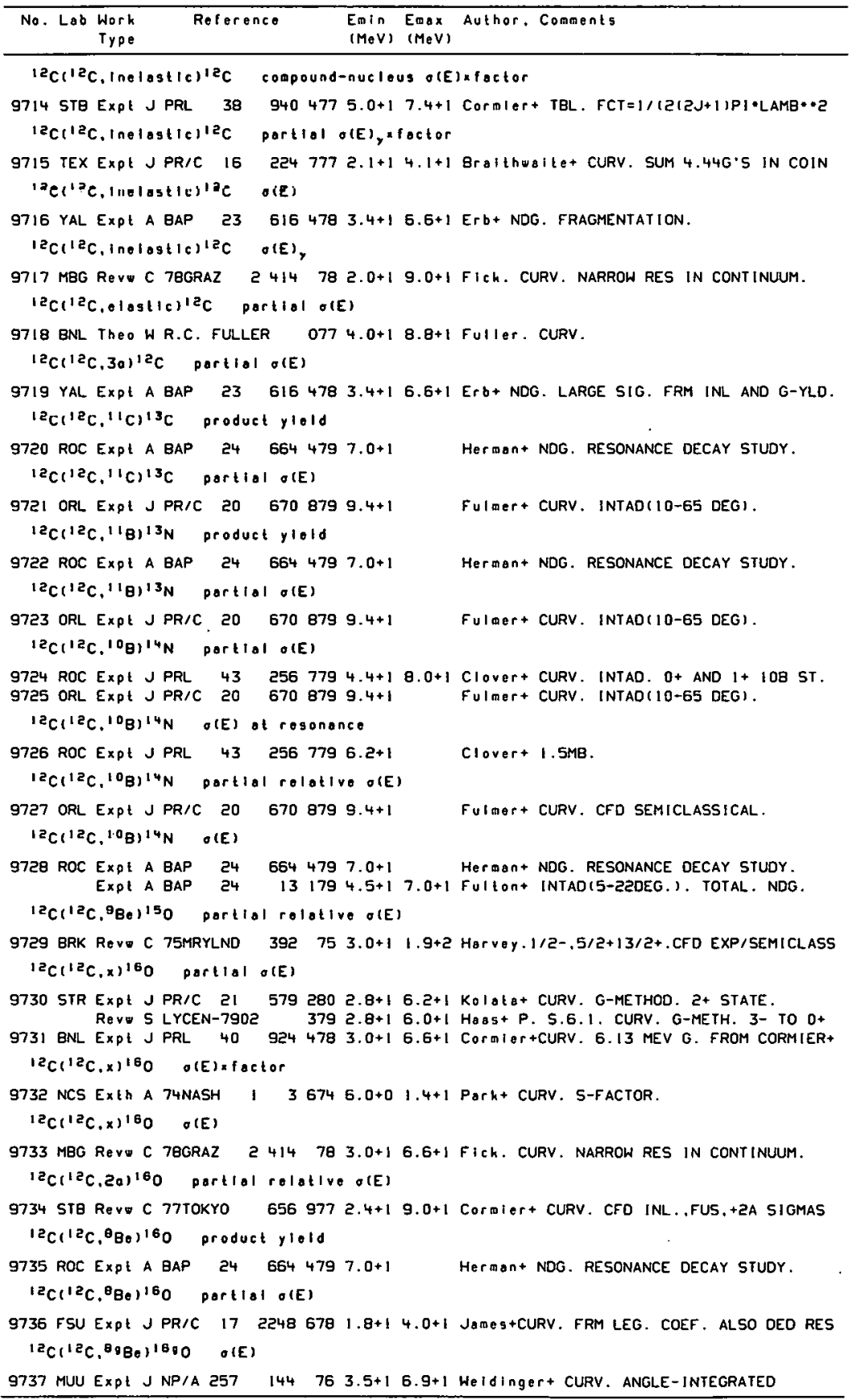




\begin{tabular}{|c|c|c|c|c|c|c|}
\hline No. Lab & $\begin{array}{l}\text { Work } \\
\text { Type }\end{array}$ & Reforence & $\begin{array}{l}\text { Emin } \\
\left(M_{e} V\right)\end{array}$ & $\begin{array}{l}\text { Exax } \\
(M \circ V)\end{array}$ & Author, & Comments \\
\hline
\end{tabular}

$12 C\left(1{ }^{2} C, a\right)^{20} \mathrm{Ne}$ partlal relativo o(E)

9738 BOC Exlh J NP/A 318125479 NDG Kuhlmonnt CURV. J=0-6. EX=18MEV. H-F.

${ }^{12} \mathrm{C}\left(\mathrm{I}^{2} \mathrm{C}, \mathrm{a}\right)^{20} \mathrm{Ne}$ partlal o(E) a foctor

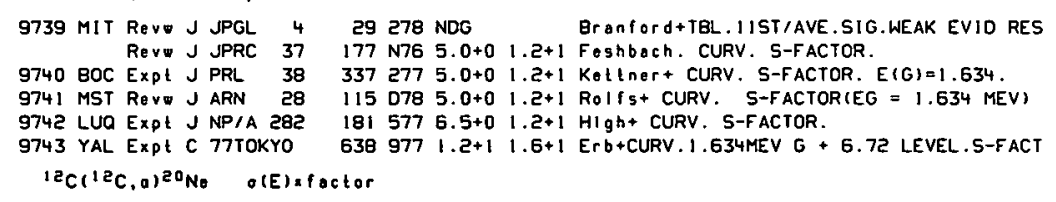

9744 UEN Expt J PR/C 15950377 Q.0+0 1.6+1 Golster+ CURV. S-FACTOR.

9745 YAL EXPt C 77TOKYO $6389771.2+11.6+1$ Erb + CURV. S-FACTOR.

9746 YAL Exth C 77TOKYO $6399771.3+11.4+1$ Korotky+ CURV. S-FCT. FROM ELASTIC SCAT

${ }^{12} \mathrm{C}\left({ }^{2} \mathrm{C}, 0\right)^{20} \mathrm{Ne}$ porttal o(E)

9747 LUO Expt J NP/A $282 \quad 1815776.5+01.2+1 \mathrm{HIgh}+\mathrm{TBL}$.

9748 UEN Expt J PL/B 67 262 477 B.0+0 $1.6+1$ Galster+ CURV. INTAD AO, A3, +SUM AO-A5 Expt J PR/C 15 950377 8.0+0 $1.6+1$ Galster+CURV.G.S. $4.97 L E V$. SUM OF AD-A5

9749 BRC Expt P CEA-N-2037 $95678 \quad 1.1+1 \quad 1.2+1$ Cocut TEL. L=0, 2, 4 . ALSO ANG. DIST.

9750 TAM Expt J PL/B $67 \quad 399477 \quad 1.3+12.5+1$ Volt+ CURV. INTAD. FIRST 5 ST . + SUM OF Expt J PL/B $67 \quad 3994771.2+1 \quad 2.4+1$ Volt+ CURV. INTAD. $0+(1)$ AND $2+(2)$ Expt J PL/B $67 \quad 3994771.4+12.4+1$ VoIt+ CURV. INTAD. SUM OF 6-10 STATES.

9751 YAL Expt C 76ANL-2 $25494761.3+1 \quad 1.9+1$ Erb+ CURV. A0-A3 GRPS. SUM OF A0-A3. Expt J PRL $37670766.5+01.1+1$ Erb + CURV. AO. ANGLE-INTEGRATED.

9752 UEN Expt J PR/C 18 2148 N78 $2.2+13.1+1$ Treu+ CURV. INTAD. $0-5$ STATES, SUM(D-5) Expt J PR/C 18 2148 N78 $2.2+13.1+1$ Treu+ CURV. INTAD. Al1,SUMS(6-10,12-22)

9753 MIT Revw J JPRC. 37 177 N76 $2.4+14.4+1$ Feshboch. CURV. GROUND STATE. RevoJ JPRC $37 \quad 177$ N76 $1.2+14.4+1$ Feshboch. CURV. 3 STATES.

${ }^{2} \mathrm{C}\left(1{ }^{2} \mathrm{C}, 0\right)^{20} \mathrm{No}_{0}$ O(E)

9754 LUO Expt A BAP $21 \quad 656762.5+03.6+0 \mathrm{HIgh+}$ NDG. MEAS. G.S. FUSION STUDY. 9755 YAL Expt J PRL 37 670 $766.5+01.1+1$ Erb+ CURV. EXCIT. ENERGY .LE. 4.97 MEV 9756 TOK Theo C 77TOKYO $6379771.0+12.4+1$ Matsuset CURV. 5 CHANNELS.

9757 MIT ReVw J JPGL $4292781.2+12.4+1$ Bronford+ CURV. WEAK EVIDENCE FOR RESON Revw J JPRC $37 \quad 177$ N76 $1.2+12.2+1$ Foshboch. CURV.

9758 TAM Expt J PL/B $67 \quad 399477 \quad 1.4+12.4+1 v_{0} 1 t+$ CURV. INTAD. SUM OF D-11 STATES. 9759 UEN EXPt J PR/C 182148 N78 $2.2+13.1+1$ Treu+ CURV. SUM(D-22 STATES).

$12 \mathrm{C}\left({ }^{2} \mathrm{C}, a^{20} \mathrm{Ne}\right.$ partlol product yleld

9760 MIT Expt A BAP $25 \quad 481802.0+18.0+1$ LOdoux+ NDG. VERY STRONG RESONANCES.

$12 C\left(1{ }^{2} \mathrm{C}, 0\right)^{23} \mathrm{No}$ partial o(E) factor

9761 BOC Expt J PRL $38 \quad 3372775.0+0 \quad 1.2+1$ Kettner+ CURV. S-FACTOR. E $(G)=0.440$ 9762 MST Revw J ARN $281150785.0+01.2+1$ Rolfs+CURV. S-FACTOR(EG $=0.440$ MEVI 9763 LUO EXPt J NP/A $2821815776.5+01.2+1 \mathrm{HIght}$ CURV. S-FACTOR.

$12 C(12 C, p) 23^{2}$ o $O(E)$

9764 LUO Expt A BAP $21656762.5+03.6+0 \mathrm{HIgh+}$ NDG. MEAS. G.S. FUSION STUDY.

${ }^{2} \mathrm{C}\left({ }^{2} \mathrm{C}, \gamma\right)^{24} \mathrm{Mg}$ portIal OIE)

9765 ANL Expt A BAP $24 \quad 6664791.2+1 \quad 1.8+1$ Nathen+ NDG. G0-G3. 4 RESONANCES. Expt P ANL-79-40 $67 \quad 791.9+1 \quad 2.3+1$ Bow 1 es+ NOG. 4 RESON. THETA=45 OEG.

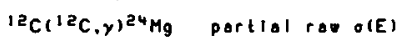

9766 BNL Expt J PRL $401252578 \quad 1.6+1 \quad 1.7+1$ Sandorf $1+$ CURV. G0, 1, AND $2+3$

$12 \mathrm{C}\left({ }^{2} \mathrm{C}, y\right)^{24} \mathrm{Mg}$ portlal o(E)xfactor

9767 BNL Expt J PRL $4012525781.0+12.2+1$ Sandorfi+CURV.G0+G AT 450EG. FCT=1/4PI

${ }^{12} \mathrm{C}\left({ }^{2} \mathrm{C}, \mathrm{x}\right) \mathrm{chorg}$ distribution (E)

9768 ANL Expt J PR/C $2013050791.5+16.2+1$ Kovar+ TBL. N,O,F.NE.NA FROM ANG. DIST. 9769 SAC Expt J NP/A $3095150782.8+15.4+1$ Conje日ud+ CURV. $Z=8-13$. INTAD. CFD. MOD Expt J BIS $224 \quad 1347772.8+15.1+1$ Harar. CURV. O,F, NE, AND NA

9770 TAM Expt J NPIA $263 \quad 491764.5+12.0+2$ Namboodir $1+$ CURV. $Z=1-12$.

9771 GRE Expt P ISN-79-0I $38791.0+21.2+2$ Conjooud+ CURV. $Z=6-11$. CFO CASCADE MOD Expt P CEA-N-2070 189978 1.0+2 $1.2+2$ Conje日ud+ CURV. CFD CASCADE MODEL.

$12 \mathrm{C}\left(\mathrm{I}^{2} \mathrm{C}, \mathrm{x}\right) \mathrm{chorg}$ distribution partlal o(E)

9772 SAC Expt P CEA-N-2026 $299775.0+1 \quad$ Conje日ud+ CURV. $Z=7-11$ FOR L=12 AND 14. 
REFERENCES (cont)

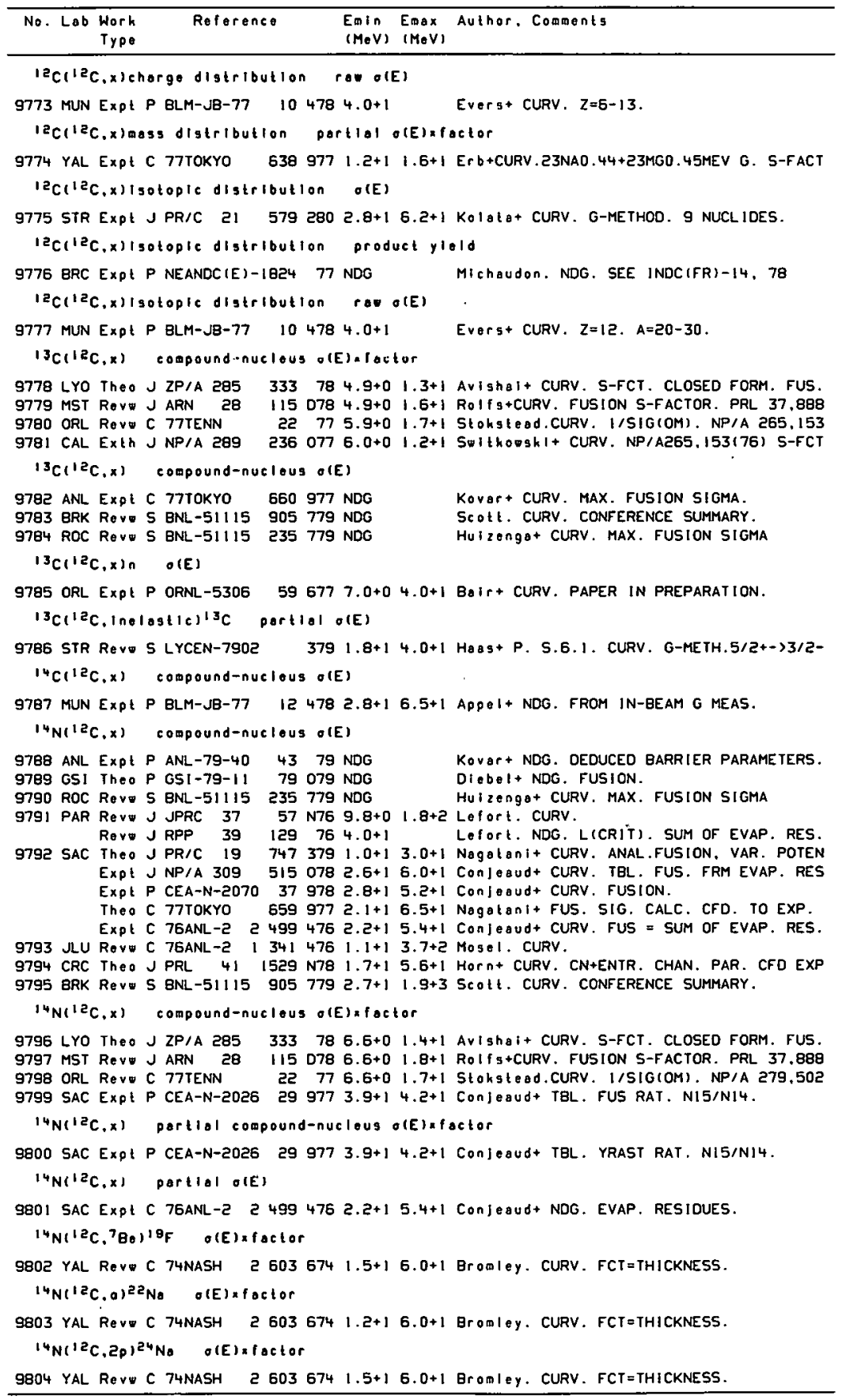


REFERENCES (cont)

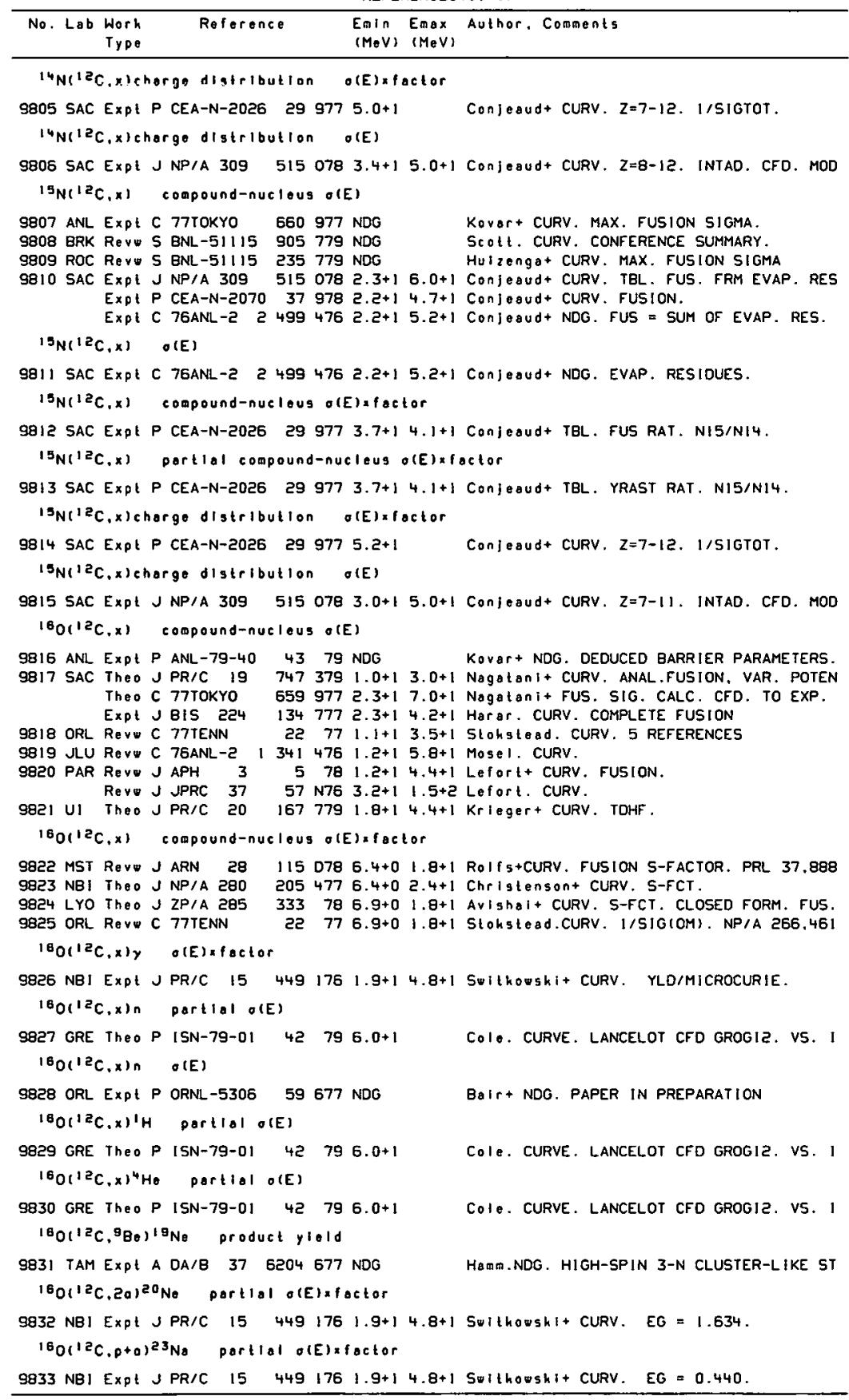


REFERENCES (cont)

\begin{tabular}{|c|c|c|c|c|c|c|}
\hline No, Lob & $\begin{array}{l}\text { Work } \\
\text { Type }\end{array}$ & Reference & $\begin{array}{l}\text { EmIn } \\
(M e V)\end{array}$ & $\begin{array}{l}\text { Emax } \\
\text { (MeV) }\end{array}$ & Author, & Coments \\
\hline
\end{tabular}

$160(12 C, n+a)^{23} M g$ portlal ofE)xfoctor

$9834 \mathrm{NBI}$ Expt JPR/C $154491761.9+14.8+150 i t h o w 5 k 1+$ CURV. $\quad E G=0.451$

$\left.1601{ }^{2} \mathrm{C} . \mathrm{x} x\right)^{24} \mathrm{Mg}$ partial o(E)

9835 CBR Expt J JP/G $31565773.0+14.3+1$ Dionferd+CIIRV, !N-BM 1.37 G+7.62-MEV LVL

$160(12 \mathrm{C}, \mathrm{a})^{24} \mathrm{Mg}$ partial olE)afoctor

9836 GER Expt J PL/B $723151781.4+12.2+1$ Treu+CURV.S-FACTOR.SUMS A0-3, $-6,-12,-23$ 9837 NBI Expt J PR/C $154491761.9+14.8+1$ Solthowski+ CURV. $\quad E G=1.369$.

$160\left({ }^{2} \mathrm{C}, 2 p\right)^{26} \mathrm{Mg}$ portlal o(E) $\times$ factor

$9838 \mathrm{NBi}$ Expl J PR/C $154491761.9+14.8+1$ Swltkowski+ CURV. EG $=1.809$.

${ }^{16} O\left(1{ }^{2} \mathrm{C}, x\right)^{26} \mathrm{Al}$ portiat ofE)

9839 CBR Expl J JP/G $31565772.3+13.2+1$ Branford+ CURV. IN-BEAM 4/7-KEV G.

$160\left({ }^{12} C, n+p\right){ }^{26} A I$ portlal $O(E) \times$ faclor

$9840 \mathrm{NB}$ ! Expt JPR/C $154491761.9+14.8+1$ Swithowshit CURV. EG $=0.418$.

${ }^{16} O\left({ }^{12} \mathrm{C}, \mathrm{x}\right) 1$ sotople distribution $O(E)$

9841 ANL Expl A BAP $21 \quad 656762.3+14.9+1$ Sperr+ NDG. DETECTED HEAVY PRODUCTS.

9842 GRE TheO P ISN-79-01 $42796.0+1$ Cole. CURV. LANCELOT CFD GROGI? + EXPT.

$170(12 \mathrm{C}, \mathrm{x})$ compound-nuclous $O(E)$

9843 ANL Expt P ANL-79-40 4379 NDG Kovar+ NDG. DEDUCEO GARRIER PARAMETERS. 9844 SAC Expl P CEA-N-2070 $379782.0+14.8+1$ Conjeaud+CURV.FUS.CFD GLAS-MOSEL. OTHERS

$170{ }^{12} \mathrm{C}, x$ lchorge distribution o(E)

9845 SAC Expt P CEA-N-2070 $379783.5+14.9+1$ Conje日ud+ CURV. CFD THEORY.

$\left(\theta_{0}\left({ }^{2} \mathrm{C}, \times\right)\right.$ compound-nuclous $O(E)$ 9847 ROC Rev S BNL-51I15 235779 NDG HuIzenga+ CURV. MAX. FUSION SIGMA

9846 PAR ReV J APH $3 \quad 5781.0+14.2+1$ Lefort+ CURV. FUSION. RevE J JPRC $37 \quad 57$ N76 $2.8+11.4+2$ Lefort. CURV.

9849 SAC Theo J PR/C I9 $7473791.0+13.0+1$ Nagalonit CURV. ANAL.FUSION, VAR. .POTEN Theo C 77TOKYO $6599772.8+17.5+1$ Nagatanit FUS. SIG. CALC. CFD. TO EXP.

9850 JlU ReVw C 76ANL-2 $13414761.1+15.6+1$ Mosel. CURV.

S851 ORL ReV $\mathrm{C}$ 77TENN $22771.2+14.2+1$ Stokstead. CURV. 5 REFERENCES

9852 UI Theo J PR/C $201677791.7+14.2+1 \mathrm{Krleger}+\mathrm{CURV}$. TDHF.

$180(12 C, x)$ o(E)

9853 ORL EXpI P ORNL-5306 59677 NDG

BaIr+ NDG. PAPER IN PREPARATION

${ }^{10} \mathrm{O}\left({ }^{2} \mathrm{C}\right.$, Inelastic) $10^{\circ} \mathrm{O}$ partial o(E)

9854 STR ReVw S LYCEN-7902 $3791.7+1$ 4.0+1 Has + P. S.6.1. CURV. G-METH. 2+ TO 0+

$10^{O}(15 C, x)^{2 \theta_{A}}$ o(E)

9855 ETH Expl C 7IALgany $527 \quad 711.3+13.7+1$ Ey 1 I + CURV. ACTIVATION METHOD.

$10^{0}\left({ }^{2} C, x\right)^{20} A I$ o(E) $x$ factor

9956 ETH EXPL C 7IALBANY $527712.8+14.0+1$ EyB + CURV. ALzB/ALZ9.

$100\left({ }^{12} C, p\right)^{20} A I$ OIE)

9857 ETH EXPL C 7IALBANY $527 \quad 711.3+13.7+1$ EYa1+ CURV. ACTIVATION METHOD.

$100(12 C, p)^{20} A L$ o(E) $x$ factor

9858 F.TH EXPt C 7IALBANY $527712.8+14.0+1$ EYal+ CURV. ALZB/ALZ9.

$19^{2} \mathrm{~F}\left({ }^{2} \mathrm{C} . x\right)$ compound-nucleus o(E)

9859 ANL EXPt C 77TOKYO 660977 NDG KOVOR+ CURV. MAX. FUSION SIGMA.

Expt C 76ANL-2 $27834761.9+14,4+1$ Sperr+ CURV. FUS = SUM OF EVAP. RES.

Expt J PRL $37321 \quad 76 \quad 1.9+14.4+1$ Sperr+ CURV. INTEG. ANG. DIST.

9860 BRK Rev S BNL-51115 905 779 NOG Stot. CURV. CONFERENCE SUMMARY.

986! ROC Rev S BNL-51115 235779 NDG HuIzenga+ CURV. MAX. FUSION SIGMA

$98625 A C$ Theo J PR/C is $7473791.0+13.0+1$ Nagatanit CURV. ANAL.FUSION, VAR. POTEN Theo C 77TOKYO $6599772.8+17.7+1$ Nagatanit FUS. SIG. CALC. CFD. TO EXP.

$9863 \mathrm{lU}$ Rev C 76ANL-2 | 341476 1.1+1 5.4+1 Mosel. CURV.

9864 CRC Ine J FRL 411529 N7B $1.5+14.9+1$ Horn+ CURV. CN+ENTR, CHAN. PAR. CFO EXP 9865 MIT ReVw C 77TENN $73771.7+15.4+1$ Negele. CURV. FRM PRL 37, 321(1976! 
REFERENCES(cont)

\begin{tabular}{|c|c|c|c|c|c|}
\hline & $\begin{array}{l}\text { Work } \\
\text { Type }\end{array}$ & Reforence & $\begin{array}{l}E \Phi I n \\
(M e V)\end{array}$ & $\begin{array}{l}E \operatorname{Eax} \\
(M \in V)\end{array}$ & Author, Comments \\
\hline
\end{tabular}

${ }^{19} \mathrm{~F}\left({ }^{12} \mathrm{C}, \mathrm{x}\right) \mathrm{lsotoplc}$ distribution o(E)

9866 ANL Expt A BAP $21656762.1+14.6+1$ Sperr+ NDG. DETECTED HEAVY PRODUCTS.

$20 \mathrm{No}\left({ }^{2} \mathrm{C}, x\right)$ chorge distribution o(E)

9867 SAC Exlh C 77TOKYO $6639774.8+19,6+1$ Conjooud + CURV. Z=5-14. INTAD. CASCADE 9868 GRE Expt P CEA-N-2070 $1909781.2+21.4+2$ Conjoaud+ TBL. CURV. CFO THEORY.

$2{ }^{\circ} \mathrm{Ne}\left({ }^{2} \mathrm{C}, \mathrm{x}\right) \mathrm{chorg}$ distribution direct-interoction o(E)

9869 GRE EXPI P CEA-N-2070 $1909781.2+21.4+2$ COnjeBud+ TBL. CURV. CFD THEORY.

$20 \mathrm{Ne}\left({ }^{2} \mathrm{C}, \mathrm{x}\right) \mathrm{s}$ otopic distribution $\sigma(E)$

9870 NOT Expt A BAP $24 \quad 8519792.0+15.4+1$ De-Young+ NDG. FROM G-YLO.

9871 GRE Theo P [SN-79-0] $42796.7+1$ Cole. CURV. LANCELOT CFD EXPT.

20 Ne $(12 C, x) i g o t o p i c$ distribution product ylold

9872 ORL Expt A BAP $24 \quad 8249793.2+14.8+1$ Shopira+ NDG. EVAP. RES.

$2{ }^{4} \mathrm{Mg}\left({ }^{2} \mathrm{C}, x\right)$ compound-nuctous $O(E)$

9873 WZI Thoo J PR/C $1921966791.7+11.2+2$ Pelte+ CURV. COUPLEO-CHANNELS.

9874 ANL Expt P ANL-79-40 $45792.0+16.0+1$ Daneshyar+ CURV. FROM EVAPORATION RESIO Expt P ANL-78-65 $413782.0+16,0+1$ Daneshyar+ CURV. FUSION CFD BASS MODEL

9875 SAC Expt P CEA-N-2070 $419782.5+13.4+1$ Gary+ CURV. FUSION.

${ }^{24} \mathrm{Mg}\left({ }^{\prime 2} \mathrm{C}, \mathrm{x}\right)$ partial diroct-1nteraction o(E)

9876 WZI Theo J PR/C $1921966795.1+1 \quad$ Pollo+ CURV. AS FUNCTION OF $L$.

$24 \mathrm{Mg}\left({ }^{2} \mathrm{C}, \mathrm{x}\right)$ partlal $\sigma(E)$

$9877 \mathrm{HEI}$ EXPl J NP/A $2942551784.2+1 \quad 8.4+1$ Novotny+CURV.2-NUCLEI EXC.REAC.TOT CFD Expl J NP/A 294 255 $1784.2+18.4+1$ Novotny+ CURV. TOT OF OBS. CFD CALC TOT

${ }^{24} \mathrm{Mg}\left({ }^{2} \mathrm{C}, \mathrm{abs}\right.$ orption) o(E)

9878 ANL Expt P ANL-78-65 41478 2.0+1 6.0+1 Daneshrar+ CURV. O.M. CALC.

$24 \mathrm{Mg}\left({ }^{12} \mathrm{C}, \mathrm{total}\right) \quad O(E)$

9879 HEI Expt J NP/A $2942551781.5+18.4+1$ Novotny+ CURV. CALC. TOT CFO TO EXP. 9880 WZI Theo J PR/C $1921966791.7+11.2+2$ Pelle+ CURV. COUPLED-CHANNELS.

${ }^{24} \mathrm{Mg}\left({ }^{12} \mathrm{C}\right.$.scottoring) ${ }^{24} \mathrm{Mg} \quad$ O(E)

9881 STR Revw 5 LYCEN-7902 379 2.2+1 3.5+1 HaAs + P. 5.6.1. CURV, G-METH.

${ }^{4} \mathrm{Mg}\left({ }^{2} \mathrm{C}, \mathrm{Inelastic}\right)^{24} \mathrm{Mg}$ o(E)

9882 HEI Expt J NP/A $2942551784.2+18.4+1$ Novolny+ CURV. INT. E/A. CFO CALC. TOT.

${ }^{24} \mathrm{Mg}\left({ }^{2} \mathrm{C}, x\right)^{28} \mathrm{SI} \quad \mathrm{O}(\mathrm{E})$

9883 STR ReVw S LYCEN-7902 379 2.2+1 3.5+1 Hoas+ P. 5.6.1. CURV. G-METH.

$24 \mathrm{Mg}\left({ }^{2} \mathrm{C}, \times\right)^{31} \mathrm{P} \quad O(\mathrm{E})$

S884 STR REVW S LYCEN-7902

${ }^{24} \mathrm{Mg}\left({ }^{12} \mathrm{C}, \times\right)^{34} \mathrm{~S} \quad \sigma(E)$

S895 STR ReVw S LYCEN-7902

$2{ }^{4} \mathrm{Mg}\left({ }^{12} \mathrm{C} . x\right)^{34} \mathrm{Cl} \quad O(E)$

9886 STR ReVw S LYCEN-7902 379 2.2+1 3.5+1 HAOS+ P. S.6.1. CURV. G-METH.

$26 \operatorname{Mg}(12 \mathrm{C}, x)$ compound-nucleus o(E)

9887 ANL Expt A BAP $24 \quad 6664792.0+16.0+1$ Daneshvart NDG. FROM EVAP, RFS. Expl P ANL-79-40 4579 2.0+1 6. $0+1$ Donoshvar+ NUG. FROM EVAPORATION RESID.

$27 A\left({ }^{12} C, x\right)$ compound-nueleus o(E)

9888 ANL EXpt C 77TOKYO 660977 NDG KOVAr+ CURV. MAX. FUSION SIGMA.

9889 DUB Theo J YF $2711915782.5+11.8+2$ Toneev+ CURV. NEW MOOEL CFD EXPT Theo C 77TOKYO $6659771.5+1 \quad 1.8+2$ Schmidt+CURV. PHENOM. MODEL CFD TO EXP Theo 5 JINR-P7-1045? $771.5+11.6+2$ Toneev+ CURV.

Theo J SNP 27631 7日 $2.5+1$ 1. $6+2$. TRANSLATION OF YF $27,119 !$

9990 PAR Revw J RPP $39129763.1+1 \quad 1.2+2$ Lefort. TBL. SUM OF EVAP. RES.

Revo C 74NASH 2 BI $6744.4+1$ Lefort+ 1040ME PR/C 6.2133/74NASH.1.115

Revw C 74NASH $2816741.8+2$ Lefort+ 834MB. PR/C 6,2!33/74NASH,1,115

9891 IMP Expt J ASI 2 15I $3784.5+17.2+1 \mathrm{Chl}-\mathrm{Chang}+\mathrm{TBL}$. CURV. FUS=SUM EVAP RES

9892 YAL Expt C 77TOKYO $6649774.5+1$ 8.0+1 Betts+ CURV. FUS SIG. CFD. OTHERS+TOT. EXPt C 76ANL-2 $24434764.5+17.0+1$ Belts + TRL. FUS = SUM OF EVAP RES. 
REFERENCES ( c on ) )

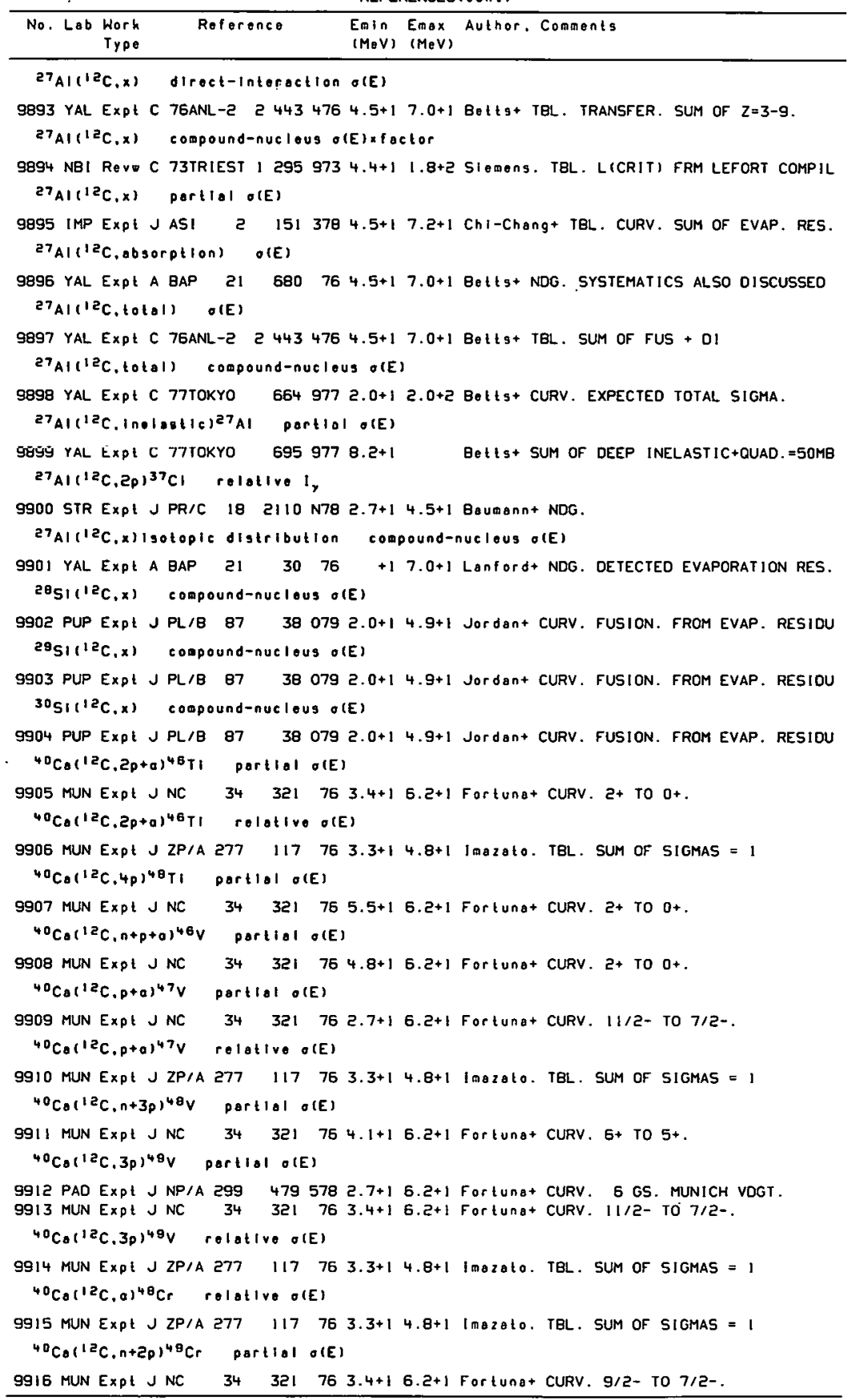


REFERENCES ( c on t)

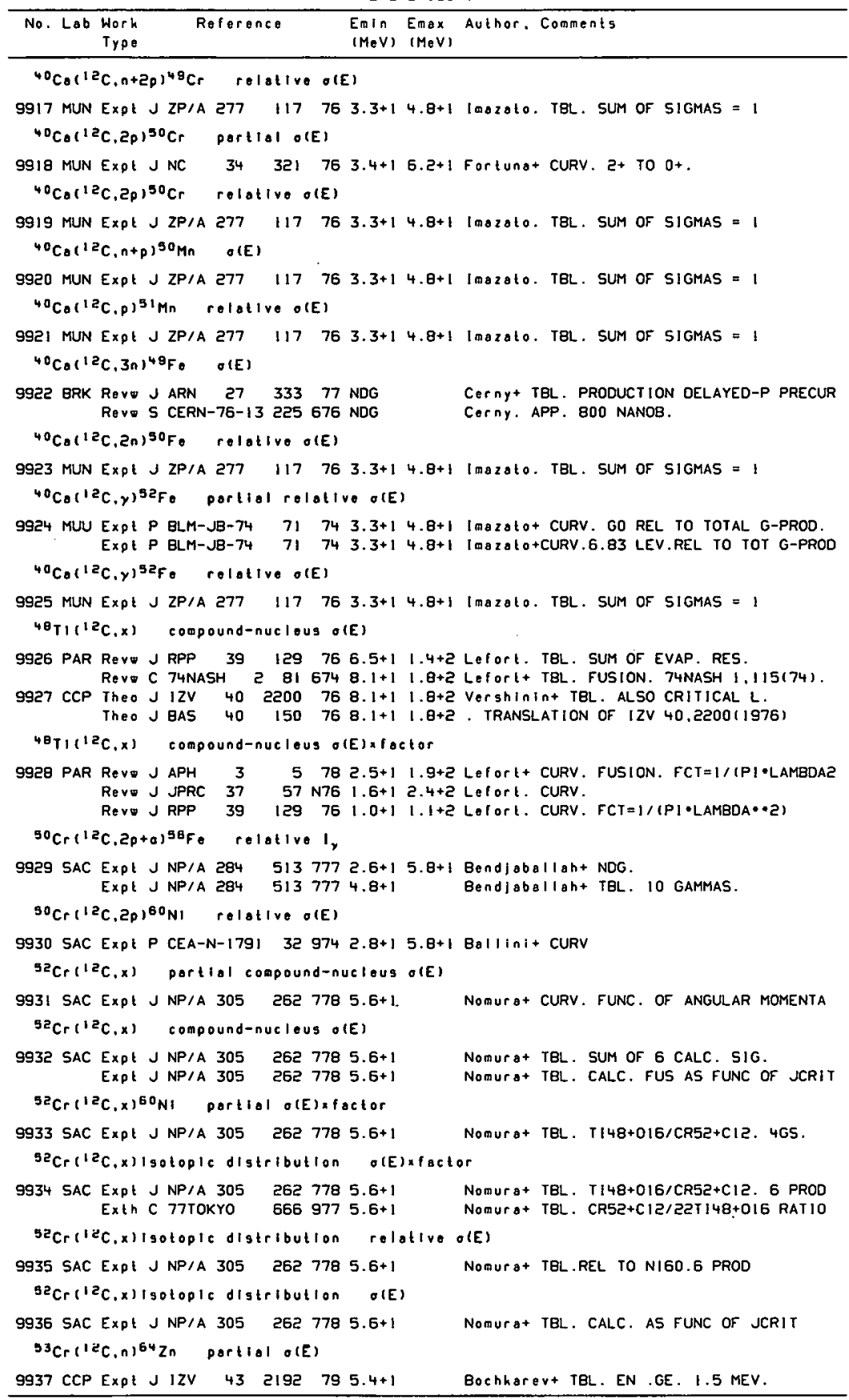




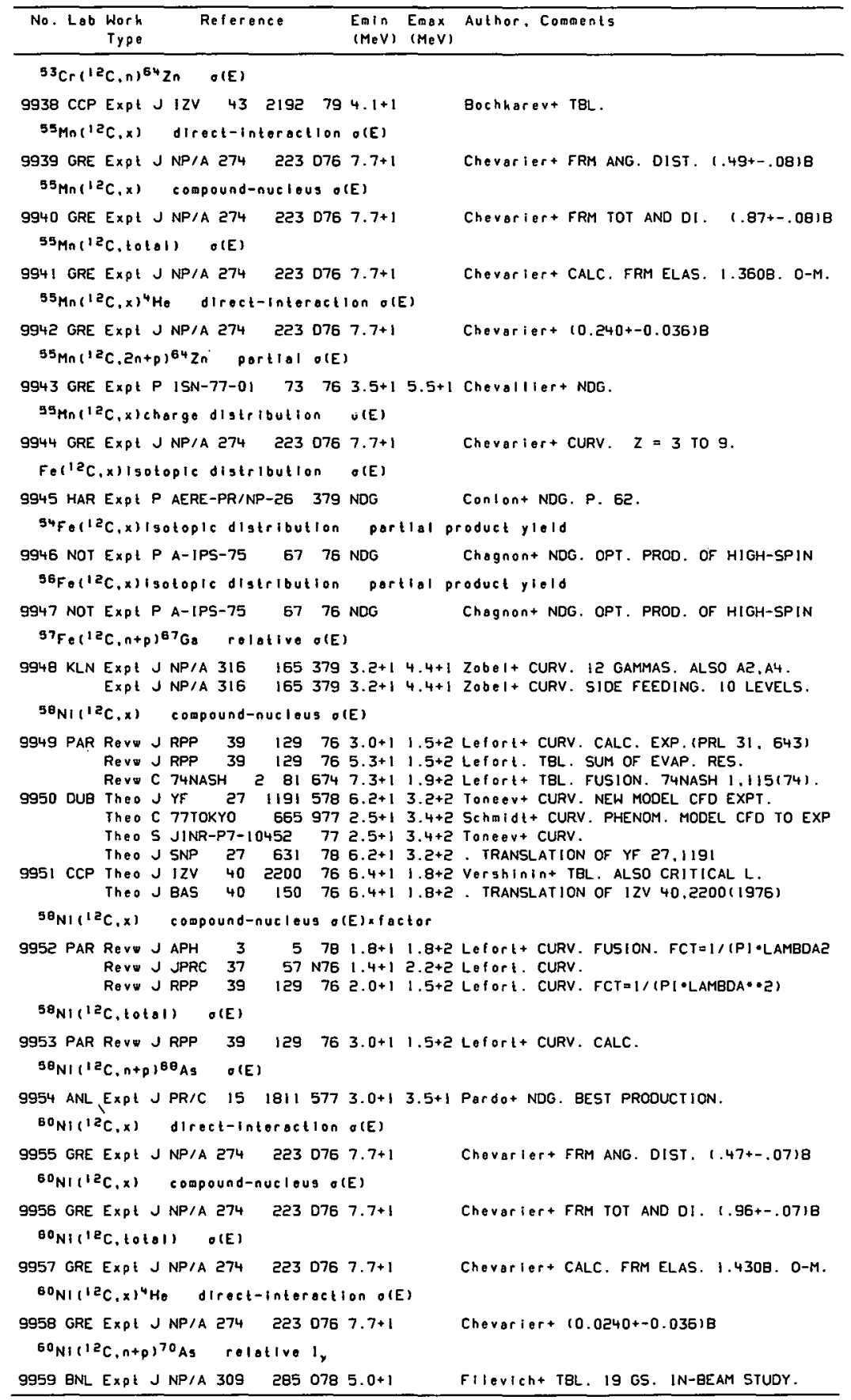


REFERENCES $(c$ on $t)$

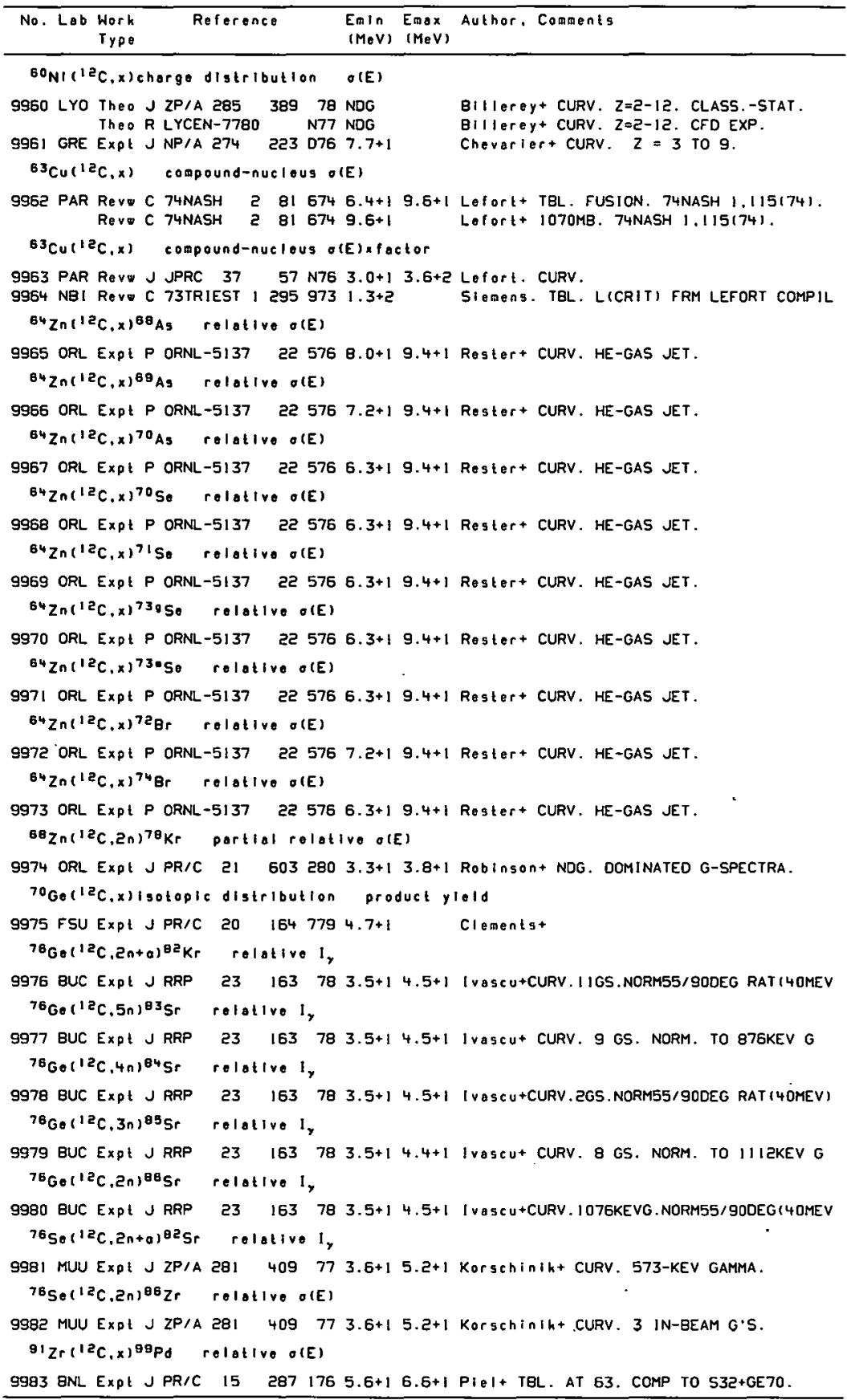


REFERENCES (cont)

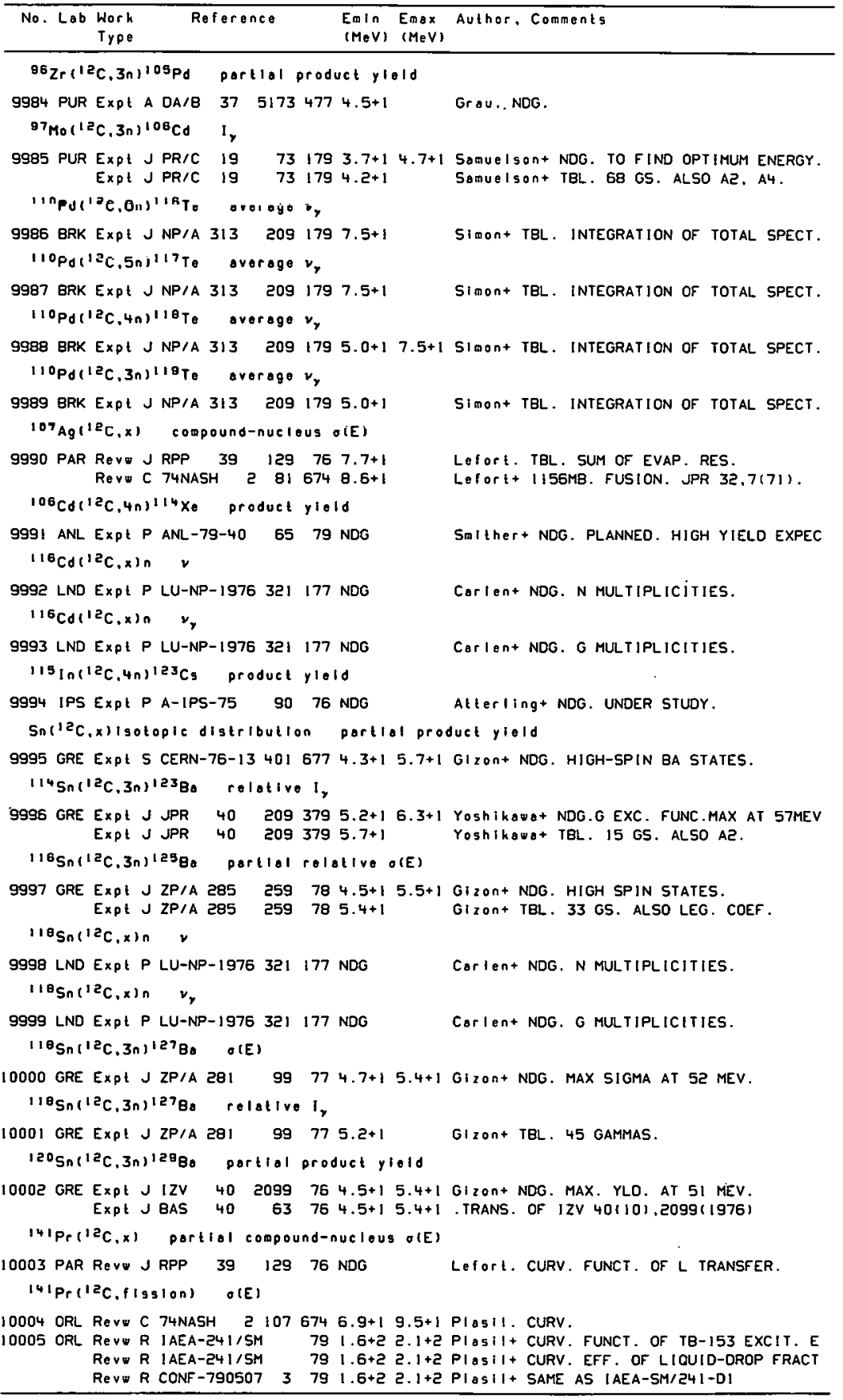


REFERENCES (cont)

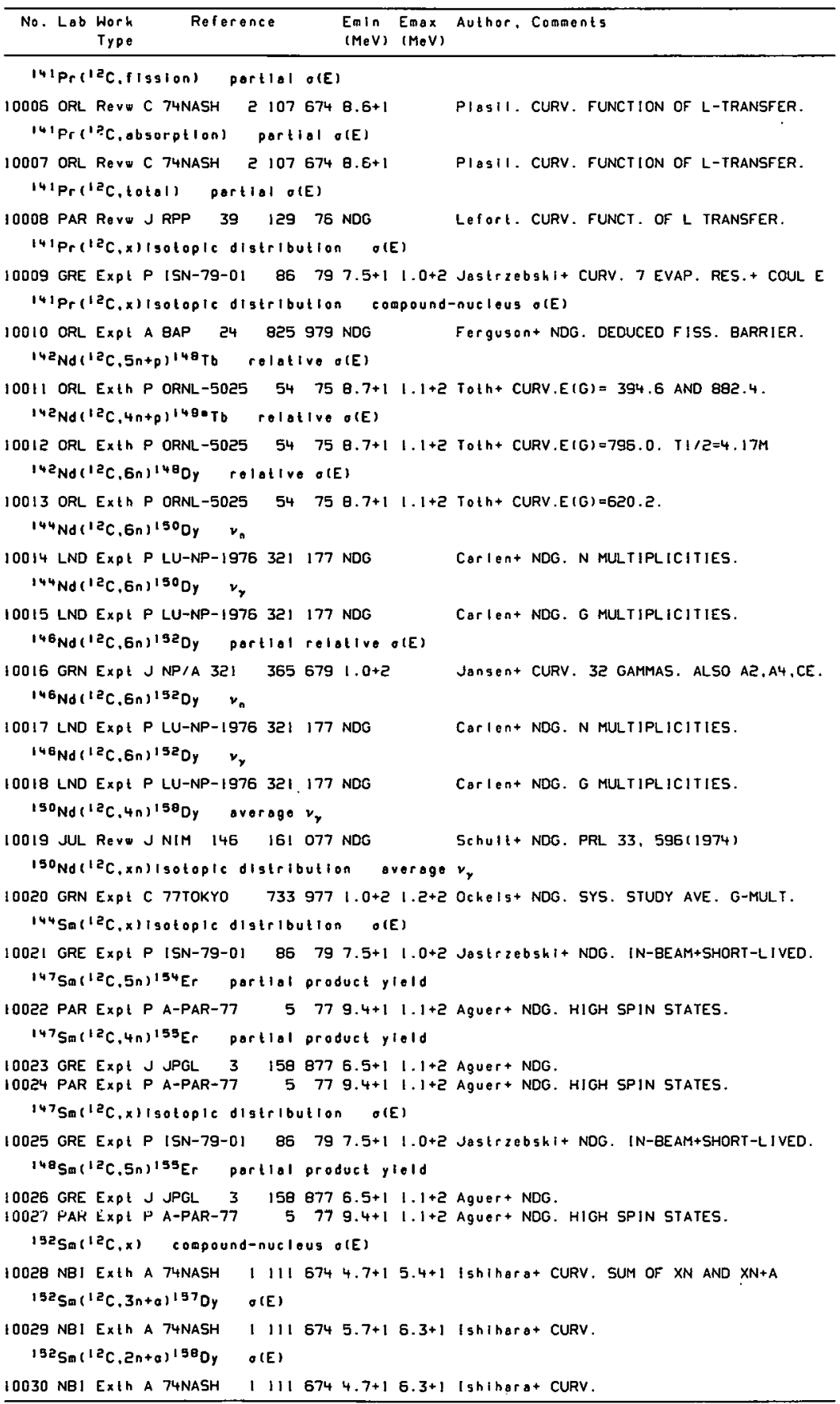


REFERENCES ( c ont)

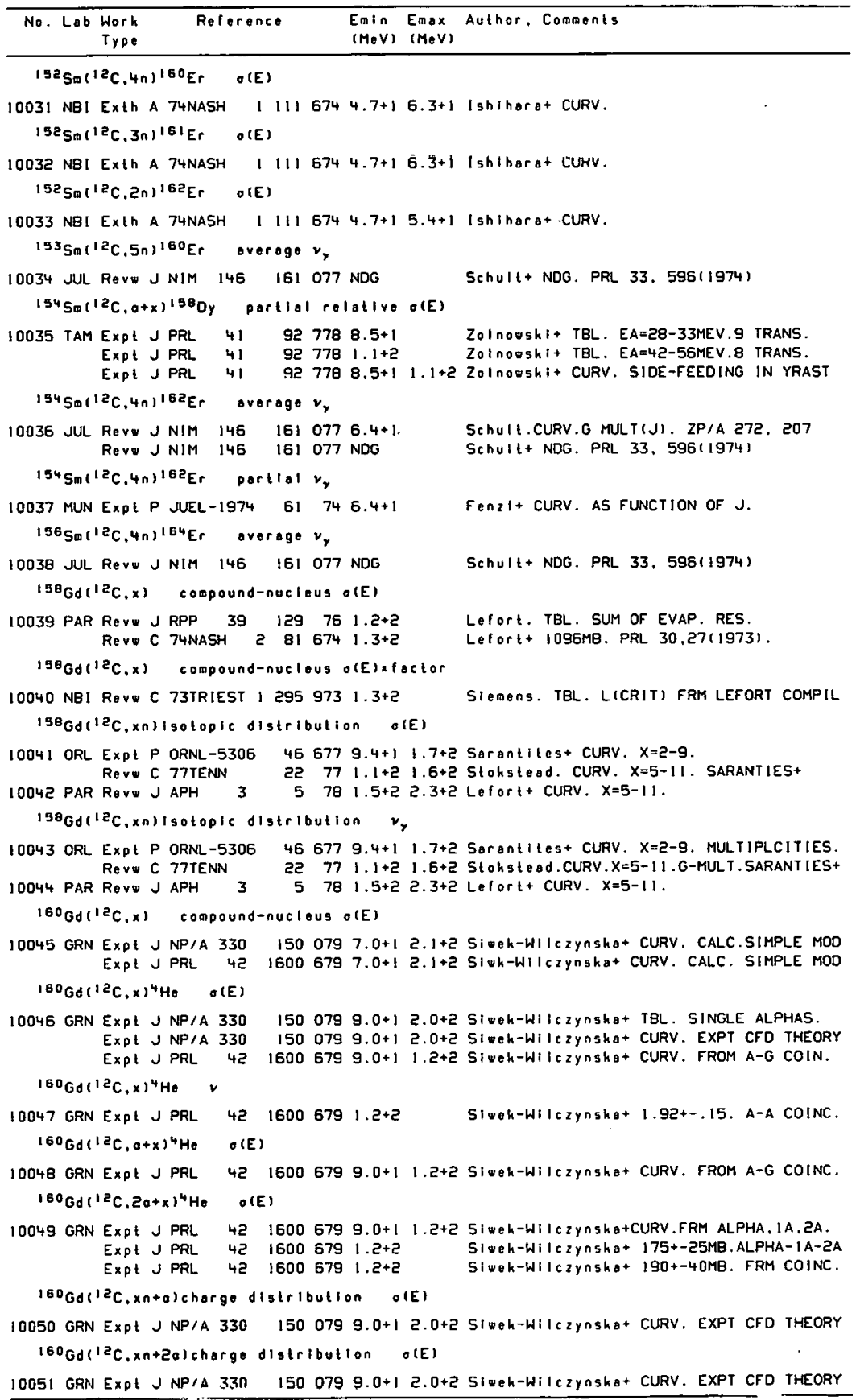


REFERENCES( c on ()

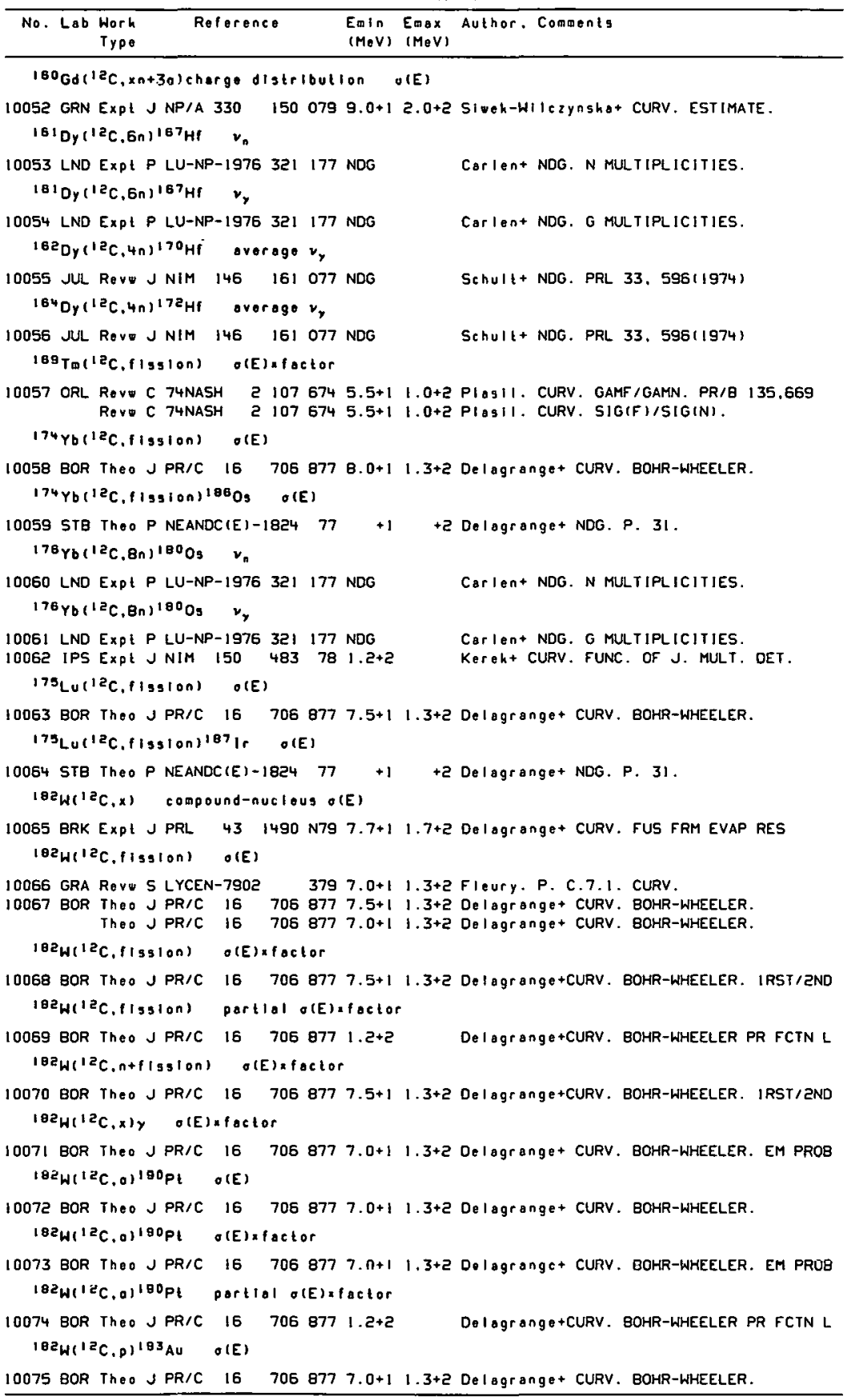


REFERENCES ( c ont)

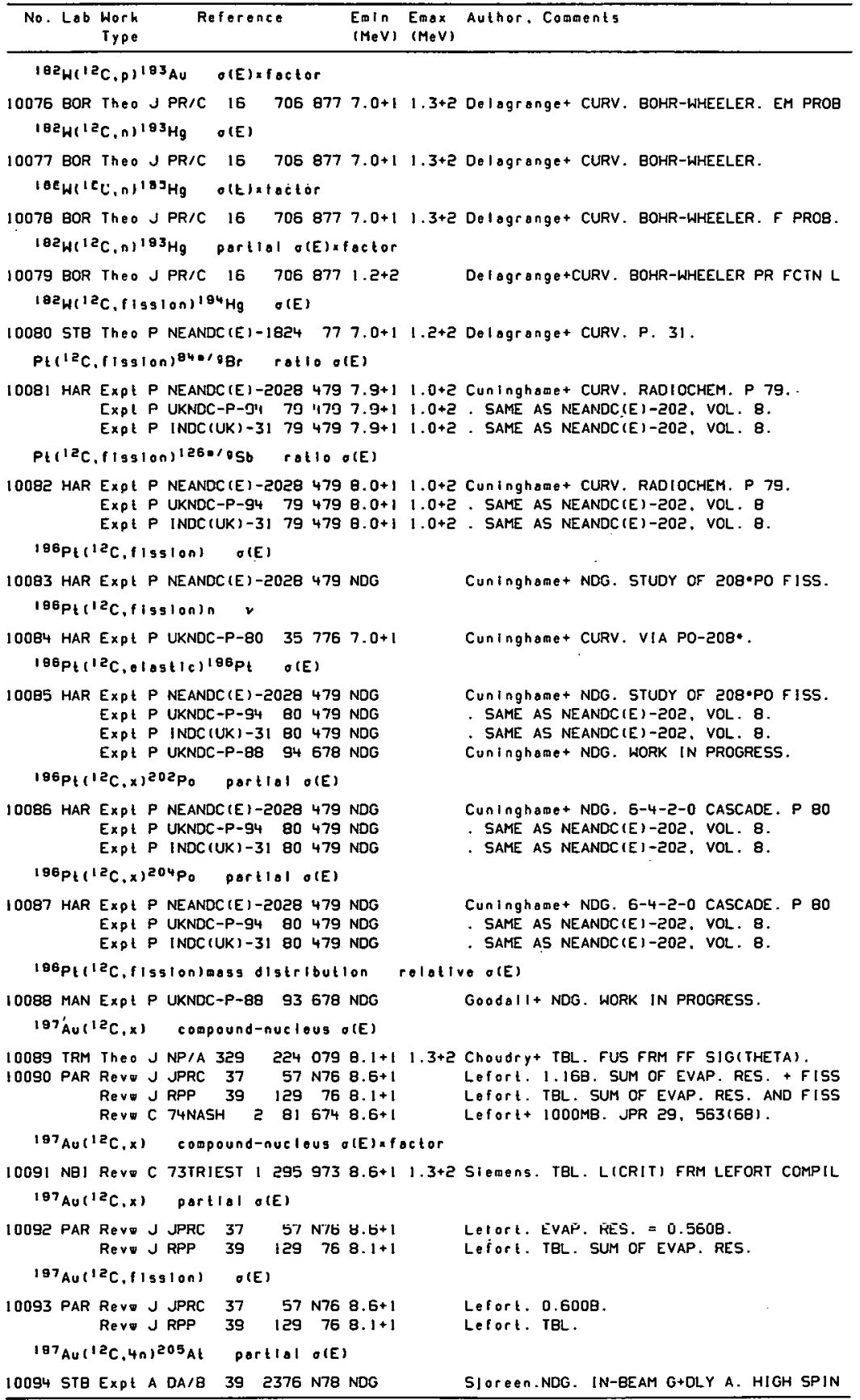


REFERENCES $($ cont $)$

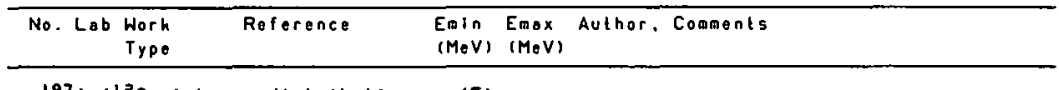

197 Au ( $12 C, x)$ chargo distribution o(E)

10095 KFK COMP B PH-OAT $1510791.3+2$ 10096 YAL EXPt J PR/C \& $11099731.3+2$ Expt 4 EXFORBO021.076 $771.3+2$

Muenzel+ TBL. FROM EXFOR.

EYOI+ TBL. $Z=3-5$. INTEG. OVER E/ANGLE.

3 ENTRIES.

$197 \mathrm{Au}(12 \mathrm{C}, \mathrm{x})$ isotopic distribution Independent o(E)

10097 KFK COMP B PH-DAT $1510791.3+2 \quad$ MuEnze1+ TBL. FROM EXFOR.

10098 YAL EXPt J PR/C $811099731.3+2 \quad E Y A \mid+T B L$. CURV. $Z=79-93 . A=195-203.23150 M$. Expt 4 EXFOREOOZI. $771.3+2 \quad 37$ DATA POINTS.

197 Au( $\left.{ }^{12} C, x\right)$ Isoloplc distribution cumulative o(E)

10099 KFK COMP B PH-DAT 15 I $0791.3+2$ Muenzel+ TBL. FROM EXFOR.

10100 YAL EXPE J PR/C B $11099731.3+2 \quad$ EYAI+TBL. CURV. $Z=79-84 . A=192-204.13150 M$. Expt 4 EXFORBOD21. $771.3+2 \quad 13$ DATA POINTS.

$197 A u(12 C, x)$ isotopic distribution o(E)

10101 BRK Theo A BAP $23 \quad 27178+4$ Menchaca-Rocha+ NDG. PERIPHERAL REACT.

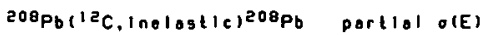

10102 BNL Revv S BNL-51115 $4057791,1+24.8+2$ Sandor $1+$ CURV. PEAK SIG FOR 11-MEV GDR

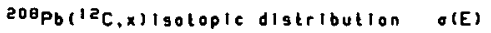

10103 BRK theo A BAP $2327178+4$ Menchaca-Rocha+ NDG. PERIPHERAL REACT.

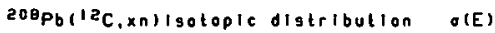

$10104 \mathrm{JCL}$ Expt J NP/A $217253736.5+19.6+1$ Nomura+ CURV. $x=3-5$. ALPHA DETECTION.

${ }^{209} \theta 1\left({ }^{2} C, x\right)$ compound-nucleug o(E)

10105 IMP Theo J PHE 2534 N78 $6.4+18.5+1$ Shu-We + + CURV. COMPLETE FUS. SEMI-CLASS 10106 IMP Expl J ASI 2 I5! $3797.0+17.3+1$ Chi-Chang+ TBL. CURV. FUS=EVAP. RES.+F 10107 TRM Theo J NP/A 329 224 079 B.1+1 9.8+1 Choudry+ TEL. FUS FRM FF SIGITHETAl. 10108 PAR Rev C 74NASH $2816748.5+1 \quad$ Lefort+1150MB. NP/A 192, 405(72).

$20{ }^{0} 1(12 C, x)$ partiol o(E)

10109 IMP Expt J ASI $2 \quad 1513797,0+17,3+1$ Chi-Chang+ TBL. CURV. SUM OF EVAP. RES. Expl J PHE I $69776.1+17.3+1$ Wen-Ging. TBL. EVAP. RES. CFD OTHER

${ }^{209} B(1)^{2} C . f(s \operatorname{sion}) \quad \sigma(E)$

10110 IMP Theo J PHE 2534 N78 6.4+1 8.5+1 Shu-We 1+ CURV. SEMI-CLASSICAL.

10111 IMP Expl J ASI 2 I51 $3787.0+17.3+1$ Chi-Chang+ TEL. CURV. CFD OTHER.

208Bi(laciabsorption) o(E)

10112 IMP Theo J PHE 2534 N78 6.4+1 B.5+1 Shu-Wei+ CURV. SEMI-CLASSICAL.

$20{ }^{3}$ i (12C.figsionin

10113 MAN Exth J JIN $3992 ! .777 .5+11.0+2$ Branquinho+ TBL. EST. FRM MOST PROB. $Z$.

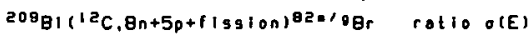

10114 HAR Expt P UKNDC-P-88 92678 8.5+1 9.4+1 GoOdaII+ NDG. PO208* FISSION.

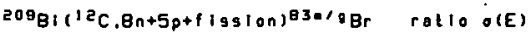

10115 HAR Expt P UKNDC-P-88 92678 8.5+1 9.4+1 Goodall+ NDG. POZOB. FISSION.

${ }^{209} B(1)^{2} C, 8 n+5 p+f(s s i o n) 120 m / 0 S b$ rallo olE)

$10 ! 16$ HAR EXgt P NEANDCIE)-2028 479 8.5+1 $1.1+2$ Cuninghamet CURV. RADIOCHEM. P 79.

Expt $P$ INDC(UK)-31 $794798.5+11.1+2$. SAME AS NEANDC $(E)-202, V O L .8$.

Expl $P$ UKNDC-P-g4 $794798.5+1 \quad 1.1+2$. SAME AS NEANDC(E)-202, VOL. 8

Expt P UKNDC-P-88 92678 8.5+1 9.4+1 Goodal1+ NDG. PO200. FISSION.

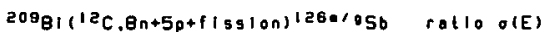

10117 HAR EXPl P NEANDC $(E)-20284797.5+11,1+2$ Cuninghame + CURV. RADIOCHEM. P 79.

Expt $P$ UKNDC-P-94 $794797.5+1 \quad 1.1+2$. SAME AS NEANDCIEI-202, VOL. 8

Expl P INDC(UK)-31 $794797.5+1 \quad 1.1+2$. SAME AS NEANDC $(E)-202$, VOL. 8.

Expt P UKNDC-P-88 ge 678 8.5+1 9.4+1 Goodal1+ NDG. POZ08 * FISSION.

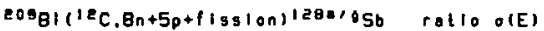

10118 HAR Expt P UKNDC-P-88 92678 8.5+1 9.4+1 Goodall+ NDG. PO208* FISSION.

$\left.{ }^{209} \mathrm{~B} 1\left({ }^{12} \mathrm{C}, x\right)^{2}{ }^{\circ} \mathrm{B}\right) \quad O(E)$

10119 PAR Expl J PR/C $1812989786.8+18.7+1$ Gardes+ TBL. 2PI ION. CHMBRS FOR A + G 
REFERENCES (cont)

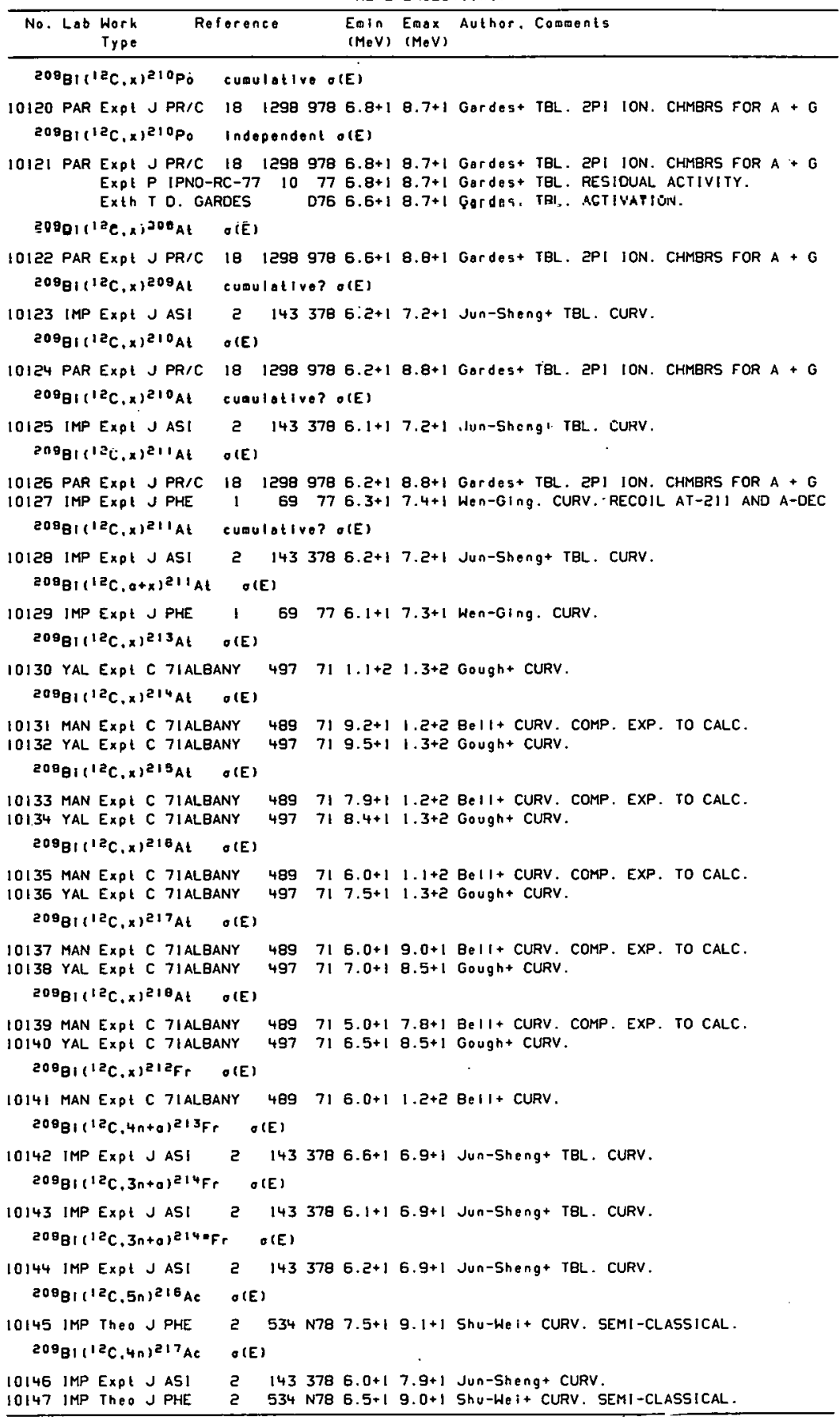


REFERENCES (c on t)

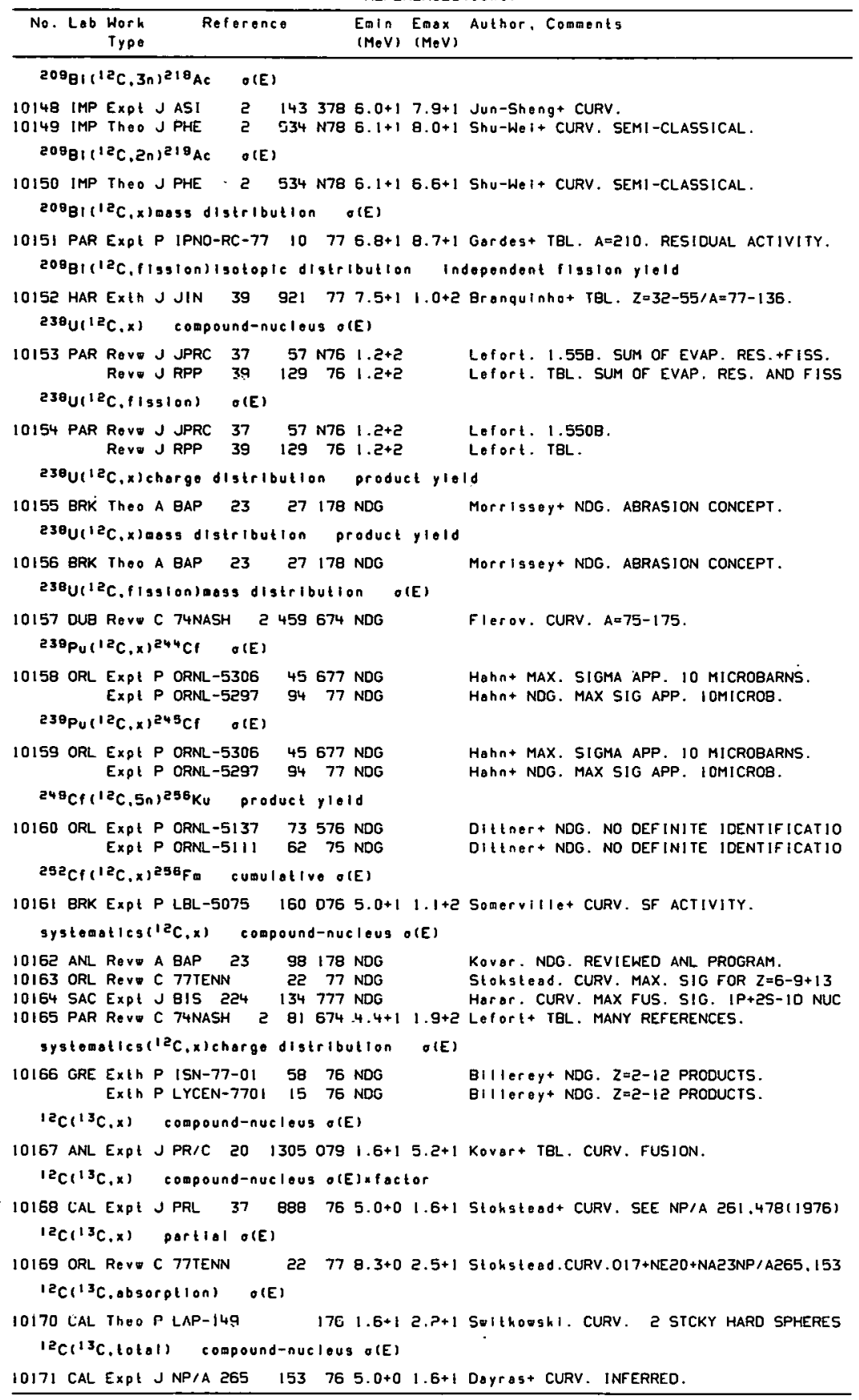


REFERENCES ( cont)

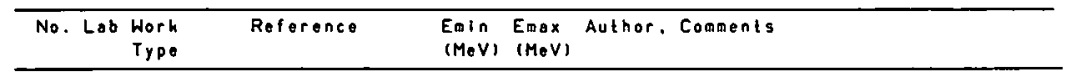

\footnotetext{
${ }_{15} \mathrm{C}(13 \mathrm{C}, x) \mathrm{O}$ o(E)x foctor

10172 YAL EXPl C 77TOKYO $6419771.5+11.6+1$ WIIlet+ CURV. S-FACTOR.

${ }^{12} \mathrm{C}\left({ }^{13} \mathrm{C}, x\right) \mathrm{O}$ o(E)
}

10173 ORL EXPt P ORNL-5137 $875767.0+03.0+1$ Bair+ CURV.

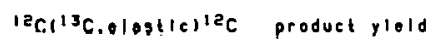

10174 KSU Expt A BAP $236164781.8+13.2+1$ Legg+ NDG. R-MATRIX ANAL.

$12 \mathrm{C}\left({ }^{13} \mathrm{C}, 20\right)^{170}$ cumulotive? o(E)

10175 ORL Reve C 77TENN 2277 8.8+0 2.5+1 Stoksteod. CURV. FRM NP/A 265. 153(76)

10176 CAL Expl J NP/A $265153761.0+13.3+1$ Dayros + CURV. MEASURED GAMMAS.

$12 C(13 \mathrm{C}, n+0)$ enNe cumulative? o(E)

10177 CAL EXPl J NP/A $265153765.0+01.6+1$ Dayros + CURV. MEASURED GAMMAS.

10178 ORL RevI C 77TENN $22771.0+12.5+1$ Stoksteod. CURV. FRM NP/A 265, 153(76)

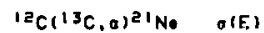

$10179 \mathrm{CAL}$ EXPl J NP/A $285153765.0+01.6+1$ Dayrag + CURV. MEASURED GAMMAS.

$12 C\left({ }^{3} \mathrm{C} . n+p\right)^{23} \mathrm{Na}$ cumulalive? o(E)

$10180 \mathrm{CAL}$ Expl J NP/A $265153765.0+0 \quad 1.6+1$ Dayras + CURV. MEASURED GAMMAS.

10181 ORL Reve C 77TENN $22778.8+02.5+1$ Stokstood. CURV. FRM NP/A 265, 153(76)

${ }^{12} \mathrm{C}\left({ }^{13} \mathrm{C}, \mathrm{p}\right)^{24} \mathrm{Na} \quad \mathrm{O}(\mathrm{E})$

$10182 \mathrm{CAL}$ Exot J NP/A $265153765.0+01.6+1$ Dayros + CURV. MEASURED GAMMAS.

${ }^{12} \mathrm{C}\left({ }^{13} \mathrm{C}, \mathrm{n}\right)^{24} \mathrm{Mg} \quad-(\mathrm{E})$

10183 CAL EXPt J NP/A $265153765.0+01.6+1$ Dayras + CURV. MEASURED GAMMAS.

10184 PEN Expt J PRL 37 279 $761.6+1 \quad$ Gellert $11.29+-0.261 M B$. GS+1.368-STATE

${ }^{1} \mathrm{C}\left({ }^{13} \mathrm{C}, \mathrm{x}\right) \mathrm{chorg}$ distribution o(E)

10185 ANL Expl J PR/C $2013050791.6+15.2+1$ Kovar+ TBL. N,O,F,NE, NA, MG FROM ANG.

${ }^{13} C\left({ }^{13} \mathrm{C}, \mathrm{total}\right)$ O(E)

10186 LUO Expl J NP/A $3332731806.1+01.4+1$ Chatterjee+ TEL. CURV. FROM G SINGLES.

${ }^{13} \mathrm{C}\left({ }^{13} \mathrm{C}\right.$, totol) $O(E) \times 1$ loctor

10187 LUO Expt J NP/A $3332731806.1+01.4+1$ ChatterJee + CURV. S-FACTOR.

Expl J NPIA 333 273 IBD $6.3+01.4+1$ Chatterjee+ CURV. RATIO TOTALIEN.

${ }^{13} \mathrm{C}\left({ }^{13} \mathrm{C}, \mathrm{X}\right) \mathrm{n} \quad \sigma(\mathrm{E})$

10188 ORL EXPt P ORNL-5306 59677 NDG BAIr+ NDG. PAPER IN PREPARATION Expt A BAP $226284778.0+04.0+1$ Del-Campo+ NOG. 200KEV STEPS. Expt P ORNL $-5137 \quad B 75767.0+03.0+1$ Bair+ CURV.

${ }^{13} \mathrm{C}\left(1{ }^{3} \mathrm{C}, 2 \mathrm{2n}\right)^{24} \mathrm{Mg} \quad \sigma(E)$

10189 LUO Expt J NP/A $3332731806.3+01.4+1$ Chotterjeet TEL. CURV. FROM G SINGLES.

${ }^{13} \mathrm{C}\left({ }^{13} \mathrm{C}, 2 \mathrm{Cn}\right)^{24} \mathrm{Mg} \quad \sigma(E) \times$ factor

10190 LUD EXPt J NP/A $3332731806.3+01.4+1$ Chatterjeet CURV. 5-FACTOR

Expt J NP/A $3332731806.3+01.4+1$ ChatterJee+ CURV. RATIO TOTAL/ZN.

${ }^{14} \mathrm{~N}\left({ }^{13} \mathrm{C}, x\right)$ compound-nucleus o(E)

10191 SAC EXPL P CEA-N-2070 $379782.9+15.4+1$ CONJeaud+ CURV. FUSION.

$1{ }^{4} N\left({ }^{3} \mathrm{C}, x\right)$ chorgo distribution olE)

10192 SAC EXPt P CEA-N-2070 $379784.4+15.4+1$ Conjeoud+ CURV. CFD THEORY.

${ }^{16} \mathrm{O}\left({ }^{13} \mathrm{C}, x\right) \cap \quad \sigma(E)$

10193 ORL EXPt P ORNL-5306 59677 NDG Bair+ NOG. PAPER IN PREPARATION Expt P ORNL-5137 B7 $5767.0+03.0+1$ Bair+ NOG.

$170(13 \mathrm{C}, x)$ compound-nucleus o(E)

10194 SAC Expt P CEA-N-2070 $379782.1+15.3+1$ COnjeaud+CURV.FUS.CFO GLAS-MOSEL. OTHERS

${ }^{17} \mathrm{O}\left({ }^{13} \mathrm{C}, x\right)$ cherge distribution o(E)

10195 SAC Expt P CEA-N-2070 $379783.8+15.3+1$ Conleaud+ CURV. CFD THEORY.

${ }^{10} O\left(^{13} \mathrm{C}, x\right) \cap \quad O(E)$

10196 ORL EXPt P ORNL-5306 59677 NDG

BaIr+ NDG. PAPER IN PREPARATION 


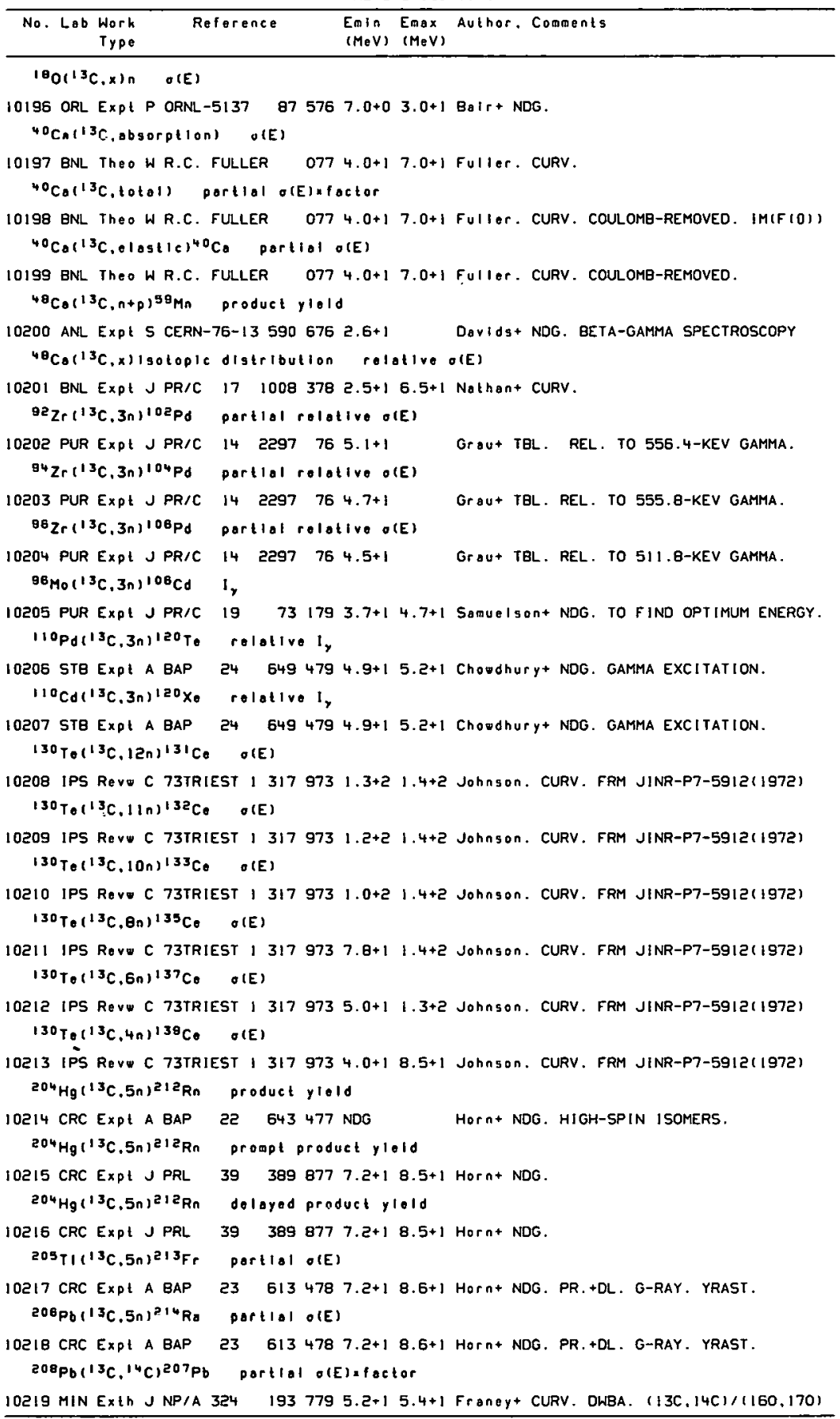




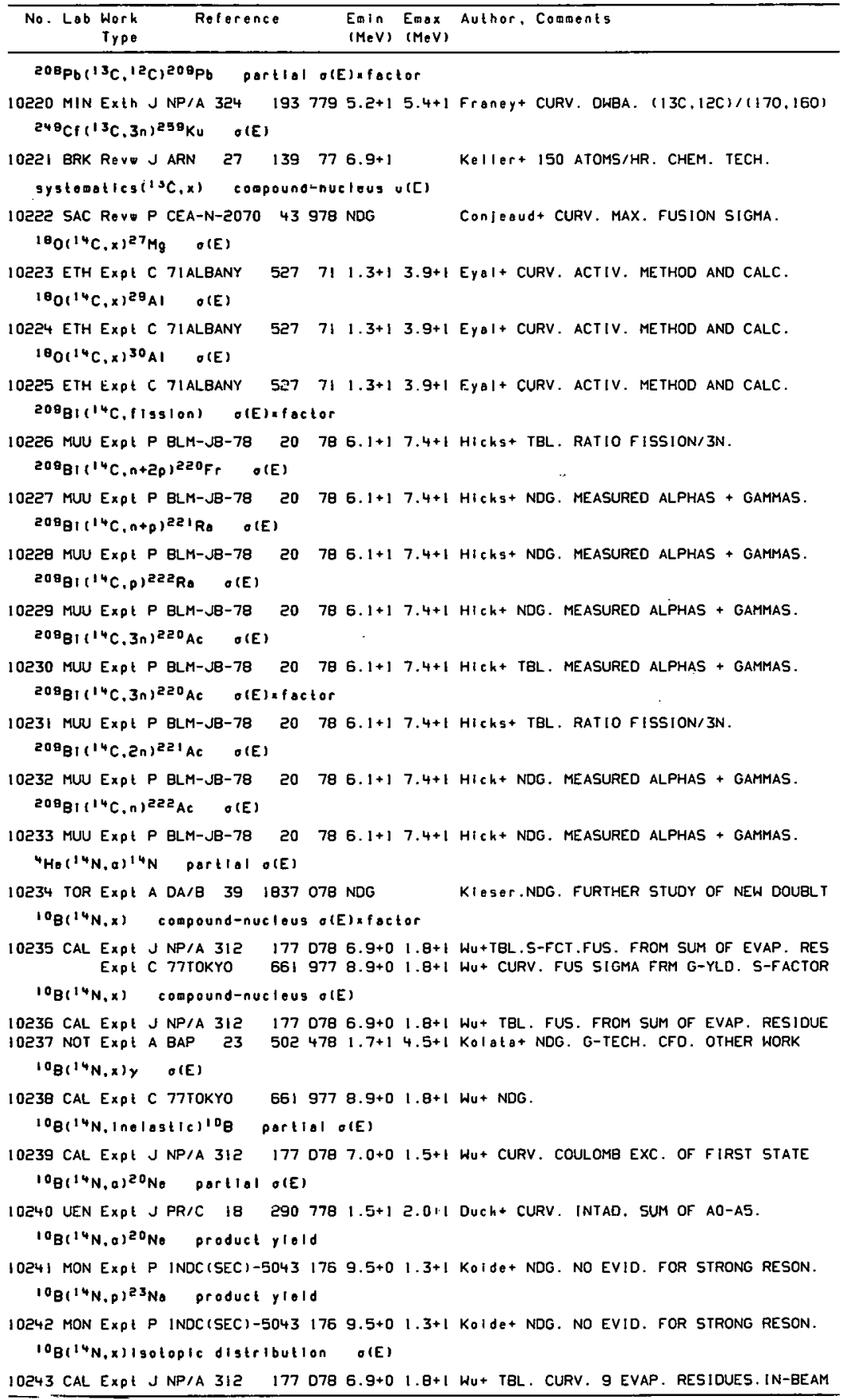


REFERENCES $(\cos t)$

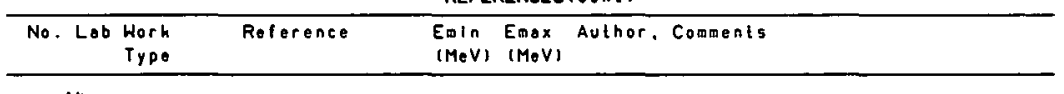

$$
C\left(1^{4} N, x\right) \text { O } O(E)
$$

10244 FSU Thoo A 74NASH $1526741.5+17.5+1 \mathrm{Holub}+$ NDG. STAT. EVAP. CODE.

$C\left({ }^{14} N, x\right)^{\prime} H \quad O(E)$

10245 FSU ThEO A 74NASH I $526741.6+17.5+1$ Holub+ NDG. STAT. EVAP. CODE.

$C\left({ }^{14} \mathrm{~N}, x\right)^{4} \mathrm{He} \quad O(E)$

10246 FSU ThOO A 74NASH I $526741.6+17.5+1$ Holub+ NDG. STAT. EVAP. CODE.

C(14N, $x)^{7}$ B. thlck torget yleldxfactor

10247 FSU Expl J PL/B 43375373 3.5+1 5.8+1 HolUb+ CURV. ACTIV. FCT=THICKNESS $C(14 N, x)^{2 e}$ Ne thick torget yloldxfoctor

10248 FSU Expl J PL/B $433753731.6+15.8+1$ Holub + CURV. ACTIV. FCT=THICKNESS $C\left({ }^{14} N, x\right)^{24} \mathrm{Na}$ thick torget yieldafoctor

10249 FSU Expt J PL/B $43 \quad 3753731.6+15.8+1$ HolUb+ CURV. ACTIV. FCT=THICKNESS

$12 \mathrm{C}\left(1^{4} \mathrm{~N}, \mathrm{X}\right)$ compound-nuclous o(E)

10250 BRK REV S BNL-51115 g05 779 NDG SCOtt. CURV. CONFERENCE SUMMARY.

10251 CAL Exp.t J NP/A $2795024776.1+01.7+1$ Solthoshit CURV. TBL. FRM SUM EVAP RES

10252 ANL Expt J PR/C $2013050793.3+15.2+1$ Kovor+ TBL. CURV. FUSION.

Expt C 77TOKYO 660977 NDG Kovar+ CURV. MAX. FUSION SIGMA.

10253 ORL Expt J PR/C $1921706793.4+1 \quad 1.8+2$ Del-Campo+ TBL. CURV. FUSION. INTAD.

10254 ORL ReY R IAEA-241/SM $793.4+11.8+2$ PIOS $11+$ CURV. FUSION. CFD EXPT. Revo R CONF-790507 $3793.4+1 \quad 1.8+2$ PIas $11+$ SAME AS IAEA-SM/241-DI

10255 MIT Revi C 77TENN $73774.0+12.5+2$ Negele. CURV. FRM PL/B 67, 134(1977)

10256 ORL Theo J PL/B $67 \quad 1343774.0+12.4+2$ Maruhn-Re 2 an it CURV. TOHF THEORY.

10257 ORL Exth J PRL $3615296764.3+1 \quad 1.8+2$ Stoksload+ CURV. FUS. FROM EVAP. RESID. Rev C 7TTENN $22774.3+1$ 1.8+2 Stokstead. CURV. PRL 36, 1529(76)

Expt P ORNL-5137 $335764.3+11.8+2$ Stokgtad+ CURV. FUSION DEO. FROM PROD.

Expt C 76ANL-2 $27954764.4+11.8+2$ Stohstad+ CURV. FUS = SUM OF EVAP. RES.

10258 BRK Expl $\checkmark$ PL/B $702890771.6+22.5+2$ Stokstod+ CURV. INTAD. EVAP. RESIDUES.

${ }^{12} C\left({ }^{4} N, x\right)$ direct-interaction $O(E)$

10259 ORL Expt J PR/C $1921706794.4+1 \quad 1.8+2$ Del-Campo+ TBL.

$1{ }^{2} \mathrm{C}\left({ }^{4} \mathrm{~N}, \mathrm{x}\right)$ compound-nucleus o(E)x tactor

10260 CAL Expt J NP/A $2795024776.1+01.7+1$ SUithoskit CURV. S-FCT. EVAP RES SUM Expt J PRL 37 B8 $765.0+01.6+1$ Slohstad+ CURV. NUCLEAR S-FACTOR.

${ }^{2} C(14 N, x)$ compound-nuclous overage $O(E)$

10261 ORL ExpL P ORNL-5!37 $335764.0+1 \quad 1.8+2$ stohstod+ (812+-27)MB FOR E GE 40 MEV.

$12 C(14 N, t \circ t a 1)$ ote

10262 ORL Expt J PR/C $1921706793.4+1 \quad 1.8+2$ Del-Campo+ TBL. OPT. MODEL.

${ }^{12} \mathrm{C}\left({ }^{14} \mathrm{~N}\right.$, totol) portiol o(E)

10263 TAM Exth J PR/C $16 \quad 22490776.7+1$ 10264 YAL REv C 74 NASH $23276747.8+1$

Slokstead+ CURV. INTAD. FUNCTION OF $J$

$$
12 C(14 N, x) Y \quad O(E)
$$

10265 GRE Expt P ISN-79-01 $39795.9+17.0+1 \mathrm{Cole}+\mathrm{TBL}$. FROM 0-DEG. MASS SPECTRA.

${ }^{12} \mathrm{C}\left({ }^{14} \mathrm{~N}, \mathrm{X}\right)^{\prime} \mathrm{H}$ portlal O(E)

10266 TAM Exth J PR/C $16 \quad 2249 \quad 077 \quad 6.7+1$ Stohstead+ CURV. INTAD. FUNCTION OF $s$

$12 \mathrm{C}\left({ }^{14} \mathrm{~N}, \times\right)^{4} \mathrm{H}_{0}$ partial $\sigma(\mathrm{E})$

10267 TAM Exth J PR/C I6 $22490776.7+1$

IEC(14N, $x)^{4}$ He coopound-nuelous o(E)

10268 TAM Exth J PR/C $16 \quad 2249077$ B.7+1

${ }^{12} \mathrm{C}\left(1{ }^{4} \mathrm{~N}, n+x\right)^{4} \mathrm{He}$ compound-nuclous o(E)

10269 TAM Exth J PR/C IG $22490778.7+1$

$12 \mathrm{C}\left({ }^{14} \mathrm{~N}, p+x\right)^{4} \mathrm{H}_{6}$ coopound-nucleus o(E)

10270 TAM Exth J PR/C $16 \quad 2249077 \quad 8.7+1$

Stokstead+TBL. STAT. MOD. TWO-SIEP INTAD

${ }^{12} \mathrm{C}\left({ }^{14} \mathrm{~N}, x\right)^{6} \mathrm{LI}$ product ylold

10271 JCL Exp\& P A-JCL-77 $390779.1+1$

Motobayash + NDG. ANAL. IN PROGRESS 


\begin{tabular}{|c|c|c|c|c|c|}
\hline No. Lo & $\begin{array}{l}\text { Work } \\
\text { Typo }\end{array}$ & Reforence & $\begin{array}{l}\text { Enin } \\
(M \circ V)\end{array}$ & $\begin{array}{l}\text { Emax } \\
(M \circ V)\end{array}$ & Author. Comments \\
\hline
\end{tabular}

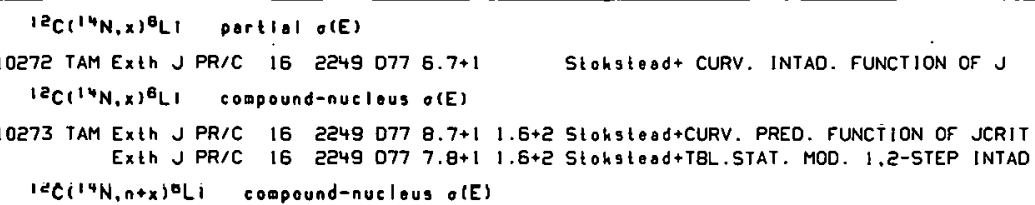

10274 TAM Exth J PR/C $16 \quad 22490778.7+1$

${ }^{12} C\left({ }^{4} N, p+x\right)^{8} L I$ compound-nuclous o(E)

10275 TAM Exih J PR/C IG $22490778.7+1$

${ }^{12} \mathrm{C}\left({ }^{14} \mathrm{~N}, a+x\right)^{6} \mathrm{LI}$. compound-nuclous o(E)

Stokstead+TBL. STAT. MOD. TWO-STEP INTAD

Stoksleod+TBL.STAT. MOD. TWO-STEP INTAD

10276 TAM Exth J PR/C 16 2249 $0777.8+1 \quad 1.6+2$ Stokstead+TBL.STAT. MOD. TWO-STEP INTAO

$12 C\left({ }^{4} N, x\right)^{7} L$ I compound-nuclous $O(E)$

10277 TAM Exth J PR/C 162249 D77 8.7+1 1.6+2 Stokstead+CURV. PREO. FUNCTION OF JCRIT Exth J PR/C 162249077 B.7+1 Stokstebd+TBL.STAT. MOD. 1.2 GTCR INTAD

$12 \mathrm{C}\left(1{ }^{14} \mathrm{~N}, n+x\right)^{7} \mathrm{LI}$ compound-nuclous o(E)

10278 TAM Exth J PR/C $16 \quad 22490778.7+1$

$12 c(14 N, p+x)^{7} L I$ compound-nuclous o(E)

10279 TAM Exth J PR/C $16 \quad 2249077$ 日.7+1

${ }^{2} \mathrm{C}\left({ }^{4} \mathrm{~N}, a+x\right)^{7} \mathrm{~L} 1$ compound-nuclous o(E)

10280 TAM Exth J PR/C I6 $22490778.7+1$

${ }^{12} \mathrm{C}\left({ }^{14} \mathrm{~N}, x\right)^{7} \mathrm{Be}$ portlal o(E)

10281 TAM Exth J PR/C $16 \quad 2249077 \quad 6.7+1$

${ }^{12} \mathrm{C}\left({ }^{14} \mathrm{~N}, x\right)^{7} \mathrm{Be}$ compound-nuclous $\sigma(E)$

10282 TAM Exth J PR/C 162249077 B.7+1 $1.6+2$ Stokstead+CURV. PRED. FUNCTION OF JCRIT Exth J PR/C 162249077 B.7+1 Stokstead+TBL.STAT. MOD. 1.2-STEP INTAD

$12\left({ }^{14} N, n+x\right)^{7} B$ e compound-nueleus o(E)

10283 TAM Exih J PR/C $16 \quad 22490778.7+1$

Stohstead+TBL.STAT. MOO. TWO-STEP INTAO

$12 \mathrm{C}\left({ }^{4} \mathrm{~N}, p+x\right)^{7} \mathrm{Be}$ compound-nucleus o(E)

10284 TAM Exth J PR/C $16 \quad 22490778.7+1$

$I^{2} C\left(1{ }^{4} \mathrm{~N}, a+x\right)^{7} \mathrm{Be}$ compound-nucleus o(E)

10285 TAM Exth J PR/C $16 \quad 2249077$ B.7+1

Stokstead+TEL.STAT. MOD. TWO-STEP INTAD

Stokstead+TBL, STAT. MOD. TWO-STEP INTAD

${ }^{12} \mathrm{C}\left({ }^{4} \mathrm{~N}, x\right)^{9} \mathrm{Be}$ compound-nuelous $\sigma(E)$

10286 TAM Exth J PR/C $1622490778.7+1 \quad 1.6+2$ Stokstead+CURV. PREO. FUNCTION OF JCRIT Exih J PR/C $16 \quad 22490778.7+1$

Stokstead+TBL. STAT. MOD. I.2-STEP INTAD

$12 \mathrm{C}\left({ }^{14} \mathrm{~N}, n+x\right)^{8} \mathrm{Be}$ compound-nuelous o(E)

10287 TAM Exth J PR/C $16 \quad 22490778.7+1$

Stokstegd+TBL. STAT. MOD. TWO-STEP INTAD

$I^{2} C\left({ }^{4} N, p+x\right)^{9}$ o compound-nucteus o(E)

10288 TAM Exth J PR/C $16 \quad 2249077$ 8.7+1

$12 C\left({ }^{14} N, a+x\right)^{8} B$ compound-nucleus o(E)

10289 TAM Exth J PR/C $16 \quad 22490778.7+1$

${ }^{2} \mathrm{C}\left({ }^{14} \mathrm{~N},{ }^{9} \mathrm{Be}\right) 17^{7} \mathrm{~F} \quad \sigma(E)$

10290 YAL ReVU C 74NASH $23276749.0+1$

$12 \mathrm{C}\left({ }^{4} N, x\right)^{10} \mathrm{~F}$ portlal o(E)

10291 YAL ReVv C 74NASH $23276742.1+13.7+1$ Stohstad. CURV. $0.937 G$.

$12 \mathrm{C}\left({ }^{14} \mathrm{~N},{ }^{7} \mathrm{Be}\right) 1 \mathrm{\theta}_{\mathrm{F}}$ partiol O(E)

10292 YAL ReVy C 74NASH $23276747.8+1 \quad$ Stohstad. CURV. FUNC. OF TOT. ANG. MOM.

$\left.12 \mathrm{Cl} 1{ }^{14}{ }^{7}{ }^{7} \mathrm{~B}_{0}\right)^{10} \mathrm{~F} \quad O(\mathrm{E})$

10293 TAM Exth J PR/C $1622490778.7+1 \quad 1.6+2$ Stokstead+ TBL. CURV. INTAD.

10294 YAL REV C 74NASH $23276749.0+1$ Stohstead. CURV. 


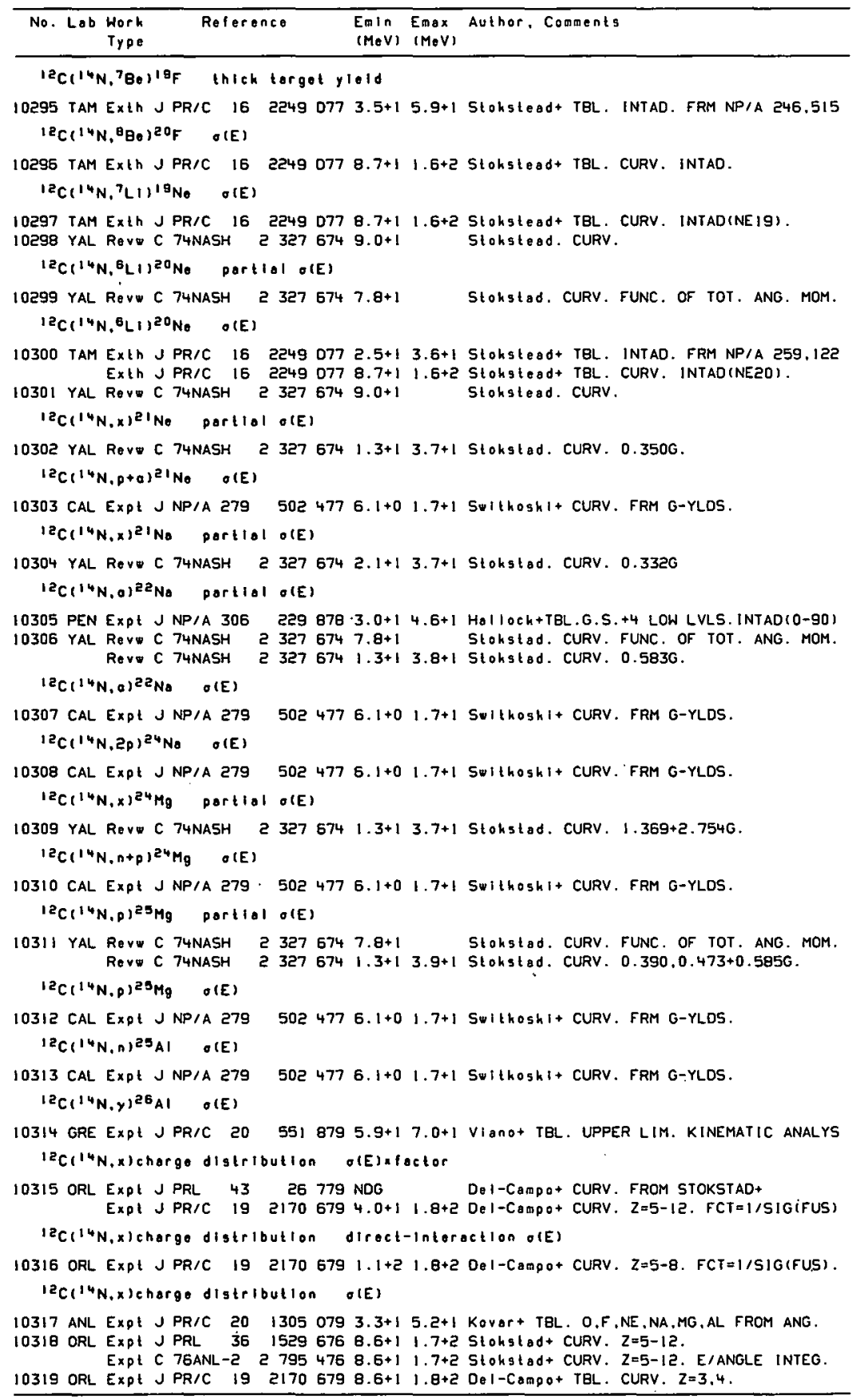


REFERENCES ( c on t )

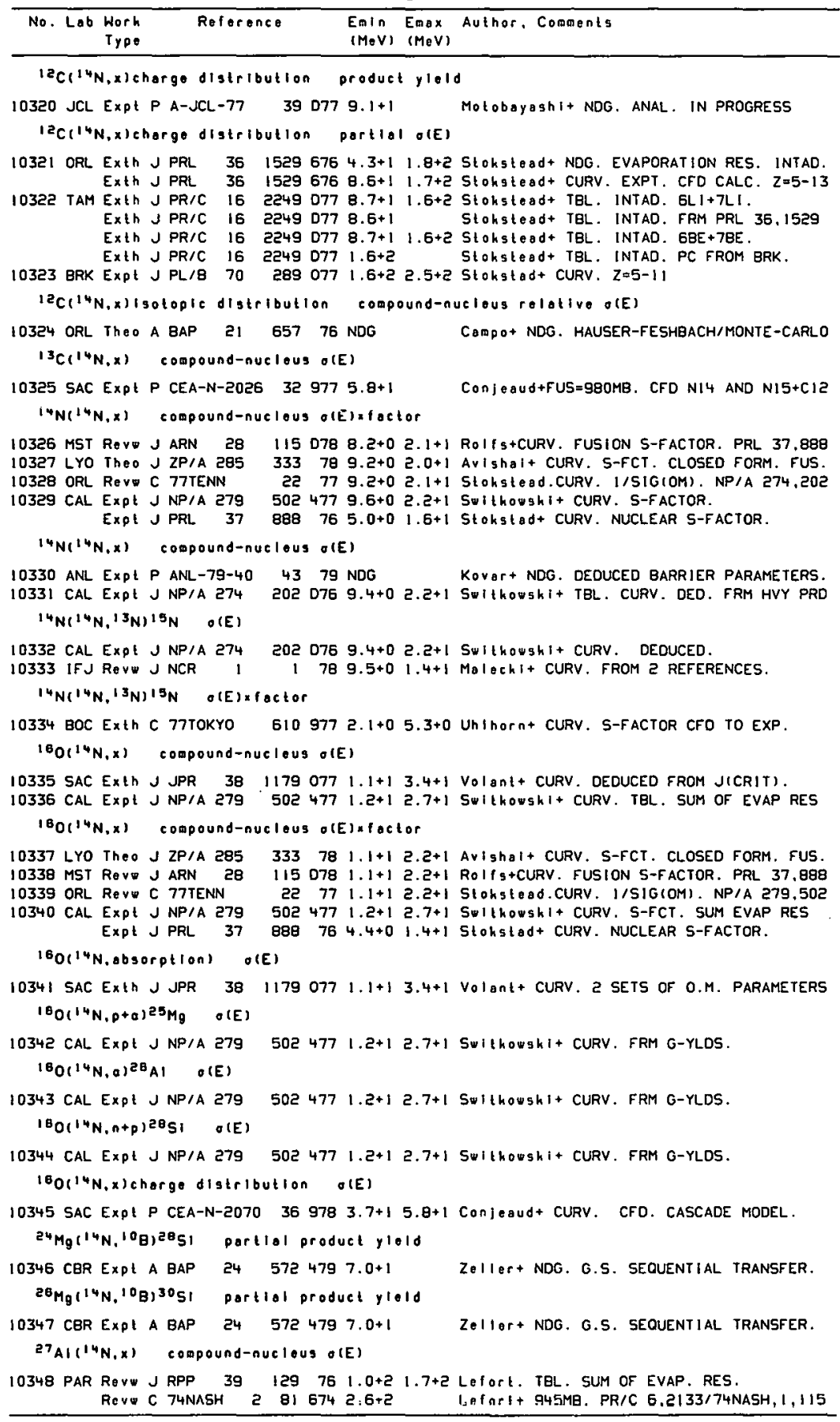




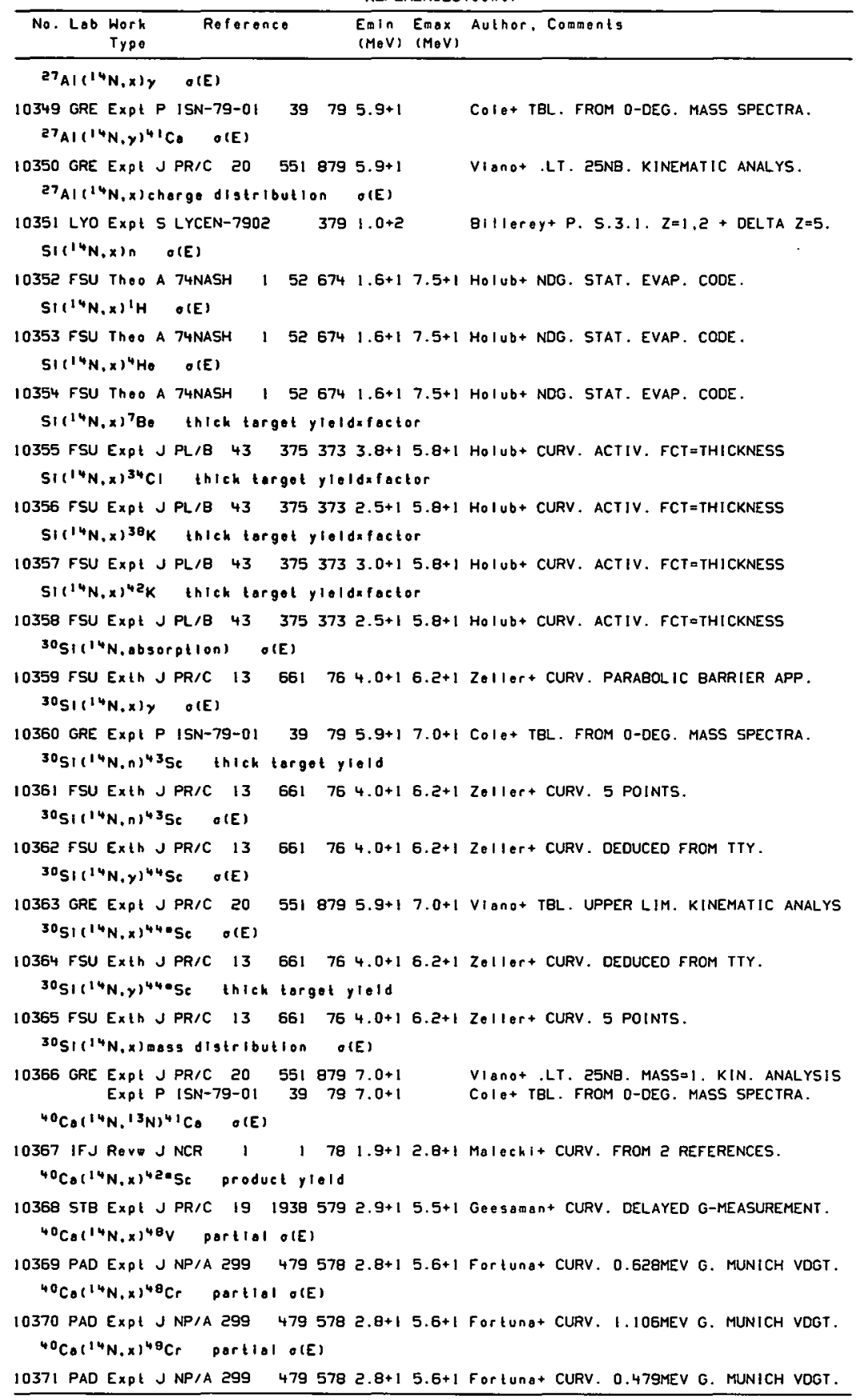


REFERENCES (cont)

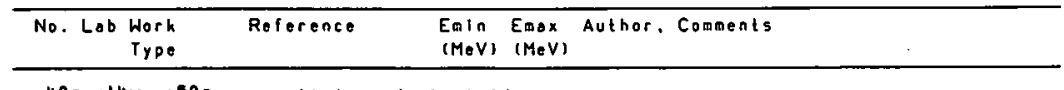

${ }^{40} \mathrm{Ca}(14 \mathrm{~N}, x)^{50} \mathrm{Cr}$ partial product ylold

$103725 T B$ Expl J PR/C $1919385792.9+13.8+1$ Geesaman+ CURV. ON-LINE. EG=783KEV.

${ }^{40} \mathrm{Co}\left({ }^{14} \mathrm{~N}, \times\right)^{50} \mathrm{Cr}$ partlot o(E)

10373 PAD Expt \ NP/A $2994795782.8+15.6+1$ Fortuna+ CURV. 0.783MEV G. MUNICH VDGT.

${ }^{40} \mathrm{Co}\left({ }^{14} \mathrm{~N}, x\right)^{51} \mathrm{Cr}$ portlial O(E)

10374 PAD Expt J NPIA $2994795782.8+15.6+1$ Fortunot CURV. $0.775 M E V$ G. MUNICH VDGT.

${ }^{40} \mathrm{Ca}\left({ }^{4} \mathrm{~N}, 3_{0}\right)^{51} \mathrm{Cr}$ partial product yield

10375 5TB Expt J PR/C $1919385792.9+13.8+1$ Geesenont CURV. ON-LINE. EG=775KEV.

${ }^{40} \mathrm{Co}_{0}\left(14 \mathrm{~N}^{\prime}, \mathrm{x}\right) 500 \mathrm{Mn}$ product yield

10376 STB Expl J PR/C $1919385792.9+15.5+1$ Geesonant CURV. DELAYED G-MEASUREMENT.

${ }^{40} \mathrm{Ca}\left({ }^{4} \mathrm{~N}, x\right) 31 \mathrm{Mn}$ partiol product yiold

$103775 T B$ Expt I PR/C. $1919385792.9+13.8+1$ Geesamant CURV. ON-LINE. 237KEV.

${ }^{40} \mathrm{Ca}\left({ }^{14} \mathrm{~N}, x\right)$ ( $1 \mathrm{Mn}$ partiol o(E)

10378 PAD Expl J NP/A $2994795782.8+15.6+1$ Fortuna+ CURV. 0.869MEV G. MUNICH VDGT.

${ }^{4} \mathrm{Ca}\left({ }^{14} \mathrm{~N}, n+2 p\right) 51 \mathrm{Mn}$ rolative I,

10379 PEN ExpI J NP/A $277 \quad 1372773.6+1 \quad$ Noe+ TBL. 12 G.S. FRM LEG. POLY. FIT

${ }^{40} \mathrm{Ca}\left({ }^{14} N, n+2_{D}\right) 51 \mathrm{Mn}$ partiol $\sigma(E)$

10380 PAD Expl J NP/A $2994795782.8+15.6+1$ Fortunat CURV. 15 GS. MUNICH VDGT.

${ }^{4} \mathrm{Ca}\left({ }^{4} \mathrm{~N}, x\right)^{52} \mathrm{Mn}$ portlel product $\mathrm{y} 1 \mathrm{l} \mathrm{Id}$

10381 STB Expt J PR/C $1919365792.9+13.8+1$ Geesomant CURV. ON-LiNE. EG=870KEV.

${ }^{40} \mathrm{Ca}\left({ }^{4} \mathrm{~N}, x\right)^{52} \mathrm{Mn}$ portlol $\sigma(E)$

10382 PAD Expt J NP/A $2994795782.8+14.2+1$ Fortuna+CURV.0.849+1.106MEV G. MUN VDGT

$\left.{ }^{40} \mathrm{Ca}\left({ }^{4} \mathrm{~N}, x\right) 5\right)^{5} \mathrm{~g}$ partlal product $y 101 d$

10383 STB Expt J PR/C $1919385792.9+13.8+1$ Goesonant CURV. ON-LINE. EG=849KEV.

${ }^{40} \mathrm{Ca}(14 \mathrm{~N}, \times) 52 \mathrm{Fo}$ part 181 O(E)

10384 PAD Expt J NP/A $2994795782.8+14.2+1$ Fortuna+ CURV. 0.732MEV G. MUNICH VDGT.

${ }^{40} \mathrm{Ca}\left({ }^{4} \mathrm{~N}, \times\right) 520 \mathrm{~F}^{\circ}$ product yleld

$103855 T$ Expt J PR/C $1919385792.9+15.5+1$ Ge samant CURV. OELAYED G-MEASUREMENT.

${ }^{50} \mathrm{Cr}\left({ }^{14} \mathrm{~N}, x\right)^{4} \mathrm{He}$ diroct-interaction $\sigma(E)$

10386 GRE Exth P ISN-77-0I $58767.1+11.0+2$ Billerey+ TBL.

Exth P LYCEN-7701 I5 76 $7.1+1$ 1.0+2 Billerey+ TBL.

$50^{\circ} \mathrm{Cr}\left({ }^{14} \mathrm{~N}, \times\right)^{8 \mathrm{LI}}$ O(E) a foctor

10387 LYO Theo J ZP/A, $285389787.1+1 \quad 1.0+2$ BIIIerey+ TBL. RATIO LIG/LI7.

${ }^{50} \mathrm{Cr}\left({ }^{14} \mathrm{~N}, x\right)^{7} \mathrm{LI} \quad \sigma(E) \times$ factor

10388 LYO Thoo J ZPIA 285 . $389787.1+1$ 1.0+2 Billerey+ TBL. RATIO LIG/LI7.

${ }^{50} \mathrm{Cr}(14 \mathrm{~N}, x)^{10} \mathrm{~B} \quad \sigma(\mathrm{E}) \times$ foctor

10389 LYO Theo J ZP/A $285389787.1+11.0+2$ Billereyt TBL. RATIO BIO/BII.

$50 \mathrm{Cr}\left(1{ }^{4} \mathrm{~N}, \mathrm{X}\right) 11 \mathrm{~B}$ o(E) $\times$ factor

10390 LYO Theo J ZP/A $285389787.1+11.0+2$ B1/lereyt TBL. RATIO BIO/BI1.

${ }^{\circ} \mathrm{Cr}\left(1{ }^{4} \mathrm{~N}, \mathrm{X}\right) \mathrm{II}^{2} \mathrm{C} \quad \mathrm{O}(\mathrm{E}) \times \operatorname{tactor}$

10391 LYO Theo J ZP/A $285389787.1+11.0+2$ Eillerey+ TBL. RATIO CIE/CI3.

${ }^{50} \mathrm{Cr}\left({ }^{14} \mathrm{~N}, \mathrm{X}\right)^{13} \mathrm{C}$ o(E) $\times$ factor

10392 LYO Theo J ZP/A $285389797.1+1 \quad 1.0+2$ BIIlerey+ TBL. RATIO CIE/CI3.

${ }^{50} \mathrm{Cr}(14 \mathrm{~N}, x)$ chargo distribution o(E)

10393 LYO Theo J ZP/A $285389787.1+11.0+2$ BIIlerey+ CURV. $Z=2-12$. CLASS. -5TAT. Theo R LYCEN-7780 N77 $7.1+1 \quad 1.0+2$ BIIlerey+ CURV. $Z=2-12$. CFD EXP.

${ }^{5} \operatorname{Cr}(14 \mathrm{~N}, \mathrm{x})$ Compound-nucleus o(E)

10394 PAR R⿴囗十 J RPP $39129761,2+2$ 2. $1+2$ Lefort. TBL. SUM OF EVAP. RES. Rovw C 74NASH $2816742.6+2$ Lefort $1224 \mathrm{MB}$. $74 \mathrm{NASH} 1.115(74)$. 


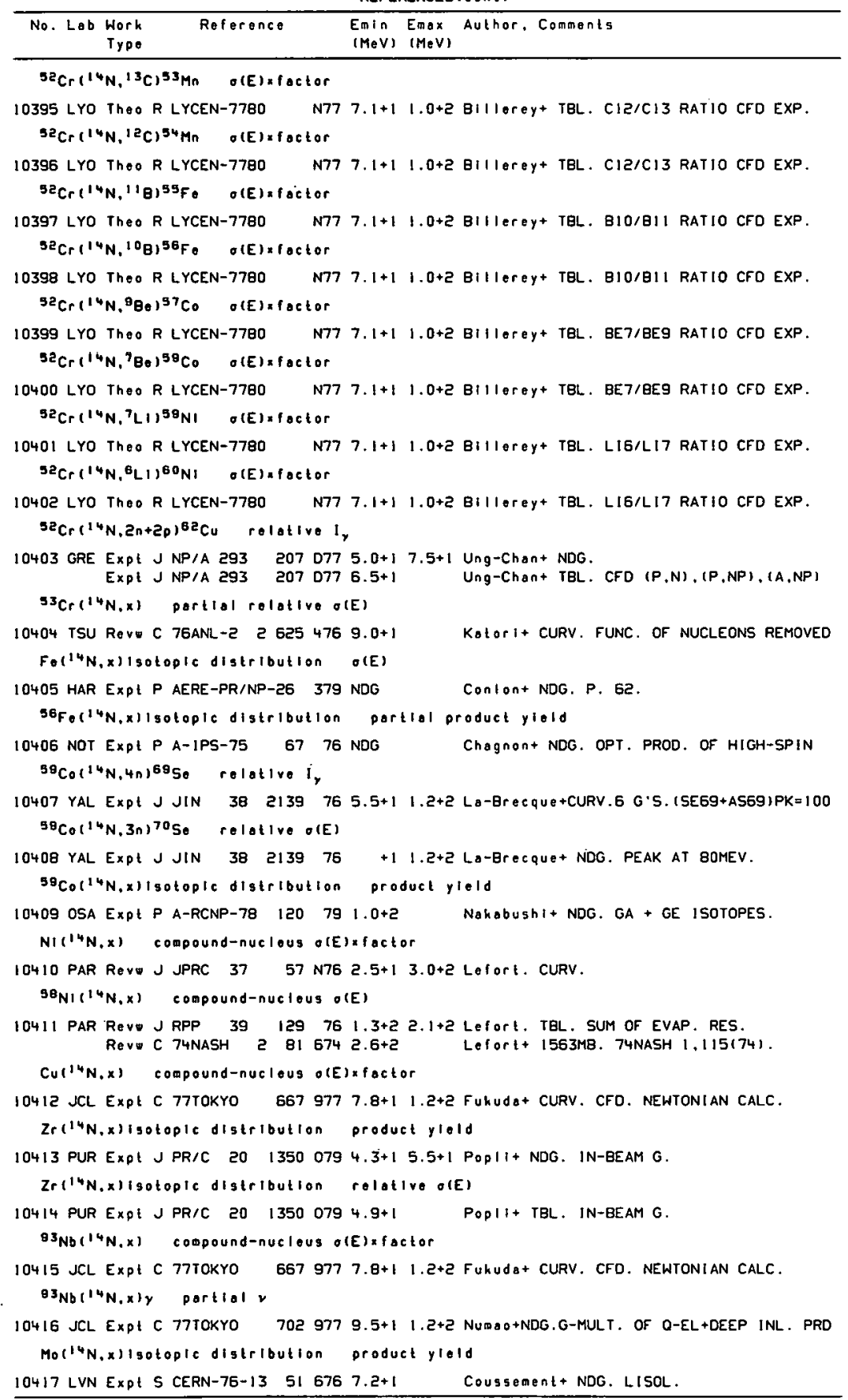




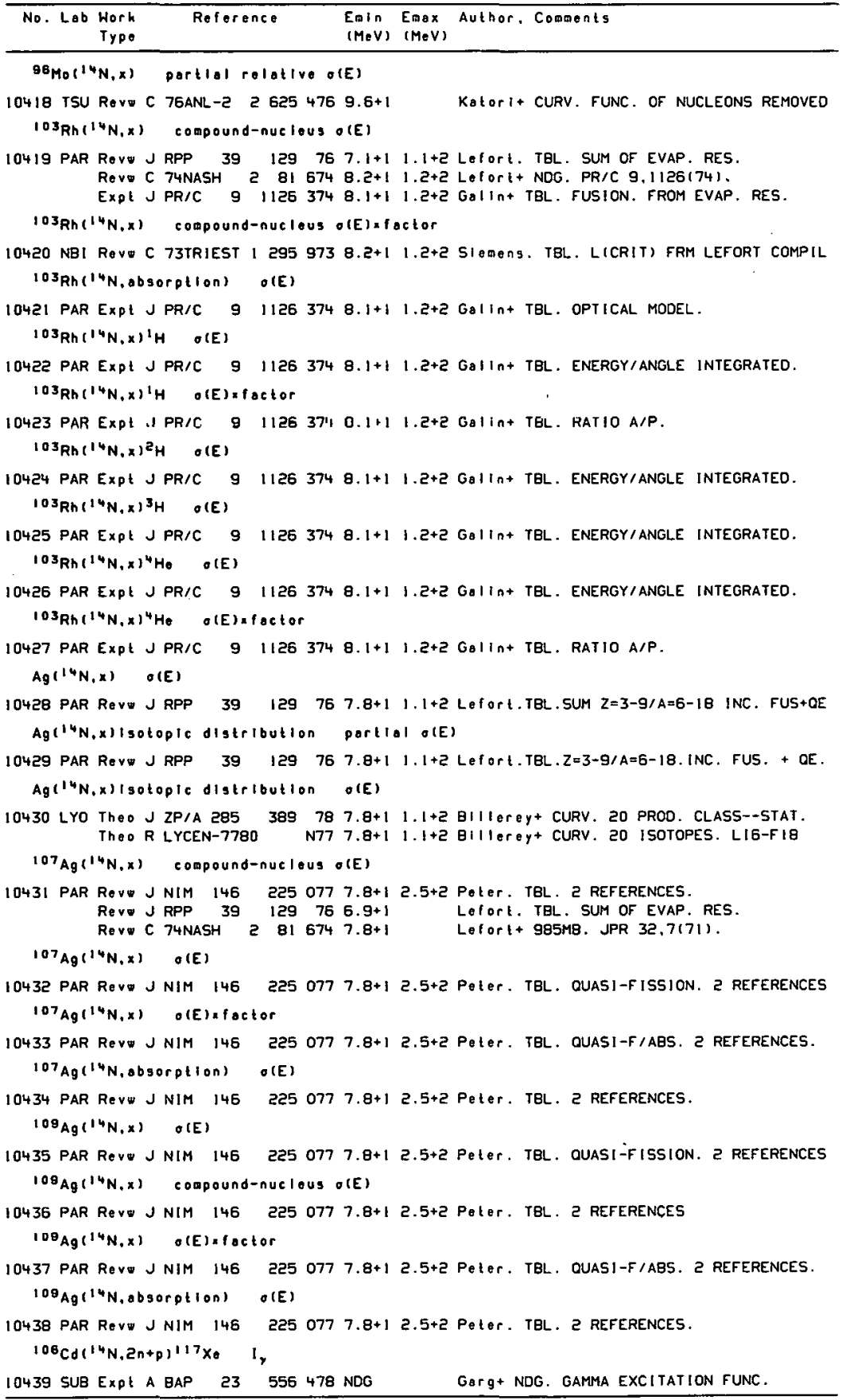




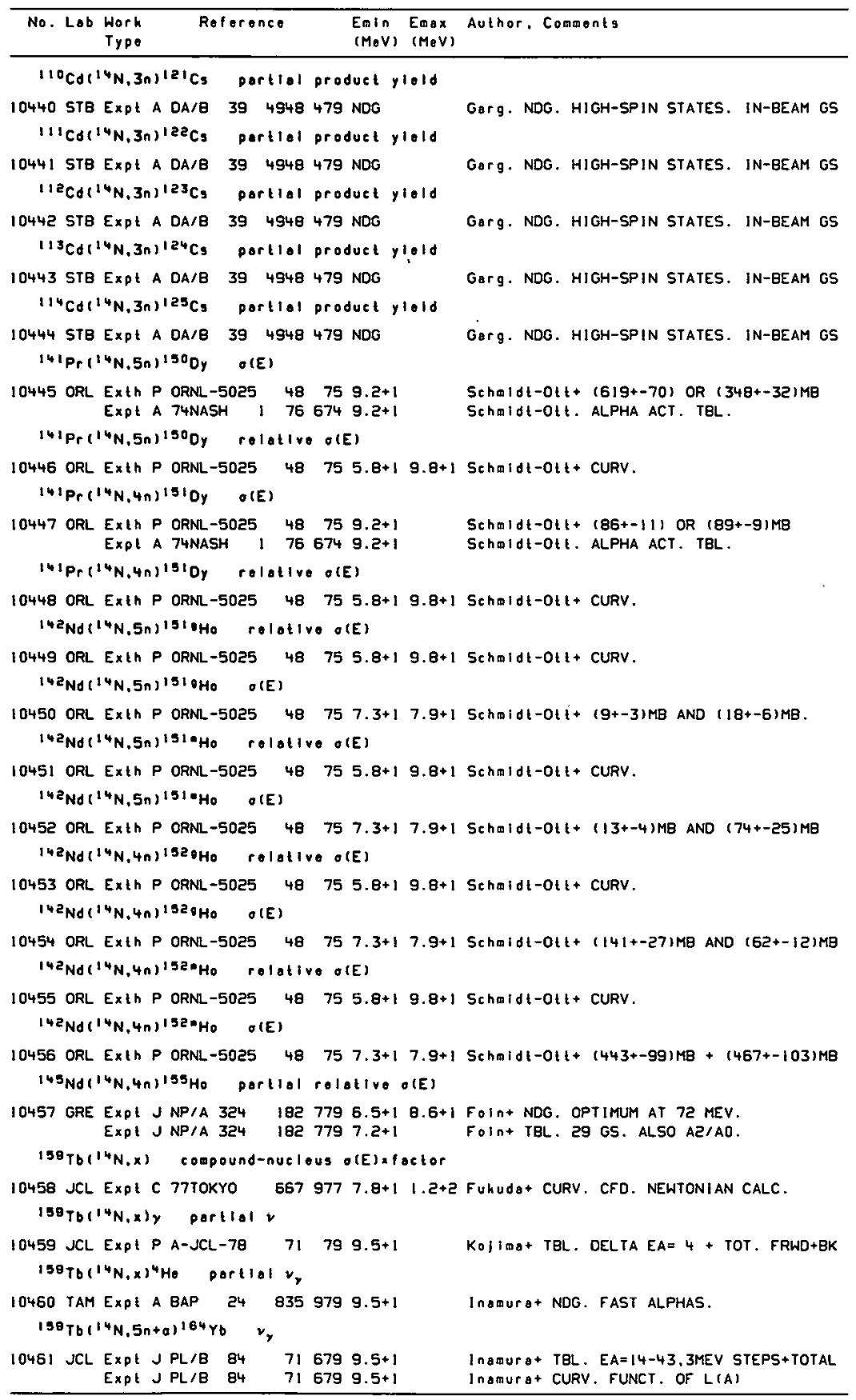




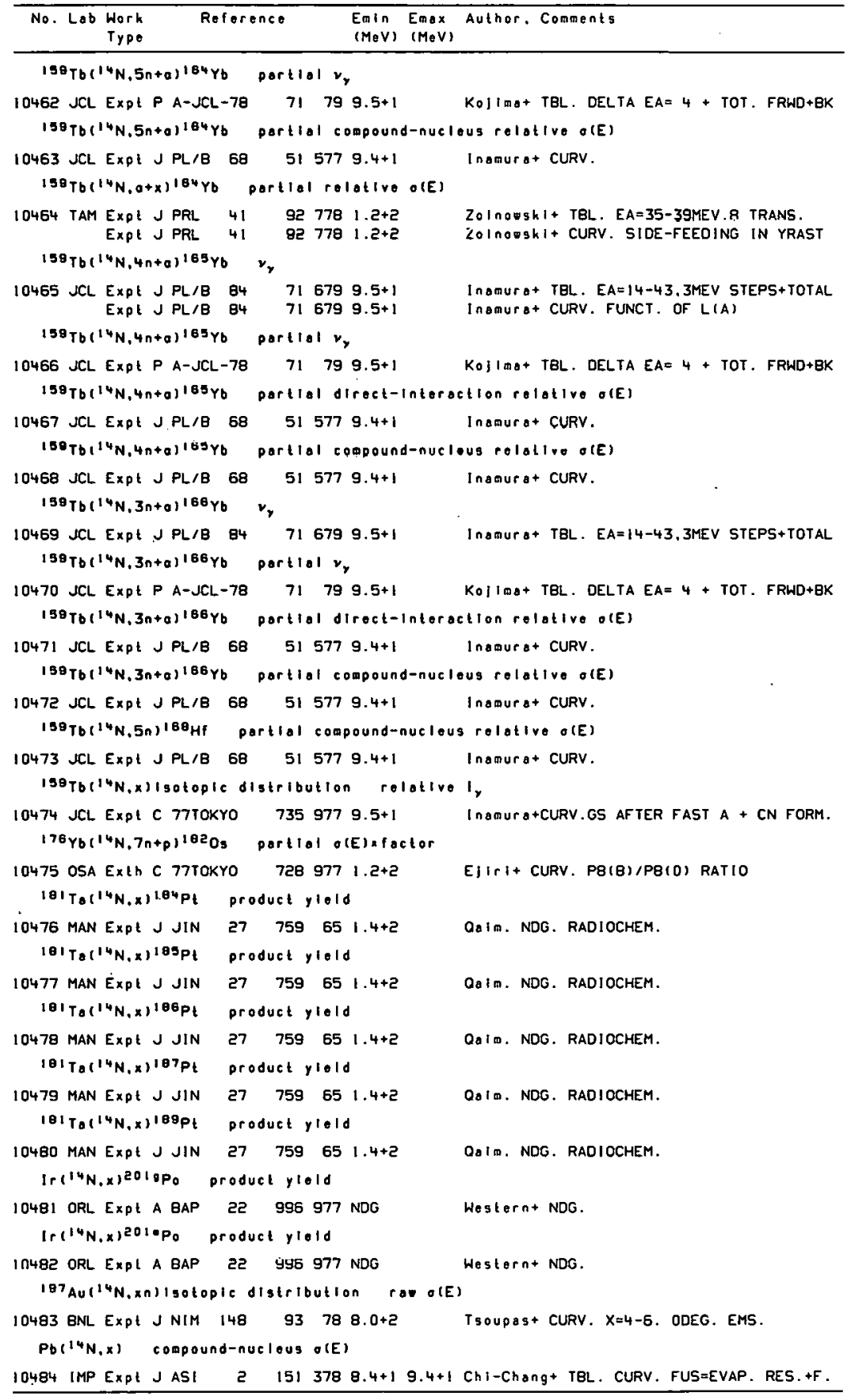




\begin{tabular}{|c|c|c|c|c|c|c|}
\hline No. Lab & $\begin{array}{l}\text { Work } \\
\text { Type }\end{array}$ & Reference & $\begin{array}{l}E m \mid n \\
(M \in V)\end{array}$ & $\begin{array}{l}\text { Emax } \\
(M e V)\end{array}$ & Author. & Comments \\
\hline
\end{tabular}

$P b(14 N, x)$ partial o(E)

10485 IMP EXPl J ASI 2151378 8.4+1 $9.4+1 \mathrm{ChI}$-Chang+ TBL. CURV. SUM OF EVAP. RES

Pbi $\left.{ }^{14} N, P I S S I O N\right)$ O(E)

10486 IMP Expt J ASI 2 15I 378 8.4+1 9.1+1 Chi-Chang+ TBL. CURV. CFD OTHER.

$207_{P b}\left({ }^{14} N, 4 n\right)^{217} A C \quad O(E)$

$10487 \mathrm{JCL}$ Expt J NP/A $217253737.0+19.6+1$ Nomurot NDG.

$207 P b\left({ }^{14} N, x\right) i s o t o p l c$ distribution $\sigma(E)$

10488 YAL EXPt C 71ALgANY 49771 NDG Gough+ NDG.

$200 \mathrm{~Pb}(14 \mathrm{~N}, \times n)$ isotopic distribution $O(E)$

$10489 \mathrm{JCL}$ Expt J NP/A $217253737.0+19.6+1$ Nomura+ NDG.

${ }^{209} B(14 N, x)$ partlol compound-nuclous o(E)

10490 PAR Exth T D. GAROES D76 $9.9+1$ Gardes. CURV. FUNC. OF L-TRANS.

$20{ }^{\circ}\left(1,\left({ }^{4} N, x\right)\right.$ portlal o(E)

10491 JAP Expl J PRL $40 \quad 694378$ B.5+1 $9.5+1$ Nomura+TBL. SUM Z=3-8.COUNT. TEL. (20-170 10492 PAR Exth T D. GARDES D76 $9.9+1$ Gardes.CURV. OUASI-ELAS.FUNC. OF L-TRANS

$20{ }^{2} B(14 \mathrm{~N}, x)$ compound-nucloug o(E)

$10493 \mathrm{JCL}$ EXpl C 77TOKYO 736977 8.5+1 9.5+1 Nowura+ HEAVY RESIDUES APP. 5MB.

$209 \operatorname{Bi}(14 \mathrm{~N}, \mathrm{X}) \quad \sigma(E)$

$10494 \mathrm{JCL}$ Expt J PRL 40694378 8.5+1 Nomuro+ TBL. Z=3 TO 8. INTAD.

Expt $J$ NPIA 217 C53 73 NDG EXPERIMENTAL DETAILS.

$20{ }^{2} \mathrm{BI}\left({ }^{4} \mathrm{~N}, \mathrm{ftgsion}\right)$ o(E) flgsion frogaents

10495 JCL Expt P A-JCL-77 $630778.5+19.5+1$ Utsunomiya+ TBL. FROM DA/DE.

$20{ }^{\circ} B\left(1{ }^{4} \mathrm{~N}, f i s \operatorname{sion}\right) \quad O(E)$

$10496 \mathrm{JCL}$ Expl J PRL $40 \quad 694378$ 8.5+1 9.5+1 Nomura+ TBL. INTAD(10-170). SI(L1).

Expt C 77TOKYO 736977 B.5+1 $9.5+1$ Nomura+ $1820+-501 \mathrm{MB}$.

Expl J NP/A 21725373 NDG . EXPERIMENTAL DETAILS.

$\operatorname{cog}_{B}\left(14{ }^{\prime} N, x\right)^{4} H_{e} \quad O(E)$

$10497 \mathrm{JCL}$ Expt J PRL 40.694378 8.5+1 9.5+1 Nomura+ IBL. COUNTER TEL. (20-170 DEG).

Expt P A-JCL-77 $630778.5+19.5+1$ Ut sunomiyo+ TBL. FROM DAIDE.

EXPL J NP/A 21725373 NDG . EXPERIMENTAL DETAILS.

$200^{0} 1\left(^{14} \mathrm{~N},{ }^{16} \mathrm{~F}\right)^{207} \mathrm{TI}$ partial o(E)

10498 PAR Theo J ZP/A $290157796.0+17.0+1$ Bimbol + CURV. SUBCOUL. TRANSFER CFO EXP $200^{6}(14 N, 150,208 \mathrm{~Pb}$ portiol o(E)

10499 PAR Thoo J ZP/A $290157795.6+17.0+1$ Bimbot+ CURV. SUBCOUL. TRANSFER CFD EXP $20{ }^{\circ} \mathrm{Bi}\left({ }^{14} \mathrm{~N}, \times\right) 21{ }^{\circ} \mathrm{BI} \quad$ O(E)

10500 PAR Expt J PR/C $1312989787.7+19.7+1$ Gerdes+ JBL. 2PI ION. CHMBRS FOR A + G Expt $P$ IPNO-RC-77 $10777.7+19.7+1$ Gardes+ TBL. RESIDUAL ACTIVITY. Exth T D. GARDES D76 $7.7+19.8+1$ Gardes. TBL. CURV. ACTIVATION.

$20{ }^{B} \mathrm{~B}(14 \mathrm{~N}, x) 210 \mathrm{PO}_{0}$ cumulative O(E)

10501 PAR Expt J PR/C 181298978 6.1PI 1.6+2 Gardes+ TBL. 2PI ION. CHMBRS FOR A + G Expt P IPNO-RC-77 $10777.5+19.7+1$ Gardes+ CURV. RESIDUAL ACTIVITY Exth T D. GARDES 076 6. $2+11.6+2$ Gardes. TBL. CURV. ACIIVATION.

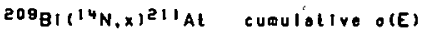

10502 PAR EXDt J PR/C 18 I298 $9786.1+1 \quad 1.6+2$ Gardes+ TBL. CPI ION. CHMBRS FOR A + $G$ Expt P IPNO-RC-77 $10776.1+1 \quad 1.6+2$ Gordes+ TBL. CURV. RESIDUAL ACTIVITY. Exth T D. GaROES $0766.2+11.6+2$ Gardes. TBL. CuRV. ACTIVATION.

$200^{0}\left({ }^{14} N, x\right)^{2} 1 I^{R} \quad O(E)$

10503 PAR Expt P IPND-RC-77 1077 6.9+1 1.6+2 Gardes+ TBL. RESIDUAL ACTIVITY. Exth T D. GARDES D76 $7.0+1 \quad 1.6+2$ GBrdes. TBL. CURV. ACTIVATION.

$20001(14 N, x) 212^{2}{ }^{\prime \prime} \quad O(E)$

10504 PAR Expt J PR/C 18' $12989786.9+1$ 1.6+2 Gardes+ TBL. 2PI ION. CHMBRS FOR A + G 
REFERENCES ( con I )

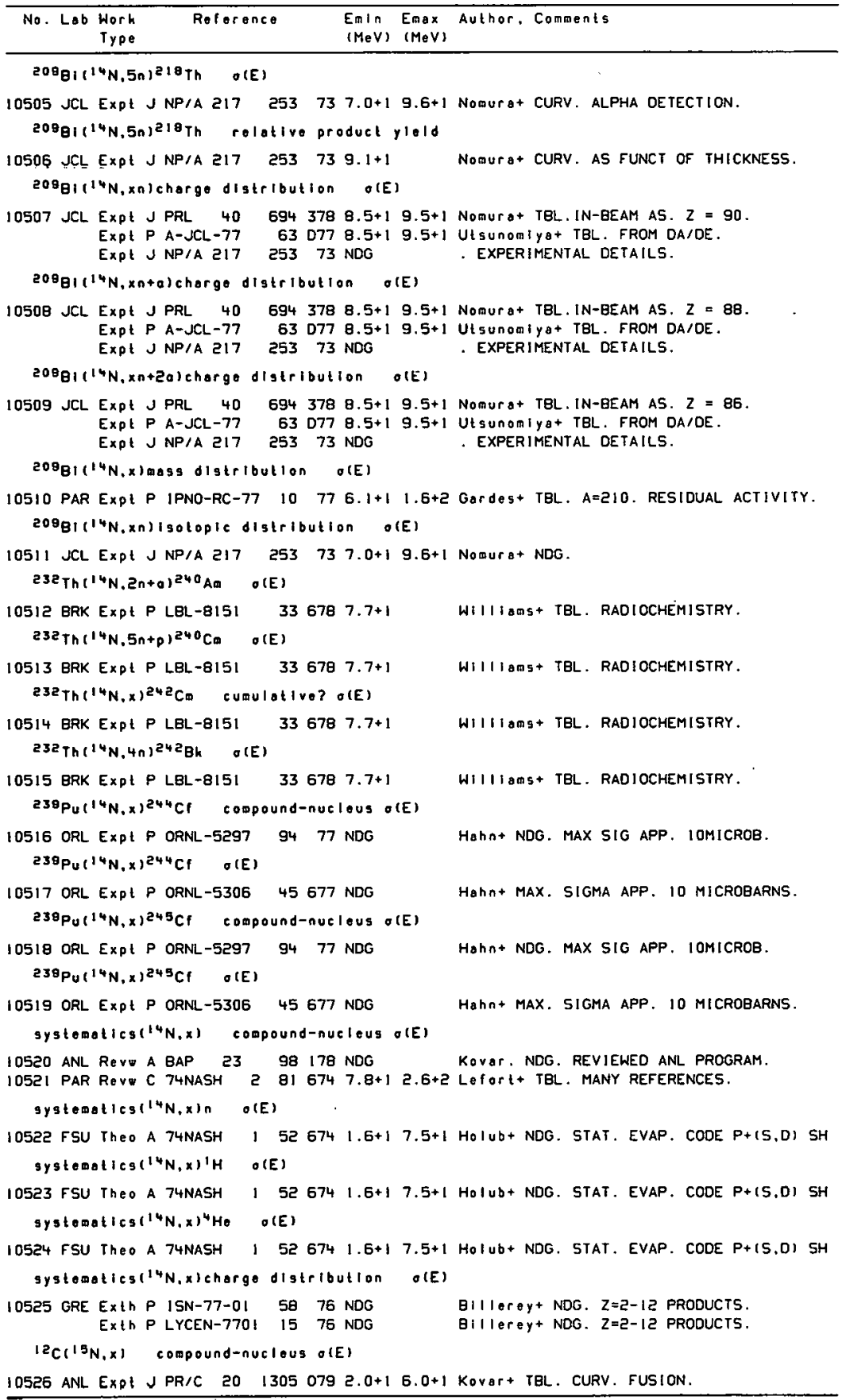


REFERENCES (cont)

\begin{tabular}{rrr}
\hline No. Lob Work & Reference & Emin Emax Author, Commonts \\
Type & & (MeV) $(M e V)$ \\
\hline
\end{tabular}

$1 e C\left(1 s_{N}, a\right)^{23} N_{0}$ compound-nuclous rolative o(E)

10527 ORL Expt J PL/B $694158772.2+03.6+0$ Gomez-Del-Campo+ CURV. RESONANCES

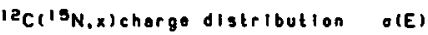

10528 ANL Expt J PR/C $2013050792.0+16.0+1$ Kovar+ TBL. O,F, NE, NA, MG, AL FROM ANG.

$180(15 N, x)$ chorge distribution o(E)

10529 SAC Expt P CEA-N-2070 $369783.6+15.5+1$ Conjeaud+ CURV. CFD. CASCADE MODEL.

$27 A \mid(15 N, x)$ compound-nuclous o(E)

10530 ANL Expt P ANL-79-40 $45 \quad 792.7+17.0+1$ Prosser+ NDG. CFD OTHER WORK. Expt A BAP $235034783.0+17.0+1$ Prosser+ NDG. SUM OF FUSION RESIDUES.

$27 A(15 N, x) i s o t o p t c$ digtribution $O(E)$

10531 ANL Expl A BAP $235034783.0+17.0+1$ Progser+ NDG. FUSION RESIOUES (Z GE 14 )

$209^{6}$ i $15 \mathrm{~N}, \mathrm{f}$ issioniags distribution proopt o(E)

10532 MAN Expt P UKNDC-P-88 94678 NDG Cuninghamet NDG. WORK COMPLETED.

$232 \operatorname{Th}\left({ }^{15_{N}} .5 n\right){ }^{242} \mathrm{Bh} \quad \sigma(E)$

10533 BRK Expt J PR/C $1917945797.6+19.1+1$ WIIIIams+ CURV. TI/2=7.0MIN.

$\left.232 T h(1)_{N}, x\right)$ Isolopic digtribution cumulative? o(E)

10534 BRK Expt J PR/C $1917945797.7+1 \quad$ WIIIIAGs+ TBL. 5 PRODUCTS.

$24{ }^{8} \mathrm{BH}\left({ }^{15} \mathrm{~N}, \mathrm{x}\right) \quad \mathrm{O}(\mathrm{E})$

10535 BRK Expl P LBL-5075 40 076 7.B+1 1.0+2 Gh10rso+ CURV. SF T1/2 APP. 20MS

$24 \theta^{B k}(15 N, x)^{258} \mathrm{Md} \quad O(E)$

10536 DUB Expt J AE $43 \quad 1559777.5+01.0+2$ Druln+ CURV.

10537 BRK Expt P LBL-5075 $400767.8+11.0+2$ Ghiorso+ CURV. SF ACTIVITY.

Expt P LBL-5075 $1600767.8+1 \quad 1.0+2$ Soderville+ CURV. SF ACTIVITY.

Rev S CERN-76-13 $5496767.8+1 \quad 1.0+2$ GhIorso. CURV.

$249^{\mathrm{Bh}}\left({ }^{13} \mathrm{~N}, 4 \mathrm{n}\right) 280^{\mathrm{K} U} \quad \sigma(\mathrm{E})$

10538 BRK Expt P LBL-5075 40 D76 7.8+1 1.0+2 Ghlorsot SF TI/2=0.3.0.1.0.085 NOT OBS.

$248 \mathrm{Bk}(15 \mathrm{~N}, 4 \mathrm{n})^{280} \mathrm{Ku}$ Including metostable? product yield

10539 DUB Expt J AE $43 \quad 1559777.5+19.3+1$ Druint

Expt R JINR-10499 $777.5+19.3+1$ Druint

Expt J SJA $437853787.5+19.3+1$. TRANSLATION OF AE $43(3), 155(1977)$

EXpt 4 EXFORAO010.002 $4787.5+19.3+1$. G DATA POINTS

$24{ }^{\circ} \mathrm{Cf}\left({ }^{19} \mathrm{~N}, 4 \mathrm{n}\right) 260105$ product yleld

10540 ORL Expl JPR/C 161146778 8.6+1 Benis+ NDG. T1/2=(1.52+-0.13)SEC.

$249^{2}\left({ }^{15} N, 4 n\right)^{260} 105 \quad 0(E)$

10541 BRK ReV J ARN 2713977 NDG Koller+3X10-33 CM2.DUBNA WORK.CHEM TECH

${ }^{2}$ C $^{160 . n)^{17}}$ F product yleld

10542 IEA Expt $P$ INDC(SEC) -5043176 NDG Rotberg. NDG. THRESHHOLD MEASUREMENT.

${ }^{9} B\left({ }^{16} 0, x\right)$ compound-nucleus o(E)

10543 CAL Exth J NP/A $2892360775.0+01.5+1$ 5oitkowgk1+ CURV. TBL. SUM OF 5 REACT.

${ }^{9}$ Be $(160, x)$ compound-nuclous o(E)afactor

10544 CAL Exth J NP/A $2892360775.0+01.5+1$ Swithowskit CURV. TBL. S-FACTOR.

10545 ORL ReV C 77TENN $22775.8+01.4+1$ Stokstead. CURV. 1/51G(OM). LAP-157(76)

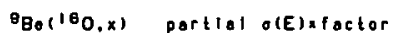

10546 CAL Exth P LAP-157 N76 5.8+0 1.9+1 5withow5hi+ CURV.S-FCTR. SUM FUS+N-TRAN

${ }^{8}$ Bo $(160, x) \quad O(E)$

10547 TNL Theo P ORO-1667-17116 79 NOS

The P A-TNL-78 $116 \quad 79 \mathrm{NDG}$

Par + NDG. LANDAU-ZENER EXCIT. MECH.

SAME AS ORO- $\$ 667-17$

${ }^{9}$ Bo( ${ }^{16} 0$, total) portlol o(E) afactor

10548 CAL Exth J NP/A $2892360775.0+01.5+1$ Solthoughi+ CURV. FUS+N-TRANSFER, S-FAC

${ }^{9} B$ (160.x) ${ }^{17} 0$ partiol relative o(E)

10549 CTH Expt JPS 1924579 8.9+0 2.0+1 Chrlstiansson+ CURV. IN-BEAM. 871-KEV G 


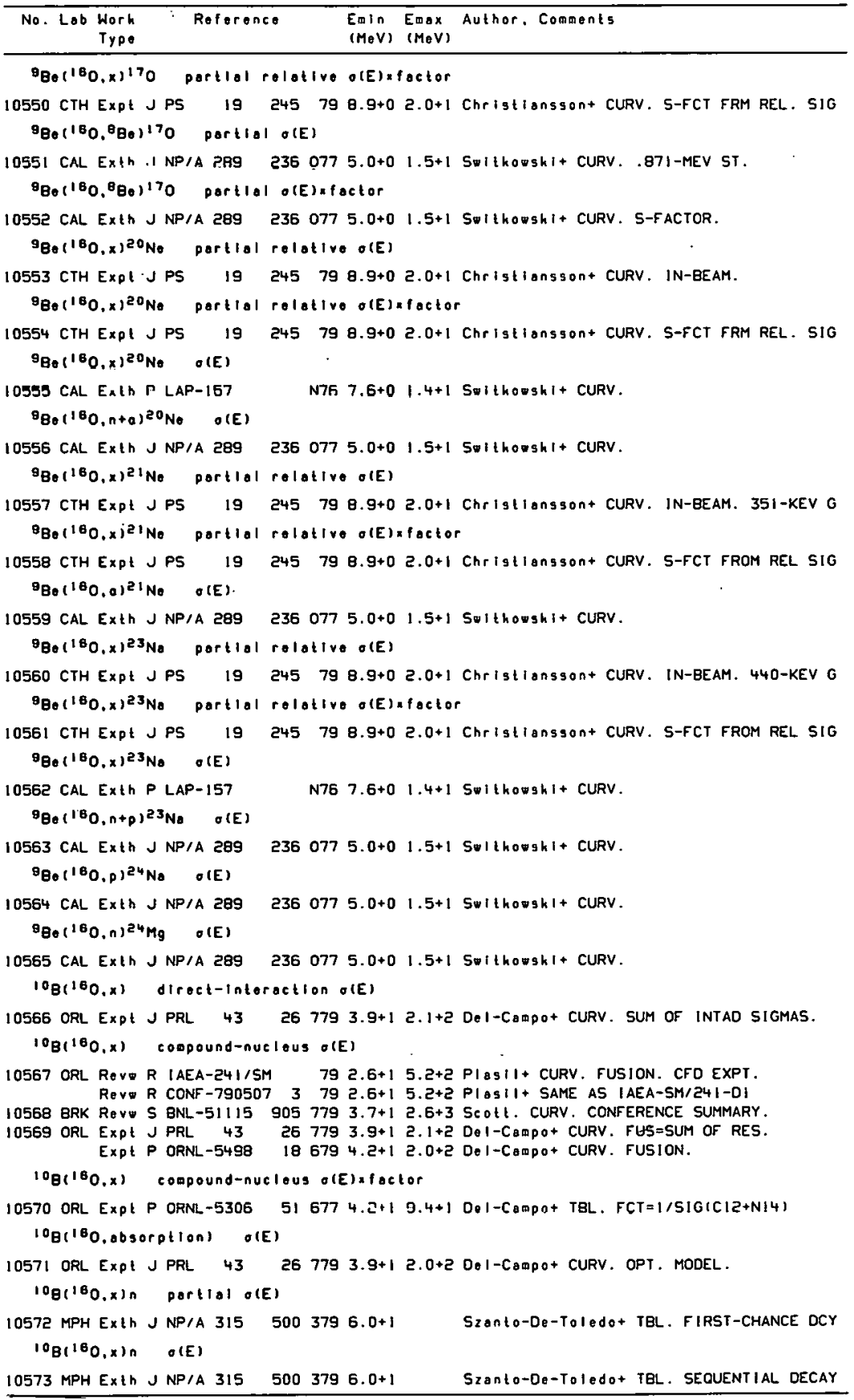


REFERENCES(cont)

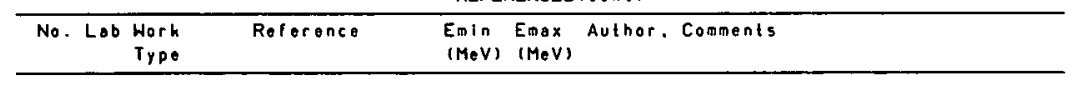

$$
{ }^{10} \mathrm{~B}\left({ }^{16} \mathrm{O}, \mathrm{x}\right)^{1} \mathrm{H} \text { partlal } \mathrm{O}(\mathrm{E})
$$

1057'1 MPII Exth JNP/A $3155003796.0+1 \quad 52$ anto-De-Toledo+ IBL. FIRST-CHANCE DCY

$$
10 \mathrm{~B}\left({ }^{15} \mathrm{O}, \mathrm{X}\right)^{1 \mathrm{H}} \text { O(E) }
$$

10575 MPH Exth J NP/A $3155003796.0+1$

${ }^{10} \mathrm{~B}\left(1^{16} \mathrm{O}, x\right)^{2} \mathrm{H}$ partlial o(E)

$10576 \mathrm{MPH}$ Exth J NP/A $3155003796.0+1 \quad 520$ to-De-Toledo+TBL.CURV. IRST-DECAY D.

${ }^{10} \mathrm{~B}\left({ }^{16} 0, x\right)^{2} \mathrm{H} \quad O(E)$

10577 MPH Exth J NP/A $3155003796.0+1 \quad$ Szonto-de-Toledo+ TBL. SEQUENTIAL DECAY

${ }^{10} \mathrm{~B}\left({ }^{16} \mathrm{O}, x\right)^{4} \mathrm{He}$ part1.1 O(E)

$10578 \mathrm{MPH}$ Exth J NP/A $3155003796.0+1 \quad 5 z 0 n t 0-0$-Toledo+ IBL. FIRST-CHANCE DCY

$100(160, x)^{4} H_{0}$ o(E)

10579 MPH Exth J NP/A $3155003796.0+1 \quad$ Szanto-De-Toledo+ TBL. SEQUENTIAL DECAY

$100\left({ }^{10} 0, x\right)^{6} \mathrm{LI}$ product yield

$10580 \mathrm{JCL}$ Expt P A-JCL-77 $390779.8+1$

$10^{\circ}(100, i n e l a s t 1 c)^{10} \mathrm{~B}$ partial o(E)

10581 CAL EXPt J NP/A 312177 D78 $1.1+11.5+1$ Wu+ CURV. COULOMB EXC. OF FIRST STATE

${ }^{10} B(160,6 L 1)^{20}{ }^{16} \quad \sigma(E)$

10582 TAM Exih J PR/C 162249077 2.0+1 3.2+1 Stokstegd+ TBL. INTAD. FRM NP/A 259,122 ${ }^{10} \mathrm{~B}(160, a)^{22} \mathrm{No}$ product ylold

10583 IEA Expt P INDC(SEC) $-50421763.6+14.0+1$ Hirato+ NDG. A-GROUPS TO LOW EXCIT. LEV $100(160 . d)^{24} \mathrm{Mg}$ portiol o(E)

10584 MPH Exth J NP/A $3155003796.0+1 \quad$ Szento-De-Toledo+ CURV. SIG(J). J=2-14.

${ }^{10} \mathrm{~B}\left({ }^{16} 0, x\right)$ chorgo distribution direct-interaction rolative o(E)

10585 ORL Expt J PRL 43 26 779 3.9+1 9.4+1. De1-C Bmpo+ 65PER-CENT OXYGEN 1SOTOPES. Expt J PRL $43 \quad 267792.0+2 \quad$ Del-Campo+ 4 CHARGES. $Z=6-9$.

10 B(160.x)chargo distribution o(E) xfoctor

10586 ORL EXPL J PRL $43 \quad 267793.9+12.0+2$ Del-Campo+ Z = 5-11. RATIO TO FUS. SIG. Expt P ORNL-5305 $516779.4+1 \quad$ Del-Campo+ CURV. Z=6-12. FCT=1/SIG(FUS)

$100(160 . x)$ chargo distribution product yleld

10587 JCL Expl P A-JCL-77 $390779.8+1 \quad$ MolobayashI+ NDG. ANALYSIS IN PROGRESS

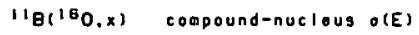

$1058 B$ BRK REV S BNL-51115 905779 NDG SEOIt. CURV. CONFERENCE SUMMARY.

$\left.\mathrm{C}^{16} 0, x\right)$ compound-nucleus o(E)

10589 UEN Exth J PL/B $64 \quad 408768.5+01.2+1$ Frohlicht CURV.

C(160, $x$ in $O(E)$

10590 FSU The A 74NASH I $526741.6+17.5+1$ Holub+ NOG. STAT. EVAP. CODE.

$C(160, x)^{\prime} H \quad \sigma(E)$

10591 fSU Theo A 74NASH $1526741.6+17.5+1$ Holub+ NDG. STAT. EVAP. CODE.

C(160.X) ${ }^{4} \mathrm{H}_{0}$ O(E)

10592 FSU The A 74NASH I $526741.6+17.5+1$ Hol Ub+ NDG. STAT. EVAP. CODE.

${ }^{12} \mathrm{C}\left({ }^{16} 0, x\right)$ compound-nucleus o(E)

10593 BNL TheO A BAP $23 \quad 615478$ NDG GoI in. NDG. DOUBLE-FOLDING POTENTIAL.

10594 BRK ReV S BNL-51115 905779 NDG SeOt. CURV. CONFERENCE SUMMARY.

10595 ROC ReVw S BNL-51115 235779 NOG HuIzenga+ CURV. MAX. FUSION SIGMA

10596 TNL Theo P ORO-1667-17109 79 NDG Hnwerdt NDG. SUB-COULOMB FUSION

Iheo P A-TNL-78 10779 NDG . SAME AS ORO-1667-17:

10597 CAL Expt J NP/A $2801894776.3+03.1+1$ Christensont TBL. CURV. SUM EV. RES.

10598 JUU Revw C 76ANL-2 I $3414761.1+11.2+2$ Mosel. CURV.

10599 WAU Expt J NP/A $3035007781.2+15.2+1$ Chan+CURV. FUS SIG FROM SUM 18 DISC. GS

10600 UEN Exth J PL/B $64 \quad 408761.5+16.5+1$ Frohlicht CURV. GLAS-MODEL MODEL.

10601 WZI Expt J PR/C $13 \quad 1527 \quad 76,1.6+13.3+1$ Eyal+ TBL. DEDUCED FROM EVAPOR. RESIDUE 10602 STB EXPt C 77TOKYO $6579772.4+14.6+1$ Cormier+ CURV. ANL FUS. CFD. PRES. INL. 
REFERENCES ( cont)

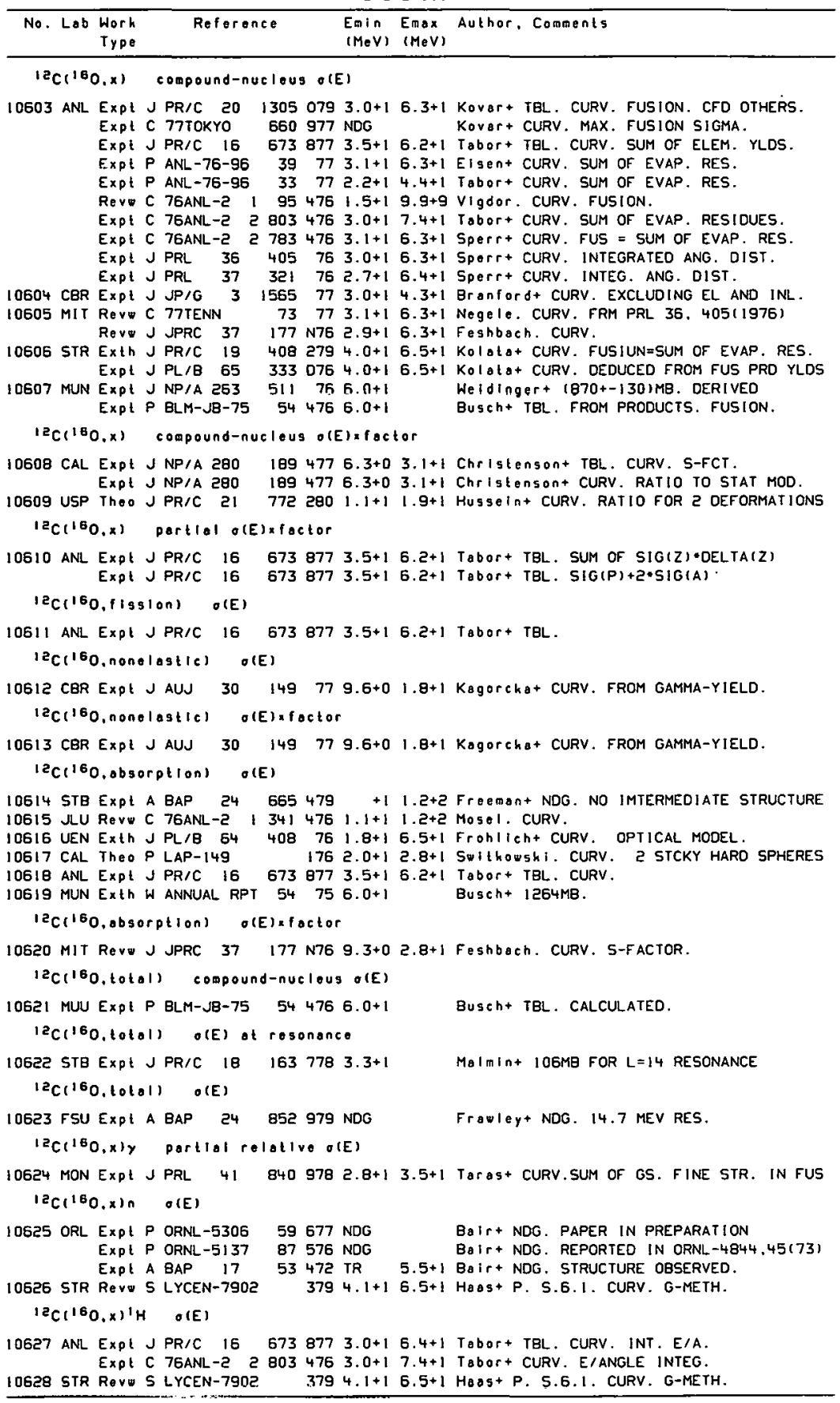


REFERENCES $(\operatorname{con} t)$

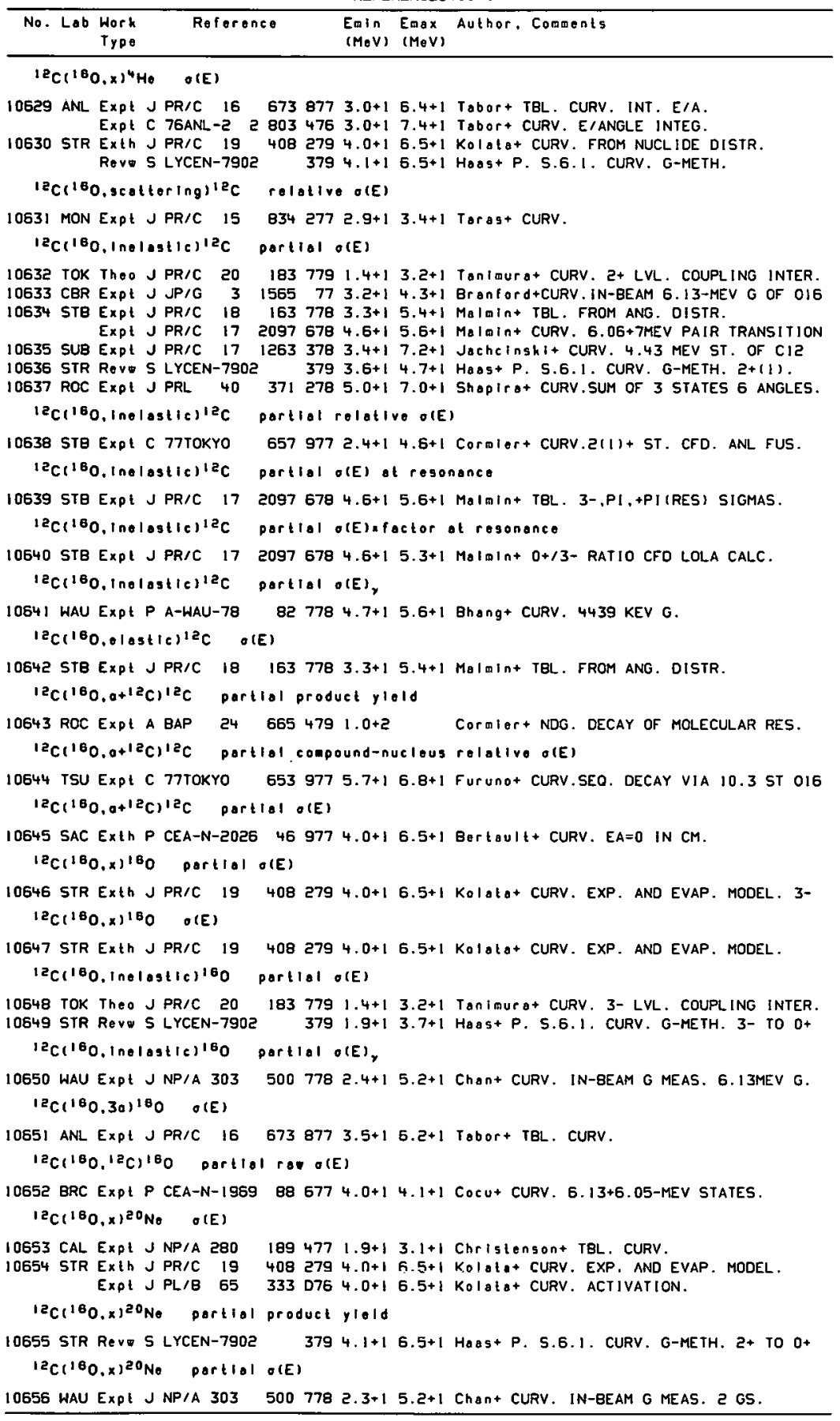


REFERENCES (con ()

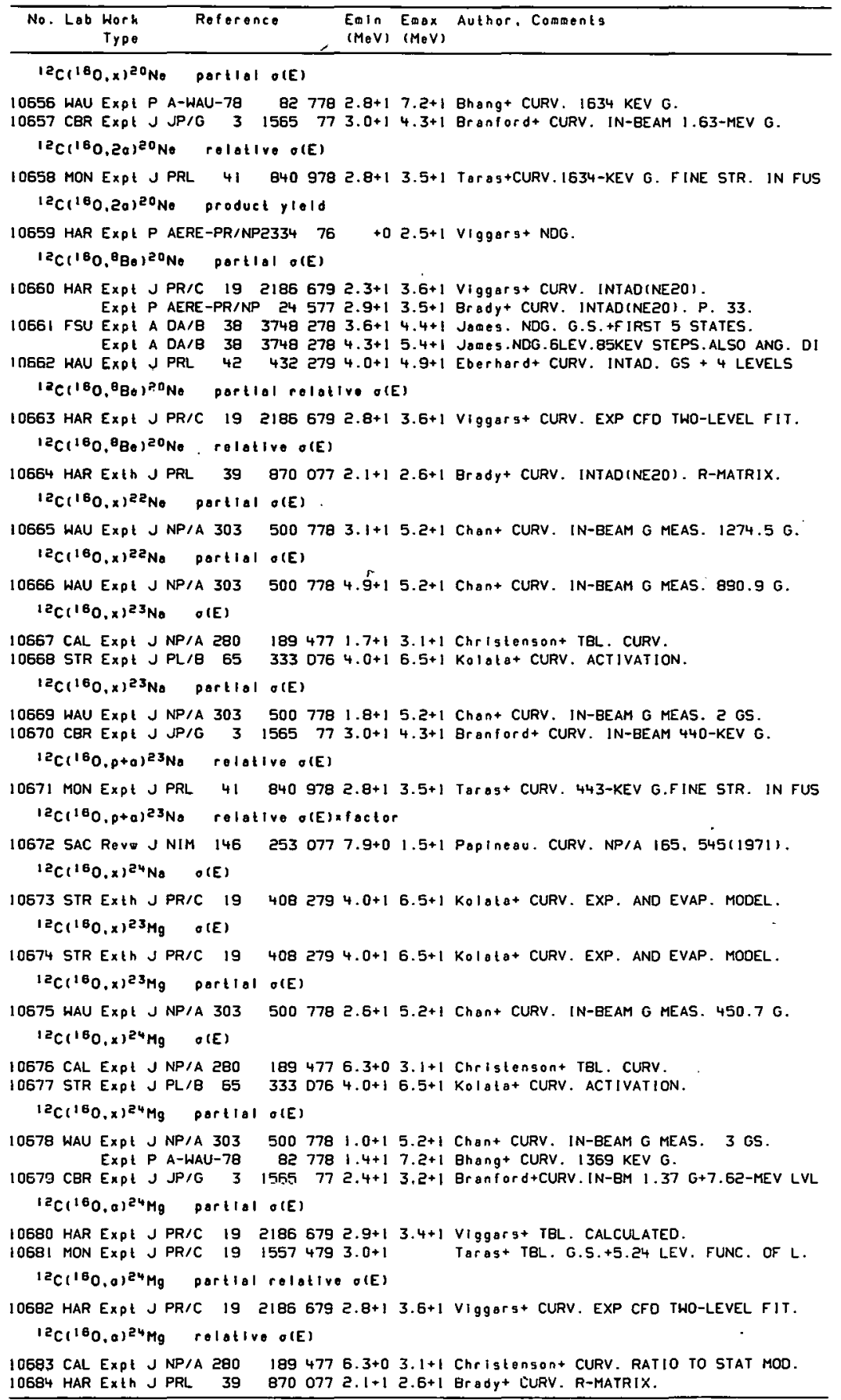




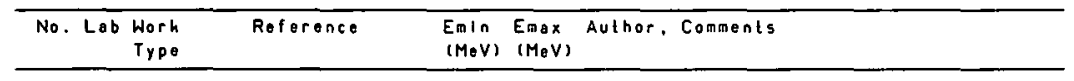

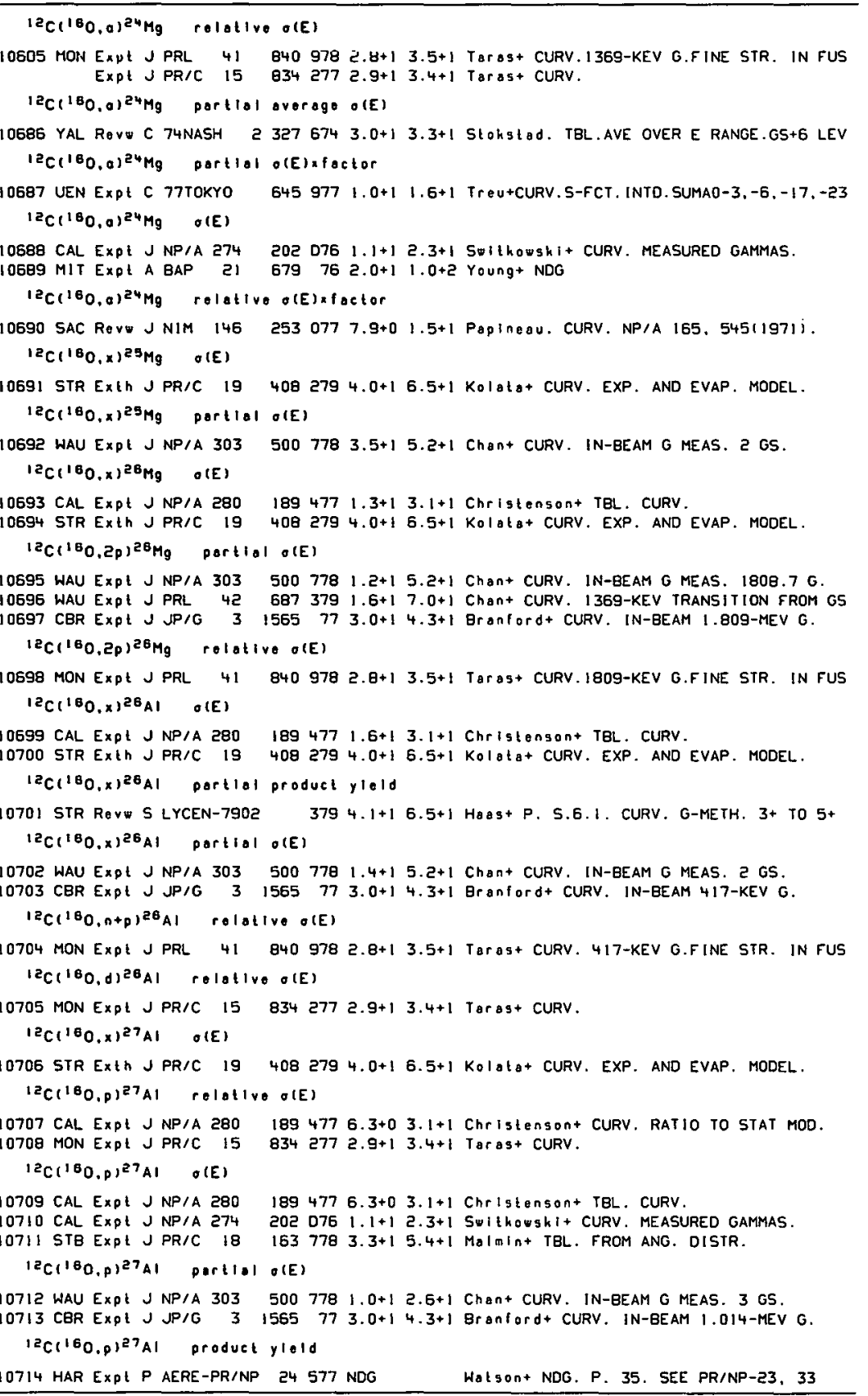




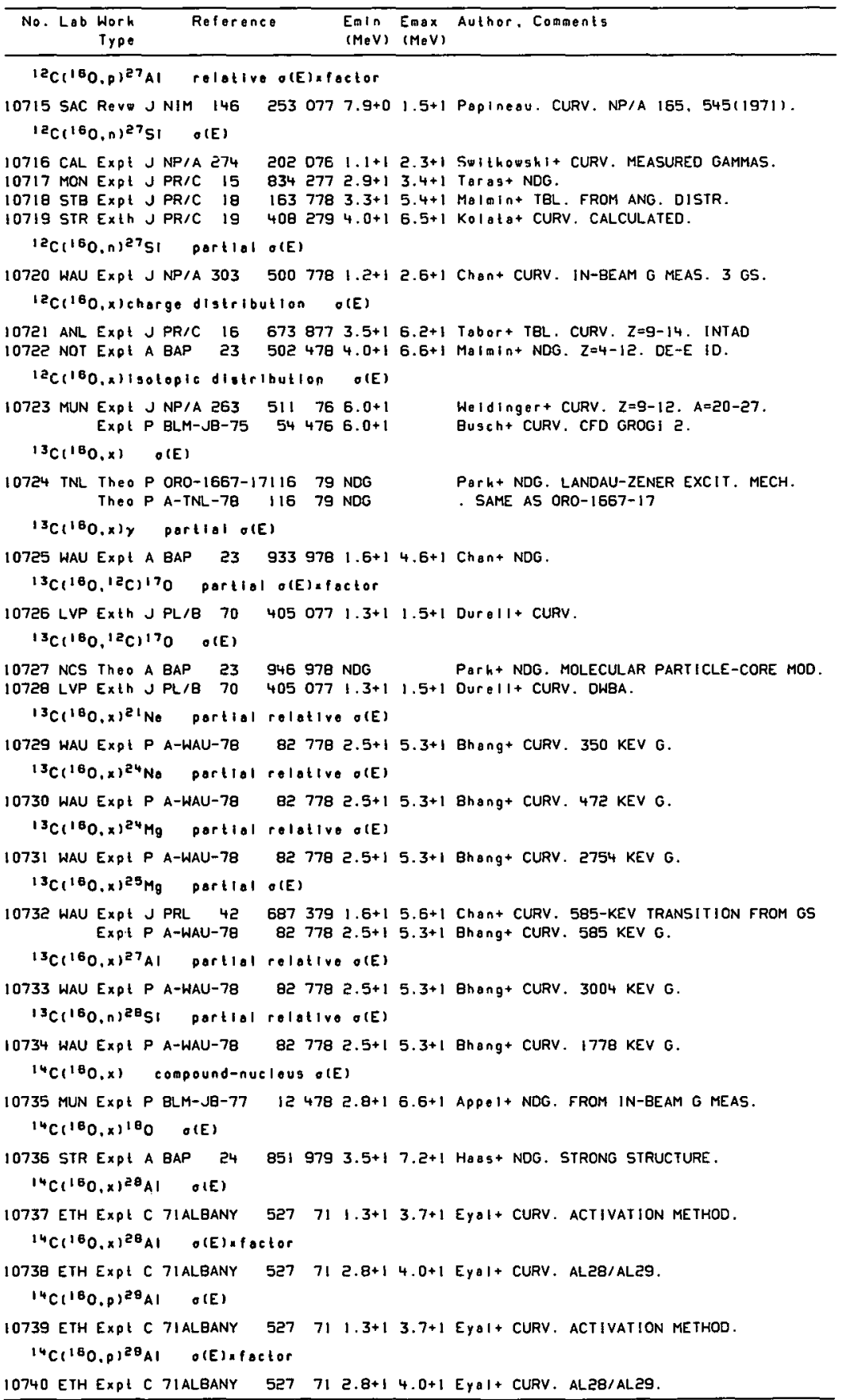


REFERENCES (cont)

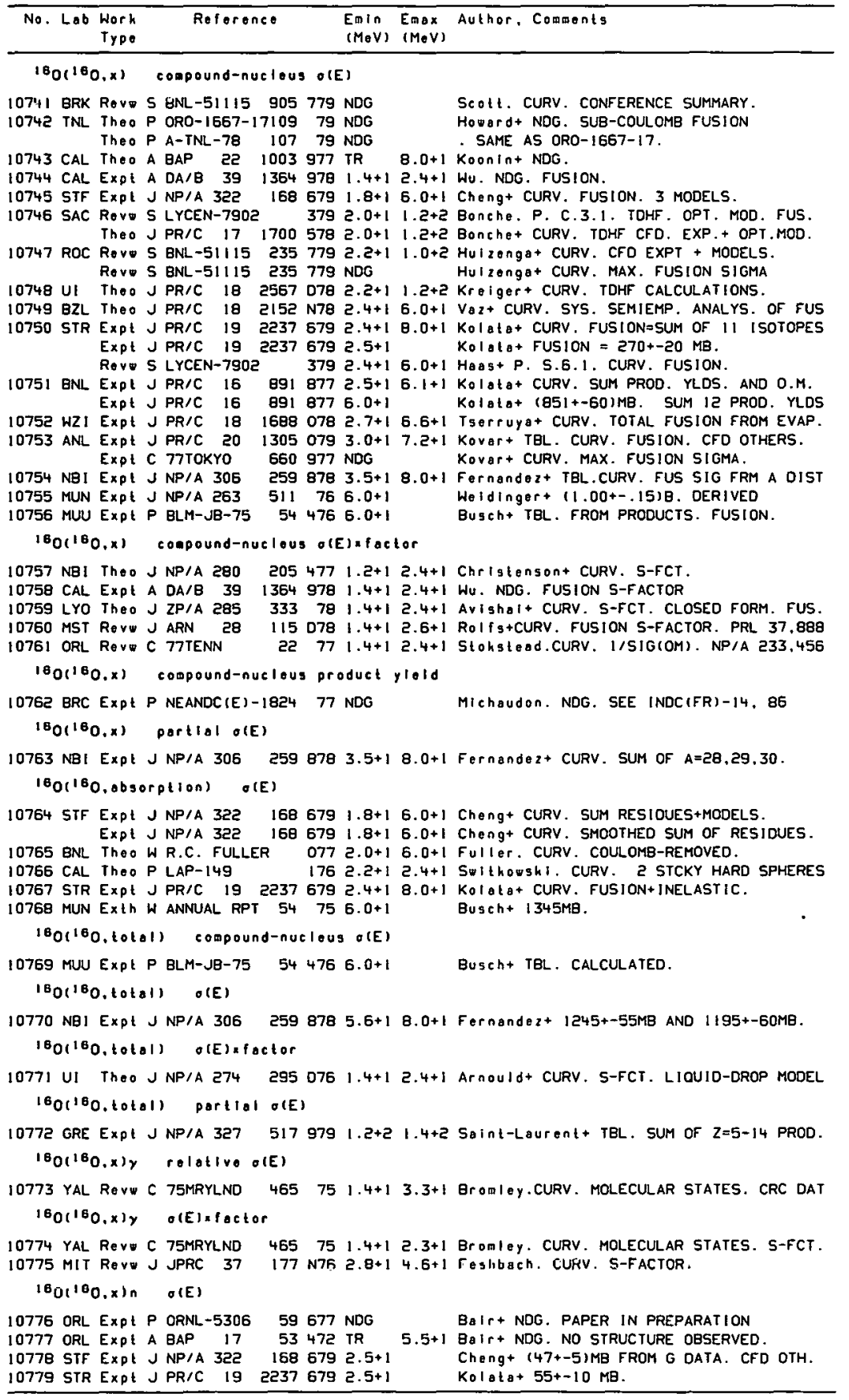




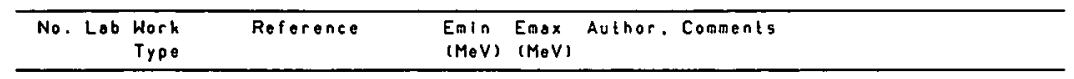

${ }^{16} \mathrm{O}\left({ }^{16} \mathrm{O}, x\right)^{1} \mathrm{H} \quad \mathrm{O}(\mathrm{E})$

10780 STF EXpl J NP/A $322 \quad 1686792.5+1$ IO7BI STR Expl J PR/C $1922376792.5+1$

$160(160 . x)^{4} \mathrm{He}$ o(E)

10792 STF Expl J NP/A $322 \quad 1686792.5+1$ 10783 STR Expl J PR/C $19 \quad 2237 \quad 679 \quad 2.5+1$

Cheng+ (139+-15)MB FROM G DATA. CFD OTH Kolota+ $270+-60 \mathrm{MB}$.

Cheng + (163+-13)MB FROM G DATA. CFD OTH Kol ot at $125+-15 \mathrm{MB}$.

$180(160, x)^{4} \mathrm{He}$ rolative O(E)

10784 YAL REV C 75MRYLND $465751.8+13.2+1$ Bromley.CURV. MOLECULAR STATES. CRC DAT

$160(160.5 \cot t e r \operatorname{lng})^{160} \quad \sigma(E)$

10795 STR ROV S LYCEN-7902 379 2.4+1 6.0+1 Hä5+ P. S.6.1. CURV. G-METH.

$160(180.1 n e l a s t l e)^{160}$ partlal o(E)

10786 WIS Theo J PL/B 97179 N79 NDG Frledmant NDG. BARRIER-TOP RES. MODEL.

10787 YAL Theo J PRL $425662793.6+18.4+1$ PhIIllps+ CURV. ORIGIN OF GROSS STRUCT.

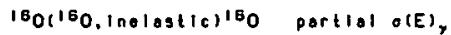

10798 STR Expl J PR/C I9 $22376792.4+18.0+1 \mathrm{Kol}$ oto+ CURV. EXTENSIVE STRUCTURE. ReY 5 LYCEN-7902 $3792.5+1$ B.0+1 HaAs+ P. S.6.1. CURV. G-METH. 3- TO $0+$

10789 BNL Expt J PR/C $16 \quad 891877$ 2.5+1 6.1+1 Kolotot CURV. FRM G YLD. 3- STATE. Expt J PR/C 16 BI B77 6.0+1 Kolotot (51.6+-3.6)MB. 3- ST. FRM G YLD

160 16 $^{16}$ inolastle) 160 o(E)

$10790 \mathrm{NBI}$ Expt J NP/A 306259 B78 $5.6+1 \quad$ Fornandez+.80+-25MB.

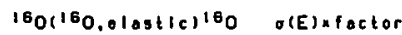

$10791 \mathrm{NBI} T$ Theo J NPIA 280205477 1.8+1 $2.9+1 \mathrm{Christenson+CURV.} \mathrm{FCT=I/SIG(MOTT)}$

$160(160.0105 t(c) 160 \quad O(E)$

10792 BNL TheO W R.C. FULLER $0772.0+16.0+1$ Fuller. CURV. COULOMB-REMOVED.

$160(160, x)^{20} \mathrm{Ne}$ partiol $\sigma(E)$

10793 STF Expl J NP/A $3221686791.8+16.0+1$ Cheng+ CURV. EG $=1.634 M E V$ FROM 9ODEG DAT

$160(160 . x)^{20} \mathrm{Ne}$ O(E)

10794 STR REV S LYCEN-7902 $3792.4+1$ 6.0+1 Haos+ P. S.6.1. CURV. G-METH.

10795 BNL Expl J PR/C 16 891 $8772.5+16.1+1$ Kolata+ CURV. FRM G YLD. Expt JPR/C 16 B91 $8776.0+1 \quad$ Kolata+ $(93.7+-6.6) M B$. FRM G YLD. 10796 MUN Exth W ANNUAL RPT $54756.0+1 \quad$ Busch + CURV. MEASURED + GROGI2 CALC.

$160(160, x)^{20} \mathrm{Ne}$ partiol o(E)*factor

10797 WZI Expt J PR/C $1816880785.4+16.0+1$ Tserruyat TEL. REL. G.S. POPULATION.

$160(160,30)^{20} N_{0} \quad O(E)$

10798 STF Expt J NP/A $3221686791.2+16.0+1$ Cheng+ CURV. SMOOTHED G DATA + MODEL.

Expt J NPIA 322 168 $6792.5+1$ Cheng+ $(2+-1) M B$ FROM G DATA. CFD OTHER.

$160(160,12 \mathrm{C})^{20} \mathrm{Ne} \quad O(E)$

10799 NBI Expt J NP/A $3062598785.6+18.0+1$ Fernondez+ 45+-8MB AND $44 M B$.

${ }^{16} O\left({ }^{16} 0, x\right)^{22} \mathrm{~N}_{0} \quad O(E)$

10800 BNL EXPt J PR/C $16 \quad 8918776.0+1$ 10801 MUN Exth W ANNUAL RPT $54756.0+1$

Kolata+ NOT OBSERVEO. FRM G YLD.

$\left.10^{10} 0, x\right)^{23} \mathrm{Na}$ partIol O(E)

10802 SIF Expt J NP/A 322 168 679 1.8+1 6.0+1 Cheng+ CURV. EG=44OKEV FROM 9/DEG DOTA

$18 O\left({ }^{16} O, x\right)^{23} N_{0} \quad O(E)$

10803 BNL EXPt JPR/C I6 $8918776.0+1$ 10804 MUN Exth W ANNUAL RPT $54756.0+1$

Kolata+ (36.3+-2. SIMG. FRM G YLD. Busch+ CURV. MEASURED + GROGIJ CALC.

$\left.1{ }^{8} O{ }^{1 B} O, x\right)^{23} \mathrm{No}$ partlal o(E) atactor

10805 WZ1 Expt JPR/C $1816880785.4+1$ 6.0+1 Tgerruyat TBL. REL. G.S. POPULATION.

${ }^{16} O\left({ }^{16} O, p+2 a\right)^{23} N_{0} \quad O(E)$

10806 5IF Expt J NP/A $3221686791.4+16.0+1$ Cheng+ CURV. SMOOTHEO G DATA + MOOEL.

${ }^{16} 0\left({ }^{16} 0, x\right)^{24} \mathrm{Mg}$ portlal O(E)

10007 STF Expt J NP/A $3221686791.8+16.0+1$ Cheng+ CURV. EG=1.369MEV FROM 9ODEG DAT 


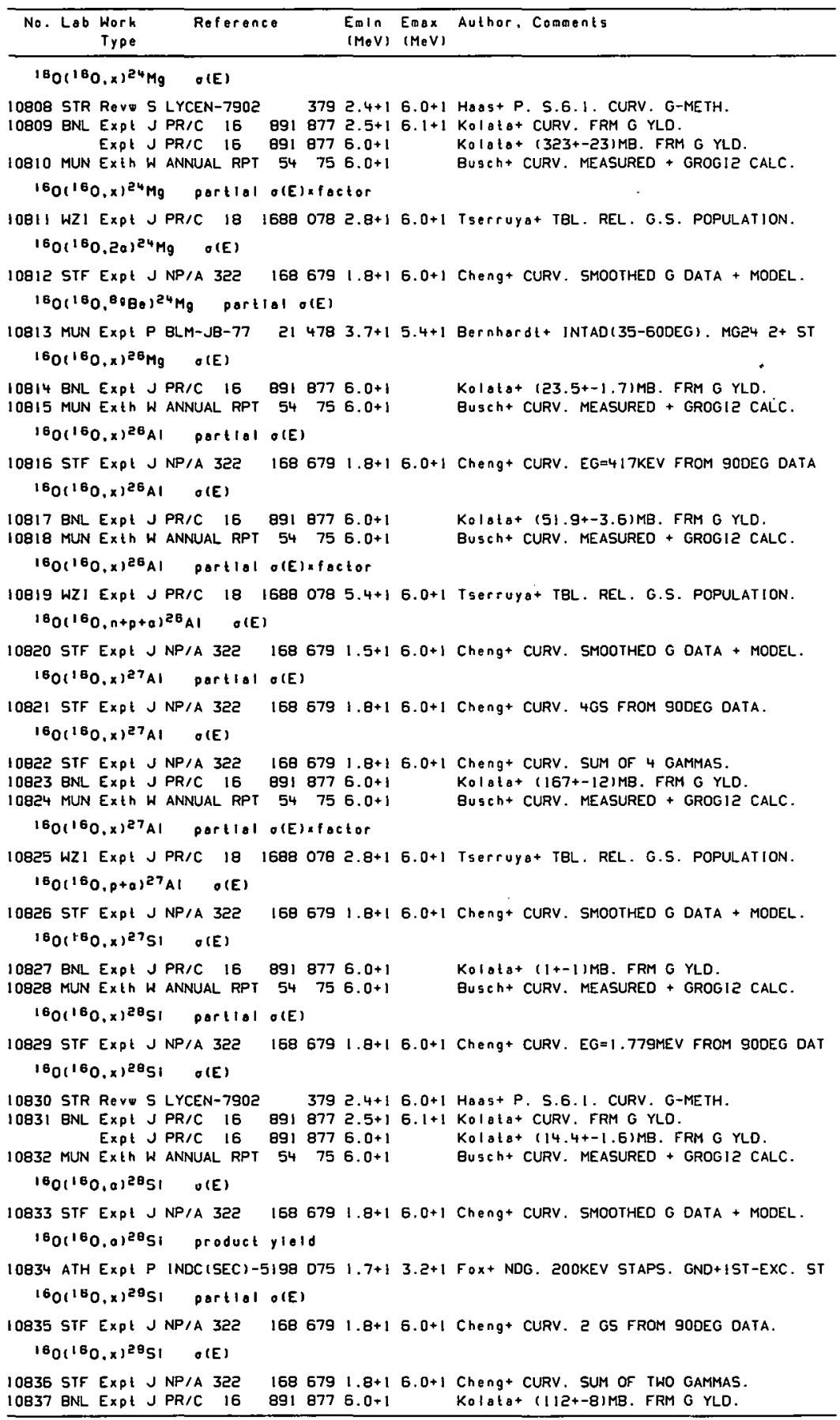


REFERENCES(cont)

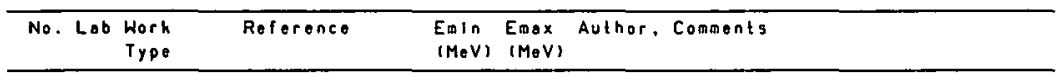

${ }^{16} 0(160, x)^{28} \mathrm{SI} \quad 0(E)$

10838 MUN Exth W ANNUAL RPT $54 \quad 756.0+1 \quad$ Busch+ CURV. MEASURED + GROGI2 CALC.

${ }^{16} 0\left({ }^{16} 0, n+2 p\right)^{29} \mathrm{SI}$ o(E)

10839 STF Expt J NP/A $3221686791.8+16.0+1$ Cheng+ CURV. SMOOTHED G DATA + MODEL.

$160(160, x)^{30} S 1$ partial o(E)atoctor

10840 WZI Expt J PR/C $1816880782.8+16.0+1$ Tserruya+ TBL. REL: G.S. POPULATION.

$160\left({ }^{16} 0, \mathrm{Cp}^{30} \mathrm{SI}\right.$ partiol o(E)

10841 STF Expl J NP/A $3221686791.8+16.0+1 \mathrm{Cheng}+$ CURV. EG=2.235MEV FROM SODEG OAT

$160\left({ }^{16} 0.50\right)^{30} \mathrm{Si} \quad \sigma(E)$

10842 STF Expt J NP/A $3221686791.8+16.0+1$ Cheng+ CURV. SMOOTHED G DATA + MODEL.

$160(160, x)^{30} \mathrm{P}$ partiol o(E)

10843 STF Expl J NP/A $3221686791.8+1$ G.0+1 Chong+ rIIRV. EG=1.264MEV FROM 9ODEG DAT Expt J NP/A $3221688791.0 \times 1$ 6.0+1 Cheng+ CURV. 3GS FROM 9ODEG DATA.

${ }^{16} \mathrm{O}\left({ }^{160} \mathrm{O}, \mathrm{X}\right)^{30 \mathrm{P}} \quad \mathrm{O}$ (E)

10844 STF Expt J NP/A 322 10845 BNL Expt J PR/C 16

$1686791.8+16.0+1$ Cheng+ CURV. SUM OF 3 GS.

$8918776.0+1 \quad \mathrm{Kol}$ oto $+(12+-2) \mathrm{MB}$. FRM G YLD.

${ }^{16} 0(160, x)^{30} \mathrm{P}$ partial o(E) * factor

10846 WZI Expt J PR/C $1816880782.8+16.0+1$ Tserruyat TBL. REL. G.5. POPULATION.

$180(160, n+p) 30 p \quad \sigma(E)$

10847 STF EXPt J NP/A 322

Expt J NP/A 322

168 $6791.8+16.0+1$ ChENg+ CURV. SHOOTHEO G DATA + MODEL.

$1686792.5+1$ Chengt $140+-31 M B$ FROM G DATA. CFO OTH.

${ }^{16} 0(160, x)^{31} p \quad \sigma(E)$

$10848 \mathrm{BNL}$ Expl JPR/C 16 891 $8776.0+1 \quad$ Kolata+ $(16+-2) M B$. FRM G YLO,

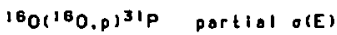

10849 STF Expt J NP/A $3221686791.8+16.0+1$ Cheng+ CURV. EG=2.234MEV FROM 9ODEG DAT Expl J NP/A $3221686791.8+16.0+1$ Cheng+ CURV. EG=1.26GMEV FROM 9ODEG DAT

$\left.1601^{16} 0, p\right)^{31 P} \quad O(E)$

10850 STF EXPt J NP/A $3221686791.8+16.0+1$ Cheng+ CURV. SMOOTHED G DATA + MODEL. 1085 ! BNL EXPL W TO BE SUBMITED $3776.0+1$ Kolota+ $116+-2) M B$. 2 GAMMAS

$\left.1{ }^{10} \mathrm{O}{ }^{18} \mathrm{O}, n\right)^{31} \mathrm{~S} \quad \mathrm{O}(\mathrm{E})$

10852 STF Expt I NP/A $3221686792.5+1 \quad$ Cheng+ LT 2 MB FROM G DATA. CFD OTHERS.

$180(180, x)$ chorge distribution O(E)

10853 ANL Expt A BAP $2210199773.0+16.0+1$ Schiffer+ NDG. $Z$. GE. 9 PRODUCTS.

10854 GRE Expt J NP/A $3275179791.2+21.4+2$ Saint-Laurent+ TBL. Z=5-14.

Expt P CEA-N-2070 $1909786.7+1 \quad 1.6+2$ Conleaud+ TBL. CURV. CFO THEORY.

Exth C 77TOKYO $6639776.0+1$ 1.2+2 Conjeaud+ CURV. $Z=5-14$. INTAD. CASCADE

$\left.160{ }^{16} 0, x\right)$ charge distribution direct-interaction $O(E)$

10855 GRE EXpL P CEA-N-2070 $1909786.7+11.5+2$ Conjeaud+ TBL. CURV. CFO THEORY.

$160(160, x)$ mass distribution $O(E)$

10856 NBI Expt J NP/A $3062598783.5+18.0+1$ Fernandez+ TBL. CURV. A=20, 23-31. $10857 \mathrm{WZI}$ Expt $J$ PR/C $1816880784.5+15.4+1$ Tserruyo+ CURV. ENERGY/ANGLE INTEGRATED

$160(160 . x) 1 s 0 t 0 p 1 c$ distrtbution o(E)

$10858 \mathrm{CAL}$ Expt A DA/B $3913649781.4+12.4+1$ Wu. NDG. PROMPT GAMMA DETECTION.

10059 STR EXpt I PR/C IS $22376792.4+1$ 8.0+1 K01ala+ CURV. 10 ISOTOPES. $2=12-15$.

10860 GRE Theo P ISN-79-01 $42796.0+1$ Cole. CURV. LANCELAT CFD GROGI2 + EXPT.

10861 MUN Expt J NP/A 26351176 6.0+1 Weidinger+ CURV. Z=9-13. A=20-29.

Expt P BLM-JB-75 $544766.0+1 \quad$ Buscht CURY. CFO GROG] 2.

$180(160, x)$ O O(E)

10862 ORL ExP! P ORNL-5306 59677 NDG BaIf+ NDG. PAPER IN PREPARATION

10863 ORL Exp: A BAP 1753472 TR $5.5+1$ Boir+ NDG. YLD. $=5.5+Y L D$ FOR O161016, XN1

20 Ne $160.1 n e l a s t i c)^{20}$ Ne product yleld

10864 STB Expt A BAP 24 $5704792.0+15.5+1$ Braun-Munzinger+ NDG. GROSS STRUCTURE. 


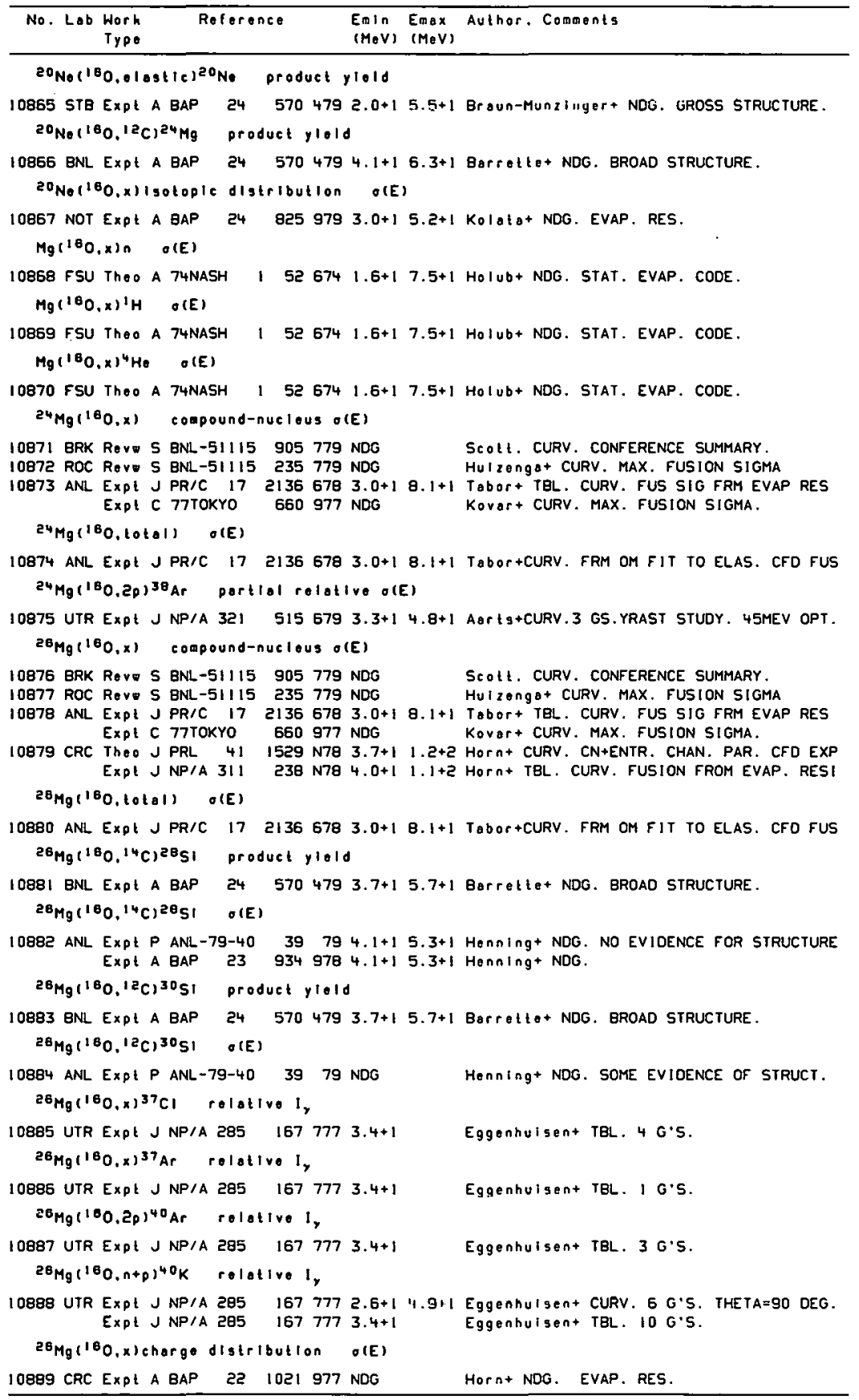


REFERENCES ( con ()

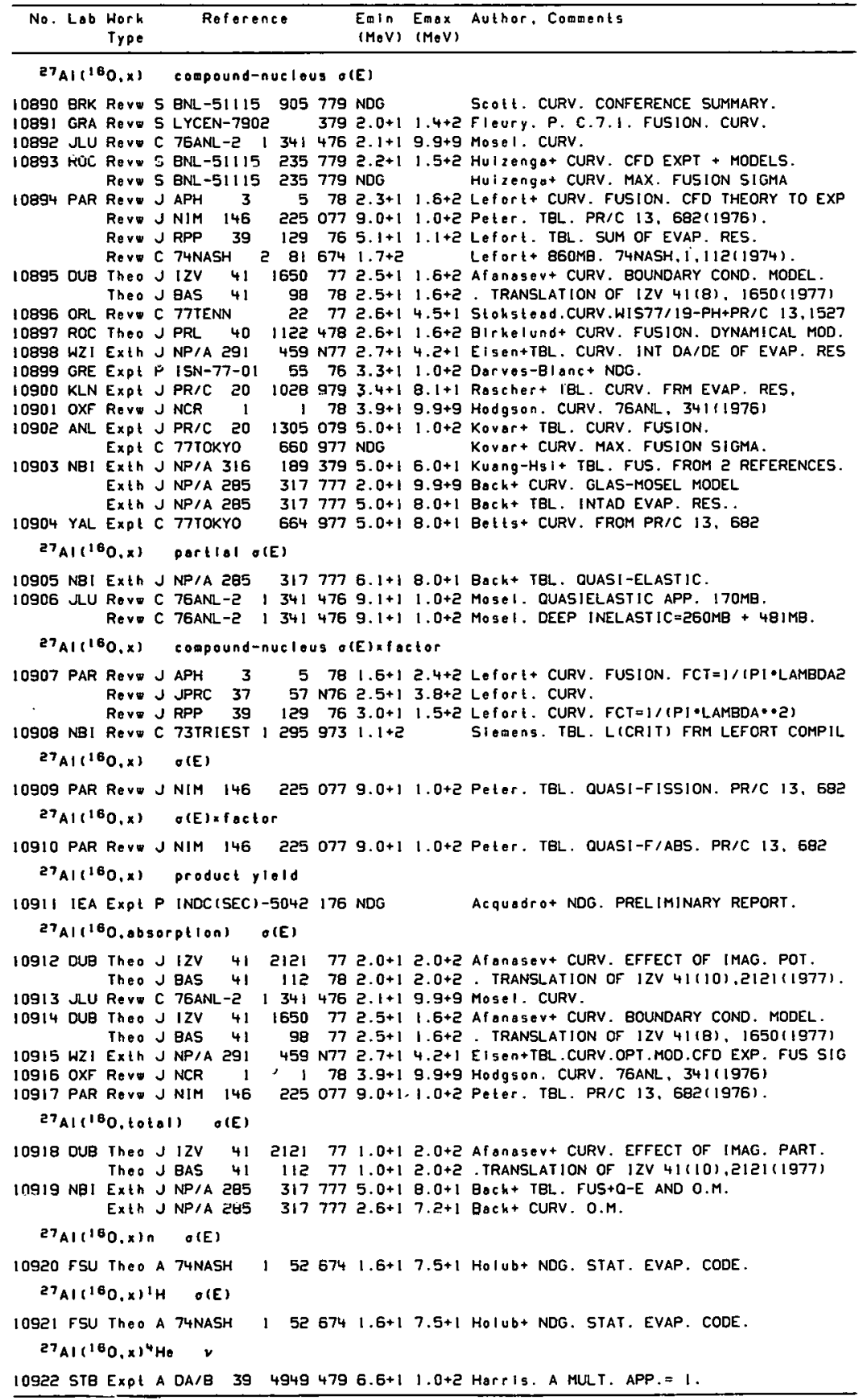




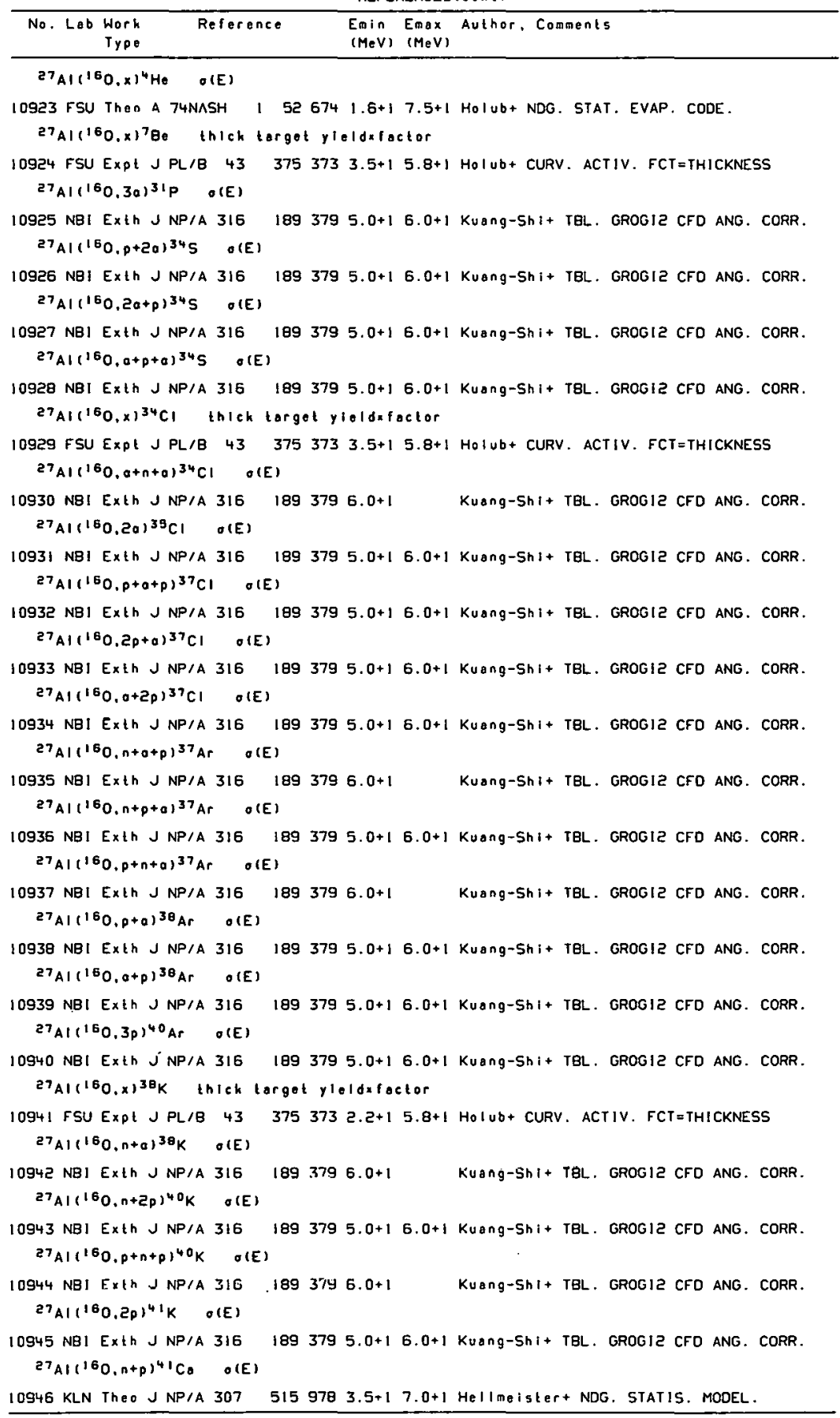


REFERENCES (con t)

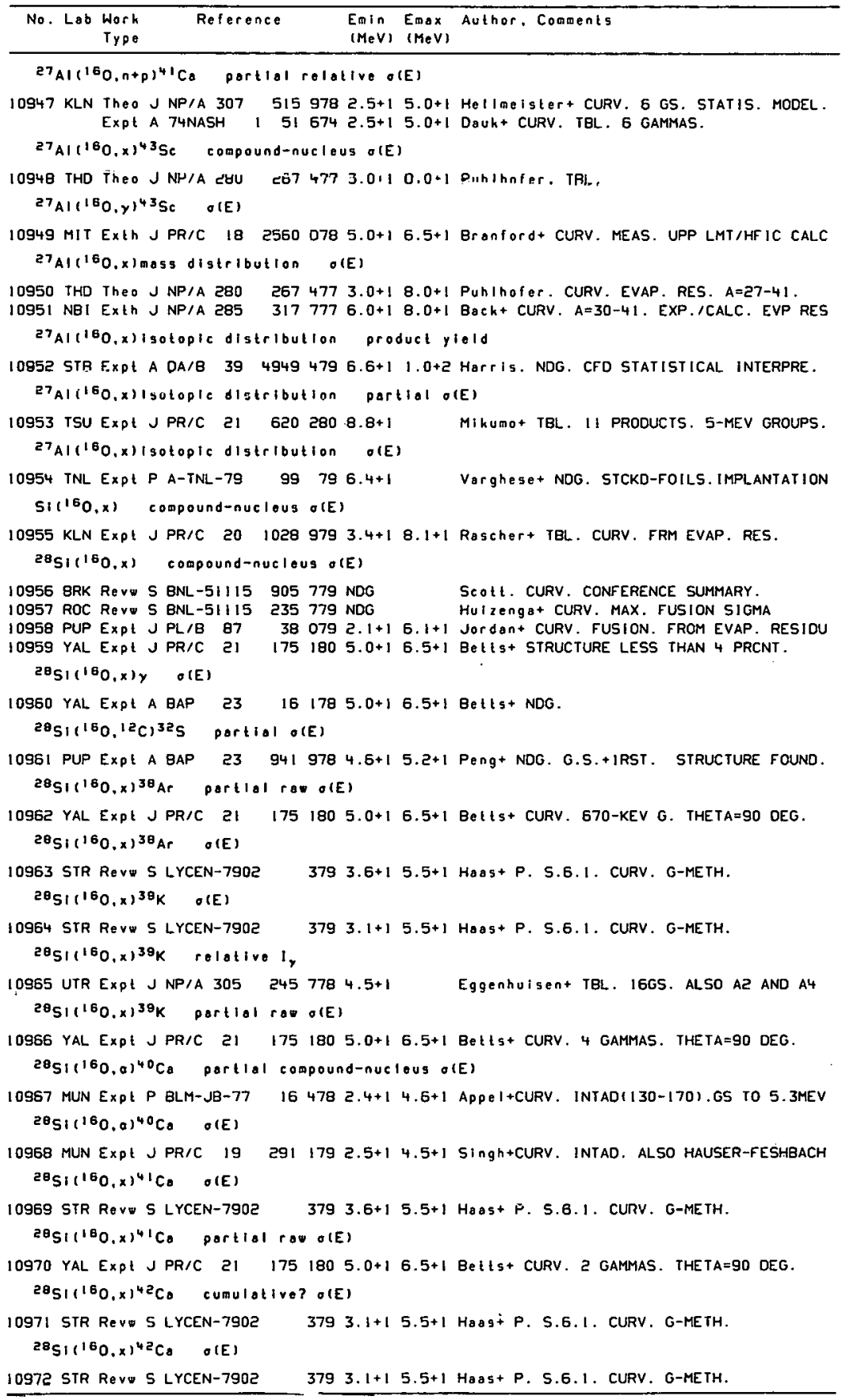


REFERENCES ( con t )

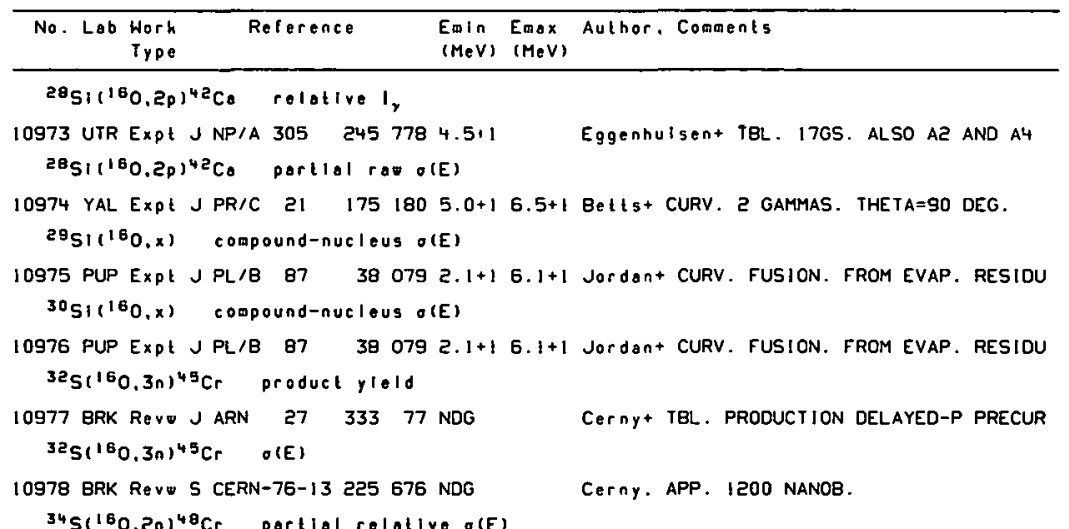




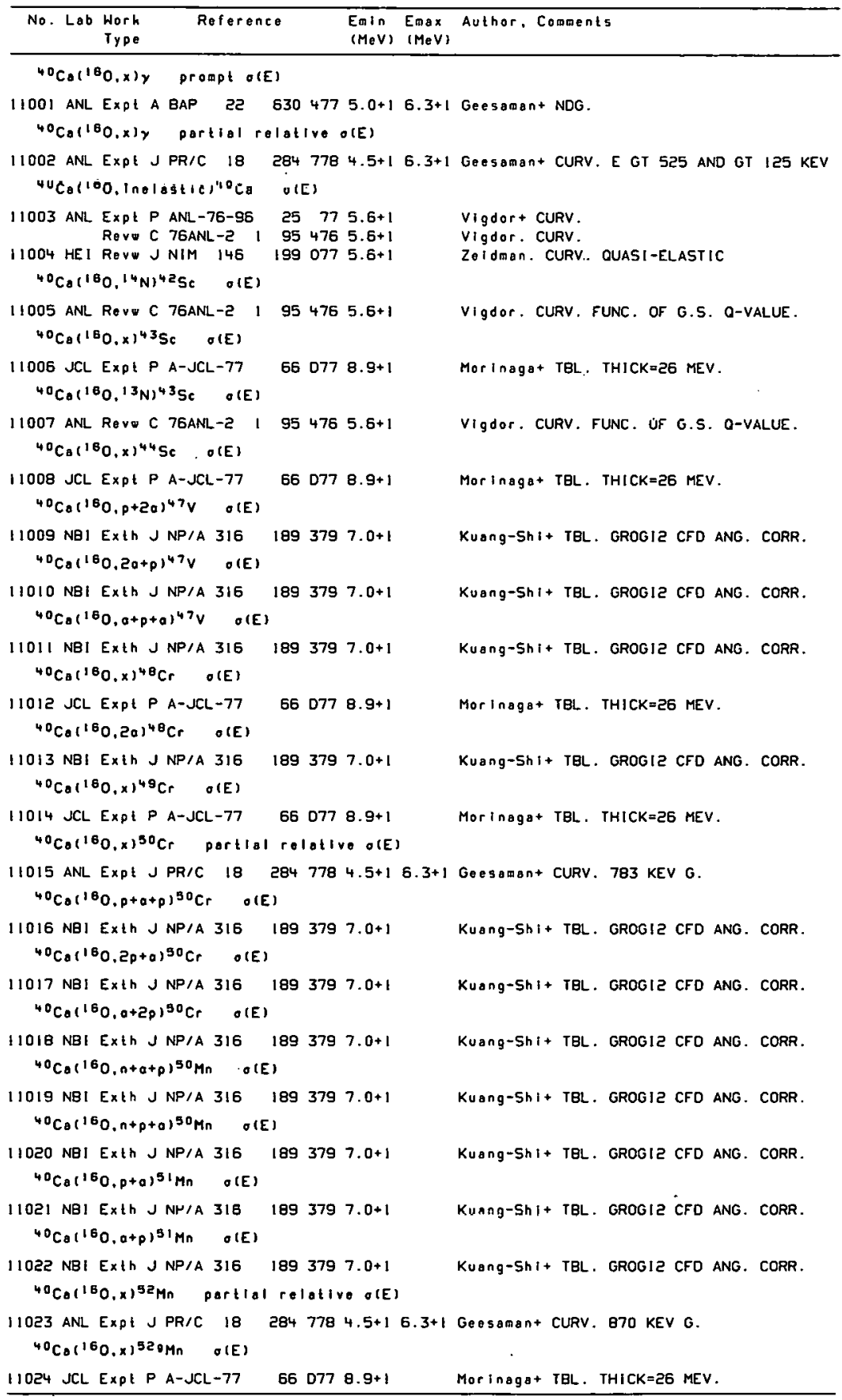




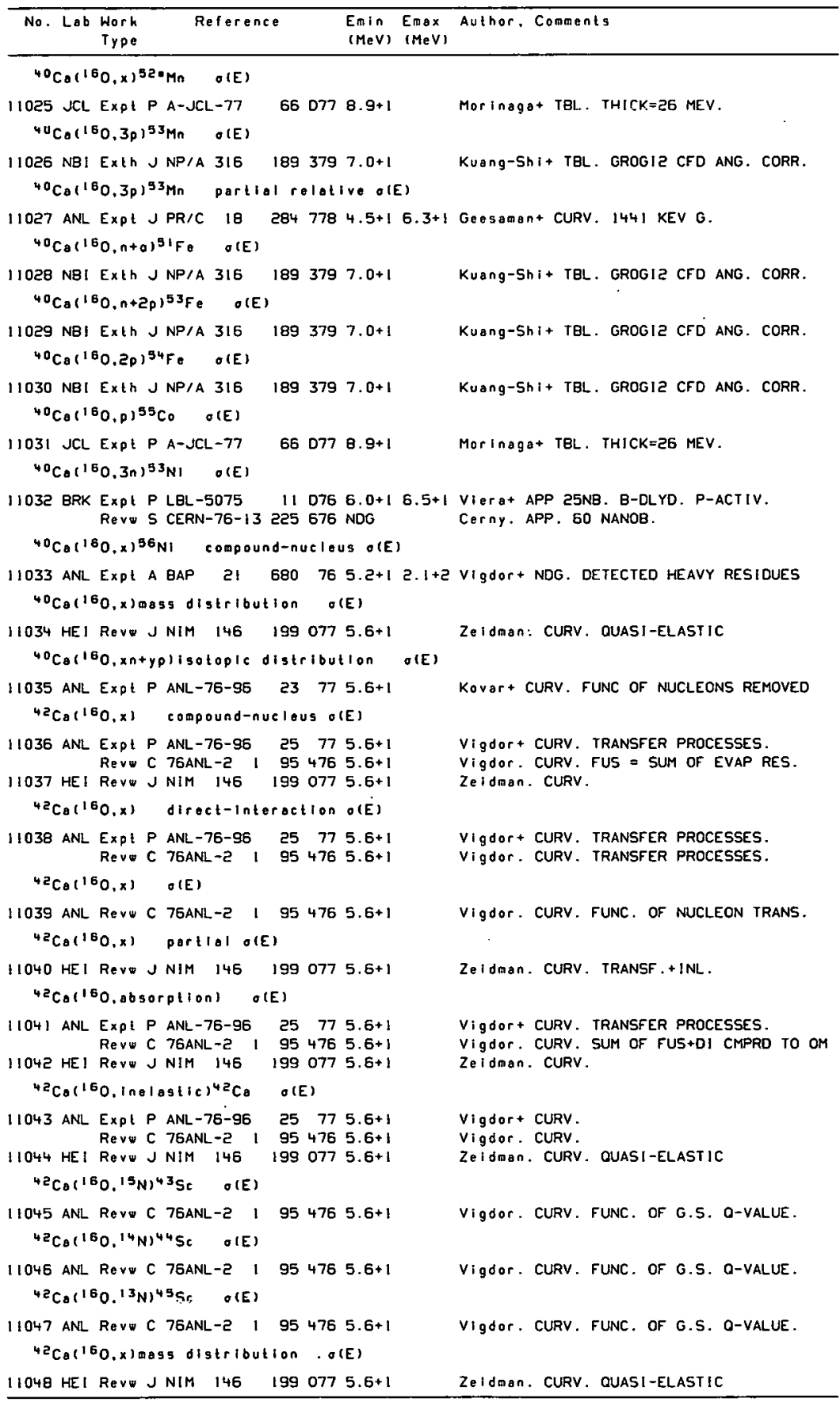


REFERENCES ( c on t)

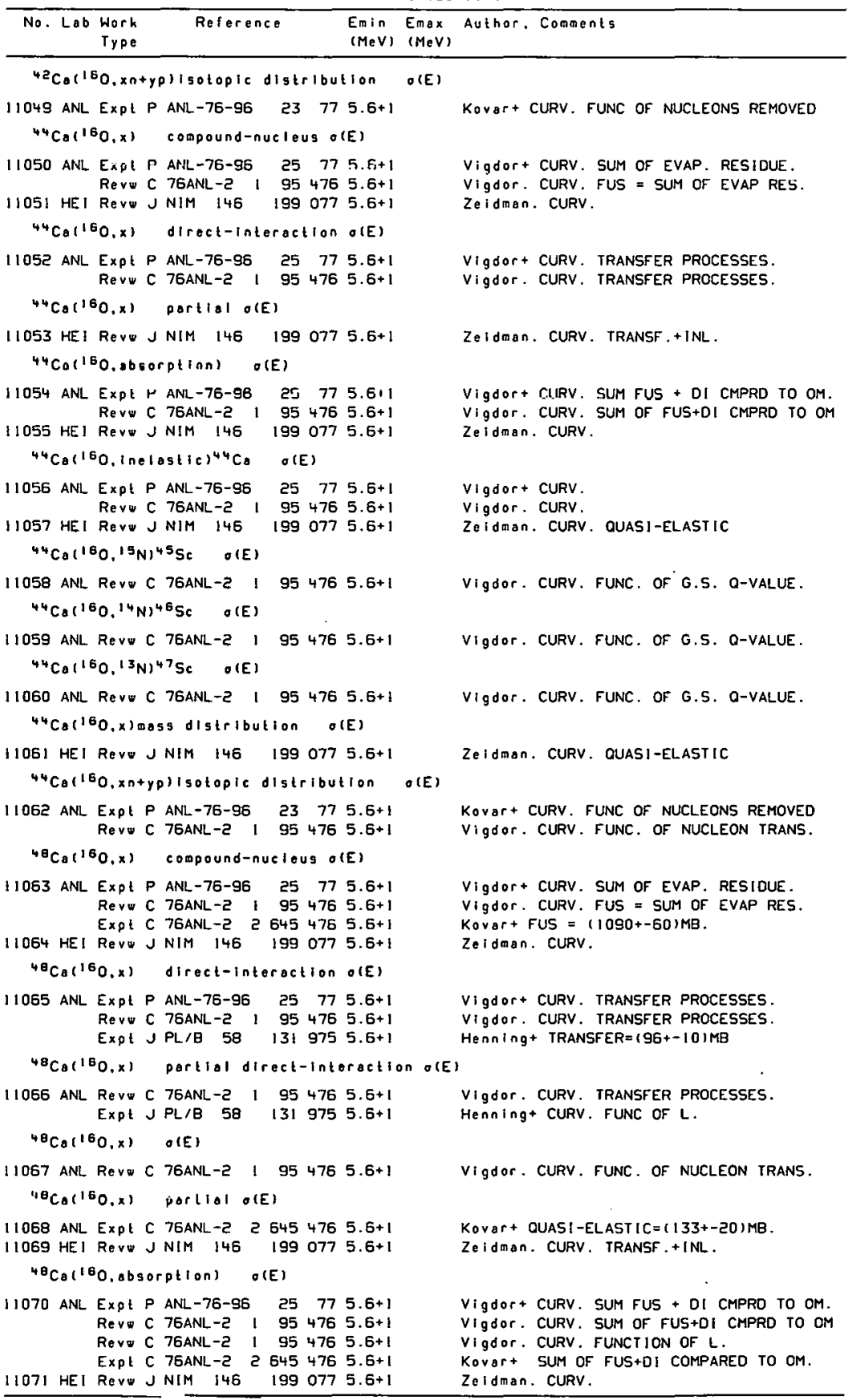




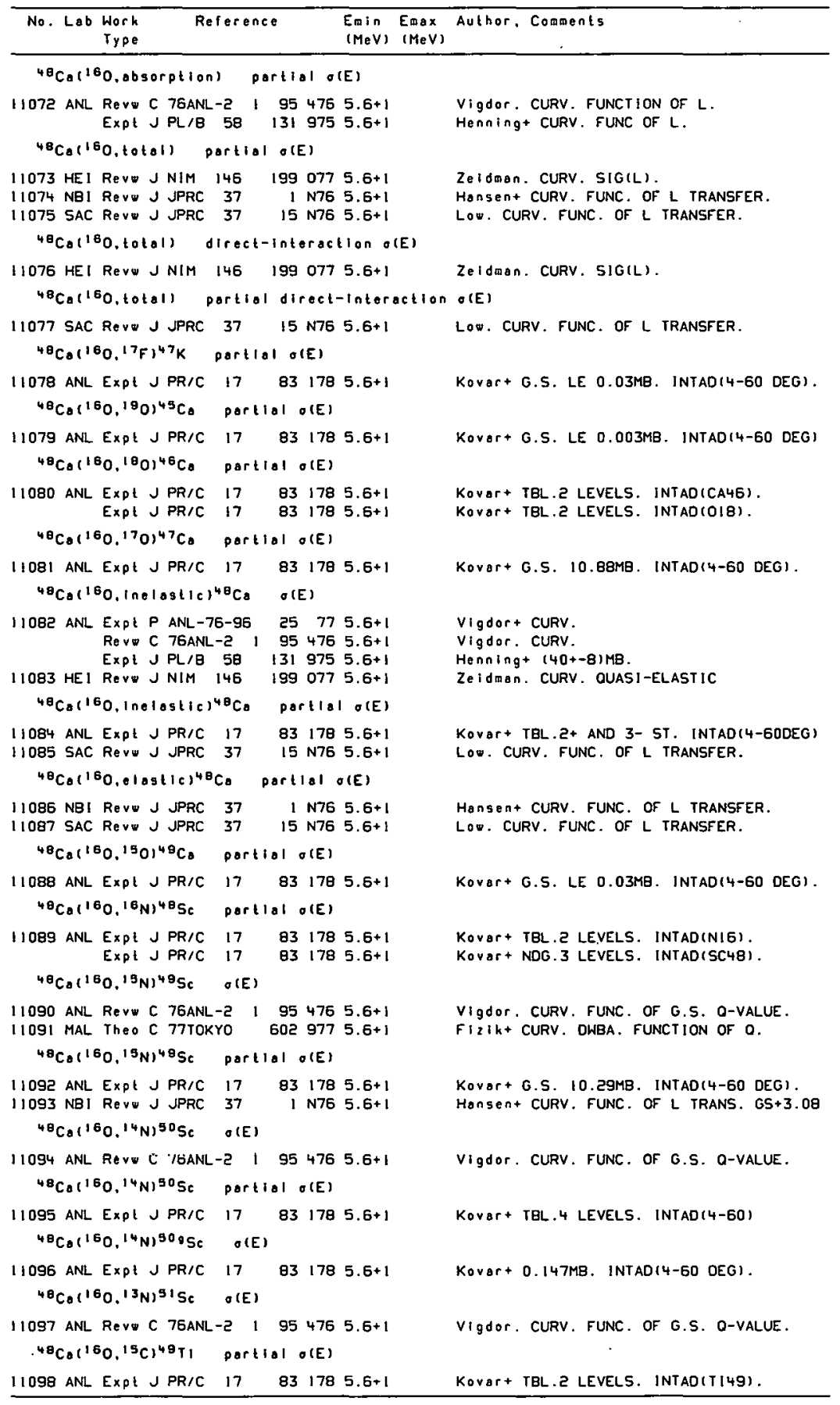




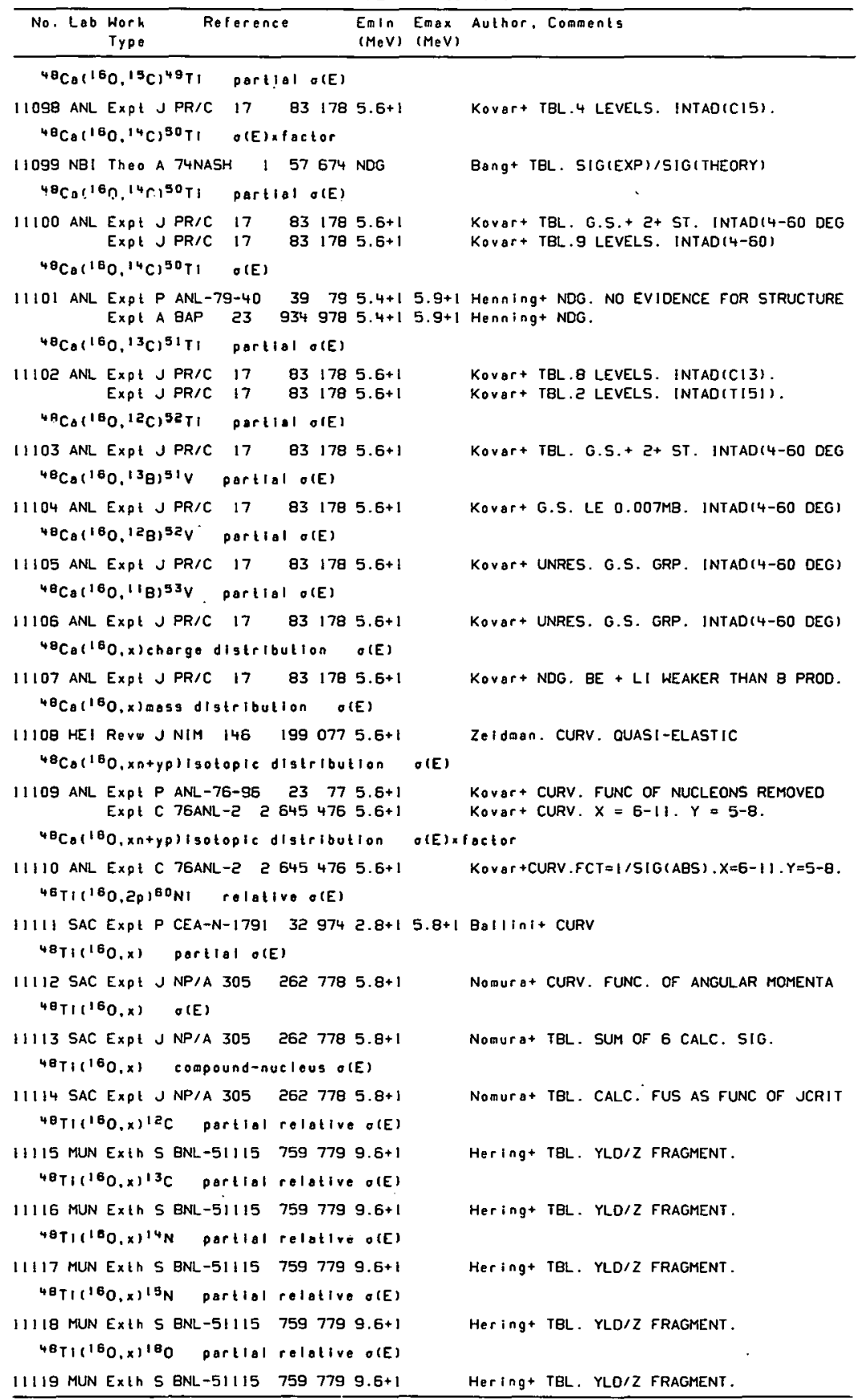




\begin{tabular}{|c|c|c|c|c|c|c|c|}
\hline & Lab & $\begin{array}{l}\text { Work } \\
\text { Type }\end{array}$ & Reference & $\begin{array}{l}\text { Emin } \\
(M e V)\end{array}$ & $\begin{array}{l}\text { Emax } \\
(M e V)\end{array}$ & Author. & Comments \\
\hline
\end{tabular}

${ }^{40} \mathrm{~T}\left(1^{16} 0, x\right)^{18} \mathrm{Ne}_{\mathrm{O}}$ partiol relative O(E)

11120 MUN Exth S BNL-5!115 $7597799.6+$ !

${ }^{40}\left(11^{160}, x\right)^{20}$ No partlol relatlve o(E)

11121 MUN Exth S BNL-51115 $7597799.6+1$

$\left.{ }^{46} T_{(16} 0 . x\right)^{21}$ Ne partial relative o(E)

11122 MUN Exth S BNL-51115 $7597799.6+1$

${ }^{40} \mathrm{~T}\left({ }^{16} 0, x\right)^{22} \mathrm{Ne}$ partiol relativo o(E)

11123 MUN Exth S BNL-5!115 $7597799.6+1$

${ }^{40} \mathrm{~T}$ i $1{ }^{16} 0$, inelastie) $48 \mathrm{TI}$ partiol relotive o(E)

11124 MUN Exth 5 BNL-51115 $7597799.6+1$ Hering+ TBL. YLD/Z FRAGMENT.

$40_{\mathrm{Ti}}(16 \mathrm{O}, x)^{50} \mathrm{Cr}$ partlal rolollvo O(E)

11125 MUN Exth $S$ BNL-51115 $7597799.6+1 \quad$ Hering + CURV. YLD/Z=6 FRAG. J=2-12.

${ }^{40} \mathrm{~T} 1(160 . x)^{50} \mathrm{Cr}$ partiol O(E)x(actor

11126 MUU Expl JPRL 43623 879 9.6+1 Puchla+ CURV. G YLD/Z=6. J(50CR)=2-12. ${ }^{40} \mathrm{Ti}\left({ }^{16} 0, x\right)^{60} \mathrm{Ni}$ partial o(E)xfactor

11127 SAC Expt J NP/A 305 262 $7785.8+1 \quad$ Nomurat TEL. TI48+016/CR52+CI2. 4GS. ${ }^{48}$ Ti $(160, x)$ lsotople disteibution o(E)xfactor

11128 SAC Expl J NP/A $3052627785.8+1 \quad$ Nomuro+ TBL. T14B+016/CR52+CI2. 6 PROD 4BTI( $\left.{ }^{16} 0, x\right) 190 t o p i c$ distribution relative olE)

11129 SAC Expl J NP/A $3052627785.8+1 \quad$ Nomura+ TBL.REL TO NI60.6 PROD ${ }^{40} T i(160, x) 1 s 0 t 0 p i c$ distribution O(E)

III30 SAC ExplJ NP/A 305 262 $7785.8+1 \quad$ Nomura+ TEL. CALC, AS FUNC OF JCRIT 40T1(160.x)lsotople distribution rolative o(E)xfactor

11131 SAC Exth C 77TOKYO $6669775.8+1 \quad$ Nomura+ TBL. CR52+C12122T148+016 RATI0 $51 v(160 . x)$ direct-interaction o(E)

11132 GRE Expt J NP/A 274 $2230767.7+1$ Chevarlert FRM ANG. DIST. (.45+- .07)B 51 Vitio, compound-nucleus o(E)

11133 GRE Expl J NP/A $274 \quad 223$ D76 7.7+1 Chevarler+ FRM TOT AND D1. (1.09+-.07)B S'vico,totoli $O(E)$

11134 GRE Expt J NP/A $2742230767.7+1$ Chevarier+ CALC. FRM ELAS. 1.540B. 0-M. 51 icio,x)y thich torget yiold

11135 ORL Expt J PR/C $1622590773.6+14.6+1$ WeIIs+ NDG. GAMMA YIELD CURVES. 31 vit $\left.{ }^{6}, x\right)^{4} H_{0}$ direct-lialoraction $O(E)$

11/36 GRE Exp! J NP/A 274 223 D76 7.7+1 Chevarler+ $10.080+-0.024 / 8$ ${ }^{91}\left({ }^{160}, n+2 p\right)^{64} \mathrm{Cu}$ thich torget y1010

11137 ATH Expt P NEANDCIORI $147753.2+14.0+1$ Paradel I Is+ NDG. MAIN CHANNEL. P. 20. sivitio $0.2 n+p)^{64} Z n$ partial thick larget ylelo

11138 ORL Expt J PR/C 16 2259 $0773.6+14.6+1$ WeIIs+ NDG. GAMMA YIELD CURVES. $51 v(160.2 n+p)^{64} Z n$ thick torget yleld

11139 ATH Expt P NEANDClOR!147 $753.2+14.0+1$ Paradeli Is+ NDG. MAIN CHANNEL. P. 20. Sivilou, n+pjos Zn thlch targot yiold

11140 ATH Expt P NEANDCIORII47 $753.2+14.0+1$ Parodellis+ NDG. MAIN CHANNEL. P. 20. 5 ivito.2nj65G thick target yloid

11141 ATH ExpI P NEANDC(OR) $147753.2+14.0+1$ Parodel $115+$ NHS. MAIN GHANHEL. P. 20. SIVIIGU,x)charge distribulion $0(E)$

11142 GRE EXPl J NP/A 274 223 076 7.7+1 Chevarter+ CURV. Z = 3 T0 9.

$\left.{ }^{53} \mathrm{Cr} 1^{160}, n+p\right)^{67} \mathrm{Ga} \quad l_{y}$

$11 ! 43$ LVP Expt J JP/G 4 1611078 3.5+1 5.1+1 AI-Naser+ NDG. G-RAY EXCIT. 
REFERENCES ( con $t$ )

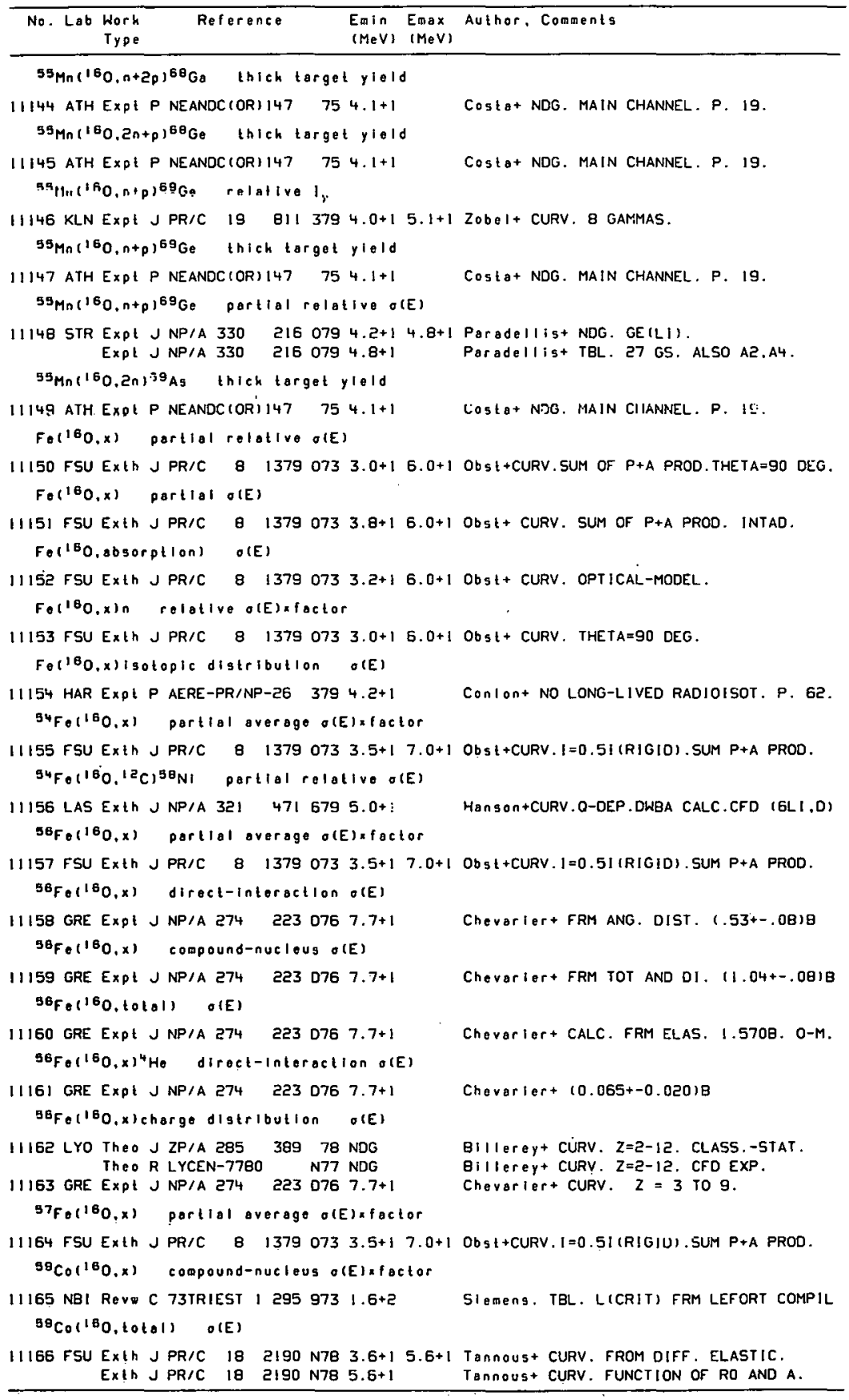




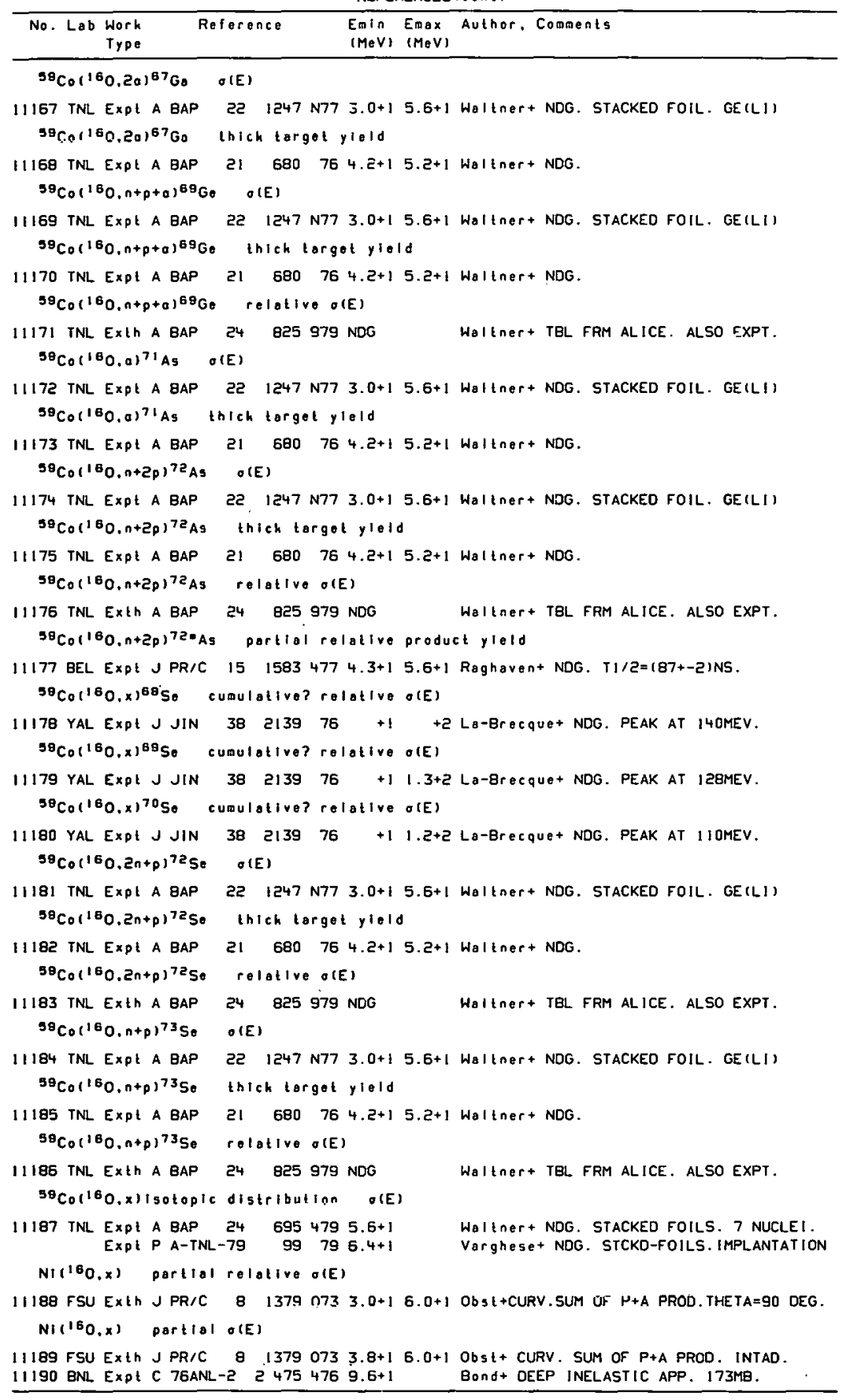


REFERENCES ( $c$ on $t)$

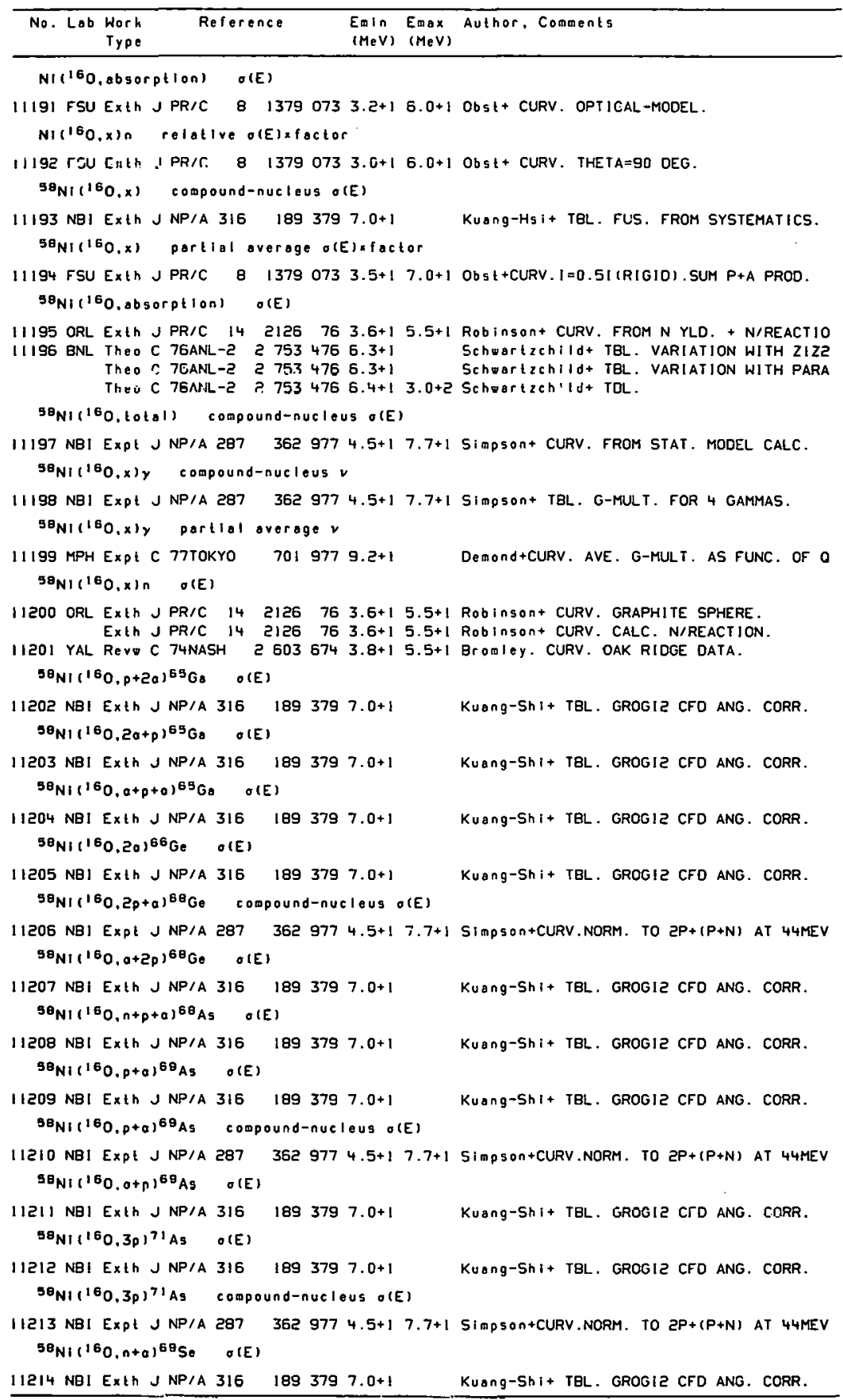


REFERENCES ( c on t )

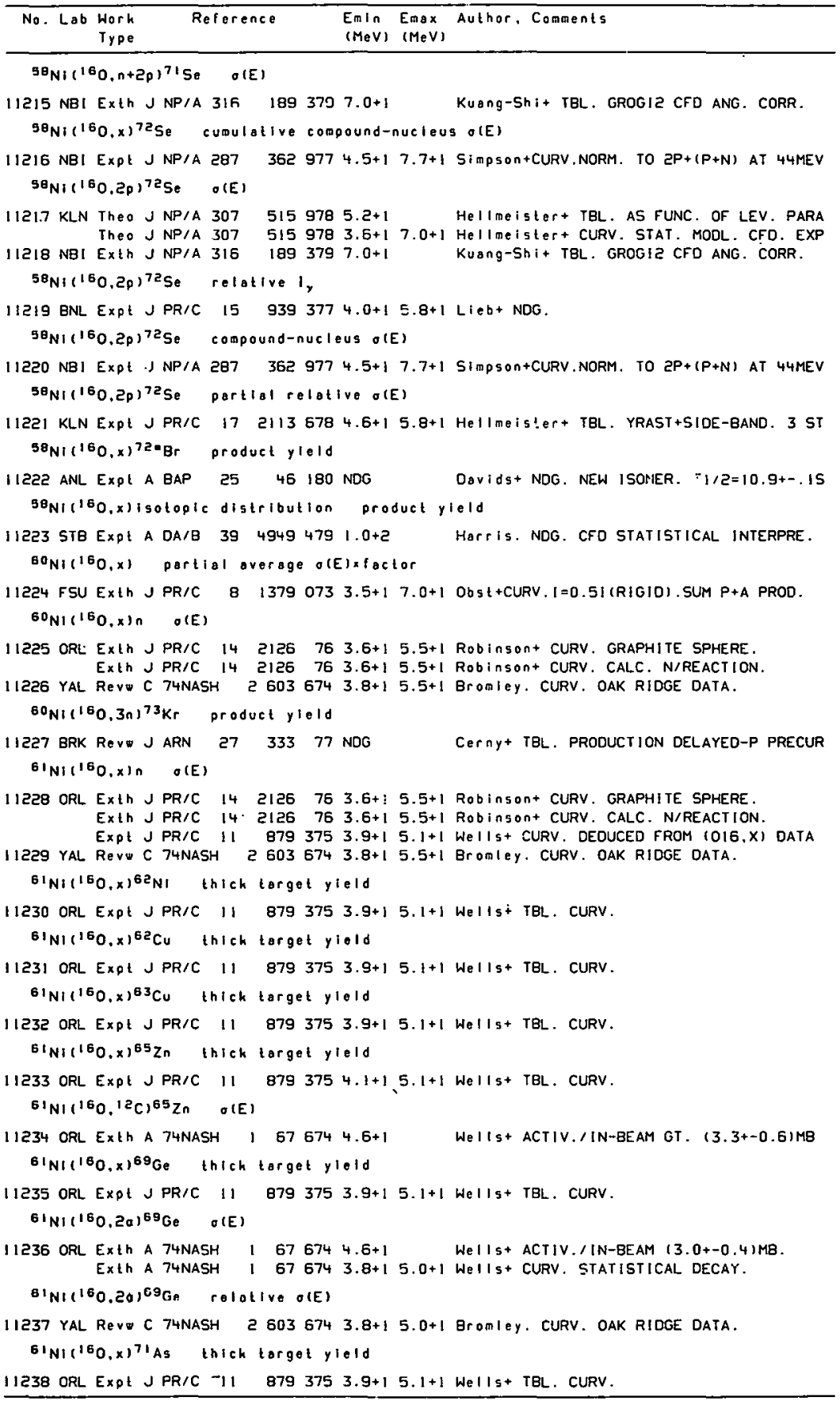




\begin{tabular}{|c|c|c|c|c|c|c|c|}
\hline No & - Lab & $\begin{array}{l}\text { Worh } \\
\text { Type }\end{array}$ & Reference & $\begin{array}{l}\text { Em in } \\
(\mathrm{MeV})\end{array}$ & $\begin{array}{l}\text { Emax } \\
(M e V)\end{array}$ & Author. & Comments \\
\hline
\end{tabular}

BINI $1180, n+p+a) 71$ AS O(E)

11239 ORL Exth A 74NASH $1676744.6+1$ WeIIs+ ACTIV./IN-BEAM $(11.6+-1.4) M B$.

BINI $(160, x)^{72}$ As thick torgot yield

11240 ORL Expt J PR/C $118793753.9+15.1+1$ WeIIs+ TBL. CURV.

${ }^{6} \mathrm{~N} 1\left({ }^{16} O, p+q\right)^{72}$ AS $O(E)$

11241 ORL Exih A 74NASH I $676743.8+15.0+1$ WeIIs+ CURV. STATISTICAL DECAY. Ex:h A 74NASH I $676744.6+1$ WeIIs+ ACTIV.IIN-BEAM $(35+-4)$ MB.

$61^{N}\left({ }^{16} 0, p+a\right)^{72}$ As relolive o(E)

11242 YAL REV C 74NASH $26036743.8+15.0+1$ Bromley. CURV. OAK RIDGE DATA.

$61 \mathrm{Ni}(160,3 p)^{74} \mathrm{As}$ thich targot yleld

11243 ORL Expl J PR/C II $8793753.9+15.1+1$ Wel Is+ IBL. CURV.

6'NI1100.3PI"AS O(E)

11244 ORL Exth A 74NASH $\quad 676743.8+15.0+1$ WeIIs + CURV. ITATISTICAL DECAY Exth A 74NASH $1676744.6+1$ Wells + ACTIV.IIN-EEAM $15.6+-1.9$ ) MB.

$61 N 1(160.30)^{74}$ AS rOIOtIVO O(E)

11245 YAL ReV C $74 \mathrm{NASH} 26036743.8+15.0+1$ Bromley. CURV. OAK RIDGE DATA.

GINI(160,x)?'se thtch targat yleld

11246 ORL Expt J PR/C I1 $8793753.9+15.1+1 \mathrm{We} 11 \mathrm{~g}+\mathrm{TBL}$. CURV.

$61 N 1(160, x)^{72}$ Se thich targot yield

11247 ORL EXPE J PR/C $118793753.9+15.1+1$ WOIIg+ TBL. CURV.

$61^{N} 1(160, n+a)^{72}{ }^{2}$ e o(E)

11248 ORL Exth a 74NASH I $676743.8+15.0+1$ Weilgt CURV. STATISTICAL DECAY. Exth A 74NASH $1676744.6+1$ WeIIs+ ACTIV./IN-BEAM $(45+-6) \mathrm{MB}$.

$61 N i(160, n+a) 725$ relative o(E)

11249 YAL REN C 74NASH $26036743.8+15.0+1$ Bromley. CURV. OAK RIDGE DATA.

$\left.{ }^{6} N{ }^{16} 0, x\right)^{73}$ Se thlek target yield

11250 ORL Expt J PR/C 1 I $8793753.9+15.1+1$ Wellst TBL. CURV.

EINI(160,a) ${ }^{73} S_{0}$ O(E)

11251 ORL Exth A 74NASH I $676744.6+1$ Hellst ACTIV. I IN-BEAM $10.7+-0.5$ IMB.

GINI(160,x) ${ }^{74}$ Se thlch torget ylold

11252 ORL Expt J PR/C II $8793753.9+15.1+1$ Wells+ TBL. CURV.

$61_{N 1}(160, n+2 p)^{74} S_{0} \quad \sigma(E)$

11253 ORL Exth A 74NASH I $676744.5+1$ Wells + ACTIV.IIN-BEAM (95+-15)MB.

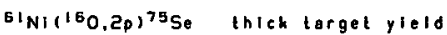

11254 ORL Expl J PR/C II $8793753.9+15.1+1$ Wells+ TBL. CURV.

$61 \mathrm{Ni}\left({ }^{16} 0.2 n+p+n\right)^{73} \mathrm{Br} \quad \sigma(E)$

11255 ORL ExIh A 74NASH $1676744.6+1$ WeIIs+ ACTIV., IN-BEAM $(145+-15) M B$

$61^{N}\left(^{18} 0, x\right)^{74}$ Or thieh target yleld

11255 ORL Expt J PR/C $11 \quad 9793753.9+15.1+1$ Wel1s+ TBL. CURV.

${ }^{1} \mathrm{Ni}\left({ }^{16} \mathrm{O} .2 \mathrm{2n}+\mathrm{p}\right)^{74} \mathrm{Br} \quad \mathrm{O}(\mathrm{E})$

11257 ORL Exth A 74NASH I $676744.6+1$ HeIIs+ ACTIV./IN-BEAM $118.1+-3.91 M B$ $6{ }^{\mathrm{Ni}}\left({ }^{160} \mathrm{O}, \mathrm{x}\right)^{75} \mathrm{Br}$ thlck target yiold

11258 ORL Expt JPR/C $118793753.9+15.1+1 \mathrm{We} / 1 \mathrm{~s}+\mathrm{TBL}$. CURV.

$6{ }^{N} \mathrm{~N}\left({ }^{16} \mathrm{O}, \mathrm{D}\right)^{76} \mathrm{Br}$ thteh target yiold

II259 ORL Expt J PR/C $118793753.9+15.1+1$ WeIIs+ TBL. CURV.

$61 \mathrm{Ni}(160.3 \mathrm{n})^{74} \mathrm{Kr}$ thick torget yield

11260 ORL Expt J PR/C $132588764.2+15.0+1$ WeIIS+ TBL. DEDUCED UPPER LIMITS.

$61 \mathrm{NI}(160,3 n)^{74} \mathrm{Kr} \quad$ O(E)

11281 ORL Exth A 74NASH I $676744.6+1$ WEII + ACTIV..IN-BEAM 17.0+-1.8IMB 
REFERENCES (con)

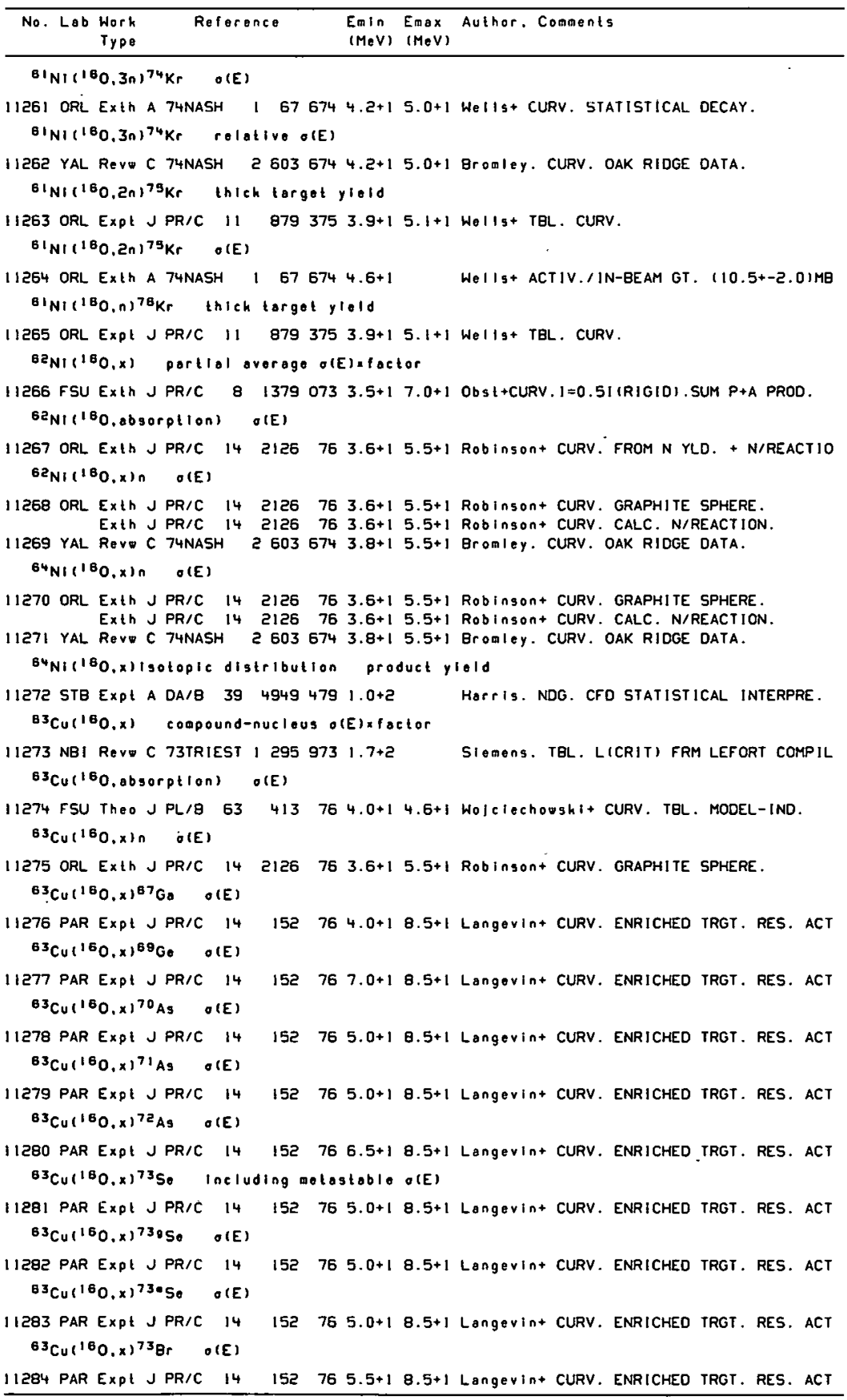


REFERENCES ( c on t)

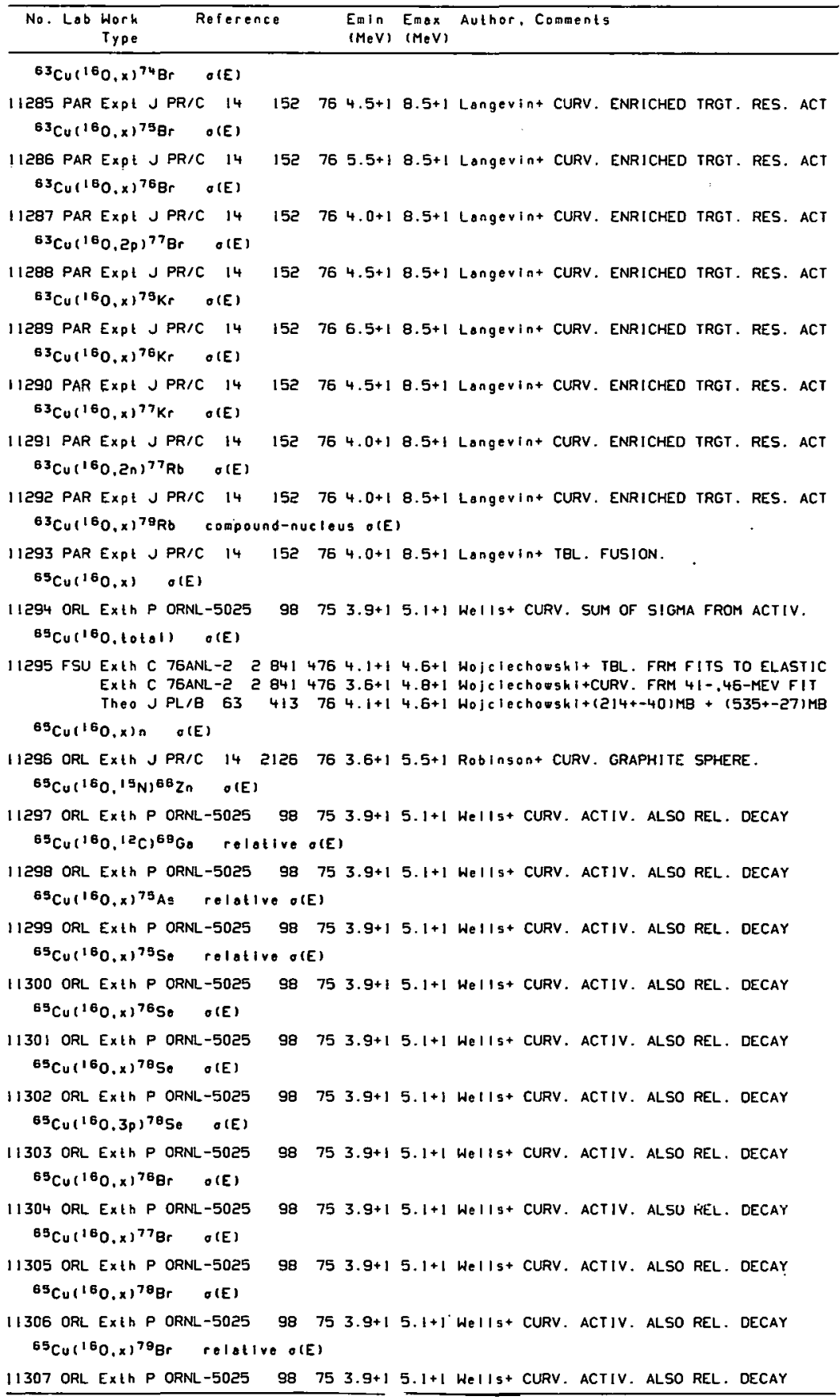




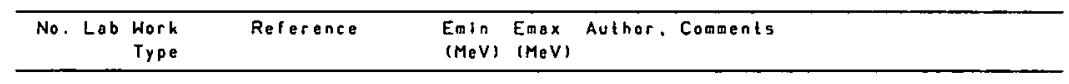

${ }^{65} \mathrm{Cu}\left({ }^{16} \mathrm{O}, \mathrm{Ep}^{\mathrm{T}}\right)^{79} \mathrm{Br} \quad \sigma(\mathrm{E})$

11308 ORL Exth P ORNL-5025 $99753.9+15.1+1$ Wel $15+$ CURV. ACTIV. ALSO REL. DECAY

${ }^{65} \mathrm{Cu}\left({ }^{16} 0.2_{n+p}\right)^{78} \mathrm{Kr}$ partial relative o(E)

$11309 \mathrm{KLN}$ Expt J NP/A $3322410794.2+15.8+1$ Hell me ister+CURV.G.5.-.OCTUPOLE-,GAMMA${ }^{65} \mathrm{Cu}\left({ }^{16} \mathrm{O}, \mathrm{x}\right)^{70_{\mathrm{Kr}}}$ O(E)

11310 ORL ExIh P ORNL. $502598753.9+15.1+1$ WeIIs+ CURV. ACTIV. ALSO REL. DECAY

${ }^{65} \mathrm{Cu}\left({ }^{16} \mathrm{O}, 3 \mathrm{n}\right)^{7 \theta_{\mathrm{Rb}}} \quad \mathrm{O}(\mathrm{E})$

11311 ORL Exth P ORNL-5025 $98753.9+15.1+1$ We $113+$ CURV. ACTIV. ALSO REL. DECAY

${ }^{65} \mathrm{Cu}\left({ }^{16} \mathrm{O}, \mathrm{Sn}^{\mathrm{n}}\right)^{78_{\mathrm{Rb}}} \quad \mathrm{O}(\mathrm{E})$

11312 ORL Exth P ORNL-5025 $98753.9+15.1+1$ Wells+ CURV. ACTIV. ALSO REL. DECAY

${ }^{65} \mathrm{Cu}\left({ }^{16} 0, x\right) 150 t 0 p l s$ distribution $O(E)$

11313 SAC EXP! P CEA-N-2070 $644784.6+1 \quad$ BoliIni+ TEL. FROM JULIAN-SUPERVAP CODE

$\left.{ }^{64} \mathrm{Zn} 1^{160} \mathrm{O}, \mathrm{x}\right) \mathrm{n} \quad \mathrm{O}(\mathrm{E})$

11314 ORL Exth J PR/C 14 2I26 $763.6+15.5+1$ Robingont CURV. GRAPHITE SPHERE.

${ }^{68} \mathrm{Zn}(160$, fissions cumulative fission ylold

11315 HEI Expt A 76ANL-2 2583476 NDG Gelbke+ NDG. WORK IN PROGRESS.

${ }^{66} \mathrm{Zn}(160, x) \cap \quad O(E)$

11316 ORL Exth J PR/C $142126763.6+15.5+1$ Robinsont CURV. GRAPHITE SPHERE.

${ }^{67} \mathrm{Zn}\left({ }^{18} 0, x\right) \cap \quad O(E)$

11317 ORL Exth J PR/C $142126763.6+15.5+1$ Roblngont CURV. GRAPHITE SPHERE.

${ }^{68} \mathrm{Zn}(160 . x \ln \quad \sigma(E)$

11318 ORL Exth J PR/C $142126763.6+15.5+1$ Robinson+ CURV. GRAPHITE SPHERE.

$70 \mathrm{Zn}(160, x) n \quad O(E)$

11319 ORL Exth J PR/C I4 $2126763.6+15.5+1$ Robinson+ CURV. GRAPHITE SPHERE.

Ge(160,x) partial rolative o(E)

11320 FSU Exth JPR/C B 1379073 3.0+1 6.0+1 Obst+CURV.SUM OF P+A PROD. THETA=90 OEG. Ge(160.xin relative o(E)x factor

11321 FSU Exth J PR/C $81379073 \quad 3.0+1$ 6.D+1 Obst+ CURV. THETA=90 DEG. Zriteo,x) partiat relativo o(E)

11322 FSU Exth J PR/C $81379073 \quad 3.0+1 \quad 6.0+1$ Obst+CURV.SUM OF P+A PROD. THETA=90 DEG. Zriteo, $x)^{16}$ relative o(E)nfactor

11323 FSU Exth J PR/C $81379073 \quad 3.0+16.0+1$ Obst+ CURV. THETA=90 DEG.

$02 z$ z $160, x)$ isotoplc distribution product yleld

11324 PUR Expl J PR/C $2013500795.4+16.6+1$ POPII+ NDG. IN-BEAM G. $92 \operatorname{Zr}\left({ }^{16} 0, x\right)$ isotopic digtribution relative o(E)

11325 PUR EXPI J PR/C $2013500796.0+1 \quad$ POPII+ TEL. IN-BEAM G. ${ }^{94} \mathrm{Zr}(160,4 n)^{106} \mathrm{Cd} \quad I$,

11326 PUR Expl J PR/C $19 \quad 731796.3+1 \quad$ Samuelsont NOG. PRELIMINARY. ${ }^{96} \mathrm{Zr}\left({ }^{10} 0,4 n\right)^{100} \mathrm{Cd}$ relative I,

11327 PUR Expt J NP/A $301 \quad 1595785.6+1 \quad$ Samuel Bont TEL. 51 GS. THETA=I25DEG.

${ }^{93} \mathrm{Nb}\left({ }^{16} \mathrm{O}, y\right)^{109} \mathrm{In} \quad \sigma(E)$

11328 MIT Exth J PR/C $1825600785.0+16.5+1$ Branford+ CURV. MEAS. UPP LMT/HFIC CALC

$9 \mathrm{Mo}(160 . x)$ compound-nucleug $O(E)$

II329 PAR ROVW J APH 3 J $781.9+2$ I.efort + TBL. FUS. COMP. EXP+CALC.

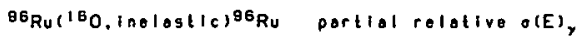

11330 LVP Expt J JP/G $31735773.3+1 \quad$ Maynard+TBL. $(2+-0+1 G$ YLO. REL TO RUIOC

${ }^{98}$ Ru ( 180, inelost 1 e) ${ }^{80}$ Ru partial relative o(E),

11331 LVP Expk J JP/G $31735773.3+1 \quad$ Maynard+TBL. $(2+-0+1 G$ YLD. REL TO RUIOC 
REFERENCES (con)

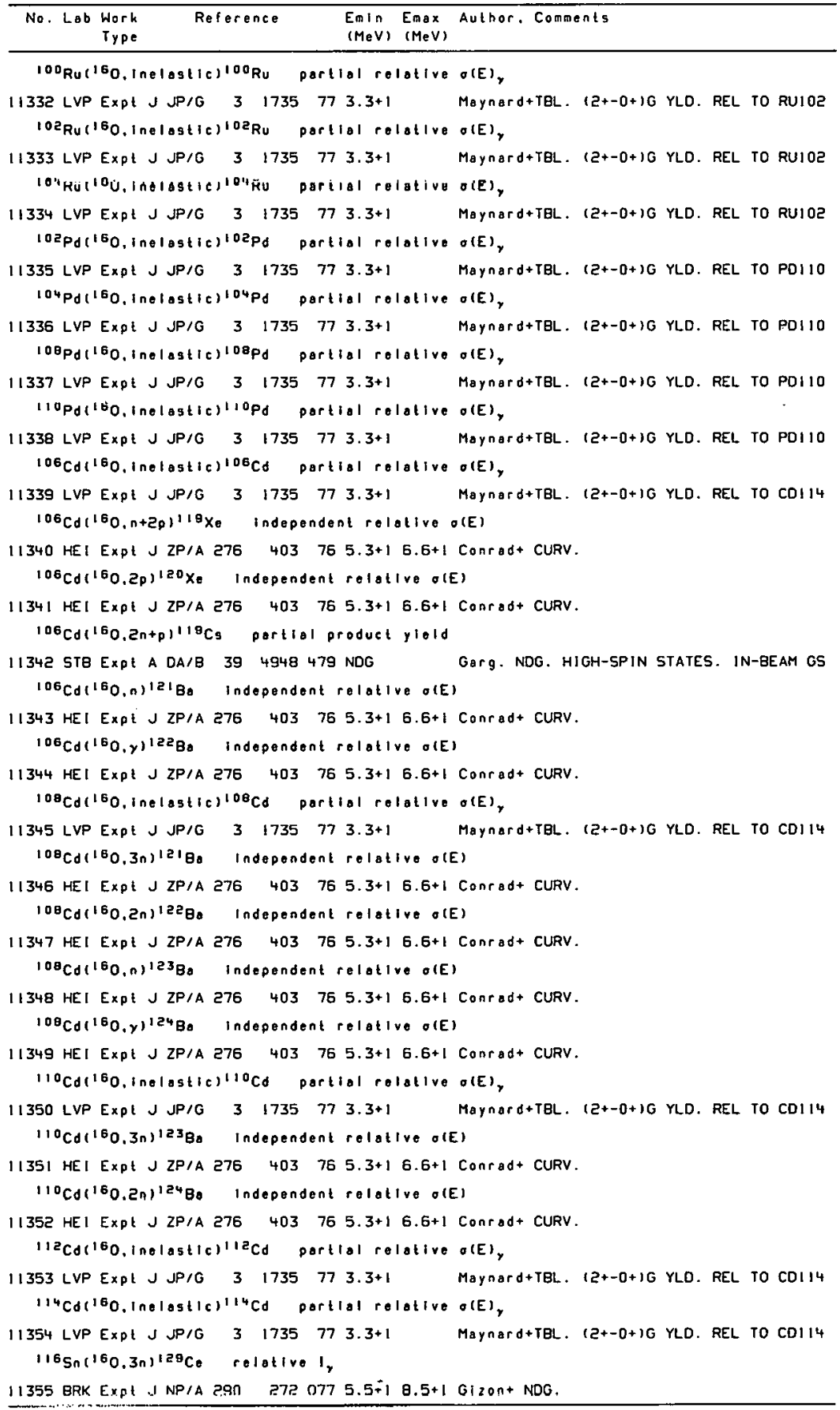




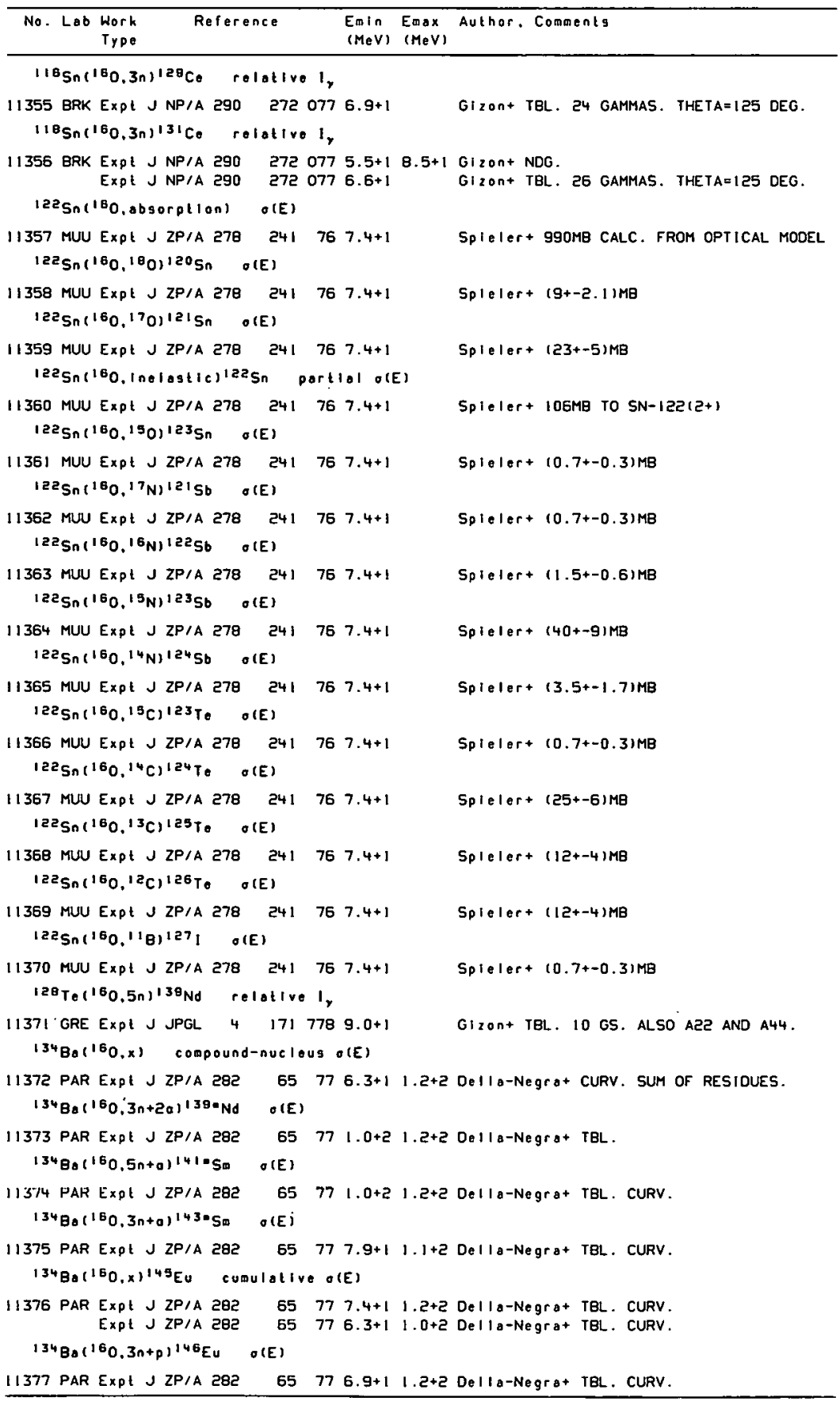




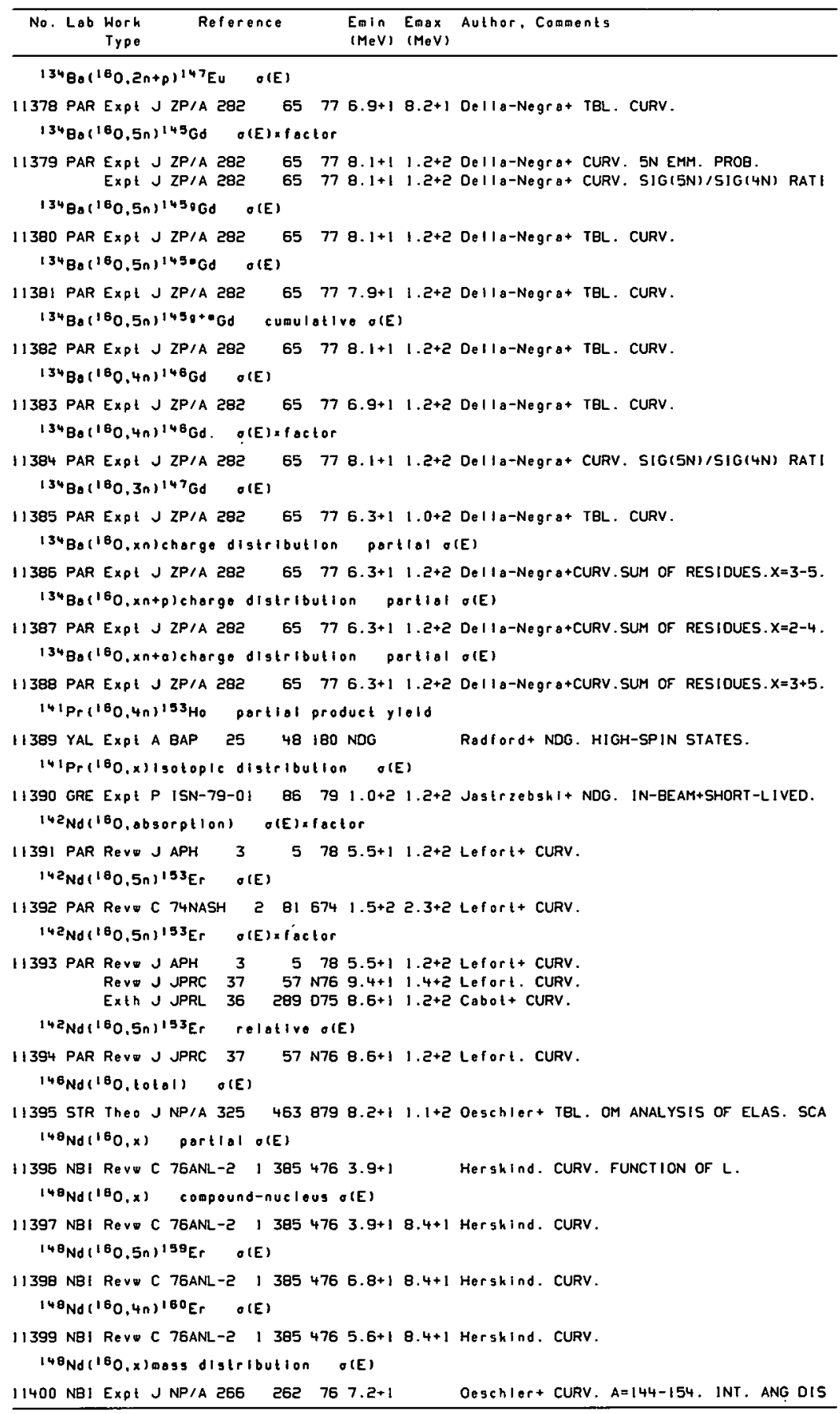




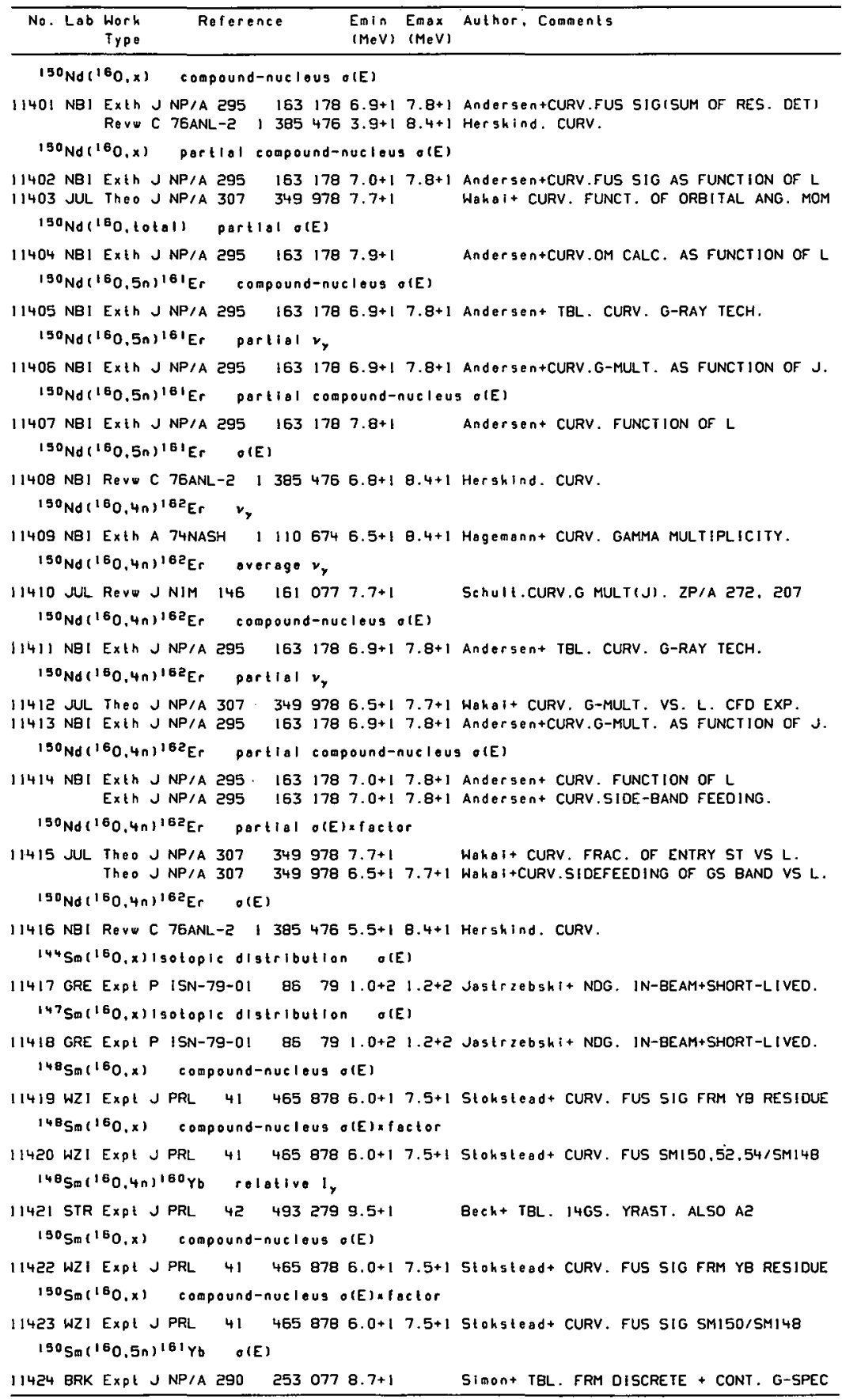


REFERENCESic on t

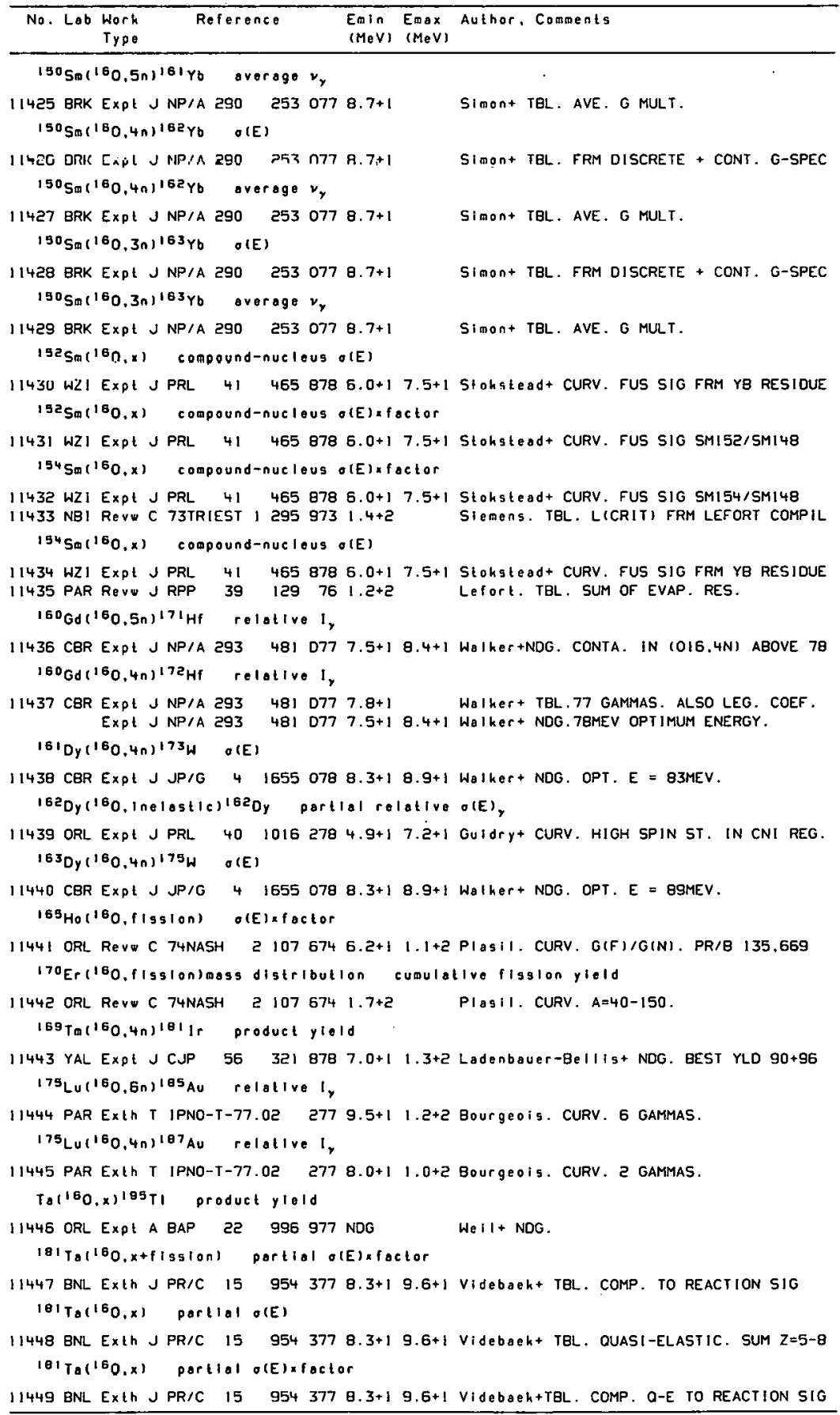




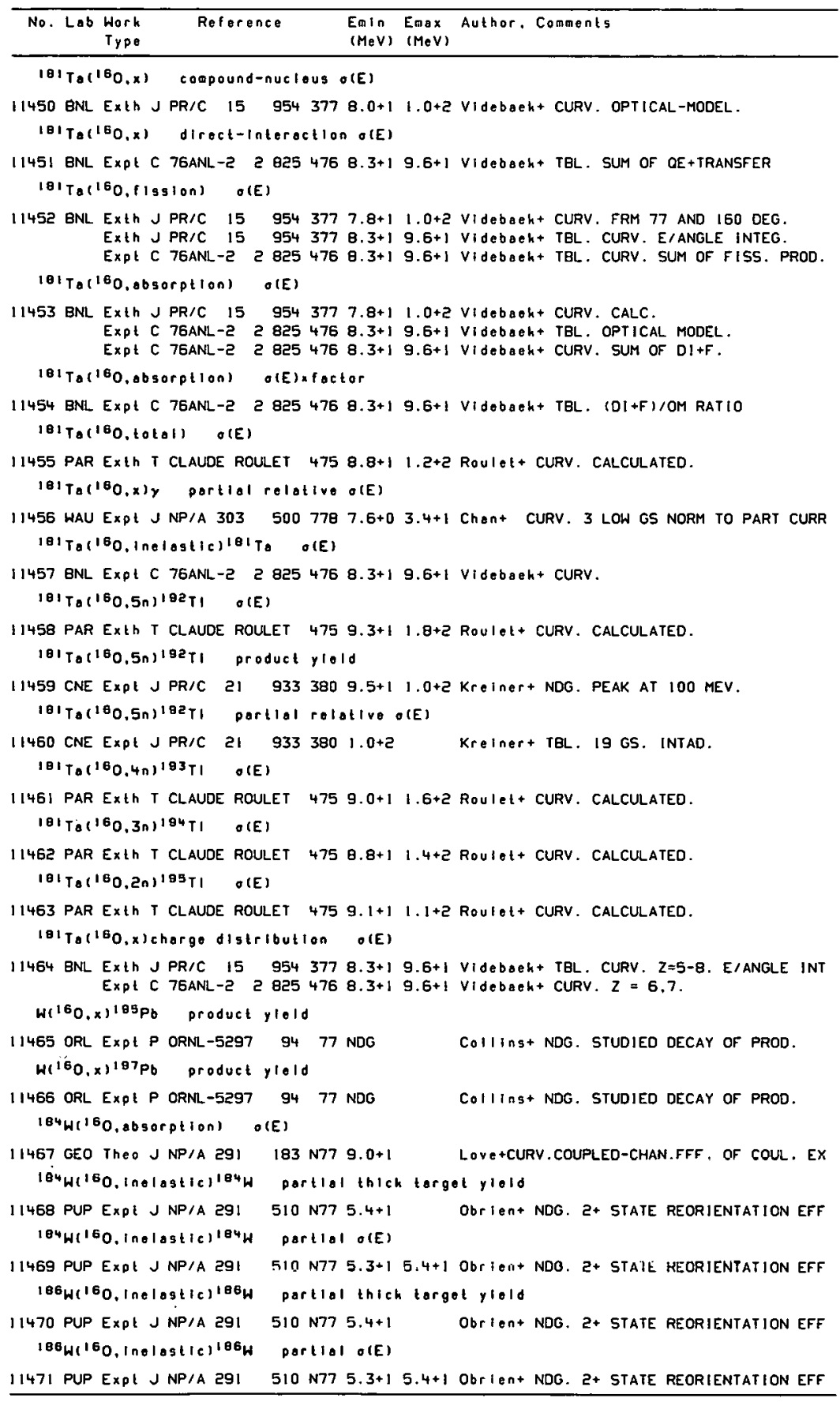


REFERENCES ( c ont)

\begin{aligned} & \hline No. Lab Work Reference Emin Emax Author, Comments \\ & Type (MeV) (MeV) \\ & \hline\end{aligned}

Re( $\left.{ }^{16} 0, x\right)^{195} \mathrm{~Pb}$ product yiold

11472 ORL Expl P ORNL-5297 $94 \quad 77$ NDG

Collins+ NDG. STUDIED DECAY OF PROD.

Re(180,x) ${ }^{197} \mathrm{~Pb}$ product yleld

11473 ORL Expt P ORNL-5297 $94 \quad 77$ NDG

Col I Ins + NDG. STUDIED DECAY OF PROD.

$1920 s\left(^{160} 0.1135(0 n)\right.$ O(E)

11474 HAR Expt P NEANDC(E)-2028 479 NDG

$1920 s(160.010 s(1 \mathrm{c}) 1920 \mathrm{~s}$ O(E)

11475 HAR ExpI P NEANDC(E)-2028 479 NDG

1920 ( $1^{16} 0$, I Issionimags distribution

relative o(E)

11476 HAR EXPt P UKNDC-P-88 93678 NOG

GoodaII+ NDG. WORK IN PROGRESS.

197 Au $(160, x)$ compound-nuclous o(E) a factor

11477 NGI Rev甲C 73TRIEST I $2959731.7+2$ SIEMENS. TBL. LICRITI FRM LEFORT COMPIL

197 AU $\left(^{16} 0, x\right)$ compound-nuclous $\sigma(E)$

11478 TRM Theo J NP/A $3292240791.2+21.4+2$ Choudty+ TBL. FUS FRM FF SIG(THETA).

197 Au(160.0+f19510n) O(E)

11479 ENL Exp! A 74NASH. I $956749.3+1$ Wegner+ VANISHINGLY SMALL SIGMA

$197 \mathrm{AU}(160.0)^{209} \mathrm{At}$ O(E)

11480 BNL EXpt A 74NASH I $856749.3+1$

Wegner+ VANISHINGLY SMALL SIGMA

197 AU $(160, x)$ chargo distribution O(E)

11481 KFK Comp \& PH-DAT $15 \quad 10791.4+21.7+2$ Muenze1+ TBL. FROM EXFOR.

11482 YAL ExplJ PR/C $811099731.4+21.7+2$ Eyal+ TBL. Z=3-7. INTEG. OVER E/ANGLE. Expt 4 EXFORBODZI. $771.4+21.7+2$. 10 ENTRIES AT TWO ENERGIES.

197 Au $(160, x)$ isotopic distribution independont o(E)

11483 KFK Comp \& PH-DAT $1510791.4+21.7+2$ Muenzel+ TBL. FROM EXFOR.

11484 YAL Expt J PR/C $811099731.4+21.7+2$ Eyal+TBL.CURV. $Z=79-83 . A=195-203.23150 M$. Expt 4 EXFORBO021. $771.4+21.7+2.74$ DATA PTS. AT TWO ENERGIES.

197 Au( $160, x)$ Isotopic distribution cumulative o(E)

11485 KFK Comp B PH-DAT $1510791.4+21.7+2$ Muenzel+ TBL. FROM EXFOR.

11486 YAL Exp! J PR/C $811099731.4+21.7+2$ Eyal+TBL.CURV.Z=79-84.A=192-205. 14 ISOM. Expl 4 EXFORBO021. $771.4+21.7+2.28$ DATA POINTS. 14 ISOMERS.

197 Au $\left(^{16} 0, x\right)$ Isotopic distribution o(E)

11487 BRK Theo A BAP $2327178+4$ Menchaca-Rocha+ NDG. PERIPHERAL REACT.

$205 T$ I $\left.{ }^{16} O, x\right) 1$ sotoplc distribution O(E)

11488 YAL Expt C 71ALBANY 49771 NDG Gough+ NDG.

$206 P b\left({ }^{16} 0, x\right)$ compound-nucleus $O(E)$

11489 GRA REY S LYCEN-7902 379 8.1+1 9.7+1 FleUry. P. C.7.1. FUSION. CURV.

$200 P_{b}(160 . x+f 1 s g i o n)$ portlal o(E)xfactor

11490 BNL Exth J PR/C I5 $9543778.0+1 \quad 1.0+2$ Videbaeh+ TBL. COMP. TO REACTION SIG

$20 P_{b}(160, x)$ partlal o(E)

11491 BNL Exth JPR/C I5 954377 B.0+1 $1.0+2$ Videbaek+ TBL. QUASI-ELASTIC. SUM Z=5-8 11492 MUN Expt J ZP/A $291179799.0+19.9+1$ Sper + TBL. QUASIELASTIC=INL+TRANSFER.

$200 \mathrm{~Pb}(160, x)$ partial $\sigma(E) \times$ factor

11493 BNL Exth J PR/C 15954377 8.0+1 1.0+2 Videbaek+TBL. COMP. Q-E IO REACTION SIG 11494 MUN EXPt J ZP/A 29117979 9.0+1 9.9+1 Spert+ TBL. QUASIELASTIC/REACTIONIOMI.

$200 \mathrm{~Pb}(16 \mathrm{O}, x)$ compound-nucleus $O(E)$

11495 GRA ReY S LYCEN-7902 $3797.2+1$ 2.2+2 Fleury. P. C.7.1. FUSION. CURV.

11496 BZL Theo J PR/C 18 2152 N78 $7.6+1$ 1.3+2 Vaz+TBL. CURV.SYS.SEMIEMP. ANLYS. OF FUS

11497 BNL Exih J PR/C 15954377 8.0+1 1.0+2 Videbaek+ CURV. OPTICAL-MODEL

11498 BER The J PR/C $20 \quad 992979$ a. $1+11.0+2 \mathrm{Krappe}+$ CURV. UNIFIED POT. CFD EXP.

11499 STB Theo J PR/C 201731 N79 $8.1+12.0+2$ Delegrange+ CURV, OM ANALYSIS.

Theo J PR/C 18 820 $8787.7+12.2+2$ Vaz 2 CURV. ABACUS -3 
REFERENCES (cont)

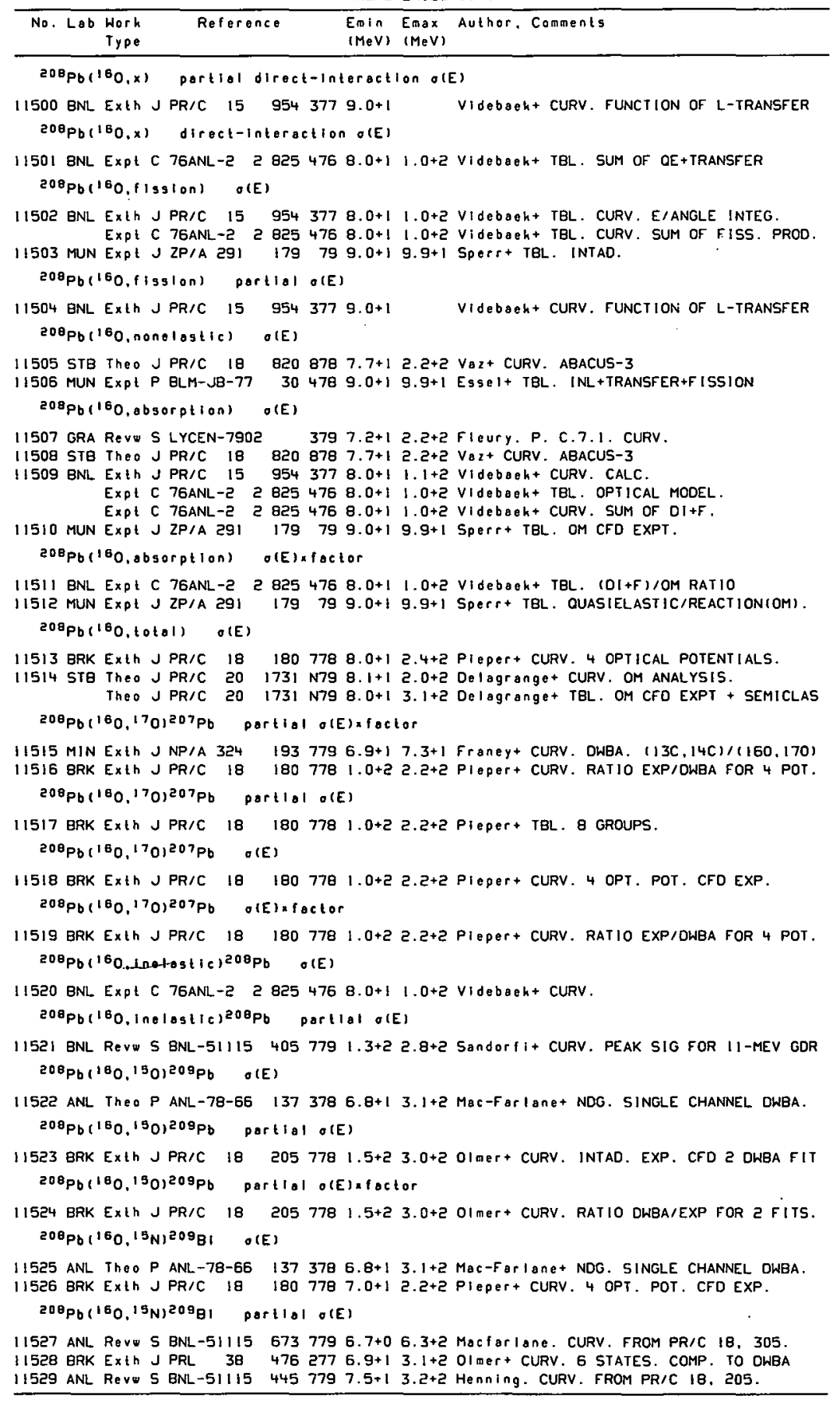


REFERENCES ( $c$ ont)

\begin{tabular}{|c|c|c|c|c|c|}
\hline No. Lab & $\begin{array}{l}\text { Work } \\
\text { Type }\end{array}$ & Reference & $\begin{array}{l}\text { Emin } \\
(M e V)\end{array}$ & $\begin{array}{l}E \max \\
(\operatorname{MeV})\end{array}$ & Author, Comments \\
\hline
\end{tabular}

$208 p_{b}\left({ }^{160} .^{15_{N}}\right)^{209} B 1$ portial o(E)

11530 MSU Revw S BNL-51115 | $7797.5+13.2+2$ Ge1bhe. CURV. PR/C 18, 305. PART. WAVES 11531 MAN ReVy J RPP $40 \quad 345771.0+2 \quad$ PhIIIIIPS. CURV. O-WINDOW. 7/2- LVL.

$200 \mathrm{~Pb}\left(100.1{ }^{10} \mathrm{~N}\right)^{209} \mathrm{~B} 1$ O(E)x foctor

11532 BRK Exth J PR/C $18 \quad 1807787.0+1$ 2.2+2 Pieper+ CURV. RATIO EXP/DWBA FOR 4 POT.

$200_{P O}\left(160,15_{N}\right)^{209}$ el portial o(E) a factor

11533 BRK Exth J PR/C $18 \quad 1807797.0+1$ 2.2+2 Pieper+ CURV. RATIO EXP/OWBA FOR 4 POT.

$208 \mathrm{~Pb}(160,150 \mathrm{~N})^{209} \mathrm{Bl}$ partial o(E)

11534 BRK Exth JPR/C I8 $1807781.0+2$ 2.2+2 Pieper+ TBL. SINGLE PROTON STATES. Exth J PR/C 18 $2057787.0+13.1+2$ OImer+ CURV. EXP. CFD 3 OWEA FITS

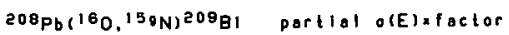

11535 BRK Exih J PR/C $181807781.0+2$ 2.2+2 Plepert TBL. RATIO FROM TWO POTENTIALS Exth J PR/C 18 $2057787.0+13.1+2$ OImer+ CURV.RATIO DWBA/EXP FOR 3 FITS. Exth J PR/C It $2057787.0113 .1+2$ Olmert CURY. INTAD. EXP. CFD 2 DWBA FIT

$200 \mathrm{~Pb}\left(160,{ }^{14} \mathrm{C}\right)^{210} \mathrm{PO}_{0} \quad \mathrm{O}(\mathrm{E})$ a factor

11536 NBI Theo A 74NASH 157674 NDG

Bang+ TEL. SIG(EXP)/SIG(THEORY)

$208 \mathrm{~Pb}(160, x)$ eharge distribution o(E)

$11537 \mathrm{BNL}$ Exih J PR/C 15954377 8.0+1 1.0+2 Videboeh+ TEL.CURV. Z=5-8. E/ANGLE INTE Expt C 76ANL-2 2 B25 $4768.0+1$ 1.0+2 Videbaek+ CURV. $Z=6.7$.

208Pb $(160, x)$ charge distribution o(E) factor

11538 BRK Exp! J PL/B 65 227 N76 1.4+2 3.2+2 Gelbke+ CURV. Z=3-7. 140MEV/315MEV. Expt JPL/B $65 \quad 227$ N76 $3.2+23.3+4$ Gelbke+ CURV. $Z=3-7$. 315MEV/33.6GEV Expt JPL/B 65 227 N76 $1.4+23.2+2$ Gelbke+ CURV. $Z=3-7$. AU197/PB208. Expt J PL/B 65 Expl J PL/B 65 227 N76 1.4+2 3.2+2 Gelbhe+ CURV. Z=3-7. ZR94/PB20B. Exol JPL/B 65 227 N76 3.2+2 Gelbke+ CURV. Z=3-7. TH232/PB208.

11539 MSU ReV S BNL-51115 $17791.4+23.2+2$ Gelbhe - CURV. Z=3-7. 14OMEV/315MEV. Revo 5 BNL-51115 I $7793.2+23.3+4$ Gelbke. CURV. $Z=3-7$. 315MEV/33.6GEV.

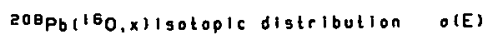

11540 BRK Theo A BAP 2327178 +4 Menchaca-Rochat NDG. PERIPHERAL REACT.

$208 \mathrm{~Pb}\left({ }^{16} \mathrm{O}, x\right) 1 \mathrm{sot} 0 \mathrm{p} i \mathrm{c}$ distribution O(E) factor

11541 BRK Expt J PL/B 65 227 N76 1.4+2 3.2+2 Gelbke+ CURV, Z=3-7. 140MEV/315MEV. Exp: JPL/B 65 227 N76 $3.2+23.3+4$ Gelbke+ CURV. $Z=3-7$. 315MEV/33.6GEV Expt J PL/B 65 227 N76 $1.4+23.2+2$ Gelbke+ CURV. $Z=3-7$. AU197/PB20B. $\begin{array}{rlllll}\text { Expt J PL/B } & 65 & 227 & N 76 & 1.4+2 & 3.2+2 \text { Gelbke+ CURV. } Z=3-7 . \\ \text { Expt J PL/B } & 65 & 227 & N 76 & 3.2+2 & \text { Gelbke+ CURV. } Z=3-7 . \text { TH232/PB208. }\end{array}$

11542 MSU Revw 5 BNL-51115 $17791.4+23.2+2$ Gelbke. CURV. $Z=3-7$. 140MEV/315MEV. Revo $S$ BNL-51115 i $7793.2+23.3+4$ Gelbke. CURV. $Z=3-7$. 315MEV/33.6GEV.

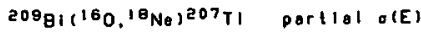

11543 PAR Theo J ZP/A $290 \quad 157796.6+18.0+1$ Bimbot+ CURV. SUBCOUL. TRANSFER CFO EXP $\left.20{ }^{8} I^{10} 0,17 F\right)^{200} \mathrm{~Pb}$ partIOI O(E)

11544 PAR Theo J ZP/A $290157797.2+1$ B. $15+1$ Bimbol+ CURV. SUBCOUL. TRANSFER CFD EXP ${ }^{209} B\left(1^{16} 0, x\right)^{210} B i \quad O(E)$

11545 PAR Expt JPR/C 181298978 8.3+1 9.9+1 Gardes+ TBL. 2PI ION. CHMBRS FOR A + $G$

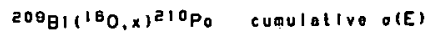

11546 PAR Expl J PR/C IB $12989787.7+19.4+1$ Gardes+ TBL. $2 P 1$ ION. CHMBRS FOR A + G $20{ }^{0}\left({ }^{16} 0, x\right)^{210} \mathrm{Po}_{0}$ independent o(E)

11547 PAR Exth T D. GAROES D76 $7.8+19.4+1$ Gardes. TBL. CURV. ACTIVATION. $20{ }^{\circ} B 1(160, x) 210 P_{0} \quad O(E)$

11548 PAR EXPt J PR/C $1812989787.7+19.4+1$ Gardos+ TBL. ZPI ION. CHMBRS FOR A + G $200^{B} 1(160, x)^{210} A t \quad o(E)$

11549 PAR Expt J PR/C 181299978 8.3+1 9.9+1 Gardes+ TBL. 2PI ION. CHMBRS FOR A + G $20{ }^{0} B\left({ }^{16} 0, x\right)^{211}$ At o(E)

11550 PAR Expt J PR/C 18 1298 $9785.8+19.9+1$ GBRdes+ TBL. 2PI ION. CHMBRS FOR A + G 
REFERENCES (cont)

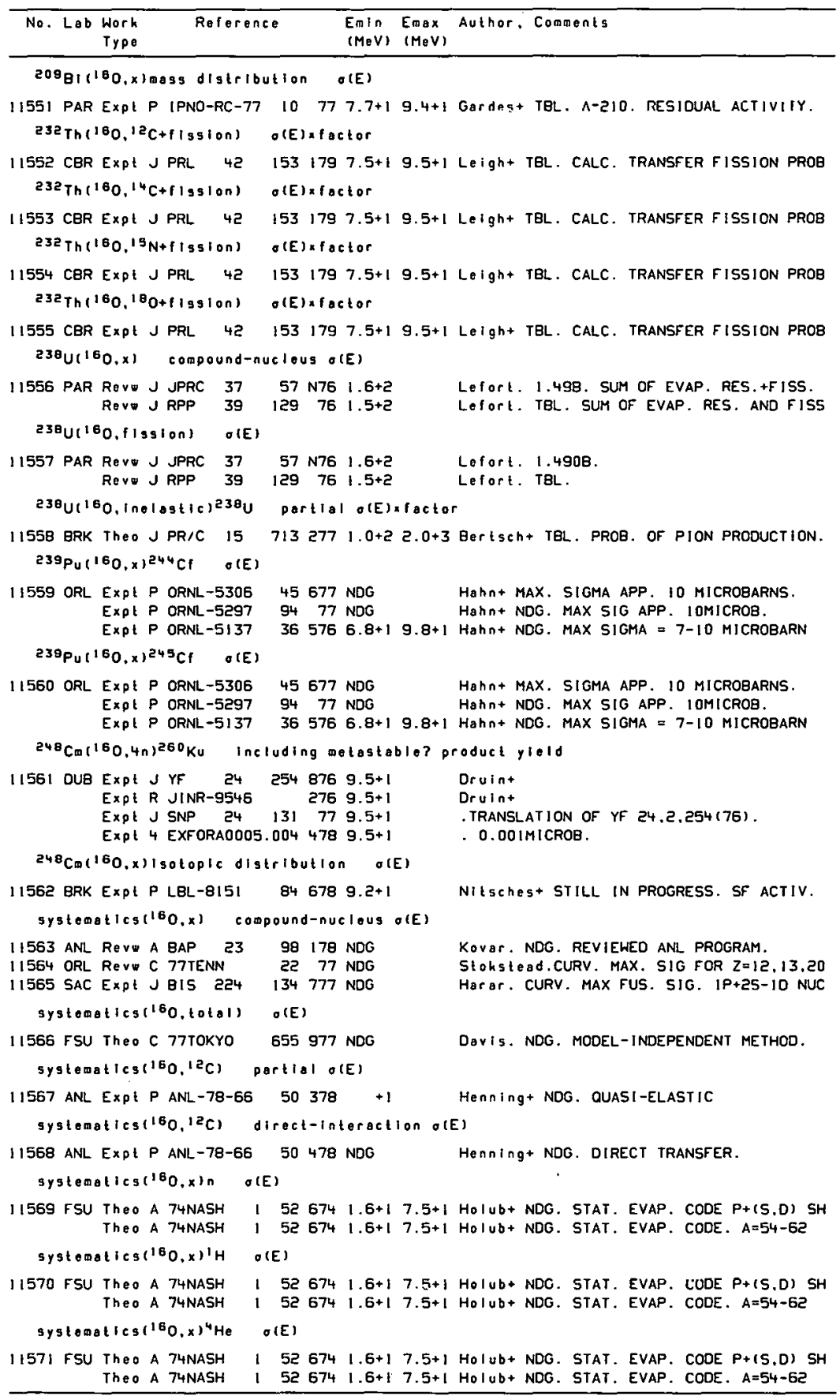


REFERENCES ( c on !)

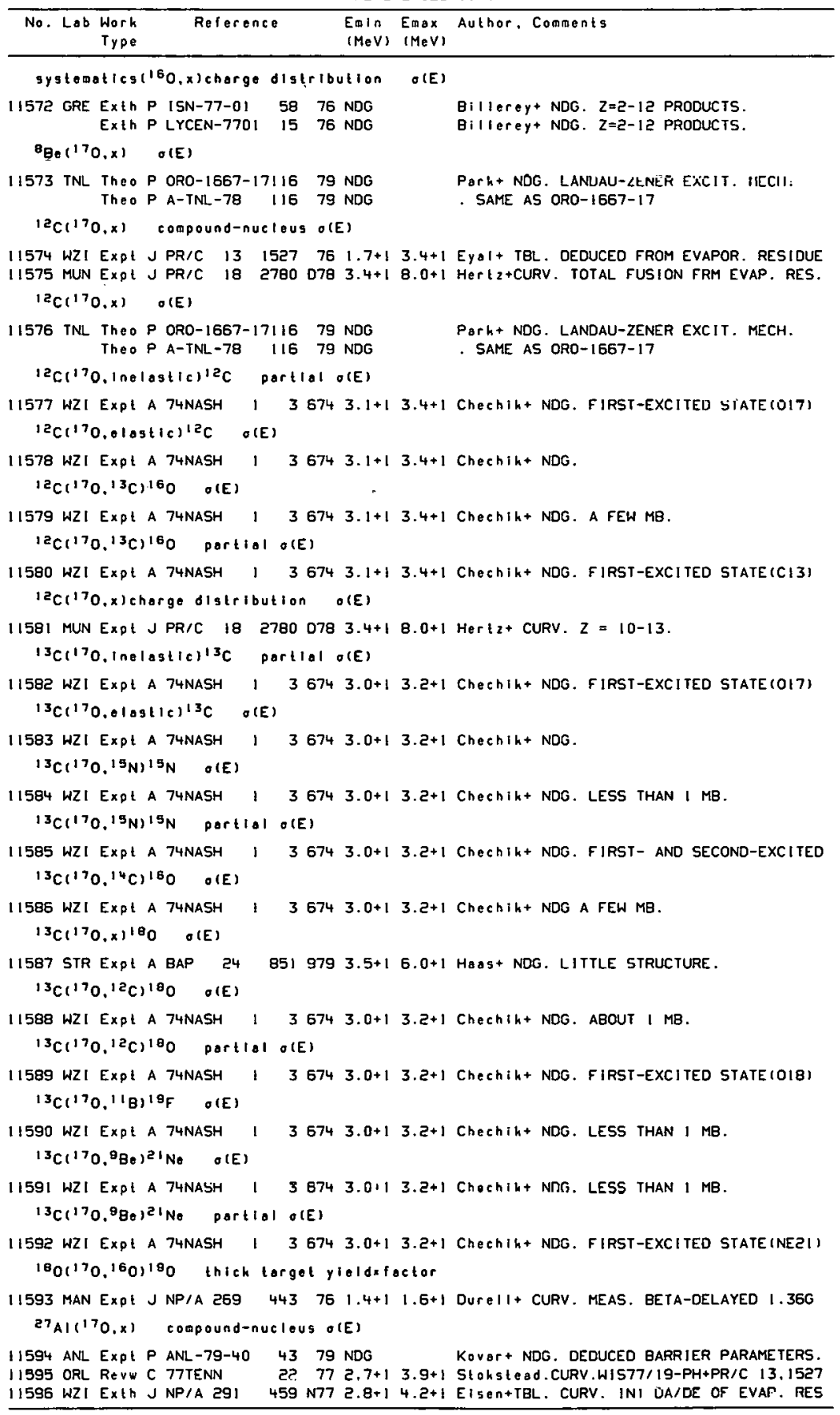




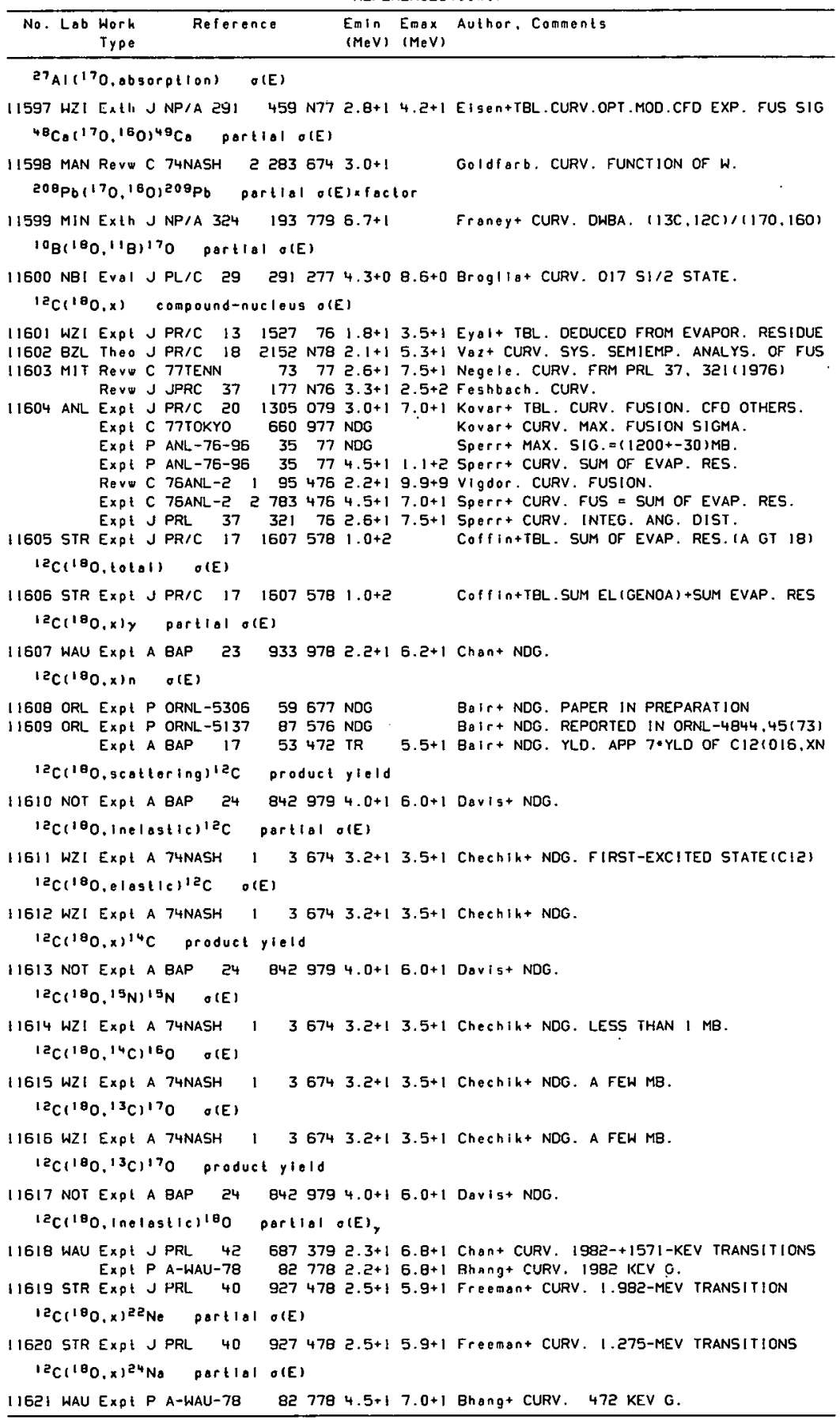




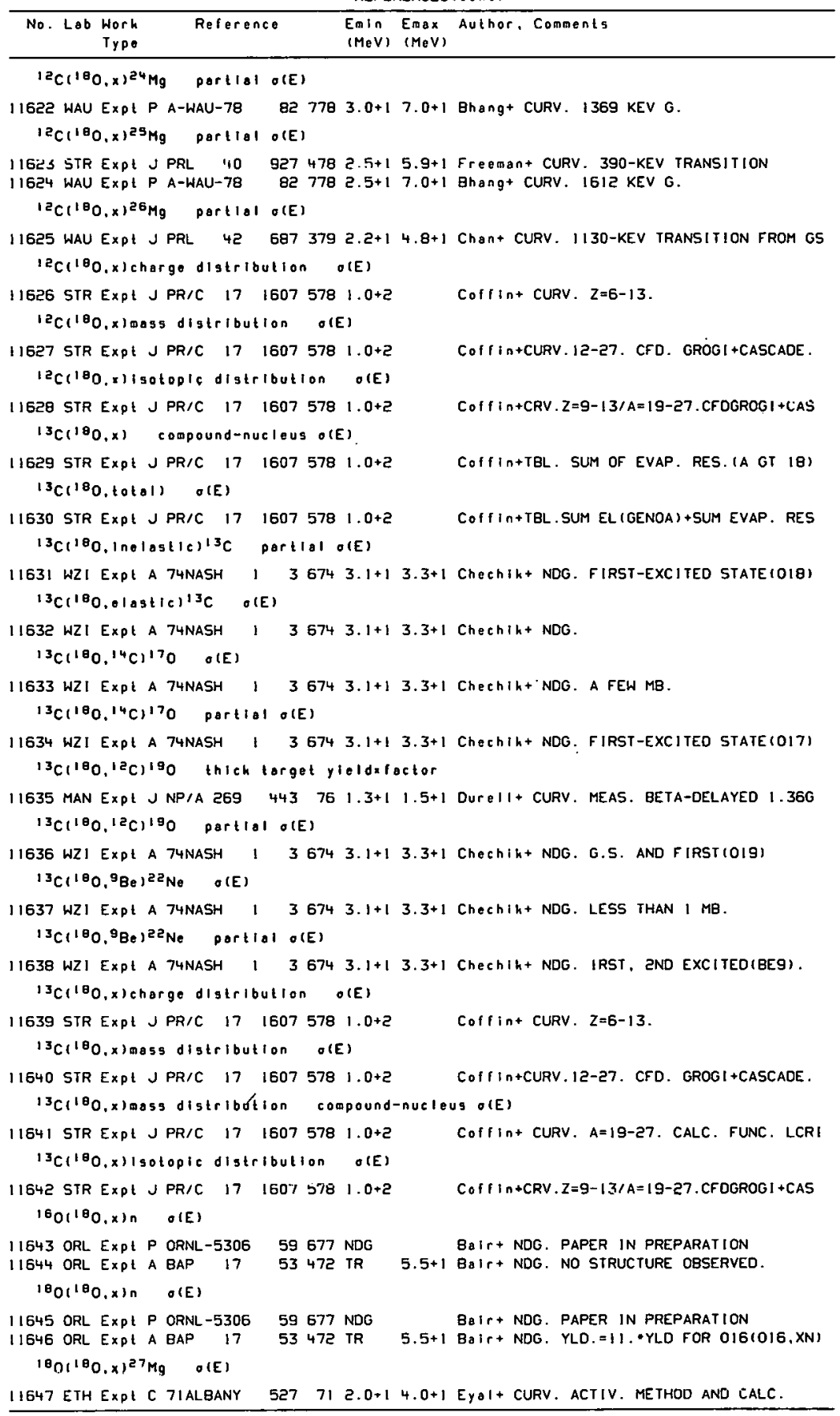




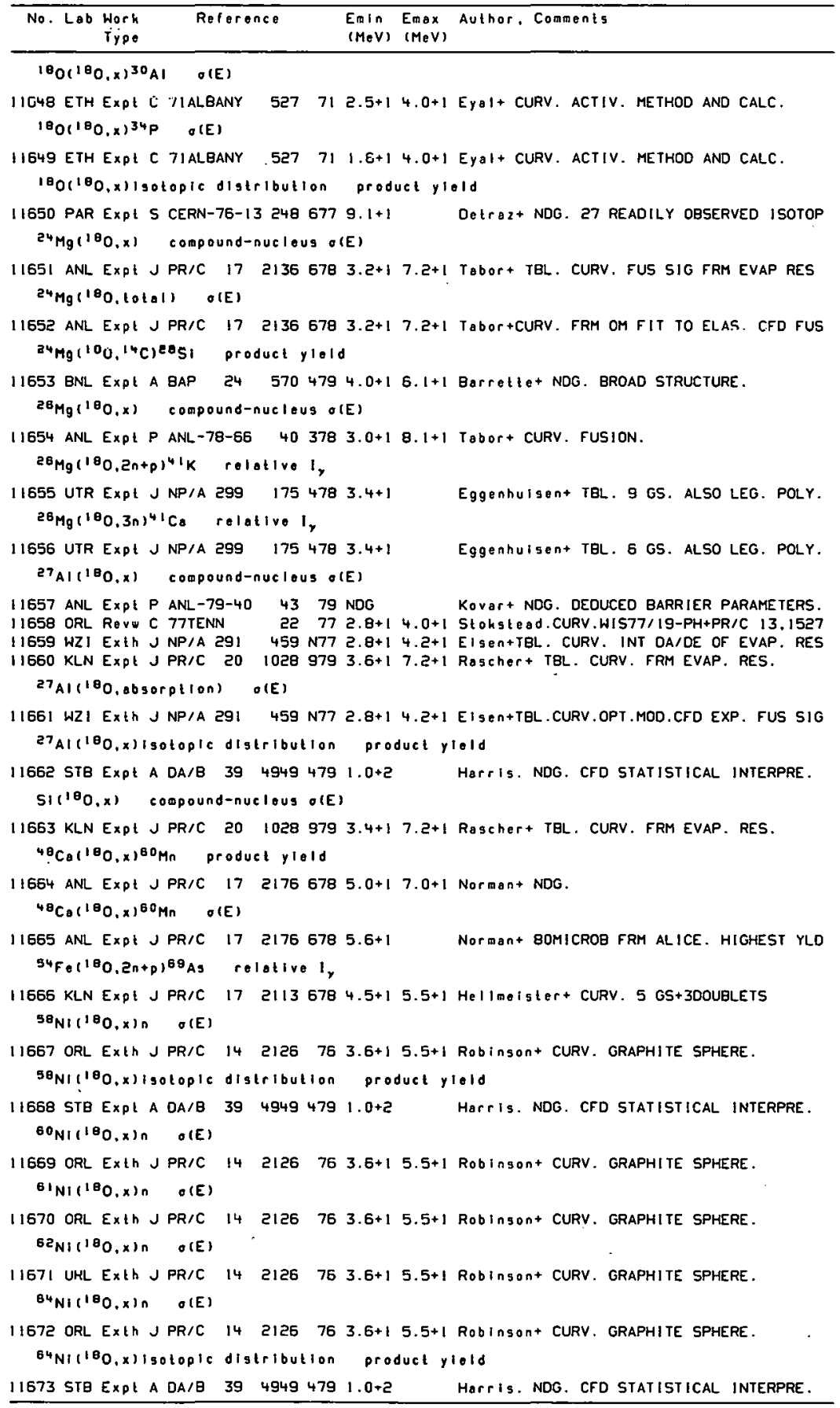




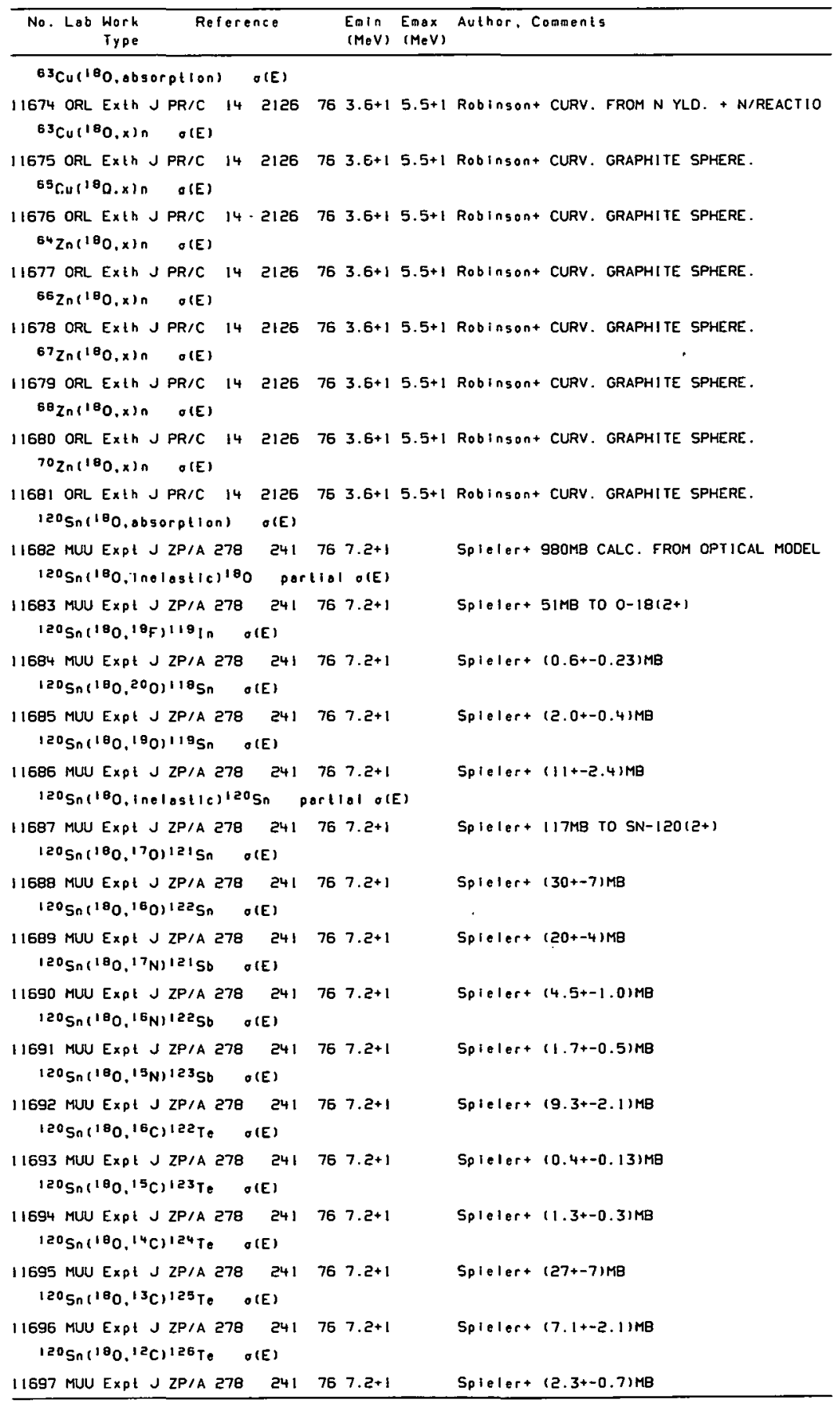


REFERENCES ( cont)

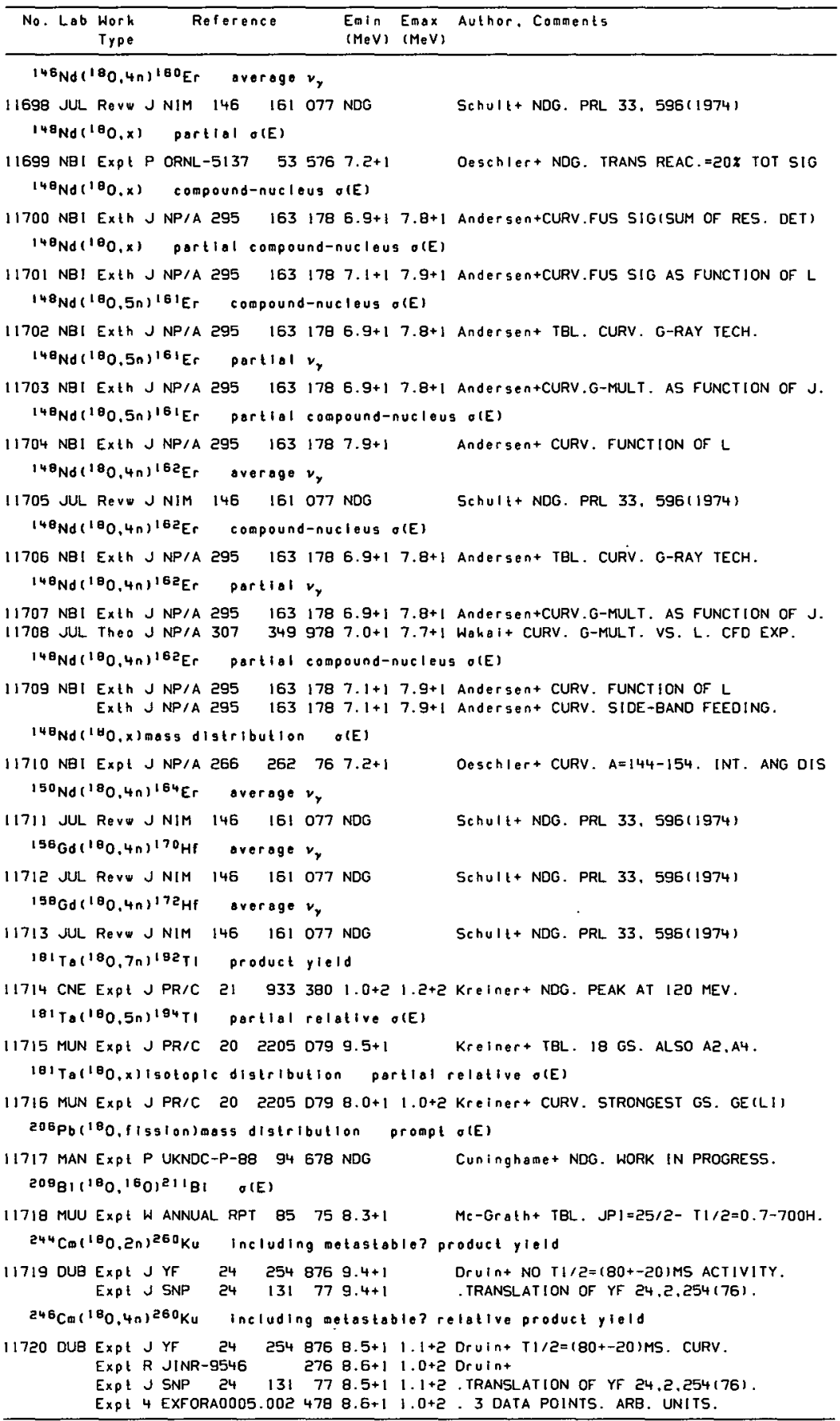




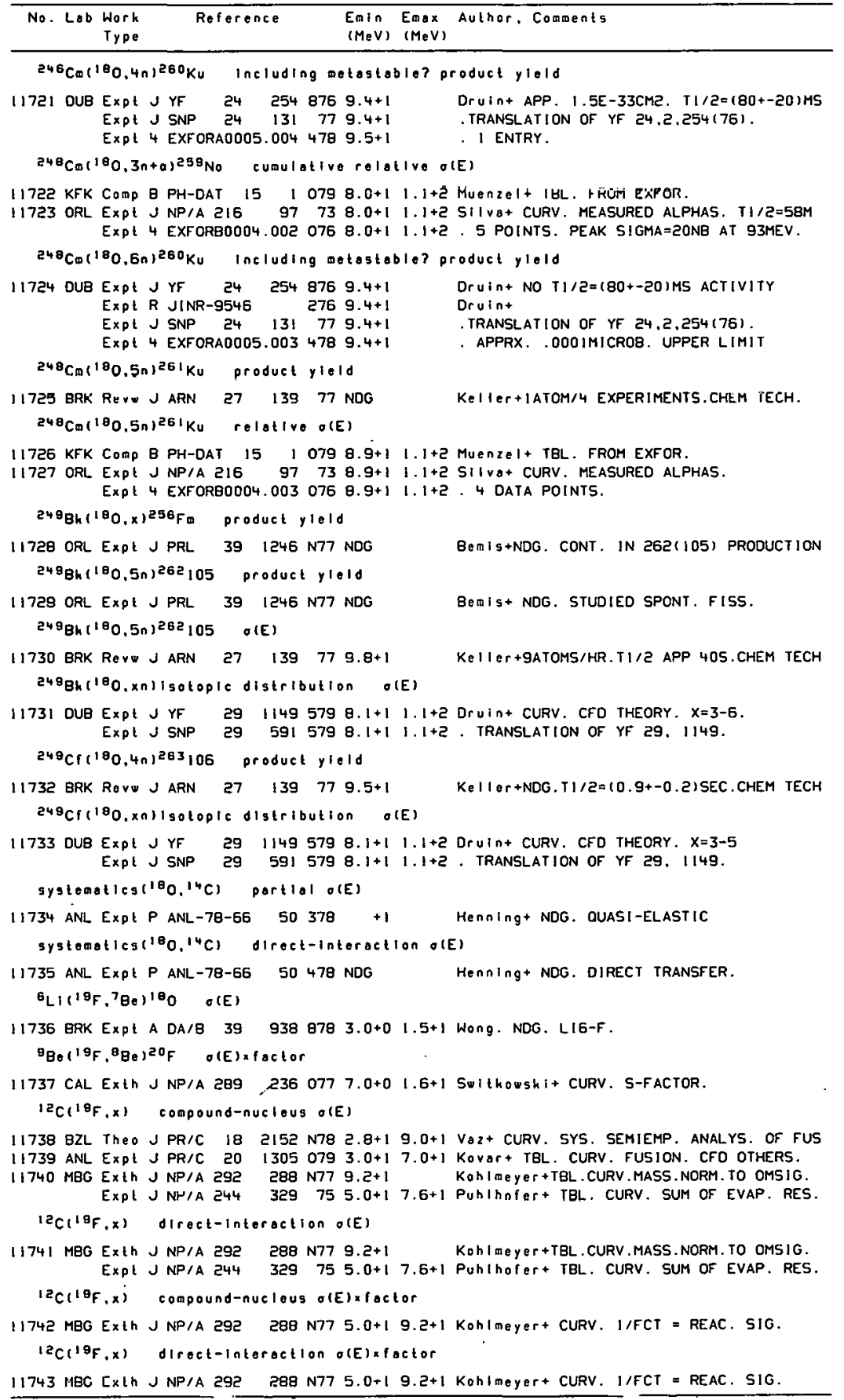




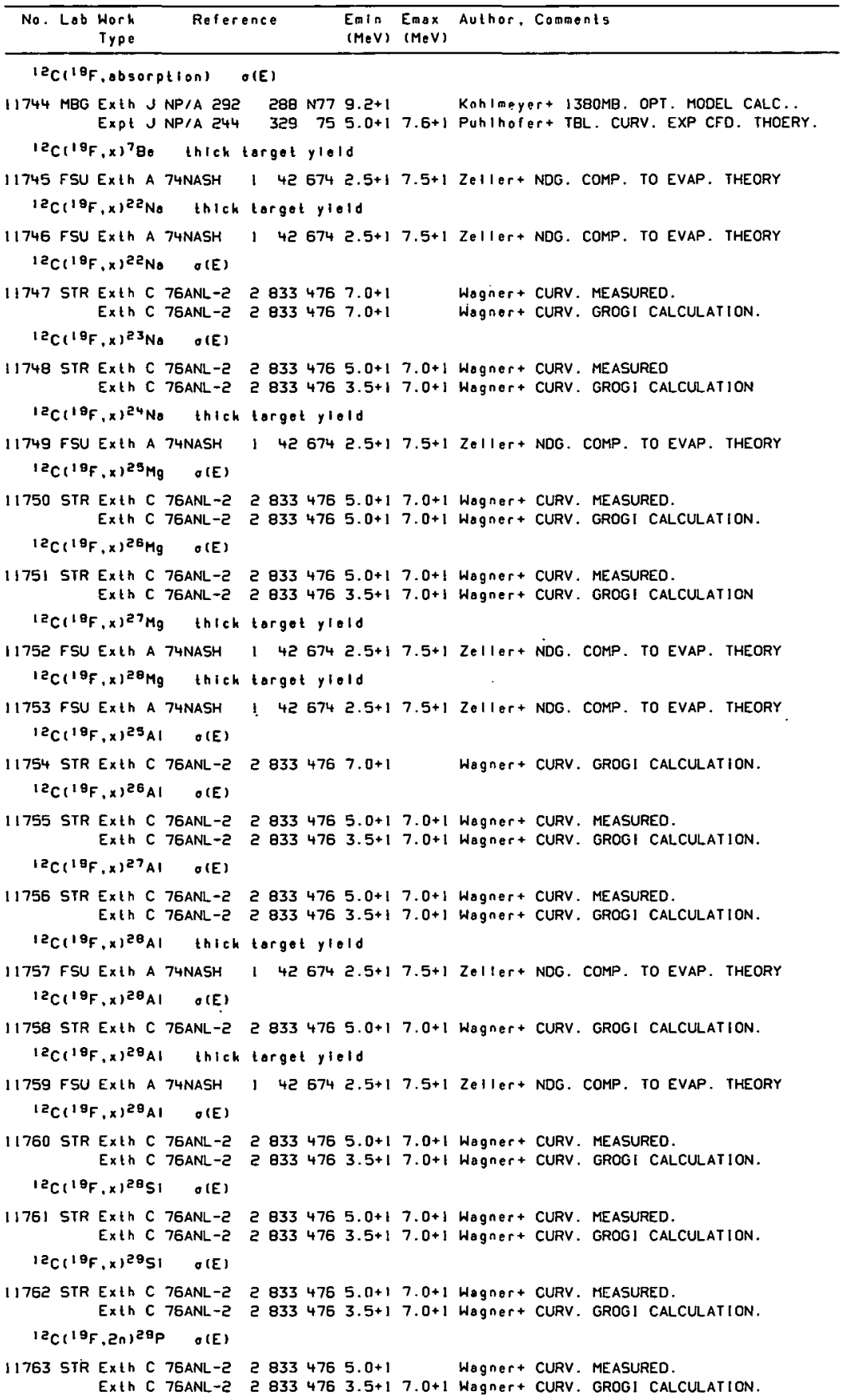


REFERENCES ( c on ( )

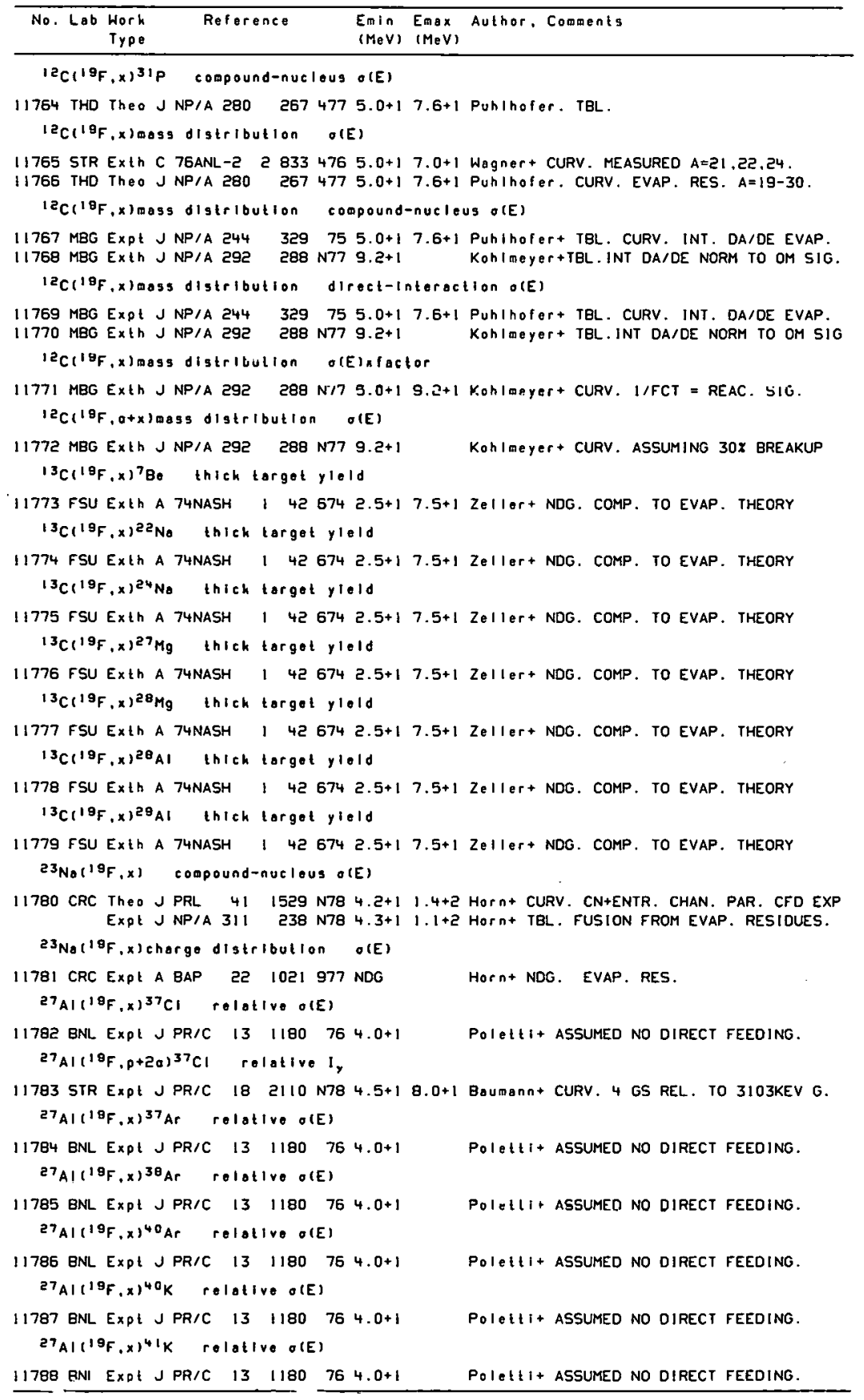




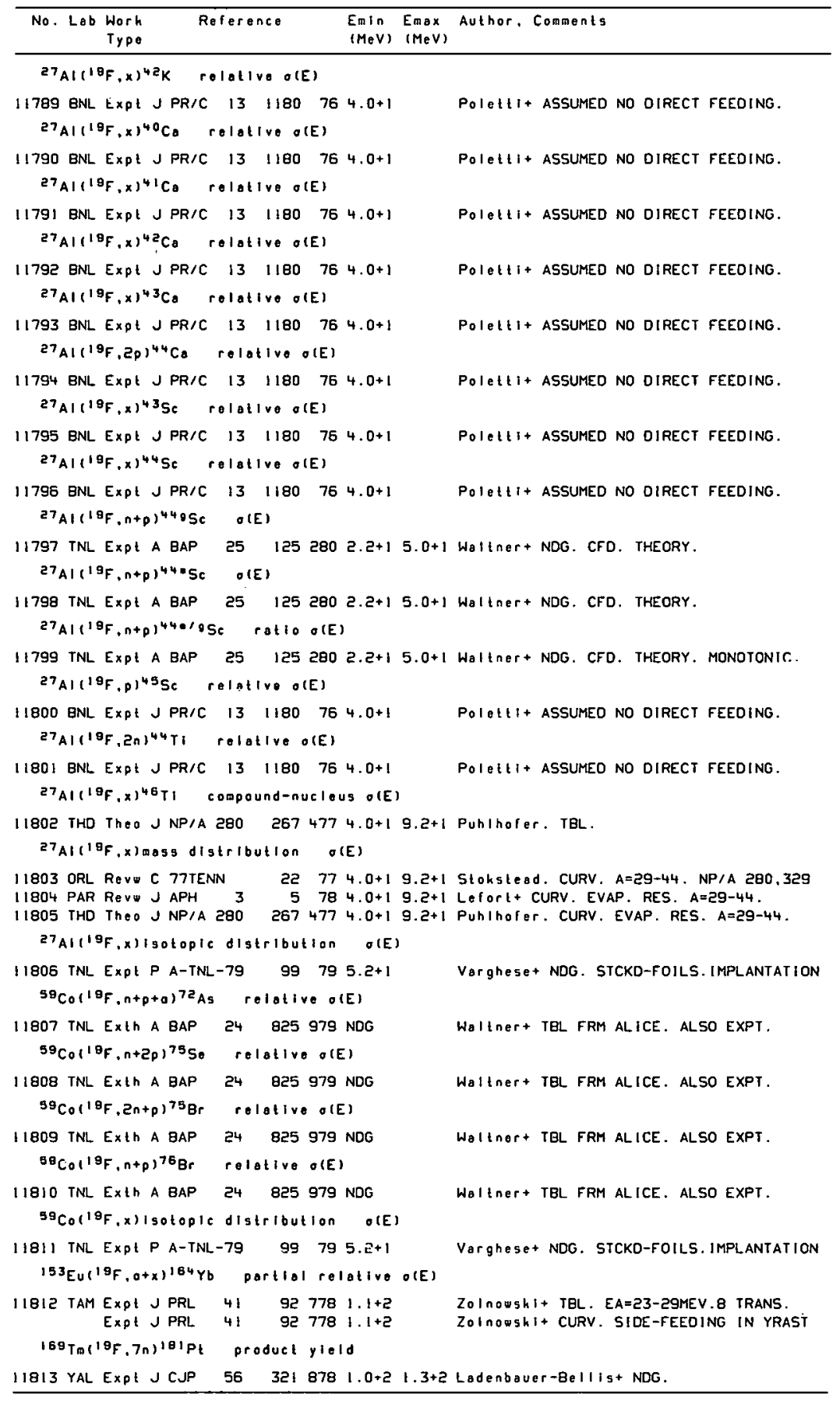




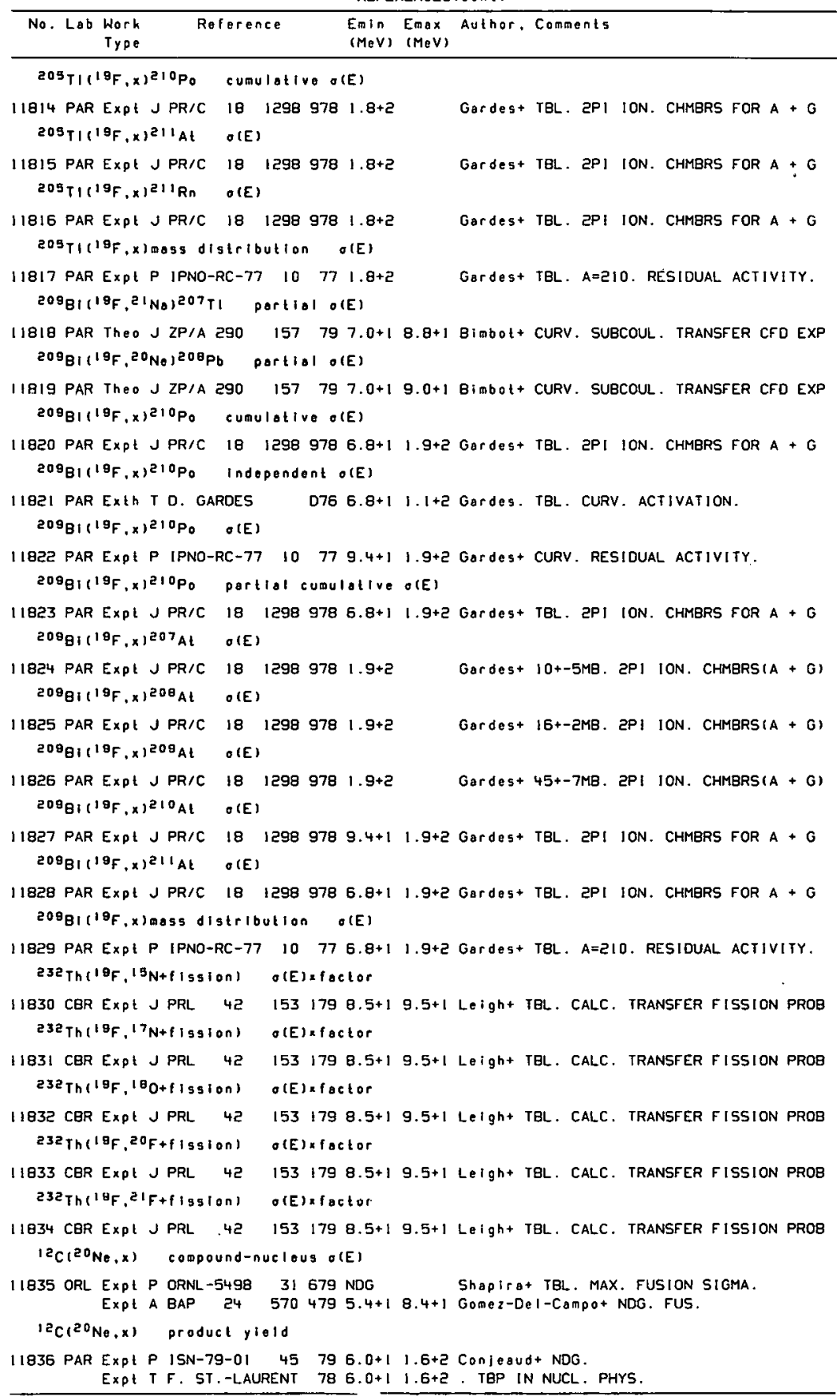


REFERENCES ( c on t )

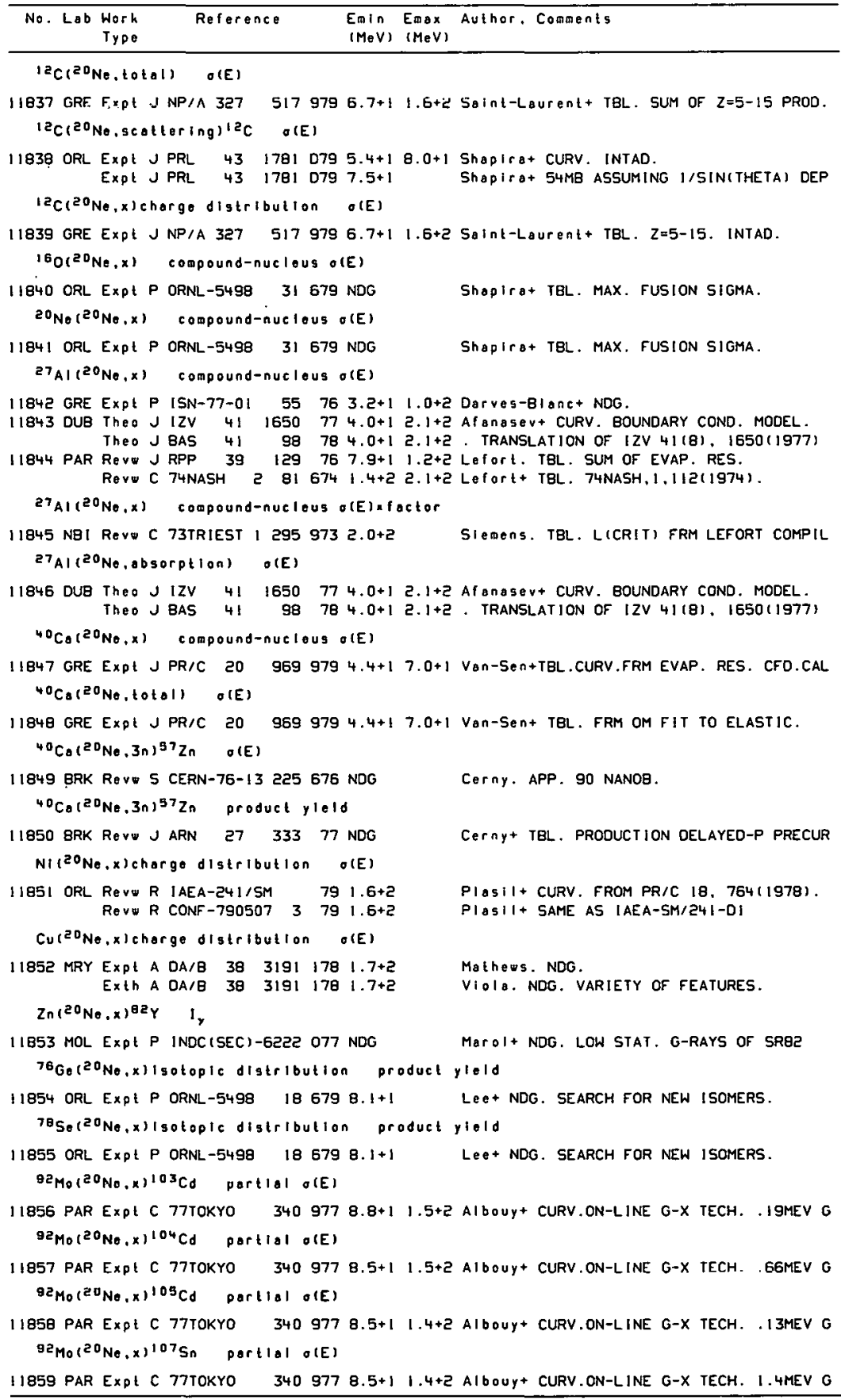




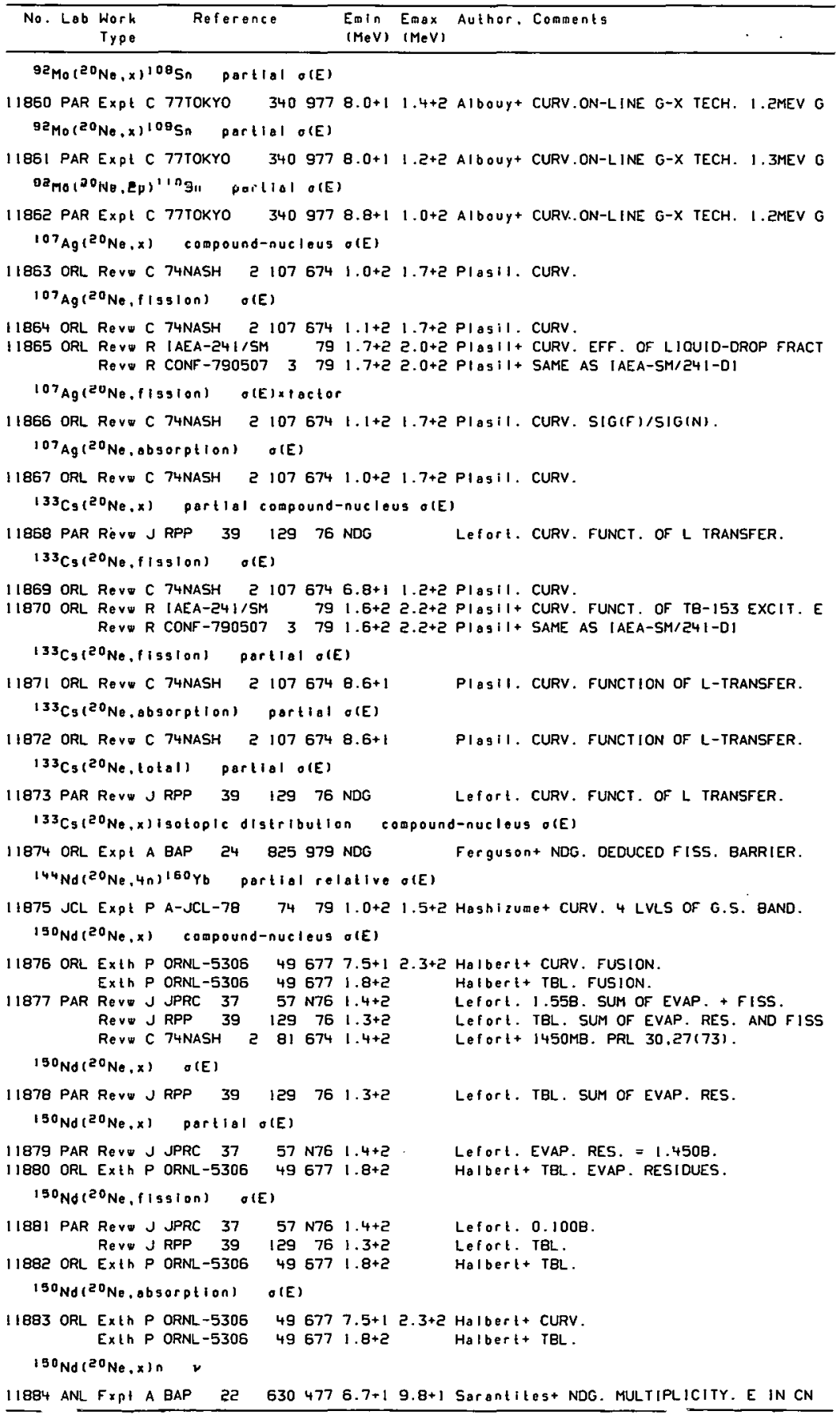


REFERENCES ( c on t)

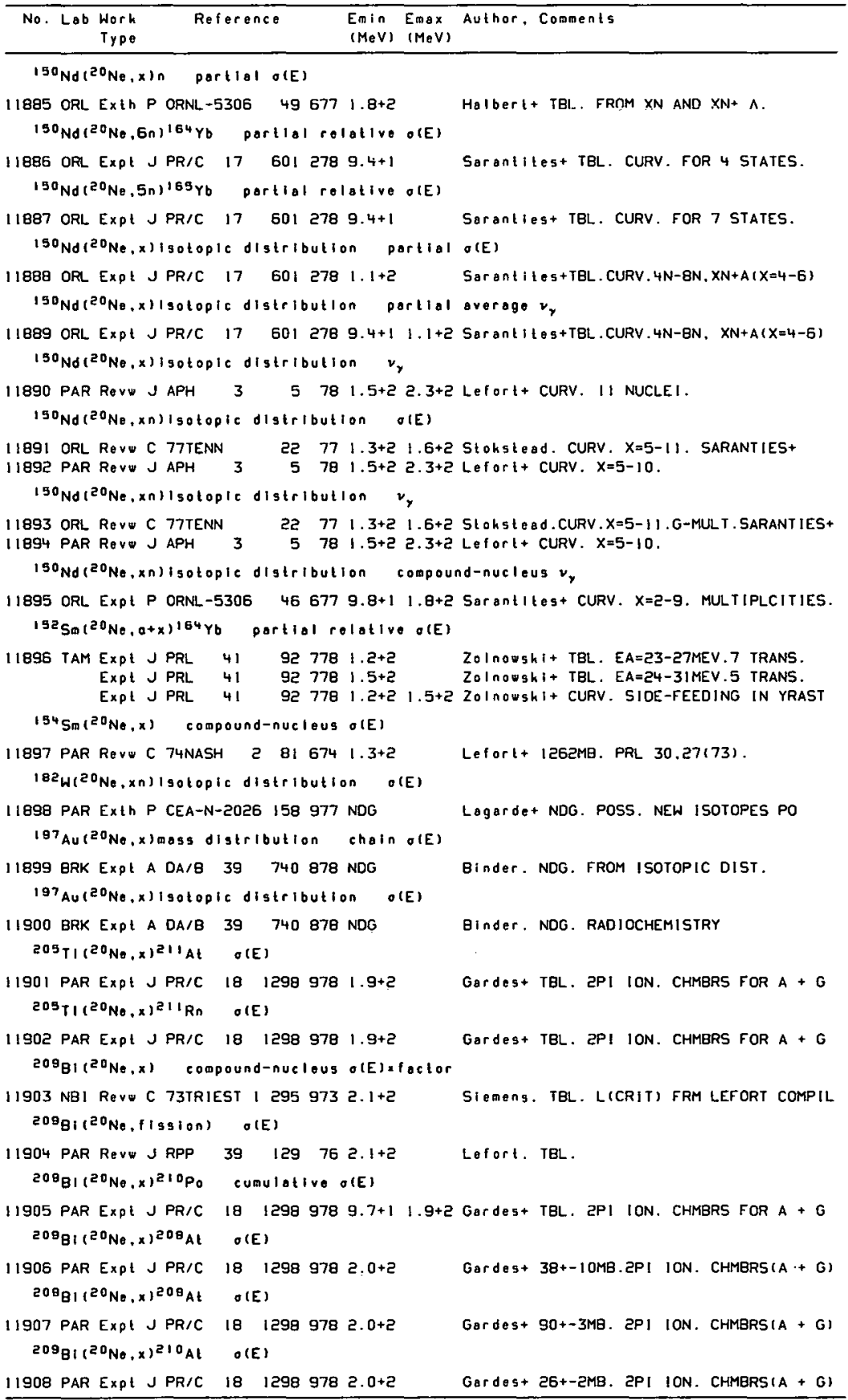




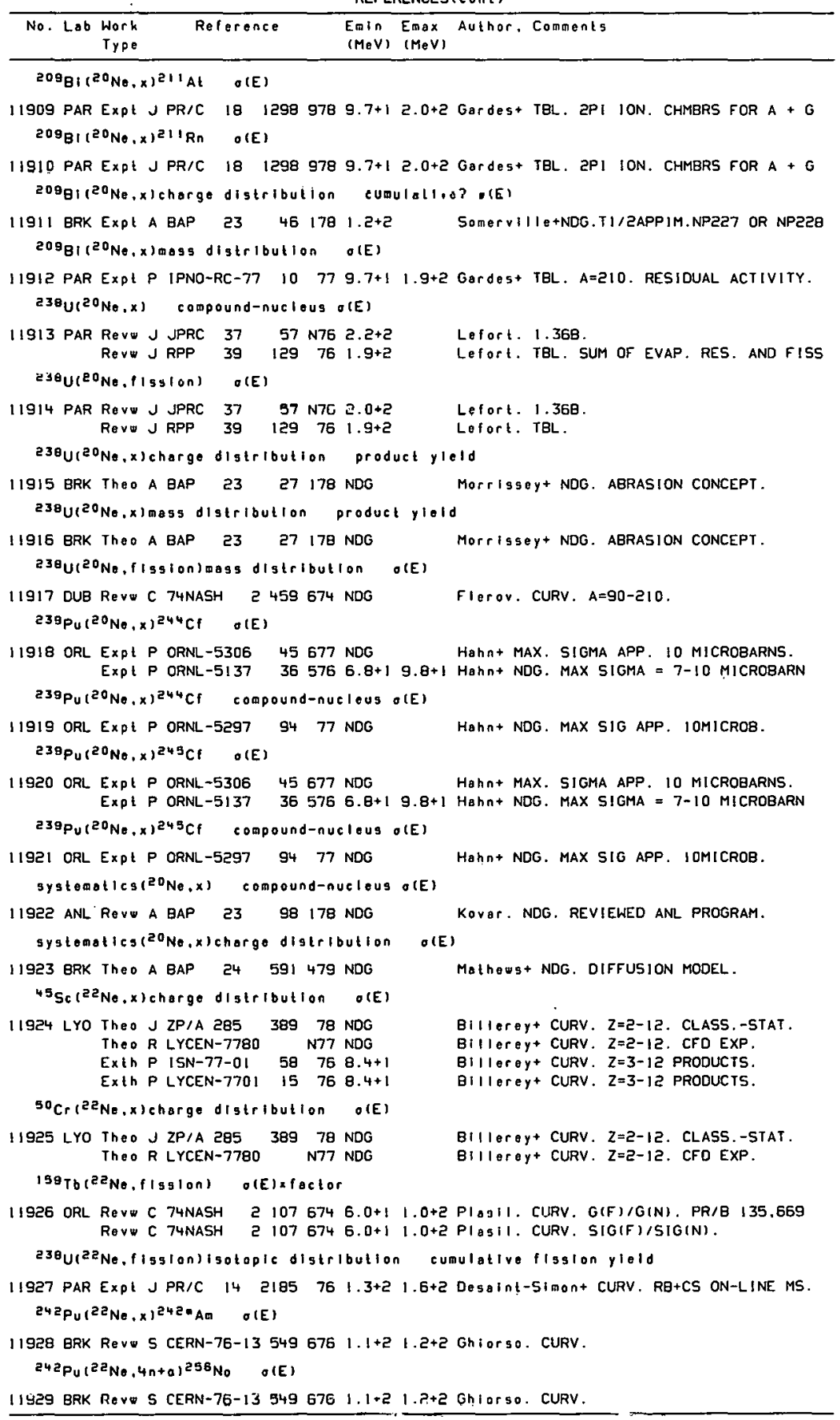




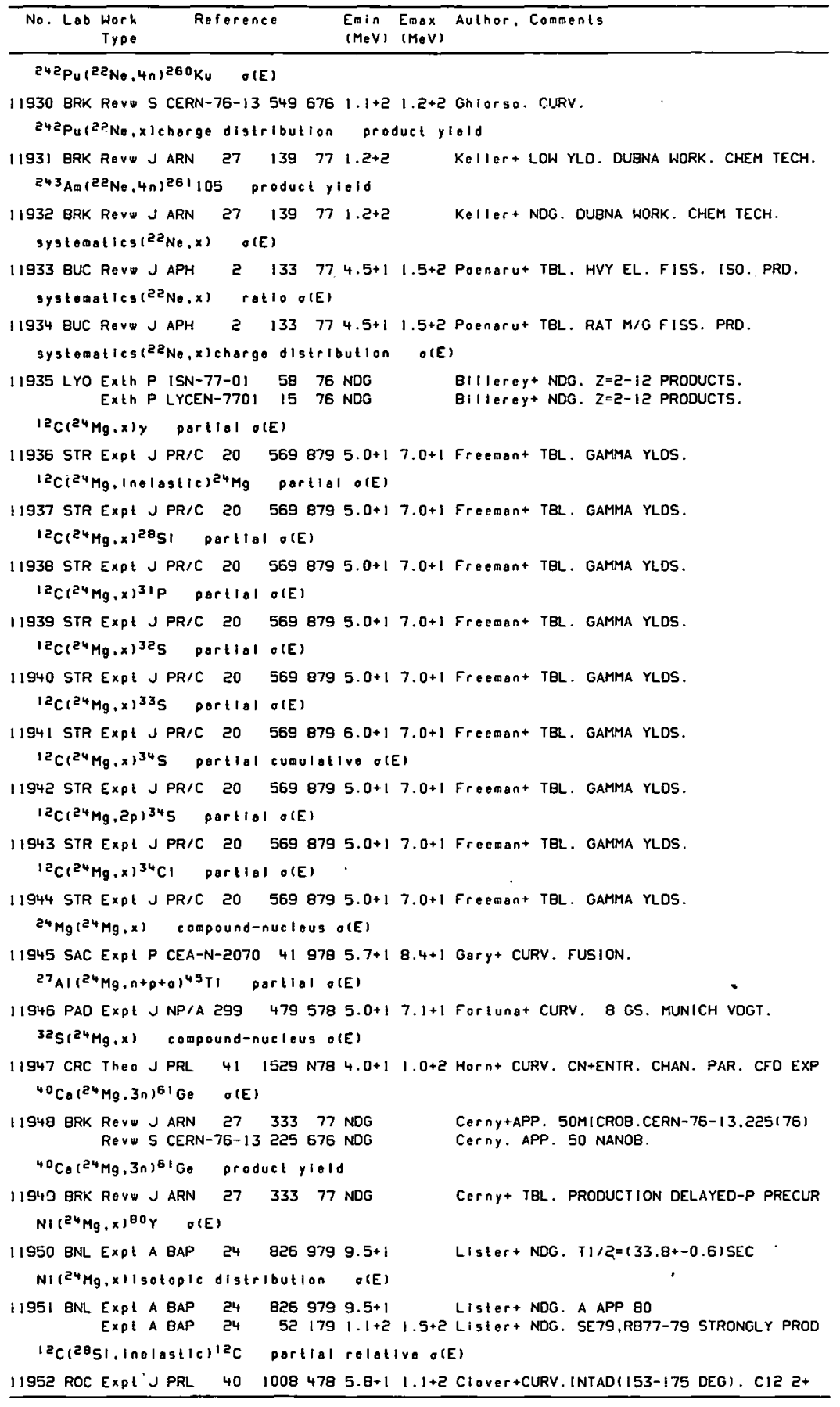




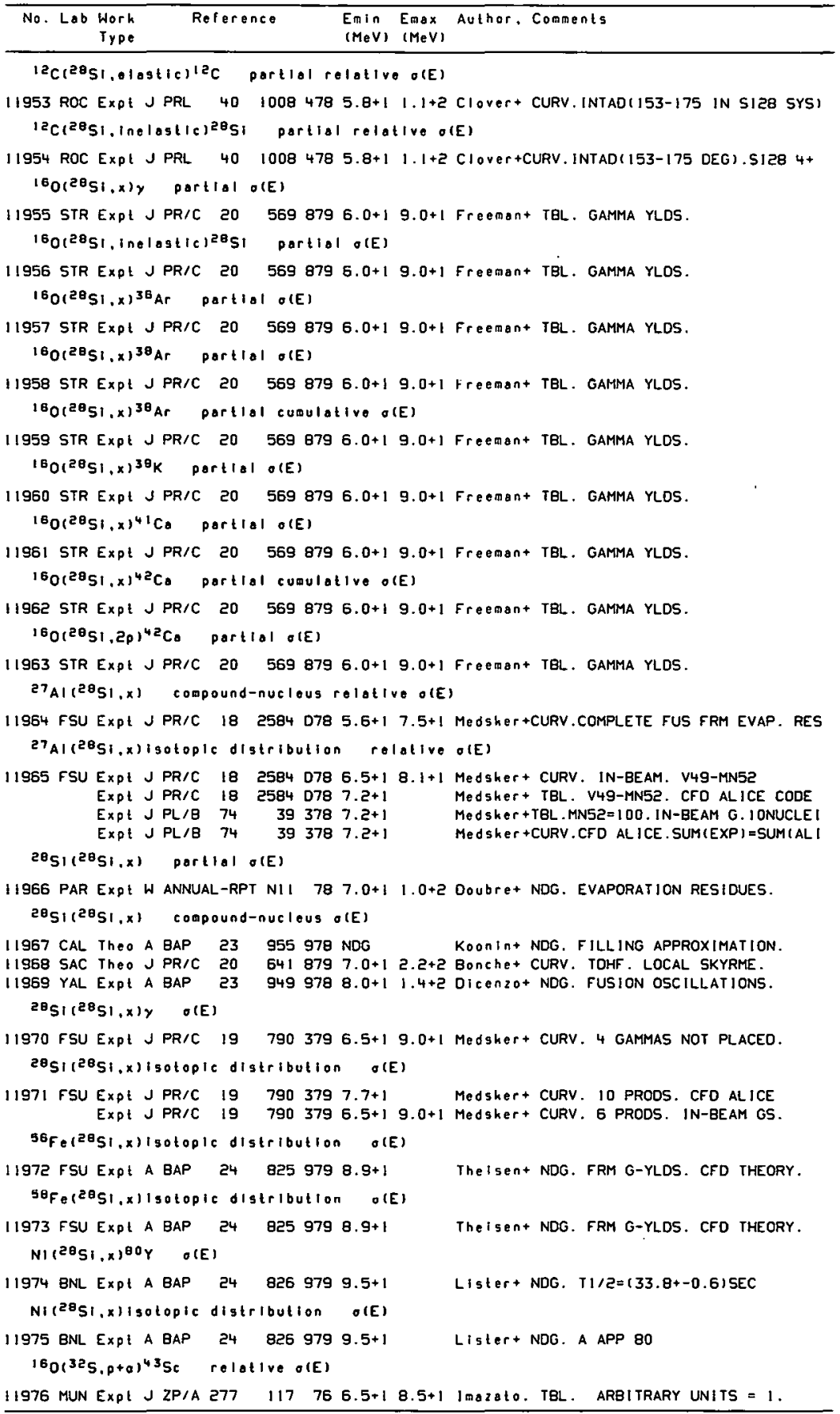


REFERENCES ( cont)

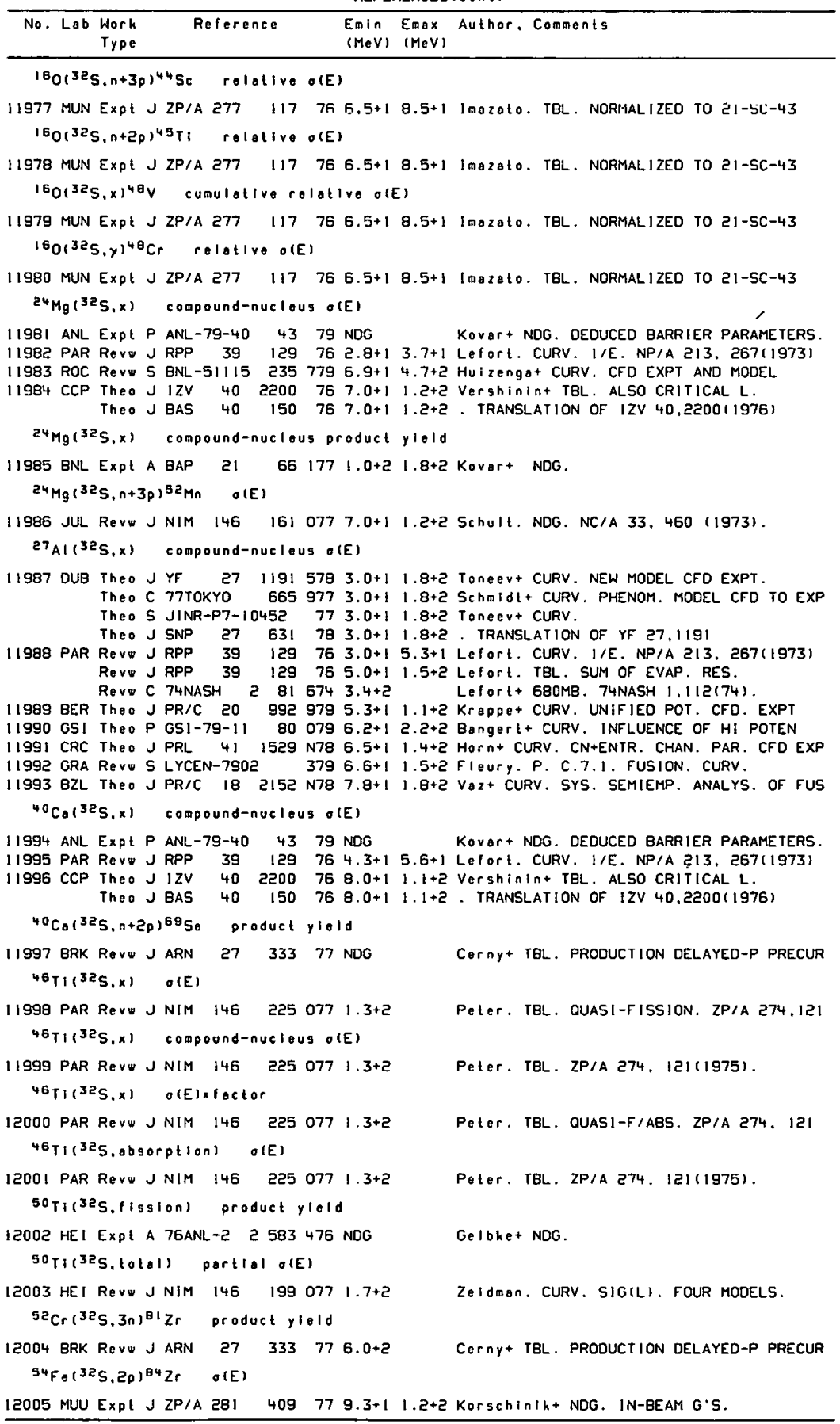




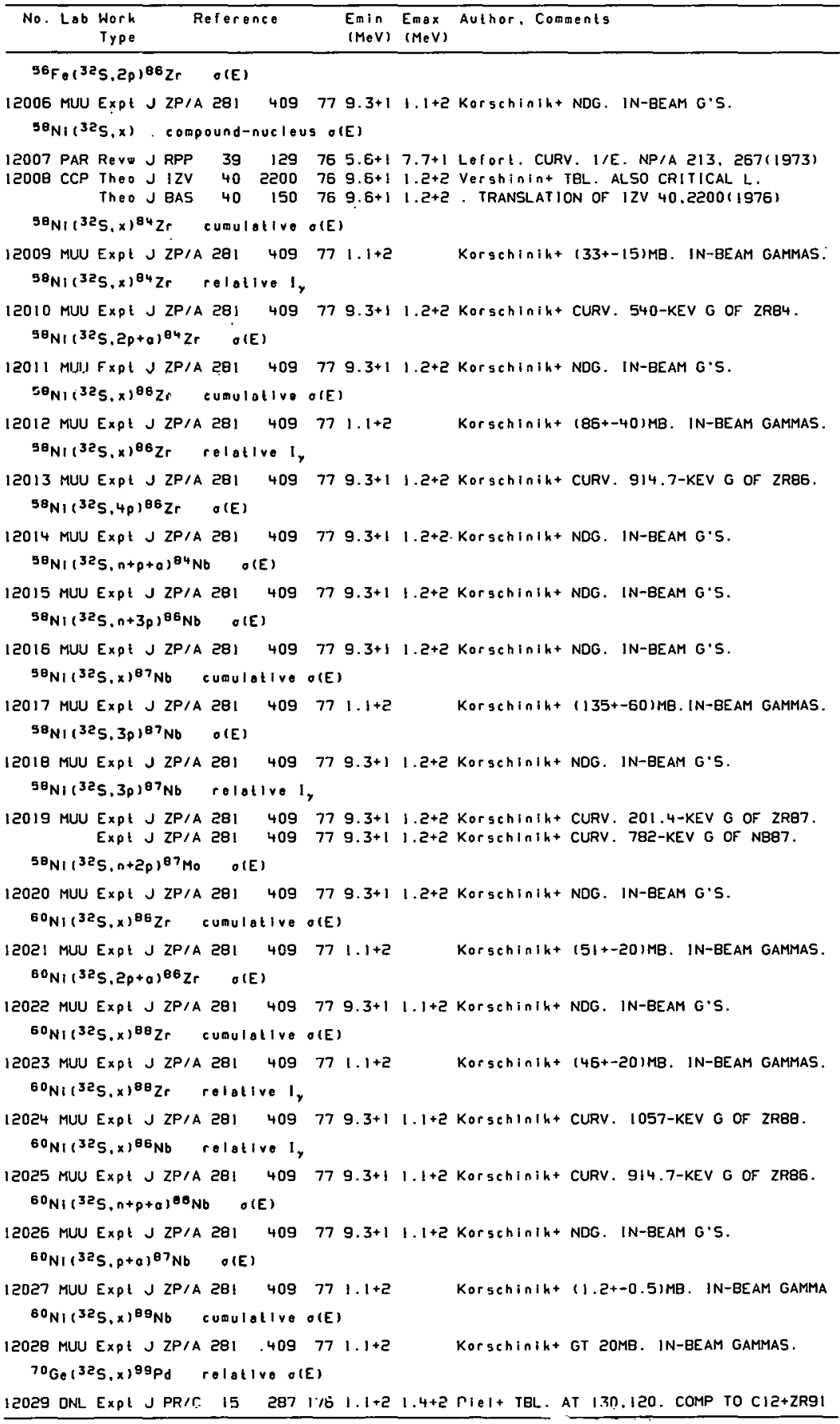




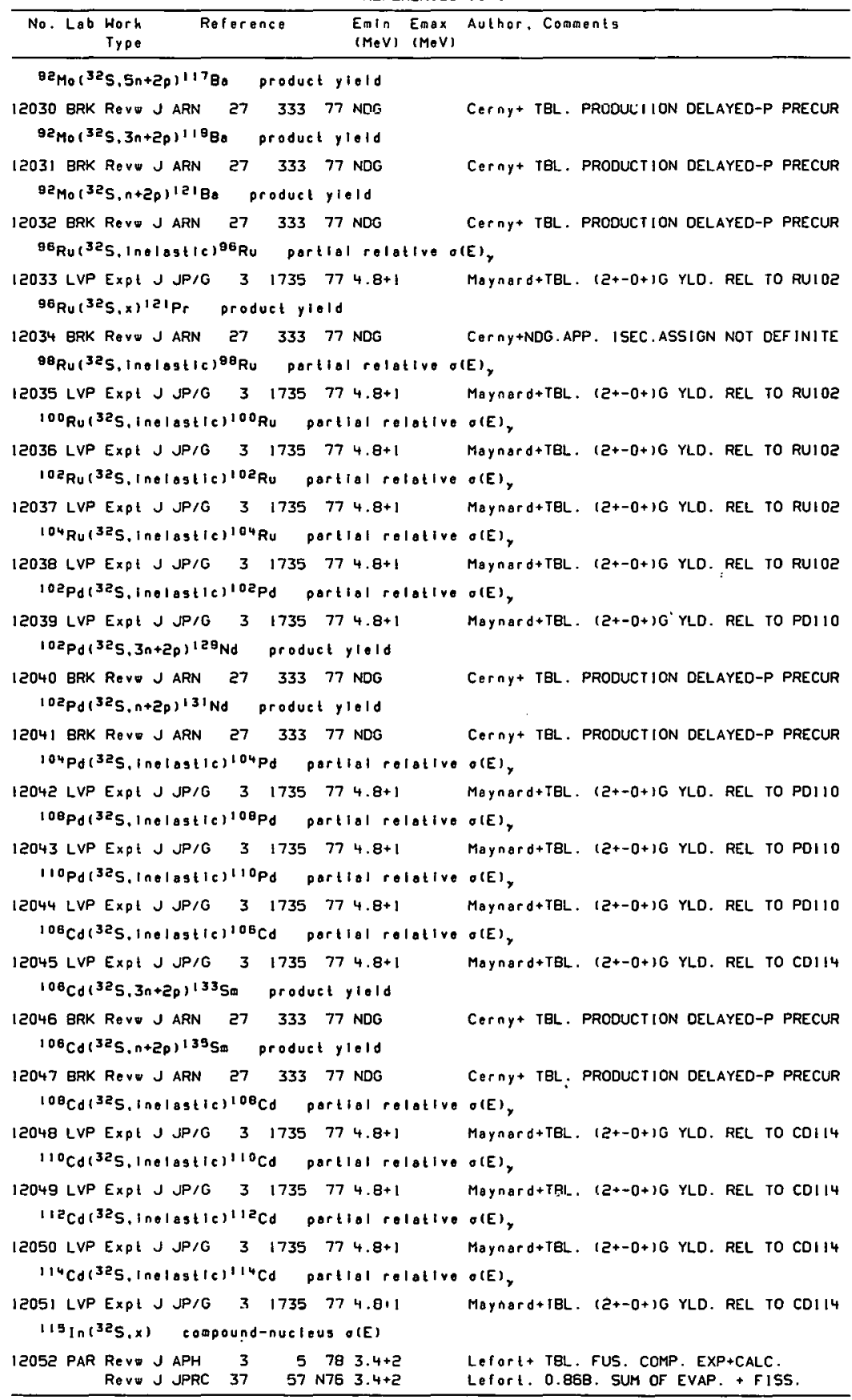




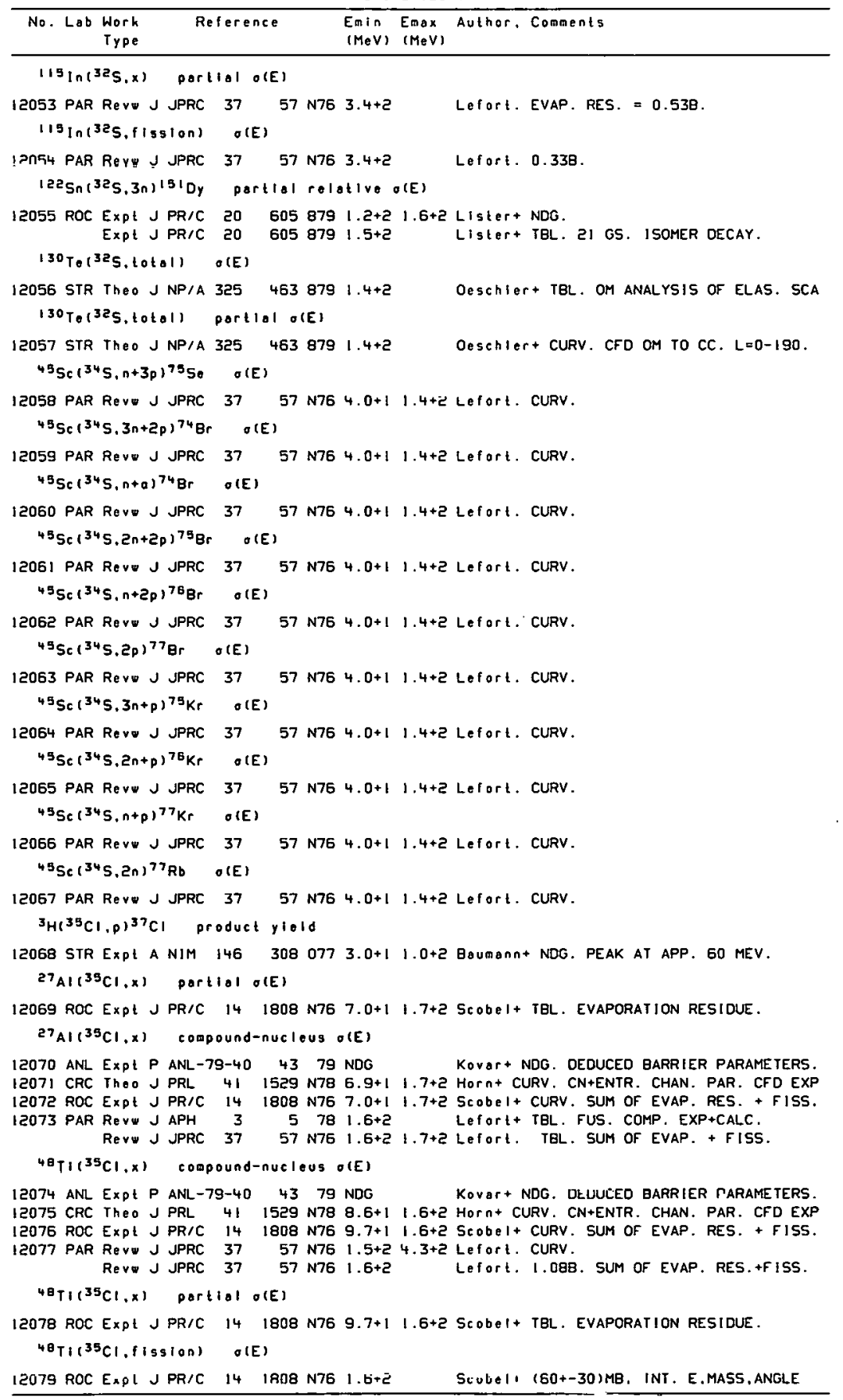


REFERENCES (cant)

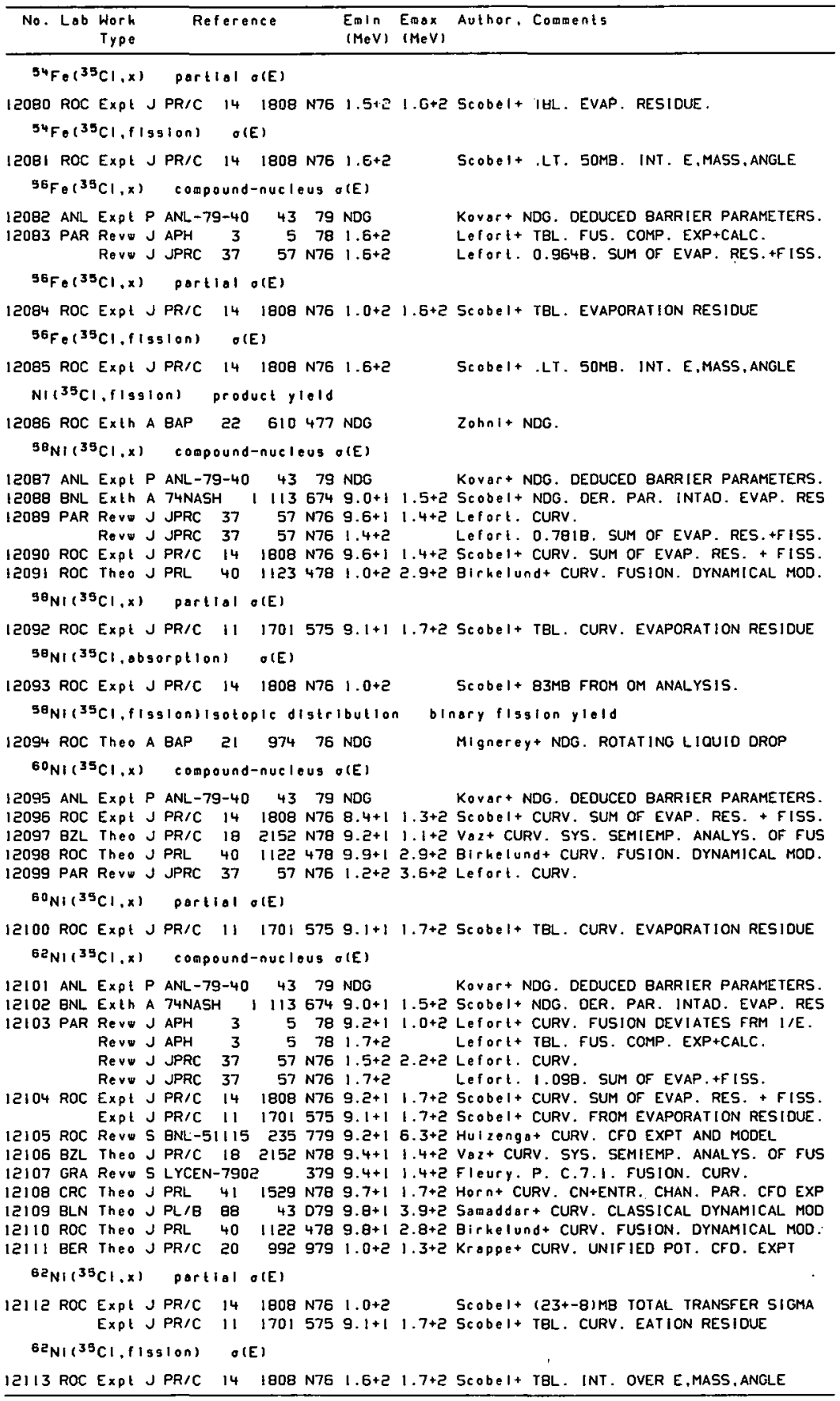


REFERENCES $(\operatorname{con}()$

\begin{tabular}{|c|c|c|c|c|c|}
\hline No. Lab & $\begin{array}{l}\text { Worh } \\
\text { Typo }\end{array}$ & Relerence & $\begin{array}{l}\text { En } 1 n \\
(M e V)\end{array}$ & $\begin{array}{l}\text { Emax } \\
(\operatorname{MeV})\end{array}$ & Author. Comments \\
\hline
\end{tabular}

$\left.6 e_{N 1}{ }^{35} \mathrm{Cl}, a b s o r p t i o n\right)$ o(E)

12114 ROC Expt J PR/C $14 \quad 1808$ N76 $1.0+2 \quad$ SCObeI+ I82MB FROM OM ANALYSIS.

${ }^{84} \mathrm{Ni}\left({ }^{35} \mathrm{Cl}, \mathrm{X}\right)$ compound-nuclous o(E)

12115 ANL Expt P ANL-79-40 4379 NDG Kovar+ NDG. DEDUCED BARRIER PARAMETERS. 12116 BNL Exth A 74NASH I $1136749.0+11.5+2$ Scobel+ NDG. OER. PAR. INTAD. EVAP. RES $12117 \mathrm{BZL}$ Theo J PR/C $182152 \mathrm{N78} 9.0+1 \quad 1.1+2 \mathrm{Vaz}+$ CURV. SYS. SEMIEMP. ANALYS. OF FUS 12118 ROC Expt J PR/C 141808 N76 9.0+1 $1.3+2$ Scobel+ CURV. SUM OF EVAP. RES. + FISS. 12119 ROC Theo J PRL $4011224789.7+12.8+2$ Birkelund+ CURV. FUSION. DYNAMICAL MOD. 12120 PAR Rev J JPRC 3757 N76 $1.2+23.4+2$ Lefort. CURV.

${ }^{6} \mathrm{NI}\left({ }^{35} \mathrm{Cl}, \mathrm{X}\right)$ partlal o(E)

I2I2! ROC Expt J PR/C $1117015759.0+11.7+2$ SCObE $1+$ TBL. CURV. EVAPORATION RESIDUE

${ }^{90} \mathrm{Zr}\left({ }^{35} \mathrm{Cl}, x\right)$ compound-nuclous o(E)

12122 ANL Expt $P$ ANL-79-40 4379 NDG Kovor + NDG. DEOUCED BARRIER PARAMETERS

12123 ROC Expt J PR/C 141808 N76 $1.2+21.6+2$ Scobel+ CURV. SUM OF EVAP. RES. + FISS. Expt J PR/C 141808 N76 $1.2+21.6+2$ Scobel+ CURV. SUM OF EVAP. RES. + FISS.

12124 PAR Revw J APH $355781.6+2$ Lefort+ TBL. FUS. COMP. EXP+CALC Reve J JPRC $37 \quad 57$ N76 $9.6+12.8+2$ Lefort. CURV. Reve J JPRC $37 \quad 57$ N76 $1.6+2$ Lefort. $0.850 B$. SUM OF EVAP. RES.+FISS

Sn( ${ }^{35} \mathrm{C} 1$, fission) product yleld

I2I ROC Exih A BAP 22610477 NDG ZOhni+ NDG.

$112 \operatorname{Sn}\left({ }^{35} \mathrm{Cl}, x\right)$ compound-nueleus o(E)

12126 ROC Theo J PRL $4011224781.4+22.6+2$ BIrkeIund+ CURV. FUSION. DYNAMICAL MOD.

$112 \operatorname{snc}{ }^{35} \mathrm{Cl}$, fissionisotopic distribution binory fission ylold

12127 ROC Theo A BAP 2197476 NDG Mignerey+ NDG. ROTATING LIOUID DROP

$1{ }^{16} \mathrm{Sn}\left({ }^{35} \mathrm{Cl}, \mathrm{x}\right)$ compound-nucleus o(E)

12128 CRC Theo J PRL $41 \quad 1529$ N7B $1.4+21.7+2$ Horn+ CURV. CN+ENTR. CHAN. PAR. CFD EXP 12139 ROC Theo J PRL $4011224781.4+22.6+2$ Birkelundt CURV. FUSION. DYNAMICAL MOD. 12130 ROC ExPl J PR/C 141808 N76 $1.4+21.7+2$ Scobel+ CURV. FUS = SUM EVAP. RES.+FISS 12131 PAR Revo J APH $3 \quad 5781.7+2 \quad$ Lefort + TBL. FUS. COMP. EXP+CALC. Revw J JPRC 3757 N76 $8.5+12.5+2$ Lefort. CURV. Reve J JPRC 3757 N76 $1.7+2$ Lefort. 0.5608 . SUM OF EVAP. RES.+FISS. 12132 ROC Revo S BNL-51115 $2357791.7+2$ Hulzenga+ TBL. CFD LCRIT.

$116 \mathrm{Sn}\left({ }^{35} \mathrm{C}(, x)\right.$ partial o(E)

12133 ROC EXPt J PR/C $14 \quad 1808$ N76 $1.4+21.7+2$ ScObe $1+$ TBL. EVAPORATION RESIDUE.

$116 \mathrm{Sn}\left({ }^{35} \mathrm{Cl}, \mathrm{ft35} 10 \mathrm{O}\right) \quad \sigma(\mathrm{E})$

12134 ORL ReVw R IAEA-241/SM $791.6+21.7+2$ PIa $11+$ CURV. CFD EXPT. SEVERAL MODELS Revw R CONF-790507 $3791.6+21.7+2$ PIasil+ SAME AS IAEA-SM/24I-DI

12135 ROC Expt J PR/C 141808 N7G $1.6+21.7+2$ Scobel+TBL. INT E, MASS, ANG.

$116 \mathrm{Sn}\left({ }^{35} \mathrm{Cl}\right.$, fisston)lsotople distribution binary fission yield

I2136 ROC Theo A BAP 2197476 NDG MIgnerey+ NDG. ROTATING LIOUID DROP

$120 \mathrm{Sn}\left({ }^{35} \mathrm{Cl}, x\right)$ compound-nuclous o(E)

12137 ROC Theo J PRL $4011224781.4+22.6+2$ Birkelund 4 CURV. FUSION. DYNAMICAL MOD. $120 \mathrm{Sn} i^{35} \mathrm{Cl}$. figsionilsotopic distelbution blinary fission yleld

I2138 ROC Theo A BAP 2197476 NDG Mignerey+ NDG. ROTATING LIOUID DROP $123 S_{n}\left({ }^{35} \mathrm{Cl}, 4 \mathrm{n}\right)^{153} \mathrm{Ho}_{0}$ portiol product yleld

12139 YAL Expt A BAP $25 \quad 48180$ NDG Radford+ NDG. HIGH-SPIN STATES.

$124 \mathrm{Sn}\left({ }^{35} \mathrm{Cl}, x\right)$ compound-nucleus o(E)

I2140 PAR ReVN J JPRC $37 \quad 57$ N76 8.2+1 $2.3+2$ Lefort. CuRV.

Reve J JPRC $37 \quad 57$ N76 $1.7+2 \quad$ Lefort. $0.632 B$. SUM OF EVAP. RES. +F ISS.

$12141 \mathrm{CRC}$ Theo J PRL 411529 N78 $1.3+21.7+2$ Horn 1 CURV. CN+ENTR. CHAN. PAR. CFD EXP

12142 ROC Theo J PRL $4011224781.4+22.6+2$ Birhelundt CURV. FUSION. OYNAMICAL MOD,

12143 ROC Expt J PR/C 141808 N76 $1.4+21.7+2$ Scobe $1+$ CURV. FUS = SUM EVAP. RES.+FISS

$124 \sin \left({ }^{35} \mathrm{Cl}, x\right)$ partIal o(E)

12144 ROC EXDt J PR/C 141808 N76 $1.4+21.7+25$ CObe $1+$ TBL. EVAPORATION RESIOUE. 


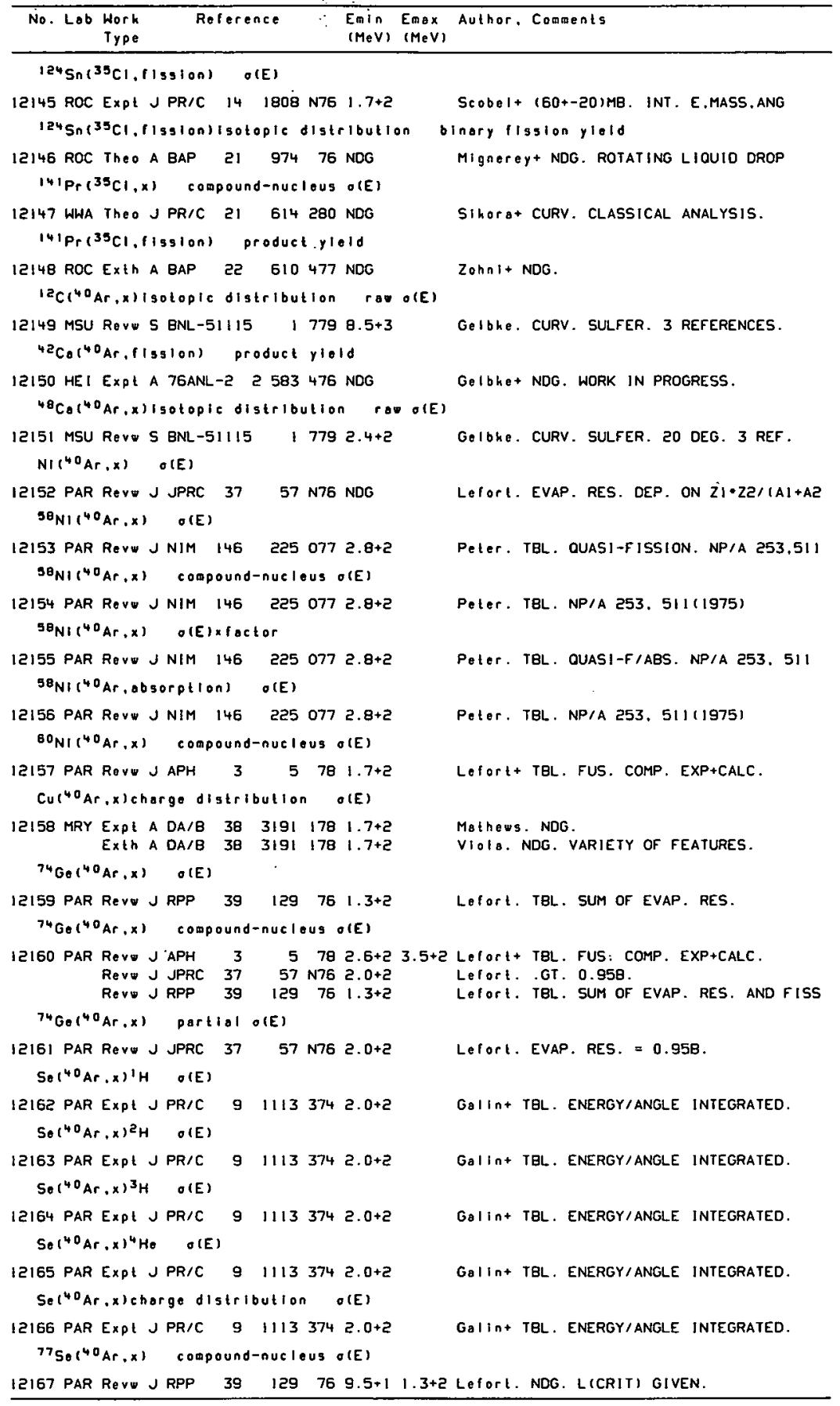




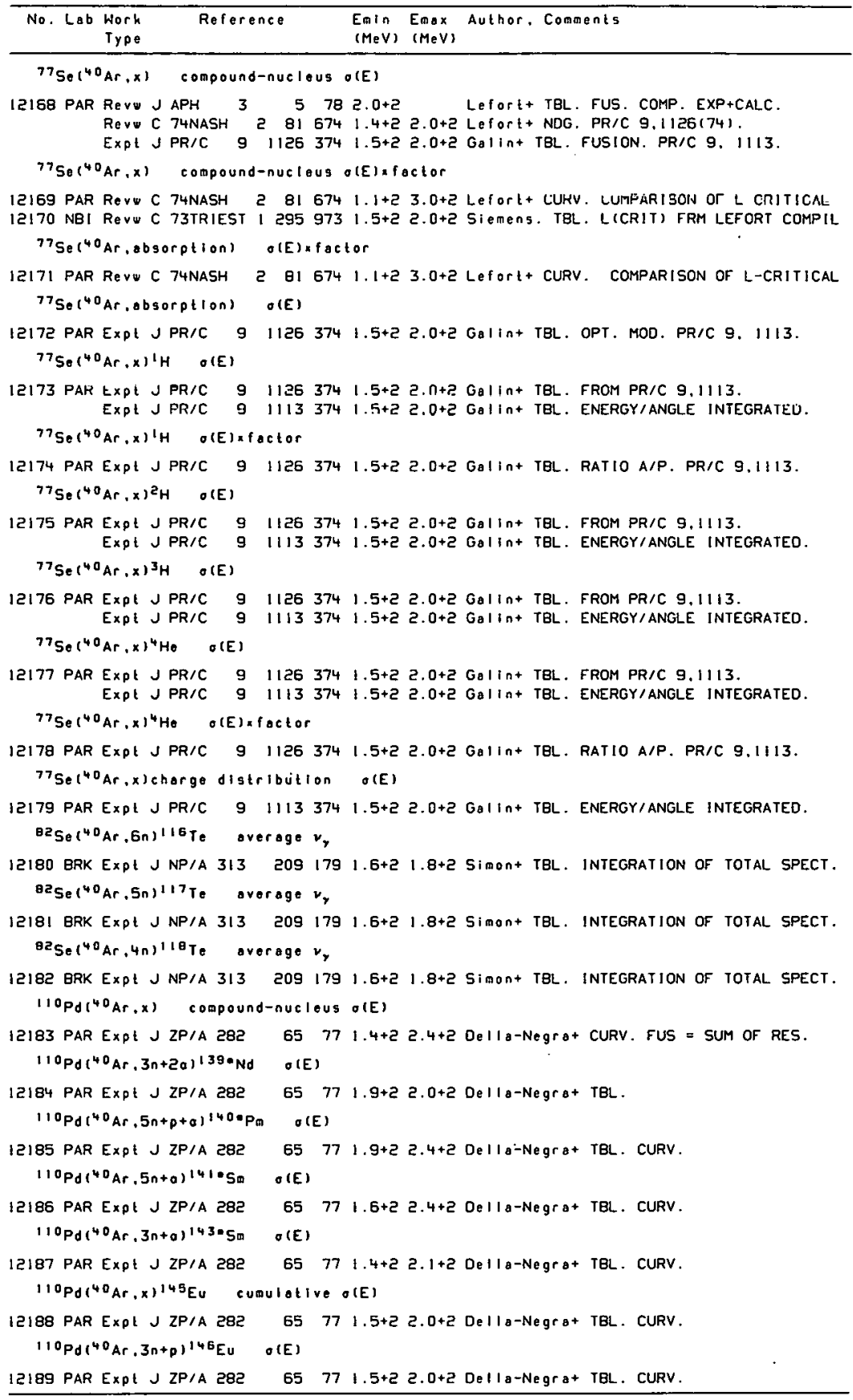


REFERENCES ( $c$ on $t$ )

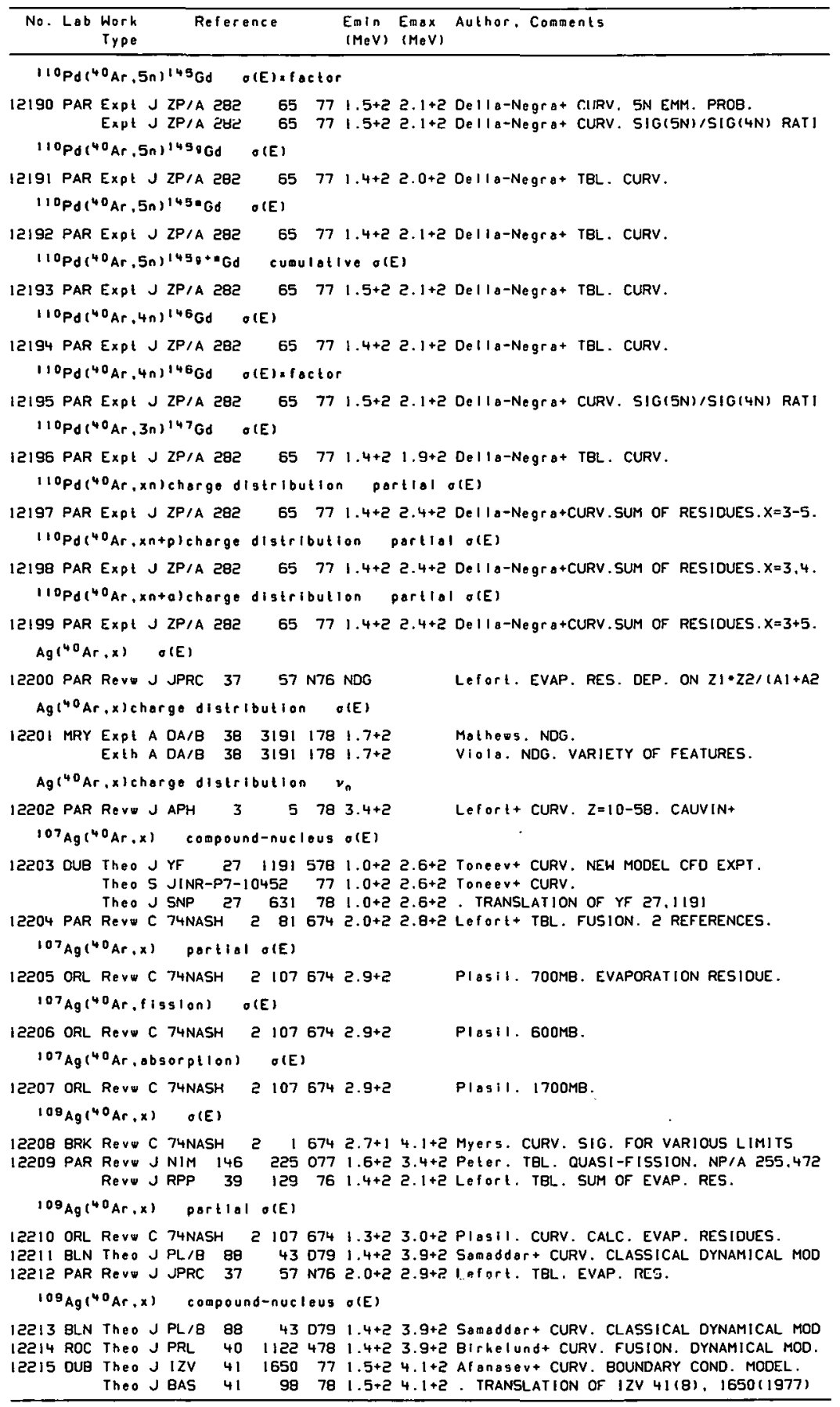


REFERENCES (cont)

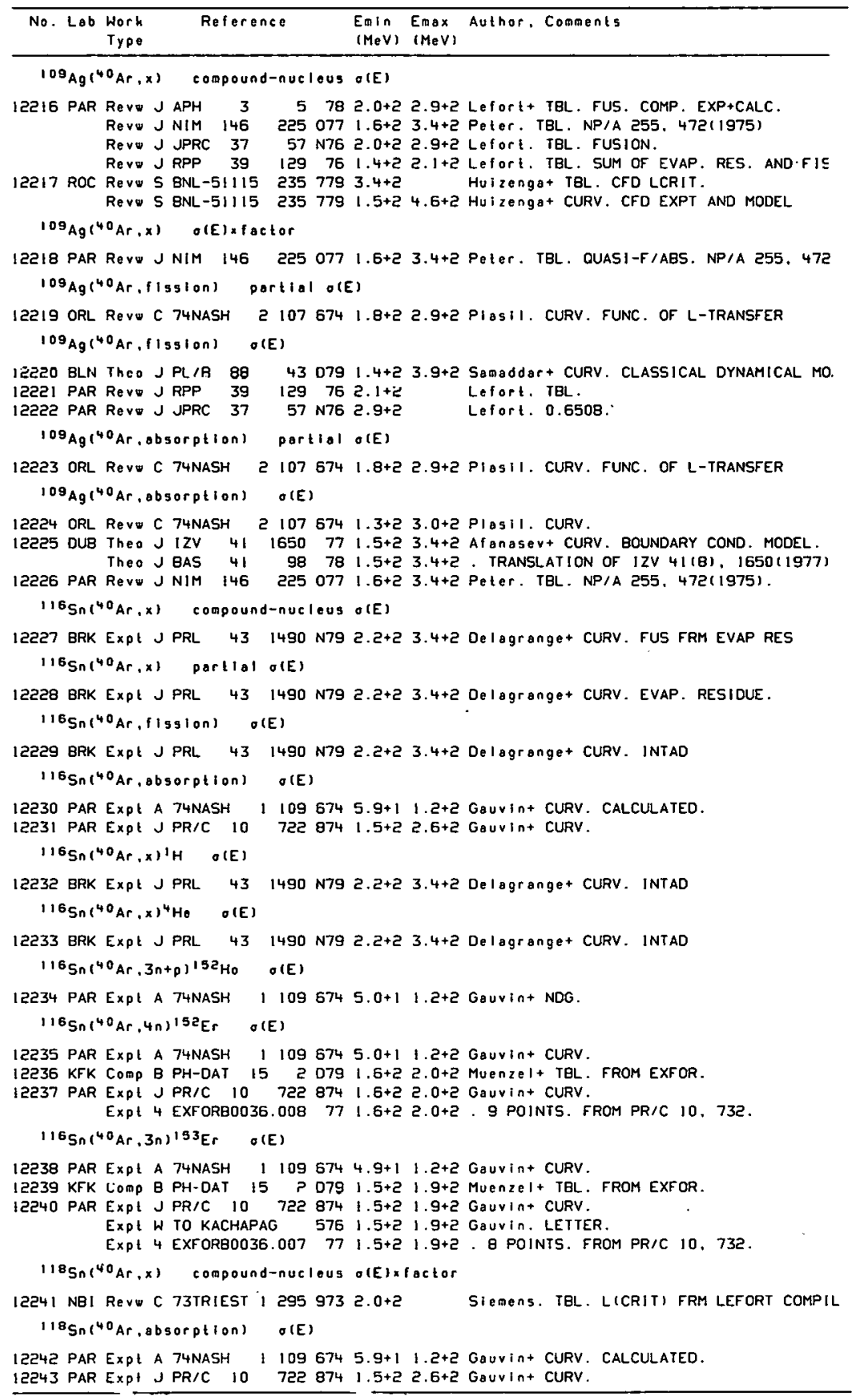


REFERENCES (cont)

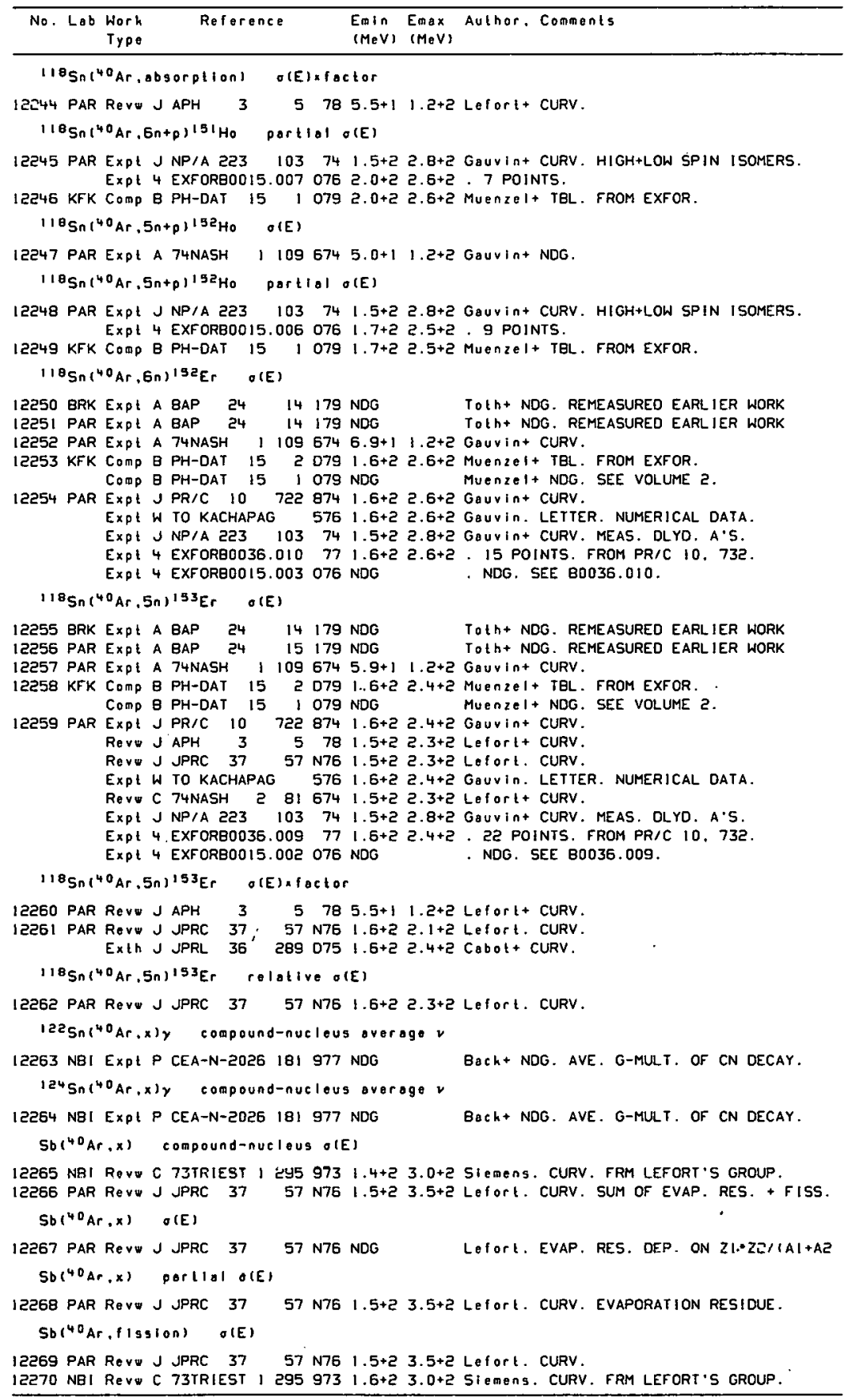


REFERENCES $(c \circ i)$

\begin{tabular}{|c|c|c|c|c|c|}
\hline No. Lab & $\begin{array}{l}\text { Work } \\
\text { Type }\end{array}$ & Reference & $\begin{array}{l}\text { EnIn } \\
(M \in V)\end{array}$ & $\begin{array}{l}\text { Emox } \\
(M e V)\end{array}$ & Author. Comments \\
\hline
\end{tabular}

Sb( ${ }^{\circ}$ Ar, absorption) O(E)

I2Z71 NBI Revo C 73TRIEST I $2959731.4+23.0+2$ SIemens. CURV. FRM LEFORT'S GROUP. 12272 PAR Revw J JPRC 3757 N76 1.5+2 $3.5+2$ Lefort. CURV.

$$
S b(40 \text { Ar, total) } O(E)
$$

$12273 \mathrm{NB}$ ! ReVw C 73TRIEST I $2959731.4+23.0+2$ SIemens. CURV. FRM LEFORT'S GROUP.

12 Isb $\left({ }^{\circ} \mathrm{Ar}, x\right)$ compound-nucleus o(E)

12274 PAR ReV J APH $3 \quad 5781.6+26.7+2$ Lefort+ CURV. FUSION. CFD THEORY TO EXP

Revw J APH $3 \quad 5782.0+22.3+2$ Lelor $t+7 B L$. FUS, COMP. EXP+CALC.

ReVu J JPRC 3757 N75 $2.0+23.0+2$ Lefort. TBL. SUM OF EVAP. + FISS

ReV J JPRC $37 \quad 57$ N76 $1.5+26.7+2$ Lefort. CURV.

Revo J RPP $39129761.5+22.2+2$ Lefort. TBL. SUM OF EVAP. RES. AND FISS

ReVUC 74NASH $2816742.0+23.0+2$ Lefort+ TBL. MANY REFERENCES.

12275 ROC Reve S BNL-51115 $2357793.0+2$ Huizengat TBL. CFO LCRIT.

$1215 b(10 A r, x)$ compound-nucleus of(E) $x$ factor

I2276 PAR ReVU C 74NASH 2 BI $6741.7+23.3+2$ Lefor $t+$ CURV. COMPARISON OF L-CR!TICAL. I2277 NBI Reve C 73TRIEST $12959732.0+23.0+2$ Siemeng. TBL. L(CRIT) FRM LEFORT COMPIL

$1215 B(40 A r, x) \quad O(E)$

12278 PAR ReVw J RPP $39129761.5+22.2+2$ Leforl. TBL. SUM OF EVAP. RES.

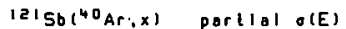

12279 PAR Revw J JPRC 3757 N76 2.0+2 3.0+2 Lefort. TBL. EVAP. RES.

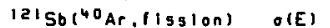

12280 PAR Revw J JPRC $37 \quad 57$ N76 $2.0+23.0+2$ Lefort. TBL.

Revw J RPP $39129761.5+2$ 2.2+2 Lefort. TBL.

laIsb( 40 Ar, absorption) O(E)*foctor

1228! PAR REVI C 74NASH $2816741.7+23.3+2$ Lefort+ CURV. COMPARISON OF L-CRITICAL.

$121 s b\left({ }^{\circ} A r, B n+2 p\right) 151 H_{0}$ partlal o(E)

12282 PAR EXPI J NP/A $223 \quad 103741.5+22.8+2$ Gauvin+ CURV. HIGH+LOW SPIN ISOMERS EXPt 4 EXFORBOOI5.011 $0762.3+25.9+2$. 5 POINTS

12283 KFK Comp 8 PH-DAT 15 I $0792.3+22.9+2$ Muenzel+ TBL. FROM EXFOR.

$1215 B(40 A r, 7 n+2 p) 152_{H O}$ partial o(E)

12284 PAR Expt J NP/A $223103741.5+22.8+2$ GaUY In+ CURV. HIGH+LOW SPIN ISOMERS. Expt 4 EXFORBO015.010 $0762.3+2$ 2.9+2. 6 POINTS

12285 KFK Comp 日 PH-DAT $15 \quad 10792.3+22.9+2$ Muenzel+ TBL. FROM EXFOR,

$121 \mathrm{Sb}(40 \mathrm{Ar}, 8 n+p) 152 \mathrm{Er} \quad O(E)$

12286 KFK COMP B PH-DAT $1510792.4+22.9+2$ Muenzel+ TBL. FROM EXFOR.

12387 PAR Expt J NP/A $223 \quad 103742.4+22.9+2$ Gauvin+ CURV. MEASURED OELAYED ALPHAS. Expt 4 EXFORBO015.009 $0762.4+22.9+2.5$ POINTS.

$1215 b(40 A r, 7 n+p) 193 \mathrm{Er} \quad$ O(E)

12288 PAR Expl J NP/A $223 \quad 103741.5+22.8+2$ GaUvin+ CURV. MEAS. DLYO. A'S. Expl 4 EXFORE0015.008 $0762.3+22.9+2$. 5 POINTS.

12289 KFK Comp B PH-DAT 15 I $0792.3+2$ 2.9+2 Muenzel+ TBL. FROM EXFOR.

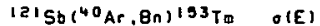

12290 PAR Expl J NP/A $223103741.5+22.8+2$ Gauvin+ CURV. MEAS. DLYD. A'S. Expl 4 EXFORB0015.005 $0762.3+25.5+2.3$ POINTS.

1229! KFK Comp B PH-DAT 15 I $0792.3+22.5+2$ Muenzel+ TBL. FROM EXFOR.

$1215 b(40 A C, 7 n 1154 \mathrm{Tm}$ pertlal O(E)

12292 PAR Expt J NP/A $223 \quad 10374 \quad 1.5+2$ 2.8+2 GauvIn+ CURV. HIGH SPIN ISOMER.

Expt 4 EXFORBO015.004 076 $2.0+22.5+2.4$ POINTS.

$12293 \mathrm{KFK}$ Comp 日 PH-DAT I5 $10792.0+22.5+2$ Muenze I+ TBL. FROM EXFOR.

$126 \mathrm{~T},\left({ }^{40} \mathrm{Ar} .6 \mathrm{SO}\right) 160 \mathrm{YO}$ a(E)

!2294 BRK EXPT J NP/A $2902530771.8+2$ SImON + TBL. FRM DISCRETE + CONT. G-SPEC

$126 \mathrm{~T} \in\left({ }^{40} \mathrm{Ar}, 6 \cap\right)^{160} \mathrm{YO}$ overoge ${ }^{2}$,

12295 BRK Expl J NP/A $2902530771.8+2$ Simon+ TBL. AVE. G MULT.

$126 \mathrm{Te}(40 \mathrm{Ar}, 5 \mathrm{Sn}) 161 \mathrm{Yb}$ O(E)

12296 ERK ExPI J NP/A $2902530771.6+21.8+251$ SON+ TBL. FRM DISCRETE + CONT. G-SPEC 


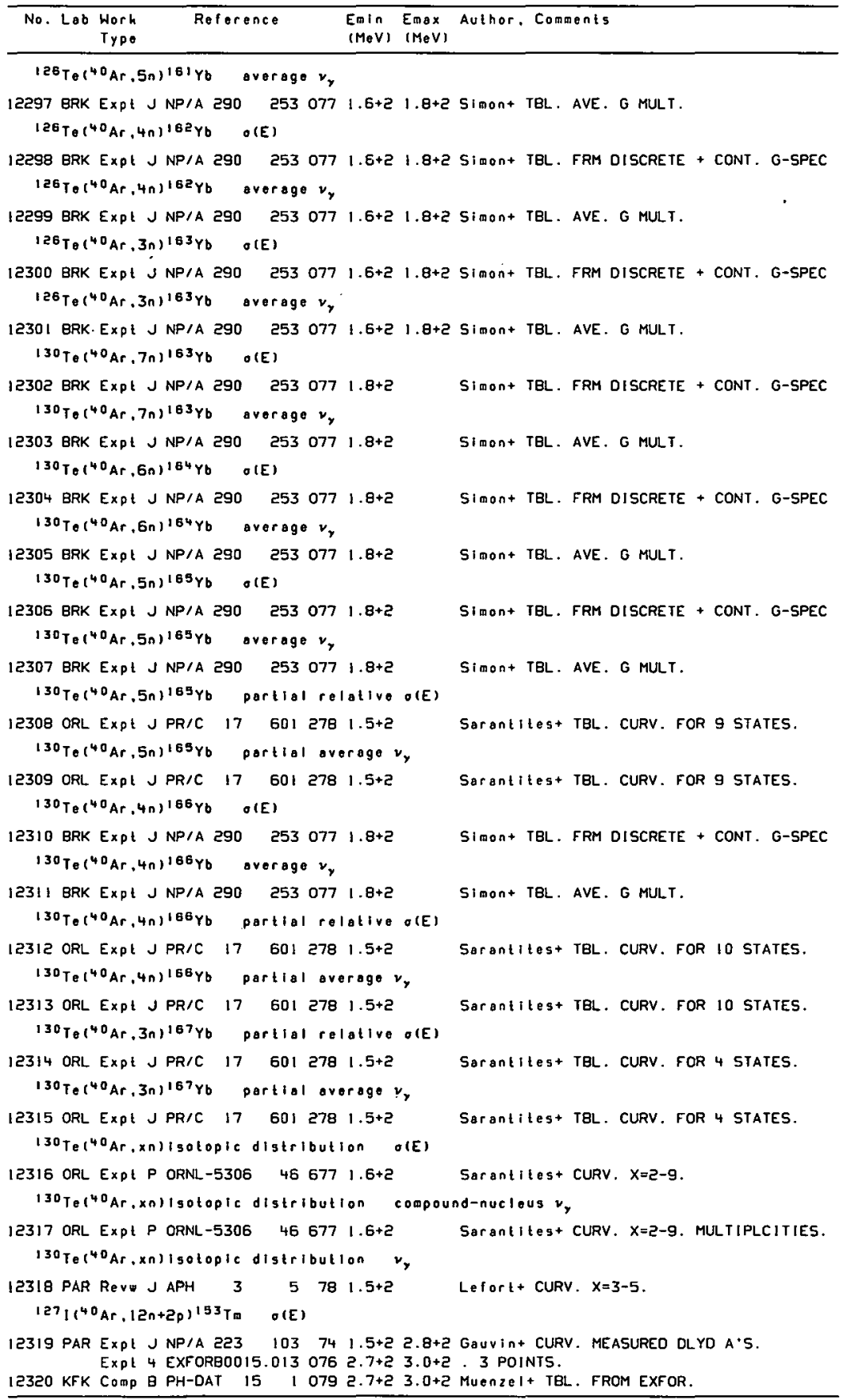


REFERENCES (cont)

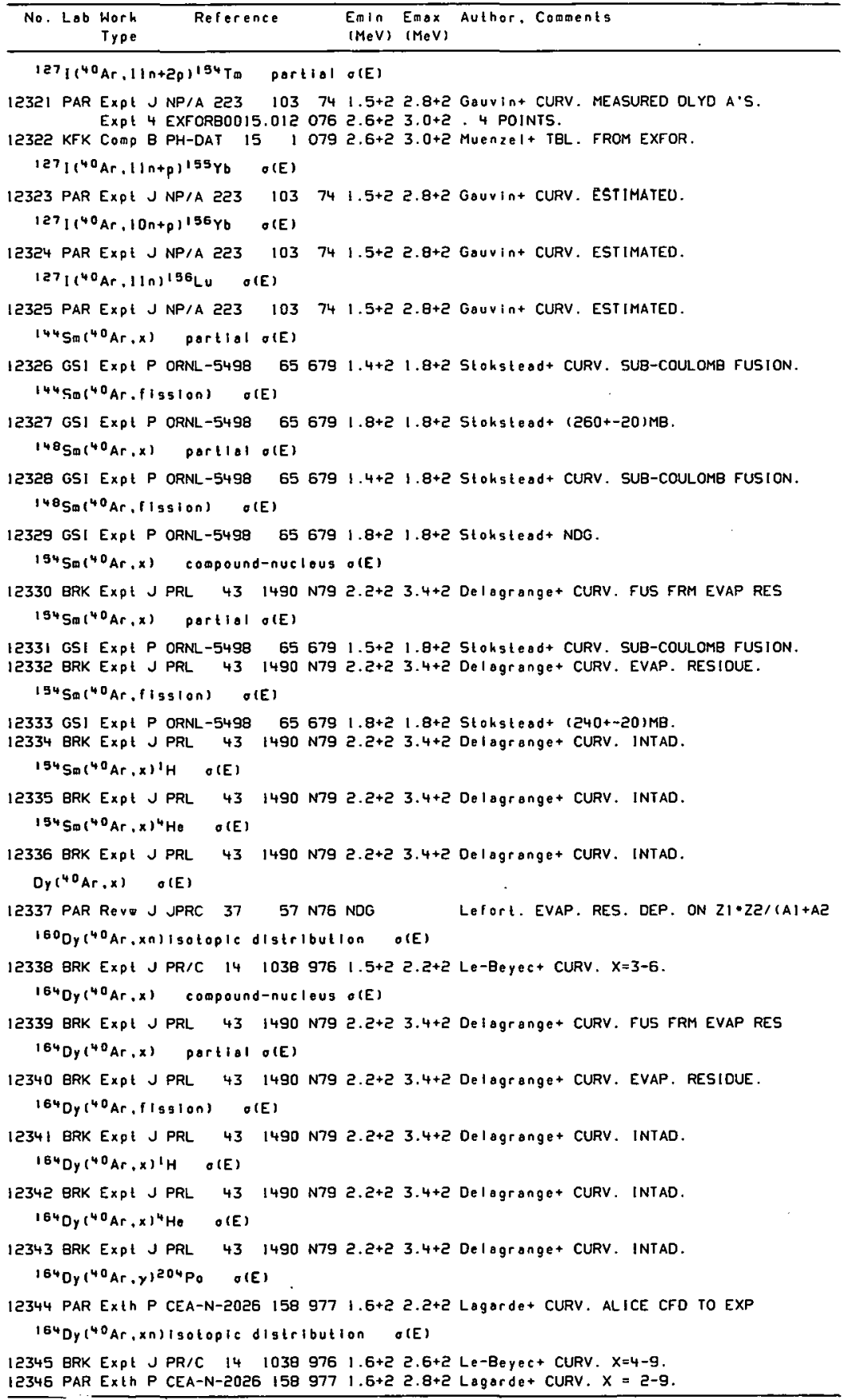


REFERENCES (cont)

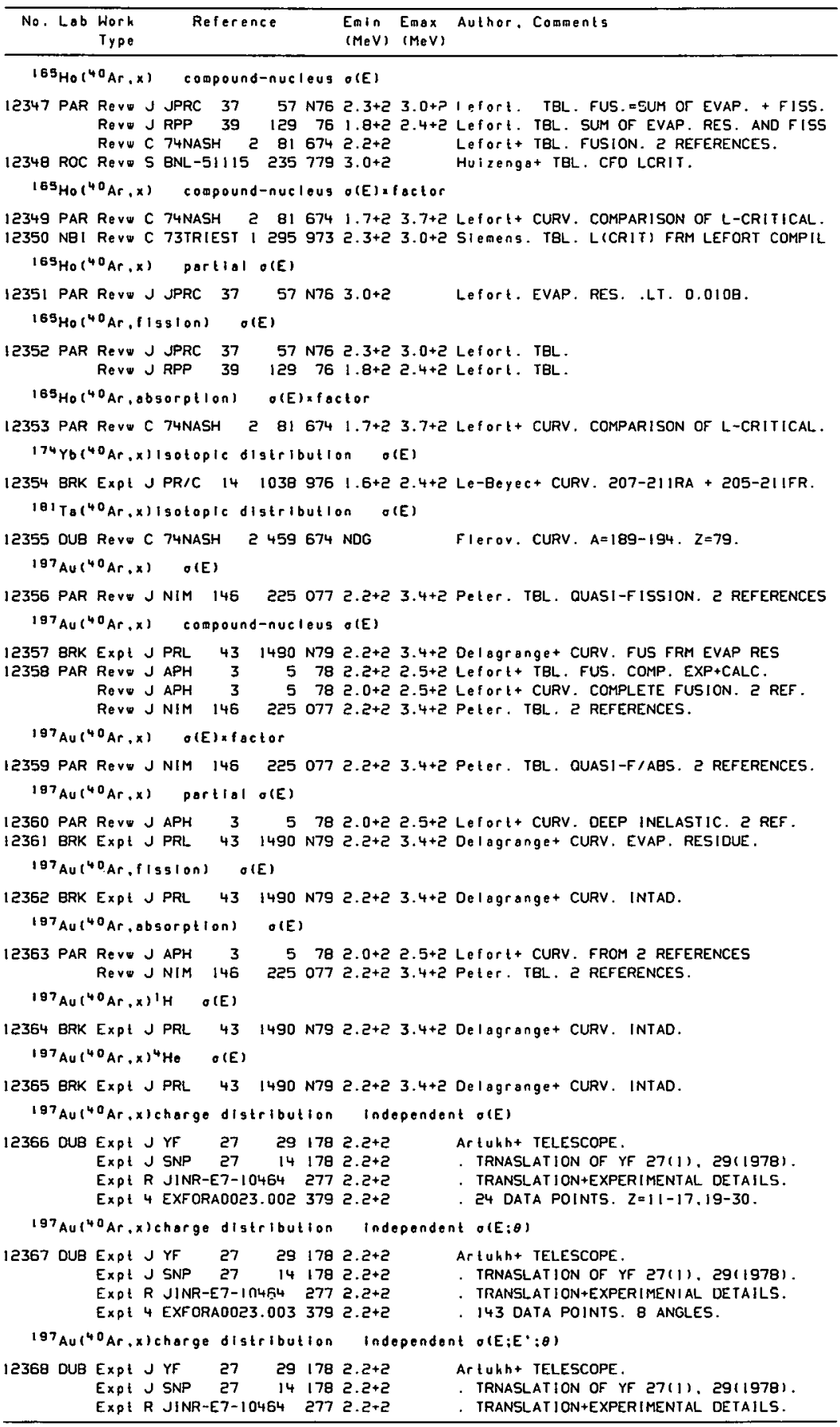




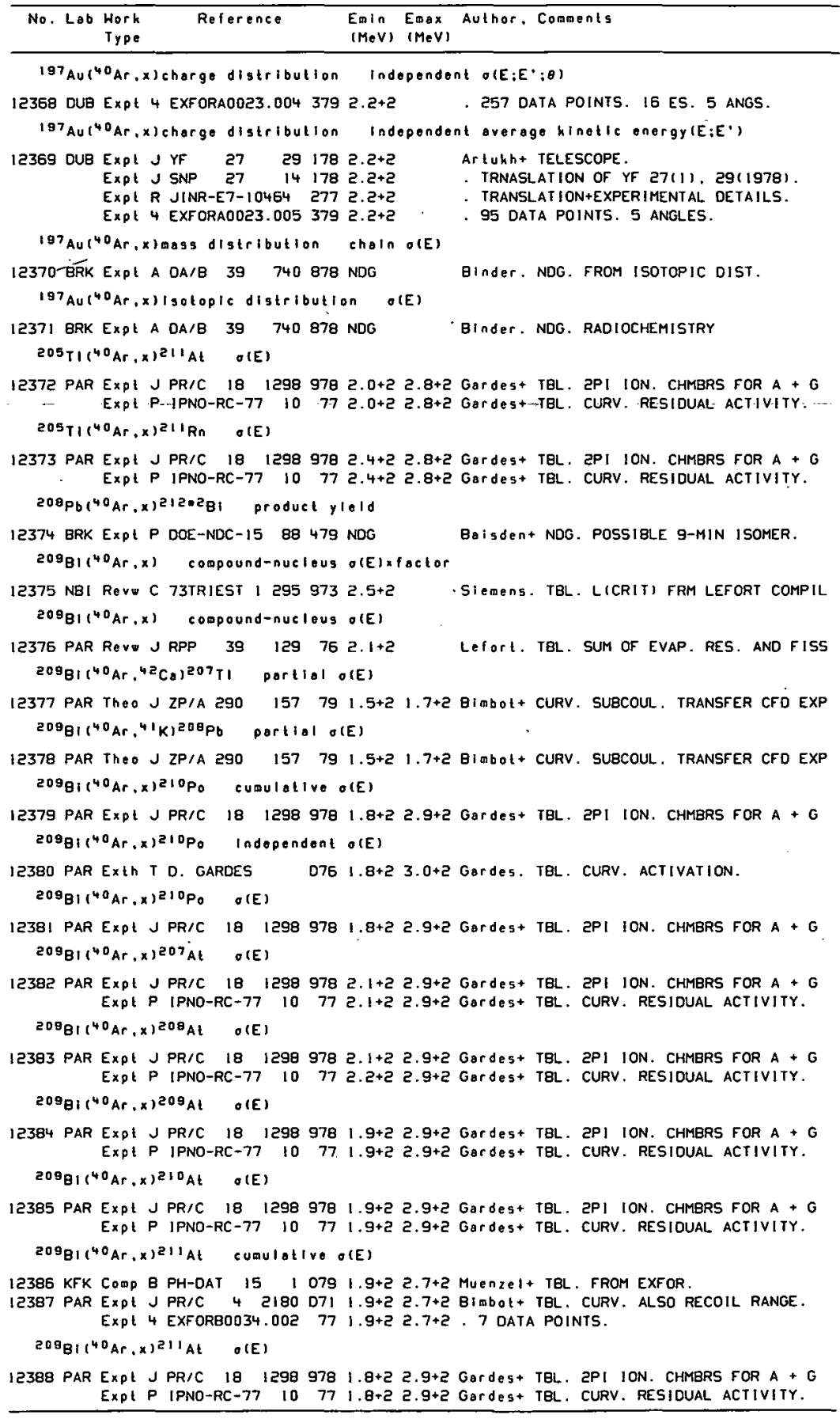




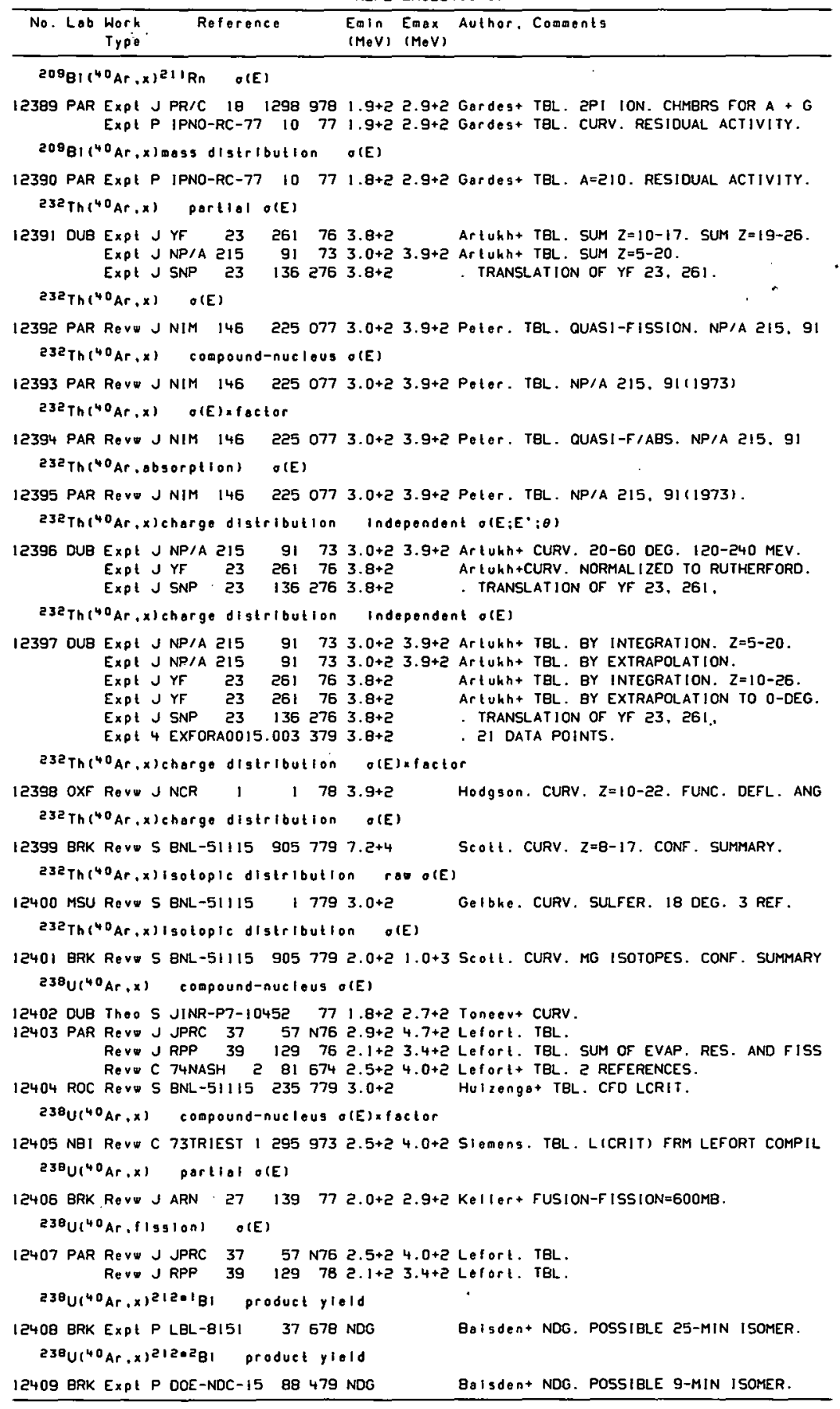


REFERENCES (cont)

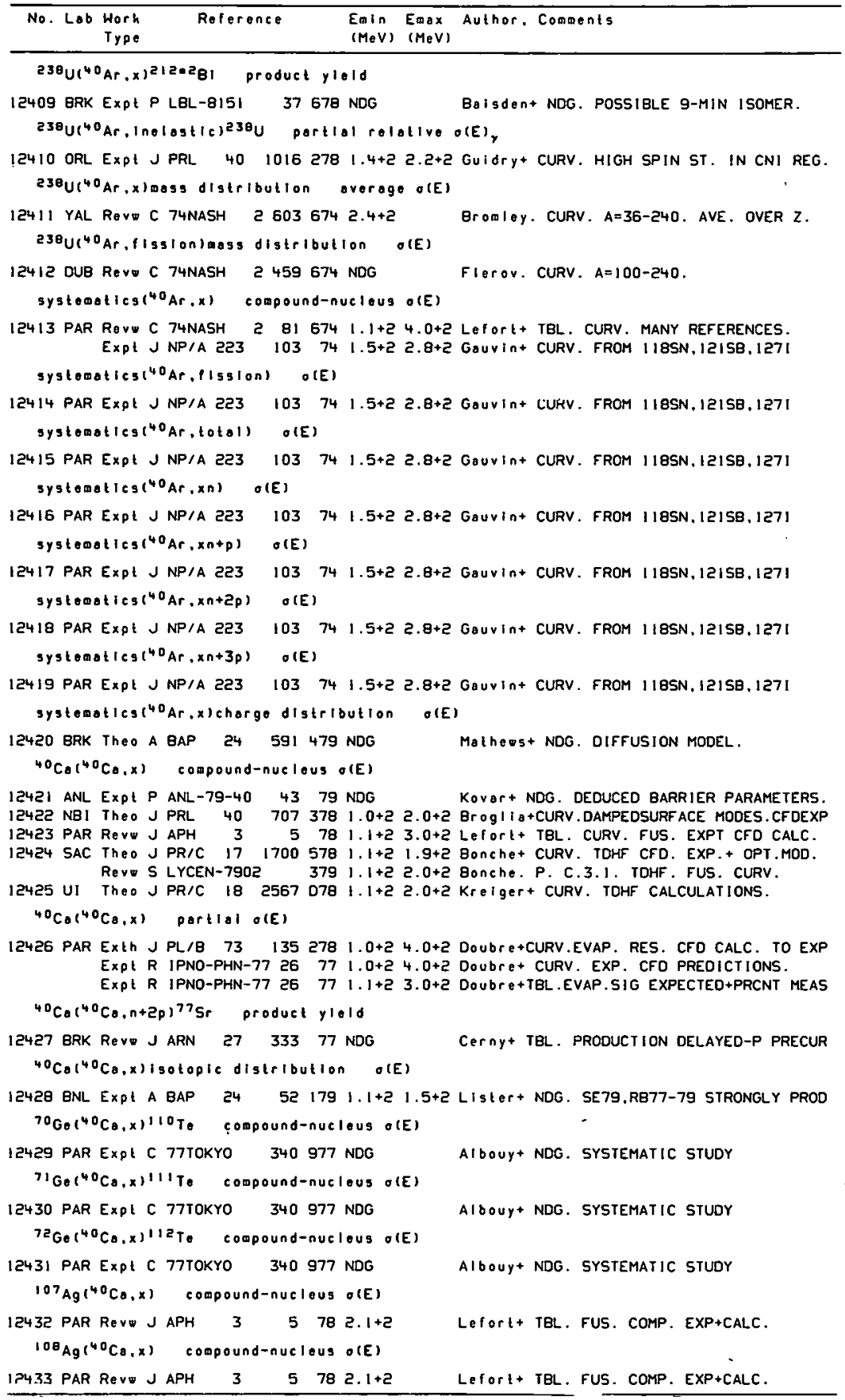


REFERENCES (cont)

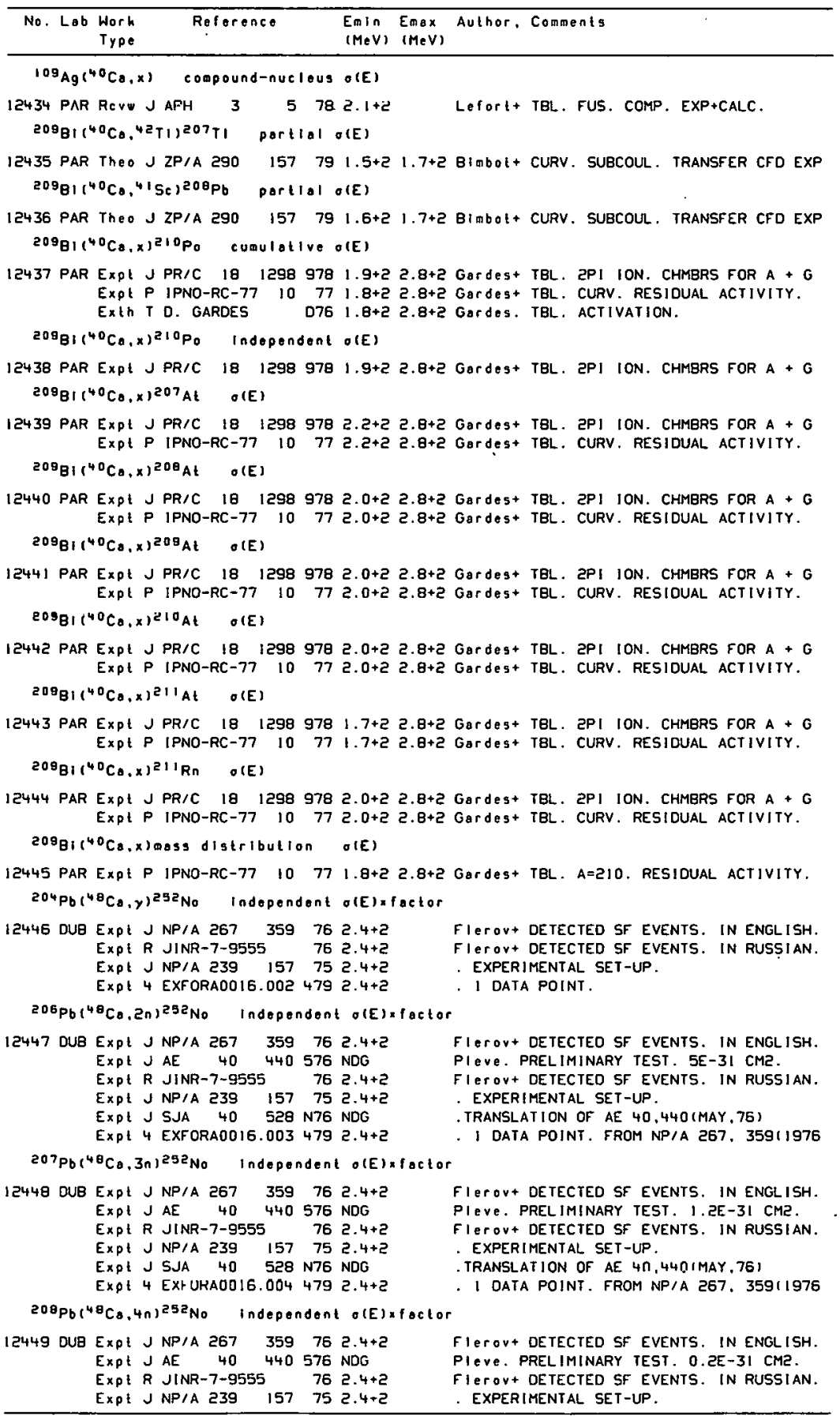




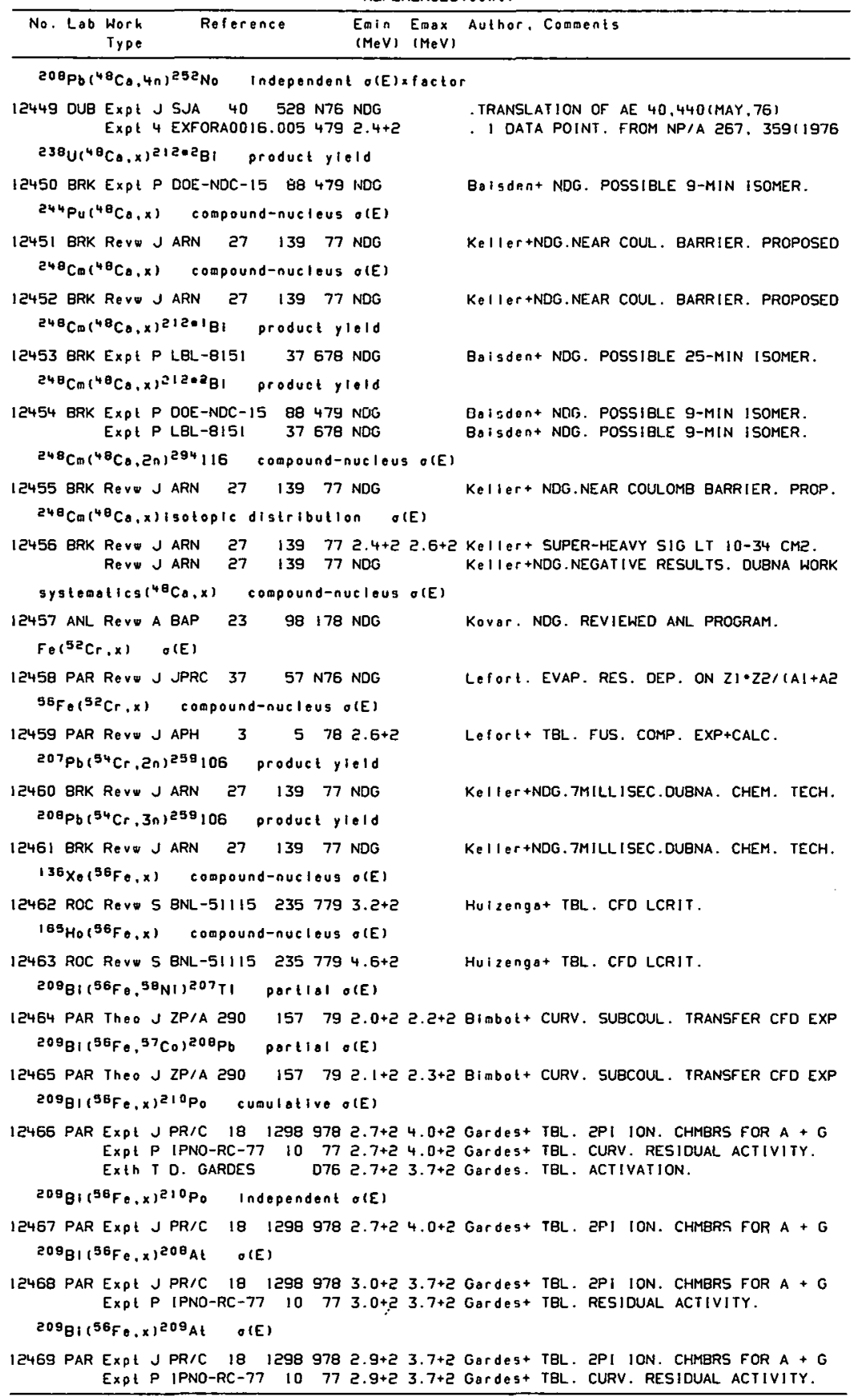


REFERENCES ( c on t )

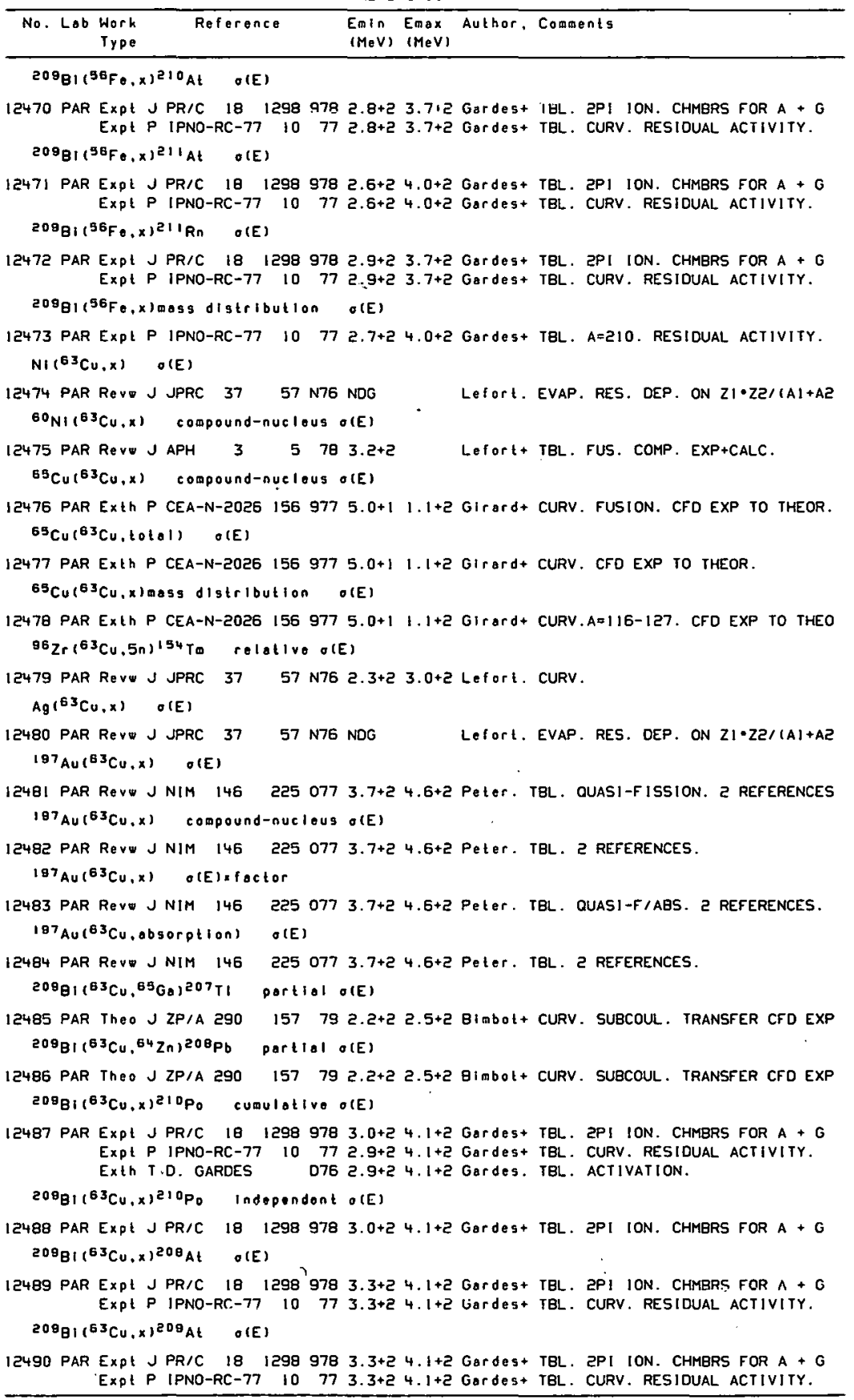




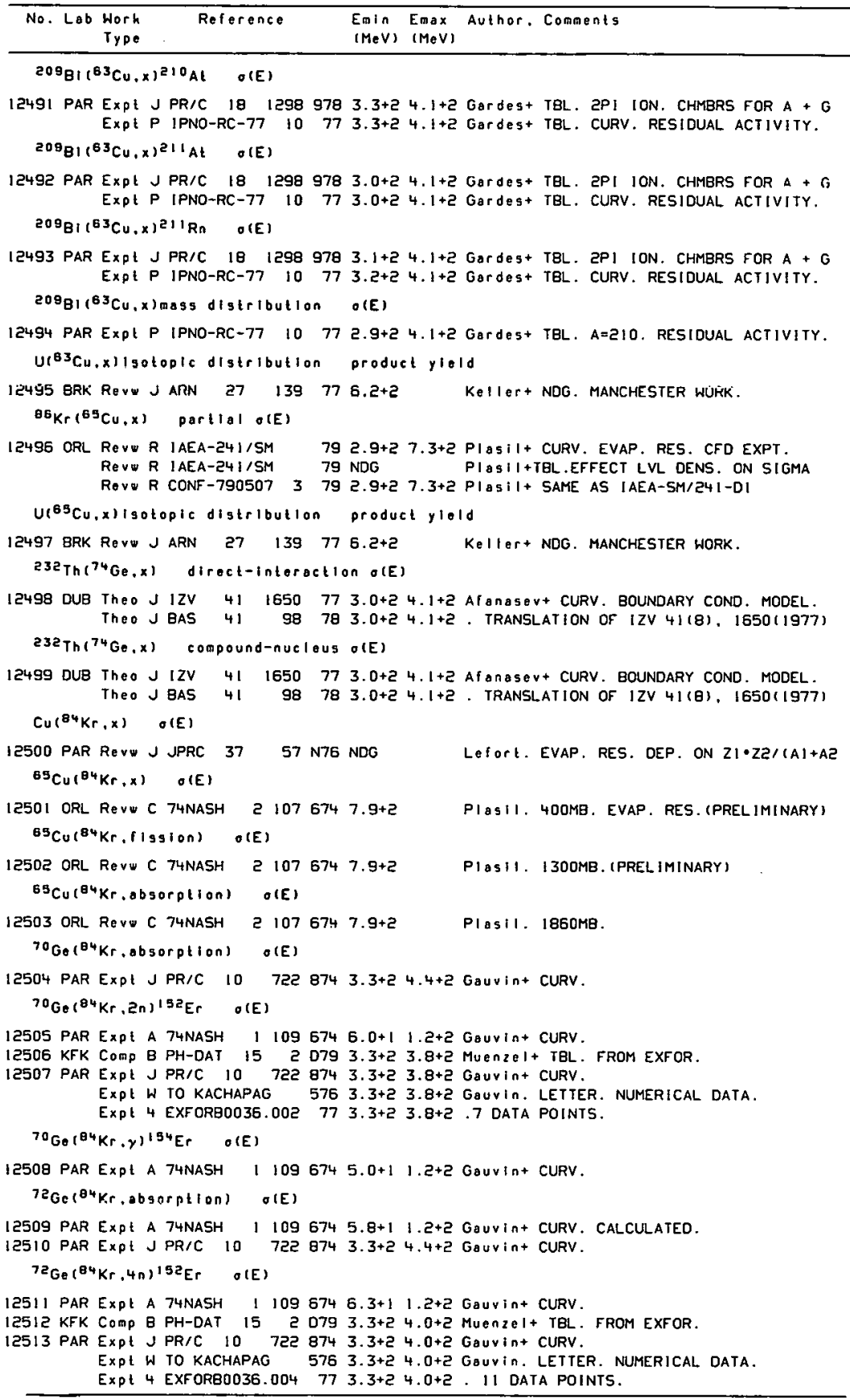


REFERENCES ( $c$ on $t)$

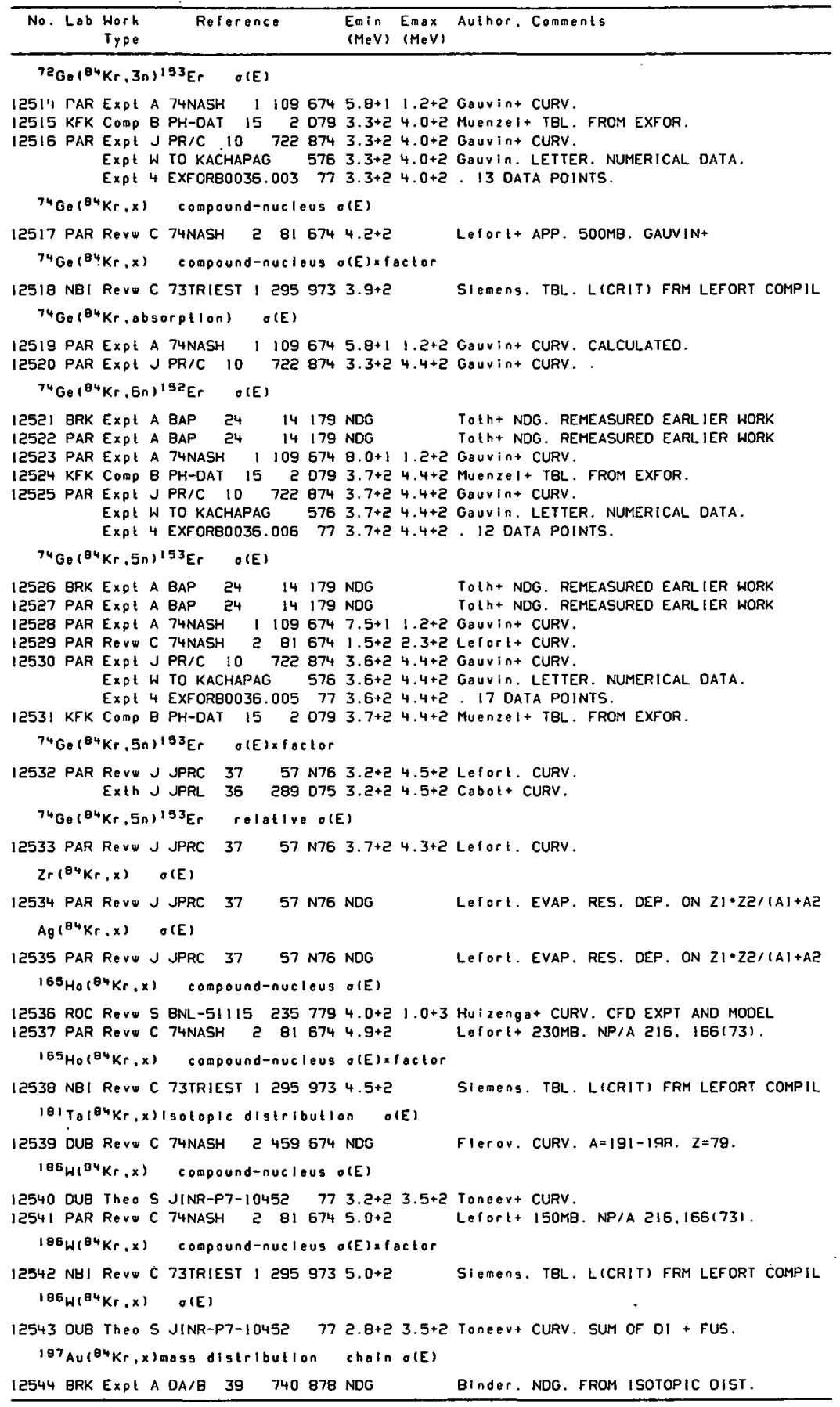


REFERENCES ( c on t

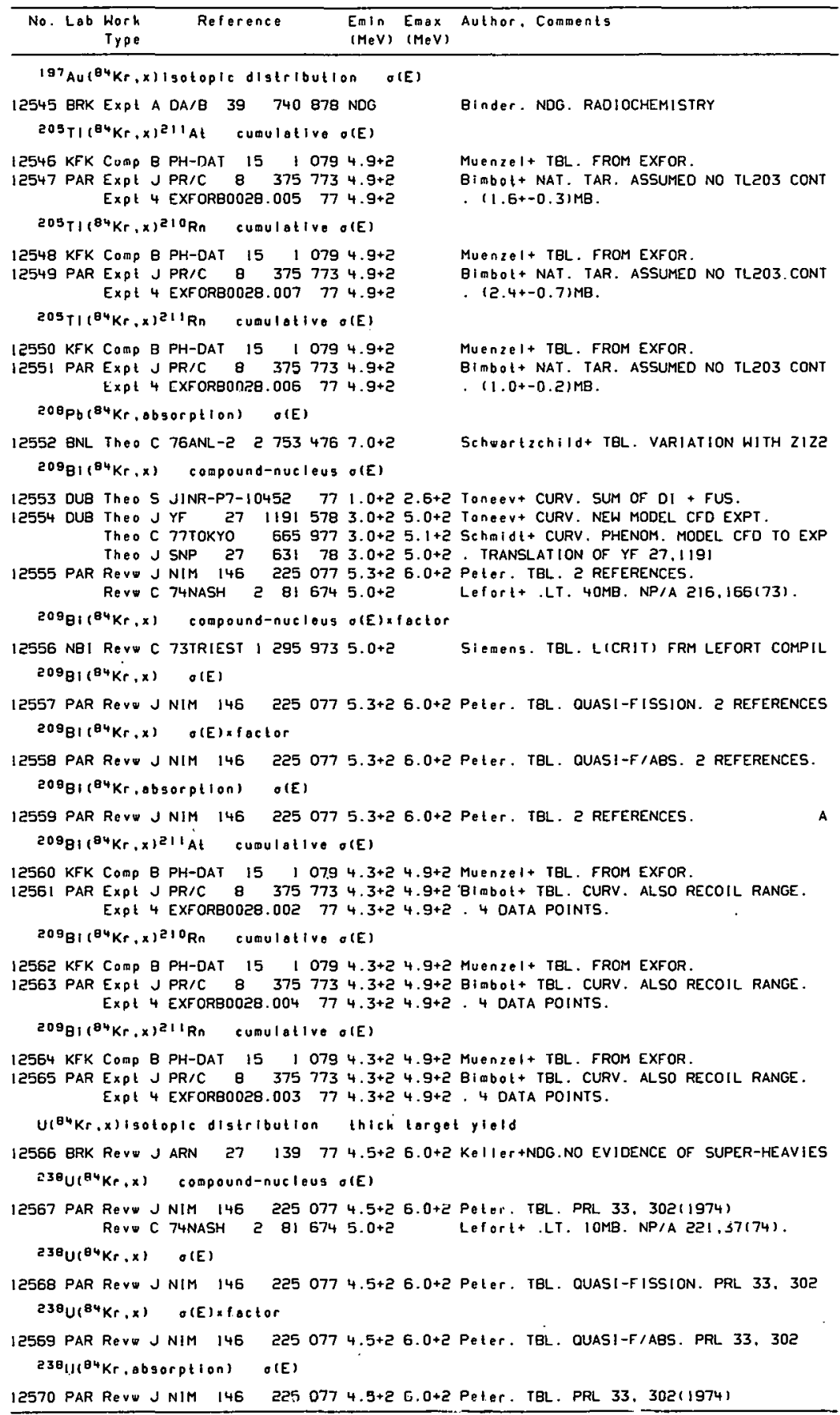


REFERENCES (cont)

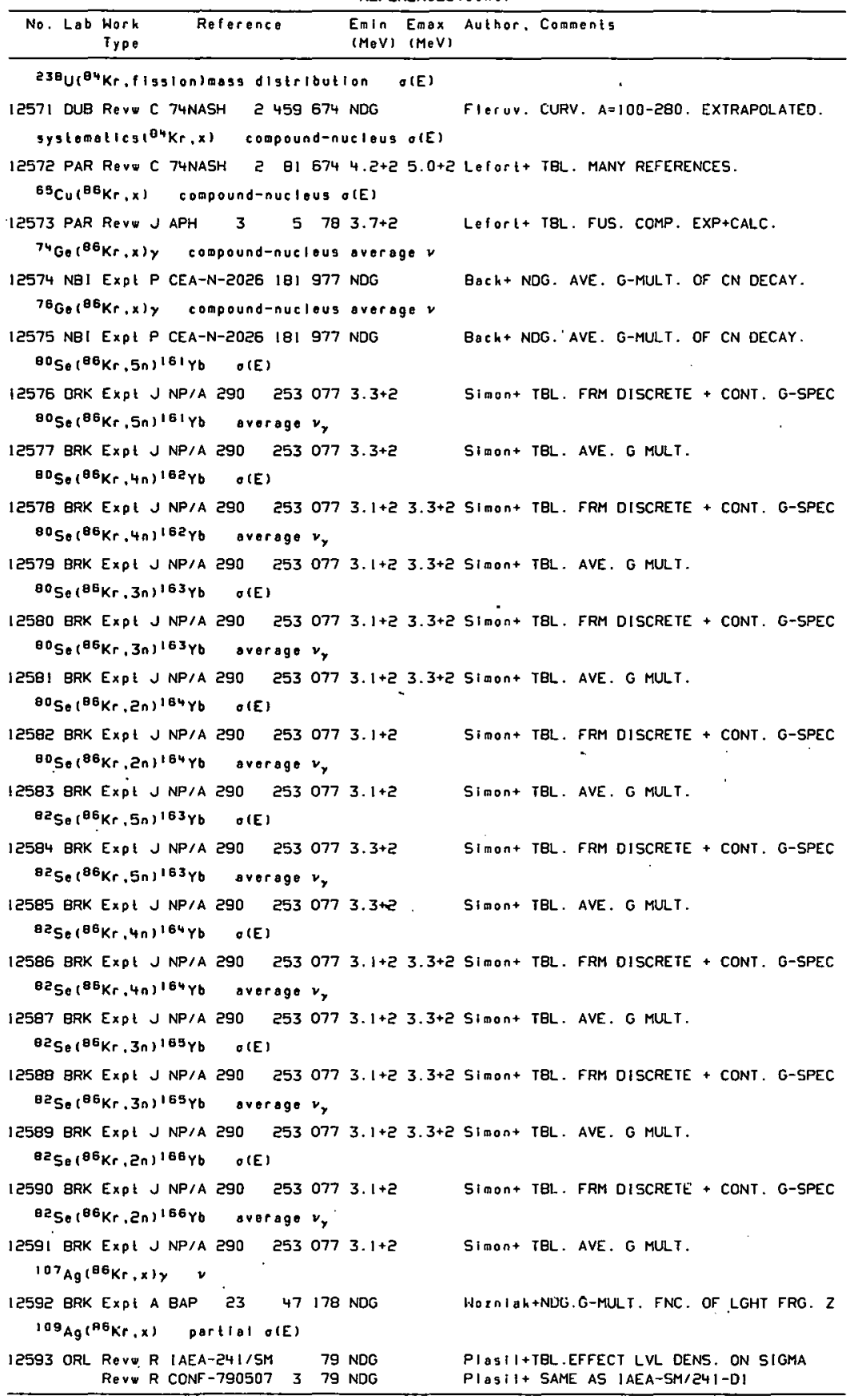




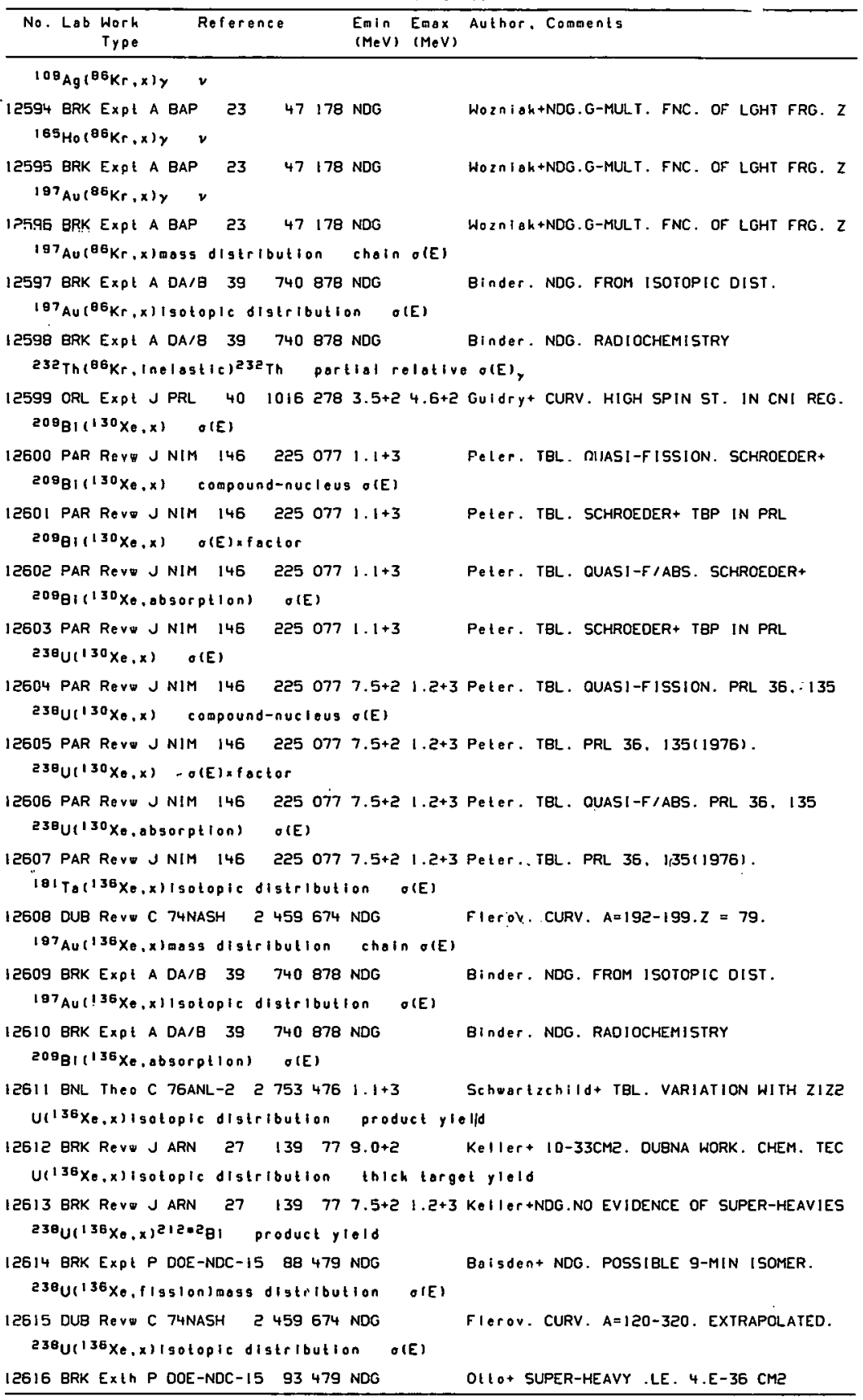




$\begin{array}{lll}1 \mathrm{H} & \text { Hydrogen } \\ 2 \mathrm{He} & \text { Helium } \\ 3 \mathrm{Li} & \text { Lithium } \\ 4 \mathrm{Be} & \text { Beryllium } \\ 5 \mathrm{~B} & \text { Boron } \\ 6 \mathrm{C} & \text { Carban } \\ 7 \mathrm{~N} & \text { Nitrogen } \\ 8 \mathrm{O} & \text { Oxygen } \\ 9 \mathrm{~F} & \text { Fluorine } \\ 10 \mathrm{Ne} & \text { Neon } \\ 11 \mathrm{Na} & \text { Sodium } \\ 12 \mathrm{Mg} & \text { Magnesium } \\ 13 \mathrm{Al} & \text { Aluminium } \\ 14 \mathrm{Si} & \text { Silicon } \\ 15 \mathrm{P} & \text { Phosphorus } \\ 16 \mathrm{~S} & \text { Sulphur } \\ 17 \mathrm{Cl} & \text { Chlorine } \\ 18 \mathrm{Ar} & \text { Argon } \\ 19 \mathrm{~K} & \text { Potassium } \\ 20 \mathrm{Ca} & \text { Calcium } \\ 21 \mathrm{Sc} & \text { Scandium } \\ 22 \mathrm{Ti} & \text { Titanium } \\ 23 \mathrm{~V} & \text { Vanadium } \\ 24 \mathrm{Cr} & \text { Chromium } \\ 25 \mathrm{Mn} \text { Manganese } & \\ 26 \mathrm{Fe} & \text { Iron } \\ 27 \mathrm{Co} & \text { Cobalt } \\ 28 \mathrm{Ni} & \text { Nickel } \\ 29 \mathrm{Cu} & \text { Copper } \\ 30 \mathrm{Zn} & \text { Zinc } \\ 31 \mathrm{Ga} & \text { Gallium } \\ 32 \mathrm{Ge} & \text { Germanium } \\ 33 \mathrm{As} & \text { Arsenic } \\ 34 \mathrm{Se} & \text { Selenium } \\ 35 \mathrm{Br} & \text { Bromine } \\ \end{array}$

$36 \mathrm{Kr}$ Krypton
$37 \mathrm{Rb}$ Rubidium
$38 \mathrm{Sr}$ Strontium
$39 \mathrm{Y}$ Yttr um
$40 \mathrm{Zr}$ Zirconium
$41 \mathrm{Nb}$ Niobium
$42 \mathrm{Mo}$ Molybdenum
$43 \mathrm{Tc}$ Technetium
$44 \mathrm{Ru}$ Ruthenium
$45 \mathrm{Rh}$ Fhodium
$46 \mathrm{Pd}$ Palladium
$47 \mathrm{Ag}$ Silver
$48 \mathrm{Cd}$ Cadmium
$49 \mathrm{In}$ Indium
$50 \mathrm{Sn}$ Tin
$51 \mathrm{Sb}$ Antimony
$52 \mathrm{Te}$ Tellurium
$53 \mathrm{I}$ Iodine
$54 \mathrm{Xe}$ Xenon
$55 \mathrm{Cs}$ Cesium
$56 \mathrm{Ba}$ Barium
$57 \mathrm{La}$ Lanthanum
$58 \mathrm{Ce}$ Cerium
$59 \mathrm{Pr}$ Praseodymium
$60 \mathrm{Nd}$ Neodymium
$61 \mathrm{Pm}$ Promethium
$62 \mathrm{Sm}$ Samarium
$63 \mathrm{Eu}$ Europium
$64 \mathrm{Gd}$ Gadolinium
$65 \mathrm{~Tb}$ Terbium
$66 \mathrm{Dy}$ Dysprosium
$67 \mathrm{Ho}$ Holmium
$68 \mathrm{Er}$ Erbium
$69 \mathrm{Tm}$ Thulium
$70 \mathrm{Yb}$ Ytterbium

$71 \mathrm{Lu}$ Lutetium
$72 \mathrm{Hf}$ Hafnium
$72 \mathrm{Ta}$ Tantalum
$74 \mathrm{~W}$ Tungsten
$75 \mathrm{Re}$ Rhenium
$76 \mathrm{Os}$ Osmium
$77 \mathrm{Ir}$ Irldium
$78 \mathrm{Pt}$ Platinum
$79 \mathrm{Au}$ Gold
$80 \mathrm{Hg}$ Mercury
$81 \mathrm{TI}$ Thallium
$82 \mathrm{~Pb}$ Lead
$83 \mathrm{Bl}$ Bismuth
$84 \mathrm{Po}$ Polonium
E5 At Astatine
$86 \mathrm{Rn}$ Radon
$87 \mathrm{Fr}$ Francium
$88 \mathrm{Ra}$ Radium
$89 \mathrm{Ac}$ Actinium
$90 \mathrm{Th}$ Thorium
$91 \mathrm{~Pa}$ Protactinium
$92 \mathrm{U}$ Uranium
$93 \mathrm{~Np}$ Neptunium
$94 \mathrm{Pu}$ Plutonium
$95 \mathrm{Am}$ Americium
$96 \mathrm{Cm}$ Curlum
$97 \mathrm{Bh}$ Berkelium
$98 \mathrm{Cf}$ Californium
$99 \mathrm{Es}$ Einsteinium
$100 \mathrm{Fm}$ Fermium
$101 \mathrm{Md}$ Mendelevium
$102 \mathrm{No}$ Nobelium
$103 \mathrm{Lr}$ Lawrencium
$104 \mathrm{Ku}$ Kurchatovium

NATIONAL NUCLEAR DATA CENTER

Brookhaven National Laboratory

Upton. New York 11973 